\title{
Beyond Prestige: \\ A ritual production model for stone tool specialization in Naqada period Egypt
}

\author{
Elizabeth Hart Skarzynski \\ Lansing, MI \\ M.A. University of Virginia, 2010 \\ B.A. University of Michigan, 2004
}

A Dissertation presented to the Graduate Faculty of the University of Virginia in Candidacy for the Degree of Doctor of Philosophy

Department of Anthropology

University of Virginia

May, 2017 
Copyright $(\mathbb{C}$ by Elizabeth Hart Skarzynski 2017 


\section{Abstract}

This dissertation looks at the organization and development of specialized production in 4th millennium BCE Egypt. At the outset of this period northeastern Africa was occupied by small-scale groups of pastoralists and early agriculturalists. By the close of the 4th millennium BC, the Nile Valley was one of the earliest instances of a society with centralized political organization, extensive labor division, and institutionalized inequalities.

This research brings data on stone tools and settlement sites to bear on competing models of specialization. Many models of specialized production have focused either on the production of symbolically meaningful goods for the elite, or the production of utilitarian goods for the masses; they have not considered the production of symbolically meaningful goods for commoners. In Egypt the prevailing theory for the development of specialized production is a prestige-goods model, where elites sponsored the production of items used to display status and which were not available to all. However, this model does not account for all specialized production. A review of the evidence for specialization in Egypt shows that some items made by specialists were quite widely used (e.g., lithic blades, black-topped red ware ceramics), and so do not fit a prestige-goods model. To explore how the production of various stone tools was organized and developed, a model of ritual production outlined by Spielmann (2002) was considered. This model recognizes that people have often increased their economic production in order to make items needed for ritual activities, such as life cycle events (e.g., birth, marriage, death) and community-encompassing rituals. These ritual activities involved many members of society, not a restricted subset. Expectations were developed for archaeological patterns of raw material choice, production locations, and find contexts that should be observed if the ritual production model can account for the development of some aspects of specialized production in 
Egypt. These expectations were evaluated based on examination of lithic artifact collections from the settlement sites of el-Mahâsna, Abydos, and Nag el-Qarmila, and comparison to published data from other sites and online museum databases.

This research showed that there were many different ways that stone tool production was organized at the intra-site and regional levels. Some of stone tools—early fishtail knives, axes, large-blade knives, and microendscrapers—-fit the ritual production model for the development of specialized production quite well. Preferences for certain raw materials were evident, and these preferences could not be accounted for by functional considerations or access to local resources. Instead, the raw material choices probably related to the symbolic significance of their colors, which can be traced from the Pharaonic back to the Predynastic periods. These tools were produced in conjunction with ritual activity areas, and the tools themselves were found in ritually significant contexts such as early 'temples', offering deposits, and tombs, as well as in more traditionally ordinary contexts such as houses, storage areas, and trash middens. Most importantly, they also had a widespread distribution not limited to the elite class. This study shows that although full-time specialized production was fostered by elites in the latter part of the Predynastic period, this process built on already multivariate and complex systems of production for stone tools, which included the production of stone tools with symbolic uses for a large cross-section of the population. 
Dissertation Committee

Patricia Wattenmaker, (chair) University of Virginia, Department of Anthropology

Adria LaViolette, University of Virginia, Department of Anthropology

Stephen Plog, University of Virginia, Department of Anthropology

Foteini Kondyli, University of Virginia, Department of Art History

Steven Rosen, Ben-Gurion University of the Negev, Near Eastern Archaeology

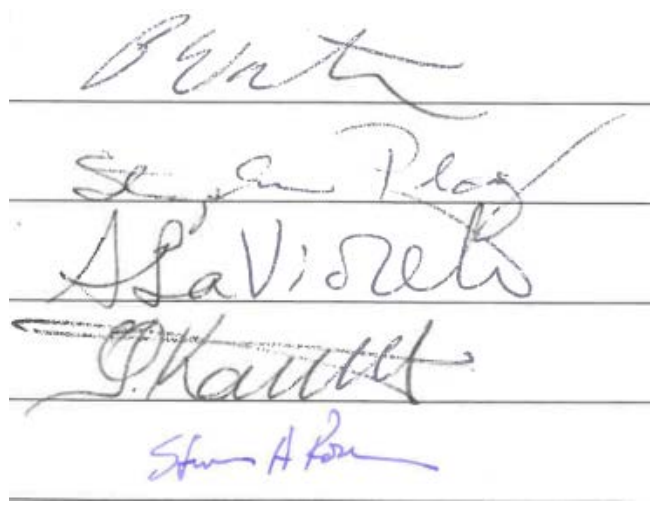

$-\mathrm{V}-$ 
This dissertation is dedicated to my many families, the Harts, the Skarzynskis, and especially the Bishay family for giving me a home away from home in Egypt. 


\section{Table of Contents}

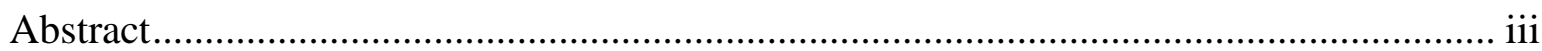

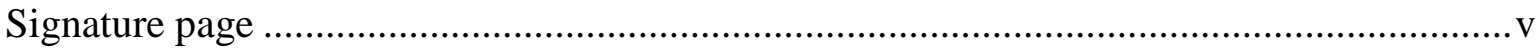

Acknowledgments ......................................................................................................... xiii

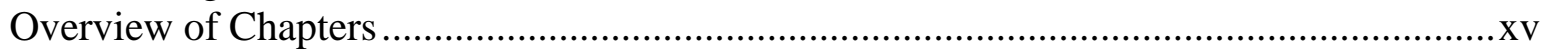

Chapter 1: The Development of Specialized Production ................................................................1

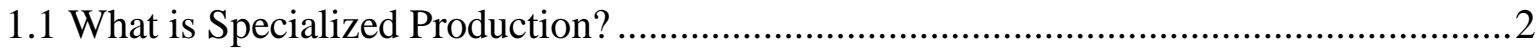

1.2 Theories for the Development of Specialized Production .................................................

1.3 Theories for the Development of Specialized Production in Egypt ................................13

1.4 A Brief Review of Evidence for Specialized Production in Egypt ................................18

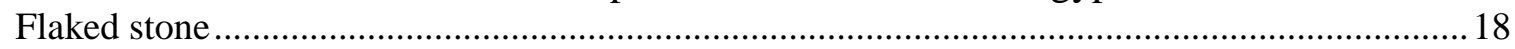

Ground stone

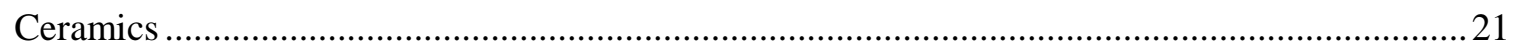

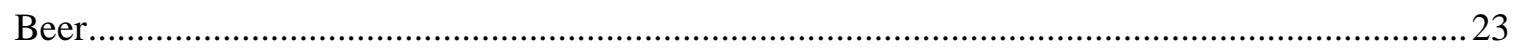

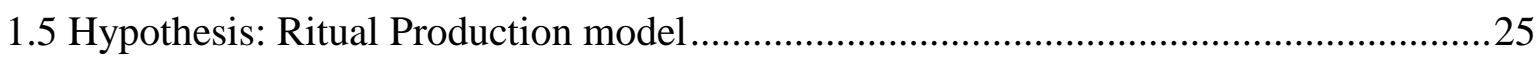

1.6 Ritual and Religion in Archaeology ………………...............................................31

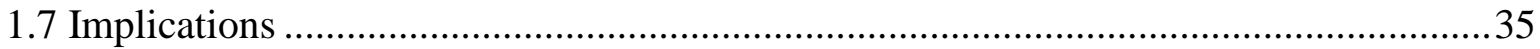

Chapter 2: Methods ..................................................................................................................................3

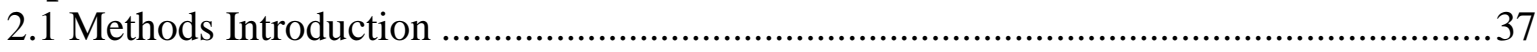

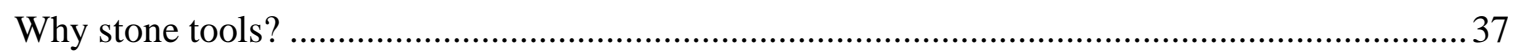

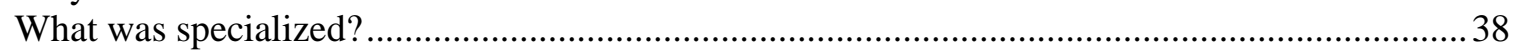

2.2 Expectations for the Ritual Production Model .................................................................39

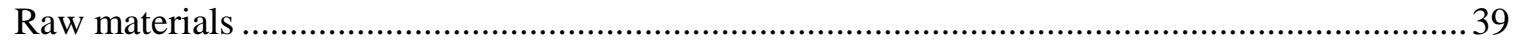

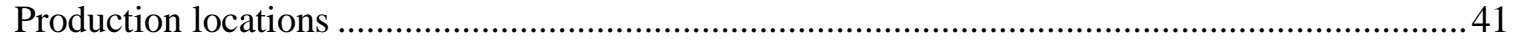

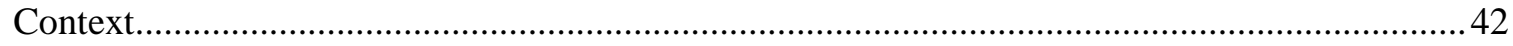

2.3 Sites Involved in the Study .................................................................................... 44

2.4 Artifact Analysis Methods.....................................................................................

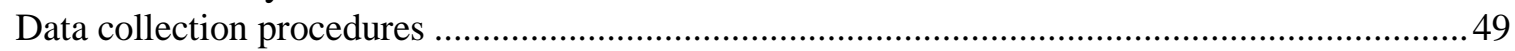

2.5 Statistical Analysis Methods ……………………….................................................51

Confidence limits of a proportion..........................................................................................51

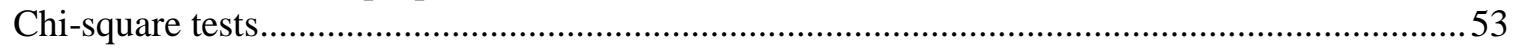

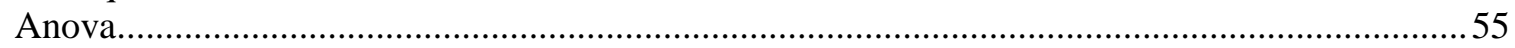

Coefficient of variation.....................................................................................................5

Chapter 3: Predynastic Settlements and their Contexts .........................................................58

3.1 Overview of Socio-Economic Changes......................................................................59

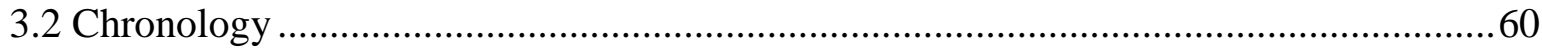

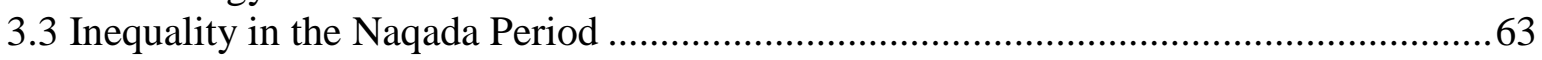

3.4 Site Descriptions: el-Mahâsna, Abydos, Nag el-Qarmila...................................................64

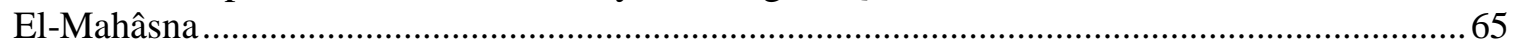

Anderson's el-Mahâsna excavations ..........................................................................................67

Alawniyeh / Beit Allam cemetery ………………………………………………………....

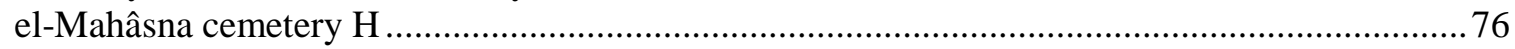

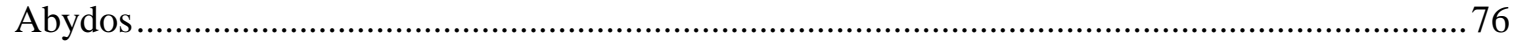

Harvey's Abydos excavations ………………………………………………………….

Other Predynastic and Early Dynastic settlement sites in the Abydos area....................................81 


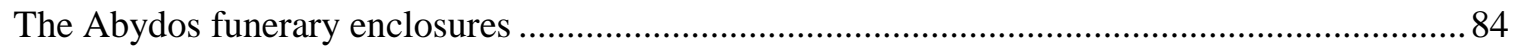

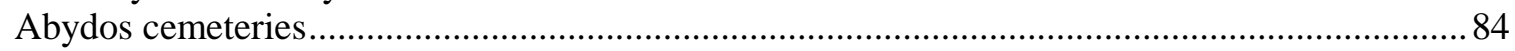

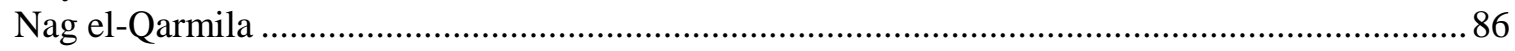

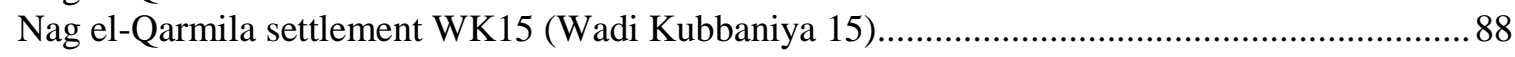

Nag el-Qarmila WK22 (Wadi Kubbaniya 22) ...........................................................................90

Nag el-Qarmila cemetery WK14 (Wadi Kubbaniya 14) .............................................................91

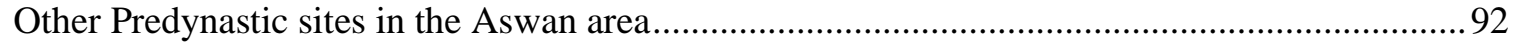

3.5 Site Descriptions: Comparative Sites .....................................................................94

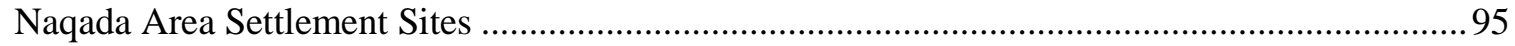

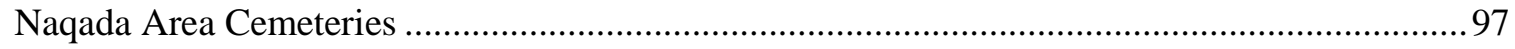

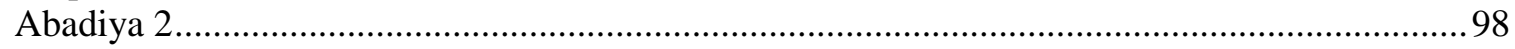

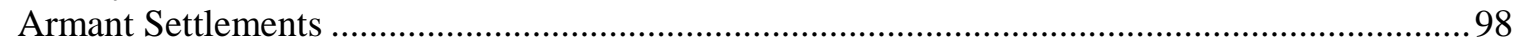

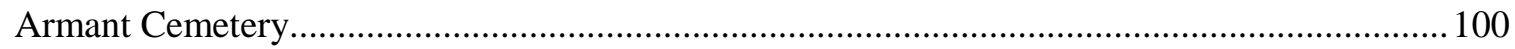

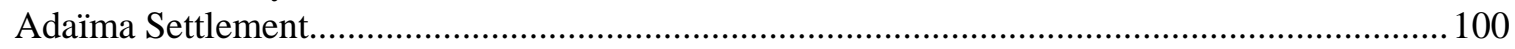

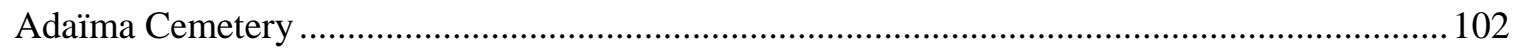

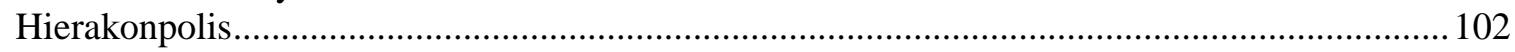

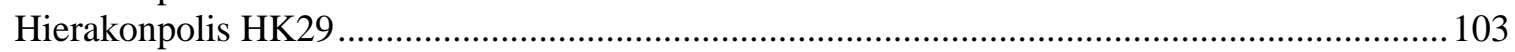

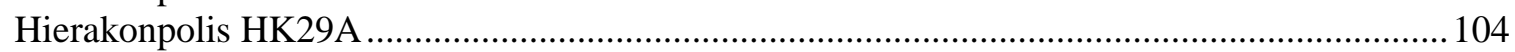

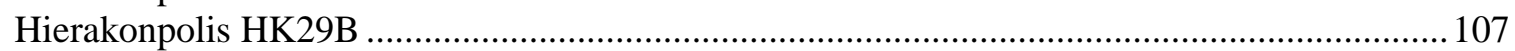

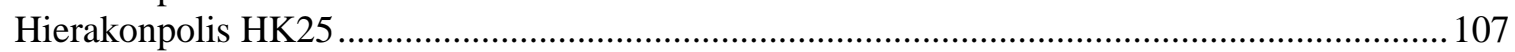

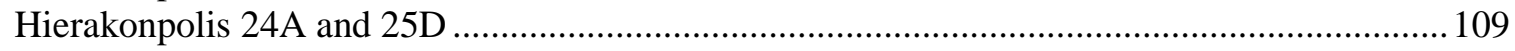

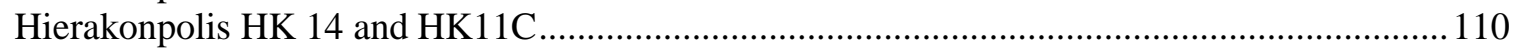

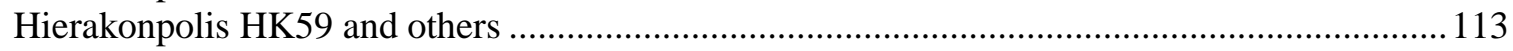

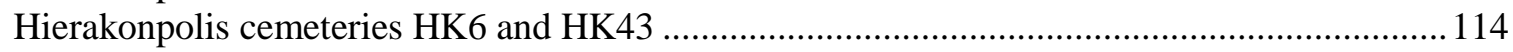

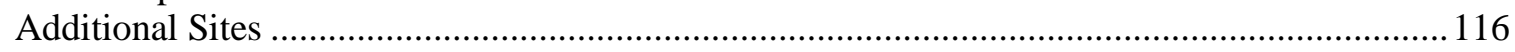

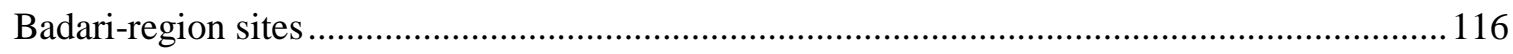

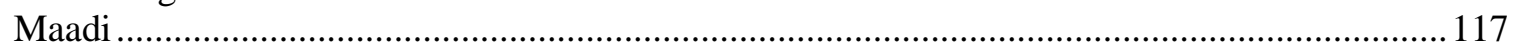

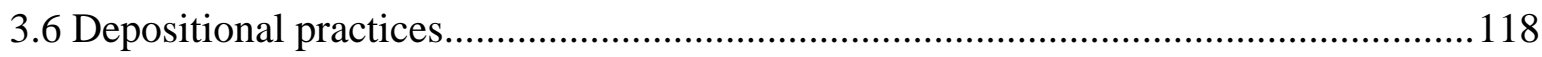

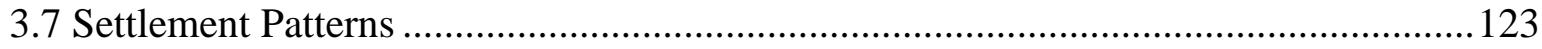

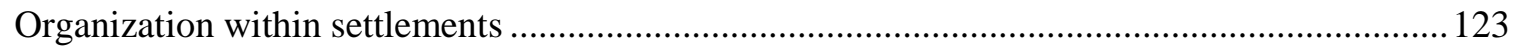

Distribution of settlements across the landscape ...................................................................127

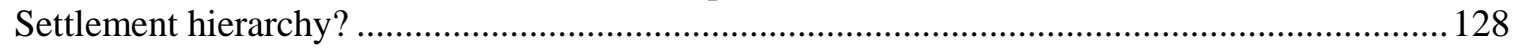

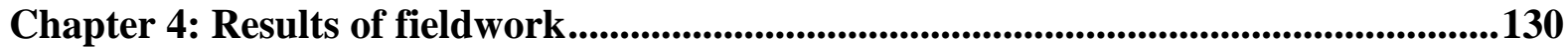

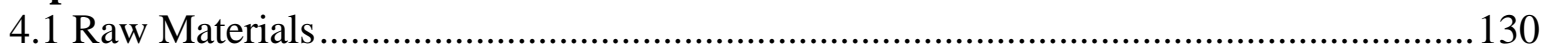

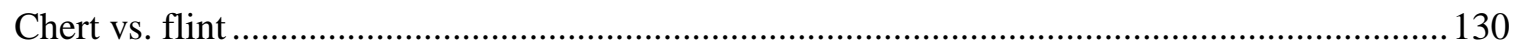

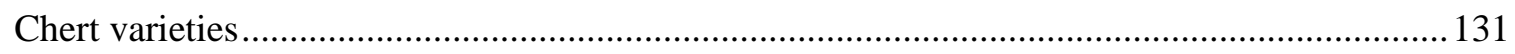

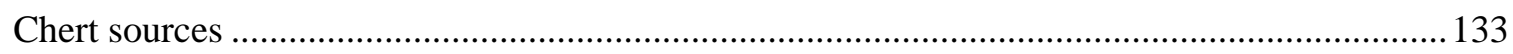

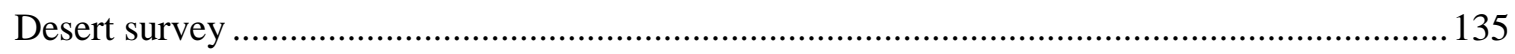

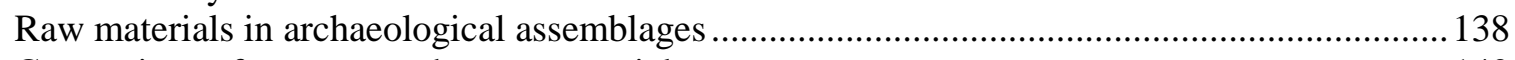

Comparison of cortex type by raw material type.................................................................. 140

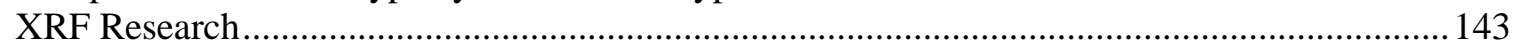

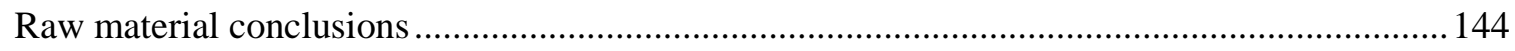

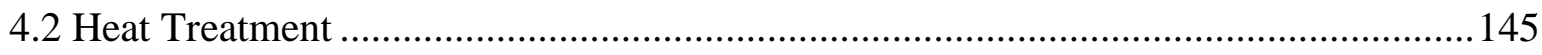

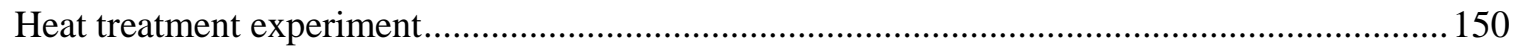

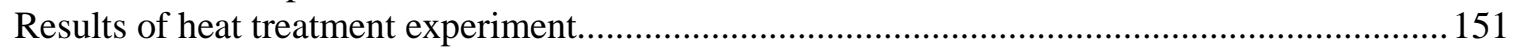

4.3 Assemblage Composition: Debitage and Tools..................................................... 153

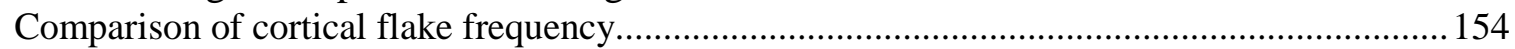

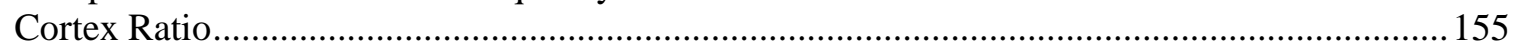

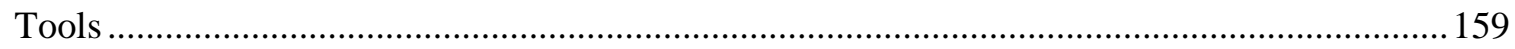

Chapter 5: Blade tool Specialization ..................................................................161 
5.1 Identifying the Organization of Production Archaeologically …................................161

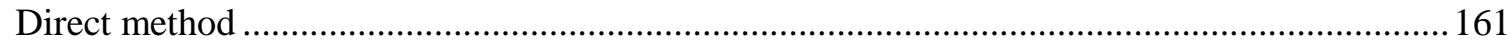

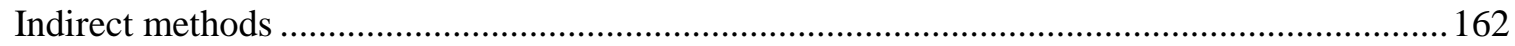

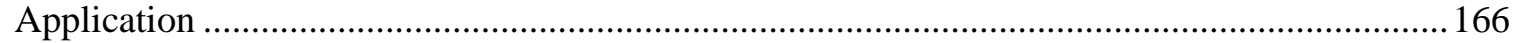

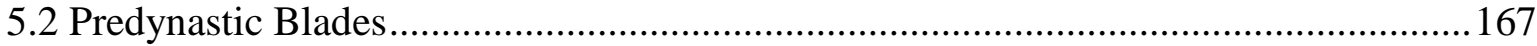

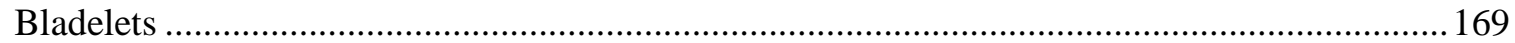

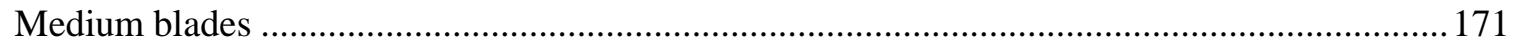

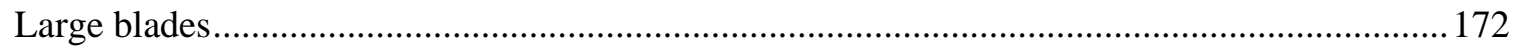

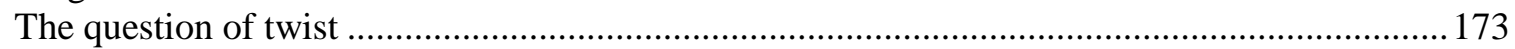

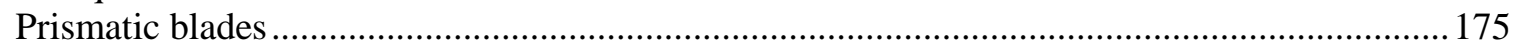

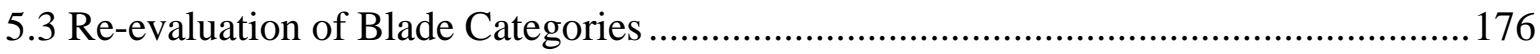

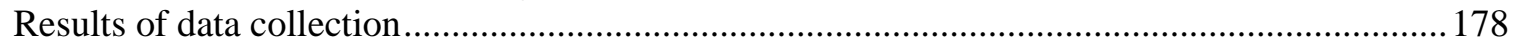

Re-evaluation of blade subtypes based on platforms and bulbs .........................................179

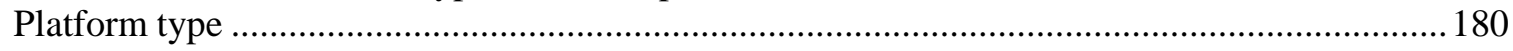

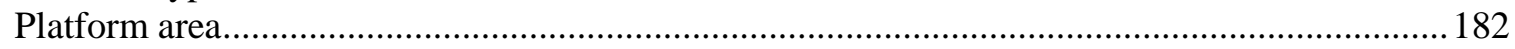

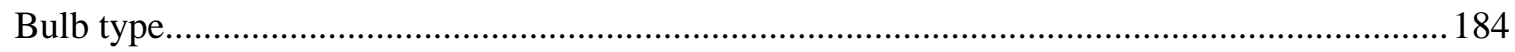

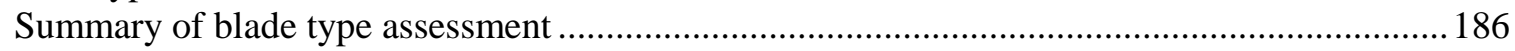

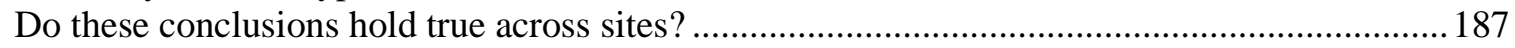

5.4 Blade Specialization at an Inter-Community Level .............................................189

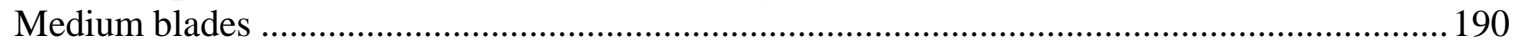

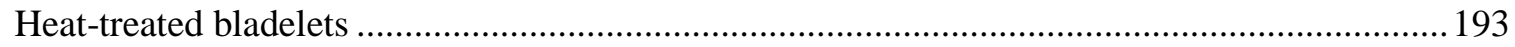

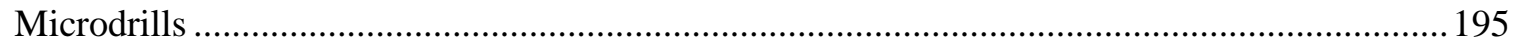

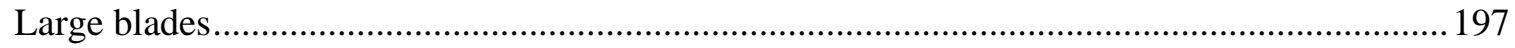

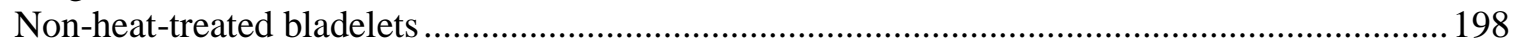

Summary of blade distribution at an inter-community level .................................................... 199

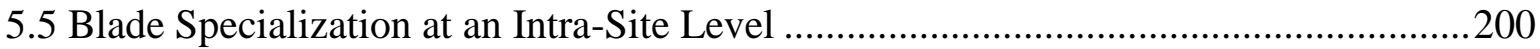

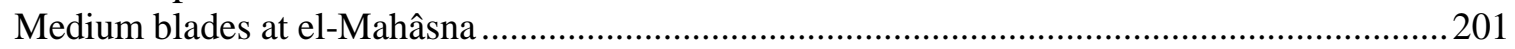

Non-heat-treated bladelets at el-Mahâsna................................................................................... 201

Heat-treated bladelets at el-Mahâsna..................................................................................202

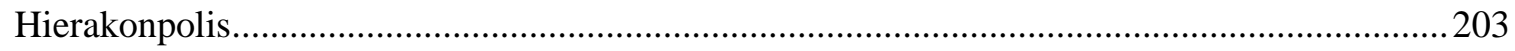

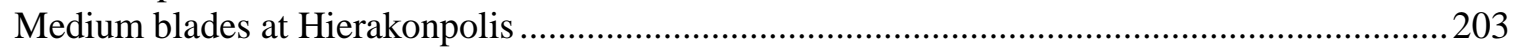

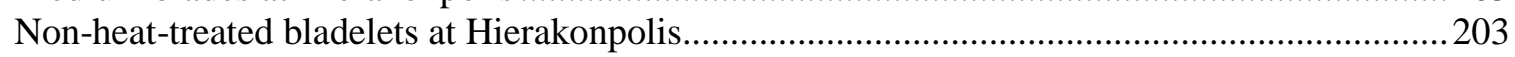

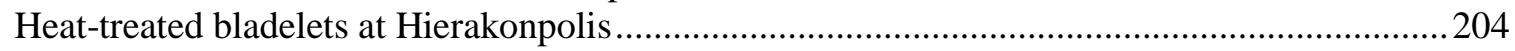

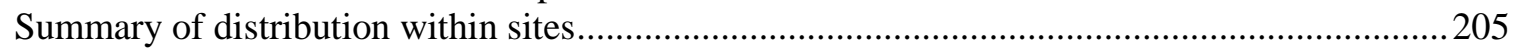

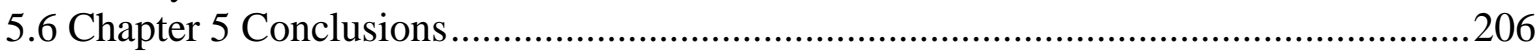

Ch 6: Bifacial Tool Specialization ...........................................................................208

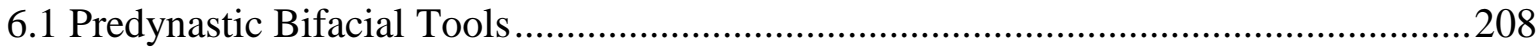

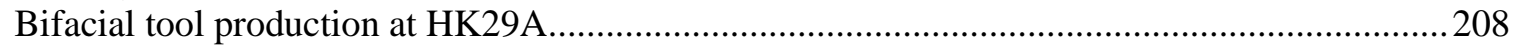

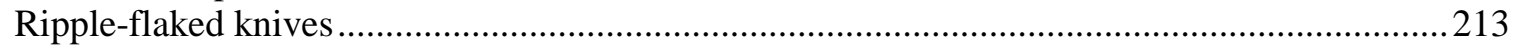

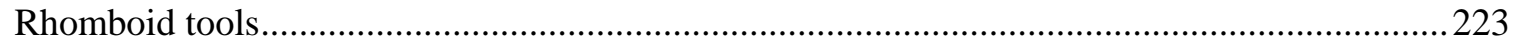

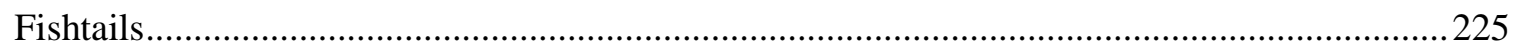

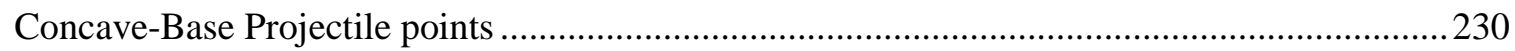

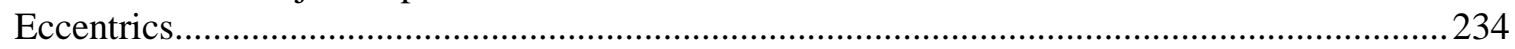

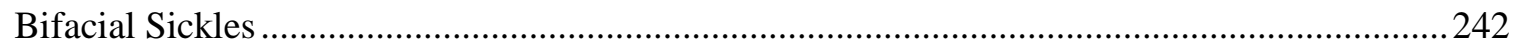

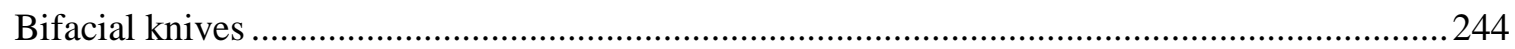

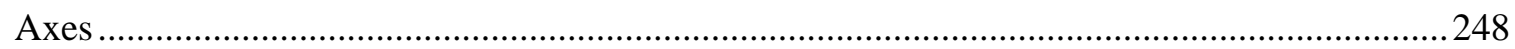

6.2 Evaluation of Specialized Production at an Inter-Community Level.........................251

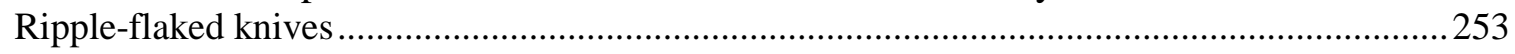

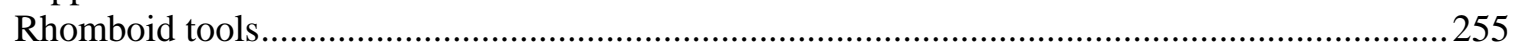

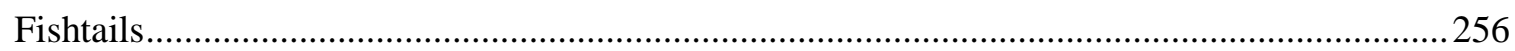




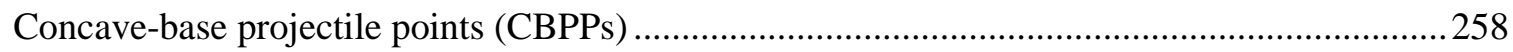

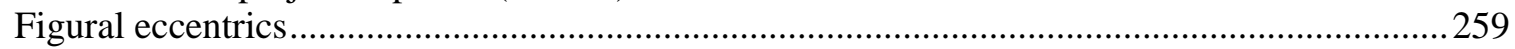

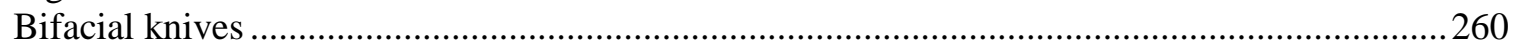

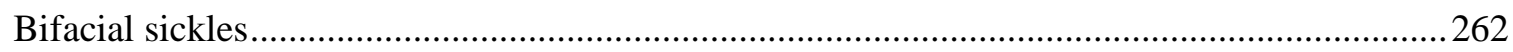

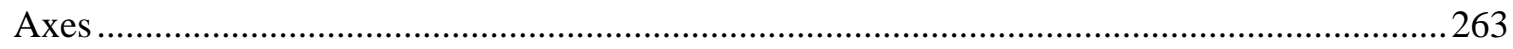

6.3 Bifacial Tool Specialization at an Intra-Site Level ...........................................264

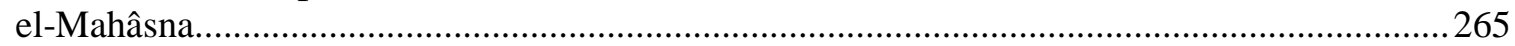

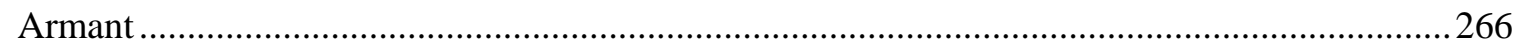

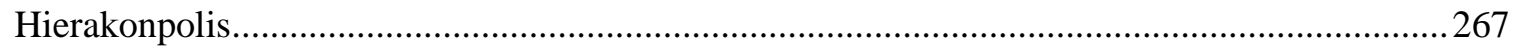

6.4 Conclusion: Production of Bifacial Tools ........................................................271

Chapter 7: Evaluation of Expectations ......................................................................2273

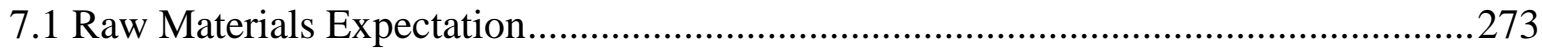

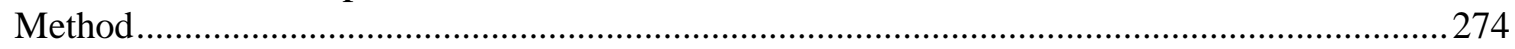

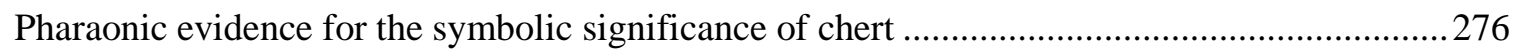

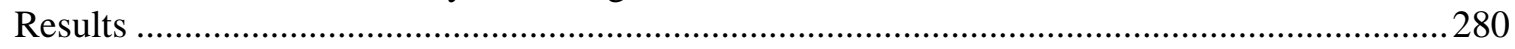

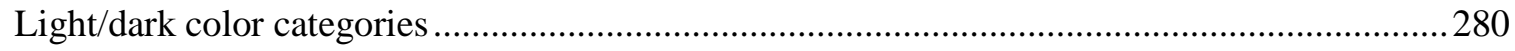

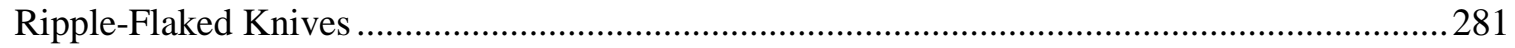

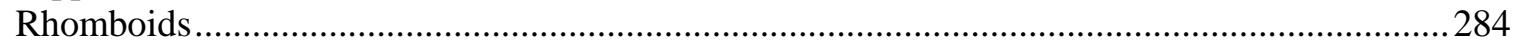

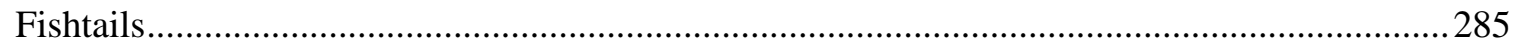

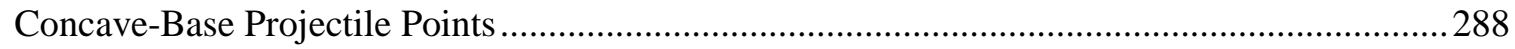

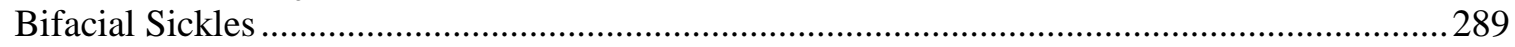

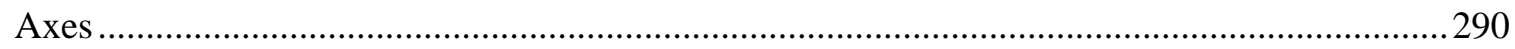

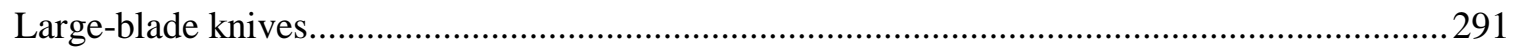

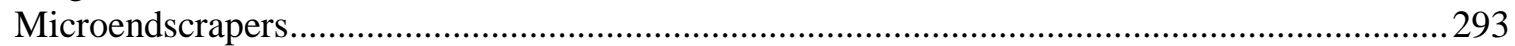

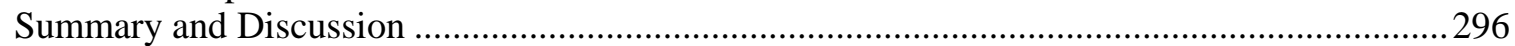

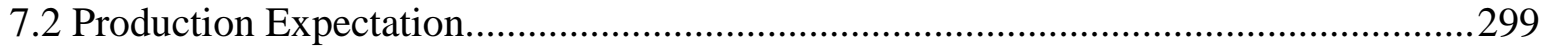

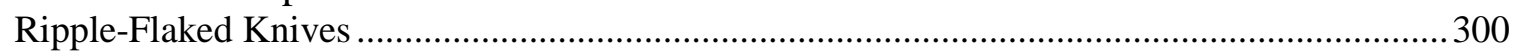

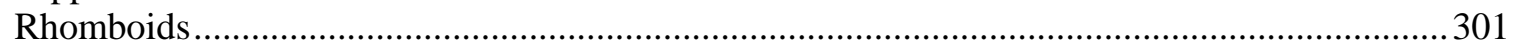

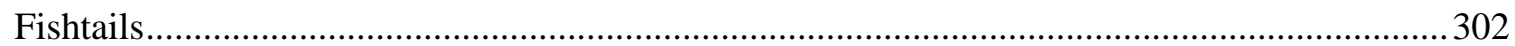

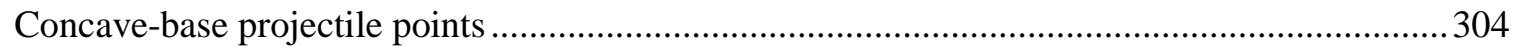

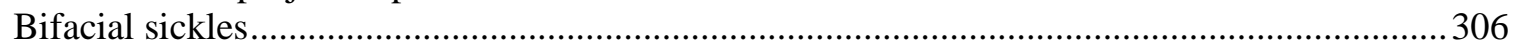

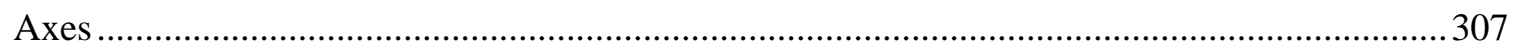

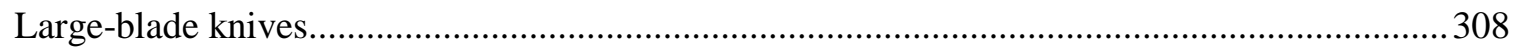

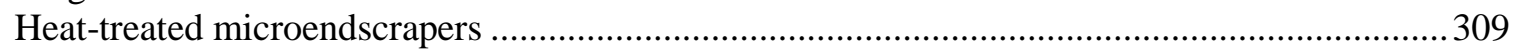

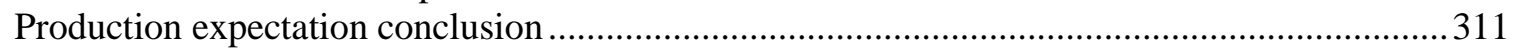

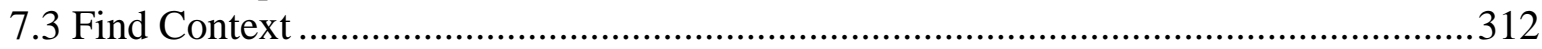

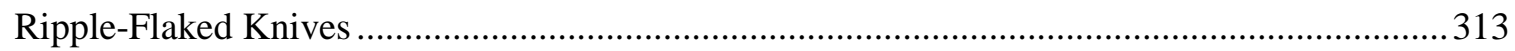

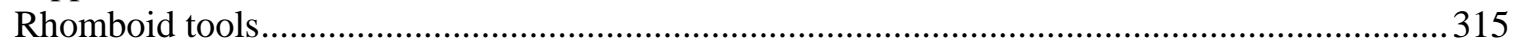

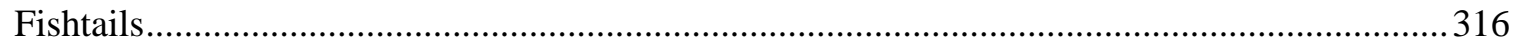

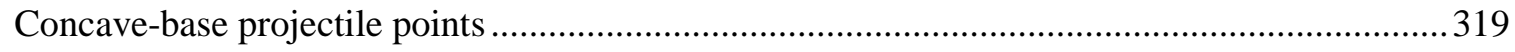

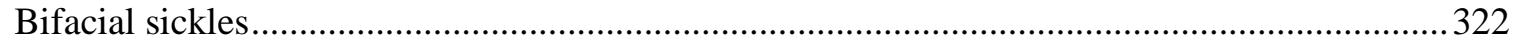

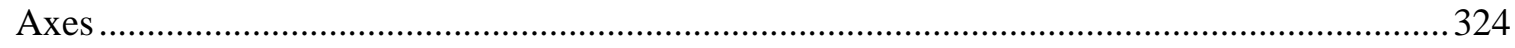

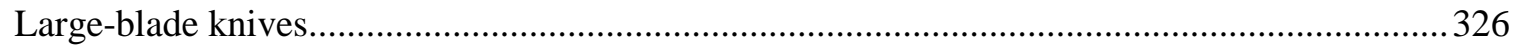

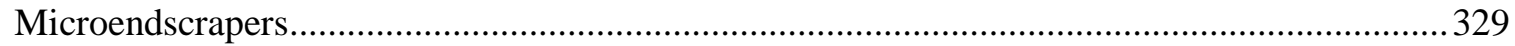

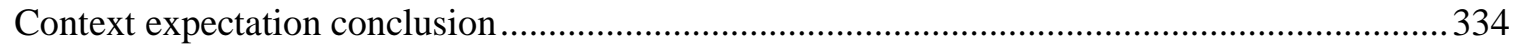

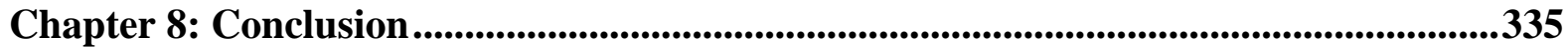

8.1 Summary of Raw Material, Production, and Find Context Patterns by Tool Class.....335

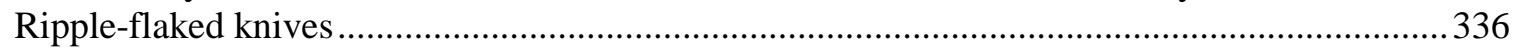

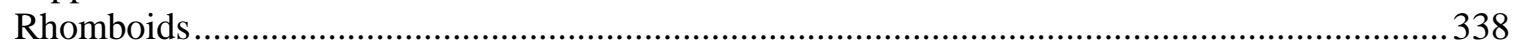

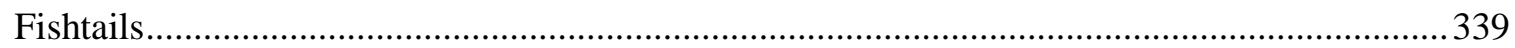




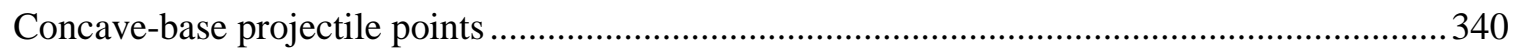

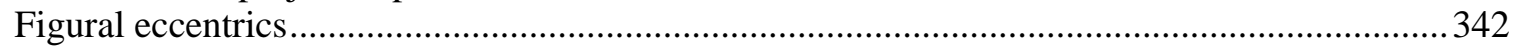

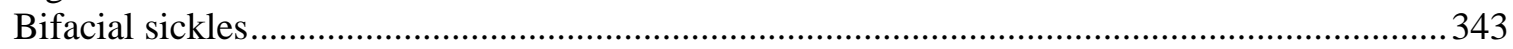

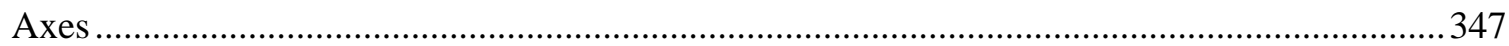

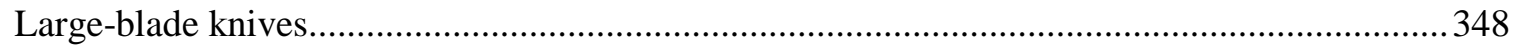

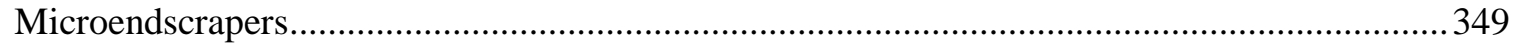

8.2 The Organization(s) of Predynastic Stone Tool Production......................................350

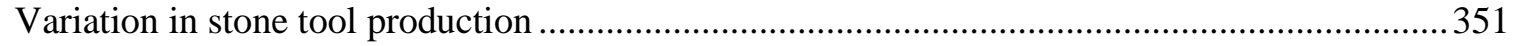

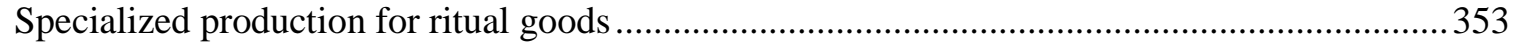

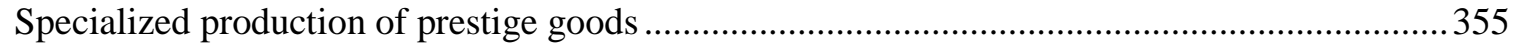

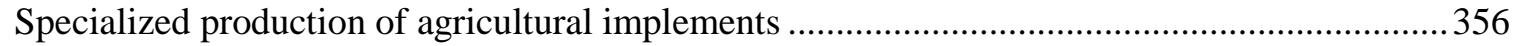

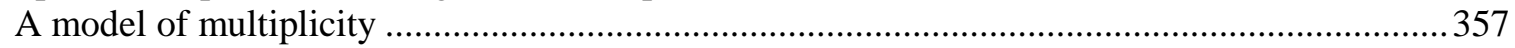

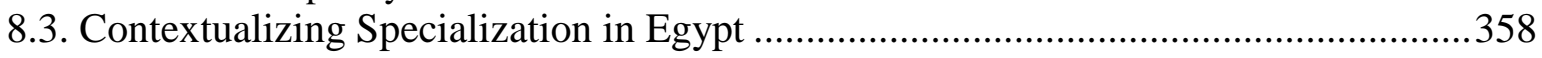

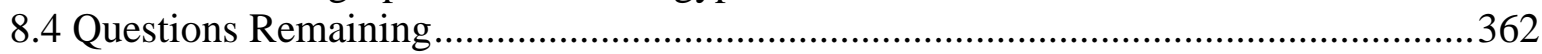

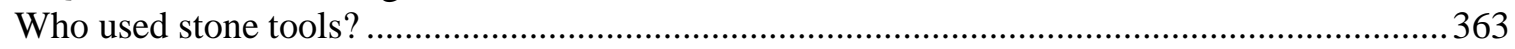

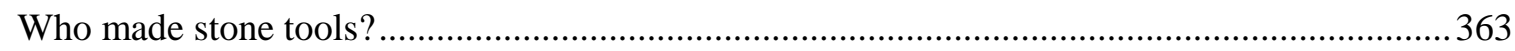

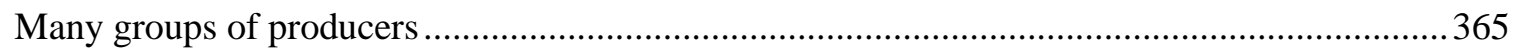

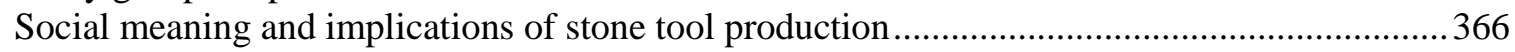

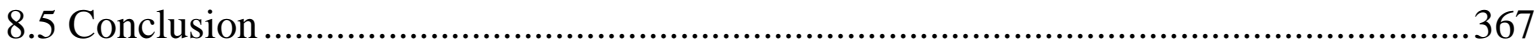

Appendix: Lithic Coding Guide .......................................................................................369

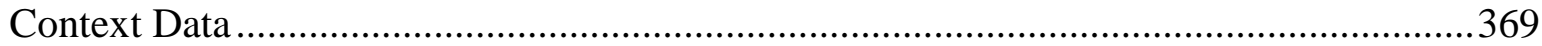

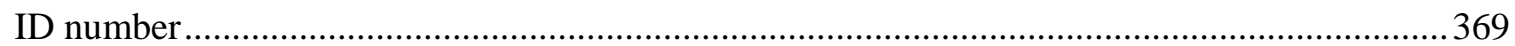

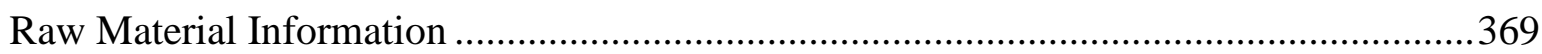

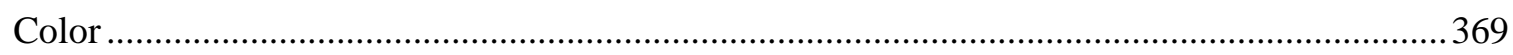

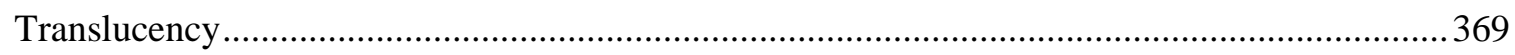

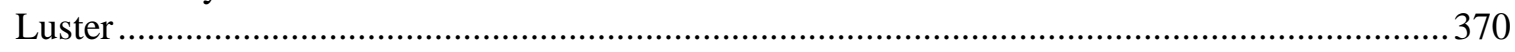

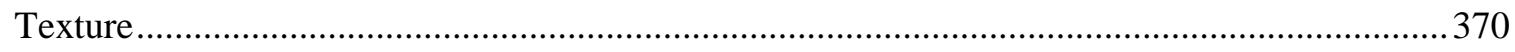

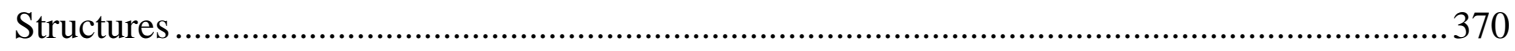

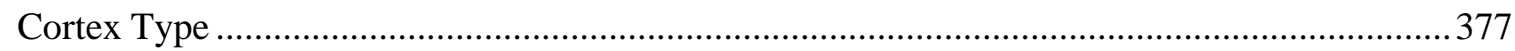

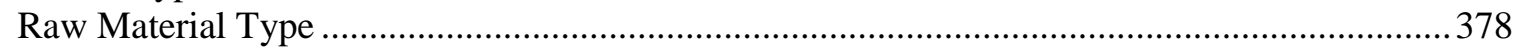

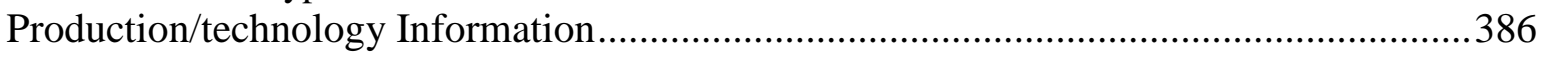

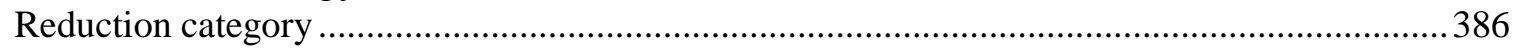

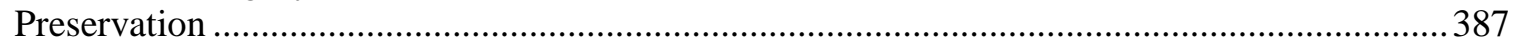

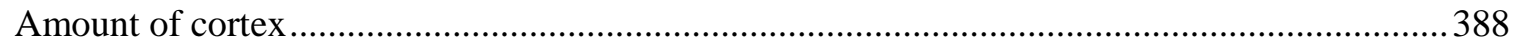

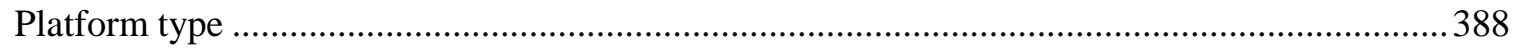

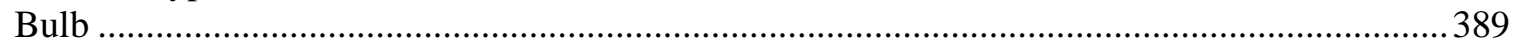

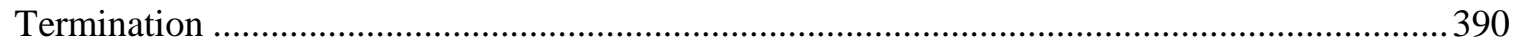

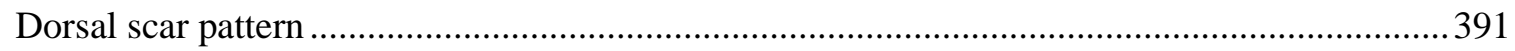

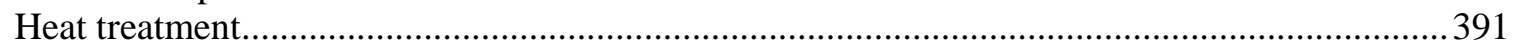

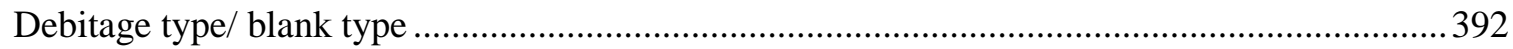

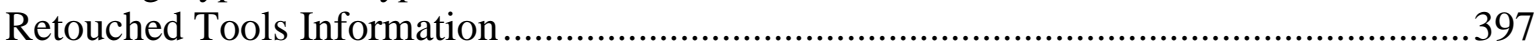

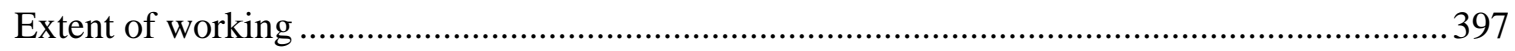

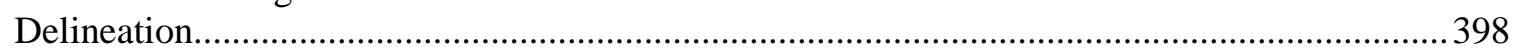

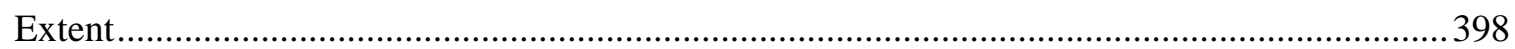

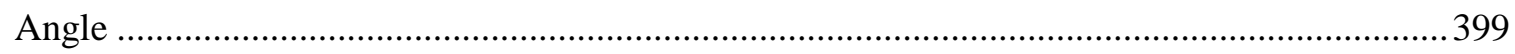

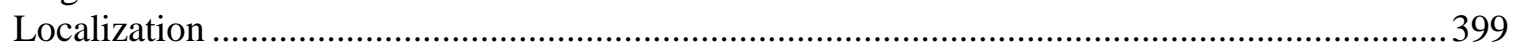

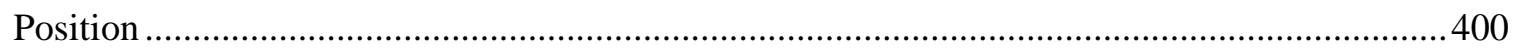

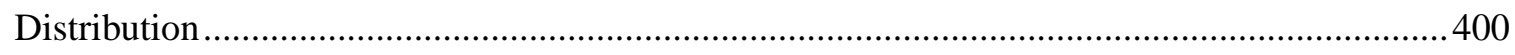

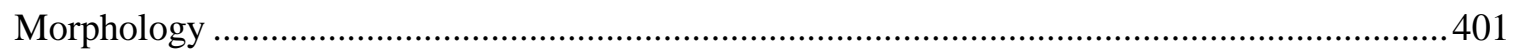

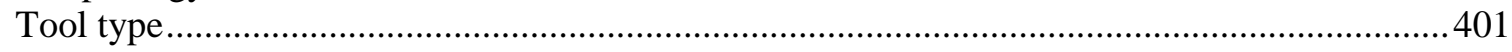




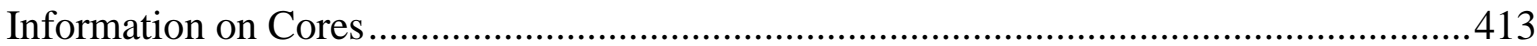

Core platforms

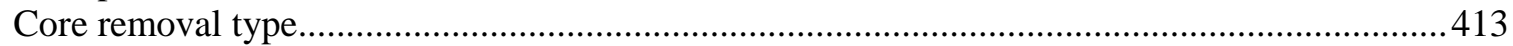

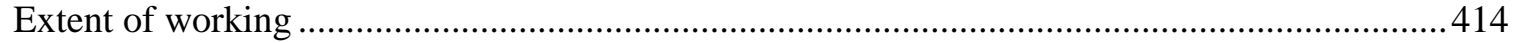

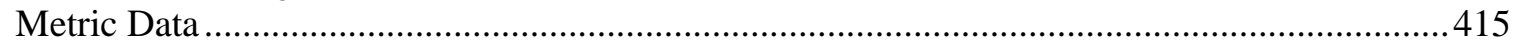

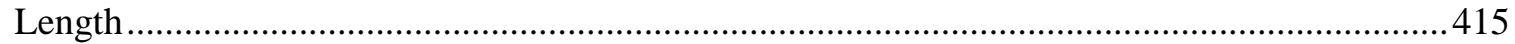

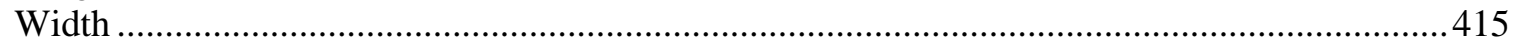

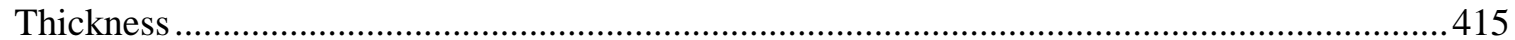

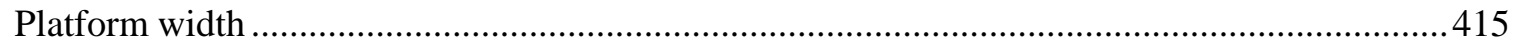

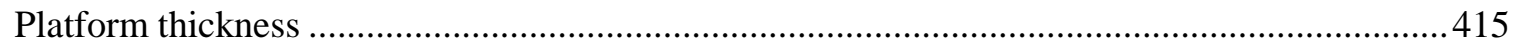

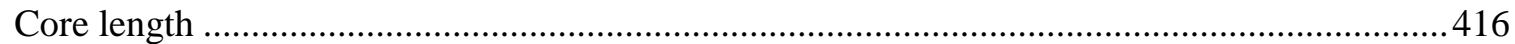

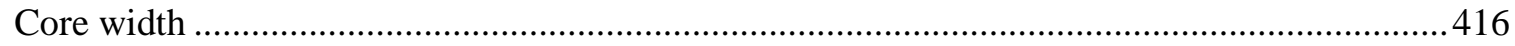

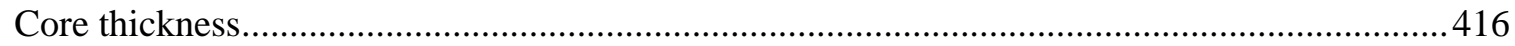

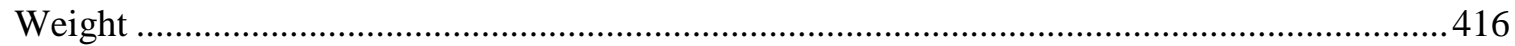

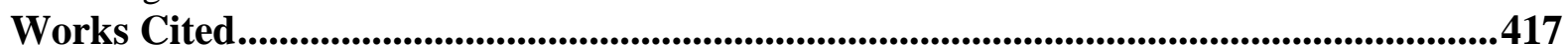

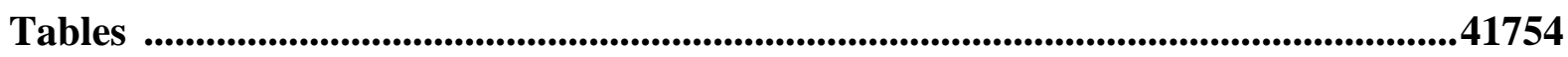

Figures........................................................................................................................................ 538 


\section{Acknowledgments}

Many thanks go to David A. Anderson, Maria Carmela Gatto, and Stephen Harvey for generously allowing me to study the lithic artifact collections that they excavated, without which this study would not exist. Thank you to Janet Richards and Matthew D. Adams for permitting me to join their teams and facilitating study seasons at Abydos, I am very grateful for the time spent there. I have been lucky to spend a substantial amount of time in the field working with and learning from Janet Richards, Matthew Adams, David Anderson, Maria Gatto, Dirk Huyge and Christiana Köhler, who all have very different and effective styles of directing teams, and I could not have asked for better role models in this respect. I hope to someday be as generous as Janet, as dedicated as Matt, as exuberant as David, as calm as Maria, as unflappable as Dirk, and as prepared as Christiana. Donatella Usai provided timely consultation on the artifact analysis in Nag el-Qarmila. Megan Cook and Darcy Hackley were paragons of patience and dedication as they drew the artifacts pictured in this study. Renée Friedman and Stan Hendricks have my heartfelt appreciation for being so generous with their resources.

I would also like to acknowledge and thank the National Science foundation and the American Research Center in Egypt for the generous grants that supported my graduate study and dissertation research.

Thank you to my committee for their direction, constructive commentary, teaching, and scholarly examples. Foetini Kondyli took a chance on me when I needed it. Steve Plog provided a measure of skepticism that made me work that much harder. Steve Rosen always went the extra mile reading my work, providing great comments, and with astoundingly fast turn-around times that I aim to emulate in my academic career. Adria could always cut right to the heart of any problems, personal or academic, often solved with more signposting! And I am especially 
grateful to Pati for being such a supportive chair, providing me an extra measure of confidence when I was unsure, and always providing me with direction and good advice.

I am so happy that I ended up with the graduate cohort that I did. I learned so much inside and outside the classroom from Julie Starr, Nathan Hedges, Dionisios Kavadias, and Victoria Kalesnikava. It was amazing to have a group of people always ready to dork-out about anthropology, overanalyze, overthink, drink wine, provide shoulders for crying on and couches for crashing on, listen to presentations, study, write, edit, and just generally be friends and scholars.

I want to thank my parents for not asking too often if I was done yet. That was awesome. And of course for being wonderful supportive sweet parents and life role models. To my friend Krishna I owe many thanks for his support and advice that always provided greater sanity and calm, I really could not have done it without you. I could never possibly give enough thanks to Nicole Bishay for moving to Egypt with me that first year (and making sure it was so much fun!) and for being an amazingly fearless person, a great friend, and always being volubly proud of me. I also must thank all of my Michigan friends for honestly not caring overly much about Ancient Egypt, beyond, 'that's pretty cool'. Success or failure, all these people will love me the same. And speaking of love, thanks Nick, for the love. 


\section{Overview of Chapters}

Through a review of theoretical approaches to the development of specialized production in archaeological literature in general, and in Egypt, Chapter 1 argues that there has often been an unnecessary divide between explanations that involve the production of symbolic goods for the elite, and those that propose the production of utilitarian goods for the masses. A discussion of existing evidence for specialization in Egypt shows that the production of some items has not been accounted for in current models. Spielmann's (2002) model of ritual production is presented as a possible explanatory framework to address these problems.

The methods for assessing whether a ritual production model helps explain the development of specialization in Egypt are given in Chapter 2. This chapter includes expectations for the ritual production model in terms of raw material use, production locations, and find contexts, and ways those expectations can be assessed archaeologically. Additionally the methods used for artifact analysis in the field and statistical analysis of the collected data are outlined.

Understanding the contexts in which stone tools and their production remains were found is essential for evaluating the ritual production model. After a discussion of chronology and inequality in the period, Chapter 3 takes an in-depth look at Naqada-period Nile Valley settlement contexts and makes the argument that Predynastic settlements were often composed of spatially separated but related localities, some of which were devoted mainly to functionally different activities, such as those pertaining to production, habitation, or ritual. Furthermore this functional differentiation may have increased over time, probably with the development of specialized production.

Chapter 4 presents basic results of the field analysis of materials from the archaeological 
sites of el-Mahâsna, Abydos, and Nag el-Qarmila, including frequencies of debitage and tool types. Particular emphasis was put on understanding the raw materials and heat treatment, because one of the expectations for evaluating the ritual production model involved raw material use. The raw-materials study showed that the local materials predominating in each site varied considerably. Furthermore, at Nag el-Qarmila much of the raw material was probably imported. The results of a heat treatment experiment provided insights for identifying heat-treated materials in Egyptian archaeological assemblages, and implications for considering how heat-treatment may have factored into specialized production.

Chapters 5 and 6 address the question of which Predynastic Egyptian tools should be considered specialized by comparing the distribution of the production remains to the distribution of the tools for different types of blade and bifacial tools. In Chapter 5, after a discussion of methods for identifying specialized production, the known blade types in Predynastic Egypt and their descriptions are given. An analysis of the attributes of bladelets and medium sized blades indicates that they should be considered separate types. The study of the distribution of production remains and tools, at the intra-site and regional levels, for five blade types showed that there was substantial variation in how blade production was organized. Lithic production cannot be divided simply into two production categories of ad hoc production by everyone vs. specialized production by a small group of sponsored professionals. There was an array of ways the production of blades was organized, ranging from diffuse production in many parts of all sites (which cannot be considered specialized), to highly concentrated production in only one or a few locations throughout the region (which definitely should be considered specialized).

The same methods of assessing the organization of production at inter- and intra- site 
levels were applied to bifacial tool types in Chapter 6. The production of most, but not all, bifacial tool types examined here should be considered specialized, but, like blades, their production was organized in a variety of ways.

Chapter 7 addresses the expectations for the ritual production model. The archaeological patterns for raw material use, production locations, and find contexts of flaked stone tools made by specialists were compared to those expected for the ritual production model. This research showed that for certain tools—axes, large-blade knives, and microendscrapers—-there was a preference for a raw material type or color which could not be explained by functional considerations or access to local raw material sources. Instead the raw material choices may have been related to the symbolic significance of the colors. The cosmological meanings of certain colors are well known for the Pharaonic period—red (desert, chaos), and black (fertile Nile Valley/order)—and the argument that these colors were also symbolically meaningful during the Predynastic was made based on finds of unusual, paired red and black ceramics in Predynastic ritual contexts.

The analysis of stone-tool production contexts shows that for each tool class where data were available, specialized production occurred in conjunction with ritual activity areas some or all of the time. Furthermore, the analysis of stone-tool find contexts showed that fishtail knives, concave-base projectile points, bifacial sickles, axes, large-blade knives, and microendscrapers, were not restricted to a subset of the population. They were found in multiple habitation areas within settlements, at settlements of multiple scales, and in cemeteries dedicated to people of different status. Therefore these tools were not restricted to use by an elite subset of the population, and cannot fit with the prestige-goods model for the development of specialized production. Furthermore the contexts in which these artifacts were found challenge 
interpretations of the stone tools as purely utilitarian or purely symbolic items, as they were found in both ritually significant contexts such as early 'temples', offering deposits, and tombs, as well as in more ordinary contexts such as habitation, storage, and midden areas.

All together the data for certain tool classes - early fishtails, axes, large-blade knives, and microendscrapers—-fit the ritual production model for the development of specialized production. These tools were produced by specialists, and used in ritual (and other) activities by a large sector of the population. The case of fishtails was particularly telling because it was the only tool class that covered the full time span of the Naqada period, and could be stylistically dated to earlier and later types. The differences in the archaeological patterns between the earlier and later types indicated a shift from a ritual production model toward a pattern of production and use that matches a prestige goods system. This study shows that although full-time specialized production was fostered by elites in the latter part of the Predynastic period to produce prestige goods, this process built on already multivariate and complex systems of stonetool production, which included the production of stone tools with symbolic uses for a large cross-section of the population. 


\section{Chapter 1: The Development of Specialized Production}

An example of one of the most intriguing items from Predynastic Egypt is shown in Figure 1.1. Known as "fishtail knives" or "fishtails," the function of these items is not immediately clear, to the extent that they are sometimes shown upside-down in catalogs or displays, and they have generated staunch and disparate interpretations of their use. More fascinating than the fishtails themselves are the people who made and used them. Studies of specialized production are at heart ways to examine the variable connections between people who make things and people who use things. This study aims to explore the organization of production for stone tools in Predynastic Egypt, and in so doing enrich the known repertoire of ways humans interact in the world, in history, and in prehistory.

The following discussion of what constitutes specialized production, and its parameters of variability, sets the stage for a review of theories for the development of specialization. Critiques of models based on efficiency or political economy have led to the possibility of ritual and religion as factors in the development of specialized production. A review of models for the development of specialized production in Egypt shows that they have followed these same broad theoretical trends focusing on explanations of adaptive efficiency and political economy, yet problems remain in applying them to the archaeological record in Egypt. Spielmann’s (2002) ritual production model provides a possible explanation for the development of some specialized production in Egypt. Following that, a short discussion of ritual and religion in archeology clarifies some terms involved in this study. The significant implications of this research are discussed at the close of this chapter. 


\subsection{What is Specialized Production?}

Definitions of what constitutes specialized production have varied widely. Flad and Hruby (2007) reviewed definitions of specialized production, and organized them into two groups: those that use a more broad definition, and those that single out a specific subset. More restrictive definitions only consider production specialized if some or all of the producer’s subsistence was provided through exchange of their product (ibid.:3). Examples include Evans (1978), Arnold and Muns (1994), and Longacre (1999). This is often identified archaeologically as full-time production (Costin 1991:16), meaning that the producer spends all of their productive time making their specific product, and none of it on other more direct subsistence pursuits. In focusing on production in exchange for subsistence and full-time production, these kinds of definitions for specialized production emphasize quantity—of products, and of time spent producing — as a primary factor in distinguishing specialized production.

Alternatively, broader definitions of specialized production do not put exchange for subsistence or full-time production as the boundary point of a qualitatively different kind of production. Flad and Hruby (2007:2) define specialization broadly as "simply production that leads to exchange, thereby integrating the society in which it occurs.” The focus here is on who is making things relative to the people who use them, not on how much or what kinds of work a producer is doing. Flad and Hruby cite Rodgers (1966), Clark and Parry (1990), and Costin (1991) as examples of this kind of broad definition for specialized production. Other examples include Wattenmaker’s (1998:3) definition: “the production of... goods for consumption by social groups other than those that produced them,” and that of Costin (2001:275-276, 2015:1) where, above a household level "fewer people make a class of objects than use it." These latter examples show that for scholars using the broader definition, the qualitative difference allowing 
something to be considered specialized production is in terms of who things are exchanged to, not the quantitative level of the exchange. This line is drawn somewhere above the individual and below that of the whole society, such as household or social group as in the examples above.

Ultimately of course the definition of specialized production that one uses depends on the research questions being explored. For example Zeder $(1988,1991)$ defined specialized economy, rather than specialized production, as when there is differentiation not only between people who make different products, such as pottery or stone tools, but that the production processes themselves are divided among different people, such as raw material acquisition, product finishing, and distribution. This even more particular and restrictive definition was appropriate for her research into the organization of an early urban economy.

For the purposes of this dissertation, specialized production is defined as where, above a household level, a class of objects is used by people other than those who made them. The producers themselves may still use the class of items in addition to others. The production and associated exchange is assumed to be a reoccurring pattern not limited to one-time or rare idiosyncratic events. This definition includes tribute relationships where any number of people make goods for the elite few, an example which would have been excluded by Costin’s (2001) definition above.

Here, “above a household level” means that items were used by people not in the household of those who made them. It should be noted that a household is understood to be a culturally defined and variable unit, not necessarily following the model of a western nuclear family (Yanagisako 1979). Furthermore, the use of households as a criterion for assessing specialized production is in no way meant to reduce them to homogeneous units. It is recognized than households themselves are made of differentially positioned people (e.g., by gender, age) 
who may have ties outside the household and may be at odds with each other within it (Hendon 1996; Robin 2003). Here "household" is the boundary for understanding specialization because there is evidence that a household was a meaningful social and economic unit, that is archaeologically identifiable. Based on textual evidence for Pharaonic Egypt (e.g., the Heqanakht Papayri), households were a meaningful conceptual unit that could include a nuclear family, extended family members, servants, or other dependents, and the household was linked to specific domestic structures (Allen 2002; Lehner 2000; Moreno Garcia 2012; Spence 2015). The built structures utilized by households could be individual houses, such as at Wah-sut (Abydos) and Lahun (Petrie 1890, 1891; Spence 2015), or tight clusters of structures such as at Amarna and Tell el-Dab’a (Beitak 2010; Kemp 2006). The association of households with individual or tightly grouped structures also can be identified for the Predynastic period. At Adaïma the house structures were grouped into clusters scattered throughout the settlement, and in the cemetery clusters of graves were found that had different ethnic affiliations. Based on the clustering of graves by ethnic groups, the house clusters were interpreted as household, family, or household groupings (Buchez 2011a, see Ch. 3). At Hierakonpolis there is evidence for an individual house with a fenced-off yard that may have included small outbuildings (Hoffman 1980b). That these structures probably reflected a household is indicated by comparisons to the elite cemetery HK6. There, some graves, such as the Tomb 16 complex, were organized in a way that probably represents an elite household with placement according to social position or job in the household (Friedman et al. 2011c). Dogs (guard dogs?) were at the front of the complex, while women and children were at the back, and the household head was in the center (ibid.). All the burials were connected with above-ground fencing and superstructures that may have been modeled on the actual residences (ibid.). Therefore Predynastic households should correspond to 
individual house structures, or spatially clustered groups of house structures identified in the archaeological record.

This dissertation specifically involves craft production, rather than production of comestibles. Here, "craft" is pragmatically intended to mean tangible, often durable, portable things (Costin 2007:146). Such items often leave extensive remains. The above definition of specialized production can be operationalized by looking at the number of producers relative to consumers, and the geographic distribution of production locations relative to the products, taking into account disposal practices and taphonomic processes (see Ch. 5.1 for more details on methods for identifying specialized production archaeologically).

While the definition of specialized production is intentionally broad, it is distinct from ad hoc production where people make what they need in an unplanned or unpatterned way, and it differentiates from production that might be planned or patterned but stays within a household and does not circulate widely enough to affect farther reaching social relationships. The above definition of specialized production notably does not stipulate that producers were entirely economically dependent on their specialized production for subsistence.

Definitions of specialization that include economic dependence can predetermine an economic motivation for specialized production, whereas this study specifically tests a theory where ritual activities were the motivation for production, although of course ritual and economic activities need not be mutually exclusive. Additionally, a broad definition, by being more inclusive, is better suited to an analysis of intensification or change, rather than presence or absence. Flad and Hruby (2007:6) note that more recent studies do not focus only on defining what is specialized and what is not, but rather focus on understanding the range of factors 
affecting different configurations of production, and the latter aim is the goal of this dissertation.

Besides these basic definitional issues, which as noted depend on the research questions involved, studies of specialized production have done very well at demonstrating the many ways specialized production can vary. One of the most effective ways of understanding the variability in specialized production was formulated by Costin (1991). She reviewed previous studies of specialized production and defined four parameters for analyzing the organization of production. These were: Context, which describes the degree to which the production was sponsored by elites or governments, and varies from independent to attached; Concentration, which characterizes the spatial distribution of production locations from dispersed to nucleated; Scale, which involves both the size of the production unit and the social composition of its members ranging from small kin-based production to factory level production; and Intensity which is a measure of how much time producers worked their trade, varying from part-time to full-time.

Since then Costin herself and others have built on this formulation, pulling apart some aspects and assumptions and adding many more variables. For instance scale can clearly be differentiated to parameters of the amount of output and the 'relationships among workers,' and intensity should also include a consideration of intermittent or seasonal labor (Flad and Hruby 2007:6). Other parameters or aspects of variability for specialized production can include the degree to which the production of different crafts are integrated (Shimada 2007), the degree to which the production process itself was differentiated (Martinon-Torres et al. 2014; Zeder 1988) who the producers and consumers are, e.g. in terms of class (Inomata 2001) or gender (Costin 2015), and understanding the meaning of production (Hruby 2007; Inomata 2001; Wells 2006), among many others. When these parameters are considered against theories of the development of specialized production, they can reveal new avenues for research. 


\subsection{Theories for the Development of Specialized Production}

Archaeological theories for the development of specialized production have been tied up with issues of political formations and class relationships and largely have not considered the symbolic uses of goods by commoners, nor seen ritual and religion as a possible basis for changes in the economy. The following discussion focuses on a number of factors which will help elucidate these issues: what kinds of items were made by specialists (subsistence goods utilitarian goods, prestige or luxury goods); who the goods were for (elites, non-elites, everyone); the relationship of specialized production to political formations and inequalities; and the active locus or motivator for the change.

As with most studies of the economy, models of the development of specialized production have been influenced to different degrees by the writings of Adam Smith and Karl Marx. Smith (1776) and other classical economists saw increasing division of labor (specialization) as a natural process spurred by rational calculating individuals. Division of labor allows more efficient production, therefore rational individuals would naturally want to specialize, thereby producing surplus product of their "particular occupation.” This surplus could then be exchanged to further their own interests. While looking out for their own economic interests through exchange and the accumulation of surpluses, individuals created more value in the world, in turn improving society as a whole. In Smith's theory there are no restrictions on the kinds of goods made by specialists or on who the products are for. How much a specialist may produce and accumulate is only limited by individual ambition and ability, and inequalities may arise from these personal differences. If left unchecked, competition between producers will lead to greater efficiency in the production process and for the economy overall. Politics and 
government policies are seen only as an inhibition to increasing specialization, preventing competition from causing increases in efficiency.

There are manifold critiques of Smith’s stance, both within traditional economics and within anthropology (Wilk and Cligget 2007), but just a few chief critiques will be mentioned here. In stating that people should focus on a particular occupation-in other words, that they should specialize-Smith's theory shows that there were already particular occupations to be had, that some specialization already existed. This existing specialization built into Smith’s theory gives away that the model was based on the particular economic formation of a certain time, yet it was assumed to apply universally across space and time. Another problem with the model is that it presumes that one has the right to the products one makes, and that the products are completely alienable, able to be exchanged freely, which is not the case in all societies for all goods (Mauss 1954).

Marx (1867) offered a radically different theory for the development of the division of labor, one where politics were central and changes were historically situated. Marx saw differences in the division of labor as tied to the creation and maintenance of different classes. Changes in the division of labor was a method for some groups to gain power over others, therefore making the development of specialization an issue of politics. Marx saw the increasing division of labor as a historical process, with different configurations of producers, consumers, rights, and obligations in different periods. People were limited in their actions by the circumstances of their time. Changes were generated by the conflicting interests of different groups who were defined by their positions in the production process. Changes over time occurred in terms of who had the right to the goods made by producers. By incrementally divesting producers from the rights to their goods, economic dependencies and class divisions 
were created. Useful summaries of Smith’s and Marx’s views can be found in Wilk and Cligget (2007:50-54) and Morehart and De Lucia (2015:7-8), among many others.

V. Gordon Childe (1950) popularized questions related to specialized production in archaeology, largely through his idea of the "Urban Revolution.” Childe drew on Marxist ideas in that he saw differences between people's production activities as playing a major role in determining the general character of societies. In Childe's model the Neolithic Revolution created the possibility of surplus subsistence. The existence of small surpluses allowed part-time specialists to emerge. In the examples Childe gave, such part-time specialists undertook specialized activities to enhance their own prestige and quality of life (ibid.:8). Childe did not discuss the uses or meanings of the produced goods, whether utility, luxury, prestige, or religious. Also the products of specialists were presumably available to anyone with sufficient surpluses to exchange for them. A combination of technological change and opportunities afforded by certain environmental contexts (namely irrigation and river transport) allowed some societies to develop substantial surpluses and support full-time specialists in multiple capacities. Centralized institutions developed to manage the support of all these specialists, and class divisions were seen as existing between those who produced food and those who did not, as well as between those who managed the surplus and those who were dependent on it (craft specialists). In Childe's model full-time specialization, not part-time specialization, is seen as a critical threshold for larger changes to the structure of society.

Childe's connection of specialized production to centralization and inequality was very influential in subsequent archaeological discussions of complexity, which were chiefly concerned with looking at the relationships between these factors. Childe's model notably lacked the Marxist element of social conflict as the engine of change (Smith 2009:7). Additionally, 
Childe's model was inadvertently akin to Smith's model in that benefits accrued to everyone involved. In Childe’s view the benefits included security and relief from 'irksome' intellectual tasks (ibid.:11). In the parallels to both Smith and Marx were the seeds of archaeological models for the development of specialized production to come.

Service (1962) advocated a managerial model for the development of specialized production and hierarchical social organization. In this model specialized production occurred when sedentary agriculture was practiced in areas which had differentially distributed natural resources. He argued that it is much more efficient, and therefore mutually beneficial, for each group to specialize in producing things based on their local resources. A managerial class would arise to manage the redistribution of products. In this model specialized communities mainly produced subsistence and utilitarian items such as food crops, fish, domesticated animals, and associated implements like pottery, basketry, and woven items. Everyone in the society used the specialized products including producers (the lower class in the hierarchy) and the managerial (upper) class. Eventually items and services "of no use to society at all in the economic sense" (such as "more wives, private shamans, ... weavers and potters who produce only for his household") were also supported as the productive level of the society as a whole was raised (Service 1962:139). However the utilitarian goods and services were the primary object of production in the model. This is clearly a model where efficiency and mutual benefit are key elements of the development of specialized production (like Smith), and (like Childe) the fulltime specialization of producers is critical to the model because it allows a level of exchange which transforms the nature of society. Subsequent archaeologists critiqued adaptationist approaches like Service’s for taking whole populations in functioning systems as the unit of analysis, and for not considering the agency of the people involved with the changes, changes 
that can result from conflict and negotiation within populations (Brumfiel 1992).

Other subsequent models looked at how specialization might increase due to gains from efficiency, when there were scheduling conflicts rather than problems with the natural distribution of resources. Blanton et al. (1982) argued that increased demands for agricultural goods as taxes or tribute in already centralized societies led to agricultural intensification, leaving agriculturalists less time to produce the utilitarian craft goods they needed for that work and for life: a scheduling conflict. Since specialists could produce such goods more efficiently, specialized production of utilitarian goods for the large base of agricultural workers was adopted in order to leave more of those people free to focus on meeting the high subsistence demands. Zeder $(1988,1991)$ similarly saw scheduling conflicts in the production of herd animal products as a motivation for specialization to increase, differentiating stages within individual production processes. Like Service's model, specialization in these cases was adopted due to its increasingly efficient production and the specialized products were mainly subsistence goods.

Models where specialization developed for the mutual benefit of all involved have some inherent problems. Later studies found that it was rarely if ever the case that districts in a polity were organized to focus on specialized production of one item, and moreover, that what goods that did travel to the center were often prestige goods and food that were not distributed among the whole population but just to those in direct support of the court (Earle 1977; Peebles and Kus 1977). These findings contributed to a different kind of theory on the development of complexity and specialized production.

Political models (e.g., Brumfiel and Earle 1987; Brumfiel and Fox 1994; Peregrine 1991) posit that self-interested individuals and factions intervened in the economy in order to create and/or maintain inequalities and political alliances. This could be done by way of developing a 
prestige-goods economy. Elites would support the livelihood of specialists who could produce high quality goods that other members of society would not be able to acquire. These highquality, specialist-made goods were symbols of status and power along with other rare or imported materials. Displaying such items was a way for elites to highlight the differences between themselves and commoners. At the same time these prestige goods could be exchanged (through gifting, trade, etc.) to build alliances with followers or between groups in different communities or regions. Explicitly influenced by Marx, this model sees changes to social organization and production arising out of conflict, but the model can include conflict between different horizontal groups, not just class conflict. In reaction to previous models which looked at societies as cohesive units, this model allowed for the agency of individuals, particularly aggrandizers, and brought politics to the forefront of analyses of change. This kind of prestige goods and competition model became a highly productive framework for the analysis of production (Blanton et al. 1996; Clark and Blake 1994; Diehl 2000; Earle 2002, 1997; Feinman et al. 2000; Hayden 1995; Peregrine 1991; Rowlands and Frankenstein 1998), but eventually political economy and aggrandizers became overemphasized as the locus for broad social and economic changes (Pauketat 2007).

Explanations of the development of specialized production due to gains from efficiency or the actions of aspiring elites do not exhaust the possible range of reasons for why specialization might develop. When the models are organized according to whom the specialist goods are for, and the purpose of the goods (Table 1.1), it becomes clear that the symbolic uses of goods by commoners have not often been considered as an explanation for specialization. Although the categories of symbolic vs. utilitarian and elite vs. non-elite are not so clear-cut in reality, the table shows that many formulations of specialized production posit either production 
of utilitarian goods for the many, or symbolically meaningful goods for the few elite (Table 1.1; Brumfiel and Fox 1994; Costin 1991:11-12; Schortman and Urban 2004:196). A notable exception is Wattenmaker (1998) who argued that non-elites in the developing urban contexts of northern Mesopotamia adopted the use of specialized goods for conveying social messages, such as group identity. However her explanation is geared for urban contexts and does not cover the entire range of symbolic reasons non-elites might produce and use specialized goods.

Besides political economy and adaptationist/efficiency/managerial approaches, one of the most potent alternate perspectives on specialization looks at the role of ritual and religion in production. Wells (2006:288) proposed the idea of a "ritual economy" as the process of how world view and belief are materialized. The materialization approach crosscuts different sociopolitical distinctions, making room for other activities besides political process to be significant for large-scale changes. Most important for this dissertation project is a model offered by Spielmann (2002) who argues that the ritual activities and symbolically laden social transactions that involve many or all members of society can be a significant source of economic intensification. Spielmann's model is discussed in more detail below (Ch. 1.5). A review of work on the development of specialized production in Egypt will highlight why Egypt is an apt case study for the ritual production model.

\subsection{Theories for the Development of Specialized Production in Egypt}

Theories for the development of specialized production in Egypt broadly mirror the theoretical trends discussed above involving explanations focused on efficiency, management or the pursuit of prestige. All the models focus on the specialized production of prestige goods, though in different circumstances. However none of these models account for the development 
of specialized production for non-prestige goods. This is probably because most of the discussions of specialization in Egypt have been couched in studies of state formation, and so have focused on political economy. Note that not all authors in this section use explicit definitions of what constitutes specialized production, nor the same definition of specialized production as was described for this study.

Hassan (1988) offered a risk-management adaptationist model for state formation, and discussed specialization as part of that process. In this model specialized production developed out of necessity to provide prestige goods. In this model, sedentism and the adoption of agriculture led to a need to mitigate the risk of local environmental fluctuations. Therefore an elite political stratum arose to act as managers, organizing intra- and inter-community pooling and exchange of staple agricultural resources (subsistence goods). The larger the area controlled, the more efficiently risks might be managed. However, for leaders to manage larger areas effectively, they had to gain support from the other leaders already present in other places. This was done by display of their power and status through the use of prestige items, creating a demand for luxury and exotic goods, and stimulating trade and specialized craft production to make those goods. Thus Hassan’s model involved elements of Service’s (1962) managerial model, but where risk due to differences in micro-environments was managed rather than differences in the distribution of resources. Unlike Service's model, prestige goods for the elite were the objects of specialized production, not utilitarian and subsistence goods. However prestige goods production was still seen as linked to a process of providing adaptive benefits for all involved, and as such still assumes that everyone works together for mutual benefit and does not account for the possibilities of conflict, competition or differing goals.

A model of state formation offered by Kemp (2006) bears similarity to conflict-based 
political economy models, and includes considerations for specialization. In Kemp’s model, it is assumed that territoriality and an expansionary dynamic were inherent in settled agricultural societies, and that that there were always a few aggrandizers in any population. The combination of these factors led to competition for land and resources. Competition occurred between individuals, lineages, and eventually polities through exchanges and conflict. The results of the competitions were greatly influenced by random variables such as the location of resources or the presence of charismatic leaders. The 'winners' became elites and leaders, and their success was made evident and reinforced through display of prestige items, which fostered the development of specialized production for prestige goods. This model bears some features similar to the political economy models discussed above, including self-interested aggrandizers and the use of prestige goods by elites to re-enforce their status. Besides the problems of assuming that territoriality and expansion are universal, the role and motivation of the producers has not been highlighted because it was not a focus of the model. Despite the attempt to acknowledge potential sources for individual agency and conflict, the model raises the question of why producers would go along with the changes to the economy and become specialized producers.

In a discussion of state formation, Köhler (2010) offered another model for the development of specialized production. In this view specialized production is thought to have developed in regional centers where, through population growth or aggregation, there was sufficient demand to support full-time specialists. In areas with higher population densities, producers had the opportunity to expand their household industries and establish full-time industries which supplied local markets (ibid.:39). The demand was by emerging elites for prestige goods to display their status. This model draws on elements of a commercial model, 
where increases in specialization are part of spontaneous economic growth, assisted by gains in efficiency from advances in technology, and with little political involvement (like that of Adam Smith, see section 1.2). Köhler's model bears similarity to a commercial model in highlighting population growth and demand as important factors, and assuming that the economic change or growth is spontaneous, needing only opportunity. However in this view elites eventually became interested in sponsoring and controlling the economy and production of prestige goods as well as long-distance trade as a way to maintain their status, so the model also involves elements of the political models.

The above models are primarily concerned with state formation. In contrast, Takamiya (2004), looked specifically at the question of the development of specialized production. She argued for a political model for the development of specialized production based on a review of the evidence in the Nile Valley focusing on three types of goods: stone, beer, and ceramics. While items from each of these classes of goods could be considered specialized, she found that the ones which could be considered luxury or prestige goods were produced by full-time specialists, while more utilitarian items were produced by part-time specialists. Comparing the data to models outlined by Brumfiel and Earle (1987), Takamiya argued that this scenario fit a political model of attached production at the behest of emerging elites for personal advancement, rather than a commercial model of specialized production (see above) or an adaptationist model where political elites intervene in the economy for economic management that is beneficial to all (like that of Service or Hassan).

Dissatisfied with the importance of agriculture in models of the development of complexity, Wengrow $(2001,2006)$ proposed that the expanding influence of emerging elites and political leaders came not from control over economic resources, but from control over 
meanings in the symbolic realm, exercised through new forms of ritual interaction with the dead. Here he highlights that in Egypt, pastoralism and mobility were adopted before agriculture, arguing that the advent of extensive burial grounds by mobile groups was the factor that connected people to places, not their agricultural fields. Critically, this focus on burial grounds also allowed emergent elites to develop and disseminate new forms of ritual practice and sumptuary codes. The grave goods involved in these new practices operated along the lines of Rowlands and Frankenstein's (1998:337) notion of a prestige-goods system. In Wengrow’s (2006:75-76) view, items obtained through long-distance trade as well as items made locally through sophisticated manufacturing processes lent political advantage to those who could obtain and use such items as funerary gifts. Therefore competing emergent elites sought to gain control over trade and fostered specialized production. Although this model places more emphasis on meaning and symbolism than previous models, it can still be seen as a model where the locus of change is in the activities of ambitious individuals, as with the political models discussed above, and prestige is the goal rather than ritual and religious participation.

These models of specialized production in Egypt harbor the same issues as the models discussed previously for examples outside of Egypt. All of the models of specialized production in Egypt focus on the production of prestige goods for the elite, thus implicitly supporting a dichotomy between symbolically meaningful goods for the elite (to display status creating and maintaining inequalities) and utilitarian goods for others, and do not consider the possibility of symbolically meaningful goods for commoners. Part of the reason for the over-emphasis of prestige goods may be because of the historical focus on cemeteries, where wealthy burials stand out. 
Additionally, these models do not account for all of the evidence for specialized production. As discussed by Takamiya (2004), there is evidence for the part-time specialized production of non-prestige goods, yet all the models put the explanatory emphasis on the prestige or luxury goods. The production of non-prestige goods does deserve theorizing, particularly because such items were used by a large portion of the population. If we are to understand specialization we must take into account non-prestige goods because they can be a significant factor in the economy, and as will be shown throughout this study, there is considerable evidence for specialized production of non-prestige goods that these models have not addressed.

\subsection{A Brief Review of Evidence for Specialized Production in Egypt}

A brief review of the evidence for specialized production shows in more detail which goods have so far been identified as specialized. The main classes of goods discussed are flaked stone tools, ground stone, ceramics, and beer.

\section{Flaked stone}

Stone tools constitute some of the best evidence for specialized production. Kelterborn (1984) analyzed Predynastic ripple-flaked knives (Figure 7.1) to understand how they were produced. He analyzed 20 ripple-flaked knives, and through replication experiments created at least 50 knives. Kelterborn concluded that ripple-flaked knives were highly standardized in terms of production technique, showed an extremely high skill-level, took a substantial amount of time to produce ( 23 hrs per tool), and thus must have been produced by specialists. Furthermore, 
ripple-flaked knives are a relatively rare class of stone tools, with less than 100 known, ${ }^{1}$ and they are sometimes found with elaborately decorated ivory knife handles. The rarity of these items and the motifs on the knife handles indicate that they could be considered prestige goods (Hendrickx 2011d:94; Midant-Reynes 1987; Takamiya 2004:1030). Ripple-flaked knives date mainly to the NIId period. ${ }^{2}$ Analyses of specialization based on skill are discussed in Ch. 5.1, and Ripple-flaked knives are discussed in more detail in Ch. 6.1.

Another line of evidence for the specialized production of stone tools is the find of a bifacial tool workshop. Large quantities of debitage, mainly from the production of bifacial tools, were found in association with a ritual structure at Hierakonpolis HK29A (Friedman 2009b; Holmes 1992). There was also evidence for the production of groundstone vessels and beads including the tools used to work these items and fragments of the items themselves. The main phase of this activity was during the NIID2-NIIIA. Holmes (1992a:43) argued that the stone-tool production was full-time, attached specialization, although she did not use the same terminology. She based this assessment on the "industrial scale" (quantity) of the lithic debris relative to other areas of Hierakonpolis, the "carefully chosen" raw material types, and the systematic reduction. More discussion of this workshop is given in Ch. 6.1.

Pre-dating the evidence for the specialized production of some bifacial tools is evidence for the specialized production of blades. Ginter et al. (1996) inferred that specialized blade production was evident at site MA21/83Armant. There the oldest and middle phases date to the

\footnotetext{
${ }^{1}$ Midant-Reynes (1987) listed 49 RFKs from tombs, based on published excavations. To that can be added a few discovered in excavations since then, plus ones purchased by museums from unknown sites. However, there is possible overlap between ripple-flaked knives in museums with unknown proveniences, and the ones known from excavation reports, since the provenience trail can sometimes be lost over time. Therefore it is difficult to say exactly how many have been found. At least 57 distinct ripple-flaked knives were identified during this study in museum collections and publications.
} 
NI period (Ginter and Kozlowski 1994). During those phases, cores (a byproduct of production) were found throughout the site. In the next phase, which dates slightly later, the frequencies of cores in the settlement drop and blade workshops were identified near the local flint outcrops (ibid.:72-73, Ginter et al. 1996:178). Additionally the frequency of wholly cortical flakes (which are often taken as an indicator of primary in situ production) also drops, and the blades themselves were longer and thicker with more frequent platform preparation than in the previous phases. It is likely that only a portion of the population worked out at the quarries since there is only limited evidence for activities in the desert compared to the evidence for activities along the cultivation. Thus the number of producers relative to the number of consumers was quite different, fitting the definition of specialized production. This is supported by the changes in blade production technology evidenced by the changes in blade attributes, which could indicate an increased level of expertise necessary for production. Since the blades were found throughout the settlement they do not appear to be used by only a subset of society and so cannot really be characterized as prestige goods.

\section{Ground stone}

Hendrickx (2011d) argued that the production of ground stone vessels should be considered specialized, because their quality indicates sophisticated workmanship. However, as will be discussed in Ch. 5.1, quality and skill are not considered here sufficient evidence for specialized production. Specialized production is rather a differential relationship between who the producers are and who the consumers are (see Ch. 1.1). However, other evidence supports

\footnotetext{
${ }^{2}$ See Chapter 3.1 for details on the chronology of the Naqada period.
} 
the interpretation that groundstone vessels were made by specialists. The distribution of the vessels and the raw materials indicate that there may have been only one production location for basalt vessels, with a much larger range of distribution. The basalt for these vessels comes from a source near Maadi in Lower Egypt, and the quantity of basalt vessels is substantially higher at Maadi than any other site, which Mallory-Greenough (2002:88) takes as an indication of production near or in Maadi. Furthermore the basalt vessel forms mimic the forms of LowerEgyptian pottery providing additional support for the finding that they were made in lower Egypt (ibid.). The stone vessels are found throughout Egypt, from Buto in the North to Hierakonpolis in the south, during the Predynastic period. Taken together, these lines of evidence indicate that groundstone vessels were produced by specialists.

Basalt ground stone vessels can be considered prestige goods since there are only a limited number of vessels known, 200-250 dating to the NI-NII period and coming from 77 known graves along with provenienced sources (Mallory-Greenough 2002: Table 4, 86). Furthermore they are found in wealthier graves. Since ground stone vessels are known from the NI, they are certainly the earliest example of a prestige good considered here.

\section{Ceramics}

There is also evidence for specialized production of ceramics, some of which could be considered prestige goods, and some of which would not. Hendrickx (1996a:44-47, 2011d:95) discussed marl clay pottery noting that there are fewer types than with other kinds of clay vessels, and a more difficult technical process for production, which together suggest specialization. A large number of these vessels were produced from the middle of the NII onward (Hendrickx 2011d:95, 2006b:79; Needler 1984:202), yet production sites have not been 
identified for Decorated ware pottery, which may be an indication that they were not numerous. Hendrickx (2011d) makes a distinction between the Decorated Marl Ware (a specific kind of pottery, not just any pottery with decoration) as 'objects of great value', and the non-decorated marl wares for daily use. The uniformity of style in the paintings on Decorated Ware vessels is considered an added indication of specialized production (Aksamit 1992; Needler 1984:2002; Takamiya 2004:1031). Like Hendrickx, many scholars consider the Decorated Marl Ware vessels prestige or luxury goods (Needler 1984:2002; Takamiya 2004:1031,1033). Early types of decoration imitated stone vessels, which as discussed above may also have been prestige goods. Later the decoration involved elaborate motifs including humans, animals, boats, and architecture. Notably the Decorated Marl Wares are mainly found in cemeteries. While much attention is given to the Decorated Marl Wares, it is important to also remember the other kinds of marl wares Hendrickx mentioned: those for daily use which occur in large quantities, and were also likely produced by specialists, but are not considered prestige or luxury goods.

A number of pottery kilns for fine untempered Black-topped and Polished-red Wares were located in the wadi behind Hierakonpolis and date to the NIc-IIa (Friedman 1994:635-648, 883-884; Geller 1984; Hoffman 1982). Friedman (1994:646-648, 2000a) argued that the fine, untempered pottery was made by specialists based on the uniformity in form and technique for these wares across the country, the quantities produced, and the remote location of the production sites at both Hierakonpolis and at Armant. Furthermore, she found that although these pottery types were similar in all sites in the region, there were minor regional differences in shape and slip that indicated that they were not all produced at one centrally organized site. Friedman did not deal with the question of whether the specialization was full or part-time production. Takamiya (2004:1031) argued that they were produced by part-time specialists due 
to the early date. While the Hierakonpolis workshops were located very near the elite cemetery HK6, the ceramic forms made there were found in both cemetery and settlement contexts (Friedman 1994:646). Furthermore these kinds of pots are found commonly in all settlements of this period, so they should not be considered prestige goods.

A pottery kiln for Coarse Ware ceramics dating to NI-NIIa was found associated with a house at Hierakonpolis HK29 (Friedman 1994:653; Hoffman 1980b). Production was likely parttime because other economic activities, such as animal husbandry, were also evident. Similar kiln structures have been found throughout the settlements at Hierakonpolis (Hoffman 1982). Friedman (1994:662-3) proposed that production was for the local Hierakonpolis area, noting that potmarks found frequently at the kiln were also present in other areas of the settlement. This also indicates a level of production beyond household consumption. It is unlikely that these pots were distributed beyond the region, because Friedman (1994, 2000a) found regional differences in tempers, manufacturing technique, and surface treatment for Coarse Ware pottery. That regional diversity disappeared by NIIc. The ubiquity of Coarse Ware ceramics indicates that they should not be considered prestige goods.

\section{Beer}

Large beer-production facilities have been identified at Tel el-Farkha, el-Mahâsna, Abydos, Naqada, Hierakonpolis, and possibly other sites (Baba 2009a; Cichowski 2008; Geller 1992a,b; Kubiak-Martens and Langer 2008; Peet 1914; Peet and Loat 1913; Takamiya and Endo 2009). The earliest, at Hierakonpolis locality HKIIC Op B, dates to NIC-IIB (3762-3537 cal BCE) (Nekhen News 2011b), and most date to the NII or slightly later (Table 1.2).

The sizes of the facilities and amounts produced imply specialized production. The 
facilities consist of rows of large vats (diameters range from about 50 to $85 \mathrm{~cm}$ ) with various materials used to create a superstructure around the vats. Openings for feeding the fires were left at the bases, and the open space between vats in the interior of the structure indicates that they were likely all used at once. The sizes range from three (Tel el-Farkha) to 35 (Abydos) vats per structure, and most sites have multiple structures. Geller (1992a) estimates that each of the six vats at HK24A could hold about 65 liters, giving a figure of 390 liters of beer at a time for that facility. A site like Abydos, with hundreds of vats, would have produced staggering quantities of beer at a time. The facilities could have been run by a relatively small number of producers, yet the large quantities combined with the fact that ancient beer spoiled quickly (Dietler 2006), indicates that there must have been a small number of producers and large number of consumers, thus meeting the definition of specialized production.

Geller (1992a,b) argued that this beer production should be considered full-time elitesponsored specialized production because maintaining the appropriate heat in these large facilities requires skill and knowledge, the scale would have required a great deal of coordination to collect the raw materials and distribute the products, and a large investment would have been required to produce such quantities. Conversely, Takamiya (2004) argued for parttime use of the facilities because, if they were used full-time and year-round, one would expect to see vessels to contain and distribute the beer on a similar scale, yet distinct 'beer jars' were not present until the mid-First Dynasty (NIIIC2) (Hendrickx et al. 2002). So far no one has been able to determine if the facilities were used periodically or year-round. Whether the production of beer was full- or part-time, the quantities produced combined with the spoilage rate of ancient beer indicate that it must have been consumed by a large number of people at once. Therefore it 
should not be considered a prestige good, which by definition would only be consumed by a select group of people.

In summary, there is evidence for specialized production of both prestige goods and nonprestige goods. The prestige goods include ripple-flaked knives and possibly other bifacial tools, ground stone vessels, and Decorated Ware ceramics. Most of these date to the latter half of the Naqada period, and some are thought to be produced by specialists who were sponsored by elites. The non-prestige goods include lithic blades, Black-topped, Polished-red, and Coarse Ware ceramics, and beer. These all date earlier than most of the prestige goods.

The data on the prestige goods fit well with the models for the development of specialized production discussed above which almost all posit a prestige goods economy as developing at the behest of elites to create and maintain inequalities. Such models are not being questioned here as they work well for some of the data. However the review of the data shows that other goods were also produced by specialists, and those goods cannot be considered prestige goods. Therefore there must have been other factors at work besides the prestige goods economy that also influenced the organization and intensification of production in Predynastic Egypt.

\subsection{Hypothesis: Ritual Production model}

On the question of the development of specialization in Egypt, an explanation for economic intensification discussed by Spielmann (2002) can account for more of the evidence of specialized production by recognizing that some production may have been geared toward addressing the symbolic needs of the many, not just the elite. Spielmann argued that in small- 
scale societies, "one of the most salient sources of increased economic production [is] the individual and communal ritual participation and performance of members of entire populations" (ibid.:195), rather than efficiency or the actions of aspiring elites. It is important to point out that this idea includes social transactions such as those for lifecycle events (e.g., birth, marriage, death), as well as community-encompassing rituals. Spielmann termed this model a "ritual mode of production."

Spielmann drew on ethnographic data to illustrate that there are many instances of specialized production in small-scale societies. She defined small-scale societies as "several hundred to several thousand people in size and characterized by relatively uncentralized political systems" (Spielmann 2002:195). One example of specialized activity comes from the Chimbu area of Papua New Guinea where small groups of men worked for five months at a time at a timbered quarry $30 \mathrm{ft}$ deep, for large bridewealth stone axes. Twenty more shafts indicated the scale and sustained practice (Strathern1969; Vial 1940). An archaeological example comes from the Ohio Hopewell, where a relatively mobile population that normally lived in small hamlets, congregated for communal rituals at geometric earthworks and produced thousands of copper ear spools (Ruhl and Seeman 1997). Additionally, Spielmann cites Damon and Wagner (1989) on the mortuary practices in the Papua New Guinea Massim for the time, effort, and resources used for community mortuary rites. These are just a few examples to show that intensified economic production can occur for ritual activities. Although the ritual production model was originally formulated for small-scale societies, ritual activities are found in all societies, so the model could apply to larger-scale societies as well.

There are four main parts of the model that should be highlighted because they are archaeologically actionable. 1) In the ritual production model, goods produced by specialists are 
used during rituals and certain social interactions. 2) The production of these items is related to their use in those activities. 3) The specialist-produced goods cannot be considered either prestige goods or utilitarian items, but rather straddle both concepts. 4) The items produced by specialists are used by many people, not just a few. These four aspects of the model are all of course interrelated.

Concerning the first point, 1), in the ritual production model the items which are made through specialized production are what Spielmann terms "socially valued goods." Spielmann (2002:95) defined social valuables specifically as "objects that are critical for ritual performance and necessary for a variety of social transactions." This is not to say that any item that has some value is included in this category, only the ones used for ritual performance and symbolically laden social transactions such as those surrounding lifecycle events. Perhaps a better term would be "socio-ritual valuables." An example Spielmann cited which demonstrates that much of the value of such items comes from their use in ceremonial obligations and exchanges was Mary Douglas's (1958) discussion of raffia cloth among the Lele people in the (now) DRC. There the raffia cloth was valued not for its use as cloth, but for its exchange value in gifts and ceremonial payments, gaining greater value the more it was circulated.

A second part of the model, 2), is that the production of these socio-ritual valuables is related to their use in ritual activities. Not only is their production motivated by the need for the items in ritual events and transactions, but in some cases production is directly associated with community wide ritual events, such as the Hopewell example mentioned above. Production during ritual events where people aggregate offers certain advantages, solving the problem of how, "hundreds and sometimes thousands of people in small-scale societies [become] provisioned with large quantities of socially valued goods" (Spielmann 2002:197) without corvée 
labor or full-time specialists. The aggregation of people during such events may facilitate procurement of raw materials and sharing of knowledge about production methods. Additionally the inverse—-widely dispersed production—may not entail the degree of exchange and interaction necessary to make the qualities of the goods (and their associated meanings) a more standardized, society-wide phenomenon. Moreover, production associated with ritual events affords the opportunity for additional symbolic meanings to be associated with the place or time of production which add value to the goods.

Another important point in the ritual production model, 3), is that socio-ritual valuables challenge the dichotomy between prestige and utilitarian goods because, as ethnographic examples show, they have symbolic meaning and utilitarian functions. They are everyday items which have significant symbolic meaning, and as such can be used in both daily life and in critical ceremonial contexts, carrying meaning in both spheres, and existing on a continuum. They are ordinary objects which can be set apart in some way, such as source, elaboration or even quantity. An example is Melanesian axes, where "the very largest axes are unequivocally ceremonial items; their form is unsuitable for subsistence use. Ordinary axes, however, may circulate as bridewealth (Berde 1983; Strathern 1965), or men may wear them in the context of communal ceremony" (Spielmann 2002:202, citing Malinowski 1934). Archaeologists have argued for many years that the distinction between luxury goods and utilitarian goods is problematic. For instance, Wattenmaker (1998) used data from the mid-3rd millennium BCE site of Korban Hoyuk in Turkey to show that even though some goods were 'utilitarian' and used in non-elite households they were also valued for their symbolic significance and social messages. Vaughn (2004) discussed a case in an Early Nasca village where seeming luxury goods--highly valued polychrome pottery vessels that had restricted production and were a primary means of 
presenting Nasca ideology--circulated to the whole community including both high- and lowstatus households.

A final aspect of the ritual production model, 4), is that many or most members of a community can be involved in the use of socio-ritual valuables, since the use of such items includes lifecycle events such as birth, marriage, and death as well as larger community scale ritual activities. The use of such items is not limited to a certain social group such as emerging elites. Rather, the demand for socio-ritual valuables is widespread and sustained, enough to affect the approach to production.

Spielmann's model can be thought of as an explanation for the development of specialization, where specialist-produced goods are made to meet the symbolic needs of the many. This is substantially different from most existing theories of specialization where symbolic needs (such as display and prestige) are associated with elites, and subsistence needs are associated with commoners. Table 1.1 demonstrates how Spielmann's model significantly differs from others.

In the ritual production model, the ritual cycle motivates and coordinates the production and use of socio-ritual valuables. Their use by many members of society, and the recurring nature of ritual activities, creates a sustained demand requiring economic intensification. The production of such items by specialists allows a sufficient quantity of items with a sufficiently comparable form (and therefore meaning) to be produced to meet the needs for these activities.

One critique if the ritual production model is that many societies produce items for ritual purposes, but it does not always result in economic change. For the case of Egypt, what makes the production of socio-ritual valuables potentially transformative to the ancient economy, are 
the circumstances in Egypt during the 4th millennium BCE. Spielmann expects the ritual production model to be particularly relevant in situations of increasing social and ritual obligations, and the context of the 4th millennium BCE in the Nile Valley was likely one such circumstance. Beginning around 5300 cal BCE the aridity of the deserts began increasing substantially (Bubenzer and Riemer2007; Kuper and Kröpelin 2006; Nicoll 2001). People responded by expanding their mobile ranges and eventually moving into the Nile Valley. By the early 4th millennium BCE there were settled communities throughout the Nile Valley (Figure 1.2). This influx of people into the Nile Valley along with the adoption of settled agriculture set the stage for new forms of interaction. Among them were certainly trade, warfare, and political maneuvering. Increased ritual interactions and activities are another likely avenue of relationships between groups and individuals.

Furthermore an increase in ritual activity during the Predynastic is not surprising given that Ancient Egypt has such an extremely rich record of ritual activity. Extensive ritual activity is documented in the written and pictographic record of the Pharaonic period, but that ritual activity extends back into the Predynastic. Many Pharaonic-period rituals have their roots in the Predynastic, as many scholars have taken pains to trace (e.g., Baines and Lacovara 2002; Hendrickx et al. 2009; Morris 2016; Patch and Eaton-Krauss 2011). It is exactly this importance of ritual activities to ancient Egyptians that led Wengrow (2006) to argue that a shift in the relations with the dead was significant in Egypt's development, with finite funeral ceremonies replaced by elaborate ones that required more and more symbolically significant items in the grave, and provisioning of the dead continuously after burial. If there is a place in the world where ritual activities might have contributed to change, it would be Egypt. Since the development of specialized production for many goods is not accounted for in current theories, it 
is necessary to take a closer look at the patterns of production to understand whether the ritual

production model does apply in Egypt. Expectations that can be used to evaluate the applicability of the ritual production model for interpreting the data from the Nile Valley during the 4th millennium BCE are given in Ch. 2.2.

\subsection{Ritual and Religion in Archaeology}

Ritual is rarely considered in studies of lithic artifacts. This is probably because so much research on lithic artifacts is based in the paleolithic past where the point at which humans or their ancestors developed symbolic thought is instead research question, worthy of rigorous interrogation. However, this dissertation focuses on a time period at the brink between prehistory and history, in a place where ritual and religious activity are well documented through text, representation, and archeology. Ritual and religion were central aspects of Ancient Egyptians’ lives, and likely had a great deal of effect on what people did and why.

The study of ritual and religion in archeology is an active field of research. Perspectives include: overviews or collected works aiming to focus the theme(s) (Insoll 2004, 2011; Kyriakidis 2007, Rowan 2012; Whitley and Hays-Gilpin 2008), studies of religious change (Graham et al. 2013; Shaw 2013a,b); ritual and religion as a source of power or social strategies (Adams 2004; Grau Mira 2016; Janusek 2006; Kovacevich 2007), material expression of religion (Droogan 2013; Fogelin and Schiffer 2015; Wells 2006), the process of ritualization (Bradley 2005; Humphry and Laidlaw 2007) and of course the intersection of ritual and production (Austin 2015; Hruby 2007; Inomata 2001; Spielmann 2002; Wright and Loveland 2015). Many of these works wrestle with finding archaeologically actionable definitions of religion and ritual. Discussions of what constitutes 'religion' can get quite complicated, 
historically particular, linguistic, and are beyond the scope of this work. Suffice it to say that many archaeologists studying religion recognize that the idea of religion is a culturally situated, historically bounded term with specific limits that can be separated from other areas of life and opposed to secular thought and activities (Graham et al. 2013; Insoll 2004:6; Shaw 2013a). Therefore 'religion' is often thought to be an insufficient term for past societies that often did not draw the same boundaries as are implied by the modern western notion of 'religion'. Archaeologists often prefer terms such as 'worldview' (Graham et al. 2013) or 'cosmology' (Plog and Heitman 2010) to religion, since these terms better convey the idea that life was not necessarily divided into secular and religious spheres but that ideas of spirituality or the divine, personhood, how people should interact and in what circumstances, relationships between the living and the dead, decision making, identity, and understandings of cause and effect, could all be intertwined.

Ritual activities are one of the primary ways the above concepts can be made manifest within a worldview. The idea of ritual itself requires definition, since it encompasses a number of features. Based on discussions by a number of authors, especially Kyriakidis (2007) the following elements are here used to characterize 'ritual': Rituals are repeated, 'set' actions that are transformative and that derive from the worldview of a specific group of people.

Rituals are first and foremost actions, activities, or practices, and as such are quite amenable to archaeological study. These actions can include language and communication. Religion is often considered the realm of belief and thought, and rituals are one aspect of the accompanying action stemming from these thoughts and beliefs. Since rituals are what people actually do, rather than what they think, rituals are quite suitable for archaeological study. While some scholars problematize the association of action with ritual and thought with religion (e.g. 
Fogelin 2007; Rowan 2012), it is simply a practical analysis.

Rituals are repeated, though the periodicity and the people involved can vary. For instance a person may only get baptized once in their life, but the ritual is repeated with many different people; a harvest festival may be repeated once every year; or an animal mummy may be offered at no prescribed time but as often as deemed necessary.

Furthermore, at least some of the actions which make up rituals are 'set' (Kyriakidis 2007) or 'invariant' (Bell 1997), or give the impression of being so (e.g. through formal or traditional action, language, and/or material objects). Despite the nature of rituals as actions which are perceived as set, there is room for change and manipulation. Rituals can be 'set' to different degrees, or rather there are elements of varying importance in any ritual activities (Humphry and Laidlaw 2007). The perception of ritual as traditional, precedent-based action is part of what gives the ritual authority (Bell 2007, 1997; Humphrey and Laidlaw 2007). It is this interplay or tension between the perception of rituals as set activities and their variable implementation, which allows people to deploy them strategically (Bell 1992; 2007).

The transformative aspect of rituals is what separates them from activities which are simply done over and over again in the same way. Rituals do something. They send people into the afterlife, bestow names, communicate with gods, promote harvests, demonstrate devotion, carry prayers, create alternate states of consciousness etc. In doing something ritual activities are tied with notions of cause and effect. Kyriakidis (2007) discusses the importance of perspective that comes with ideas of cause and effect.

The idea of perspective in cause and effect brings up a central consideration for rituals, which is that they stem from the worldview. The rituals take place in the framework of a worldview, they are actions that stem from and combine with thought or belief (Insoll 2004:10- 
12). By considering ritual as part of worldview or cosmology rather than religion, there is less of a need to separate between secular and religious ritual, a separation which seems inappropriate for Ancient Egypt. As mentioned above, worldview relates notions of cause and effect, the divine or sacred, attitudes toward when and how people should act, and many other facets of life. Thus there is clearly room for domestic as well as larger-scale ritual activities.

All of the above aspects combine to make rituals mark themselves as somehow set apart, special, giving them meaning and import. Additionally, besides the overt proximate intentions of a what a ritual does, rituals also embody aspects of solidarity and differentiation. The solidarity building aspect of ritual activities has been theorized since Durkheim (1915), but acknowledgment of the differing positionality of people involved in (and excluded from) rituals has also drawn attention to their divisive aspects (e.g., Hastorf 2007). The many tensions and oppositions inherent in ritual activities — thought and action, stasis and change, special and quotidian, solidarity and differentiation—are what make them so important to understanding past human lifeways and change.

A final clarification needs to be mentioned for this particular study of ritual production. A distinction should be made between production as performance (the process of production being ritualized), and production activities that are motivated by or coordinated by ritual activities. This study is not concerned with whether or not the actual sequence of steps to make objects was ritualized (e.g.. Carter 2007; Hruby 2007; Nikolaidou 2007), but rather is concerned with looking at whether things were made for their symbolic meanings (related to worldview) and uses in ritual activities. 


\subsection{Implications}

This study of production in the Egyptian Nile Valley during the 4th millennium BCE has important implications for understanding Egypt's past, including the different people or groups involved in large-scale changes, and the significance of ritual activities for such changes. One of the primary implications of this research is the recognition that all people, not just elite, can have uses for symbolically meaningful goods. In so doing this work challenges the dichotomy between prestige goods (symbolic items associated with status), and utilitarian goods (items without significant symbolic meaning beyond their functional uses).

A related implication concerns who is involved in large-scale changes. The ritual production model underlines the involvement of other people besides ambitious aggrandizers in large-scale changes such as the development of specialized production. Similarly, concerning arenas for generating change, the ritual production theory emphasizes that ritual activities can be a significant source of economic change.

Another important aspect of the model being tested is that it does not assume that people only produce items “in excess of biological need” (Morehart and De Lucia 2015) when required to by adaptive necessity (risk aversion) as with managerial models, or when coerced or persuaded into it, as with political models. Nor does this research swing to the opposite end of the spectrum and assume that people will always naturally produce as much as they possibly can whenever not limited by outside factors (classical economic and commercial models). Rather the ritual production model makes room for motivated production activity undertaken for reasons internal to the individuals and groups involved in those activities.

Furthermore, this theory grants that ritual activities have significant cultural content, and are not just venues for the production of "false consciousness that masks reality and operates in 
the interests of the elite" (Inomata 2001:324). The widespread participation of many people implies that the ritual activities are not infinitely malleable. Despite Egypt's rich and historically deep ritual record, in considerations of state formation or complexity, the ritual realm is often treated as a straightforward venue for legitimization, and little else.

In summary, the central question addressed in this research is to understand how specialized production developed during the Naqada period in Egypt. This question requires examination of craft production activities of a wide range of the population. The hypothesis presented here is that some specialized production developed to make socially valued goods that were used for rituals and social transactions. The following chapter on methods outlines some expectations for how the ritual production model can be evaluated archaeologically. Patterns in raw materials, production locations, and the contexts of specialist-produced goods can be used to evaluate whether or not the ritual production model should be applied in Egypt. 


\section{Chapter 2: Methods}

\subsection{Methods Introduction}

To test whether the hypothesis that the ritual mode of production accounts for the development of some specialized production in Naqada period Egypt, I developed a series of expectations based on the preceding discussion of the ritual production model (Ch. 1.5). These expectations concern patterning in raw materials, production locations, and find contexts. This chapter begins with a discussion of why stone tools are an appropriate artifact class for this

study, and sets up the first problem for this research: identifying what was produced by specialists. Section 2.2 lays out the expectations for the ritual production model, and addresses how these patterns can be assessed archaeologically. A brief introduction of the sites involved in this study demonstrates that there are known Predynastic Egyptian contexts appropriate for evaluating the model (which are discussed more fully in Ch3). This is followed by an overview of the methods that were used for analyzing lithic artifacts, and the chapter concludes with a discussion of the main statistical methods involved in comparing the data.

\section{Why stone tools?}

Chipped stone artifacts are an ideal class of artifacts for addressing the hypothesis of ritual production as a mode of increasing specialized production in Predynastic Egypt. Some of the earliest evidence for specialized production in Egypt concerns stone tools (see Ch. 1.4). Furthermore, stone tool production reaches a particularly excellent level of craftsmanship in the later Naqada period, as was the case for ripple-flaked knives. Chipped stone artifacts were found 
frequently in both settlements and cemeteries, so there is ample data on this class of artifacts. Most importantly, stone survives extremely well in the archaeological record, including stone tools themselves, and the debitage (production remains) which provide evidence of the process and location of production. All of these factors are critical for understanding the development and organization of production.

\section{What was specialized?}

The expectations for evaluating whether the ritual production model may apply to the development of specialization during the Naqada period revolve around an assessment of the patterns of raw material use, production locations, and find contexts of items made by specialists. Therefore the first step is to assess which items were made by specialists. Although certain chipped stone artifacts have uncontroversially been identified as objects of specialized production, such as the ripple-flaked knives, others are less well understood. Therefore Chapters 5 and 6 are devoted to understanding which flaked stone artifacts were produced by specialists. The methodology for this analysis is discussed at the outset of Ch. 5. In short, the method employed was essentially an analysis of the spatial distribution of production remains relative to the spatial distribution of tools, at both intra-site and regional levels. This method was used to assess the production patterns of heat-treated artifacts, six types of blades, and eight types of bifacial tools. The results indicated that there was a surprising array of different ways production of stone tools was organized, many of which could be considered specialized. Once certain tools could be identified as resulting from specialized production, their patterning could be compared to the expectations for the ritual production model. 


\subsection{Expectations for the Ritual Production Model}

Table 2.1 shows the expectations for archaeological patterns that should be observable if specialized production developed according to the ritual production model as outlined in Ch. 1.5. Table 2.2 compares these expectations to other general models of specialized production and their associated archaeological patterns that would contradict the ritual production model.

\section{Raw materials}

If the ritual mode of production is applicable then the raw materials of specially produced goods could be significant to their symbolic meaning. For example, the more distant, relatively inaccessible and symbolically charged sites may be chosen because the source sets them apart from other goods (Spielmann 2002:198-99). Procurement of special raw materials takes more investment which can be an indication of the added value. However the raw material needs to be available in sufficient amounts to meet the widespread demand for the goods. Therefore extremely long-distance raw materials, which could only be obtained in small amounts, are not expected to have been chosen for socio-ritual valuables. Another possibility is that if the raw material was procured from a site with important associations, such as a holy place or other symbolically charged site, then those associations could be indexed by the raw material. If the raw material is significant to the meaning of the good, whether for its symbolic associations with place or for other reasons, then there should be an association between the type of raw material and the type of specialist produced good. If there is an association between raw material and tool type, other non-symbolic considerations for raw material choice should be ruled out, such as functional considerations, or local availability relative to production locations.

The most common material for flaked stone tools in Egypt by far is chert (see Ch. 4.1 for 
a discussion on the terminology chert vs. Flint). However chert is variable, and not evenly distributed across Egypt (see Ch. 4.1). Chert varieties are notoriously difficult to distinguish with any method, whether macroscopic, microscopic, or non-visual, like x-ray diffraction. Nonetheless the variability in Egyptian chert is readily apparent to lithic analysts and laymen alike (Figure 4.1). Most researchers divide their chert assemblages into groups based on macroscopic characteristics (e.g., Ginter et al. 1996; Kabacinski 2012; Kindermann 2010; Midant-Reynes and Prost 2002; Pawlik 2006; Riemer 2011; Rizkana and Seeher 1988). Due to issues with the non-macroscopic methods discussed in Chapter 4 such as reliability, time, and money, macroscopic methods were used to distinguish chert varieties.

Luedtke (1992) described five macroscopic variables for cherts: color, translucency, texture, luster, and inclusions. Additionally, cortex is affected by secondary movement from its natural context (gravity, wind, water, patina development), and can point to whether chert came from a primary or secondary deposit. These characteristics were recorded for a sample of 600 artifacts from el-Mahâsna and Nag el-Qarmila in January and March-April, 2012. A detailed description of how each characteristic was assessed is given in the Appendix. Inclusions such as fossils and banding were documented with a Proscope high resolution digital microscope to facilitate comparison between sites and publication of the raw material groupings.

Groupings of chert varieties were created by laying out a sample of the artifacts and grouping them based on observations of the macroscopic variables as defined by Luedtke, and taking into account previous researchers' definitions (Figure 4.2). A type collection consisting of 1-3 examples of each chert grouping was set aside to facilitate comparison and assignment of artifacts to specific chert groups during analysis. Cortex type and inclusions were the most 
reliable and replicable variables for differentiating cherts. Color, translucency, texture, and luster were all useful, but grade into each other.

Determination of local vs. non-local materials was done by examining the proportions of the chert types at each site, with the expectation that the most common material was local for each area, unless there were indications otherwise. Additionally, I conducted a survey of chert deposits in the high desert area immediately behind Abydos and in the el-Mahâsna area to observe the local materials there (Ch. 4), and considered some other possible sources for certain varieties mentioned in archaeological literature.

In Ch. 7.1 the frequency of each raw material variety was calculated for a sample of artifacts from each specialist produced tool class. If there was an association between tool type and raw material then one raw material variety was expected to be dominant. Some tool classes clearly were made from one or a few raw material varieties only, whereas others were made from many different chert varieties. Additionally, the distribution of raw material types by site relative to known sources of that material was also analyzed, to check whether any of the variability in raw material type could be accounted for by the use of local raw materials.

\section{Production locations}

If the ritual model of production applies to specialization in Egypt, then remains from specialized production could be associated with ritual contexts. Production of items may have been organized around ritual events for a few reasons. Production in close association with ritual activities could again add extra meaning or value to the goods. Moreover, if items were produced during events which brought many people together, there would be certain advantages such as the collection or sharing of raw materials and production knowledge. Additionally such 
production would be part-time rather than full-time due to the periodic nature of ritual activities. Part-time production not associated with ritual contexts does not rule out the possibility of the ritual production model, since socio-ritual valuables may need to be prepared before ritual events, but finds of production remains from specialist produced tools in ritual contexts would certainly support the model.

The locations of production for each tool type can be determined by looking at the specific kinds of debitage associated with each tool type, and the contexts in which it is found. Chapters 5 and 6 give descriptions of each tool type, including the kinds of debitage that might be associated with each. Determining whether tool production was part time or full time is more tricky. Estimates of the amount of time required to produce an artifact, the quantities of finished tools known or the quantities of production remains in a given location, periodicity evident in the production contexts, or even the difficulty of the production technology all factored into an evaluation of whether an artifact was made full-time or part-time.

\section{Context}

The context in which specialist-produced goods were found can address a number of aspects of the ritual production model. First, since socio-ritual valuables are necessary to ritual activities, then if the ritual production model applies specialist produced goods should be found in at least some ritual contexts such as temples, ritual activity areas, offering deposits, or burials. Second, since socio-ritual valuables bridge the divide between utilitarian and symbolic goods, being used in daily life and on ceremonial occasions, they should also be found in other contexts which do not have an overtly ritual character, such as domestic contexts, middens, production areas, or storage areas. Luckily examples of all of the contexts mentioned above are now known 
for Predynastic Egypt due to increased focus on settlement sites. The specific sites and contexts drawn on for this study are briefly mentioned below in section 2.3, and discussed fully in Ch. 3 .

Third, since socio-ritual valuables would have been used by many people throughout a society, not just by a select group, then they should be (socially) widespread. A wide social distribution could be indicated in a number of ways. Finds of specialist-produced goods in settlements of all scales, not just paramount or regional centers, would indicate a wide distribution, since settlements of different scales may imply functional or social differences that could affect access to artifacts. In Chapter 3 the sites involved in this study are classified into high level, mid-level, and low level sites according to population size (as indicated by area of the settlement and number of graves in the associated cemeteries), and the diversity of functions evident in the settlements (administration, ritual activities, intensive trade, and large-scale production activities for beer, pottery, stone tools, ground stone, copper, or grain processing).

A widespread distribution of specialist produced goods is also assessed by looking at whether or not such items were found in multiple contexts within individual settlements, not concentrated on one house or structure within a settlement. Additionally the social distribution of specialist produced tools is also assessed by looking at whether they occur in elite vs. non-elite cemeteries or graves of different wealth/status.

As is evident in Table 2.2, which compares the expectations for the ritual production model to equivalent expectations for other models of specialist production, every aspect of each model is not perfectly distinct. However, overall each model has a different set of expectations. Any one of line of evidence is not in itself irrefutable evidence for the ritual production model, but a number of them together would indicate that a ritual mode of production was a major factor 
in economic intensification during the Naqada period. If the archaeological patterns associated with specialist produced tools match up with those expected for the ritual production model, then a compelling case will exist indicating that specialized production began in Egypt to meet the symbolic needs of the many for objects critical for ritual performance and social transactions.

\subsection{Sites Involved in the Study}

The expectations for production locations and find contexts both rely on understanding the kinds of contexts in which artifacts were found, and assessing a wide variety of contexts. Historically, much of the data about Ancient and Predynastic Egypt has come from cemetery evidence. This is not only because the finds of gold, jewels, and intact artifacts are more common in cemeteries, but also because settlements are harder to locate. Predynastic settlement sites lack telltale standing architecture because the structures were usually built from wattle and daub. Fortunately, a number of relatively recent excavations have focused on locating and excavating Predynastic settlement sites, providing the opportunity to take into account production sites and living areas. These new contexts provide data on the activities of a larger range of social statuses, not just the ones that have left ample material records in their graves.

Data for this study were collected from three sources: new analysis of archaeological assemblages, published literature on Predynastic assemblages, and online museum databases. Lithic artifacts were analyzed from three recently excavated settlement sites: El-Mahâsna, Abydos, and Nag el-Qarmila (Figure 2.2). These sites are of different scales, from a small farming village to an emerging political center. Furthermore, they include domestic and ritual contexts. The sites are briefly introduced here, and detailed information about each of the sites, including excavation methods, is in Ch. 3.4. 
The main body of new material analyzed for this project comes from el-Mahâsna, which is a mid-sized settlement site located $\sim 10 \mathrm{~km}$ north of the politically important site of Abydos. El-Mahâsna was briefly excavated by John Garstang (1903), and more recently by David Anderson (2006, 2007, 2011). The architectural, ceramic, and faunal evidence from Anderson’s excavations have already been analyzed extensively (Anderson 2006; Rossel 2007). The remains date to NIC-NIID (see Ch. 3.2 for chronology details). El-Mahâsna is integral to the present study because Anderson's excavations revealed the existence of a ritual structure (Anderson 2011), in addition to domestic structures, and middens (Anderson 2006). The associated cemetery was excavated by Ayrton and Loat (1911) and published material is available for reference.

Abydos was a regional political center, as is indicated by the series of Predynastic burials culminating in the tombs of the first kings of Egypt and their associated funerary enclosures which are the earliest examples of monumental architecture in Egypt. However little is known of the associated settlement(s). Stephen Harvey recently uncovered a portion of a Predynastic settlement in the Abydos area while excavating Eighteenth Dynasty remains (Harvey 1998:163164), and a sample of the lithic materials were analyzed for this study. The site dates to the NINIIAB. Materials from Abydos provide an intra-regional comparison of raw materials, tool types, and production techniques to those found at el-Mahâsna.

Artifacts were also analyzed from Nag el-Qarmila, a small, multi-component farming village in the Aswan region. Rescue excavations have been underway since 2007 under the direction of Maria Gatto (Gatto 2014; Gatto et al. 2009a,b), and artifact analysis is ongoing. The site dates to NIC-NIIA/B, and consists of three spatial components: the settlement with identified habitation contexts, a cemetery, and a storage area on a nearby ridge. It is unusual for such a 
small settlement to be found and thoroughly excavated, and it provides an important window on differences or similarities in production activities and artifact distributions compared to larger sites. The earliest currently known depiction of a pharaoh was recently found just south of Nag el-Qarmila, indicating that the area must have been politically important at least in the later part of the Naqada period (Hendrickx et al. 2012).

The interpretations of different contexts within el-Mahâsna, Abydos, and Nag el-Qarmila were made by the excavators and authors through analysis of architectural remains, stratigraphic relationships, and ceramic and faunal artifacts (Anderson 2006, 2007, 2011; Gatto et al. 2009; Harvey, pers. comm.) (see Ch. 3.4 for more details). These interpretations of context were made independent of the lithic artifact analysis.

In addition to the data from el-Mahâsna, Abydos, and Nag el-Qarmila, this study draws extensively on published lithic artifact assemblages from the following Predynastic sites, listed from north to south: the Naqada Khattara sites (Holmes 1989), Abadiya 2 (Vermeersch et al. 2004), Armant (Ginter and Kozlowski 1994; Ginter et al. 1996), Adaïma (Midant-Reynes and Buchez 2002), and Hierakonpolis (Hikade et al. 2008; Holmes 1989; Takamiya and Endo 2011). Each of these sites has been excavated with modern collection techniques (Ch. 3.1), and has been dated through ceramic relative chronology, in some cases with absolute dating techniques as well. The contexts include habitation areas, production areas, animal pens, middens, and ritual activity areas. Most have associated cemeteries. The sites are described in detail in Ch. 3.5.

Publications are inherently limited in how many photographs, drawings, and measurements of specific tools they can include; therefore I also consulted online museum databases to gather a larger sample of the specific kinds of tools made through specialized production, particularly for looking at the raw material expectation. The total sample includes 
artifacts from 39 museums and storerooms spread over nine countries and three continents (Table 7.2). More information is given in Ch. 7.1.

This project focuses on the Nile Valley sites for a few reasons. Foremost, it is already well known that there is some regional variation within the valley itself during the Naqada period (Holmes 1989), so other sites beyond the Nile Valley in Egypt were not included, to reduce variability due to regional differences, whether stylistic or environmental. Furthermore, the Nile Valley itself seems to be the prime locus for the development of a suite of characteristics along with specialization, such as writing, social stratification, and kingship. Therefore the main goal of this project was to understand influences on the development of specialized production in the Nile Valley itself, rather than looking at issues between larger regions.

\subsection{Artifact Analysis Methods}

The artifact assemblages studied included flake, blade, and bifacial tool technologies. Much experimentation has been done by archaeologists to understand how ancient flaked stone tools were produced, and how they and their byproducts can be identified in the archaeological record (e.g., Ahler 1983; Crabtree 1972; Desrosiers 2012; Domanski et al. 2009; Tixier 1984; Whittaker 1994; Wilke and Quintero 1996). Most lithic-reduction experimenters emphasize that there are no smoking gun attributes associated with a given reduction technique. Rather they stress that among a given population of tools and debitage reduced in a particular way, certain

attributes will occur frequently. This observation highlights the utility of analyzing attributes, not just types of artifacts. Additionally, typing can mask variability, sometimes forcing continuous factors into artificial groups (Dibble 1995). However, typologies continue to be a time-efficient 
method of analysis, and when widely used or well defined, they are a good way to communicate much data between researchers.

I used a combined attribute and typology approach for this project. I analyzed each artifact individually, including both debitage and tools. Raw material type, flake type, tool type, and core type were recorded along with more specific attributes such as the amount of cortex, platform faceting, flake scar pattern, and metric measurements. Types were recorded as an attribute category for each artifact, rather than first sorting the artifacts by type, then recording additional attributes. This system allowed type categories to be re-evaluated as necessary (Ch. 5.3), and facilitated analysis of variation within type groups (Ch. 7.1).

No single reference typology exists for Predynastic or Pharaonic Egyptian stone tools, let alone debitage. Over the course of a preliminary research season in 2009 at el-Mahâsna, I developed a coding guide specific to the Predynastic Egyptian material and the questions asked here. The main references used to create the coding guide were: Andrefsky 2005, Debénath and Dibble 1994, Holmes 1989, Inizan et al. 1999, Luedtke 1992, Midant-Reynes and Prost 2002, Rosen 1997, and Tixier 1963. Additionally, a type collection for identifying heat-treated materials was created through experimental heating of chert (Ch. 4). The Appendix gives a detailed description of each characteristic examined and definitions of the choices in each category. The basic classes of observations were: context data, raw material data, production/technology data, tool data, and metric information. Since this study is concerned with identifying production locations as well as distributions of tools, all lithic artifacts (within the selected samples) were analyzed from each site in order to examine the different production technologies present, the products, and their chains of production. 


\section{Data collection procedures}

The lithic artifacts in this study were analyzed in Egypt over the course of two study seasons: January-May 2012, and January-May 2013. I analyzed the el-Mahâsna and Abydos material at the Abydos American excavation house where the materials are stored. I studied the material from Nag el-Qarmila in the official Kom Ombo storage magazine for the Aswan region. I conducted a preliminary analysis at Abydos on the el-Mahâsna materials in January 2009, however all the data presented here is from the 2012 and 2013 study seasons. Support for the 2013 season came from a fellowship through the American Research Center in Egypt. For information on how the artifacts were collected in the field, see Chapter 3.4.

Artifact analysis proceeded according to the following process. First the artifacts were washed by Egyptian workmen, using soft brushes and water, and laid out to dry. Then pieces below $1.5 \mathrm{~cm}$ were sorted out, counted, and weighed. At the same time non-cultural material was sorted out and discarded. There had to be recognizable flake scar attributes, (e.g., bulb, ripples point of initiation, erailure scar, etc.) for an item to be classified as an artifact (cultural material). ${ }^{3}$ Artifacts equal to and above $1.5 \mathrm{~cm}$ and tools smaller than $1.5 \mathrm{~cm}$ were individually labeled with the bag number and a sequential number of the artifact in the bag. The labels were written with a .4mm-tipped Sakura Identi pen, and the ink can be removed with acetone. Flakes were labeled on the ventral side, and tools were labeled on the ventral side if one was discernible, or in the least conspicuous place.

Subsequently, each labeled artifact was individually analyzed for type and attributes (see

\footnotetext{
${ }^{3}$ Shatter/angular debris is cultural material that does not have the usual recognizable flake scar attributes. Shatter/angular debris was differentiated from natural material based on the raw material types, evidence of heat treatment, and freshness of the surfaces (natural debris was much more rolled and damaged that the man-made artifacts).
} 
the Appendix). The data were entered using E4 software, ${ }^{4}$ which brings up one question at a time with associated menu choices and automatically enters the answers into an access database. This software helps to standardize the entries and reduce entry error. Metric measurements were made with metal digital calipers that had a USB attachment to directly feed the measurements into the computer, thus reducing typing error and saving time.

Although the artifacts were analyzed in multiple locations, effort was made to use comparable lighting conditions. Analysis was carried out indoors near windows with natural sunlight. Additionally small desk lamps provided angled light for highlighting certain features. A microscope was also used to examine small details. The advantage of using a microscope instead of a hand loop (10x) magnification is not only in the greater magnification, but in the ability to stabilize or rotate the artifacts as needed.

After analysis all artifacts were photographed in indirect sunlight (shade). Most photographs were taken around midday, but the time of day sometimes varied. Grey background was chosen to minimize contrast, and each photo included a color scale for standardizing the color adjustments on the photographs. The photography surface and the camera were both leveled using a bubble level to minimize distortion from the camera angle. Cores and tools were photographed individually from as many sides or angles as necessary. The dorsal and ventral sides of the debitage were photographed in groups according to type within each bag. Additionally a Proscope high-resolution digital microscope was used to photograph detailsparticularly raw material inclusions, edge retouch, and usewear. The magnification is 30x unless otherwise noted.

\footnotetext{
${ }^{4} \mathrm{~A}$ free software program designed for data entry by Dibble and McPherron, available on: www.oldstoneage.com.
} 
Representative and unusual examples of debitage, core, and tool types were chosen for illustration. Darcy Hackley and Megan Cook illustrated the selected items following conventions in Addington (1986). Additionally, Darcy Hackley made drawings of intricately flaked tools directly in digital format using photographs as the base and referencing the actual artifact while drawing.

\subsection{Statistical Analysis Methods}

A number of statistical methods were used to analyze the collected data, including Confidence intervals of proportions, Chi-square, analysis of variance (Anova), and coefficient of variation (CV). All of these statistics are relatively common in archaeological publications. Nonetheless, a discussion of each follows, including what kinds of data they apply to and how they are calculated. The discussions were drawn mainly from Thomas (1986), Diez et al. (2012), and University of Virginia Stats Lab materials. In some cases the discussion of the statistic is reiterated in the data chapters the first time a method is used, for clarity.

The statistics were calculated using the "R” software (Dalgaard 2008) "Introductory Statistics with R.” Many of the charts and graphs were also built using R software. All of the tables were made in Excel or PowerPoint. Throughout the study the "alpha” significance level used was .05, which, although an arbitrary cut-off level, is standard in archaeological research and makes the results more easily comparable to other studies.

\section{Confidence limits of a proportion}

Confidence limits of a proportion were used to answer the question of whether variability 
in proportions among assemblages is meaningful, a method of hypothesis testing (Diez et al. 2012:173-175). Confidence limits of a proportion can be used to compare nominal/categorical data rather than interval data, by calculating an error range for a proportion. For instance, if the proportions of scrapers in three tool assemblages are $5 \%, 10 \%$, and $12 \%$, are these proportions really different, or are they essentially similar? This same question could be answered with Ttests, which give the probability that two proportions are the same. However, it is not a good idea to run many T-tests, as that increases the chance of getting a significant result when there should not be one. The advantage of confidence intervals is that you can compare more than two proportions at a time, and present them in easily interpretable figures.

The confidence limits of a proportion are an error range for that proportion. Archaeological data are essentially samples, rather than entire populations. All of the material under question probably does not enter the archaeological record in the first place, and then postdepositional processes affect preservation, and finally archaeologists rarely collect the entirety of a given site. Therefore a proportion is an estimate or sample of what the real value is. Since archaeological data are samples, there is the possibility of (random) sampling error.

Given a certain sample, the confidence interval indicates the range of values for the actual population, where getting the given sample is a likely result. If the cut-off level for significance is $95 \%$ is, then if the sample was repeated 100 times and the confidence intervals calculated for each, 95 times out of 100, the actual population would be in the resulting range. There still remains $5 \%$ of the time where the actual population would not be within a given range.

If the confidence intervals of multiple samples do not overlap then it is very likely that the actual underlying populations are different. To put it in standard hypothesis-testing terms: 
The null hypothesis is that the proportions are the same. The alternative hypothesis is that the two proportions are really different. If the two confidence intervals do not overlap, then there is sufficient evidence to reject the null hypothesis that the two proportions are essentially similar.

The binomial confidence intervals are calculated based on the binomial probability distribution, using the sample size and an actual estimate to determine the probability distribution. Binomial distribution probabilities were calculated by statisticians running trial and error tests (e.g., for a sample size of 20, there is a very low probability that heads will turn up 19 times, and a very high probability that heads will turn up 10 times). Sample size inversely affects the interval size. So a large sample will result in a small range, and a small sample will result in a large range. The observed proportion affects the location of the peak of the binomial distribution bell curve, and therefore the probability associated with that proportion, given the sample size. In this study, intervals were calculated using R code written by Fraser Neiman for the ClopperPearson binomial confidence intervals. The code uses 100,000 tests based on the parameters input for sample size and observed count, and calculates cumulative probabilities. It gives a more conservative error range (in other words, a larger error range) than some other formulas for binomial confidence intervals.

The confidence intervals are very useful for this study because some of the samples sizes are large while others are smaller. Additionally, the confidence intervals are amenable to visual display that can be easily and quickly interpreted.

\section{Chi-square tests}

Chi-square tests were used to look for associations among sets of nominal data. Chisquare tests can be used for a kind of hypothesis testing where the null hypothesis is that the two 
variables are independent of each other. The alternative hypothesis is that the outcome of one variable is dependent on the second variable. A Chi-square test compares whether the observed distribution varies significantly from the null hypothesis distribution, which is the theoretical Chi-square curve for a given number of categories and the counts in each category.

A Chi-square test works by making a contingency table of counts of each possibility of a given variable, which are grouped according to a second set of variables, for instance the bulb types found for each different blade type. Then the expected count in each cell is calculated by taking the sum of the number of observed occurrences in that row, multiplied by the sum of the number of observed occurrences in that column, and dividing by the total number of occurrences in the entire contingency table. The difference between the actual observed counts and the expected counts is figured simply by subtracting the observed from the expected in each cell (they are then squared to remove any negative numbers), and divided by the expected to account for different weights (count sizes) across the groups (cells).

The validity requirements (assumptions) for a Chi-square test are that the two variables are independently measured or defined, and that the expected counts are all above one, and not more than 20\% are below five (Thomas 1986:298). If these validity requirements are met, then a probability value can then be calculated. This probability value answers the question of how likely it is that the difference between the observed and expected is due to random chance. This is the 'p' value. Sampling distributions for the Chi-square probability curve have been defined by statistics researchers who did many trials with different combinations of the numbers of variables, for instance a $2 \times 2$ contingency table or a 3 × 5 contingency table (which relates to the degrees of freedom), and different sample sizes (Thomas 1986:286). These experiments helped define the validity requirements for the Chi-square test, and help to define the probability of 
variation due to random chance. A very low p value indicates that it is very unlikely that the difference is due to random chance. If the probability is below a certain cut off then the null hypothesis can be rejected. The cut-off value for significance, or alpha value, used throughout this study is .05.

\section{Anova}

For continuous data, such as metric data, an analysis of variance statistic (Anova) can be useful for comparing the means of multiple groups. Anova is appropriate to use when comparing means across more than two groups, rather than pairing the data in all possible ways and doing multiple T-tests, because there is always a small possibility that a significant result is due to chance. When multiple tests are run the possibility of getting a significant result when one does not really exist is compounded (Diez et al. 2012:236). Anova combats compounding error by checking only whether the means across all groups are equal or not.

The validity requirements (assumptions) of an Anova test are that the observations are independent, that the data approximate a normal distribution, and that the groups have similar variability. QQ plots were used to examine whether the data approximate normal. In a QQ plot the quantiles (the fraction of the material below the $n^{\text {th }}$ value) are plotted against the quantiles of a normal distribution. If the data are normal then the resulting plot should be close to a straight line. If the data are not normal then a log transformation can be applied to the data, which usually normalizes the data. Again the log data can be checked for normality using another QQ plot. The variances of the data were calculated in $\mathrm{R}$, and then tested for similarity using Bartlett's test for homogeneity of variances. With Bartlett's test the null hypothesis is that the variances are equal, and the null is rejected if the p-value is below .05. 
The null hypothesis for most Anova tests is that the means are equal. The alternative hypothesis would then be that at least two of them are different from each other. The null can be rejected if the probability calculated is equal to or below .05. If it is found that the null should be rejected, the question remains as to which are significantly different from each other? A post-hoc test called Tukey's Honestly Significant Difference can be done after an Anova test in order to determine which means are different. Tukey’s HSD uses data from the Anova (Mean Square Within) to calculate what a significant difference would be (HSD), and then compares each pair of differences in mean to the HSD. ${ }^{5}$

\section{Coefficient of variation}

The coefficient of variation (CV) was used to explore the degree of standardization or variability of tools in Chapter 6 . The coefficient of variation compares the degree of variability within a sample or population. It is derived by calculating the mean and the standard deviation, then dividing the standard deviation by the mean. The result is multiplied by 100 so that it can be expressed as a percentage.

The standard deviation itself is a measure of dispersion (variability) around a mean, but if the means of two groups being compared are very different, then the standard deviations will accordingly also be different. The magnitude of the standard deviation is related to the magnitude of the mean. Groups with a smaller mean will have a smaller standard deviation, even if the amount of variability is actually the same. For instance, a group of tools with an average

\footnotetext{
${ }^{5}$ For the exact formula and more information on Tukey's HSD see Tukey (1949) or http://faculty.ucmo.edu/dkreiner/psy2120websitestuff/psy2120oldexams/ documents/StatSheet12_TukeysHSD.pdf
} 
length of $4 \mathrm{~cm}$ will have a smaller standard deviation than a group of tools with an average length of $40 \mathrm{~cm}$. Dividing by the mean controls for differences in the amplitude of the mean, thereby making the amount of variability comparable across groups with means of any size.

Eerkens and Bettinger (2001) used observations on the degree of variability that humans can perceive in order to set a limit for the degree of standardization that humans could produce. This limit is a CV of $1.7 \%$. They also discussed examples of items produced without aid of measuring devices which are considered quite standardized, and these typically range closer to 25\% (ibid.:496). Additionally, they generated CVs of random data to set a constant for extreme variability, which worked out to a CV of 57.7\%. 


\section{Chapter 3: Predynastic Settlements and their Contexts}

Specialized production developed over the 4th millennium BCE in Egypt. This chapter provides the background information on Predynastic Egypt including chronology, evidence for inequality, settlement contexts, deposition practices, and settlement patterns. The find contexts of specialist produced tools and their contexts of production are referred to in later chapters to evaluate the expectations for the ritual production model.

The overview of chronology includes differences between relative dating schemes, and their correlated absolute dates. Many of the models for specialized production in Egypt involve different roles for elites and non-elites, therefore a brief overview of the evidence for inequalities during the Predynastic period is given below.

Lithic artifacts were analyzed from three settlements sites to understand the ways production of lithic artifacts was organized, and to evaluate the expectations for the ritual production model. The descriptions of these three settlements and the history of research at each are given here. The information from these three sites was also compared to published information on lithic artifacts from a number of other sites. Therefore brief descriptions of the comparison sites follow. This overview of the settlements includes information about and interpretation of individual contexts, which allows for an analysis of disposal practices and natural taphonomic processes, concluding that the artifacts found in a given context were most likely related to the use of the features therein, rather than from later trash dumping. Finally a discussion of the settlement patterns shows how individual contexts were organized within a site and how sites were distributed across a region. 


\subsection{Overview of Socio-Economic Changes}

Worldwide, Egypt is one of the cases where pastoralism was adopted before agriculture. This is significant because scholars argue that pastoralism affected the development of Egyptian culture even after the adoption of agriculture (Buchez 2011a; Gatto 2014; Wengrow 2006). By the 6th millennium BCE, pastoralism was evident through the presence of domesticated cattle and ovicaprid bones in campsites from the Red Sea to Chad (McDonald 1998). By the beginning of the 5th millennium BCE the climate had begun shifting toward greater aridity (Bubenzer and Riemer2007; Kindermann et al. 2006; Kuper and Kröpelin 2006; Nicoll 2001; Riemer et al. 2013; Wendorf et al. 2001). By 3500 BCE deserts were only suitable for transit and occupation at a few oases. People responded by expanding mobile ranges and moving south or into the Nile Valley. Evidence for agriculture was first identified at sites in the Delta and the Fayoum, dating to the 5th millennium BCE, and from there the evidence for agriculture shifted south over time. This north-south trend along with the appearance of a 'bundle' of agricultural domesticates including emmer wheat, barley, lentils and flax, implies that agriculture was adopted from the Levant (Wetterstrom 1993).

The Badarian is a regional culture in the middle Nile Valley (Abydos area and north) that dates to 4400-3800 BCE with evidence for agriculture and a material culture that directly links to the subsequent Naqada culture (Hendrickx and Vermeersch 2001; Holmes and Friedman 1994). Regional surveys of settlement sites indicate settled agricultural communities existed throughout the Nile Valley by 3800 BCE (Hassan 1988; Patch 1991; for all sites see Hendrickx and van den Brink 2002). Regional differences in ceramic (Friedman 1994) and lithic (Holmes 1989) material culture existed during the early part of the 4th millennium BCE, and became more unified over the course of the millennium. The people of the 4th millennium also made drastic changes to 
their lifestyles, leading to the rise of Pharaonic Egypt by the beginning of the 3rd millennium BCE. The major changes included: uniform material culture throughout Nile Valley and delta made by specialists; centralized administrative organization evident through the development of writing, bureaucratic titles, and tax collection; and a pervasive system of social stratification centered around the pharaoh, marked most powerfully by monumental architecture in settlements and cemeteries.

\subsection{Chronology}

A summary of the chronological divisions of the 4th millennium BCE is necessary for an analysis of the development of specialized craft production. Named for the site of Naqada, the Naqada period is a chronological designation for a set of related material culture (known as the Naqada culture). This material culture spanned most of the time period from 4000 BCE into the early 3rd millennium BCE. Geographically, it began in the Nile Valley in Upper Egypt (from Aswan to Asyut), and eventually expanded into Lower Egypt and the oases. The term "Predynastic" is a looser but somewhat interchangeable term for the Naqada period, but can also encompass the Lower Egyptian early 4th millennium cultural sequences (Buto-Maadi culture), and the Badarian period material which is found in middle Egypt in the latter half of the 5th millennium BCE (Hendrickx 2006b:55-57).

The Naqada period is divided into three main parts based on the relative chronology of the ceramics. This was first and famously accomplished by Petrie, who worked with data from the cemeteries of Naqada, Ballas, and Diospolis Parva (a.k.a. Abadiya and Hu), and seriated the grave assemblages to provide Sequence Dates (Petrie 1921; Petrie and Mace 1901; Petrie and Quibell 1896). The three partitions, from earliest to latest, were known as the Amratian, Gerzean, 
and Semainian, and the divisions were based on the occurrence or absence of different pottery types. Petrie observed that the White Cross-Lined Wares and Decorated Wares never occurred in the same graves, and that the changes in the wavy handles on some pottery could be put into a logical order. Scholars have been continually revising Petrie’s chronology (Buchez 2011a,b; Kaiser 1957; Hendrickx 1996a, 2006b, 2011b; Hartmann 2011a,b). Table 3.1 presents the basic correlations between different dating schemes. Based primarily on cemetery data from Armant, Kaiser (1957) revised Petrie’s chronology by taking into account horizontal spatial differences within the cemetery. Kaiser observed three spatial clusters of ceramic types: Black-Topped Ware, Rough Ware, and Late ware, and divided the Naqada period into three stages or 'Stufe': I, II, III, with I the earliest and III the latest. Those three main periods were further subdivided and marked by lowercase letters, so that Ia is earlier than Ic and so on. Kaiser's scheme became widely used, but Hendrickx (1996, 2006b) offered further refinements to Kaiser’s chronology by using a similar method but basing his analysis on multiple cemeteries. Chronological designations based on Hendrickx's scheme are written with uppercase letters (e.g., IC or IID) rather than lowercase letters to differentiate from Kaiser’s chronology.

Kaiser and Hendrickx used the pottery types defined by Petrie, which had some inherent problems (Buchez 2011b; Hendrickx 1996a), but they could not refine the typological categories because they did not have access to the original materials. Buchez developed new typological categories based on material from the site of Adaïma, and used a serration process to examine chronology rather than spatial distribution. Her studies definitively demonstrated that the phase IId1 could not be distinguished from IIc, which means that phase IID2 can just be considered IID. This latter point is an important consideration for understanding ripple-flaked knife production which dates mainly to the NIID period. 
Hartmann (2011a,b) refined the dating of the early part of the Naqada period based on finds from the Abydos Umm el-Qa'ab cemetery. Drawing data from 650 graves of the NI/II period, she made a new classification of vessels according to fabric, ware, and shape. She also included some vessels from early excavations in order to correlate the results with Petrie's types and chronology. Hartmann recommends treating the entire period of NIA-NIIB as the Naqada I period, with two main phases, IA and IB, that have additional subdivisions (see Figure 3.1).

These dating schemes were based on cemetery materials, and as more work has focused on settlements it has become clear that settlement ceramic assemblages are made up mainly of vessel fragments, and that the ceramic types are represented in different proportions than in cemeteries. Many authors have worked to correlate the settlement materials to the existing dating schemes (Friedman 1994; Patch 1991). Throughout this dissertation referenced dates follow those given by the original authors, the majority of whom draw on either the Kaiser chronology (lowercase letters) or Hendrickx's Naqada chronology (uppercase letters). Table 3.1 can be used to compare the dates.

There are two important points for understanding Naqada chronology. First, the Naqada III overlaps with the Early Dynastic period, because the latter was often identified through writing and evidence for kings, while the former was based on pottery finds. The second point, is that there are more differences between NIIAB/NIIab and NIIBC/NIIbc than there are between NIC/NIc and NIIA-B/ NIIa (Hendrickx 1996a:39, 2011c). Effectively, these differences divide the Naqada period before Aha into an earlier part (NI-NIIAB), and a later part (NIICD-IIIB).

Absolute dating based on radiocarbon dates for these relative time periods has been beset with problems including the unreliability of calibration curves for the NIII period and the inability to export carbon material out of Egypt for testing after the 1980s. A few projects 
nonetheless were able to offer some absolute dates (Hassan 1988; Savage 1998), and a $C^{14}$ dating facility is now open in Cairo. The conventional range for the Predynastic period has been from around 3900 BCE to around 3050 BCE. However, due to the above-mentioned problems with dating, Dee et al. (2013) used Bayesian statistical modeling methods to refine the radiocarbon dates from new samples taken from museum collections (focusing on short-lived remains) and measurements available in published material. Notably this revised chronology indicates that the Predynastic period and all its associated socio-economic and political changes took place over a shorter time span than had been previously thought, from around 3700 to 3075 BCE (Table 3.1).

\subsection{Inequality in the Naqada Period}

Since current theories argue that specialization developed in Egypt in relation to elite demand for prestige goods, it is necessary to briefly present evidence for elites and inequality during this period. In Egypt this issue has mainly been assessed through cemetery evidence. W. Anderson (1992) analyzed Badarian graves and argued that some vertical differentiation was evident. She found statistical differences between and within cemeteries, showing that luxury goods were segregated by area in some cemeteries, that there was a bimodal distribution in the quantity of goods per grave, and that sub-adults could be found with numerous ceramics. Bard (1988, 1994) worked with data at Armant and Naqada, and argued for increasing differentiation from NI to NII, with a two-tiered social hierarchy in the NIICD, and more variability in differentiation during the NIII. Wilkinson (1996) worked with data from a number of cemeteries, and came to similar conclusions using different methods. ${ }^{6}$ There were actually fewer differences

\footnotetext{
${ }^{6}$ However some authors have noted problems with the way Wilkinson grouped Petrie's pottery types.
} 
among graves in some cemeteries in the NIII. Both Bard and Wilkinson attribute this to the suppression of local elites, as more encompassing socio-political structures formed. Savage (1997) provided a detailed analysis of cemetery evidence that considered both horizontal and vertical differentiation at Naga-ed-Dêr. He argued for the presence of descent groups as the best explanation for grave clustering, and showed that each grouping had changing economic fortunes over time. Bard also demonstrated that the social position or wealth of a person was more often expressed by including large quantities of similar objects, rather than through the inclusion of a few rare or exceptional items.

The advent of cemeteries specifically reserved for elites indicates that the developing inequalities became sustained. Hierakonpolis has the earliest cemetery dedicated to elite burials, which dates to the NIC-NIIB. As will be discussed in more detail below (Ch. 3.5), there are tombs with wooden- post superstructures, central tombs surrounded by subsidiary human burials (arguably sacrificed), wild and domestic animals (elephant, hippo, baboons, hartebeest, wild and domestic cattle, goats, dogs, cats), caches of artifacts, and a destroyed human statue in stone (Adams 2000a; Friedman 2011c). Cemetery T at Naqada dates to the NII-III, and has large bricklined tombs with large quantities of artifacts including rare imported artifacts (Davis 1983; Petrie and Quibell 1896). The Umm el-Qa'ab cemetery at Abydos dates back to before even the NI (Hartmann 2011a). By the NIId it was reserved for elites, and the cemetery eventually became the burial place for the First Dynasty kings (Dreyer 1992).

\subsection{Site Descriptions: el-Mahâsna, Abydos, Nag el-Qarmila}

The description of the settlements and the history of research at each are given here, along with details about the excavation methods and contexts from which the materials analyzed 
here derived. Associated settlement sites and cemeteries are also briefly discussed to provide context.

\section{El-Mahâsna}

The settlement site of el-Mahâsna (26 15’16” N, 31 50’26” E) is located 10km north of Abydos, on the west bank of the Nile, in the low desert just on the edge of the modern cultivation (Figure 3.1). The site is situated on a small rise. The Predynastic settlement site itself extends for about 7.6ha ${ }^{7}$ (Anderson 2006, 2007, 2011), and probably was originally larger since much of the surrounding area has become farmland. Later cemetery remains intrude on the settlement and extend farther to the north.

El-Mahâsna was originally excavated by John Garstang in 1900-1901 (Garstang 1903).

Garstang's work focused on a wide embayment in the high desert cliffs, immediately north of the Abydos embayment. Garstang and company located a number of sites, including the Predynastic settlement of el-Mahâsna, and the Predynastic cemetery of Alawniyah (Beit Allam). Garstang identified the settlement area based on exposed artifacts and darker soils characteristic of decayed mud architecture. He divided the settlement area into two parts, labeled S1 and S2, due to a natural topographic depression which can still be seen today. Garstang (ibid.:5) and Anderson (2006:29) both concluded that the two areas are parts of the same overall settlement, and indeed, a feature of many Predynastic settlements is that they can have somewhat separated localities as part of the whole (Ch. 3.7). Garstang's excavations in the S2 area revealed preserved

\footnotetext{
${ }^{7}$ Patch (1991:404-408) gave a much smaller size estimate from her survey, only .36 and .3 ha for the site's two parts, but Anderson's excavations have revealed Predynastic remains in a larger and basically contiguous area.
} 
stubs of wattle and daub architecture in situ, post holes with actual wooden posts still in them, and intact ceramics, all of which demonstrate the excellent preservation of the site. However Garstang did note some areas of sebakh ${ }^{8}$ digging. Other interesting finds from the site included a cache of oddly-shaped natural flint nodules, and a series of kilns which Geller (1992a,b) later argued were beer brewing facilities.

Garstang's work documented that after the settlement was abandoned, the area was reused as a cemetery, with tombs dating from the Early Dynastic through the Old Kingdom and later. Some of the tombs had been excavated previously, possibly by de Morgan (Garstang 1903:2; J. de Morgan 1896, 1897). The tombs avoided the main settlement area, but it is unknown whether this was due to coincidence, the soil type, the existence of visible remains, or because the site was still remembered.

In 1982-1983 Diana Craig Patch (1991) surveyed the Abydos region for Predynastic-Old kingdom remains, including the el-Mahâsna Predynastic settlement site. Her survey aimed at dating the sites in order to understand the chronological development of urbanism in the area. Patch worked using a combination of pedestrian survey and surface collection of diagnostic sherds in stratified random samples. As with Garstang, the el-Mahâsna Settlement site was again separated into two parts, S83-40 (which included Garstang’s S2 and later the Pharaonic cemetery) and S83-41 (Garstang’s S1) ${ }^{9}$ (ibid.:404-408). Patch noted that the density of surface materials was sparser at S83-41 compared to S83-40. Patch (ibid.:404) dated the site(s) to the IcIId2.

\footnotetext{
${ }^{8}$ Sebakh is the Arabic term for the rich soil that built up in ancient settlements. Farmers in the 19th century would dig up the sebakh to use as fertilizer on their fields, destroying many ancient settlements in the process.
} 
David A. Anderson $(2006,2007,2011)$ conducted new excavations at the site over the course of three field seasons from 1995-2000 as part of his dissertation research, and has continued analyzing the material and undertaking new excavations since. Anderson's dissertation focused on comparing the applicability of two models for the development of complexity in Egypt. To do this Anderson's team carried out systematic surface collections, excavations, and analyzed the ceramics, faunal remains, special finds, and evidence for architecture and stratigraphy (Anderson 2006, 2007; Rossel 2007). Like Garstang, Anderson’s work uncovered extremely well-preserved habitation remains including preserved posts and matting, basketry, hearths, floor surfaces, and intact pottery. These impressive finds are unfortunately now threatened by modern farmland expansion (Anderson 2006:23). Anderson (2006, 2011:3) dated the Predynastic settlement remains to the NIC-IID. The lithic material analyzed for this dissertation project came from Anderson’s excavations.

\section{Anderson's el-Mahâsna excavations}

El-Mahâsna is integral to the present study because Anderson's excavations revealed the existence of domestic structures (Anderson 2006) and a ritual structure (Anderson 2011), both of which are contexts that will be used to evaluate the ritual production model. Anderson excavated nine 'Blocks' of units, totaling $405 \mathrm{~m}^{2}$ (Figure 3.2). The Blocks were placed to explore factors identified during the surface survey, and each Block was composed of a number of adjacent 3x3m excavation units. All the Blocks varied in size based on the materials and features present. Each unit was excavated in arbitrary $10 \mathrm{~cm}$ levels within natural or cultural stratigraphic

\footnotetext{
${ }^{9}$ Patch is not sure if S83-41 corresponds to S1, but Anderson (2006) believes it does based on his
} 
loci. All the material was dry screened through $4 \mathrm{~mm}$ mesh. The Op-Locus-Lot recording system was used at el-Mahâsna. Each excavation unit was called an operation and given an 'Op’ number in the order of their excavation. During excavation individuals features or stratigraphic layers were then assigned sequential 'Locus' numbers. Locus numbers were not repeated in different Ops, instead each locus number is unique across the whole site. Each portion of contiguous soil removed at a time was assigned a 'Lot' number sequentially in each unit. A 'Lot' is a threedimensionally defined volume of soil and can either consist of an entire locus (such as a small hearth), or an arbitrary division of the locus, (such as the top $10 \mathrm{~cm}$ of a stratigraphic layer). For example Op5, Locus 44 might be a thick layer of loose sandy soil spreading across the whole unit. The first $10 \mathrm{~cm}$ removed would be assigned the next available 'Lot' number for the Op, e.g. 4. Materials collected from each individual lot were given unique tracking numbers called “MAP” numbers (tag numbers). ‘MAP’ stands for Mahâsna Archaeological Project. In this example all the lithic artifacts from Op 3-Locus 15-Lot 4 would be given a MAP number, say MAP\#1317, all the ceramics the next MAP number, MAP\#1318, and so on. For more details on the Op-Locus-Lot and MAP\# system, see Anderson (2006:46-51). After excavation Anderson analyzed the stratigraphy and features and assigned each lot to a habitation phase, and assigned dates to the habitation phases based on the associated diagnostic ceramics.

Figure 3.2 shows the location of each of the excavation blocks, and Table 3.2 presents the basic interpretations of each excavation block. For this study, lithic artifacts were analyzed from two of the domestic contexts (Blocks 1 and 4) and the ritual activity area (Block 3), each described below. Time did not permit analysis of the entire lithic assemblage from each block. Two units were selected per block and fully analyzed: Block 1 Ops 4 and 5; Block 3 Ops 20 and

observations of the site. 
23; Block 4 Ops 25 and 28. Additionally a few other MAP numbers were randomly selected in each block, and special finds were analyzed. Figure 3.3 shows the location of the analyzed Ops in each excavation block.

\section{Evidence for habitation contexts}

Anderson identified habitation areas in Blocks 1, 4, and 8. Furthermore Anderson (2006: 242-247) devised an 'elite index score' to compare the excavated areas at el-Mahâsna based on expenditure of effort in construction and presence of wealth items. Block 3 clearly stood out from all of the other blocks, while Blocks 1, 4 and 8 had comparable scores. Blocks 1 and 4 were selected for lithic analysis because the faunal material had already been analyzed and contributed to the interpretation of the contexts.

The excavations in Block 1 revealed evidence for a structure (Figure 3.4) made of reeds and wood posts with mats tied onto them (ibid.:77). The structure (or one part of the structure) was defined by walls on at least three sides, had internal divisions and a compact floor surface, and was at least $4 \mathrm{~m} \times 2.75 \mathrm{~m}\left(8.86 \mathrm{~m}^{2}\right)$ in size. One side of this structure was formed by a long (13m) wall which extended further north and south and which may define a larger structure or one side of a fenced area. Another structure just south of the first was identified only by remains of its mud plaster floor surface adjacent to the long wall (ibid.:78).

Other features and artifacts attest to the domestic character of the activities. The presence of a number of hearths, some with faunal remains indicate cooking activities. High proportions of basins and jars in the ceramic assemblage suggest subsistence production and storage activities (ibid.:159). A storage pit which was capped with a piece of limestone and contained a lower grinding stone and two flake cores (MAP\#s 1313.1, 1313.2) also complements the ceramic 
evidence for subsistence production and storage. The lithics analyzed from this op date from the Ic to IIa-b periods.

Identification of Garstang’s dig-house allowed Garstang’s maps to be rectified with modern maps. Block 4 was located adjacent to an area of Garstang’s excavations. Posts and somewhat ephemeral living surfaces were found in Block 4 (Figure 3.6). Although there was no clear patterning to the posts, Anderson (2006:128) thinks they may be associated with a structure described by Garstang, based on proximity. Quite a few ash features were also found in this block including hearths, ash deposits, and middens/areas of trash disposal, which Anderson interpreted as food processing activities in an outdoor area adjacent to a structure (ibid.:132). Finds of faunal remains, particularly large amounts of turtle bone, support this interpretation. The analyzed contexts in this Op date from the Ic-IIa-c periods.

\section{Evidence for the ritual activity area}

Analysis of ceramic, faunal, and architectural data distinguished Block 3 from the other excavated contexts at el-Mahâsna. The excavations revealed evidence for a structure (Figure 3.7). The architecture and artifacts associated with this structure date to the NIC-NIIAB, possibly into NIIC (Anderson 2011:12, 14). The Block 3 structure was located prominently on a rise, at the highest part of the site. The Block 3 structure showed a higher investment in architecture compared to other blocks, with significantly larger posts (Anderson 2006:117-119). The size of the structure is unknown, since its limits were not reached during the excavation of the $162 \mathrm{~m}^{2}$ block, which itself indicates that it must have been a rather large structure. A layer of clean sand was found underneath the structure, possibly intentionally placed there, as is seen with later Pharaonic temples (Anderson 2011:14). 
The ceramic inventory of Block 3 also stood out from the other excavation blocks, with statically significant higher proportions of imported ceramics (Anderson 2006:170). Higher proportions of White-Cross Lined and Decorated Ware ceramics, the two kinds of ceramics that bear figural decoration, were also more frequent in Block 3, though the statistical significance is only moderately higher due to the overall low frequency of such materials at the site (Anderson 2006:175-179, 260, 2011:19, ).

Most notably, 22 human and animal unfired clay figurines and figurine fragments were found in Block 3 (Figure 3.5). Some of the cattle figurines have incision marks on the neck which may represent slaughtering cuts (Anderson 2011:15). Anderson argued that the anthropomorphic (female) figurines were made to be broken. They were constructed from separate individually modeled parts which were not scored to increase adhesion, nor were the parts modeled around sticks to hold them together, like some other known Predynastic figurines. The el-Mahâsna figurines broke along the weak join lines. Furthermore, the (refitting) parts of the figurines were not found adjacent to each other, but scattered around inside the structure, showing that they were not broken after deposition (ibid.:18). Pre-depositional wear on the figurines indicates that they were not production rejects, but artifacts utilized during the life of the structure.

Faunal analysis showed that Block 3 had the highest number of wild animals remains, including gazelle, Barbary sheep, hippo, and antelope, with over $60 \%$ of the wild desert animal bones coming from Block 3 (ibid.:20). Remains from wild aquatic animals were also associated with Block 3, and included crocodile, and many turtles (Anderson 2006:208-209). In a study that compared faunal remains from 12 different Predynastic sites Linseele et al. (2009) showed that while hunting was iconographically important, hunted animal remains were actually quite rare in 
Predynastic settlements, and could not have constituted a major part of subsistence strategies. Additionally, higher proportions of very large catfish and Nile perch suggest that the best catches were slaughtered or consumed in conjunction with the Block 3 ritual activity area (Anderson 2011:22).

A higher, moderately statistically significant proportion of cattle remains ( $35 \%$ of the domestic mammal assemblage) were found in Block 3. There was also a higher proportion of young cattle remains, and a much higher statistically significant proportion of forelimbs of cattle and other domestic mammals. Young cattle and cattle forelimbs probably constituted preferred beef types for Predynastic Egypt, due to the tenderness of meat form young animals and since the forelimbs of cattle were depicted as a quintessential part of offering ceremonies during the Pharaonic period (Ikram 1995).

Complementing the evidence for the slaughter of wild animals and choice domesticates are the artifacts associated with hunting and/or warfare that were found in Block 3. These include fragments from a ground-stone mace head, bifacial knife fragments, concave-base bifacial projectile points, and an ivory projectile point. Anderson argued that the mace head was more likely a ceremonial or symbolic item than a strictly utilitarian one, since the hole in it would have only supported a very small shaft that would have broken upon any impact. Smiting enemies with a mace is of course one of the most classic and enduring elements of Pharaonic royal imagery dating from before Narmer though the entirety of Pharaonic history.

Other artifacts might be associated with weaving, such as bone awls, copper and bone needles, and spindle whorls. Sixteen of the 28 spindle whorls from el-Mahâsna came from Block 3, and five of the rest came from Block 2 (Anderson 2006:233), which Anderson thinks is an outdoor activity area associated with Block 3. Anderson suggested that the concentration of 
weaving implements in Block 3 may be evidence of specialized production, although he acknowledges there is no way to determine what sorts of items were produced, which could be anything from fishing nets (which would complement the faunal remains), to fine textiles which might be considered luxury goods. Other notable artifacts found in Block 3 are sealing fragments, which were only found in Block $3(n=3)$, and do not have any seal impressions on them.

Anderson argued that the architectural, ceramic, and faunal evidence indicate that the structure should be considered an elite ritual activity area. The findings certainly also fit the definition of ritual activity used here, as repeated, set actions that are transformative and that derive from the worldview of a specific group of people (Ch. 1.6). That the activities which took place in el-Mahâsna Block 3 were to some degree repeated or set can be shown through Block 3’s similarity to a Predynastic ceremonial enclosure at Hierakonpolis HK29A. That structure was unusually large compared to other Hierakonpolis domestic structures, the ceramic assemblage included unique forms known only from that site and a nearby cemetery, and the faunal assemblage also includes an unusually high concentration of wild animals, choice domesticates, and large fish. The Hierakonpolis enclosure is discussed in more detail below (Ch. 3.5). The similarities between the two loci indicate that comparable activities were repeated in separate areas of the country. Moreover there is evidence for the same seasonal periodicity in the activities. Four of the seven faunal bones of dorcas gazelle found in el-Mahâsna Block 3 were from very young animals. Consideration of the mating and birth periods indicates that the animals were probably slaughtered in May-June, which is just before the annual Nile flood would have begun (Anderson 2011:20-21). A number of species found at HK29A such as Nile Perch, Nile Oyster, and gazelles would have been easier to obtain when the flood was very low, 
as would have been the case in June, just before the flood waters arrived (Linseele et al.

2009:134). There are also some differences between the two assemblages, such as the presence of figurines at el-Mahâsna but not Hierakonpolis, and unique ceramic vessel forms found only at Hierakonpolis. The similarities and differences highlight the tension between rituals as 'set' or precedented action and the ability of those involved to modify them.

The activities which took place in Block 3 reflect some dominant themes in the Predynastic worldview. In Pharaonic Egypt cattle were shown ubiquitously in scenes of temple offerings, where the left front foreleg was the 'choice cut' offered to the gods (Ikram 1995:129). By comparing the distributions of skeletal elements from a Middle Kingdom mortuary temple and associated town at Abydos to those from el-Mahâsna, Rossel (2007) argued that cattle were a preferred high status-food often found in ritual contexts and that cattle were valued and used similarly during both the Predynastic and Middle Kingdom periods. Furthermore a number of authors have argued based on many lines of evidence, that women and cattle (and birds) were particularly salient features of the Predynastic iconographic record, and were often associated with fertility, renewal, strength, and eventually divinities and royalty (Hassan 1988, 1992a, 2004; Hendrickx 2002; Wengrow 2001). The finds of female figurines and cattle figurines support the connection of these features of Predynastic Egyptian worldview to the activities in Block 3. Moreover, markings (tattoos?) on one female figurine consist of shallow incised dots which may represent grain seeds, zigzag wavy lines similar to the later hieroglyphic symbol for water, and an incised and colored pubic triangle (Figure 3.5). Anderson (2011:18) argued that the three together show a focus on female and agricultural fertility. Another significant theme known from the Predynastic iconographic record is that of warfare/hunting (Hendrickx 2011a, 2012). Many artifacts found in Block 3 (mace head, projectile points, bifacial knife fragments) can be 
associated with warfare/hunting and show the enactment of activities related to this important aspect of the Predynastic worldview in Block 3.

Hendrickx et al. (2009) explicitly related the hunting and slaughter activities associated with HK29A, along with Predynastic evidence for female figurines, to Old Kingdom 'Acacia house' ritual activities, which are known from textual references. Known from the beginning of the Old Kingdom onward, the 'Acacia house' was a structure associated with a temple. Textual references indicate that butchers were associated with the 'Acacia house', as were dancers who performed at funerary ceremonies and occasionally at royal jubilees. Therefore the activities associated with the 'Acacia house', and by extension the ritual activities indicated at HK29A and el-Mahâsna Block 3, can be understood as mediating liminal boundaries between life and death.

\section{Alawniyeh / Beit Allam cemetery}

Garstang (1903:5, Pl 1) excavated a cemetery near the modern villages of Alawniyeh and Beit Allam during his 1900-1901 season (Figure 3.1). Originally containing an estimated 200300 graves, the cemetery was already plundered when Garstang reached it, and he only excavated 45 graves, 23 of which were recorded in relative detail. Garstang related the cemetery to the settlement site of el-Mahâsna based on the similarity of pottery types (ibid.:5-6). However the cemetery is 3 km SE of the el-Mahâsna settlement, and Ayrton and Loat (1911) later excavated a cemetery much closer to el-Mahâsna (see below). Clay models of stone tools and bird-headed humans were striking finds from this cemetery. Little remained of the cemetery 
when Patch (1991:134,396-397) surveyed it in the early 1980s, designating the site S83-29. ${ }^{10}$

She dated the site to the Ib- IIb period based on Garstang’s published material.

\section{el-Mahâsna cemetery $H$}

Ayrton and Loat (1911) excavated another cemetery even closer to el-Mahâsna, called Cemetery H, in 1909. The cemetery was also surveyed by Patch (1991:409) during her regional survey, and designated S83-42. The cemetery is located about 1km southwest of the el-Mahâsna settlement site (Anderson 2007:33; Patch 1991:134, Map 4). Extensively looted, Ayrton and Loat (1911:2) estimated that it originally contained approximately 600 graves, and they excavated around 300 of them, although details were only given for 135 tombs (Thomas 2004:1042). Because this cemetery was one of the better recorded for its time, its contents have featured in a number of studies of chronology and/or social inequality (Hendrickx 1996a; Kaiser 1957; Kemp 1982; Wilkinson 1996). Wilkinson’s (1996) study of cemetery H showed initial increases in wealth disparity followed by declining differences before the cemetery was moved to a new location in the First Dynasty. ${ }^{11}$

\section{Abydos}

The main Predynastic and Early Dynastic sites in the Abydos area include the famous

\footnotetext{
${ }^{10}$ Note that Hendrickx and van den Brink (2002) list the cemetery twice, once as Mahâsna/Alwaniyeh and referring to page numbers for Patch's Mahâsna settlement site which had later Early Dynastic tombs built over it, and once as Beit Allam with page numbers for Patch's S83-29. This confusion probably stems from Garstang alternately referring to the site as "near Alawniyeh" or "near Beit Allam", and Patch designating the site Beit Allam, while including the Predynastic Mahâsna settlement in an overall entry for the NIII and later cemetery.

${ }^{11}$ See Thomas (2004) for problems with the cemetery data.
} 
Umm el-Qa'ab cemetery near the high desert cliffs (Dreyer et al. 1996, 1998, 2000, 2003), the funerary enclosures of the First and Second Dynasties (Adams and O’Connor 2003; Bestock 2008, 2009; O’Connor 2009:158-181), the Early Dynastic town site in Kom es-Sultan (Petrie 1902,1903) and a number of small settlement sites (Figure 3.8). For the present study, lithic artifacts were analyzed from a Predynastic settlement site found underneath the much later pyramid complex of Ahmose, which will be described in detail. Following that brief descriptions of the other sites in the Abydos area provide context and are important for the discussion of settlement patterns later in this chapter.

The Abydos ATP settlement site is located in the low desert, near the cultivation, on the southern side of the high desert embayment which delineates the Abydos area $\left(26^{\circ} 10^{\prime} 32.2206^{\prime \prime}\right.$, $\left.31^{\circ} 56^{\prime} 18.4128^{\prime \prime} \mathrm{E}\right)$ (Figure 3.8). The settlement is underneath the Ahmose pyramid complex (Figure 3.9). The site was first noticed and partially excavated by RandallMacIver and Mace (1902:76) in the course of the New Kingdom excavations. They documented 1-3 feet of thickness for the Predynastic layer. Patch (1991:376-377) also recorded the surface material in her survey of the Abydos region. Denoting the site S83-3, she estimated the size of the site at $7800 \mathrm{~m} 2 / .78 \mathrm{ha}$, and based on surface collection of the ceramic material, dated it to the Naqada Ic-IIc. This site is important because it is the earliest known Predynastic settlement in the Abydos area.

\section{Harvey's Abydos excavations}

In 1993, Stephen Harvey (1998:146-147) excavated some Predynastic remains in the course of his dissertation research on the New Kingdom Ahmose pyramid complex, and continued excavations from 2002-2006. He surmised that the new kingdom remains were built 
directly on top of the Predynastic settlement, in some cases with little or no preparation. However the site was not completely disturbed by the New Kingdom activities since Harvey's team found many intact ceramic vessels from different parts of the site, and even more fragile remains such as basketry. Hearths and other features were also found in situ emphasizing that much of the settlement was preserved. Harvey (pers. comm.) also noted many examples of White Cross-lined Ware pottery, which date to the NI-NIIAB, and no pottery clearly indicative of a later NII date. Rita Hartmann (pers. comm.) briefly looked at some of the intact vessels in 2013, and dated them to the NIB to NIC/IIA (Hartmann chronology, see Ch.3.2). These estimates are slightly earlier than Patch’s dates for the site, but generally the estimates correspond.

The Predynastic materials were rich in the eastern portion of the Ahmose pyramid temple, particularly near Temple A (Harvey, pers. comm.). The lithic materials analyzed for this study come from that area, and were excavated during Harvey’s 1993 season. The excavation methods used was similar to the Op-Locus-Lot system described above for el-Mahâsna (ibid.). However the excavation units (Ops) were each $10 \mathrm{~m}$ x10m in size, and Lot numbers were only unique to each unit. The tracking numbers given to each tag for artifacts and samples were designated ATP numbers (Ahmose-Tetisheri Project). All of the material was dry sieved through $4 \mathrm{~mm}$ screens.

A sample of lithic materials were analyzed from two different Ops (Ops 8 and 19, Figure 3.10). The material analyzed here was only a small portion of the total from each Op. Materials from lower lots were chosen to limit any potential mixing with the New Kingdom layers. Bifacial sickles from Op 11 are also mentioned in the analysis. Details given below derive from the field notebooks and communications with Stephen Harvey. 
Op 8 was mainly located in an area between the New Kingdom constructions. Some of the Predynastic remains were found directly after removal of the surface layer. The Predynastic stratum consisted of loose yellow sand with a high percentage of charcoal pieces and lithic artifacts. The find of an intact Black-topped jar with many repair holes (Figure 3.15b) indicated that this was a Predynastic layer. In the SW corner of the unit a number of features were found together, including the above mentioned in situ intact jar, a hearth (Locus 4), a possible floor surface, and a mud(?) bin filled with chaff (Locus 6). The three features were all on the same level as the mouth of the intact jar, and in the Predynastic sandy matrix (Figure 3.11). The exact date of the three features is not clear since they were found directly below the surface material, and could have been added later, but the excavator interpreted them as a Predynastic activity area. After removal of these materials, and $\sim 40 \mathrm{~cm}$ below the rim of the first Predynastic jar, a stack of two Predynastic jars covered by a large jar fragment was found (ATP 4609), with two zoomorphic figurine fragments (ATP 2515 and 2516, Figure 3.13) and another chaff deposit nearby. Two more vessels were found, one to the north (ATP 3077), and one to the east (ATP 3076) (Figure 3.12). Excavation stopped after this material was removed, but the Predynastic deposits likely continue. At least $50 \mathrm{~cm}$ of Predynastic material were removed in total.

The Predynastic remains in Op 19 were found sealed underneath a New Kingdom floor. The matrix of the stratum is exactly like that of Op 8: loose tan sand with a high percentage of charcoal fragments. Fewer features were identified here than in Op 8, but the Predynastic levels were not excavated as deeply. A complete but crushed pot was found in the center of the unit, and a small hearth was located in the SE corner (not excavated) (Figure 3.14). The total thickness of the excavated Predynastic material ranged from about $30 \mathrm{~cm}$ in the NE to $10 \mathrm{~cm}$ in the SW. Two zoomorphic figurine were found in the upper layers of this Op (Figure 3.13). 
A few other Predynastic finds from the site should also be mentioned. Quite a bit of Predynastic material was found in Op 11. A cache of at least 8 complete vessels placed inside baskets was found just underneath a New Kingdom wall in Op 11 (Figure 3.17a-c), along with a zoomorphic figurine (Figure 3.13). In the same Op three bifacial sickles (Figure 7.8) were found together in a Predynastic strata which was under a New Kingdom floor surface. Unfortunately time did not permit analysis of lithic materials from Op 11. Five additional figurine fragments were found in Ops 4, 11, 27, and 24, including one that may be torso of female. A ceramic vessel fragment with an incised drawing of a bovid came from Op 18. A concave base projectile point was found in the surface layer of Op 1 (Figure 7.7), and a broken rhomboidal slate palette was excavated in Op 13.

The exact nature of the contexts in Ops 8 and 19 are somewhat difficult to determine at this point. Quite a few pieces of pottery with repair holes (Figure 3.15a,b) were noted by Harvey (1998:164), including some from Op 8. Repaired pottery was also mentioned by RandallMacIver and Mace (1902:76) although they excavated a different part of the site. Pottery without wear, along with special types of pottery, were observed at the HK29A ritual structure (Friedman 2009b:85-88); therefore the presence of the repaired pottery in Op 8 accords better with an interpretation of the area as something other than a ritual activity locus. However it should be noted that at el-Mahâsna vessel fragments with drilled holes were found in Blocks 1-4, including habitation contexts and the ritual activity area, with no statistically meaningful difference in their frequencies, which were quite low (0.1-.26\% of the ceramic material) (Anderson, pers. comm.). A number of the vessels found in Op 8 were relatively large open-mouth jars (e.g., Figure 3.16a,b), which could be related to storage and/or cooking. The chaff features in Op 8 may indicate animal feeding or food processing in the later phase of Op 8. The presence of the cattle 
figurines in Ops 8 and 19 does not necessarily help to interpret the context, since clay figurine fragments were found in domestic contexts at el-Mahâsna, ${ }^{12}$ and in the ritual activity area (in a much higher frequency) (Anderson 2006: 216-230). However the presence of at least 5 bull figurines, some with possible cut-marks or blood lines on their necks all within a 30m x 30m area, is somewhat rare, even if those in Op 19 were not in situ. Two figurine fragments were found some distance away, in Op 4. A study of the Abydos ATP faunal remains will certainly help to clarify these contexts. For now they are interpreted as general settlement contexts with activities relating to food processing and storage, habitation, and some ritual activities.

\section{Other Predynastic and Early Dynastic settlement sites in the Abydos area}

Two Predynastic settlement sites were found by Peet and Loat (Peet 1914; Peet and Loat 1913), both with facilities interpreted as beer brewing kilns. One of these sites was near the later Seti temple and the other was on the north site of the Abydos embayment near cemetery D (Figure 3.8). The one near the Seti temple was described as roughly circular and $\sim 900 \mathrm{~m}^{2} / .09$ ha in size. Patch (1991:437) designated the site S83-61, and dated it to the IId-IIIa1 period, though it is not clear whether this was based on Peet's publication or her surface surveys, which revealed that the site is now extensively disturbed. In the center of the site 'powdered mud' infused the sand and was thought to derive from wattle and daub constructions, though no architecture nor post holes were found (Peet 1914:2). Over 300 flaked stone microdrills and drills were also found in the center of the site, and along with pieces of agate and carnelian. Together these may indicate bead production. Other evidence for production activities included

\footnotetext{
${ }^{12}$ These included a cattle figurine fragment and two unidentifiable figurine fragments from Block 4, and
} 
grinding stones, and spindle whorls. Clay models of bread loaves(?) and a clay sealing fragment (ibid.:5), are possibly evidence of administration or tracking of the production activities. On the outskirts of the site were two large hearths containing bone (40 cm deep x 5-6m wide). Cattle, sheep/goat, and fish bones were common, and donkey, dog, cat and gazelle were each represented by one or a few bones (ibid.:6-7). Also on the edge of the settlement was a feature originally thought to be a grain parching kiln, but later convincingly interpreted as a beer brewing facility (Geller 1992a,b; Ch. 1.4). This structure incorporated at least 23 vats and certainly indicates beer production on a large scale.

Even more extensive brewing facilities were found in the area of cemetery D (Peet and Loat 1913:1-7). Here 8 different sets of vats were found below the remains of later Pharaonic tombs and mastabas. The best preserved set contained at least 35 vats. Peet and Loat (ibid.:6-7) dated the cemetery D kilns to the Predynastic period generally. By the Old Kingdom period beer consumption had increased to the point that it was a staple food, as is indicated by its prevalence in offering scenes and the equal prevalence of 'beer jars' in settlement sites. Therefore, the cemetery D facilities may be contemporary with or slightly later than the Seti temple ones, since they are much more extensive and the increase in size may relate to increasing beer consumption. It is not known whether other settlement remains or evidence of other activities existed adjacent to the brewing structures, but it seems likely since brewing facilities have never been found isolated from a settlement (Table 1.2).

Nearby in an area known as Kom es-Sultan Petrie $(1902,1903)$ excavated a town site which dates from the NIII/Dynasty 0- the Second Dynasty (Adams 1999:108; Petrie 1902:10). This early settlement was underneath successive layers of later habitation remains which formed

one unidentifiable fragment from Block 1 (Anderson 2006:230). 
a tell ${ }^{13}$ (mound). In the early town Petrie excavated a 'temple zone' of enigmatic mud brick structures under or near the later Osiris temples. Outside of the temple area Petrie also found a living site consisting of small mud brick houses. Hearths and abundant ceramic and lithic artifacts were associated with these houses, which were extensively affected by the water table and years of flooding. Petrie excavated a small portion of the and noted that it continued underneath the later town remains. Petrie observed changes in town development over the period. First the excavated are contained habitation remains, then it became a cemetery for a time, then it was again an area for housing.

Patch (1991:426) identified another possible Predynastic-Early Dynastic settlement site called S83-54 near the modern town of Salmani. It was located on the northernmost end of the Abydos embayment, north of the breweries excavated by Peet (1914) in cemetery D (Figure 3.8). The site is underneath an abandoned Islamic period town. Patch was only able to note the presence of Predynastic ceramics. Since a Predynastic cemetery was located nearby (el-Sayed 1979), Patch thought that this site might be the related settlement.

Egyptian excavations south of the Seti temple have revealed a settlement and Early Dynastic cemetery (el-Aref 2016; Habachi 1939; Hossein 2011; Patch 1991:414, 448). Additionally Patch (1991:423, S83-52) located a possible raw material collection and tool processing location on the edge of the high desert with extensive lithic debitage and a some nondiagnostic Predynastic(?) sherds. However the lithic reduction activities and in this area near and on the high desert cliffs have a very long time span dating back to the Middle Paleolithic (Olszewski et al. 2005, 2010), so the relationship between the lithic reduction activities and Predynastic ceramics remain to be understood.

\footnotetext{
${ }^{13}$ The tell had been eaten away by sebakh digging, leaving the earlier layers accessible in many areas.
} 


\section{The Abydos funerary enclosures}

At the edge of the low desert just behind the Kom es-Sultan settlement are a series of Early Dynastic mud brick funerary enclosures (Adams and O’Connor 2003; Bestock 2008, 2009; O’Connor 2009:158-181). These structures are the temple component of the royal tombs which were located further back in the desert. Each consists of a large, high wall enclosing an open space and a smaller temple building. Some also had a smaller perimeter wall. The pyramid complex around the Third Dynasty step pyramid of Djoser incorporates many of these features, but with an added pyramid, showing that the Abydos enclosures were the direct precursors to later royal funerary monuments. Enclosures have been identified for most of the First Dynasty kings, and the last two kings of the Second Dynasty. Only the last enclosure, that belonging to Khasekhemwy remains standing. The rest were intentionally destroyed (sent into the afterlife?) probably in association with funerary ceremonies. The structures were very large.

Khasekhemwy's funerary enclosure was the largest: $10,395 \mathrm{~m}^{2}$ in area with walls $11 \mathrm{~m}$ high and 5m thick (O’Connor 1999:102). These structures are the earliest examples of monumental architecture in Egypt. The First Dynasty enclosures were surrounded by human sacrificial subsidiary burials ('cemetery S’/ S83-59: Patch 1991:434-435; Peet 1914:30-34; Petrie 1925). Funeral processions probably linked the town, enclosures and the royal cemetery.

\section{Abydos cemeteries}

There are many cemeteries and tombs in the Abydos area, the most important of which is Umm el-Qa'ab. Due to its time span, excavation history, and spectacular finds, the cemetery has factored into many studies of chronology, social inequality, the development of the state, and the 
development of writing. Umm el-Qa'ab is located about $1.5 \mathrm{~km}$ into the low desert from the edge of the cultivation, at the mouth of a wadi leading out of the high desert (Figure 3.8). The cemetery actually consists of three parts: cemetery $\mathrm{U}$ with the earliest graves, cemetery B with graves of Dynasty 0 rulers, and the royal cemetery with tombs of all the First Dynasty rulers and the last two of the Second Dynasty. The cemeteries were excavated a number of times around the beginning of the 20th century (Amelineau 1899; Naville 1914; Peet 1914; Petrie 1900, 1901, 1902), and most recently by the German Institute since the 1970s (e.g., Dreyer 1992, 2008, 2009, 2010; Dreyer et al. 1996, 1998, 2000, 2003, 2006, 2011). The cemetery is large, with over 600 tombs in cemetery $\mathrm{U}$, another 5 large tombs with 34 subsidiary graves in cemetery $\mathrm{B}$, and nine royal tombs accompanied by over 800 subsidiary burials. Umm el-Qa'ab shows a long duration with basically continuous development from the beginning of the NI into the First and Second Dynasties (Hartmann 2011a). In the very earliest period (NIA-C ${ }^{14}$ ) graves clustered around a few of the oldest burials which were each located some distance apart from each other. During the NIC-IIA just a few of these groups remained in use, and eventually all burials clustered around one of them (ibid.:931-932). By the late NIIC period cemetery U was reserved for large elite burials (ibid.:934; Dreyer 1999:121).

One of the most important discoveries was the partially intact tomb U-J, which bore evidence for foreign trade and the early development of writing (Dreyer 1992, 2011). Small bone or ivory labels were found in the tomb, each with a few very early hieroglyphs, likely recording the amounts and sources of the goods in the tomb. One chamber of the tomb was filled with jars made in the style of Levantine pottery. Items from other parts of Umm el-Qa'ab are particularly

\footnotetext{
${ }^{14}$ Here Hartmann's phases were converted to Hendrickx's Naqada chronology to facilitate comparison with other dates given throughout this study.
} 
significant for this dissertation, including spectacular flaked-stone swords, ripple-flaked knives, and fishtail knives (Dreyer 1999, 2010; Hikade 1997). The Umm el-Qa’ab cemetery remained important to Egyptians during the Pharaonic periods, eventually being considered the burial place of Osiris. Its status as a pilgrimage site is attested to by numerous inscriptions and the mounds of Pharaonic offering pottery which give the site its name in Arabic: Mother of Pots.

A number of other cemeteries have been found in the Abydos area (Figure 3.8). They cover all the periods from NIA-NIII. Most are only known from turn of the century publications, and accordingly less detailed information is known about these cemeteries. Lithic artifacts from some of these cemeteries are mentioned in this study, including one concave base projectile point which was found by the Abydos Middle Cemetery project (AMC, University of Michigan, directed by Janet Richards). This area corresponds to cemeteries E/G which extend under the Old Kingdom remains currently being excavated by the AMC project.

\section{Nag el-Qarmila}

Nag el-Qarmila ( $\left.24^{\circ} 14^{\prime} 9.5856 " \mathrm{~N}, 32^{\circ} 51^{\prime} 40.2258 " \mathrm{E}\right)$ is located on the west bank of the Nile, $\sim 17 \mathrm{~km}$ north of the modern town of Aswan (Figure 3.18). It is situated in the mouth of a small wadi that branches off of the north side of the larger Wadi Kubbaniya. The Predynastic remains are in the low desert adjacent to the cultivation (Figure 3.19) (Gatto et al. 2009c). The overall site has three components: the habitation area (WK15), a storage area on a nearby terrace (WK22), and the cemetery (WK14) (Figure 3.20). A road cuts through the site to the east, and its construction likely impacted the remains. Additionally Predynastic pottery has been found in surface survey of the nearby cultivated land, so the cultural materials probably extended farther east toward the Nile. The sites date to the NIC-IIB (Dee et al. 2013 chronology) and the 
cemetery was re-used during the NIICD (Gatto 2014:100, pers. comm.). Radiocarbon dates corroborate these relative dates (Gatto et al. 2009a).

Nag el-Qarmila is an important site for many reasons. It is the only settlement site dating to the NIC-IIA ever studied south of Hierakonpolis (Gatto 2011:866). The settlement and cemetery are also undeniably related to each other. Additionally, Nag el-Qarmila is a rare example of a small farming village. Such villages are often assumed to have existed, but are less rarely located and studied. This site also gives insight into cultural interaction on a border region. Nubian ceramics make up just under $10 \%$ of the pottery from the settlement showing local Nubian influence in a Naqada culture settlement (Gatto 2014). Nubian character is also detectable in the use of quartz/chalcedony, and small pebbles for flaked stone tools, and in features of the later burials at the cemetery. The spread of Naqada culture into this region and the diachronic interaction between Nubian and Naqada culture took place in conjunction with the emergence of social stratification and centralization and resulted in complex 'cultural entanglement' for this area that was materialized in a number of ways (Gatto 2014). Nubian identity was materialized most explicitly in tombs assemblages, while hybridization of domestic objects, combining Naqadian and Nubian elements, occasionally occurred among the settlements artifacts. Additionally aspects of material culture which are purely local and unique to this area, different form either Naqadian or Nubian examples, have also been identified.

Nag el-Qarmila was found during a 2005-2006 survey of the area by the Aswan-Kom Ombo Archaeological Project (AKAP), directed by Maria Carmela Gatto (Gatto and Giuliani 200). Rescue excavations began in 2007 because the site was in danger of destruction due to the expansion of modern villages and farmland, and continued through 2012. The site has now been substantially built over. The Nag el-Qarmila localities were excavated in 'areas', which could 
range from 5m x 5m to any size deemed appropriate for the terrain. Each 'area' was divided into 1x1m 'squares' and given an alphanumeric code (e.g., C1, C2, etc) in order to provide relatively tight horizontal control of proveniences. Feature and level numbers were assigned consecutively within each area, but the materials from each square were bagged separately. The levels consisted of natural or cultural strata which were excavated in arbitrary $10 \mathrm{~cm}$ levels within the natural/cultural layers, although most strata in the settlement were not thick enough to merit the arbitrary vertical divisions. Each bag of collected material within a square was assigned an unique tracking number (AKAP\#) according to material (e.g., AKAP 1042 lithics, AKAP 1043 ceramics, etc). All of the excavated material was screened through 4mm or smaller mesh. Analysis of the ceramics, lithics, osteological remains, and other artifacts is ongoing.

\section{Nag el-Qarmila settlement WK15 (Wadi Kubbaniya 15)}

The settlement WK15 is located on the north side of the wadi, and is $~ 5000 \mathrm{~m}^{2} / .5 \mathrm{ha}$ size (Gatto et al. 2009a:193). Four 'areas' have been excavated within WK15 to date (Figure 3.20). These excavations reveled many intact features including hearths, post holes, in situ pottery, plastered pits, a child burial, and more. The features indicate that there was little postdepositional disturbance of the site. However some parts of the settlement were impacted by (ancient?) silt extraction, and construction of modern facilitations including the road, houses, boundary walls, and gardens.

Lithic artifacts were analyzed from Area A (here) and Area B (Gatto et al. 2009a; Usai 2012). Area B (Gatto et al. 2009a) is in a the central portion of the settlement. Three levels of activity were identified in this area. The uppermost consisted only of two hearths. In the middle layer a living surface of compact sand and ash was in the NW corner of the Area. Resting on top 
was a shell bracelet fragment and portion of a ceramic model boat covered in ocher (Figure 3.21). Two meters to the SE an intact vessel with repair holes was set into the ground (Figure 3.22). The earliest layer in Area B (Figure 3.23) contained a stratified sequence of four hearths, with a fragment of a pot in situ next to them. Just to the east were two mud-lined pits, which may have been large post holes or storage pits. Adjacent to these features, and to the south was a floor surface of compact silty soil, and at least four post holes. It was not possible to define a precise architectural pattern outlining the structure. The remains indicate the presence of a structure with an associated cooking and storage area. Additional artifacts from area B are another boat model fragment, a copper ring or earring, upper and lower grinding stones, ceramic fragments, lithic tools and debitage, and faunal remains. Boats in Predynastic iconography are related to the solar cycle (D. Darnell 2007, 2009; Huyge 2002; Lippiello 2012), and these figurines can be considered indications of ritual activities. However on the whole Area B can be interpreted as a habitation area with evidence for food preparation and storage, and possibly some domestic ritual activities.

Area A is situated in what was probably on the outer edge of the settlement, to the south (Figure 3.20). The surface may have been affected by erosion or deflation. There were two main levels of activity evident in area A. The features in uppermost level (Figure 3.24), consisted of multiple hearths, a large in situ stone mortar (Figure 3.25), and a small pit filled with stones. Notable associated artifacts included an upper grinder, a hammerstone (pestle?), a spindle whorl, a fragment of a sherd with a repair hole, and a sherd with post-firing incisions. The lower layer (Figure 3.26) of actives consisted of a few artifact concentrations and a child burial (Figure 3.27) on the south side of the unit. The burial was underneath a burnt surface (feature 2) but not directly associated with it because the two features were separated by sand layers. A raptor talon 
was found nearby, and a transverse arrowhead was found in the hard concreted soil that around the body, but it is not clear whether the projectile was a grave good or incidental. This child was about 1 year old +/- 4 months, and has the earliest identified case of scurvy in Egypt (Pitre et al. 2016). The practice of burying infants in settlements is known from other Predynastic habitation sites (e.g., Adaïma, Midant-Reynes and Buchez 2002; Badari sites, Brunton and CatonThompson 1928; Maadi, Caneva et al 1989:288; Naqada, Petrie and Quibell 1896:2).

The frequency of lithic cores was much higher in area A (see Ch. 4.3), as was the frequency of piece esquilles which are either cores or wedging tools (Bradbury 2010; LeBlanc 1992; Shott 1999). Either way, the lithic remains, along with the hearths, grinding stones, spindle whorl, and the lack of any clear structural features or midden deposits indicate that this area which was likely used for production activities, at least in the later phase.

\section{Nag el-Qarmila WK22 (Wadi Kubbaniya 22)}

WK22 is located on the rocky natural sandstone terrace south of WK15 (Figure 3.20). It was at first thought to be a cemetery (Gatto et al. 2009), but excavations in 2012 revealed that the area is better interpreted as a storage area, later re-used for a few burials, including children. The ceramics found at the site date it to NIC-IIAB, contemporary with the settlement and the early phase of the cemetery. Covering at least $225 \mathrm{~m}^{2}$, the main features identified at WK22 were a number of pits that varied in size and depth (Figure 3.28). Some were quite small and shallow. Some pits cut through earlier ones, showing that the area was used over time. Only three of the 14 pit features contained human remains. Some of the bones were from young individuals, and the bodies were highly disturbed and only found in the upper layers of the pits. No bones were found at the bottom of the pits as is usual for small bones disturbed primary burials. Two of the 
pits were filled with very clean loose sand, almost entirely devoid of artifacts. This sand is likely intentional fill and not wind accumulation. Charred grains of emmer wheat, barley, and weed seeds (Gatto 2014:100) were found near the bottom of one of the deepest pits in this clean sand layer. Very few ceramics or lithics were found at all compared to the settlement WK15, and cemetery WK14. The finds of grains, grinding stones, the general paucity of artifacts, and the variability in size and depth of the pits led to an interpretation of WK15 as a storage area. All of the lithic artifacts from WK22 (numbering only 50 in total) were studied as part of this dissertation. The analysis (Ch. 4.3) showed that there was a very high percentage of tools, and a low frequency of cores and debitage.

\section{Nag el-Qarmila cemetery WK14 (Wadi Kubbaniya 14)}

The cemetery WK14 is located just north of the WK15 settlement area, in the slopes of a sandy river terrace (Figure 3.20). As of 2012, excavations indicate that the cemetery covered at least $3250 \mathrm{~m}^{2} /$.33ha. At least 30 burial pits have been excavated, 22 more were identified, and the cemetery must have contained more. Erosion and ancient plundering have affected the burials. Two phases of use were detectable in the cemetery. The first phase corresponds in date to the settlement at WK15, and consists of small pit burials. This first phase is present in the northwestern part of the cemetery, on the sandy river terrace slopes (Area A and Trench 9). The second phase dates slightly later (NIICD-NIIIA1), and in this phase there is a much higher frequency of Nubian ceramics, along with Nubian tomb characteristics such as slab coverings and lateral niches. These later tombs extend from the northeast to the southwest of the cemetery, and almost encroach on the early settlement, which was most likely abandoned by that time. The earlier phase of the cemetery must be associated directly with the inhabitants of the settlement 
because of the very close proximity and the contemporaneous time periods (Gatto et al. 2009a:195-201). Ceramics and other artifacts such as palettes and items of personal adornment were found in the cemetery, some in situ, again emphasizing the difference between the cemetery and WK22. A few lithic artifacts were recovered from general stratigraphic layers in the cemetery and occasionally from the fill of the tombs. Unfortunately time did not permit analysis of these materials. No striking lithic artifacts, such as the bifacial knives, were noted during excavation of the cemetery.

\section{Other Predynastic sites in the Aswan area}

A few more sites should be mentioned to put Nag el-Qarmila in its regional context. The site of Elephantine was located on an island in the first cataract of the Nile, about $17 \mathrm{~km}$ south of Nag el-Qarmila (Figure 3.18). This cataract formed a natural border that was also a political border during Pharaonic Egypt. The position of Elephantine in the middle of the Nile made it a strategic site for observing and controlling river traffic. As such, during the Pharaonic period Elephantine was an important border town, involved in trade and administration, with a fortress built there by the middle of the First Dynasty that lasted at least through the Old Kingdom (Kaiser 1999). Elephantine was also an important religious site. A regional temple of unusual design focused on a cleft in the rocks on Elephantine, and probably related to the arrival of the Nile flood. Subsequent temples dedicated to Satet and eventually to Khnum were built over this same spot, into the Greco-Roman Period.

The island was inhabited during the Predynastic period beginning by the IId/IIIa (Kopp 2006:90), which is slightly later than the Nag el-Qarmila settlement site. A limestone baboon figurine and the earliest examples of faience figurines were found at the rock niche that was the 
focus of later temples, indicating that this area was already a site of ritual activities by the NIIIA2/b (early Dynasty 0). Production remains were located in the area in front of rock cleft ( 10m away), with indications of ceramic and possibly copper production (ibid.: 90, Figure 10). Contemporary burials are also known on the island. Few lithic artifacts were recovered from the settlement area ( $\mathrm{N}=193)$, and these cover four stratigraphic periods from NIIIA2-NIIIC1 (Kopp 2006:16, 80). Due to the paucity of material plus the relatively late date of the site, it is not included in the overall comparison of lithic assemblages from settlement sites.

Nag el-Hamdulab is a rock art site located on the west bank of the Nile, 6km North of Elephantine and $11 \mathrm{~km}$ south of Nag el-Qarmila (Figure 3.18). The rock art at this site dates to late Dynasty 0 and shows how politically important the area was by that time (Hendrickx et al. 2012:1073). The rock art consists of a series of tableaux spread around the mouth of a wadi. The content and technical style indicate that they were all made at the same time and should be read together (ibid.:1073). These tableaux contain the earliest known images of a pharaoh, along with scenes of cattle hunting/herding, boats processions (at least one of which carries a shrine), animal sacrifice, female dancers alongside bulls, and beer production (ibid.: 1073-1080). Additionally, there is a short early hieroglyphic inscription referring to the 'Following of Horus' (Darnell 2015) which, in the Early Dynastic period, was the biennial procession of the King throughout the country to collect taxes and dole out judgment. The iconography includes many motifs known from Dynastic jubilee scenes (Williams and Logan 1987), blended with equivalent ones known for the Predynastic (such as Hierakonpolis Tomb 100), and other late Predynastic iconographic elements. The composition is an early representation of tax collection and the economic power of the pharaoh, and it is by no coincidence located in this natural and cultural borderland. Finds of charcoal, lithic artifacts, and Predynastic sherds among the modern village 
remains directly in front of Nag el-Hamdulab indicate that a settlement was located there (Gatto 2014:111,112).

A number of other settlement, cemetery, and rock art locales are known in the Aswan region, as identified in survey by the Aswan Kom-Ombo archaeological project, along with a few known from earlier archaeological work (Gatto 2014:110-114) (Figure 3.18). Gatto notes that there is a trend toward nucleation over the course of the Predynastic period in this area.

\subsection{Site Descriptions: Comparative Sites}

Due to an increasing interest in settlement studies, primarily over the last few decades, there is now published data on lithic artifacts from a number of Predynastic settlement sites. Brief descriptions of the sites, their contexts, collection strategies, and any associated cemeteries provide the necessary information for comparing the sites to each other and to the analyzed material from el-Mahâsna, Abydos, and Nag el-Qarmila. The specific contexts will be referenced for comparing the contexts of finds to expectations for the ritual production model. The main sites drawn on for comparative analysis were: Naqada sites (South Town, North town, KH3, KH4, KH7), Abadiya 2, Armant (MA21/83 and MA21a/83), Adaïma, and Hierakonpolis (localities 11C, 14, 24A, 25, 25D, 29, 29A, 29B). All of the sites are in the Nile Valley, were excavated relatively recently with thorough collection strategies, and have published material on the lithic artifacts. The sites will be discussed below, in order from north to south. Figure 2.2 shows their locations. 


\section{Naqada Area Settlement Sites}

The Naqada settlement sites are located 20km north of Luxor, on the west bank of the Nile (Figure 2.2). These sites are each located approximately $2 \mathrm{~km}$ apart, and are known as the Naqada-Khattara sites. The sites were excavated by Hassan and Hays as part of the Predynastic of Egypt project (Hassan 1981a; Hays 1976; Holmes 1989). Deposits were up to 1m thick (Hays 1976:552). All of the excavated material was screened (Holmes 1989:33). North Town and KH4 were surface collections only, so little is known about the contexts and therefore they are not included in the main comparative analysis, but reference was occasionally made to pertinent examples of artifacts from these sites. The lithic artifacts from the Naqada sites were analyzed by Holmes (1989).

North town is a large site (4ha) excavated by Petrie and Quibell (1896:2) and surveyed by Hassan and Hays (Holmes 1989:197). It dates to the late Naqada period, likely NIIcd into the NIII (Holmes 1989:197; Hendrickx and van den Brink 2002:378). No indications of mud brick architecture were found at the site, but small trenches were probably from reed walls or fences. Hearths, grinding stones, spindle whorls, and storage pits were identified at the site (Petrie and Quibell 1896:2). Finds of fire-bars supporting a large ceramic vat indicate that beer production took place here (Geller 1992). Holmes’ (1989:34) analyzed materials come from four 5m x 5m surface collection units.

South Town (Aka. Zawaydah) is a large settlement site (3ha) that dates mainly to the later Naqada period, NIIcd-NIII (Barocas et al. 1989:298-300; Hassan 1999:670; Hendrickx and van Den Brink 2002:378; Holmes 1989:195). Petrie and Quibell (1896:54,Pl85) found a very large mud brick wall or structure at the site, which unfortunately has not be re-located by subsequent 
excavations, but was probably in the northern part of the site. Remains of small trenches and post holes from wattle and daub constructions were identified. Administrative and ritual artifacts including seals, tokens, and over 100 human and animal figurine fragments were found in the southeastern part of the site (Barocas et al. 1989; Di Pietro 2011; Fattovich et al. 2007). The large mud-brick wall and the administrative artifacts, along with the site's association with an elite cemetery and a very large regular cemetery has led many scholars to consider it a politically important site, the paramount site in the Naqada region. Holmes analyzed lithic materials from 10 randomly selected $1 \mathrm{~m} \times 1 \mathrm{~m}$ units from six larger test trenches spread across the site, and reanalyzed artifacts from Petrie’s excavations which were housed in the Petrie Museum, University College London.

KH4 is the next settlement south of South Town, and it covers 2ha. It dates to the early Naqada period, I-IIab (Hassan and Matson 1989). Two 10m x 10m units were surface collected and the top few cm screened, but the site was not excavated (Holmes 1989:33, 194) so little is known about the contexts of the finds.

KH3 is about 3ha in size and dates to the early Naqada period, I-IIab, with an absolute date around 3830+/-75 B.C.E ${ }^{15}$ (Hassan and Matson 1989:309). Excavations revealed a number of 'household complexes’ each consisting of a habitation area and an animal pen (Holmes 1989:192). Features found in the habitation portions consisted of hearths, complete ceramic vessels, and posts from structures. Part of one such complex excavated in Area B had a hearth (with dung (fuel), seeds, and bone), and two lithic processing concentrations, all spread over a $12 \mathrm{~m}^{2}$ area. In Area X/XI hearths, grinding stones, a cache of complete pots, and a child burial

\footnotetext{
${ }^{15}$ Note that this does not take into account the different methods used by Dee et al. 2013 , so the dating or relative chronology may be slightly different.
} 
were found in the habitation portion, and dark sediments rich in dung characterized the animal pen area. The lithic artifacts analyzed by Holmes in Area B came from three $2 \mathrm{~m}$ x 2m squares, G54, G56, and I54, which include both the habitation and animal pen areas (Holmes 1989:33, 193-194). The analyzed materials from Area X/XI came from forty 2m x 2m squares excavated down 10cm (ibid.:33).

Site KH7 is smaller than the other sites, only around .21ha, and it also dates to the early Naqada period I-IIab, but may be slightly older within that sequence than KH3 or KH4. Investigations included four $5 \mathrm{~m} \times 5 \mathrm{~m}$ surface collections and one $3 \mathrm{~m} \times 2 \mathrm{~m}$ test unit that revealed a large pit filled with ashy charcoal rich material (Holmes 1989:33, 194). Holmes analyzed the lithic materials from the test pit and the surface collections. She noted that there was a lower density of lithic material at this site compared to the others (ibid.:195).

\section{Naqada Area Cemeteries}

Petrie and Quibell (1896) excavated three cemeteries near South Town: Cemeteries B, T, and the "great new race cemetery" which hereafter is referred to as the main cemetery. Cemetery T was a spatially separated cemetery reserved for a small number of elite burials, and included a later First Dynasty mastaba tomb known as the 'royal' tomb, which may have been the tomb of someone from the royal family. The tombs in this elite cemetery were large and some had mudbrick lining. The cemetery dates to NIIB-IIIB (Hendrickx and van den Brink 2002:360). In contrast, the main cemetery contained around 2000 graves, and is the largest known cemetery in Predynastic Egypt. Bard (1994) demonstrated that cemetery T, B, and the western and eastern portions of the main cemetery were differentiated based on types and quantities of grave goods. Cemetery T was the richest and most exclusive of all, but the main-west cluster was richer and 
more exclusive than the main-east cluster, and B was somewhat similar to main-west. The main cemetery covers a long time span, from NIA-IIIC1 (Hendrickx and van Den Brink 2002:360). Through a lifelong effort, Baumgartel (1970) reconstructed the grave assemblages based on Petrie's notes and visits to museum collections. Combined with the work of Payne (1987) who edited and updated this information, much is known about the grave assemblages from these Naqada cemeteries.

\section{Abadiya 2}

Abadiya 2 is a small site (at least .12ha) located a few kilometers south of KH7 in the Naqada region. Vermeersch et al. (2004) carried out rescue operations at the site before it was destroyed by the expansion of farmland. Two test units were excavated, each $12 \mathrm{~m}^{2}$, one in the north part of the site and one in the south. The units were excavated in arbitrary $10 \mathrm{~cm}$ levels, with the material was screened through $4 \mathrm{~mm}$ mesh. The deposits were about $50 \mathrm{~cm}$ thick consisting of loose yellow silty sand with charcoal, lithics, ceramics, and bone throughout, along with occasional patches of dung. Few features were identified and the deposits were interpreted as general settlement midden, affected by bioturbation. The site was dated to the NI-IIA/B. No cemetery is specifically associated with this site.

\section{Armant Settlements}

The settlement site of Armant is located on the west bank of the Nile, about $10 \mathrm{~km}$ south of modern Luxor, at the edge of the low desert. The site consists of two separate localities, MA21/83 and MA21a/83, on either side of a small wadi that may have held a seasonal pond. Ginter and Kozlowski (1994:41) considered the two localities part of one overall settlement site. 
This is supported by the find of a long fence or palisade wall (Area 300) 90m north of the two localities. It may have encircled the site or delineated a portion of it. Hendrickx and van den Brink (2002:379) give a combined size of .25ha for the Armant localities, but if the area with palisade wall is also factored in the site may have been over .5ha in area, based on Ginter and Kozlowski’s maps. Both localities date to the early part of the Naqada period, with absolute dates ranging from 4100-3600 BCE ${ }^{16}$ about NI-IIAB. MA21/83 and MA21a/83 were among a series of at least 15 Predynastic sites identified in an area of about $8 \mathrm{~km}$ by Ginter et al. (1985).

MA21/83 was divided into northern and southern portions for their analyzes, and MA21a/83 was excavated in three separate trenches. Ginter and Kozlowski (1994:38-45) detailed the features and interpreted the activities which took place in each of the excavated areas. The northern sector of MA21/83 was a subsistence activity and storage area consisting of hearths and storage pits, with light structures that may have been dwellings or windbreaks. The southern sector of MA21/83 contained multiple habitation structures with stone foundations and associated features including storage pits, hearths, a basket, and other pit features. Bifacial tool production was also concentrated in this area (Ginter et al. 1996). MA21a/83 Trench I was a subsistence activity area with storage pits, storage containers, and hearths. Trench II was also a subsistence activity area with flat hearths and pit hearths, and two huts made from posts that may have been dwellings. Trench III was midden zone, including refuse pits.

\footnotetext{
${ }^{16}$ Not calibrated to the shorter Naqada period dates given by Dee et al. 2013.
} 


\section{Armant Cemetery}

Mond and Meyers (1937) excavated the Armant cemetery, which is about 300 meters away from MA21/83, across a small wadi (Ginter et al. 1985: Fig 1). It should be noted that Ginter and colleagues also identified other Predynastic settlement localities nearby this cemetery, just 200 meters away, MA18/83 and MA19/83 (ibid.). The cemetery consists of three different parts: two large Dynasty 0 tombs, a few Predynastic tombs in area 1300, and the main cemetery in area 1400-1500, which contained 169 burials.

The location of the burials shifted over time, from south to north. This spatial difference allowed Kaiser (1957) to make his revisions of Petrie’s chronology. The graves in the main cemetery 1400-1500 date from Ic to IIIa2 (Bard 1994:54), showing that the cemetery continued to be used after MA21/83 and MA21a/83 were abandoned.

Bard (1994) analyzed the graves in the Armant cemetery as part of a study of the development of inequality. She found that the quantity of grave goods and the size of grave pits increased over time (ibid.:61-68). She also found that burials could only be differentiated into two basic hierarchical groups, a richer one and a poorer one, based on grave size and quantity of pottery. Bard (1994:72) considered Armant a small farming village lacking more than a twotiered internal hierarchy.

\section{Adaïma Settlement}

Adaïma is located on the west bank of the Nile, about $60 \mathrm{~km}$ upriver from Luxor and ( 25 km north of Hierakonpolis). The whole spread of archaeological remains including settlement and cemeteries is over 35 ha in size. However the habitation areas occupy only a portion of the total archaeological zone. The settlement was occupied from NI-III, but the main 
time period was NI-NIIC, after which the settlement dwindled (Buchez 2011a).

The northern portion of the settlement is an area devoted to grain processing and storage. A series of perpendicular wall trenches for wattle and daub fencing surrounded 73 shallow wide pits that ranged in size from 13-145 cm, averaging 45cm wide, and only around 8cm deep (Midant-Reynes 1999:128). Seeds of wheat and barley were recovered from pit fillings.

The southern area contained the main habitation zone. Excavation of one portion of this habitation zone reveled structures made from wattle and daub as was evident from the arrangement of post holes in elongated oval shapes. The best preserved of the structural footprints measured 4.3m x1.2m (Midant-Reynes and Buchez 2002:37, 129). Associated features, generally outside the structures, included hearths, storage jars, pot emplacements, grinding stones, and trash pits. Four infant burials were found in this habitation area. Not only in this excavated area, but throughout the whole southern portion of the site distinct habitation or household areas with sets of structures or dwellings could be identified, with different 'units' located 100-400m apart, and each rebuilt in place over time (Buchez 2011a:33, 36). These were interpreted as family groupings. This interpretation is supported by evidence of grave grouping in the cemeteries (see below).

Lithic artifacts were analyzed from the southern habitation area, excavation block 1001 and extensions, which consisted of $395 \mathrm{~m}$ x 5m excavation units, totaling $975 \mathrm{~m}^{2}$ (Midant-Reynes and Prost 2002). The deposit was up to 90 cm deep (Midant-Reynes and Buchez 2002:141-148). The materials were dry screened through $5 \mathrm{~mm}$ mesh (ibid.:12). 


\section{Adaïma Cemetery}

Two cemeteries were associated with the site of Adaïma, known as the West cemetery and the East cemetery (Crubézy et al. 2002; Debono 1971; Garstang 1907; de Morgan 1912; Needler 1984; Sauneron 1974). They are located only a few hundred meters away from the settlement. Badly plundered and excavated by archaeologists numerous times, the cemeteries may have originally contained around 1500 burials (Midant-Reynes 1999:128). The West cemetery was used from the NI through the Third Dynasty (Buchez 2011a:32). The East cemetery was used only for child burials from the NIIC on. The graves were organized into clusters that probably represent family groupings based on the distribution of Nubian pottery (ibid.). There was also some segregation of the cemeteries by class, with the wealthiest graves in a distinct area of the West cemetery. After the NIID, wealthy graves were not apparent, and the overall number of burials began to decline. This is interpreted as movement of the population, especially the wealthiest people, out of the area entirely (Buchez 2011a:35,38).

\section{Hierakonpolis}

Hierakonpolis is located on the west bank of the Nile, approximately half way between the modern cities of Luxor and Aswan (Figure 2.2). The overall site of Hierakonpolis consists of many different components spread for about $1.5 \mathrm{~km}$ along the edge of the cultivation and extending at least $2 \mathrm{~km}$ back into the mouth of Wadi Abu Suffian (Figure 3.29). The main large settlement area lies in the low desert along the edge of the cultivation, and there is another settlement $2 \mathrm{~km}$ back in into wadi near the elite cemetery. An Early Dynastic and later settlement was located in an area now within the cultivation. This later settlement is called Kom el-Ahmar or Nekhen, and is known for the temple there where deposits of votive offerings (the 'main 
deposit') were found, many of which date to the late Predynastic period, including the famous Narmer Palette. Hierakonpolis has been proposed to be an early urban center and the seat of one of the earliest forms of kingship in the Nile Valley. Here the discussion is confined to the main localities with substantial published information on Predynastic lithic artifacts (localities 11C, 14, 24A, 25, 25D, 29, 29A, 29B).

\section{Hierakonpolis HK29}

HK29 is the designation for a large area of Predynastic habitation remains along the edge of the cultivation (Figure 3.29). This should not be confused with the ritual center at HK29A (see below). Hoffman (1980a) excavated a habitation area in HK29, with a dwelling, a fenced yard, and a pottery production kiln. Hoffman dated the site to the Ib-Ic period (ibid.:129), and radiocarbon dates yielded an average estimated date around 3500BCE (Hassan 1984), which should be somewhere around the NIC-IIAB considering the revised chronology of Dee et al. (2013). The dwelling was semi-subterranean and composed of wattle and daub with 8 wooden posts. Features in the house include an oven, a large storage pot, and an upright pottery slab that might have functioned as a heat shield for the oven (Hoffman 1980:131-132). A few small wattle and daub outbuildings were also present in the enclosed fenced area, along with the kiln, which was about 5x6m in size (Holmes 1989:287). Faunal remains associated with these habitation remains include cattle, sheep/goat, and pig (ibid.). The lithic artifacts discussed in the comparative analysis here come from the kiln area square 10L10 (Holmes 1989) and the house area square 17L13 (Holmes 1996). All of the material was collected by screening through 6mm mesh (Hoffman 1980:124). 


\section{Hierakonpolis HK29A}

HK29A is located in the central area of the Hierakonpolis settlement in the low desert along the alluvium (Figure 3.29). It is a large enclosure which has been interpreted as a ritual or ceremonial site (Friedman 1996, 2009b). Many of the aspects are similar to those from elMahâsna Block 3 (Ch. 3.4). Because this is a critical context for this dissertation, the argument that it is a ritual activity area is discussed in some detail here.

The two main phases of use at HK29A date to the NII(A)B-C, and NIID2- ${ }^{17}$ NIIIA (Friedman 2009b:79). The structure consists of an unusually large oblong courtyard (13m x 45m) (ibid.:81). Other known structures from this period rarely exceed six meters, often smaller (Tristant 2004:123-125). The walls and floors show a series of renewals. At least four episodes of floor plastering indicate the long use-life and care of the structure (Friedman 2009b:91). The walls were originally constructed of posts, some extremely large with post holes $1.5 \mathrm{~m}$ in diameter (Friedman 1996:24), and later the walls were replaced with mud brick (Friedman 2009b:95). The large size of the posts and the structure itself indicate a substantial labor investment. At one end of the courtyard a mud brick platform evokes the scenes of a king on a platform as shown on the Narmer mace head, and a tag of Den. At the other end of the courtyard a single post hole in the center of the floor brings to mind contemporary depictions of temples with flag/standard pole(s) at one end. The HK29A structure is surrounded or flanked by a large palisade wall, HK29B (Hikade 2011; Hikade et al. 2008). This wall may have enclosed HK29A and a nearby columned hall HK29B, perhaps forming a ceremonial precinct.

The ceramic assemblage associated with the structure was quite substantial but only

\footnotetext{
${ }^{17}$ Since the sub-phase IId1 is no longer thought to be valid (Buchez 2011b; see Ch 3.2), the date of the second phase should be considered NIID-IIIA.
} 
incorporated a limited range of forms. These include unique vessel types with distinctive shape and surface treatment that are only found at this ceremonial precinct and the elite HK6 cemetery: polished black egg-shaped vessels and matte red flaring collared rim jars (Friedman 2009b:8588; Hendrickx and Friedman 2003). Friedman also noted a lack of wear in the ceramic assemblage. The contexts and lack of wear support the idea that the vessels had a ritual function (Friedman 2009b:88). Additionally, like at el-Mahâsna, fragments of Decorated Ware pottery and imported pottery were also more frequent in HK29A than other areas of the settlement (ibid.).

In a study comparing faunal remains from HK29A to those from other Hierakonpolis localities and other Predynastic settlement sites, Linseele et al. (2009) demonstrated that the assemblage at HK29A was quite unusual for a Predynastic site. HK29A had a high frequency of hunted/wild animals compared to other sites. Those wild animals include Barbary sheep, cat, crocodile, gazelle, hare, hartebeest, hippopotamus, and turtle. There was a higher proportion of crocodile remains than at any other Predynastic site (ibid.:118). HK29A also had higher proportions of deep water fish than other sites, many of which are very large and required concerted coordinated efforts to obtain (ibid.:115-116). The diversity of so many wild animals in once place is also unique compared to other Predynastic sites besides HK6 elite cemetery and elMahâsna Block 3. Linseele et al. emphasize that many of these animals were iconographically significant in Predynastic Egypt and that they derive from both the Nile Valley and the deserts.

Other well represented iconographically important animals were domesticated dog and cattle. Dog bones were unusually frequent here, and some bore cut marks indicating that they were skinned or eaten. Dogs were directly correlated to elites in Predynastic iconography, such as on the Hunter's Palette where hunters (elites) wore dog tails (Baines 1993; Hendrickx 
2006a).The cattle remains were not only quite prevalent at HK29A, but were on the whole younger than elsewhere at Hierakonpolis, and on average larger (Linseele et al. 2009:127). There is evidence that the HK29A structure may have been used for activities related to the arrival of the annual flood, since a number of species such as Nile Perch, Nile Oyster, and gazelles would have been easier to obtain when the Nile flood was very low, as would have been the case in June, just before the flood arrived (Linseele et al. 2009:134). On the other hand, the few aquatic bird species identified such as heron and egret would have been available in the fall, during the decline of the flood (ibid.:120). These potentially seasonal species were found in both the earlier and later phases of use at HK29A (ibid.: Table 2). These data do not definitively solve the question of seasonal use, but they do point to repeated periodic use.

Other notable artifacts are flat or slightly concave slabs of clay thought to be model bread loves, possibly tokens or symbolic offerings (Friedman 2009b:96). Incised sherds are examples of early ostraca. A number of ceramic spindle whorls were also found at HK29A, like at elMahâsna Block 3.

There was also ample evidence at HK29A for the large-scale production of bifacial tools (including knives and projectile points), along with beads, and ground stone (Holmes 1992a). The evidence is discussed in detail in Ch. 6.1. The lithic production must have made up an integral activity associated with the enclosure. 143kg of lithic material were collected from the 1985-1986 excavations of a 600m² area in HK29A (Holmes 1992a:39). Holmes analyzed 46\% of this material by weight, about 54,000 pieces (ibid.). 


\section{Hierakonpolis HK29B}

About 40m north of HK29A, in an area designated HK29B, the remains of a palisade wall were found (Hikade 2011; Hikade et al 2008). About 50m of the wall have been uncovered. There were two construction phases, and earlier one with very large post holes $(70-110 \mathrm{~cm})$ and a later one consisting of a narrower trench (Hikade 2011). The remains mainly date to the NIID, with some earlier and later material (ibid.), so it is largely contemporary with the HK29A structure. The orientation of the palisade wall is the same as that of the HK29A enclosure and the columned hall in HK25 (see below), both of which are structures for ritual activities. Therefore it is possible that this palisade enclosed a ceremonial complex within the greater Hierakonpolis settlement (Hikade 2011:105). Hikade’s analyzed lithic material comes from five 5m x 5m squares excavated during the 2005/2006 seasons (Hikade et al. 2008:155). The deposits were sometimes shallow, only 5-10 cm thick, but the post holes were much deeper, cut into the sterile soil (ibid.).

\section{Hierakonpolis HK25}

A columned hall was found in HK25 (Hikade 2011, 2008; Hikade et al. 2008), located about 80 meters northwest of HK29B. The hall measures at least 20m x 8m and was built of at least 50 ‘columns’ or posts arranged in a grid and set into a thick mud floor (Hikade 2011:9396). Some post holes contained the remains of wooden posts up to $40 \mathrm{~cm}$ thick. A thick layer of clean sand was laid underneath the floor surface, and similar practices have been identified in other temple sites such as el-Mahâsna Block 3, and later Pharaonic temples. Remains of roofing materials were found above the floor. The structure has the same orientation as HK29A and HK29B, and a similar date, mainly in the NIID and NIIB-C (ibid.). The only other known 
Predynastic columned halls in Egypt are at H6, the Hierakonpolis elite cemetery. The large size and investment in the structure, its use of columns (which is known from later temples and contemporary funerary structures), and the layer of clean white sand below the floor all indicate that this should be considered a ritual structure.

Analysis of the associated artifacts gives some idea of the ritual activities that took place at this structure. At least 776 fragments bifacial tools, along with over 200 small natural ringshaped flint nodules, and fragments from 10 mace heads were found in this area, and all were burnt (Friedman and Nagaya 2013). The bifacial tools represented included at least 24 fishtail knives, 17 bifacial knives, 3 rhomboid lances, and 15 concave-base arrowheads (ibid.). Stylistically, these tools date to the NI-IIA, earlier than the rest of the remains associate with the structure, so they could be heirlooms (Hikade et al. 2008). Finely flaked bifacial tools such as these are actually quite rare in settlements, so to find so many, all treated in the same way indicates that they were all likely deposited as part of a distinctive activity. Distribution analysis shows that each type was centered in a slightly different part of the structure, indicating that each type was probably burned separately (Friedman and Nagaya 2013). The floor of the structure and the sand directly above it contained very few finds (Hikade 2011:105). The burnt deposits were presumably above the relatively clean material. It is hard to believe that such a unique burnt artifact assemblage is completely unconnected to such a unique building. Perhaps the burnt assemblage relates to activities associated with the 'closing' of the structure.

The ceramic assemblage parallels the dates and the ritual nature of the activities in HK25. The ceramics mainly date to the NIID and NIIB-C. The vessels include the distinct ceramic forms only otherwise found at HK29A and the elite cemetery HK6 (Hikade 2011:102): polished black egg-shaped vessels and matte red flaring collared rim jars (Friedman 2009b:85-88; 
Hendrickx and Friedman 2003). Ceramics in the NE part of the excavated area, where all the burnt bifacial tools were found, included White Cross-Lined wares and date to the NI-IIA. Also found were sherds incised with images of plants and in one case a bird. The incisions were definitely made after the vessels was broken, so they are early ostraca.

The lithic material analyzed by Hikade comes from ten $5 \mathrm{~m}$ x 5m units, which had deposits over 50cm thick (Hikade et al. 2008). Hikade reported the burnt bifacial tool fragments separately from the rest of the assemblage.

\section{Hierakonpolis 24A and 25D}

HK24A and 25D are located in the low desert along the edge of the cultivation, on the north side of the depression formed by the final extent of Wadi Abu Suffian (Figure 3.29). Just across this depression to the south are the settlement remains containing HK29, HK29A, and HK25. HK24A is a beer production facility, consisting of at least six vats arranged in two rows with evidence for heating (Geller 1992a,b). Black residue from the interior of the vats contained emmer wheat grains, and residue analysis revealed compounds identified with "all phases of the biosynthetic fermentation system” (Geller 1992a:21). Geller (1992b:118-119) gave a relative date of Ib-IIa for the structure. However the C14 absolute date was calibrated to 3500-3400 BCE, which according to Dee et al. 2013's scheme is closer to NIIA-B (Table 3.1). The lithic artifacts analyzed by Holmes (1996) come from a 5m x 5m unit that included most of the beermaking structure, square 360L420, unit 2.

HK25D is only 100 meters away (separate from the above described Hk25), and consisted of a silty platform with six shallow depressions of fire-reddened silt (Geller 1992b:9598). Mud-crusted sherds were dense on top of the platform and may be related to the ephemeral 
superstructure(s). Emmer wheat grains and charred wood were found in the depression features. Geller interpreted the area as a bread baking facility due to the light heat indicated and the proximity to the beer production area, which may have used bread in the beer recipe. Geller (1992b:102) dated the remains to the IIa period. The lithic artifacts come from the 5m x 5m square (502.5L387.5) in which this baking platform was found (Geller 1992b:95; Holmes 1996). All the excavated materials from both HK24A and HK25D were screened through 6mm mesh (Geller 1992b:95)

A circular mud brick structure lined with mud, likely a storage facility, was found at HK24B (Takamiya 2011b; Takamiya and Shirai 2010). Outside this structure was a layer of chaff and straw from barley and emmer wheat. Another beer production facility, even larger than the HK24A structure, was found adjacent to the circular silo. The facilities date to the mid Naqada II period (ibid.). These finds confirm that the activities in the HK24A-24B-25D area focused on grain based food production.

\section{Hierakonpolis HK 14 and HK11C}

HK14 and HK11C are a settlement area about 2km from the edge of the cultivation, in Wadi Abu Suffian, on the southeast side of the wadi (Figure 3.29). The settlement is across from and just east of the elite cemetery HK6. HK14 is an extension of HK11 into the wadi (Fairservice 1971-1972:Fig 1; Harlan 1982). The presence of White Cross-Lined Ware sherds dates the locality to the I-IIa. In 1969 Hoffman (1971-2) excavated a test pit in the area. He did not encounter any features, and interpreted the area as a midden accumulation. Artifacts include ceramics, lithics, well preserved faunal remains, coprolites, and a wooden arrowhead. The material was hand collected, not screened, but effort was made to collect as many artifacts as 
possible (Hoffman 1971-72:49). Holmes (1996) analyzed the lithic material from this area.

HK11C was a settlement site with living areas, animal pens, and zones of large-scale production activities. The lithic artifacts from HK11C discussed in this study come from Operation A (a beer production kiln) and excavations by Harlan in an animal pen area. However each of the main excavated areas in HK11C are discussed below to show the complex array of activities which took place in this settlement.

Harlan (1982; 1985; Khalifa 2012) excavated a mound in HK11C and identified a 2mdeep midden (Mound A/Test A). Thin lenses of material resulting from repeated dumping episodes were visible in the profile. The material consisted of general domestic refuse dating to the early Naqada period. Harlan identified four similar mounds throughout HK11C, which based on surface inspection also appeared to be middens. Square 0N6E was a 5m x 5m unit excavated by Harlan (1982) with a mud brick feature interpreted as a trough for watering and feeding animals, and a nearby fence. Reeds, branches, and barley were found on the trough, and a concentration of wheat and chaff was also found in the unit. The material was collected by screening through 6mm mesh and flotation (Harlan 1982:15). Holmes (1996) analyzed lithic material from sq 0N6E. Harlan also excavated a 'kiln’ which was later investigated more thoroughly by Takamiya (see below).

Excavations by Watrall $(2000,2001)$ farther to the northeast (area G) identified multiple phases of habitation in a house structure and fenced yard or animal pen area, dating from NICIIB (Watrall 2001). The two primary phases of occupation each contained a floor surface with wattle and daub structural elements, a fence, and a stone lined hearth outside the floor surface. Pot emplacements and in situ upper and lower grinding stones were also associated with some phases. A small trash deposit in a pit separated these two phases. After the later phase, part of the 
area was re-paved, and finds of dung indicate that the area was used as an animal pen. In the latest phase, NIIC, a large pit was dug through all the layers and used as a refuse dump.

Other areas of HK11C do not have a domestic character but instead were focused on large scale production activities. Some of the more unusual remains are from squares C3-4 and C10-11 (Baba 2010, 2011, 2012, 2013). In both of these locations large areas (11.5 x were walled off by low mud brick walls — the earliest known use of mud brick in a Predynastic settlement. The interiors were filled with ash. The interior of structure in C3-4 was excavated revealing a number of hearths below the ash. Finds of numerous fish bones and cattle bones suggest cooking. What's more, the skeletal elements represented were opposite of those found in HK29A (Baba 2013:13), head and small extremities, not the bones associated with the meaty parts. This area may have been a large-scale food preparation area for feasting done elsewhere. Outside of one of these walls over 1000 small 'potato sized' worked pieces of sandstone were found, and the excavators suggest that they may have been used as counters, keeping track of all the food being processed in this area. The remains date to the NIIC-D.

Other evidence for large-scale production in HK11C area comes from squares A6-A7 (operation A), and squares B4-B5 (Operation B). The activities in Operation B (Baba 2006, 2007, 2008a,b; Friedman 2004c) were mainly devoted to pottery production. In the earliest phase of this area there was a combined pottery and beer production kiln (Baba 2007:26-27) that dates to the NIC-IIB (Nekhen News 2011b:24). During a second phase of use a platform of burnt mud and potsherds was built over the earlier kiln, and used for firing pottery. Adjacent to the kiln were wattle and daub structures or fences, and 5+ caches of pottery tools containing over 1000 worked sherds for making pottery (Baba 2007, 2008b). This second phase dates to the latter half of the NII period, NIIC-D. 
Another beer production kiln was located in Operation A (Takamiya and Baba 2004; Takamiya and Aoki 2005; Takamiya and Endo 2007, 2011a). Part of the kiln was first excavated by Harlan in 1979 and was thought to be a shallow pit-updraft kiln, but later was determined to be part of the larger structure in squares A6-A7 (Takamiya and Baba 2004:19; Takamiya and Aoki 2005:18-19). The kiln consisted of at least 8 vats supported by firebars and a superstructure composed of fragmentary ceramic plates and mud. It dates to the NIIB-NIIC (Takamiya and Endo 2011a:729). Many phases of use were evident in the layers adjacent to the kiln, with wattle and daub structures, floor surfaces, and layers of sheep dung found underneath the layer of soot that marked the brewery surface level (Takamiya and Endo 2007:19, 2011a:728). The lithic artifacts from Operation A area were studied by Takamiya and Endo (2011a), with only 450 of the over 3000 pieces coming from the layers below the brewery surface.

\section{Hierakonpolis HK59 and others}

A series of pottery production sites were found in Wadi Abu Suffian on the low cliffs to the northwest above the elite cemetery HK6 (Friedman 1994:635-649; Geller 1984). These were localities 67, 59, 59A, 40, and 30. Although no lithic artifacts from these sites are discussed in this study, these sites are important for understanding settlement organization and specialized production. The sites date to the NIc-IIa (Friedman 1994:883-884), and consisted of ceramic sherd concentrations which were overwhelmingly dominated by untempered Black-Topped and Red-Polished wares. Many ceramic wasters were found among the sherds. Geller (1992:87) found a water-smoothed pit in a test excavation and Friedman (1994:636) suggests it may have been used for shaping pottery or preparing clay. Friedman (1994:646-648) argued that these kinds of ceramics were made by specialists based on the uniformity in form and technique for 
these ceramic wares across the country, and the remote location of the production sites at both Hierakonpolis and at Armant.

\section{Hierakonpolis cemeteries HK6 and HK43}

A number of cemeteries existed at Hierakonpolis (Figure 3.29), including HK6, the elite cemetery, and HK43, a non-elite cemetery. HK6 (Friedman 2008a,b, 2010, 2011; Friedman et al. 2011a,b) is located about $2 \mathrm{~km}$ from the edge of the cultivation, in Wadi Abu Suffian, distinctly separated from the rest of the cemeteries at the site. The main phase of use dates to the NIC-IIB with tomb complexes and substantial above ground architecture; and the cemetery was re-used again in the NIII period. An animated timeline available at the Hierakonpolis-online website very clearly demonstrates the different construction phases. Over 72 tombs have been identified so far, many of which were not for humans (see Table 6.9 for full references).

During the main phase of use at the cemetery central tombs were surrounded by satellite graves of family members and/or courtiers, who may even have been sacrificed, prefiguring the subsidiary burials around the Abydos royal tombs (Friedman 2011c:39). The tombs were also surrounded by wild and domestic animals, and the graves were situated within complexes of above-ground architecture. In one part of the cemetery a number of columned halls were built with no associated substructures — possibly the forerunners to the First Dynasty mud brick funerary enclosures at Abydos. A large wattle-and-daub plastered and painted enclosure wall surrounded the cemetery.

Offering deposits and artifacts have been found associated with the above-ground architecture, including the earliest known life size statue, which was later crushed into small fragments. Two offering caches in one of the columned hall structures each contained a fishtail 
knife, ground stone and flaked stone animal figurines, trapezoidal and bifacial projectile points, and unusual forms of ceramic vessels (Friedman 2006:7-8). Other spectacular finds include the earliest funerary masks, made of ceramic. Tomb 16 included hundreds of ceramic vessels (Friedman 2011c).

Of the over 100 animals found at HK6, the wild animals included hippopotamus, elephant, baboon, wild donkey, aurochs (wild bull), hartebeest, and wild cat. The domestic animals included cattle, sheep, goat and dog (Linseele et al. 2009:110-111). Friedman (2011c:40) interprets the wild animals as part of a display of power perhaps related to control over chaos, and also possibly related to a ruler's capacity to take on the abilities of the animals, as is paralleled in late Predynastic iconography that shows the king alternately depicted as human, as a bull, or as a falcon.

In contrast, the non-elite cemetery at HK43 had many more graves, but fewer grave goods and architectural investment. The cemetery is located in the low desert at the edge of the cultivation at the southern most end of the Predynastic remains (Figure 3.29), in a non-secluded location compared to HK6. There were at least 452 graves of NIIAB date in this cemetery (Friedman 2008a:20), and probably many more (thousands?) but the cemetery was heavily disturbed by modern activities. Rescue operations indicate that less than half of the tombs still retained grave goods, but some of the goods might be missing due to plundering. The burials that did contain grave goods usually had only 1-3 ceramic pots (ibid.). The tombs were very small, only large enough to fit a flexed body, and no indications of superstructures have been found. A few of the better preserved tombs contained lithic artifacts such as a fishtail knife with a reed handle (Friedman 2004b). Despite the relative dearth of material goods associated with these graves, they were certainly not without indications of lively funerary practices. The earliest 
evidence for mummification in Egypt comes from this cemetery, where some bodies had padding and wrapping around the hands and neck (Maish and Friedman 1999). Some burials were found with cut marks to the neck and the heads placed elsewhere in the burial (ibid.). Five cases of scalping have been identified through skulls with up to hundreds of cut marks (Dougherty 2004). More generally, the graves in the cemetery were placed in dense rings situated around blank central areas that contained concentrations of pottery, perhaps related to funerary feasts and rituals (Friedman 2008a).

\section{Additional Sites}

Besides the above sites which are comparable because of their overall cultural milieu and collection strategies, two more sites were pertinent to this study because of the availability of information on the lithic artifacts: the Badari sites, which are mainly known from museum collections, not modern excavations, and Maadi, which is in the lower Egyptian cultural sphere.

\section{Badari-region sites}

The Predynastic Badari sites are a series of settlements on the east bank of the Nile, over $300 \mathrm{~km}$ south of Cairo, and about $100 \mathrm{~km}$ downriver from Abydos. These settlements were excavated by Brunton in the 1920s and 1930s (Brunton and Caton-Thompson 1928). Brunton did not leave detailed information about the size of the settlements, but they do seem to vary, including many small sites and a few larger ones (Hendrickx and van den Brink 2002:375-376). Nor are there many plans of the settlement remains, but Brunton did mention floor surfaces, low mud walls, posts, post holes, stacks of pottery, and hearths. Firebars were found at site 3000/6, indicating that there may have been a beer brewery there. 
Brunton also excavated many sites dating to the earlier Badarian period in the same area, some of which were occupied continuously from the Badarian through the later Naqada period. By the Naqada III period, the sites were abandoned and re-used as cemeteries. One of the sites with continuous occupation was Hemmamiya (North Spur), which was excavated in detail by Caton-Thompson (Brunton and Caton-Thompson 1928). She found a series of small circular mud structures ranging mainly from .9-2.75m in size. Caton-Thompson thought that some might be houses, but others have interpreted them as storage facilities or animal pens (Holmes 1999:186; Holmes and Friedman 1994:124). Wattle and daub fences were also found in the upper layers of the site. Holmes analyzed materials from Brunton’s and Caton-Thompson’s excavations which are now housed in the University College London Petrie Museum. These artifact collections cannot be considered complete or representative samples due to selective collection and the vagaries of what actually ends up in a museum. However the presence certain lithic items is useful information.

\section{Maadi}

Maadi is a large settlement site (4ha) located at the apex of the Nile Delta, on the eastern side of the Nile (Seeher 1999b). Culturally it is a type site for the lower Egyptian culture, and the site dates to the equivalent of the Naqada NI- NIIb. Maadi is noted for the abundant evidence trade with the Levant, including imported pottery, stone tools (tabular scrapers), copper, and houses built in a style similar to that of the Chalcolithic Beersheba culture (Braun 2011; Hartung et al. 2003; Rizkana and Seeher 1989). Ironically, the site is now threatened and affected by expansion of a modern suburb which is a current enclave for foreigners. The ancient contexts identified in the Maadi settlement certainly included habitation contexts evidenced by the 
structural remains, including post holes, wattle-and-daub architecture, and subterranean

dwellings. However no specific activity zones were identified due to the spread of the settlement over time (ibid.:547). One cemetery was found 150m from the settlement, and another larger one was located $1 \mathrm{~km}$ to the south. Rizkana and Seeher (1988) published much of the lithic material from the settlement site.

\subsection{Depositional practices}

The method for evaluating the ritual production model involves looking at the archaeological contexts of artifacts. Such a method raises the question of whether the artifacts found in a given context are actually related to the features in that context. Evidence for disposal practices and taphonomic processes indicates that on the whole, artifacts do relate to the contexts in which they were found.

There is evidence that 'trash' was often disposed of very near to the structures it derived from, particularly for ritually significant 'trash'. At HK29A much of the material associated with this structure comes from a series of pits immediately adjacent to and outside (river side) of the North wall. The unusual nature of the assemblages in the pits with their high frequencies of wild animals and distinct ceramics complements the unusual shape and size of the HK29A structure. More importantly, the assemblages in the pits are comparable to the assemblages excavated in the structure itself indicating that they are all related (Friedman 2009b). The consistency of the assemblages indicates that they derived from clean-up episodes that took place on a regular basis (ibid.:85). The relative chronology of the pottery in the pits shows that they were filled from the east to the west (ibid.:88).

This practice of disposing items used in ritual activities near to their associated 'temple' 
was not limited to Hierakonpolis. A similar practice can be observed at el-Mahâsna. Block 2 was located just east (river side) of the ritual activity area in Block 3. Block 2 is on the very edge of the low desert escarpment as it begins to slope down to the cultivated fields below. The main stratum was made of inter-fingered lenses of material with more or less ash and charcoal that probably resulted from small deposition events over time (Anderson 2006:90). The only substantial feature associated with these layers was a large hearth located in the north part of Block 2. Below this layer was a collapsed fence. Artifacts had accumulated next to the fence before it collapsed, and Anderson interpreted it as a division of outdoor space (fence). Block 2 had the highest density of ceramics of any block, many of which were small and possibly trampled. Furthermore, the artifact assemblages of Blocks 2 and 3 bear some important similarities, with each area having relatively high frequencies of (beer?) drinking cups, the forelimbs of mammals (the 'choice cut'), Synodontis catfish (coordinated deep-water fishing), and spindle whorls (Anderson 2006:163, 191-194, 200, 233). Anderson (2006:162, 246) interpreted Block 2 as an outdoor activity area with trash disposal, associated with Block 3. This evidence shows that at el-Mahâsna, as at Hierakonpolis HK29A, the refuse from a ritual activity area was deposited near the structure.

Special disposal of ritually charged items is also known from later Pharaonic contexts. At the temple in the Hierakonpolis dynastic town site of Nekhen, numerous caches of objects were found associated with the temple. They were deposited singly and in groups, and while it is not clear whether they were votive offerings dating to the original use of the temple, or heirloom temple furniture collected over the years, they were certainly groups of artifacts which could not be deposited just anywhere (Whitehouse 2002). The main deposit included elaborately decorated palettes like the Narmer Palette, ivory figurines and handles, mace heads, faience objects, and 
more (Adams 1974; Quibell and Green 1902). Similarly at Abydos, Petrie (1903) found a cache of ivory and glazed figurines near the temple remains dating to the early First Dynasty. Examples of temple caching are known from other sites including Elephantine (Dreyer 1986) Tel Ibrahim Awad (van Haarlem 2001), and Tel el-Farkha (Chłodnicki et al. 2012).

Special disposal was not limited only to the rather distinct and obviously symbolic objects like figurines and decorated palettes. During the Pharaonic period, the leftover materials from mummification, known as embalming caches, which included bandages, natron, resins, and even ceramic vessel fragments, were often buried in or near the tomb of the deceased (EatonKrauss 2008).

In domestic contexts, trash pits, trash dumps, and midden accumulations were located in houseyards, between structures, or in open portions of the settlement more often than in abandoned structures. In the habitation section of the Adaïma settlement numerous trash deposits were identified, including ashy concentrations, deposits of organic material, and concentrations of mixed material, often in wide shallow pits (Midant-Reynes and Buchez 2002:66-69). These were mainly around and outside of the remains of structures as outlined by post holes (ibid.:126, 130). At KH7 a trash pit filled with ashy material was identified (Holmes 1989:194). Its exact relationship to other structures is not known, since no other features were identified, but the lack of features indicates that the pit was not in a house structure. At Armant MA21/83 pits of unknown function nearby house structures in the southern sector of the site may have been trash pits.

Other evidence indicates that trash was also dumped nearby houses, in addition to being dumped in pits. Hassan (1978, 1981a; Hays 1976) carried out microarchaeological analysis of archaeological remains and soil from the Naqada Khattara sites, particularly at KH3. This 
involved taking soil samples and characterizing all artifacts larger than $1 \mathrm{~mm}$, and sorting and characterizing the soil and artifacts in the 1-.01mm range (Hassan 1978:208-209). Hassan deduced that trash deposits were interspersed with dwellings in the Naqada sites (Hassan 1999:670). At Armant MA21a/83, trench II contained dwellings, and the trash midden deposits in the nearby Trench III were interpreted as deriving from the dwelling area in II (Ginter and Kozlowski 1994:41). At HK11C multiple deep midden accumulations were found in areas without structural remains, and were likely designated dumping areas that were used over a long period of time (Harlan 1982).

In the settlement contexts surveyed here there was only one case of trash dumping in an abandoned structure. At area G of HK11C Watrall (2001) recorded a large pit dug through two earlier house floor surfaces associated with wattle-and-daub architecture. However this pit was well defined and clearly differentiable from the earlier materials.

The environment and materials of Predynastic houses differ substantially from the tell accumulations that many archaeologists are familiar with. Rather than mud brick structures which could stand open when abandoned and were often built close together, in Predynastic Egypt the houses were most often built out of light materials on sandy surfaces (e.g. Maadi, Mahâsna, Naqada-Khattara sites, Armant, Adaïma, Nag el-Qarmila). ${ }^{18}$ Collapsed houses would have formed layers or piles rather than convenient pits.

Moreover there would have been ample room for disposal around the houses and around the habitation areas. Outdoor spaces (yards?) were often associated with the houses, such as the animal pen areas found at Naqada KH3, the fenced area adjacent to HK29, the outdoor space in 
el-Mahâsna Block 4 found by Anderson and associated with a structure excavated by Garstang, the structures and large fence in Block 1 at el-Mahâsna, and the structure with adjacent cooking area at Nag el Qarmila WK15. Similarly at each of the Armant localities habitation and subsistence production zones were identified. These data show that the houses were not packed in on each other, which would have limited the options for refuse disposal. At HK 11C multiple areas specifically reserved for trash dumping were identified, which must have accumulated over many years (Harlan 1982). All of this indicates that the artifacts found associated with domestic structures probably did not derive from the refuse of other houses.

More to the point, post-and-mat architecture and sandy surfaces make it quite likely that artifacts could easily be lost among the sands and reeds where they were used. In fact there is direct evidence for accumulations of artifacts against the reed fencing. Anderson (2006:95) found examples of this at el-Mahâsna, as did Watrall (2001) in HK11C.

Furthermore most sites were only minimally disturbed by natural taphonomic processes that could have resulted in substantial horizontal and vertical movement of artifacts. The existence of fragile features such as hearths, floor surfaces of compact sand, and even light fencing with the knots of the matting still in place, indicates that the sites were well preserved. The exception that proves the rule is Abadiya II where there was clear evidence of extensive bioturbation, seen in the contact between sterile soil and cultural layers, as well as in the return of aberrant $\mathrm{C}^{14}$ dates (Vermeersch et al. 2004). However there was also little evidence for in situ features such as hearths or floor surfaces at this site.

\footnotetext{
${ }^{18}$ The HK29 house (Hoffman 1980) was characterized as semi-subterranean and is somewhat unique, probably related to the presence of a silt layer at Hierakonpolis. The Hemmamiya mud circles have been
} 
There was certainly surface deflation at many sites, such as at Nag el-Qarmila (Gatto et al. 2009) but once past the surface levels other layers were intact. In the dry desert environment rain events were rare, and strong rain events that would lead to alluvial transportation of artifacts even rarer. When present, finds such as the refitting of figurine fragments from Block 3 indicate that any horizontal movement must have been minimal.

In sum depositional practices and natural taphonomic processes do not inhibit an association between artifacts and features from the same contexts. The Predynastic Egyptian contexts are well suited to a methodology that involves understanding artifacts based on comparisons of their find contexts.

\subsection{Settlement Patterns}

\section{Organization within settlements}

The above site descriptions indicate that many Predynastic settlements consisted of a number of distinct localities spread across the landscape. Some of these localities were for functionally different activities. For example, at Nag el-Qarmila there was a habitation area (WK15), and a separate storage area (WK22) 150m away. At Adaïma there was a habitation area in the southern portion of the site, and an area devoted to grain processing and storage in the northern portion of the site. Hierakonpolis is the best and most complicated example of functionally differentiated areas spread across a larger landscape. In the earliest phase, NINIIA(B) there were habitation areas at HK11C square G and HK29 (the latter included a pottery kiln), and by the end of this period there were dedicated production facilities at HK11C

\footnotetext{
interpreted as storage features or animal pens, rather than houses (Holmes and Friedman 1994).
} 
Operation B (beer and ceramics), at HK 24/HK25D (beer and bread), and at HK59/59A (ceramics). In the Naqada IIBC period many of these production areas continued, another beer production facility was added in HK11C (operation A), and a ritual precinct was founded at HK29A and HK25. By the Naqada IID period there was also large scale food production (meat and fish) occurring at HK11C squares C3-4 and C10-11, and the ritual precinct was bordered by a large palisade wall. In sum, Hierakonpolis always consisted of multiple localities spread across a large area, and these became more functionally distinct over time. The existence of an elite cemetery and a non-elite cemetery at two of the furthest points of the overall settlement along with the functional interdependence of the parts (e.g. only one ritual area) indicate that these localities should be considered one overall settlement, and not a number of independent sites that happen to be near each other.

Similarly, the Abydos Predynastic settlement sites should also be understood as a series of separate but interrelated localities. Certainly by Dynasty 0/Dynasty 1 there is a habitation area (possibly with a temple) close to the alluvium (Kom es-sultan), one or two beer production facilities slightly farther back in the low desert, an elite cemetery far back toward the entrance to the high desert, and a ritual activity area (funerary enclosures) between the settlement and cemetery (Figure 3.8).

On the other hand there is evidence that localities with the same functional activities could be repeated in one overall settlement. Examples include the above mentioned HK29 and HK11C square G, both with habitation areas, approximately 1.5-2km apart. Armant MA21/83 and $21 \mathrm{a} / 83$ constitute two domestic habitation areas separated by $25 \mathrm{~m}$ and a natural dip in the topography, yet they were both surrounded by a palisade wall. A similar situation was apparent at el-Mahâsna where two localities, Garstang's S1 and S2, were separated by a small natural 
depression. Both of the el-Mahâsna localities contained habitation remains. The multiple, basically contemporary beer production facilities at Hierakonpolis and Abydos are another example of repeated but separate areas devoted to the same activities.

The site of Maadi does not fit this pattern, as no specific activity zones were identified at the site, and it seemed to spread and shift over time (Seeher 1999b:547). However it is not surprising that Maadi exhibits a different pattern of internal settlement organization since it is in a different environmental region (Lower Egypt) and belongs to a different cultural milieu (Buto-Maadi culture). The Naqada-Khattara sites are the only Nile Valley sites so far known without documented separate localities. However this kind of settlement patterning for Predynastic upper Egyptian sites was not known at the time when the sites were surveyed, and so any outlying localities may not have been identified. An understanding of Predynastic settlement sites as composed of related but spatially separated localities can also explain the density of 'sites' identified by Ginter et al. (1985) in the Armant region. They located 15 Predynastic sites in a span of $8 \mathrm{~km}$. That would be a site every $500 \mathrm{~m}$ if they were distributed evenly. Actually the sites can be grouped into at least three stretches, with each group spreading over 1-2km, and with a space of $1-3 \mathrm{~km}$ between each group. ${ }^{19}$ It seems quite likely that each of these 'sites' might actually have been one of a series of related localities.

This kind of 'internal' settlement organization makes it very hard to estimate and compare site size. For some sites the area of an individual locality is given, which can be very small, only tenths of a hectare. For others the overall spread including much relatively blank space is included. And in many cases expansion of modern villages and farmland have made it

${ }^{19}$ Group 1: 21,20,19,18,18A,17, and Mond and Myers' site 1000 (following Buchez 2011); Group 2:16,15,14,14A; Group 3:8, 7(A,B,C), 6, 5, 4. 
impossible to understand whether there were originally more 'parts' to a site. Therefore caution should be used whenever comparing Predynastic site sizes.

The locations and distance of the different parts of a settlement are likely related to the features of the landscape to some degree. The sites were often arranged around wadi mouths or embayments. At Nag el-Qarmila and Armant the habitation areas were just above the lowest point in a wadi mouth, perhaps facilitating access to water, at least during the flood season. At Nag el-Qarmila the storage area was placed on a sandstone shelf which allowed for more durable storage structures than the edge of the sand dune where the main habitation was located. Similarly at Adaïma the grain processing area was on the firmer silt and gravel terrace, while the habitation remains were located in a sandy area. At Abydos and Hierakonpolis the elite cemeteries were set back from the settlement by $1-2 \mathrm{~km}$, in or near to the wadi itself. The sizes of wadi mouths vary considerably across Egypt, thus affecting the space available for a related series of localities. Hierakonpolis and Abydos may be examples of wadi mouths or embayments which were just the right size: quite large with room for plenty of population in comparison to places like Nag el-Qarmila, but small enough to be delineated. Patch (1991) noted that the Abydos embayment was actually the smallest in the Abydos area. El-Mahâsna, just north of Abydos, was not set within one of these naturally delimited areas. Instead it was at the edge of the cultivation in an extremely large desert embayment, with the high desert $~ 6 \mathrm{~km}$ behind the site. It is unknown how this more open environmental context would have affected the organization of the settlement, since so much of the surrounding area has been turned into farmland. Buchez (2011a) noted that Adaïma was a more spatially distinct unit than the Armant settlements, and thought that this was because Adaïma was situated in a large sandy plain-a kind of inverse relationship to available space so that localities clustered closer together in areas 
where the landscape did not delineate the settlement area. The Armant sites constitute a third environmental landscape. There each 'site' or locality was situated on the hillocks or promontories between wadi mouths, rather than in the wadi mouths. However the wadi mouths are small and very close together in this area, being only 100-250m wide according to Ginter et al.'s (1985) maps. They also do not appear to cut back into embayments, but to end rather linearly which hinders the identification of potentially related clusters. A similar scenario occurs at Badari where Predynastic sites were found rather linearly on spurs that were only $50-100 \mathrm{~m}$ apart (Brunton and Caton-Thompson 1928; Holmes and Friedman 1994).

\section{Distribution of settlements across the landscape}

Whether individual sites or clusters of localities, Predynastic settlements tended to space out across the landscape about $2 \mathrm{~km}$ apart in the early part of the period. Later in the Naqada period there was nucleation of sites into larger settlements, as is now well documented in multiple regions of the country.

Patch (1991) conducted a large-scale regional survey charting the location and date of settlements and cemeteries in the Abydos region. She found that in the early part of the period (Ic-IIc) settlements were located evenly across the landscape, except immediately around Abydos where there was greater spacing between the Abydos settlement group and the sites to the north and south. This spacing may have been related to the preeminence of the site, or may even have been intentionally maintained. After the Naqada IIc period there was a reduction in the number of sites, which, based on growth of certain cemeteries, Patch argued was due to the population nucleating into fewer sites (Abydos, Mahâsna, and Nag ed-Deir), rather than from people moving onto the alluvium. Similar patterns were observed in the Naqada region, where 
the Naqada I-II period sites were found spaced approximately every $2 \mathrm{~km}$ throughout the area, but the later sites were fewer and larger (South Town and North Town) (Hassan 1981a; Holmes 1989). Buchez (2011a) also argued for population nucleation based on evidence of the depopulation of farming villages at Adaïma and Armant. She observed that at Armant the number of settlement localities decreased over time, and at Adaïma the number of domestic units in the site also decreased over time. Meanwhile a gradual decease in population occurred in the cemeteries after the NIIC, with a precipitous drop in population after the NIIIA1. Since the presence of large wealthy graves also dropped, she argued that the changes were a result of people, especially wealthy people, moving to the more preeminent sites. Gatto (2014) also documented settlement nucleation in the Aswan region, with a reduction in both the number of settlements and the number of cemeteries over time.

\section{Settlement hierarchy?}

The nucleation of settlements begs the question of whether there was a settlement hierarchy in the Predynastic period. This gets into issues of state-level organization and/or urbanism, which depend on an analysis of the interrelatedness of the sites, and such an analysis is outside the scope of the present project. However the above analysis of settlement contexts and settlement patterns do indicate that there were differences in population size and in the kinds of activities that occurred at sites. These differences are important to the present study because of the need to evaluate whether artifacts made by specialists were used by large portions of the population, or only by certain subsets. It is conceivable that access to artifacts might be related to the kind of site, such as whether that site was a large center or a small farming village. Therefore I divided the sites into a three-part scale: highest-order sites which are large sites in terms of 
area/population and have indications of many different kinds of activities (habitation, designated ritual activity areas, administration, trade, production of ceramics, beer, or other items), and in some cases the known, continuing historical importance of the site; mid-level sites which are not quite as large and have indications of a smaller range of activities; and low-order sites, which are small and devoted mainly to habitation and subsistence.

This scheme is not meant to imply any specific relationships between the sites, such as urban-hinterland relationships, but is simply a way to look at whether there were differences in access to goods based on where people lived. Table 3.3 shows how the sites were assigned based on the information provided in sections 3.4 and 3.5, and the above parameters. 


\section{Chapter 4: Results of fieldwork}

\subsection{Raw Materials}

Special focus was put on the analysis of raw materials to understand patterns of raw material use and to evaluate the expectation for the ritual production model of an association between raw material type and tool type. The most common material for chipped stone tools in Egypt was chert (Harrell 2012). Quartzite, agate, rock crystal, carnelian, silicified limestone, and obsidian are other raw materials sometimes used for chipped stone tools, but by far the majority of lithic artifacts were made from chert.

\section{Chert vs. flint}

A note on terminology. In English, 'chert' and 'flint' are both terms that have been used to refer to microcrystalline quartz. The distinctions between the two terms for microcrystalline quartz can be based on color, quality, geological source, or geographical source (Whittaker 1994:70). However there is no standard definition of the difference between the two, resulting in discrepancies between researchers. The same problem applies to raw material definitions in Egypt. As Harrell (2012:3) stated, both terms have been "variously and inconsistently defined" in Egypt. Hikade (2013:22) also observed that the distinction is arbitrary in studies of Egyptian materials, with the implicit distinction being that chert is coarser and greyer, while flint is finer and brown. Complicating the matter are terms from other languages, which may not map onto English terms in the same ways. French uses the term silex which translates to flint, but there is no translation for the term chert, while German has hornstein and feuerstein. 
Luedtke (1992:6) describes the distinction between chert and flint as originating from Britain and specific to geological formations there. She stresses that the definitions do not translate well to the rest of the world. During the study undertaken here, it was apparent that there is so much variety in the macroscopic properties microcrystalline quartz rocks in Egypt (Figure 4.1), that it would be difficult to formulate and apply a dichotomy between chert and flint consistently. Therefore, this study will use only the term chert, following Harrell (2012) who treats the terms chert and flint as synonymous in Egypt, and Luedtke (1992) who uses the term chert to refer to all microcrystalline quartz rocks.

\section{Chert varieties}

There are three main geological contexts in which chert can be found in Egypt: in its primary context in the high desert limestone plateaus and cliff faces as tabular veins or nodules; in secondary context as nodules or chunks which have eroded out into gravitation screes or on desert plateau surfaces; or in secondary context as surface or buried material which has been washed down from the wadis through gravitational and/or alluvial action. Limestone cliffs with primary deposits of cherts are present in Egypt north of Esna, but chert is available throughout the country in secondary deposits, such as gravel terraces (Harrell 2012; Said 1990 ).

Many varieties of chert are present in Egypt (Figure 4.1). Attempts to classify chert varieties or types have increased in recent years (Briois 2002; Ginter et al. 1996; Hikade 2013; Kabacinski 2012; Kindermann 2010; Midant-Reynes and Prost 2002; Nagaya 2011; Pawlik 2006; Rizkana and Seeher 1988). Researchers divide their chert assemblages into groups based on visual characteristics. However every researcher defines the groups differently, ranging from 3 to 36 defined varieties of chert per study. 
Without actual physical examples, it is difficult to replicate chert groupings between researchers for a number of reasons. For one, it is probable that the chert varieties present in any given site differ, and second, the level of detail given in chert descriptions varies widely. Color photographs are helpful when possible (e.g. Hikade 2013; Kindermann 2010; Nagaya 2011). During a recent workshop at the IFAO on lithic industries in Egypt (Midant-Reynes et al. 2014) interest was expressed in forming a type collection of chert varieties to be housed at the IFAO, which would be very useful for standardizing raw material studies.

In the meantime, for this study 14 chert varieties were defined based on the macroscopic properties of color, luster, texture, translucency, structures, and cortex, observed in the materials from el-Mahâsna and Nag el-Qarmila (Ch. 2.2). To be as explicit and replicable as possible, systematic descriptions using those six characteristics are given in the Appendix, along with color photos. The groupings were formed in a multi-step process. First previous definitions of chert varieties were consulted. Then while in the field, during study seasons in 2009 and 2012, hundreds of labeled artifacts were laid out and grouped according to the above mentioned properties (Figure 4.2). This process was done for the materials from el-Mahâsna and Nag elQarmila to account for differences in assemblages across Egypt. Then a type collection with representative samples was set aside at each site for reference when analyzing individual artifacts. Structures and cortex were the most helpful features for differentiating materials because they were the most discretely defined, identifiable, and replicable characteristics. Color, luster, texture, and translucency were also helpful, but are all relative characteristics which grade into each other.

Although 14 separate groups were defined, some relationships between the groups should be noted. The first four groups are all beige, mostly opaque cherts separated for 
differences in texture and structures. Groups 5, 6, and 7 are all medium to dark browns, mostly opaque, with very fine texture, separated for their structures. Groups 8-11 are all semitransparent and with small white (probably calcareous) mottles throughout, separated for differences in color, and in some cases structures or cortex. Types 12 and 13 are both rare and distinctive, and 14 is an 'other' category.

Among the research described here and in the above mentioned publications on raw materials, certain chert types or varieties are readily identifiable, and have been singled out by many researchers. These are the groups called here type '4.Beige with pink bands', type '7.Dark gray and brown', and type '13.Caramel.' The fact that a number of researchers have recognized and defined these same varieties is significant because it underlines the validity of the groupings, and efforts at sourcing would probably be most productive if focused on these materials. This brings up the question of how well these chert varieties correspond to actual raw material sources.

\section{Chert sources}

Remains of cortex on lithic artifacts indicate that chert was obtained from both primary and secondary contexts (including surface material, gravel terraces, and gravitational screes) during the Predynastic period in Egypt (e.g. Ginter et al. 1996; Rizkana and Seeher 1988). Only a few specific chert quarries have been identified in Egypt (Briois 2002; Briois and Midant-Reynes 2015; Friedman and Youngblood 1999; Ginter et al. 1996; Harrell 2012). Large-scale chert quarrying occurred by the Early Dynastic period (Köhler et al. 2017). However smaller-scale quarrying could have taken place anywhere in the high desert limestone formations north of Esna, and collection of surface materials probably took place throughout the Nile Valley. There 
is only one documented source of chert dating to the Predynastic period, although many more likely existed. Surprisingly it is near Hierakonpolis, south of the main chert-bearing limestone formations, and consists of chert cobbles in a gravel matrix (Friedman and Youngblood 1999; Harrell 2012, pers. comm.). Ginter et al. (1996) also matched samples of cherts found in primary and secondary context near Armant to archaeological materials in the settlements.

The problems associated with linking specific artifacts to specific quarries complicate the possibilities for sourcing raw materials. Luedtke’s (1992) discussion of chert formation showed that chert is an extremely variable material, and highlights some of the difficulties for sourcing. Unlike obsidian which forms all at once from the cooling of a relatively homogenous lava flow, each individual chert nodule is formed separately through chemical precipitation. The first step in chert formation is the precipitation of silica out of a silica solution in water. Silica can also be derived from silica-secreting organisms. Precipitation is affected by temperature, pressure, $\mathrm{pH}$, impurities, the concentration of silica, and the presence of nuclei for formation. Microcrystalline quartz is likely to form when silica concentrations are low and impurities are abundant. The actual formation of chert takes place in many steps that can involve compaction, cementation, chemical alternation, replacement, and recrystalization. There is not a single overall process, but multiple paths through which chert can form. All of the above factors can affect color, opacity, grain size, inclusions such as minerals and carbonates, final water content, and porosity. Thus there is great variability in chert across the world, within a region, and even within a single chert source.

Furthermore, chert in Egypt is often found in horizontal beds in the limestone, layered one on top of another, each of which formed in vastly different time periods (e.g. Ginter et al. 1996). The result is that multiple 'kinds' of chert are all located in a single geographic area, that 
might be considered a single ‘source'. Conversely, natural alluvial and gravitational transportation can carry eroded chert far from its primary source, so a nodule may have been obtained by people at one place, even though it originated at a primary source farther away. Such secondary deposits could contain multiple varieties of chert from different original sources. An additional complication is that many artifacts do not retain cortex making it difficult to determine if the material came from a primary or secondary source.

Nonetheless, personal observations made while visiting sites across Egypt indicated that the chert at one archaeological site often seemed predominantly different from chert at another archaeological site. Therefore a three pronged approach was taken to understanding raw material sources: desert survey for primary sources, comparison of the chert varieties found in the assemblages from multiple sites, and comparison of the cortex types associated with each raw material type. Additionally, a small proof-of-concept study of XRF for chert artifacts was carried out (see below).

\section{Desert survey}

A pedestrian survey for raw material sources near Abydos was undertaken on February 11, 2013. Figure 4.3 shows the relationship of the main Abydos survey area to Abydos and elMahâsna. I examined exposed materials and collected information on geological context, nodule shape, cortex description, Munsell color, inclusions, chert variety, metric measurements, and volume. Chert nodules, pieces, and artifacts were abundant throughout the studied area. Time and permissions were not available for survey in the high desert behind el-Mahâsna, however some raw material examples were observed on the desert surface around Beit Khallaf, which is just north of el-Mahâsna. The Beit Khallaf desert surface provides a likely comparison for the 
desert surface around el-Mahâsna, which cannot be studied now since it is completely covered in farming fields. No primary sources of chert exist around Nag el-Qarmila, however a few examples of secondary chert gravels from Wadi Kubbaniya were studied. The 42 total entries are given in Table 4.1.

Figures 4.4- 4.10 show the geological context of the survey area around Abydos. The Umm el-Qa'ab cemetery is located in an embayment formed by the high desert, and near the mouth of a wadi that cuts into the high desert plateaus (Figure 3.8, 4.4, 4.5). The archaeological settlement areas are 1 - 2 kilometers from the high desert. Chert was observed on the surface of the high desert (Figures 4.9-4.10), on the erosional slopes in front of the cliff faces in the wadi (Figures 4.6, 4.8), and in primary context in exposed sections of the cliff faces (Figures 4.114.12).

Three raw material varieties_-'1.Indistinct beige', '3.Beige less-fine’, and '8. Translucent brown’-were observed in primary context (Figure 4.13). No quarries were observed in the surveyed area, but the materials could have been easily removed from exposed outcrops. Furthermore the presence of the raw materials indicates at least that they were locally available, even if the exact source was not located during this survey. The types '1.Indistinct beige', ‘8.Translucent brown’, and ‘4.Beige with pink bands’ were also found commonly in secondary context on the high desert surface, in slopes coming down from the high desert cliff faces, and washed down from the wadi onto the low desert surface (Figure 4.14). Additionally examples of '2.Beige-fine’, ‘10.Pink-gray’, and silicified limestone were also found in secondary contexts. Figure 4.15 shows examples of each of the raw material types found in this survey.

In sum, two chert varieties were commonly available and local to Abydos: ' 1 . Indistinct beige’, and ‘8.Translucent brown’. Type ‘4.Beige with pink bands’ was also a commonly 
available local material, at least in secondary context, and its abundance indicates that it is highly likely that primary chert outcrops of this variety are probably nearby, but just were not found in this survey.

Beit Khallaf is just 7km north of el-Mahâsna, and provides a good example of the geology of the area in lieu of el-Mahâsna, because both are 4-6 km from the chert-bearing high desert cliffs and both are located in the flat low desert plain. Figure 4.16 shows the desert surface around Beit Khallaf, and Table 4.1 includes examples of raw materials from Beit Khallaf. Abundant material of the type '8.Translucent brown' was noted all across the desert surface in the form of gravel nodules, fist sized and slightly larger. The type '9.Translucent brown with pink gravel cortex' was also found there (Figure 4.17), and is likely a subtype of 8. Type 9 was originally separated from type 8 in case the dark pink color of the cortex was related to heat treatment. However finds of nodules with dark pink cortex on the desert surface at Beit Khallaf indicates that this dark pink color can be due to natural processes. Furthermore, it was noted that on some nodules only the upper exposed portion was dark pink, while the cortex of the side face down was tan (Figure 4.18, upper right), which verifies that the color change occurred naturally. Such two-colored nodules also show that the surface around Beit Khallaf has been stable for quite some time.

Additionally, nodules of type '1. Indistinct beige' and some gravels of a coarser material, probably silicified limestone, were identified (Figure 4.18). However types 8 and 9 seemed to be the most abundant. Therefore, by extension it is possible to surmise that surface nodules of gravel chert, particularly type ‘8.Translucent brown’ would have been an easily accessible local chert source at el-Mahâsna. 
No systematic survey took place around Nag el-Qarmila because that far south in Egypt the main stone type in the area is sandstone rather than limestone, so there should not be any primary sources of chert in the area. However a few small nodules were noted in Wadi Kubbaniya (Table 4.1, Figure 4.19). These were quite small, much smaller than fist-sized, and the cortex indicates extensive transportation from their original source including alluvial transportation. The types were ‘1.Indistinct beige’ and '8.Translucent brown’. These finds indicate that some small nodules would have been locally available to the inhabitants of Nag elQarmila. However the presence of artifacts larger than these nodules in the artifact assemblages shows that material must have also been obtained from farther afield.

To summarize, there was plenty of local chert available at Abydos and el-Mahâsna. Types 1, 2, 3, 4, 8, and 10 were probably all locally accessible for the people of Abydos, and possibly also el-Mahâsna since it is not very far away. Types 8 and 9 surely were locally available chert types at el-Mahâsna. Conversely, at Nag el-Qarmila some small nodules of types 1 and 8 were available, but at least some of the chert used there must have been imported.

\section{Raw materials in archaeological assemblages}

Raw material types were recorded according to the groups defined in the Appendix. Table 4.2 shows the frequencies of raw material types at el-Mahâsna, Abydos, and Nag elQarmila. At each site a different chert variety is more prevalent than any other. At el-Mahâsna type '8.Translucent brown' is the most common chert variety, with over $37 \%$ of the material belonging to that group. Notably that is a type of gravel chert. At Abydos the type "4.Beige with 
Pink Bands' had the highest frequency, at over 25\% of the material. And at Nag el-Qarmila, type '6.Brown fossil', ${ }^{20}$ stood out as the most common (10.4\%) of the identifiable chert varieties. Moreover, the 95\% binomial confidence limits for each of these cases (Figures 4.21-4.22) indicate that the differences can be considered reliable. Another difference between the sites is in the use of non-chert materials. Table 4.3 shows the comparison of the frequencies of chert materials to non-chert materials. A significantly higher percentage of non-chert materials was present at Nag el-Qarmila (Figure 4.23).

These data show that the most common raw material type varies by site, and that the overall composition of the raw materials varies by site. Therefore, the sources of raw material were likely different for each site. This information can then be combined with the observations from the desert survey for raw materials. The desert survey indicated that chert types 8 and 9 were probably locally available at el-Mahâsna, and indeed type 8 was the most common material used in the archaeological assemblage at el-Mahâsna. At Abydos type 4 was the most common raw material in the archaeological assemblage, and type 4 was also found to be present in the desert just behind Abydos. The clear conclusion is that much of the material used at el-Mahâsna and Abydos came from local sources. This cannot be said for Nag el-Qarmila. There, type 6 was the most abundant identifiable raw material, and it was not observed among the local material, underlining the inference that at least some raw materials were imported into Nag el-Qarmila. These conclusions on the sourcing of raw materials at the three sites are supported by the analysis of the cortex, below.

\footnotetext{
${ }^{20}$ Note that the name "Brown fossil" is given only because the structures look like small fossils, but the actual mineral content of the structures has not yet been identified. They are probably foraminifera.
} 


\section{Comparison of cortex type by raw material type}

Analysis of the cortex associated with each raw material type can help identify the source of the materials based on the kinds of weathering present. If the material came from a primary source it would have unmodified chalky cortex. If the material came from a lag deposit (moved by gravity, wind, etc. and often exposed to the sun), it might show light weathering such as light pitting, abrasion, and discoloration. Materials that have been transported by extensive alluvial action have extremely smooth and shiny cortical surfaces. There are also examples of cortex that result from a combination of these forces, such as the gravels on the low desert surface which have washed down from the wadis through a combination of gravity, wind, and occasional water transportation. Additionally some pieces simply have patina which is essentially a process of chemical weathering.

Table 4.4 shows the proportion of cortex types in the overall assemblages of el-Mahâsna, Abydos and Nag el-Qarmila. The numbers were calculated from all pieces above $1.5 \mathrm{~cm}$, including tools, cores, and complete and fragmentary debitage, where the cortex type was recorded. The most interesting finding is that there is a high proportion of unmodified material at Nag el-Qarmila. The 95\% binomial confidence intervals for the proportion of unmodified cortex at each site are given in Figure 4.24, showing that there is a significantly higher amount of unmodified cortex at Nag el-Qarmila than at el-Mahâsna or Nag el-Qarmila. Unmodified cortex most likely comes from a primary source, possibly even quarried, since there was little evidence of weathering on the cortex. This finding corresponds well with the idea that much of the material at Nag el-Qarmila was imported, implying that the material was imported from a primary context, such as a chert quarry.

The fact that Nag el-Qarmila was obtaining chert from a primary context is significant 
because Nag el-Qarmila is just a small farming village, compared to el-Mahâsna and Abydos which were larger sites with more diverse activities, in the case of Abydos possibly an emerging political center, and both el-Mahâsna and Abydos were associated with extensive cemeteries that had indications of emerging social stratification. Nonetheless, the inhabitants of Nag el-Qarmila were well connected or well-traveled enough to obtain a fair amount of chert from somewhere north of Esna, where the primary deposits of chert are located. By extension the inhabitants of Nag el-Qarmila may also have been able to connect into wider exchange networks for other items besides chert, such as specialist produced goods.

An examination of the cortex types of individual raw material categories indicates the probable sources for some chert varieties. Tables 4.5-4.15 show the frequencies of cortex types for each raw material, at each of the three sites studied here. For types '2. Beige Fine', and '4Beige with Pink Bands' the most common cortex types were the lightly weathered cortex from lag deposits, and the unmodified chalky cortex from primary contexts. Possibly this material was collected from near outcrops of the primary sources which would have a mix of unmodified and more weathered pieces. This scenario accords well with the results of the desert survey where both types were found in lag deposits scattered around the desert. Although the primary sources were not located, they may be nearby. Type ‘5.Medium brown’ also fits the pattern of material collected from near a primary outcrop, because both unmodified and lightly weathered cortex types are the most common for type 5 . However this material was not identified during the Abydos desert survey, so it probably comes from elsewhere.

On the other hand, Types ‘6.Brown fossil' and '7.Dark gray and brown' were likely quarried. Where the cortex was present it was predominantly the chalky unweathered cortex of primary deposits. Furthermore, over 50\% of the material for both of these types did not retain 
any cortex, which fits well with the scenario of some initial processing outside of the settlement site, perhaps decortication at the quarries.

A third source of chert is evident for types ‘8.Translucent brown', '9. Translucent brown with pink gravel cortex’, and '12.Pink-purple-red family.' For these groups the gravel and lag cortex types were the most common. Of course type '9. Translucent brown with pink gravel cortex’ was defined by the gravel cortex, but it seems to be a subset of 8 , which also predominantly had gravel cortex. The combination of gravel and lag cortex types indicates collection from desert surfaces_ - either near the sites, or farther away_or from gravel deposits. Given the observations at Beit Khallaf, desert surface collection in the vicinity of the site seems the most likely option, especially at el-Mahâsna. One caveat is that for type 8 at Nag el-Qarmila the most common cortex type was unmodified chalky cortex, (although some gravel and lag cortex was also present). However this fits with the overall pattern of high amounts of unmodified cortex from Nag el-Qarmila, which is likely due to the fact that there simply were not as many surface or secondary deposits available in the area.

Type ‘10.Pink-gray’ deserves a special mention. It would seem to fit with the last group of material collected from the desert surface, since the most common cortex types are gravel and lag cortex at el-Mahâsna and Abydos, and unmodified cortex at Nag el-Qarmila. Furthermore, this fit with the last group is underscored because type 10 has properties similar to the types 8,9 , and 12, such as translucency and white calcareous(?) mottles. However, at all three sites type 10 has an extremely high percentage of non-cortical pieces, ranging from $65 \%$ to almost $80 \%$ of the type 10 material. This factor alone indicates a different pattern of initial processing, which must have taken place outside of the site. Since types 8, 9 and 12 do not have high percentages of 
non-cortical material, it is hard to imagine them being collected at the same places but treated so differently.

Types '1.Indistinct beige' and '3.Beige less-fine’ showed no clear pattern in the frequencies of cortex types. The sample sizes for types '11. White translucent', and '13.Caramel' were too small for any patterns to be discerned.

\section{XRF Research}

All the above raw material groupings were made based on the macroscopically observable properties. Such designations are to some degree subjective, although every effort was made to make the groups standardized and replicable. Therefore, an X-ray Fluorescence (XRF) element analysis was tested this season to determine whether XRF has potential to aid in raw material grouping and possibly, sourcing for Egyptian cherts.

Three artifacts were tested by Gregory Dale Smith of the Indianapolis Museum of Art using a Bruker Tracer III-V Portable XRF. Each artifact was analyzed for 180 seconds, with no filter, and no vacuum, at $40 \mathrm{keV}$ voltage with an $\mathrm{Rh}$ anode. Two of the artifacts were of the same type of raw material, to look at whether or not they gave similar results, and the third was a different raw material variety, to see whether the pieces returned differentiable results. The two similar artifacts were both characterized as type ‘7.Dark gray and brown’ but came from different locations in the Abydos region: MAP 2528 from el-Mahâsna and AMC 12.48 from the Abydos Middle cemetery, and the third piece was: MAP 2021.14 type '8.Translucent brown' (Figure 4.25).

The results for two comparisons are shown in Figures 4.26-4.27. The two pieces of type '7.Dark gray and brown' returned basically similar results with peaks in the same places, but to 
differing degrees. On the other hand, the comparison between the type '8.Translucent brown' and one of the type '7.Dark gray and brown' showed peaks in different places. Thus the XRF does have some potential to help identify or separate chert groups. However it must be cautioned that other surface modification such as desert varnish or patina can affect the results and must be taken into account before any conclusions are drawn on tests of actual artifacts. Samples of raw materials with fresh surface breaks might provide better results for characterizing the material itself.

\section{Raw material conclusions}

It is difficult to look at a single artifact and determine whether the raw material was found locally or was brought in from elsewhere. However the study of large collections of artifacts allowed some inferences to be made on which materials were obtained locally and which were not. Knowledge of the raw material provenience forms a basis for understanding patterns of production and exchange.

The desert survey combined with the analysis for the most common chert materials in each settlement assemblage indicates that at el-Mahâsna type '8.Translucent brown’ was likely a local material. Investigation of the cortex types indicated that this material was probably collected from the desert surface or lag deposits. At Abydos these same analyses showed that type '4.Beige with pink bands' was the local material which was likely collected from around outcrops of the primary sources. An additional important point is that although el-Mahâsna and Abydos were relatively near to each other ( $10 \mathrm{~km})$, they each mainly drew on different chert sources. 
Such sourcing of local materials was not the case at Nag el-Qarmila. There the most common identifiable chert variety was type '6.Brown fossil', which was likely an imported material that came from a primary source, and was possibly quarried. Indeed much of the chert at Nag el-Qarmila probably was imported, as indicated by the high frequency of unmodified chalky cortex coming from primary deposits but the lack of primary sources south of Esna, which is $\sim 170 \mathrm{~km}$ to the north. The importing of chert into Nag el-Qarmila indicates that the inhabitants must have had been able to obtain resources from a relatively wide geographical area.

There is evidence that el-Mahâsna and Abydos also imported some chert. The raw material type '7.Dark gray and brown’ was probably imported to all three sites, as indicated by its high frequency of chalky cortex, and high frequency of non-cortical pieces. Furthermore this type was only present in relatively low frequencies at el-Mahâsna and Abydos. Other types that occur only in low frequencies, such as type '11.White translucent' and type '13.Caramel' may also have been imported.

\subsection{Heat Treatment}

Heat treatment is the process of heating stones, usually cherts, up to high temperatures. This process effects the mechanical properties of the chert, making the stones easier to flake (Domanski and Webb 2007; Luedtke 1992). It is a particularly useful preparation for pressure flaking because not as much pressure or force is necessary to drive off the flakes after they have been heat-treated (Crabtree and Butler 1964; Patterson 1981). Additionally, sharper edges and fewer step and hinge terminations are reported by flintknappers (Whittaker 1994:73). Heat treatment also tends to change the visual properties of the material, which may have been a desired effect (Kennoyer et al. 1991:55). Humans have been heating chert for a very long time, 
some researchers suggest as far back as 100,000 years ago, but it is found commonly after the Paleolithic in many parts of the world (Domanski and Webb 2007).

The process of heating chert involves slow heating, sometimes to specific temperatures, and care to avoid thermal shock. It certainly required some level of expertise (see heat treatment experiment below). Ethnographic accounts of heat-treatment in western Australia (Akerman 1979: 146-147) give some idea of how chert might have been heated anciently. Thin, shaped preforms were prepared, then a fire built in a large sandy pit, and one the ground around the pit. The resulting coals were removed, and the preforms embedded in fresh sand in the pit, and then topped with the coals from the fire. After up to two days, when the coals had completely cooled, the preforms were removed and final flaking ensued.

It can be difficult to identify heat-treated chert in archaeological assemblages because chert is such a variable material. Since most assemblages contain multiple varieties of chert, it can be difficult to know whether an individual piece was heat altered or is just a different variety of chert. The differences in minerals, carbonates, water content, and porosity can result in diverse macroscopic changes during heat treatment, including color, translucency, luster, and texture (Luedtke 1992). Reddening is the most widely known change in heated chert. However, reddening depends on the presence of certain trace minerals, and does not consistently manifest with heat treatment (Domanski and Webb 2007). Because cherts are not all similar, the effects that heat treatment causes also vary. Chert can also become heated naturally, if geological strata were exposed to heat, changing these same properties.

A few aspects of what happens to chert on the microscopic level during heating are clear, but the overall process is debated. Water is driven off during heating, but the amount depends on crystal size and porosity. Non-silica minerals can undergo oxidation causing color changes 
depending on the trace minerals present. Unfortunately the specifics of what happens to affect the mechanical properties of chert remain unresolved. Domanski and Webb (1992; 2009) argue that the chert re-crystallizes during heating, changing the shape, size, and interlocking of the crystals. They argue that fractures propagate along the boundaries between crystals, so there would be different possible paths for fracture propagation in heated chert, potentially offering more direct paths for the force and fracture to travel along. Their findings however, were not supported by a study by McCutcheon and Kuener (1997; c.f. Domanski et al.2009). Others argue that heating increases cracks or microflaws within grains in the material, allowing fractures to occur more easily (Luedtke 1992:104). Until this issue is sorted out it cannot directly aid our understanding and identification of heat-treated material in archaeological assemblages.

Besides macroscopic visual identification, a number of other methods have been attempted for identifying heat-treated chert in archaeological assemblages. Borradaile et al. (1993) tested samples from a chert source in northern Ontario and found changes in magnetic susceptibility and saturation were measurable before and after heating, and could be used to identify heat-treated chert in archaeological assemblages. This method is only appropriate for cherts containing iron oxides. DeForest (2006) developed an experimental method for differentiating heated and non-heat-treated chert based on striking the piece of stone and using computer software to measure the sound waves. Heat-treated rocks did have differences in sound intensity than unheated specimens. However these experiments were done comparing the sound generated from pieces of exact same size and weight dimensions and no outline was offered for how to apply this method to an actual archaeological assemblage with many different sizes and shapes of artifacts and multiple varieties of chert. Domanski et al. (2009) could see clear textural differences in scanning electron microscope images of their experimentally heated and raw 
Polish flint. However they used this method to understand the fracture mechanics, and were not recommending it as a method to differentiate heated cherts in archaeological assemblages. Dunnell et al. (1994) and Griffiths et al. (1986) have used electron spin resonance to detect heat treatment, and Godfrey-Smith et al. (2005) tried thermoluminescence as another method for assessing whether stones were heated. However, all these non-visual methods for assessing heat treatment have many drawbacks. They require specialized equipment, so artifacts need to be brought to the equipment or the equipment needs to be brought to the artifacts. In Egypt artifacts are not allowed to be transported out of the country at this time, and it is difficult, expensive, restricted or sometimes impossible to bring the appropriate equipment into Egypt. Moreover, these methods can also be time consuming, which is not ideal if the goal is to evaluate each artifact in an assemblage of thousands.

Given these issues with non-visual methods, the most practical way to identify heattreatment in archaeological assemblages still seems to be through macroscopic visual identification. To overcome the problem of chert variability the main recommendation is to test the local varieties of chert and determine what they look like when heated (Luedtke 1992).

In Egypt, heat treatment has not been reported in many assemblages until just recently, mainly because few people were looking for it. With good quality chert available heat treatment does not seem necessary. As discussed above in section 4.1 there was a range of coarser and finer grained cherts and other flakeable stone materials in Egypt, and it is not likely that these different qualities and kinds of cherts were evenly distributed across Egypt. For instance, south of Esna the desert is composed of sandstone rather than limestone containing chert. Moreover, availability of high quality chert could have been affected by social factors, such claims to specific resources, or prioritizing of other activities, like agriculture, over travel to distant 
resources. Therefore Ancient Egyptians may have had reasons to heat treat more easily accessible cherts, or even change the properties of cherts that might otherwise be considered high quality.

Indeed, Ancient Egyptians did heat treat their chert. Diana Holmes (1989) was the first to argue for the presence of heat-treated material in Predynastic assemblages. In her dissertation research she examined lithic material from Badari, Naqada, and Hierakonpolis, and identified material she thought was heat-treated. She also reported on a heat treatment experiment she carried out on cherts from Luxor, which supported her argument that there were heated materials present in Predynastic assemblages. Subsequently, she examined some of the lithic artifacts from Maadi and confirmed that heat-treated material was also present in the collection there, although the authors did not recognize it as such at the time of publication (Holmes 1992b).

Other researchers have only recently begun to build on Holmes' finding. In an article on lithics from Hierakonpolis HK11C, Takamiya and Endo (2011) specifically researched and reported the presence of heat treatment at the site. They analyzed materials from the same sites as Holmes and were certainly aware of her work. Additionally, in the publication on Adaïma, Midant-Reynes and Prost (2002) mention heat-treated materials. Kabacinski also described some heat-treated materials from the Lower Egyptian site of Tel el-Farkha.

Holmes described heat-treated chert as reddish, glossy, and with a light speckling. Takamiya and Endo (2011) and Midant-Reynes and Prost (2002) used this same basic definition. However, preliminary research into raw materials for this study revealed naturally occurring pinkish toned chert with white inclusions (Figure 4.28). This observation raised the question of whether this is the only variety of chert that turns really red during heat treatment. An indication that there is indeed some variability in the macroscopic changes to Egyptian chert during heating 
comes from Holmes. She heated a few nodules from near Maadi and noted that the results were somewhat different from the Luxor samples (Holmes 1992b). Considering Luedtke's conclusions on the variability in chert, and the observations made here, heat-treatment experiments were conducted to make a type collection that would facilitate identification of heat-treated material in archaeological assemblages.

\section{Heat treatment experiment}

Six varieties of visually different cherts were tested. To ensure variability in the nodules tested, chert was obtained from two different sources, Abydos and Elkab. These different regions are separated by about $300 \mathrm{~km}$ and are on different sides of the Nile. The nodules vary in color and texture, and most come from lag deposits while a few show signs of alluvial transportation. Nine nodules were partially reduced. Each nodule was given an alphanumeric code, and each flake from each nodule was labeled with a subscript, e.g. A1.1, K2.1, K1.2 etc. Flakes from each rock were kept aside as control samples, and photographs were taken before and after heating. The following attributes were recorded for heated samples before and after firing, and for control samples: ease of flaking, volume, Munsell color, cortex Munsell color, luster, texture, and inclusions. Phil Geib ${ }^{21}$ did all the flaking and rated the ease of flaking for each based on his personal assessment.

Thirty one pieces were heated, consisting of flakes and cores or core fragments. The specimens were heated in a portable kiln. Some were embedded in sand and others were placed directly in the kiln. Five different tests were run (Table 4.16, 4.17). In each test, the pieces were

${ }^{21}$ University of Nebraska 
heated slowly, between 10 to $40^{\circ} \mathrm{C}$ per hour. The temperature was raised to a maximum temperature of 300,350 , or $400^{\circ} \mathrm{C}$. The maximum temperature was held for $6-7$ hours. Then the pieces were allowed to cool slowly, to avoid breakage from thermal shock. After the tests were finished additional flakes were removed from each heated piece in order to examine differential visual properties.

\section{Results of heat treatment experiment}

There are a few important results from these tests. First, while all cherts got somewhat darker with heating, some cherts did not redden. Figure 4.30 presents some pieces before and after heat treatment, showing the variety of color change. Second, the surface that was exposed during heating became more matte or dull, but the scars of subsequent removals were glossier. This result was expected, as increased luster of surfaces flaked after heating is the most frequently reported change (Domanski and Webb 2007; Luedtke 1992). Luster difference is a bit difficult to capture with photography, but Figure 4.29 shows a few good examples. Surprisingly, the glossiness was sometimes subtle.

And finally, an important finding was that the cherts had different optimal maximum temperatures (Table 4.18, Figure 4.31). Optimal maximum temperature was measured by how easy the piece was to pressure flake after being heated to a certain temperature without burning. Some pieces reached optimal ease of flaking at $300^{\circ} \mathrm{C}$ while others burnt at the same temperature. The same goes for $350^{\circ} \mathrm{C}$ and $400^{\circ} \mathrm{C}$. A difference of 50 degrees could make or break a chert.

These findings have implications for the identification of heat treatment in the field, and for understandings of how heat treatment might fit into the development of specialized 
production. This experiment indicates that differential luster is a better indicator for heat treatment than a reddish color. According to these experiments, researchers that want to definitively identify heat-treated artifacts should look for an earlier flake scar or surface that is matte with a later cross-cutting scar that is glossier. Figure 4.29 shows examples of the differential luster of flake scars before and after heating from these experiments, and Figure 4.32 shows an archaeological example of two refitting artifacts where the oldest flake surface is matte and the more recently flaked surface is glossier. It should be noted that artifacts can also become glossy from wear due to exposure to wind and sand, often referred to as desert gloss. However, the patterns of glossy and matte flake scars is inverted for desert gloss pieces compared to heattreated pieces: on artifacts with desert gloss the more recent scars will be matte while the older scars will be glossier. Additionally glossiness resulting from heat treatment is differentiable from desert gloss because pieces with desert gloss often have slightly worn ridges, causing them to look waxy. It is also important to note that with heat-treatment reddening did occur on a number of the samples, so red color can help identify heat treatment in some pieces.

Regarding the speckling mentioned by Holmes, it is likely part of the raw material, not an effect of heat treatment. Raw materials with small white inclusions were observed naturally occurring in Egypt, like that seen in Figure 4.33. There may be an association between speckled raw materials and heat treatment because such materials may have been chosen for heat treatment to improve the flaking results of the non-homogeneous material.

The results of these experiments also indicate that heat treatment would be a good candidate for specialized production because the process is not just a matter of throwing rocks into a campfire. People heat treating chert in Ancient Egypt would have needed a fine control of temperature- within 50 degrees Celsius, and a good knowledge of the varieties of cherts, and to 
match them to the right temperatures. Similar arguments of skill with temperature control, and access to resources for fires have been used as part of the argument for specialized beer production (Geller 1992).

\subsection{Assemblage Composition: Debitage and Tools}

Table 4.20 gives the frequencies of tools, cores, debitage categories, and debris from elMahâsna, Abydos, and Nag el-Qarmila compared to other Predynastic settlement sites/localities in the Nile Valley. Note that the percentages of flakes vary depending on how many debitage categories were differentiated. Debris is included in the overall counts for comparability to existing publications. Figures 4.35-4.36 show examples of cores and debitage.

On the whole, the assemblages are all basically comparable except for the sites from the ceremonial area of Hierakonpolis, and Nag el-Qarmila WK22. Hierakonpolis HK29A, 29B, and 25 stand out for having extremely low proportions of flakes, high proportions of debris, and relatively low proportions of tools. At HK29A and HK29B the bifacial thinning flake proportions are high, and at HK29A the cores are few. Notably HK29A, HK29B, and HK25 are all together part of a ritual precinct in Hierakonpolis (Friedman 1996, 2009b; Hikade 2008; 2011). As discussed above, Holmes (1992a) argued that specialized production of finely flaked bifacial tools occurred in the HK29A structure, and she used such evidence as the extremely high proportions of chips and thinning flakes in her argument.

The storage area at Nag el-Qarmila WK22 also has a somewhat different profile of reduction categories. WK22's tool and debitage profile clearly shows that it was not a place where much lithic reduction occurred. WK22 has the highest percentage of tools by far and the second lowest percent of debris. The debris at WK22 consisted of only seven pieces that were all 
flake fragments- none of the debris consisted of piece below $1.5 \mathrm{~cm}$ or angular debris, both of which can usually be considered indicators of on-site production. WK22 also had an overall low number of lithics. The WK22 and the Hierakonpolis ceremonial precinct assemblages highlight the general comparability of the other settlement sites in terms of lithic reduction. Chapter 5 looks at comparisons of blade types between assemblages in more detail, and Chapter 6 looks at bifacial tool production in depth.

\section{Comparison of cortical flake frequency}

Another way to look at production is via the cortex. Cortex can be removed early in the production sequence, and an area with a high proportion of cortical flakes may very well be a locus of primary production. For instance, at Armant there was a very high proportion of wholly cortical flakes in the early phase of the southern sector of MA21/83, which aided in identifying the area as a location where at least some stages of lithic production took place. Problems with this method are known (see cortex ratio, below), but many publications give this information, so it is presented here for comparison.

Information was available on the proportions of cortical flakes from 15 sites, including el-Mahâsna, Abydos, and Nag el-Qarmila. Definitions of cortex categories varied slightly from publication to publication, so the data could only be divided into two groups: "primary flakes" defined as those with more than 50\% cortex, and "secondary flakes" defined as those with less than 50\% cortex, including flakes with no cortex. ${ }^{22}$ Table 4.19 and Figure 4.34 show that, of the fifteen comparable sites, the percentage of primary (mostly cortical) flakes ranges mainly 
between 7-16\%. Only el-Mahâsna stands out with 25\% primary flakes. The 95\% confidence limits of the proportions indicate that the high proportion at el-Mahâsna is indeed a real difference, and not likely due to sampling error. This finding may indicate higher levels of primary production took place at el-Mahâsna, and may be related to the use of surface gravel materials available directly around the site as argued in Ch. 4.1.

\section{Cortex Ratio}

Another way to look at cortex and production is with the "Cortex Ratio.” Most strategies for examining cortex in an assemblage use a three-stage typology for recording cortex: primary

(mostly cortical), secondary (partially cortical), and tertiary (non-cortical) (Andrefsky 2006:115). One problem with the three-stage typology method is that it quantifies the numbers of pieces with and without cortex. However, different methods or intensities of reduction can create higher or lower counts of cortical flakes (and different sizes of flakes) (Andrefsky 2006:104). These counts result in percentages of cortical vs. non-cortical pieces that would vary due to the technologies employed, not just due to the stage or amount of reduction done on site, even if the entire reduction processes were present, or even if the same quantities and sizes of nodules were used. This insight is significant for understanding lithic processing in Predynastic Egypt because flake, blade, and biface technologies were all utilized, each of which may produce different numbers of cortical detachments. These considerations make it difficult to determine whether assemblages are really comparable since it is not clear what the expected frequencies of cortex are, and whether the data collected deviates from what is expected.

${ }^{22}$ Adaïma could not be added to the comparison because they used different categories: wholly cortical, 
Dibble et al. (2005) developed a method for determining an expected amount of cortex for an assemblage and for evaluating whether the assemblage has less or more cortex than expected. This method compares the expected amount of cortical material that should be found in a given area if all processing was carried out at therein, to the actual observed amount. The resulting ratio will show if the expected amount is present (a ratio close to 1), if there is more cortex than expected $(>1)$, or if there is less cortex than expected $(<1)$. This method also takes into account the sizes of cores and flakes, which are not accounted for in considerations based only on raw frequencies. So far, Dibble et al.'s method has been applied mainly to Paleolithic sites and/or considerations of mobility (Douglass and Holdaway 2011; Douglass et al. 2008; Lin et al. 2009; Marwick 2008; Phillipps 2006). A high Cortex Ratio (more cortex than expected) means that products have been moved away from the production area, and a low Cortex Ratio might mean that present items were moved from an initial processing area. Since the question of specialized production also involves determining where items were found relative to where they were produced, data necessary for calculating the Cortex Ratio were collected from the three sites examined here. This data includes weight, length, width, and percentage of cortex for individual artifacts.

Cortex Ratios for el-Mahâsna and Nag el-Qarmila were calculated, since el-Mahâsna stood out from all other sites as having more cortex based on the raw percentages, and Nag elQarmila was comparable to all the other sites in terms of the percentages of cortical material. The Abydos site sample was substantially smaller than these two sites, so its Cortex Ratio was not calculated. Nor could Cortex Ratios be determined for other published sites since the cortex

partially cortical, and no cortex (Midant-Reynes and Prost 2002:301-318). 
was not recorded in fine enough intervals to give good estimates of observed cortical surface area.

Tables 4.21-4.24 show the calculations used for determining the Cortex Ratio at elMahâsna, and Nag el-Qarmila. The expected quantity of cortex was determined by first calculating the volume of lithic material present. The volume of material was determined from the weight of all the lithic pieces, divided by density. Density was calculated by measuring the weight and volume of displaced water for 89 raw material samples of chert from Egypt. Next, the surface area expected for that volume was estimated based on the average size and shape of nodules of that raw material. The estimated average nodule volume and surface area were calculated based on the average nodule size and shape, using geometric formulas appropriate to the shape. Estimated nodule shape was based on observations of partially reduced cores in the assemblage as well as visits to local raw material sources. The volume of the total assemblage was divided by that average nodule volume, resulting in an estimated number of original nodules. The estimated number of nodules was then multiplied by the estimated surface area for an average nodule resulting in the expected amount of cortical surface area in the assemblage, since cortex is effectively surface area. Dibble et al.'s (2005:549) experiments with the reduction of different nodule shapes and sizes found the method to be robust enough to be applicable even if there is some variation in the nodule shapes and sizes.

The observed amount of cortex in the assemblage was determined by multiplying the length and width of each lithic artifact that was larger than $1.5 \mathrm{~cm}$, to obtain the individual surface area. The percentage of surface area covered by cortex was recorded in intervals: $0 \%, 1$ $10 \%, 10<-40 \%, 40<-60 \%, 60<-90 \%, 90<-99 \%, 100 \%$. The surface area for each artifact was multiplied by the mid-point of its cortex interval, then all of the members of a assemblage were 
added together to find the observed surface area of a given assemblage. Finally, the observed amount of cortex was divided by the expected amount to arrive at the Cortex Ratio.

Since the estimated nodule size and shape greatly affect the outcome of the Cortex Ratio, each Cortex Ratio was calculated four times, using two different shapes of nodules (cubic/rectilinear, and right cylinder), and two different methods for determining the average nodule size and quantity. In the first method, average nodule size was determined from looking at the largest tested cores found on the site, and the largest dimensions of length, width, and thickness found in the assemblage. All of the tested nodules at el-Mahâsna were smaller than the dimensions of the largest artifacts. The largest dimensions of all artifacts were used as average nodule size since overestimating the nodule size affects the expected amount of cortex less than underestimating the average nodules size (Dibble et al. 2005). There is an exponential relationship between the volume and surface area of a geometric shape as the shape gets smaller. For the second method, Dibble et al. recommended basing the estimated number of nodules in the assemblage on the number of cores found in the assemblage, and calculating the average nodule size from that and the total volume.

The cortex ratio was higher at el-Mahâsna than at Nag el-Qarmila (Tables 4.21-4.24). However both were above 1, meaning that there was more cortex than expected at both sites. This was surprising for Nag el-Qarmila since the general impression after analysis was that there was not a lot of cortex there. At both el-Mahâsna and Nag el-Qarmila, varying the presumed nodule shape did not alter the results greatly. On the other hand, the two different methods for estimating average nodule size did produce substantially different results. Nonetheless, in all cases, the Cortex Ratios remained above 1, greater than expected, and in all cases the cortex ratio of el-Mahâsna was farther above 1 than that of Nag el-Qarmila. 
These results confirm that el-Mahâsna is substantially different from other sites in terms of cortex, and indicate that there is more cortex than should be expected for the volume of chert material found there. This could be interpreted in two ways: 1) nodules were reduced on-site at el-Mahâsna and much of the non-cortical products left the site by some means, or 2) cortical material was brought in from elsewhere. Option 1 is the more likely explanation, because it is not clear why cortical material would be brought to the site.

\section{Tools}

Table 4.25 gives the tool types found at el-Mahâsna, Abydos and Nag el-Qarmila compared to other Nile Valley settlement sites. Retouched pieces and burins are the most common tool types. From their lack of patterning, ubiquity, and simple production technique both should be considered ad hoc tools. Bifacial tools are very rare. More specifics on bifacial tool types in settlements are given in Chapter 6.

The Mahâsna and Abydos assemblages are very comparable to other Nile Valley tool assemblages. The presence of axes at el-Mahâsna is notable since it is the only site with axes outside of the Naqada region in this survey. As will be discussed in Chapter 6, Garstang (1903) found many more axes in his excavations at el-Mahâsna.

The Nag el-Qarmila tool assemblage had a few features different from the other tool assemblages. There was a very high percentage of piece esquilles (Figure 4.52). Usai (Gatto et al. 2009:201; Usai 2012) also noted a high percentage of these tools in Nag el-Qarmila Area B. Piece esquilles are produced through bipolar reduction, and it is debated whether they should be considered wedging tools or cores for very small flakes (Bradbury 2010; LeBlanc 1992; Shott 1999). Considering that the raw material study (Ch. 4.1) indicated that much of the raw material 
for lithic artifacts had to be imported to Nag el-Qarmila, the piece esquilles may have been a way to maximize the output of the raw materials present, however this suggestion should be studied in more detail in the future. The frequency of transverse arrowheads was also high at Nag elQarmila, and may relate to hunting. One of the tools in the 'other' category was a lunate, also probably used for a similar purpose. Lunates were also found in Area B (Gatto et al. 2009a:201; Usai 2012), and their presence probably relates to the Nubian influence at the site (Gatto 2014). Other pieces found at el-Mahâsna but not included in Table 4.25 (such as surface finds, special finds from other blocks besides 1,3, or 4, or items from disturbed contexts from Blocks 1 and 3) include a fishtail knife fragment, a bifacial knife fragment, a bifacial sickle, a bifacial tool preform, a burnt concave base projectile point fragment, a circular scraper, six sickle blades, five denticulates, four fragments of blade knives, and an unidentifiable unifacial tool fragment. Drawings and photographs of a sample of tools from all three sites are given in Figures 4.374.52. 


\section{Chapter 5: Blade tool Specialization}

Chapters 5 and 6 address the question of which Predynastic Egyptian tools should be considered specialized by comparing the distribution of the production remains to the distribution of the tools for certain types of tools. Section 5.1 is a review of methods for assessing specialized production, and this is followed by on overview of the known blade types in Predynastic Egypt (5.2-5.3). The distribution of production remains and tools for the different blade types at an inter-site level is given in section 5.4 shows that there were a variety of ways blade production was organized. A look at the distribution data within sites in section 5.5 shows that there was also variability in the distribution of production elements within sites for some blade types.

\subsection{Identifying the Organization of Production Archaeologically}

\section{Direct method}

The first step in understanding how craft specialization developed in Predynastic Egypt is to determine which items were produced by specialists. The identification of specialized goods depends on the definition of specialized production. As discussed in Ch. 1.1, here specialized production is defined as where, above a household level, a class of objects is used by people other than those that made them. Accordingly, a primary way specialized production can be identified is through the distributions of the production remains compared to the distributions of the products (Costin 1991:3, 2001:276; Tosi 1984; Wattenmaker 1994). The products of specialists should be found beyond the production areas, either within a community, or at a 
regional level. For instance, if bifacial knives were made by specialists within a given community the production remains might be located in one household only, but the knives themselves would be found in multiple households. Or if there was regional specialization, all the production remains of bifacial knives might be located in one settlement, but the knives would be found in multiple settlements within the larger region. Evidence of a differential distribution of tools compared to the distribution of production remains would be direct evidence for specialized production. This method of assessing the organization of production works very well for stone artifacts which have durable, plentiful, and easily identifiable production remains (e.g. Charlton et al. 1993; Hartenberger and Runnels 2001; Hogberg 2009; Schafer and Hester 1991; Wright and Loveland 2015).

\section{Indirect methods}

One problem with the distribution method for assessing the organization of production is that if production areas were highly concentrated or very few, they may be difficult to locate archaeologically. Therefore it is useful to have other strategies for determining whether a class of objects was produced by specialists. Indirect methods have been used to identify specialized production by looking at skill, and standardization. These characteristics only indicate specialized production because of the implied relationship between producers and consumers.

A high degree of skill required to make an item can be indication of specialization (e.g. Charlton et al. 1991:103; Costin and Hagstrum 1995:623; Geller 1992; Kelterborn 1984; Sheets 1978). An advanced skill-level evident in an object's production implies that only a small number of people had the expertise and ability required to make the object. If such goods were distributed widely, then there must have been few producers relative to the number of 
consumers, and the item could be considered specialized according to the definition used here. However it should be noted that a lack of production skill required for an object does not rule out specialized production, nor does a high degree of skill (or even more vaguely 'quality') always equate to specialized production. Increases in specialization can sometimes result in objects which require only simple skills in their production, or result in less elaborate or high-quality products, such as when efficiency or quantity was the production goal. This was the case for ceramic bread molds in Old Kingdom Egypt and Uruk period Mesopotamia (Chazan and Lehner 1990). These bread molds were mass produced (by specialists) and were not technically difficult to make, and were notably somewhat crude rather than high-quality. Conversely, there are cases where items with elaborate decorations, which may be interpreted as requiring more skill, have been made in ways that should not be considered specialized. For instance pottery decoration in 6th millennium BCE Mesopotamia, could be quite elaborate. However the designs were all slightly different, made by many people and may reflect each producer's individual creativity, social positioning, ways of combining meanings related to motifs and functions (Wengrow 200l). In this example, with increasingly specialized production decorations actually became less elaborate over time.

Skill, quality, or time investment, are not sufficient characteristics for considering an item specialized. More time and even skill may be invested to make elaborate, high-quality but unique products in less concentrated forms of production, where time and efficiency were not as important. On the other hand mass production can result in quite simple items. Costin (2001:282) notes that the idea that high skill levels are a characteristic of specialists has not been tested. It is certainly possible to imagine situations where jobs were inherited and the people who execute those jobs have varying levels of skill. Skill necessary for production or overall 'quality' can 
only be considered indicators of specialization when accompanied with an argument that those who used an item were different from or more numerous than those that produced it. A high degree of skill can be considered an indicator of specialization if it seems likely that few people had the time or talent to master the technologies involved. Replication experiments have the potential to demonstrate skill levels. In this study skill level was assessed by reference to literature on experimental recreation of technologies.

Standardization as an indirect indicator of specialization comes from the idea that specialized items would have been made by a smaller number of people, therefore there would be fewer chances for variability stemming from idiosyncratic differences, training differences, personal preference, degree of ability etc (Costin and Hagstrum 1995:622) and from the idea that specialists make their wares over and over again, and this repetition (high rate of production or routinization) would also lead to decreases in variability (Longacre 1999). Archaeological and ethnoarchaeological studies specifically aimed at understanding whether standardization is a correlate of specialized production have verified that standardization does often result from specialized production, with the caveats that the analysis of standardization stays within boundaries of quite specific typologies and time periods (Blackman et al. 1993; Longacre et al. 1988; Roux 2003).

A few additional factors are pertinent for assessing specialization through standardization. Standardization can be affected by how important standardization is to the people making and using the item. If it is very important that a product be standardized, (for whatever reason such as function, preference etc.), then people may try harder to reach that goal, resulting in overall higher level of standardization (Longacre 1999). An example of a functional reason an item might need to be standardized might be sickle inserts which have to be of a 
specific size to fit into sickle hafts. Social reasons items might need to be standardized is that similarity of products might be a matter of pride or status for the producers, or items of similar style might be necessary to convey social messages such as affiliation or status. In the latter case standardization may even be a reason why specialized production was pursued (Wattenmaker 1994). Additionally, the degree of variability that can be tolerated for something to still be considered standardized can vary culturally (Roux 2003:778-779). However, Eerkens and Bettinger (2001) have outlined the physical parameters for what humans can perceive as the same in terms of size or magnitude.

Another consideration is the degree to which the technical production process affects standardization rather than the number or producers or their experience. Quintero and Wilke (1995) argue that the blade production technology in the PPNB facilitated the ability to make large numbers of generally standardized blades in terms of shape and size. Some techniques of ceramic production might result in more standardized items, such as wheel made compared to mold made (Arnold and Nieves 1992), although some authors have found that similar degrees of standardization in pottery production can be reached using different production techniques (Roux 2003).

Therefore, to use standardization as an method for assessing whether or the degree to which an item was specialized there are two points which must be considered: what was standardized (the process, the raw material choice, the form, or the metric measurements); and where that standardization was coming from (the technology, the requirements of the product, or the number of producers and their training and ability).

A difference or change in technology can also potentially indicate that a class of items was made by specialists. A contrast in the technology used to produce a class of tools over time, 
or a contrast in the technology used to make different types of tools could indicate specialization if there are major differences in the skill levels and knowledge required. For instance, the presence of both ad hoc tools, clearly reflecting low skill levels, and another tool type reflecting high skill levels, would offers indirect support for specialized production. Such an assertion a could be bolstered by experimental data.

\section{Application}

In this study, the primary method used for assessing and describing the organization of production was analysis of the spatial distribution of production remains and tools (products), looking at inter- and intra-site distributions of artifacts in the Nile Valley — the direct method. Reference to issues such as standardization and skill were only relied upon when primary data from production locations was not available.

The spatial distribution method has the potential to show different degrees or configurations of production. Just a few examples include: Production which should not be considered specialized according to the definition used here Ch. 1.1, such as where the distribution of tools and their production remains overlap entirely- either within a site or on a larger scale; Diffuse production where a product is made in every community but only by certain people within that community; Very concentrated production where an item is made in only one or a very few locations but distributed widely; Tribute like production where an item is made in one or multiple places but only used in one or other places.

The rest of Chapter 5 and Chapter 6 deal with assessing these spatial distribution patterns for a number of different classes of stone tools. Although some researchers have argued that certain stone tools were produced by specialists during the Predynastic period (Ginter et al. 1996; 
Holmes 1992; Kelterborn 1984) these studies have only focused on a few tool types. This study expands the existing discussions of specialized production of stone tools in Predynastic Egypt by looking at the organization of production for heat-treatment, and multiple kinds of blade types and bifacial tool types.

As discussed in Ch. 1.1 there are many other factors or parameters besides spatial distribution that can contribute to understanding past systems of production. Throughout Chapter 7 a number of other parameters of specialized production are layered onto the basic understanding of the spatial patterns for the organization of production. These include the relative quantities of items produced, understandings of the contexts in which the items were produced and used, and who was using the items, and whether production was full-time, parttime, or periodic.

\subsection{Predynastic Blades}

In the following sections a number of specific blade types are described and the organization of production is evaluated for each. Section 5.2 provides the definitions of blades types known from existing literature on Predynastic Egypt. Section 5.3 deals with the question of whether blades and bladelets should really be considered separate types by evaluating attribute data collected from el-Mahâsna, Abydos, and Nag el-Qarmila, and includes that indeed, they should. Having (re-)established the blade type categories, section 5.4 assesses the degree of specialization for each blade type by looking at the distributions of production remains and tools, drawing on data from the three sites studied here along with data available in the literature on Predynastic Egypt. This analysis shows that there were many different ways blade production 
was organized, and that a simple dichotomy between items made by sponsored specialists and ad hoc production does not capture the diversity of Predynastic stone tool organization.

Blades result from a reduction strategy whereby flaking follows scar ridges that are roughly parallel to the flaking axis. The detached pieces produced (blades) generally have relatively parallel margins, although some may converge at the distal end. They have one or two dorsal ridges forming a triangular or trapezoidal cross section. Blades are usually relatively thin, and generally have a uniform thickness (Andrefsky 2005:165). Because identifying these characteristics can be somewhat arbitrary in practice, Tixier (1963:36) defined a blade as any detached piece where the length along the striking axis is equal to or more than twice the width. Most publications on Predynastic lithic artifacts follow this rule of thumb (e.g. Ginter and Kozlowski 1994:48; Holmes 1989:445). Blades can be produced through a variety of technologies, including different kinds of percussion flaking, or pressure flaking (Dessrosiers 2012; Pelegrin 2012; Whittaker 1994; Wilke and Quintero 1996).

A number of different blade subtypes exist in the literature on Predynastic Egypt. Bladelets, medium-sized blades, and large blades are differentiated based on metric measurements. These can then be further separated into even more subtypes based on characteristics such as raw material, heat-treatment, and twist. In this section the signature characteristics of Predynastic Egyptian bladelets, medium blades, and large blades are described, along with their associated cores, and any details known about how they were produced. Additionally, descriptions are also given for twisted blades and trapezoidal blades. The characteristics for all the blade subtypes are summarized in Table 5.1. 


\section{Bladelets}

There is some variability in how bladelets are defined. For instance, at Adaïma bladelets were defined as blades below $1.5 \mathrm{~cm}$ in width and 3cm in length (Midant-Reynes and Prost 2002:305), while at Maadi Rizkana and Seeher (1988:21) mention that "the usual separation between blades and bladelets at $5 \mathrm{~cm}$ length is not realistic" for their assemblage. Others follow Tixier's (1963) definition of bladelets as small blades with a maximum width of $1.2 \mathrm{~cm}$ (Ginter and Kozlowski 1994:48; Holmes 1989:445), and Tixier's definition is also followed here to facilitate comparison because it is the most commonly used definition for bladelets. Figure 5.1 shows examples of bladelets from el-Mahâsna. Observation of bladelets in the assemblages analyzed for this dissertation showed that they tend to be thinner than regular blades, and have very small platforms. Table 5.2 shows the average dimensions for bladelets from the sites analyzed here. The average bladelet from these sites is just over $3 \mathrm{~cm}$ long, $1-1.12 \mathrm{~cm}$ wide, and $.33-.45 \mathrm{~cm}$ thick.

Bladelet cores have a length similar to bladelet dimensions. The removals are parallel (part of the process of creating ridges for subsequent removals), and a few authors have noted that the bladelet core scars tend to have rather regular scars (Holmes 1989:111, 293; Rizkana and Seeher 1988:17). Analysis of the materials presented here left the impression that bladelet cores tend to be single platform with regular scars and a conical shape (Figures 4.35-36). The tendency for bladelet cores to be single platform has been observed at other sites (e.g. Holmes 1989:111, 293; Hikade et al. 2008:179, 181; Midant-Reynes and Prost 2002:317; Rizkana and Seeher 1988:17, Pl2) and the conical shape is often depicted in the associated drawings.

The category "bladelet” can encompass multiple production trajectories. At Hierakonpolis three different trajectories of bladelet production have been defined (Holmes 
1992a; Takamiya and Endo 2011). One trajectory is specifically for making micro-drills. The raw material chosen for microdrill production is a course gray chert. In this case the choice of raw material is definitely functional as the coarse material would have stronger edges and be more abrasive, properties which aid the drilling process. The microdrills were found abundantly at HK29A. A second specific kind of blade production identified at Hierakonpolis is heat-treated bladelet production, where bladelets were produced from heat-treated cores. The final kind of bladelet production defined at Hierakonpolis is where bladelets were made from non-heat-treated cores of regular (non-coarse) chert raw materials. Additionally, bladelets with a longitudinal twist are also known from Predynastic sites. These will be discussed in the twisted blades section below.

Rizkana and Seeher (1988:16-17) offered a reduction sequence for bladelet and small blade production from gravel chert at Maadi. First, small roundish gravel nodules "rarely larger than a fist" were selected. Then one end was removed with a "heavy blow", resulting in an oblique break and creating the platform. As a result of this oblique break, one side of the core had an obtuse angle, the other an acute angle, the latter of which was used for removing the blades. The core was worked from the acute platform angle end only, and no further preparation was necessary. Hikade (1996:35) measured the platform angles on 24 bladelets and bladelet tools form Abydos cemetery U and found them to be 60-70 degrees. Holmes (1989:290-291, 1992:42) and Rizkana and Seeher (1988:19) suggested that small blades and bladelets may have been produced from soft hammer percussion, indirect percussion, or pressure flaking. Hikade (1996:35) offered indirect percussion as a possible removal technique based on the diffuse bulbs in some of the examples from Abydos cemetery U. Midant-Reynes and Prost (2002:317) proposed that soft hammer percussion may have been used because punctiform platforms are 
most common among the bladelets compared to other flake groups. No refitting or replication experiments have been reported out for any kind of bladelet production.

Bladelets spanned most of the Naqada period. In Lower Egypt they disappeared around NIId (Hikade 1996:35; Schmidt 1992a:34). There may have been some regional variation in the frequency of bladelets. They were quite common in Maadi and at Lower Egyptian sites, their frequency defining the Buto-Maadi lithic industry (Rizkana and Seeher 1988; Schmidt 1992a, 1999). Although not as frequent, they were also present in Upper Egyptian assemblages (e.g. Mostagedda: Holmes 1989; Hierakonpolis: Holmes 1989, 1992).

\section{Medium blades}

Here the term "medium blades" is used to designate the blades which fall in neither the bladelet nor large blade metric categories. These were the most common Predynastic Egyptian blades. Holmes (1992a:41) and others noted that medium blades were not particularly regular (e.g. Holmes 1992a:41; Rizkana and Seeher 1988:19; Takamiya and Endo 2011:733;

Vermeersch et al. 2004:229). Figure 5.2 shows some examples of medium blades. Unfortunately, little dimensional data just for these blades was available, since the metric data for medium blades and bladelets are sometimes combined. Table 5.3 shows the metric data for medium blades from el-Mahâsna, Abydos, and Nag el-Qarmila. The average size tends to be 3.6-4.4cm long, $1.5-1.8 \mathrm{~cm}$ wide, and $.5-.7 \mathrm{~cm}$ thick.

Some idea of how medium blades were produced has been gathered from the cores and the blades themselves. Predynastic medium blade cores tend to have a single platform and removals in a single direction, but some cores also have 90 degree platforms, or opposite platforms. Single platform blade cores are the most common kind of core at Armant and Maadi 
(Ginter and Kozlowski 1994:72; Rizkana and Seeher 1988:16-17). From HK29 Holmes

(1989:293) reports that the medium blade cores are all single platform, exhausted, and with steep platform angles. At Adaïma there are many mixed flake and blade cores that are multiplatform and globular, but they also have a consistent proportion of single platform blade and bladelet cores (Midant-Reynes and Prost 2002:317). Holmes (1989:111) proposed that medium blades from the Badari area may have been produced through indirect or soft hammer percussion.

\section{Large blades}

Some blades are so much larger than medium blades that they must have been made with a distinctive production technology. Large blades were the blanks for the tools known as ‘Gerzean blade knives’ (Baumgartel 1960:40, 41), ‘Hemamieh knives’ (Schmidt 1992a:32-33), or 'Blade knives’ (Holmes 1989:402), and ‘Endscraper knives’ (Baumgartel 1960:37) which were found quite often in cemeteries, but also in some settlements (Brunton and CatonThompson 1928: 45-47; Holmes 1989:118; Payne 1993:156-158; Takamiya and Endo 2011:739). Table 5.4 gives the dimensions of some examples of large blades from settlements and Figure 7.7 shows examples of large blade tools. Lengths ranged from $10-17+\mathrm{cm}$, widths $2.5-$ 4.4cm, and thicknesses $1.5-3+\mathrm{cm}$. These dimensions are noticeably larger than the medium blades from the sites analyzed here (Table 5.3), which rarely reached more than $8 \mathrm{~cm}$ in length, and average closer to 4 or 5cm in length. Additionally, Holmes (1989: 402) gave dimensions for 'blade knives' as $8.2-15 \mathrm{~cm}$ in length. Therefore large blades are here defined as wide and thick blades whose length is over $8 \mathrm{~cm}$, usually $10 \mathrm{~cm}+$.

Baumgartel described large-blade knives as the typological markers for the Naqada II period. Looking at patterns of retouch, Schmidt (1992a:32-33) found that the retouch pattern 
typical of Baumgartel's Gerzean blade knives was present in Tel Iswid from the NIIc period on, but that less-standardized versions were present in earlier periods at Tel Iswid and in upper Egyptian sites. However Schmidt’s observations were not correlated to blade size. The settlements with the above examples of large blades fit with a Naqada II date for the knives.

No description of cores specifically for these large blades was found in the literature, but presumably the dimensions of the cores should approximate the dimensions of the blades in terms of length. However, a possible problem with finding large blade cores is that such cores may have been re-purposed and further exploited to make smaller blades (or even flakes) after they were reduced to a size too small for large blades. Alternatively such blade cores may have been reduced in the desert near raw material sources and not transported to settlements.

No production process has been proposed for large blades. The size of the large blades indicates that these were unlikely to have been produced through pressure (see Pelegrin (2012) for pressure flaking techniques and associated maximum widths), leaving direct or indirect percussion as possibilities.

\section{The question of twist}

Blades of each of the above defined dimensions (bladelets, medium blades, and large blades) may have the added attribute of a longitudinal twist. That is, when looking down the length of the blade from the platform, the distal end is rotated. Rizkana and Seeher (1988:19) found that twist was a common feature of the longer blades (up to $20 \mathrm{~cm}$ in length) and note that a twist is “typical of large blades of Predynastic date.” Indeed Baumgartel (1960:34,40) considered large twisting blades to be the hallmark of the Naqada II period. However twisted versions of smaller blades are also known. Schmidt (1996) described the lithics of the Buto- 
Maadi culture as a twisted blade and bladelet industry, and gave data on the twist direction of bladelets, (medium) blades, and large blades at Tell Ibrahim Awad (Schmidt 1992a:81). In the field analyses conducted for this study, twisted versions of both medium blades and bladelets were identified.

A question remains as to the relationships between twisted and non-twisted versions of blades of all dimensions, and how different or similar their production processes were. Rizkana and Seeher (1988:19) conjectured that twisted blade cores would be pyramidal shaped with corresponding scars that ran obliquely from the upper left to the lower right of the core face. However they identified no such cores at Maadi, even though most of the blades there had this distinct twist. Thus the question of how twisted blades and bladelets were produced remains open.

Outside of Egypt, Pelegrin (2012) noted from his experimental reproductions that pressure blades produced by holding the core in the hand, rather than stabilizing it on the ground or with a device, often had a slight twist. Pelegrin also noted that with increased stability of the core, the blades were more regular. Accordingly, it is conceivable that the Egyptian twisted blades might have been made using a method where the core was not particularly stable.

Data on twists indicate that there may be some differences in production. Rizkana and Seeher (1988:19) reported that at Maadi blades and bladelets blades were invariably twisted anticlockwise. Schmidt (1992b:81) found the same consistently counter-clockwise twist at Tell Ibrahim Awad in the layers transitional between the Predynastic and Early Dynastic periods. However study of the direction of twist for a sample of medium blades and bladelets from elMahâsna indicated both clockwise (20.2\%) and counter-clockwise twist (17.24\%), with 51.23\% showing no twist, and $11.33 \%$ indeterminate. Furthermore, the degree of twist can vary. Rizkana 
and Seeher (1988:19) reported that "a difference of 20-40 between the plane of the distal and the proximal end is common," but that some twist as much as $70-80^{\circ}$. They also noted that many blades curved to the right in plan view.

Other differences in production may relate to heat-treatment. Schmidt (1996:280) argued that nearly all of the twisted bladelets of Buto and Tell el-Iswid were glossy, and therefore likely heat-treated. Heat treatment was often done to make pressure flaking easier (Domanski and Webb 2007; Luedtke 1992), so it is possible that the heat-treated bladelets were detached using a pressure technique. However, as discussed above, larger blades could also be twisted. Detaching very long blades with a pressure technique is much more complicated (Clark 2012; Pelegrin 2012). Therefore it is likely that there were differences between the production processes for twisted large blades and twisted bladelets, and possibly even for twisted medium blades.

Twisted blades of all kinds cover a range of time in the Predynastic period. Maadi, and its twisted blades and bladelets, date to the NI-NIIb (Seeher 1999b), while at Buto the end of the twisted bladelet industry is in Buto layer III (Schmidt 1996), which is equivalent to the Late Predynastic/ Naqada III (von der Way 1999:210). The large twisted blades and blade knives are characteristic of the Naqada II period (Baumgartel 1960:40).

\section{Prismatic blades}

Prismatic or trapezoidal blades with very straight parallel margins and ridges were made in Egypt during the Pharaonic period. Barket and Yohe (2011:31) studied trapezoidal percussion blade production from Wadi el-Sheikh. On the few complete trapezoidal blades that they were able to observe the blades ranged from around $8-12 \mathrm{~cm}$ in length, and mainly had punctiform platforms. Recent research at Wadi el-Sheikh shows that the blades were very narrow ranging 
from $.6 \mathrm{~cm}$ to $2.4 \mathrm{~cm}$, and averaging around $1.2 \mathrm{~cm}$ (Hart et al. In prep.). The cores for producing these blades are very distinctive, with an acute platform angle of around $40^{\circ}$. They are also thin, often with cortex remaining on both sides, likely as a result of using the tabular material found in Wadi el-Sheikh. The core sizes found by Barket and Yohe (2011:35) were 17 to 19cm in length, 4 to $8 \mathrm{~cm}$ in width, and 3 to $5 \mathrm{~cm}$ in thickness.

Barket and Yohe reconstructed a reduction sequence for trapezoidal blades. A bifacial crest was prepared and then removed to create a long flat platform. Another crest was prepared on the flaking face to facilitate the blade removals that would follow. The blades were detached

unidirectionally, from one face of the core only. In cases where the raw material was already in a suitable shape for removing blades, the crests were not prepared.

Recent finds at a particular locality in Wadi el-Sheikh (L20) where trapezoidal blades and cores have been found date the site to the Early Dynastic through Old Kingdom periods (Köhler et al. 2017), and similar material has been found in a nearby Early Dynastic-Old Kingdom settlement site (Pawlik 2006). During the Naqada II period more regular blades become common throughout the Nile Valley and delta (Ginter et al. 1996; Hikade 2010:8; Holmes 1989:392). Often these were used for sickle elements (e.g. Kindermann 2008). By the early Dynastic period prismatic blades were produced in large quantities at workshops adjacent to desert quarry sites (Köhler et al. 2017).

\subsection{Re-evaluation of Blade Categories}

Despite the seeming distinctness of the blade types described above, some authors have questioned whether Predynastic blades and bladelets should really be treated as separate types 
(Holmes 1989; Takamiya and Endo 2011). After all, the division between blades and bladelets was originally organized by Tixier (1963) who was looking at assemblages from the Epipaleolithic of the Maghreb, a different cultural context. Were Predynastic medium blades and bladelets made through separate production processes, or was there essentially one process that resulted in both smaller and larger blanks? In other words, are medium blades and bladelets actually different types? Holmes (1989:290,297) and Takamiya and Endo (2011:733) state that blades and bladelets from Hierakonpolis grade into each other in terms of size, and they therefore suggest that they are one reduction technology. However it is not clear how they came to these conclusions as no statistical data were presented. Conversely, preliminary observations of the elMahâsna material left the impression that medium blades and bladelets had different bulb and platform characteristics, which could indicate that they were made in distinctive ways.

Furthermore, typological questions also surround the division of blades/bladelets into twisted and non-twisted forms. Do twisted blades and bladelets really result from production strategies distinct from those of non-twisted blades and bladelets? Or are these perceived differences in form merely byproducts of their production process, perhaps unintentional on the part of the producers?

Since these typological/technological questions exist, before analyzing whether their production processes can be considered specialized, it is critical to first assess whether each of these blade subtypes is really different from the others, or whether they should be combined into fewer groups. In order to test the blade categories, the blades analyzed from el-Mahâsna, Abydos, and Nag el-Qarmila were separated into the above blade types according to certain relevant attributes during the primary data collection analysis, then later other attributesattributes thought to vary depending on the production process—-were used to analyze whether 
the members of each group were essentially similar or different. The attributes used to classify the blades into types during the data collection analysis were: width, presence of twist, and the presence of straight parallel margins and ridges. Then the similarities or differences among these groups were assessed using statistical evaluations of platform types, platform areas, and bulb types. The premise is that items resulting from distinct production processes would have different attributes in terms of the platforms and bulbs.

\section{Results of data collection}

Some information regarding the results of the initial classification of blade types from the three analyzed sites is necessary before moving on to the re-evaluation of blade types. Table 4.20 gives the overall frequency of blade debitage (of all types combined) at each of the three sites in this study. Nag el-Qarmila (AKAP WK15), has a much smaller percentage of blades than elMahâsna or Abydos. Table 5.5 shows the frequency of the blade types identified among the debitage across the three sites analyzed here. Table 5.6 shows the frequencies of blade types identifiable as tool blanks. Both medium blade and bladelet debitage were present at all three sites (Table 5.5). Medium blades were the most common type of blade debitage in all cases, with percentages ranging from $44-52 \%$ of the blade debitage. Tools made on medium blanks were found at all three sites, and comprised the most common type of blank for blade tools (Table 5.6). Tools made on bladelets were found at el-Mahâsna and Nag el-Qarmila, but not at Abydos.

Tools made on twisted medium blades were found at el-Mahâsna, and Nag el-Qarmila, but not at Abydos (Table 5.6). Tools made on twisted bladelets were only found at el-Mahâsna. However, twisted blades and twisted bladelets were present among the debitage at all three sites, counting for roughly 5-15\% of the blade types (Table 5.5). The relative degree of twist was 
recorded for a sample of 91 twisted blades from el-Mahâsna (Table 5.7). Approximately 12\% were only slightly twisted, and only 3 (3.3\%) were very twisted.

Tools made on large blades were identified at el-Mahâsna and Abydos (Table 5.6). However no debitage from any of the three sites could be reliably identified as large blades

(Table 5.5). The only cores that could have produced detachments of large blade dimensions were found at el-Mahâsna, but they had only flake removals without any signs of blade removals. This evidence suggests that while the large blades tools were used at el-Mahâsna and Abydos, they were not manufactured in any of the excavated areas. Because there are few examples of large blades, the following evaluation of the distinctness of blade subtypes will focus only on the medium blade, bladelet, twisted blade, and twisted bladelet types.

\section{Re-evaluation of blade subtypes based on platforms and bulbs}

The analysis of how distinct the blade type categories are is first carried out using data from one site only, in order to control for any differences in production by site. The analyzed material comes from el-Mahâsna, because it had the largest sample size, and largest range of blade types present. Below, platform types, platform area, and bulb types are each evaluated across blade type categories for comparability. Each attribute is described, along with the statistical method for evaluating the attribute in question, and the results given. A summary of the results showing that medium blades and bladelets should be considered separate types is given after presenting all the results of the attribute assessments. Following that, data from Abydos and Nag el-Qarmila are analyzed showing that the conclusions drawn regarding blade types at el-Mahâsna can be extended to the other sites. 


\section{Platform type}

A platform on a blade is the area of the core surface where force was applied, that still remains on the detached piece (Figure 5.4). The different types of platforms mainly refer to the number of facets on the platform (for complete descriptions see the Appendix). The platform type categories used here are commonly found in, and comparable to, other publications dealing with Egyptian lithic artifacts (Close 1980; Ginter and Kozlowski 1994; Holmes 1989; MidantReynes and Prost 2002). Often platform faceting correlates with different kinds of reduction strategies (Debénath and Dibble 1994:13-14; Tomka 1989:146-147). Therefore differences in platform faceting among blade types may indicate different reduction strategies.

Table 5.8 shows that there is some variability in platform type frequencies across blade types. A chi-square test was used to assess whether there was a relationship between platform type and blade type. The null hypothesis was that the platform type is independent of blade type (no relationship between the variables). The alternative hypothesis was that platform type was related to the blade type. The null hypothesis would be rejected if the probability calculated from the chi-square statistic was equal to or below .05. The validity requirements for a chi-square test are as follows: The variables should be independently measured and defined, and the generated expected counts should all be above one, with fewer than 20\% below five (Thomas 1986:298).

A chi-square test of eight platform types (cortical, flat, dihedral, multifaceted, linear, broken, other, indeterminate) by the four blade types (medium blade, bladelet, twisted medium blade, twisted bladelet) was determined not to be valid because the sample sizes were too small: many of the expected counts were below one and over $50 \%$ were below five. However, a valid chi-square test was performed on a contingency table looking at the platform types of only the medium blade and bladelet categories. The results were a Pearson’s chi-square statistic of 
18.5122, with 7 degrees of freedom, and a probability of .009861. This probability is well below .05 , so the null hypothesis that platform type is independent of blade type should be rejected. In other words there is a better than $99 \%$ probability that the variation in platform frequencies noted on medium blades and bladelets is not just due to chance. Therefore platform type does vary by blade type, which is what would be expected if medium blades and bladelets resulted from different production processes.

However, there is some indication that this variation in platform type may be related to platform size. In this case the linear and the multifaceted bladelet platform chi-square values were furthest from zero, thus causing the most influence, as might be guessed by looking at the frequencies. There is a high frequency of linear platforms among bladelets, and a low frequency of multifaceted platforms among bladelets (Table 5.8). These frequencies are probably related to platform size, since a smaller platform is less likely to pick up any faceting (resulting in a low occurrence of multifaceted platforms), and linear platforms are by definition minute, so the platform area may be affecting the variability of platform type.

The question still remains regarding how the platform types of the twisted medium blades and twisted bladelets compare to each other and to the medium blades and bladelets. Although the significance of the variation in platform type frequencies could not be evaluated via a chisquare test for the twisted blades and twisted bladelets, it was still possible to assess whether the variation in frequencies of platform type was significant by comparing the binomial confidence intervals $^{23}$ of select platform types. The binomial confidence intervals were calculated for the flat platforms since the flat platforms showed the most variation across blade types. Despite this variation, the binomial confidence intervals mostly overlap (Figure 5.6), showing that the 
frequencies of flat platforms cannot be statistically differentiated at the $95 \%$ confidence level for medium blades, bladelets, and twisted medium blades. While flat platforms are significantly more common among the twisted medium blades than among the medium blades, the frequency of flat platforms for twisted medium blades cannot be completely differentiated from bladelets and twisted bladelets.

In sum, the chi-square test indicates that blades and bladelets may very well have a different spectrum of platform types. Additionally, the confidence intervals of the frequencies of flat platform types indicate that flat platforms are more common among twisted medium blades than among regular medium blades, giving some indication that these two types may come from distinct production methods, however, as is shown below the overall relationship between medium blades and medium twisted blades is somewhat ambiguous.

\section{Platform area}

Since the differences in platform type are likely related to platform area, platform area was also analyzed. Platform area was obtained by multiplying the measurement of platform width by the measurement for platform thickness (Figure 5.4). A box plot of the platform areas by blade type shows variation in median platform areas between blade types (Figure 5.8). The means also show variation, with the mean thickness of medium blades much larger than the other blade types (Table 5.9).

A one-way analysis of variance (ANOVA) test was used to compare the means of the platform areas of the different blade types. ANOVA is an appropriate test to use when comparing

\footnotetext{
${ }^{23}$ See Chapter 2: Methods.
} 
means across more than two groups. The requirements of an Anova test are that the observations are independent, that the data approximate a normal distribution, and that the groups have similar variability. To examine whether the data approximate normal, the quantiles were plotted against the quantiles of a normal distribution (a qq plot). If the data are normal then the resulting plot should be close to a straight line, but these did not. Therefore the log of the data was computed in order to transform them to a normal distribution. QQ plots of the resulting data show that they approximate a normal distribution (Figure 5.7). The variances of the transformed data are given in Table 5.9, and show that the variances are (basically) similar. Nonetheless, a Bartlett's test for homogeneity of variances was run on the log transformed platform areas. With Bartlett's test the null hypothesis is that the variances are equal, and the null is rejected if the p-value is below .05 . The Bartlett's test of the log platform areas returned a p-value of 0.1114 , so the null hypothesis that the variances are homogeneous holds, and the assumptions for the ANOVA are met.

The null hypothesis for the ANOVA was that the means of platform area for all blade types were equal. The alternative hypothesis was that at least two of them were different from each other. The null would be rejected if the probability calculated was equal to or below .05. Table 5.10 gives the ANOVA results. The p-value, or the probability that the null hypothesis is true, is far smaller than .05, therefore the null should be rejected-not all of the means are essentially the same, at least two of them are significantly different.

The goal here is to understand which, if any of the blade subtypes have statistically different attributes, which could indicate that they likely come from different production processes. Therefore knowing that some of the means are significantly different from each other is not enough, but an understanding of which means are significantly different is necessary. A glance at the means (Table 5.9) shows that the average platform area of medium blades is double 
that of any other blade subtype. A post-hoc test called Tukey’s Honestly Significant Difference was done in order to verify which means are different. Tukey’s HSD uses data (Mean Square Within) from the Anova to calculate what a significant difference would be (HSD), and then compares each pair of differences in mean to the HSD. ${ }^{24}$ Table 5.11 shows the results of the Tukey's HSD including the pairwise differences and the corresponding probabilities. A probability below .05 would indicate a significant difference in the means. According to this, the medium blades and bladelets have significantly different mean platform areas.

Since these tests indicate that the platform areas of medium blades and bladelets are significantly different, then it is reasonable to conclude that blades and bladelets were made through different production processes that resulted in different platform areas. Again, as with platform types, none of the other blade subtypes could be differentiated from each other based on the platform areas.

\section{Bulb type}

A bulb of force (also known as a bulb of percussion) is a protrusion on the ventral side of a flake just below the platform, reflecting the Hertzian cone resulting from conchoidal fracture (Figure 5.5). Bulbs types were defined according to the size and prominence of a bulb relative to the rest of the flake. There were four main categories: diffuse (no bulb), moderate, prominent, and small prominent, along with other and indeterminate (for specific definitions see the Appendix). The formation and size of a bulb of force is thought to relate to the technique of flake

\footnotetext{
${ }^{24}$ For the exact formula and more information on Tukey's HSD see: Tukey 1949, Or http://faculty.ucmo.edu/dkreiner/psy2120websitestuff/psy2120oldexams/documents/StatSheet12_Tuk eysHSD.pdf
} 
detachment including hammer type, force, and application of force (Andrefsky 2005:20). For example, pressure flakes tend to have short fairly pronounced bulbs (Inizan et al. 1999:63). Direct percussion with a soft hammer (such as an antler) rather than a hard hammer (such as stone) produced flakes with relatively thinner bulbs of percussion in an experimental setting (Pelcin 1997a).

Table 5.12 shows that there is variation in bulb types when blades were grouped according to blade subtype. A chi-square test was run to assess whether there was a relationship between bulb type and blade type. The chi-square test of the six bulb types (prominent, moderate, diffuse, small prominent, other, indeterminate) by the four blade types was determined not to be valid because the sample sizes were too small- many of the expected counts were below one and over 50\% were below five. However, a valid chi-square test was run on a contingency table looking at the bulb types of the medium blade and bladelet categories only. The results were a Pearson's chi-square statistic of 21.3332, with 5 degrees of freedom, and a probability of .0007006. This probability is well below .05, so the null hypothesis that platform type is independent of blade type is rejected. In other words there is an over 99\% probability that the variation in bulb type frequencies is not just due to chance. This suggests that bulb type is related to blade type, or to put it in other words, the blade types have statistically different bulb types. By extension, this variation in bulb type likely means that the blades were made in distinctive ways, and so should be considered different types.

Since the chi-square test could not be run with the data from twisted blades and twisted bladelets, the significance of the variability in bulb types for twisted blades and twisted bladelets was tested using binomial confidence limits. Moderate bulbs showed the most variability between categories, so the confidence limits of the frequency of moderate bulbs for each blade 
type was analyzed. The binomial confidence intervals of the proportions of moderate bulbs (Figure 5.9) shows clearly that the proportions of moderate bulbs on medium blades and twisted medium blades are similar, and the proportions of moderate bulbs on bladelets and twisted bladelets are similar, and that the two groups (medium blades/twisted medium blades versus bladelets/twisted bladelets) are different from each other. The twisted bladelet category is a very small sample so it has a much larger confidence interval that overlaps slightly with the twisted medium blades, but otherwise the pattern is clear. These confidence intervals provide some indication that in terms of bulbs, bladelets and twisted bladelets are similar, while medium blades and twisted medium blades are similar.

These data support the findings from the above platform analyses that blades and bladelets have statistically distinct attributes and so likely result from different methods of production, and should therefore be considered distinct types. However this bulb analysis additionally indicates that twisted medium blades may be differentiable from twisted bladelets.

\section{Summary of blade type assessment}

Table 5.13 summarizes the outcomes of the above tests. It is very clear is that in almost every case the medium blades and bladelets have significantly different attributes, and should be considered different types. ${ }^{25}$ The relationship between bladelets and twisted bladelets is also quite clear, but in contrast the results reveal that their characteristics consistently overlapped, so they should be grouped together. The logical extension of these two conclusions, that medium 
blades should be differentiated from twisted bladelets, was reasonably well supported by these tests.

However, the relationship of twisted medium blades to each of the other blade subtypes was not as clear cut. The platform areas of the twisted medium blades were smaller than those of the medium blades, and quite similar to those of the twisted bladelets, but the bulb types of twisted medium blades were more similar to medium blades than to either bladelets or twisted bladelets. Therefore, in the following section examining which items were specialized, bladelets and twisted bladelets will form one group, medium blades will be treated as a second, separate group, and twisted medium blades will be omitted from the assessment.

\section{Do these conclusions hold true across sites?}

Having completed the analysis of data from el-Mahâsna, the next issue to consider is whether these conclusions about blade types are generalizable to other sites. This is done by comparing these same attributes across sites. The analyses below show that the attributes of medium blades do not differ significantly across sites. The sample sizes of the other three blade subtypes were too small for meaningful statistical comparisons, but since the medium blades compare favorably across sites, then it is assumed that the other blade types probably also are similar.

The proportions of platform types and bulb types among medium blades from elMahâsna were compared to those of Abydos and Nag el-Qarmila. The 95\% confidence intervals

\footnotetext{
${ }^{25}$ The fact that the frequencies of flat platforms cannot be differentiated between the medium blades and bladelets does not negate this conclusion for two reasons. First, the chi-square is a better measure because it compares all of the platform type categories, rather than just one. Second, it is entirely
} 
were used to assess whether any differences might be due to random chance, or whether the variations reflect real differences in the populations. For platform types, cortical platforms showed the widest range of variation (Table 5.14), and so the confidence limits were compared. Despite this variation, the $95 \%$ confidence limits for the frequency of cortical platforms from each of the sites overlap (Figure 5.10), indicating that the differences are not meaningfully different. Since all of the other platform types show less variation, and have the same sample sizes, their confidence intervals will all overlap as well.

The metric platform areas were also compared between the sites. Box plots of the platform areas of medium blades (Figure 5.12) shows that while the mean and median are slightly higher at el-Mahâsna the data are similar in terms of median, spread and skew. An ANOVA was run to test the null hypothesis that the means of the platform areas from all three sites are essentially similar. The platform areas were adjusted through a log transformation so that the data are normally distributed and variances are homogeneous (Figure 5.13). The Anova of the log transformed platform areas returned a p value of 0.573 , well over .05 , so we cannot reject the null hypothesis that all three means are equal. Therefore, in terms of platform sizes medium blades cannot be distinguished across the three sites.

Similarly, the proportions of bulb types (Table 5.15) for medium blades is notably similar at all three sites. Moderate bulbs showed the most variability, however the confidence intervals (Figure 5.11) indicated that this variability is not necessarily significant. Therefore, medium blades cannot be differentiated across sites in terms of bulb types.

possibly that two different production methods may produce some attributes in similar frequencies. That is why multiple attributes were analyzed. 
Given that the data were comparable for platform type, platform area, and bulb type, the conclusions regarding blade subtype groupings can be extended to other Predynastic sites besides el-Mahâsna. To re-cap, medium blades and bladelets are clearly distinct groups, and twisted bladelets can be considered part of the bladelet group. Twisted medium blades are somewhat ambiguous, and so will not be included in the following analyses. All in all that leaves two main blade groups, medium blades and bladelets, along with large blades, to be considered in the rest of this chapter.

\subsection{Blade Specialization at an Inter-Community Level}

Specialized production of blade subtypes is assessed here through an examination of the distribution of production remains and tools across settlement sites, then, in section 5.6 within sites, for the blade types: large blades, medium blades, and bladelets. The category of bladelets can be further subdivided into heat-treated bladelets, non-heat-treated bladelets, and microdrill bladelets because the differences in the raw material choice or preparation of the raw material.

Comparing sites is somewhat challenging because data were reported or collected with different degrees of specificity or different foci. Therefore presence/absence tables were generated to compare the settlement site data, which enabled a more extensive comparison than a frequency/proportion comparison would have. While the focus is on the settlement data, information on lithic artifacts in the associated cemeteries was also consulted when available. A few terms should be explained. The word "blanks" refers to un-retouched blades or bladelets which could have been discarded production debris, unfinished tools, or pieces that could have been used as tools without further modification. Along with cores, “core rejuvenation pieces” 
(e.g. core tablets), “crested blades,” and "plunging blanks" ${ }^{26}$ are all specific kinds of production remains ${ }^{27}$ It should be pointed out that empty spaces in the presence/absence tables do not necessarily indicate that the item was definitely not present, but rather, only that it was not reported. There are multiple reasons why such information might be omitted. For example, during the original research a given item type may not have been differentiated from other items such as when bladelets are not differentiated form blades, or the data may not have been pertinent to the original publication topic, such as the presence of blades with plunging terminations for a publication focusing on tool use. In the presence/absence tables, a solid black circle indicates that the specified item was definitely identified at the given site. A red " $\mathrm{X}$ " indicates that the item was looked for, but specifically reported as not present. Some of the presence/absence information was determined from the published drawings of the artifacts in addition to the text and tables, or from reference to museum collections (in the case of certain items from cemeteries). Finally a few notes worth bearing in mind: Systematic data on debitage and tools from North Town was not given in Holmes (1989), but the presence of a few items mentioned may be significant, and so North Town was included here.

\section{Medium blades}

Table 5.16 shows that for medium blades, most types of production remains and tools were present at almost all sites. Congruously, medium blade blanks and blade tools were also found in all of the corresponding cemeteries where data were available (Abydos cemetery $\mathrm{U}$ (Hikade 2000); Naqada cemeteries (Holmes 1989); Armant cemeteries (Mond and Meyers

\footnotetext{
${ }^{26}$ Plunging blanks having a thick and often curving termination that removed part of the end of a core.
} 
1937); Adaïma cemeteries (Crubézy et al. 2002); Hierakonpolis HK6 (Adams 2000a; Friedman et al. 2011a,c). A closer look at the settlement sites where some production elements are missing indicates that despite these missing elements it is still likely that blade production occurred at those sites. At el-Mahâsna, Abydos, and Nag el-Qarmila no core rejuvenation pieces for medium blades were reported, but that is because all core rejuvenation pieces were treated as a single category and not separated into blade core rejuvenation pieces and flake core rejuvenation pieces. This means that it is not known whether blade-core rejuvenation pieces were present at those sites—-they very well may have been. At Hierakonpolis, locality HK 11C did not have any blade cores, but other remains from medium blade production were found there, such as crested blades. Similarly, at HK29 there were no crested blades but there were blade cores. Since at this point the aim is to compare findings among sites and across regions, the Hierakonpolis localities can be grouped together, in which case certainly all the elements of medium blade production were well represented at Hierakonpolis. In the Naqada region, North Town materials were not fully reported in the publication, so the absences there cannot be considered reliable. South Town did have cores in addition to blanks, a strong indicator that blade production occurred there. KH3B did not have any production remains besides blanks (which can be used as tools), so, at best, the jury is still out on whether or not there was blade production at KH3B, and it is perhaps the exception that proves the rule. In all, the data indicate that blade production was found at most sites and cannot be considered specialized at an inter-community level.

The presence/absence data has two drawbacks. This kind of information does not allow for a closer comparison of frequencies and proportions, so the quantity or frequency of production may vary among the sites. For instance the medium blade production at Naqada

\footnotetext{
${ }^{27}$ See the Appendix for definitions.
} 
KH3B may have been of a low intensity compared to other sites, which would account for why few production remains have been located in the archaeological record. The second problem is that there may be variability in the kinds of medium blades produced at each site. For instance at Maadi a difference was discerned between larger medium blades produced from quarried nodules and other medium blades produced from gravels at the site (Rizkana and Seeher 1988:16-17). Conversely, at Hierakonpolis the medium blades produced were described as small (Holmes 1996:95). This variability in the medium blades from different sites indicates that there were probably producers in each community, producing slightly different blades, thereby providing more support for the conclusion that medium blade production was not specialized at an inter-site level.

Furthermore, the data on tool types (Tables 5.17- 5.18) also suggest that medium blade tool production was not specialized at an inter-community level for two reasons. First, many of the tools produced on these blades are ad hoc, such as burins, retouched pieces, notches and perforators. Burins are often made on broken tools, a form of re-use, and also exist in such a large variety of "types" that they seem to be an as-needed tool rather than systematically produced. ${ }^{28}$ Tools classified as "retouched pieces" by definition exhibit non-standardized retouch that does not fit into another type category. Burins and retouched pieces are the most consistently represented pieces made on medium blade blanks both in the presence/absence data and in frequency data. The only sites where the presence of burins on blades is unknown are those where the medium blade blanks were not reported. Furthermore, in the sites where 
frequency data were available for tools made on blades, burins and retouched pieces are almost always the first and second most frequent tool types (in either order). Notches and perforators are tools that also rather variable and unstandardized in the Predynastic period.

Second, the range and frequency of tools produced on medium blades are basically comparable (Tables 5.17- 5.18). If a site or sites were producing tools for the other sites, we might expect the frequency of those tools to vary by site, depending on access. However, among tools made on blades the range of tool types present is basically similar at all sites. ${ }^{29}$ The only sites that are missing a number of the tool types are those where the blanks were not specifically reported for some of the tools classes (North Town, Armant, HK29A, HK29B, HK25). On the whole, the data on medium blade debitage and tools indicate that medium blade production was not specialized at an inter-community level.

\section{Heat-treated bladelets}

Table 5.19 shows the presence/absence distribution of heat-treated bladelet tools and production remains across sites. It is immediately clear that this distribution is quite different from that of medium blades. Heat-treated bladelet tools and the production remains for making those tools were present at only a few sites (Badari, el-Mahâsna, Adaïma, and Hierakonpolis). At el-Mahâsna every stage of production for making heat-treated bladelet tools was found (Figure 5.3), including angular debris, debitage, a core of bladelet dimensions with a prepared crest

\footnotetext{
${ }^{28}$ For example, the following "types" of burins were found at HK29 (Holmes 1989:3): Single burin on a: break, oblique straight truncation, straight truncation, other truncation, retouched edge, unretouched edge, backed blade, other; Double burin on:1 break, 2 breaks, 1 burin on a break, burins not on breaks, other; Dihedral burins; Angle dihedral burins; Multiple dihedral burins; Multiple burins on: 1 break, on 2 breaks; Burins not on breaks.
} 
(Figure 4.37), reduced bladelet cores, core trimming elements, heat-treated bladelet blanks, and bladelets tools. At other sites, heat-treated bladelet tools were present, but the production remains were glaringly absent from those sites (Abydos, Naqada sites, Nag el-Qarmila). Therefore, it is likely that heat-treated bladelet tools were not produced at all sites, but rather that some sites obtained heat-treated bladelet tools from elsewhere.

At the Naqada sites, although no heat-treated bladelet tools or production remains were found in the settlements, the tools themselves were reported from the cemeteries ${ }^{30}$ (Holmes 1989:276-282). At Nag el-Qarmila, besides having no remains related to the production of heattreated bladelet cores, the overall proportion of heat-treated material was quite low (1.6\%) compared to el-Mahâsna (12.4\%) and Abydos (8.5\%). Abydos is an interesting case because there was no evidence for heat-treated bladelet production nor heat-treated bladelet tool use even though it is very near el-Mahâsna where there was extensive evidence for heat-treatment, including the production of heat-treated bladelet tools. ${ }^{31}$ Therefore at the Naqada sites and at Nag el-Qarmila (AKAP- WK15) the tools were present where the production materials were not. A relatively small sample size may be a factor for Abydos, but since no categories of heat-treated bladelet production or use were present at all, it seems likely that something different was going on there compared to el-Mahâsna.

The range of tools produced on heat-treated bladelets (Table 5.20) was also strikingly different from those made on medium blades. For heat-treated bladelets, the tool production was

\footnotetext{
${ }^{29}$ Blade knives are essentially a specific kind of backed blade so those categories can be considered together.

${ }^{30}$ The overall number of heat-treated tools in cemeteries is likely underrepresented in the literature because heat-treatment has not been assessed for stone tool assemblages until recently. Many bladelets and bladelet tools are known from cemeteries which have not been assessed for heat treatment.
} 
geared toward one specific kind of tool—microendscrapers — a finding that aligns with expectations for specialized production. Microendscrapers (Gilead 1984; Holmes 1989) were the most commonly found tool type made on heat-treated bladelets. Along with those were some ad hoc tools such as retouched pieces, and occasionally other tools such as sickle blades or truncations. All in all, production of heat-treated bladelet tools, specifically microendscrapers, was clearly specialized at the inter-community level.

\section{Microdrills}

Microdrills present a third system of production (Table 5.21). Microdrills were made on coarse-grained bladelets, and few other tools were made on these coarse bladelets except for microdrills (Hikade et al. 2008; Holmes 1989, 1992a:41), indicating a production process geared toward a single tool type. In this case the microdrill production was concentrated in one area: Hierakonpolis. The use of the tools was also limited to Hierakonpolis. Note that no mention of microdrills was found in the assessment of cemetery lithics for this study. By the definition of specialized production used here, microdrills cannot be considered specialized. They were not used beyond the areas where they were made. However, microdrills may have been employed in the production of other specialized items.

Microdrill production was connected to bead drilling. Unfinished stone beads and carnelian debris were found at HK29A, where many microdrills were found. Experiments using flaked stone microdrills on carnelian pieces produced wear patterns that matched those found on the HK29A microdrills, indicating that they were indeed used to drill beads (Nagaya 2014). In

\footnotetext{
${ }^{31}$ Other heat-treated material was present at Abydos, but it was not related to bladelet production.
} 
Nekhen (Kom el-Ahmar), the Early Dynastic to Old Kingdom town site on the floodplain in Hierakonpolis, Quibell and Green (1902:11-12) found a deposit with “an enormous number of exceedingly small pointed flint implements... and with them were many broken carnelian pebbles, some chipped into the form of rough beads, one or two of which showed signs of the commencement of the boring operation.” This description matches the descriptions of microdrills and bead remains from HK29A. These microdrills from Nekhen were found adjacent to an outer wall of the temple enclosure and Quibell and Green date them to the Old Kingdom. Additionally, microdrills were reported from a site at Abydos dating to the late Naqada period (Peet 1914:3-6), where they were also found with unworked carnelian and agate, and carnelian beads. The Abydos microdrills were probably produced on the spot, since much flaking debris was found associated with them, and cores were present. Concentrations of microdrills have been found in the Early Dynastic layers at Tel el-Farkha in the Nile Delta, where they were also produced and used in the same spot as evidenced by their production remains and heavy wear on the drills (Kabacinski 2012:339-344). However these later examples differed in some details of their production (raw material choice and treatment) from those discussed here. Jordeczka and Mrozek-Wysocka (2012:289) suggest the drills were used to make beads, but note that can't be confirmed until trace analysis is done.

The Abydos example (and possibly the Tel-el-Farkha example) not only shows that microdrills were consistently used for bead production, but it also shows that the microdrills should not be considered only a regional phenomenon. The microdrills have been found in multiple regions, but in each case they were made and used in the same area. Perhaps a distinction between 'specialized' and 'specific' is useful here: Microdrills were not a subject of specialized production (which involves exchange), although they were made and used for 
specific purposes by a specific set of producers (who were probably making other items which would be considered specialized).

\section{Large blades}

Large blades show a distribution which indicates that they were made through specialized production since the products, but not the production remains, were present in most of the settlement sites studied here (Table 5.22). The one exception is Badari where there were two refitting unretouched blanks of large blade dimensions, and a single platform blade core of a quite similar raw material (Holmes 1989: 111, 118; Figure 7.7). The blade core dimensions were on the small side for large blades $(\mathrm{L}=10.7 \mathrm{~cm})$, but it could have been very reduced or used to produce smaller blades. Unretouched large blades were also found in South Town, but these had wear showing that the blanks were used as tools (Payne 1993:156-158). These findings indicate that for most sites the large-blade knives must have been produced outside of the settlement. This interpretation is supported by a study at Adaïma, where raw material analysis indicated that a number of 'backed blade knives' were imported into the settlement, not made there (Briois and Midant-Reynes 2008:25).

While the quantities of large-blade knives found in settlements was usually very few, large blades knives were found quite commonly in cemeteries, including cemeteries associated with each of the settlements studied here except for Nag el-Qarmila: el-Mahâsna Cemetery H (Ayrton and Loat 1911); Abydos cemetery U (Hikade 2000); Naqada cemeteries (Holmes 1989); Armant cemeteries (Mond and Meyers 1937), Hierakonpolis cemeteries (Quibell and Green 1902: 48-49 Pl61) and others (Table 5.22; Table7.18). Their presence in numerous cemeteries emphasizes the spatial disparity between their production in few sites, and use in many sites. 
The range of tools made on these large blade blanks was limited to a few kinds of knives. The knives have been classified as 'Hemamija A' knives (more standardized retouch pattern), 'Hemamija B' knives/other blade knives (less standardized retouch pattern), or end-scraper knives (based on the shape of the retouch at one end) (Baumgartel 1960:37, 40-41; Holmes 1989:402-403; Schmidt 1992a: 32-33). The width of the knife may also be taken into account (Kabacinski 2012). It is very clear that the large blades were primarily used to make knives, and this restriction of tool types made on the large blades is in line with specialized production.

The distribution of the large blades extends well beyond the areas where they were made. Production of these large blades did not take place at most of the sites where they were used. Therefore it is possible to conclude that they were produced by specialists. However some question remains as to precisely how the specialization was organized. The large blades could have been made in some communities, such as Badari, and exchanged to other communities throughout the region or even to other regions. However it is not possible to say at this point how many communities produced large blades, only that it was likely very few. Additionally, large blades may have been made out at desert quarry sites since there were certainly raw material size requirements for these tools, and special raw materials may have been selected.

\section{Non-heat-treated bladelets}

Nothing conclusive can be said about the patterns of production for non-heat-treated bladelets at the inter-community level (Table 5.23). Heat-treated bladelets were not differentiated from non-heat-treated ones at the Armant, HK29B, and HK25 settlements. The same goes for the cemetery data. Bladelet blanks were present in Abydos cemetery U (Hikade 1998, 2000) and in Hierakonpolis HK6 (Droux and Friedman 2014), and microendscrapers have been identified at 
Abydos cemetery U (Hikade 1998, 2000) and Armant (Mond and Meyers 1937) but it was not indicated whether any of these examples were heat-treated or not. From the settlement evidence it is clear that there was production of non-heat-treated bladelets at el-Mahâsna and at the Hierakonpolis localities, both of which also have evidence for heat-treated bladelet production. At Maadi, a site in Lower Egypt, there was a substantial amount of bladelet production; however it is not known how much of it was non-heat-treated since Holmes identified some heat-treated material from Maadi (Holmes 1992b). Most likely, only some of the material was heat-treated since at other sites where heat-treated bladelets were present there were also non-heat-treated bladelet remains.

There was no clear focus on the production of a certain tool type from non-heat-treated bladelets (Table 5.25). Retouched pieces (unpatterned tools) were the most commonly reported tool type, but other tool types have been reported sporadically. The represented tool types were somewhat similar to those reported for heat-treated bladelet tools, with some microendscrapers, denticulates, and truncations. On the whole there is not enough data to support any conclusions on the organization of production for non-heat-treated bladelets.

\section{Summary of blade distribution at an inter-community level}

In summary, the distribution of blade tools and production remains across sites shows that the organization of blade production was actually quite complex-there were multiple diverse ways tools were produced by different groups of people across the Nile Valley—not just simple homogeneous production. There were many different types of blade production, and they were all organized differently in terms of the sites of production and use.

Medium blades cannot be considered specialized at the inter-community level since they 
were made and used in all settlements studied. On the other hand, the production of large blades was specialized at the inter-community level. The large blade production was geared toward producing blade knives, only a few of these large-blade knives were recovered in each settlement, their production did not take place in most communities where they have been identified, and the number of production locations was likely quite few, possibly even at desert quarry sites. The heat-treated bladelet production constitutes a third kind of organization, where these items were produced in a number of settlements, but not all of them, yet the products reached all areas. There was not enough information on non-heat-treated bladelets to really discern their production pattern. They were at least made in the same sites that made heat-treated bladelets, possibly in others as well. And finally, microdrills showed another entirely different pattern of tool production, one which cannot be considered specialized according to the definition used here, because they were not exchanged. Microdrills were made in the same places where they were used, although what people made with the microdrills may have been specialized. All in all, the variety of blade production systems shows that Predynastic lithic production was anything but a homogeneous or simplistic affair.

\subsection{Blade Specialization at an Intra-Site Level}

There is sufficient data from el-Mahâsna and Hierakonpolis to look for differences in specialization within sites. Specialization was assessed through differences in the distribution of tools and debitage for different reduction sequences: medium blades, heat-treated bladelets, and non-heat-treated bladelets. As per the data in the preceding section 5.4, these blade types had evidence of production remains in settlements, and were either not specialized at the inter 
community level or could be even more finely specialized within the sites where they were produced.

\section{Medium blades at el-Mahâsna}

Table 5.24 shows the frequency of medium blade production remains and tools across three blocks at el-Mahâsna. The percentages do not vary greatly. There is sufficient data to calculate the confidence intervals (Figures 5.14- 5.15), which show that no real difference can be distinguished in the distribution of blade tools and production remains in el-Mahâsna. This information indicates that blade production should not be considered specialized within elMahâsna.

\section{Non-heat-treated bladelets at el-Mahâsna}

The non-heat-treated bladelet production was concentrated in Block 3. All the non-heattreated bladelet cores and crested blades were found in Block 3 (Table 5.26). However, the sample size is rather small (all under 30), and the confidence limits for the percentages from different blocks overlap (Figure 5.16), indicating that the differences may not be meaningful. Therefore the concentration of production remains in Block 3 is not conclusive, and for now it is not possible to determine whether non-heat-treated bladelet production was specialized within elMahâsna. However at the 80\% confidence limits the proportion of non-heat-treated bladelet cores in block 3 is statistically different from the other blocks. While not conclusive, this evidence is certainly intriguing. 


\section{Heat-treated bladelets at el-Mahâsna}

Table 5.27 shows the counts of identifiable heat-treated bladelet debitage and tools at elMahâsna. Heat-treated bladelet production remains were present in Blocks 1 and 3, but not in Block 4. Block 3 had more cores, and the core in Block 1 is a prepared core with no actual bladelet removals (Figure 4.37). The sample size is small, and the $95 \%$ confidence limits for the percentages of cores all overlap, indicating that the differences could simply be due to sampling error. However, at the 85\% confidence level the percentages are basically distinct (Figure 5.17) which means that the patterns are suggestive. While it is not possible to conclude that heattreated bladelet production was definitely specialized within el-Mahâsna, there is some variability in the distribution of production remains which may be important, and, together with the data from Hierakonpolis (see below) may indicate a slightly more intricate way of organizing the production.

Given that the sample sizes were so small for the non-heat-treated bladelet and heattreated bladelet data they were also analyzed as a combined group since the only difference in production may be in the raw material treatment. When the heat-treated and non-heat-treated bladelet data from el-Mahâsna were combined, production was clearly concentrated in Block 3 (Table 5.28). Six of the seven bladelet cores were found in Block 3, and only one (the prepared core) was from Block 1, while no bladelet cores of any kind were found in Block 4. The confidence intervals show that these percentages can be differentiated at the $90 \%$ confidence level (Figure 5.18), which, although slightly lower than the usual 95\% significance cut off, indicates that it is quite likely that there was significantly more bladelet production in Block 3, whether heat-treated or not. 


\section{Hierakonpolis}

Hierakonpolis provides the opportunity to compare across localities. Each locality is a kind of "site" in and of itself, with sample sizes the same order of magnitude as the el-Mahâsna sample was before being divided into blocks. The Hierakonpolis localities are all within $2 \mathrm{~km}$ of each other, and some are even closer. They were likely all integrated into a larger settlement complex composed of diverse parts (Friedman 2011c; also see Ch. 3.7). Analyzing the distribution of materials across localities at Hierakonpolis is a way of looking at production within a large multi-component site. The following figures are more specified versions of the presence/absence tables reported in Tables 5.19-5.25. When possible actual counts are also given.

\section{Medium blades at Hierakonpolis}

As with el-Mahâsna, medium blade production at Hierakonpolis does not appear to be specialized at the intra-site level (Table 5.29). All of the localities had elements from many stages of medium blade production, and many kinds of tools were made on medium blades. The comparison of the counts and percentages from HK11C and HK29 show that they were practically identical (Tables 5.30 -5.31).

\section{Non-heat-treated bladelets at Hierakonpolis}

Cores, blanks, and (likely) tools from non-heat-treated bladelet production were present in all three localities (Table 5.32). Thus it seems there is no discernible difference in production. Unfortunately, there were not enough specific quantities available for statistical comparison. 


\section{Heat-treated bladelets at Hierakonpolis}

Cores, blanks, and tools associated with heat-treated bladelet production were present at three Hierakonpolis localities $^{32}$ (Table 5.33). It should also be noted that Holmes (1992) clearly demonstrates that the main focus of production at HK29A was finely flaked bifacial tools, along with bead production. There was some heat-treated bladelet tool production there, but not necessarily more than at HK11C or HK29.

Before concluding that heat-treated bladelet production was the same across these three localities at Hierakonpolis, some additional data regarding heat-treated chunks (angular debris) should be considered. "Chunks” are lithic debitage that result from random shattering of a rockeither through force during flaking or through heat shattering. They could be considered the 'slag' of lithic reduction. Chunks were not included in the tables on heat-treated bladelets because heat-treated chunks could result from the production of any kind of heat-treated tool, not just bladelets. On the other hand, heat-treated chunks certainly would be a part of the early production of heat-treated bladelets. HK11C had a high percentage of heat-treated chunks, 10.9\% of all heat treated material (calculated from Takamiya and Endo 2011:Table 1), compared to el-Mahâsna (7.9\%) and Abydos (2.2\%). Unfortunately the percentages of heat-treated chunks from the other Hierakonpolis localities was not available for comparison. Despite this high percentage of heat-treated chunks, there was only a low percentage of heat-treated cores at HK11C (1.2\%). For comparison, at el-Mahâsna, heat-treated cores accounted for 7.45\% of all of

\footnotetext{
${ }^{32}$ Specific counts of heat treated material for all tool and debitage types were not available, so statistical comparison was not possible. But where present, the reported frequencies from HK11C and HK29 were commensurate, since the sample sizes were similar.
} 
the heat-treated material. ${ }^{33}$ Considering the high ratio of chunks to cores at HK11C, it seems likely that some cores are missing from HK11C. It is possible that the cores were prepared there (heated, and then possibly flaked to produce an initial crest) and then distributed to other localities or sites, where they could have been reduced as needed. It is important to remember that HK11C was a workshop area where other crafts involving heating took place, including beer production, ceramic production, and food production (Ch. 3.5). This situation of preparing cores for others to then reduce could be considered a form of within-site specialization. Furthermore, a similar case can be made for el-Mahâsna.

At el-Mahâsna there was variability in the distribution of bladelet cores across the site, which may indicate that not only was the heat treating segment of production specialized, but perhaps also core preparation. At el-Mahâsna a prepared but unreduced core was found in Block 1, whereas in Block 3 there were a number of (reduced) heat-treated bladelet cores. While these data are qualitative and not based on a large sample, variability in the distribution of key early production elements (chunks and cores) may indicate that indicated that the entire production process may not simply have been carried out in each area, but organized in a more complex way across the site at both el-Mahâsna and Hierakonpolis.

\section{Summary of distribution within sites}

Medium blade production certainly was not specialized within the sites studied here. Conversely, heat-treated bladelet production did show some variability in the distributions of cores and chunks within sites, which indicates that specialization in the production process may

\footnotetext{
${ }^{33}$ This includes other core types besides bladelet cores.
} 
have been developing at el-Mahâsna and Hierakonpolis, although whether such hypothesized specialists produced only prepared cores for exchange, or the final bladelets tools as well is indeterminate. An additional pattern worth pointing out is that the distributions of the non-heat treated bladelet production elements and the heat-treated bladelet production elements tend to overlap.

\subsection{Chapter 5 Conclusions}

From the above analysis two tool types can be considered specialized: large-blade knives, and microendscrapers. Large-blade knives appear to be specialized at the inter-site level since the knives themselves are found in many sites, but few if any of the associated production remains have been found in any Predynastic settlement sites. Presumably they were made in only very few areas and/or near the raw material sources in the desert.

The production of heat-treated microendscrapers was definitely specialized at the intercommunity level: production remains from these tools were only present in some settlements, but the microendscrapers reached all areas. Furthermore at the within-site level the preparation of the cores, in terms of heating them and possibly preparing the crests, may also have been done by specialists. Additionally it should be mentioned that microendscrapers were made on both heattreated and non-heat-treated bladelets, and, in the cases where sufficient data are available, nonheat-treated bladelet tool production overlapped spatially with the heat-treated bladelet tool production. However the distribution of the production remains for non-heat-treated microendscrapers was not as clearly specialized as with the heat-treated microendscrapers, which would make sense if it was only the core preparation that was specialized within sites. The best interpretation that can be offered at this point is the probably both were made together, or in the 
same basic reduction stages, with certain nodules being heat-treated as deemed necessary by the ancient knappers.

Neither medium blade production nor the production of microdrills could be considered specialized according to the definition here: as where, above a household level, a class of objects is used by people other than those who made them. In both cases the tools were produced where they were used (the distribution of production remains overlapped with the distribution of tools) so as far as we can tell archaeologically they were made by the same people that used them, without wider exchange. However the details of their production nonetheless differed in terms of concentration, quantity, and tool type: Medium blades were relatively common item made in multiple areas in many communities, and used for many different kinds of tools. On the other hand the bladelets for microdrills were much rarer, only made in certain communities and only microdrills were made on these coarse bladelets.

These patterns are visually summarized in Table 6.35. These findings are significant because they show that Predynastic blade production was quite diverse, not limited only to asneeded household production nor even limited to a simple division between ad hoc production and specialized production. Rather the distribution of blade tools and production remains across and within sites shows that lithic production was organized in an array of ways including multiple forms of production that can be considered specialized and multiple forms of production that is not considered specialized here. Evaluation of the ritual production model depends on analyzing patterns of specialist produced tools. Therefore, only the data concerning large-blade knives and microendscrapers will be used to evaluate the ritual production model, since those are the most clearly specialized blade tools. 


\section{Ch 6: Bifacial Tool Specialization}

Investigations into specialized production of Predynastic stone tools have so far mainly focused on bifacial tools. It has already been argued, quite convincingly, that ripple-flaked knives were produced by specialists (Kelterborn 1984; see below). Additionally, Holmes (1992a) argued that at HK29A the large quantities of lithic remains indicated that bifacial tools were produced there by specialists. However, many different kinds of bifacial tools were made and used during the Naqada period in Egypt, and no clear case has been made about the organization of production for many other kinds of bifacial tools.

In section 6.1, descriptions are given for the HK29A bifacial tool production area, and eight different bifacial tool types (RFKs, rhomboid tools, fishtails, concave-base projectile points, figural eccentrics, bifacial knives, bifacial sickles, and axes). Each discussion includes a description of the tool type, date, production methods, and use (including symbolic meaning, use-wear, discard). Sections 6.2 and 6.3 contain discussions assessing the degree of specialization for each tool type. Specialization was evaluated by looking at the distribution of production remains and tools first on an inter-site level (Ch. 6.2), and then on an intra-site level (Ch. 6.3). The results of this evaluation indicate that, as with the blades, Predynastic Egyptians had many approaches to organizing the production of their tools.

\subsection{Predynastic Bifacial Tools}

\section{Bifacial tool production at HK29A}

Large scale production of bifacial tools and beads was identified at HK29A (Holmes 
1992a). The evidence that HK29A was a ceremonial enclosure was laid out in Chapter 3.5, based on its unusual size and shape, investment in materials and repair over time, its unusual and unique ceramic assemblage, and the unusual faunal assemblage with a focus on rare wild animals. Large amounts of lithic material were associated with this enclosure. Here the evidence for this production and the argument that it represents attached specialized production is discussed in more detail.

\section{Bifacial tool production evidence}

$143 \mathrm{~kg}$ of lithic artifacts were recovered from approximately 600sq m of HK29A during excavations in 1985-86 (Holmes 1992a:37,39). Holmes sorted and classified just under half of that, about 54000 pieces. ${ }^{34} 68.6 \%$ of the material is “debris-like debitage, largely consisting of chips” (ibid.:39). Chips are flakes and flake fragments less than $1.5 \mathrm{~cm}$ in dimension. Holmes’ interpretation was that most of the debris came from bifacial tool reduction.

Of the remaining $31.4 \%$ of the material, almost half of it consisted of biface thinning debitage $^{35}-15.4 \%$ of the total assemblage (ibid.:39, 1996:196). Probably even more of the material actually resulted from the biface reduction process, but did not have all of the specific markers of a thinning flake, and so were classified as plain flakes (Holmes 1992a:39).

The main raw material among the bifacial tool debitage was a fine-grained beige chert, but $13 \%$ of the biface thinning debitage was of an unusual cream or orange chert (ibid.:39). The

\footnotetext{
${ }^{34}$ It is not clear whether the number 54,000 applies to the analyzed material or is an estimate of the number of pieces in the total weight of the material. Following Takamiya and Endo (2011:740) 54,000 seems to refer to the analyzed material, and the total estimate must have been over 100,000 .
} 
cream and orange material was also found among the chips, but not among the blades, flakes, or cores. Holmes argued that this shows careful selection of raw material for bifacial tool production. One raw material source has been located near Hierakonpolis (Friedman and Youngblood 1999; Harrell 2012). However this material is not described and it is unknown whether the chert is the beige described for these bifacial tools.

Takamiya and Endo $(2008,2011)$ examined more of the lithic artifacts from the original excavation of the HK29A enclosure, plus newly excavated material. They confirmed Holmes' findings of an assemblage predominated by biface thinning debitage, and also found a number of tabular flint nodules with initial flake removals that were likely the blanks for bifacial tools.

\section{Date}

Newer excavations at the HK29A ceremonial structure in 2002 and 2008 helped clarify the dating of the material and the trends in lithic tool production over time (Friedman 2009b). Materials from the refuse pits along the NE side of the courtyard (from the 1980s excavations) were dated to NIIB-C based on the ceramics (ibid.:85). The 2002 excavations of square 150L40SW (a little farther to the south, along the same wall) uncovered more refuse pits, and 1350 lithic artifacts were collected from that 25 sq meter unit (ibid.:89). This gives a density of 54 lithic artifacts per square meter. Additionally there was some indication that this unit contained material dating slightly earlier, to NIIAB. Sixty-five percent of the lithic material recovered from the 2002 excavation unit was biface debitage (ibid.:89), showing that biface production was already the main focus of lithic reduction at that time. However the quantity and

\footnotetext{
${ }^{35}$ Holmes called this biface thinning flakes and blades- meaning that some of the biface thinning debitage were of blade like proportions (twice as long as wide) but she classified them as from biface
} 
density was substantially lower than in the following period. No microdrills were found, indicating that bead production also came later.

In the next phase NIID2- ${ }^{36}$ NIIIA, lithic production increased in both quantity and diversity. Square 140L50 (presumably also a 5x5m unit), excavated in 1986 and dated to NIId contained over 9,000 lithics (Takamiya and Endo 2008:8) which works out to over 360 pieces per square meter. In addition to the increase in bifacial tool production there was evidence of bead production. In this later phase $34 \%$ of the tools were microdrills for bead production. Beads and unfinished beads were also found. Crescent drills and partial stone vessels indicate that stone vessel manufacture that took place there or nearby (Friedman 2009b:98).

\section{Tool types produced}

The production remains were from what Holmes (1992a:39) termed “finely-flaked” bifacial tools. No complete bifacial tools were found, but broken projectile points and other bifacial tool fragments, possibly knives, were recovered. Holmes (1992:41, 43, Fig. 5) illustrated an item which she called a bifacial knife, however Friedman (2009b:89, footnote 36) argued it may be part of a rhomboid lance. Holmes (1992a:41) thought some of the projectile points may be production rejects, making it extremely likely that they were produced in the enclosure. All I all, the "finely-flaked” bifacial tools produced at HK29A likely consist of projectile points, and possibly also ripple-flaked knives, fishtails (which can have ripple-flaking and whose hafts,

thinning, not from blade production.

${ }^{36}$ Since the sub-phase IId1 is no longer thought to be valid (Buchez 2011b; see Ch 3.2), the date of the second phase should be considered NIID-IIIA. 
when broken look like bifacial knives), rhomboid tools, and other bifacial knives. Other bifacial tools cannot be ruled out.

\section{Specialization}

Holmes (1992a) argued that the evidence suggested a temple workshop of full-time specialists, in other words, full-time attached specialization (Takamiya 2004). This assessment was based first and foremost on the large quantity of lithic debitage and debris relative to other areas of Hierakonpolis. Secondly, Holmes (1992a:43) argued that the special selection of certain chert varieties for certain tools indicates a level of standardization in production. Furthermore, if the tools produced were the ripple-flaked knives, fishtails, rhomboid knives, concave-base arrows, etc, then there was a degree of standardization in the products, since these are the most stylistically standardized lithic tools from the period. The quantity of remains also led Holmes to conclude that the specialists worked full-time, and the location of the remains in a ceremonial enclosure was taken to indicate that elites supported the specialists.

The most compelling evidence that the HK29A lithic production activities should be considered specialized production according to the definition used here (Ch. 1.1), is that the quantity of debris relative to the paucity of broken or finished tools. Much of what was made there "was destined for use elsewhere" (Holmes 1992a:44). Holmes, Friedman (2009b), and Takamiya and Endo (2011) all reasonably concluded that many of the tools went to the elite cemetery at HK6 and likely beyond. 


\section{Ripple-flaked knives}

\section{Description}

Ripple-flaked knives (RFKs) are bifacially worked tools where one face was completely ground, and the other face was covered by very precise systematic ripple-flaking (Figures 6.16.6, 7.1). Ripple-flaking is a kind of retouch with "large, flat, parallel, lamellar” removals (Holmes 1989:408), where the spacing of the flakes is very regular, leaving even, parallel scars that look like ripples. Ripple-flaked knives have tiny microserrations along the knife edge, and the ground side usually has marginal edge retouch. These knives are elongated with a straight to slightly concave back and a slightly convex cutting edge. The haft end is usually rounded, but some have short tangs. A few RFKs have been found with elaborately decorated handles of ivory or gold. Midant-Reynes and Tixier (1981:380) gave average dimensions of the ripple-flaked knives as about $20-30 \mathrm{~cm}$ long, $4.5-6.5 \mathrm{~cm}$ wide, and $.7-1 \mathrm{~cm}$ thick. These match closely to dimensions of 22 RFKs studied here (Table 6.18), that had lengths between $14.9-30 \mathrm{~cm}$, widths of 4.6-8.3cm, and thicknesses of .5-.9cm (see Ch. 6.2). Other Predynastic bifacial tools are known which have ripple-flaking (Figure 6.7), but only knives of this shape, with one ground face, are considered ripple-flaked knives.

\section{Date}

RFKs existed only for a short period of time, dating mainly to the NIId (Dreyer 1999:213; Midant-Reynes 1987:212). From a technical point of view their appearance is a culmination of foregoing technologies (ibid.), so they can be considered to have had a long slow development. Conversely, RFKs disappeared rather suddenly, around about the same time as the D-ware pottery, both largely absent in the NIII. However a few RFKs have been found in later 
graves, such as the Abu Zaidan knife with ornate handle found in a grave which possibly dates to the Naqada IIIB, or the RFK fragments found in the tomb of Djer (First Dynasty) (Petrie 1902:Pl 14; Pitt Rivers Museum object 1901.40.23). Such pieces may have been heirlooms, buried well after they were originally produced (Huyge 2004:823). Additionally the use of elaborate decorated knife handles may date toward the later end of the time span for RFKs, perhaps late NIId-NIII, given the stylistic dating of the handles and the provenienced examples (MidantReynes 1987:220; Needler 1984:270; Whitehouse 2002:432).

\section{Production}

RFKs have the best understood, and most elaborate, production process of any Predynastic Egyptian lithic tools, largely thanks to the replication experiments done by Kelterborn (1984) and the research by Midant-Reynes (1987; Midant-Reynes and Tixier 1981). These scholars and others (Bradley 1972), all agree on the major aspects of production.

Kelterborn examined twelve complete and eight incomplete RFKs from European museum collections, and based on these observations, carried out more than 50 full replication experiments, in order to understand the production process. He concluded that RFK production likely employed many different reduction techniques, including percussion, grinding, pressure flaking, and microflaking. The six basic production stages that Kelterborn outlined are as follows (from Kelterborn 1984:439):

1- "Obtaining the blank": quarrying, testing, and selection of raw materials. The archaeological knives Kelterborn studied had very high quality raw materials without visible grains, and were often glossy and pinkish (ibid.:441). He emphasized that the chert must have been carefully selected and tested for quality. Kelterborn's description suggests heat treatment: 
glossiness and pinkish tones. However, Kelterborn said that heat-treatment of the specimens he studied could not be determined one way or the other (ibid.:439), and after examining a few specimens from the University of Cambridge, Bradley (1972:3) did not find any evidence for heat treatment. On the other hand, the grinding (see below step 3) may have removed any possibility for observing differential luster. Kelterborn himself made the replications on heattreated material and on window glass.

Kelterborn did not guess whether tabular nodules or flakes were used as blanks, although others (Bradley 1972:3, Midant-Reynes and Tixier 1981:381) supposed tabular slabs were used for blanks. Kelterborn estimated that the size parameters for the blanks should have been approximately 20-30cm long, 10-20cm wide, 5-10cm thick. (Estimated time: 3 hrs)

2-Making the preform: Hard and soft hammer percussion and possibly some pressure flaking were used to create the shape of the preform from the blank. Percussion flake scars often remain on the back (ground) face, and sometimes on the front faces, particularly in the region where the haft would have been. This stage and the next have to strike a balance: percussion reduction is faster but more likely to break the piece, grinding is safer but far more time consuming. The percussion preform size should end up about 1.5-2cm thick. (Estimated time: 2 hrs)

3-Grinding the preform: On all observed archaeological knives the back faces were fully ground and the front faces of over $90 \%$ of the examples Kelterborn examined had small traces of grinding, showing that the preforms had been fully ground on both sides. Experimental ground preforms ended up 19-27cm long, 5-6.5cm wide, and .6-.9cm thick. The edges were also ground to a $75^{\circ}$ angle to create the right platform for pressure flake removals. (Estimated time: 12 hrs) 
4- Front surface ripple-flaking: Precision pressure flaking was carried out to remove relatively large ellipsoid flakes, starting at the hafted end and moving counter clockwise. The apparent "perfection” (regularity) can only be reached on a ground preform. Kelterborn (1984:443) says the secret to this flaking is getting "the right combination of depth and spacing of the pressure point, and the suitable amount of inclination (about $10^{\circ}$ ) of the flake detachment force, resulting in just the right amount of flake overlapping.” These experiments show that the flakes were not long and thin as the remaining scars would suggest, but rather C-shaped, with one rounded side and one straighter side. The maximum size of replicated flakes was $4.5 \mathrm{~cm}$ long x $1.1 \mathrm{~cm}$ wide. (Estimated time: about 2 hours)

5- "Marginal retouching": Extremely fine pressure flaking was used to remove the high areas remaining between the bulbar portions of each ripple-flake scar. (Estimated time: about 2hrs)

6-Serration of the cutting edge: Extremely fine grinding and pressure flaking with a very fine tip was done to form the microserrations. These were bifacially flaked from both the front and back faces, creating about 10-13 teeth per cm (ibid.:449). Bradley (1972:4) found a maximum of 16 and an average of 11 serration flakes removed per $\mathrm{cm}$. (Estimated time: around 4hrs)

The tools used for the experimental replications were few and simple: a pressure flaker with a copper point, a wooden block with a cleft for holding the knife, and a small grinding stone for preparing the platforms. Presumably during the earlier stages hard and soft percussors would have been used, along with a larger stone for grinding the faces of the stones. 
The techniques used with these simple tools to carry out the ripple-flaking required great skill. Kelterborn pointed out the difficult aspects of making these knives: keeping the line where the flakes from opposing sides met centered, and keeping a steady even rhythm and spacing to the flake removals. Kelterborn (1984:449-451) also described a few different recovery techniques he was able to observe when problems or mistakes occurred during ripple flaking. The ability to recover from the (relatively few) mistakes made underlines rather than takes away from the skill and repertoire of the knappers.

Moreover, the knives took quite some time to produce. The total time for the replicated knives took 17-25 hours. Midant-Reynes and Tixier (1981) reproduced a knife in only 10 hours, citing just 5 hours for grinding instead of Kelterborn's 12, and not including raw material procurement time. Either way it was clearly a time consuming process and much of the time can be spent in the grinding stage.

As the above production outline shows, many different techniques, each with a different skill level, were involved in the production of RFKs: hard hammer percussion, soft hammer percussion, grinding, pressure flaking, ripple flaking, and microserration. Hence Kelterborn proposed that production of RFKs was probably spread across a number of people-a kind of segmented production. Another important point regarding the multiplicity of techniques that were combined to make RFKs is that each of these techniques was known previously and used to produce other tools (Midant-Reynes 1987). Ripple flaking itself was the only new technology, however it was also done on some fishtails (Figure 6.11(2)), and a few other tools (Figure 6.7).

The RFKs' combined suite of flaking techniques (grinding- ripple flakingmicroserration) fell out of use by the First Dynasty (ibid.). However, grinding remained in use for technically impressive, prestigious knives at least through the First Dynasty, since it is 
evident on the large sword-like knife from the tomb of Khasekhemwy (Hikade 1997:88). The production of elaborate stone vessels took off at the same time that RFK production decreased (Hendrickx 2011:95-96; Midant-Reynes 1987:214), and also used at least similar principles of grinding and polishing. So in a way, RFKs left a legacy of ground stone (prestige?) items.

\section{Use}

Almost all provenienced RFKs have been found in cemeteries (see Ch. 7.3), and the primarily funerary context of the knives could mean that they were made specifically to be included in burials. However Whitehouse (2002) finds that unlikely due to the high quality of workmanship and the content of the decorated handles, which seems intended for display (during life). Supporting this interpretation is the fact that most knives were not buried intact (see below)- they were either broken or had their handles removed before burial, an unlikely scenario if they were intended as purely funerary objects.

One of the best ways to understand the use or purpose of RFKs is to look at the depictions on the knife handles (Figures 6.3-6.6). There are seven known decorated handles associated with RFKs , and up to ten other decorated handles (Ciałowicz 1992; Delange 2000, 2009; Dreyer 1999; Midant-Reynes 1987; Needler 1984; Whitehouse 2002; Williams and Logan 1987). Most are of carved ivory and one RFK handle is embossed gold. Much has been written about the symbolism, art, composition, and interpretation of these pieces (Asselberghs 1961; Ciałowicz 1992; Davis 1992; Delange 2000, 2009; Dreyer 1999; Hendrickx 2006a; Huyge 2004; Kelley 1983; Midant-Reynes 1987; Needler 1984; Whitehouse 2002; Williams and Logan 1987). The main themes depicted relate to control over chaos, dominance, and royalty. 
Rows of animals were a primary theme depicted on these knives, and they relate to control over chaos and domination. The animals include elephants, storks, giraffe, dogs, bovines, sheep, donkeys, oryx(?), badgers, fish, ibises(?), lions, jackals, and others along with fantastical creatures. ${ }^{37}$ The order of the first few rows, and certain combinations of animals, was repeated on many handles and other media showing that particular constructed meanings were intended, not simple depictions of the natural world. At the end of many rows is a contrasting image, such as a dog, feline, rosette, or even fish. There is a connection between dogs and elite hunters (Baines 1993; Hendrickx 2006a), so dogs found at ends of the rows of animals represented the elites in terms of a controlling force, as did the other controlling motifs capping the rows. Rosettes, a vegetal motif, were shown on many knife handles (Figure 6.6), where they appeared either between curved serpents or at the end of rows of animals. The symbol of the rosette was borrowed from Mesopotamia where it was associated with dominant figures (Smith 1992: 241242), and in Egypt came to be associated with Egyptian royalty. Other scenes depicted domination and control over chaos more explicitly, such as the Gebel Arak knife handle which has the 'master of animals' motif and scenes of humans fighting (Figure 6.3). The royal themes on the Metropolitan Museum knife handle are even clearer, showing a person in a boat wearing the white crown with a rosette in front of his face, and what are likely kneeling prisoners on the other side. These themes all relate to the main job of Ancient Egyptian kings: to maintain order over chaos, and this idea persisted as a part of Pharaonic kingship ideology (Kemp 2006:92-99).

Dualities apparent in the knives constitute additional evidence of the symbolic nature of the knives. Duality was an important aspect of Ancient Egyptian iconography. The knife handles display elements of duality in their composition, including pairing and mirror imaging, such as

\footnotetext{
${ }^{37}$ Some identifications are disputed.
} 
the coiled snakes on the Gebel Tarif knife. However, the symbolism and meanings incorporated into ripple-flaked knives did not stop at the end of the haft. Duality was embodied in the chert knives themselves with the different treatment of the two faces: one flaked and one ground. When the production process is taken into account, these faces can be related to desert vs. Nile symbolism, another theme apparent in Predynastic and Ancient Egyptian iconography. Stone grinding did not just involve rubbing one stone against another, but sand was often added to help speed the process along and to create a finer grain (Stocks 2003: 109-111). Sand is indexically related to the desert, associating the ground side of the knives with the desert. In turn the shiny "ripple-" flaked side could reference the water of the Nile. If such is the case then the boats of the Gebel Arak knife handle literally ride on the water, while the wild animals on the opposite face stand in the desert. Given the common association of the desert with chaos in Predynastic Egypt and later, then the theme of control over chaos would be built into the knives themselves, not just the handles, and underlines the highly symbolic nature of these items.

These high-brow ideological themes show that the knives were undeniably laden with symbolic meaning. However the rows of animals literally marching toward the blade of some knives suggests that they may have been used in animal slaughter, in addition to symbolic uses for display, raising the question of whether the knives were also used for cutting.

\section{Cutting?}

Although RFKs had clear symbolic meanings and value, were they nonetheless also used for cutting? Scholars disagree. Usewear analysis of RFKs was conducted by M. Christensen, with conflicting results. In a 1993 publication Christensen et al. concluded that RFKs were used for cutting plant materials based on the identification of silica on the knives. This work was 
based on a model of usewear analysis where trace elements of the worked material build up on the tool creating a polish. However, many now agree that abrasion is a more likely cause of usewear polish (Odell 2001:51-53; Rosen et al. 2014). In a later publication Christensen concluded that RFKs were not used (Delange 2009:9).

Other opinions on the usewear of RFKs were based on the microserrations. MidantReynes (1987:186) considered the microserrations (not to mention the ripple-flaking and grinding) to be aesthetic rather than functional. Conversely, Kelterborn (1984:449) opined that the tiny microserrations are "visually ineffective" and therefore must be functional. In Bradley's (1972:5) opinion, the thinness and serration of the knives would be good for light cutting. He did not find any macroscopically visible wear or breakage on the pieces he examined in Cambridge, but he noted that does not rule out microwear. Similarly, Stevenson (2009:113) examined the serrated edges of the RFKs from Gerzeh under 10x magnification and found no evidence of wear. At this point, there is no consensus for whether or not the knives were used for cutting.

\section{Discard}

Many RFKs are broken rather than complete. Many examples displayed in museums have been discreetly repaired by conservators. In Midant-Reynes’ (1987:200) survey of RFKs the condition of the knife was reported in 35 cases: 24 were broken or fragmentary (69\%) and 11 were complete (31\%). The question is whether the pieces were broken post-depositionally, broken during their use in life and then buried in a tomb, or whether they were intentionally broken for cosmological reasons before burial.

Post-depositional breakage is the least likely explanation. Bradley (1972:5) noted that many of the RFKs have similar kinds of breaks (e.g. Figure 6.2). Similarly, of the 15 RFKs 
recovered from the Abusir-el Meleq cemetery, all were "broken in the same manner” (Seeher 1999a:100). Such similar breaks would have to be the result of being used in the same way or intentionally broken in the same way, rather than random post-depositional breakage. Furthermore, Graves-Brown (2011:209) and Stevenson (2011:72) both point out that plundering is an unlikely explanation for the breakage because broken knives have been found in graves where the other artifacts remained intact. Similarly, at Tel el-Farkha a sword-like bifacial knife and a RFK were found together in a deposit with two gold covered statues (Ciałowicz 2007). The bifacial knife was intact while the RFK was broken in several places (illustrations in Kabacinski 2012). If the knives were broken while being used in life, then associated wear would be expected, along with worn but unbroken knives. However, as discussed above the knives show few unambiguous signs of use, even though many are broken. Thus intentional breakage is left as the most likely explanation.

Intentional breakage of burial items was practiced during the Pharaonic era to render dangerous representations harmless. Similarly RFKs may have been broken before being put into a tomb in order to protect the deceased. Additionally, most knives were found without their handles. Whitehouse (2002:432) suggested that handle removal may have been part of “decommissioning” the knives, rendering them practically and symbolically unusable, i.e. another form of breakage. Either way, intentional breakage and inclusion in burials, or the inclusion of these items in burials despite being broken underlines the symbolic significance of the objects. 


\section{Rhomboid tools}

\section{Description}

Elongated bifacial tools that widen in middle and taper to the ends are known as rhomboid knives or lances or daggers (Figure 6.8, 7.2). Because these terms are used interchangeably and imply functions that are not securely known for this case, these items will be referred to as rhomboid tools. Some of the rhomboid tools are more pointed in the middle while others are more curved, and the tips can be rounded, ogival or pointed (Holmes 1989:408). The flaking of one end is usually less well executed, indicating where the haft would be. They are symmetrical in shape along the longitudinal and sometimes latitudinal axes. Rhomboid tools are very thin and they often have microserrations along the edges, except along the haft area. Baumgartel gave sizes between $27-41 \mathrm{~cm}$ length, by $4-5 \mathrm{~cm}$ width, and a maximum thickness of .6cm, and the 18 rhomboids studied here had dimensions ranging between $18-41 \mathrm{~cm}$ in length, 3.5-6.4cm in width, and .3-.9cm in thickness (see section 6.2, Table 6.19). So far no systematic study has been specifically devoted to the rhomboid tools.

\section{Date}

Baumgartel (1960) classed rhomboid tools with the Naqada I period tools. However it is likely that their use may have extended slightly later. Petrie dated rhomboid tools from SD 3245 based on his excavations at Diospolis Parva (Petrie 1901:23, PL 4), which corresponds to NIA-IIB (Table 3.1). A rhomboid tool was found recently in a partially preserved tomb at Hierakonpolis HK6, which dates to the NIIA-B (Droux and Friedman 2014). The early date of rhomboid knives makes them much earlier than RFKs and contemporary with the earlier fishtails. 


\section{Production}

The production of rhomboid tools involved many of the same suite of techniques as RFKs, fishtails, and CBPPs, including: hard and soft hammer percussion, grinding, pressure flaking, and microserration. Rhomboids are very thin, and the signs of grinding (Midant-Reynes 1987:189), indicate that their preforms were likely ground down to size. They may have followed a very similar production process as RFKs, except without ripple-flaking.

Use

Like RFKs, rhomboid tools have been found mainly in cemeteries (Baumgartel 1960:32; Ch. 7.3). And as with RFKs there is some question as to whether these tools were used primarily for display or whether they were also used for cutting, spearing, or stabbing. There is little clear evidence for use-wear. Baumgartel (1960:32) deemed them too thin to withstand much pressure or any form of hard usage. Spurrell (1896:57) considered the tools "all for show" due to the microdenticulation. However, no study of use-wear has been conducted. Some wooden model rhomboid tools exist, with red or black painted designs on them (Petrie 1920:25) possibly indicating blood, although the wavy and striped patterns could have other interpretations.

Rhomboid tools found in cemeteries were usually broken. For instance Brunton (1937:72, 90, PL40) found a broken rhomboid tool laying in pieces in front of the face of the tomb occupant. The pieces were widely scattered and some small pieces were missing, showing it was not broken in place. The burial was covered by matting and undisturbed. This intentional breakage points to the existence of some symbolic meaning beyond a practical use for cutting. 


\section{Fishtails}

\section{Description}

"Fishtails" are bifacial tools shaped like an elongated triangle where the pointed end is the handle and the wide end is the working end (Figures 1.1, 6.9-6.11, 7.3). The wide has an indentation, either a softly concave 'u shape' or sharply indented ' $V$ shape', creating the fishtail look. There is often a very fine microserration inside the indent and extending around the sides down to where the haft or handle portion would begin (Figure 6.9). The flaking of the haft end is usually not as well executed as that of the forked end. Van Walsem (1978) documented over 150 examples known, and since then more have been found in excavations. A sample of 63 complete fishtails yielded lengths ranging from $7.7-20 \mathrm{~cm}$, widths of $3.2-10 \mathrm{~cm}$, and thicknesses of .32-.9cm (see Ch. 6.2, Table 6.20).

A few fishtails have been found with the hafts intact. One fishtail in the Cairo museum was hafted with a gold handle ${ }^{38}$ (Currelley 1913: 272, Pl 47). A probably more typical example comes from the HK43 non-elite cemetery at Hierakonpolis and was hafted in a reed handle with a leather band holding it on (Friedman 2004). Additionally there was a leather sheath covering the forked blade, and another fishtail, now housed in the Metropolitan Museum (MMA20.5), also preserves a sheath on the forked end. Model fishtail knives often depicted the haft (Figure 6.13).

${ }^{38} \mathrm{~J}$. P. Corteggiani, The Egypt of the Pharaohs at the Cairo Museum (Paris, 1986), 21 -2, has argued that this knife (purchased at Gebelein in I 906) is probably genuine (Roth 1992). 


\section{Date}

Fishtails mainly date from the Naqada I to late Naqada IId or NIII (Hikade 2003:139). They were most common in the late NI- early NII (Hikade et al. 2008:185-186). Some later examples exist, such as the Early Dynastic fishtail found at Abydos by Petrie ${ }^{39}$ (1902:Pl 51), but they are certainly rare by that point. It is also worth noting that fishtails were produced before RFKs, and then they were both produced simultaneously during the late NII (Hikade 2003:149). Fishtails changed stylistically over time, and can serve as chronological markers. The earlier fishtails have the softer and wider ' $U$ ' shaped fork, and date from NI to NIIc or d, while the later fishtails have the sharper ' $\mathrm{V}$ ' shaped fork (Figures 6.10-6.11) and date from NIIa or b to NIId, possibly into NIII (Hikade 2003:139-140; van Walsem 1978:242). The later specimens with the $\mathrm{V}$ shaped fork also tend to have more parallel sides rather than the converging sides of the earlier fishtails. Additionally, there is a style of fishtails with a more pronounced haft. Van Walsem dated these to NIIc-d, but Hikade placed these a bit earlier, transitional between the other two types (see Ch. 7.1). After the Predynastic period fishtails likely evolved into the pš̌-kf, an implement used for the opening of the mouth (rebirth/mummification) ceremony (Roth 1992; van Walsem 1978; cf. Hikade 2003).

\section{Production}

Fishtails were made in many varieties of chert, along with other raw materials, and there may have been some change over time in raw material choice (see Ch. 7.1). Bradley (1972) did not see any sign of heat-treatment on the few fishtail knives he examined in the Cambridge 
museum. However, it is not outside the realm of possibility considering that heat-treatment facilitates the use of fine bifacial pressure flaking such as is observed on the fishtails. Most fishtails were probably made from tabular cores, as was suggested by Bradley (1972) and Hikade (2003). This idea is supported by an assessment of cortex on fishtails. Out of the 101 fishtails inventoried in Table 7.8), three had cortex on at least one face and another 21 had cortex remaining on the very bottom of the haft (e.g. Figure 6.11(1)). ${ }^{40}$ The placement of the cortex seems more in line with thin tabular nodules than of flakes.

Bradley (1972) examined a few fishtail knives from the Cambridge Museum of Archeology and Ethnography and constructed the following production sequence. First the core margins were prepared via alternate flaking. Then the piece was bifacially thinned with direct percussion, probably using a soft hammer. Next came surface grinding limited to the distal (fishtail) half of the piece. Then the distal (fishtail) half of the piece was pressure flaked continuously around the edge (not back and forth alternating edges). Last the microserrations were all done on one face, then the piece was turned over and all the serrations done on the second face. There was an average of 13 serrations per centimeter.

However it is quite possible that the production process for fishtail knives had a degree of variability. Van Walsem (1978:243) noted that the quality of workmanship for fishtails varied widely, as did their size. Some fishtails of the later style were ripple-flaked (Figure 6.11 (2)). Unlike the RFKs, when ripple-flaking was used on fishtails both sides were ripple-flaked rather than just one. Another indicator of production variability is a fishtail from Maadi that was made on a blade, ground on one side, and only bifacially edged, not fully bifacially thinned (Rizkana

${ }^{39}$ Hikade (2003) questions whether this object should be in the same category as the Predynastic fishtails. 
and Seeher 1985: 243,247). Roth (1992:128) suggested it was a locally made imitation of the Upper Egypt fishtails.

Use

The exact use of fishtails is a topic of debate. Many authors (Brunton 1935:216-217; Massoulard 1936; Petrie 1914; and others, see Roth 1992; van Walsem 1978:197) have made a connection based on similarity of form between Predynastic fishtail tools and the later $p s \check{s}-k f$ implement which was a central part of model tool sets used for ritual purposes (Figure 6.12). Dating mainly from the Old Kingdom, examples are known into the New Kingdom (Roth 1992). They were used in the opening of the mouth ritual which "served to give power to the mummy, or a statue of the deceased, so that it might continue to live in the hereafter” (van Walsem 1978:194). This ritual is documented textually from the Old Kingdom period through the Roman period (ibid.:193). The pš̌-kf tools were made from limestone, alabaster, and polished chert (Roth 1992). The connection between Predynastic fishtails and the $p s \check{\text { s}}-k f$ is additionally supported by the translation of the word “pš̌-kf” itself, which means either stone that divides or divided stone (van Walsem 1978:202-203), a description that fits the chert fishtails well. Therefore the use of the fishtails might be similar to, or a precursor of, the use of the $p s \check{\text { - }} k f$. Based on the use of the psš-kf, van Walsem (1978) interpreted the earlier Predynastic fishtails as wedges used to hold a corpse's jaw closed during the Protodynastic period. Noting the impracticality of these carefully sharpened tools for such a task, Roth (1992) offered a different explanation. She interpreted the opening of the mouth ceremony as recounted in the

\footnotetext{
${ }^{40}$ For many, only one face is visible in published documents, so more may retain cortex.
} 
pyramid texts as a ritual of rebirth, and from this she surmised that the fishtail knives were used during births to cut the umbilical cord. Conversely, Hikade (2003) rejected the connection of fishtails to $p s \check{s}-k f$ sets and the opening of the mouth ceremony due to the problems of projecting meanings backward in time. Instead he interpreted fishtails as status symbols for men in outstanding community positions, because fishtails have been found in some graves that also have implements of hunting and warfare. Unfortunately Hikade's interpretation is not compelling because it is based on a small selective sample of tombs, with no statistical verification. The main theme running through all these interpretations, and what all authors could probably agree on, is that fishtails were symbolically significant items.

Hester (1976) carried out a usewear analysis of six fishtail knives from the Naga ed-Deir cemetery. She found heavy intentional dulling on the haft ends. One specimen from tomb $\mathrm{N} 7120^{41}$, had a re-worked edge where one of the tangs had broken off, and the micro-serrations on the rest of the knife had been worn away (ibid.:349), indicating that it was probably used in some way that caused wear. Additionally, a number of model fishtails were painted red on the forked end, which may represent blood (Petrie 1920:25) (Figure 6.13).

It is possible that many were intentionally broken. The tip or one tang of many specimens is missing. While such breaks may have happened accidentally during use, or even occurred during production, one fishtail found in Abydos cemetery U tomb 178 (Hikade 2003) had two notches carefully worked on the outer edge of one tang that must have been intentionally put there (Figure 6.9). Hester (1972) noted from her review of Quibell (1905) that many fishtails were found broken through the middle. Similar breakage patterns might result either from use in a similar way or intentional breakage. Additionally, just as Whitehouse (2002) suggested for the 
RFKs, the handles missing from most fishtails could have been intentionally removed as a means of breaking or immobilizing them to minimize the danger to the deceased tomb occupants (Friedman 2004:9).

\section{Concave-Base Projectile points}

\section{Description}

Concave-base projectile points (CBPPs) ${ }^{42}$ are triangular shaped tools with two long barbs that form a hollow or concave notch for hafting in the middle (Figure 4.48, 6.14, 7.4). There is some variability to their shape in terms of how elongated the point is, whether the sides are straight or curved, the depth of the notch, the curvature of the barbs, and the shape of the bottom of the barbs (Baumgartel 1960:28). There have been attempts to divide the CBPPs into subtypes, (Caton-Thompson and Gardner 1934:27-28; Ciałowicz 1990), and there was certainly some variation over time. Hikade (2001:119-120) described earlier points, such as "Merimde points" and "Fayoum points" as less elongated and with shorter barbs/ shallower notches compared to the Naqada period examples. The Naqada examples also sometimes had fine lammellar retouch (ibid.) from pressure flaking. Rizkana and Seeher (1988:33) pointed out that at many sites CBPPs with notches of different depths existed alongside each other. The sizes of concave-base projectile points examined during this study (Ch. 6.2, Tables 6.22-6.23), ranged from 3.4cm $10 \mathrm{~cm}$ in length, $1.7-3.1 \mathrm{~cm}$ in width, and $.4-.8 \mathrm{~cm}$ in thickness.

The method of hafting is known because there is an extant example of a CBPP mounted on a foreshaft in the Ashmolean Museum (Clark et al. 1974:361). Unfortunately the provenance

\footnotetext{
${ }^{41}$ Lowie Museum accession \# 6-3102.
} 
and date are unknown. Clark et al. think it is likely that the example is from a much later period than the Predynastic, and likely from Nubia. Seen in X-ray, the chert point is $3.4 \mathrm{~cm}$ long (ibid.), so it is smaller than many Predynastic examples. Regardless, it is the only known example of a hafted CBPP. The tool was set onto a slotted shaft and completely covered by mastic leaving only the tip and lateral edges uncovered.

Date

CBPPs can date early as the 5th millennium BCE in Merimde (Eiwanger 1999), and continued to be used well after the Naqada period, into the 1st millennium AD in areas outside Egypt, particularly Nubia (Clark et al. 1974). However, as mentioned above, the style during the Naqada period was distinctive, and in Egypt proper CBPPs were most common in the late NIearly NII (Hikade et al. 2008:185-186), and fell out of use by the Early Dynastic period (Clark et al. 1974:358; Hikade 2001:123). Possible explanations for the decline of CBPPs include changes in hunting practices, the increasing use of metals, and changes in lithic reduction techniques.

\section{Production}

A number of chert varieties were selected for making CBPPs (see Ch. 7.1). Based on study of materials from HK29A, Takamiya and Endo (2008) reconstructed a process whereby concave-base projectile points and other bifacial tools were made from tabular pieces of chert. Hikade (2001: 121) also implied that CBPPs were made from tabular nodules. Holmes (1989:274) noted one example from Petrie’s South Town collection which was made on a thin

\footnotetext{
${ }^{42}$ Also referred to as hollow-base projectile points.
} 
patinated piece of raw material, and was likely unfinished. However some CBPPs were also made on flake or blade blanks, such as a possibly unfinished one from Maadi (Rizkana and Seeher 1988:32, Pl 68). Examples from the Naqada area include some CBPPs made on flakes and one made on a thin nodule (Holmes 1989:247-249).

In Takamiya and Endo’s (2008) model, raw material selection was followed by edge trimming the nodule using hard hammer percussion, then the blank was shaped and thinned by soft hammer percussion, and final stylization applied with pressure retouch. A few CBPPs have been found with signs of grinding (Midant-Reynes 1987:189), although most examples are usually entirely flaked on both sides leaving no place to see grinding marks, so it is difficult to determine if this was a regular practice. As Nagaya (2011) pointed out, the same technology and skills used in making these concave-base projectile points was also applied to the production of flaked figural eccentrics (see below).

Use

CBPPs have also been found in both cemeteries and settlements (Table 7.12). Some CBPPs were definitely used as projectiles. Classic impact scars are visible on some Merimde points, and debitage from resharpening or re-shaping was found in the settlement (Hikade 2001:119). For the Naqada period, a triangular projectile point with a slightly concave base from South Town also had wear indicative of use (Holmes 1989:275).

There is some question as to whether CBPPs were used as arrowheads or lance heads, because they are rather large (Hikade 2001:111). Clark et al. (1974) classified the preserved specimen in the Ashmolean Museum as an arrowhead. Additionally, due to the size and weight, Rizkana and Seeher (1988:32) suggested that they were arrowheads for close distance hunting, 
not designed to fly very far, and Hikade (2001:123) also added that a special kind of bow could have been used.

It is possible that the projectile points were used in conjunction with poison. Poison has been identified on Egyptian bone arrowheads (Clark et al. 1974:342), so it may have been used on other projectiles. A test for similar poison on the mastic from the Ashmolean hafted concavebase projectile was negative, however Clark et al. pointed out that it was a very small sample tested with a limited array of methods, so it does not rule out the possibility of poison. Small indentations in the mastic indicate that the projectile point was possibly wrapped, which is a known method for keeping poison moist (ibid.:366).

CBPPs were also used in a symbolic way. At a columned hall (structure 07) in HK6 a number of concave-base projectile points were recovered from the NE corner of the structure, along with a number of other objects, including ostrich eggshells, ivory objects (clappers?), a hippopotamus figurine, and a fragmented falcon statuette (Droux and Friedman 2007:8,16; Friedman 2010:69-70). Some of the CBPPs were unusually large, almost $10 \mathrm{~cm}$ in length. Friedman suggested that the large size and context indicates that these CBPPs were over-sized votive offerings not intended for use. Along similar lines, clay models of CBPPs have been found in cemeteries (Garstang 1903:7, Pl3).

Though no systematic study of CBPP breakage patterns has been made, the impression from research here is that they were often found broken, usually with a tang or tip missing. However complete ones are also known. A study of the breakage patterns of CBPPs to determine the variability in location of the break and whether the break was from use, production, incidental occurrences, or was intentional, would help to identify production areas and specify how they might have been used, whether for hunting/warfare, as ritual items, or both. 


\section{Eccentrics}

\section{Description}

Among Predynastic Egyptian lithic artifacts, the eccentrics constitute one of the best examples of creativity, skill, and blatantly symbolic use. Naqada period knappers produced an array of figures in chipped stone. These included an antelope(s), arrows, Barbary sheep, birds, bovine heads, crocodiles, dogs, a donkey, a giraffe, hippos, a hartebeest, a human(s), ibex, a scorpion, serpents, and other figures which are more difficult to identify (Figures 6.17-6.24). The figure depicted, size, and production techniques all varied widely. Of 20 figures for which metric data were available, the sizes ranged from $3.3 \mathrm{~cm}-23 \mathrm{~cm}$ in length.

A study by Hendrickx et al. (2003) gave an in-depth look into Egyptian figural eccentrics. However a number of new figures have since been found, particularly at Hierakonpolis. Therefore, a new inventory was compiled adding on to Hendrickx et al.'s list and focusing on production attributes. Table 6.1 lists 53 figural eccentrics, ${ }^{43}$ and Table 6.2 lists additional ones which are probably not authentic. ${ }^{44}$ Given that an attempt was made to be exhaustive, the low quantity of these items shows that figural eccentrics were rarer than any other class of bifacial tools, particularly since they covered a comparatively long time span.

\footnotetext{
${ }^{43}$ In addition to the items listed in Table 6.1, a number of fragments which probably come from eccentrics have been found at HK6 (Friedman 2013:7; Friedman et al. 2017). Additionally five lithic items dubbed "bow-ties" have been found at the HK6 cemetery could be considered eccentrics (Droux 2011:17; Friedman et al. 2017; Nagaya 2011). Alternatively, the very slightly asymmetrical objects could also be a type of transverse arrowhead, with the notches used for hafting. Arrowheads and eccentrics alike have been found as offerings in HK6.
} 


\section{Date}

The figural eccentrics have been found in contexts dating from the early NII into the Early Dynastic period. Some of the ones found in later contexts may have been produced earlier and retained as heirlooms. The earliest eccentrics mainly come from Hierakonpolis. At HK 6 a chipped stone figure of a human (dwarf) was found in a tomb complex containing dating to the NIC-IIA, and the human figurine was likely an offering relating to a dwarf burial in that tomb complex (Friedman 2011:4-6). Similarly, tomb 24 is dated to NIIAB (Friedman 2009b:7), and contained not only an elephant burial but also a chipped stone figure of an elephant head, likely related and so of the same date (Friedman 2006:7-8).

Many other animal eccentrics were found at Abydos, and came from later contexts. Petrie recovered a bovine head from the tomb of Djer (First Dynasty) (Petrie 1902: pl XIV), and a crocodile from the Osiris temple at Abydos, which Petrie dated to the Second Dynasty (Hendrickx et al. 2003:10). A number of additional crocodiles and a serpent were found in the settlement under/around the Osiris temple at Abydos, one of which Petrie dates it to the reign of Djer (ibid.:22) with the rest found at earlier levels below that piece, but all within the Naqada III (Petrie 1902, 1903). Hendrickx et al. (2003:10) suggested that a long thin piece from Umm elQa’ab Tomb U-178 which dates (to phase Ia2 which corresponds roughly to NIB (Hartmann 2011a:932-933, 935) may be a figural eccentric because of its slight curvature. However, the piece is more likely from a CBPP, which accords better with the date. Another piece from a First Dynasty context is a bull head from the royal mastaba tomb of Neithhotep at Naqada, which

\footnotetext{
${ }^{44}$ Scharff (1929: 68, figure 47) reported that flaked-stone (bird) figures bought in Luxor in the 1920's were locally made fakes.
} 
Hendrix et al. (2003:10) feel should be considered an heirloom based on the style and association with other early Predynastic artifacts.

Recently, an undulating bifacial object was found at Kom al-Ahmar/Sharuna, near Wadi el-Sheikh, in middle Egypt (Pawlik 2006:557). While the date of the object was not given, the site has contexts dating at least as early as the Second Dynasty, and into the Old Kingdom and later. The object is similar to the serpents, however production of chipped stone bracelets is known from Wadi el-Sheikh and one was found at the Kom al-Ahmar/Sharuna (ibid.), so the undulating piece could be related to jeweler production instead of figural eccentrics. Petrie also reported a chipped stone figure in the shape of a hippopotamus from Kahun, a Twelfth Dynasty site (Petrie et al. 1890:30 Pl VIII). However, since there is a vast temporal gulf between that hippopotamus and the eccentrics which have secure proveniences, it seems likely that the Kahun hippopotamus is not in its original context, but either was a Predynastic item mixed into a later context, or was unrelated to the Predynastic-Early Dynastic eccentric production.

\section{Production}

Egyptian figural eccentrics were executed in a variety of production techniques with variable quality ranging from rather simple unifacially edged silhouettes of animals, to quite finely thinned and pressure-flaked fully bifacial pieces. At least four were made on flakes. ${ }^{45}$ It is not possible to determine if any were made on thin nodules or tabular slabs because cortex rarely remains. Of the 53 (authentic) figures inventoried here, 25 are bifacial, and 13 are edge retouched (including unifacial and/or bifacial edge retouching). The production technique of 15

${ }^{45}$ BM.EA.30411; BrM.09.889.291; HK.6.2005.1; OIM.10534; See Table 6.1 for descriptions. 
eccentrics was indeterminate because images of one or both sides were not available. These details show that there was variability in how the eccentrics were produced.

Indeed, variability characterizes the eccentrics. Many different animals or figures were depicted, with the same one rarely represented more than a few times. Among figures of the same animal, individual examples were not always made the same way in terms of both production technique and/or style/shape of the figure. This variability is an important consideration for the present study because it indicates that there were probably multiple producers, which is significant for understanding the organization of their production (Ch. 6.2). A few of the different kinds of animals or figures are discussed below to illustrate the degrees of variability or similarity.

The three or four bovine heads were all made differently (Figure 6.20). One was made through very fine bifacial reduction and is perfectly symmetrical (RMAH.E.6185A). The other two specimens were less well worked and are noticeably asymmetrical (BM.EA.32124, FMcGC.1). All three have horns with different shapes, sizes and angles, along with different head shapes. The fourth is only known from a cursory description and its current whereabouts are unknown (Capart 1905:153; Hendrickx et al. 2003).

There are five probable crocodiles (Figures 6.15-6.17) among the collection of figures, and all show a great range of style, making the identification somewhat dubious for the most abstract ones. The unifying feature seems to be an open mouth. Additionally, some have a ridged back, though these could be executed differently. There is also variability in how they were made, two are bifacial, and two are only edge-retouched. The production techniques of the last are difficult to determine from available materials.

The two scorpions are different in both reduction technique and shape. One is bifacial 
(MrM.98.88) and the other (Unknown.10) was only edge-retouched. The pincers are depicted differently and the bifacial piece has a smooth body while the other scorpion has distinct ridges on the sides.

Much more uniform are the ibex, which were made with similar production techniques but show some minor differences in style (Figure 6.23). All were bifacially worked. The ibexes from HK6 are extremely similar, in shape and reduction quality. However, the legs of the ibex in the Berlin museum are quite different in style, with a thick back leg and a protuberance on the foreleg.

Like the ibex, the five hippos show similarities in production, but still many differences in style (Figure 6.21). ${ }^{46}$ All were bifacially worked, but the one from Kahun is of slightly lower quality. Noticeably, they are all of rather dark raw materials, and the raw materials of the two in the Hearst museum are so similar that they seem to be a set. Four have rounded bellies and a slight protrusion marking the tail. The Kahun and HK6 hippos have rounded snouts, the hippo from a private collection has a square snout with rounded corners, and one of the Hearst museum pieces has a square snout. The other from the Hearst Museum has an additional appendage or is shown with its mouth open. The square snouted Hearst Museum one has clear bumps on top of the snout indicating wrinkles above its nose- a feature of many Predynastic hippos (Hendrickx and Depraetere 2004). Though the PAHMA catalog cards note them as possible fakes, the detail above the nose, the raw material type, and general reduction style argue for authenticity.

There are at least three dogs (Table 6.1, Figure 6.24), and they show similarities in style but differences in production. They were all depicted in a similar position with their front paws 
out, leaning back with butt up and bellies down, although the exact shapes vary. A quadruped from HK 6 (HK6.2006.3) has similar features and may also be a dog. ${ }^{47}$ Unlike the ibex and hippos, the dogs vary in production technique from fully bifacial to only worked along the edges.

The five serpents (Table 6.1, Figure 6.22) form a rather cohesive group in that they are all squiggly and of not particularly high quality. It is difficult to determine from such thin pieces whether they are worked only along the edge or across the whole face. None of them stand out as being particularly well made. They are all likely all fragmentary.

Use

These items clearly had symbolic uses. However it is difficult to understand the selection of figures chosen, and to derive insight on how they were used from that selection, because the eccentrics do not fit into a clear category from a modern point of view. The figures include both wild and domesticated animals; both Nile and desert animals; both edible animals and animals which show no signs of having been eaten during the Predynastic. Furthermore, both humans and animals were represented (although the human dwarf might still fit into an "other” category); and all manner of taxa are present including mammals, reptiles, fish, insects, and birds.

All the animals represented among the eccentrics were important iconographic elements of the period (Hardtke (2012); Hendrickx 2002, 2006a; Hendrickx and Depraetere 2004; Hendrickx et al. 2009; Huyge 1998). Underlining their ritual and religious significance is the fact that many of the animals identified among the chipped stone figures were also present among the

\footnotetext{
${ }^{46}$ One from the Hearst museum is identified there as an elephant, but given its similarity to the other hippopotami, and dissimilarity to the more convincingly elephantine piece from Hierakonpolis, it is here classified as a hippopotamus.
} 
faunal remains identified at ritually significant sites, as shown in Table 6.3. The overlap between the animal figures and the faunal remains from ritually significant sites is not just coincidence because, except for the domesticates, wild hunted animals are all very rare at Predynastic sites (Linseele et al. 2009:120) yet they show up in disproportionately large quantities in these ritually significant areas (Anderson 2011; Friedman 2009b; Linseele et al. 2009; Rossel 2007).

Hendrickx et al. (2003:14) proposed that the meaning or use of the flint figures may not be uniform. They broke down the potential meanings as follows: Those coming from elite cemeteries could be symbols of politico-religious power; some figures may have had an apotropaic and eventually religious significance (crocodiles, scorpions, serpents); while others may represent models of (victual?) offerings (birds, fish). Victual offerings, should probably be ruled out since most of the birds were probably fakes, and both birds and fish could be iconographically important animals (e.g. Horus, or Narmer [catfish]).

Recent excavations at the elite Hierakonpolis HK6 cemetery indicate that the figural eccentrics there were used as some sort of offerings (which does not rule out that the nature of the offerings may relate to religious/political power). All of the eccentrics there were found near the surface rather than inside graves (Adams 2001:6; Friedman 2010:70). Many have been found in the corners of above-ground structures or groups of structures, often with specific caches of artifacts. The locations of these items in the corners may relate to delineation and protection (Friedman et al. 2017). A good example of eccentrics in an offering deposit comes from structure E8. There, in the NW corner, a cache of artifacts was found in a shallow depression sealed in place by plaster melt from the reed walls (Friedman 2006:7, 2010:71). The cache consisted of an animal eccentric shaped like a dog or gazelle, a full-size model steatite fishtail knife, 20

\footnotetext{
${ }^{47}$ The excavators propose that it could be a gazelle.
} 
transverse arrowheads, 2 tanged projectile points, a large piece of obsidian, and an unusual ceramic vessel. In the NE corner of the same structure parallel artifacts were found consisting of a fragment(?) of an elephant shaped eccentric, a fishtail knife, 60 transverse arrowheads, 2 concave-base projectile points, and the same kind of unusually shaped ceramic vessel (ibid.:8). An actual elephant was buried in the center of the tomb complex, so the choice of an elephant as one of the animal eccentrics does not seem coincidental. Other figural eccentrics at HK6 were also found in the corners of tomb complexes or structures (Droux and Friedman 2007:8; Friedman 2005:4-6; Friedman et al. 2017), including a human figure that is likely a dwarf found in the NW corner of a complex that contained a dwarf burial (Friedman 2011). At Hierakonpolis, over the course of the Predynastic there is evidence for a shift toward representations replacing the actual item (Friedman 2009b:95-96). The figural eccentrics might be seen as part of this shift toward representation in addition to, or eventually in place of, the real thing.

The use of eccentrics was not limited to cemetery offerings. Chipped stone figural eccentrics were also found in settlements. Of the 23 excavated pieces, nine were from settlements. The settlement pieces showed less variability in figure type than cemetery eccentrics with four (probable) crocodiles, two dogs, two serpents, and a fragment of an unidentifiable quadruped. It is also important to note that only one of the settlement eccentrics came from a temple, and the rest from a domestic settlement context.

Although the samples were small, there is a discernible difference between the settlements and cemeteries in terms of preservation. Among the pieces with secure proveniences from cemeteries, six are complete, six broken, and two indeterminate. From the settlements, two are complete, five are broken or fragmentary, and two are indeterminate but look fragmentary. The difference in breakage rates may indicate that they were used in different ways in 
settlements and cemeteries. Unlike the RFKs, fishtails, and rhomboid tools discussed above a case cannot be made for intentional breakage, since of the 42 where the preservation could be determined, 66.7\% ( $\mathrm{N}=28)$ were complete.

All in all, the figural eccentrics were primarily religious/ideological objects. Proposed meanings/uses include offerings in cemeteries, symbols of politico-religious power, and apotropaic elements, none of which need be mutually exclusive. Their uses may have varied depending on who was using them, when, and where. Many of the types of animals represented became associated with gods in the Pharaonic period.

\section{Bifacial Sickles}

\section{Description}

Bifacial sickles are fully bifacially thinned tools that have denticulations along one edge (Figures 4.49, 7.5). These elongated tools were made in a few main shapes: bi-pointed, pointed on one end and squared on the other, or squared on both ends. The degree of convexity of the sides can vary, and some are symmetrical along the longitudinal axis while others have a straighter back and convex working edge. Bifacial sickles can be relatively large tools, the bipointed ones sometimes reaching over $20 \mathrm{~cm}$ in length. The widths of bifacial sickles studied here (Table 6.25) ranged from $1.3-4.2 \mathrm{~cm}$, and thicknesses from $.5-1.3 \mathrm{~cm}$. They are one of the main kinds of bifacial tool types found in settlements.

Date

Bifacial sickles date from the Neolithic period at least through the Naqada III. Bifacial 
sickles were quite common at Merimde, Badarian sites, and Fayoum Neolithic sites (Baumgartel 1960:25,29; Brunton and Caton-Thompson 1928:36-37; Caton-Thompson and Gardner 1934:21). Stylistically the Badarian sickles are quite different, shorter and more convex in the middle with larger sickle teeth (Homes and Friedman 1994:132). Bifacial sickles continued to be used in the Naqada I-II (e.g. Rizkana and Seeher 1988:34-35), and have been found in the uppermost level at Adaïma (Midant-Reynes and Prost 2002:353-354) which dates to early NIII (ibid.:20). Bifacial sickles decreased in frequency over time, which likely related to the increase of sickles made on blades with only edge retouch from the NII onward (Rizkana and Seeher 1985:249). Bifacial sickles were certainly gone by the Old Kingdom (Graves-Brown 2011:425). Occasionally, denticulated bifacial tools have been found in the Dynastic periods, such as a First Intermediate Period “knife sickle” from Ayn Asil (Midant-Reynes 1998). However they were not common.

\section{Production}

Bifacial sickles were made in a wide variety of raw materials (Ch. 7.1). Some of them may have been heat-treated. Midant-Reynes and Prost (2002:354) noted one "bifacial piece with regular denticulations” from Adaïma that was heat-treated. Holmes and Friedman (1994:132) suggested that a bifacial sickle from Badarian-Amratian transition level was heat treated. Others observed in this study were not heat-treated or were indeterminate. They could be made on flakes or nodules. Holmes (1989:163) noted one bifacial sickle that was probably made on a blade blank. Given that some bifacial sickles have very large dimensions, around $20 \mathrm{~cm}$, it is likely that some were also made on nodules, rather than flakes. However, bifacial sickles were fully flaked leaving little cortex remaining that would indicate a nodule blank.

Bifacial sickles were probably made using soft-hammer percussion, or a combination of 
hard- and soft-hammer percussion, along with pressure flaking. Holmes (1989:162-163) noted some variation in the technique for forming the denticulations of the six examples she studied from Predynastic sites in the Badari region, although most denticulations were made through some kind of pressure retouch.

\section{Use}

Bifacial sickles have sickle gloss clearly indicating that they were used to cut plant material. Some tools with the same morphological features as bifacial sickles have been found without sickle gloss. They simply may not have been used long enough to develop the gloss, or the tools could have been used for multiple purposes. Many, even the ones without sickle gloss, also often have broken serrations.

There is no indication of intentional breakage. Some broken examples are extant such as examples from Predynastic sites in the Badari area (Holmes 1989:162) but many more have been found complete except for minor wear, such as examples from Maadi (Rizkana and Seeher 1988: Pl 73).

\section{Bifacial knives}

\section{Description}

A bifacial knife is a tool that has been bifacially thinned across most of both faces, has a single cutting edge, and an asymmetric shape (Holmes 1989:405; Midant-Reynes and Prost 2002:374) (Figures 4.50; 6.25). Predynastic bifacial knives had a wide range of morphologies that varied in terms of the degree of curvature of the back, the tip shape, the width, and the 
presence or shape of a handle or haft. Some knives have a more standardized shape and some researchers have defined subclasses such as 'comma shaped knives' and 'standard Gerzean knives' (Holmes 1989:405-406). The sizes can be quite variable, ranging from knives as small as 7cm long (Holmes 1989:161), to swords over 51cm long by the NIIIB (Kabacinski 2012:332).

\section{Date}

Bifacial knives existed well before the Naqada period (e.g. Kindermann 2003), and continued to be made after. While the morphology of knives was quite variable in all the periods (Hikade 2013; Holmes 1989; Kabacinski 2012), some trends are characteristic for different time periods. The subclasses defined by Holmes (1989) are distinctive for the Predynastic period. Pronounced handles become more common in the Early Dynastic and later (Kabacinski 2012; Petrie 1902; Schmidt 1992a,b), though hafting areas are often discernible on Predynastic knives by the less controlled retouching (Holmes 1989: 265, 405; Midant-Reynes and Prost 2002:374). Bifacial knives became more prevalent in lithic tool assemblages in the Early Dynastic and continued to become increasingly pervasive throughout the 3rd millennium BCE (Graves-Brown 2011; Hikade 2013:115). Bifacial knives of gigantic proportions $\left(40 \mathrm{~cm}^{+}\right)$mainly date to the Early Dynastic (Graves-Brown 2011:109, 452-453; Hikade 1997, 2010:9).

\section{Production}

A wide range of raw materials were used for bifacial knives (Holmes 1992a; Kabacinski 2012; Takamiya and Endo 2008, 2011). Holmes (1992:41) did not find indications of heattreatment among bifacial thinning flakes and bifacial tool fragments from HK29A. Those from the el-Mahâsna materials analyzed here were not heat-treated. One bifacial knife fragment from 
Adaïma was classed as a type of raw material thought to be heat-treated (Midant-Reynes and Prost 2002:360). The most evidence for heat-treatment of bifacial knives so far comes from Tel el-Farkha, where there is limited and declining indication of heat treatment of knives ${ }^{48}$ (Kabacinski 2012:324,339).

Often the blank for bifacial tools is difficult to discern if the piece was fully flaked, however there is evidence that bifacial knives could be made on tabular nodules or on large flakes. Naqada period knives made on large flakes or blades have been identified at Tell el Farkha (Kabacinski 2012:328, Fig 5), Hemmamiya (Holmes 1989:79) and Adaïma (MidantReynes and Prost 2002:320). Knives made on nodules or thin tabular pieces come at least from Badari, Hemmamiya, and South Town (Holmes 1989). Furthermore, Takamiya and Endo (2008, 2011) identified tabular flint nodules with a few preliminary flake removals among the material from HK29A, which they argued were the blanks for the bifacial tools produced at the site.

The quality of flaking for bifacial knives can vary from "irregularly flaked and shaped to extremely finely retouched and precisely shaped” (Holmes 1992:405), and this variation likely indicates that, at least to some extent, multiple flaking techniques were used, although skill in execution of the techniques could factor into the variation as well. Probably a range and/or combination of techniques were used including hard and soft hammer percussion and pressure flaking. Holmes (1989:265) identified one non-ripple flaked knife with signs of grinding, from South Town (UC.5331). Additionally, Kabacinski (2012:331) found two knife fragments with signs of polishing from Tel el-Farkha, so grinding was part of the production process for some bifacial knives.

\footnotetext{
${ }^{48}$ Some of the knives were not fully bifacial but made on blades.
} 


\section{Use}

There is much evidence for usewear on bifacial knives. Some bifacial knives have been found with sickle gloss (e.g. Midant-Reynes and Prost 2002:354), though it is possible that the gloss may be from woodworking rather than reaping (Graves-Brown 2011:179). Furthermore knives are often found broken, especially in settlements (Holmes 1989, 1992; Kabacinksi 2012:329-333; Midant-Reynes and Prost 2002:353-354). It is not uncommon for broken knives to be reworked, such as examples from el-Mahâsna studied by the author in University of Pennsylvania Museum. Other times bifacial knives were simply reworked into knives of a different form (e.g. Schmidt 1992b:86, Fig 9.53), which may contribute to the diversity of forms in this class of tools.

However the fact that the knives were used for practical purposes does not mean that they lacked symbolic significance. From the Old Kingdom and into the New Kingdom bifacial knives were often depicted in cattle slaughter scenes (Eggbrecht 1973; Graves-Brown 2011:173-179; Hikade 2010:9). Cattle slaughter has been associated with temple activities in the Pharaonic Period through archaeological and representational evidence (Graves-Brown 2011:177; Rossel 2007), and this same association likely extends back to the Predynastic. The faunal assemblages in the ritual activity areas of HK29A (Linseele et al. 2009:127) and el-Mahâsna Block 3 (Anderson 2006:186-195; Rossel 2007:206-207) had higher quality cattle remains (in terms of age, size, and cuts of meat) and/or higher proportions of cattle compared with surrounding contexts. Graves-Brown (2011:173-179) argued that the knives used in cattle slaughter were important elements of the ritual, and there is evidence that bifacial knives were used for the cattle slaughter at least into the Middle Kingdom. 


\section{Axes}

\section{Description}

Predynastic axes are bifacial flaked stone tools with an oval or expanding form, and a distal rather than lateral working edge (Figures 6.26, 7.6). The cross-section of axes is convex on both faces. Some but not all Predynastic axes often have the scar from a transverse blow across the end that created a sharp edge. This blow forms a distinctive type of debitage known as a “tranchet” or “axe-preparation” flake (Figure 4.40) (Holmes 1990:9). Predynastic axes can range from approximately 4 to $15 \mathrm{~cm}$ in size (e.g. Holmes 1989:247, 1990), however on average they tend to be rather small, only around 6-7cm long (Table 6.29). Fifty-eight axes studied here had lengths ranging from $4.2-10.5 \mathrm{~cm}$, width of $3.3-8 \mathrm{~cm}$, and thicknesses of $1.5-3 \mathrm{~cm}$ (see Ch. 6.2).

Holmes (1990) has conducted the most detailed study of axes, on examples from settlement sites in the Naqada region. Predynastic axes were a major factor in identifying regional variation among Nile Valley lithic assemblages dating to the early Predynastic period (Holmes 1989a,b). A distinctive characteristic of the Naqada region was that axes were much more common there than in the others. The prevalence of axes in collections from that area has been borne out by subsequent studies (e.g. Ginter and Kozlowski 1994; Vermeersch et al. 2004).

\section{Date}

Axes appeared in conjunction with Neolithic activities in many parts of the world (Barkai 2011:445). In Egypt, early forms of these axes have been found in Tarifian and Badarian sites, as well as in the Kharga oasis (Holmes 1990). Many examples are known from Naqada I and II sites, and the latest Naqada-type axes may come from Adaïma (ibid.). Axes certainly continued to be made in the Dynastic period in different forms, but perhaps less frequently, in conjunction 
with the increasing prevalence of copper axes (Graves-Brown 2011:473-475). However the Dynastic axes lack the distinctive tranchet scar, are longer, and tend to have a higher width to thickness ratio (ibid.). In the Middle Kingdom, some chipped stone axes were more proportional in their width and length ratios and had pronounced spurs or lugs for hafting, which imitates the metal axes of the time (ibid.).

\section{Production}

Axes were often made on small and/or thin nodules, and occasionally on large flakes (Holmes 1989:240, 274; 1990:5). The axes tend to be fully bifacial, but sometimes patches of cortex remain. Holmes (1990:7) did some experimental replication of axes using chert from the Naqada area and concluded they were likely made with hard hammer percussion, although she also noted that some may have been finished with soft hammer percussion. Very few axes show traces of grinding. Holmes (1989:273) only noted one axe (UC5351 from South Town) which had indications of grinding, but this grinding occurred after the flaking as a finishing modification rather than as a preparation for flaking.

Sixty-seven percent of the axes studies by Holmes had a tranchet scar. Based on signs of wear such as microchipping, Holmes (1990:9-10) concluded that the tranchet blow was a form of preparation, not re-sharpening. This was based on analysis of the order of flake removals and measurements of the axe preparation flakes relative to the size of the axes themselves, which showed that the tranchet flakes were larger than the average width of the axes.

\section{Use}

Axes are commonly found in settlements, and so are thought to relate to daily-life 
activities. Based on early excavations, axes seemed to be one of the most common tool types found in settlements (e.g. Baumgartel 1960:35). However comparison of modern Predynastic settlement excavations to museum collections shows that this was simply a matter of collection bias- preference for bifacial tools (Holmes 1990:3). For instance, Garstang (1903:7-8; Pl 3,5) specifically reported on and pictured axes and hoes from the el-Mahâsna settlement, but only three axes were counted among the artifacts studied here from Anderson’s el-Mahâsna excavations. $^{49}$

Use-wear studies of axes with tranchet blows from other parts of the world have concluded that such tools likely were used for light woodworking (Barkai 2011:445; Yerkes et al. 2003). This interpretation seems reasonable for at least some Naqada axes. The average edge angle for axes is around $65^{\circ}$ (Holmes 1990:10), which is thick enough to stand up to some strong work but sharp enough to still be a cutting edge. Holmes (1987) conducted a use-wear study of a few Predynastic artifacts, and for two axes she initially proposed a woodworking use. Holmes also observed edge rounding and sheen on two additional axes (ibid.). However she also noted problems due to the presence of patina and desert varnish, and in the end concluded that the function of Naqada axes remains unknown (Holmes 1990:10).

Phil Geib and I examined 12 axes housed in the University of Pennsylvania museum in June 2011. John Garstang collected these artifacts from the El- Mahâsna settlement site in 19001901. Figure 7.6 shows the variability in size and shape of these objects. The average dimensions of the axes were comparable to those published by Holmes (1990) (Tables 6.26-6.28). Two of

\footnotetext{
${ }^{49}$ The size of the area excavated by Garstang is unknown, but was likely larger than the volume of material excavated by Anderson and analyzed here.
} 
the axes had a macroscopically visible wear pattern which can be identified as soil polish ${ }^{5 \odot}$ (E9681, E9682). Soil polish is described as "distinctive, flat, fluted polish with numerous cometshaped pits” (Yerkes et al. 2003:1054) (Figures 6.26-6.27): therefore, the two artifacts (E9681, E9682) were likely used as hoes. Additional support for hoeing is that micro-fractures were also observed near the edge, on the side of the artifact opposite the soil polish. Chopping the artifact into the soil and pulling it would likely result in microfracturing to the far side and soil polish on the near side. Additionally, another example with an even more developed soil polish was found by and mentioned in Garstang (1903:7 PL V).

The category 'axe' is based on morphology and production characteristics, and is not meant to specify function. Therefore the category 'axe' may actually include tools used for chopping and tools that may have been used for hoeing.

All of the 49 Naqada axes in Holmes' (1990) sample were complete. Of the 15 axes from el-Mahâsna three were broken or fragmentary. Five of the 49 axes studied by Holmes showed evidence of re-working (ibid.:10). Re-working or re-sharpening may account for some of the range of dimensions and small average size of Predynastic axes.

\subsection{Evaluation of Specialized Production at an Inter-Community Level}

Below, specialized production of bifacial tool types is evaluated on the inter-site level (Ch. 6.2) and at the intra-site level (Ch. 6.3) by comparing the distributions of tools and production remains. Tables of the tool types and production remains were constructed for this

\footnotetext{
${ }^{50}$ The identification of this wear pattern as resulting from soil was made by Phil Geib, based on similarity to such patterns on Mayan agricultural tools
} 
analysis. Additionally the relative degree of standardization was assessed through a look at the coefficient of variation (CV) for metric attributes ${ }^{51}$ (see Ch. 2.5 for an explanation of the CV).

Tables 6.6-6.7 show the bifacial tool types from the Naqada period Nile Valley settlement sites focused on in this study: el-Mahâsna, Abydos, Naqada sites, Armant, Adaïma, Hierakonpolis, and Nag el-Qarmila. These sites all have sufficient published data on lithic artifacts to calculate percentages, and thus compare between the sites.

Many of the studies represented in Tables 6.6-6.7 were not the first or the largest collections from a given site, so Table 6.8 shows the counts of bifacial tools from other publications relating to each site. However in many cases the total counts of lithic artifacts collected are unknown, so percentages could not be calculated. Furthermore many are from much older excavations, where collection was not as comprehensive as it is these days. Therefore this information is best seen as presence/ absence data.

Since some of the bifacial tool types studied here were mainly found in cemeteries, Table 6.9 shows the counts of bifacial tools in cemeteries associated with the settlement sites studied here. Again, collection strategies varied, and total counts of lithic artifacts found were not always available, so percentages were not calculated. The total number of graves gives some idea of the sample sizes.

Table 6.5 shows that bifacial thinning flakes were found in all settlements, but the frequencies differ. Axe preparation flakes, also shown in Table 6.5, are the only debitage type assessed here that relates to a specific tool type. Summary tables were made for each individual

\footnotetext{
${ }^{51}$ The variability of tools described here does not come near the limits and typical ranges discussed by Eerkens and Bettinger (2001) for considering something standardized, which is a COV around 2-5\%. The least variable tools here range instead around $12-15 \%$. However here the question is not whether or not the tools were standardized, but rather which were the most standardized for their time.
} 
bifacial tool type, showing the presence/absence of the tools and the production remains together (Tables 6.10-6.17).

\section{Ripple-flaked knives}

Ripple-flaked knives were definitely made by specialists. Previous studies dealing with RFKs agree on this point (Bradley 1972:4; Hikade 2010:7, Holmes 1989: 338; Kelterborn 1984:452; Midant-Reynes 1987:222, 1992:200; Takamiya 2004:1030). However, reasoning and details can vary. Most scholars base their assessment on the obvious degree of skill involved. After replicating 50 RFKs, Kelterborn’s (1984:452) conclusion was that: "Such a degree of conceptual sophistication and manual perfection can only be reached by highly specialized master craftsmen who must be ranged among the world's best.” Kelterborn’s (1984:452) only reference to standardization was his conclusion that there must have been formalized education and training in order to account for the uniform quality of the knives. Holmes (1989:338) based her assessment of specialization on the standardization of the style and manufacturing techniques. The flakes were always removed in a counter-clockwise direction (ibid.:442; MidantReynes 1987:190), which is a kind of standardization of the production process.

Because of the high levels of knowledge, skill, and aesthetic achievement Takamiya (2004:1034) classed the producers of RFKs as full-time specialists. Furthermore, Takamiya posited attached specialization for the producers of the RFK knives, figuring that the royal and elite symbolism evident on the RFK knife handles can be considered evidence that the specialists were supported by emerging leaders (ibid.:1035).

In the present study, the definition of specialization (Ch. 1.1) is where items were used by other people beyond those who made them (above a household level), which is here assessed by 
looking at distributions. Kelterborn's assessment of skill does fit with this definition because it hard to believe that the kind and degree of skill evident in the knives' production would be found among more than a very small subset of the population, while the knives themselves were found spread throughout the country (Tables 6.9-6.10). RFKs reached as far north in Egypt as Minshat abu Omar and Tel el Farkha (in the Delta) and even as far as Azor in the Levant (Braun 2014:39). To the south, RFKs were found at Hierakonpolis in Egypt and Qushtamna in Nubia (Midant-Reynes 1987). In contrast to this broad distribution, there were probably only one or a few production locations for RFKs. Because of the standardization of the knives, Holmes (1989: 338) posited that there may have been only one workshop for RFKs in the Egypt. From his examination of the raw materials used for RFKs, Kelterborn (1984:441) thought that more than one source of material was used for the knives, which may indicate that different materials were brought to one workshop or that there were multiple workshops. Only one candidate for an actual RFK production area has been found, the above described locality HK29A at Hierakonpolis. Kelterborn detailed what kinds of production remains should be expected from every stage of RFK production. So far none have been specifically identified and correlated with Kelterborn's analysis, but finding them would help identify RFK production locations with greater certainty. Overall, the distribution is much wider than the possible production areas, and the knives were certainly specialized.

Metric data on ripple flaked knives (Table 6.18) shows that besides being made with standardized production techniques, they were also among the most standardized bifacial tools in terms of size. Twenty-two complete RFKs surveyed here had an average length of $23.75 \mathrm{~cm}$, with a standard deviation of 3.75 and a coefficient of variation of $15.79 \%$. The average thickness for 18 RFKs was $5.87 \mathrm{~cm}$, with a standard deviation of .832 and a CV of $14.17 \%$. Thickness were 
available for 10 knives, which averaged $.66 \mathrm{~cm}$, with a standard deviation of .106, and a CV of $16.06 \%$. Only the concave-base projectile points (see below) were more standardized in width and thickness, but this probably relates to their smaller size and functional requirements rather than to the skill and number of the producers.

Tables 6.6-6.10 show that the RFKs were found in cemeteries, and while they were present in many cemeteries they were not found in all cemeteries. Some of the cemeteries without RFKs in Table 6.9 do not date to the right period, but others do (el-Mahâsna cemetery H) showing that despite having wide distribution, RFKs, have not been found in all cemeteries. The quantities of RFKs are small compared to the number of graves in each cemetery, which points to their restricted distribution. All in all, RFKS had concentrated production and wide geographical distribution (to only a limited number of people), and were used/discarded in a select venue (cemetery). They clearly should be considered specialized at an inter-site level.

\section{Rhomboid tools}

Tables 6.8, 6.9, and 6.11 show the distribution of rhomboid tools and production remains among the sites in this study. The distribution pattern for rhomboids is notably similar to that of RFKs. Rhomboids were also primarily found in cemeteries. The geographic distribution was not concentrated, and in fact was wider than is reflected in Table 6.11 because rhomboid tools have also been recovered from other sites in the regions covered here, such as Mesaid ${ }^{52}$. There is no clear evidence of where rhomboid tools were produced. Again a likely production place for rhomboids is HK29A where one tool fragment may be from a rhomboid tool (Friedman

\footnotetext{
${ }^{52}$ Examples in the Boston Museum of Fine Arts: 13.3766, 11.256, 11.253, 11.23
} 
2009b:89, footnote 36; Holmes 1992:41,43, Fig. 5). However the earliest phase of the HK29A remains dates to NIIB-C, which would be on the late side for rhomboid tools.

Rhomboids were quite variable in terms of their metric attributes (Table 6.19). The average thickness was .65cm (standard deviation .189, coefficient of variation 29.08\%) for seven examples where thickness data were available. The average length for 15 rhomboids was 26.68cm (standard deviation 8.16, CV 30.58\%). Width was the least variable characteristic. Fifteen rhomboids had an average width of $4.74 \mathrm{~cm}$, with a standard deviation of .916, and a CV of $19.32 \%$. The variability in length may relate to the availability of raw materials of sufficient size to make the really large rhomboids.

The metric data show that on average, rhomboid tools are extremely thin, just as thin as RFKs, especially considering their length, which speaks to the skill of their producers. This thinness indicates a degree of flintknapping skill not likely had by many people. Furthermore rhomboid tools involved many of the same production techniques as RFKs, except for the ripple flaking. Since RFKs must have been produced by specialists at the inter-site level, and rhomboid tools bear many similarities to fishtails and RFKs in production techniques and thinness, and were similar to RFKs in terms of distribution (being mainly found in cemeteries), it is likely that rhomboid tool production was specialized, probably at an inter-site level.

\section{Fishtails}

Tables 6.8, 6.9, and 6.12 show that fishtails, although much more common in cemeteries, were also found in settlements, so their use/discard was not as restricted as RFKs. Neither the settlement nor the cemetery distribution of the tools was concentrated in any particular region. Even though Naqada has the highest number of fishtails, it also has a much higher number of 
graves. In cemeteries, the fishtails were actually the most common bifacial tool type, being found in almost all of the cemeteries represented in Table 6.9, and at many of those cemeteries' fishtails are the most numerous of any bifacial tool type.

No production sites for fishtails have been specifically identified, except that HK29A is a likely production area. No debitage has been specifically associated with fishtails, nor have any preforms been securely identified. Since fishtails were made in many different raw material types (see Ch. 7.1), it is quite possible that there were multiple production locals for them. The example from Maadi which was possibly locally made (Roth 1992:128) supports this idea, as does the variability in quality of the fishtails (Van Walsem 1978:243).

Metric data were collected on 63 examples that were complete enough for accurate dimensions (Table 6.20). When these data were analyzed according to the shape type (Table 6.21), it confirmed van Walsem's (1978:243) assessment that fishtail lengths increased over time with the average length of Type 1 fishtails at $12.23 \mathrm{~cm}$, and the average length of Type 2 fishtails at $16.16 \mathrm{~cm}$. But more importantly for this study, the data showed that the variability in lengths, as measured by the coefficient of variation, decreased over time from $19.94 \%$ to $13.63 \%$. This means that the lengths became more standardized over time, as would be expected for increasing specialization. Additionally this trend confirms that style 3 should be placed between styles 1 and 2 chronologically. There was not enough thickness data to look at the variability of thickness over time, and the widths relate more to the style of the object than to factors affecting standardization.

Since the production techniques for making fishtails was very similar to the production of RFKs, particularly for those fishtails with grinding and/or actual ripple-flaking, and there is very good evidence for inter-site specialization for RFK production, it is very likely that at least the 
later fishtails with ripple-flaking were produced by specialists at the inter-site level. By extension, the production of the earlier fishtails was probably either specialized or developing into specialized production. The decreasing variability in length supports this conclusion indicating that fishtails were probably made by fewer and fewer specialists over time, despite their wide geographic distribution. In sum, fishtails had a wide geographical distribution, but involved production techniques that were not likely practiced by all, and became more standardized over time. Therefore it is likely that fishtails were produced by specialists at an inter-site level.

\section{Concave-base projectile points (CBPPs)}

Tables 6.6-6.9, 6.12 show that CBPPs were more common in settlements, but also could also be found in cemeteries, including model CBPPs. The distribution of the tools was geographically widespread, not concentrated. In this case it is clear that there were multiple production areas. Unfinished CBPPs have been identified at Naqada KH4 (Holmes 1989:248249), South Town (Holmes 1989:274) and at HK29A (Holmes 1992:41). Whether they were produced at other sites besides these is unknown.

It is difficult to judge from this data alone whether or not they were made by specialists. Again, the production techniques used—pressure flaking, microserration, and possibly grinding to prepare and thin the blank—-were also features of RFKS, fishtails, and rhomboid tools, all types that are likely produced by specialists. Microserration was not here identified on other tool types besides these. Furthermore this suite of techniques is significant, because the Naqada period CBPPs were different in style and technology compared to those produced during earlier periods. Naqada period CBPPs have deeper notches, finer barbs, and more lammellar retouch 
(Hikade 2001:119-120). This change could related to increasing specialization in the organization of production.

The metric data for CBPPs (Tables 6.22-6.23) show that they were actually the thinnest

tools, having an average thickness of about .6cm, but this probably relates to their smaller size. CBPPs were also the most standardized bifacial tools in terms of thickness (CV=15.41\%) and width ( $\mathrm{CV}=12.65 \%)$, but the lengths were quite variable. The standardization of the thickness and width might have to do with the hafting requirements rather than (or in addition to) the number and skill of the producers.

All in all, CBPPs had a wide geographical distribution, with use focused on but not limited to settlements, production at least in multiple sites but it is indeterminate if production occurred in all sites, and there was a technological change toward a suite of production techniques which were also used on other tools types that were made by specialists. Additionally there was a very high degree of standardization in metric characteristics which was comparable to other tools made by specialists. Altogether, it is likely that specialists produced CBPPs. Whether that production was on an inter-regional, inter-site, or intra-site level cannot be conclusively stated at the moment.

\section{Figural eccentrics}

As was mentioned in section 6.1, the distribution of figural eccentrics was uneven, focused mainly on Hierakonpolis and Abydos. Finds of figural eccentrics from other sites are significantly later in date, have had their authenticity called into question, have insecure proveniences, or were likely heirlooms.

Although no unfinished figural eccentrics have been identified to indicate production 
locations, a number of lines of evidence indicate that there were probably multiple production locations, specifically Hierakonpolis and Abydos. The variability in figures depicted, and the variability in production style ${ }^{53}$ and quality within the same figure type (Ch. 6.1), indicate that there were multiple producers and possibly more than one production locality. One of those is certainly Hierakonpolis, where so many of the figural eccentrics come from, and where there is a known location for the production of bifacial tools at HK29A. Note that the Hierakonpolis figural eccentrics date mainly to the NIC-IIAB period, and the earliest phase of bifacial tool production at HK29A dates to NIIB-C, or possibly slightly earlier (Friedman 2009b:85-97), with the main phase of industrial-scale production even later, NIID-IIIA (ibid.:93).

A second location of production was likely at Abydos, where the most figural eccentrics were found after Hierakonpolis. However, the Abydos figures, dating to the NIII and First Dynasty, were later than the majority of the Hierakonpolis figures. Given the temporal and spatial differences, with many of the provenienced early figures coming from Hierakonpolis and the later ones coming from Abydos, the main location of production and/or use shifted over time. In this case the animals seem to mainly be used, and probably made, in one site at a time, which does not fit the definition of specialized production at the inter-site level. See section 6.3 below for a discussion of specialization at the intra-site level.

\section{Bifacial knives}

Bifacial knives have been found at many Naqada period settlements and cemeteries (Tables 6.6-6.9, 6.15). Production of bifacial knives has been identified at both el-Mahâsna and

\footnotetext{
${ }^{53}$ It would not be appropriate to compare the variability in metric characteristics since the eccentrics
} 
HK29A, through the presence of unfinished knives and thinning flakes.

Figure 6.28 shows an unfinished bifacial tool in the University of Pennsylvania's collection that Garstang found during his excavations at el-Mahâsna. Based on the size and shape, it is most likely a preform or roughout for a bifacial knife or dagger. The el-Mahâsna preform is made from a type of chert (type '1.Indistinct beige') that was quite common at the site, and about $10 \%$ of the thinning flakes from the analyzed material of Anderson's excavations were the same variety of chert. Therefore at el-Mahâsna there is a case of local production for local use. Whether knives such as the one made at el-Mahâsna were also exchanged farther is unknown.

At HK29A, Takamiya and Endo (2008:9) identified a bifacial knife that was broken during the production process, confirming that bifacial knives were made at Hk29A. Bifacial knives were also found in other parts of the site localities (localities 11, 29, and 25D), so some of the bifacial knife production at HK29A could have been for use in Hierakonpolis. In both the elMahâsna and Hierakonpolis cases, there was production of knives for use within the same community where they were produced, which would not be considered specialized at an intersite level.

However there was evidence from other sites that bifacial knives were obtained from outside communities and not produced locally. At Adaïma, Briois and Midant-Reynes (2008:27) argued based largely on raw materials that bifacial knives were brought into Adaïma from elsewhere and not made on site. ${ }^{54}$ At Tel el-Farkha Kabacinski (2012:339) divided the Naqada

had such diverse form.

${ }^{54}$ However the article is somewhat confusing, because the charts imply that there were no bifacial knives on the site besides the sickles and fishtails, but other publications from Adaïma show that bifacial knives were found there. 
period and Early Dynastic knives into two distinct groups. The first group consisted of small, poor quality, poorly standardized, locally made knives, and the second group was composed of large wide knives with distinct handles, imported from outside the site. The locally made knives were more numerous than the imported ones. By definition the imported knives must have been made by specialists, and Kabacinski implied that the locally made knives were not made by specialists, although the possibility for variation in the production remains within the site was not addressed.

It is also important to point out that the category of bifacial knives can be quite variable in terms of morphology, quality of flaking, and production technique (Holmes 1989:405; Ch. 6.1). Due to this morphological variability, no attempt at a comparison of metric characteristics was made. In sum, at least some bifacial knife production was specialized at the inter-site level, but not all. Since this category combines both specialized and non-specialized tools, it will not be used to evaluate the expectations for a ritual production model of specialization in Chapter 7.

\section{Bifacial sickles}

Tables 6.6-6.9, and 6.16 show that bifacial sickles were present in many sites, including both settlements and cemeteries. At most settlements only a few bifacial sickles have been recovered ( $n=1-3)$. There is no direct evidence for where bifacial sickles were produced. No unfinished pieces have been identified, and there are no specific forms of debitage associated with these tools besides bifacial thinning flakes, which were present in low amounts at all sites.

Information on the raw materials constitutes the best evidence for production locations. Looking at raw materials of bifacial thinning flakes present at Adaïma compared to various tool classes, Briois and Midant-Reynes (2008:27) indicated that bifacial sickles and other bifacial 
knives were imported into the site, which implies some sort of specialization. A similar situation was evident at Abydos (ATP). There one bifacial sickle was made of type '1.Indistinct beige' raw material and only 1 thinning flake of that raw material was found at the site (3\% of the thinning flakes). Two bifacial sickles were made from type '7.Dark gray and brown’ material, and only two thinning flakes were found of the equivalent material.

Conversely at el-Mahâsna there was one bifacial sickle made of raw material type '10.Pink-gray' material, and $26 \%$ of the thinning flakes were of this same material indicating that the sickle could have been made in el-Mahâsna. In a comparable situation, the bifacial sickle from Nag el-Qarmila (AKAP) was made of raw material type '7.Dark gray and brown', an 22\% of the thinning flakes were also of that material.

The metric data indicate the bifacial sickles were relatively variable in terms of their widths and thicknesses (Tables 6.24-6.25), with CVs of 24.84\%, and 29.74\%, respectively. Only width and thickness were examined because bifacial sickles come in bi-pointed and single point varieties, and many had breaks at one end. This relative variability could indicate multiple producers. All in all, bifacial sickles had a wide geographical distribution, and were probably produced at multiple sites, but not at every site, indicating that there was some intra-site specialization in the production of bifacial sickles.

\section{Axes}

Axes were a major factor in defining regional differences among lithic artifact assemblages in the Nile Valley (Holmes 1989). Holmes' analysis showed that axe production was much more prevalent in the Naqada region. Tables 6.6-6.9, 6.17 show a very different pattern of production than any of the previous bifacial tool classes. Axe production is very 
obvious in the Naqada-Armant region, where all kinds of production remains and as axes were present in the settlements and cemeteries. However the presence of axe and preparation flakes in a few other sites indicates that some axe production also went on in other sites. The lack of identifiable axe roughouts or preforms probably indicates that axe production was much less frequent outside of the Naqada area, which fits well with Holmes' interpretation of regional variation.

The metric data on axes (Tables 6.26-6.29) indicate that they were relatively variable tools in all dimensions, with CVs ranging around $20-25 \%$. The axe data also show that the average dimensions were slightly different between sites, although this could relate to collection bias for the samples that come from museum collections, as excavators might have been more inclined to both recognize and keep the larger axes. Width was the least variable characteristic and length the most variable, which makes sense if axes were resharpened by adjusting the working end. The range of variability was comparable at all sites, which could indicate that there were not substantial differences in the organization of production between the Naqada-Armant region and other areas. The major difference seems to be in the quantity of axe production in the Naqada-Armant region, not in the characteristics of the axes or how they were produced. All in all, there is evidence for production of axes in almost every site where they were used, so axes cannot be considered specialized at an inter-site level, but see below for specialization at the intra-site level.

\subsection{Bifacial Tool Specialization at an Intra-Site Level}

There were sufficient data from three sites (el-Mahâsna, Armant, and Hierakonpolis) to analyze intra-site differences in bifacial tool production. For the most part, bifacial tool 
production could only be analyzed as a whole, rather than as individual tool classes, because of the similarity of the production remains, and because some of the tools described above were only rarely found in settlements. The distribution of bifacial production remains and tools was used to assess whether there were any differences in the organization of bifacial tool production at each site, by looking at differences in frequencies. The significance of the differences were checked using confidence limits (see Ch. 2.5 for an explanation of confidence limits).

\section{el-Mahâsna}

There was a slight but defined concentration of bifacial tool production remains in Block 3 at el-Mahâsna. Bifacial thinning flakes were present in low percentages in each of the three blocks (Table 6.30), however the frequency was higher in Block 3. The 95\% confidence limits of the percentage of bifacial thinning flakes for Block 3 does not overlap with those from Blocks 1 and 4 Figure 6.29). Therefore the differences are not simply due to chance or sample size but probably reflect a real difference in the underlying population. Additionally, the only axe preparation flake (Table 6.30), and a bifacial tool preform (CBPP) (Table 6.33), were also found in Block 3.

There was some variability in the distribution of bifacial tools with a higher amount in Block 3 (Table 6.33). Each block had non-standardized miscellaneous bifacial tools, but almost all of the more standardized identifiable types, such as CBPPs, axes, and a knife, were found in Block 3. However this variability in the frequencies of bifacial tools could not be differentiated to the $95 \%$, or even $85 \%$ confidence levels (Tables 6.31-6.32). Therefore, even though there was a higher percentage of bifacial tools in Block 3, this difference is not statistically meaningful, and the concentration of bifacial tools in Block 3 cannot be confirmed. 
The definition of specialization used here is that items were used by others than just those that make them. Since there were more bifacial tool production remains in Block 3, but no statistical difference in the distribution of bifacial tools, then it is likely that there was production of bifacial tools in Block 3 for use in other areas. This more intensive bifacial tool production in Block 3 means that there was a degree of intra-site specialization in the production of bifacial tools. Since a preform for a concave base projectile point (Figure 4.47) and an axe preparation flake were found in Block 3, then some of the specialized bifacial tool production in Block 3 was likely to make axes and CBPPs.

\section{Armant}

Additional evidence for intra-site specialization of axe production comes from site of Armant MA 21/83. There, Ginter et al. (1996:177-178) found that the production of axes, and possibly other bifacial tools, was concentrated in the southern sector of the site in all phases. The flakes from bifacial tool production were distinctly concentrated in that area, and the full sequence of bifacial tool production took place in the "specialized sector." Additionally, many preforms were found at the site (Ginter and Kozlowski 1994). This spatial concentration of bifacial tool production did not change over the different phases of the site even though blade production shifted from being carried out in the settlement, to workshops in the desert (Ginter et al. 1996). They identified the differential distribution of flakes from the production of bifacial tools by simply plotting the quantities of such flakes on a map of $1 \times 1 \mathrm{~m}$ units. This method however did not take into account differences in sample size. For consistency, the data provided in Ginter and Kozlowski (1994) were analyzed in the same manner as was done for el-Mahâsna (above) and Hierakonpolis (below). Table 6.34 shows the percentages of bifacial thinning flakes 
from the two sectors at Armant MA21/83, and Figure 6.30 gives the upper and lower 95\% confidence limits. The data support Ginter et al.'s findings, showing that there was a statistically higher portion of thinning flakes in the southern sector of Armant.

Table 6.35 gives the data for the frequencies of different tool types (including preforms) in the two areas of Armant MA21/83. Figure 6.31 gives the percentages and upper and lower 95\% confidence intervals for the preform data, confirming that there were significantly more

preforms in the southern sector. ${ }^{55}$ The percentage of bifacial tools (Table 6.36), however, was the same in both areas.

These data clearly show that bifacial production was concentrated in the southern sector of the site, as indicated by bifacial thinning flakes and bifacial preforms, while the bifacial tools themselves were found relatively evenly beyond that area. Ginter and Kozlowski classified most of the preforms as axe preforms, showing that this bifacial tool production focused on axes. This constitutes evidence of within-site specialization in the production of axes.

\section{Hierakonpolis}

While it is already very clear that specialized bifacial tool production was taking place at HK29A (Holmes 1992), Hierakonpolis still provides an opportunity to see how that production area compares to other localities at the site. In this case the comparison is between more widely disbursed localities, rather than different parts of a single, well defined site.

Unsurprisingly, clear differences in the frequencies of bifacial thinning flakes are 
apparent among localities at Hierakonpolis (Table 6.5) ${ }^{56}$, and the 95\% confidence intervals bear out these differences (Figure 6.32). HK29A has the highest frequencies by far, and there are moderate amounts at HK29B, HK25, and parts of HK11C, while the lowest amounts occur at HK25D, HK14, HK24, and other parts of HK11C. These data also show that adjacent localities could have very dissimilar frequencies of thinning flakes. Parts of HK11C were quite different, ${ }^{57}$ and HK25 and 25D can be statistically distinguished, even with a small sample size at 25D. All this points to quite a bit of intra-site differentiation in bifacial tool production. Like at elMahâsna, the only axe-preparation flakes were found in the ritual precinct, in HK29Bv(Table $6.5)$.

On the other hand, the distribution of bifacial tools across Hierakonpolis localities (Table 6.7) showed some variation, but could not be separated statistically. The 95\% confidence intervals overlapped in all cases (Figure 6.34), meaning that they could come from similar underlying populations and the differences could be due to chance, sample size, or other factors.

A few points are interesting to note for individual tools classes. Like at el-Mahâsna Block 3, a greater variety of bifacial tool types were present at Hk29A compared to surrounding areas, and CBPPs were found only at those ritually significant locations within each site. It should also be noted that HK29 10L10 had a higher percentage of drills than in other areas, which may be evidence of some other kind of production (stone vessels?).

\footnotetext{
${ }^{55}$ One caveat is that nothing was classified as 'unidentifiable bifacial tools', so it is likely some of what would have been classified at other sites a miscellaneous or unidentifiable bifacial tool, here was put in the general bifacial preform category. However there is no way to know how these compare, and many of the preforms were identified specifically as axe preforms, which was not possible at other sites, so the conclusion still should be tenable.

${ }^{56}$ No data on bifacial thinning flakes was available from HK29 10L10, so the data from Hk29 17 L13 was used instead (Holmes 1996). The rest of this dissertation uses the HK10L10 data.

${ }^{57}$ Although they were also analyzed by different researchers, which cause some differences.
} 
The case for intra-site specialization of bifacial tool production is very clear at Hierakonpolis. There was unmistakable variability in the production remains across Hierakonpolis, with bifacial tool production of course concentrated on HK29A. Localities 29B and 25 also had relatively high frequencies of production remains, which I find significant because these sites potentially make up a precinct of ritual activities along with HK29A. The high frequencies of thinning flakes at HK11C indicate that more intensive bifacial tool production may have occurred there as well, which makes perfect sense since the area was also the site for production of ceramics, beer, and food (Baba 2013; Friedman et al. 2009, 2011).

One last consideration for Hierakonpolis is whether there was intra-site variability in the production of the figural eccentrics. Some of the fine bifacial eccentrics from Hierakonpolis were certainly made by the same people who produced other specialized items. Nagaya (2011) analyzed eccentrics and tools from Hierakonpolis and related the production of certain animal figures to certain tools and raw material types. For instance, the Hierakonpolis ibex can be seen as a variation on the concave base projectile points, using many of the same production techniques and a similar base form (Figure 7.4). To that could be added the crocodile (Figure 6.17) which seems to have the base shape of a dagger or rhomboid with additional adjustments.

However the eccentrics had a much more limited distribution than other similar items made by specialists, since there are no reliably provenienced eccentrics of the same date outside Hierakonpolis. Within Hierakonpolis the eccentrics were found in only two contexts, which are very different from each other: the elite cemetery HK6 where they were clearly used as offerings (Friedman 2010:70-71; Friedman et al. 2017:236), and the settlement area of HK11 (Watrall 2000). 
It is not possible to determine the exact production location for the eccentrics; they could have been made at Hk29A, but many of the figural eccentrics date earlier than HK29A. However the presence of the eccentrics of similar quality in two very different contexts within Hierakonpolis shows that they were likely used by people other than those who made them (even if it is not possible to confirm who made them), thus fitting the definition of specialization at an intra-site level.

Complicating the picture is the fact that some of the figural eccentrics could have been made by people who were not specialists at all. Many of the more simple edge retouched pieces were made with the same techniques as were used to make non-specialized tools, such as scrapers, and some bifacial and edge retouched pieces do not show the same degree of control over form as the finest examples (e.g. Figures 6.15, 6.16, 6.22, 6.24 top left). Although in some cases the provenience of these simpler eccentrics is unknown, a number of the Abydos ones have these characteristics, and are notably later in date.

Due to the very limited quantity of the figural eccentrics, and to the fact that it is not possible to confirm that they were all made by specialists, they are not considered further in Ch7 for the comparison of the specialized tool to the expectation for the ritual production model. However the eccentrics certainly constitute production for religious/ritual purposes, since they are clearly of a symbolic nature and have been found in offering contexts associated with a cemetery (HK6) and a temple or ritual area (Abydos). They are discussed further in the conclusion (Ch. 8). 


\subsection{Conclusion: Production of Bifacial Tools}

Like with blades, Predynastic knappers produced bifacial tools in a spectrum of ways. These results are summarized in Table 6.37 and Figure 6.35. RFKs and rhomboid tools were made by specialists at an inter-site level, meaning that they were not produced in all communities. These tools were principally for cemetery use. Chronologically they did not overlap, and the earlier rhomboids were more variable which may relate to the size of the tools and the availability of raw materials, but could also indicate a larger number of producers than RFKs. Fishtails were also produced by specialists and the decreasing variability in fishtails, along with changes in the production techniques indicate that the later fishtails were certainly specialized at an inter-site level.

In contrast, CBPPs were made at many sites as was indicated by the presence of preforms and unfinished CBPPs. Distribution data from el-Mahâsna and Hierakonpolis indicate that CBPPs were specialized within sites, meaning that only some people at a given site produced CBPPs. Their production drew on many of the same techniques as RFKS, rhomboids, and fishtails (such as pressure flaking, microserration and possibly grinding), and CBPPs were the least variable tools in terms of width and thickness.

Figural eccentrics were also specialized at an intra-site level, but the production and distribution of figural eccentrics was highly localized and the overall quantities produced were quite low. Some eccentrics may not have been made by specialists at all. Bifacial knives were another variable category, with the production of some knives specialized at an inter-site level, while others were not made by specialists at all.

Bifacial sickles were specialized at an inter-site level since they were clearly not produced in certain communities. However there may have been more locations for the 
production of these tools compared to RFKs, fishtails, or rhomboids, as was indicated by raw material data and the variability in metric attributes. Axes were produced in almost all (if not all) sites, but by specialists within each site. Furthermore there were regional differences in the frequencies of axe production.

On the other hand there was also non-specialized production of unstandardized bifacial tools at all sites, as indicated by the low but prevalent presence of bifacial thinning flakes and unstandardized miscellaneous bifacial tools at all sites, and in multiple areas within sites. Moreover, these unstandardized bifacial tools were often more common within settlements than the more standardized identifiable bifacial tools.

The important conclusion here is that bifacial tool production cannot be described as a simple dichotomy of specialist produced luxury goods and utilitarian tools made and used by the masses. Moreover, as will be discussed in Chapter 7, the organization of production for bifacial tools was even more varied than is reflected above, when patterns in raw material use, contexts of production, and contexts of finished tools are taken into account. 


\section{Chapter 7: Evaluation of Expectations}

Chapter 7 compares the archaeological patterns of raw material choice, production contexts, and find contexts of specialist produced tools to expectations derived from Spielmann's (2002) discussion of a ritual production model for the development of specialization (Ch. 2.2). The specialist produced tools identified in Chapters 5 and 6 were ripple-flaked knives, rhomboid tools, fishtail tools, concave-base projectile points, bifacial sickles, axes, large-blade knives, and heat-treated microendscrapers. Table 7.22 gives a summary of the findings for these expectations by tool class.

\subsection{Raw Materials Expectation}

If specialized production of some tools developed in Egypt to make symbolically meaningful goods for a large portion of the population, then the raw materials for those tools may have been chosen based on symbolic considerations. The raw material could add significance to the meaning or symbolic value of the item. For instance the difficulty of obtaining the raw material, or specific associations with the place of raw material procurement (e.g. holy place, or any sort of symbolically charged site) could be indexed by the raw material.

Additionally, a review of the Pharaonic evidence for the symbolic significance of chert showed that color may have been important to the symbolic meaning of the tools, influencing raw material choice (see below). 


\section{Method}

This expectation was evaluated by looking for an association between tool type and raw material type, and by ruling out other factors that could govern raw material choice such as functional considerations or local (easy) access to the raw material sources. Raw material and provenience data were collected for a sample of artifacts from each of the tool classes that were identified as specialized in Chapters 5 and 6 (RFKs, rhomboids, fishtails, CBPPs, bifacial sickles, axes, large-blade knives, and heat-treated microendscrapers). The goal was to have a sample of at least 30 artifacts in each category. Since the data from el-Mahâsna, Abydos, and Nag el-Qarmila did not constitute a sufficiently large sample of any of the individual tool types, additional data were also gathered from online museum data bases, or observed in person in a few cases. Museum databases were chosen as a primary source rather than publications because many more photographs—essential for examining raw materials—-were available via online museum databases than in publications which normally show artifacts in black and white line drawings. Publications were also consulted when color photographs were available and the artifacts could definitively be differentiated from those already studied in the museum databases, in order to avoid counting the same artifacts twice. For certain tool types—CBPPs and bifacial sickles—only those with provenience information were included in the sample so that the artifacts could be securely dated to the Naqada period rather than the Badarian. This resulted in a small sample size for the bifacial sickles. The rhomboids included only relatively complete examples so that they could be differentiated from other kinds of lances/daggers, also resulting in a relatively small sample.

The total sample of all tool types includes artifacts from 39 museums and storerooms spread over nine countries and three continents (Table 7.1). Thus it was not feasible to travel and 
observe them all in person given the time frame and financial scope of this research. However the same principles were used for assigning raw material types as described in Chapter 4: The primary focus was on the structures such as banding and mottles, along with the degree of translucency. Color was used only broadly and secondarily to assign artifacts to groups. Translucence could generally be deduced from photographs due to familiarity with the cherts from Egypt. Any descriptions of the raw material given with the artifact were also consulted.

A few new raw material categories had to be defined because the materials appeared quite distinctive, ${ }^{58}$ and simply did not correspond to any of the groups identified during fieldwork. This is not surprising since the examples in this chapter include more sites and a longer time span than what was studied in the field. Sometimes a specific raw material type could not be identified since many of the determinations were made from photographs, but only a general group was assigned, for instance ' $1,2,4$ ', which are all light opaque cherts with differences in texture and/or structures. Unfortunately this made statistical comparisons of the diversity of chert varieties relative to the sample size impossible, since the number of raw material types represented was not exact. However, the data are sufficient enough to determine whether one type was dominant, and to compare qualitatively between tool categories. When grouped more broadly, according to light/dark color categories the data were sufficient to do a chi-square test for association between tool type and raw material color. Additionally, the

\footnotetext{
${ }^{58}$ One was 'Other: variable translucent-opaque,' which is light in color ranging from beiges to light or medium yellowish browns, with opaque and translucent patches that occurred in swirls or large bands. Remnants of a chalky (?) white cortex were visible on four of the RFKs (BrM.09.889.120; ManM.38160; ManM.5305a-e; Pr.1900.42.2) indicating that the material likely came from a primary source. The second was 'Other: chocolate,' which is homogeneous dark brown, fine grained, and with chalky white cortex. It corresponds to a raw material described by Hikade (2013:23, PI 2c-d) as looking like chocolate.
} 
distribution of raw material types by site was analyzed, to check whether any of the variability in raw material type could be accounted for by the use of local raw materials.

Since many of the artifacts come from excavations that occurred before the 1960's, the artifacts can only be associated with general dates (e.g. Naqada period rather than NIC or NIIIA). Therefore it was not possible to look at differences in raw material choice over time for most of the tools classes. The exceptions are the RFKs and the fishtails, since both can be dated stylistically to some degree. In cases where there does appear to be a clear association between tool type and raw material, functional parameters were also considered.

\section{Pharaonic evidence for the symbolic significance of chert}

There is evidence that chert itself had symbolic significance during the Pharaonic period. Aufrere (1983) looked at the relationship between minerals and divinity. Not only were the images of gods, and the gods themselves, said to be made of gold and lapis lazuli, these stones were thought to both originate from the gods and to protected them. Even in an unworked form minerals had symbolic properties. Certain minerals were crushed and mixed with water to act as purifying agents, showing that the minerals themselves had efficacy, not just their final forms as amulets, jewelry, statues, (or even perhaps, as tools). Aufrere also argued that the variety of types and colors of stones allowed them to form a symbolic array that could invoke different aspects of gods or reference myths well known to Egyptians. Although Aufrere’s research mainly focused on the Greco-Roman period texts, he also drew on the Pyramid texts which date much farther back, to the Old Kingdom. Thus the divine significance of minerals can conceivably date back to earlier periods.

Representational and textual evidence indicate that chert in particular had symbolic 
significance. The ancient Egyptian word for chert (or flint) was ds (Graves-Brown 2011:61; Harris 1961; Midant-Reynes 1981). Ds could also mean knife, which is understandable since most Predynastic-Old Kingdom knives were made from chert. Graves-Brown (2011:176-277) showed that chert knives were used for cattle sacrifice—an important ritual act—-through the Middle Kingdom, even though metal knives were commonly available by the late Old Kingdom. Her evidence was based on the sharpening scenes depicted with cattle slaughter and the archaeological distribution of flint knives. Furthermore, all of the textual references to chert during the Pharaonic period are in religious contexts, not secular ones (ibid.:223). MidantReynes (1981) discussed examples of chert in texts about the afterlife (including the pyramid texts, coffin texts, and the book of the dead), showing that chert knives were used to defeat enemies. Additionally she showed that chert could constitute the eyes of Horus, which recalls Aufrere's arguments for the significance of minerals that made up the bodies of gods. Considering the evidence for cattle slaughter connected with ritual areas at HK29A and elMahâsna Block 3, it is conceivable that these symbolic/religious connotations associated with chert could have begun earlier, during the Predynastic period, and moreover that these considerations may have affected raw material choice for specialist produced tools.

Furthermore, the color of the chert may have been important to the symbolic meaning.

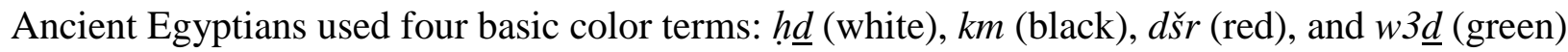
(Baines 1985). ds (chert) could be described as km, or $h \underline{h} .{ }^{59} \mathrm{~A}$ look at ancient Egyptian color terms, color pairings, and color dualities shows not only that colors often carried symbolic 
religious meanings, but also indicates how the chert artifacts studied here could be divided into groups that roughly correspond the Ancient Egyptian categories $d s \mathrm{~km}$ and $d s \underline{h} \underline{d}$.

Egyptian color terms do not align with western colors, nor do they necessarily even correspond to the idea of hue (Baines 1985; Quirke 2001). Quirke 2001 notes that even among the basic color terms the words do not refer exclusively to a color, noting that 'black' and 'white' are also the words for dark and bright, and that that 'green' also means fresh and can be applied even to things that might otherwise be considered red, such as meat. The colors black and green were both associated with plant fertility and Osiris' rebirth into the afterlife (Baines 1985:284; Pinch 2001:183). The association of green with plants is pretty straightforward, and most scholars point to the fertile black soil of the Nile for the connection between the color black and fertility. Osiris's face can be painted either black or green. The connection between black, green, fertility, and Osiris is made most plain with the New Kingdom 'Osiris corn beds', which were planters in the shape of Osiris, filled with dirt (black) and germinating grain (green), and included in tombs (Ikram and Dodson 1998:120). However the idea for these planters was probably derived from a section in the pyramid texts, showing that the connection dates back much earlier.

The most famous color dualism from Ancient Egypt is in the name of Ancient Egypt itself: Kmt, black land, named for the fertile black soil from which the Egyptian livelihood sprung. Kmt was the land of the living. This black land was opposed to dšrt, red land, the dry desert that surrounds the Nile Valley, and home to the ‘other'— dangerous wild creatures, even

\footnotetext{
${ }^{59} \mathrm{Ds}$ could also be described as tḥn, yellow, but the translation of yellow for tḥn is not always accepted and may refer instead to other properties rather than hue, such as shininess or brightness (GravesBrown 2010:142-145; Harris 1961:138-139; Midant-Reynes 1981:41-42; Quirke 2001). Additionally, the
} 
the beings of the afterlife. Given the close association between black and green, the opposition of red to black is less surprising.

The word $d s ̌ r$ (red) could best be defined as warm colors focusing on red, and has clear associations with the desert (Baines 1985:284-286). Yellows were also a part of this category, including the light yellows and tans that westerners might more readily associate with the desert. Red and yellow-gold were used interchangeably for representations of the sun disk (Pinch 2001:184). Some browns could also fall into the category of $d \check{r}$, such as in reference to cows on a late Middle Kingdom onomasticon (Harris 1961:228 228).

There is some evidence that the opposition of black and red probably extends back into the Predynastic period. The juxtaposition of red and black during the Predynastic period is clearly seen in the black-topped pottery with red bases. This of course may simply be related to how they were produced (Baba 2005, 2008b, 2009a, 2011). However there is evidence that the pairing of red and black was ritually significant. Two types of ceramic vessels with distinctive shape and surface treatment were found at Hierakonpolis: polished black egg-shaped vessels and matte red flaring collared-rim jars (Friedman 2009b:85-88; Hendrickx and Friedman 2003). These forms, intentionally made all in dull red or all in shiny black and occurring together in the same contexts, are found only in ritually significant areas of Hierakonpolis, the HK29A ceremonial enclosure, and the pillared halls in the elite cemetery $\mathrm{HK}^{60}{ }^{60}$ (Friedman 2009b:97). This intentional play on the red and black colors of the vessels in ritual contexts indicates that the

use of tḥn as yellow seems to have come into ancient Egyptian textual references later in its history, so is not salient for the current study.

${ }^{60}$ They were even specifically found paired together in one context: Two red collared jars and two black egg-shaped jars were found with foot bones of a sheep/goat in a basket or mat-lined pit at the entrance to the chapel of tomb 23 (Friedman 2009: 87). Fragments of the earliest life size statue were also found in that chapel. 
red-black opposition was likely significant during the Predynastic period.

Based on the above discussion of Egyptian color categories the raw material groups as defined here were divided into $d s \mathrm{~km}$ (black chert) and $d s \underline{h \underline{d}}$ (light chert). Given that $k m$ (black) was opposed to $h \underline{h}$ (white), but also to $d \check{r} r$ (red), and that the opposition of red and black probably extends back into the Predynastic period, the light yellowish and reddish toned chert categories were combined. Thus all the beige, pinkish toned, and lighter-medium brown categories such as types $1,2,3,4,8$, and 13 might reasonably be supposed to correspond to $d s$ $h \underline{d}$. Correspondingly, the raw material categories '7.Dark gray and brown', obsidian, and any other very dark or greenish materials could easily be considered $\mathrm{ds} \mathrm{km}$. The artful manipulation of natural color variation within a piece of raw material to depict clothing for the dwarf/human eccentric (Nagaya 2011) (Figure 6.28) shows that the Predynastic Egyptian flintknappers did pay attention to the colors and patterns in the material, and did not always treat it all as interchangeable. Therefore, in addition to looking for associations between tool type and raw material type (as defined in the Appendix), associations between tool type and light ( $d s \underline{h} \underline{d}$ ) or dark $(d s \mathrm{~km})$ raw material color categories were also analyzed.

\section{Results}

\section{Light/dark color categories}

A chi-square test ${ }^{61}$ was used to look for an association between tool type and light/dark color categories (Table 7.4). The test returned a chi-square statistic of 91.6993 with 8 degrees of freedom, and a probability of 2.2e-16. All of the expecteds were above five, so the test was valid.

\footnotetext{
${ }^{61}$ See Ch. 2.5 for more information on chi-square tests.
} 
The probability is well below the .05 significance level, so the null hypothesis that there is no relationship between tool type and light/dark raw material color categories can be rejected. Therefore there was an association between tool type and color category for at least some of the tools. The individual tool types are each discussed below, with the analysis of particular raw material varieties.

\section{Ripple-Flaked Knives}

Sufficient data were available to make raw material determinations for 44 RFKs (Table 7.2, Figure 7.1), which could be assigned to six different raw material groups (Table 7.3). The most frequent raw material type was type '4. Beige with pink bands’ (31.82\%). The type group ' $1,2,4$ ' was almost equally as abundant (27.27\%), and many of the pieces in that group are probably also type 4 , because type 4 is a high quality material. Therefore there was an overwhelming preference for raw material type 4 for RFKs.

However the fact that most of the RFKs were made from type 4 material may be related to the production location rather than symbolic considerations. The type 4 RFKs were concentrated at Abydos (Table 7.5). Of the six type 4 RFKs with provenience information, half came from Abydos. Dreyer (1999:213) argued that a fourth, the Gebel Arak knife, actually came from Abydos as well. ${ }^{62}$ Two additional pieces that could only be identified as type ' 1,2 , or 4' also came from Abydos. All the rest of the RFKs made from type 4 or type ' $1,2,4$ ' were found only singly at sites which stretch from Fayoum in the north to Qushtamna in the south (see Figure 2.2 for site locations). Since there was a source of the type 4 raw material near Abydos

\footnotetext{
${ }^{62}$ This knife is not included in Table 7.5 since its provenience is not secure.
} 
(Ch. 4.1; Hikade 2013), and there was a high concentration of RFKs made from that material at the site, then it is possible that the RFKs were produced near there, and that the raw material choice was a matter of proximity. However, it should be noted that Holmes (1989:459) also found type 4 material in secondary deposits on the west bank of Luxor, and that it has been identified among artifacts at other sites such as Hierakonpolis and Elephantine, so Abydos may not have been the only source for type 4 raw material.

Grouping the raw materials according to the light/dark color categories (ds $k m$ or $d s \underline{h} \underline{d}$ ), showed that the overwhelming majority were of light chert (84.09\%) (Table 7.4). Furthermore, these light materials only consisted of 2-3 raw material types: 4, '1,2,4', and 'Other: variable translucent-opaque'. However, the choice of the type 4 light raw material could have been due to local availability of materials, so it is not possible to conclude that the preference for light materials was due to symbolic considerations. Additionally, the raw material choice may have been limited by the availability of nodules or tabular pieces of the required size. Lengths were available for 25 RFKs, and these ranged from $14.9 \mathrm{~cm}-30.4 \mathrm{~cm}$, averaging $23.78 \mathrm{~cm}$ showing that these tools were rather long.

It should also be noted that the raw material choice for RFKs may have changed over time, shifting towards use of darker materials. Two RFKs of type 'Other: chocolate' have ivory handles (MMA.26.241.1, the “Carter knife” (Figure 7.1); UC.16294, the "University College knife”). The use of elaborate decorated knife handles is thought to happen toward the later end of the time span for RFKs, perhaps late NIId-NIII, given the stylistic dating of the handles and the provenienced examples (Midant-Reynes 1987:220; Needler 1984:270; Whitehouse 2002:432). The third RFK of raw material type 'Other: chocolate’ (UC.35723), is a small proximal 
fragment ${ }^{63}$ that was found in the tomb of Djer, also late. Additionally a dark RFK from Tel elFarkha (here designated raw material type 5) was found in a context that dates to the NIIIB (Chłodnicki et al. 2012; Ciałowicz 2007). The possibility that the piece was an heirloom cannot be ruled out, but the lesser quality of that particular piece (the ripple-flakes did not carry all the way across the face and join in the middle), combined with the unusual dark raw material could be an indication that it was a later example made after the knowledge and skill of such production was not in regular practice. If all of these dark RFKs are later, then they indicate that there was a change in raw material choice over time. It is tempting to relate this possible shift in raw material choice to the shift in Egypt's political center. The political capitol of Egypt moved from Abydos up to Memphis at about the same time. Sponsored workers could have moved in tandem, changing their access to raw material sources. However much more work would need to be done to verify this proposal, such as identifying the source of the 'chocolate' raw material, and definitively identifying production locations for the RFKs.

In summary, was some preference for lighter colored raw materials, and type 4 raw material in particular, it is not possible to conclude that RFKs meet the expectation that raw materials were chosen for symbolic reasons, because the because the raw material choice might have stemmed from simple use of raw materials available near the place of production, rather than symbolic concerns.

\footnotetext{
${ }^{63}$ It is possible that UC.35723 was burnt, which could make the raw material designation questionable. There is a small divot on the flaked side of the knife which could either be the scar of a heat spall or the remnants of a deep flaking scar from original shaping that was not fully removed with subsequent ripple-flaking. There are no signs of crazing or other heat spalls, and the material looks very much like the "other: Chocolate" pieces, so it was classed accordingly.
} 


\section{Rhomboids}

The 20 tools that could definitively be classed as rhomboids (Table 7.6, Figure 7.2) were made from only four chert raw material types ${ }^{64}$ (Table 7.3). Raw material type '7.Dark gray and brown’ was by far the most common, accounting for 55\% of the pieces. Just over half of the rhomboids were made from dark cherts ( $d s \mathrm{~km}, 60 \%$ ), and $40 \%$ were made from light materials

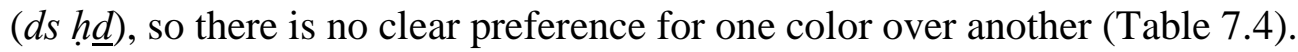

The sample available for assessing the distribution of rhomboids by raw material type was small because many came from unknown proveniences (Table 7.7). There was some clustering of type 7 rhomboids at Naqada, however no source of that material is so far known near Naqada. Ginter et al. (1996) characterized raw materials on the west bank of the Luxor area, just south of Naqada. They described a gray flint, but did not describe it as including brown portions. Holmes (1989:459-460) described material from the same Luxor area as grayish-beige to yellowish beige (Munsell 10YR 7/3). Type '7.Dark gray and brown’ has a Munsell color range of 7.5YR 4/1 - 10YR5/2 so the material described by Holmes is not the same as type 7. Therefore the clustering of type 7 rhomboids at Naqada cannot be explained as local production using local raw material sources, given current knowledge of the raw materials in the area.

Functional considerations must have limited the raw material choices. Coarse or difficult to flake materials would not have been appropriate for rhomboids which are often very finely flaked and would have required fine-grained materials. Moreover, rhomboids are quite large tools, up to $41 \mathrm{~cm}$ long (e.g. Brunton 1937:90). A sample of 18 complete rhomboids yielded lengths ranging from $17.5 \mathrm{~cm}$ to $41 \mathrm{~cm}$, with an average of $25.88 \mathrm{~cm}$. Thus rhomboids would have 
required very large nodules or tabular pieces which must have occurred only among certain raw materials.

Rhomboids do not meet the raw material expectation for the Ritual Production Model. There is no clear preference for one color over another, and size requirements probably greatly influenced the raw material choice, more so than symbolic considerations.

\section{Fishtails}

Fishtails were made from a comparatively wide variety of raw materials ${ }^{65}$ (Table 7.3 ). Of the 101 fishtails with identifiable raw material types (Table 7.8, Figure 7.3), the most common type was '7.Dark gray and brown’ (34.65\%), but fishtails were made from at least ten other raw material types as well, including non-chert materials (obsidian, ${ }^{66}$ rock crystal), so there was no clear association with one raw material type.

However fishtails were in use over a long span of time, and when broken down by time period, there was a trend for dark raw materials ( $d s \mathrm{~km} \mathrm{66 \% )} \mathrm{in} \mathrm{the} \mathrm{earliest} \mathrm{phase,} \mathrm{and} \mathrm{light} \mathrm{raw}$ materials ( $d s \underline{h} \underline{d}, 88 \%)$ in the latest phase (Table 7.4). Three main shape types have been defined for fishtails (Figure 6.23) (Massoulard 1936; van Walsem 1978), and the shape type changed over time. Stylistic types 1 and 1a with wide U-shaped forks were the earliest (NI - NIIc or d), and stylistic type 2, with sharper V-shaped forks, the latest (NIIa or b - NIId). Hikade (2003:140141, 148) had the impression that early fishtails were made on a dark gray-brown flint (type 7),

\footnotetext{
${ }^{64}$ Note the small sample size.

${ }^{65}$ However, note that the sample of fishtails was larger than any other group, which increases the likelihood of finding more raw material types if they existed.
} 
and the later fishtails were made on a "brown (honey or caramel colored) variety of flint often with light red or pinkish discoloration as concentric bands” which is equivalent to this study's type 4. (Table 7.9) shows the frequencies of raw materials by fishtail shape and confirms Hikade's impression. The $95 \%$ confidence limits for the proportions shows that type 7 is significantly more frequent for the earlier fishtails than for the later fishtails (Figure 7.11). Furthermore, van Walsem placed shape 3 fishtails coeval with the shape 2 fishtails ( NIIc-d). However, Hikade (2003:138-140) posited that shape 3 should actually be assigned a bit earlier, dating to the transition between shapes 1 and 2 . The frequencies of raw material types corroborate Hikade's proposal. When placed in that order, shape 3 fishtails fit perfectly with the trend for increasing use over time of raw material type 4, and decreasing use of raw material type 7 (Table 7.9).

Provenience information was examined to see whether any of the variability in raw materials could be accounted for by use of local resources, with the idea that if fishtails were made using a local materials, examples of that material would be more frequent near where they were produced. Tables 7.10-7.11 show the provenience information for 58 fishtails, separated into earlier and later types. The distributions for the type 4 and type 7 raw materials echo what was seen for the RFKs and rhomboids. Raw material type 4 and ' $1,2,4$ ' fishtails clustered slightly around Abydos, where there is a known source of type 4 material (Ch. 4; Hikade 2013:2), so the slightly higher frequency there could be related to production in the area. Fishtails of raw material type 7 clustered somewhat at Naqada, however the source(s) of the type 7 material is

\footnotetext{
${ }^{66}$ A few other obsidian fishtails exist besides those listed in table 7.8, (two in the Berlin Museum and two in the Egyptian Museum in Cairo), but they are probably intermediary between the Predynastic
} 
not known. The diversity of raw materials for fishtails suggests that there could have been multiple production locations for fishtails.

The variety of materials used to make fishtails indicates that functional constraints were not restrictive, and that the preference for type 4 material in the later fishtails could not have been due to functional considerations. A sample of 63 fishtails of all shape types yielded an average of $13.49 \mathrm{~cm}$. This relatively small average length means there was less restriction on the required nodule size, and that more raw material types would have fit the requirements.

It is unlikely that symbolic considerations affected the raw material choice for fishtails since so many different raw materials types were used. Additionally many fishtail models have been found, and these were made out of diverse raw materials, such as copper (Ayrton and Loat 1911:Pl22), steatite (Friedman 2010:71; Friedman et al. 2008:90), alabaster (Petrie 1920:Pl9), serpentine (Petrie 1912:24,Pl 6), sandstone (Seton-Karr 1904); clay (Quibell and Green 1902:26,51,PL67; Petrie 1920:PL28), and possibly one of black paste ${ }^{67}$ (Hartung 2011:476). Moreover the predominant variety of chert used to make fishtails changed over time, which implies either that the raw material was not integral to the meaning of the tool, since it could change, or that the meaning changed over time. The later shape 2 fishtails sometimes were made with ripple flaking, showing that with the change in raw material there was also a change in production technique. These later fishtails may have been made in workshops alongside ripple flaked knives since they used the same production techniques, and had the same predominance of type 4 or type ' $1,2,4$ ' raw materials. It is also important to point out that these later fishtails also include examples made from obsidian. Obsidian had to have been imported from quite

fishtails and old kingdom psš-kfs (van Walsem 1978:242), so they were not included here. 
distant sources (Ethiopia or South Arabia; Bavay et al. 2000), so it was a very difficult material to obtain which was only available in small quantities. In sum the raw material choice for fishtails does not meet the expectations for the ritual production model. The raw materials were originally quite variable, perhaps indicating multiple production locations, but it changed over time to a more restricted raw material choice centering on type 4, and including the use of very difficult to get raw materials, combined with production involving more difficult flaking techniques. Despite the fact that symbolic factors cannot be deduced for the raw material choice, the existence of models, which by definition are not utilitarian items, means that tools themselves must have had some symbolic significance.

\section{Concave-Base Projectile Points}

The 31 concave-base projectile points that can be dated to the Naqada period (Table 7.12, Figure 7.4) were made from eight different varieties of chert (Table 7.3). The most common raw material was type '7.Dark gray and brown’ (45.16\%), with the rest distributed across eight other raw material types, showing that while type 7 is prevalent, CBPPs were made from many raw material types. Nor was there any preference for a certain color, with half of the CBPPs made from dark cherts ( $d s \mathrm{~km}, 51.61 \%$ ), and the other half from light cherts ( $d s \underline{h} \underline{d}, 48.39 \%$ ) (Table 7.4). The variability in raw materials was facilitated by the relatively small size of CBPPs, which do not require as strict size parameters for the raw materials compared to rhomboids or RFKs. A sample of lengths for 11 CBPPs with at least one full tang preserved ranged from $3.4 \mathrm{~cm}-10 \mathrm{~cm}$, and averaged $6.46 \mathrm{~cm}$.

\footnotetext{
${ }^{67}$ Hartung interprets this piece as a model gutted fish because one end is hollow. However the piece has
} 
The distribution of the raw material types by site shows that type 7 CBPPs were quite prevalent at Hierakonpolis (Table 7.13). Eight of the 14 pieces which were made from type 7 material came from Hierakonpolis. A source of raw material was found near Hierakonpolis which consists of chert cobbles in a gravel deposit (Friedman and Youngblood 1999; Harrell 2012, pers. comm.) however the material was not described in detail. Without knowing what kinds of materials were available or commonly used at Hierakonpolis it is not possible to determine whether some of the variability in raw material choice may be accounted for by proximity to raw material sources. There was also some clustering of the type '13.Caramel' CBPPs in the Badari sites, but the local raw materials in that area are also unknown.

In sum, like fishtails, CBPPs were made from many different raw materials, so based on the method used here, it is not possible to conclude that symbolic factors affected raw material choice. Additionally, like fishtails, model CBPPs existed, made out of clay (Garstang 1903:5, PL3-4). Again the existence of models implies that the tools had some symbolic significance beyond their utilitarian functions.

\section{Bifacial Sickles}

Only 20 Bifacial sickles were found that can be dated to the Naqada period and that have identifiable raw materials (Table 7.14, Figure 7.5). Despite the small sample size, the sickles were made from seven different raw material types (Table 7.3). Type '7.Dark gray and brown’ was the most common (35.3\%). The bifacial sickles were basically evenly divided between light

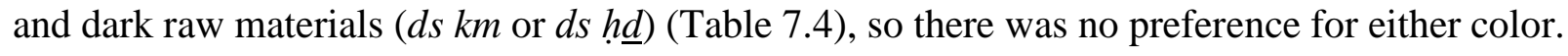

a different coloring on the forked end, which corresponds quite easily to a handle and a knife. 
Of six complete examples, the sizes ranged from $11.7 \mathrm{~cm}$ to $22 \mathrm{~cm}$ long, averaging $16.9 \mathrm{~cm}$, and no functional explanations for raw material choice were obvious.

The was no clear association between any site and any particular raw material type (Table 7.15). The find of a cache of three bifacial sickles made from two different raw material types at the Abydos settlement site (Ch. 3.4) indicates that the tools made from different raw materials were in use at the same time at the site, so the variation in raw material type cannot be only a function of time. Overall, in the case of bifacial sickles, there was no clear association between tool type and raw material type that would indicate symbolic factors contributing to raw material choice.

\section{Axes}

A sample of 82 axes with provenience information (Table 7.16, Figure 7.6) yielded 11 different raw material types ${ }^{68}$ (Table 7.3). The majority were assigned to the ' $1,2,4$ ' chert type group (41.46\%). Despite the use of many different raw material types, axes were

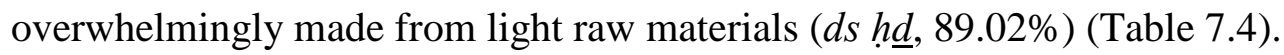

Moreover, this preference for light colored raw materials cannot be explained by a simple preference for local resources. The raw material studies in Chapter 4.1 showed that the local materials which were preferentially used could vary between sites, even if the sites were close together. Therefore, if raw material choice for axes was governed by the availability of local materials then variation in raw material choice by site should be observable. However this was

\footnotetext{
${ }^{68}$ Note that many of the axes were actually studied in person by the author, whereas for many other tool types the raw material designations were made from photographs. This may account for some of the reason why more detailed and thus more raw material distinctions could be made.
} 
not the case for axes (Table 7.17). Axes identified as type group '1,2,4' were common at two sites (Naqada and Armant), and type 1 axes were common at both el-Mahâsna and Armant. Additionally, each site had light axes made from quite a few different raw materials. This is significant because at el-Mahâsna (the only site where such data were available), most of the axes were made of raw material 1,2 , or 4 , even though the most commonly used local material was type '8.Translucent Brown.' Therefore the choice to use light materials for axes, particularly types 1,2 , and 4 does seem to be intentional.

Furthermore there were few functional constraints that would have limited axe production to lighter materials rather than darker ones. In fact the occasional production of axes from darker materials shows that darker materials could be suitable. The axes are rather small in size, a sample of 48 complete axes ranged from $4.96 \mathrm{~cm}$ to $15.9 \mathrm{~cm}$ long and averaged $7.6 \mathrm{~cm}$. Holmes (1990) reported an average length of $6.5 \mathrm{~cm}$ for 49 axes from the Naqada region. Gravel nodules were observed at Beit Khallaf (near el-Mahâsna) over $10 \mathrm{~cm}$ in size, so even small gravel nodules would have been sizable enough for axes, and indeed some were occasionally made from such materials.

Therefore the choice of light raw materials for axes cannot be explained by functional considerations nor local availability of materials. Nonetheless there was an association between tool type and raw material choice, so the expectation was met. Symbolic considerations related to color very well may have affected raw material choice for axes.

\section{Large-blade knives}

The 60 examples of large-blade knives (Table 7.18, Figure 7.7) were made from nine different raw material varieties (Table 7.3). Most of the large-blade knives were made from type 
group ' $1,2,4$ ' (40.85\%). As with the axes, the majority of the large-blade knives (84.52\%) were a light material (ds $\underline{h} \underline{d})$ (Table 7.4).

Table 7.19 gives the distribution of raw materials by site, and shows that all of the knives classed as type '1.Indistinct beige' were from Naqada. However type 1 knives may have been included in the type ' $1,2,4$ ' category at other sites. At Badari and at Naqada there was a lot of diversity in the materials used for large-blade knives, not a focus on one type which would be more in line with simple use of local raw materials. The raw material sources available near Naqada and Badari are unknown. On the whole the raw material choices for large-blade knives cannot be accounted for by access to local materials at this time, although more information about raw material sources would be helpful on this point.

A sample of 54 complete pieces ranged from $8.12 \mathrm{~cm}$ to $23.4 \mathrm{~cm}$ in length, ${ }^{69}$ averaging $13.5 \mathrm{~cm}$. Many artifacts of darker material types in that size range and larger existed (e.g. fishtails, rhomboids) so the choice for lighter raw materials could not have been restricted by the size of available materials. Certainly darker materials were sufficiently sharp and strong for use as knives since other kinds of knives or daggers were made from those materials.

Large-blade knives meet the expectation evaluated here since there is an association between raw material color and tool type. This association cannot be explained by functional considerations, or use of local materials nearby where they were made, given current information.

${ }^{69}$ These blades were defined as pieces larger than $8 \mathrm{~cm}$. 


\section{Microendscrapers}

Only 14 heat-treated microendscrapers ${ }^{70}$ were identified, coming mainly from the elMahâsna and Nag el-Qarmila assemblages, along with a few pieces from Naqada which were studied by Holmes (1989). However there are likely many more in archaeological assemblages, but images and raw material descriptions of such small and inconspicuous items rarely get published. The non-heat treated and heat-treated microendscraper production processes were likely quite closely related (Ch. 5), so the sample was expanded to include 11 additional nonheat-treated microendscrapers (Table 7.20, Figure 7.8). Table 7.3 shows that the types of raw materials used to make microendscrapers was very limited. Type ‘8.Translucent Brown’ was by far the most common raw material type (44\%). The pieces in the 'other light cherts' category (24\%) were made from raw materials that could not be differentiated between type 8 or 10 , so likely even more of them were made of type 8 material.

All of the microendscrapers are light to reddish in color, and so should all be considered $d s \underline{h} \underline{d}$ (light chert). Only three raw material types were present, all of which are quite similar. All of these types have small white mottles, are semi-translucent, and are light brown to pink in color. The type 11 pieces are quite possibly a heat-treated version of types 8 and 10, which would mean that only two basic raw material types were used to make microendscrapers (types 8, and 10). In terms of the source for these raw materials, types 8 and 11 are definitely nodular gravel chert, and the source of type 10 is not known (Ch. 4.1). Therefore, the microendscrapers were made mainly from raw material type 8 , and a few pieces were also made from another type 
that is visually strikingly similar. This shows a high degree of association between raw material type and tool type, the most of any of the specialist produced tools seen here.

Table 7.21 shows the distribution of the raw materials by site. All the type 11 pieces come from el-Mahâsna. This is not surprising since type 11 may just be a heat-treated version of type 8, and there is evidence that people at el-Mahâsna heat-treated raw materials there (Ch. 5.5). The use of these pink-brown mottled semi-translucent materials cannot be explained solely by the use of local materials. While the raw material was certainly local to el-Mahâsna, this was not the case at other sites. Heat-treatment and microendscraper production also occurred at Hierakonpolis (Holmes 1989; Takamiya and Endo 2011). Some bladelets found in HK6 tomb 72, and HK43 Burial 333 can be identified as raw material types 8 or $10^{71}$ (Figure 7.9) (Droux and Friedman 2014:4, lower photo, center, 18-19; Friedman 2003:17-19). These items were described as bladelets in this short publication, but the rounded retouched ends are visible in the photographs, so they include microendscrapers. However the main locally available material at Hierakonpolis must have been quite different from that of el-Mahâsna. A chert source composed of chert-bearing fossilized coral was discovered near Hierakonpolis and thought to be the main source of chert (Friedman and Youngblood 1999), whereas the el-Mahâsna chert probably ultimately originated in the extensive limestone deposits surrounding el-Mahâsna and found everywhere north of Esna. These two very different contexts must have yielded quite different examples of a raw material which is already noted for its variability, yet both sites used the same

\footnotetext{
${ }^{70}$ In some cases a number of microendscrapers of identical raw materials were found in the same tomb, and likely came from the same core. There were only listed as a group to not unduly weight the sample towards one raw material or another.

${ }^{71}$ Some of the lithics from tomb 72 are also clearly type 4 . Sizes and descriptions of the bulb and platform characteristics have not yet been published for this recently found tomb, so it is not yet known which should be considered bladelets according to the definitions used in this study.
} 
materials for microendsrapers. Therefore, the choice to use light brown-pinkish semi-translucent mottled raw materials was a trend over multiple sites, from Hemmamiya and Badari in the north, to Hierakonpolis in the south, and not due to simple availability of local resources.

Nor was the choice of raw materials due to functional considerations. They are very small tools, $(2.8-5.5 \mathrm{~cm}$ long, averaging $3.8 \mathrm{~cm})$, so large nodules were not required. The texture of the microendscrapers is not discernibly different from many of the other chert varieties (e.g. types $2,4,5,6,7$, or 13 ), so any of these other materials would have sufficed for the production of small sharp thin microendscrapers. It is possible that the small size of the tools made them suited to the fist-sized gravel nodules, thus saving larger materials for other tools. However raw material economy is not a persuasive argument considering the abundance of chert available at most sites throughout Egypt, if the particular variety is not important. If anything, the small white calcareous mottles found in the microendscraper raw materials could have been an impediment to predictable thin flaking. Indeed type 8 material was more difficult to flake than some other materials heated to the same temperature during the heat-treatment experiment described in $\mathrm{Ch}$. 4.2. Therefore no functional reason was identified for the choice to make microendscrapers out of the types 8 and 10 raw materials.

In conclusion, there is an association between tool type and raw material for microendscrapers. It is quite possible that the raw materials for microendscrapers were chosen for symbolic reasons, since functional explanations can be ruled out. A possible symbolic reason for choosing this kind of raw material could have been the distinctive reddish-brown coloration. Heat-treatment would have enhanced the reddish color and/or the luster of the pieces. 


\section{Summary and Discussion}

The expectation of an association between raw material type and tool type was met for three classes of tools: Microendscrapers, axes, and large-blade knives. Microendscrapers had the least variability in raw material choice, made from only two main types which were visually quite similar. Axes and large-blade knives were made from many different types as defined here,

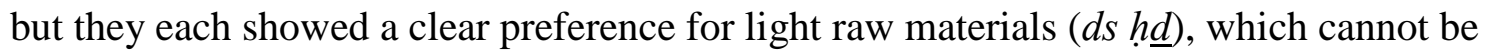
explained through functional considerations nor preference for local materials.

As for the other tool classes, RFKs and rhomboids both only had a moderate amount of variability in raw material choice. The production of RFKs focused on one raw material type in particular, but this focus may have been related to the raw materials available near the production locations, and/or the size requirements for the knives. Rhomboid raw material selection was also probably governed by the size requirements for these large tools, and there was no preference for light or dark materials for rhomboids, so symbolic considerations were probably not a factor in raw material choice.

Fishtails, CBPPs, and bifacial sickles were all made from many different raw materials, and there was no clear preference for a certain color. Therefore it is not likely that one raw material type or one color was significant to the symbolic meaning of the tools. The use of so many different raw materials may indicate that there were multiple production locations for these tools. Fishtails however showed a change in raw material choice over time, toward fewer raw material types focusing on the same raw material that was preferred for RFKs. Both the later fishtails and RFKs used the ripple-flaking technique. This change in the production of fishtails may indicate a shift toward fewer production locations.

At the beginning of this chapter a case was made that the color of the chert varieties may 
have been important to the symbolic meaning of tools made from them. And the finds of color preference for axes, large-blade knives, and microendscrapers corresponds nicely to the Pharaonic category of chert $d s \underline{h} \underline{d}$ meaning light or bright chert. Furthermore, the heat-treating of microendscrapers may have been done to increase the pinkish color and/or the luster of the pieces. The heat treatment does not seem to have been functional since microendscrapers were made of both heat-treated and non-heat-treated pieces of the same raw material, although more experimental work may clarify this point.

These findings suggest a new avenue for future research. One way to further substantiate the suggestion that the color of the raw materials was a significant part of the meanings of the tools is to look at patterns in how raw materials were deposited. The practice of depositing materials in specific patterns for symbolic reasons is known from other archaeological contexts worldwide. In Mesoamerica each of the four cardinal directions was associated with a color and specific attributes (Marcus 2007:61-63). This principle was not only depicted in motifs painted on celts and pottery, but also three-dimensionally replicated in deposits such as colored basins distributed around a dooryard (San Jose Mogote), offering caches deposited in the four corners of a structural platform (Zapotec), or even in the layout of a Tenochtitalan which was divided into four quadrants and associated with the appropriate colors (Aztec). An example of topographic locations indexed by raw materials comes from Chaco Canyon where non-structural wood corner posts in a burial chamber referenced the sacred mountain regions from which they came (Plog and Heitman 2010:7). These are but a few examples to illustrate the idea. There is some evidence for color pairings of tools in intentionally deposited Predynastic Egyptian contexts.

Tomb 72 at HK6 was a basically intact grave which contained one dark and one light 
bifacial sickle among many other fabulous objects (Droux and Friedman 2014). Similarly, a cache of three bifacial sickles was found at Abydos containing two dark and one light sickle (Ch. 3.4, Figure 7.5). That these sickles were deposited together indicates that the differences in chert color were not due to changes in the raw material source over time. Additionally, a number of offering deposits (some sealed, some disturbed) have been found in the corners of the columned structures in HK6. In the NE corner of Structure 7 a number of CBPPs were found. Most of these CBPPs were made from dark cherts and at least one was made from a light chert (Figure 7.4). Similarly, in the Block 3 ritual activity area in el-Mahâsna, two CBPPs were found, one dark and one light (Figure 7.4). The pairing of light and dark stone tools could echo the color pairing of red and black pottery in ritual locations at Hierakonpolis (Friedman 2009b:85-88; Hendrickx and Friedman 2003). This suggestion that light and dark (red and black) materials might have been intentionally paired should be researched further, through collection of more detailed information on the raw materials of items in intact contexts.

Besides raising new questions for study, this analysis of the raw materials of specialized tools has demonstrated the utility of studying raw materials. Such studies can provide information about production and distribution patterns, change over time, and even the symbolic uses of tools. 


\subsection{Production Expectation}

If specialized production of some tools developed in Egypt to make symbolically meaningful goods, then it is likely that the production of those tools would have been associated with ritual activities. The production may have been organized around ritual events, when people aggregated together, which would have offered advantages such as facilitating the procurement of raw materials, or sharing of knowledge. Additionally production associated with ritual activities or ritually important locations could have added meanings to the tools indexing the place or time of production. This expectation was evaluated by looking for production remains in ritual contexts ${ }^{72}$. Such contexts include the HK29A, HK29B, and HK25 ritual precinct at Hierakonpolis, the ritual activity area in Block 3 at el-Mahâsna, and the numerous cemeteries throughout Egypt. See Chapter 3.4 and 3.5 for more details on these contexts. Chapters 5 and 6 looked at the spatial distribution of production remains relative to products at inter- and intra-site levels to understand the organization of production. This chapter is concerned with looking at production remains relative to the interpretations of their contexts.

It should be noted that production associated with ritual activity areas has often been interpreted as indications of sponsored production (e.g. Holmes1992; Takamiya 2004). One way that production associated with ritual aggregation may be differentiated from such sponsored production is that the former is by definition part-time, seasonal or periodic. Therefore the intensity of the activities (sensu Costin 1991, 2001) will also be considered below.

\footnotetext{
${ }^{72}$ Ritual contexts judged as such from other lines of evidence such as the ceramics, faunal remains, and architecture.
} 


\section{Ripple-Flaked Knives}

No production locations for RFKs have been definitively identified. However there was indirect evidence that pertains to RFK production locations and intensity. The standardization of the production process has led researchers to conclude that production was limited to one or very few locations (Holmes 1989:338; Kelterborn 1984:452). Although the exact dimensions of the knives varied, the consistency of the direction of flake removals (ibid.:442; Midant-Reynes 1987:190) points to a standard production process.

The analysis of RFK raw materials in the previous section (Ch. 7.1), indicated that RFKs were possibly produced near Abydos. The most commonly used RFK raw material type was available and used near Abydos, and the RFKs made from that raw material type clustered around Abydos. Abydos’ political importance is clear from the exceptional graves, such as the burials of Egypt's first Kings, or the earlier tomb UJ, which had evidence of far distant trade connections and early writing. If RFKs were produced near Abydos their production could have had political overtones in addition, to or instead of religious ones, especially considering that many of the knife handles had motifs of warfare and domination (Ciałowicz 1992; Delange 2000, 2009; Dreyer 1999; Midant-Reynes 1987; Needler 1984; Williams and Logan 1987). Accordingly, production of RFKs at Abydos could easily fit a model of sponsored production.

An additional or alternative production location for RFKs was the HK29A ritual enclosure at Hierakonpolis. As discussed in Ch. 6.1, large quantities of remains from bifacial tool production were associated with this enclosure. The enclosure was used during two periods, NIIAB-C and NIID2- ${ }^{73}$ NIIIA (Friedman 2009b). RFKs date to the NIId (Dreyer 1999:213;

\footnotetext{
${ }^{73}$ Since the sub-phase IId1 is no longer thought to be valid (Buchez 2011b; see Ch 3.2), the date of the second phase does correspond to the dates of the RFKs.
} 
Midant-Reynes 1987:212), so they could have been made there during the early part of the second stage of that workshop. The technique of ripple flaking has been identified among HK29A remains (e.g. Takamiya and Endo 2008:8), but no actual RFK fragments with one ground side and one flaked side have been reported.

The issue of seasonal vs. continuous use of the HK29A structure is somewhat unresolved. There is some faunal evidence that suggests the HK29A ritual structure was used during multiple seasons throughout the year (Linseele et al. 2009, see Ch. 3.5). More importantly, the quantities of lithic remains from the later phase of the workshop, when RFKs would have been made, indicate a full-time production level (Holmes 1992), rather than seasonal or periodic production that would be more in line with production associated with ritual aggregation.

Therefore, while the production location(s) for RFKs is not definitively known, neither of the two possible production sites discussed here fit well with the expectations for the ritual production model. Instead they indicate sponsored production, possibly in a full time capacity, not one related to ritual cycles.

\section{Rhomboids}

At least some rhomboid tools were almost certainly made at the HK29A ritual enclosure. A fragment of a probable rhomboid tool was found there (Friedman 2009b:89; c.f. Holmes 1992:41), along with large amounts of bifacial thinning flakes. Rhomboid tools primarily date to NI-IIAB (Ch. 6.1), the tail end which overlaps with the beginning of the earlier phase of the HK29A ritual structure (NIIAB-C) (Friedman 2009b). Moreover, Hierakonpolis was the only site where rhomboids have been found in a settlement (Friedman and Nagaya 2013:22; Needler 1984:114-115, 265-266). More rhomboids (n=8) come from the Hierakonpolis settlement and 
cemeteries than any other site, more even than were found at Naqada with its very large cemetery (Table 6.3, 6.4, 7.6). However it should be noted that since the HK29A structure was only in use at the end of the period in which rhomboids were made, it cannot be the only production area for rhomboid tools.

In the previous section, Ch. 7.1, the analysis of rhomboid raw materials and their distribution showed some clustering of the most common raw material type around Naqada. However note that this was a rather small sample and no source of that material has been identified near Naqada. The dates of South Town are slightly later than the dates of the rhomboid tools, but many other earlier settlements existed in the Naqada area.

The rhomboids do meet the expectation of production associated with a ritual location, since some of their production likely comes from the early phase of HK29A ritual enclosure. In the earlier phase of the structure the lithic artifact density was not as high as in the later phase (Friedman 2009b, see also Ch. 6), so the production was not necessarily full-time, but very well could have been periodic or associated mainly with ritual events.

\section{Fishtails}

Fishtails were likely made at multiple sites. The variability in quality and size of the fishtails (Van Walsem 1978:243; Ch. 7.1) and in the raw materials used (Ch. 7.1, Table 7.4)) indicate that fishtail production probably occurred in multiple locations. Some of this variability comes from changes in raw material choice over time. Type '4.Beige with pink bands' was quite common for later fishtails, and might be associated with a shift in the place(s) of production.

One probable fishtail production location was the HK29A ritual enclosure. The fishtails date from the NI to late NIId or NIII (Hikade 2003:139), and most scholars agree that they 
continue as the $p s \check{\text { š }} k f$ instrument, in a slightly altered form. Therefore fishtails were certainly coeval with both phases of lithic reduction at the HK29A ritual enclosure: NII(A)B-C and NIID2-NIIIA (Friedman 2009b). Although no broken fishtails or fishtail preforms have been found associated with the site, there was a high frequency of bifacial thinning flakes, unidentifiable fragments from bifacial tools, and as mentioned above, a fragment of a tool that shows some ripple-flaking. Later fishtails (shape type 2) had similar ripple-flaking (e.g. Figure 7.6 MMA.24.2.13).

As discussed with RFKs, HK29A was a ritual activity area, which would seem to fit the production expectation. However, in the later period, coeval with the later fishtails, the scale of production was quite high, indicating full-time production, which does not fit the expectation for production in the context of ritual aggregation, at least. On the other hand, the earlier HK29A context did not have the same density of remains, indicating that stone tool production did not occur as intensively in there in the NII(A)B-C. Since there was also some evidence for seasonal use of the structure (Linseele et al. 2009; Ch. 3.5), then it is likely that production in the earlier phase may have been more periodic, and that the production context for earlier fishtails does fit the expectation.

Overall the production context of fishtails may have been variable and/or changed over time. Fishtails were probably made in multiple locations, as was indicated by the variability in their style, size, quality, and raw material types. At least some early fishtails were probably produced at the HK29A ritual structure in its early phase, which does conform to the expectation for the ritual production model. However they were also likely produced there during the second phase, which does not fit well with the model since the high level of production has been 
interpreted as sponsored full-time production. This shows change over time over time in the production of fishtails.

\section{Concave-base projectile points}

Evidence for the production of CBPPs came from HK29A, South Town, and Naqada Khattara site KH4, all in the form of unfinished CBPPS. These included both ritual and nonritual contexts. At the HK29A ritual enclosure Holmes (1992:41) found a number of bifacial tool fragments which she thought were projectile points broken during manufacture, along with more complete fragmentary specimens that may have broken during manufacture, or may have been finished pieces that broke later. Takamiya and Endo (2008:8) indicated that CBPPs were probably among the tools manufactured at HK29A. The finds of CBPP fragments have not been correlated to the different phases of activity at HK29A, but because CBPPs themselves date to the late NI-early NII (Hikade et al. 2008:185-186), they were likely produced during the earlier phase of bifacial tool production at HK29A (NIIAB-C). As mentioned above, production of tools in the early phase of HK29A corresponds well to the expectation of part-time or periodic production associated with ritual activities in the ritual production model, because of the lower density of artifacts during that phase.

Additionally, the distribution of CBPP raw material types by site shows that eight of the 13 examples which were made from type "7. Dark gray and brown” material came from Hierakonpolis (Table 7.13). However it is not known whether there was a source for this material near Hierakonpolis. Additionally it should be noted that while Holmes (1992) and Takamiya and Endo $(2008,2011)$ emphasized the preferential use of orange and cream colored raw materials for bifacial tool production at HK29A, those materials made up only 13\% of the bifacial tool 
manufacture debitage in HK29A (Holmes 1992:8). Holmes (ibid.) also noted that beige and dark brown materials were used for bifacial tools, thus supporting the notion that raw material type 7 CBPPs were made at HK29A. Furthermore, besides the 13 CBPPs with identifiable raw materials known from Hierakonpolis, at least 15 more burnt CBPPs were found at HK25, which was an extremely high concentration of CBPPs in one site (Tables 6.1-6.4, 7.12).

Petrie found an unfinished CBPP in his excavations at South Town (Naqada) (Holmes 1989:274; Petrie and Quibell 1896:Pl 55). Petrie’s work revealed extensive mud brick architecture, but there was no other information about the context of the find. Subsequent excavations at the site by Barocas and others (Barocas et al. 1989; Di Pietro 2011; Fattovich et al. 2007) found evidence for administrative and ritual artifacts including seals, tokens, and figurine fragments. However the Italian excavations took place in the Southeast part of the settlement, and Petrie's excavations took place in the North and Northwest part of the site, so the CBPP production cannot be directly associated with the ritual and administrative activities in the area. Nonetheless, the existence of CBPP production, ritual activities and administration at such a prominent site, especially considering the evidence from Hierakonpolis where CBPPs were very likely manufactured in association with ritual activities, suggests a possible relationship.

Three unfinished CBPPs came from Naqada KH4 (Holmes 1989:, Holmes (1989:247) along with an additional finished but broken specimen. The site was only surface collected, so little is known about the kinds of contexts that existed at the site. KH4 was a relatively substantial size for a Predynastic site, at 2 ha, but was smaller than other nearby sites including KH3, South Town and North town. There was no evidence for overt or concentrated administrative or ritual activities. The botanical and faunal remains, and other finds, indicate that KH4 was an agricultural village. Therefore the production of CBPPs cannot be connected to 
ritual activities there, given current data. A possibly unfinished CBPP was also found at Maadi, another site with no clear evidence of concentrated ritual activities (Rizkana and Seeher 1988:32 Pl 68).

Thus it is likely that CBPPs were produced in a number of contexts. These contexts included a ritual context (HK29A), a possible a context of sponsored production which may have included ritual activities (Naqada South Town), a settlement not securely connected with either ritual or political activities (KH4), and a town known for its trading activities (Maadi). For the early phase of HK29A and especially for KH4 part time production of CBPPs seems reasonable based on the lower density of lithic remains compared to the later phase of HK29A. Therefore the evidence accords well with the expectations from the ritual production model.

The connection between lithic tool production and the ritual activities at HK29A was very clear for CBPPs. Both relate to hunting and animal slaughter, which had highly symbolic ritual connotations and status building effects (Hendrickx 2013). So in this case it is very clear how the production of CBPPs would have been structured by these ritual activities, although production occurred in other places as well, so it was either not limited to these activities or could have been associated with smaller-scale hunting and ritual activities that did not leave as many traces.

\section{Bifacial sickles}

There is no data for the precise production context of bifacial sickles. There was a moderate amount of variability in the raw material choices compared to the other specialized tool types (Table 7.3). Additionally there was fair amount of variability in size and shape, but this was difficult to quantify since some have clear indications of being re-worked. All in all, there 
was nothing to rule out their production at ritual sites like HK29A or el-Mahâsna Block 3, since thinning flakes of the appropriate raw materials were found in both locations. But there was nothing to confirm that these tools were made there, so for now this expectation cannot be evaluated.

\section{Axes}

Axe production was associated with both ritual and non-ritual contexts. Axes were produced in sites throughout the Naqada region including Armant 21/83, Armant 21a/83, the Naqada Khattara sites, and Abadiya (Tables 4.20, 6.1, 6.3). The evidence for axe production included thinning flakes, axe preparation flakes, and axe preforms. At Armant MA21/83 axe production remains were more prevalent in the southern part of the site (Ginter et al. 1996:177178) which was primarily a habitation context (Ginter and Kozlowski 1994:38-45; Ch. 3.5).

However, there was also evidence for axe production in association with ritual contexts at HK29A and el-Mahâsna Block 3. The only reported axe preparation flakes from any of the Hierakonpolis localities studied here came from HK29B in the ritual precinct, Table 4.20). HK29B and HK29A also had significantly higher amounts of bifacial thinning flakes than other areas of Hierakonpolis (Table 6.22), although no axe preforms were specifically identified. Axes themselves were found in the settlement and cemetery (Ch. 7.3). HK29B dates to the second half of the Naqada II period (Friedman et al. 2011b:115), which bridges the end of the first phase of use at HK29A and the beginning of the second phase of use there so it is not clear whether axe production should be associated with part-time or full time bifacial tool production.

At el-Mahâsna, only one axe preparation flake was recovered, and it was found in Block 3 the ritual activity area. Block 3 also had a higher percentage of bifacial thinning flakes than the 
other blocks (Tables 6.17-6.18), and there was a preform for an unidentified tool which could be an axe. Finished axes were found in Block 3 and in other areas of the settlement. The densities and quantities of lithic tools production remains were only slightly higher than other parts of the site, so the production should not be considered full-time.

On the whole, axe production is best characterized as part-time production in conjunction with ritual and non-ritual activity areas, but at Hierakonpolis may have been associated with more intensive bifacial tool production during the later part of the Naqada period.

\section{Large-blade knives}

The only possible production place so far identified for large-blade knives was at Badari 3000/6, where two refitting large blades matched the raw material of a blade core (Figure 7.7). However, little is known about the context of these production remains. Brunton left very few notes about the contexts he excavated except to say that they "showed remains of settlements" (Brunton and Caton-Thompson 1928:45). At settlement 3000/6 he did recover a few intact jars full of interesting items such as ivory tags, a disk mace head, pendants, a rhomboid slate palette, resin, malachite, ochre, horns, shells, pebbles and a tusk shaped like a human head that has a loop on top, which some have likened to an early version of the white crown (ibid.: Pl 53; Hendrickx et al. 2015). Additionally there may have been a beer production facility at Badari 3000/6 since some of the distinctive fire bars associated with such structures were found there (Geller 1992a). The only other sites with beer production facilities so far identified were Abydos, el-Mahâsna, Hierakonpolis, and Tel-el Farkha, each associated with substantial evidence for ritual activities and/or elite political activities. In the end though it is not possible to say one way 
or another whether any production of large-blade knives at Badari 3000/6 was associated with a ritual activity area or not.

\section{Heat-treated microendscrapers}

The production remains for heat-treated bladelet tools were associated with both ritual and non-ritual contexts at the sites where context information was available. Production of heattreated microendscrapers occurred in a number of communities including Badari, el-Mahâsna, Adaïma, and Hierakonpolis, but not at others, such as Naqada region sites or Nag el-Qarmila (Table 5.24). At both el-Mahâsna and Hierakonpolis, where intra-site data were available, production remains from the process of making heat-treated bladelets were found in multiple areas of the sites, including, but not limited to, the ritual contexts. There was variability in the distribution of the specific kinds of production remains (chunks, cores, prepared cores) which indicated that the entire production process may not simply have been carried out in each area, but organized in a more complex way across the site (see Ch. 5.6). These data show that heattreated microendscraper production was sometimes associated with ritual contexts, and in other cases not.

More evidence of microendscraper production in association with ritual activities came from the cemetery finds. There were indications that some stages of the production of heattreated bladelets were carried out in relation to burials or funerals (ritual activities). Bladelets that refit together were found in tombs in the Hierakonpolis and Abydos cemeteries (Droux and Friedman 2014:4-5; Hikade 1996) (Figures 7.9, 7.10). These bladelets were made from the kinds of raw materials preferred for microendscrapers (see Ch. 7.1). The fact that the bladelets refit shows that they all came from the same core, and that they were all likely made at once for the 
burial, otherwise time and life would have scattered the pieces into different depositional contexts. According to Hikade $(1998,2000)$ a third of the bladelets from Abydos cemetery U were made into microendscrapers. The exact location where the bladelet production and/or microendscraper finishing retouch took place, whether at the burial site or elsewhere, is not pertinent for this discussion. What matters is that the bladelet production was motivated by a ritual event—a burial or funeral. Furthermore this practice of producing bladelets and/or microendscrapers probably occurred at other places besides Hierakonpolis and Abydos, as will be discussed in section 7.3.

The production of microendscrapers was probably part-time, perhaps eventually becoming full-time. At el-Mahâsna (which dates to the NIc-IIc) the microendscraper production remains, and the lithic production remains in general, were not numerous enough to be considered full time, and the same probably goes for other sites like Badari or Adaïma where no dense concentrations of lithic remains have been noted. The later phase of lithic production at HK29A probably derived from full-time production (Holmes 1992; Friedman 2009b; Ch. 6.1), but it is not clear whether the heat-treated microendscrapers there were associated with the early and/or late phase of use. However microendscraper production likely also occurred at HK11C, Operation A, which by the later NII period was a dedicated production area for many items including beer and ceramics, and may very well have constituted full-time production.

Microendscrapers certainly meet the expectation for the ritual production model. Their production was often associated with ritual activities in settlements and cemeteries, and part-time production in the earlier phase of the Naqada period. 


\section{Production expectation conclusion}

In conclusion, for every case where sufficient data were available, specialized production of lithic tools took place in association with ritual contexts at least some of the time. Rhomboids, fishtails, CBPPs, axes, and microendscrapers all fit the expectation for production associated with ritual activities, on a part-time basis. Moreover the association between the production of specialized tools and ritual contexts was not limited to one site, but occurred over multiple sites, including Hierakonpolis, el-Mahâsna, and possibly Naqada and Badari, showing that this was a real pattern in production practices.

However in some cases (fishtails, CBPPs, axes, and microendscrapers) the tools were also made in other contexts which cannot be associated with ritual activities. Upon reflection, the fact that a number of these specialist produced tools were made in both ritual and non-ritual contexts sits quite well with the ritual production model. As discussed in Ch. 1.5 the ritual production model concerns production of items which have both utilitarian uses and symbolic value. Therefore it makes sense that they could be produced in multiple kinds of contexts.

Over time, production levels increased from part-time production to full-time production (Takamiya 2004), here this trend is evident from the increase in the density of lithic debitage with the later phase of the HK29A ritual enclosure. This trend can be seen clearly for fishtails, which were made over the longest time span of any of the tool classes, and have their own independent stylistic measure of dating. The variability in raw material types used for fishtails decreased over time, probably indicating fewer production locations. Additionally there was a change in technology with the adoption of ripple flaking, which also probably indicates a reduction in the number of producers/production locations since it was such a difficult technique. Microendscrapers may eventually have been made by full-time specialists since their production 
remains occurred in areas with high levels of production activities at Hierakonpolis (HK11C and HK29A).

\subsection{Find Context}

In the ritual production model outlined by Spielmann (2002), the ethnographic examples she drew on showed that the socio-ritual valuables made by specialists were often used in both ritual contexts and more ordinary contexts. These items blur the boundary between symbolic goods and utilitarian goods, often being used for both purposes. Therefore the goods made by specialists should be found in both ritual and non-ritual contexts.

Ch. 3.4 and 3.5 gave evidence for the interpretation of many Predynastic contexts which can be used to evaluate this expectation. Examples of ritual contexts include the HK29A ceremonial enclosure, the HK25 columned hall, el-Mahâsna Block 3, the temple area of Kom esSultan, and cemeteries. Examples of contexts which were not primarily or overtly used for ritual purposes include habitation areas (el-Mahâsna Blocks 1 and 4, Abydos ATP, Naqada KH3B, Armant MA 21/83, Adaïma 1001, Hierakonpolis HK29, HK11C area G, Nag el Qarmila WK15), middens (HK14, HK11C mound A, Armant MA21a/83 trench 3), storage areas (Nag el-Qarmila WK22), animal pens (Hemmamiya North Spur, HK11C ON6E), or production areas (Adaïma 1000, HK24A, HK25D, HK11C Op A).

Additionally, in the ritual production model specialist goods are used by many people, not just by a subset designated according to emerging class, wealth, or other factor. So the second expectation examined here was whether specialist produced tools are found in widespread rather than more restricted contexts. One way this was evaluated was by looking at whether the tools were found in settlements of all scales. It is conceivable that access to artifacts 
might be related to the kind of site people lived in, such as whether that site was a large center or a small farming village. In Ch. 3.7 the settlements were divided into a three-part scale based on the area/populations and the diversity of activities apparent at that site (Table 3.4). The cemeteries were assumed to correspond to the scale of their associated settlement. The expectation for widespread distribution was also evaluated by looking at whether the tools were found in multiple household contexts within a settlement, rather than concentrated in only one (a more restricted distribution of the tools). The presence of specialist tools in burials or cemeteries of different status at a given site was also taken as an indicator of a widespread rather than more restricted distribution of the artifacts. It is important to point out that while the determinations of which tool types were produced by specialists involved looking at the spatial or geographic distribution of the tools vs. their production remains (Ch. 5 and 6), this expectation looks at what those contexts were, i.e. the social distribution, of the tools themselves.

\section{Ripple-Flaked Knives}

RFKs were overwhelmingly found in ritual contexts. Those ritual contexts included burials, where they were primarily found, and a cache of goods at the Tel el-Farkha settlement, which also included two golden statues and an over-sized bifacial knife (Ciałowicz 2012; Kabacinski 2012). One possible RFK was also found in a domestic context. ${ }^{74}$ An RFK reworked into a scraper was found in the Hemmamiya settlement (Brunton and Caton-Thompson

\footnotetext{
${ }^{74}$ A fragment of a RFK was reported from a trash pit dug through domestic layers in HK11C area G (Watrall 2000). However it was later deemed to not be a true RFK with one flaked side and one ground side (R. Friedman, pers. comm.)
} 
1928:77, Pl81). However, the Hemmamiya piece is a bit too wide to be from an actual RFK, ${ }^{75}$ and may have originally derived from another kind of knife with ripple flaking. ${ }^{76}$ Alternatively it may be from an unfinished example that broke during production, never having become an RFK. Overall, true RFKs were found only in ritual contexts.

RFKs were found in cemeteries of all scales, but were most common in highest-order sites like Abydos (see Tables 6.4, 7.4, and 7.5). They were present in the highest-order cemeteries of Abydos and Naqada, and in some but not all mid-level cemeteries, like Adaïma, but not at el-Mahâsna. Occasionally RFKs have been found in some small cemeteries. For instance, an RFK comes from Armant, but the cemetery was in use after the settlement discussed here was abandoned, so it was not clear whether it should be considered a low-level site or a mid-level site. An RFK was found at Harageh cemetery G, which was a small cemetery, and so it may represent a lower order site (Engelbach and Gunn 1923:7; Hendrickx and van den Brink 2002:352).

RFKs have also been found in graves which exhibit different degrees of wealth. RFK fragments were found in the royal tomb of Djer. At Gerzeh an $\mathrm{RFK}^{77}$ comes from the largest tomb in the cemetery (Stevenson 2009:113). At Abu Zaidan tomb 32 contained three RFKS and could certainly be considered among the wealthiest if not the wealthiest grave in the cemetery in terms of size and quantity and variety of objects (Needler 1984:124-125, 392). However, looking at overall data on tombs with RFKs Midant-Reynes (1987:202) concluded that many graves with

\footnotetext{
${ }^{75}$ The Hemmamiya piece is $4 \mathrm{~cm}$ wide, and shows ripple flaking from one direction only. If it was from a true RFK, with ripple flaking from both lateral edges, the original knife would have been at least $8 \mathrm{~cm}$ wide. A sample of 25 complete RFKs with width measurements showed that only one example was $8 \mathrm{~cm}$ wide, the rest ranged from $2.4-\mathrm{cm}$, averaging $5.5 \mathrm{~cm}$ wide.

${ }^{76}$ E.g. Boston Museum of Fine Arts 03.1388, or University of Pennsylvania Museum E.1114.

${ }^{77}$ Pitt Rivers Museum Knife 1911.33.1. Also see notes there by Stevens.
} 
RFKs could not be classified as elite, based on dimensions, architecture, and offerings, nor would she place them in a lower strata of society according to those same criteria. At Naqada, RFKs were found in the main (non-elite) cemetery. However Bard (1994) demonstrated that the western cluster of graves in the Naqada main cemetery was wealthier and more exclusive than the eastern cluster based on types and quantities of grave goods. RFKs were only found in the wealthier western cluster. Thus the overall cemetery data indicates that RFKs were restricted to tombs of upper and middle status/wealth.

All in all, while the RFKs were found in cemeteries of all scales, the RFKs do not meet the expectation for find contexts well because they were only found only in ritual contexts, and they were mainly found in graves with middle or high degrees of status or wealth.

\section{Rhomboid tools}

Rhomboids have only been found in ritual contexts. These contexts were: the cluster of burnt bifacial tools at the HK25 columned hall (Hikade et al. 2008; Nagaya and Friedman 2013), the HK29A ceremonial enclosure (Friedman 2009b:89), and burials. Two rhomboid tools were collected by H. de Morgan from somewhere in the Hierakonpolis the settlement (Needler 1984:114-115, 265-266) but these probably also came from HK25 considering that they were burnt and broken, just like the bifacial tools from HK25.

Rhomboid tools were found most commonly in the highest order and mid-level sites, but also in some lower order sites (Tables 6.3, 6.4, 7.6, 7.7). The Abydos, Hierakonpolis, and especially Naqada cemeteries represent the highest-order sites. Additionally rhomboids were found at the Mesaeed cemetery (Table 7.6), Abadiya Cemetery B (Petrie and Mace 1901:Pl 5), and Hiw Cemetery U (Hikade 2010; Petrie and Mace 1901), which were all medium- large 
cemeteries of 400-700+ graves each. A number of rhomboids came from Mostagedda cemetery 1800 (Brunton 1937:90, Pl40, tombs 1803, 1847, 1854), which was a small to medium-sized cemetery of around 170+ graves. One was found at Armant cemetery 1400-1500 (Mond and Meyers 1937:Pl20), which is here considered a lower order site.

Whether or not rhomboid tools occurred graves of different status was more difficult to determine. They definitely have been found in very high status graves, such as Tomb 72 at Hierakonpolis, which may have belonged to an early ruler (Droux and Friedman 2014:7). Additionally, two rhomboids were also found as heirlooms in the 'royal' tomb at Naqada (Hendrickx 1994:52-55, 2002:283). On the other hand they were also found in regular non-elite cemeteries, in both the western and eastern clusters of the main cemetery at Naqada (1241, 1437, 1676), where they were found with other stone tools, pottery, and sometimes stone vessels or pallets.

Overall the contexts or social distribution of rhomboid tools was similar to that of the RFKs. They occurred mostly in the highest order sites and definitely in elite graves, and sometimes in medium level cemeteries and graves which may not be the most elite, but rarely in the lowest order cemeteries and not at all in poorer graves.

\section{Fishtails}

Fishtails have been found in both ritual and non-ritual contexts. Fishtails were certainly prevalent in cemeteries, where they occur as grave goods (Table 6.9). Additionally they have been found in offering caches associated with a columned hall (Structure E8) in Hierakonpolis cemetery HK6 (Friedman 2006:7-8). In terms of ritual contexts in settlements, fishtails have been found among the group of burnt bifacial tools at the HK25 columned hall (Hikade et al. 
2008; Nagaya and Friedman 2013). Additionally, at least one fishtail was found associated with the late Predynastic votive offerings of the Hierakonpolis Nekhen temple 'main deposit' (Quibell 1900: $\mathrm{Pl24).}$

Fishtails have also been found in a number of other contexts which were primarily domestic in character. Two fishtails were excavated from habitation contexts at the site of Hemmamiya, which has evidence for storage, animal pens, and debatably, houses (CatonThompson 1928:77,96,107 Pl.71-72, 79-80). Two fishtails also come from the settlement site of el-Mahâsna. One (PR.1901.42.107) was from Garstang’s “Settlement 2” area, and Anderson (2006:30, Fig 3.10) thinks Garstang’s excavations there were located west of the ritual activity area (Figure 3.2) and contained wattle and daub structural remains. Another fishtail fragment, found by Anderson (MAP.301.1), was on the surface at the southern end of the site, which was the opposite end of the settlement as the Block 3 ritual activity area, in an area that corresponds to Garstang's “Settlement 1" (Anderson, pers. comm.). Another fishtail fragment was located on the surface at Adaïma, where only habitation and grain production contexts have been identified (Midant-Reynes and Prost 2002:367, no 431, Pl 27.1). Hoffman found a fishtail fragment on the surface of HK29, an area with a dwelling, fenced yard, and pottery production remains (Holmes 1989:318).

The examples above show that the fishtails came from settlements of all scales including Hierakonpolis for the highest order sites, el-Mahâsna and Adaïma for mid-level sites, and Hemmamiya for the low order sites. Correspondingly, fishtails are known from cemeteries of all scales (Tables 6.4 and 7.8), including the Abydos, Naqada, and Hierakonpolis cemeteries (highorder sites), el-Mahâsna, Mesaeed, Abadiya, and Hu cemeteries (mid-level sites), and Armant cemetery and Harageh Cemetery H (Engelbach and Gunn 1923:7, Pl 7) (low-order sites). 
Fishtails were quite numerous and these examples do not cover all cemeteries where fishtails have been found, but are just examples to show that they were found in cemeteries of all scales.

Furthermore, the two fishtails from Hemmamiya were from different parts of the site, and the two fishtails from el-Mahâsna were from widely separated areas of the site, showing that their distribution within a settlement was not confined to a single context or household. Additionally fishtails were included in graves of different status. At Hierakonpolis multiple fishtails were uncovered in the elite cemetery HK6, and one was associated with painted Tomb 100 (Quibell and Green 1902:21) also an elite grave. At least one fishtail was included in a grave of the non-elite 'working class' cemetery (HK43) (Friedman 2004b). At Naqada two fishtails were found in cemetery $\mathrm{T}$ (tomb 22), the elite cemetery, while many also came from the nonelite main cemetery. Even in the Naqada main cemetery, the fishtails were found from graves of diverse wealth. They ranged from graves with only a few pots, to tombs with all sorts of items, such as tomb 271 which in addition to a fishtail had ivory figures, a clay figure, 16 ceramic pots, 3 stone vases, tags, resin, a slate amulet, malachite, coral, and cloth with stucco and paint (Baumgartel 1970; Payne 1987; Petrie 1896). Bard's analysis showed that the western cluster of graves in the main Naqada cemetery was slightly wealthier and more exclusive than the Eastern cluster, but fishtails were found in both clusters. ${ }^{78}$ These data indicate that people with a range of wealth or social affiliation used fishtail tools, not only a restricted set of people.

In sum fishtails meet the expectations derived from the ritual production model for context. The contexts show that fishtails were associated with ritual activities but could also be a 
part of other contexts which were not primarily ritual in nature, and they were used by many people, not just a subset, at least as far as status and settlement scales are concerned.

\section{Concave-base projectile points}

Finished CBPPs have been found in both ritual and non-ritual contexts (Table 6.1-4, 6.19, 7.12-13). The ritual contexts of CBPPs include the Block 3 ritual activity area at el-Mahâsna, the HK29A ceremonial enclosure (Holmes 1992; Takamiya and Endo 2008), the nearby HK25 columned hall where they were among the group of burnt and broken bifacial tools (Hikade et al. 2008; Nagaya and Friedman 2013), and the temple area of Hierakonpolis Kom el-Ahmar/Nekhen town site (Adams 1974:xv, 39, Pl27). CBPPs have also been found in different capacities in cemeteries, such as the offering deposits associated with structures 7 and 8 in the Hierakonpolis HK6 cemetery (Friedman 2012:69-71), and as grave goods in tombs, e.g. HK6 tomb 72 (Droux 2014:4-7, 18-19), or tomb 178 in Abydos cemetery U (Hikade 2000).

In addition, CBPPs come from contexts which were not predominantly associated with ritual activities. Examples include ones found among the habitation structures in Adaïma Block 1001 (Midant-Reynes and Prost 2002:366, Pl 25) and Naqada KH3 (Holmes 1989:247). Two were found in Predynastic levels of the living/storage/animal pen remains at Hemmamiya (Caton-Thompson 1928:98, Pl 71,79; Holmes 1989:83), and one comes from a general settlement context at Elkab (Claes et al. 2014:85). A CBPP from Armant MA21a/83 was found in Trench I which was a subsistence activity area with storage pits and hearths (Ginter and Kozlowski 1994:43).

\footnotetext{
${ }^{78}$ The grave inventories are given in Baumgartel (1970) and Payne (1987). Bard gave the grave numbers
} 
How widespread the CBBPs were in terms of social distribution was gauged by looking at their presence in settlements and cemeteries of different scales, multiple non-ritual contexts in settlements, and/or graves of different status. Finished CBPPs have been found in sites of all scales. The highest order sites include the settlements of Naqada South Town (Baumgartel 1960:28, Pl 1; Petrie and Quibell 1896:Pl 72) and Hierakonpolis (above), and the cemeteries of Abydos and Hierakonpolis (Table 7.12). Mid-level sites with finished CBPPs include the settlements of Badari 3000/6, el-Mahâsna, Adaïma, and Maadi (Tables 6.1, 6.3, 7.12; Rizkana and Seeher 1988:32, Pl 68). Examples of CBPPs from Naqada Khattara sites, Armant, and some Badari sites (Holmes 1989) show that they were also present in the low-order settlements. Examples from Hemmamiya cemetery 1700, and Badari cemetery 3900 show that their distribution extended to these lower-order cemeteries as well. ${ }^{79}$

Within settlements there was conflicting evidence for whether CBPPS were concentrated within contexts associated with certain groups, or more widespread. At Hierakonpolis the CBPPs were concentrated in ritual contexts (HK25, and HK29A) and were not found in other kinds of contexts, ${ }^{80}$ let alone in multiple non-ritual contexts. However at el-Mahâsna there was some evidence that the CBPPs were not quite as concentrated. Two of the three el-Mahâsna CBPPs

for the cemeteries as: Western cluster tombs 56-499 \& 1200-1299; Eastern cluster 500-1195 \& 13011953.

${ }^{79}$ Brunton thought these dated to the Badarian, but he assigned the date based on the presence of the CBPPs, which we now know extend into the Predynastic. These cemeteries are otherwise Predynastic (Holmes 1989:171).

${ }^{80}$ Two CBPPs found by de Morgan in the Hierakonpolis settlement (Needler 1984:262-263) probably came from the HK25 columned hall since they were burnt and broken, like all the bifacial tools from that locality. Friedman and Nagaya (2013) similarly argued that burnt and broken bifacial tools collected by Petrie probably came from HK25. 
from Anderson's excavations ${ }^{81}$ were found in Block 3, the ritual activity area (Table 6.19).

However, Anderson (pers. comm.) also located a third one (MAP 395) on the surface adjacent to Block 4, and Garstang (1903:7, Pl3) found a few CBPPs in the "settlement 2" portion of elMahâsna which is likely adjacent to Block 4 (Anderson 2006:30, Fig 3.10). Finds of CBPPs from other settlement sites either occurred singly, or were not associated with the level of data that would enable comparison between contexts.

Additionally, there were examples of CBPPs from very elite wealthy tombs and from less exceptional tombs. The examples from Abydos Cemetery U tomb 178 (Hikade 2000) and Hierakonpolis HK6 tomb 72 (Droux 2014:4-7, 18-19 ) were certainly from elite burials. On the other hand the example from Badari tomb $3920^{82}$ was from an intact grave of a male whose only other graves goods were two rough bowls of type R3f(6) (Brunton and Caton-Thompson 1928:52).

On the whole the CBPPs meet the expectation for a widespread social distribution since they were found in settlements of all scales, in graves of different status, and in multiple contexts at some settlements. However their concentration in ritual contexts at Hierakonpolis may indicate that their use varied either by location or over time.

\footnotetext{
${ }^{81}$ This includes all the blocks excavated by Anderson, not just blocks 1,3, and 4, because any CBPP would have been tagged separately as a special find (Anderson pers. comm.).

${ }^{82}$ Brunton originally dated the tomb to the Badarian based on the shape of the CBPP, but Brunton himself questioned the date, and Holmes (1989:171) pointed out that all of the rest of the graves in that cemetery were Naqada period. Hendrickx and van den Brink (2002:355) dated the cemetery to NIICNIID2.
} 


\section{Bifacial sickles}

Bifacial sickles came from both ritual and non-ritual contexts. Burials were the only ritual contexts with bifacial sickles. Examples come from Naqada Tomb 1906 (Holmes 1989:278), Armant Tomb 1413 (Mond and Meyers 1937:26, Pl8), Adaïma Tomb 326 (Midant-Reynes and Prost 2002:354, 379, Pl 16), Hemmamiya cemetery 1700 (Brunton and Caton-Thompson 1928:Pl 57), and Hierakonpolis HK 6 (Tomb 72, Droux 2014:4-7, 18-19).

In settlements bifacial sickles were found in non-ritual contexts. At el-Mahâsna a bifacial sickle was found in Block 5, an outdoor activity and trash disposal area (Anderson 2006:133137). Three bifacial sickles were excavated at Armant 21/83 (Ginter and Kozlowski 1994:152153). Two came from the southern area, which included habitation structures and a concentration of remains from bifacial tool production. The third came from an unspecified feature. Three bifacial sickles were found in a cache in ATP Op 11. At least five bifacial sickles were found in the domestic habitation section of Adaïma, the 1001 Block plus extensions (Midant-Reynes and Prost 2002: 344-354), and more have been found on the surface (ibid.) and in the settlement excavations by H. De Morgan (Needler 1984:83-87). A bifacial sickle was found in test pit 2 at Elkab which was in a domestic area with floor surfaces, hearths, and a nearby workshop using many burins (Claes et al. 2014:85; Kindermann pers. comm.). A bifacial sickle came from Nag el-Qarmila WK 15 Area A, a domestic and/or production area (Table 6.1, Figure 4.49). Two bifacial sickles were found at Hemmamiya near the circular mud structures, one stylistically dates to the Naqada period, and the other came from a Badarian/Amratian transition level but is more Badarian in style (Holmes and Friedman 1994:132).

Bifacial sickles were used in sites of all scales, showing a wide distribution. Highestorder sites with bifacial sickles include the Abydos and Naqada South Town settlement sites 
(Table 6.3), and the Hierakonpolis HK6 and Naqada cemeteries (Table 7.14). The examples from the el-Mahâsna, and Adaïma settlement sites, paralleled by an example in the Adaïma cemetery, constitute evidence for the presence of bifacial sickles in mid-level sites. The Nag el-Qarmila bifacial sickle, two examples from the small site of Abadiya 2 (Vermeersch et al. 2004:236-237, Table 6.1), and the examples from the Armant settlement and cemetery show that they were also used in low-order sites.

At sites where multiple bifacial sickles were found they were not concentrated in a specific house or area. At el-Mahâsna one bifacial sickle was found by Anderson in Block 5, and at least one by Garstang (1903:Pl. 5), so they must have come from different areas. The bifacial sickles from the habitation area in block 1001 at Adaïma came from different areas of the excavation block, not focused on one of the structures. Additionally, more bifacial sickles were collected during earlier excavations, and while their proveniences are unknown, they likely represent an even larger spread of the bifacial sickles. At Armant bifacial sickles were found at both locality MA21/83 (above) and at MA21a/83 Trench 3, a refuse zone associated with habitation structures in MA21a/83 Trench 2 (Ginter and Kozlowski 1994:160-163). The presence of bifacial sickles in multiple contexts at these sites shows they were not restricted to one group but were used by many. This is not surprising since the presence of sickle gloss indicates that bifacial sickles were used for harvesting grain, which was one of the staple foods for Naqada period society, and was carried out by many people.

Despite this seemingly humble use for bifacial sickles, two were nonetheless included in Tomb 72 in the elite Hierakonpolis HK6 cemetery (Droux and Friedman 2014:4-7, 18-19). Bifacial sickles have also been found in other tombs which do not stand out as particularly elite, such as Tomb 1906 from Naqada main cemetery (Holmes 1989:278), Armant tomb 1413 (Mond 
and Meyers 1937), and Adaïma Tomb 326 (Midant-Reynes and Prost 2002: 354, 379, Pl 16). The inclusion of bifacial sickles in a variety of tombs shows that they had a widespread social distribution.

The bifacial sickles meet the context expectations for the ritual production model for the most part. However they were not as strongly associated with ritual activities as some of the other specialists produced tools, since they were found as burial goods but not in other overtly ritual contexts.

\section{Axes}

Axes have been found in ritual contexts, and more often in habitation contexts. Three axes were found in the Block 3 ritual activity area at el-Mahâsna (Table 6.19). Axe preparation flakes were found at HK29B, and some bifacial tools were also found there, unfortunately their types were not given (Hikade et al. 2008). Additionally axes were included as grave goods in burials (Table 6.9, 7.16). At the Naqada main cemetery tombs 178 and 350 contained axes, another axe was found in a pot in an unspecified burial, and one was found on the surface of Cemetery B (Baumgartel 1970; Holmes 1989:274; Spurrel 1896:56). Mond and Meyers (1937:48) reported axes from cemeteries 700 and 1300 at Armant. Axes were also part of grave assemblages outside the Naqada region. At Hierakonpolis, in the same cemetery as the Painted tomb 100, Quibell and Green found an axe inside a jar in a burial (Quibell and Green 1902:48, Pl60, 17). An axe comes from a grave in Badari cemetery 25/5500, ${ }^{83}$ but the dating is somewhat dubious.

\footnotetext{
${ }^{83}$ Provenience given in the Petrie museum online catalog, UC.9839.
} 
Axes were found commonly in household contexts, production areas, and middens. Examples include those from Naqada KH3B a domestic area with evidence for cooking storage, and flint working (Holmes 1989:192-193), and KH3X/XI, another domestic area interpreted as a household unit including an occupation area with hearths and grinding stones, and an animal pen area (ibid.:194). Other examples of axes from habitation contexts include ones from Armant (Ginter and Kozlowski 1994, see below), Adaïma (Midant-Reynes and Prost 2002:38, 130, 369), and midden deposits at Abadiya (Vermeersch et al. 2004:218, 236, 272).

The finds of axes in settlements of all scales show that the distribution of axes was widespread, not restricted to certain groups (Tables 6.1-6.4, 7.16). Highest-order settlement sites with axes include Naqada South Town and Hierakonpolis, and their corresponding cemeteries. El-Mahâsna, Adaïma and Badari 3000/6 are examples of mid-level sites with axes. Lower-order sites with axes include the Naqada Khattara sites, Armant, and Abadiya.

There was ample evidence showing that axes were a part of multiple contexts within settlements, not concentrated in specific areas. Axes were found in all of the excavated areas at Armant MA21/83 and MA 21a/83, and these contexts included habitation areas, subsidence and storage areas, and middens (Ginter and Kozlowski 1994:38-45, 152-153, 162-163). Similarly, axes were also found in both of the excavated areas at Abadiya, which were interpreted as habitation middens located over 20m apart (Vermeersch et al. 2004:218). As mentioned above, axes came from at least two different household units at Naqada KH3: area B and area X/XI (Holmes 1989:192-193,201). At el-Mahâsna Anderson’s excavations located axes in the ritual activity area in Block 3, but Garstang (1903) collected quite a few axes from other areas of the settlement. At Adaïma one axe was reported from the excavations of Block 1001, and another was found elsewhere in the settlement, on the surface (Midant-Reynes and Prost 2002:369). 
Finally, it should also be noted that while no axes were reported from Holmes' (1989, 1992, 1996) or Takamiya and Endo’s (2008, 2011) analysis at Hierakonpolis, H. De Morgan collected a number of axes from the main Hierakonpolis settlement area (Needler 1984:114-117), although the contexts of these finds are not known. While axes have been found in cemeteries, there was not sufficient information about the particular burials to determine whether they show a distribution across emerging class boundaries or social groups.

In general axes were found in both ritual and domestic contexts, and were used by a large range of people, being found in settlements of all scales and in multiple domestic contexts within settlements. Thus they meet the expectations for context derived from the ritual production model.

\section{Large-blade knives}

While large-blade knives were found most commonly in burials, which were of course ritual contexts, they were also found in habitation contexts (Table 7.18). Examples of burials include the many from tombs at Naqada, (Holmes 1989:278, Petrie and Quibell 1896: Pl 73), along with examples from el-Mahâsna, Mesaeed, Badari, and Gerzeh. The examples given in Table 7.18 are only a sample of the whole, not an exhaustive list since these tools were relatively common grave goods. Large-blade knives have been found in many other cemetery sites, such as Abydos (Hikade 2000), Hierakonpolis (Quibell and Green 1902:48-49, P161), Armant (Mond and Meyers 1937:Pl 15, 20), Mostagedda (Brunton 1937: Pl 40-41), Abadiya cemetery B and Hiw cemetery U (Petrie 1901: Pl8) and more.

However some large-blade knives also came from settlements contexts. One was found at Hierakonpolis HK11C, squares A6-A7, a beer production area (Takamiya and Endo 2011:739). 
Fragments of large-blade knives were found in the habitation sector of Adaïma (Midant-Reynes and Prost 2002:344-346, 391, 419). A fragment (ATP.1990.36) was found in the Abydos ATP settlement site in Op 8, which had features generally relating to food processing, storage, and habitation. Others were found in settlements but in less clear or unknown contexts, including an example found in a disturbed surface area at el-Mahâsna (MAP\#2176; Anderson, pers. comm.), and numerous examples from Petrie's South Town excavations (Baumgartel 1960:40; Holmes 1989:265; Payne 1993:176-177). At Badari 3000/6 some large-blade knives were found among caches of objects in three pots (3165, 3167, 3284, Brunton and Caton-Thompson 1928: 45-47). The pots contained remarkably similar inventories. Each had large-blade knives, other flakes or blades, ivory tags, a slate pallet, animal bones/horns, shells, pebbles, hair, minerals or natural stones, and in some cases other items such as a copper needle or pieces of wood. It is unknown whether these were ritual offering deposits such as those found in the corners of the HK6 cemetery structures, or whether they were collections of standard household goods.

Besides demonstrating that large-blade knives occur in both overtly ritual contexts and in other contexts, the above examples also show that they were found in settlements and cemeteries of all scales, rather than only in the highest- or lowest-order sites. Their occurrence at many scales indicates that they must have had a widespread social distribution. Large blades knives were found in both the settlements and cemeteries at the highest order sites of Abydos, Naqada South Town, and Hierakonpolis, and at the mid-level settlements and cemeteries of Mahâsna, Adaïma and Badari 3000/6. The examples from the cemetery of Armant, and examples from small cemeteries such as cemetery 3800, constitute lower-order sites.

Furthermore, there was some evidence that within settlements blade knives were found in multiple contexts, supporting a widespread rather than restricted social distribution. A large 
blade knife was found at Hierakonpolis HK11C (above), while other large-blade knives found by H. de Morgan came from the main settlement area close to the cultivation (Needler 1984:115117). Therefore large-blade knives certainly came from multiple contexts in Hierakonpolis. The examples from recent work at Adaïma were found in the habitation area, Block 1001 and extensions (Midant-Reynes and Prost 2002:344-346, 391, 419), and H. de Morgan recovered large-blade knives from other parts of the Adaïma settlement (Needler 1984:84-86, 277).

The blade knives from cemeteries also show a widespread social distribution. Bard demonstrated that four different cemetery clusters at Naqada were differentiated based on types and quantities of grave goods (Bard 1994). These four cemeteries or grave clusters were: T, B, main West, and main East. Bard found that the main-west cluster was richer and more exclusive than the main-east cluster, but that cemetery $\mathrm{T}$ was the richest and most exclusive of all, with $\mathrm{B}$ somewhere in the middle, somewhat similar to main-west. A look at the distribution of largeblade knives across these cemeteries using data from Baumgartel (1970) and Payne (1987), showed that large-blade knives were found in Cemeteries B, main-west and main-east ${ }^{84}$. Although not recorded in the most elite cemetery large blade knife use did cross cut finer social distinctions.

In summary the large-blade knives meet the expectations for context. They occurred in both ritual and habitation contexts, and were used by many people throughout society.

\footnotetext{
${ }^{84}$ Bard gave the grave numbers for the cemeteries as: Western cluster tombs 56-499 \& 1200-1299; Eastern cluster 500-1195 \& 1301-1953.
} 


\section{Microendscrapers}

Microendscrapers have been found in both ritual contexts and in other contexts that did not have an overt ritual character. The ritual contexts included burials and ritual areas in settlements. Numerous microendscrapers were identified in Abydos cemetery U and the Naqada main cemetery (Hikade 1998, 2000; Holmes 1989:278; Table 7.20). As will be discussed below, items identified as small bladelets at the Hierakonpolis, Adaïma, and Maadi cemeteries probably also included microendscrapers. Outside of cemeteries, microendscrapers were also identified in ritual contexts in settlements. Three were found in the ritual activity area in el-Mahâsna Block 3, consisting of $<2 \%$ of the tools. At the Hierakonpolis ceremonial precinct microendscrapers were identified in each of the excavated areas HK29A (4.5\%, Holmes 1992:42, 1996:197), HK29B (2.5\%, Hikade et al. 2008:180), and HK25 (1.77\%, ibid.:182).

However, as is expected for the ritual production model, microendscrapers were also used in other settlement contexts as well. At el-Mahâsna they were present in the habitation contexts of Block 1 ( $n=4, \sim 2 \%$ of tools) and Block 4 ( $n=2, ~ 1 \%$ of tools). Holmes (1996) identified one microendscraper at Hk11C 0N6E, an animal pen area (.76\% of tools), and from the house structure at HK29 square 17L13 she identified 14 microendscrapers, (5.83\% of tools). An example from Hemmamiya was found among general settlement layers in area $\mathrm{H}$ (Brunton and Caton-Thompson 1927:114, pl 80, no 83), and at least one microendscraper was found at Adaïma in the area of the house structures, Block 1001 (Midant-Reynes and Prost 2002:323, 356, 398). Three microendscrapers (1.5\% of tools) were identified in the analysis of materials from Nag el-Qarmila WK15 Area A, which was an area used for food production, lithic reduction, and a child burial. Although a Lower Egyptian site, it should also be mentioned that many microendscrapers came from the Maadi settlement (Rizkana and Seeher 1988). 
Besides occurring in both ritual and non-ritual contexts, the microendscrapers showed a widespread distribution, as was expected for the ritual production model. They were present in settlements of all scales. The Hierakonpolis town site along with the Abydos and Naqada cemeteries represent highest order sites. The above el-Mahâsna and Adaïma examples, along with those from Badari 3000/6 $(n=19,5.8 \%$ Holmes 1989:10) show that microendscrapers were used in mid-level sites. The microendscrapers from Hemmamiya and Nag el-Qarmila represent examples from low-order sites. There is little evidence for microendscrapers in mid and low level cemeteries, but, as is discussed below, this may relate to early collection strategies than actual under representation.

The widespread rather than concentrated social distribution of bladelets was also underlined by their occurrence in multiple domestic contexts, and in cemeteries of different status. The above described examples from el-Mahâsna Blocks 1 and 4, along with the examples form HK11C and Hk29 show that microendscrapers were not restricted to single households within settlements. As discussed below, the finds of (probable) microendscrapers in the HK6 elite cemetery, in tomb 72 which was thought to belong to an early ruler (Droux and Friedman 2014:7), and in HK43 the non-elite cemetery shows that status was not the only guiding factor in the use of microendscrapers. It is also worth noting that the HK6 tomb 72 owner is probably male, while the HK43 tomb 333 owner was female, showing microendscrapers were used by males and females.

In sum, microendscrapers fit the expectations for context quite well since they were found in ritual and non-ritual contexts, and were widely used throughout society by people of different status and gender, and in settlements of all scales. 
The use of microendscrapers in cemeteries deserves more attention, to show that they were probably more common than has so far been noted, and to better understand patterning that might relate to their use and ritual nature. The frequency and importance of the small and lessimposing microendscrapers has been overshadowed by more striking lithic tools like RFKS, rhomboids, fishtails and large-blade knives. However microendscrapers can actually be one the most prevalent kinds of lithic grave goods. Such was the case at Abydos cemetery U where Hikade $(1998,2000)$ found that microendscrapers were the most common tool type, accounting for almost a third of all tools. They were also found in a number of tombs at Naqada (Table 7.20). Additionally, bladelets were found in tombs at Hierakonpolis HK6 (Tomb 72) and HK43 (Tomb 333) (Droux and Friedman 2014:4, 18-19; Friedman 2003:17-19). The rounded retouch on the ends is visible in the photographs showing that they were microendscrapers. At Adaïma a fragment of a lustrous bladelet was found in tomb 116 of the West cemetery (Crubézy et al. 2002:268-273). However it could very well be from a microendscraper since the retouch is limited to small areas on these tools. All of the above examples are from recent excavations, and in some cases relatively intact graves. Therefore it seems likely that these small tools were overlooked in early excavations or thought to be 'waste,' debitage or debris not worth mentioning. Detailed excavation, recording, and publication of all chert materials associated with burials will probably show that microendscrapers were more common than has hitherto been recognized.

Identifying these materials among grave goods is important because they may reveal some aspects of funerary practice and provisioning. Multiple examples of these items were often found together in tombs along with unretouched bladelets, and a number of them either refit or were made of identical raw materials, showing they were struck from the same core. Examples 
include those from Abydos cemetery U (Figure 7.10; Hikade 1996), Naqada tombs 144, 471, 1786, and 1233, HK6 tomb 72, and probably HK43 tomb 333. The close association of items from the same core indicates that there was little time between production and deposition, and that they were made specifically for the burial. Were some of them used before the burial, creating the small fine "retouch"? Only finds of additional examples can help clarify the funerary practices surrounding these microendscrapers and bladelets.

Furthermore, these microendscrapers and bladelets were associated with grinders, pigments, ivory combs, and other items in a number of graves. Tomb 72 at the elite HK6 Hierakonpolis cemetery was partially disturbed in antiquity, with some of the body missing, but most of the grave goods were left in place. Twenty-nine flint bladelets and small blades (including microendscrapers) were found "arranged around the corner of the tomb" and in close association with 3-4 hippopotamus tusks (Droux and Friedman 2014: 4-5). The hollowed out and perforated tusks had traces of yellow ochre inside showing that they were pigment containers. Very nearby were two stone palettes, a number of pebble grinders, malachite, and a number of ivory combs, one with a donkey-shaped top. Many other objects were also found in the tomb, in the center and in the North corner, including an ivory figurine of a bearded man. Friedman et al. (2017) suggested that the placement of the bladelets in the corner of the tomb parallels the placement of eccentrics in the corners of structures and tomb complexes at HK6 which they think was related to rituals of protection.

The inventory of objects found in a basket in Tomb 333 at Hierakonpolis HK49 the nonelite cemetery, was strikingly similar. There a slate palette was found propped against a basket. Inside the basket were three matching bladelets (probably microendscrapers), two pebble grinders, the pigments galena and red ochre, an ivory comb, and a pendant with the face of a 
bearded man (Friedman 2003:17-19), all of which parallel finds from Tomb 72. Other items also found in the basket were stone pendants, animal bone pointed tools, gaming pieces (?), shell, plant remains (dill, tamarix leaflets, tubers of tiger nuts and nut grass, herb fruits, herbs, carbonized wood, juniper, cedar/fir/cyprus, and bread), and a leather bag with clay cones. The excavator suggested that the basket was a magical or medical kit, based on ethnographic parallels of the uses of some of the plant remains for medicinal purposes and incense (Fahmy 2003:20). Additionally the unusual number of children interred around the tomb may have indicated that the occupant was considered a "protective presence" (Friedman 2003:19).

Similar sets of finds in other tombs show that the pattern or 'set' was not confined to Hierakonpolis. Naqada Tomb 144 included microendscrapers bladelets, many of identical raw materials, along with malachite, ochre, galena, a pebble grinder, bone 'pins' (one with a bird head), small natural pebbles, a siltstone pendant, and a few other items (Baumgartel 1970; Payne 1987; Petrie Museum online database). Naqada tomb 1233 had a similar inventory. At Abydos cemetery U, 20 microendscrapers of identical raw material and four bladelets of another raw material (Figure 7.10) were found together on the floor of Tomb 127, in a decayed organic container (Hikade 1996:35, 2003:146). Also found were tokens or gaming pieces, which may be equivalent to the gaming pieces (?) or clay cones found in HK43 tomb 333, a fishtail knife, a ripple-flaked knife, decorated and plain ivory handle fragments (Dreyer 1999), dice sticks (“wurfel-stabe”), and pieces of gold thread (Hikade 2003:145-146). While no pigments, grinders or palettes were reported, the tomb was heavily disturbed and the grave inventory is probably not complete. Considering the vagaries of time, looting, early excavation and recording practices, and the inconspicuous nature of the microendscrapers, the set of items occurring with microendscrapers was probably more prevalent than just these examples. The similar use of these 
tools in at least three cemeteries separated by geography and status indicates that this 'set' was well defined, repeatedly used, and significant for the afterlife.

\section{Context expectation conclusion}

In summary, all of the tool types fit the context expectations, except for RFKs and rhomboid tools, which were only found in ritual contexts, and showed a distribution focusing on tombs of upper status or wealth. The remaining tool types, fishtails, CBPPs, bifacial sickles, axes, large-blade knives and microendscrapers were all found in ritual contexts and in more ordinary contexts. Additionally all were used by a large portion of society since they were found in sites of all scales, multiple habitation contexts within settlements, and in graves of different status or wealth. The only exception was the CBPPs which at Hierakonpolis were concentrated on the ritual structures. All of the expectations for each tool type are summarized in Table 7.22. Chapter 8.1 provides a discussion of expectations for raw material use, production locations, and context in tandem for each tool type relative to the models for the development of specialized production. 


\section{Chapter 8: Conclusion}

\subsection{Summary of Raw Material, Production, and Find Context Patterns by Tool Class}

This study compared archaeological patterns for raw material use, production locations, and find contexts of flaked stone tools made by specialists to the patterns expected for the ritual production model (summarized in Table 7.22). For the raw material expectation, this research showed that for certain tools—axes, large-blade knives, and microendscrapers—a certain raw material type or color was preferentially chosen for these tools. Furthermore, these raw material choices could not be explained solely by functional considerations, or by simple access to local raw material sources. Instead the raw material choices may have been related to the symbolic significance of the colors. The cosmological meanings of certain colors are well known for the Pharaonic period-- red (desert, chaos), and black (fertile Nile Valley/order)-- and finds of unusual, paired red and black ceramics in Predynastic ritual contexts indicate that these colors were also symbolically meaningful during that period (Ch. 7.1).

The analysis of stone tool production contexts showed that for each tool class where data were available, specialized production occurred in conjunction with ritual activity areas some or all of the time. Furthermore, the analysis of stone-tool find contexts showed that most of the tools—fishtail knives, concave-base projectile points, axes, large-blade knives, and microendscrapers - were not restricted to a subset of the population. Rather, they were found in multiple habitation areas within settlements, at settlements of multiple scales, and in cemeteries dedicated to people of different status. Even the figural eccentrics (Ch. 6) were found in both elite and non-elite contexts. Therefore these tools were not restricted to use by an elite subset of 
the population, and do not fit with the prestige-goods model for the development of specialized production. Furthermore, the find contexts challenge interpretations of the stone tools as either purely utilitarian or symbolic items, since many classes of tools were found in ritually significant contexts such as early 'temples', offering deposits, and tombs, as well as in more traditionally ordinary contexts such as habitation, storage, and trash midden areas.

All together the data for certain tool classes—early fishtails, axes, large-blade knives, and microendscrapers—fit the ritual production model for the development of specialization. Interpretations of each tool class are discussed below based on their patterns of raw material use, production contexts, find contexts, and information on possible ritual uses.

\section{Ripple-flaked knives}

Ripple-flaked knives fit best with a prestige-goods model of production where elites sponsored the production of goods that were symbols of status and power, to create and maintain status differences and build alliances. It was not possible to conclude that symbolic considerations were a factor in raw material choice. Instead raw material choice may have been related to the materials available near where the tools were produced, as was indicated by clustering of RFKs of certain raw material type near a known source of that material. The production of RFKs likely took place in at least one ritual structure (HK29A). The production levels evident at that structure, combined with the time estimates and degree of technical skill involved in their manufacture, make it probable that the producers were involved in stone tool 
production full-time. ${ }^{85}$ Motifs on the RFK handles referred to warfare, hunting, domination, and royalty, showing that the use of these tools was related to themes of power, supporting the idea that RFK production was sponsored by elites.

The distribution of RFKs was less restricted than expected given their high degree of workmanship and high-brow motifs. RFKs were certainly found in wealthy and even royal tombs, but also in more modest graves. They were found in cemeteries of all scales, including low-order sites. However they were more common in the high and mid-level sites, and wealthier cemeteries.

Their presence in cemeteries of all scales, and in tombs with more modest amounts of goods, can be reconciled with the evidence for extreme technical skill and ideological themes of royalty and power, by taking into consideration the alliance-building aspects of a prestige goods system. RFKs could have been elements of the prestige goods economy as alliance-building gifts from higher-ups to socio-politically aspiring followers. An analogy can be made to the Pharaonic period where the king would donate items made in the capital with high degrees of workmanship, such as tomb stelae or statues, to prominent officials. Such goods often listed or emphasized the person's interaction with royalty. The royal symbolism found on the RFK knife handles could be seen as a parallel practice. This interpretation of RFKs as alliance-building elements of the prestige goods economy resolves the conundrum of an items with royal symbolism and high skill ending up in wealthy and not-so-wealthy graves.

\footnotetext{
${ }^{85}$ Although the overall low number of RFKs indicate that they would have worked on other tools in addition to RFKs.
} 


\section{Rhomboids}

Rhomboids were somewhat similar to RFKs in their contexts of production and use, and are another class of tools that largely fits with a prestige-goods model of production. No symbolic associations based on color could be discerned for the raw materials of rhomboids, which were made in almost equal numbers from light and dark raw materials. Only a limited number of chert varieties were used for rhomboids, and this probably related to practical necessities related to the size of nodules needed to make these large tools, rather than preferences due to symbolic considerations. Rhomboids were very likely produced at the HK29A ritual enclosure in the early phase of its use. While there was certainly a concentration of bifacial tool production there in the early phase, the artifact densities were not as high as in the later phase and did not necessarily entail sponsored full-time production, but could have occurred seasonally in conjunction with ritual events. This aspect of their production does not align perfectly with the prestige-goods model, but other factors help indicate their importance as status items. The most striking aspects of rhomboids are their large size and shape. The rhomboid shape has been symbolically connected to male power through early depictions of men with pointed beards and conical headgear that formed a pronounced rhomboid shape (Figure 8.1). Hendrickx et al. (2015) interpret such headgear as an early version of the white crown of Pharaonic Egypt. This association, along with their distribution and use in exclusive contexts, supports the interpretation of rhomboid tools as prestige items. Like RFKs, rhomboid tools were quite notably only found in ritual contexts, primarily burials, but also in at least one ritual structure, and never in mundane contexts. The distribution of rhomboid tools was also similar to RFKs in that they were found in cemeteries of all scales, but more frequently in the highest-order sites and wealthy graves. 


\section{Fishtails}

Fishtail knives were ritually significant items that largely met the expectations for the ritual production model, but also showed change over time. Fishtail knives were found in ritual contexts including tombs, offering caches, and temples, and in non-ritual contexts among general settlement remains. Their widespread distribution in cemeteries and settlements of all scales, in multiple habitation contexts within settlements, and in cemeteries of different status showed that they were used by many members of society, and were not restricted to the upper class. Fishtails were also more numerous than RFKs or rhomboids. Their broad social distribution and higher frequency argue against the idea that they were primarily prestige goods.

There is no doubt that these items were symbolically meaningful. However there is some question as to what that symbolic meaning was. Hikade (2004) argued that they were status items based mainly on a small sample from one cemetery (Abydos), but the widespread distribution of the tools shown here indicates that they do not fit well with that interpretation. Roth (1992) argued that fishtail knives were part of rituals of birth and re-birth, potentially used to cut the umbilical cord at birth, thus ‘opening the mouth' of the baby so it could take in its own food. In the Old Kingdom period ‘opening of the mouth’ rituals evidenced by text and psš-kf artifacts were used to transition the mummy/spirit to the afterlife. Psš- $k f$ means 'chert divider' or 'divided chert' (ibid.:116), and these instruments had a shape very similar to fishtail knives. Furthermore the presence of many model fishtails during the Predynastic period along with later fishtail/psš-kf amulets underlines the importance of their use as ritual items.

The archaeological patterns of Predynastic fishtails changed over time. The raw material study indicated that the variability in raw material choice decreased over time, which may be 
related to a decrease in the number of production areas. Fishtail sizes also became less variable over time, which also points to a decrease in the number of production areas. Additionally, fishtails were very probably produced at the HK29A ritual enclosure, and the intensity of bifacial tool manufacture there increased over time. This higher intensity of work has been interpreted as sponsored production, an aspect that matches well with a prestige goods system. Additionally a number of later-style fishtails were made from obsidian, which is a long-distance trade material that was difficult to obtain and very rare in Predynastic Egypt. Use of obsidian accords well with the idea of a prestige goods system where people would have used materials that were not available to much of the population.

Thus this ritually important item also became a status item during the NIID/early NIII with concomitant changes in the organization of its production and raw material selection. The overall quantity of fishtails made in the later style also decreased somewhat compared to the earlier style. Out of 110 fishtails with an identifiable shape, $61 \%$ were the earlier style shapes (1 and 1/a), and 39\% were the later style shapes (2 and 3). However the continued use of fishtails in the form of $p s \check{s}-k f$ instruments and psš-kf amulets into the Old Kingdom and later periods indicates that the ritually important aspect of the tools proved more durable than their meaning as a status item.

\section{Concave-base projectile points}

Concave-base projectile points (CBPPs) met most of the expectations for the ritual production model, but like fishtails, they showed some variability in their patterning that may indicate change over time or regional differences. There was no indication of symbolic considerations in the raw material choice for CBPPs, because many different varieties and colors 
of chert were used. This variety points to multiple production locations. The existence of multiple production locations for CBPPs was definitively indicated by finds of unfinished examples at a number of sites. Some of these production contexts were ritual activity areas, and the intensity of production was likely part-time. Finished CBPPs were found in both ritual and non- ritual contexts indicating that they were used or at least could be deposited in both settings. The distribution of CBPPs showed that they were used by people in settlements of all scales. CBPPs were not found very commonly in graves, but at a regional level they were included in burials with different degrees of wealth. At el-Mahâsna the CBPPs were not concentrated only in the ritual activity area, but were found in other parts of the site as well. These features agree with the ritual production model: specialist produced goods were used by a cross-section of the population, not a restricted subset, and were produced for use in ritual activities, but not restricted to them. Additionally the production was not necessarily sponsored by elites because it was part-time or periodic, but production may have been facilitated by ritual aggregation, since production remains were sometimes found in ritual contexts.

However the data from Hierakonpolis were somewhat different. There CBPPs were only found in the elite cemetery and in ritual contexts in the settlement. An understanding of the use of CBPPs helps clarify why they have different archaeological patterns at Hierakonpolis. CBPPs were almost certainly involved in activities or rituals related to hunting. Hunting was one of the main iconographic themes during the Predynastic period (Hendrickx 2013), but the faunal evidence indicates that hunted animals were not a major factor in subsistence (Linseele et al. 2009). Rather, hunted animals were important for their symbolic associations, including control over chaos, adoption of powerful forces, and relationships to divinities. Some of these symbolic associations carried over into the dynastic era. 
Hendrickx (2006a, 2012) and others (Hendrickx and Eykerman 2010; Hendrickx et al. 2009) discussed the connection between elites and hunting, arguing that (group) hunting gave elites access to more varied food, weapons, social networking, and opportunities for display and spectacle when they returned from the hunt with dead or live animals. This connection to hunting as an elite activity associated with display would seem to support the prestige-goods model for CBPP production. However overall the CBPPs showed a more socially widespread distribution than an activity restricted to elites would imply, so they do not fit perfectly with the prestigegoods model. At Hierakonpolis where there was extensive faunal evidence for hunting wild animals found in ritual and elite contexts, the CBPPs were more restricted in distribution. So the best explanation is that CBPP production was motivated by their use in ritual hunting activities, and that over time or in some areas these activities became associated with or even restricted to high-status individuals. A detailed look at whether the different styles of CBPPs relate to changes over time, regional differences, or to re-shaping of the artifacts could help clarify how they were used and how that use changed.

\section{Figural eccentrics}

The archaeological patterns of figural eccentrics (Ch. 6) did not correspond to all of the expectations for the ritual production model, nonetheless they sit very well with the idea of production for ritual purposes. The context of production for figural eccentrics is unknown, but possibly includes the HK29A ritual area, which would accord well with the model. The overall quantity of eccentrics indicates that they themselves could not have been made at full-time production levels, which is also expected in the ritual production model. However, since some of the eccentrics bear similarities to other bifacial tools, they were probably produced in 
conjunction with other items. Their find contexts indicate that they were used by people of different classes since they were found in both elite (HK6) and non-elite contexts such as the HK11 settlement, and domestic areas of Abydos Kom es-Sultan. The differences in quality of production have also been cited as a possible indication of use by different classes (Hendrickx et al. 2003:13). However the localized nature of the eccentrics and their overall low quantity do not match the expectation of widespread use in the ritual production model. Nonetheless the production and use of these items was very clearly motivated by their use for purposes due to their symbolic forms, and the very good evidence for their use as offerings and/or boundary markers in the elite Hierakonpolis cemetery HK6. Additionally at Abydos' Kom es-Sultan one eccentric was found in a temple or ritually significant area. Another proposed use of these eccentrics was for apotropaic purposes.

Overall the eccentrics constitute a clear case of production for ritual purposes, which in some cases did not necessarily exclude connotations of power and prestige. The important point about these items is that their ritual/ religious use seems to be more integral than their use for purposes of prestige and power, since the later contexts do not have as clear ties to notions of prestige and power. In other words, their use as religious or apotropaic items lasted longer.

\section{Bifacial sickles}

The evidence for the specialized production of bifacial sickles does not sit well with either the ritual production model or the prestige-goods model. The data indicating that bifacial sickles were made by specialists showed that at some sites with bifacial sickles, there were few to no thinning flakes of the same raw materials found at the sites, so the tools were likely produced at other sites by people other than those who used them. Additionally, there were 
stylistic and technological differences between the earlier Badarian bifacial sickles and the Predynastic ones, which support the idea of a change in the organization of production.

The manufacture of bifacial sickles does not fit with the ritual production model for a few reasons. First, no symbolic considerations in raw material choice could be discerned: there was no association between this tool type and any raw material type or color. Additionally the only ritual contexts they were found in were burials, and those did not occur frequently (but neither were they extremely rare). Bifacial sickles were certainly used by a broad cross-section of the population, since they were found in settlements and cemeteries of all scales, multiple domestic contexts within settlements, and in graves with different degrees of wealth, which rules out the possibility that they were produced as prestige items. No information about their precise production locations could be identified, so this parameter cannot help clarify the understanding of bifacial sickle production.

Since the production of these items does not fit with either of the models focused on here, there must have been other factors besides prestige display or use in ritual activities that contributed to the increasing specialization of bifacial sickles. The specialized production of bifacial sickles in the Predynastic period can be better understood when contextualized in a longer-term picture of sickle production.

Badarian bifacial sickles were smaller, more numerous and their denticulations were not as fine. They were also possibly more variable than the later Predynastic versions, which could indicate that their production was more diffuse. Predynastic sickles had finer denticulations than the Badarian versions, and their production was likely more concentrated. This technological change in the denticulations and the increasing concentration of production indicate increasing specialization in production. 
The bifacial sickles of the Badarian and Predynastic periods are thick sturdy tools which can be re-sharpened and re-used over time. A substantial amount of work went into preparing each tool before use, including procuring the appropriate sized raw material, bifacial shaping of a preform, and final finishing (possibly with pressure retouch) to form the denticulations. Once made, these bifacial tools could be used for a long time, and re-sharpened as needed (e.g. Kelly 1988:718, 720). Indeed evidence of resharpening after sickle gloss had developed was observed on bifacial sickles in this study, such is in Figure 4.49 (AKAP.1552.1, right image) where the resharpening flaking cuts through the sickle gloss. The Predynastic bifacial sickles are often larger than the Badarian versions, showing increasing investment in this system of substantial up-front input into a tool that could be used for a long time.

However at the same time, in the Naqada period production of sickle blades for harvesting grain also began. ${ }^{86}$ They are relatively rare in the early Naqada period and become more common in the later Naqada period (see Table $4.25,<1 \%$ of tools in early Naqada sites are sickle blades; at later Naqada sites sickle blades tend to account for 2-3\% of the tools). These sickle blades were made on medium blade blanks (Table 5.18), and it is quite possible that the blanks were initially used without denticulation, and that denticulation resulted from later resharpening as was the case in the Levant (Rosen et al. 2015; Vardi et al 2010) (although this suggestion should be verified in a future study). Whether or not the blade blanks were predenticulated or denticulated during resharpening, production of medium blade blanks for sickle segments does not require as much work or investment as production of bifacial sickles. Sickles

\footnotetext{
${ }^{86}$ Sickle blades appeared slightly earlier in the Delta (Debono and Mortensen 1990) but in the Nile valley they do not appear until the Naqada period. Holmes (1989:179-180) does not consider sickle blades a part of the Badarian tool industry. The ones mentioned by Brunton from Mostagedda sites could be from later contexts (ibid).
} 
on blades were certainly the more efficient tool to produce, requiring much less flaking time per sickle segment and possibly more cutting edge per weight of material. However, these sickle blade segments were small and thin, so their potential for resharpening was probably more limited than for bifacial sickles, so they would not last as long and would have been replaced more often.

As discussed in Chapter 5, production of medium blade blanks was not specialized, but rather was quite diffuse, and took place in multiple areas of many sites, so any household could have produced blade blanks as needed. However by the Early Dynastic period, production of sickle blade segments was highly specialized as is evidenced by the large-scale production of sickle blades at quarry sites like Wadi el-Sheikh (Köhler et al 2017; Briois and Midant-Reynes 2014, 2015), and the attributes and dimensions of the blades themselves were different than the earlier blades, and more standardized (Kindermann 2008; Kobusiewicz 2015:16-17, Fig.1-2).

Therefore three stages in the production of agricultural tools can be discerned from the Badarian to the Early Dynastic period: 1) diffuse production of a high-investment long-lasting bifacial sickles in the Badarian; 2) increased investment in this system of long-lasting highinvestment tools in the (early) Predynastic, with more concentrated production and larger bifacial sickles that had finer denticulations, and at the same time diffuse production of low-investment short-term sickle blades began; 3) finally, production of the low-investment short-term sickle blades became highly concentrated and specialized in the Early Dynastic period, and totally replaced the production of the high-investment long-lasting bifacial sickles.

The suggested scenario of the long term phases of development for sickles is meant only as a preliminary overview and should be explored though more study of Badarian-Old Kingdom sickles, paying particular attention to changes in technology, the stage at which denticulation was 
added to sickle blades, and the metric measurements. The important point for this study is that increasing specialization was influenced not only by motivations associated with ritual uses and prestige, but at the same time there was a third influence related to increasing needs for agricultural tools for a large cross-section of the population, discussed further in section 8.2.

\section{Axes}

The archaeological patterns of raw material use, production, and find contexts for axes corresponded to those expected for the ritual production model. While many raw material types were used to produce axes, there was an overwhelming preference for light-colored raw materials. This preference was probably rooted in symbolic considerations since issues like local access to materials and functional constraints did not greatly affect the raw material choice. Production remains from axes, and axes themselves, occurred in both ritual contexts and in domestic contexts. However non-ritual contexts were more prevalent for both production remains and finished axes. Axes had an undeniably widespread distribution, found frequently in settlements of all scales and multiple household contexts within settlements. This widespread distribution and the frequency of axes indicate that they do not correlate well with the prestigegoods model of production, so another model for why and how axes became produced by specialists must be sought. Axes were undoubtedly used for utilitarian purposes such as chopping or hoeing as was indicated by macroscopic wear on the tools. However the color preference, and finds of axe-production remains and finished axes in ritual contexts (like el-Mahâsna Block 3 and some burials), indicate that they were not without additional symbolic meanings. Since there were indications of soil polish on some axes, they were probably agricultural tools, and may have been involved in agricultural ceremonies. One such agricultural ceremony was depicted on 
the Scorpion macehead. It is also worth noting that Barkai (2011) has argued that (PPNA) axes in the Levant had a symbolic meaning as cultural markers of a Neolithic way of life.

\section{Large-blade knives}

The archaeological patterns of large-blade knives matched the expected patterns for the ritual production model quite well. Large-blade knives were very likely tools whose production was related to their use in ritual activities as well as in daily life. Light-colored raw materials were chosen for large-blade knives, and this choice cannot be explained by functional considerations nor simple use of local resources, given current data. Instead, the color may have had symbolic meanings that added value to the tool or were important for the meaning of the tool. The production location of the large-blade knives is not known, but their production certainly did not take place in every settlement. Badari 3000/6 was the only identified possible production location. Unfortunately little is known about the contexts there, but ritual activities were hinted at by the existence of artifacts relating to beer production and caches of symbolically meaningful goods including figurines, ivory tags, mace heads and palettes. More importantly, the find contexts of large-blade knives themselves clearly show that they were used for ritual purposes. Large-blade knives were predominantly found in burials, and they were relatively common, known in far greater quantities than items like ripple-flaked knives, so they must have been an important element in funerals and/or preparations for the afterlife. In addition, they were found in cemeteries of all scales and different degrees of wealth or status, so they were used by many members of society. The knives however were not produced only as grave goods. They have also been found in quite a few settlements, sometimes broken, sometimes complete.

All in all, these data correspond to a pattern of specialized production for use in ritual 
activities by many members of society. Large-blade knives could be an integral element in funerary proceedings, as indicated by their prevalence in burials, but they were also used during life possibly even for other ritual events and for more quotidian activities, as their presence in settlements shows.

\section{Microendscrapers}

Microendscrapers met all of the expectations for the ritual production model. They were preferentially made from one main type of raw material, and that choice could not be explained by access to local resources because it was a pattern across many sites, nor by functional considerations since these small items could have been made out any of the many types of fine grained chert varieties available. Instead their raw material choice of pinkish cherts may have been for symbolic associations with the color, and the heat-treatment may have increased the reddish color and glossiness of the tools. The tools were found in settlements of all scales, in multiple household contexts within settlements, and in graves of different status or wealth, all of which show that they were used by a large portion of society (socially widespread) and not restricted to high-status people. Their production in association with ritual contexts and the finds of these items in ritual contexts including burials and ritual structures in settlements, indicates that they must have had symbolic value. Their symbolic value and use is hinted at by finds of sets of artifacts in burials at different sites. Along with microendscrapers these sets included many symbolically significant items like palettes, pigments, figures of bearded men, ivory items with animal shapes on top, tokens or gaming pieces, and in at least one case with good preservation, plant remains. While the pigments, hair combs, pins, and pendants could relate to personal adornment, these items were not found on the body but away from it, sometimes in 
containers. The exact nature of ritual activities suggested by these kits is difficult to determine. The existence of these sets in the grave of a female in a non-elite cemetery and a male in an elite cemetery indicate that they addressed issues pertinent to many people.

Heat-treated bladelet and microendscraper production was more common in Lower Egypt where it was not thought to be specialized (Rizkana and Seeher 1988; Schmidt 1992a). However if production of heat-treated bladelets for microendscrapers was adopted in Upper Egypt, it makes sense that the production might have been organized and implemented in a different way than in lower Egypt, adopted by the emerging specialists to make certain items which could be used in ritual activities.

\subsection{The Organization(s) of Predynastic Stone Tool Production}

What do the observed patterns of raw material use, production contexts and find contexts mean for understanding how specialized production was organized and changed over the Predynastic period? The findings from the preceding chapters indicate two significant points. The first is that the production of stone tools was organized in a variety of ways. The second is that the development of stone tool production cannot be accounted for through any single model. Many factors contributed to the development of specialized production. As discussed at the outset of this dissertation (Ch. 1.2-1.5), models for the development of specialized production in different areas of the world have addressed a number of possible factors contributing to the development of specialized production such as gains from efficiency, elite actions aimed at obtaining prestige goods, and motivations related to socially and ritually significant activities/uses of goods. Below, after a review of the variability in the organization of stone tool production, the specialized production of goods for ritual uses, prestige, and agricultural uses in 
Egypt are discussed. This section closes with the consideration that many factors contributed to the development of specialized production in Egypt.

\section{Variation in stone tool production}

This study reached conclusions about the organization of production for blade and bifacial stone tools, as discussed in Chapters 5 and 6. Medium blades and bifacial tools of unstylized forms were produced in a completely diffuse manner, in all settlements, with little indication of specialization. Most unifacial flake tool classes were also produced in an unspecialized way since they show little uniformity of style, were found commonly in all settlements, and their production remains (cores, and flakes), were distributed across the same areas.

Other tools were made and used in workshops without ever being distributed more widely. These included microdrills on coarse raw materials, used to make beads. While the people who used the drills were specialists, and the beads could be considered specialized, the drills themselves cannot be considered specialized since they were made and used in the same workshops without ever being distributed more widely. Burins are another example of a tool that could be produced and used in a workshop for making something else. At Elkab 70 burin spalls and numerous burins were found in one layer of a test pit, and may indicate that there was a workshop in the area for working wood or other semi-hard items (Claes et al. 2014:85).

The interesting case of the bifacial animal eccentrics was another type of good with a different organization of production, one with a lot of variability. These items were made and used in only some sites, they were probably produced by specialists within some of those sites, but also may have included items made by non-specialists. 
Even within the tool classes which were produced by specialists there was variation in the specifics of some of the parameters of production. Some tools (axes, CBPPs) were made in many communities but were produced by only certain people in those communities, as is indicated by more concentrated distributions of production remains within the settlements. Additionally axes were made in larger quantities at some sites compared to others.

Production of heat-treated microendscrapers also took place in multiple communities, but not in all communities. Additionally there may have been variability in the distribution of different stages of production, with the heat-treatment and core preparation stages of the process probably done by specialists who made prepared 'cores', but the blade removals and the retouching of the tools may have been carried out by other people. Early fishtails and bifacial sickles were probably made in even fewer communities than microendscrapers, but there were still probably multiple production locations, as indicated by the diversity of raw materials

Other tools were regionally specialized, with only one or a very few production locations across Upper Egypt. These include the RFKs, and large-blade knives. There were no definitively identified production areas for these tools, and, high skill levels required for production (especially in the case of RFKs), indicating that they probably had a very restricted or concentrated production. It should also be pointed out that there was a trend for increasingly concentrated production over time, since the later tool types—RFKs, later fishtails, and largeblade knives—-had the most concentrated production.

There was evidence that the development of specialization was not only a matter of increasing skill. The changes in lithic production did include inventing some new techniques that involved a high degree of skill, such as ripple flaking or microserration, but other techniques used by specialists, such as basic bifacial flaking and heat treatment, were practiced rather 
widely. Over time, the number of people who did this production decreased. So the process of increasing specialization was not just one of inventing new skills that could not be done by all, but involved a re-alignment of time and effort, a narrowing of skills. Furthermore, many of the tools made by specialists, even the most complicated ones, involved some techniques that did not require a lot of skill, such as grinding. This underlines the fact that the process of specialization was more about who does what, than who can do what.

Overall, the rather simple method of looking at the distribution of production remains vs. the distribution of tools at inter- and intra-site levels resulted in quite an array of ways that lithic production was organized for the Predynastic period. A simple dichotomy between ad hoc unspecialized tools and highly technical specialized tools does not capture the diversity of production strategies. More importantly, this study shows that although full-time specialized production was fostered by elites in the latter part of the Predynastic period, this process built on existing multivariate and complex systems of production for stone tools.

\section{Specialized production for ritual goods}

The majority of specialist-produced flaked stone tools and their production remains were consistently associated with ritual areas. Accordingly, the tools cannot be regarded as purely utilitarian items. Rather, the tools must have had some sort of symbolic associations.

Previous theories for the development of specialized production in Egypt have posited that there was a prestige goods system, where production of status items was sponsored by elites. Such items are by definition restricted and not widely available; that is where they get their ability to signal status. However, this study has demonstrated that many tools produced by specialists cannot be considered status items. The socially widespread distributions of fishtails, 
CBPPs, axes, large-blade knives, and microendscrapers simply do not fit the definition of prestige goods. Therefore their production cannot be explained with a model that specialized production developed to make status items for elites.

The problem can be resolved by expanding the possible symbolic meanings connected with specialized tools beyond status to also include cosmological meanings—i.e. those relating to worldview or religion. To simply associate symbolic meanings of artifacts only with status, and disregard the possibility for cosmological meanings associated with the shapes, designs, and uses of the tools sells the richness of Predynastic Egyptians' lives quite short. Moreover, it denies that the content of ritual activities motivated by worldview can have any effect on the course of events or how people acted in them. And really, Egypt is nothing if not a story of worldviews materialized in lasting ways.

Symbolic meanings related to status and those related to worldview were not necessarily distinct or easily disentangled in Predynastic Egypt. It is undeniable that ritual and prestige can be intertwined. The point here is not to attempt to parse them out or set up an untenable dichotomy. Rather, the point is to emphasize that status was not the only thing that Predynastic Egyptians signaled in their symbolic world. There was an array of cosmological views, and these were a part of many people's lives, activities, and motivations, not only related to displaying status via materials, techniques, or products not available to all.

Therefore a ritual production model can help explain the specialized production of some stone tools. This model was built on observations that people often intensify production in order to make things with symbolic meanings for important ritual events and exchanges. Grave goods, bride wealth payments, or gifts associated with weddings, are just a few of the most familiar kinds of examples. Furthermore, these items did not necessarily need to be used only for their 
symbolic meanings, excluding practical functions. Rather they can and did often include everyday items which were nonetheless critical to have in certain contexts. Production by specialists can increase the comparability of form (such as shape, raw materials, etc.) which facilitates comparable meaning — we all have the same items, and we are all doing the same things. Reasons why people in Predynastic Egypt opted for increasing materialization of ritual activities are discussed in section 8.3, below.

\section{Specialized production of prestige goods}

With all that said, the observations that some lithic tools did correspond to expectations for the prestige-goods model of production must also be factored in. Some tools—RFKs, rhomboids, later fishtails, and possibly CBPPs—-fit well with the idea that they were produced by specialists as status items since they had more restricted distributions, and in some cases were made in contexts with higher levels of production that probably correspond to full-time sponsored production. Additionally RFKs and rhomboids were found almost entirely in cemeteries, unlike the tools discussed above which were found in a variety of contexts both ritual and domestic, indicating that RFKs and rhomboids had a different overall pattern of use than the other stone tools.

The specialized production of bifacial stone tools as prestige goods increased over time. Rhomboids were an early type of tool that fits this model for the most part, although their production may not have been full-time. CBPPs were another early tool type, and could be considered status items with restricted distribution and use in Hierakonpolis, but not in other areas. RFKs were a later tool type that certainly can be considered a prestige good. Fishtails were the only tool class that spanned the entirety of the Naqada period, and there were differences 
between the early and late types that indicated a shift toward a pattern of production and use matching a prestige goods system. However it is notable that the later sponsored production of fishtails was still associated with ritual activities, so even with change over time and an increasing focus on status, the ritual context of production remained significant.

\section{Specialized production of agricultural implements}

A surprising and important finding from this study was that one tool class, bifacial sickles, did not fit with either a ritual production or a prestige-goods model (Ch. 8.1). There was no indication of symbolic uses for the raw material types, their production locations could not be identified so they could not be definitively associated with ritual production areas, and they were only found in limited ritual contexts. Additionally the tools were certainly not prestige goods because they were found with a socially widespread distribution.

Besides ritual and prestige uses, the other main type of model for the development of specialized production discussed in Chapter 1 related to increases in efficiency, which could occur for a number of reasons such as scheduling conflicts, or differential distribution of resources. However a desire for increased efficiency in tool production does not fully explain the changes in sickle production here. With an increasing amount of people settling in the Nile Valley and focusing on agriculture, there was increased specialization of bifacial sickles from the Badarian to the Predynastic (Ch. 8.1). These tools took a substantial amount of effort to produce, and this increase in specialization is best understood as increased investment in the product to make the durable long-lasting bifacial sickles used by a wide cross-section of the population. In other words, production first became more time-consuming, not less, with larger tools and finer serrations. Later, there was a shift toward more efficient production: concentrated production of 
the easier to produce sickle blades took place at quarry sites in the Early Dynastic and later periods. Presumably economies of scale were in play, so at that point there was increased efficiency in production. However, there was a trade-off, because the sickle blades did not last as long, and had to be replaced more often, so more sickle-blades were also required overall.

Explanations for this shift toward more efficient production in the Early Dynastic and later periods could involve greater demands on time, demands for agricultural taxes, or even state sponsorship of production. Certainly it is possible that the quarrying and production of sickle blades in the Early Dynastic and later were organized by the state, because there are indications of large-scale organization at Wadi el-Sheikh where many such blades were produced (Hart 2017; Kohler et al. 2017). If this is the case, it would rule out a commercial model of production where independent producers responded to local needs. Specialized production in general was well established by the Early Dynastic period, so the switch to mass production of sickle blades rather than bifacial sickles can be seen as a secondary change in specialized production. In all, increasing specialization of sickles related, at minimum, to rising agricultural production and growing needs for agricultural tools.

\section{A model of multiplicity}

The finding that no single model accounted for all specialized production is a significant result. This study began by asking whether a ritual production model as outlined by Spielmann (2002) was applicable to the Predynastic Egyptian data, which served as a heuristic starting point highlighting which features of the archaeological record could prove to be significant. These features (raw material use, production location, and find contexts) showed that in addition to tool types that largely fit with the prestige-goods and ritual production models studied here, sickle 
production responded to different factors related to agricultural production.

Therefore a larger finding of this study was that there were many influences that contributed to the development of specialized production during the Predynastic period in the Egyptian Nile Valley. A unimodal explanation does not account for all of the data. Instead a multi-modal perspective is required, along with recognition that there were numerous simultaneous influences on economic life. Furthermore, it is important to note that these factors included symbolic considerations along with practical ones. Cosmological views, prestige display, and increasing use of agricultural resources all contributed to changes in the organization of stone tool production and the development of specialization.

\subsection{Contextualizing Specialization in Egypt}

To better understand why there were so many influences contributing to specialized production in Predynastic Egypt, and why ritual uses were among them, these developments need to be placed in the overall context of what was happening in Egypt at the time. The development of specialized production in Predynastic Egypt took place at a time when many facets of life were changing for the Predynastic Egyptians, in terms of environment, demographics, and the subsistence economy.

Rising aridity in the deserts from the late 6th millennium BCE onward led more and more people to settle in the Nile Valley (Bubenzer and Riemer2007; Kindermann et al. 2006; Kuper and Kröpelin 2006; Nicoll 2001; Riemer et al. 2013; Wendorf et al. 2001). In the 6th millennium BCE, the number of known habitation sites in Egypt are few, and these were primarily located in the deserts, where the subsistence economy was based on mobility with both foraging and some limited herding of domesticated animals (Linseele et al. 2014; Riemer 2007). Some settlements 
may have existed in the Nile Valley in the area that is now beneath the alluvium, but these have not been identified archaeologically. In the 5th millennium BCE, people increasingly relied on domesticated animals, and a few settlements began to appear using domesticated crops introduced from the Near East, first in the Delta and the Fayoum, then later in the Nile Valley (Linseele et al 2014; Wetterstrom 1993). By the early Naqada period in the 4th millennium BCE, there were many agricultural settlements throughout the Nile Valley and the Delta. These increases in population and the number of settlements probably resulted from a combination of sources, including people moving into the Nile Valley from the deserts, into the Delta from the Near East, and internal demographic growth. It is also important to note that while there were many more people in the Nile Valley during the 4th millennium BCE compared to earlier periods, the total numbers of people were still relatively low, and came nowhere near the subsistence carrying capacity of the land (Butzer 1976:101-103; Hassan 1981b; 1988; 1992b).

At the same time that people were moving into the Nile Valley and adopting domesticates, burial practices were also changing. Cemeteries became larger with increasing amounts of material associated with burials. Cemeteries may have served to tie people to specific pieces of land through a focus on bodies as the mediating symbolic factor in identity and social frameworks (Wengrow 2006:69-71).

Overall Predynastic Egyptians were faced with numerous new scenarios that affected how people interacted: new places, more numerous communities, and being more attached to specific locations. Here I argue that Predynastic Egyptians pursued a diverse array of strategies for social interaction in the face of these new scenarios.

New political formations and increasing inequality are two such approaches to, or results of, these circumstances, and they have already been discussed in the framework of theories of 
state formation and complexity (Ch. 1). Warfare is another form of interaction visible in the Predynastic period. Evidence for warfare is certainly prominent in the iconographic record with smiting scenes such as found on the Narmer palette and in Hierakonpolis tomb 100. The increasing materialization of personal identity (Wengrow 2006) can also be understood as an new approach to social interaction in the changing circumstances of the time.

In addition, new ritual activities, and/or new materialization of ritual activities, would have been another approach to meeting the transformations in the Predynastic period. As was discussed in Chapter 1.6, ritual activities provide a venue for building solidarity, forging identities and divisions, and constructing a base of authority. These likely included communally encompassing rituals such as agricultural ceremonies, and more individualistic rituals such as those associated, for example, with birth, marriage, and of course death. Evidence for ritual activities was mentioned intermittently throughout this study, but evidence for a few specific rituals is described below to illustrate the idea of increasing ritual activity and/or increasing materialization of ritual activities.

Depictions of women in prescribed poses are shown on Decorated Ware ceramics, and these women along with boats, animal skins, and trees are associated with the funerary realm (Graff 2009). The Decorated Ware ceramics themselves were primarily found in funerary contexts, and their imagery gives some hint about the funerary rituals. Hendrickx et al. (2009) have drawn parallels between these images and Predynastic and Pharaonic figurines, which can be linked to textual references about "women of the acacia house” who played music and danced at funerals, and were linked to animal butchery.

Other rituals associated with death are evident from offerings left on the surface or in shallow pits in cemeteries, such as those at HK6 (Friedman 2006, 2010; Friedman et al. 2017). 
Some of the offerings were comprised of flaked-stone eccentrics, model fishtail knifes, projectile points and ceramic vessels. Others included incised ostrich eggshells. These offerings may have been associated with rituals for founding or protecting the tomb complex because they were situated in the corners of the tomb complex (ibid.).

Although less well-known, Predynastic Egyptians also participated in rituals associated with life, not just death. Roth (1992) argued that there were elaborate rituals associated with birth and re-birth based on remains in graves and later texts. The Scorpion Macehead depicts an agricultural ceremony, with the King standing by a canal wielding a hoe, presumably to inaugurate a water channel into the fields (Ciałowicz 1992, 1997; Millet 1990; Quibell 1900). Agricultural ceremonies are also attested through remains in the ritual activities areas of HK29A and el-Mahâsna Block 3, which both have seasonality data indicating that activities may have related to the Nile flood and the agricultural cycle (Anderson 2011:20-21; Linseele et al. 2009:134; see Ch. 3.4-3.5). Presumably an array of ritual activities took place in temples such as these. Temples were depicted iconographically on palettes, tags, seals, and other media (Friedman 1996; Hendrickx 1996b; Millett 1990; Spencer 2010).

The development of specialized production of objects for use in rituals and other activities fits with the idea of increasing ritual activities and/or increasing materialization of ritual activities. Escalating use of materials in ritual activities would have generated demand for the objects necessary to appropriately carryout these activities. This demand could be met by specialists because specialization can increase comparability of form (in terms of shape, style, size or materials) which facilitates comparable meaning, which is the significant factor for symbolic items.

However it is important to recognize that this situation does not exclude avenues for 
variability. The analysis of stone tool production by tool type and the techniques involved indicated that production was organized in a number of ways (Ch. 5 and 6). Different kinds of rituals and activities could have generated diverse requirements which did not all need to be met in the same way.

Moreover, specialized production would have offered advantages to production of other classes of goods in addition to those used in rituals. For instance more concentrated production would have facilitated the ability to control production or distribution of prestige goods. Or, a change in the organization of production toward greater concentration-i.e. fewer producersmight have allowed those producers to develop different techniques, skills, and knowledge not attainable by larger numbers of people in a more diffuse form of production, as may have been the case for changes in bifacial sickle production.

No single one of these benefits of specialization need be primary. Rather, as specialized production was increasingly pursued to make any item, the different possibilities and advantages of specialized production would have become apparent to the Predynastic Egyptians, and could have been applied to a range of items (not just flaked stone tools, but also ground stone, ceramics, and beer). The situations of Predynastic life in the Nile Valley generated many new scenarios and circumstance and Predynastic Egyptians did not respond in only one way, but always pursued diverse strategies.

\subsection{Questions Remaining}

While this study has accounted for more of the data related to specialized production, it has also raised new questions, particularly about who the producers were and the social significance of production activities. 


\section{Who used stone tools?}

Through the analysis of the find contexts of tools, this study addressed who used the tools in terms of class, scale of settlement where they lived, and in some cases, gender. For instance at Hierakonpolis microendscrapers were found in the grave of a young elite male and in the grave of an elderly non-elite female. The concave-base arrowheads were used by hunters from many settlements, and eventually or in some areas used by elites who hunted. The animal eccentrics were probably utilized mainly by elites in Hierakonpolis, but they were also found in a non-elite settlement area, and later at Abydos, where they were also probably used by non-elites (for other tool classes see section 8.1).

\section{Who made stone tools?}

The question of who produced the tools however, deserves more discussion, since it can be particularly difficult to assess who was working in a particular place (Costin 2001:299). An assumption built into the prestige-goods model is that the producers were not elites, but that elites sponsored others to do the production. This notion stems from a latent association between manual labor and lower status. There are nonetheless many historic and archaeological examples where production of craft items was associated with elite producers. For instance, in Maya society, Inomata (2001) argued that elites often produced textiles and scribal art objects like codices and stelae, based on finds of the associated production remains (pigment grinders, shell ink pots, spindle whorls, bone needles, axes, and chisels) in elite residences at the rapidly abandoned site of Aguateca. Another example of Maya elite production is in the manufacture of jade ritual/prestige goods such as elaborately carved ornaments, plaques, and jewelry. The later 
stages of production were carried out by the elites who controlled the ritual knowledge necessary to make the objects potent (e.g. Kovacevich 2006:184-185; ), although others were involved in earlier stages of jade production or in production of less elaborate jade goods (Kovacevich 2006, 2007; Rochette 2009).

It is worth considering whether elites might have been the producers of concave-base projectile points in Egypt. If they were used during desert hunting expeditions, they could have been damaged during the hunting. Hunters would have needed to bring many of these rather large projectile points, been able to re-work them, or had specialist assistants along with them. One advantage of larger bifacial projectile points is that they can be re-worked if needed, changing the morphology (e.g. Frison 1968; Flennikan and Wilke 1989). Indeed CBPPs come in a wide variety of shapes and styles which have largely defied classification according to chronological variation. If they were re-worked during hunting expeditions, the elites doing the hunting may have needed to be able to re-work or even produce these tools.

A second production consideration associated with desert expeditions is raw material acquisition. Venturing into the desert was a risky endeavor and apparently associated with privilege at least some of the time. Yet more than hunting took place in the deserts. Some raw materials must have been collected or quarried from desert sites (e.g. the imported materials found at Nag el-Qarmila with primary cortex: Ch. 4.1), and some tools may have even been prepared or produced near the raw material collection points (e.g. potentially large-blade knives). Might trips to the desert have incorporated a number of tasks, such as hunting, quarrying, and production or preparation of certain tools? Desert raw material collection may have been embedded within hunting trips or vice versa. Either all of these tasks would have been undertaken by elite hunters, or the expeditions might have included non-elite members. 


\section{Many groups of producers}

While these suggestions about the class of producers for certain tools are interesting and merit additional consideration, it is clear that there must have been a number of different groups of people who produced stone tools, and that these groups sometimes overlapped or worked together Figure 8.2.

People in many or all households produced some of the tools they needed such as many unifacial tools (notches, denticulates, endscrapers, retouched pieces), unpatterned bifacial tools, and medium blades. It is not possible at this point to say how this production may have been divided up across members of a household. Did each person produce tools for their own tasks? For example, women preparing fish could have made small scrapers for scaling, or people constructing a house might have made notches for shaving reeds. Alternatively, one person in a household might have been responsible for preparing most of the stone tools necessary for daily life.

In addition there was another group of producers in each of the (or most) communities: a smaller more concentrated subset of people who made axes, because axe production remains have been found concentrated in certain parts of settlements. Similarly, in only some communities there was a subset of people who made heat-treated prepared bladelet cores, and possibly also bifacial sickles, although these later two were not necessarily the same people since they entailed different techniques and skill sets.

Another set of techniques and skills was necessary for the production of the thin finely shaped and often microdenticulated bifacial tools_-rhomboids, fishtails, CBPPs, and animal 
eccentrics — and these were probably made by an even smaller group of producers working at fewer sites.

Many of these bifacial tools were probably made together by the same people, sine similar skills and techniques were involved. Additional evidence that this was the case comes from the eccentrics, since some are basically variations on CBPPs and rhomboids (see Ch. 6.1). However even this idea of overlapping production is slightly more complicated. Finds of unfinished CBPPs and the variability in raw material and metric measurements of fishtails, indicate that they were probably made in more sites than some of the other fine bifacial tools, such as rhomboids and animal eccentrics. Later, RFKs and later fishtails, were probably only made in only one or two sites. These ideas about the distribution and overlap of producers are summarized in Figure 8.2.

\section{Social meaning and implications of stone tool production}

The suggestion that there were many groups of producers with different levels of skill, making tools for daily needs, prestige, ritual uses, and agricultural production, implies that what it meant to be a stone tool producer also varied. The diffuse and regular production of items of daily life may not have set people apart, but production of other items, carried out by fewer people, must have created a distinction for them. Moreover, there were not just two groups of stone tools producers, but many (see above). Furthermore, it is already possible in the Predynastic period to discern three foci of stone tool production: the temple (ritual), the palace (elites), and the fields (agricultural tools), and these arenas remained significant throughout the Pharaonic period. The various groups of producers and multiple kinds of production indicate that there were many roles or opportunities available for Predynastic Egyptians, but these were 
differentially distributed across settlements, and certain communities had more kinds of stone tools producers than others. Therefore stone tool production was already contributing to the growing complexity developing in Egypt over this time.

Some stone-tool production probably granted increased opportunities for participation in ritual and social events, since production often took place in ritual structures. This participation may have facilitated a degree of agency for those people to negotiate what occurred and what was involved in the ritual events. Furthermore it is likely that producers of different skill levels or tasks worked together, at least occasionally. For instance the production of RFKs drew on several skill sets (Kelterborn 1984; Midant-Reynes and Tixier 1981). Another example is bead production at HK29, which consisted of making microdrills, shaping the beads, and grinding the beads. There was also bifacial tool production at HK29A which draws on a very different set of techniques. Similarly, in the ritual activity area in Block 3 at el-Mahâsna there was evidence that bifacial tool production and bladelet production both occurred there. These finds of evidence for multiple skill sets and production types occurring together in ritual contexts, further underscores the idea that the ritual events brought producers together, giving many people a chance to participate in and influence ritual events.

\subsection{Conclusion}

The original question posed here was whether the ritual production model may be applicable to Predynastic Egypt. This study argues that indeed, increased demand for objects which could be used in ritual and other activities likely did affect the organization of stone tool production. This perspective contributes to archaeological research which has already considered numerous other explanations for the development of specialized production (Ch. 1.2). The 
application of the ritual production model to Egypt has invited a reconsideration of assumptions about the source of demand for specialist-produced goods. Ritual uses must be included among other considerations such as status or needs for increased efficiency in production.

The findings that ritual activities affected the organization of production moves ritual activities from a secondary process only significant from an etic perspective for its role in legitimizing leaders, to a primary process that can affect large socio-economic changes. This should not be surprising for Egypt because later, with pyramid construction, Egypt is perhaps the quintessential example of worldviews causing people to move heaven, and especially earth, to materialize those views in lasting ways.

Moreover, by showing that the goods used by specialists were socially widespread, this study also contributes to an understanding of who was involved in large-scale changes. Internal motivations for change were not limited to elites or aggrandizers, but a range of people could contribute to such changes through increasing the demand for ritual goods, and by participating in their production.

This study went beyond the original ritual production model which was the focus of the research and demonstrated that the development of specialized production was likely affected by a number of factors, including ritual, prestige, and changes in subsistence strategy. For Egypt, this research can serve as a basis for looking at how each of these aspects affected specialized production over time, since stone tools continued to be made by specialists for thousands of years after the Predynastic. More broadly, this research encourages the framing of future research questions to include multi-modal explanations for large socio-economic processes, taking into account the complexity of (pre-)history and highlighting the multiplicity of influences on people in the past. 


\section{Appendix: Lithic Coding Guide}

This coding guide was developed based on the following sources: Andrefsky (2005); Debénath and Dibble (1994); Holmes (1989); Inizan et al. (1999); Luedtke (1992); MidantReynes and Prost (2002); Rosen (1997); Tixier (1963); and a lithic technology course taught by Phil Geib at the University of Virginia in 2011. The procedure for analyzing the artifacts is described in Chapter 2.4.

\section{Context Data}

\section{ID number}

The ID number assigned to each artifact comprised the abbreviation for the project name,

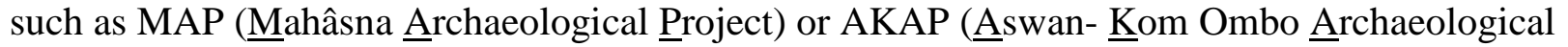
Project), plus the tag number of the artifact bag, plus a subscript which was assigned consecutively to each artifact in a bag starting with one. The combined tag number plus subscript creates a unique identifier such as MAP.3008.1 or AKAP.1552.4. The ID numbers were written on the ventral surface of the artifact below the bulb, or in an inconspicuous area so that the artifacts could be identified in photographs and relocated. The tag number links to data about the context such as Block, Locus, and Habitation Level, (or Area, Square, and Level, depending on the project).

\section{Raw Material Information}

Chert varies in the macroscopic properties of color, luster, texture, translucency, inclusions and cortex type (Luedtke 1992). These attributes were recorded for a sample of $\sim 600$ artifacts, and were used to create raw material categories within the cherts. Structures and cortex type proved to be the most useful and clearest characteristics for raw material grouping, so those two characteristics along with overall raw material type were assessed for all analyzed material.

\section{Color}

Colors were recorded based on comparison to a Munsell soil color chart.

\section{Translucency}

Translucency was assessed by holding each artifact about $5 \mathrm{~cm}$ in front of a desk lamp and rated on a four-step scale of translucency. Artifacts varied in thickness, which affects the translucency, so the options take into account the thickness of the object and the translucency near the edges.

Opaque: The material is opaque even when held to light, regardless of thickness. 
Mostly opaque: The material is opaque to the naked eye, and slightly translucent when held to light, especially at thinner parts.

Semi-translucent: The material is translucent when held to a light.

Translucent: The material is translucent to the naked eye, very translucent when held to the light.

Indeterminate: Transparency cannot be assessed. For example, the piece may be covered by a thick patina.

Other: See comments.

\section{Luster}

Artifacts were held under a desk lamp (to improve comparability of conditions) and rated on a three-step scale for luster.

Glossy: Luster was noticeable even before the artifact was put under a lamp. Obsidian and heat treated materials are good examples of glossy artifacts.

Semi-glossy: The artifact has a slight luster, but it was not very pronounced. It is glossier under direct light from a lamp.

Matte: No luster, even under direct lamp light.

Indeterminate: Luster cannot be assessed. For example, the piece may be covered by a thick patina.

Other: See comments.

\section{Texture}

Texture was assessed by the feel of the flaked surfaces, compared to type examples. The medium to very fine gradients apply to cherts.

Glassy: Very smooth texture, such as with minerals or obsidian.

Very fine: Not as smooth as glass, but still very smooth, such as with the finest cherts. Fine: The texture of typical Egyptian chert.

Medium: A coarser texture for chert. Silicified limestones and sandstones here.

Coarse: The texture is actually bumpy, like a porphyry.

Indeterminate: Texture cannot be assessed, often because it is obscured by patina.

Other: Additional description recorded in the notes.

\section{Structures}

Structures are patterns or materials in the fabric of the chert, such as banding or fossils. They are often occur due to replacement of features in the sediments during formation or from the process of formation itself (Luedtke 1992: 71-72). Structures were assessed by examining the artifact with 0-40x magnification and were categorized based on the most dominant pattern or inclusion. Representative and unusual examples were photographed with a digital microscope at 30x magnification. Representative examples were set aside for reference when assessing other 
materials. The designations are descriptive, awaiting identifications of the structures via thinsections.

Pink bands: Pink bands that range from dark to medium pink, and can vary in thickness and number.

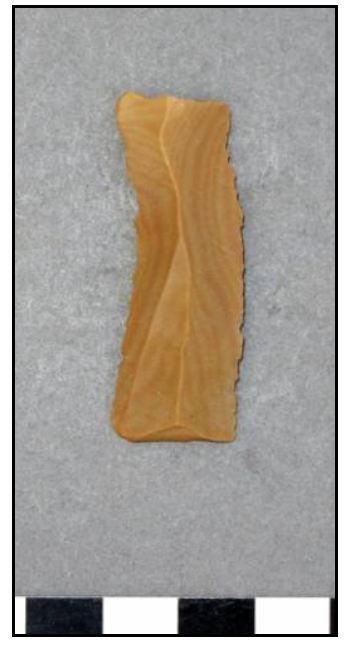

Figure I. Pink bands. MAP.1045.90.

Other bands: Bands of any color other than pink, most often light cream-white.

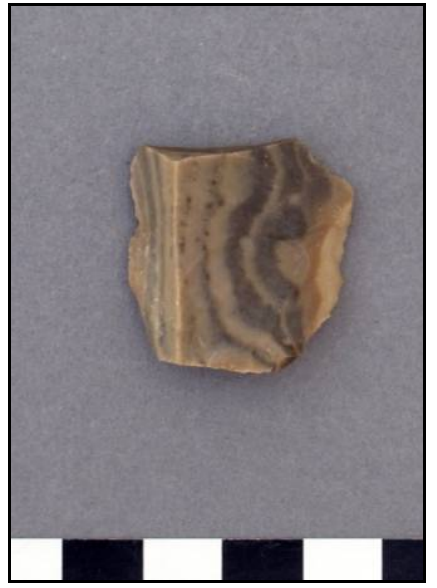

Figure II. Other Bands. MAP.2439 
Red mottles: Tiny red to brown specs, with reasonably well defined outlines. Possibly iron oxide (limonite). The specs are not very frequent or common in terms of the overall composition of the material matrix.

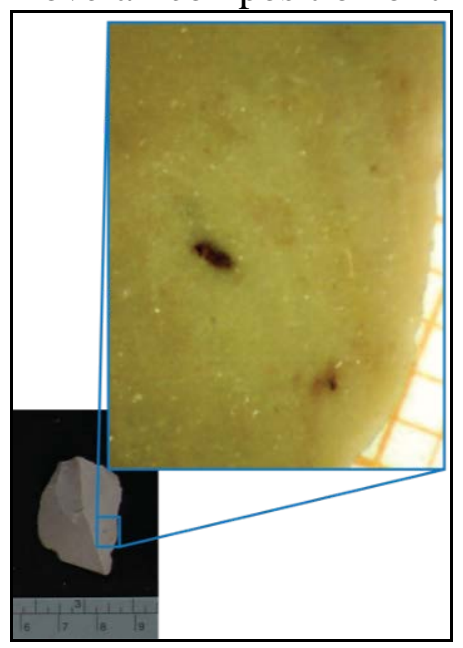

Figure III. Red mottles. MAP.2497.7

Black mottles: Tiny black specs, with reasonably well defined outlines. The specs are not very frequent or common in terms of the overall composition of the material matrix.

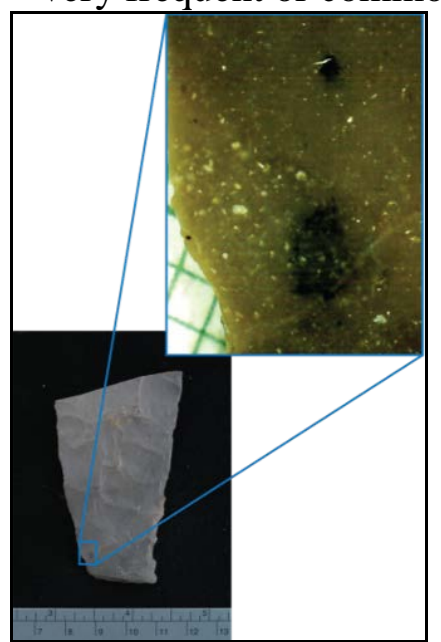

Figure IV. Black mottles. MAP.401. 
White mottles: Tiny white specs which can have well defined or slightly diffuse outlines. They are usually quite numerous when present. Sometimes they are arranged in bands. This was the most common type of structure.

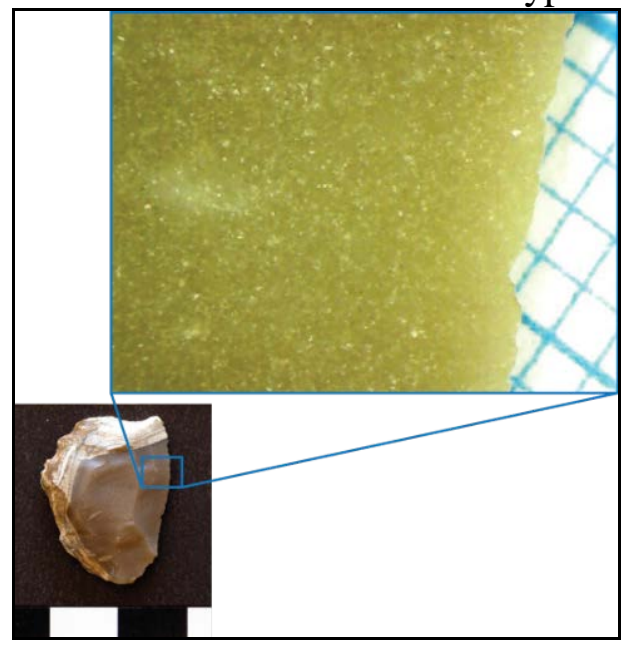

Figure V. Small white mottles. AKAP.2349.5.

Black mossy mottles: Black mottles that have a mossy shape, but are somewhat well defined at the edges. They are usually not very frequent or common in terms of the overall composition of the material matrix.

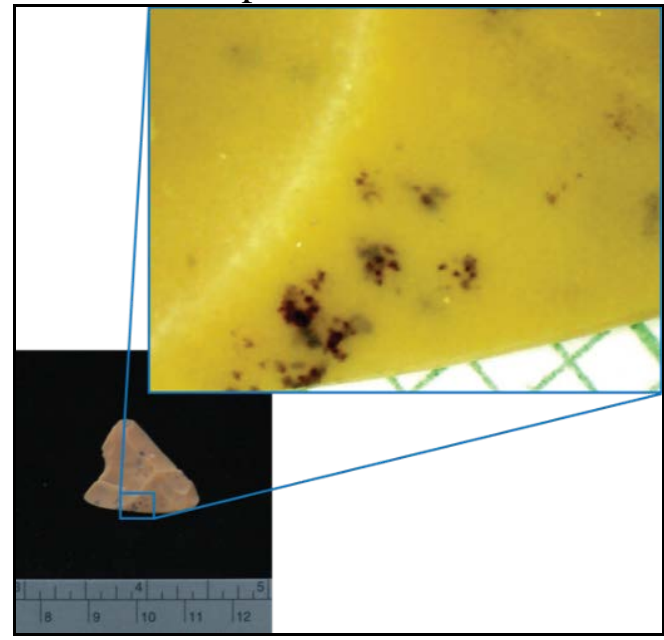

Figure VI. Black mossy mottles. MAP.3278.38. 
White mossy mottles: These structures are white, often with diffuse outlines, and often running into each other giving a mossy appearance. They are usually quite numerous when present. They can be mixed with the more well-defined round mottles or the tiny white specs. Sometimes they are arranged in bands. The structures of this type in artifact \# MAP 3278.39 were tested for carbonates using lemon juice (ph2) under a microscope. A localized reaction occurred, so these structures are likely calcareous.

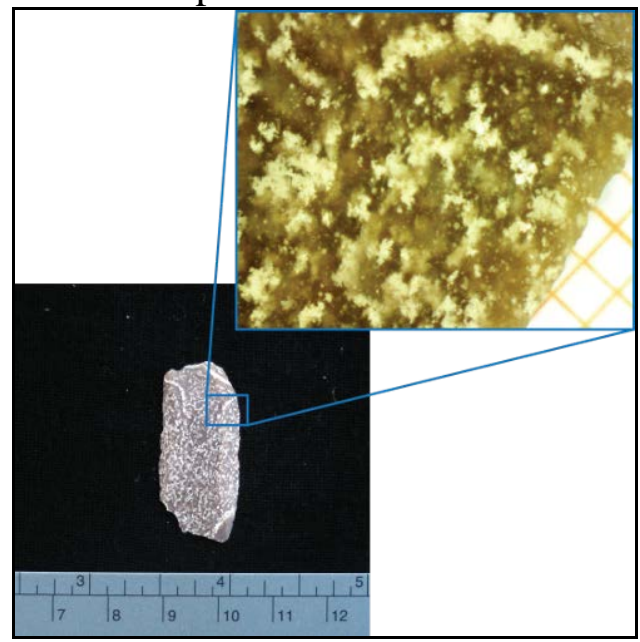

Figure VII. White mossy structures. MAP.2497.36.

Opaque round white structures: These structures are white, round, and have very well defined outlines. They often occur in clusters. They may be oolites, which are "tiny spheres of inorganic origin that form primarily in marine environments where strong bottom currents agitate sediments... Calcium carbonate precipitate[s] in concentric layers around a sand grain or fossil nucleus $1-2 \mathrm{~mm}$...The resulting oolites can become silicified..." (Luedtke 1992:71).

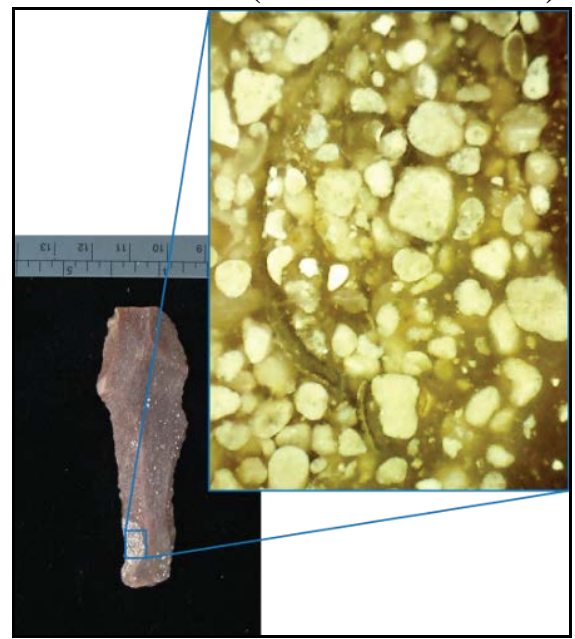

Figure VIII. Opaque round white structures. MAP.304. 
Tan diffuse round structures: These structures are very distinctive consisting of small round circles that are diffuse, opaque, and tan.

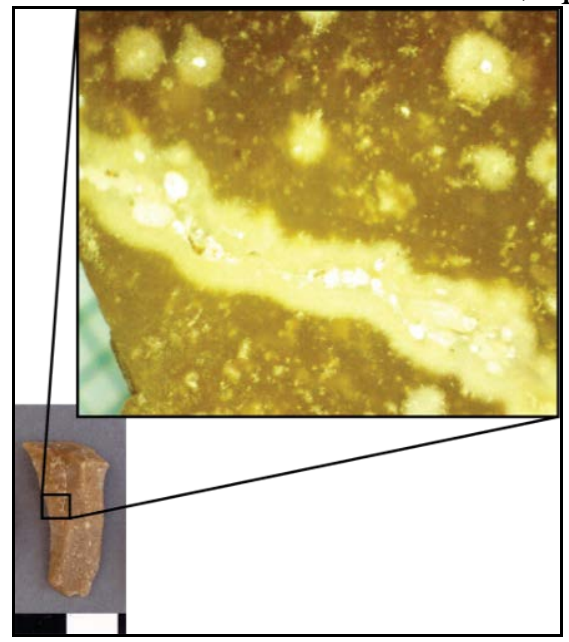

Figure IX. Tan diffuse round structures. ATP.4289.16.

Foraminifera: These are structures can reasonably be identified as foraminifera, singlecelled organisms that form shells, and in cherts can become silicified or exist as casts. Preliminary identifications include miliolids, and nummulitids.

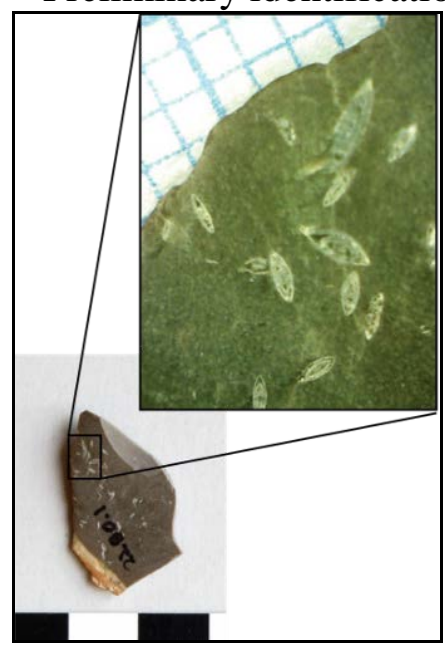

Figure X. Foraminifera structures (probably nummulites). AKAP.2280.1. 
Eroding spheres: Small white spheres that erode out and leave holes.

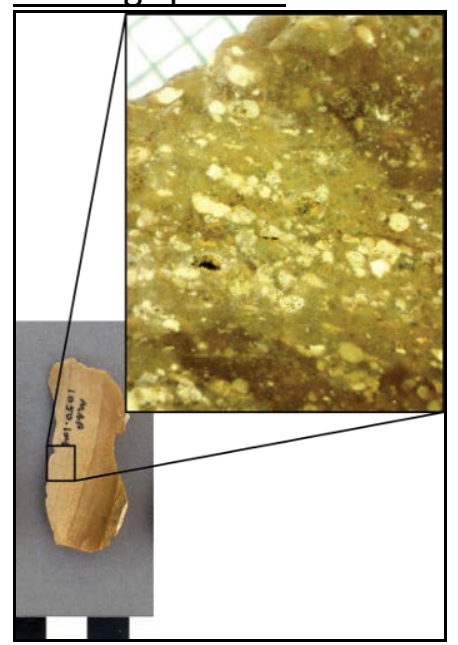

Figure XI. Eroding spherical structures. MAP.1050.104.

Mixed elongated structures: These structures are probably a mixture of fossils, including bivalve shell fragments and other unidentified structures.

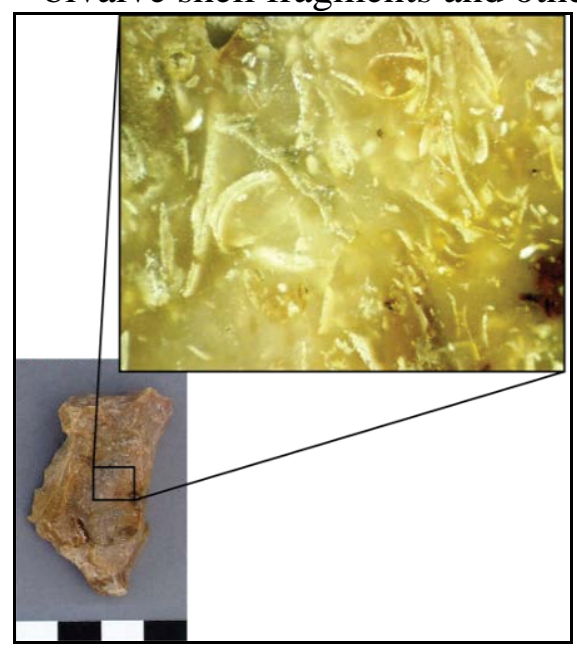

Figure XII. Mixed elongated structures. MAP.1022.132.

Phenocryst: A relatively large and usually conspicuous crystal distinctly larger than the grains of the fabric.

None: no structures are identifiable in the fabric.

Other: Structures do not conform to any of the above categories, see comments.

Indeterminate: Structures cannot be determined, likely because the surface is obscured. 


\section{Cortex Type}

The wear and characteristics of cortex can indicate whether a nodule was obtained from a primary deposit where the chert formed, or in a secondary deposit (Luedtke 1992). Materials with unmodified cortex probably came from a primary deposit. Materials with cortex that has some pitting from erosion and gravity transport and color change of the cortex from chemical weathering and/or build up could have been collected or quarried from a secondary deposit. Such materials do not have evidence of smoothing from alluvial transportation. Alluvial transportation can result in potting and smoothing of the cortical surface. The stone would have been obtained from a secondary deposit. Other stones collected from secondary deposits may have cortex that displays signs of multiple weathering events, such as pitting from erosion and/or alluvial movement, color change from chemical weathering and/or mineral build up, and smoothing from water action. These are often referred to as gravel nodules.

\section{UNMODIFIED}

Chalky: Cortex is white and chalky, not weathered.

Chalky + patina: Cortex is white and chalky without pitting, and with a layer of patina.

The patina may have developed after it was quarried.

Chalky pitted: Light pitting shows that the cortex was subject to some wear, but the cortex is still chalky indicating it was not extensively exposed.

Natural fissure planes: The surface of the chert does not have a cortex per se, but has a different texture that results from fracturing by heat, or salt.

\section{TRANSPORT BY EROSION \& GRAVITY}

Chalky pink-orange: Cortex is chalky but a thin layer of pink to orange material has developed. The color change probably resulted from chemical weathering and/or build up, likely from exposure after eroding out of a primary deposit.

Weathered white to tan heavy patina: Cortex color varies from white to tan, with some pitting and signs of wear, and a heavy patina.

Dark pitted: The cortex color has changed to a dark color (often dark pink) and there is pitting, with no evidence of alluvial smoothing.

\section{ALLUVIAL TRANSPORT}

Completely smoothed: Any pitting has been eroded away, the surface is extremely smooth and shiny.

\section{CHEMICAL WEATHERING}

Patina: A patina has developed on a non-cortical surface. No determination can be made about quarrying activity.

\section{MULTIPLE}

Black or brown pitted smoothed: Cortex has pits and some smoothing, and is black or brown in color.

Dark-pink pitted smoothed: Cortex has pits and some smoothing, and is dark pink in color, which could result from sun exposure or heat-treatment. 
Other pitted smoothed: Cortex has pits and some smoothing, and is any other color besides black or Dark pink, such as light tan.

\title{
OTHER
}

Indeterminate: Cortex is present, but it cannot be categorized, for instance if there is only a very small amount of cortex.

Other: The cortex wear does not fit well in any of these categories, see comments.

N/A: No cortex is present.

\section{Raw Material Type}

Chert was by far the most numerous raw material type, and it was divided into varieties based on macroscopic properties including color, luster, texture, opacity, and with particular reference to structures and cortex, and are described in detail below. Published descriptions of other researcher's chert varieties were consulted, but without a type collection to reference, there is likely to be some variation.

\author{
Agate/chalcedony \\ Chert (a.k.a. flint, see Ch. 4.1) \\ 1. Indistinct Beige \\ 2. Beige- fine \\ 3. Beige- less fine \\ 4. Yellow-Beige with pink bands \\ 5. Medium Brown \\ 6. Fossiliferous Brown \\ 7. Dark Gray and Brown \\ 8. Translucent Brown Family \\ 9. Translucent Brown with Pink Gravel Cortex \\ 10. Pink-gray Translucent \\ 11. Pink-Purple-Red Family \\ 12. Translucent White \\ 13. Caramel \\ 14. Other Chert \\ 15. Indeterminate Chert \\ Limestone \\ Obsidian \\ Quartzite \\ Sandstone \\ Silicified limestone \\ Silicified sandstone \\ Other Stone (not one of the types listed above) \\ Indeterminate (Indeterminate whether the stone is chert or another kind of stone)
}

\section{Indistinct Beige:}

Color: Yellow-brown - Beige. 7.5- 10 YR 5-7/ 4-3 (Munsell color names: Brown, light brown, pale brown, very pale brown, light yellowish brown) 
Texture: Variable

Luster: Variable

Translucency: Opaque (to mostly opaque)

Structures: Variable- banding, mottles, etc

Cortex: Various pitted-smoothed cortexes and lightly weathered cortexes

Geologic origin: Gravel and lag deposits, although some primary deposits are evident

Comparable descriptions: Briois (2002) (Adaïma): “2”- Gray or brown silex, sometimes banded, often grainy, poor quality. Midant-Reynes and Prost (2002) (Adaïma): "Silex

Diverse"- various shades of brown and gray. Takamiya and Endo (2011)

(Hierakonpolis): Standard flint, beige, light brown, dark brown.

Image: Figure XIV

\section{Beige-Fine:}

Color: Beige 7.5-10 YR 5-7 / 3-4 (Munsell color names: Brown, light brown, pale brown, very pale brown, light yellowish brown)

Texture: Fine to very fine

Luster: Semi-glossy

Translucency: Opaque to mostly opaque

Structures: Red mottles, white mottles

Cortex: Chalky and lightly weathered cortexes

Geologic origin: Lag deposits and primary contexts

Comparable descriptions: Kabacinski (2012) (Tel el-Farkha): "Variation I”- smooth cortex, light brown to brown (7.5YR5/3-4, 6/2-4, 7/2), non-transparent, homogeneous, practically free from intrusions, sometimes striped. Nagaya (2011) (Hierakonpolis):

"Group 1"- beige/light brown flint.

Image: Figure XIV

\section{Beige Less-Fine:}

Color: This variety of chert can range from quite light beige to quite dark brown. 10YR 5-8/ 2-3 (Munsell colors: brown, grayish brown, pale brown, very pale brown, light brownish gray, light gray), but variable.

Texture: Fine to medium

Luster: Matte

Translucency: Mostly opaque to semi opaque

Structures: Variable- banding, color variation, etc

Cortex: Various pitted-smoothed cortexes and lightly weathered cortexes

Geologic origin: Mainly gravel and lag deposits

Comparable descriptions: Holmes (1992) (Hierakonpolis), Takamiya and Endo (2011):

“coarse gray flint”. Pawlik (2006) (Kom al-Ahmar, Middle Egypt): “RM 4”- dark Gray, coarse, matte.

Image: Figure XIV

\section{Beige with pink bands:}

Color: 7.5-10 YR 5-7 / 3-4 (Munsell colors: Brown, light brown, pink, pale brown, very pale brown, light yellowish brown)

Texture: Fine to very fine 
Luster: Any

Translucency: Semi-opaque, mostly opaque, opaque

Structures: Pink bands, often concentric

Cortex: Chalky pink-orange, and pitted smoothed were common.

Geologic origin: Lag and primary deposits. Observed in lag deposits around Abydos.

Also identified in a gravel deposits at Elkab.

Possible source: One source of this material is likely located near Abydos. Secondary deposits of the material were found during the desert survey (Ch 4). Additionally certain tools (RFKs, Fishtails) made from this material have been found most frequently around Abydos (Ch7). Holmes (1989:459) also described a similar material available in lag deposits at the entrance to the Valley of the Kings, on the west bank in Luxor.

Comparable descriptions: Briois (2002) (Adaïma):“3”- Light brown silex, beige rosé, often banded, very fine grain. Hikade (2000):18- Light brown, very dense flint with pinkish bands or stripes, known near Abydos, some ripple-flaked knives made of this material. Hikade (2013) (Elephantine): "RM 4"- fine homogenous, light brown to light grayish with pinkish stripes or lenses, available in the desert near Abydos. MidantReynes and Prost (2002) (Adaïma): “No 2”- light brown with pink banding. Takamiya and Endo (2011) (Hierakonpolis): "light brown flint with pinkish stripes".

Image: Figure XIV

\section{Medium Brown Homogeneous:}

Color: 7.5-10 YR 4-6/2-3(4) (Munsell colors: brown, light brown, dark grayish brown, grayish brown, light grayish brown, pale brown, dark yellowish brown, yellowish brown)

Texture: Fine to very fine

Luster: Semi-glossy

Translucency: Mostly opaque

Structures: White mottles, some fine bands, some red or black mottles

Cortex: Chalky or lightly modified.

Geologic origin: Primary and lag deposits

Comparable descriptions: Briois (2002) (Adaïma): “1”- Brown, opaque, fine grain. Midant-Reynes and Prost (2002) (Adaïma): "No1”- homogeneous dark brown fine grained fine beige-cream cortex.

Image: Figure XV

\section{Brown with fossils/foraminifera:}

Color: 7.5-10YR-2.5Y 4-5/1-2 (Munsell colors: Brown, gray, dark gray, grayish brown, dark grayish brown)

Texture: Fine

Luster: Semi-glossy

Translucency: Opaque, mostly opaque, semi opaque

Structures: Macroscopic foraminifera

Cortex: Chalky, some lightly modified

Geologic origin: Mainly primary contexts, some lag deposits 
Comparable descriptions: Midant-Reynes and Prost (2002) (Adaïma): “N $\mathrm{N}^{0} 3$ ”- Dark brown fossiliferous silex, identified in local limestone formations to the South west of Adaïma. Pawlik (2006) (Kom al-Ahmar, Middle Egypt): "RM 11”- greenish, semiopaque, microfossil intrusions.

Notes: Note that the name Brown fossil was chosen because the structures look like small fossils, but the actual mineral content of the structures has not yet been identified. They are probably foraminifera.

Image: Figure XV

\section{Dark gray and brown:}

Color: 7.5-10 YR-2.5Y 4-5/1-2 (Munsell colors: Brown, gray, dark gray, grayish brown, dark grayish brown)

Texture: Fine to very fine

Luster: Semi-glossy

Translucency: Mostly opaque, opaque

Structures: Variable color and small black mottles. Dark gray and medium brown colors occur together with sharp boundaries between them.

Cortex: Chalky, and a very few have lightly modified cortex. Note that many pieces do not retain any cortex (Ch 4).

Geologic origin: Primary contexts

Comparable descriptions: Briois (2002) (Adaïma): “\#1”- Brown, sometimes dark in the middle, light brown or gray, opaque, fine grain. Hikade (2013) (Elephantine): "RM 3"Dark gray-brown to greenish, heterogeneous, probably mined. Kabacinski (2012) (Tel el-Farkha): "Variation II": Dark-gray and Brown (7.5YR 3/2-4, 4/1-4,5/1-3), translucent or semi translucent, diverse, from nearly homogenous to calcareous inclusions, sometimes lightly striped or milky. Midant-Reynes and Prost (2002) (Adaïma): "No 4"- Brown-black, two distinct hues with a sharp boundary, homogenous, fine grained. Nagaya (2011) (Hierakonpolis): “Group 2”- Dark brown/black, often with lighter veins. Pawlik (2006) (Kom al-Ahmar, Middle Egypt): "RM 3"- Middle brown to black, fine grained, semi-lustrous, semi-opaque, homogeneous. Rizkana ad Seeher (1988) (Maadi): "Nodular"- Dark-brown to dark-gray or blackish- gray, mined cortex not much altered, can be larger than $10 \mathrm{~cm}$. Image: Figure XV

\section{Translucent Brown Family:}

Color: Variable, slightly reddish browns. This type can have variable color blending and banding. 5-10 YR (mostly 7.5YR) 4-6/ 1-6 (mostly 3-4) (Munsell colors brown, light brown, reddish brown, light reddish brown, pale brown).

Texture: Fine

Luster: Semi-glossy

Translucency: Semi-translucent

Structures: White mottles, white mossy mottles

Cortex: Pitted-smoothed cortex

Geologic origin: Gravel and lag deposits

Comparable descriptions: Briois (2002) (Adaïma): “\#4”- Translucent flint in shades of beige, blonde, light brown, pink or purple, mottled and fine grained, in small nodules. 
Hikade (2013) (Elephantine): “RM 2”- Nodules from the desert surface with brown, black, or reddish brown weathered cortex.

Image: Figure XVI

\section{Translucent brown with pink gravel cortex:}

Color: Reddish brown- honey colored. 7.5-10 YR 4-6/4-6 (Munsell colors brown, strong brown, light brown, reddish yellow, yellowish brown, light yellowish brown, dark yellowish brown).

Texture: Fine

Luster: Semi-glossy

Translucency: Semi-translucent to mostly opaque

Structures: White mottles, white mossy mottles

Cortex: Pink to brown pitted and smoothed cortex

Geologic origin: Gravel deposits

Comparable descriptions: Briois (2002) (Adaïma): “4”- Translucent flint in shades of beige, blonde, light brown, pink or purple, mottled and fine grained, in small nodules. Rizkana and Seeher (1988) (Maadi): “Gravel Flint”- Alluvial transported pebbles, peck marks, dark reddish brown to med brown, honey colored brown.

Notes: This is a subset of 8 , with a distinctive cortex.

Image: Figure XVI

\section{Pink-gray translucent:}

Color: Pink-gray 2.5-10 YR 5-7/ 2-3 (Munsell colors light gray, pinkish gray, reddish gray, weak red, dark reddish gray, reddish brown, light reddish brown, light brownish gray, pale brown).

Texture: Fine to very fine

Luster: Semi-glossy to glossy

Translucency: Semi-translucent to translucent

Structures: Opaque round white, white mottles, white mossy mottles,

Cortex: Variable. Note that many pieces do not retain any cortex (Ch 4).

Geologic origin: Variable, mainly lag deposits, but some gravel and some primary contexts. Note that many pieces had no cortex.

Comparable descriptions: Briois (2002) (Adaïma): “4”- Translucent flint in shades of beige, blonde, light brown, pink or purple, mottled and fine grained, in small nodules. Ginter et al. (1996) (Armant): Pink transparent flint with opaque light intercalations (structures), non-local to Theban area, might come from the north.

Notes: Similar to 8, but with distinctive pink to light gray color, and sometimes with opaque round white structures that were not usually present in 8.

Image: Figure XVI

\section{White translucent:}

Color: White 7.5 YR 8/1

Texture: Fine to very fine

Luster: Semi-glossy to glossy

Translucency: Translucent to semi-translucent

Structures: Bands 
Cortex: Chalky

Geologic origin: Primary deposit

Comparable descriptions: Holmes (1992) and Takamiya and Endo (2011)

(Hierakonpolis): Notes: Only 2 examples identified in this study.

Image: Figure XV

\section{Pink-purple-red Family:}

Color: 10R-7.5YR 3-6 / 1-3 (Munsell colors weak red, dark reddish brown, reddish gray, dark reddish gray, dark gray, pinkish gray)

Texture: Variable

Luster: Variable, but tends to be glossy

Translucency: Variable

Structures: White mottles, white mossy mottles

Cortex: Various colors of pitted-smoothed cortex, a few more lightly modified pieces Geologic origin: Mainly Gravel deposits

Comparable descriptions: Briois (2002) (Adaïma): “4”- Translucent flint in shades of beige, blonde, light brown, pink or purple, mottled and fine grained, in small nodules. Holmes (1992), and Takamiya and Endo (2011) (Hierakonpolis): pinkish-orangepurplish shades, glossy, with fine speckling.

Notes: Similar to 8, 9, and 10, this group could be strongly heat-treated versions of varieties 8,9 , and/or 10 . However heat treatment was assessed separately from assignment to this category, according to the method described in chapter 4.

Image: Figure XVI

\section{Caramel:}

Color: Bright yellowy-orange-caramel 7.5YR 6-/6 (Munsell color reddish yellow) Texture: Very fine

Luster: Semi-glossy

Translucency: Semi-translucent

Structures: Occasional mossy black mottles

Cortex: lightly modified, very few examples with cortex

Geologic origin: lag deposit (?)

Comparable descriptions: Holmes (1992) (Hierakonpolis): fine grained translucent orange, used for bifacial tools. Kindermann (2010) (Djara): “127”-light brown to yellow (Caramel).

Image:

14. Other Chert: Any chert which does not fit into any of the above varieties, and is very distinctive.

15. Indeterminate Chert: A chert that does not clearly fit into one of the above varieties, but is between a number of them. 


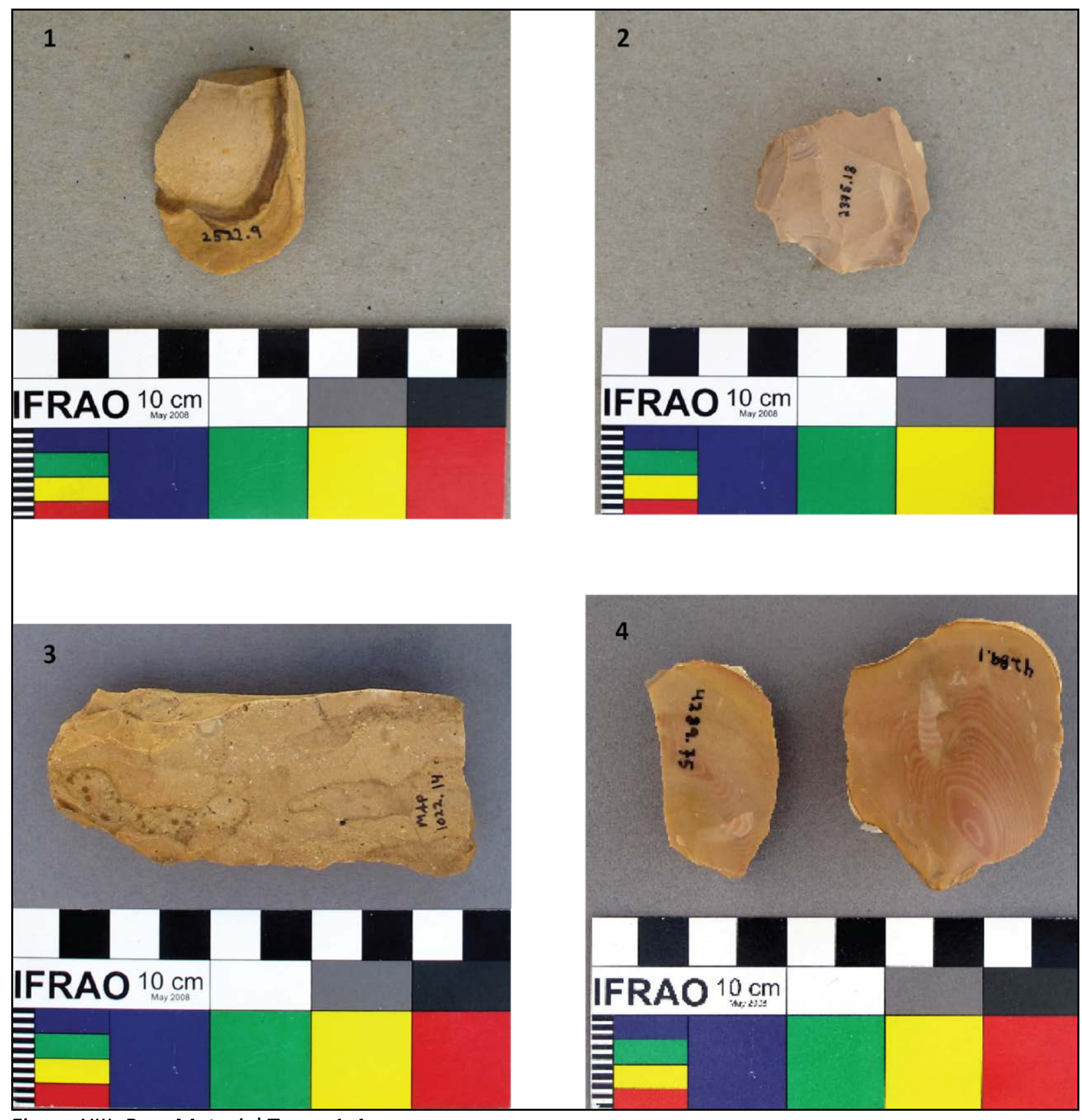

Figure XIII. Raw Material Types 1-4. 


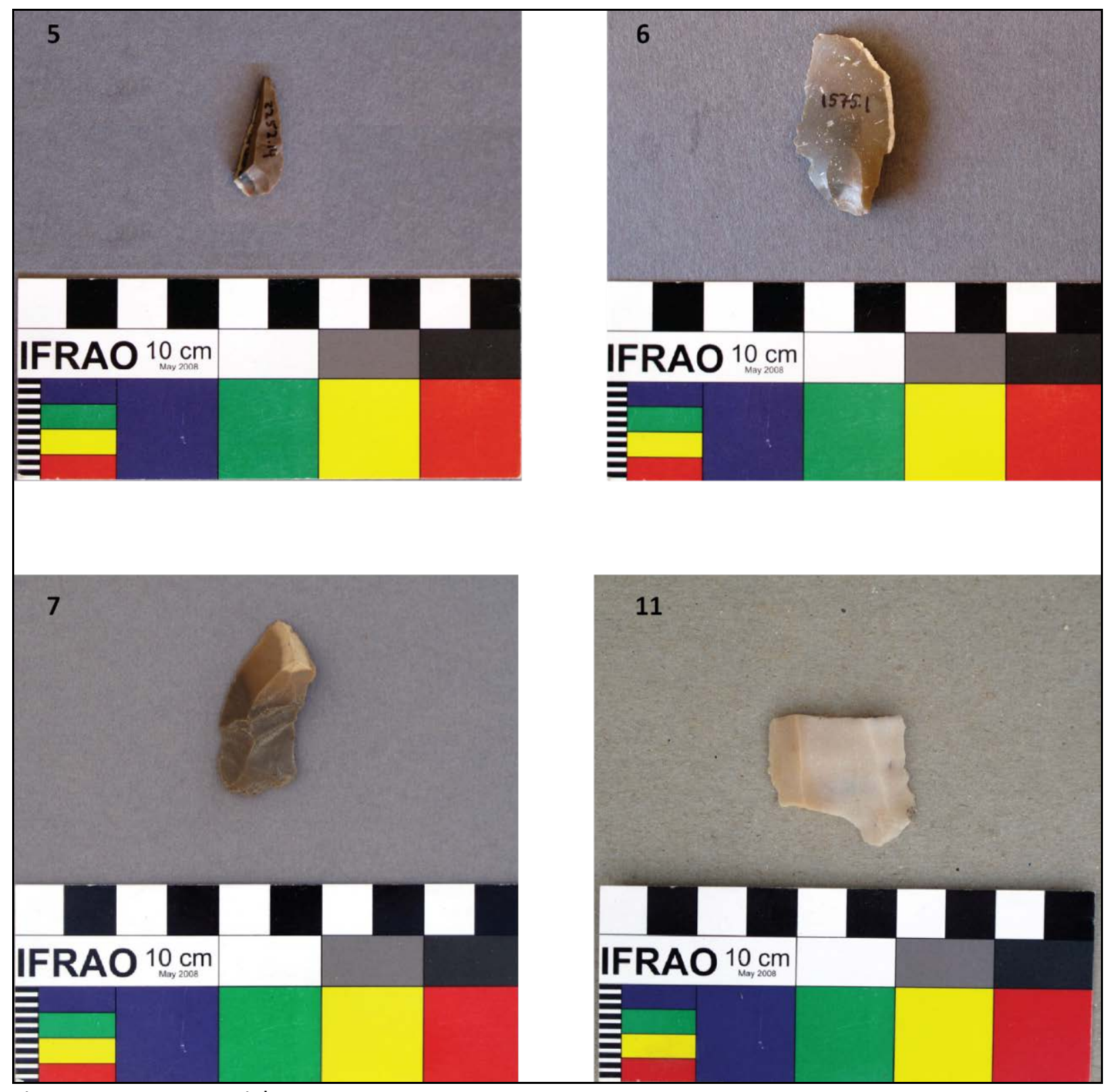

Figure XIV. Raw material types 5-7, 11. 


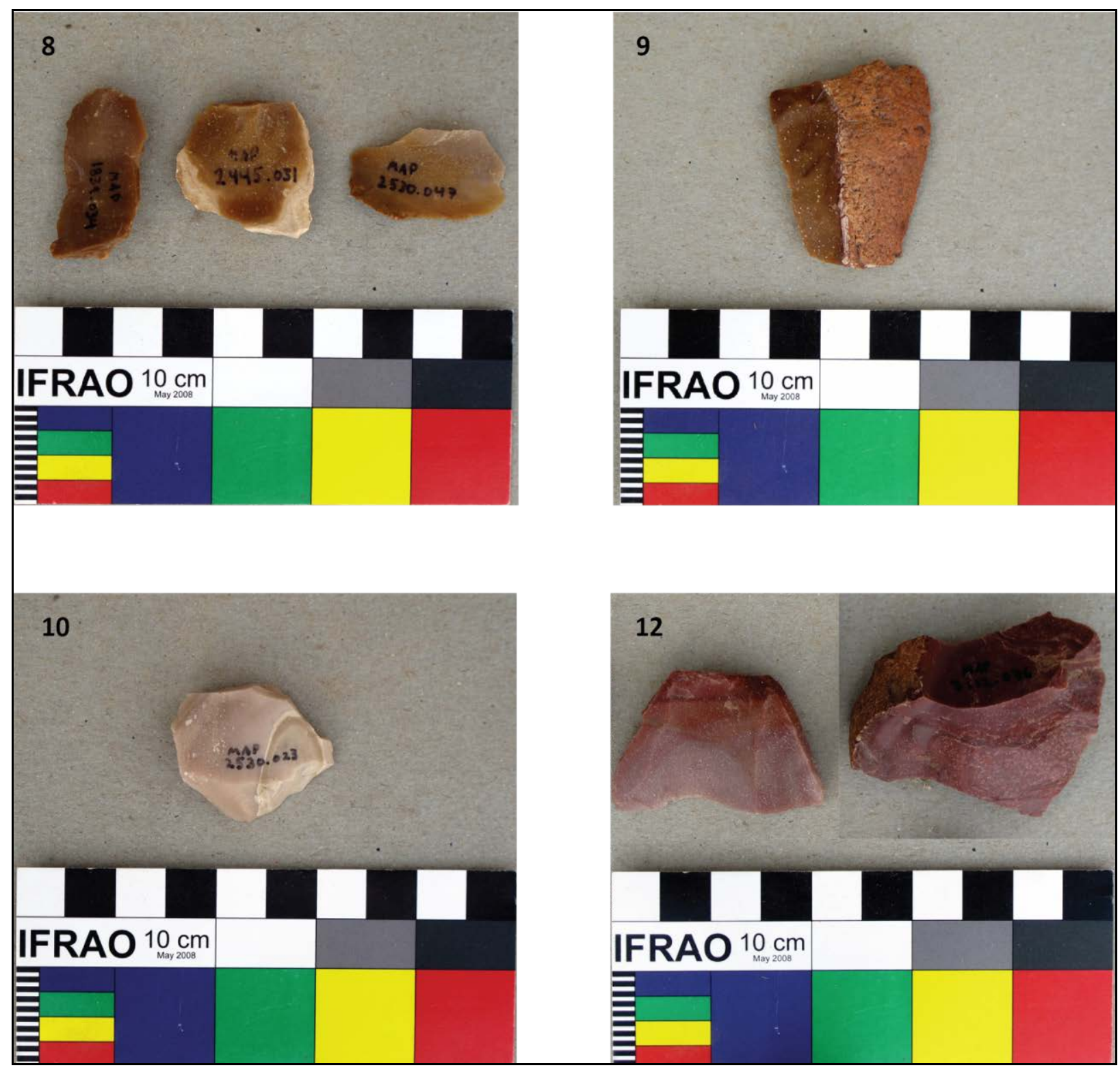

Figure XV. Raw material types 8-10, 12. Note that 12 has had parts of the same original image edited together.

\section{Production/technology Information}

\section{Reduction category}

Reduction category refers to whether an item was a tool, core, debitage or angular debris. This information was used to analyze the spatial distribution of production remains. Debris (debitage smaller than $1.5 \mathrm{~cm}$ ) were sorted out and recorded separately. 
Tool: A tool is a piece of stone that has patterned macroscopically observable retouch scars from flake removals. Flake scars were considered patterned if there were even sized removals or removals focused along a useable edge. If the scars were small and distributed randomly and intermittently around the flake edges the removals were not considered retouch.

Core: A block of raw material from which flakes of any type were detached (Tixier 1974:14). A core is a source for flake blanks for tools manufacture. To be considered a core a piece needed to have identifiable flake scars. Distinguishing between cores and tools was based on familiarity with Predynastic tools and core types.

Core/tool: This category is for items with flake scars where it is not clear whether the mass itself was the intended tool or whether it was a source of flakes (Debénath and Dibble 1994:12; Kelly 1988). This category also includes initially struck or tested cobbles, and cores that may have been subsequently used as tools. Ultimately they were grouped with tools.

Debitage: Debitage, or 'flakes' can broadly be defined as any piece detached from a mass of stone, with clear dorsal and ventral faces, and which is not modified further (Andrefsky 2005:76, 255; Cotterel and Kamminga 1987:676). In addition to the dorsal and ventral surfaces, other characteristic flake features include: a striking platform, a bulb of percussion, ripples, and dorsal flake scars. Debitage includes a variety of characteristic types, such as blades, bifacial thinning flakes, burins, axe preparation flakes etc. For coding purposes, flake fragments were included in this category as long as some flake characteristics were evident, and the preservation was recorded separately.

Angular Debris: Pieces that do not have clear dorsal/ventral characteristics, nor flake scars that could classify them as cores or tools (Debénath and Dibble 1994:11; Rosen 1997:24). They result from shattering of a stone upon impact or as a result of exposure to rapid temperature changes. These items can also be referred to as "chunks" (Holmes 1989:446).

Indeterminate: Any item that did not fit in the above categories. See comments.

\section{Preservation}

Preservation identifies whether an item is whole or broken, and which portion of it is present. The categories were based on Debénath and Dibble (1994).

Complete: The item is not broken, margins are intact. There can be small nicks or damage on the margins, but such would not greatly affect measurements.

Proximal: Any broken flake which has the platform preserved, or broken tools made on flakes where the platform is still identifiable. Fragmentary bifacial tools are proximal if the handle or haft portion is present.

Medial: The middle section of a flake or tool. For flakes, both the platform and the distal termination would be missing. Flakes that terminate in a step fracture would be indistinguishable from breaks and could be included in the proximal or medial categories. 
Distal: Flakes or tools with a distal termination is but missing the platform, or fragmentary Bifacial tools missing the haft/handle portion.

Corefrag: Any broken core.

Vertical split: A flake that has broken vertically, with a lateral half missing. The platform and distal terminations are both partially preserved, but width measurements would be inaccurate.

Indeterminate: Any item that cannot be put into one of the above categories for some reason.

\section{Amount of cortex}

This question assed the amount of surface area covered by cortex on an artifact. These assessments were done by estimating/visualizing quadrants on the flake or tool made on a flake. For cores and bifacial tools, estimations of percentage were based on total surface area, not just one face (and surface area will be calculated differently). The cortex categories were combined with metric size data to assess the amount of cortex in an assemblage relative to the expected amount following Dibble et al. (2005). Comparisons of cortical flakes counts between assemblages that do not consider the preservation and size of the flakes are problematic because different reduction techniques may produce different counts of cortical pieces (Dibble et al 2005).

The publications on Predynastic sites often use different definitions of cortex categories. For example, Holmes $(1989: 449,456)$ defined primary pieces as flakes with $50 \%$ or more of the surface covered in cortex, and everything else as secondary. Close (1980:50) also defined primary flakes as those with $50 \%$ or more cortex, but made a distinction between secondary flakes (25-50\% cortex) and tertiary flakes (0-25\% cortex). At Adaïma Midant-Reynes and Prost (2002) separated debitage into categories of those with cortex and those without cortex. At Armant, Ginter and Kozlowski (1994) separated flakes into three groups: wholly cortical, partly cortical, and no cortex. The system here with more divisions allows the artifacts to be compared to assemblages that defined cortex groupings differently.

$\underline{0}$ : No cortex whatsoever

1-10: The piece retains only a very small amount of cortex

11-40: Less than half of the surface has cortex

41-60: Around half of the surface has cortex

61-90: More than half of the surface has cortex, but it is not totally cortical

91-99: Only a very small amount of the surface is not covered by cortex

100: The surface is completely covered by cortex

Indeterminate: It is not possible to determine whether the piece has cortex or not

\section{Platform type}

This category describes the platform surface, mostly referring to the number of facets on the platform of a flake. This category is used to consider and compare the reduction technique. Often platform faceting correlates with different kinds of reduction strategies (Debénath and 
Dibble 1994:13-14; Tomka 1989:146-147). For instance bifacial thinning flakes tend to have faceted platforms. The categories used here are commonly found in, and comparable to, other publications dealing with Predynastic lithics (Close 1980; Ginter and Kozlowski 1994; Holmes 1989; Midant-Reynes 2001; Midant-Reynes and Prost 2002; Nelson 1999). Some publications refer to platforms as "Butts" (e.g. Vermeersch et al. 2004; Tixier and Newcomer 1974).

Cortical: The platform is entirely covered by cortex.

Flat (a.k.a. "lisse" or "plain" or "unfaceted"): The platform is a single flake scar surface.

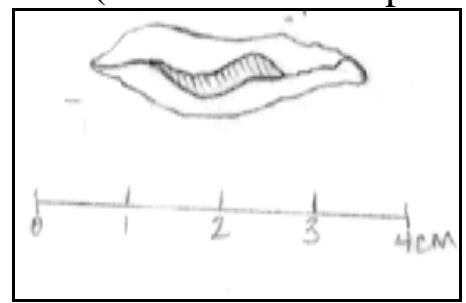

Figure XVI. Flat platform. MAP.2931.15.

Dihedral: The platform consists of parts of two flake scars that intersect, sometimes at a high angle.

Multifaceted (aka "faceted"): The platform has three or more flake scars, multiple ridges.

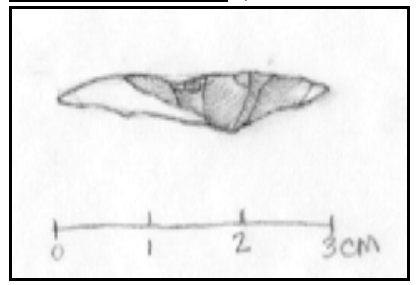

Figure XVII. Multifaceted platform. MAP.1050.28.

Gull-winged: A faceted platform with an undulating shape like that of gull wings (Inizan et al. 1999: 136).

Punctiform (a.k.a. "pointed" or "thinned"): An extremely narrow or small platform (Debénath and Dibble1994:14; Holmes 1989:427).

Linear: The platform is just an edge, width and thickness measurements would not be very precise.

Broken: The platform type is indeterminate because part of the platform has been broken or crushed, possibly during production (Holdoway and Stern 2004:120).

Other: The platform is present but it does not fit clearly into one of the above categories.

For instance a fully abraded platform would be recorded here.

Indeterminate: The platform type cannot be identified for some reason.

N/A: Not applicable.

\section{Bulb}

This question refers to the size and prominence of a bulb of force relative to the rest of the flake. The formation and size of a bulb relates to the technique of flake detachment including hammer type, force, and application of force (Andrefsky 2005:20). For example, pressure flakes tend to have short fairly pronounced bulbs (Inizan et al. 1992:63). Direct percussion with an 
Antler (rather than a stone billet) produced flakes with thinner bulbs of percussion among flakes with a small platform thickness, in an experimental setting (Pelcin 1997).

Diffuse: No bulb is present, the ventral surface is flat.

Moderate: In profile view some protrusion can be seen but it is gradual and not prominent.

Small prominent: The bulb is well defined but short/small (Holmes 1989: 291).

Prominent: In profile view the bulb is well defined and large (the bulb accounts for much of the flake's weight).

Ventral convex: The whole ventral side of the flake is convex. The bulb takes up the entire ventral surface.

Other: The bulb is present but does not fit any of the above categories.

Indeterminate: The bulb type cannot be determined, for instance if it was removed by flaking.

N/A: Not applicable.

\section{Termination}

Termination refers to the shape of the distal portion of the flake. The termination is formed by the way the flaking force exited the core, which itself relates to a combination of initiation type, core surface morphology, force, platform thickness, and platform angle (Cotterell and Kamminga 1987:698-703; Holdoway and Stern 2004:132). Feathered terminations are often ideal, but some technologies intentionally produce other terminations such as hinges or overshot flakes (Bradley et al. 2010; Cotterell and Kamminga 1987:701). The categories below derived from Cotterell and Kamminga (1987), Inizan et al. (1999), and Holdoway and Stern (2004). Stepped terminations also occur, but these are indistinguishable from breaks, and would be coded as incomplete flakes.

Feathered: The dorsal and ventral surfaces come together evenly at an acute angle. Hinged: The ventral surface of the flake curves out to meet the dorsal face, usually ending in an more or less right angle or sometimes carrying over onto the dorsal face. Plunging (aka "overshot" or "outrepassée"): The opposite of a hinged termination, these flakes remove part of the distal end of the object being worked. "The fracture plane curves markedly away from the surface of the core and continues into the core, removing part of the base of the core and resulting in a $\mathrm{J}$ shape when viewed in longitudinal cross section" (Holdoway and Stern 2004:132). Plunging flakes can be helpful for estimating nodule/core size, since they show the full length of the core at the time they were removed.

Axial: Axial terminations result when the force travels straight through a core, such as with a wedging initiation in a bipolar reduction technique.

Other: The termination is present but does not fit into any of the above categories. Indeterminate: The distal end is present, but is unclear for some reason.

N/A: Not applicable. 


\section{Dorsal scar pattern}

Dorsal scars were classified according to where the scars initiated relative to the axis of the flake. These are the scars that were present on the core before the flake was struck off, not scars resulting from retouch. Dorsal scar pattern information is used for assessing the kind of core and reduction strategy that the flake derived from, in conjunction with other production characteristics. Many Predynastic publications separate the flakes and blades into types based on the direction of dorsal flake scars (Close 1980:49-50; Midant-Reynes and Prost 2002; Vermeersch et al. 2004).

Proximal: Scars are unidirectional starting from the proximal end of the piece. Distal: Scars are unidirectional starting from the distal end of the piece. Opposite: Scars start at both the proximal and distal ends of the piece. Lateral: Scars were initiated lateral relative to the axis of the piece Proximal +lateral: Scars come from both the proximal and lateral directions. Distal +lateral: Scars come from both the distal and lateral directions. Multidirectional: Scars initiate from many/all directions.

Centrifugal: Flakes initiate on a ridge in the center of the flake. Other: Flake scars are present, but they do not fit any of the above categories. Indeterminate: There are flake scars present, but the pattern cannot be determined for some reason.

N/A: Not applicable.

\section{Heat treatment}

A primary reason to heat treat chert is to effect the mechanical properties of the chert. Controlled heating can make flaking easier to flake, but it also makes the pieces more likely to break. The changes in the visual properties may also have been a desired effect. Heat treatment can be identified through the increased luster and/or color change that occurs with heating. See Chapter 4.2 for a discussion of heat treatment and identification of heat treatment. The categories below were based mainly on luster differences but heat treatment could also be identified though comparison to the experimentally heated collection.

No: The piece is definitely not heat-treated. There is no difference in luster between earlier surfaces and later flake scars. Often the piece is matte rather than lustrous, even on flake scars.

Yes: More recent scars are glossier (more lustrous) than older scars, and/or color and luster changes match experimentally heat-treated materials of the same raw material variety.

Burned: The piece has thermal spalls, crazing, and/or rough breaks. There is also often an extreme color change to black, white, or grey.

Indeterminate: Heat treatment cannot be determined, for instance if the piece has a desert varnish, is burnt, or has a patina. This category also applies to pieces where there is no discernible difference in luster between earlier and later flake scars (which would 
definitively indicate heat-treatment), but the scars are semi-glossy to glossy which could result from heat-treatment.

N/A: Not applicable.

\section{Debitage type/ blank type}

The debitage type category applies to any piece above $1.5 \mathrm{~cm}$ that was detached from a larger chunk of material, and cannot be considered a core or debris. This category was also used to classify the blank types for tools. The production of different tool types can often be associated with certain debitage types. The presence of debitage types is useful for identifying tool production locations. The list of debitage types considered in this study is followed by a description of each type. The characteristics given for each debitage type are the ideal versions. An individual flake would not necessarily have all of the characteristics ideal for a given category. Rather, the attributes are characteristics that are commonly found in an assemblage reduced in a certain way.

Flake: Flakes have a length to width ratio of less than two, which differentiates them from blades. Flakes can be any shape, such as expanding, contracting, or blocky. Flakes can result from core reduction aimed to make blanks for tools, or they can be byproducts of other reduction processes. Therefore they can be quite variable in size. The dorsal scar pattern can be single direction, rotated, or multi-directional.

Cortical flake: Any flake with over 50\% cortex was characterized as a cortical flake. Such flakes usually do not have enough characteristic features to identify them with a specific reduction technique or reduction process because they have few dorsal flake scars. They may be the result of early stages in a tool production process.

Flake from a blade core: A piece of flake dimensions but with a dorsal scar pattern consisting of unidirectional evenly spaced straight thin flake scars. Such pieces were likely a result of accidents or core maintenance techniques associated with blade production rather than flake production, or from repurposing a blade core into a flake core.

Blade (medium blade): Blades result from a reduction strategy where flaking follows scar ridges parallel to the flaking axis and the flakes produced have parallel margins and relatively uniform thickness. In order to standardize blade identifications, Tixier (1963:36) defined blades as flakes where the length is (equal to or) more than twice the width. Blades should have parallel sides, although some may converge at the distal end. They can have one or two dorsal ridges forming a triangular or trapezoidal cross section. Blades are usually relatively thin, and usually have a uniform thickness (Andrefsky 2005:165). Blades can be removed through percussion or pressure flaking. Medium blades have a width over $1.2 \mathrm{~cm}$ to differentiate them from bladelets (below). See Ch 5.2 for more on blade types. 
Bladelet: Any piece with a length to width ratio of 2 or more and a width smaller than 1.2cm (1963:36). Bladelets generally have uniform width and thicknesses along with dorsal flake scars and ridges parallel to the flaking axis. Platforms also tended to be small. See Ch 5.2 for a more detailed discussion of bladelets.

Crested blade (a.k.a. lame à crête): Crested blades are of medium blade dimensions, have a triangular cross section, and dorsal scars that begin in the center of the dorsal ridge and extend out past the lateral margins. A "crested blade" results from shaping the edge of a blade core into a ridge to prepare it for removal of the first blade (Inizan et al. 1999:137-138). The detached ridge is the crested blade, and its removal sets up straight parallel ridges for subsequent blade removals. The second and third blades removed from a core prepared in such a way may also have signs of the initial shaping, and are called secondary crested blades and tertiary crested blades (Figure 5.7), and were noted in the comments. Unlike the removal of a utilized tool edge, there should not be use wear on the dorsal ridge.

Crested bladelet: A crested blade (see above) of bladelet dimensions.

Large blade: Large blades are quite a bit larger than medium blades in length, width and thickness, and must have resulted from a different production technology. Blades were classified as large blades if they had a length over $10 \mathrm{~cm}$ (if complete) or a width over $2.5 \mathrm{~cm}$ and a thickness over $1.5 \mathrm{~cm}$ (if broken). See Ch 5.2 for more on large blades.

Prismatic blades: Prismatic blades with ridges and margins that are very straight and parallel to the flaking axis were made during the Pharaonic period, at least as early as the Early Dynastic period (Köhler et al. 2017). Prismatic blades from Wadi el-sheikh had lengths that ranged from 8-12cm (Barket and Yohe 2011:32), and were very narrow with widths that ranged from $.6-2.4 \mathrm{~cm}$ and averaged $1.2 \mathrm{~cm}$ (Hart et al. in prep). See Ch 5.2 for more on prismatic blades. 
Table I. Debitage types.

\begin{tabular}{|c|c|}
\hline Debitage Type & Associated Tool Type \\
\hline Flake & Many \\
\hline Cortical flake & Many \\
\hline Flake from a blade core & \multirow{7}{*}{ Blade production } \\
\hline Blade & \\
\hline Bladelet & \\
\hline Crested blade & \\
\hline Crested bladelet & \\
\hline Large blade & \\
\hline Prismatic blade & \\
\hline Alternate & \multirow{4}{*}{ Bifacial tool production } \\
\hline Thinning & \\
\hline Ripple "C" & \\
\hline $\begin{array}{l}\text { Axe preparation flake / } \\
\text { tranchet }\end{array}$ & \\
\hline Burin spall & Burin production \\
\hline Tool Maintenance & \multirow{4}{*}{ Many } \\
\hline Core tablet & \\
\hline Core rejuvenation & \\
\hline Maintenance/ recovery & \\
\hline Bipolar & Scaled pieces \& other tools \\
\hline
\end{tabular}




\begin{tabular}{|l|c|}
\hline Side-blow & Side-blow tools \\
\cline { 1 - 1 } Hammer spall & \multirow{2}{|c|}{ Many } \\
\cline { 1 - 1 } Bulb removal & \\
\hline Nodule & \\
\hline
\end{tabular}

Thinning flake: Thinning flakes are curved in longitudinal section because they follow the shape of the biface. In plan they often expand toward the distal end because they are being removed from a flat surface instead of following strong ridges which would shape the direction of flake removal. They are generally thin. Dorsal flake scars are usually multidirectional since thinning flakes are usually removed from a bifacial preform, and the platforms are often multifaceted for the same reason. Platforms are often small due to the need to have very controlled placement and application of force.

Ripple "C" flake: A special subset of thinning flakes that result from the ripple-flaking was identified by Kelterborn (1984:442) through experimental recreations of rippleflaked knives. Preforms for ripple-flaked tools were ground very thin before flaking, so one of their most distinctive features is a ground surface on the dorsal side of the flake. Made through pressure flaking each ripple "C" flake follows a ridge left by the previous removal. Kelterborn described these flakes as being relatively large, oval flakes with a curved longitudinal profile, a pronounced bulb, a slightly curved single central ridge in the shape of a "C", and a ground platform with a platform angle around 75 degrees.

Bulb removal flake: Sometimes the bulb of a flake was removed in order to thin a tool. Such flakes are distinctive because the dorsal surface consists of a bulb from part of a ventral surface. The flakes look like they have two ventral surfaces. They are often biconvex with the axis of flaking rotated 90 degrees relative to the original flake axis.

Alternate flake: Alternate flakes result from removal of a square edge, in order to make a bifacial edge so that a piece can be thinned or shaped (Yerkes and Kardulias 1993:97). These are called alternate flakes because they are removed alternately from one face then the other. The key features are the flake shape relative to the orientation of removal: the flake extends far to one side relative to the flaking axis (most of the flake mass is off-center from the flaking axis). The flakes are wide rather than long. Additionally the dorsal face of the flake carries a ridge or corner making a triangular cross-section.

Axe preparation flake (a.k.a. tranchet): Axe-preparation flakes result from a transverse blow along the distal margin of an axe in order to create an acute-angled sharp edge (Holmes 1989: 456-458, 1990). The flake will have a triangular cross section, and the two faces that form the dorsal surface should show bifacial flaking from shaping the axe. 
Burin spall: A burin spall is a flake that removes a thin edge rather than a face of a tool or core. It is struck off parallel to the edge, leaving a scar that meets with the dorsal and ventral surfaces of a flake at 90 degree angles. Burin spall removals create a strong working edge. Burin spalls are triangular in cross section, and often extremely narrow.

Tool maintenance: A burin spall that removes a retouched (and utilized) tool edge is classified as a tool maintenance flake.

Core tablet: A core tablet results from the removal of an entire core platform and is often done to rejuvenate a core platform surface. The core platform is struck an angle perpendicular to previous removals. Core tablets often have a scalloped edge(s) in planview due to the scars where previous flakes have been removed perpendicular to the core tablet dorsal face. The cross section can be relatively square, with parallel dorsal and ventral surfaces joined by perpendicular margins.

Core rejuvenation: A core rejuvenation flake is like a core tablet, but only part of the core platform was removed, creating a triangular rather than square cross section.

Maintenance flakes (recovery flakes): A flake with a deep hinge or series of compounded step terminations on the dorsal surface is a maintenance or recovery flake. Such flakes were removed in order to correct a mistake or smooth the surface of a core or preform.

Bipolar Flake: A bipolar flake is a wedging initiated percussion flake that is detached by striking a core that is resting on an anvil. These flakes have platforms that are crushed, or missing, and there may be crushing at both ends. Bipolar flakes have no bulbs because they are wedging (axial) initiations rather than conchoidal fractures. The flakes are straight or wedge-shaped in the longitudinal section.

Side blow: Side-blow flakes are characteristic of Egyptian Neolithic sites (Banks 1984:175; Caton-Thompson and Gardner 1934:21; Kindermann 2010:43-44), and have sometimes been found in Predynastic sites (Vermeersch et al. 2004). Side blow flakes are flakes struck from a nodule that is held so that it is wider than it is long. Since it is struck that way the platforms are very wide and thick and the flakes are short. A number may be removed in a row directly behind each other resulting in a dorsal flake scar with a central concavity (from the bulb of the previous removal) and giving the flake a swooping appearance.

Hammer spall: A hammer spall results when a flake detaches unintentionally from the hammer stone rather than from the nodule being reduced. Therefore they are often of a non-chert raw material. There is usually crushing and incipient fractures on the platform of the flake from previous use as a hammer stone. They have characteristics of hard hammer percussion such as being large with prominent bulbs. 
Nodule: This category was used to describe tool blanks when cortex could be observed on two faces of the tool showing that it must have been made from a nodule.

Other: Any distinctive flake which is definitely different from the above categories.

Indeterminate: Any flake that cannot securely be attributed to one of the above categories, such as fragments.

N/A: Not applicable.

\section{Retouched Tools Information}

Retouched tools have patterned macroscopically observable scars from flake removals that were made as part of making or re-working a tool. The seven categories used to describe the retouch after the 'extent of working' category follow Inizan et al. (1999). See also Holmes (1989: 450-456).

\section{Extent of working}

The extent of working codes how intensively a tool was shaped. This information is useful for categorizing the tool types, and for understanding how much investment there was in their production.

Edge retouched- unifacial: The retouch was confined to the edge (not invasive) on only one face of the piece.

Edge retouched- alternate: The retouch was confined to the edge (not invasive), on more than one edge, but on different faces.

Edge retouched-alternating: The retouch was confined to the edge (not invasive), on both faces of an edge, but without overlapping.

Edge retouched- bifacial: The retouch was confined to the edge, on both faces of an edge, and the retouch on both faces meet at the edge.

Invasive unifacial retouch: There is retouch on only one face of the tool, and the scars are invasive, reaching most of the way or all the way to the middle of the piece.

Invasive bifacial retouch: There are invasive scars on at least one face of the piece, and invasive or edge retouch on the other face, that forms a bifacial edge.

Bifacial: There are invasive scars on both faces of a tool.

Bifacially thinned and stylized: There are invasive scars covering most or all of both faces of the tool, and it has been worked into a distinctive shape clearly associated with a well-defined bifacial tool type.

Other: There are retouch or shaping scars that do not fit with any of the above categories. Indeterminate: The extent of working cannot be securely attributed to one of the above categories, such as for fragments.

N/A: Not applicable. 


\section{Delineation}

140).

Delineation describes the shape of an edge created by retouch, in plan view (ibid:87,138,

Rectilinear: The retouched edge is rectilinear.

Convex: The retouched edge curves outward.

Concave: The retouched edge curves inward.

Denticulated: A series of adjacent notches.

Notch: A single notch or multiple notches that are not adjacent (e.g. on different edges). Cran: A retouched edge that is partially straight and curves outward at one end.

Shoulder: The retouched edge forms a small convex protrusion, with retouch continuing on one side.

Nose: The retouched edge forms a small convex protrusion, with retouch continuing on either side of the protrusion.

Tongue: The retouched edge forms a long protrusion, with retouch continuing on either side of the protrusion.

Tang: The retouched edge forms a protrusion, which is opposite a working edge and was used for hafting.

Long narrow tang: A longer tang.

Combination: Any combination of the above

Irregular: The retouched edge is variable.

Other: The retouched edge shape cannot be categorized according to the above descriptions.

Indeterminate: The retouched edge shape cannot be determined, for instance if it is broken.

N/A: Not applicable, (e.g. burins).

\section{Extent}

Extent characterizes the invasiveness of the retouch (ibid:141; Holmes 1989:) invasiveness.

Non-invasive (short): the retouch is confined to the edge, around a quarter of the way to the center of the piece from the edge, or less.

Slightly invasive (long): the retouch reaches from about a quarter of the way to the center of the piece, to around half way to the center of the piece.

Invasive: the retouch reaches over half way to the center of the piece.

Combination: The retouch does not predominantly fit into one of the above categories but a combination (described in the notes).

Other: The retouch scars cannot be categorized according to the above descriptions (e.g. burins). 
Indeterminate: The extent of the retouch scars cannot be determined, for instance if the piece is broken.

N/A: Not applicable.

\section{Angle}

Angle describes the angle of the removals relative to the opposite face the angle formed by removals relative to the face from which they were initiated (Inizan et al. 1999:129).

Flat (low): Very acute, 25 degrees or less.

Semi-abrupt: 45 degrees

Abrupt: 90 degrees.

Combination: The retouch scars are not predominantly one of the above categories but a combination (described in the notes).

Other: The retouch scars cannot be categorized according to the above descriptions (e.g. burins). Indeterminate: The angle of the retouch scars cannot be determined N/A: Not applicable.

\section{Localization}

Localization describes the location of removals along the edges relative to the orientation of the piece (ibid:144-145). For tools on debitage blanks the descriptions applies to a piece oriented so that the platform is near the viewer and the dorsal side is up. For tools on nodular blanks the descriptions follow congenital orientations for the tool, usually with the working edge farthest from the viewer and the haft or handle nearest.

\section{Proximal}

Distal

$\underline{\text { Right lateral }}$

Left lateral

Combination: The specifics are described in the notes.

Other: The retouch scars cannot be categorized according to the above descriptions.

Indeterminate: The localization of the retouch scars cannot be determined, for instance if the piece is broken.

N/A: Not applicable. 


\section{Position}

Position describes the location of the removals relative to the faces of the piece (ibid:151152).

Direct: The scars of the removals are on the dorsal side of the piece, and were initiated on the ventral side of the piece.

Inverse: The scars of the removals are on the ventral side of the piece, and were initiated on the dorsal side of the piece.

Alternate: The removal scars are on multiple edges of the piece, but on different faces.

Alternating: The removals are on both faces of a piece, on the same edge, but were not initiated in the same place (not bifacial).

Bifacial: There are removals on the dorsal and ventral faces, initiating at the same place along the edge. In other words the removals meet at the edge forming an angle less than 90 degrees.

Crossed: The removals were initiated on both faces of the piece, forming a face perpendicular to the dorsal and ventral faces (e.g. backing retouch).

Combination: The removals are not predominantly one of the above categories but a combination (described in the notes).

Other: The removals cannot be categorized according to the above descriptions (e.g. burins).

Indeterminate: The position of the removals cannot be determined, for instance if the piece is broken.

N/A: Not applicable.

\section{Distribution}

Distribution describes how much of the edge is occupied by retouch (ibid:140-141).

Total: The retouch occurs along the entire length of an edge.

Partial: The retouch is continuous, but does not occupy the entire length of an edge. Discontinuous: The retouch is not continuous along the edge, there are one or more spaces in between the removals.

Combination: The retouch scars are not predominantly one of the above categories but a combination, for instance total on one margin, but partial on another.

Other: The retouch scars cannot be categorized according to the above descriptions. Indeterminate: The retouch scars cannot be determined, for instance if the piece is broken.

N/A: Not applicable. 


\section{Morphology}

Morphology describes the general shape of the retouch removals (Inizan et al. 1999:146)

Scaled: The retouch scars are wide and short, resembling fish-scales. Their terminations can be feathered or slightly hinged.

Stepped: Wide short removals, with stepped and/or hinged terminations. These types of scars result from bending rather than conchoidal initiations. Often the scars are compounded on top of each other on thick blanks.

Parallel: The retouch scars are longer than they are wide, and they are parallel to each other, with feathered terminations or slightly hinged terminations.

Sub-parallel: The retouch scars are longer than they are wide, but the scars are not quite parallel to each other, going in slightly different directions, usually with feathered terminations or slightly hinged terminations.

Combination: The retouch scars are not predominantly one of the above categories but a combination (described in the notes).

Other: The retouch scars cannot be categorized according to the above descriptions (e.g. burins).

Indeterminate: The retouch scars cannot be determined, for instance if the piece is broken.

N/A: Not applicable.

\section{Tool type}

The following list of tool types was derived primarily from a number of works dealing with 4th millennium B.C.E. Egyptian lithics: Baumgartel 1960; Hikade (2001); Holmes (1989, 1990); Kabacinski (2012); Midant-Reynes and Prost (2002); Rizkana and Seeher (1985, 1988); Schmidt (1999, 1996, 1992a,b). Kindermann (2010) worked on mid-Holocene Egyptian stone tools and clarified some of the boundaries between tool types. Much research on Predynastic and earlier lithics in Egypt was influenced by Tixier's (1963) work in the Epipaleolithic of the Maghreb. Inizan et al. (1999) provide a glossary of definitions, much of which is based on Tixier's work, but updated and in English. Tixier's work stemmed from and was influenced by European Paleolithic research (Bordes 1961), and a more recent development of that line of research that is also in English is Debénath and Dibble (1994). Another useful source was Rosen (1997) which deals with stone tools for the post-Paleolithic Levant, since that material was chronologically and geographically close to the Predynastic Egyptian lithic stone tools. Also Tixier did not cover bifacial tools, while most of the rest of the above mentioned sources do.

The blank type was recorded separately, so there was no need to make specific tool types related to the blank (reducing the number of tool types from Tixier's list). The total number of tool types is 53, but not all were identified in the assemblages from el-Mahâsna, Abydos, and Nag el-Qarmila. 
Table II. Tool types.

\begin{tabular}{|c|c|c|}
\hline Retouch Intensity & Tool Class & Tool type \\
\hline \multirow{19}{*}{ Edge retouched tools } & \multirow{5}{*}{ Burins } & Burin \\
\hline & & Multiple burin \\
\hline & & Dihedral burin \\
\hline & & Burin on a retouched edge \\
\hline & & Nucleiform burin \\
\hline & \multirow{3}{*}{ Notches \& Denticulates } & Notch \\
\hline & & Denticulate \\
\hline & & Sickle blade \\
\hline & \multirow{5}{*}{ Scrapers } & Endscraper \\
\hline & & Microendscraper \\
\hline & & Circular scraper \\
\hline & & Sidescraper \\
\hline & & Tabular Scraper \\
\hline & \multirow{5}{*}{ Truncations } & Truncation \\
\hline & & Double truncation \\
\hline & & Backed truncation \\
\hline & & Backed double truncation \\
\hline & & Truncation knife \\
\hline & Geometrics & Transverse arrowhead \\
\hline
\end{tabular}




\begin{tabular}{|c|c|c|}
\hline & & Lunate \\
\hline & Backed pieces & Backed piece \\
\hline & \multirow{3}{*}{ Perforators } & Perforator \\
\hline & & $\begin{array}{l}\text { Drill/double-backed } \\
\text { perforator }\end{array}$ \\
\hline & & Microdrill \\
\hline & Unpatterned & Retouched piece \\
\hline \multirow{10}{*}{ Invasively retouched pieces } & \multirow{3}{*}{ Knives } & $\begin{array}{l}\text { Hemmamiya Type A blade } \\
\text { knives }\end{array}$ \\
\hline & & $\begin{array}{l}\text { Hemmamiya Type B Blade } \\
\text { knives }\end{array}$ \\
\hline & & El-Omari knives \\
\hline & Planes & Plane \\
\hline & \multirow{5}{*}{ Worked nodules } & Worked tabular slab \\
\hline & & Chopper \\
\hline & & Pounder \\
\hline & & Grand perçoir \\
\hline & & Chisel \\
\hline & Scaled pieces & Piece esquille \\
\hline \multirow{4}{*}{ Bifacial Tools } & \multirow{3}{*}{ Drills } & Bifacial drill \\
\hline & & Crescent drill \\
\hline & & Winged drill \\
\hline & Axe & Axe \\
\hline
\end{tabular}




\begin{tabular}{|c|c|c|}
\hline & \multirow{4}{*}{ Varia } & Bifacial triangle \\
\hline & & Preform \\
\hline & & Bifacially retouched piece \\
\hline & & Core/tool \\
\hline \multirow{9}{*}{$\begin{array}{l}\text { Bifacially thinned and } \\
\text { stylized tools }\end{array}$} & \multirow{2}{*}{$\begin{array}{l}\text { Projectile points } \\
\text { (symmetrical) }\end{array}$} & $\begin{array}{l}\text { Concave-base projectile } \\
\text { point }\end{array}$ \\
\hline & & Tanged point \\
\hline & \multirow{3}{*}{$\begin{array}{l}\text { Daggers/lances } \\
\text { (symmetrical) }\end{array}$} & Fishtails \\
\hline & & Rhomboids \\
\hline & & Daggers \\
\hline & \multirow{3}{*}{ Knives (asymmetrical) } & Bifacial knife \\
\hline & & Bifacial sickle \\
\hline & & Ripple-flaked knife \\
\hline & Eccentrics & Eccentrics \\
\hline Other & Indeterminate & Unidentifiable tool fragment \\
\hline
\end{tabular}

\section{EDGE RETOUCHED PIECES}

The edge retouched pieces are generally unifacial, unless otherwise noted.

\section{BURINS}

A burin is a tool with a scar from a flake removed parallel to a flake margin, rather than off of the face of the tool. The scar meets with the dorsal and ventral surfaces of a flake at $\sim 90$ degree angles, leaving a very sturdy working edge. Burins are a very common type of Predynastic tools, and more divisions can be made for burins (e.g. Holmes 1989:398-399).

Burin: A tool with a single burin spall facet. Tixier type 19. Multiple burin: A tool with two or more non-adjacent burin spall facets. Tixier types 20, 27. 
Dihedral burin: On dihedral burins the scar of the first burin spall removed becomes the striking platform for the second burin spall, and forms an angle less than 90- degrees. Tixier types 17-18.

Burin on a retouched tool: A tool where a burin spall or spalls was removed from a retouched edge, or a tool that has retouching that is not adjacent to the burin. This kind of tool can be further specified with details on the type of retouching, such as a burin on a truncation, burin on a scraper, or burin on a piece with continuous retouch. Tixier types 21-26, 29-33.

Nucleiform burin: A burin or burins on a very thick blank. Tixier type 28.

\section{NOTCHES AND DENTICULATES}

Notch: A notch is a tool with one or more non-adjacent concavities formed by retouch. Sometimes they are found on pieces with other retouch. Tixier types: 73, 74, 76.

Denticulate: A tool is a denticulate when there are two or more adjacent notches (Tixier 1963:117-124). The denticulations can be regular or irregular, with the details noted in the comments. Tixier types $75,77$.

Sickle blade: A blade or bladelet with regular denticulations along one or two lateral margins and sickle gloss. (Holmes 1989:402; Midant-Reynes and Prost 2002:375; Rosen 1997:44-60). Any truncations or backing were described in the comments. It should be noted that the denticulations can be formed by unifacial retouch or bifacial retouch that is confined just to the edges forming the denticulations Tixier type78.

\section{SCRAPERS}

As described by Tixier (1963:54) scrapers have continuous regular retouch, the inclination of the retouch at the edge is usually flat to semi abrupt, but can be resharpened to abrupt scalar retouch. Rosen (1997: 86-87) describes scrapers from the Levant as made on flakes from amorphous cores with no preparation. The retouch is varied from rather ephemeral to somewhat invasive and less regular than on Paleolithic scrapers.

Endscraper: An endscraper is a scraper with the retouched edge on the distal margin, although the retouch may extend farther around the piece, and the scraper edge is more or less regularly rounded (Tixier 1963:54). Endscrapers are differentiated from truncations because truncations form at least one corner with a lateral margin. Furthermore, the angle of the retouch for endscrapers is generally flat to semi-abrupt, whereas truncations are semi-abrupt to abrupt, and the working edge for endscrapers is the retouched edge, but the working edge for truncations is the lateral margin (Holmes 1988:400). Additionally, truncations are usually on blades rather than flakes, while scrapers can be made on either kind of blank. Endscrapers may vary in the details of the delineation, angle, position, and morphology. The thickness of the piece and any additional retouch was noted. Tixier types 1, 2, 4, 5, 6, 7,8 , 9, 10, 11.

Microendscraper: "Nibbling retouch on the distal and sometimes lateral edges of bladelets superficially resembling miniature endscrapers on blades" (Rosen 1997:65). Although termed microendscrapers, the small nature of the tools and the retouch on the distal end makes these tools somewhat ambiguous between endscrapers and truncations 
(Rizkana and Seeher 1988:27; Holmes 1989:403). Many authors have noted that these bladelets often have more or less ephemeral retouch along a lateral margin in addition to the distal retouch (Hikade 2004:65-66; Holmes 1989:403; Rizkana and Seeher 1988:27). These were sometimes made on heat-treated bladelets, and Holmes (1989:403) termed these "glossy bladelet tools", but most authors use the term microendscrapers, which is followed here. See also Hikade 1996; Midant-Reynes and Prost 2002:376; Schmidt 1999).

Circular scraper: A scraper whose retouched edge extends around at least 75 percent of the entire tool margin. They are circular to oval in shape, and some are D-shaped Holmes 1989:396). They can be any size. Tixier type 3.

Sidescraper: A tool with continuous regular retouch along the lateral margin, usually slightly convex with a flat to semi-abrupt angle, is a sidescraper. Following Kindermann (2010:43) sidescrapers were differentiated form edge-retouched knives based on dimensions, with side scrapers having a length to width ratio of less than 2, and knives a length to width ratio of 2 or more. Tixier type 106.

Tabular scraper: Tabular scraper production is a well known phenomena in the Levant (Rosen 1997:71-80), but they have also been found in Egypt (Rizkana and Seeher 1988:29-31). Tabular scrapers were made on large flat flakes with cortex covering most of the dorsal surface. They were made in shape varieties. They often have pronounced bulbs of percussion and can have flat or faceted platforms. On some pieces the cortex is ground very flat and smooth. Some examples have bulbar thinning. None were identified in the three assemblages studied here.

\section{TRUNCATIONS}

Tixier (1963:124-127) defined truncations as abrupt rectilinear continuous retouch at the distal or proximal end of blade or bladelet (only rarely on a flake). The retouch can sometimes be semi-abrupt. The retouch forms at least one angle with the lateral margins the blade or bladelet, which distinguishes the tools from endscrapers (see endscrapers above for more on differentiating endscrapers and truncations). Abrupt retouch on a lateral margin would be considered a backed piece. The delineation of the retouched edge could be straight or convex (but usually straight). A concave delineation would be considered a notch. The orientation of the truncated edge relative to the blade axis (horizontal, oblique) was noted in the comments.

Truncation: A piece with one truncation at either the distal or proximal end. Tixier type 80, 81.

Double Truncation: Also called a bitruncated blade (Rosen 1997:60-65), or a rectangle (Schmidt 1999:35), a double truncation is a blade or bladelet with truncations at both the distal and proximal ends. The "razor blades" of the Pharaonic period would be a subtype of the double truncations, on wide blades and with very neat retouch, but none were observed during this study.

Backed truncation: A blade or bladelet with a single truncation at one end and backing retouch along one lateral margin.

Backed Double truncation: A blade or bladelet with truncation at both the distal and proximal ends, and backing retouch along one lateral margin. 
Truncation knife: A truncation knife (Holmes 1989:146, 400), also known as "blade knives of the Badarian Type" (Schmidt 1996:281), is an oblique convex truncation on a blade or bladelet which forms a corner with one lateral margin (the working edge) and is rounded at the other corner. The truncation is usually proximal. The angle of the retouch may be semi abrupt or even flat. The end opposite the truncation is usually retouched round, sometimes ogival. One lateral edge is often retouched with very small to small scars along some or all of the edge.

\section{GEOMETRICS}

Tixier (1963:127) defined geometrics as tools of small or very small dimensions, made on a blade or bladelet with two abrupt retouch. The tools have a geometric outline in plan, such as rectangle, half circle, trapeze, triangle, or scalene triangle. At least one edge is not modified, presumably the cutting or working edge. Functionally geometrics were projectile points or parts of composite tools. Although there were many distinct types of tools in the geometric tool class for the Epipaleolithic of the Maghreb, they were less common in Egypt during the 4th millennium B.C.E. so only two types are considered here.

Transverse arrowheads: Transverse arrowheads generally have proximal and distal oblique truncations forming a small tool that is trapezoidal in outline. One lateral margin was longer and unworked, forming the cutting edge. Holmes (1989:X) notes that the proximal or distal edge may be a break instead of a retouched edge. These tools were usually made on blades or bladelets, but could also be made on thinning flakes (Takamiya and Endo 2008). Tixier types 83-93.

Lunate: A very small tool where the proximal end, distal end, and one lateral margin have been retouched into a semi-circular shape leaving one lateral edge unworked as a cutting edge. Tixier type 82.

\section{BACKED PIECES}

Backed pieces: Inizan et al. (1999:130) define backed pieces as those with continuous regular retouch that extends along the length of blank (on a lateral margin). This retouch should be abrupt enough not to create a new cutting edge, and could be made through direct or bipolar retouch (Rosen 1997:60; Tixier 1963:84). Backing is more common on blades or bladelets than flakes. For Paleolithic collections Debénath and Dibble (1994:100) emphasize that backing has to be across from a cutting edge, and this rule will be followed here (for instance a tool with continuous abrupt retouch across from a cortical edge would be included with retouched pieces, not backed pieces). Blade knives can be differentiated from backed pieces based on the presence of more standardized shaping and the presence of more invasive retouch (see blade knives below). Holmes (1989:400) observed are few backed pieces in Predynastic assemblages, accordingly Tixier's many separations of backed blade types are not required here. Tixier types 34-37, 39-40.

\section{PERFORATORS/DRILLS}

Perforators, also known as borers, piercers, awls, and drills, are a group of tools which can be made from unifacial edge retouch but can also have alternate or 
alternating edge retouch. Although defined morphologically, they often show usewear indicative of perforating or perforating and twisting .

Perforator: Flake, blade, or bladelet presenting a projection generated by bilateral retouch forming a single or double shoulder (Tixier 1963:63). The retouch can be flat to abrupt. Tixier type 12, 13.

Microdrill: Microdrills are tools made on bladelets with drill tips shaped by small retouch on both lateral edges (Holmes 1989:399). The retouch sometimes continues along the entire length of the edge. Microdrills are small, those from HK29A ranged from 1.8$5.8 \mathrm{~cm}$ long and averages $2.02 \mathrm{~cm}$ long (ibid). None were identified in the three assemblages studied here.

Drill: A double backed perforator (a.k.a double backed perforator or mèche de foret), has a long slender bit, is made with abrupt retouch, the lateral margins are mainly parallel, and does not necessarily have a shoulder or shoulders (Tixier 1963:66).The edges can be formed by burin blows, or they can be made on burin spalls. There is often usewear at the tip. Tixier type 16.

\section{UNPATTERNED}

Retouched piece: A unifacially retouched tool which is complete, but does not fit any of the above categories. The retouch is often irregular and does not have any clear design (Holmes 1989:417). Here this category includes Tixier type 105, piece with continuous retouch. These are considered ad-hoc tools.

\section{INVASIVELY RETOUCHED/THINNED PIECES}

\section{KNIVES}

Hemmamiya type A blade knives (a.k.a. blade knives, end-scraper knives) (Baumgartel 1960:37,40; Holmes 1989:402; Schmidt 1989:85,1992a:32, 1996:281-282, 1999:32): These tools are made on blade blanks, and show a standardized retouch pattern with the knife point at the proximal end, often formed by bifacial retouch, with the bulb removed by flat (invasive) retouch. There is semi-abrupt to abrupt backing retouch along the right lateral margin (dorsal side up, proximal end toward the viewer) on the dorsal side forming a straight back. Often the backing continues onto the distal end forming a rounded handle which looks like a scraper end. The working edge (on the left lateral margin) is straight or slightly convex, and there is often flat (semi-invasive?) retouch on the ventral side of this edge, which may be from re-sharpening. These knives were usually made on large twisted blades. Schmidt (1996) designated the knives that conform precisely to these parameters Hemmamiya knives type A, and called this standardized retouch pattern "Seitenbezogenheit." Holmes (1989:402) gave a size range of $8-15 \mathrm{~cm}$ in length.

Hemmamiya type B blade knives (AKA other blade knives, endscrapers on blades): Tools made on blades with lateral backing retouch and additional retouch (such as invasive retouch removing a bulb or flat ventral edge retouch), but that do not conform precisely to the retouch patterns or shape outlined for Hemmamiya type A knives, are Hemmamiya Type B blade knives. If a blade only has backing retouch it would be classed as a backed blade. Schmidt (1996:281) also writes that some items classed as endscrapers on blades at Maadi (Rizkana and Seeher 1988:27) should be in this type B 
blade knife category. Kabacinski 2012 gave a size range of 5-15 cm in length for examples from Tel el-Farkha.

el-Omari knife: These knives have backing retouch forming a convex edge, a straight cutting edge, and a tang-like handle defined by retouch (Schmidt 1996:283).

Plane ( A.K.A rabot-plane, adze): Planes are made on thin nodules or flakes, and are expanding (trapezoidal) in plan-view shape. The working edge is the broad distal edge. They have a plano-convex cross section, one face is very flat, either naturally, or through thinning retouch, and the other is convex. The working edge is located closer to the flat face. The convex face often has invasive retouch, or complete facial thinning. The retouch on the working edge is semi-abrupt to abrupt. Holmes (1989:405); MidantReynes and Prost (2002:378).

\section{WORKED NODULES}

Worked tabular slab: A thin natural piece of material (nodule or natural spall) that has been worked into a tool, often with bifacially edged or invasive bifacial retouch (Holmes 1989). The retouch does not cover the entire tool, and cortex remains. To differentiate them from choppers the width to thickness ratio should be less than 2 .

Chopper: a Chopper is an approximately fist-sized nodule (approximately round in plan, and thick) with some flakes removed forming an broad edge. The edge can be bifacial or unifacial. Much of the cortex remains on the nodule. Choppers can be distinguished from cores by indications of use on the working edge (Rosen 1997: 98).

Pounder: This tool type had to be defined based on the find of one such item at elMahâsna (Figure 4.51). This thick tool with an expanding shape was partially shaped or thinned from both ends, leaving much of the surface covered in cortex. The working edge was a broad flat face perpendicular to the axis of the tool, which bore many pecking marks from shaping and/or use. The tool may have been used as a pounder or pestle.

Grand Perçoir: As Tixier (1963:66) describes, these are large pointed implements that are partially bifacially worked and made on a nodule. the proximal end is wide, differentiating them form picks/chisels. Holmes (1989:399) and Midant-Reynes and Prost 2002:375-376) drop Tixier's "Caspien" designation and note that the perforator tip need not have a triangular cross section as Tixier specified. Tixier type 15. None of these tools were found in the three sites studied here.

Chisel ( A.K.A pick): These are elongated narrow tools with a narrow or pointed working edge that could be made on a nodule or large flake (Midant-Reynes and Prost 2002:378). They are coarsely made and can be trihedral or bifacial (Rosen 1997:93). Chisels can be differentiated from axes, because axes have a broader working edge, and are not as elongated or narrow as chisels. It seems reasonable to suggest a length: width ratio greater than 2 to separate chisels and axes. None of these tools were found in the three sites studied here.

\section{OTHER}

Piece esquille (Scaled pieces): Piece esquilles are produced through bipolar reduction, and it is debated whether they should be considered wedging tools or cores for very small flakes (Bradbury 2010; LeBlanc 1992; Shott 1999). The flakes usually come off bifacially from both ends and can be invasive. The 
resulting items are usually rectangular or square in shape (Tixier 1963:146). The bipolar reduction often causes crushing at both ends. Tixier type 104.

\section{BIFACIAL TOOLS}

\section{DRILLS}

Bifacial drill: Holmes (1989:412,414) defined a bifacial drill as an elongated tool (with no shoulder) that has a thick cross section, and wear at one end. Such tools were shaped by full bifacial retouch. This differs from a double-backed perforator/meche de foret which has steep edge retouch blunting the piece (Kindermann 2010:43). None were identified in the three assemblages studied here.

Crescent drill: Holmes (1989:415, 416) described crescent drills as thick bifacially worked tools with a concave upper margin and a convex lower margin that is the working edge. They were used for drilling soft stone into vessels and usually date to the Early Dynastic and Old Kingdom, but have also been found in Predynastic sites (Hierakonpolis and Naqada). Midant-Reynes and Prost (2002:374) note that the crescent drills and winged drills may be related. None were identified in the three assemblages studied here.

Winged Drill: Holmes (1989:412, 415) described winged drills as relatively thick bifacially worked Y-shaped tools where the drill bit tends to have a circular cross section, and wear. the drill bit is thicker than the wings. See also crescent drills. None were identified in the three assemblages studied here.

\section{AXES}

Axe: Axes are bifacial tools that are bi-convex in cross section with a wide working edge at the distal extremity. They are relatively thick and their shape in plan view is generally oval, rectangular, or expanding toward the working edge (see Holmes 1990 for more shape details). The working edge of some axes was prepared by a transverse blow (tranchet), creating a smooth sharp cutting edge. The working edge is generally in the middle of the tool in cross section (Rosen 1997:93), but those with a tranchet blow may be skewed more toward one face. Predynastic axes can be relatively small averaging around 6cm in length (Holmes 1990). See chapter 6.1 for more details about axes.

\section{VARIA}

Bifacial triangle: Holmes (1989:412) describes these as triangular tools (in plan view) that are fully bifacially thinned and have a triangular shape with slightly rounded corners. Two of the edges may be slightly convex. These are rare items which could be preforms rather than finished tools.

Preform: A preform is defined here as a bifacial piece with relatively fine (rather than course) scars from thinning, and that is somewhat thicker than the bifacially thinned and stylized tools. The edges in profile view may be wavy rather than retouched to be straight, indicating that the final retouching has not been completed. To some degree classifying something as a preform depends on familiarity with the other bifacial tool types. 
Bifacially retouched piece ( A.K.A Miscellaneous bifacial tool): A bifacially retouched tool which is complete, but does not fit any of the bifacial tool categories. The retouch is often irregular and course and there is no clear design or pattern to the shape (Holmes 1989:417). They may be relatively thick.

Core/tool: The category core/tool is for items that could be either a core (a source of flakes) or a tool.

\section{BIFACAILLY THINNED AND STYLIZED TOOLS}

\section{PROJECTILE POINTS}

Concave-base projectile point: These are relatively small symmetric fully bifacially retouched and shaped tools which are quite thin. They are triangular in shape and two long barbs form a deep notch or hollow base, which was used for hafting (Clark 1975). The lateral edges may be straight, slightly convex. The barbs can be straight or slightly convex, and the shape of the wing tips can be pointed or square. the distance from the interior of the notch to the tip of the point can vary. Many have very fine microdenticulation along the outer margins. (See Chapter 6.1, Hikade 2001 for more details).

Tanged projectile point: Small pointed symmetrical thin bifacially retouched tools with a tang formed by retouch at the base. The curvature of some examples from Maadi indicate that they may have been made on blades (Rizkana and Seeher 1985:243). See Hikade (2001) for subtypes and more details. None were identified in the three assemblages studied here.

DAGGERS/LANCES(elongated symmetrical bifacial tools)

Fishtail tools: These tools are shaped like an inverted triangle, with a U or V-Shaped notch or fork at the top, which is the working edge. They are fully bifacially thinned, usually with fine flat scars. The haft area often has coarser flake scars. They have microdenticulation in the forked area and along the lateral margins which stops above the haft area. Some later versions have ripple flaking on both faces. The shape of the fork is a chronological indicator. See Chapter 5.1 for more details.

Rhomboid tools: Rhomboid tools are elongated bifacially thinned tools that can be extremely long, over 40 cm (Baumgartel 1960:32). They are also quite thin, especially considering their length. They are symmetrical along the vertical and horizontal axes. Rhomboid tools are slightly wider in the center, usually with a rounded corner in the middle of each lateral edge. The tips are usually rounded rather than pointed. They have fine microdenticulations all along the edges except for in the hafting area, which can have slightly courser retouch. See Chapter 5.1 for more details.

Dagger: A dagger is an elongated symmetrical thin bifacial tool. Similar to rhomboid tools they are smaller, with a pointed tip, and convex lateral margins without the marked corner of rhomboids. A rarer kind of tool, they are not always included among Predynastic tool type lists. Examples: Figure 6.7; Petrie and Quibell 1896:Pl 72 (56).

\section{KNIVES}

Bifacial knives: Following Midant-Reynes and Prost (2002:374) and Holmes (1989:405) bifacial knives are asymmetrical bifacially thinned tools with a straight or slightly 
convex back. The working edge is slightly convex and curves back to meet the back forming an acute or right angle. The proximal end can be rounded or have a handle. Holmes (ibid) notes that the handle is often formed by indenting from one side only and that truly 'handled' knifes are not common until the Early Dynastic period. The Predynastic bifacial knives are generally thin and the working edge can have fine microdenticulation. They can have edge retouch that follows the same "Seitenbezogenheit" pattern described for blade knives (Schmidt 1989:85,1992a:32, 1996:281-282, 1999:32). The forms and quality of the thinning and retouch can vary widely. Kabacinski 2012 differentiated bifacial knives based on their width. See Chapter 5.1 for more details.

Bifacial sickles: Bifacial sickles are fully bifacially thinned tools that have denticulations along one edge. Some can be symmetrical, but with denticulations along only one margin, while on others the denticulated side can be more convex than the opposite margin. The three shapes are bipointed, pointed on one end and squared on the other, or squared on both ends. The bipointed ones are often larger. These can be differentiated form bifacial knives with denticulations because they lack the rounded or handled proximal end of knives, and the denticulations on bifacial sickles are larger than those on the knives. Bifacial sickles were probably used for reaping or for sawing plant material as they often have sickle sheen. See Chapter 5.1 for more details.

Ripple-flaked knife: Ripple flaked knives follow the same shape as described above fro bifacial knives, but they have a very distinctive type of ripple flaking on one face, and a ground surface on the other face. The ripple-flaking is extremely uniform flat parallel pressure flaking carried out from each lateral edge and meeting in the center of the face. Ripple flaked knives were hafted, and some have small tangs for hafting. See Chapter 5.1; Holmes 1989; Kelterborn 1984; Midant-Reynes and Tixier 1981; Midant-Reynes 1987 for more details.

\section{OTHER}

Eccentrics: Eccentrics are flaked stone items which have been shaped into images of figures, usually animals but also including humans, arrows, and unidentifiable figures. Many examples are fully bifacially retouched with very fine flaking, but edge retouched examples also exist. See Chapter 5.1; Friedman 2000; Hendrickx et al. 2003; for more details.

Unidentifiable tool fragment: A fragment of a tool that is not preserved to a sufficient degree for the appropriate type to be identified. The retouch description, such as unifacial edge retouch or bifacial retouch, is recorded in the notes. 


\section{Information on Cores}

\section{Core platforms}

The core platform category indicates the number of flaking platforms and their relationship to each other (Close 1977:54-56).

Single: The core has at least 3 scars going in the same direction off of a single platform. Opposite platform-one face: Scars initiate from two platforms, at opposite ends of a core, on the same face.

Opposite platform-different face: Scars initiate from two platforms at opposite ends of a core, but on different faces.

90-degree: Scars initiate from two platforms, roughly perpendicular to one another, on the same face.

90-degree-different face: Scars initiate from two platforms, roughly perpendicular to one another, but on different faces.

Multiplatform: Scars initiate from three or more platforms, in many areas of the core (unpatterned), going in many directions.

Discoidal: Discoidal cores have a circular outline and an asymmetrical biconvex section. Flakes were removed by centripetally directed blows (Inizan et al 1999:61).

Bipolar: Flakes were removed by placing the core on a hard surface (anvil) and striking it with a hammer, usually leaving flake scars on two opposite ends of the core, and sometimes on two faces. There tends to be and crushing on the platforms. The flakes scars may be short and wide.

Initial: A nodule or naturally fractured stone fragment with only one or two flakes scars. Other: The scar patterning on the core is distinctive, but does not fit any of the above categories.

Indeterminate: There are flake scars present, but the pattern cannot be determined for some reason, for example if the piece is a core fragment.

N/A: Not applicable.

\section{Core removal type}

Core removal type describes the kind of detached piece removed from the core, based on the patterning and length of flake scars, and the shape of the scar for the last piece removed.

Flake: Flake removals.

Blade: Blade removals.

Bladelet: Bladelet removals.

Blade/Bladelet: Indeterminate the removals were either blades or bladelets, but it is not possible to determine which.

Mixed: The core has scars that are like from different kinds of removals, such as flakes and blades. 
Other: The scar patterning on the core is distinctive, but does not fit any of the above categories.

Indeterminate: The type of removal cannot be determined, for instance if the core is a fragment.

N/A: Not applicable.

\section{Extent of working}

Extent of working describes the degree of core exploitation, based on Holmes (1989:424).

One area: The removals are confined to a small area, and do not cover the whole face of the core.

One face: The removals cover most or all of one face of the core.

Two faces: The removals cover most of two faces, which can be adjacent or not

Around an edge: This category is mainly for single platform cores where the removals cover multiple faces, but the faces are not distinct facets. The core removals go around the edge of a platform, such as with pyramidal cores.

Entirety: The removals cover all faces, or more than $75 \%$ of the core.

Exhausted: Any core for which further exploitation does not seem readily feasible. The core may be small, and the platform angles may be inappropriate for more removals. Other: The scar patterning on the core is distinctive, but does not fit any of the above

categories. Indeterminate: The extent of working cannot be determined, for instance if the core is a fragment.

N/A: Not applicable. 


\section{Metric Data}

All metric information was entered with digital calipers that connect directly to the computer, making for fast and accurate metric data collection.

\section{Length}

The length of tools and debitage was measured as the maximum length along the axis orientation. For debitage that was from the point of percussion to the most distal point following flake orientation (Debénath and Dibble 1994:17-18). For tools the maximum length followed the longitudinal tool orientation if flake characteristics were not identifiable.

\section{Width}

The width of tools and debitage was measured as the maximum width perpendicular to the length (Debénath and Dibble 1994:17-18). This measurement could be at any point along the flake, not just in the middle

\section{Thickness}

The thickness of tools and debitage was measured as the maximum thickness perpendicular to the plane of flaking, or for tools without flake characteristics (such as bifacial tools), perpendicular to the plane of length and width measurements.

\section{Platform width}

For any piece of debitage or tool retaining a platform, the width of the platform was measured as the maximum distance of the platform following the direction of one lateral margin to the other. In other words, in the same direction as flake width.

\section{Platform thickness}

For any piece of debitage or tool retaining a platform, the thickness of the platform was measured as the maximum distance from the interior (ventral) side of the platform to the exterior (dorsal) side of the platform at the thickest part of the platform. In other words, in the same direction as flake thickness. 


\section{Core length}

The core length was measured as the maximum distance along the face (or primary face) of the core, usually approximately perpendicular to the platform and parallel to the flaking face (Andrefsky 2005:145-146; Holmes 1989:420).

\section{Core width}

The core width was measured as the maximum width perpendicular to the core length.

\section{Core thickness}

The core thickness was measured as the maximum thickness perpendicular to the plane of core length-width measurements. Usually the thickness back from the main flaking face.

\section{Weight}

The weight of all lithic artifacts was measured in grams. 


\section{Works Cited}

Adams, Barbara. 1974. Ancient Hierakonpolis. Warminster: Aris \& Phillips.

. 2000a. Excavations in the Locality 6 cemetery at Hierakonpolis, 1979-1985. Oxford, England: Archaeopress.

— 2000b. "Some problems solved in the locality 6 cemetery." Nekhen News. 12: 4-6.

—. 2001. "Locality 6 in 2000: Amazing revelations." Nekhen News. 13: 4-7.

Adams, Ellen. 2004. "Power and ritual in Neopalatial Crete: a regional comparison". World Archaeology. 36 (1): 26-42.

Adams, Matthew D. 1999. "Abydos, North." In Encyclopedia of the archaeology of ancient Egypt, edited by Kathryn A. Bard, 106-109. London: Routledge.

Adams, Matthew D., and David O'Connor. 2003. " The royal mortuary enclosures of Abydos and Hierakonpolis." In The treasures of the pyramids. edited by Z. Hawass, L. Accomazzo, V. Manferto De Fabianis, and P. Piacco, 78-85. Cairo: The Amercian University in Cairo Press.

Addington, Lucile R. 1986. Lithic illustration: drawing flaked stone artifacts for publication. Chicago: University of Chicago Press.

Ahler, Stanley A. 1983. "Heat Treatment of Knife River Flint". Lithic Technology. 12 (1): 1-8.

Aksamit, J. 1992. "Petrie's type D 46 D and remarks on the production and decoration of Predynastic Decorated pottery." In Cahiers de la Céramique Egyptienne, edited by P. Ballet, 17-21. Cairo: Institut français d'archéologie orientale.

Allen, James P. 2002 The Heqanakht Papyri. New York: Metropolitan Museum of Art.

Amélineau, Emile Clément 1899. Les Nouvelles Fouilles d'Abydos 1895-1899 I. Paris: Ernest Leroux.

Anderson, David. 2006. "Power and competition in the Upper Egyptian Predynastic; a view from the Predynastic settlement at el Mahasna, Egypt.” Thesis (PhD), University of Pittsburgh.

_ 2007. "Zoomorphic Figurines from the Predynastic Settlement at el-El-Mahâsna.” In The archaeology and art of ancient Egypt: essays in honor of David B. O'Connor, Hawass, Z.A. and Richards, J.E., eds. Cairo: Conseil Suprême des Antiquités de l'Égypte. pp. 33-54.

- 2011. Evidence for Early Ritual Activity in the Predynastic Settlement at Mahasna. In Egypt at its origins 3: proceedings of the third international conference "Origin of the state: predynastic and early dynastic Egypt", London, 27th July-1st August 2008, edited by R.F. Friedman, and P. N. Fiske, 329. Leuven: Peeters.

Amelineau, Emile Clément. 1899. Les Nouvelles fouilles d'Abydos I (1895-1899). Paris: Ernest Leroux. 
Anderson, Wendy. 1992. "Badarian Burials: Evidence of Social Inequality in Middle Egypt During the Early Predynastic Era". Journal of the American Research Center in Egypt. 29: 51-66.

Andrefsky, William. 2005. Lithics: Macroscopic Approaches to Analysis. Cambridge [England]; New York: Cambridge University Press.

Arnold, Jeanne E., and Ann Munns. 1994. "Independent or Attached Specialization: The Organization of Shell Bead Production in California." Journal of Field Archaeology. 21:473-489.

Arnold, Dean E. and Alvaro L. Nieves. 1992. "Factors Affecting Ceramic Standardization." In Ceramic production and distribution: an integrated approach, edited by G.J. Bey, 93-113. Boulder u.a: Westview Press.

Asselberghs, Henri. 1961. Chaos en beheersing; documenten uit aeneolithisch Egypte. Leiden: E.J. Brill.

Aufrère, Sydney. 1983. "Caractères Principaux et Origine Divine des Minéraux." Revue d'Égyptologie. 34:3-21.

Austin, Robert J. 2015. "The ritual uses of lithic raw materials during the Woodland Period, Fort Center, southern Florida". Journal of Field Archaeology. 40 (4): 413-427.

Ayrton, Edward Russell, and W. L. S. Loat. 1911. Pre-dynastic cemetery at El Mahasna. London: Egypt Exploration Fund.

Baba, Masahiro. 2005. "Understanding the HK potters: Experimental firings."Nekhen News. 17: 20-21.

—. 2006. "The pottery kilns at HK11C revisited." Nekhen News. 18:19.

—. 2007. " Okey-dokey ! Big pots and more kilns at HK11C." Nekhen News. 19:26-27.

_ 2008a. "Pottery-making tools: Worked sherds from HK11C square B4, Hierakonpolis." In Egypt at its Origins 2: Proceedings of the International Conference "Origin of the State. Predynastic and Early Dynastic Egypt", Toulouse (France), 5th - 8th September 2005. Edited by B. Midant-Reynes, 720. Leuven: Peeters.

. 2008b. "More big pots: HK11C square B5 in 2008." Nekhen News. 20: 18-19.

. 2009a. "HK11C square B4-B5: Kiln site." In Friedman, R.F. et al. "Report on the 2006-2007 season of the Hierakonpolis Expedition." Annales du Service des Antiquites de L'Egypte, Cairo. 83:199201.

2009b. "One more big pot: HK11C operation B in 2009." Nekhen News. 21: 23-24.

. 2010. "Hitting the wall: Testing anomalies at HK11C." Nekhen News. 22: 20-21.

. 2011. "Up against the walls at HK11C." Nekhen News. 23: 22-24.

. 2012. "A harvest of potatoes: Excavation at HK11C in 2012." Nekhen News. 24: 10-11.

. 2013. "Fishy business ? Excavations at HK11C in 2013." Nekhen News. 25: 12-13. 
Baines, John. 1985. "Color Terminology and Color Classifications: Ancient Egyptian Color Terminology and Polychromy." American Anthropologist. 87:282-297.

— 1993. "Symbolic roles of canine figures on early monuments." Archéo-Nil. 3: 57-74.

Baines, John, and Peter Lacovara. 2002. "Burial and the dead in ancient Egyptian society: respect, formalism, neglect." Journal of Social Archaeology. 2 (1): 5-36.

Bard, Kathryn. 1988. A Quantitative Analysis of the Predynastic Burials in Armant Cemetery 14001500. The Journal of Egyptian Archaeology. 74: 39-55.

. 1994. From farmers to pharaohs: mortuary evidence for the rise of complex society in Egypt. Sheffield, England: Sheffield Academic Press.

Barkai, Ran. 2011. "PPNA stone and flint axes as cultural markers: technological, functional, and symbolic aspects." In The state of the stone: terminologies, continuities and contexts in Near Eastern lithics : proceedings of the sixth PPN Conference on Chipped and Ground Stone Artefacts in the Near East, Manchester 3rd-5th March 2008, edited by Elizabeth Healey, Stuart Campbell, and Osamu Maeda, 443-448. Berlin: Ex Oriente.

Barket, Theresa M., and Robert M. Yohe. 2011. "A technological evaluation of the flint blade-core reduction sequence at Wadi el-Sheikh, middle Egypt.” Lithic Technology. 36 (1): 27-38.

Barocas, Claudio, Rodolfo Fattovich, and Maurizio Tosi. 1989. "The Oriental Institute of Naples expedition to Petrie's South Town (Upper Egypt), 1977-1983: An interim report." In Late prehistory of the Nile Basin and the Sahara, edited by Lech Krzyżaniak and Michał Kobusiewicz, 295-301. Poznań: Poznań Archaeological Museum.

Baumgartel, Elise J. 1960. The cultures of prehistoric Egypt II. London: Published on behalf of the Griffith Institute, Ashmolean Museum.

—. 1970. Petrie's Naqada excavation: a supplement. London: Quaritch.

Bavay, Laurant, and Stan Hendrickx. 2000. [Notices d'exposition.] In Pierres égyptiennes ...: chefsd'oeuvre pour l'éternité, edited by Christina Karlshausen, Thierry de Putter, and Johan Buelens. : 118120, 128-135, 139-145. Mons: Faculté polytechnique de Mons.

Behrmann, A. 1989. "Das Nilpferd in der Vorstellungswelt der Alten Ägypter. Teil I, Katalog." Europaïsche Hochschulschriften. Reihe XXXVIII. Archäologie. Bd. 22. Frankfort/Main.

Bavay, Laurant, Thierry de Putter, Barbara Adams, Jacques Navez, and Luc André. 2000. "The origin of obsidian in Predynastic and Early Dynastic Upper Egypt." Mitteilungen des Deutschen Archäologischen Instituts, Abteilung Kairo. 56: 5-20.

Bell, Catherine M. 1992. Ritual theory, ritual practice. New York u.a: Oxford Univ. Press. 1997. Ritual: perspectives and dimensions. New York, N.Y.: Oxford University Press. 


\footnotetext{
- 2007. Response: Defining the need for a definition. In The archaeology of ritual, edited by K. Evangelos, 277-288. Los Angeles: Cotsen Institute of Archaeology, University of California, Los Angeles.
}

Berde, Stuart. 1983. "The Impact of Colonization on the Economy of Panaeti." In The Kula: New Perspectives on Massim Exchange,edited by J.W. Leach, and E. Leach, 431-443. Cambridge: Cambridge University Press.

Berman, Lawrence Michael, and Kenneth J. Bohač. 1999. "The Predynastic and Early Dynastic periods." In Catalogue of Egyptian art: the Cleveland Museum of Art, edited by Lawrence Michael Berman, and Kenneth J. Bohač, 77-121. Cleveland, OH: Cleveland Museum of Art.

Bestock, Laurel D. 2008. "The Early Dynastic funerary enclosures of Abydos." Archéo-Nil. 18: 42-59.

Bienkowski, Piotr, and Angela Tooley. 1995. Gifts of the Nile: Ancient Egyptian arts and crafts in Liverpool Museum. London: HMSO.

- 2009. The development of royal funerary cult at Abydos two funerary enclosures from the reign of Aha. Wiesbaden: O. Harrassowitz.

Bietak, Manfred. 1996. Haus und Palast im alten Ägypten. Wien: Verlag der Österreichischen Akademie der Wissenschaften.

_ 2010. "Houses, Palaces, and the Development of Social Structure in Avaris." In Cities and Urbanism in Ancient Egypt, edited by M. Bietak, E. Czerny, and I. Forstner-Müller, 11-68. Vienna: Verlag der österreichischen Akademie der Wissenschaften.

Blackman, M. James., Gil J. Stein, and Pamela B. Vandiver. 1993. “The Standardization Hypothesis and Ceramic Mass Production: Technological, Compositional, and Metric Indexes of Craft Specialization at Tell Leilan, Syria.” American Antiquity 58 (1):60-80.

Blanton, Richard E., et al. 1982. Monte Alban's hinterland, part I: the prehispanic settlement patterns of the central and southern parts of the valley of Oaxaca, Mexico. Ann Arbor: University of Michigan, Museum of Anthropology.

Blanton, Richard E., Gary M. Feinman, Stephen A. Kowalewski, and Peter N. Peregrine. 1996. "A DualProcessual Theory for the Evolution of Mesoamerican Civilization." Current Anthropology. 37 (1): 114.

Borradaile, G., S. Kissin, J. Stewart, W. Ross, and T. Werner. 1993. "Magnetic and Optical Methods for Detecting the Heat Treatment of Chert.” Journal of Archaeological Science 20 (1): 57-66.

Bradbury, Andrew. 2010. "Bipolar Reduction Experiments and the Examination of Middle Archaic Bipolar Technologies in West-Central Illinois". North American Archaeologist. 31 (1): 67.

Bradley, Bruce A. 1972. "Predynastic Egyptian flint implements - An inductive technological sequence." Newsletter of Lithic Technology, 1,3: 2-5.

Bradley, Richard. 2005. Ritual and domestic life in prehistoric Europe. London: Routledge. 
Braun, Eliot. 2011. "Early interaction between peoples of the Nile valley and the Southern Levant." In Before the pyramids. The origins of Egyptian civilization. OIMP 33, edited by E. Teeter, 104-122. Chicago: University of Chicago Press.

- 2014. "Reflections on the context of a late dynasty 0 Egyptian colony in the southern Levant: Interpreting some evidence of Nilotic material culture at selected sites in the southern Levant (ca. 3150 BCE - ca. 2950 BCE)." In The Nile Delta as a centre of cultural interactions between upper Egypt and the southern Levant in the 4th millenium BC, edited by Agnieszka Maczyńska, 37-56. Poznań: Poznań Archaeological Museum.

Briois, François. 2002. "L'Industrie Lithique." In "Travaux de l'Institut Français d'Archéologie Orientale en 2001-2002. Adaïma," edited by B.Mathieu. Bulletin de l'Institute Français d'Archéologie Orientale du Caire. 102:457-461.

Briois, François, and Midant-Reynes, Béatrix. 2008. "Lithic Industries from Adaima. Between Farmers and Craftsmen." In Egypt at its Origins 2: Proceedings of the International Conference "Origin of the State. Predynastic and Early Dynastic Egypt", Toulouse (France), 5th - 8th September 2005. Edited by B. Midant-Reynes, 21-31. Leuven: Peeters.

—. 2014. "Sur les traces de Georg August Schweinfurth. Les sites d'exploitation du silex d'époque pharaonique dans le massif du Galâlâ nord (Désert Oriental)". Bulletin de l'Institute Français d'Archéologie Orientale du Caire. 114: 73-98.

. 2015. "Wadi Sannur." In: Rapport d'activite' 2014-2015. Supplément au Bulletin de l'Institute Français d'Archéologie Orientale du Caire. 115: 49-55.

Brumfiel, Elizabeth, 1992. "Distinguished Lecture in Archeology: Breaking and Entering the Ecosystem Gender, Class, and Faction Steal the Show." American Anthropologist. 94 (3):551-567.

. 1987. "Elite and utilitarian crafts in the Aztec state." In Specialization, Exchange and Complex Societies, edited by E. Brumfiel and T. Earle, 102-118. Cambridge: Cambridge University Press.

Brumfiel, Elizabeth, and Timothy Earle. 1987. "Specialization, Exchange and Complex Societies: An Introduction." In Specialization, Exchange and Complex Societies, edited by E. Brumfiel and T. Earle, 1-19. Cambridge: Cambridge University Press.

Brumfiel, Elizabeth, and Fox, John W. 1994. Factional competition and political development in the New World. New directions in archaeology. Cambridge: Cambridge University Press.

Brunton, Guy. 1935. "Pesesh-kef amulets" Annales du Service des Antiquités de l'Égypte 35:21-217.

—. 1937. Mostagedda and the Tasian culture. London: B. Quaritch Ltd.

_. 1948. Matmar. London: B. Quaritch.

Brunton, Guy, and Gertrude Caton-Thompson. 1928. The Badarian civilisation and predynastic remains near Badari. London: British school of archaeology in Egypt.

Brunton, Guy, and G. M. Morant. 1937. Mostagedda and the Tasian culture. London: B. Quaritch Ltd. 
Caton-Thompson, Gertrude, and E. W. Gardner. 1934. The desert Fayum. [London]: Royal Anthropological Institute of Great Britain and Ireland.

Bubenzer, Olaf, and Riemer, Heiko. 2007. "Holocene climatic change and human settlement between the central Sahara and the Nile Valley: Archaeological and geomorphological results." Geoarchaeology. 22 (6): 607.

Buchez, Nathalie. 2011. "Adaïma (Upper Egypt): The stages of state development from the point of view of a 'village community'." In Egypt at its origins 3: proceedings of the third international conference "Origin of the state : predynastic and early dynastic Egypt", London, 27th July-1st August 2008., edited by Renée F. Friedman and Peter N. Fiske, 31-40. Leuven: Peeters.

_ 2011b. "A reconsideration of Predynastic chronology: The contribution of Adaïma." In Egypt at its origins 3: proceedings of the third international conference "Origin of the state : predynastic and early dynastic Egypt", London, 27th July-1st August 2008., edited by Renée F. Friedman and Peter N. Fiske, 939-951. Leuven: Peeters.

—_. 2011c. "Chalcolithique final (ou moyen ?), Nagada IIC-D/IIIA." Archéo-Nil. 21: 51-64.

Budge, E. A. Wallis. 1909. A guide to the Egyptian collections in the British Museum. London: Printed by order of the Trustees.

Butzer, Karl W. 2002. Early hydraulic civilization in Egypt: a study in cultural ecology. Prehistoric Archaeology and Ecology. Chicago: University of Chicago press.

Cahn, Jean-David. 2005. "Krokodil." In Tiere und Mischwezen V. Katalog 17, edited by Jean-David Cahn, $n^{\circ}$ 4. Basel: Jean-David Cahn.

Caneva, Isabella, Marcella Frangipane, and Alba Palmieri. 1989. " Recent excavations at Maadi (Egypt)." In Late prehistory of the Nile Basin and the Sahara, edited by Lech Krzyżaniak and Michał Kobusiewicz, 287-293. Poznań: Poznań Archaeological Museum.

Capart, Jean. 1905. Primitive art in Egypt. London: H. Grevel.

Carter, Tristan. 2007. "The Theatrics of Technology: Consuming Obsidian in the Early Cycladic Burial Arena." In Rethinking Craft Specialization in Complex Societies: Archaeological Analyses of the Social Meaning of Production, edited by Z. X. Hruby and R. K. Flad, 88-107. Archeological Papers of the American Anthropological Association 17. Arlington, Va: American Anthropological Association.

Casini, Maria. 1988. "Figurine in selce, ritagliate a forma di animali e loro probabile valore totemico." Bollettino del Centro Camuno di Studi Preistorici. 24: 127-132.

Charlton, Thomas H., Deborah L. Nichols, and Cynthia Otis Charlton. 1991. "Aztec Craft Production and Specialization: Archaeological Evidence from the City-State of Otumba, Mexico.” World Archaeology. 23 (1): 98-114.

Chazan Michael, and Mark Lehner. 1990. "An Ancient Analogy : Pot Baked Bread in Ancient Egypt and Mesopotamia." Paléorient. 16(2): 21-35.

Childe, V. Gordon. 1950. 'The Urban Revolution.’ Town Planning Review. 21:3-17. 
Chłodnicki, Marek, Krzysztof M. Ciałowicz, and A. Mączyńska. 2012. Tell el-Farkha I: excavations 1998-2011. Poznań : Poznań Archaeological Museum.

Ciałowicz, Krzysztof M. 1990. "L'étude des armes égyptiennes dans les époques prédynastique et archaïque. La typologie des pointes de flèches et de javelots". Folia Orientalia, 27: 63-79.

1992. "La composition, le sens et la symbolique des scenes zoomorphes predynastiques en relief: les manches de couteaux". In Followers of Horus: Studies Dedicated to Michael Allen Hoffman, 19441990, edited by Friedman, Renée F., and Barbara Adams, 247-258. Oxford [England]: Oxbow Books.

2007. Ivory and gold. Beginnings of the Egyptian art. Discoveries in Tell el-Farkha (the Nile Delta). Poznań: Poznań Prehistoric Society.

2012."Early Egyptian objects of art." In Tell el-Farkha I: excavations 1998-2011, edited by M. Chłodnicki, K. M. Ciałowicz, and A. Mączyńska. 201-243. Poznań : Poznań Archaeological Museum.

Cichowski, K. 2008. "The Brewery Complex from Tel el-Farkha. Archaeological aspects of the discovery." In Egypt at its origins 2: proceedings of the International Conference "Origin of the State. Predynastic and Early Dynastic Egypt",edited by B. Midant-Reynes, 33-40. Leuven:Peeters.

Claes, Wouter, Stan Hendrickx, Ann Devillers, Elizabeth Hart, Karin Kindermann, Morgan De Dapper, and Salima Ikram. 2014. "From the early Old Kingdom to the Badarian. Preliminary report on the 2012 excavation campaign in the settlement area of Elkab." In: The Nile Delta as a Centre of Cultural Interactions between the Upper Egypt and the Southern Levant in the $4^{\text {th }}$ Millennium BC, edited by A. Mączyńska, 13:73-93. Studies of African Archaeology. Poznań: Poznań Archaeological Museum.

Clark, John Desmond, Preston S. Staley, and James L. Phillips, and. 1974. "Interpretations of Prehistoric Technology from Ancient Egyptian and other Sources. Part I : Ancient Egyptian Bows and Arrows and their relevance for African Prehistory". Paléorient. 2 (2): 323-388.

Clark, John E. 2012. "Stoneworkers’ Approaches to Replicating Prismatic Blades.” In The emergence of pressure blade making: from origin to modern experimentation, edited by P. M. Desrosiers, 43-135. New York: Springer.

Clark, John E., and Michael Blake. 1994. "The Power of Prestige: Competitive Generosity and the Emergence of Rank Societies in Lowland Mesoamerica." In Factional Competition and Political Development in the New World, edited by E. M. Brumfiel, and J. W. Fox, 258:28. Cambridge: Cambridge University Press.

Clark, John E., and William J. Parry. 1990. Craft Specialization and Cultural Complexity. Research in Economic Anthropology 12:289-346.

Cleyet-Merle, J.- J. and Vallet F. 1982. "Egypte." In Catalogue sommaire illustré des collections du Musée des Antiquités Nationales de Saint-Germain-en-Laye. 1 : Archéologie comparée, 68-165. Paris: Éd. de la Réunion des Musées Nationaux.

Close, Angela E. 1980. "Methods of analysis of lithic artifacts.” In Loaves and fishes: the prehistory of Wadi Kubbaniya, edited by F. Wendorf, R. Schild, \& A. E. Close, 49-54. Dept. of Anthropology, Institute for the Study of Earth and Man, Southern Methodist University. 
Costin, Cathy L. 1991. "Craft Specialization: Issues Defining, Documenting, and Explaining the Organization of Production.” In Archaeological Method and Theory Vol. 3, edited by M. Schiffer, 156. Tuscon: University of Arizona Press.

. 2001. “Craft Production Systems.” In Archaeology at the Millennium: a Sourcebook, edited by G.M. Feinman and T.D. Price, 273-327. New York: Kluwer Academic/Plenum.

_ 2007. "Thinking about Production: Phenomenological Classification and Lexical Semantics." In Rethinking Craft Specialization in Complex Societes: archeological analyses of the social meaning of production, edited by Z. X. Hruby, and R. K. Flad, 143-162. Arlington, VA: American Anthropological Association.

.2015. "Craft Specialization." In The International Encyclopedia of Human Sexuality, edited by P. Whelehan and A. Bolin, Malden, MA: Wiley-Blackwell.

Costin, Cathy L., and Melissa B. Hagstrum. 1995. "Standardization, Labor Investment, Skill, and the Organization of Ceramic Production in Late Prehispanic Highland Peru.” American Antiquity 60 (4): 619-39.

Crabtree, Don E. 1972. An introduction to flintworking. Pocatello: Idaho State University Museum.

Crabtree, Don E. and Butler, B. Robert. 1964. "Notes on Experiments in Flintknapping: 1- Heat Treatment of Silica Materials.” Tebiwa 7 (1): 1-6.

Crubézy, Éric, Thierry Janin, and Béatrix Midant-Reynes. 2002. Adaïma 2. La nécropole prédynastique. Le Caire: Institut Français d'Archéologie Orientale.

Currelly, Charles Trick. 1913. Catalogue générale des antiquités égyptiennes du musée du Caire, nos. 63001-64906. Stone Implements. Cairo.

Dalgaard, Peter. 2002. Introductory statistics with R. New York: Springer.

Damon, Frederick, and Roy Wagner. 1989. Death Rituals and Life in the Societies of the Kula Ring. DeKalb: Northern Illinois University Press.

Darnell, Deborah. 2007. "The Deserts." InThe Egyptian World, edited by T.A. Wilkinson, 2948. Routledge, London:

— 2009. "Iconographic Attraction, Iconographic Syntax, and Tableaux of Royal Ritual Power in the Pre-and Proto-Dynastic Rock Inscriptions of the Theban Western Desert." Archéo-Nil. 19: 83-107.

Darnell, John Coleman. 2015. "The Early Hieroglyphic Annotation in the Nag el-Hamdulab. Rock Art Tableaux, and the Following of Horus in the Northwest Hinterland of Aswan." Archéo-Nil. 25:19-43.

Davis, Whitney. 1983. "Cemetery T at Naqada." Mitteilungen des Deutschen Archäologischen Instituts, Abteilung Kairo. 39: 17-29. 
-1992. Masking the blow: the scene of representation in late prehistoric Egyptian art. Berkeley: University of California Press.

Debénath, André, and Harold L. Dibble. 1994. Handbook of paleolithic typology. Philadelphia: University Museum, University of Pennsylvania.

Debono, Fernand. 1971. "Recherches préhistoriques dans la région d'Esna." Bulletin de l'Institute Français d'Archéologie Orientale du Caire. 69:246-251.

Debono, Fernand, and Bodil Mortensen. 1992. El Omari: a Neolithic settlement and other sites in the vicinity of Wadi Hof, Helwan. Mainz am Rhein: P. von Zabern.

Dee, M., David Wengrow, Andrew Shortland, Alice Stevenson, Fiona Brock, Linus Girdland Flink, and Christopher Bronk Ramsey. 2013. "An absolute chronology for early Egypt using radiocarbon dating and Bayesian statistical modelling." Proceedings. Mathematical, Physical, and Engineering Sciences. 469 (2159).

DeForest, David Scott. 2006. “Assessing “lithic Sound” to Predict a Rock’s Ease of Flaking.” MA thesis, University of Missouri-Columbia.

Delange, Elisabeth. 2000. Le couteau dit "du Djebel el-Arak". Dossiers de l'Archéologie. 257: 52-59.

— 2009. Le poignard égyptien dit "du Gebel el-Arak". Paris: Somogy.

Derriks, Claire. 2012. "Flint in the shape of a hippopotamus." In Ancient Egypt: masterpieces from collectors and collections : 10th edition of the Brussels Ancient Art Fair, BAAF, edied by J. Billen, 2829. Brussels: Brussels Ancient Art Fair.

Desrosiers, Pierre M. 2012. The emergence of pressure blade making: from origin to modern experimentation. New York: Springer.

Dibble, Harold L. 1995. Middle paleolithic scraper reduction: Background, clarification, and review of the evidence to date. Journal of Archaeological Method and Theory. 2 (4): 299-368.

Dibble, Harold L., Utsav A. Schurmans, Radu P. Iovita, and Michael V. McLaughlin. 2005. “The Measurement and Interpretation of Cortex in Lithic Assemblages.” American Antiquity 70 (3):545-60.

Diehl, Michael William. 2000. Hierarchies in action: Cui bono? Carbondale: Center for Archaeological Investigations, Southern Illinois University, Carbondale.

Dietler, M. 2006. "Alcohol: Anthropological/Archaeological Perspective." Annual Review of Anthropology. 35: 229-249.

Diez, David M., Christopher D. Barr, and Mine Çetinkaya-Rundel. 2012. OpenIntro statistics. Lexington, KY: CreateSpace.

Di Pietro, Grazia Antonella. 2011. Miscellaneous artefacts from Zawaydah (Petrie's South Town, Naqada). In Egypt at its origins 3: proceedings of the third international conference "Origin of the state : predynastic and early dynastic Egypt", London, 27th July-1st August 2008., edited by Renée F. Friedman and Peter N. Fiske, 59-79. Leuven: Peeters. 
Domanski, Marian, and John Webb. 1992. "Effect of heat treatment on siliceous rocks used in prehistoric lithic technology". Journal of Archaeological Science. 19 (6): 601-614.

—. 2007. “A Review of Heat Treatment Research.” Lithic Technology 32 (2): 153-94.

Domanski, Marian, John Webb, Robert Glaisher, Jan Gurba, Jerzy Libera, and Anna Zakoscielna. 2009. "Heat Treatment of Polish Flints.” Journal of Archaeological Science 36: 1400-1408.

Douglas, Mary. 1958. "Raffia Cloth Distribution in the Lele Economy." Africa. 28:109-122.

Douglass, Matthew J., and Simon J. Holdaway. 2011. “Changing perspectives in Australian archaeology, part IV. Quantifying stone raw material size distributions: investigating cortex proportions in lithic assemblages from western New South Wales.” Technical Reports of the Australian Museum, Online. 23: 45-57.

Douglass, Matthew J., Simon J. Holdaway, Patricia C. Fanning, and Justin I. Shiner. 2008. "An Assessment and Archaeological Application of Cortex Measurement in Lithic Assemblages." American Antiquity. 73 (3):513-26.

Dougherty, Sean P. 2004. "A little more off the top." Nekhen News. 16: 11-12.

Dreyer, Günter. 1986. Elephantine VIII: der Tempel der Satet : die Funde der Frühzeit und des alten Reiches. Mainz am Rhein: Zabern.

. 1992. "Recent discoveries in the U-cemetery at Abydos." In The Nile Delta in Transition 4th 3rd Millennium B.C.: Proceedings of the Seminar Held in Cairo, 21-24 October 1990, at the Netherlands Institut, edited by E. C. M. Van den Brink, 293-300. Tel Aviv, Israel: Israel Exploration Society.

. 1999. "Motive und Datierung der dekorierten prädynastischen Messergriffe.” In L'art de l'Ancien Empire égyptien: actes du colloque organisé au Musée du Louvre par le Service culturel les 3 et 4 avril 1998, edited by Ziegler, Christiane, Nadine Palayret, and Michel Baud, 195-226. Paris: Documentation française.

2005-2006." Annales du Service des Antiquités de l'Égypte. 82: 49-59.

. 2009. "Report on the 21st campaign of reexamining the royal tombs of Umm el-Qaab at Abydos 2006/2007." Annales du Service des Antiquités de l'Égypte. 83: 165-175.

. 2010. "Report on the 22nd campaign of reexamining the royal tombs of Umm el-Qaab at Abydos 2007/2008." Annales du Service des Antiquités de l'Égypte. 84: 143-156.

Dreyer, Gunter, Eva-Maria Engel, Ulrich Hartung, Thomas Hikade, E. Christiana Köhler, and Frauke Pumpenmeier. 1996. " Umm el-Qaab. Nachuntersuchungen im frühzeitlichen Königsfriedhof 7./8. Vorbericht." Mitteilungen des Deutschen Archäologischen Instituts, Abteilung Kairo. 52: 11-81. 
Dreyer, Gunter, Ulrich Hartung, Thomas Hikade, E. Christiana Köhler, Vera Müller and Frauke Pumpenmeier. 1998. "Umm el-Qaab. Nachuntersuchungen im frühzeitlichen Königsfriedhof 9./10. Vorbericht." Mitteilungen des Deutschen Archäologischen Instituts, Abteilung Kairo. 54: 77-167.

Dreyer, Gunter, A. von den Driesch, Eva-Maria Engel, Rita Hartmann, Ulrich Hartung, Thomas Hikade, and others. 2000. "Umm el-Qaab. Nachuntersuchungen im frühzeitlichen Königsfriedhof 11./12. Vorbericht." Mitteilungen des Deutschen Archäologischen Instituts, Abteilung Kairo. 56 : 43-129.

Dreyer, Gunter, Rita Hartmann, Ulrich Hartung, Thomas Hikade, Heidi Köpp, Claudia Lacher, Vera Müller, Andreas Nerlich, and Albert Zink. 2003. " Umm el-Qaab. Nachuntersuchungen im frühzeitlichen Königsfriedhof 13./14./15. Vorbericht." Mitteilungen des Deutschen Archäologischen Instituts, Abteilung Kairo. 59:67-138.

Dreyer, Gunter, A. Effland, U. Effland, Eva-Maria Engel, Rita Hartmann, D. Hartung, and C. Lacher. 2006. " Umm el-Qaab. Nachuntersuchungen im frühzeitlichen Königsfriedhof. 16./17./18. Vorbericht." Mitteilungen des Deutschen Archäologischen Instituts, Abteilung Kairo. 62: 67-129.

Dreyer, Gunter, A.I. Blöbaum, Eva-Maria Engel, Heidi Köpp, and Vera Müller. 2011. " Umm el-Qaab. Nachuntersuchungen im frühzeitlichen Königsfriedhof 19./20./21. Vorbericht." Mitteilungen des Deutschen Archäologischen Instituts, Abteilung Kairo. 67: 53-92.

Droogan, Julian. 2014. Religion, material culture, and archaeology. London: Bloomsbury Academic.

Droux, Xavier. 2008. "Heading west at HK6." Nekhen News. 20: 14-15.

. 2009. "The tale of tomb 30." Nekhen News. 21:15.

. 2011. "Where's the beef ? The surprise of tomb 49." Nekhen News. 23: 16-17.

— 2014. "A Tomb Fit for a King? The Discovery of Tomb 72 at HK6." Nekhen News. 26:4-7.

Droux, Xavier, and Renée F. Friedman. 2007. "The columned hall at HK6 and other wonders." Nekhen News. 19: 7-9.

Dunnell, R. C., P. T. McCutcheon, M. Ikeya, and S.Toyoda. 1994. "Heat treatment of Mill Creek and Dover cherts on the Malden Plain, southeast Missouri.” Journal of Archaeological Science 21:79-89.

Durkheim, Émile. [1915] 1965. The Elementary Forms of the Religious Life. New York: Free Press.

Ead Ltd. 1999. Charles Ede Ltd. Antiquities Catalogue168.

Earle, Timothy, K. 1977. "A reappraisal of redistribution: complex Hawaiian chiefdoms." In Exchange Systems in Prehistory, edited by T. Earle, and J. Ericson, 213-29. New York: Academic Press.

. 1997. How chiefs come to power: the political economy in prehistory. Stanford, Calif: Stanford University Press.

. 2002. Bronze Age economics: the beginnings of political economies. Boulder, Colorado: Westview Press. 
Eaton-Krauss, Marianne. 2008. "Embalming Caches". The Journal of Egyptian Archaeology. 94: 288293.

Ede, Ltd. 1999. "Egyptian Pre-dynastic flint animals." In Charles Ede Ltd. Antiquities, no.5. Catalogue 168. London.

Eerkens, Jelmer W., and Robert L. Bettinger. 2001. "Techniques for Assessing Standardization in Artifact Assemblages: Can We Scale Material Variability?" American Antiquity. 66 (3): 493.

Eggebrecht, Arne. 1973. Schlachtungsbräuche im alten Ägypten und ihre Wiedergabe im Flachbild bis zum Ende des Mittleren Reiches. München, Univ., Phil. Fak. II, Diss. v. 1966.

Eiwanger, Josef. 1999. "Merimde Beni-salame." In Encyclopedia of the archaeology of ancient Egypt, edited by Kathryn A. Bard, 603-608. London: Routledge.

El-Aref, Nevine, 2016. "Metropolis discovered in Abydos in the Upper Egypt's governate of Sohag." Ahram Online, November 24, 2016. Accessed on November 24, 2016.

http://english.ahram.org.eg/NewsContent/9/40/250686/Heritage/Ancient-Egypt/Metropolis-discoveredin-Abydos-in-the-Upper-Egypt.aspx

Elsasser, Albert B., and Vera-Mae Fredrickson. 1966. Ancient Egypt: an exhibition at the Robert H. Lowie Museum of Anthropology of the University of California, Berkeley, March 25-Oct. 23, 1966. Berkeley: University of California.

El-Sayed, Abdullah. 1979. "A Prehistoric cemetery in the Abydos area." Mitteilungen des Deutschen Archäologischen Instituts, Abteilung Kairo. 35: 249-301.

Engelbach, Reginald, and Battiscombe G. Gunn. 2006. Harageh. Worcester, U.K.: Yale Egyptology.

Evans, Robert K. 1978. "Early Craft Specialization: An Example for the Balkan Chalcolithic." In Social Archaeology: Beyond Subsistence and Dating, edited by C. L. Redman, et al., 113-129. New York: Academic Press.

Fahmy, Ahmed, G. 2003. "A fragrant mixture: Botanicals from the basket in B333." Nekhen News. 15: 20.

Fairservis, Walter Ashlin. 1971-2. "Preliminary report on the first two seasons at Hierakonpolis: part I". Journal of the American Research Center in Egypt. 9: 7-27.

Fattovich, Rodolfo, Sabina Malgora, Rosanna Pirelli, and Maurizio Tossi. 2007. "Explorations at South Town by the Naples Oriental Institute (1977-1986)." In The International Conference on heritage of Naqada and Qus region: Monastery of the Archangel Michael, Naqada, Egypt, 22-28 January 2007, edited by H. Hanna, 46-56. Cairo:

Feinman, Gary M., Kent G. Lightfoot, and Steadman Upham. 2000. "Political Hierarchies and Organizational Strategies in the Puebloan Southwest." American Antiquity.65 (3): 449-470.

Felgenhauer, Annette. 1996. Ägyptische und ägyptisierende Kunstwerke: vollständiger Katalog. Kassel: Staatliche Museen. 
Firth, C.M. 1912. The Archaeological Survey of Nubia : report for 1908-1909 2 2. Cairo: Government Press.

Flad, Rowan K., and Zachary X. Hruby. 2007. "Specialized Production in Archaeological Contexts: Rethinking Specialization, the Social Value of Products, and the Practice of Production.” In Rethinking Craft Specialization in Complex Societies: Archaeological Analyses of the Social Meaning of Production, edited by Z. X. Hruby and R. K. Flad, 1-19. Archeological Papers of the American Anthropological Association 17. Arlington, Va: American Anthropological Association.

Flannery, Kent, V. 1972. "The Cultural Evolution of Civilizations." Annual Review of Ecology and Systematics. 3:399-426.

Flenniken, J. Jeffrey, and Philip J. Wilke. 1989. "Typology, Technology, and Chronology of Great Basin Dart Points". American Anthropologist. 91 (1): 149-158.

Fogelin, Lars. 2007. "The Archaeology of Religious Ritual." Annual Review of Anthropology. 36: 55-71.

Fogelin, Lars, and Michael B. Schiffer. 2015. "Rites of passage and other rituals in the life histories of objects". Cambridge Archaeological Journal. 25 (4): 815-827.

Frankfort, H. "The cemeteries of Abydos: Work of the season 1925-1926. II. Description of tombs." Journal of Egyptian Archaeology. 16: 213-219.

Friedman, Renée F. 1994. Predynastic settlement ceramics of Upper Egypt: A comparative study of the ceramics of Hemamieh, Nagada and Hierakonpolis. Thesis (PhD), University of California, Berkely.

J. Spencer, 16-35. London: British Museum Press.

. 2000a. "Regional diversity in the Predynastic pottery of Upper Egyptian settlements." In Rece
research into the Stone Age of Northeastern Africa, edited by L. Krzyzaniak, K. Kroeper, and M. Kobusiewicz, 171-186. Poznań: Poznan Archeological Museum.

—. 2000b. "Figures in flint." Nekhen News. 12: 14.

— 2003. "A basket of delights: The 2003 excavations at HK43." Nekhen News. 15: 18-19.

—_. 2004a. "Farewell to HK43." Nekhen News. 16: 4-5.

—. 2004b. "He's got a knife ! Burial 412 at HK43." Nekhen News. 16: 8-9.

— 2004c. "Predynastic kilns at HK11C: One side of the story." Nekhen News. 16: 18-19.

—_. 2005. "Excavating Egypt's early kings." Nekhen News. 17: 4-6.

— 2006. "Bigger than an elephant. More surprises from HK6." Nekhen News. 18: 7-8.

_ 2007. "From pillar to post at Hierakonpolis." Nekhen News. 19:3.

—_. 2008a. "The cemeteries of Hierakonpolis." Archéo-Nil. 18: 8-29. 
. 2008b. "Excavating Egypt's early kings: Recent discoveries in the elite cemetery at Hierakonpolis." In Egypt at its Origins 2: Proceedings of the International Conference "Origin of the State. Predynastic and Early Dynastic Egypt", Toulouse (France), 5th - 8th September 2005, edited by B. Midant-Reynes, 1157-1194. Leuven: Peeters.

_. 2009a. "HK6 checklist." Nekhen News. 21: 7.

— 2009b. "Hierakonpolis Locality HK29A: The Predynastic Ceremonial Center Revisited.” Journal of the American Research Center in Egypt. 45: 79-103.

_ 2010. "The early royal cemetery at Hierakonpolis: An overview." In Recent discoveries and latest researches in Egyptology. Proceedings of the First Neapolitan Congress, edited by F. Raffaele, M. Nuzzolo, and I. Incordino, 67-86. Wiesbaden: Harrassowitz.

—_. 2011a. "Perseverance pays off: Answers from tomb 16 at HK6." Nekhen News. 23: 4-6.

—. 2011b. "Dog days: Tomb 48." Nekhen News. 23: 13.

—. 2011c. "Hierakonpolis.” In Before the pyramids. The origins of Egyptian civilization. OIMP 33, edited by E. Teeter, 33-44. Chicago: University of Chicago Press.

—. 2012. "A big pink pot." Nekhen News. 24: 6-7.

_. 2013. "In a corner at HK6 - at last!" Nekhen News. 25: 4-7.

Friedman, Renée, and Xavier Droux. 2015. "Complex Arrangements: HK6 in 2015." Nekhen News. 27:46.

Friedman, Renée, and Kazuyoshi Nagaya. 2013. "In the Footsteps of Petrie? The HK25 Deposit Revisited." Nekhen News. 25:21-22

Friedman, Renée, and Youngblood, Dawn. 1999. “Concession survey”. Nekhen News. 11: 7-8.

Friedman, Renée, Masahiro Baba, Hitoshi Endo, Izumi Takamiya et al. 2009. "Report on the 2006-2007 season of the Hierakonpolis Expedition.” Annales du Service des Antiquités de l'Égypte. 83:191-234.

Friedman, Renée, Masahiro Baba, Veerle Linseele, Kazuyoshi Nagaya, Fred Hardtke, and Richard Jaeschke. 2011a. "Report on the 2009 season of the Hierakonpolis expedition." Annales du Service des Antiquités de l'Égypte. 85: 141-164.

Friedman, Renée, Thomas Hikade, Masahiro Baba, J. Bunbury, A. Graham, Richard Jaeschke, Veerle Linseele. 2011b. "Report on the 2008 season of the Hierakonpolis expedition." Annales du Service des Antiquités de l'Égypte. 85: 115-139.

Friedman, Renée, Thomas Hikade, Masahiro Baba, J. Major, and J. Paulson. 2008. "The 2005-2006 field season of the Hierakonpolis Expedition." Annales du Service des Antiquités de l'Égypte. 82: 89-111. 
Friedman, Renée, Amy Maish, Ahmed Gamal Fahmy, John C. Darnell, and Edward D. Johnson. 1999. "Preliminary report on field work at Hierakonpolis: 1996-1998." Journal of the American Research Center in Egypt. 36: 1-35.

Friedman, Renée, Wim Van Neer, Bea De Cupere, and Xavier Droux. 2017 (in press). "The Elite Predynastic Cemetery at Hierakonpolis HK6: 2011-2015 progress report." In Egypt at its origins 5: Proceedings of the Fifth International Conference "Origin of the state. Predynastic and Early Dynastic Egypt", Cairo,13th-18th April 2014, edited by B. Midant-Reynes, Y.Tristant, E.M. Ryan . Orientalia Lovaniensia analecta, Leuven: Peeters.

Friedman, Renée, Wim Van Neer, and Veerle Linseele. 2011c. The elite Predynastic cemetery at Hierakonpolis: 2009-2010 update. In Egypt at its origins 3: proceedings of the third international conference "Origin of the state : predynastic and early dynastic Egypt", London, 27th July-1st August 2008., edited by Renée F. Friedman and Peter N. Fiske, 157-191. Leuven: Peeters.

Friedman, Renée, Ethan Watrall, Jana Jones, Ahmed Gamal Fahmy, Wim Van Neer, and Veerle Linseele. 2002. "Excavations at Hierakonpolis." Archéo-Nil. 12: 55-68.

Frison, George C. 1968. "A functional analysis of certain chipped stone tools". American Antiquity. 33: 149-155.

Gaballa, G. A. N.D. Nubia Museum. Aswan. Nubia Museum.

Galerie Nefer. 1990. "Bird (Chipped flint.)" Nefer. 8: nº 29.

Garstang, John. 1902. "A Predynastic pot-kiln recently discovered at Mahâsna, in Egypt." Man. 2: 38-40.

—. 1903. Maḥ̂asna and Bêt Khallâf. London: B. Quaritch.

. 1907. "Excavations at Hierakonpolis, at Esna and in Nubia." Annales du Service des Antiquités de l'Egypte. 8:132-148.

Gatto, Maria Carmela. 2011. "Egypt and Nubia in the 5th-4th millennia BC: A view from the First Cataract and its surroundings." In Egypt at its origins 3: proceedings of the third international conference "Origin of the state : predynastic and early dynastic Egypt", London, 27th July-1st August 2008., edited by Renée F. Friedman and Peter N. Fiske, 859-877. Leuven: Peeters.

2014. "Cultural Entanglement at the Dawn of the Egyptian History: A view from the Nile First cataract region." Origini. 36:93-123.

Gatto, Maria Carmela, and Serena Giuliani. 2006. "Survey between Aswan and Kom Ombo." Egyptian Archaeology. 29: 6-9.

Gatto, Maria Carmela, Morgan De Dapper, Rainer Gerisch, Elizabeth Hart, Stan Hendrickx, Tomasz Herbich, Hannah Joris, Hans-Åke Nordström, Mindy Pitre, Sara Roma, Dawid Święch and Donatella Usai. 2009a. "Predynastic settlement and cemeteries at Nag el-Qarmila, Kubbaniya." Archéo-Nil 19:186-206.

Gatto, Maria Carmela, John C. Darnell, Morgan de Dapper, Carla Gallorini, Rainer Gerisch, Serena Giuliani, Elizabeth Hart, Stan Hendrickx, Tomasz Herbich, Hannah Joris, Ilka Klose, Colleen M. 
Manassa, Marcel Marée, Hans-Åke Nordström, Mindy Pitre, Gillian Pyke, Dietrich Raue, Sara Roma, Pamela Rose, Dawid Święch and Donatella Usai. 2009b. "Archaeological investigation in the AswanKom Ombo region (2007-2008)." Mitteilungen des Deutschen Archäologischen Instituts Kairo. 65:947.

Gatto, Maria Carmela, Morgan de Dapper, Merel Eykerman, Rainer Gerisch, Hannah Joris, Claire Newton, Stan Hendrickx. 2009c. "Landscape reconstruction of the Predynastic site at Nag el-Qarmila, (Upper Egypt)." Sahara. 20: 63-68.

Geller, Jeremy. 1984. The predynastic ceramics industry at Hierakonpolis, Egypt. Thesis (M.A.)-Washington University, Dept. of Anthropology. Ann Arbor, Mich: U.M.I.

- 1992 a. Predynastic beer production at Hierakonpolis, Upper Egypt: archaeological evidence and anthropological implications. Thesis (Ph. D.)-- Washington University, Dept. of Anthropology. Ann Arbor, Mich: U.M.I.

. 1992b. "From Prehistory to History: Beer in Egypt.” In The followers of Horus: studies dedicated to Michael Allen Hoffman, 1944-1990, edited by R. F. Friedman, and B. Adams, 19-26. Oxbow monograph, 20. Oxford: Oxbow Books.

Geller, Jeremy, and Friedman, Renée. 2009. "Excavations at HK24B." In Report on the 2006-2007 season of the Hierakonpolis Expedition, Friedman, R.F. et al. Annales du Service des Antiquites de L'Egypte, Cairo. 83: 197-199.

Gilead, Isaac. 2013. "The Micro-Endscraper: A New Tool Type of the Chalcolithic Period". Tel Aviv. 11(1): 3-10.

Ginter, Bołeslaw, and Janusz Krysztof Kozłowski. 1994. Predynastic Settlement near Armant. Heidelberg: Heidelberger Orientverlag.

Ginter, Bołeslaw, Janusz Krysztof Kozłowski, and Maciej Pawlikowski. 1985. "Field report from the survey conducted in Upper Egypt in 1983." Mitteilungen des Deutschen Archäologischen Instituts, Abteilung Kairo. 41: 15-42.

1996. "Raw Material Procurement in the Tarifian and in the Nagada Culture: a case study from the Nile valley in Upper Egypt.” In Interregional contacts in the later prehistory of northeastern Africa, edited by L. Krzyżaniak, K. Kroeper, and M. Kobusiewicz, 165- 179. Poznań: Poznań Archaeological Museum.

Godfrey-Smith, Dorothy I., Luc Bouchet-Bert, Peter H. von Bitter, and Peter L. Storck. 2005. “Thermal Activation Characteristics and Thermoluminescence of Chert from the Red Wing, Ontario Region, and Its Putative Heat Treatment in Prehistory.” Geochronometria. 24: 13-20.

Gould, Richard A. 1981. "Brandon revisited: A new look at an old technology." In Modern material culture: the archaeology of us, edited by Richard A. Gould and Michael B. Schiffer, 269-282. New York: Academic Press.

Graff. Gwenola. 2009. Les peintures sur vases de Nagada I - Nagada II. Nouvelle approche sémiologique de l'iconographie prédynastique. Egyptian Prehistory Monographs 6. Leuven: Leuven University Press. 
Graham, Elizabeth A., Scott E. Simmons, and Christine D. White. 2013. "The Spanish conquest and the Maya collapse: how 'religious' is change?" World Archaeology. 45 (1): 161-185.

Grau Mira, Ignacio. 2016. "Forging communities: coalitions, identity symbols and ritual practices in Iron Age eastern Iberia". World Archaeology. 48 (1): 110-124.

Graves-Brown, C. A. 2011. The ideological significance of flint in Dynastic Egypt. Thesis (Ph.D.)-University College London (University of London), 2011.

Griffiths, D.R., C.A. Bergman, C.J. Clayton, K. Ohnuma, V. Robins, and N.J. Seeley. 1987.

"Experimental Investigation of the Heat Treatment of Flint." In The Human Uses of Flint and Chert, edited by G. de G. Sieveking and M. H. Newcomer, 43-52. Cambridge: Cambridge University Press.

Habachi, Labib. 1939. "A First Dynasty cemetery at Abydos." Annales du Service des Antiquités de l'Egypte. 39:767-781.

Hardtke, Fred. 2012. "Wadi el Pheel. The wadi of the elephant." Nekhen News. 24: 18-19.

Harlan, John Fredrick. 1982. "Excavations at locality 11C." In The Predynastic of Hierakonpolis: An Interim Report, edited by M. Hoffman, 14-25. Giza, Egypt; Macomb, Ill.: Cairo University Herbarium, Faculty of Science ; Dept. of Sociology and Anthropology, Western Illinois University.

1985. Predynastic settlement patterns: A view from Hierakonpolis. Dissertation (PhD.), Washington University. U.M.I.

Harrell, James A. 2012. "Utilitarian Stones." UCLA Encyclopedia of Egyptology, 1(1). UCLA: Department of Near Eastern Languages and Cultures.

Harris, John Richard. 1961. Lexicographical Studies in Ancient Egyptian Minerals. Berlin: Akademie Verlag.

Hart, Elizabeth, E. 2017. "Large-scale Chert Quarries at Wadi el-Sheikh, Egypt" Talk presented at the Annual Meeting of the Society for American Archaeology, Vancouver, B.C., Canada, March 30-April 2, 2017.

Hart, Elizabeth, E. Christiana Köhler, and Michael Klaunzer. In preparation. "Lithic technologies at Wadi el-Sheikh."

Hartenberger, Britt, and Curtis Runnels. 2001. "The Organization of Flaked Stone Production at Bronze Age Lerna". Hesperia. 70 (3).

Hartmann, Rita. 2011a. "The chronology of Naqada I tombs in the Predynastic cemetery U at Abydos." In Egypt at its origins 3: proceedings of the third international conference "Origin of the state : predynastic and early dynastic Egypt", London, 27th July-1st August 2008., edited by Renée F. Friedman and Peter N. Fiske, 917-938. Leuven: Peeters.

. 2011b. "Some remarks on the chronology of the early Naqada culture (Naqada I / early Naqada II) in Upper Egypt." Archéo-Nil. 21: 21-32. 
Hartung, Ulrich . 2011. "Nile mud and clay objects from the Predynastic cemetery U at Abydos (Umm elQa'ab)." In Egypt at its origins 3: proceedings of the third international conference "Origin of the state : predynastic and early dynastic Egypt", London, 27th July-1st August 2008., edited by R. F. Friedman and P. N. Fiske, 467-496. Leuven: Peeters.

Hartung, Ulrich, Mohammed Abd el-Gelil, Angela von den Driesch, Gamal Fares, Rita Hartmann, Thomas Hikade, and Christian Ihde. 2003. "Vorbericht über neue Untersuchungen in der prädynastichen Siedlung von Maadi." Mitteilungen des Deutschen Archäologischen Instituts, Abteilung Kairo. 59: 149-198.

Harvey, Stephen P. 1998. The cults of King Ahmose at Abydos. Thesis (PhD)., University of Pennsylvania.

Hassan, Fekri A. 1978. "Sediments in Archaeology: Methods and Implications for Palaeoenvironmental and Cultural Analysis". Journal of Field Archaeology. 5 (2): 197-213.

1981a. The Predynastic of Egypt: Subsistence-Settlement Studies in the Nagada-Khattara Region. Final Report to the National Science Foundation, Washington D.C.

1981b. "The origins of the Egyptian civilisation. A working model." Annales du Service des antiquités de l'Egypte. 65: 135-148.

1984. "Radiocarbon chronology of Predynastic Naqada settlements, Upper Egypt." Current Anthropology. 25: 681-683.

1988. "The Predynastic of Egypt." Journal of World Prehistory. 2(2):135-185.

1992a. "Primeval goddess to divine king. The mythogenesis of power in the early Egyptian state." In The Followers of Horus. Studies dedicated to Michael Allen Hoffman, edited by R. Friedman, and B. Adams, 307-322. Oxford: Oxbow Books.

. 1992b. "Town and village in ancient Egypt: Ecology, society and urbanization." In The archaeology of Africa. Food, metals and towns, edited by T. Shaw, P. Sinclair, B. Andah, and A. Okpoko, 551-569. London : Routledge.

1999." Nagada (Naqada)." In Encyclopedia of the archaeology of ancient Egypt, edited by Kathryn A. Bard, 555-557. London: Routledge.

2004. "Between man and goddess: The fear of nothingness \& dismemberment." In Egypt at its Origins: studies in memory of Barbara Adams, edited by S. Hendrickx, and B. Adams, 779-799. Orientalia Lovaniensia Analecta, 138. Leuven: Peeters.

Hassan, Fekri A., and Richard G. Matson. 1989. " Seriation of Predynastic potsherds from the Nagada region (Upper Egypt)." In Late prehistory of the Nile Basin and the Sahara, edited by Lech Krzyżaniak and Michał Kobusiewicz, 303-315. Poznań: Poznań Archaeological Museum.

Hastorf, Christine, A. 2007. "Archaeological Andean Rituals: Performance, Liturgy, and Meaning." In The archaeology of ritual, edited by K. Evangelos,77-108 . Los Angeles: Cotsen Institute of Archaeology, University of California, Los Angeles. 
Hawkes, Christopher. 1954. "Archaeological Theory and method some suggestions from the Old World." American Anthropologist. 56:155-168.

Hayden, Brian. 1995. "Pathways to Power: Principles for Creating Socioeconomic Inequalities." In Foundations of Social Inequality, edited by D. T. Price and G.M. Feinman, 15-86. New York: Plenum Press.

Hays, T.R. 1976. "Predynastic Egypt: Recent Field Research". Current Anthropology. 17 (3): 552-554.

Hendon, Julia A. 1996. "Archaeological Approaches to the Organization of Domestic Labor : Household Practice and Domestic Relations." Annual Review of Anthropology 25: 45-61.

Hendrickx, Stan. 1994. "Antiquités préhistoriques et protodynastiques d'Egypte." Guides du département égyptien 8. Bruxelles: Musées Royaux d'Art et d'Histoire.

. 1996a. " The relative chronology of the Naqada culture: Problems and possibilities." In Aspects of Early Egypt, edited by A. J. Spencer, 36-69. London: British Museum Press.

1996b.

. 2002. "Bovines in Egyptian Predynastic and Early Dynastic iconography." In Droughts, food, and culture ecological change and food security in Africa's later prehistory, edited by Fekri A. Hassan, 275-318. New York: Kluwer Academic/Plenum Publishers.

. 2006a. "The dog, the Lycaon pictus and order over chaos in Predynastic Egypt." In Archaeology of early Northeastern Africa: in memory of Lech Krzyżaniak, edited by Kroeper, Karla, Marek Chłodnicki, Michał Kobusiewicz, and Lech Krzyżaniak, 723-749. Poznań: Poznań Archaeological Museum.

. 2006b. "Predynastic - Early Dynastic chronology." In Ancient Egyptian Chronology, edited by E. Hornung, R. Krauss, and D. A. Warburton, 55-93, 487-488. Handbook of oriental studies. Section 1, the Near and Middle East, v. 83. Leiden, Boston: Brill Academic Publishers. 237-263.

2009. "Hunting and social complexity in predynastic Egypt". Bulletin Des Séances. 57 (2/4):

2011a. " Iconography of the Predynastic and Early Dynastic periods." In Before the pyramids. The origins of Egyptian civilization, edited by Emily Teeter, 75-81. OIMP 33. Chicago: University of Chicago Press.

. 2011b "Naqada IIIA-B, a crucial phase in the relative chronology of the Naqada culture." Archéo-Nil. 21: 65-80.

—. 2011c. "The Chronology Workshop." In Egypt at its origins 3: proceedings of the third international conference "Origin of the state : predynastic and early dynastic Egypt", London, 27th July-1st August 2008., edited by Renée F. Friedman and Peter N. Fiske, 911-915. Leuven: Peeters.

.2011d. "Crafts and craft specialization." In Before the pyramids. The origins of Egyptian civilization. OIMP 33, edited by E. Teeter, 93-98. Chicago: University of Chicago Press. 
- 2013. "Hunting and social complexity in Predynastic Egypt." Academie Royale des Sciences d' Outre-mer, Bulletin des Séances / Koninklijke Academie voor Overzeese Wetenschappen, Mededelingen der Zittingen, 57, 2-4 (2011): 237-263.

Hendrickx, Stan, and David Depraetere. 2004. "A theriomorphic Predynastic stone jar and hippopotamus symbolism." In Egypt at its Origins: studies in memory of Barbara Adams, edited by S. Hendrickx, and B. Adams, 801-822. Orientalia Lovaniensia Analecta, 138. Leuven: Peeters.

Hendrickx, Stan, and Meryl Eyckerman. 2010. "Continuity and change in the visual representations of Predynastic Egypt." In Recent discoveries and latest researches in Egyptology. Proceedings of the First Neapolitan Congress, edited by F. Raffaele, M. Nuzzolo, and I. Incordino, 121-144. Wiesbaden: Harrassowitz.

Hendrickx, Stan, and Renée F. Friedman. 2003. " Chaos and order: A Predynastic "Ostracon" from HK29A." Nekhen News. 15: 8-9.

Hendrickx, Stan, Kathryn E. Piquette, Merel Eyckerman, Karine Madrigal, and Carolyn Graves-brown. 2015. "The Origin and Early Significance of the White Crown." Mitteilungen des Deutschen Archäologischen Instituts, Abteilung Kairo. 70/71(2014/2015):227-238.

Hendrickx, Stan, and Edwin C.M. Van Den Brink, 2002. "Inventory of Predynastic and Early Dynastic cemetery and settlement sites in the Egyptian Nile Valley." In Egypt and the Levant : Interrelations from the 4th through the Early 3rd Millennium B.C.E., edited by E. C. M. van den Brink, and T. E. Levy, 346-399. London, New York: Leicester University Press.

Hendrickx, Stan, and Pierre M Vermeersch. 2001. "Prehistory: From the Palaeolithic to the Badarian culture (c. 700,000-4000 BC)." In The Oxford history of ancient Egypt, edited by Ian Shaw, 17-43. Oxford: Oxford University Press.

Hendrickx S., Darnell J.C., and Gatto M.C. 2012. "The earliest representations of royal power in Egypt: The rock drawings of Nag el-Hamdulab (Aswan)." Antiquity. 86 (334): 1068-1083.

Hendrickx, S., Faltings, D., Op de Beeck, L., Ruae, D., and Michiels, C. 2002. Milk, Bread and Beer Technology during the Early Dynastic Period. Mitteilungen des Deutschen Archäologischen Instituts. Abteilung Kairo. 58:277-304.

Hendrickx, Stan, Dirk Huyge, and Barbara Adams. 2003. "Le scorpion en silex du Musée royal de Mariemont et les silex figuratifs de l'Egypte pré- et protodynastique." Les Cahiers de Mariemont. 28-29 (1997-1998): 6-33.

Hendrickx, S., Dirk Huyge, and Barbara Adams. 2003. "Le scorpion en silex du Musée royal de Mariemont et les silex figuratifs de l'Egypte pré- et protodynastique." Les Cahiers de Mariemont. 28-29 (1997-1998): 6-33.

Hendrickx, Stan, Heiko Riemer, Frank Förster, and John C. Darnell. 2009. Late Predynastic / Early Dynastic rock art scenes of Barbary sheep hunting in Egypt's Western Desert. From capturing wild animals to the women of the 'Acacia House'. In Desert animals in the eastern Sahara: status, economic significance, and cultural reflection in antiquity: proceedings of an interdisciplinary ACACIA workshop held at the University of Cologne December 14-15, 2007, edited by H. Riemer, 189-244. Köln: Heinrich-Barth-Inst. 
Hendrickx, Stan., and Edwin C.M. Van Den Brink. 2002. "Inventory of Predynastic and Early Dynastic cemetery and settlement sites in the Egyptian Nile Valley." In Egypt and the Levant : Interrelations from the 4th through the Early 3rd Millennium B.C.E., edited by E. C. M. van den Brink and T. E. Levy, 346-399. London, New York: Leicester University Press.

Hester, Thomas R. 1976. "Functional analysis of ancient Egyptian chipped stone tools: The potentials for future research." JFA, 3: 346-351.

Hierakonpolis Expedition. "Tomb 72". Hierakonpolis-online.org. Web. Accessed Sept. 2015. http://hierakonpolis-online.org/index.php/explore-the-predynastic-cemeteries/hk6-elite-cemetery/tomb72

Hikade, Thomas. 1996. "Die lithischen Kleinfunde." In "Umm el-Qaab. Nachuntersuchungen im frühzeitlichen Königsfriedhof 7./8. Vorbericht," Dreyer, G. et al., Mitteilungen des Deutschen Archäologischen Instituts, Abteilung Kairo. 52: 33-39.

1997. "Ein aussergewöhnliches Silexmesser aus Abydos." Mitteilungen des Deutschen Archäologischen Instituts, Abteilung Kairo. 53: 85-89.

1998. "Lithik." In "Umm el-Qaab. Nachuntersuchungen im frühzeitlichen Königsfriedhof 9./10. Vorbericht," Dreyer, G. et al., Mitteilungen des Deutschen Archäologischen Instituts, Abteilung Kairo. 54: 115-123.

. 2000. "Friedhof U. Lithik." In "Umm el-Qaab. Nachuntersuchungen im frühzeitlichen Königsfriedhof 11./12. Vorbericht," Dreyer, G. et al., Mitteilungen des Deutschen Archäologischen Instituts, Abteilung Kairo. 56: 81-86.

. 2001. "Silex-Pfeilspitzen in Agypten". Mitteilungen des Deutschen Archäologischen Instituts, Abteilung Kairo. 57: 109-126.

. 2003. Getting the ritual right - Fishtail knives in Predynastic Egypt. In Egypt - temple of the whole world: studies in honour of Jan Assmann. Meyer, S., edited by Sibylle Meyer, 137-151. Leiden u.a: Brill.

—_. 2008. " Grand design in the sacred compound." Nekhen News. 20: 4-5.

—. 2010. “Stone Tool Production.” UCLA Encyclopedia of Egyptology, 1(1). Web. Accessed 2013. http://www.escholarship.org/uc/item/7pb3h0h1

. 2011. "Origins of Monumental Architecture: Recent Excavations at Hieraonpolis HK29B and HK25." In Egypt at Its Origins 3: Proceedings of the Third International Conference "Origin of the State : Predynastic and Early Dynastic Egypt”, London, 27th July-1st August 2008, edited by R. F. Friedman, and P. N. Friske, 81-108. OLA 205. Leuven: Peeters.

\section{. 2013. The Lithic Industries on Elephantine Island during the 3rd Millennium BC. Wiesbaden:} Harrassowitz. 
Hikade, Thomas, Gillian Pyke, and D’arne O’Neill. 2008. “Excavations at Hierakonpolis HK29B and HK25 - The campaigns of 2005/06.” Mitteilungen Deutschen Archaologischen Instituts Abteilung Kairo. 64: 27-30.

Hodder, Ian. 1986. Reading the past: current approaches to interpretation in archaeology. Cambridge: Cambridge University Press.

Hoffman, Michael A. 1971-2. "Preliminary Report on the First Two Seasons at Hierakonpolis: Part IV: Test Excavations at Locality 14." Journal of the American Research Center in Egypt. 9:49-66.

. 1980a. Egypt before the pharaohs: the prehistoric foundations of Egyptian civilization. New York: Knopf.

1980b. "A Rectangular Amratian House from Hierakonpolis and its Significance for Predynastic Research." Journal of Near Eastern Studies 39 (2): 119-137.

1982. The Predynastic of Hierakonpolis: An Interim Report. Giza, Egypt; Macomb, Ill.: Cairo University Herbarium, Faculty of Science ; Dept. of Sociology and Anthropology, Western Illinois University.

Högberg, Anders. 2009. Lithics in the Scandinavian Late Bronze Age: sociotechnical change and persistence. Oxford: Archaeopress.

Holmes, Diane L. 1987. "Problems encountered in a high-power microwear study of some Egyptian Predynastic lithic artefacts." In The human uses of flint and chert: proceedings of the Fourth International Flint Symposium, held at Brighton Polytechnic, 10-15 April 1983, edited by G. de G. Sieveking, and M. H. Newcomer, 91-96. Cambridge [Cambridgeshire]: Cambridge University Press.

. 1989. The Predynastic Lithic Industries of Upper Egypt : A Comparative Study of the Lithic Traditions of Badari, Nagada, and Hierakonpolis. Oxford, England: British Archaeological Reports.

. 1989b. "Inter-regional variability in Egyptian Predynastic lithic assemblages." In Late prehistory of the Nile Basin and the Sahara, edited by Lech Krzyżaniak and Michał Kobusiewicz, 243-

253. Poznań: Poznań Archaeological Museum.

1990. "The Flint axes of Nagada, Egypt: Analysis and Assessment of a Distinctive Predynastic Tool Type.” Paleorient. 16 (1):1-21.

. 1992a. "Chipped stone-working craftsmen, Hierakonpolis, and the rise of civilization in Egypt.” In The Followers of Horus. Studies dedicated to Michael Allen Hoffman, edited by R. Friedman, and B. Adams, 37-44. Oxford: Oxbow Books.

_ 1992b. "The Evidence and Nature of Contacts Between Upper and Lower Egypt During the Predynastic: A View from Upper Egypt.” In The Nile Delta in Transition 4th - 3rd Millennium B.C.: Proceedings of the Seminar Held in Cairo, 21-24 October 1990, at the Netherlands Institut, edited by E. C. M. Van den Brink, 301-16. Tel Aviv, Israel: Israel Exploration Society.

. 1996. "Lithic assemblages from Hierakonpolis and interregional relations in Predynastic Egypt.” In Interregional contacts in the later prehistory of northeastern Africa, edited by L. Krzyżaniak, K. Kroeper, and M. Kobusiewicz, 193-202. Poznań: Poznań Archaeological Museum. 
- 1999. " El-Badari district Predynastic sites." Encyclopedia of the archaeology of ancient Egypt, edited by Kathryn A. Bard, 161-164. London: Routledge.

Holmes, Diane L., and Friedman, Renée. 1994. "Survey and test excavations in the Badari region, Egypt." Proceedings of the Prehistoric Society. 60:105-142.

Hossein, Yasser Mahmoud. 2011. "A new Archaic period cemetery at Abydos." In Egypt at its origins 3: proceedings of the international conference "Origin of the state. Predynastic and early dynastic Egypt", London, 27th July - 1st August 2008, edited by R. Friedman, 269-280. Leuven: Peeters.

Hruby, Zachary X. 2007. Ritualized Chipped-Stone Production at Piedras Negras, Guatemala. In Rethinking Craft Specialization in Complex Societies Archaeological Analyses of the Social Meaning of Production, edited by Z. X. Hruby, and R. Flad, 68-87. Archeological Papers of the American Anthropological Association,. Arlington, VA: Blackwell Publishing.

Humphrey, Caroline, and James Laidlaw. 2007. "Sacrifice and Ritualization." In The archaeology of ritual, edited by K. Evangelos, 255-276. Los Angeles: Cotsen Institute of Archaeology, University of California, Los Angeles.

Huyge, Dirk. 1998. "Giraffes in ancient Egypt." Nekhen News. 10: 9-10.

2002. "Cosmology, ideology and personal religious practice in ancient Egyptian rock art." In Egypt and Nubia: gifts of the desert, edited by R.F. Friedman, 192-206. London: British Museum Press.

. 2004. "A double-powerful device for regeneration: The Abu Zaidan knife handle reconsidered.” In Egypt at its Origins: studies in memory of Barbara Adams, edited by S. Hendrickx, and B. Adams, 823-836. Orientalia Lovaniensia analecta, 138. Leuven: Peeters.

Ikram, Salima. 1995. Choice cuts: meat production in ancient Egypt. Leuven: Peeters.

Ikram, Salima, and Aidan Dodson. 1998. The mummy in ancient Egypt: equipping the dead for eternity. London: Thames \& Hudson.

Inizan, Marie-Louise, Michèle Reduron-Ballinger, Hélène Roche, and Jehanne Féblot-Augustins. 1999. Technology and terminology of Knapped Stone: followed by a multilingual vocabulary Arabic, English, French, German, Greek, Italian, Portuguese, Spanish. Nanterre: Cercle de Recherches et d'Etudes Préhistoriques.

Inomata, Takeshi. 2001. "The Power and Ideology of Artistic Creation: Elite Craft Specialists in Classic Maya Society." Current Anthropology. 42 (3): 321-349.

Insoll, Timothy. 2004. Archaeology, ritual, religion. London: Routledge.

- 2011. The Oxford handbook of the archaeology of ritual and religion. Oxford: Oxford University Press.

Janusek, John Wayne. 2006. "The changing 'nature' of Tiwanaku religion and the rise of an Andean state". World Archaeology. 38 (3): 469-492. 
Jórdeczka, Maciej, and Małgorzata Mrozek-Wysocka. 2012. "Stone working. Tools and workshops." In In Tell el-Farkha I: excavations 1998-2011, edited by Chlodnicki, Marek, Krzysztof M. Cialowicz, and Agnieszka Maczynska, 279-296. Posnan: Archaeological Museum.

Kabacinski, Jacek. 2012. "Selected aspects of lithic production.” In Tell el-Farkha I: excavations 19982011, edited by Chlodnicki, Marek, Krzysztof M. Cialowicz, and Agnieszka Maczynska, 323-344. Posnan: Archaeological Museum.

Kaiser, Werner. 1957. "Zur inneren Chronologie der Naqadakultur." Archaeologia Geographica. 6:69-77.

. 1999. "Elephantine." In Encyclopedia of the archaeology of ancient Egypt, edited by Kathryn A. Bard, 283-289. London: Routledge.

Julius Kaplan. 2005. Predynastic Egyptian pottery: in the collection of the Robert V. Fullerton Art Museum, California State University, San Bernardino. San Bernardino: Robert V. Fullerton Art Museum California State University, San Bernardino.

Kelley, A.L. 1983. A review of the evidence concerning early Egyptian knife handles. In A tribute to Professor Ronald J. Williams. The Ancient World Egyptological Miscellanies 6, edited by J.K. Hoffmeier \& E.S. Meltzer, 95-102. Chicago: Ares Publishers.

Kelterborn, Peter. 1984. “Toward replicating Egyptian Predynastic flint knives.” Journal of Archaeological Science. 11: 433-453.

Kemp, Barry J. 1982. "Automatic Analysis of Predynastic Cemeteries: A New Method for an Old Problem". The Journal of Egyptian Archaeology. 68: 5-15.

- 2006 [1989]. AncientEgypt. Anatomy of a civilization. $2^{\text {nd }}$ ed. Routledge, London.

Kenoyer, Jonathan Mark, Massimo Vidale, and Kuldeep Kumar Bhan. 1991. “Contemporary Stone Beadmaking in Khambhat, India: Patterns of Craft Specialization and Organization of Production as Reflected in the Archaeological Record.” World Archaeology, 23 (1): 44-63.

Khalifa, Eman. 2012. "Residue analysis at HK11C mound A." Nekhen News. 24: 12.

Kindermann, Karin. 2003. "Djara: Prehistoric links between the desert and the Nile." In Egyptology at the dawn of the Twenty-first Century : proceedings of the Eighth International Congress of Egyptologists, Cairo, 2000, edited by Zahi Hawass, and Lyla Pinch Brock, 272-279. Cairo: American University in Cairo Press.

. 2008. "Lithic technology and production sequences: Recent investigations from Tell elFara'in/Buto.” Paper presented at Egypt at its Origins: The Third International Colloquium on Predynastic and Early Dynastic Egypt, British Museum, London, 27th July - 1st August.

- 2010. Djara: zur mittelholozänen Besiedlungsgeschichte zwischen Niltal und Oasen (AbuMuharik-Plateau, Ägypten). 1 1. Köln: Heinrich-Barth-Inst.

Kindermann, Karin , Olaf Bubenzer, Stefanie Nussbaum, Heiko Riemer, Frank Darius, Nadja Pöllath, and Ursula Smettan. 2006. "Palaeoenvironment and Holocene land use of Djara, Western Desert of Egypt." Quaternary Science Reviews. 25: 1619-1637. 
Kobusiewicz, Michał. 2015. The production, use and importance of flint tools in the archaic period and the old kingdom in Egypt. Oxford: Archaeopress.

Köhler, E. Christiana. 2004. "On the origins of Memphis. The new excavations in the Early Dynastic necropolis at Helwan." In Egypt at its Origins: studies in memory of Barbara Adams, edited by S. Hendrickx, and B. Adams, 295-315. Orientalia Lovaniensia analecta, 138. Leuven: Peeters

_ 2010. "Theories of State Formation." In Egyptian Archaeology, edited by W. Wendrich, 36-54. Chichester, U.K. ; Malden, MA : Wiley-Blackwell.

Köhler, E. Christiana, Elizabeth Hart, and Michael Klaunzer. 2017 "Wadi el-Sheikh: A new archaeological investigation of ancient Egyptian chert mines". PLOS ONE, 12(2): e0170840. doi:10.1371/journal.pone.0170840

Kopp, Peter. 2006. Elephantine XXXII: die siedlung der Naqadazeit. Archäologische Veröffentlichungen, 118 Mainz: Philipp von Zabern.

Kovacevich, B. 2006. Reconstructing Classic Maya economic systems production and exchange at Cancuen, Guatemala. Thesis (Ph. D. in Anthropology) Vanderbilt University.

- 2007. Ritual, crafting, and agency at the classic Maya kingdom of Cancuen. In Mesoamerican Ritual Economy: archaeological and ethnological perspectives, edited by E. C. Wells, and K. L. DavisSalazar. Mesoamerican worlds. Boulder: University Press of Colorado. pp. 67-114.

Kubiak-Martens, L. and J.J. Langer, 2008. "Predynastic Beer Brewing as Suggested by Botanical and Physicochemical evidence from Tel el-Farkha, Eastern Delta." In Egypt at its Origins 2: Proceedings of the International Conference "Origin of the State. Predynastic and Early Dynastic Egypt", Toulouse (France), 5th - 8th September 2005. Edited by B. Midant-Reynes, 427-442. Leuven: Peeters.

Kuper, Rudolph, and Stefan Kröpelin. 2006. "Climate-controlled Holocene occupation in the Sahara: motor of Africa's evolution". Science (New York, N.Y.). 313 (5788): 803-7.

Kyriakidis, Evangelos. 2007. The archaeology of ritual. Los Angeles: Cotsen Institute of Archaeology, University of California, Los Angeles.

LeBlanc, Raymond. 1992. "Wedges, Pieces Esquillees, Bipolar Cores, and Other Things: An Alternative to Shott's View Of Bipolar Industries". North American Archaeologist. 13 (1): 1-14.

Lehner, Mark. 2000. "Fractal house of Pharaoh: Ancient Egypt as a Complex Adaptive System, a Trial Formulation." In Dynamics in Human and Primate Societies: Agent-Based Modeling of Social and Spatial Processes, edited by T. A. Kohler, 275-353. New York: Oxford University Press.

Lin, Sam C. H. , Matthew J. Douglass, Simon J. Holdaway, and Bruce Floyd. 2010. “The Application of 3D Laser Scanning Technology to the Assessment of Ordinal and Mechanical Cortex Quantification in Lithic Analysis.” Journal of Archaeological Science. 37 (4): 694-702.

Linseele, Veerle, Wim Van Neer, and Renée Friedman. 2009. "Special Animals from a Special Place? The Fauna from HK29A at Predynastic Hierakonpolis". Journal of the American Research Center in Egypt. 45: 105. 
Linseele, Veerle, Wim, Van Neer, Sofie Thys, Rebecca Phillips, René Cappers, Willeke Wendrich, Simon Holdaway. 2014. "New archaeozoological data from the Fayuim "Neolithic" with a critical assessment of the evidence for early stock keeping in Egypt." PLOS ONE. 9(10): e108517.

Lippiello, Lauren Elizabeth. 2012. Landscapes of ancient Egyptian religion: rock art as indicator for formal ritual spaces during the formative stage of the Egyptian state. Thesis (PhD), Yale University.

Longacre, William A., 1999. "Standardization and Specialization: What's the Link?" In Pottery and people: a dynamic interaction, edited by J. M., Skibo, and G. M. Feinman, 44-58. Salt Lake City: University of Utah Press.

Longacre, William A., Kenneth L. Kvamme, and Masashi Kobayashi. 1988. "Southwestern Pottery Standardization: An Ethnoarchaeological View from the Philippines.” The Kiva 53, (2): 101-12.

Luedtke, Barbara E. 1992. An archaeologist's guide to chert and flint. Los Angeles: Institute of Archaeology, University of California.

Lundt, Marquardt. 2008. "Egyptian Flintwork, Part I: "SHT SF.W"- Chipping Butcher Knives in Ancient Egypt." Chips. 20 (3):4-13.

Maish, Amy, and Renée Friedman. 1999. "Pondering Paddy: Unwrapping the mysteries of HK43." Nekhen News. 11: 6-7.

Malinowski, Bronislaw. 1934. "Stone Implements in Eastern New Guinea." In Essays Presented to C. G. Seligman, edited by E.E. Evans-Pritchard, R. Firth, B. Malinowski, and I. Schapera, 189-196. London: K. Paul, Trench, Trubner.

Mallory-Greenough, Leanne M. 2002. "The geographical, spatial, and temporal distribution of Predynastic and First Dynasty basalt vessels." Journal of Egyptian Archaeology. 88: 67-94.

Marcus, Joyce. 2007. "Rethinking Ritual." In The Archaeology of Ritual, edited by E. Kyriakidis, 43-76. Cotsen Institute of Archaeology, UCLA, Los Angeles.

Martinón-Torres, Marcos, Xiuzhen Janice Li, Andrew Bevan, Yin Xia, Kun Zhao, and Thilo Rehren. 2014. "Forty Thousand Arms for a Single Emperor: from chemical data to the labor organization behind the bronze arrows of the Terracotta army." Journal of Archaeological Method and Theory 21(3): 534562.

Marwick, Ben. 2008. "What Attributes Are Important for the Measurement of Assemblage Reduction Intensity? Results from an Experimental Stone Artifact Assemblage with Relevance to the Hoabinhian of Mainland Southeast Asia.” Journal of Archaeological Science. 35 (5): 1189-1200.

Marx, Karl. 1867 [1952]. Capital. Chicago: Encyclopaedia Britannica.

Massoulard, Emile. 1936. "Lances fourchues et Peseshkaf. A propos de deux acquisitions récentes du Musée du Louvre." RdE, 2: 135-163.

Mauss, Marcel. 1954. The gift: the form and reason for exchange in archaic societies. London: Cohen \& West. 
McCutcheon, P. T., and S. M. Kuehner. 1997. "From macroscopic to microscopic: Understanding prehistoric heat treatment of stone tools.” In Siliceous Rocks and Culture, edited by A. Ramos-Millan, and M. A. Bustillo, 447-462. Editorial Universidad de Granada,Granada, Spain.

McDonald, Mary M.A., 1998. "Early African pastoralism: view from Dakhleh Oasis (South Central Egypt)." Journal of Anthropological Archaeology. 17:93-99.

McNamara, L. and Xavier Droux. 2006. "When is a tomb not a tomb ?" Nekhen News. 18: 9-10.

McNamara, Liam, and Xavier Droux. 2006. "When is a tomb not a tomb?" Nekhen News. 18: 9-10.

Midant-Reynes, Béatrix. 1981. "Les noms du silex en égyptien." Revue d'Égyptologie. 33: 39-45.

— 1987. "Contribution à l'étude de la société prédynastique: le cas du couteau "ripple-flake" ”. Studien zur altägyptischen Kultur. 14: 185-224.

1998. Le Silex de 'Ayn-Asil Oasis de Dakhla-Balat. Documents de Fouilles de l'IFAO 34. Cairo: Institut Français d'Archéologie Orientale du Caire.

. 1999. "el-Adaïma." In Encyclopedia of the archaeology of ancient Egypt, edited by Kathryn A. Bard, 114-115. London: Routledge.

Midant-Reynes, Béatrix, François Briois, and C. Jeuthe. 2014. Program for "Table Ronde on Lithic Industries in Egypt form the Neolithic to the Dynastic Period: towards a new perspective." Unpublished.

Midant-Reynes, Béatrix, and Nathalie Buchez. 2002. Adaïma. 1, Economie Et Habitat. Le Caire: Institut français d'archéologie orientale.

Midant-Reynes, Béatrix, and Dominique Prost. 2002. “Le matériel lithique.” In Adaïma. 1, Economie Et Habitat, edited by B. Midant-Reynes, and N. Buchez, 291-419. Le Caire: Institut français d'archéologie orientale.

Midant-Reynes, Béatrix, and Tixier, Jacques. 1981 “Les Gestes de l’Artisan Égyptien.” Recherche. 120:380-381.

Millet, N. B. 1990. "The Narmer Macehead and Related Objects". Journal of the American Research Center in Egypt. 27: 53-59.

. 1991. "The Narmer Macehead and Related Objects: [Correction]". Journal of the American Research Center in Egypt. 28: 223-225.

Mond, Robert, and Oliver Humphrys Myers. 1937. Cemeteries of Armant, I. London: Egypt Exploration Society, H. Milford, Oxford University Press.

Morehart, Christopher T., and Kristin De Lucia. 2015. "Surplus: the politics of production and the strategies of everyday life: An Introduction." In Surplus: the politics of production and the strategies of everyday life, edited by C. T. Morehart, and K. De Lucia, 3-43. Boulder : University of Colorado Press. 
Moreno García, Juan Carlos. 2012. "Households." In UCLA Encyclopedia of Egyptology, edited by E. Frood and W. Wendrich. Los Angeles.

http://digital2.library.ucla.edu/viewItem.do?ark=21198/zz002czx07

de Morgan, Henri. 1912. "Report on Excavations made in Upper Egypt during the Winter 1907-1908." Annales du Service des Antiquités de l'Egypte. 12:25-38.

Morris, Ellen. 2016. "Middle Kingdom Clappers, Dancers, Birth Magic, and the

Reinvention of Ritual." Talk given at the 67th Annual Meeting of the American Research Center in Egypt, April 15-17, Atlanta, GA.

_ 2006. "Lo, nobles lament, the poor rejoice" : state formation in the wake of social flux." In After collapse: the regeneration of complex societies, edited by Schwartz, Glenn M., and John J. Nichols, 5871. Tucson: University of Arizona Press.

Musée des antiquités nationales. 1982. Archéologie comparée. Paris: Ministère de la culture, Editions de la Réunion des musées nationaux.

Nagaya, Kazuyoshi. 2011. "Focus on flint: Artisans from the elite cemetery." Nekhen News. 23: 18-19.

—. 2013. "Fish and (flint) chips: An experimental approach." Nekhen News. 25: 14-15.

_. 2014. "Piercing Insights: Experiments in Predynastic Craftsmanship." Nekhen News. 26:16-17.

Naville, Edouard. 1914. The cemeteries of Abydos. Part I. 1909-1910. Part I. 1909-1910. London: Egypt Exploration Fund.

Nekhen News. 2011a. "Group 2." (Image, center left). Nekhen News. 23:19.

_ 2011b. Oldest in the world! Nekhen News. 23:24.

—. 2012. "Where is the Beef?" Nekhen News. 24:7.

Needler, Winifred. 1984. Predynastic and archaic Egypt in the Brooklyn Museum. Brooklyn, N.Y.: Brooklyn Museum.

Nicoll, K. 2001. Radiocarbon chronologies for prehistoric human occupation and hydroclimatic change in Egypt and northern Sudan. Geoarchaeology. 16(1):47-64.

Nikolaidou, Marianna. 2007. "Ritualized Technologies in the Aegean Neolithic?" In Rethinking Craft Specialization in Complex Societies Archaeological Analyses of the Social Meaning of Production, edited by Z. X. Hruby, and R. Flad, 183-208. Archeological Papers of the American Anthropological Association,. Arlington, VA: Blackwell Publishing.

O'Connor, David B. 1999. "Abydos, Early Dynastic funerary enclosures." In Encyclopedia of the archaeology of ancient Egypt, edited by Kathryn A. Bard, 93-95. London: Routledge.

2009. Abydos: Egypt's first pharaohs and the cult of Osiris. London: Thames \& Hudson. 
Odell, George H. 2001. "Stone Tool Research at the End of the Millennium: Classification, Function, and Behavior". Journal of Archaeological Research. 9 (1): 45-100.

Olszewski, Deborah I., Harold L. Dibble, Shannon P. McPherron, Utsav A. Schurmans, Laurent Chiotti, and Jennifer R. Smith. 2010. "Nubian Complex strategies in the Egyptian high desert". Journal of Human Evolution. 59 (2): 188-201.

Olszewski, Deborah I., Harold L. Dibble, Utsav A. Schurmans, Shannon P. McPherron, and Jennifer R. Smith. 2005. "High Desert Paleolithic Survey at Abydos, Egypt". Journal of Field Archaeology. 30 (3): 283-303.

Patch, Diana Craig. 1991. The origin and early development of urbanism in ancient Egypt: a regional study. Thesis (PhD), University of Michigan.

Patch, Diana Craig, and Marianne Eaton-Krauss. 2011. Dawn of Egyptian art. New York: Metropolitan Museum of Art.

Patterson, L.W. 1981. “Fracture Force Changes from Heating and Edge Grinding.” Flintknapper's Exchange 4 (3): 6-9.

Pauketat, Timothy R. 2007. Chiefdoms and other archaeological delusions. Lanham: AltaMira Press.

Pawlik, Alfred F. 2006. "The Lithic industry of the Pharaonic site Kom al-Ahmar in Middle Egypt and its relationship to the flint mines of the Wadi al-Sheikh.” In Stone age - mining age, edited by G. Körlin, and G. Weisgerber, 545-561. Bochum: Deutsches Bergbau-Museum.

Payne, J. Crowfoot. 1987. Appendix to Naqada excavations supplement. London: Egypt Exploration Society.

1993. Catalogue of the Predynastic Egyptian Collection in the Ashmolean Museum. Oxford: Griffith Institute, Ashmolean Museum.

Peebles, Christopher S., and Susan M. Kus. 1977. "Some Archaeological Correlates of Ranked Societies." American Antiquity. 42 (3): 421-448.

Peet, T. Eric. 1914. The cemeteries of Abydos. Vol. 2, 1911-1912 ; London: Egypt Exploration Fund.

Peet, T. Eric, and W. L. S. Loat. 1913. The cemeteries of Abydos. Vol. 3, 1912-1913 ; London: Egypt Exploration Fund.

Pelcin, Andrew W. 1997a. "The Effect of Indentor Type on Flake Attributes: Evidence from a Controlled Experiment.” Journal of Archaeological Science. 24: 613-621.

Pelcin, Andrew W. 1997b. "The Effect of Core Surface Morphology on Flake Attributes: Evidence from a Controlled Experiment.” Journal of Archaeological Science. 24: 749-756.

Pelegrin, Jacques. 2012. "New Experimental Observations for the Characterization of Pressure Blade Produciton Techniques.” In The emergence of pressure blade making: from origin to modern experimentation, edited by P. M. Desrosiers, 465-500. New York: Springer.

Peregrine, Peter. 1991. "Some Political Aspects of Craft Specialization." World Archaeology. 23(1):1-11

Petrie, William Matthew Flinders. 1890. Kahun, Gurob, and Hawara. London: K. Paul, Trench, Trübner. 
- 1891. Illahun, Kahun, and Gurob. Warminster, Eng: Aris \& Phillips.

- 1900. The royal tombs of the First Dynasty. 1900. Part I. Egypt Exploration Fund 18. London: Egypt Exploration Fund.

— 1901. The royal tombs of the earliest dynasties. 1901. Part II. Egypt Exploration Fund 21. London: Egypt Exploration Fund.

—. 1902. Abydos. Part I. Egypt Exploration Fund 22. London: Egypt Exploration Fund.

—. 1903. Abydos. Part II. Egypt Exploration Fund 24. London: Egypt Exploration Fund.

-1912. The Labyrinth, Gerzeh and Mazguneh. British School of Archaeology in Egypt. London: Egypt Exploration Fund.

—. 1914. Amulets. London: Constable \& Company LTD. . 1920. Prehistoric Egypt. Egypt Research Account 31. London: British School of Archaeology in
Egypt.

- 1921. Corpus of prehistoric pottery and palettes. Egypt Research Account 32. London: British school of archaeology in Egypt.

- 1925. Tombs of the courtiers and Oxyrinkhos. Egypt Research Account 37. London: British School of Archaeology in Egypt.

Petrie, William Matthew Flinders, and A.C. Mace. 1901. Diospolis Parva. The Cemeteries of Abadiyeh and Hu. 1898-9. Egypt Exploration Fund. 20. London: Egypt Exploration Fund.

Petrie, William Matthew Flinders, and James Edward Quibell. 1896. Naqada and Ballas. 1895. London: B. Quaritch.

Petrie, William Matthew Flinders, and A. H. Sayce. 1891. Illahun, Kahun and Gurob. London: David Nutt.

Petrie William Matthew Flinders, Ll Griffith, and Percy E. Newberry. 1890. Kahun, Gurob, and Hawara. London: K. Paul, Trench, Trübner.

Phillipps, Rebecca. S. 2006. Neolithic surface stone artefact assemblages from the Fayum Depression, Egypt. Thesis (MA--Ancient History) University of Auckland.

Pinch, Geraldine. 2001, "Red Things: The Symbolism of Colour in Magic." In Colour and Painting in Ancient Egypt, edited by W. V. Davies, 182-185. London: British Museum Press.

Pitre, Mindy C., Robert J. Stark, and Maria Carmela Gatto. 2016. "First probable case of scurvy in ancient Egypt at Nag el-Qarmila, Aswan". International Journal of Paleopathology. 13: 11-19. 
Plog, Stephen, and Carrie Heitman. 2010. "Hierarchy and social inequality in the American Southwest, A.D. 800-1200." Proceedings of the National Academy of Sciences of the United States of America. 107 (46): 19619-26.

Quibell, James Edward. 1900. Hierakonpolis I. London: B. Quaritch.

1905. Catalogue Général des Antiquités Egyptiennes. nos. 11.001 - 12.000 et 14.001 - 14.754. Archaic objects. Cairo.

Quibell, James Edward, and F. W. Green. 1902. Hierakonpolis: part II. London: B. Quaritch.

Quirke, Stephen. 2001, "Colour Vocabularies in Ancient Egypt." In Colour and Painting in Ancient Egypt, edited by W. V. Davies, 186-192. London: British Museum Press.

Randall-MacIver, David, and Arthur C. Mace. 1902. El Amrah and Abydos 1899-1901. London: Egypt Exploration society 23.

Redding, Richard. 2010. "Status and Diet at the Workers' Town, Giza, Egypt." In Anthropological approaches to zooarchaeology complexity, colonialism, and animal transformations, edited by Douglas V. Campana, 65-75. Oxford, UK: Oxbow Books. http://site.ebrary.com/id/10695224.

Reisner, George Andrew. 1910. The archaeological survey of Nubia report for 1907-1908. Cairo: National Printing Department.

Riemer, Heiko. 2011. El Kharafish. The archaeology of Sheikh Muftah pastoral nomads in the desert around Dakhla Oasis (Egypt). Africa Praehistorica 25. Köln: Heinrich-Barth-Institut.

Riemer, Heiko, Mathias Lange, and Karin Kindermann. 2013. "When the Desert Dried Up: Late Prehistoric Cultures and Contacts in Egypt and Northern Sudan." In The First Cataract of the Nile: one region - diverse perspectives, edited by D. Raue, 157-183. Berlin: De Gruyter.

Rizkana, Ibrahim, and Seeher, Jürgen. 1985. "The chipped stones at Maadi: Preliminary reassessment of a Predynastic industry and its long-distance relations." Mitteilungen des Deutschen Archäologischen Instituts, Abteilung Kairo. 41: 235-256.

1988. Maadi. 2, The lithic industries of the predynastic settlement. Mainz am Rhein: von Zabern.

Robin, Cynthia. 2003. "New Directions in Classic Maya Household Archaeology." Journal of Archaeological Research 11(4): 307-356.

Rochette, Erick T. 2009. The late classic organization of jade artifact production in the middle Motagua Valley, Zacapa, Guatemala. Thesis (PhD), Pennsylvania State University.

Rodgers, William B. 1966. Development and Specialization: A Case from the Bahamas. Ethnology 5(4):409-414.

Rosen, Steven A. 1997. Lithics after the Stone Age: a handbook of stone tools from the Levant. Walnut Creek, CA: AltaMira Press. 
Rosen, Steven A ., Aaron Shugar, and Jacob Vardi. 2014. "Function and Value in Sickle Segment Analysis: Odellian Perspectives." In Works in Stone, Contemporary Perspectives on Lithic Analysis, edited by M. Shott, 116-30. The University of Utah Press, Salt Lake City.

Rossel, Stine. 2007. The Development of Productive Subsitence Strategies in the Nile Valley: Zooarchaeologicl Analysis at el-Mahâsna and South Abydos, Upper Egypt. Thesis (Ph.D.) Havard University.

Roth, A.M. 1992. "The Pssh-kf and the 'Opening of the Mouth' Ceremony: A Ritual of Birth and Rebirth." Journal of Egyptian Archaeology. 78:113-147.

Roux, Valentine. 2003. "Ceramic Standardization and Intensity of Production: Quantifying Degrees of Specialization". American Antiquity. 68: 768-782.

Rowan, Yorke M. 2012. Beyond Belief: the archaeology of religion and ritual. Hoboken, N.J.: Wiley.

Rowlands, Michael, and Susan Frankenstein. 1998. "Internal structure and regional context of Early Iron Age society in south-western Germany." In Social Transformations in Archaeology: global and local perspectives, edited by K. Kristiansen and M. J. Rowlands, 324-363. London: Routledge.

Ruhl, Katharine C., and Mark F. Seeman. 1997. "Temporal and Social Implications of Ohio Hopewell Copper Ear Spool Design." American Antiquity. 63:651-662.

Sahlins, Marshall. 1972. Stone Age Economics. Chicago \& New York: Aldine, Atherton.

Said, Rushdi. 1990. The geology of Egypt. Rotterdam: Published for the Egyptian General Petroleum Corp., Conoco Hurghada Inc. and Repsol Exploracion, S.A. by A.A. Balkema.

Sauneron, S. 1974. "Les travaux de l'Institut Français d'Archéologie Orientale en 1973-1974." Bulletin de l'Institut Français d'Archéologie Orientale. 74:184-233.

Savage, S. H. 1997. "Descent Group Competition and Economic Strategies in Predynastic Egypt." Journal of Anthropological Archaeology. 16 (3): 226-268.

- 1998. AMS radiocarbon dates from the Predynastic Egyptian cemetery, N.7000, at Naga-ed-Dêr. Journal of Archaeological Science. 25(3): 235-249.

Scharff, Alexander. 1929. Die Altertümer der vor- und frühzeit Ägyptens. II Berlin: Karl Curtius.

Schmidt, Klaus. 1992a. "Tell el-Fara'in/Buto and el-Tell el-Iswid (south): The lithic industries from the Chalcolithic to the early Old Kingdom." In The Nile delta in transition: 4th.-3rd. millennium B.C. : proceedings of the seminar held in Cairo, 21.-24. October 1990, at the Netherlands Institute of Archaeology and Arabic Studies, edited by E. C. M. van den Brink, 31-42. Tel Aviv, Israel.

1992b. "Tell Ibrahim Awad: Preliminary report on the lithic industries.” In The Nile delta in transition: 4th.-3rd. millennium B.C. : proceedings of the seminar held in Cairo, 21.-24. October 1990, at the Netherlands Institute of Archaeology and Arabic Studies, edited by E. C. M. van den Brink, 7996. Tel Aviv, Israel. 
- 1996. "Lower and Upper Egypt in the Chalcolithic Period. Evidence of the lithic industries: a view from Buto.” In Interregional contacts in the later prehistory of northeastern Africa, edited by L. Krzyżaniak, K. Kroeper, and M. Kobusiewicz, 279-289. Poznań: Poznań Archaeological Museum.

Schortman, Edward M., and Patricia A. Urban. 2004. "Modeling the Roles of Craft Production in Ancient Political Economies." Journal of Archaeological Research. 12 (2): 185-226.

Scott, Gerry D. 1986. Ancient Egyptian art at Yale. New Haven, Conn: Yale University Art Gallery.

Seeher, Jürgen. 1999a. "Abusir el-Meleq." In Encyclopedia of the archaeology of ancient Egypt, edited by Kathryn A. Bard, 91-93. London: Routledge.

— 1999b. "Ma'adi and Wadi Digla." In Encyclopedia of the archaeology of ancient Egypt, edited by Kathryn A. Bard, 455-458. London: Routledge.

Service, Elman Rogers. 1962. Primitive social organization: an evolutionary perspective. New York: Random House.

Seton-Karr, Heywood Walter. 1904. "Fayoom flint implements." Annales du Service des Antiquités de l'Égypte. 5: 145-186.

Shafer, Harry J., and Thomas R. Hester. 1991. "Lithic craft specialization and product distribution at the Maya site of Colha, Belize". World Archaeology. 23 (1): 79-97.

Sharer, Robert J. 1994. The ancient Maya.(5th ed.). Stanford, CA: Stanford University Press.

Shaw, Julia. 2013a. "Archaeology of religious change: Introduction." World Archaeology. 45(1):1-11.

_ 2013b. "Archaeologies of Buddhist propagation in ancient India: 'ritual' and 'practical' models of religious change." In Archaeology of religious change, edited by J. Shaw, 83-108. London : Routledge.

Sheets, Payson D. 1978. "From Craftsmen to Cog: Quantitative Views of Mesoamerican Lithic Technology.” In Papers on the Economy and Architecture of the Ancient Maya, edited by R. Sidrys, 4071. Los Angeles: Institute of Archaeology, University of California.

Shimada, Izumi. 2007. Craft Production in Complex Societies: multicraft and producer perspectives. Salt Lake City: University of Utah Press.

Shott, Michael J. 1999. "On Bipolar Reduction and Splintered Pieces." North American Archaeologist. 20 (3): 217-238.

Smith, Adam. 1776. An Inquiry into the Nature and Causes of the Wealth of Nations. VI-II. London.

Smith, Harry S. 1992. "The making of Egypt: A review of the influence of Susa and Sumer on Upper Egypt and Lower Nubia in the 4th Millennium B.C." In Followers of Horus: Studies Dedicated to Michael Allen Hoffman, 1944-1990, edited by Renée F. Friedman and Barbara Adams, 235-246. Oxford [England]: Oxbow Books. 
Smith, Michael. 2009. "V. Gordon Childe and the Urban Revolution: a historical perspective on a revolution in urban studies." Town Planning Review. 80 (1): 3-29.

Spence, Kate. 2015. "Ancient Egyptian Houses and Households: Architecture, Artifacts, Conceptualization, and Interpretation." In Household studies in complex societies: (micro) archaeological and textual approaches : papers from the Oriental Institute Seminar Household Studies in Complex Societies, held at the Oriental Institute of the University of Chicago, 15-16 March 2013, edited by Miriam Müller, 83-99. Chicago, Illinois : The Oriental Institute of the University of Chicago.

Spencer, Neal. 2010. "Shrine." UCLA Encyclopedia of Egyptology.

Spielmann, Katherine A. 2002. "Feasting, Craft Specialization, and the Ritual Mode of Production in Small-Scale Societies." American Anthropologist. 104(1): 195-207.

Spurrel, F. C. J. 1896. "The flint implements of Naqada". In Naqada and Ballas, 1895. Petrie, W. M. F. \& Quibell, J.E., 55-58. London: B. Quaritch.

Stevenson, Alice. 2009. The Predynastic Egyptian cemetery of el-Gerzeh. Social identities and mortuary practices. OLA 186. Leuven: Peeters.

_ 2011. "Material culture of the Predynastic period." In Before the pyramids. The origins of Egyptian civilization. OIMP 33, edited by E. Teeter, 65-74. Chicago: University of Chicago Press.

Stocks, Denys A. 2003. Experiments in Egyptian archaeology. Stoneworking technology in ancient Egypt. London: Routledge.

Strathern, Marilyn. 1965. "Axe Types and Quarries: A Note on the Classification of Axe Blades from the Hagen Area, New Guinea." Journal of the Polynesian Society. 74:182-191.

. 1969. "Stone Axes and Flake Tools: Evaluations from Two New Guinea Highlands Societies." Proceedings of the Prehistoric Society. 35:311-329.

Takamiya, Izumi H. 2004. "Development of Specialization in the Nile Valley During the $4^{\text {th }}$ Millennium BC.” In Egypt at its Origins: studies in memory of Barbara Adams, edited by S. Hendrickx, and B. Adams, 1025- 1039. Orientalia Lovaniensia analecta, 138. Leuven: Peeters.

Takamiya, Izumi H., and Michiko Aoki. 2005. "Another look at HK11C squares A6-A7." Nekhen News. 17: 18-19.

Takamiya, Izumi H., and Masahiro Baba. 2004. " Kilns in square A6: The other side of the story." Nekhen News. 16: 19-20.

Takamiya, Izumi H. and Endo, Hitoshi. 2007. "Beer after sheep ? HK11C squares A6-A7 in 2007." Nekhen News. 19: 28. 20:8-9. 
- 2009. "HK11C squares A6-A7 in 2007.” In Report on the 2006-2007 season of the Hierakonpolis Expedition, Friedman, R.F. et al. Annales du Service des Antiquites de L'Egypte, Cairo. 83:202-204.

_. 2011a. "Variations in Lithic Production at Hierakonpolis: A preliminary report from the excavation of HK11C Squares A6-A7.” In Egypt at its origins 3: proceedings of the international conference "Origin of the state. Predynastic and early dynastic Egypt", London, 27th July - 1st August 2008, edited by R. Friedman, 727-744. Leuven: Peeters.

_ 2011b. "The inside story: The installation and the granary at HK24B." Nekhen News. 23: 20-21.

Takamiya, Izumi H., and Noriyuki Shirai. 2010. " In the shadow of the red mound: HK24B in 2010." Nekhen News. 22: 21-22.

Teeter, Emily. 2011. Before the pyramids: the origins of Egyptian civilization. Chicago, Ill: Oriental Institute of the University of Chicago.

Thomas, David Hurst. 1986. Refiguring anthropology: first principles of probability \& statistics. Prospect Heights, IL: Waveland Press.

Tixier, Jacques. 1963. Typologie de l'épipaléolithique du Maghreb. Paris: Arts et métiers graphiques.

— 1984. Le Debitage par Pression. Prehistoire de la Pierre Taillee: 2 Economie de Debitage Laminaire. Cercle de Recherches et d'Etudes Prehistoriques, Paris. pp. 57-70

Tixier, Jacques, and M. H. Newcomer. 1974. Glossary for the description of stone tools: with special reference to the Epipalaeolithic of the Maghreb. Pullman, Wash: Newsletter of Lithic Technology, Washington State University.

Tomka, Steve A. 1989. “Differentiating Lithic Reduciton Techniques: An Experimental Approach.” In Experiments in lithic technology, edited by D. S. Amick, and R. P. Mauldin, 137-162. Oxford, England: B.A.R.

Tosi, Maurizio. 1984. "The Notion of Craft Specializaiton and its Representation in the Archaeological Record of Early States in the Turanian Basin." In Marxist Perspectives in Archaeology, edited by M. Spriggs, 22-52. New York: Cambridge University Press.

Tristant, Yann. 2004. L'habitat prédynastique de la Vallée du Nil. Vivre sur les rives du Nil au Ve et IVe millénaires. Oxford, England: B.A.R. (1287).

Tukey, J. W. 1949. “Comparing individual means in the analysis of variance.” Biometrics. 5: 99-114.

Usai, Donatella. 2012. "Report on 2012 Field Season with AKAP". Unpublished.

Van Haarlem, Willem. 2001. "Tell Ibrahim Awad." Egyptian Archaeology. 18: 33-35.

Van Neer, Wim, and Veerle Linseele. 2008. "More animal burials from the elite cemetery." Nekhen News. 20: 12-13. 
Van Walsem, René. 1978. "The pss-kf. An investigation of an ancient Egyptian funerary instrument." OMRO, 59-60: 193-249.

Vardi, Jacob, Amir Golan, Daniel Levy, and Isaac Gilead. 2010. "Tracing sickle blade levels of wear and discard patterns: a new sickle gloss quantification method." Journal of Archaeological Science. 37 (7): 1716-1724.

Vaughn, Kevin J., 2004. "Households, Crafts, and Feasting in the Ancient Andes: The Village Context of Early Nasca Craft Consumption." Latin American Antiquity. 15(1): 61-88.

Vermeersch, Pierre M., Wim van Neer, and Stan Hendrickx. 2004. "El Abadiya 2, a Naqada I site near Danfiq, Upper Egypt.” In Egypt at its Origins. Studies in Memory of Barbara Adams, edited by S. Hendrickx, R. Friedman, K. M. Cialowicz, and M. Chlodnicki, 213-276. Orientalia Lovaniensia Analecta 138. Peeters, Leuven.

Vial, L G. 1940. "Stone Axes of Mount Hagci I, New Guinea." Oceania. 11:158-163.

von der Way, Thomas. 1999. "Buto (Tell el-Fara'in).” In Encyclopedia of the archaeology of ancient Egypt, edited by K. A. Bard, 180-184. London: Routledge.

Walker, Alan S. 1996. Animals in ancient art from the Leo Mildenberg collection. Part III. Mainz am Rhein: P. von Zabern.

Watrall, Ethan C. 2000. “Excavations at locality HK 11.” Nekhen News. 12:11-12.

—. 2001. "Tales of trash: Excavations at HK11." Nekhen News. 13: 8-9.

Wattenmaker, Patricia. 1998. Household and state in upper Mesopotamia: specialized economy and the social uses of goods in an early complex society. Smithsonian series in archaeological inquiry. Washington, DC: Smithsonian Institution Press.

Welch, B. L. 1951. “On the comparison of several mean values: an alternative approach.” Biometrika, 38: 330-336.

Wells, E. Christian. 2006. "Recent Trends in Theorizing Prehispanic Mesoamerican Economies." Journal of Archaeological Research 14(4): 265-312.

Wendorf, Fred., Romuald Schild, and Associates. 2001. The Archaeology of Nabta Playa. New York: Kluwer Academic/Plenum.

Wendrich, Willeke. 2000. "Basketry." In Ancient Egyptian materials and technology, edited by P. T. Nicholson, and I. Shaw. 254-267. Cambridge: Cambridge University Press.

Wengrow D. 2001. "Rethinking 'Cattle cults' in early Egypt: Towards a Prehistoric perspective on the Narmer palette." Cambridge Archaeological Journal. 11: 91-104.

—. 2006. The Archaeology of Early Egypt. Cambridge. 
Wetterstrom, Wilma. 1993. "Foraging and farming in Egypt: The transition from hunting and gathering to horticulture in the Nile Valley." In The Archaeology of Africa: food, metals, and towns, edited by Thurstan Shaw, 165-226. London: Routledge.

Whitehouse, Helen. 2002. "A decorated knife handle from the 'Main Deposit' at Hierakonpolis." Mitteilungen des Deutschen Archäologischen Instituts, Abteilung Kairo. 58 (2002): 425-446.

Whitley, David S., and Kelley Hays-Gilpin. 2008. Belief in the past: theoretical approaches to the archaeology of religion. Walnut Creek, Calif: Left Coast Press.

Whittaker, John C. 1994. Flintknapping: Making and Understanding Stone Tools. Austin: University of Texas Press.

Whittaker, John, Kathryn Kamp, and Emek Yilmaz. 2009. "çakmak revisited: Turkish flinknappers today.". Lithic Technology. 34 (2): 93-110.

Wilk, Richard R., and Lisa Cliggett. 2007. Economies and Cultures: foundations of economic anthropology. New York: Westview Press.

Wilke Philip. J., and Leslie A. Quintero. 1995. "Evolution and Economic Significance of Naviform Coreand-Blade Technology in the Southern Levant." Paléorient. 21(1):17-33.

1996. "Naviform Core-and-Blade Technology; Assemblage Character as Determined by Replicative Experiments.” In Neolithic chipped stone industries of the Fertile Crescent: proceedings of the First Workshop on PPN Chipped Lithic Industries, Seminar für Vorderasiatrische Altertumskunde, Free University of Berlin, 29th March-2nd April, 1993, edited by S. Kozlowski, and H. G. Gebel, 3360. Berlin: Ex oriente.

Wilkinson, Toby A. H. 1996. State formation in Egypt: chronology and society. Oxford: British Archaeological Reports

Williams, Bruce, Thomas J. Logan, and William J. Murnane. 1987. "The Metropolitan Museum Knife Handle and Aspects of Pharaonic Imagery before Narmer". Journal of Near Eastern Studies. 46 (4): 245-285.

Wright, Alice P., and Erika Loveland. 2015. "Ritualised craft production at the Hopewell periphery: new evidence from the Appalachian Summit." Antiquity. 89 (343): 137-153.

Yanagisako, Sylvia Junko. 1979. "Family and Household: The Analysis of Domestic Groups." Annual Review of Anthropology 8(1):161-205.

Yerkes, Richard W., Ran Barkai, Avi Gopher, and Ofer Bar Yosef. 2003. "Microwear analysis of early Neolithic (PPNA) axes and bifacial tools from Netiv Hagdud in the Jordan Valley, Israel". Journal of Archaeological Science. 30 (8): 1051-1066.

Zeder, Melinda A. 1988. "Understanding urban process through the study of specialized subsistence economy in the near East." Journal of Anthropological Archaeology 7:1-55.

1991. Feeding cities: specialized animal economy in the ancient Near East. Washington: Smithsonian Institution Press. 


\section{$\underline{\text { Tables }}$}

A note on reading the tables. Much of the information in the following tables involves comparing data from different publications and different researchers. In some cases the exact same categories or definitions were not used. Unless otherwise noted, a blank space means that the item in question was not reported, which can mean either that they didn't look for that item, or that they did and it was not there. Zeros mean that an item was specifically reported as a possibility, but that it definitely was not present. 


\section{Chapter 1 Tables}

Table 1.1. Models for the development of specialized production organized by the kinds of goods made by specialists, and who those goods were for.

\begin{tabular}{|c|c|c|}
\hline & Elites & Commoners \\
\hline $\begin{array}{l}\frac{\underline{U}}{\overline{0}} \\
\text { है } \\
\text { ฝे }\end{array}$ & $\begin{array}{l}\text { Elite activities models: } \\
\text { Political model: political elites employ } \\
\text { specialization and exchange to create social } \\
\text { inequality and build alliances } \\
\text { (Brumfiel \& Earle 1987) } \\
\text { Prestige goods economy: Political advantage } \\
\text { gained by control of external trade and } \\
\text { specialized goods (emphasis on external } \\
\text { trade) } \\
\text { (Rowlands \& Frankenstein 1998) }\end{array}$ & $\begin{array}{l}\text { Symbolic goods models: } \\
\text { Social model: Non-elites use specialized } \\
\text { goods for social messages in addition to } \\
\text { practical functions } \\
\text { (Wattenmaker 1998) } \\
\text { Ritual production: Specialized goods } \\
\text { produced for ritual participation and social } \\
\text { transactions (Spielmann 2002) }\end{array}$ \\
\hline 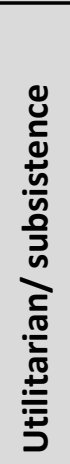 & & $\begin{array}{l}\text { Efficiency models: } \\
\text { Managerial model: Efficient use of } \\
\text { differentially distributed resources } \\
\text { (Service 1962) } \\
\text { Scheduling conflicts: Increased government } \\
\text { demands for agriculture leaves households } \\
\text { little time to make other items } \\
\text { (Blanton et al. 1982) }\end{array}$ \\
\hline
\end{tabular}

Table 1.2. Dates and sizes of beer brewing facilities. For more information on chronology, see Ch 3.2.

\begin{tabular}{|c|c|c|c|}
\hline Location & Date & Size & Publication \\
\hline Abydos, Cemetery D & Gerzean & $\begin{array}{l}8 \text { structures, } 35 \text { vats each (?) } \\
19+m \times 2.6+m\end{array}$ & Peet 1913 \\
\hline Abydos, Seti temple & Gerzean & 23 vats, $14.9 \mathrm{~m} \times 3 \mathrm{~m}$ & Peet 1914 \\
\hline $\begin{array}{l}\text { Hierakonpolis, HK11C, } \\
\text { B4-B5, lower level }\end{array}$ & $\begin{array}{l}\text { NIC-IIB, 3762- } \\
3537 B C\end{array}$ & $\begin{array}{l}\text { 5+ vats, } \\
\sim 8 \mathrm{~m} \times \sim 7 \mathrm{~m} \times .3-.45 \mathrm{~m}(\mathrm{H})\end{array}$ & $\begin{array}{l}\text { Baba 2007, 2008b, 2009a,b, } \\
2011\end{array}$ \\
\hline $\begin{array}{l}\text { Hierakonpolis, HK11C, } \\
\text { A6-A7 }\end{array}$ & mid-NII & 8 vats, $7 \mathrm{~m} \times 3.5 \mathrm{~m}$ & $\begin{array}{l}\text { Hoffman 1982; Takamiya \& } \\
\text { Endo } 2009\end{array}$ \\
\hline Hierakonpolis, HK24A & NII, 3425-3379BC & 6 vats & Geller 1992 \\
\hline Hierakonpolis, HK24B & mid-NII & 16 vats, $6.5 \mathrm{~m} \times 5 \mathrm{~m}$ (interior) & $\begin{array}{l}\text { Geller \& Friedman 2009; } \\
\text { Takamiya \& Shirai } 2010\end{array}$ \\
\hline Mahasna, S1 & Predynastic & Isolated \& groups of $2-3$ vats & Garstang 1902; Geller 1992 \\
\hline Naqada, North Town & NIlcd, Gerzean & $\begin{array}{l}1 \text { vat, possibly other } \\
\text { structures }\end{array}$ & $\begin{array}{l}\text { Petrie \& Quibell 1896; } \\
\text { Geller } 1992\end{array}$ \\
\hline Tel el-Farkha, W47 & NIId & 3 vats & Cichowski 2008 \\
\hline Tel el-Farkha, W192 & NIIC & 11 vats & Cichowski 2008 \\
\hline Tel el-Farkha, W201 & NIIb & 2 rows & Cichowski 2008 \\
\hline Tel el-Farkha, W200 & NIIC & 2 rows, 4 vats each & Cichowski 2008 \\
\hline
\end{tabular}




\section{Chapter 2 Tables}

Table 2.1. Summary of the ritual production model. Description of aspects of the model is on the left (gray) and expectations for archaeological patterns are in the columns to the right.

\begin{tabular}{|c|c|c|c|}
\hline $\begin{array}{c}\text { The Ritual Production } \\
\text { Model }\end{array}$ & Raw Materials & Production Location & Find Contexts \\
\hline $\begin{array}{l}\text { 1-Necessary to ritual } \\
\text { performance and } \\
\text { symbolically laden social } \\
\text { transactions }\end{array}$ & $\begin{array}{l}\text {-Symbolic meanings } \\
\text { associated with the } \\
\text { RM, affecting RM } \\
\text { choice. (Correlation } \\
\text { between Rm \& Tool } \\
\text { type) }\end{array}$ & & $\begin{array}{l}\text {-Specialist made items } \\
\text { are at least sometimes } \\
\text { found in ritual } \\
\text { contexts }\end{array}$ \\
\hline $\begin{array}{l}\text { 2-Ritual performances } \\
\text { and symbolically laden } \\
\text { social transactions } \\
\text { motivate the production }\end{array}$ & & $\begin{array}{l}\text {-Production } \\
\text { associated with } \\
\text { ritual contexts (adds } \\
\text { meaning and } \\
\text { facilitates } \\
\text { production) } \\
\text {-Production can be } \\
\text { only part time }\end{array}$ & \\
\hline $\begin{array}{l}\text { 3-The items cross the } \\
\text { divide between purely } \\
\text { utilitarian and purely } \\
\text { symbolic }\end{array}$ & $\begin{array}{l}\text {-RM sets the items } \\
\text { apart from others } \\
\text { through symbolic } \\
\text { meanings and/or } \\
\text { materials which are } \\
\text { difficult to get } \\
\text { (Correlation } \\
\text { between Rm \& Tool } \\
\text { type) }\end{array}$ & & $\begin{array}{l}\text {-Specialist made items } \\
\text { are found in both } \\
\text { ritual \& non-ritual } \\
\text { contexts }\end{array}$ \\
\hline $\begin{array}{l}\text { 4-The items were used } \\
\text { by many people not just } \\
\text { a specific subset }\end{array}$ & & & $\begin{array}{l}\text { Specialist produced } \\
\text { items are found in: } \\
\text {-settlements of all } \\
\text { scales; } \\
\text {-multiple contexts } \\
\text { within a settlement } \\
\text { (not concentrated) } \\
\text {-graves of different } \\
\text { wealth/status }\end{array}$ \\
\hline
\end{tabular}


Table 2.2. Expectations for the ritual production model compared to expectations for other general models of the development of specialized production.

\begin{tabular}{|c|c|c|c|}
\hline & Raw Materials & Production location & Find Contexts \\
\hline 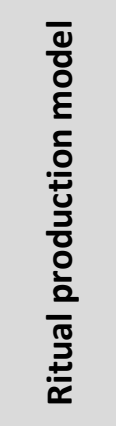 & $\begin{array}{l}\text {-RM adds to the symbolic } \\
\text { meaning of the object. Results } \\
\text { in a correlation between } \mathrm{Rm} \\
\& \text { Tool type }\end{array}$ & $\begin{array}{l}\text {-Production associated } \\
\text { with ritual contexts } \\
\text {-Production can be part } \\
\text { time }\end{array}$ & $\begin{array}{l}\text { Specialized goods } \\
\text {-found in ritual and non } \\
\text { ritual contexts } \\
\text {-found in settlements of all } \\
\text { scales, multiple contexts } \\
\text { within a settlement, and } \\
\text { graves of different } \\
\text { status/wealth } \\
\text { (used by many) }\end{array}$ \\
\hline 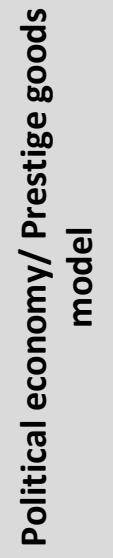 & $\begin{array}{l}\text {-RM can be specifically chosen } \\
\text { because it is not accessible by } \\
\text { all }\end{array}$ & $\begin{array}{l}\text {-Production in discrete } \\
\text { (controllable) areas, } \\
\text { possibly associated with } \\
\text { elite and/or ritual } \\
\text { contexts, along with } \\
\text { administrative artifacts } \\
\text {-full time production } \\
\text { (workers sponsored by } \\
\text { elites) }\end{array}$ & $\begin{array}{l}\text {-Specialized goods found } \\
\text { primarily in non-ordinary } \\
\text { contexts (burials, ritual } \\
\text { contexts, administrative } \\
\text { contexts) } \\
\text {-specialized goods } \\
\text { concentrated in primary } \\
\text { centers, concentrated in } \\
\text { limited contexts within } \\
\text { settlements, and/or in } \\
\text { elite/wealthy graves (used } \\
\text { by restricted groups) }\end{array}$ \\
\hline 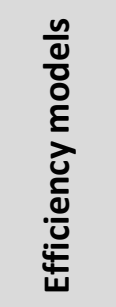 & $\begin{array}{l}\text {-RM chosen for ease of access } \\
\text { near the place of production }\end{array}$ & $\begin{array}{l}\text {-Production locations not } \\
\text { associated with ritual } \\
\text { contexts } \\
\text {-full time production }\end{array}$ & $\begin{array}{l}\text {-not in ritual contexts } \\
\text { (utilitarian goods) } \\
\text {-found in settlements of all } \\
\text { scales, multiple contexts } \\
\text { within a settlement } \\
\text { (used by many) }\end{array}$ \\
\hline 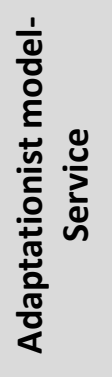 & $\begin{array}{l}\text {-specialization based on } \\
\text { geographic distribution of raw } \\
\text { materials, every place } \\
\text { specialized based on its } \\
\text { available materials }\end{array}$ & $\begin{array}{l}\text {-Production locations } \\
\text { based on the local } \\
\text { availability of raw } \\
\text { materials } \\
\text {-Different places each } \\
\text { focus on one kind of } \\
\text { technology } \\
\text {-full time production }\end{array}$ & $\begin{array}{l}\text {-not in ritual contexts } \\
\text { (utilitarian goods) } \\
\text {-found in settlements of all } \\
\text { scales, multiple contexts } \\
\text { within a settlement } \\
\text { (used by many) }\end{array}$ \\
\hline
\end{tabular}




\section{Chapter 3 Tables}

Table 3.1. Correlation of relative and absolute chronologies for the Predynastic and Early Dynastic periods, derived mainly from Buchez (2011b), Hartmann (2011), Hendrickx (2006, 2011b,c), and Dee et al. (2013). Köhler (2004) offers revisions for the NIIIC and divisions of the NIIID (not pictured here). Note that traditionally the Amratian goes only up to SD 37, but here it is put up to the NIIB/C transition to mark that there are more differences between NIIB and NIIC than between NIC and NIIA (Hendrickx 2011c). The heavy lines indicate the three major chronological divisions. ${ }^{1}$ These ranges mark the transition between the indicated phases, and are the $68 \%$ highest posterior density ranges (Dee et al. 2013). ${ }^{2}$ These ranges mark the ranges for the accession dates for the indicated ruler, and are the $68 \%$ highest posterior density ranges (Dee et al. 2013).

\begin{tabular}{|c|c|c|c|c|c|c|c|c|}
\hline 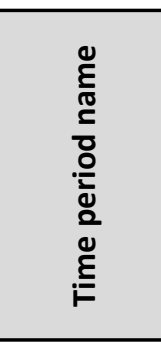 & 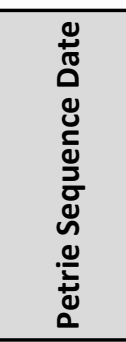 & 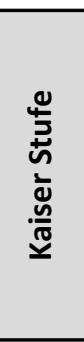 & 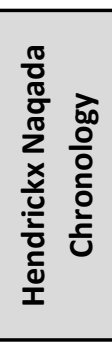 & 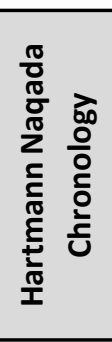 & & U. & 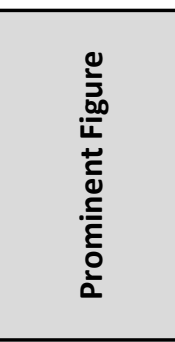 & 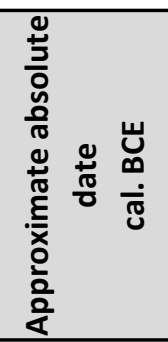 \\
\hline $\begin{array}{c}\text { 2nd } \\
\text { Dynasty/ } \\
\text { Archaic/ } \\
\text { Early } \\
\text { Dynastic }\end{array}$ & $83-86$ & & NIIID & & & & $\begin{array}{c}\text { Hetep- } \\
\text { sekhemwy } \\
\text { to } \\
\text { Khasekhem } \\
\text { wy }\end{array}$ & \\
\hline $\begin{array}{c}\text { 1st } \\
\text { Dynasty/ }\end{array}$ & $81-82$ & IIIc3 & NIIIC2 & & \multirow{2}{*}{\multicolumn{2}{|c|}{$\begin{array}{l}\text { Late ware most } \\
\text { frequent }\end{array}$}} & Djet - Qaa & $\begin{array}{c}\text { Qaa }^{2}: \\
\text { 2906-2886 }\end{array}$ \\
\hline $\begin{array}{l}\text { Archaic/ } \\
\text { Early } \\
\text { Dynastic }\end{array}$ & 79 & IIIc2 & \multirow[t]{2}{*}{ NIIIC1 } & & & & $\begin{array}{c}\text { Hor Aha - } \\
\text { Djer }\end{array}$ & $\begin{array}{c}\text { Aha }{ }^{2}: 3111 \\
-3045\end{array}$ \\
\hline \multirow{6}{*}{$\begin{array}{l}\text { Proto- } \\
\text { Inostic / } \\
\text { ynasty } 0\end{array}$} & \multirow{3}{*}{$77-78$} & IIIc1 & & & \multirow{5}{*}{\multicolumn{2}{|c|}{$\begin{array}{l}\text { Wavy-Handled most } \\
\text { frequent \& Late Ware } \\
\text { common }\end{array}$}} & Ka-Narmer & \\
\hline & & $111 \mathrm{~b} 2$ & \multirow{2}{*}{ NIIIB } & & & & Iri-hor & \\
\hline & & IIIb1 & & & & & & \\
\hline & \multirow{3}{*}{$63-76$} & Illa2 & NIIIA2 & & & & Abu-Zeidan & \\
\hline & & & NIIIA1 & & & & Tomb U-J & \multirow{2}{*}{$\begin{array}{l}\text { NIIID to } \\
\text { NIIIIA }{ }^{1} \text { : } \\
3352-3297\end{array}$} \\
\hline & & Illa1 & \multirow[t]{2}{*}{ NIID } & & \multirow{4}{*}{\multicolumn{2}{|c|}{$\begin{array}{l}\text { Decorated } \\
\text { Ware } \\
\text { with } \\
\text { figures }\end{array}$}} & & \\
\hline \multirow{7}{*}{ Gerzean } & \multirow{3}{*}{$\begin{array}{c}40 / 45- \\
62 / 63\end{array}$} & IId 2 & & & & & & \\
\hline & & IId1 & \multirow{2}{*}{ NIIC } & & & & & \\
\hline & & IIc & & & & & & \multirow{3}{*}{$\begin{array}{c}\text { NIIB to } \\
\text { NIIC }^{1}: \\
3498-3413\end{array}$} \\
\hline & \multirow{3}{*}{$\begin{array}{c}38- \\
40 / 45\end{array}$} & $\| \mathrm{h}$ & \multirow{2}{*}{ NIIB } & IC & \multirow{6}{*}{$\begin{array}{l}\text { Black- } \\
\text { Topped } \\
\text { Ware }\end{array}$} & \multirow{2}{*}{$\begin{array}{c}\text { Decorate } \\
\text { d }\end{array}$} & & \\
\hline & & 110 & & IB2 & & & & \\
\hline & & Ila & NIIA & & & \multirow{4}{*}{$\begin{array}{l}\text { White } \\
\text { Cross- } \\
\text { Lined } \\
\text { Ware }\end{array}$} & & \multirow{4}{*}{$\begin{array}{c}\text { NIB to } \\
\text { NIC }^{1}: \\
3690 / 3605 \\
\end{array}$} \\
\hline & \multirow{3}{*}{$\begin{array}{c}30- \\
37 / 38\end{array}$} & IC & NIC & $\frac{\mathrm{IB1}}{\mathrm{IA} 3}$ & & & & \\
\hline \multirow[t]{3}{*}{ Amratian } & & $\mathrm{Ib}$ & NIB & IA2 & & & & \\
\hline & & la & NIA & & & & & \\
\hline & & & & IA1 & & & & \\
\hline
\end{tabular}


Table 3.2. Summary of excavation Blocks at Mahâsna, (Anderson 2006).

\begin{tabular}{|c|l|l|l|}
\hline Block & \multicolumn{1}{|c|}{ Description } & \multicolumn{1}{c|}{ Date } & \multicolumn{1}{c|}{ Reference } \\
\hline 1 & Habitation structure \& midden deposits & Ic-Ilab & Anderson 2006:89 \\
\hline 2 & $\begin{array}{l}\text { Outdoor area with large hearth \& trash disposal } \\
\text { (probably associated with the structure in Block 3) }\end{array}$ & Ic-Ilc & $\begin{array}{l}\text { Anderson 2006:89-97; 162, } \\
246\end{array}$ \\
\hline 3 & Ritual activity area with a partially exposed structure & Ic-Ilc & Anderson 2006:97-123 \\
\hline 4 & Domestic activity area & Ic-Ilb & Anderson 2006:123-132 \\
\hline 5 & Outdoor activity \& trash disposal area like Block 2 & Ic-Ila-c & Anderson 2006:133-137, 246 \\
\hline 6 & Area disturbed by Garstang's habitation & Modern & Anderson 2006:137-138 \\
\hline 7 & Garstang's Excavation house & Modern & Anderson 2006:138 \\
\hline 8 & Habitation structure & Ic-Ilb & Anderson 2006:139-146 \\
\hline 9 & Partial habitation structure? & Ind. & Anderson 2006:147-149 \\
\hline
\end{tabular}

Table 3.3. Classification of settlement sites according to population size and functional variability, for comparing the distribution of artifacts. Cemetery estimates for Abydos, Naqada, and Hierakonpolis are combined from a number of cemeteries. All sites include habitation, subsistence, and disposal activities. See Ch. 3.4 \& 3.5 for site descriptions and references. * See section 3.7 for issues regarding area comparisons.

\begin{tabular}{|c|c|c|c|c|c|}
\hline Site & Date & Area* & Cemetery size & Activities & Classification \\
\hline Abydos & NI-NIII & Unknown & $>2000$ & $\begin{array}{l}\text { Administration, ritual, Beer, } \\
\text { beads? }\end{array}$ & High Level \\
\hline $\begin{array}{l}\text { Naqada } \\
\text { South Town }\end{array}$ & NIIc-NIII & 3ha & $>2000$ & $\begin{array}{l}\text { Administration, ritual, } \\
\text { (beer North town) }\end{array}$ & High Level \\
\hline $\begin{array}{l}\text { Hierakonpoli } \\
\mathrm{s}\end{array}$ & NI-NIIIA & $\begin{array}{l}\text { Very } \\
\text { large }\end{array}$ & $>2000$ & $\begin{array}{l}\text { Administration(?), ritual, } \\
\text { beer, pottery, stone tools }\end{array}$ & High Level \\
\hline Maadi & NI-NIIb & 4 - 13ha & $\sim 500$ & $\begin{array}{l}\text { Trade, groundstone, } \\
\text { copper?, }\end{array}$ & Mid Level \\
\hline $\begin{array}{l}\text { Badari } \\
3000 / 6\end{array}$ & NI-NIID & Unknown & ? & Beer & Mid Level \\
\hline Mahâsna & NIC-NIID & 7ha & $\sim 600$ & Ritual, Beer & Mid Level \\
\hline Adaïma & NI-NIII & $<35$ ha & 1500 & Grain processing & Mid Level \\
\hline $\begin{array}{l}\text { Hemmamiya } \\
\text { North Spur }\end{array}$ & $\begin{array}{l}\text { Badarian- } \\
\text { NI-NIIB or C }\end{array}$ & $<1$ ha & $?$ & & Low Level \\
\hline $\begin{array}{l}\text { Naqada- } \\
\text { Khattara }\end{array}$ & NI-NIIab & $<1-3 h a$ & $?$ & & Low Level \\
\hline Abadiya 2 & NI-NIIA/B & $<1$ ha & $?$ & & Low Level \\
\hline Armant & NI-NIlab & $<1$ ha & $\sim 200$ & & Low Level \\
\hline $\begin{array}{l}\text { Nag el } \\
\text { Qarmila }\end{array}$ & NIC-IIAB & $<1$ ha & $>52$ & & Low Level \\
\hline
\end{tabular}


Chapter 4 Tables 
Table 4.1 (part 1). Raw material survey data. For raw material category descriptions, see Appendix.

\begin{tabular}{|c|c|c|c|c|c|c|c|c|c|c|c|c|c|c|}
\hline $\begin{array}{c}\text { Nodule } \\
\text { ID }\end{array}$ & $\begin{array}{c}\text { UTM } \\
\text { coordi- } \\
\text { nates E }\end{array}$ & $\begin{array}{c}\text { UTM } \\
\text { coordi- } \\
\text { nates } \mathbf{N}\end{array}$ & $\begin{array}{r}\text { Elevation } \\
\text { (M ASL) }\end{array}$ & Location & $\begin{array}{c}\text { Geological } \\
\text { context }\end{array}$ & $\begin{array}{c}\text { Nodule } \\
\text { shape }\end{array}$ & $\begin{array}{c}\text { Cortex } \\
\text { Description }\end{array}$ & Munsell & $\begin{array}{c}\text { RM } \\
\text { category }\end{array}$ & Length & Width & $\begin{array}{c}\text { Thick- } \\
\text { ness }\end{array}$ & Weight & $\begin{array}{r}\text { Volume } \\
(\mathrm{ml})\end{array}$ \\
\hline A1 & & & & Abydos & $\begin{array}{l}\text { Secondary- } \\
\text { Wadi Wash }\end{array}$ & Cube & & $\begin{array}{l}\text { 10YR 6/3 \& } \\
10 Y R 7 / 2\end{array}$ & 2 & 190.68 & 103.75 & 46.45 & 1058 & \\
\hline A2 & & & & Abydos & $\begin{array}{l}\text { Secondary- } \\
\text { Wadi Wash }\end{array}$ & Irregular & & 10YR $7 / 2$ & 1 & 105.39 & 56.71 & 34.04 & 217.7 & 90 \\
\hline$A 3 b$ & 391069 & 2894773 & $\begin{array}{l}112.6 \\
+/-6.6\end{array}$ & Abydos & $\begin{array}{l}\text { Secondary- } \\
\text { Wadi Wash }\end{array}$ & Cube & $\begin{array}{l}\text { Light weather } \\
\text { \& patina }\end{array}$ & 10YR 6/4 & 4 & 81 & 61.16 & 40.71 & 289.2 & 115 \\
\hline A4a & 391316 & 2894639 & & Abydos & $\begin{array}{l}\text { Secondary- } \\
\text { Wadi Wash }\end{array}$ & Cube & Other & 7.5YR 5/6 & 1 & 52.91 & 46.86 & 21.9 & 77.6 & 30 \\
\hline$A 4 b$ & 391316 & 2894639 & & Abydos & $\begin{array}{l}\text { Secondary- } \\
\text { Wadi Wash }\end{array}$ & Sphere & $\begin{array}{l}\text { Lightly } \\
\text { weathered }\end{array}$ & 7.5YR 4/1 & 8 & 58.12 & 41.54 & 40.92 & 138.9 & 50 \\
\hline A5 & 391319 & 2894625 & $\begin{array}{l}138.3 \\
+/-5.1\end{array}$ & Abydos & $\begin{array}{l}\text { Secondary- } \\
\text { Wadi Wash }\end{array}$ & Irregular & & 7.5YR 4/1-2 & 8 & 45.74 & 34.15 & 24.66 & 43 & 20 \\
\hline A6a & 391311 & 2894592 & $\begin{array}{l}143.1 \\
+/-5.1\end{array}$ & Abydos & $\begin{array}{l}\text { Secondary- } \\
\text { Wadi Wash }\end{array}$ & $\begin{array}{l}\text { Right } \\
\text { cylinder }\end{array}$ & $\begin{array}{l}\text { Light weather } \\
\text { \& patina }\end{array}$ & 5YR 6/1 & 10 & 130.74 & 52.61 & 45.53 & 468.3 & 180 \\
\hline$A 6 b$ & 391311 & 2894592 & $\begin{array}{l}143.1 \\
+/-5.1\end{array}$ & Abydos & $\begin{array}{l}\text { Secondary- } \\
\text { Wadi Wash }\end{array}$ & $\begin{array}{l}\text { Right } \\
\text { cylinder }\end{array}$ & $\begin{array}{l}\text { Lightly } \\
\text { weathered }\end{array}$ & $2.5 Y R$ 5/1 & 10 & 139.09 & 74.93 & 82.61 & 1200 & 495 \\
\hline A7 & 391117 & 2894269 & $\begin{array}{l}161.5 \\
+/-8.5\end{array}$ & Abydos & $\begin{array}{l}\text { Secondary- } \\
\text { Lag }\end{array}$ & Cube & $\begin{array}{l}\text { Chalky \& lightly } \\
\text { weathered }\end{array}$ & not recorded & 1 & 70.92 & 49.96 & 38.06 & 214.7 & 80 \\
\hline A8 & 391034 & 2894134 & $\begin{array}{l}155.9 \\
+/-7.8\end{array}$ & Abydos & $\begin{array}{l}\text { Secondary- } \\
\text { Lag }\end{array}$ & $\begin{array}{l}\text { Right } \\
\text { cylinder }\end{array}$ & $\begin{array}{l}\text { Chalky \& lightly } \\
\text { weathered }\end{array}$ & 10YR 6/1 & 1 & 118.96 & 96.6 & 67.54 & 1000 & 400 \\
\hline A9 & 391022 & 2894104 & $\begin{array}{l}156.5 \\
+/-6.1\end{array}$ & Abydos & Primary & $\begin{array}{l}\text { Right } \\
\text { cylinder }\end{array}$ & $\begin{array}{l}\text { Lightly } \\
\text { weathered }\end{array}$ & 7.5YR 4/1 & 8 & 81.14 & 62.91 & 47.24 & 302.4 & 115 \\
\hline A10 & 391036 & 2893943 & $\begin{array}{l}161.7 \\
+/-22.6\end{array}$ & Abydos & Primary & Cube & Chalky & 7.5YR 4/1 & 8 & 47.06 & 23.44 & 18.84 & 29.5 & 10 \\
\hline$A 11 b$ & & & & Abydos & Secondary & $\begin{array}{l}\text { Right } \\
\text { cylinder }\end{array}$ & $\begin{array}{l}\text { Lightly } \\
\text { weathered }\end{array}$ & 10YR 6/1-2 & 1 & 91.38 & 68.64 & 50.25 & 500 & 195 \\
\hline A12a & 391094 & 2893870 & $\begin{array}{l}180.1 \\
+/-24.2\end{array}$ & Abydos & Primary & Irregular & $\begin{array}{l}\text { Lightly } \\
\text { weathered }\end{array}$ & 10YR 4/1 & 1 & 74.04 & 36.91 & 35.77 & 141 & 50 \\
\hline$A 12 b$ & 391094 & 2893870 & $\begin{array}{l}180.1 \\
+/-24.2\end{array}$ & Abydos & Primary & Cube & $\begin{array}{l}\text { Chalky \& lightly } \\
\text { weathered }\end{array}$ & 10YR 4/1 & 1 & 62 & 39.4 & 23.22 & 70.7 & 25 \\
\hline $\mathrm{A} 12 \mathrm{C}$ & 391094 & 2893870 & $\begin{array}{l}180.1 \\
+/-24.2\end{array}$ & Abydos & Primary & Cube & $\begin{array}{l}\text { Chalky \& lightly } \\
\text { weathered }\end{array}$ & 10YR 4/1 & 1 & 51.87 & 41.01 & 24.58 & 71.4 & 25 \\
\hline
\end{tabular}


Table 4.1 (part 2). Raw material survey data. For raw material category descriptions, see Appendix. R= Rock crystal/ quartzite.

\begin{tabular}{|c|c|c|c|c|c|c|c|c|c|c|c|c|c|c|}
\hline $\begin{array}{c}\text { Nodule } \\
\text { ID }\end{array}$ & $\begin{array}{c}\text { UTM } \\
\text { coordi- } \\
\text { nates E }\end{array}$ & $\begin{array}{c}\text { UTM } \\
\text { coordi- } \\
\text { nates } \mathbf{N}\end{array}$ & $\left|\begin{array}{r}\text { Elevation } \\
\text { (M ASL) }\end{array}\right|$ & Location & $\begin{array}{c}\text { Geological } \\
\text { context }\end{array}$ & $\begin{array}{c}\text { Nodule } \\
\text { shape }\end{array}$ & $\begin{array}{c}\text { Cortex } \\
\text { Description }\end{array}$ & Munsell & $\begin{array}{c}\text { RM } \\
\text { category }\end{array}$ & Length & Width & $\begin{array}{l}\text { Thick- } \\
\text { ness }\end{array}$ & Weight & $\begin{array}{r}\text { Volume } \\
(\mathrm{ml})\end{array}$ \\
\hline A13 & 391049 & 2893736 & $\begin{array}{l}177.7 \\
+/-11.6\end{array}$ & Abydos & Primary & Cube & Chalky & 10YR 5/1 & 3 & 76.61 & 45.77 & 10.97 & 52.8 & 12 \\
\hline A14 & 391154 & 2893496 & $\begin{array}{l}209.4 \\
+/-16.2\end{array}$ & Abydos & Primary & $\begin{array}{l}\text { Right } \\
\text { cylinder }\end{array}$ & $\begin{array}{l}\text { Chalky \& lightly } \\
\text { weathered }\end{array}$ & 7.5YR 4/1 & 8 & 63.67 & 43.74 & 39.54 & 161.8 & 55 \\
\hline A15 & 391145 & 2893497 & & Abydos & Secondary & Cube & $\begin{array}{l}\text { Lightly } \\
\text { weathered }\end{array}$ & 5YR 5/2 & 4 & 49.15 & 28.09 & 20.74 & 39.2 & 10 \\
\hline A16 & 391316 & 2893413 & $\begin{array}{l}258.4 \\
+/-5.4\end{array}$ & Abydos & Primary & $\begin{array}{l}\text { Cube, } \\
\text { irregular }\end{array}$ & $\begin{array}{l}\text { Chalky \& lightly } \\
\text { weathered }\end{array}$ & 10YR 4/1 & 1 & 123.8 & 63.43 & 41.45 & 471.9 & 180 \\
\hline A17 & 391454 & 2894011 & $\begin{array}{l}298.6 \\
+/-5.4\end{array}$ & Abydos & $\begin{array}{l}\text { Secondary- } \\
\text { Lag }\end{array}$ & $\begin{array}{l}\text {-Cube, } \\
\text { irregular }\end{array}$ & $\begin{array}{l}\text { Chalky \& lightly } \\
\text { weathered }\end{array}$ & 7.5YR 6/4 & 4 & 58.98 & 50.71 & 42.55 & 229.6 & 85 \\
\hline A18 & 391510 & 2894019 & $\begin{array}{l}299.9 \\
+/-12.8\end{array}$ & Abydos & $\begin{array}{l}\text { Secondary- } \\
\text { Lag }\end{array}$ & $\begin{array}{l}\text {-Cube, } \\
\text { irregular }\end{array}$ & $\begin{array}{l}\text { Natural fissure } \\
\text { planes }\end{array}$ & & $\mathrm{R}$ & 82.52 & 29.48 & 26.1 & 96.1 & 30 \\
\hline A19 & 390875 & 2893824 & $\begin{array}{l}296.3 \\
+/-4.9\end{array}$ & Abydos & Secondary & $\begin{array}{l}\text { Right } \\
\text { cylinder }\end{array}$ & $\begin{array}{l}\text { Pitted \& } \\
\text { smoothed }\end{array}$ & $\begin{array}{l}\text { 10YR 6/1 \& } \\
10 Y R 7 / 3\end{array}$ & 8 & 83.17 & 65.69 & 41.29 & 299.7 & 115 \\
\hline$A 20 b$ & 391078 & 2893277 & $\begin{array}{l}251 \\
+/-6\end{array}$ & Abydos & $\begin{array}{l}\text { Secondary- } \\
\text { Lag }\end{array}$ & $\begin{array}{l}\text {-Right } \\
\text { cylinder }\end{array}$ & $\begin{array}{l}\text { Lightly } \\
\text { weathered }\end{array}$ & 10YR 6/2 & 1 & 122.15 & 41.66 & 36.66 & 276.2 & 105 \\
\hline$A 20 c$ & 391078 & 2893277 & $\begin{array}{l}251 \\
+/-6\end{array}$ & Abydos & $\begin{array}{l}\text { Secondary- } \\
\text { Lag }\end{array}$ & Cube & $\begin{array}{l}\text { Chalky \& lightly } \\
\text { weathered }\end{array}$ & 10YR 7/2 & 4 & 64.5 & 42.52 & 30.89 & 140.9 & 52 \\
\hline$A 20 d$ & 391078 & 2893277 & $\begin{array}{l}251 \\
+/-6\end{array}$ & Abydos & $\begin{array}{l}\text { Secondary- } \\
\text { Lag }\end{array}$ & Sphere & $\begin{array}{l}\text { Chalky \& lightly } \\
\text { weathered }\end{array}$ & $7.5 Y R$ 4/2 & 8 & 53.57 & 44.78 & 37.51 & 114.2 & 40 \\
\hline$A 21$ & & & & Abydos & $\begin{array}{l}\text { Not } \\
\text { Recorded }\end{array}$ & Cube & $\begin{array}{l}\text { Chalky \& lightly } \\
\text { weathered }\end{array}$ & 7.5YR 5/4 & 8 & 106.54 & 86.03 & 33.37 & 424 & $\begin{array}{r}\sim 125- \\
150\end{array}$ \\
\hline A23 & & & & Abydos & $\begin{array}{l}\text { Not } \\
\text { Recorded }\end{array}$ & Irregular & $\begin{array}{l}\text { Chalky \& lightly } \\
\text { weathered }\end{array}$ & $\begin{array}{l}10 \text { YR } 5 / 2 \& \\
10 \text { YR } 7 / 2\end{array}$ & 1 & 124.87 & 48.89 & 29.99 & 297.2 & 110 \\
\hline A25 & & & & Abydos & $\begin{array}{l}\text { Not } \\
\text { Recorded }\end{array}$ & Irregular & $\begin{array}{l}\text { Light weather } \\
\text { \& patina }\end{array}$ & 10YR 5/4 & 1 & 71.53 & 49.9 & 37.33 & 137.3 & 55 \\
\hline A26 & & & & Abydos & $\begin{array}{l}\text { Not } \\
\text { Recorded }\end{array}$ & Cube & $\begin{array}{l}\text { Chalky \& lightly } \\
\text { weathered }\end{array}$ & 7.5YR 6/4 & 4 & 51.84 & 34.12 & 18.01 & 62.6 & 25 \\
\hline A27 & & & & Abydos & $\begin{array}{l}\text { Not } \\
\text { Recorded }\end{array}$ & Cube & $\begin{array}{l}\text { Chalky \& lightly } \\
\text { weathered }\end{array}$ & 7.5YR 5/3 & 4 & 43.09 & 37.02 & 11.08 & 22.6 & 10 \\
\hline
\end{tabular}


Table 4.1 (part 3). Raw material survey data. For raw material category descriptions, see Appendix. SL= silicified limestone.

\begin{tabular}{|c|c|c|c|c|c|c|c|c|c|c|c|c|c|c|}
\hline $\mid \begin{array}{c}\text { Nodule } \\
\text { ID }\end{array}$ & $\mid \begin{array}{c}\text { UTM } \\
\text { coordinat } \\
\text { es E }\end{array}$ & $\begin{array}{c}\text { UTM } \\
\text { coordinat } \\
\text { es N }\end{array}$ & $\mid \begin{array}{c}\text { Elevatio } \\
n \text { (M } \\
\text { ASL) }\end{array}$ & Location & $\begin{array}{c}\text { Geological } \\
\text { context }\end{array}$ & $\begin{array}{l}\text { Nodule } \\
\text { shape }\end{array}$ & $\begin{array}{c}\text { Cortex } \\
\text { Description }\end{array}$ & Munsell & $\begin{array}{c}\text { RM } \\
\text { category }\end{array}$ & Length & Width & $\begin{array}{l}\text { Thick- } \\
\text { ness }\end{array}$ & Weight & $\begin{array}{r}\text { Volume } \\
(\mathrm{ml})\end{array}$ \\
\hline A32 & & & & Abydos & $\begin{array}{l}\text { Not } \\
\text { Recorded }\end{array}$ & Cube & $\begin{array}{l}\text { Light weather } \\
\text { \& patina }\end{array}$ & $\begin{array}{l}10 Y R 7 / 3 \& \\
10 Y R 5 / 3\end{array}$ & 4 & 110.98 & 33 & 17.81 & 120.4 & 45 \\
\hline A 35 & & & & Abydos & Secondary & Sphere & & $7.5 Y R \quad 4 / 3$ & 8 & 10.09 & 4.87 & 4.22 & 150 & 100 \\
\hline A 38 & & & & Abydos & Secondary & Sphere & & 10YR 5/2 & 8 & 9 & 7.27 & 4.88 & 510 & 125 \\
\hline A 41 & & & & Abydos & Secondary & Cube & & 10YR 6/2-4 & 1 & 6.27 & 6.25 & 3.03 & 190 & 100 \\
\hline BK1 & & & & $\begin{array}{l}\text { Beit } \\
\text { Khallaf }\end{array}$ & $\begin{array}{l}\text { Secondary- } \\
\text { Wadi } \\
\text { Wash }\end{array}$ & Sphere & $\begin{array}{l}\text { Pitted \& } \\
\text { smoothed }\end{array}$ & 10YR 6/2 & 8 & 90.12 & 69.75 & 63.65 & 550 & 240 \\
\hline BK2 & & & & $\begin{array}{l}\text { Beit } \\
\text { Khallaf }\end{array}$ & $\begin{array}{l}\text { Secondary- } \\
\text { Wadi } \\
\text { Wash }\end{array}$ & $\begin{array}{l}\text { Cube, } \\
\text { irregular }\end{array}$ & $\begin{array}{l}\text { Dark-pink } \\
\text { pitted \& } \\
\text { smoothed }\end{array}$ & $\begin{array}{l}\text { 10YR } 5 / 2 \& \\
10 Y R 6 / 6\end{array}$ & 8 & 88.8 & 50.8 & 35.02 & 241 & 95 \\
\hline BK3 & & & & $\begin{array}{l}\text { Beit } \\
\text { Khallaf }\end{array}$ & $\begin{array}{l}\text { Secondary- } \\
\text { Wadi } \\
\text { Wash }\end{array}$ & $\begin{array}{l}\text { Cube, } \\
\text { irregular }\end{array}$ & $\begin{array}{l}\text { Dark-pink } \\
\text { pitted \& } \\
\text { smoothed }\end{array}$ & $\begin{array}{l}2.5 \text { YR } 4 / 3 \text { to } \\
10 \text { YR } 5 / 3\end{array}$ & 9 & 85.63 & 79.01 & 37.99 & 384 & 150 \\
\hline BK4 & & & & $\begin{array}{l}\text { Beit } \\
\text { Khallaf }\end{array}$ & $\begin{array}{l}\text { Secondary- } \\
\text { Wadi } \\
\text { Wash }\end{array}$ & $\begin{array}{l}\text { Right } \\
\text { cylindar }\end{array}$ & $\begin{array}{l}\text { Pitted \& } \\
\text { smoothed }\end{array}$ & 10YR 6/2 & SL & 118.66 & 107.73 & 40.39 & 500 & 300 \\
\hline BK5 & & & & $\begin{array}{l}\text { Beit } \\
\text { Khallaf }\end{array}$ & $\begin{array}{l}\text { Secondary- } \\
\text { Wadi } \\
\text { Wash }\end{array}$ & $\begin{array}{l}\text { Cube, } \\
\text { irregular }\end{array}$ & $\begin{array}{l}\text { Pitted \& } \\
\text { rsmoothed }\end{array}$ & 10YR $7 / 2$ & 1 & 114.38 & 61.14 & 44.91 & 486.9 & 185 \\
\hline Wk1 & & & & $\begin{array}{l}\text { Wadi } \\
\text { Kubbaniya }\end{array}$ & $a^{\text {Secondary }}$ & Cube & $\begin{array}{l}\text { Completely } \\
\text { smoothed }\end{array}$ & 10YR 6/3 & 1 & 59.75 & 40.17 & 16.95 & 70.6 & 25 \\
\hline WK2 & & & & $\begin{array}{l}\text { Wadi } \\
\text { Kubbaniya }\end{array}$ & $a^{\text {Secondary }}$ & Irregular & $\begin{array}{l}\text { Pitted \& } \\
\text { smoothed }\end{array}$ & 10YR 6/4 & 8 & 47.19 & 35.61 & 19.82 & 28.4 & 10 \\
\hline
\end{tabular}


Table 4.2. Comparison of counts and percentages of raw material varieties. The most prevalent type at each site is highlighted in bold.

\begin{tabular}{|c|c|c|c|c|c|c|}
\hline & \multicolumn{2}{|c|}{ MAP } & \multicolumn{2}{|c|}{ ATP } & \multicolumn{2}{|c|}{ AKAP } \\
\hline & Count & $\%$ & Count & $\%$ & Count & $\%$ \\
\hline 1. Indistinct beige & 521 & 12.36 & 43 & 7.93 & 106 & 9.19 \\
\hline 2. Beige (Fine) & 91 & 2.16 & 18 & 3.32 & 98 & 8.49 \\
\hline 3. Beige (Less-Fine) & 200 & 4.74 & 20 & 3.69 & 5 & 0.43 \\
\hline 4. Yellow-Beige with Pink Bands & 98 & 2.33 & 136 & 25.09 & 5 & 0.43 \\
\hline 5. Medium Brown & 48 & 1.14 & 2 & 0.37 & 57 & 4.94 \\
\hline 6. Brown Fossil & 2 & 0.05 & 0 & 0 & 120 & 10.4 \\
\hline 7. Dark Gray and Brown & 18 & 0.43 & 2 & 0.37 & 70 & 6.07 \\
\hline 8. Translucent Brown Spectrum & 1585 & 37.6 & 122 & 22.51 & 85 & 7.37 \\
\hline $\begin{array}{l}\text { 9. Translucent Brown with Pink } \\
\text { Gravel Cortex }\end{array}$ & 290 & 6.88 & 28 & 5.17 & 3 & 0.26 \\
\hline 10. Pink-Gray Translucent & 380 & 9.02 & 69 & 12.73 & 69 & 5.98 \\
\hline 11. Whitish Translucent & 2 & 0.05 & 0 & 0 & 0 & 0 \\
\hline 12. Pink-Purple-Red Family & 292 & 6.93 & 30 & 5.54 & 0 & 0 \\
\hline 13. Caramel & 20 & 0.47 & 2 & 0.37 & 0 & 0 \\
\hline 14. Other Chert & 198 & 4.7 & 24 & 4.43 & 15 & 1.3 \\
\hline Indeterminate Chert & 188 & 4.46 & 23 & 4.24 & 255 & 22.1 \\
\hline Burnt Chert & 129 & 3.06 & 7 & 1.29 & 76 & 6.59 \\
\hline Indeterminate Stone & 75 & 1.78 & 7 & 1.29 & 15 & 1.3 \\
\hline Agate & 0 & 0 & 1 & 0.18 & 15 & 1.3 \\
\hline Silicified Limestone & 36 & 0.85 & 3 & 0.55 & 0 & 0 \\
\hline Silicified Sandstone & 7 & 0.17 & 3 & 0.55 & 17 & 1.47 \\
\hline Sandstone & 1 & 0.02 & 1 & 0.18 & 0 & 0 \\
\hline Quartzite & 3 & 0.07 & 0 & 0 & 85 & 7.37 \\
\hline Other Stone & 31 & 0.74 & 1 & 0.18 & 58 & 5.03 \\
\hline TOTAL & 4215 & 100.01 & 542 & 99.98 & 1154 & 100.02 \\
\hline
\end{tabular}

Table 4.3. Comparison of the proportions of non-chert materials.

\begin{tabular}{|l|r|r|r|r|r|r|}
\hline & \multicolumn{2}{|c|}{ MAP } & \multicolumn{2}{c|}{ ATP } & \multicolumn{2}{c|}{ AKAP } \\
\hline & \multicolumn{1}{|c|}{ Count } & $\%$ & Count & $\%$ & Count & $\%$ \\
\hline Cherts & 4062 & 96.37 & 526 & 97.05 & 964 & 83.54 \\
\hline Other stones & 153 & 3.63 & 16 & 2.95 & 190 & 16.46 \\
\hline Total & 4215 & 100 & 542 & 100 & 1154 & 100 \\
\hline
\end{tabular}


Table 4.4. Proportions of recorded cortex types found at el- Mahâsna, Abydos and Nag el-Qarmila. 'Other' includes natural fissure planes, thermoclastic fracture surfaces, and types not clearly classifiable into the other groups. Indeterminate pieces often only retained very tiny amounts of cortex.

\begin{tabular}{|l|r|r|r|r|r|r|}
\hline & \multicolumn{2}{|c|}{ MAP } & \multicolumn{2}{c|}{ ATP } & \multicolumn{2}{c|}{ AKAP } \\
\cline { 2 - 7 } & \multicolumn{1}{|c|}{ Count } & \multicolumn{1}{c|}{$\boldsymbol{\%}$} & \multicolumn{1}{c|}{ Count } & \multicolumn{1}{c|}{$\%$} & \multicolumn{1}{c|}{ Count } & \multicolumn{1}{c|}{$\%$} \\
\hline Chemical- Patina & 131 & 3.9 & 10 & 1.88 & 11 & 2.11 \\
\hline Alluvial- Smooth shiny cortex & 43 & 1.28 & 12 & 2.26 & 63 & 12.07 \\
\hline Multiple- Gravel cortex & $\mathbf{1 0 6 3}$ & $\mathbf{3 1 . 6 7}$ & 97 & 18.23 & $\mathbf{2 8}$ & 5.36 \\
\hline Gravity/wind- Lag deposits & 444 & 13.23 & $\mathbf{1 2 7}$ & $\mathbf{2 3 . 8 7}$ & $\mathbf{4 2}$ & 8.05 \\
\hline Unmodified- Chalky & 132 & 3.93 & 58 & 10.9 & $\mathbf{1 7 6}$ & $\mathbf{3 3 . 7 2}$ \\
\hline Other & 186 & 5.54 & $\mathbf{3 8}$ & 7.14 & $\mathbf{2 4}$ & 4.6 \\
\hline Indeterminate & 334 & 9.95 & 38 & 7.14 & 50 & 9.58 \\
\hline No cortex & 1024 & 30.5 & 152 & 28.57 & 128 & 24.52 \\
\hline Total & 3357 & 100 & 532 & 99.99 & 522 & 100.01 \\
\hline
\end{tabular}

Table 4.5. Proportions of recorded cortex types for the chert variety '1.Indistinct Beige'.

\begin{tabular}{|l|r|r|r|r|r|r|}
\hline \multirow{2}{*}{1 1. Indistinct Beige } & \multicolumn{2}{|c|}{ MAP } & \multicolumn{2}{c|}{ ATP } & \multicolumn{2}{c|}{ AKAP } \\
\cline { 2 - 7 } & Count & \multicolumn{1}{c|}{ \% } & \multicolumn{1}{c|}{ Count } & \multicolumn{1}{c|}{ \% } & \multicolumn{1}{c|}{ Count } & \multicolumn{1}{c|}{$\%$} \\
\hline Chemical- Patina & 28 & 6.32 & 0 & 0 & 2 & 2 \\
\hline Alluvial-Smooth shiny cortex & 5 & 1.13 & 2 & 4.65 & 0 & 0 \\
\hline Multiple- Gravel cortex & 98 & 22.12 & 5 & 11.63 & 1 & 1 \\
\hline Gravity/wind- Lag deposits & 78 & 17.61 & 7 & 16.28 & 5 & 5 \\
\hline Unmodified- Chalky & 25 & 5.64 & 7 & 16.28 & 22 & 22 \\
\hline Other & 20 & 4.51 & 3 & 6.98 & 0 & 0 \\
\hline Indeterminate & 46 & 10.38 & 5 & 11.63 & 6 & 6 \\
\hline No cortex & 143 & 32.28 & 14 & 32.56 & 64 & 64 \\
\hline Total & 443 & 99.99 & 43 & 100.01 & 100 & 100 \\
\hline
\end{tabular}

Table 4.6. Proportions of recorded cortex types for the chert variety '2.Beige Fine'.

\begin{tabular}{|l|r|r|r|r|r|r|}
\hline \multirow{2}{*}{ 2.Beige Fine } & \multicolumn{2}{|c|}{ MAP } & \multicolumn{2}{c|}{ ATP } & \multicolumn{2}{c|}{ AKAP } \\
\cline { 2 - 7 } & \multicolumn{1}{c|}{ Count } & \multicolumn{1}{c|}{ \% } & \multicolumn{1}{c|}{ Count } & \multicolumn{1}{c|}{ \% } & \multicolumn{1}{c|}{ Count } & \multicolumn{1}{c|}{$\%$} \\
\hline Chemical- Patina & 1 & 1.49 & 0 & 0 & 2 & 2.08 \\
\hline Alluvial-Smooth shiny cortex & 0 & 0 & 1 & 5.56 & 0 & 0 \\
\hline Multiple- Gravel cortex & 3 & 4.48 & 0 & 0 & 0 & 0 \\
\hline Gravity/wind- Lag deposits & 10 & 14.93 & 8 & 44.44 & 5 & 5.21 \\
\hline Unmodified- Chalky & 11 & 16.42 & 3 & 16.67 & 31 & 32.29 \\
\hline Other & 3 & 4.48 & 0 & 0 & 1 & 1.04 \\
\hline Indeterminate & 5 & 7.46 & 2 & 11.11 & 3 & 3.13 \\
\hline No cortex & 34 & 50.75 & 4 & 22.22 & 54 & 56.25 \\
\hline Total & 67 & 100.01 & 18 & 100 & 96 & 100 \\
\hline
\end{tabular}


Table 4.7. Proportions of recorded cortex types for the chert variety '3.Beige less fine'. Percentages not calculated for samples below 25 .

\begin{tabular}{|c|c|c|c|c|c|c|}
\hline \multirow[b]{2}{*}{ 3. Beige Less-Fine } & \multicolumn{2}{|c|}{ MAP } & \multicolumn{2}{|c|}{ ATP } & \multicolumn{2}{|c|}{ AKAP } \\
\hline & Count & $\%$ & Count & $\%$ & Count & $\%$ \\
\hline Chemical- Patina & 10 & 5.21 & 0 & & 0 & \\
\hline Alluvial-Smooth shiny cortex & 6 & 3.13 & 2 & & 1 & \\
\hline Multiple- Gravel cortex & 60 & 31.25 & 7 & & 0 & \\
\hline Gravity/wind- Lag deposits & 50 & 26.04 & 1 & & 0 & \\
\hline Unmodified-Chalky & 1 & 0.52 & 0 & & 0 & \\
\hline Other & 8 & 4.17 & 3 & & 0 & \\
\hline Indeterminate & 18 & 9.38 & 1 & & 1 & \\
\hline No cortex & 39 & 20.31 & 6 & & 3 & \\
\hline Total & 192 & 100.01 & 20 & & 5 & \\
\hline
\end{tabular}

Table 4.8. Proportions of recorded cortex types for the chert variety '4.Beige with Pink Bands'. Percentages not calculated for samples below 25 .

\begin{tabular}{|c|c|c|c|c|c|c|}
\hline \multirow{2}{*}{ 4. Beige w Pink Bands } & \multicolumn{2}{|c|}{ MAP } & \multicolumn{2}{|c|}{ ATP } & \multicolumn{2}{|c|}{ AKAP } \\
\hline & Count & $\%$ & Count & $\%$ & Count & $\%$ \\
\hline Chemical- Patina & 3 & 4 & 4 & 2.94 & 0 & \\
\hline Alluvial-Smooth shiny cortex & 1 & 1.33 & 0 & 0 & 0 & \\
\hline Multiple- Gravel cortex & 2 & 2.67 & 1 & 0.74 & 0 & \\
\hline Gravity/wind- Lag deposits & 15 & 20 & 65 & 47.79 & 1 & \\
\hline Unmodified-Chalky & 13 & 17.33 & 30 & 22.06 & 3 & \\
\hline Other & 2 & 2.67 & 9 & 6.62 & 0 & \\
\hline Indeterminate & 3 & 4 & 7 & 5.15 & 0 & \\
\hline No cortex & 36 & 48 & 20 & 14.71 & 1 & \\
\hline Total & 75 & 100 & 136 & 100.01 & 5 & \\
\hline
\end{tabular}

Table 4.9. Proportions of recorded cortex types for the chert variety '5.Medium Brown'. Percentages not calculated for samples below 25.

\begin{tabular}{|c|c|c|c|c|c|c|}
\hline \multirow{2}{*}{ 5. Medium Brown } & \multicolumn{2}{|c|}{ MAP } & \multicolumn{2}{|c|}{ ATP } & \multicolumn{2}{|c|}{ AKAP } \\
\hline & Count & $\%$ & Count & $\%$ & Count & $\%$ \\
\hline Chemical- Patina & 1 & 3.23 & 0 & & 2 & 4 \\
\hline Alluvial-Smooth shiny cortex & 0 & 0 & 0 & & 0 & 0 \\
\hline Multiple- Gravel cortex & 1 & 3.23 & 1 & & 1 & 2 \\
\hline Gravity/wind- Lag deposits & 5 & 16.13 & 1 & & 5 & 10 \\
\hline Unmodified-Chalky & 5 & 16.13 & 0 & & 20 & 40 \\
\hline Other & 1 & 3.23 & 0 & & 0 & 0 \\
\hline Indeterminate & 5 & 16.13 & 0 & & 5 & 10 \\
\hline No cortex & 13 & 41.94 & 0 & & 17 & 34 \\
\hline Total & 31 & 100.02 & 2 & & 50 & 100 \\
\hline
\end{tabular}


Table 4.10. Proportions of recorded cortex types for the chert variety '6.Brown Fossil'. Percentages not calculated for samples below 25.

\begin{tabular}{|l|r|r|r|r|r|r|}
\hline \multirow{2}{*}{ 6.Brown Fossil } & \multicolumn{2}{c|}{ MAP } & \multicolumn{2}{c|}{ ATP } & \multicolumn{2}{c|}{ AKAP } \\
\cline { 2 - 7 } & \multicolumn{1}{c|}{ Count } & \% & Count & \% & Count & \multicolumn{1}{c|}{$\%$} \\
\hline Chemical- Patina & 0 & & 0 & 0 & 0 \\
\hline Alluvial-Smooth shiny cortex & 0 & & 0 & 0 & 0 \\
\hline Multiple- Gravel cortex & 0 & & 0 & 0 & 0 \\
\hline Gravity/wind- Lag deposits & 0 & & 0 & 4 & 3.74 \\
\hline Unmodified- Chalky & 0 & & 0 & & 39 & 36.45 \\
\hline Other & 0 & & 0 & 0 & 0 \\
\hline Indeterminate & 0 & & 0 & 5 & 4.67 \\
\hline No cortex & 2 & & 0 & 59 & 55.14 \\
\hline Total & 2 & & 0 & 107 & 100 \\
\hline
\end{tabular}

Table 4.11. Proportions of recorded cortex types for the chert variety '7.Dark Gray and Brown'. Percentages not calculated for samples below 25 .

\begin{tabular}{|c|c|c|c|c|c|c|}
\hline \multirow{2}{*}{ 7.Dark Gray and Brown } & \multicolumn{2}{|c|}{ MAP } & \multicolumn{2}{|c|}{ ATP } & \multicolumn{2}{|c|}{ AKAP } \\
\hline & Count & $\%$ & Count & $\%$ & Count & $\%$ \\
\hline Chemical- Patina & 0 & & 0 & & 0 & 0 \\
\hline Alluvial-Smooth shiny cortex & 0 & & 0 & & 0 & 0 \\
\hline Multiple- Gravel cortex & 0 & & 0 & & 0 & 0 \\
\hline Gravity/wind- Lag deposits & 0 & & 0 & & 4 & 6.25 \\
\hline Unmodified-Chalky & 2 & & 1 & & 17 & 26.56 \\
\hline Other & 1 & & 0 & & 0 & 0 \\
\hline Indeterminate & 1 & & 1 & & 6 & 9.38 \\
\hline No cortex & 13 & & 0 & & 37 & 57.81 \\
\hline Total & 17 & & 2 & & 64 & 100 \\
\hline
\end{tabular}

Table 4.12. Proportions of recorded cortex types for the chert variety '8.Brown Translucent'.

\begin{tabular}{|l|r|r|r|r|r|r|}
\hline \multirow{2}{*}{ 8. Brown Translucent } & \multicolumn{2}{c|}{ MAP } & \multicolumn{2}{c|}{ ATP } & \multicolumn{2}{c|}{ AKAP } \\
\cline { 2 - 7 } & \multicolumn{1}{c|}{ Count } & \multicolumn{1}{c|}{ \% } & \multicolumn{1}{c|}{ Count } & \multicolumn{1}{c|}{ \% } & \multicolumn{1}{c|}{ Count } & \multicolumn{1}{c|}{ \% } \\
\hline Chemical- Patina & 66 & 4.97 & 5 & 4.1 & 2 & 2.44 \\
\hline Alluvial-Smooth shiny cortex & 12 & 0.9 & 4 & 3.28 & 1 & 1.22 \\
\hline Multiple- Gravel cortex & 400 & $\mathbf{3 0 . 1 4}$ & 34 & $\mathbf{2 7 . 8 7}$ & 3 & 3.66 \\
\hline Gravity/wind- Lag deposits & 177 & 13.34 & 27 & $\mathbf{2 2 . 1 3}$ & 6 & 7.32 \\
\hline Unmodified- Chalky & 50 & 3.77 & 8 & 6.56 & 11 & 13.41 \\
\hline Other & 58 & 4.37 & 6 & 4.92 & 3 & 3.66 \\
\hline Indeterminate & 118 & 8.89 & 10 & 8.2 & 5 & 6.1 \\
\hline No cortex & 446 & 33.61 & 28 & 22.95 & 51 & 62.2 \\
\hline Total & 1327 & 99.99 & 122 & 100.01 & 82 & 100.01 \\
\hline
\end{tabular}


Table 4.13. Proportions of recorded cortex types for the chert variety '10.Pink-Gray'.

\begin{tabular}{|l|r|r|r|r|r|r|}
\hline \multirow{2}{*}{ 10. Pink-Gray } & \multicolumn{2}{|c|}{ MAP } & \multicolumn{2}{c|}{ ATP } & \multicolumn{2}{c|}{ AKAP } \\
\cline { 2 - 7 } & \multicolumn{1}{c|}{ Count } & \multicolumn{1}{c|}{ \% } & \multicolumn{1}{c|}{ Count } & \multicolumn{1}{c|}{ \% } & \multicolumn{1}{c|}{ Count } & \multicolumn{1}{c|}{$\%$} \\
\hline Chemical- Patina & 7 & 2.19 & 0 & 0 & 1 & 1.61 \\
\hline Alluvial-Smooth shiny cortex & 0 & 0 & 0 & 0 & 0 & 0 \\
\hline Multiple- Gravel cortex & 15 & 4.69 & 4 & 5.8 & 1 & 1.61 \\
\hline Gravity/wind- Lag deposits & 29 & 9.06 & 7 & 10.14 & 1 & 1.61 \\
\hline Unmodified- Chalky & 9 & 2.81 & 2 & 2.9 & 5 & 8.06 \\
\hline Other & 18 & 5.63 & 8 & 11.59 & 1 & 1.61 \\
\hline Indeterminate & 18 & 5.63 & 3 & 4.35 & 4 & 6.45 \\
\hline No cortex & 224 & 70 & 45 & 65.22 & 49 & 79.03 \\
\hline Total & 320 & 100.01 & 69 & 100 & 62 & 99.98 \\
\hline
\end{tabular}

Table 4.14. Proportions of recorded cortex types for the chert variety '12.Pink-Purple-Red Family'. Percentages not calculated for samples below 25 .

\begin{tabular}{|l|r|r|r|r|r|r|}
\hline \multirow{2}{*}{ 12. Pink-Purple-Red Family } & \multicolumn{2}{|c|}{ MAP } & \multicolumn{2}{c|}{ ATP } & \multicolumn{2}{c|}{ AKAP } \\
\cline { 2 - 7 } & \multicolumn{1}{|c|}{ Count } & \multicolumn{1}{c|}{ \% } & Count & \% & Count & \% \\
\hline Chemical- Patina & 0 & & 0 & 0 & & \\
\hline Alluvial-Smooth shiny cortex & 0 & & 0 & 0 & & \\
\hline Multiple- Gravel cortex & 2 & & 12 & 40 & & \\
\hline Gravity/wind- Lag deposits & 3 & & 3 & 10 & & \\
\hline Unmodified- Chalky & 0 & & 1 & 3.33 & & \\
\hline Other & 0 & & 1 & 3.33 & & \\
\hline Indeterminate & 0 & & 2 & 6.67 & & \\
\hline No cortex & 3 & & 11 & 36.67 & & \\
\hline Total & 8 & & 30 & 100 & & 0 \\
\hline
\end{tabular}

Table 4.15. Proportions of recorded cortex types for the chert variety '13.Caramel'. Percentages not calculated for samples below 25 .

\begin{tabular}{|l|r|r|r|r|r|r|}
\hline \multirow{2}{*}{ 13. Caramel } & \multicolumn{2}{c|}{ MAP } & \multicolumn{2}{c|}{ ATP } & \multicolumn{2}{c|}{ AKAP } \\
\cline { 2 - 7 } & Count & \% & Count & $\%$ & Count & $\%$ \\
\hline Chemical- Patina & 1 & & 0 & & & \\
\hline Alluvial-Smooth shiny cortex & 0 & & 0 & & & \\
\hline Multiple- Gravel cortex & 0 & & 0 & & & \\
\hline Gravity/wind- Lag deposits & 2 & & 0 & & & \\
\hline Unmodified- Chalky & 2 & 2 & 0 & & & \\
\hline Other & 2 & & 0 & & & \\
\hline Indeterminate & 8 & & 0 & & & \\
\hline No cortex & 17 & 2 & & & \\
\hline Total & & 2 & & & 0 \\
\hline
\end{tabular}


Table 4.16. Details of the five heat treatment tests.

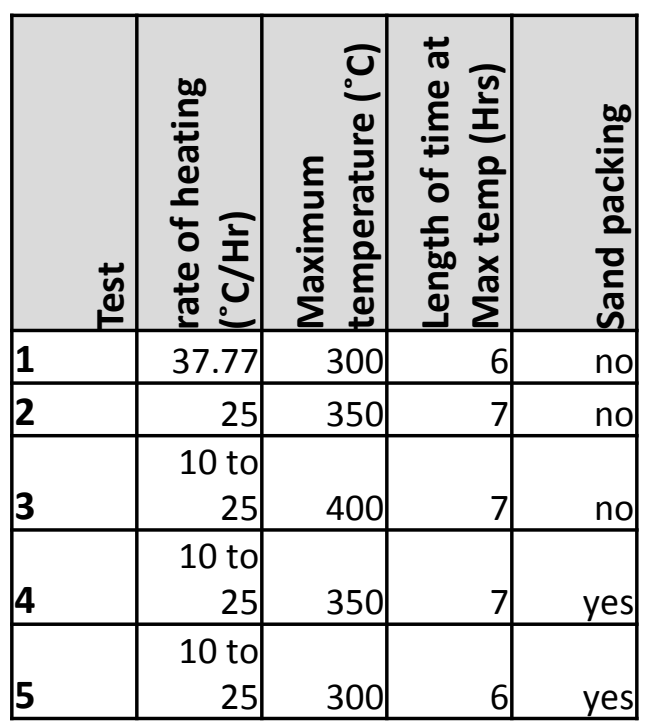

Table 4.17. List of tests for individual pieces.

\begin{tabular}{|c|c|c|c|}
\hline ID & Test & ID & Test \\
\hline A1.1 & 2 & K3.5 & 2 \\
\hline A1.2 & 1 & K4.1 & 3 \\
\hline A1.3 & $1 \& 4$ & K5.1 & 1 \\
\hline A1.4 & 1 & K5.2 & 1 \\
\hline A1.6 & 1 & K5.4 & 2 \\
\hline A1.7 & 2 & K6.1 & 3 \\
\hline $\mathrm{A} 1.8$ & 3 & K6.2 & 3 \\
\hline A2.2 & 1 & K7.1 & 1 \\
\hline A2.3 & 1 & K7.2 & 1 \\
\hline A2.4 & 2 & K7.4 & 1 \\
\hline A2.5 & 3 & K7.5 & 4 \\
\hline K1.2 & 1 & K8.1 & 1 \\
\hline $\mathrm{K} 1.3$ & 2 & K8.2 & 1 \\
\hline K1.4 & 5 & K8.3 & 1 \\
\hline K3.1 & 1 & K8.7 & 2 \\
\hline K3.3 & 1 & & \\
\hline
\end{tabular}

Table 4.18. Ratings of the ease of flaking after materials were heated to different temperatures. Great = easy to pressure flake. All ratings were made by Phil Geib according to his personal assessment of the ease of flaking.

\begin{tabular}{|c|c|c|c|c|}
\hline Nodule & No heat & 300 & 350 & 400 \\
\hline $\mathrm{A} 1$ & & Ok & GREAT & Burnt \\
\hline $\mathrm{A} 2$ & & Ok & Ok & GREAT \\
\hline K1 & & GREAT & Burnt & \\
\hline $\mathrm{K} 3$ & GREAT & Burnt & Burnt & \\
\hline $\mathrm{K} 4$ & OK & & & Burnt \\
\hline K5 & & \multicolumn{2}{|c|}{ GREAT } & \\
\hline K6 & OK & & & Burnt \\
\hline K7 & & Ok & GREAT & \\
\hline $\mathrm{K} 8$ & & GREAT & & \\
\hline
\end{tabular}

Table 4.19. Comparison of the percentage of complete flakes and blades with more than $50 \%$ cortex. These numbers do not include tools, angular debris, cores, or "characteristic debitage" (e.g. burins, core rejuvenation flakes, biface thinning flakes). The numbers on the left refer to Figure 4.34.

\begin{tabular}{|l|l|r|r|r|r|}
\hline & \multicolumn{1}{|c|}{ Site } & \multicolumn{1}{c|}{$\mathbf{N}$} & \multicolumn{1}{c|}{ UCL } & \multicolumn{1}{c|}{ \% } & \multicolumn{1}{c|}{ LCL } \\
\hline 1 & MAP & 1679 & 27.71 & 25.55 & 23.54 \\
\hline 2 & ATP & 229 & 18.17 & 13.1 & 9.39 \\
\hline 3 & KH3B & 2416 & 9.49 & 8.32 & 7.29 \\
\hline 4 & KH3XXI & 4641 & 16.96 & 15.88 & 14.86 \\
\hline 5 & KH7 & 437 & 9.92 & 7.09 & 5.06 \\
\hline 6 & South town & 1090 & 11.15 & 9.27 & 7.69 \\
\hline 7 & MA21/83 & 6047 & 10.29 & 9.53 & 8.81 \\
\hline 8 & MA21a/83 & 12206 & 8.16 & 7.68 & 7.22 \\
\hline 9 & HK11C & 2514 & 9.08 & 7.96 & 6.97 \\
\hline 10 & HK11C & 736 & 18.45 & 15.63 & 13.2 \\
\hline 11 & HK14 & 142 & 18.48 & 11.97 & 7.69 \\
\hline 12 & HK24A & 352 & 17.67 & 13.64 & 10.48 \\
\hline 13 & HK25D & 77 & 27.14 & 16.88 & 10.31 \\
\hline 14 & HK29 & 1489 & 16.54 & 14.64 & 12.95 \\
\hline 15 & AKAP & 421 & 11.91 & 8.79 & 6.47 \\
\hline
\end{tabular}


Table 4.20. Percentages of tools and debitage at Naqada period Nile valley settlement sites. Blank spaces indicate that the category was not counted separately. Debris includes flake fragments, $<1.5 \mathrm{~cm}$ pieces, and shatter. *The percents given for el-Mahâsna include 14 tools and 1 flake from surface collections, and 4 special find tools from units 2 and 5,. + Noted, but not counted as a separate category. ${ }^{1}$ Holmes $1989,{ }^{2}$ Vermeersch et al. $2004,{ }^{3}$ Ginter $\&$ Kozlowski 1994, ${ }^{4}$ Midant-Reynes \& Prost 2002, ${ }^{5}$ Holmes 1996, 6 Takamiya \& Endo 2011, 7 Hikade et al. 2008,8 Usai 2012

\begin{tabular}{|c|c|c|c|c|c|c|c|c|c|c|c|c|c|c|c|c|c|c|c|c|c|}
\hline Site & 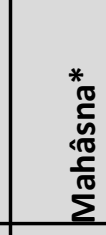 & $\begin{array}{l}\text { 은 } \\
\text { 음 } \\
\frac{0}{0} \\
\frac{2}{\alpha}\end{array}$ & 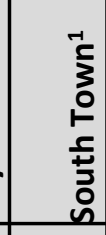 & 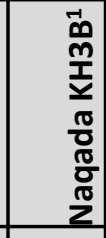 & 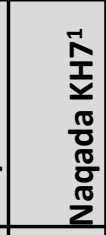 & 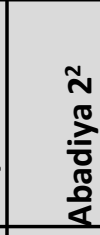 & 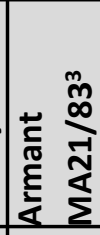 & 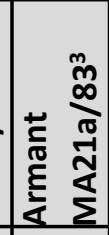 & : & 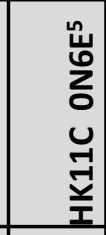 & 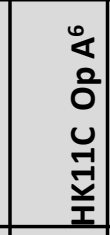 & 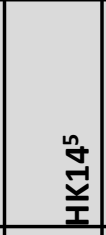 & 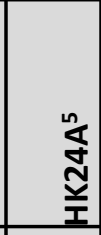 & $\begin{array}{l}\text { 号 } \\
\text { ํㅗㅁ } \\
\text { 至 }\end{array}$ & $\begin{array}{r}\text { 윽 } \\
\text { 음 } \\
\text { ్ㅗ } \\
\text { 포 }\end{array}$ & 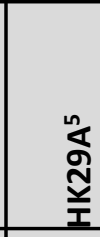 & $\begin{array}{l}\hat{\infty} \\
\text { స్ } \\
\text { 至 }\end{array}$ & 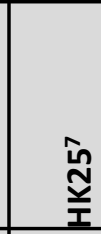 & 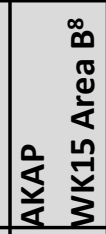 & 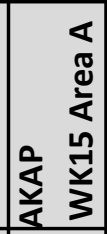 & 这 \\
\hline Time period & $\begin{array}{l}\text { NIC- } \\
\text { IIC }\end{array}$ & $\begin{array}{l}\mathrm{NI}- \\
\mathrm{IIAB}\end{array}$ & $\begin{array}{l}\text { NIIC- } \\
\text { IIII }\end{array}$ & $\begin{array}{l}\mathrm{NI}- \\
\mathrm{IIAB} \\
\end{array}$ & $\begin{array}{l}\mathrm{NI}- \\
\mathrm{IIAB}\end{array}$ & $\begin{array}{l}\mathrm{NI}- \\
\mathrm{IIAB}\end{array}$ & $\begin{array}{l}\mathrm{NI}- \\
\mathrm{IIAB}\end{array}$ & $\begin{array}{l}\mathrm{NI}- \\
\mathrm{IIAB}\end{array}$ & $\begin{array}{l}\mathrm{NI}- \\
\mathrm{NIII}\end{array}$ & $\begin{array}{l}\mathrm{NI}- \\
\mathrm{IIAB}\end{array}$ & NIIB-D & $\begin{array}{l}\mathrm{NI}- \\
\mathrm{Ila}\end{array}$ & $\begin{array}{l}\text { NIb- } \\
\text { llab }\end{array}$ & NIla & $\begin{array}{l}\text { NIC- } \\
\text { IIAB }\end{array}$ & $\begin{array}{l}\text { NIIA-C; } \\
\text { NIIIA }\end{array}$ & $\begin{array}{l}\text { NIIB-C, } \\
\text { NIID }\end{array}$ & NIIC-D & $\begin{array}{l}\text { NIC- } \\
\text { IIAB }\end{array}$ & $\begin{array}{l}\text { NIC- } \\
\text { IIAB }\end{array}$ & $\begin{array}{l}\text { NIC- } \\
\text { IIAB }\end{array}$ \\
\hline $\begin{array}{l}\# \text { Artifacts } \\
\text { (withdebris) }\end{array}$ & 6267 & 609 & 2206 & 5055 & 623 & 21090 & 11326 & 19831 & 12023 & 1190 & 3677 & 214 & 711 & 183 & 3557 & 54000 & 10451 & 7481 & 2595 & 2042 & 50 \\
\hline \# Tools & 660 & 79 & 177 & 194 & 48 & 546 & 743 & 1206 & 826 & 131 & 307 & 16 & 35 & 9 & 468 & 1944 & 279 & 225 & & 204 & 17 \\
\hline Tools & 10.53 & 12.97 & 8.02 & 2.91 & 7.7 & 2.5 & 6.56 & 6.13 & 6.85 & 11.01 & 8.35 & 7.48 & 4.92 & 4.92 & 13.16 & 3.6 & 2.67 & 3.01 & 7 & 9.99 & 34 \\
\hline $\begin{array}{l}\text { Cores ( } \& \text { core } \\
\text { remnants) }\end{array}$ & 3.57 & 1.97 & 1.04 & 0.57 & 1.12 & 0.88 & 3.27 & 1.35 & 2.94 & 1.01 & 1.06 & 3.74 & 0.42 & 1.09 & 1.07 & 0.3 & 1.21 & 1.98 & 1 & 1.57 & 2 \\
\hline Flakes & 19.24 & 28.41 & 44.02 & 44.29 & 59.23 & 20.77 & 45.47 & 39.64 & 21.66 & 46.05 & 46.29 & 57.01 & 41.07 & 34.43 & 56.84 & 6.6 & 5.27 & 5.72 & 42 & 15.48 & 32 \\
\hline Blades & 5.11 & 7.39 & 5.39 & 3.5 & 10.91 & 2.43 & 7.98 & 6.71 & 3.11 & 11.18 & 15.28 & 9.34 & 5.62 & 5.47 & 13.52 & 4.8 & 5.59 & 5.51 & 3 & 2.11 & 2 \\
\hline Burin spalls & 0.88 & 0.82 & & & & 1.36 & 0.5 & 0.87 & 0.53 & 1.01 & 0.41 & 0 & 0.84 & 1.09 & 2.78 & 0.5 & 0.96 & 0.31 & 1 & 1.47 & 2 \\
\hline $\begin{array}{l}\text { Rejuvenation } \\
\text { pieces }\end{array}$ & 0.88 & 1.81 & 0.54 & 0.1 & 0.16 & 0.18 & 0.53 & 0.11 & & 0 & & 0.47 & 0.14 & 0 & 0.31 & 0.1 & 0.06 & 0.05 & & 0.78 & 2 \\
\hline Crested blades & 0.05 & 0.16 & & & & 0.05 & 0.36 & 0.17 & 0.25 & & & & & & 0 & & 0.01 & 0.05 & & 0.05 & 0 \\
\hline $\begin{array}{l}\text { Axe prep } \\
\text { flakes }\end{array}$ & 0.02 & 0 & 0.14 & 0.26 & 0.16 & 0.21 & 0.31 & 0.57 & & & & & & & 0 & & 0.03 & 0 & + & 0 & 0 \\
\hline $\begin{array}{l}\text { Thinning } \\
\text { flakes }\end{array}$ & 1.79 & 4.6 & & & & $\dagger$ & 4.35 & 6.48 & 0.03 & 4.62 & 6.8 & 0 & 2.81 & 2.19 & & 15.4 & 8.66 & 5.91 & & 1.76 & 0 \\
\hline Other debitage & 10.29 & 12.15 & 0.05 & 0.45 & 1.28 & 5.24 & 1.3 & 0.77 & 9.64 & & 1.39 & & & & 2.98 & & & & & 9.41 & 12 \\
\hline Debris & 47.65 & 29.72 & 40.8 & 47.91 & 19.42 & 63.77 & 29.36 & 37.21 & 54.99 & 25.12 & 20.42 & 21.96 & 44.17 & 50.82 & 9.33 & 68.6 & 75.53 & 77.41 & 47 & 57.4 & 14 \\
\hline TOTAL & 100.01 & 100 & 100 & 99.99 & 99.98 & 97.39 & 99.99 & 100.01 & 100 & 100 & 100 & 100 & 99.99 & 100.01 & 99.99 & 99.9 & 99.99 & 99.95 & 101 & 100.02 & 100 \\
\hline
\end{tabular}


Table 4.21. Calculations for Cortex ratio at el-Mahasna using largest artifact dimensions as estimate of average nodule size.

\begin{tabular}{|c|c|c|}
\hline MAP & Cube & Rt cylinder \\
\hline$\#$ of artifacts & 4013 & 4013 \\
\hline Weight (g) & 51225.56 & 51225.56 \\
\hline Density $\left(\mathrm{g} / \mathrm{cm}^{\wedge} 3\right)$ & 2.42 & 2.42 \\
\hline Total Volume $\left(\mathrm{cm}^{\wedge} 3\right)$ (weight / density) & 21167.59 & 21167.59 \\
\hline Average nodule size $(\mathrm{cm})$ & $10.7 \times 8.1 \times 5.6$ & $h=10.7, r=3.425$ \\
\hline Average nodule shape & cube & $\begin{array}{r}\text { Rt cylinder } \\
\left(\mathrm{V}=\mathrm{pi}^{*} \mathrm{r}^{\wedge} 2^{*} \mathrm{~h}\right) \\
\end{array}$ \\
\hline Estimated avg nodule volume & 485.35 & 394.3254 \\
\hline Estimated surface area per avg nodule $\left(\mathrm{cm}^{\wedge} 2\right)$ & 383.9 & 315.0261 \\
\hline $\begin{array}{l}\text { Estimated \# of nodules (Total volume / est avg } \\
\text { nodule volume) }\end{array}$ & 43.61 & 53.6805 \\
\hline \begin{tabular}{|l} 
Expected surface area $\left(\mathrm{cm}^{\wedge} \mathbf{2}\right)$ \\
(est avg SA* est \# nodules) \\
\end{tabular} & 16741.88 & 16910.76 \\
\hline $\begin{array}{l}\text { Observed surface area }\left(\mathrm{cm}^{\wedge} \mathbf{2}\right) \\
\text { SUM of (SA* mid point of cortex amount) }\end{array}$ & 224836.8 & 224836.8 \\
\hline Ratio & 13.43 & 13.3 \\
\hline
\end{tabular}

Table 4.22. Calculations for Cortex ratio at el-Mahasna using the observed number of cores as the estimate for the number of nodules represented.

\begin{tabular}{|c|c|c|}
\hline MAP & Cube & Rt cylinder \\
\hline \# of artifacts & 4013 & 4013 \\
\hline Weight (g) & 51225.56 & 51225.56 \\
\hline Density $\left(\mathrm{g} / \mathrm{cm}^{\wedge} 3\right)$ & 2.42 & 2.42 \\
\hline Total volume (total weight / density) & 21167.59 & 21167.59 \\
\hline Observed \# of cores & 224 & 224 \\
\hline $\begin{array}{l}\text { Estimated nodule volume } \\
\text { (Total volume / observed \# of cores) }\end{array}$ & 94.49816 & 94.49816 \\
\hline Shape & $\begin{array}{r}\text { Cube } \\
\left(\mathrm{SA}=6 \mathrm{~V}^{\wedge} 2 / 3\right) \\
\end{array}$ & $\begin{array}{r}\text { Rt cylinder } \\
\left(\mathrm{SA}=4 \mathrm{pi}(\mathrm{V} / \mathrm{pi})^{\wedge} 2 / 3\right) \\
\end{array}$ \\
\hline Estimated surface area per nodule & 124.4802 & 121.5418 \\
\hline $\begin{array}{l}\text { Expected surface area } \\
\text { (SA per nodule } x \# \text { of nodules) }\end{array}$ & 27883.56 & 27225.35 \\
\hline Observed SA & 224836.8 & 224836.8 \\
\hline Ratio & 8.06 & 8.26 \\
\hline
\end{tabular}


Table 4.23. Calculations for Cortex ratio at Nag el-Qarmila WK15 using largest artifact dimensions as estimate of average nodule size.

\begin{tabular}{|c|c|c|}
\hline AKAP & Cube & Rt cylinder \\
\hline \# of artifacts & 757 & 757 \\
\hline Weight (g) & 2277.7 & 2277.7 \\
\hline Density $\left(\mathrm{g} / \mathrm{cm}^{\wedge} 3\right)$ & 2.42 & 2.42 \\
\hline $\begin{array}{l}\text { Total volume }\left(\mathrm{cm}^{\wedge} \mathbf{3}\right) \\
\text { (weight / density) }\end{array}$ & 941.1983 & 941.1983 \\
\hline Average nodule size $(\mathrm{cm})$ & $7.28 \times 5 \times 2.67$ & $7.28 \times 5 \times 2.67$ \\
\hline Shape & cube & Rt cylinder $\left(V=\mathrm{pi}^{*} \mathrm{r}^{\wedge} 2^{*} \mathrm{~h}\right)$ \\
\hline Estimated avg nodule volume & 97.188 & 84.3109 \\
\hline Est surface area per avg nodule $\left(\mathrm{cm}^{\wedge} 2\right)$ & 138.3752 & 110.9862 \\
\hline $\begin{array}{l}\text { Estimated \# of nodules } \\
\text { (total volume / avg nodule volume) }\end{array}$ & 23.43602 & 27.01549 \\
\hline $\begin{array}{l}\text { Expected surface area }\left(\mathrm{cm}^{\wedge} \mathbf{2}\right) \\
\text { (est avg SA* est \# nodules) }\end{array}$ & 3242.964 & 2998.346 \\
\hline $\begin{array}{l}\text { Observed surface area } \\
\text { (SUM of (SA * mid point of cortex } \\
\text { amount)) }\end{array}$ & 5695.978 & 5695.978 \\
\hline Ratio & 1.76 & 1.9 \\
\hline
\end{tabular}

Table 4.24. Calculations for Cortex ratio at Nag el-Qarmila using the observed number of cores as the estimate for the number of nodules represented.

\begin{tabular}{|c|c|c|}
\hline AKAP & Cube & Rt cylinder \\
\hline \# of artifacts & 757 & 757 \\
\hline Weight (g) & 2277.7 & 2277.7 \\
\hline Density $\left(\mathrm{g} / \mathrm{cm}^{\wedge} 3\right)$ & 2.42 & 2.42 \\
\hline $\begin{array}{l}\text { Total volume (cm^3) } \\
\text { (total weight / density) }\end{array}$ & 941.1983 & 941.1983 \\
\hline Observed \# of cores & 17 & 17 \\
\hline $\begin{array}{l}\text { Estimated nodule volume } \\
\left(\mathrm{cm}^{\wedge} 3\right) \text { (Total volume / } \\
\text { observed \# of cores) }\end{array}$ & 55.36461 & 55.36461 \\
\hline Shape & cube $\left(S A=6 V^{\wedge} 2 / 3\right)$ & rt cylinder (S=4pi(V/pi)^2/3) \\
\hline $\begin{array}{l}\begin{array}{l}\text { Est surface area per nodule } \\
\left(\mathrm{cm}^{\wedge} 2\right)\end{array} \\
\end{array}$ & 87.15776 & 85.10037 \\
\hline \begin{tabular}{|l} 
Expected surface area \\
(SA per nodule $x \#$ of nodules)
\end{tabular} & 1481.682 & 1446.706 \\
\hline Observed SA & 5695.978 & 5695.978 \\
\hline Ratio & 3.84 & 3.94 \\
\hline
\end{tabular}


Table 4.25. Tool types. All \%s were re-calculated from published counts. \%s for sites with <30 tools were not calculated, except for WK22 (not reported elsewhere). HK25 counts do not include burnt bifacial artifacts. MAP frequencies only include material from Blocks 1,3,4. See Table 4.20 for references.

\begin{tabular}{|c|c|c|c|c|c|c|c|c|c|c|c|c|c|c|c|c|c|c|c|}
\hline Site & $\begin{array}{l}\frac{\pi}{5} \\
\frac{1}{\pi} \\
\frac{C}{\pi} \\
\sum\end{array}$ & $\begin{array}{l}0 . \\
\frac{1}{4} \\
\text { o. } \\
\frac{0}{0} \\
\frac{0}{2} \\
\end{array}$ & 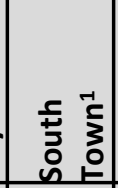 & 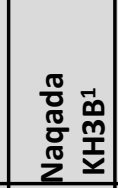 & $\begin{array}{l}\frac{\pi}{0} \\
\frac{\pi}{0} \\
\frac{\pi}{1} \\
z \\
2\end{array}$ & 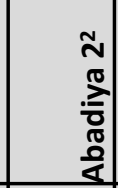 & 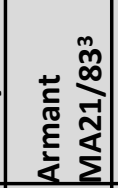 & 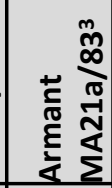 & $\begin{array}{l}\stackrel{+}{0} \\
: \frac{\delta}{\pi} \\
\frac{\pi}{0} \\
\end{array}$ & 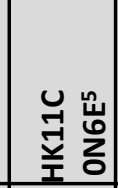 & 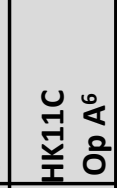 & 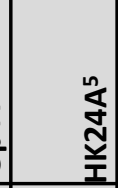 & 류ํ & 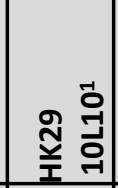 & 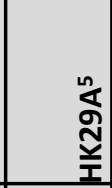 & $\begin{array}{l}\hat{\tilde{O}} \\
\text { ָे } \\
\text { İ }\end{array}$ & 总 & 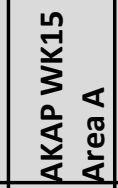 & $\frac{\sqrt{3}}{\frac{3}{2}}$ \\
\hline Time period & $\begin{array}{l}\text { NIC- } \\
\text { IIC } \\
\end{array}$ & \begin{tabular}{|l|}
$\mathrm{NI}-$ \\
$\mathrm{IIAB}$ \\
\end{tabular} & \begin{tabular}{|l|} 
NIIC- \\
$\mathrm{III}$
\end{tabular} & \begin{tabular}{|l|}
$\mathrm{NI}-$ \\
$\mathrm{IIAB}$
\end{tabular} & $\begin{array}{l}\mathrm{NI}- \\
\mathrm{IIAB} \\
\end{array}$ & \begin{tabular}{|l|} 
NI- \\
IIAB
\end{tabular} & \begin{tabular}{|l|}
$\mathrm{NI}-$ \\
$\mathrm{IIAB}$
\end{tabular} & \begin{tabular}{|l|}
$\mathrm{NI}-$ \\
$\mathrm{IIAB}$ \\
\end{tabular} & \begin{tabular}{|l|} 
NI- \\
NIII
\end{tabular} & \begin{tabular}{|l|}
$\mathrm{NI}-$ \\
$\mathrm{IIAB}$
\end{tabular} & NIIB-D & $\begin{array}{l}\text { NIb- } \\
\text { Ilab }\end{array}$ & $\begin{array}{l}\text { NIC- } \\
\text { IIAB } \\
\end{array}$ & \begin{tabular}{|l|} 
NIC- \\
IIAB
\end{tabular} & $\begin{array}{l}\text { NIIA-C; } \\
\text { NIIIA }\end{array}$ & \begin{tabular}{|l|} 
NIIB-C, \\
NIID \\
\end{tabular} & NIIC-D & \begin{tabular}{|l|} 
NIC- \\
IIAB
\end{tabular} & $\begin{array}{l}\text { NIC- } \\
\text { IIAB } \\
\end{array}$ \\
\hline \#Tools & 660 & 79 & 177 & 194 & 48 & 546 & 743 & 1206 & 826 & 131 & 307 & 35 & 240 & 468 & 1921 & 279 & 225 & 204 & 17 \\
\hline Notches & 10 & 7.59 & 11.86 & 13.4 & 8.33 & 4.03 & 7.67 & 7.25 & 7.87 & 9.92 & 3.9 & 17.14 & 3.33 & 4.3 & 3.7 & 0.36 & 0.89 & 5.39 & 17.65 \\
\hline Denticulates & 9.09 & 10.13 & 3.95 & 3.61 & 2.08 & 4.95 & 11.31 & 9.4 & 8.11 & 2.29 & 1.6 & 0 & 2.92 & 2.8 & 1.1 & 6.09 & 2.66 & 2.94 & 17.65 \\
\hline Sickle blades & 0.45 & 0 & 3.39 & 0.52 & 0 & 0.37 & 0.27 & 0.33 & & 0 & 2 & 0 & 0 & 0 & 0.2 & & & 0 & 0 \\
\hline Endscrapers & 6.06 & 8.86 & 16.38 & 16.49 & 16.67 & 6.23 & 9.02 & 7.16 & 5.21 & 4.58 & 14 & 2.86 & 10 & 4.9 & 4.6 & 4.3 & 6.22 & 3.43 & 5.88 \\
\hline $\begin{array}{l}\text { Sidescraper \& } \\
\text { other scrapers }\end{array}$ & 2.58 & 5.06 & 0 & 0 & 0 & 0.73 & 4.16 & 4.93 & 1.69 & 0 & 12 & 0 & 2.5 & 1.1 & 1.9 & 18.6 & 16 & 0.98 & 0 \\
\hline Truncations & 4.7 & 5.06 & 2.26 & 2.58 & 2.08 & 0.18 & 1.88 & 3.79 & 1.69 & 4.58 & 2 & 0 & 3.75 & 4.1 & 5.3 & 1.08 & 0.89 & 1.96 & 11.76 \\
\hline $\begin{array}{l}\text { Micro- } \\
\text { endscrapers }\end{array}$ & 1.97 & 0 & & & & & & & & 0.76 & & 0 & 5.83 & & 4.5 & 2.51 & 1.78 & 3.43 & 0 \\
\hline Backed pieces & 0.76 & 1.27 & 1.69 & 0.52 & 0 & & 0.67 & 0.66 & 0.73 & 1.53 & & 0 & 1.67 & 1.3 & 1.9 & 0.72 & 0 & 0.98 & 0 \\
\hline Transv. Arrowh & 0 & 0 & & & & 0.18 & 0.27 & 0.74 & & 0 & & 0 & 0 & 0.9 & 0.6 & 0.36 & 0 & 1.96 & 0 \\
\hline $\begin{array}{l}\text { Retouched } \\
\text { pieces }\end{array}$ & 24.24 & 21.52 & 27.12 & 22.68 & 12.5 & 42.13 & 15.34 & 20.44 & 21.8 & 35.88 & 52 & 25.71 & 19.17 & 31 & 12.8 & 29 & 24 & 29.9 & 11.76 \\
\hline Burins & 17.42 & 27.85 & 25.99 & 29.38 & 45.83 & 26.56 & 15.75 & 22.34 & 17.4 & 27.48 & 7.2 & 34.29 & 32.08 & 25 & 24.5 & 10.8 & 16.89 & 14.71 & 23.53 \\
\hline Piece esquille & 0 & 0 & 0 & 0 & 0 & & & & 2.54 & 0 & & 0 & 0 & & 0.1 & 1.43 & 4.89 & 17.16 & 5.88 \\
\hline Perforators & 5.3 & 3.8 & 2.82 & 2.58 & 6.25 & 3.3 & 5.79 & 5.51 & 14.2 & 0 & & 5.71 & 3.33 & 2.1 & 1 & 4.66 & 4.89 & 8.33 & 0 \\
\hline Micro drill & 0 & 0 & & & & & & & & 0 & & 2.86 & 1.25 & 1.7 & 34.3 & 15.8 & 15.11 & 0 & 0 \\
\hline Winged drills & 0 & 0 & & & & & & & & 0 & & 0 & 0.42 & 3 & 0.1 & & & 0 & 0 \\
\hline Other drills & 0.3 & 0 & & & & & 1.08 & 1.4 & 0.97 & 0 & 1 & 0 & 2.5 & 0 & 0 & & & 1.47 & 0 \\
\hline Blade knives & 1.06 & 1.27 & & & & & 1.62 & 1.23 & 1.69 & & 0.3 & & & 0 & & & & 0 & 5.88 \\
\hline Planes & 0.45 & 0 & 0 & 0.52 & 0 & & 1.08 & 0.25 & & & & & & 0 & & & & 0 & 0 \\
\hline Axes & 0.45 & 0 & 0 & 3.61 & 2.08 & 8.42 & 5.92 & 2.8 & & & & & & 0 & & & & 0 & 0 \\
\hline Proj. points & 0.3 & 0 & & & & & & 0.25 & 0.97 & 0 & & 0 & 0 & & 0.5 & & & 0 & 0 \\
\hline Other BFC tools & 0.76 & 0 & 3.39 & 2.06 & 4.17 & 2.56 & 7.31 & 3.05 & & 2.29 & 3.3 & 2.86 & 1.25 & 4.5 & 1.4 & 4.3 & 5.78 & 0.98 & 0 \\
\hline Other & 13.48 & 7.59 & 1.13 & 2.06 & 0 & 0.36 & 4.72 & 4.62 & 13.2 & 2.29 & 1 & 5.71 & 0.83 & 1.5 & 1.4 & & & 5.39 & 0 \\
\hline Unidentifiable & 0.61 & 0 & & & & & 5.79 & 3.22 & 1.94 & 8.4 & & 2.86 & 9.17 & 12 & & & & 0.98 & 0 \\
\hline TOTAL & 99.98 & 100 & 99.98 & 100.01 & 99.99 & 100 & 99.65 & 99.37 & 100 & 100 & 100 & 100 & 100 & 100 & 99.9 & 100 & 100 & 99.99 & 99.99 \\
\hline
\end{tabular}




\section{Chapter 5 Tables}

Table 5.1. Summary of characteristics for types of blades.

\begin{tabular}{|c|c|}
\hline Blade Subtype & Characteristics \\
\hline Bladelets & $\begin{array}{l}\text { - Length equal to twice the width, or longer } \\
\text {-Maximum width } 1.2 \mathrm{~cm} \\
\text {-Parallel edges and ridges } \\
\text { - Generally shorter and less thick than 'blades' } \\
\text { - Cores often show regular removals } \\
\text { - Non-heat treated flint }\end{array}$ \\
\hline $\begin{array}{l}\text { Bladelets for } \\
\text { microdrills }\end{array}$ & $\begin{array}{l}\text { - Length equal to twice the width, or longer } \\
\text {-Maximum width } 1.2 \mathrm{~cm} \\
\text {-Parallel edges and ridges } \\
\text {-Made on a coarse grey flint }\end{array}$ \\
\hline $\begin{array}{l}\text { Heat-treated } \\
\text { bladelets }\end{array}$ & $\begin{array}{l}\text { - Length equal to twice the width, or longer } \\
\text {-Maximum width } 1.2 \mathrm{~cm} \\
\text {-Parallel edges and ridges } \\
\text {-Made on heat treated flint }\end{array}$ \\
\hline Medium Blades & $\begin{array}{l}\text { - Length equal to twice the width, or longer } \\
\text {-Width greater than } 1.2 \mathrm{~cm} \\
\text { - Roughly parallel edges \& ridges }\end{array}$ \\
\hline Large Blades & $\begin{array}{l}\text { - Length equal to twice the width, or longer } \\
\text {-Width greater than } 1.2 \mathrm{~cm} \text {, closer to } 3 \mathrm{~cm} \\
\text { - Length over } 10 \mathrm{~cm} \\
\text {-Very thick }\end{array}$ \\
\hline $\begin{array}{l}\text { Twisted } \\
\text { Large blades/ } \\
\text { Medium blades/ } \\
\text { bladelets }\end{array}$ & $\begin{array}{l}\text { - Length equal to twice the width, or longer } \\
\text {-Size dimensions correspond to those for large blades, medium blades, or } \\
\text {-Twist around the longitudinal axis }\end{array}$ \\
\hline $\begin{array}{l}\text { Trapezoidal } \\
\text { blades }\end{array}$ & $\begin{array}{l}\text { - Length equal to twice the width, or longer } \\
\text {-Narrow width ranging from } .6 \mathrm{~cm} \text { to } 2.4 \mathrm{~cm} \text {, averaging } \sim 1.2 \mathrm{~cm} \\
\text { - Length around } 8-12 \mathrm{~cm} \\
\text {-Very straight parallel margins and dorsal ridges }\end{array}$ \\
\hline
\end{tabular}

Table 5.2. Average dimensions of complete bladelets from elMahasna, Abydos, and Nag el-Qarmila.

\begin{tabular}{|l|r|r|r|}
\hline Site & \multicolumn{1}{|c|}{ MAP } & \multicolumn{1}{c|}{ ATP } & \multicolumn{1}{c|}{ AKAP } \\
\hline $\mathrm{N}$ & 42 & 4 & 4 \\
\hline Mean Length $(\mathrm{cm})$ & 3.05 & 3.16 & 3.01 \\
\hline Length Range & $1.83-5$ & $2.51-4.43$ & $2.47-4.22$ \\
\hline Mean Width $(\mathrm{cm})$ & 1.02 & 1.12 & 1.11 \\
\hline Width range & $.53-1.19$ & $.98-1.19$ & $1.02-1.15$ \\
\hline Mean Thickness $(\mathrm{cm})$ & .45 & .34 & .41 \\
\hline Thickness range & $.19-1.18$ & $.27-.41$ & $.27-.72$ \\
\hline
\end{tabular}


Table 5.3. Average dimensions of complete medium blades from el-Mahasna, Abydos, and Nag el-Qarmila. *1 outlier was $10.8 \mathrm{~cm}$.

\begin{tabular}{|l|r|r|r|}
\hline Site & \multicolumn{1}{|c|}{ MAP } & \multicolumn{1}{|c|}{ ATP } & \multicolumn{1}{|c|}{ AKAP } \\
\hline $\mathrm{N}$ & 171 & 39 & 20 \\
\hline Mean Length $(\mathrm{cm})$ & 4.4 & 4.3 & 3.64 \\
\hline Length Range & $2.65-7.18^{*}$ & $2.63-6.34$ & $1.76-7.4$ \\
\hline Mean Width $(\mathrm{cm})$ & 1.78 & 1.81 & 1.86 \\
\hline Width range & $1.21-3.53$ & $1.22-3.08$ & $1.21-2.98$ \\
\hline Mean Thickness $(\mathrm{cm})$ & 0.72 & 0.66 & 0.53 \\
\hline Thickness range & $.22-2$ & $.27-1.59$ & $.24-1.24$ \\
\hline
\end{tabular}

Table 5.4.Dimensions of large blades from Nile valley settlement sites.

\begin{tabular}{|l|r|r|r|r|}
\hline Publication & \multicolumn{1}{c|}{$\begin{array}{c}\text { Holmes } \\
\text { 1989:118 }\end{array}$} & Analyzed here & $\begin{array}{c}\text { Payne } \\
\text { 1993:156-158 }\end{array}$ & $\begin{array}{c}\text { Takamiya \& } \\
\text { Endo 2011:739 }\end{array}$ \\
\hline Site & \multicolumn{1}{|c|}{ Badari } & el-Mahâsna & $\begin{array}{c}\text { Naqada South } \\
\text { Town }\end{array}$ & HK11C \\
\hline $\mathrm{N}$ & 3 & 1 & 7 & 13 \\
\hline Mean Length (cm) & 15.4 & 12.7 & 11.03 & 13.5 \\
\hline Length range & $13.7-17.4$ & $\mathrm{n} / \mathrm{a}$ & $9.5-15.3$ & 2.7 \\
\hline Mean Width (cm) & 3.2 & 4.4 & 2.6 & \\
\hline Width range & $3-3.5$ & $\mathrm{n} / \mathrm{a}$ & $2.2-3.5$ & \\
\hline Mean Thickness (cm) & 2.3 & 1.5 & & \\
\hline Thickness range & $1.8-3.2$ & $\mathrm{n} / \mathrm{a}$ & & \\
\hline & 2 blanks \& 1 & Blade Knife & Blanks & Blade knife \\
\hline
\end{tabular}

Table 5.5. Counts and percentages of blade type categories among the debitage. The counts are of complete and proximal examples of each type. Medial and distal pieces are counted under fragments. The sample from el-Mahâsna includes all the analyzed material from el-Mahâsna, not just that from Blocks 1, 3, \& 4.

\begin{tabular}{|l|r|r|r|r|r|r|}
\hline \multirow{2}{*}{ Artifact type } & \multicolumn{2}{|c|}{ MAP } & \multicolumn{2}{c|}{ ATP } & \multicolumn{2}{c|}{ AKAP WK15 } \\
\cline { 2 - 7 } & \multicolumn{1}{|c|}{ Count } & \multicolumn{1}{c|}{ \% } & Count & \multicolumn{1}{c|}{$\%$} & \multicolumn{1}{c|}{ Count } & \multicolumn{1}{c|}{$\%$} \\
\hline Medium blades & 223 & 44.33 & 32 & 52.46 & 32 & 47.06 \\
\hline Bladelets & 60 & 11.93 & 3 & 4.92 & 7 & 10.29 \\
\hline Crested blades & 1 & 0.2 & 1 & 1.64 & 1 & 1.47 \\
\hline Crested bladelets & 2 & 0.4 & 0 & 0 & 0 & 0 \\
\hline Large blades & 0 & 0 & 0 & 0 & 0 & 0 \\
\hline Twisted blades & 27 & 5.37 & 9 & 14.75 & 3 & 4.41 \\
\hline Twisted bladelets & 10 & 1.99 & 1 & 1.64 & 1 & 1.47 \\
\hline Fragments & 180 & 35.79 & 15 & 24.59 & 24 & 35.29 \\
\hline Total N & 503 & 100 & 61 & 100 & 68 & 100 \\
\hline
\end{tabular}


Table 5.6. Counts and percentages of the identifiable tools blanks for blade types. Note that the counts for ATP and AKAP WK15 are below 30, so the percentages are not as reliable.

\begin{tabular}{|c|c|c|c|c|c|c|}
\hline \multirow{2}{*}{ Tool Blank type } & \multicolumn{2}{|c|}{ MAP } & \multicolumn{2}{|c|}{ ATP } & \multicolumn{2}{|c|}{ AKAP WK15 } \\
\hline & Count & $\%$ & Count & $\%$ & Count & $\%$ \\
\hline Medium Blades & 80 & 84.21 & 8 & 88.89 & 10 & 55.56 \\
\hline Bladelets & 10 & 10.53 & 0 & 0 & 6 & 33.33 \\
\hline Crested blades & 1 & 1.05 & 0 & 0 & 0 & 0 \\
\hline Crested bladelets & 0 & 0 & 0 & 0 & 0 & 0 \\
\hline Large blades & 1 & 1.05 & 1 & 11.11 & 0 & 0 \\
\hline Twisted blades & 3 & 3.16 & 0 & 0 & 1 & 5.56 \\
\hline Twisted bladelets & 0 & 0 & 0 & 0 & 1 & 5.56 \\
\hline TOTAL & 95 & 100 & 9 & 100 & 18 & 100.01 \\
\hline
\end{tabular}

Table 5.7. Counts and percentages of the relative degree of twist for a sample of 91 twisted medium blades \& twisted bladelets from el-Mahâsna.

\begin{tabular}{|l|r|r|}
\hline Degree of twist & \multicolumn{1}{|c|}{ Count } & \multicolumn{1}{c|}{ \% } \\
\hline Slightly twisted & 11 & 12.09 \\
\hline Twisted & 77 & 84.62 \\
\hline Very twisted & 3 & 3.3 \\
\hline Total & 91 & 100.01 \\
\hline
\end{tabular}

Table 5.8. Counts and percentages platform types by blade types.

\begin{tabular}{|c|c|c|c|c|c|c|c|c|}
\hline \multirow{2}{*}{ Platform type } & \multicolumn{2}{|c|}{$\begin{array}{c}\text { Medium } \\
\text { blades }\end{array}$} & \multicolumn{2}{|c|}{ Bladelets } & \multicolumn{2}{|c|}{$\begin{array}{l}\text { Twisted } \\
\text { medium } \\
\text { blades }\end{array}$} & \multicolumn{2}{|c|}{$\begin{array}{c}\text { Twisted } \\
\text { bladelets }\end{array}$} \\
\hline & Count & $\%$ & Count & $\%$ & Count & $\%$ & Count & $\%$ \\
\hline Cortical & 32 & 11.72 & 8 & 10.96 & 1 & 3.03 & 0 & 0 \\
\hline Flat & 134 & 49.08 & 39 & 53.42 & 25 & 75.76 & 5 & 41.67 \\
\hline Dihedral & 9 & 3.3 & 4 & 5.48 & 1 & 3.03 & 1 & 8.33 \\
\hline Multifaceted & 27 & 9.89 & 1 & 1.37 & 4 & 12.12 & 2 & 16.67 \\
\hline Linear & 8 & 2.93 & 9 & 12.33 & 0 & 0 & 0 & 0 \\
\hline Broken & 18 & 6.59 & 5 & 6.85 & 1 & 3.03 & 3 & 25 \\
\hline Other & 9 & 3.3 & 2 & 2.74 & 0 & 0 & 0 & 0 \\
\hline Indeterminate or not recorded & 36 & 13.19 & 5 & 6.85 & 1 & 3.03 & 1 & 8.33 \\
\hline TOTAL & 273 & 100 & 73 & 100 & 33 & 100 & 12 & 100 \\
\hline
\end{tabular}


Table 5.9. Platform area data for blade types, showing that there is variability in the means and that the log transformed variances are basically similar. Note that one outlier was removed from the medium blade category (platform area $=251.23$ )

\begin{tabular}{|l|r|r|r|r|}
\hline & $\begin{array}{c}\text { Medium } \\
\text { blade }\end{array}$ & Bladelet & \multicolumn{1}{c|}{$\begin{array}{l}\text { Twisted } \\
\text { blade }\end{array}$} & $\begin{array}{l}\text { Twisted } \\
\text { bladelet }\end{array}$ \\
\hline $\mathrm{N}$ & 187 & 47 & 31 & 9 \\
\hline Mean & 30.168 & 9.067 & 14.859 & 10.643 \\
\hline SD & 27.99 & 14.14 & 13.83 & 12.96 \\
\hline Variance & 783.41 & 199.8 & 191.27 & 167.95 \\
\hline LOG & & & & \\
Variance & 1.268 & 2.112 & 1.131 & 1.167 \\
\hline
\end{tabular}

Table 5.10. ANOVA of platform area results showing a $p$ value less than .05 , so at least two of the means are significantly different.

\begin{tabular}{|l|r|r|r|r|l|}
\hline $\begin{array}{c}\text { Platform } \\
\text { Areas } \\
\text { ANOVA }\end{array}$ & Df & $\begin{array}{c}\text { Sum } \\
\text { Sq }\end{array}$ & Mean Sq & F value & $\operatorname{Pr}(>$ F) \\
\hline Blade type & 3 & 83.7 & 27.895 & 20.31 & $6.71 \mathrm{e}-12$ \\
\hline Residuals & 270 & 370.8 & 1.373 & & \\
\hline
\end{tabular}

Table 5.11. Results of Tukey's HSD test. The probability that the means of the blades and bladelets are similar is 0 .

\begin{tabular}{|l|r|r|r|r|}
\hline \multicolumn{1}{|c|}{ TukeysHSD } & \multicolumn{1}{c|}{ diff } & \multicolumn{1}{c|}{ lwr } & \multicolumn{1}{c|}{ upr } & \multicolumn{1}{c|}{ p adj } \\
\hline Twisted bladelet-Bladelet & 0.337668 & -0.76461 & 1.439942 & 0.8580907 \\
\hline Twisted medium blade-Bladelet & 0.762078 & 0.061134 & 1.463022 & 0.0270813 \\
\hline Medium blade-Bladelet & 1.413979 & 0.919663 & 1.908294 & 0 \\
\hline Twisted medium blade-Twisted bladelet & 0.424411 & -0.72267 & 1.57149 & 0.7742181 \\
\hline Medium blade-Twisted bladelet & 1.076311 & 0.042474 & 2.110147 & 0.037702 \\
\hline Medium blade-Twisted medium blade & 0.6519 & 0.064421 & 1.239379 & 0.0229354 \\
\hline
\end{tabular}

Table 5.12. Counts and percentages of bulb types by blade types.

\begin{tabular}{|c|c|c|c|c|c|c|c|c|}
\hline \multirow[t]{2}{*}{ Bulb type } & \multicolumn{2}{|c|}{ Medium blades } & \multicolumn{2}{|c|}{ Bladelets } & \multicolumn{2}{|c|}{$\begin{array}{c}\text { Twisted medium } \\
\text { blades }\end{array}$} & \multicolumn{2}{|c|}{ Twisted bladelets } \\
\hline & Count & $\%$ & Count & $\%$ & Count & $\%$ & Count & $\%$ \\
\hline Prominent & 5 & 2.53 & 1 & 1.92 & 0 & 0 & 0 & 0 \\
\hline Moderate & 90 & 45.45 & 7 & 13.46 & 16 & 50 & 1 & 9.09 \\
\hline Diffuse & 68 & 34.34 & 27 & 51.92 & 12 & 37.5 & 6 & 54.55 \\
\hline Small prominent & 20 & 10.1 & 13 & 25 & 4 & 12.5 & 3 & 27.27 \\
\hline Other & 9 & 4.55 & 2 & 3.85 & 0 & 0 & 1 & 9.09 \\
\hline Indeterminate & 6 & 3.03 & 2 & 3.85 & 0 & 0 & 0 & 0 \\
\hline TOTAL & 198 & 100 & 52 & 100 & 32 & 100 & 11 & 100 \\
\hline
\end{tabular}


Table 5.13. Summary of the tests on the comparability of blade type categories. Color coding is to facilitate reference of the same pairings. Parentheses indicate that the conclusion is not very strong.

\begin{tabular}{|l|l|l|}
\hline \multicolumn{1}{|c|}{ Test } & \multicolumn{1}{|c|}{ Not significantly different } & \multicolumn{1}{c|}{ Significantly Different } \\
\hline Platform Chi sq & & Blades - Bladelets \\
\hline Flat Platform Cls & $\begin{array}{l}\text { Bladelets-Twisted bladelets } \\
\text { Twisted Blades- Twisted Bladelets } \\
\text { Blades - Bladelets } \\
\text { Bladelets- Twisted Blades } \\
\text { Blades- Twisted Bladelets }\end{array}$ & Blades - Twisted Blades \\
\hline $\begin{array}{l}\text { Platform area } \\
\text { Anova }\end{array}$ & $\begin{array}{l}\text { Bladelets-Twisted bladelets } \\
\text { (Twisted Blades - Twisted bladelets) }\end{array}$ & $\begin{array}{l}\text { Blades - Bladelets } \\
\text { (Bladelets- Twisted Blades) } \\
\text { (Blades- Twisted Blades) }\end{array}$ \\
(Blades- Twisted Bladelets)
\end{tabular}

Table 5.14. Counts and percentages of platform types for medium blades from three sites.

\begin{tabular}{|l|r|r|r|r|r|r|}
\hline \multirow{2}{*}{ Platform type } & \multicolumn{2}{c|}{ MAP } & \multicolumn{2}{c|}{ ATP } & \multicolumn{2}{c|}{ AKAP } \\
\cline { 2 - 7 } & \multicolumn{1}{c|}{ Count } & \multicolumn{1}{c|}{ \% } & \multicolumn{1}{c|}{ Count } & \multicolumn{1}{c|}{ \% } & \multicolumn{1}{c|}{ Count } & \multicolumn{1}{c|}{$\%$} \\
\hline Cortical & 32 & 11.72 & 6 & 17.65 & 1 & 2.56 \\
\hline Flat & 134 & 49.08 & 19 & 55.88 & 17 & 43.59 \\
\hline Dihedral & 9 & 3.3 & 3 & 8.82 & 1 & 2.56 \\
\hline Multifaceted & 27 & 9.89 & 2 & 5.88 & 5 & 12.82 \\
\hline Linear & 8 & 2.93 & 1 & 2.94 & 3 & 7.69 \\
\hline Broken & 18 & 6.59 & 2 & 5.88 & 6 & 15.38 \\
\hline Other & 9 & 3.3 & 1 & 2.94 & 2 & 5.13 \\
\hline Indeterminate & 36 & 13.19 & 0 & 0 & 4 & 10.26 \\
\hline TOTAL & 273 & 100 & 34 & 99.99 & 39 & 99.99 \\
\hline
\end{tabular}

Table 5.15. Counts and percentages of bulb types for medium blades from three sites.

\begin{tabular}{|l|r|r|r|r|r|r|}
\hline \multirow{2}{*}{ Bulb type } & \multicolumn{2}{|c|}{ MAP } & \multicolumn{2}{c|}{ ATP } & \multicolumn{2}{c|}{ AKAP } \\
\cline { 2 - 8 } & \multicolumn{1}{c|}{ Count } & \multicolumn{1}{c|}{$\%$} & \multicolumn{1}{c|}{ Count } & \multicolumn{1}{c|}{$\%$} & \multicolumn{1}{c|}{ Count } & \multicolumn{1}{c|}{$\%$} \\
\hline Prominent & 5 & 2.53 & 0 & 0 & 0 & 0 \\
\hline Moderate & 90 & 45.45 & 16 & 47.06 & 12 & 31.58 \\
\hline Diffuse & 68 & 34.34 & 14 & 41.18 & 13 & 34.21 \\
\hline Small prominent & 20 & 10.1 & 3 & 8.82 & 3 & 7.89 \\
\hline Other & 9 & 4.55 & 0 & 0 & 0 & 0 \\
\hline Indeterminate & 6 & 3.03 & 1 & 2.94 & 10 & 26.32 \\
\hline TOTAL & 198 & 100 & 34 & 100 & 38 & 100 \\
\hline
\end{tabular}


Table 5.16. Presence/absence of medium blade production elements and tools across sites.

\begin{tabular}{|c|c|c|c|c|c|c|c|c|c|c|c|c|c|c|}
\hline $\begin{array}{c}\text { Publicati } \\
\text { on }\end{array}$ & 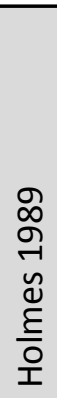 & $\frac{\dddot{U}}{\frac{0}{I}}$ & $\begin{array}{l}\frac{0}{U} \\
\frac{\pi}{1}\end{array}$ & 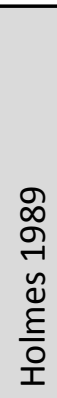 & 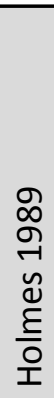 & 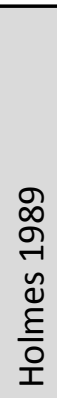 & 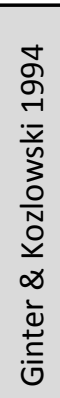 & 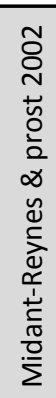 & 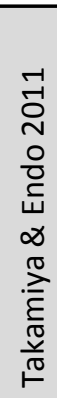 & 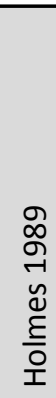 & 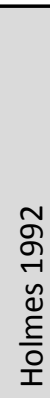 & 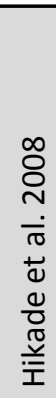 & 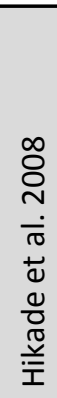 & $\frac{\varrho}{\frac{0}{I}}$ \\
\hline Site & $\begin{array}{l}\frac{1}{\pi} \\
\frac{\pi}{0} \\
\mathbb{0} \\
\infty\end{array}$ & $\sum$ & 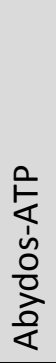 & $\begin{array}{l}\infty \\
\dddot{m} \\
\frac{1}{1} \\
\frac{1}{0} \\
\frac{0}{0} \\
\frac{0}{0} \\
\frac{\pi}{z}\end{array}$ & 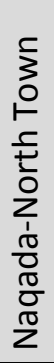 & 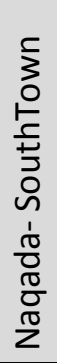 & 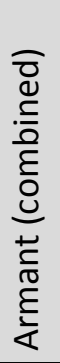 & $\frac{\pi}{\frac{\pi}{\pi}}$ & 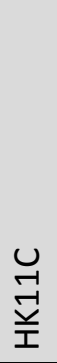 & 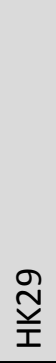 & $\begin{array}{l}\underset{\nwarrow}{\mathbb{~}} \\
\text { 至 }\end{array}$ & 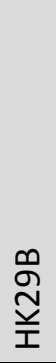 & 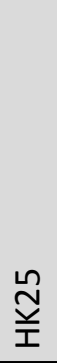 & 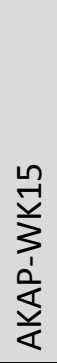 \\
\hline $\begin{array}{c}\text { Total \# of } \\
\text { artifacts } \\
\text { W/ } \\
\text { debris }\end{array}$ & $\frac{\pi}{z}$ & $\begin{array}{l}\hat{\sigma} \\
\text { }\end{array}$ & 오 & นึก & 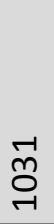 & ஜ & 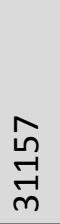 & 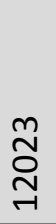 & $\hat{\hat{n}}$ & $\stackrel{\hat{n}}{\mathrm{n}_{n}}$ & $\begin{array}{l}8 \\
8 \\
\text { ஸे }\end{array}$ & 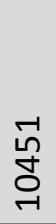 & $\stackrel{\substack{\infty \\
N}}{N}$ & ঙั \\
\hline Cores & $\bullet$ & $\bullet$ & $\bullet$ & $X$ & $X$ & $\bullet$ & $\bullet$ & $\bullet$ & $X$ & $\bullet$ & $\bullet$ & $\bullet$ & $\bullet$ & $\bullet$ \\
\hline $\begin{array}{l}\text { Core re- } \\
\text { juvenatio } \\
\text { n }\end{array}$ & $\bullet$ & & & & & & & $\bullet$ & & & & & & \\
\hline $\begin{array}{l}\text { Plunging } \\
\text { blades }\end{array}$ & & & • & & & & • & • & & & & & & $\bullet$ \\
\hline $\begin{array}{l}\text { Crested } \\
\text { blades }\end{array}$ & $\bullet$ & • & • & & & & • & $\bullet$ & $\bullet$ & $X$ & & $\bullet$ & $\bullet$ & $\bullet$ \\
\hline $\begin{array}{l}\text { Blade } \\
\text { Blanks }\end{array}$ & $\bullet$ & $\bullet$ & • & $\bullet$ & & • & $\bullet$ & $\bullet$ & $\bullet$ & • & $\bullet$ & $\bullet$ & $\bullet$ & $\bullet$ \\
\hline Tools & • & $\bullet$ & $\bullet$ & $\bullet$ & $\bullet$ & $\bullet$ & $\bullet$ & $\bullet$ & $\bullet$ & $\bullet$ & $\bullet$ & $\bullet$ & $\bullet$ & $\bullet$ \\
\hline
\end{tabular}


Table 5.17. Presence/absence of specific tool types for medium blades across sites. For references see Table 5.16. *Blank types were not specified for all tools.

\begin{tabular}{|c|c|c|c|c|c|c|c|c|c|c|c|c|c|c|}
\hline Site & 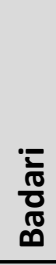 & $\sum_{\Sigma}^{\frac{Q}{\alpha}}$ & $\begin{array}{l}0 \text { 는 } \\
\frac{1}{1} \\
\frac{0}{0} \\
\frac{0}{0}\end{array}$ & $\begin{array}{l}\infty \\
m \\
\frac{1}{1} \\
\frac{1}{j} \\
\frac{0}{0} \\
\frac{0}{\pi} \\
\frac{0}{\pi} \\
\frac{\pi}{2}\end{array}$ & 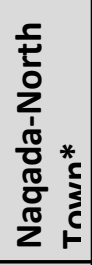 & 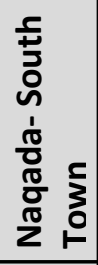 & 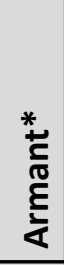 & $\frac{\text { O্ }}{\frac{5}{\pi}}$ & $\begin{array}{l}\underset{-1}{\mid} \\
\underset{\mid}{\text { I }}\end{array}$ & $\begin{array}{l}\text { ิㅗ } \\
\text { İ }\end{array}$ & 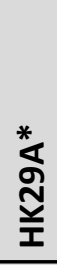 & $\begin{array}{l}\text { * } \\
\text { Оू } \\
\text { ָे }\end{array}$ & 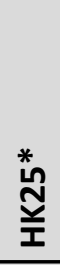 & 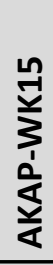 \\
\hline $\begin{array}{l}\text { Total \# of } \\
\text { tools }\end{array}$ & $\frac{\mathbb{z}}{z}$ & $\hat{N}$ & $\stackrel{n}{n}$ & హ్ & 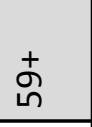 & $\underset{\neg}{\curvearrowright}$ & 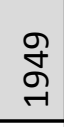 & $\underset{\sim}{\infty}$ & مิ & 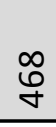 & $\begin{array}{l}\text { ৪ } \\
\text { i } \\
\text { 2 }\end{array}$ & $\underset{\sim}{\stackrel{9}{N}}$ & $\stackrel{\Perp n}{N}$ & à \\
\hline Backed & $\bullet$ & $\bullet$ & & & & $\bullet$ & & $\bullet$ & & $\bullet$ & & $\bullet$ & & \\
\hline $\begin{array}{l}\text { Blade } \\
\text { knives }\end{array}$ & $\bullet$ & $\bullet$ & & & & & & & & & & & & \\
\hline Burins & $\bullet$ & $\bullet$ & $\bullet$ & $\bullet$ & & • & $\bullet$ & $\bullet$ & $\bullet$ & $\bullet$ & & & & $\bullet$ \\
\hline $\begin{array}{l}\text { Dent- } \\
\text { iculates }\end{array}$ & $\bullet$ & $\bullet$ & $\bullet$ & - & & $\bullet$ & & $\bullet$ & $\bullet$ & $\bullet$ & & $\bullet$ & $\bullet$ & \\
\hline $\begin{array}{l}\text { End- } \\
\text { scrapers }\end{array}$ & $\bullet$ & $\bullet$ & & $\bullet$ & & $\bullet$ & $\bullet$ & • & $\bullet$ & $\bullet$ & & & & \\
\hline Notches & $\bullet$ & $\bullet$ & & $\bullet$ & & $\bullet$ & & $\bullet$ & $\bullet$ & $\bullet$ & & $\bullet$ & $\bullet$ & \\
\hline Other & & $\bullet$ & & & & & - & $\bullet$ & $\bullet$ & - & & & & $\bullet$ \\
\hline $\begin{array}{l}\text { Perfor- } \\
\text { ators }\end{array}$ & $\bullet$ & $\bullet$ & & $\bullet$ & & $\bullet$ & $\bullet$ & • & • & $\bullet$ & & & & $\bullet$ \\
\hline Retouched & - & - & - & - & & $\bullet$ & - & $\bullet$ & $\bullet$ & $\bullet$ & & - & - & - \\
\hline $\begin{array}{l}\text { Sickle } \\
\text { blades }\end{array}$ & $\bullet$ & $\bullet$ & & & $\bullet$ & $\bullet$ & & $\bullet$ & $\bullet$ & & $\bullet$ & & & \\
\hline $\begin{array}{l}\text { Trun- } \\
\text { cations }\end{array}$ & $\bullet$ & $\bullet$ & $\bullet$ & $\bullet$ & & $\bullet$ & $\bullet$ & $\bullet$ & $\bullet$ & $\bullet$ & & & & $\bullet$ \\
\hline
\end{tabular}


Table 5.18. Counts and percentages of specific tool types made on medium blades across sites. For references see table 5.16. *The reported number is the minimum number of tools made on blade blanks from that site because not all blanks types were identified.

\begin{tabular}{|c|c|c|c|c|c|c|c|c|c|c|c|c|}
\hline Site & \multicolumn{2}{|c|}{ MAP } & ATP & \multicolumn{2}{|c|}{$\begin{array}{l}\text { Naqada- } \\
\text { КH3B }\end{array}$} & \multicolumn{2}{|c|}{$\begin{array}{l}\text { Naqada- South } \\
\text { Town }\end{array}$} & \multicolumn{2}{|c|}{ HK11C } & \multicolumn{2}{|c|}{ НК29 } & $\begin{array}{l}\text { AKAP- } \\
\text { WK15 }\end{array}$ \\
\hline \# of tools & \multicolumn{2}{|c|}{767} & 75 & \multicolumn{2}{|c|}{194} & \multicolumn{2}{|c|}{177} & \multicolumn{2}{|c|}{307} & \multicolumn{2}{|c|}{468} & 97 \\
\hline & Count & $\%$ & Count & Count & $\%$ & Count & $\%$ & Count & $\%$ & Count & $\%$ & Count \\
\hline Backed & 1 & 1.49 & 0 & 0 & 0 & 3 & 4.41 & 0 & 0 & 5 & 4.1 & 0 \\
\hline Blade knives & 5 & 7.46 & 0 & 0 & 0 & 0 & 0 & 0 & 0 & 0 & 0 & 0 \\
\hline Burins & 17 & 25.37 & 4 & 19 & 57.58 & 19 & 27.94 & 6 & 6.82 & 28 & 22.95 & 5 \\
\hline Denticulates & 11 & 16.42 & 2 & 1 & 3.03 & 5 & 7.35 & 1 & 1.14 & 5 & 4.1 & 0 \\
\hline Scrapers, end & 1 & 1.49 & 0 & 2 & 6.06 & 4 & 5.88 & 16 & 18.18 & 8 & 6.56 & 0 \\
\hline Notches & 4 & 5.97 & 0 & 2 & 6.06 & 2 & 2.94 & 2 & 2.27 & 5 & 4.1 & 0 \\
\hline other & 2 & 2.99 & 0 & 0 & 0 & 0 & 0 & 3 & 3.41 & 4 & 3.28 & 1 \\
\hline Perforators & 1 & 1.49 & 0 & 2 & 6.06 & 7 & 10.29 & 1 & 1.14 & 3 & 2.46 & 1 \\
\hline retouched & 11 & 16.42 & 1 & 6 & 18.18 & 12 & 17.65 & 49 & 55.68 & 54 & 44.26 & 1 \\
\hline Sickle blades & 3 & 4.48 & 0 & 0 & 0 & 11 & 16.18 & 4 & 4.55 & 0 & 0 & 0 \\
\hline truncations & 11 & 16.42 & 1 & 1 & 3.03 & 5 & 7.35 & 6 & 6.82 & 10 & 8.2 & 1 \\
\hline Total & 67 & 100 & 8 & $33 *$ & 100 & $68 *$ & 99.99 & 88 & 100.01 & $122 *$ & 100.01 & 10 \\
\hline
\end{tabular}

Table 5.19. Presence/absence of production remains and tools for heat-treated bladelets across sites. For references see Table 5.16, plus Abydos cemeteries: Hikade (1998, 2000); and Adaima cemetery: Crubezy et al. (2002:268-273).

\begin{tabular}{|c|c|c|c|c|c|c|c|c|c|c|c|}
\hline Site & $\begin{array}{l}\frac{1}{\pi} \\
\frac{\pi}{0} \\
\frac{\pi}{0} \\
0\end{array}$ & $\frac{2}{\Sigma}$ & 㔖 & 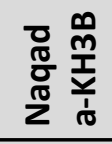 & $\begin{array}{l}\frac{\sigma}{\pi} \\
\frac{\pi}{\pi} \\
\frac{\pi}{2} \\
2\end{array}$ & $\begin{array}{l}\frac{\delta}{\pi} \\
\frac{\pi}{0} \\
\frac{\pi}{2}\end{array}$ & $\begin{array}{ll}\frac{E}{\pi} \\
\frac{\pi}{0} \\
\frac{\pi}{\alpha}\end{array}$ & $\begin{array}{l}\text { U. } \\
\stackrel{\vec{I}}{I}\end{array}$ & 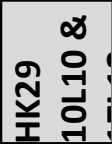 & ฐ্ণ & 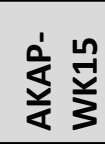 \\
\hline $\begin{array}{l}\text { Total \# of } \\
\text { artifacts W/ } \\
\text { debris }\end{array}$ & $\frac{\pi}{z}$ & 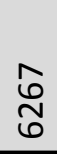 & ஜి & ํํำ & مे & ֻั & 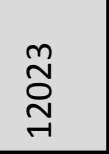 & $\hat{n}$ & 岗 & 8 & ‡ั \\
\hline Cores & $\bullet$ & $\bullet$ & & $x$ & $x$ & $x$ & $\bullet$ & $\bullet$ & $\bullet$ & $\bullet$ & $x$ \\
\hline \multicolumn{12}{|l|}{$\begin{array}{l}\text { Core re- } \\
\text { juvenation }\end{array}$} \\
\hline $\begin{array}{l}\text { Plunging } \\
\text { bladelets }\end{array}$ & & $\bullet$ & $x$ & & & & & & & & $x$ \\
\hline $\begin{array}{l}\text { Crested } \\
\text { bladelets }\end{array}$ & & $\bullet$ & $x$ & & & & & & & & $x$ \\
\hline Blanks & $\bullet$ & $\bullet$ & $x$ & & & & $\bullet$ & $\bullet$ & $\bullet$ & $\bullet$ & $x$ \\
\hline $\begin{array}{l}\text { Tools } \\
\text { (settlements) }\end{array}$ & $\bullet$ & $\bullet$ & $x$ & $x$ & $x$ & $x$ & $\bullet$ & $\bullet$ & $\bullet$ & $\bullet$ & $\bullet$ \\
\hline $\begin{array}{l}\text { Tools } \\
\text { (cemeteries) }\end{array}$ & & & $\bullet$ & & - & & $(\bullet)$ & & $(\bullet)$ & & \\
\hline
\end{tabular}


Table 5.20. Presence/absence of specific tool types for heat-treated bladelets across sites. For references see Table 5.16. *Blank types were not specified for all tools.

\begin{tabular}{|c|c|c|c|c|c|c|c|c|c|c|c|c|c|c|}
\hline Site & $\begin{array}{l}\frac{2}{\pi} \\
\frac{\pi}{\pi} \\
\frac{0}{0} \\
0\end{array}$ & $\frac{a}{\Sigma}$ & $\begin{array}{l}0 \\
\frac{0}{4} \\
\tilde{n} \\
\frac{0}{0} \\
\frac{0}{0} \\
\frac{0}{\alpha}\end{array}$ & $\begin{array}{l}\infty \\
m \\
\frac{m}{1} \\
\frac{1}{0} \\
\frac{\pi}{0} \\
\frac{\pi}{0} \\
\frac{\pi}{2}\end{array}$ & 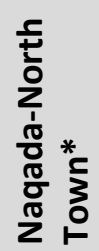 & 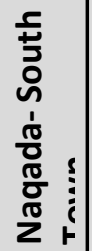 & 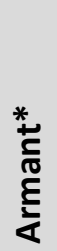 & $\frac{\mathbb{0}}{: \frac{\delta}{\pi}}$ & $\begin{array}{l}u \\
\stackrel{-}{-} \\
\text { 至 }\end{array}$ & ָั & 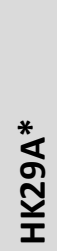 & $\begin{array}{l}\stackrel{*}{\mathscr{0}} \\
\text { ్ } \\
\text { 至 }\end{array}$ & 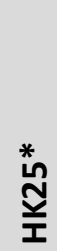 & $\begin{array}{l}\text { n } \\
\stackrel{y}{3} \\
\frac{1}{1} \\
\frac{1}{5} \\
\frac{5}{\alpha}\end{array}$ \\
\hline $\begin{array}{l}\text { \# of total } \\
\text { tools }\end{array}$ & $\frac{\pi}{z}$ & $\hat{\sigma}$ & $\stackrel{n}{N}$ & హ్ & ถึ & $\underset{ન}{\stackrel{f}{f}}$ & શ્ & $\underset{\infty}{\infty}$ & în & $\underset{\mathscr{\sigma}}{\infty}$ & \& & 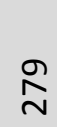 & $\stackrel{\sim}{N}$ & à \\
\hline Backed & & & & & & & & & & & & & & \\
\hline $\begin{array}{l}\text { Blade } \\
\text { knives }\end{array}$ & & & & & & & & & & & & & & \\
\hline Burins & & & & & & & & & & & & & & \\
\hline $\begin{array}{l}\text { Dent- } \\
\text { iculates }\end{array}$ & & & & & & & & & & & & & & \\
\hline $\begin{array}{l}\text { Scrapers, } \\
\text { micro-end }\end{array}$ & $\bullet$ & - & & & & & & - & & - & - & - & - & - \\
\hline Notches & & & & & & & & & & & & & & \\
\hline Other & & & & & & & & & & & $\bullet$ & • & $\bullet$ & \\
\hline $\begin{array}{l}\text { Perfor- } \\
\text { ators }\end{array}$ & & & & & & & & & & & & & & \\
\hline Retouched & & & & & & & & • & & & & & & • \\
\hline $\begin{array}{l}\text { Sickle } \\
\text { blades }\end{array}$ & & • & & & & & & & - & & & & & \\
\hline $\begin{array}{l}\text { Trunc- } \\
\text { ations }\end{array}$ & & & & & & & & & & $\bullet$ & & & & $\bullet$ \\
\hline
\end{tabular}


Table 5.21. Presence/absence of production remains and tools for microdrill bladelet tools across sites. For references see Table 5.16 .

\begin{tabular}{|c|c|c|c|c|c|c|c|c|c|c|c|c|c|c|}
\hline Site & $\begin{array}{l}\frac{1}{\pi} \\
\frac{\pi}{0} \\
\frac{\pi}{0} \\
0\end{array}$ & $\sum^{\frac{0}{\pi}}$ & 量 & $\begin{array}{l}\frac{1}{0} \\
\frac{\pi}{0} \\
\frac{0}{0} \\
2\end{array}$ & 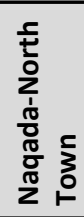 & 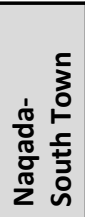 & 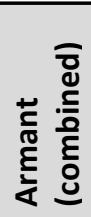 & $\frac{\pi}{\frac{\pi}{\pi}}$ & $\begin{array}{l}u \\
\underset{-1}{\vec{I}} \\
\text { 王 }\end{array}$ & 疍 & 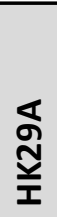 & $\begin{array}{l}\text { ஜิ } \\
\text { ָे } \\
\text { 至 }\end{array}$ & 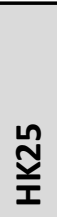 & $\frac{d}{\frac{d}{\alpha}}$ \\
\hline $\begin{array}{l}\text { Total \# of } \\
\text { artifacts } \\
\text { W/ debris }\end{array}$ & $\frac{\pi}{z}$ & ڤ్ర & 옴 & 㞫 & $\stackrel{\vec{n}}{\stackrel{-}{-1}}$ & 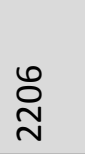 & 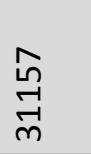 & 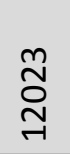 & $\underset{n}{\hat{n}}$ & 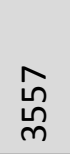 & $\begin{array}{l}8 \\
8 \\
\text { ஸे }\end{array}$ & ํㅠㅁ & 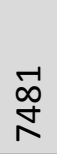 & ঙั \\
\hline Cores & & $x$ & $x$ & & & & & & & & $\bullet$ & & $\bullet$ & $x$ \\
\hline $\begin{array}{l}\text { Core re- } \\
\text { juvenation }\end{array}$ & & & & & & & & & & & & & & \\
\hline $\begin{array}{l}\text { Plunging } \\
\text { bladelets }\end{array}$ & & $x$ & $x$ & & & & & & & & & & & $x$ \\
\hline $\begin{array}{l}\text { Crested } \\
\text { bladelets }\end{array}$ & & $x$ & $x$ & & & & & & & & & & & $x$ \\
\hline Blanks & & $x$ & $x$ & & & & & & & $\bullet$ & $\bullet$ & & & $x$ \\
\hline Tools & & $x$ & $x$ & & & & & $x$ & & • & • & • & • & $x$ \\
\hline
\end{tabular}

Table 5.22. Presence/absence of production remains and tools for large blades across sites. For references see Table 5.16.

\begin{tabular}{|c|c|c|c|c|c|c|c|c|c|c|c|c|c|}
\hline Site & $\begin{array}{l}\frac{1}{\pi} \\
\frac{\pi}{0} \\
\frac{0}{0} \\
0\end{array}$ & $\frac{\frac{0}{\pi}}{\sum}$ & 은 & $\begin{array}{l}\frac{\delta}{\pi} \\
\frac{\pi}{0} \\
\frac{\pi}{2}\end{array}$ & $\begin{array}{l}\frac{1}{0} \\
\frac{\pi}{0} \\
\frac{\pi}{0} \\
\frac{\pi}{2} \\
\frac{\pi}{2} \\
0\end{array}$ & 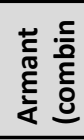 & $\frac{\xi}{\frac{E}{\pi}}$ & $\begin{array}{l}\stackrel{U}{-} \\
\stackrel{\vec{I}}{I}\end{array}$ & $\frac{\text { ్ㅗ․ }}{\text { 至 }}$ & $\begin{array}{l}\text { ্ָ } \\
\text { I্ }\end{array}$ & 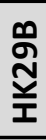 & 站 & 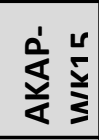 \\
\hline $\begin{array}{l}\text { Total \# of } \\
\text { artifacts } \\
\text { W/ debris }\end{array}$ & $\frac{\varangle}{z}$ & $\hat{\mathscr{U}}$ & 욤 & ํํํ & ֻัญ & $\begin{array}{l}\hat{\text { Ln }} \\
\stackrel{\mathrm{m}}{\mathrm{n}}\end{array}$ & 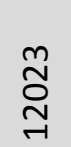 & $\hat{\hat{\sigma}}$ & กิก & 요 & $\begin{array}{l}\stackrel{-1}{0} \\
\text { f }\end{array}$ & $\stackrel{\substack{\infty \\
N}}{N}$ & ঙั丶 \\
\hline Cores & (?) & $x$ & $x$ & $x$ & $x$ & & $(X)$ & $x$ & $x$ & & & & $x$ \\
\hline $\begin{array}{l}\text { Core re- } \\
\text { juvenatio } \\
\text { n }\end{array}$ & & & & & & & (X) & & & & & & \\
\hline $\begin{array}{l}\text { Plunging } \\
\text { blades }\end{array}$ & & $x$ & $x$ & & & & $(X)$ & $x$ & & & & & $x$ \\
\hline $\begin{array}{l}\text { Crested } \\
\text { blades }\end{array}$ & & $x$ & $x$ & & & & $(X)$ & $x$ & & & & & $x$ \\
\hline Blanks & $\bullet$ & $x$ & $x$ & & $\bullet$ & & (X) & $x$ & & & & & $x$ \\
\hline Tools & $\bullet$ & • & • & & $\bullet$ & & $\bullet$ & $\bullet$ & & & & & $x$ \\
\hline
\end{tabular}


Table 5.23. Presence/absence of production remains and tools for non-heat-treated bladelets across sites. For references see Table 5.16. NS1= item was present, but not specified whether the item was Heat-treated or not; NS2= blades not separated from bladelets; NS3= blanks of tool types not specified.

\begin{tabular}{|c|c|c|c|c|c|c|c|c|c|c|c|c|}
\hline Site & 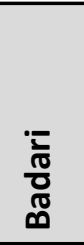 & $\sum_{\Sigma}^{0}$ & 是 & $\begin{array}{l}\frac{1}{0} \\
\frac{\pi}{0} \\
\frac{\pi}{0} \\
\frac{\pi}{2}\end{array}$ & 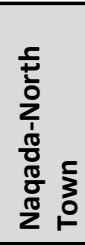 & 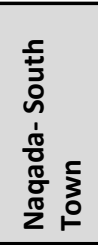 & 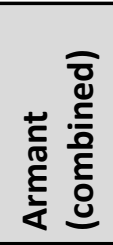 & $\frac{\pi}{\frac{\delta}{5}}$ & 荘 & 空 & $\begin{array}{l}\text { ్ָ } \\
\text { 至 }\end{array}$ & 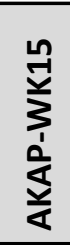 \\
\hline $\begin{array}{l}\text { Total \# } \\
\text { of } \\
\text { artifacts } \\
\text { W/ } \\
\text { debris }\end{array}$ & $\frac{\nwarrow}{z}$ & $\begin{array}{l}\hat{\omega} \\
\tilde{\sigma}\end{array}$ & 옹 & ㄴํํ & 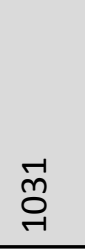 & ֻั & $\begin{array}{l}\hat{n} \\
\stackrel{-}{-} \\
\text { n }\end{array}$ & 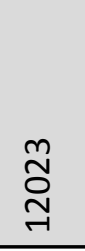 & $\hat{\widehat{b}}$ & 命 & 8 & ָั \\
\hline Cores & $x$ & $\bullet$ & $x$ & $x$ & $\bullet$ & $x$ & $\begin{array}{l}\text { NS1 } \\
\& 2\end{array}$ & $\begin{array}{l}\text { NS1 } \\
\& 2\end{array}$ & $\bullet$ & $\bullet$ & $\bullet$ & $x$ \\
\hline $\begin{array}{l}\text { Core re- } \\
\text { juvenation }\end{array}$ & & & & & & & & & & & & \\
\hline $\begin{array}{l}\text { Plunging } \\
\text { bladelets }\end{array}$ & & $\bullet$ & $x$ & & & & & & & & & $x$ \\
\hline $\begin{array}{l}\text { Crested } \\
\text { bladelets }\end{array}$ & NS2 & $\bullet$ & $x$ & & & & NS2 & & & $x$ & & $x$ \\
\hline Blanks & $x$ & $\bullet$ & $\bullet$ & & & & NS1 & $\bullet$ & $\bullet$ & $\bullet$ & $\bullet$ & $\bullet$ \\
\hline Tools & NS3 & $\bullet$ & $x$ & & & & NS3 & $\bullet$ & $\bullet$ & NS3 & NS3 & $\bullet$ \\
\hline
\end{tabular}

Table 5.24. Counts and percentages of medium blade production remains and tools found in three blocks at el-Mahâsna. Complete \& proximal pieces.

\begin{tabular}{|l|r|r|r|r|r|r|}
\hline \multirow{2}{*}{ Artifact Type } & \multicolumn{2}{|c|}{ Block 1 } & \multicolumn{2}{c|}{ Block 3 } & \multicolumn{2}{c|}{ Block 4 } \\
\cline { 2 - 7 } & Count & $\%$ & Count & $\%$ & Count & $\%$ \\
\hline Cores & 3 & 2.91 & 4 & 3.28 & 5 & 5.43 \\
\hline Crested blades & 0 & 0 & 1 & 0.82 & 1 & 1.09 \\
\hline Plunging blanks & 9 & 8.74 & 8 & 6.56 & 2 & 2.17 \\
\hline Blade blanks & 68 & 66.02 & 89 & 72.95 & 66 & 71.74 \\
\hline Tools & 23 & 22.33 & 20 & 16.39 & 18 & 19.57 \\
\hline Total & 103 & 100 & 122 & 100 & 92 & 100 \\
\hline
\end{tabular}


Table 5.25. Presence/absence of specific tool types for non-heat-treated bladelets across sites. For references see Table 5.16. *Blanks types were not specified for all tools.

\begin{tabular}{|c|c|c|c|c|c|c|c|c|c|c|c|c|c|}
\hline Site & $\begin{array}{l}\bar{\Sigma} \\
\frac{\pi}{0} \\
\frac{0}{\pi} \\
0\end{array}$ & $\sum^{\frac{0}{10}}$ & 证 & $\begin{array}{ll}\frac{\pi}{0} & 0 \\
\frac{\pi}{\pi} & 0 \\
0 & 0 \\
\frac{\pi}{2} & 3 \\
\end{array}$ & $\begin{array}{l}\frac{\pi}{0} \\
\frac{\pi}{\pi} \\
\frac{1}{0} \\
\frac{\pi}{2} \\
2\end{array}$ & 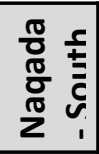 & 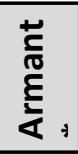 & $\frac{\mathbb{\pi}}{\frac{1}{\pi}}$ & 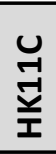 & $\begin{array}{l}\text { ิㅗ․ } \\
\text { 至 }\end{array}$ & 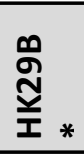 & $\begin{array}{l}\stackrel{*}{\stackrel{*}{N}} \\
\text { 至 }\end{array}$ & $\frac{\text { d }}{\text { 市 }}$ \\
\hline $\begin{array}{l}\text { \# of total } \\
\text { tools }\end{array}$ & $\frac{\varangle}{\Sigma}$ & $\hat{\imath}$ & $\stackrel{n}{\sim}$ & హે & 오 & $\underset{-}{\curvearrowright}$ & જ્ & $\underset{\infty}{\stackrel{\infty}{N}}$ & $\hat{\text { ô }}$ & $\underset{\wp}{\infty}$ & $\underset{\sim}{\stackrel{9}{N}}$ & $\stackrel{\stackrel{n}{N}}{N}$ & à \\
\hline Backed & & & & & & & & $\bullet$ & & & & & \\
\hline $\begin{array}{l}\text { Blade } \\
\text { knives }\end{array}$ & & & & & & & & & & & & & \\
\hline Burins & & & & & & & & & & & & & \\
\hline $\begin{array}{l}\text { Dent- } \\
\text { iculates }\end{array}$ & & & & & & & & • & & & & & \\
\hline $\begin{array}{l}\text { Scrapers, } \\
\text { microend }\end{array}$ & & $\bullet$ & & & & & & & & & & & • \\
\hline Notches & & & & & & & & & & & & & \\
\hline Other & & & & & & & & $\bullet$ & & & & & \\
\hline Perforators & & & & & & & & & & & & & \\
\hline Retouched & & • & & & & & & $\bullet$ & • & & & & $\bullet$ \\
\hline $\begin{array}{l}\text { Sickle } \\
\text { blades }\end{array}$ & & & & & & & & & & & & & \\
\hline Truncations & & & & & & & & • & & & & & \\
\hline
\end{tabular}

Table 5.26. Counts of production remains and tools for non-heattreated bladelets from three blocks at el-Mahâsna. Complete \& proximal pieces. The tool in Block 3 is a microendscraper, and the tools in Block 4 are a microendscraper and two retouched pieces.

\begin{tabular}{|l|c|c|c|}
\hline \multicolumn{1}{|c|}{ Artifact Type } & Block 1 & Block 3 & Block 4 \\
\hline Cores & 0 & 3 & 0 \\
\hline Crested blades & 0 & 1 & 0 \\
\hline Plunging blanks & 1 & 1 & 2 \\
\hline Bladelet blanks & 28 & 17 & 11 \\
\hline Tools & 0 & 1 & 3 \\
\hline Total & 29 & 23 & 16 \\
\hline
\end{tabular}




\begin{tabular}{|l|c|c|c|}
\hline \multicolumn{1}{|c|}{ Artifact Type } & Block 1 & Block 3 & Block 4 \\
\hline Cores & 1 & 3 & 0 \\
\hline Crested blades & 1 & 0 & 0 \\
\hline Plunging blanks & 1 & 0 & 1 \\
\hline Blanks & 7 & 3 & 5 \\
\hline Tools & 3 & 1 & 1 \\
\hline Total & 13 & 7 & 7 \\
\hline
\end{tabular}

Table 5.27. Counts of production remains and tools for heat-treated bladelets from three blocks at el-Mahâsna. Complete \& proximal pieces. All the tools are microendscrapers.

\begin{tabular}{|l|c|c|c|}
\hline Artifact Type & Block 1 & Block 3 & Block 4 \\
\hline Cores & 1 & 6 & 0 \\
\hline Crested blades & 1 & 1 & 0 \\
\hline Plunging blanks & 2 & 1 & 3 \\
\hline Bladelet blanks & 35 & 20 & 16 \\
\hline Tools & 3 & 2 & 4 \\
\hline Total & 42 & 30 & 23 \\
\hline
\end{tabular}

Table 5.28. Counts of production remains and tools for all bladelet remains combined from three blocks at elMahâsna. Complete \& proximal pieces.

Table 5.29. Presence/absence of production remains and tools for medium blades from Hierakonpolis localities. Complete \& proximal pieces. For references see table 5.16.

\begin{tabular}{|c|c|c|c|c|c|}
\hline Site & $\begin{array}{l}\text { HK } \\
11 \mathrm{C}\end{array}$ & $\begin{array}{l}\text { HK } \\
29\end{array}$ & $\begin{array}{l}\text { HK } \\
29 A\end{array}$ & $\begin{array}{l}\text { HK } \\
\text { 29B }\end{array}$ & $\begin{array}{l}\text { HK } \\
25\end{array}$ \\
\hline $\begin{array}{l}\text { Total \# of } \\
\text { artifacts } \\
\text { W/ debris }\end{array}$ & $\hat{\hat{n}}$ & ஸn & $\begin{array}{l}\text { ○ } \\
\text { ஸे }\end{array}$ & 공 & 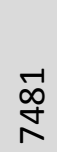 \\
\hline Cores & $x$ & • & $\bullet$ & $\bullet$ & $\bullet$ \\
\hline $\begin{array}{l}\text { Crested } \\
\text { blades }\end{array}$ & $\bullet$ & $x$ & & • & • \\
\hline $\begin{array}{l}\text { Blade } \\
\text { blanks }\end{array}$ & • & $\bullet$ & • & $\bullet$ & • \\
\hline Tools & • & $\bullet$ & • & • & • \\
\hline
\end{tabular}

Table 5.30. Counts and percentages of production remains and tools for medium blades from Hierakonpolis HK11C and HK29. The percentages of HK11C were calculated without the "several" crested blades because the exact count was not known. However those few would probably only make the percentages even more similar. For references see table 5.16.

\begin{tabular}{|l|l|l|l|l|}
\hline \multicolumn{1}{|c|}{ Site } & \multicolumn{2}{c|}{ HK11C } & \multicolumn{2}{c|}{ HK29 } \\
\hline & Count & $\%$ & Count & $\%$ \\
\hline Cores & 0 & 0 & 5 & 0.82 \\
\hline $\begin{array}{l}\text { Crested } \\
\text { blades }\end{array}$ & several & & 0 & 0 \\
\hline $\begin{array}{l}\text { Blade } \\
\text { blanks }\end{array}$ & 356 & 79.29 & 481 & 79.11 \\
\hline Tools & 93 & 20.71 & 122 & 20.07 \\
\hline TOTAL & 449 & 100 & 608 & 100 \\
\hline
\end{tabular}


Table 5.31. Counts of specific tools types made on blades from HK11C and HK29. *Blanks types were not specified for all tools. Because of that the percentages were not calculated. For references see Table 5.21.

\begin{tabular}{|l|l|l|}
\hline \multicolumn{1}{|c|}{ Site } & $\begin{array}{l}\text { HK } \\
\text { 11C }\end{array}$ & HK 29* \\
\hline \# of total tools & 307 & 468 \\
\hline \# of blade tools & 88 & 122 \\
\hline Backed & 0 & 5 \\
\hline Blade knives & 0 & 0 \\
\hline Burins & 6 & 28 \\
\hline Denticulates & 1 & 5 \\
\hline Scrapers, end & 16 & 8 \\
\hline Notches & 2 & 5 \\
\hline Other & 3 & 4 \\
\hline Perforators & 1 & 3 \\
\hline Retouched & 49 & 54 \\
\hline Sickle blades & 4 & 0 \\
\hline Truncations & 6 & $(10)$ \\
\hline
\end{tabular}

Table 5.32. Presence/absence data for non-heat-treated bladelets from Hierakonpolis localities. The tool from HK11C is a retouched piece. *Blanks of tool types not specified. For references see Table 5.16

\begin{tabular}{|c|c|c|c|}
\hline Site & $\begin{array}{l}u \\
\ddot{H} \\
\underline{y}\end{array}$ & $\begin{array}{l}\text { 오․ } \\
\text { 至 }\end{array}$ & $\begin{array}{l}\text { ฐั } \\
\frac{1}{1}\end{array}$ \\
\hline $\begin{array}{l}\text { Total \# of } \\
\text { artifacts W/ } \\
\text { debris }\end{array}$ & $\hat{\mathfrak{n}}$ & $\begin{array}{r}\hat{\mathrm{n}} \\
\stackrel{\mathrm{n}}{\mathrm{n}}\end{array}$ & $\begin{array}{l}8 \\
\text { ஓ } \\
\text { ஸे }\end{array}$ \\
\hline Cores & $\bullet$ & $\bullet$ & $\bullet$ \\
\hline Core rejuvenation & & & \\
\hline Plunging bladelets & & & \\
\hline Crested bladelets & & $x$ & \\
\hline Blanks & $\bullet$ & $\bullet$ & $\bullet$ \\
\hline Tools & $\bullet$ & $*$ & $*$ \\
\hline
\end{tabular}

Table 5.33. Presence/absence data for heattreated bladelets from Hierakonpolis localities. For references see Table 5.16

\begin{tabular}{|c|c|c|c|}
\hline $\begin{array}{l}\text { Hierakonpolis } \\
\text { Heat-treated } \\
\text { Bladelets }\end{array}$ & $\begin{array}{l}\text { U } \\
\stackrel{\text { I }}{\text { I }}\end{array}$ & 疍 & $\begin{array}{l}\text { ๙̃ } \\
\text { 壬 }\end{array}$ \\
\hline $\begin{array}{l}\text { Total \# of } \\
\text { artifacts W/ } \\
\text { debris }\end{array}$ & $\hat{\mathfrak{n}}$ & 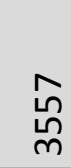 & $\begin{array}{l}8 \\
\text { O } \\
\text { ம }\end{array}$ \\
\hline Cores & $\bullet$ & $\bullet$ & $\bullet$ \\
\hline $\begin{array}{l}\text { Core } \\
\text { rejuvenation }\end{array}$ & & & \\
\hline $\begin{array}{l}\text { Plunging } \\
\text { bladelets }\end{array}$ & & & \\
\hline Crested bladelets & & & \\
\hline Blanks & $\bullet$ & $\bullet$ & $\bullet$ \\
\hline Tools & $\bullet$ & $\bullet$ & $\bullet$ \\
\hline
\end{tabular}


Chapter 6 Tables 
Table 6.1 (part 1). Figural eccentrics.

\begin{tabular}{|c|c|c|c|c|c|c|c|c|c|c|c|c|}
\hline Item ID & Figure & Site & Acquisition & Context & Context Detail & Date & \begin{tabular}{|c}
$\begin{array}{c}\text { Preserv- } \\
\text { ation }\end{array}$ \\
\end{tabular} & Reduction & \begin{tabular}{|c|} 
Length \\
$(\mathrm{cm})$
\end{tabular} & \begin{tabular}{|l|} 
Width \\
$(\mathrm{cm})$
\end{tabular} & \begin{tabular}{|l|} 
Thick \\
$(\mathrm{cm})$ \\
\end{tabular} & \begin{tabular}{|c|} 
Ref. \\
$\#$
\end{tabular} \\
\hline ÄM.15708 & $\begin{array}{l}\text { Model } \\
\text { arrowhead }\end{array}$ & & & & & & Complete & Bifacial & & & & 1 \\
\hline ÄМ.15709 & $\begin{array}{l}\text { Model } \\
\text { arrowhead }\end{array}$ & & & & & & Complete & Bifacial & & & & 2 \\
\hline ÄМ.15711 & $\begin{array}{l}\text { Model } \\
\text { arrowhead }\end{array}$ & & & & & & Complete & Bifacial & & & & 3 \\
\hline ÄM.15712 & Barbary sheep & & Purchased & & & & Broken & Ind-probably bifacial & & & & 4 \\
\hline ÄМ.15774 & Hartebeest & & Purchased & & & & Complete & Bifacial & & & & 5 \\
\hline ÄM.15775 & Nubian ibex & & Purchased & & & & Complete? & Bifacial & & & & 6 \\
\hline BM.EA.49284 & indeterminate & $\begin{array}{l}\text { Abydos- Um el- } \\
\text { Qa'ab }\end{array}$ & Excavated & Cemetery & & & Broken & Bifacial & 10.3 & $1.8+$ & 3 & 7 \\
\hline BM.EA.30411 & Antilope & & & & & $\mathrm{NII}$ & Complete & Edge (bi or unifacial) & 7.4 & 5 & $.4(?)$ & 8 \\
\hline BM.EA.32124 & Bovine head & & & & & & Complete & Ind-probably bifacial & 7.5 & 5.2 & 0.6 & 9 \\
\hline BM.EA.37269 & Crocadile & Abydos & Excavated & Settlement & $\begin{array}{l}\text { Settlement Osiris } \\
\text { temenos, IVl } 50\end{array}$ & $\begin{array}{l}\text { NII or } \\
\text { Djer }\end{array}$ & Complete & Bifacial & 15.7 & 4.6 & 0.7 & 10 \\
\hline BrM.09.889.291 & $\begin{array}{l}\text { Quadruped } \\
\text { (dog?) }\end{array}$ & $\begin{array}{l}\text { Hierakonpolis- } \\
\text { Kom el-Ahmar }\end{array}$ & Excavated & Settlement & \begin{tabular}{|l|} 
"kitchen \\
middens"
\end{tabular} & & Broken & Edge (bi \& unifacial) & $>4.7$ & $>2.9$ & $1-3$ & 11 \\
\hline EM.43085 & Fish & Saqqara & Excavated & Cemetery & & & Ind & Bifacial & & & & 12 \\
\hline FMcGC.1 & Bovine head & & & & & & Ind & & & & & 13 \\
\hline HK.6.1980.1 & Hippo & HK6 & Excavated & Cemetery & \begin{tabular}{|l|} 
Surface Near \\
tomb 1. Reg. 179
\end{tabular} & & Complete & Bifacial & & & & 14 \\
\hline HK.6.1999.1 & Giraffe head & HK6 & Excavated & Cemetery & \begin{tabular}{|l|} 
Surface. \\
Reg.\#519
\end{tabular} & \begin{tabular}{|l|} 
Prob. \\
NIC-IIA
\end{tabular} & Complete & Bifacial & & & & 15 \\
\hline HK.6.2000.1 & Nubian Ibex & HK6 & Excavated & Cemetery & $\begin{array}{l}\text { Surface near } \\
\text { tomb 23 Reg.532 }\end{array}$ & NIIB & Complete & Bifacial & & & & 16 \\
\hline HK.6.2005.1 & Ram head & HK6 & Excavated & Cemetery & $\begin{array}{l}\text { T. } 23 \text { enclosure, } \\
\text { NE section }\end{array}$ & NIIAB & Complete & Edge (bi \& unifacial) & & & & 17 \\
\hline HK.6.2006.1 & Quadruped & HK6 & Excavated & Cemetery & $\begin{array}{l}\text { Structure E8, NW } \\
\text { corner }\end{array}$ & NIIAB & Broken & Bifacial & & & & 18 \\
\hline
\end{tabular}


Table 6.1 (part 2). Figural eccentrics. *Based on Hendrickx \& Depraetere 2004.

\begin{tabular}{|c|c|c|c|c|c|c|c|c|c|c|c|c|}
\hline Item ID & Figure & Site & Acquisition & Context & Context Detail & Date & $\begin{array}{c}\text { Preserv- } \\
\text { ation }\end{array}$ & Reduction & $\begin{array}{c}\text { Length } \\
(\mathrm{cm})\end{array}$ & \begin{tabular}{|l|} 
Width \\
$(\mathrm{cm})$
\end{tabular} & \begin{tabular}{|l|} 
Thick \\
$(\mathrm{cm})$ \\
\end{tabular} & \begin{tabular}{|c|} 
Ref. \\
$\#$
\end{tabular} \\
\hline HK.6.2006.2 & Elephant head & HK6 & Excavated & Cemetery & $\begin{array}{l}\text { Structure E8, NE } \\
\text { corner }\end{array}$ & NIIAB & Broken (?) & Bifacial & & & & 19 \\
\hline HK.6.2007.1 & Nubian Ibex & HK6 & Excavated & Cemetery & $\begin{array}{l}\text { Modern pit near } \\
\text { SE corner of Str.7 }\end{array}$ & & Broken & Bifacial & & & & 20 \\
\hline HK6.2007.2 & Nubian Ibex & HK6 & Excavated & Cemetery & Near Tomb 42 & & Fragment & Bifacial & & & & 21 \\
\hline HK.6.2011.1 & $\begin{array}{l}\text { Human } \\
\text { (Dwarf?) }\end{array}$ & HK6 & Excavated & Cemetery & $\begin{array}{l}\text { T16 enclosure, } \\
\text { NW section. }\end{array}$ & $\begin{array}{l}\text { NIC-IIA } \\
\text { orlater }\end{array}$ & Complete & Bifacial & & & & 22 \\
\hline HK6.2011.2 & Donkey & HK6 & Excavated & Cemetery & $\begin{array}{l}\text { Shallow pit along } \\
\text { the NE of T49 }\end{array}$ & & Complete & Bifacial & & & & 23 \\
\hline HK.11.2000.1 & Quadruped & HK11 & Excavated & Settlement & Square G & NIC-IIC & Broken & Bifacial & & & & 24 \\
\hline HK.11.2000.2 & Dog & HK11 & Excavated & Settlement & $\begin{array}{l}\text { Square C4- trash } \\
\text { pit }\end{array}$ & & Broken & Ind. (bifacial?) & & & & 25 \\
\hline KAS.96.24.1 & $\begin{array}{l}\text { Serpent?/ } \\
\text { jewelery }\end{array}$ & $\begin{array}{l}\text { Kom el Ahmar } \\
\text { /Sharuna }\end{array}$ & Excavated & Settlement & & & Broken & Bifacial & 11 & \begin{tabular}{|r|}
$1.1 \mathrm{to}$ \\
2.2 \\
\end{tabular} & \begin{tabular}{|r|}
.6 to \\
.85 \\
\end{tabular} & 26 \\
\hline $\begin{array}{l}\text { MANStGL.56.53 } \\
2\end{array}$ & Dog (?) & Hierakonpolis & & & & & & & & & & 27 \\
\hline $\begin{array}{l}\text { MANStGL.71.08 } \\
6\end{array}$ & Human (?) & Kom Auchim & $?$ & & & & & & & & & 28 \\
\hline MrM.98.88 & Scorpion & & & & & & Complete & Bifacial & 11.9 & 3.5 & 0.8 & 29 \\
\hline OIC.E.7477 & Crocadile (?) & Abydos & Excavated & Settlement & $\begin{array}{l}\text { Settlement Osiris } \\
\text { temenos }\end{array}$ & NIII-D1 & Ind & Ind & & & & 30 \\
\hline OIM.E.10534 & Bovine(?) & & & & & & Complete & Edge (bifacial) & 6 & 3.75 & & 31 \\
\hline PAHMA.5.210 & $\begin{array}{l}\text { Hippo or } \\
\text { Elephant }\end{array}$ & & Purchased? & & & $\begin{array}{l}\text { Prob. } \\
\text { NI-IIA* }\end{array}$ & $\begin{array}{l}\text { Complete } \\
\text { (repaired) }\end{array}$ & Bifacial & & & & 32 \\
\hline PAHMA.5.211 & Hippo & & Purchased? & & & \begin{tabular}{|l|} 
Prob. \\
NI-IIA*
\end{tabular} & Complete & Bifacial & & & & 33 \\
\hline RMAH.E.6185A & Bovine head & Naqada & Excavated & Cemetery & Royal tomb & D1 & Broken & Bifacial & 17.1 & 10.4 & & 34 \\
\hline UC.15166 & Gazelle (?) & & & & & & Complete & Edge (bi or unifacial) & 7.2 & & & 35 \\
\hline
\end{tabular}


Table 6.1 (part 3). Figural eccentrics. Where the reduction type is indeterminate, it is often because photographs of one or both faces were not available. The main reference for each entry is the relevant online museum database. Additional sources are noted. See Table 7.1 for museum list and abbreviations.

\begin{tabular}{|c|c|c|c|c|c|c|c|c|c|c|c|c|}
\hline Item ID & Figure & Site & Acquisition & Context & Context Detail & Date & $\begin{array}{c}\text { Preserv- } \\
\text { ation }\end{array}$ & Reduction & \begin{tabular}{|c|} 
Length \\
$(\mathrm{cm})$
\end{tabular} & $\begin{array}{l}\text { Width } \\
(\mathrm{cm})\end{array}$ & \begin{tabular}{|l|} 
Thick \\
$(\mathrm{cm})$ \\
\end{tabular} & \begin{tabular}{|c|} 
Ref. \\
$\#$
\end{tabular} \\
\hline UC.15167 & $\begin{array}{l}\text { Dog (?)/ } \\
\text { Quadruped }\end{array}$ & & Purchased & & & & Complete & Edge (bi or unifacial) & 3.3 & & & 36 \\
\hline UC.15168 & Bird (flying) & & Purchased & & & & Complete & Edge (bi or unifacial) & 6.8 & & & 37 \\
\hline UC.15169 & Ind-Vulture (?) & & Purchased & & & & Complete & Edge (bi or unifacial) & 6.9 & & & 38 \\
\hline UC.15170 & Serpent & & Purchased & & & & Ind & Ind & 9.5 & & & 39 \\
\hline UC.15171 & Serpent & Coptos (?) & Purchased & & & & Broken & Ind & 8.2 & & & 40 \\
\hline UC.15172 & Ind-Turtle (?) & & Purchased & & & & Complete & Edge (bi or unifacial) & 6.6 & & & 41 \\
\hline UC.15173 & Ind & & & & & & Ind & Ind & & & & 42 \\
\hline UC.16780 & Hippo & Kahun & Excavated & Other & Lahun & $\begin{array}{l}\text { D } 12 \\
\text { (?) }\end{array}$ & Complete & Edge (bi or unifacial) & 5 & & & 43 \\
\hline UC.42705 A+B & Crocadile (?) & Abydos & Excavated & Settlement & Osiris 'temple' & & Ind & Edge (bi or unifacial) & 9.4 & 6 & 1 & 44 \\
\hline Unknown.1 & Crocadile (?) & Abydos & Excavated & Settlement & $\begin{array}{l}\text { Settlement Osiris } \\
\text { temenos }\end{array}$ & NIII-D1 & Complete & Edge (bi or unifacial) & & & & 45 \\
\hline Unknown.3 & Serpent (?) & Abydos & Excavated & Settlement & $\begin{array}{l}\text { Settlement Osiris } \\
\text { temenos }\end{array}$ & NIII-D1 & Broken & Ind & & & & 46 \\
\hline Unknown.4 & Bovine head (?) & Abydos & Excavated & Cemetery & $\begin{array}{l}\text { Umm el-Qa'ab- } \\
\text { Djer Tomb }\end{array}$ & D1 & Broken & Ind-probably bifacial & & & & 47 \\
\hline Unknown.5 & Serpent (?) & & & & & & Broken & Ind & & & & 48 \\
\hline Unknown.6 & Serpent (?) & & & & & & Broken & Ind & & & & 49 \\
\hline Unknown.8 & Ind & & & & & & Complete & Ind & & & & 50 \\
\hline Unknown.10 & Scorpion (?) & & & & & & Complete & Edge (bi or unifacial) & & & & 51 \\
\hline Unknown.12 & Crocadile & & & & & & Complete & Bifacial & 23 & 4.7 & & 52 \\
\hline Unknown.13 & Hippo & & & & & & Complete & Bifacial & 13 & 6 & & 53 \\
\hline
\end{tabular}




\section{References for Tables 6.1}

${ }_{1}^{1}$ Personal photograph

2 Personal photograph

${ }^{3}$ Personal photograph

${ }^{4}$ Hendrickx et al. 2003; Scharff 1929: 68, n 99 . (photo couleur: Tokyo 1988: 60, fig. 26, 1)

${ }^{5}$ Hendrickx et al. 2003; Scharff 1929: 67-68, $n^{\circ}$ 97. (photo couleur: Tokyo 1988: 60, fig. 26, 2)

${ }^{6}$ Hendrickx et al. 2003; Scharff 1929: 68, n 98 . (photo couleur: Tokyo 1988:

60, fig. 26, 3)

${ }^{7}$ Naville 1914. The cemeteries of Abydos I. 39, PI.XIV

8 Budge 1909: 148; Capart 1905: 153; Casini 1988: 129, fig. 92,5; Hendrickx et al. 2003

${ }^{9}$ Capart 1905: fig. 115, 32124; Casini 1988: 129, fig. 92,5; Hendrickx et al. 2003

${ }^{10}$ Budge 1909: 148; Capart 1905: fig. 115, A.294; Hendrickx et al. 2003; Petrie 1902: 12, 22 pl. XXVI, 294;

${ }^{11}$ Hendrickx et al. 2003; Needler 1984: $n^{\circ} 292$

${ }^{12}$ Casini 1988: 129, fig. 92,1; Hendrickx et al. 2003

${ }^{13}$ Capart 1905: 153; Hendrickx et al. 2003

${ }^{14}$ Adams 2000a: 70, n 83; Behrmann 1989: 16 b; Hendrickx et al. 2003

${ }^{15}$ Adams 2000b, 2001; Hendrickx et al. 2003

${ }^{16}$ Adams 2001: 6; Hendrickx et al. 2003

${ }^{17}$ Friedman 2005:4-6

${ }^{18}$ Friedman 2006:7-8, 2010:71

${ }^{19}$ Friedman 2006:7-8, 2010:71

${ }^{20}$ Friedman 2007:8

${ }^{21}$ Friedman et al. 2017

22 Friedman 2011a:4-6; Friedman et al. 2017 in press

${ }^{23}$ Droux 2011:16-17; Friedman et al. 2017 ; Nagaya 2011

${ }^{24}$ Friedman 2000b; Friedman et al. 2002; Hendrickx et al. 2003; Watrall 2000

${ }^{25}$ Friedman 2000; Hendrickx et al. 2003; Watrall 2000

${ }^{26}$ Pawlik 2006:557, fig 25

${ }^{27}$ Cleyet-Merle \& Vallet 1982: 126; Hendrickx et al. 2003
${ }^{28}$ Cleyet-Merle \& Vallet 1982: 92; Hendrickx et al. 2003

${ }^{29}$ Hendrickx et al. 2003

${ }^{30}$ Capart 1905: fig. 115, A.293; Hendrickx et al. 2003;

Petrie 1902: 12, pl. XXVI, 293

${ }^{31}$ oi.uchichcago.edu

${ }^{32}$ pahma.berkeley.edu

${ }^{33}$ Elsasser \& Fredrickson 1966 : 21; Behrman 1989: Dok. $16 a$

${ }^{34}$ Bavay \& Hendrickx 2000: 131; Hendrickx 2002: 284, fig. 16.3; Hendrickx et al. 2003

${ }^{35}$ Hendrickx et al. 2003; Petrie 1920: 11, pl. VTI, 1

${ }^{36}$ Capart 1905: fig. 115,19; Hendrickx et al. 2003; Petrie 1920: 10, pl. VII,2

${ }^{37}$ Capart 1905: fig. 115, 17; Hendrickx et al. 2003; Petrie 1920: 13, pl. VII,3

${ }^{38}$ Hendrickx et al. 2003; Petrie 1920: pl. VII, 4

39 Hendrickx et al. 2003; Petrie 1920: 13, pl. VII, 5

${ }^{40}$ Capart 1905: fig. 115, 18; Hendrickx et al. 2003; Petrie

1920: 13, pl. VII,6

${ }^{41}$ Hendrickx et al. 2003; Petrie 1920: pl. VII, 9

${ }^{42}$ Hendrickx et al. 2003; Petrie 1920: pl. VII, 10

${ }^{43}$ Capart 1905: fig. 115, K22; Hendrickx et al. 2003; Petrie et al. 1890: 30, pl. VIII,22

${ }^{44}$ Hendrickx et al. 2003

${ }^{45}$ Capart 1905: fig. 115, A.292; Hendrickx et al. 2003;

Petrie 1902: 12, pl. XXVI, 292

${ }^{46}$ Hendrickx et al. 2003; Petrie 1903: pl. X, 220

${ }^{47}$ Hendrickx et al. 2003; Petrie 1902: pl. XIV

${ }^{48}$ Hendrickx et al. 2003; Petrie 1920: pl. VII, 7

${ }^{49}$ Hendrickx et al. 2003; Petrie 1920: pl. VII, 8

${ }^{50}$ Hendrickx et al. 2003; Hoffman 1980a: 112, fig. 30, 1

${ }^{51}$ Hendrickx et al. 2003; Hoffman 1980a: 112, fig. 30, 1;

${ }^{52}$ Cahn 2005:4

53 Derricks 2012 
Table 6.2 (part 1). Figural eccentrics which are probably not authentic.

\begin{tabular}{|c|c|c|c|c|c|c|c|c|c|c|c|c|}
\hline Item ID & Figure & Site & Acquisition & Context & \begin{tabular}{|c|} 
Context \\
Detail \\
\end{tabular} & Date & $\begin{array}{c}\text { Preserv- } \\
\text { ation }\end{array}$ & Reduction & $\begin{array}{l}\text { Lengt } \\
\mathrm{h}(\mathrm{cm})\end{array}$ & $\begin{array}{l}\text { Width } \\
(\mathrm{cm})\end{array}$ & $\begin{array}{l}\text { Thick } \\
\text { (cm) }\end{array}$ & \\
\hline BM.EA.unknown & $\begin{array}{l}\text { Lizard/ } \\
\text { arrowhead }\end{array}$ & & & & & & Complete & Ind & & & & 53 \\
\hline BM.EA.65508 & Bird & & & & & & Complete & & 4 & 3 & & 54 \\
\hline BM.EA.65509 & Bird & & & & & & Complete & & 4.5 & 5 & & 55 \\
\hline CLMA.1995.40 & Bird & & & & & & Complete & Unifacial & 5.2 & 5.2 & & 56 \\
\hline FGC.2 & Bird & Hierakonpolis (?) & & & & & Complete & Edge (bi or unifacial) & & & & 57 \\
\hline FWM.E.GA.4240.1943 & Camel? & & & & & & Complete & Edge (bifacial) & & & & 58 \\
\hline FWM.E.GA.4304.1943 & Quadruped & & & & & & Complete & Edge (unifacial) & & & & 59 \\
\hline FWM.E.GA.4305.1943a & Ind. & & & & & & Complete & Edge (unifacial) & & & & 60 \\
\hline FWM.E.GA.4305.1943b & Quadruped & & & & & & Complete & Edge (unifacial) & & & & 61 \\
\hline FWM.E.GA.4306.1943 & Bird & & & & & & Complete & Edge (unifacial) & & & & 62 \\
\hline FWM.E.GA.4308.1943 & Bird & & & & & & Complete & Edge (unifacial) & & & & 63 \\
\hline FWM.E.GA.4309.1943 & Bird & & & & & & Complete & Edge (unifacial) & & & & 64 \\
\hline FWM.E.GA.4310.1943 & Bird & & & & & & Complete & Edge (unifacial) & & & & 65 \\
\hline FWM.E.GA.4311.1943 & Bird & & & & & & Complete & Edge (unifacial) & & & & 66 \\
\hline FWM.E.GA.4312.1943 & Bird & & & & & & Complete & Edge (unifacial) & & & & 67 \\
\hline FWM.4313.1943 & $\begin{array}{l}\text { Lizard/ } \\
\text { arrowhead }\end{array}$ & & & & & & Complete & Edge (unifacial) & 7.5 & & & 68 \\
\hline Gnefer.1 & Bird & & & & & & Complete & Edge (bi or unifacial) & & & & 69 \\
\hline Kassel & Bird & & & & & & Complete & Edge (bi or unifacial) & & & & 70 \\
\hline MbC.1 & Bird & & & & & & Complete & Edge (bi or unifacial) & & & & 71 \\
\hline MbC.2 & Bird & & & & & & Complete & Edge (bi or unifacial) & & & & 72 \\
\hline $\mathrm{MbC} .3$ & Bird & & & & & & Complete & Edge (bi or unifacial) & & & & 73 \\
\hline
\end{tabular}


Table 6.2 (part 2). Figural eccentrics which are probably not authentic. See Table 7.1 for museum list and abbreviations. Scharff (1929: 68, figure 47) reported that flaked-stone (bird) figures bought in Luxor in the 1920's were locally made fakes. The birds are all very uniform in shape and style, and are quite numerous, and these characteristics differ from the authentic eccentrics. The possible lizard from the Fitzwilliam museum, listed as a modern item, was obtained from the same collection as many of the birds, so may be from the same source. Given that, two other similar "lizard" figures may also be fake. The one from the British museum looks like it could be an arrowhead, but no direct parallels could be found. The only line of evidence indicating that some birds may not be fakes is the bird from the Nefer gallery, which in the photograph looks like it has significant wear over the retouch scars.

\begin{tabular}{|c|c|c|c|c|c|c|c|c|c|c|c|c|c|}
\hline Item ID & Figure & Site & Acquisition & Context & $\begin{array}{c}\text { Context } \\
\text { Detail }\end{array}$ & Date & \multicolumn{2}{|c|}{$\begin{array}{c}\text { Preserv- } \\
\text { ation }\end{array}$} & Reduction & \begin{tabular}{|c|}
$\begin{array}{c}\text { Length } \\
(\mathrm{cm})\end{array}$ \\
\end{tabular} & \begin{tabular}{|c|} 
Width \\
$(\mathrm{cm})$ \\
\end{tabular} & \begin{tabular}{|l|} 
Thick \\
$(\mathrm{cm})$
\end{tabular} & \begin{tabular}{|c|} 
Ref. \\
$\#$
\end{tabular} \\
\hline MdO.unknown & Bird & & & & & & \multicolumn{2}{|c|}{ Complete } & Edge (bi or unifacial) & & & & 74 \\
\hline MMA.26.2.148 & Bird & Dra Abu el-Naga & Purchased & & & NIII-Dyn 1 & \multicolumn{2}{|c|}{ Complete } & Unifacial & 6.9 & 5.2 & 1 & 75 \\
\hline MMA.26.2.153 & Bird & Dra Abu el-Naga & Purchased & & & NIII-Dyn 1 & \multicolumn{2}{|c|}{ Complete } & Unifacial & 7.4 & 6.1 & 1 & 76 \\
\hline MMA.26.2.256 & Bird & Dra Abu el-Naga & Purchased & & & NIII-Dyn 1 & \multicolumn{2}{|c|}{ Complete } & Unifacial & 4.4 & 3.9 & 0.9 & 77 \\
\hline Unknown.7 & Falcon (?) & & & & & & \multicolumn{2}{|c|}{ Complete } & Ind & & & & 78 \\
\hline Unknown.9 & Bird & & & & & & \multicolumn{2}{|c|}{ Complete } & Edge (bi or unifacial) & & & & 79 \\
\hline Unknown.11 & $\begin{array}{l}\text { Lizard / } \\
\text { arrowhead }\end{array}$ & & & & & & \multicolumn{2}{|c|}{ Complete } & Edge (bi or unifacial) & & & & 80 \\
\hline YPM.131127 & Bird & Coptos & & & & & \multicolumn{2}{|c|}{ Complete } & Edge (bi or unifacial) & & & & 81 \\
\hline YPM.7290.1 & Bird & Coptos & & & & & \multicolumn{2}{|c|}{ Complete } & Edge (bi or unifacial) & & & & 82 \\
\hline YPM.7290.2 & Bird & Coptos & & & & & \multicolumn{2}{|c|}{ Complete } & Edge (bi or unifacial) & & & & 83 \\
\hline YPM.7290.3 & Bird & Coptos & & & & & \multicolumn{2}{|c|}{ Complete } & Edge (bi or unifacial) & & & & 84 \\
\hline \multicolumn{3}{|c|}{ References for Table 6.2} & \multicolumn{10}{|c|}{ 63fitzmuseum.cam.ac.uk } & \\
\hline \multicolumn{3}{|c|}{53 Budge 1909: 148; Hendrickx et al. 2003} & \multicolumn{5}{|c|}{${ }^{64}$ fitzmuseum.cam.ac.uk } & \multicolumn{6}{|c|}{76 metmuseum.org } \\
\hline \multicolumn{3}{|c|}{54 britishmuseum.org } & \multicolumn{5}{|c|}{${ }^{65}$ fitzmuseum.cam.ac.uk } & \multicolumn{6}{|c|}{77 metmuseum.org } \\
\hline \multicolumn{3}{|c|}{55 britishmuseum.org } & \multicolumn{5}{|c|}{ 66fitzmuseum.cam.ac.uk } & \multicolumn{6}{|c|}{${ }^{78}$ Hendrickx et al. 2003; } \\
\hline \multicolumn{3}{|c|}{${ }^{56}$ Berman \& Bohac 1999: 120; } & \multicolumn{5}{|c|}{${ }^{67}$ fitzmuseum.cam.ac.uk } & \multicolumn{6}{|c|}{ Hoffman 1980: 112, fig. 30, 1} \\
\hline \multicolumn{3}{|c|}{ Hendrickx et al. 2003} & \multicolumn{5}{|c|}{68 fitzmuseum.cam.ac.uk } & \multicolumn{6}{|c|}{${ }^{79}$ Hendrickx et al. 2003; } \\
\hline \multicolumn{3}{|c|}{$\begin{array}{l}{ }^{57} \text { Ede 1999: } n^{\circ} 5 \text { b; Friedman 2000; } \\
\text { Hendrickx et al. } 2003\end{array}$} & ${ }^{69}$ Galerie Nef & fer 1990: $r$ & $\mathrm{n}^{\circ} 29 ; \mathrm{Her}$ & ndrickx et al & 2003 & & fman 1980: 112, fig. 30 & & & & \\
\hline $\begin{array}{l}\text { Hendrickx et al. } \\
\text { 58fitzmuseum.ca }\end{array}$ & & & $\begin{array}{l}{ }^{70} \text { Felgenhaue } \\
\text { Hendrickx et }\end{array}$ & $\begin{array}{l}\text { er 1996: } 7 \\
\text { al. } 2003\end{array}$ & $2-73, n^{\circ} 2$ & & & & $\begin{array}{l}\text { endrickx et al. 2003; } \\
\text { fman 1980: } 112, \text { fig. } 3\end{array}$ & & & & \\
\hline${ }^{59}$ fitzmuseum.ca & & & ${ }^{71}$ Hendrickx $€$ & et al. 2003 & 3; Walker & 1996: $55, \mathrm{n}$ & 79 a & & endrickx et al. 2003; Sc & ott 1986 & 6: $24-2$ & $5, n^{\circ} 2$ & \\
\hline 60fitzmuseum.ca & & & 72 Hendrickx $€$ & et al. 2003 & 3; Walker & 1996: $55, \mathrm{n}$ & $79 \mathrm{~b}$ & & endrickx et al. 2003; Sc & ott 1986 & 6: $24-2$ & $5, n^{\circ} 2$ & \\
\hline${ }^{61}$ fitzmuseum.ca & & & ${ }^{73}$ Hendrickx $\epsilon$ & et al. 2003 & 3; Walker & 1996: $55, \mathrm{n}$ & $79 \mathrm{c}$ & & eabody.yale.edu & & & & \\
\hline 62fitzmuseum.ca & & & ${ }^{74}$ Casini 1988 & 3: 130 , fig. & 93,$1 ; \mathrm{He}$ & endrickx et a & 2003 & ${ }^{84} \mathrm{pe}$ & eabody.yale.edu & & & & \\
\hline
\end{tabular}


Table 6.3. Comparison of figural eccentrics types to faunal remains in HK6, HK29A, and Mahâsna Block 3 (Anderson 2011; Friedman 2009b, 2011a:4-6 ; Linseele et al. 2009; Rossel 2007). • indicates that remains from that animal were present.

\begin{tabular}{|l|l|c|c|c|}
\hline \multicolumn{1}{|c|}{ Classification } & \multicolumn{1}{|c|}{ Figure } & HK6 & HK29A & $\begin{array}{c}\text { Mahasna } \\
\text { Block 3 }\end{array}$ \\
\hline Aquatic wild hunted & Crocadile & $\bullet$ & $\bullet$ & $\bullet$ \\
\hline Aquatic wild hunted & Hippo & $\bullet$ & $\bullet$ & $\bullet$ \\
\hline Aquatic wild hunted & Turtle? & 0 & $\bullet$ & $\bullet$ \\
\hline Aquatic wild hunted & Fish & $?$ & $\bullet$ & $\bullet$ \\
\hline & & & & \\
\hline & Antilope/ & & & \\
\hline Desert wild hunted & gazelle & & & $\bullet$ \\
\hline Desert wild hunted & Hartebeest & $\bullet$ & $\bullet$ & $\bullet ?$ \\
\hline Desert wild hunted & Barabry sheep & 0 & $\bullet$ & $\bullet$ \\
\hline Desert wild hunted & Elephant & $\bullet$ & 0 & 0 \\
\hline Desert wild hunted & Bird & $?$ & $\bullet$ & $\bullet$ \\
\hline & & & & \\
\hline Wild or domestic & Bovines & $\bullet$ & $\bullet$ & $\bullet$ \\
\hline Wild or domestic & Dog & $\bullet$ & $\bullet$ & $\bullet$ \\
\hline Wild or domestic & Donkey & $\bullet$ & $\bullet$ & 0 \\
\hline Wild or domestic & Ram & $($ sheep/goat $)$ & (sheep) & (sheep) \\
\hline & & & & \\
\hline Desert wild, not hunted & Giraffe & $\bullet$ & 0 & 0 \\
\hline Desert wild, not hunted & bex & & 0 & 0 \\
\hline Desert wild, not hunted & Serpent & & & \\
\hline Desert wild, not hunted & Scorpion & & & \\
\hline & & & & \\
\hline Human & Dwarf & & & $\bullet$ \\
\hline
\end{tabular}


Table 6.5. Percentages of tools and debitage categories at Naqada period Nile valley settlement sites. *Debris includes flake fragments, pieces less than $1.5 \mathrm{~cm}$ and angular debris. **Thinning flakes were present, but not counted as a separate category.

\begin{tabular}{|c|c|c|c|c|c|c|c|c|c|c|c|c|c|c|c|c|c|c|c|c|}
\hline Publication & $\frac{\Perp}{\frac{1}{I}}$ & $\begin{array}{l}\frac{0}{\square} \\
\frac{1}{I}\end{array}$ & 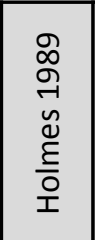 & 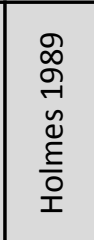 & 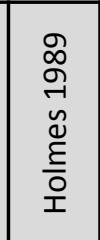 & 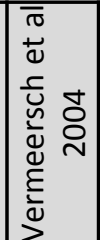 & 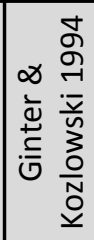 & 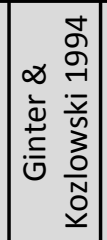 & 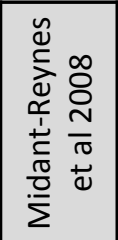 & 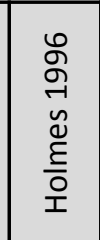 & 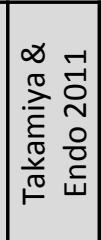 & 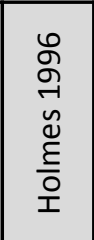 & 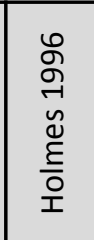 & 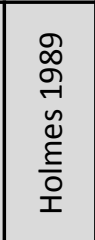 & 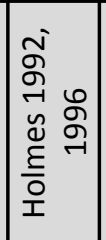 & 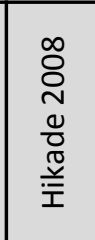 & 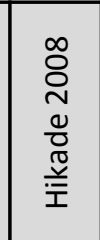 & 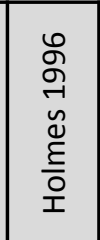 & 选 & $\frac{\Perp}{\frac{1}{I}}$ \\
\hline Site group & Mahâsna & Abydos & \multicolumn{4}{|c|}{ Naqada } & \multicolumn{2}{|c|}{ Armant } & Adaïma & \multicolumn{9}{|c|}{ Hierakonpolis } & \multicolumn{2}{|c|}{ Aswan } \\
\hline Site & $\begin{array}{l}\underset{\pi}{n} \\
\frac{\pi}{\sqrt{n}} \\
\frac{\pi}{\pi} \\
\sum \\
\sum\end{array}$ & 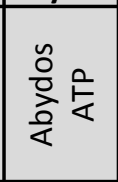 & 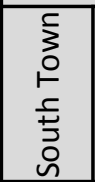 & $\begin{array}{l}\frac{\pi}{0} \\
\frac{0}{\sigma} \\
\frac{m}{I} \\
\frac{\pi}{z} \\
z\end{array}$ & $\begin{array}{l}\frac{\pi}{0} \\
\frac{\pi}{\pi} \\
\frac{\pi}{\pi} \\
z \\
z\end{array}$ & 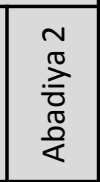 & 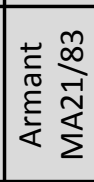 & 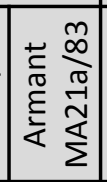 & $\frac{\mathbb{0}}{: \frac{\delta}{\pi}}$ & $\begin{array}{l}\cup \\
\stackrel{-}{\mid} \\
\stackrel{\vec{I}}{I}\end{array}$ & 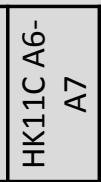 & $\stackrel{\vec{J}}{\stackrel{\vec{I}}{I}}$ & $\begin{array}{l}\stackrel{\nwarrow}{\mathbb{D}} \\
\text { 至 }\end{array}$ & 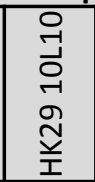 & 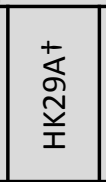 & $\begin{array}{l}\text { Фั } \\
\text { 폼 }\end{array}$ & 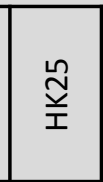 & $\begin{array}{l}\stackrel{0}{\stackrel{\overbrace{}}{\simeq}} \\
\text { 至 }\end{array}$ & 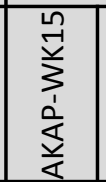 & 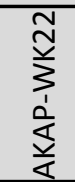 \\
\hline Time period & $\frac{\frac{u}{\dot{U}}}{z}$ & 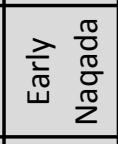 & $\begin{array}{l}\text { U } \\
0 \\
\text { \& } \\
\text { m }\end{array}$ & 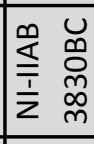 & $\frac{\infty}{\frac{\infty}{z}}$ & $\frac{\frac{m}{\dot{1}}}{\bar{z}}$ & $\overline{\bar{i}}$ & $\overline{\overline{1}}$ & $\begin{array}{l}\text { 呈 } \\
\bar{\sum} \\
\underline{u} \\
z\end{array}$ & 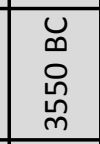 & 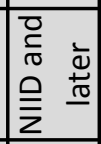 & 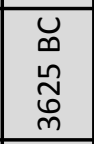 & 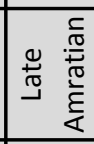 & 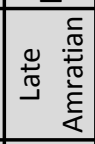 & 号 & 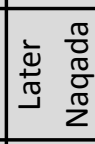 & 嘋 & 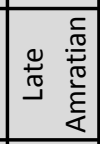 & $\begin{array}{l}\frac{m}{\vdots} \\
\bar{u} \\
\Sigma\end{array}$ & $\frac{m}{\grave{1}}$ \\
\hline Total \# artifacts & 6276 & 609 & 2206 & 5055 & 623 & 21090 & 11326 & 19831 & 12023 & 1190 & 3677 & 214 & 711 & 3557 & \begin{tabular}{|c|}
$\sim 54$, \\
000 \\
\end{tabular} & 10451 & 7481 & 183 & 2042 & 50 \\
\hline Total \#tools & 660 & 79 & 177 & 194 & 48 & 546 & 743 & 1206 & 826 & 131 & 307 & 16 & 35 & 468 & 1944 & 279 & 225 & 9 & 204 & 17 \\
\hline Tools & 10.53 & 12.97 & 8.02 & 2.91 & 7.7 & 2.5 & 6.56 & 6.13 & 6.85 & 11.01 & 8.35 & 7.48 & 4.92 & 13.16 & 3.6 & 2.67 & 3.01 & 4.92 & 9.99 & 34 \\
\hline Cores ( $\&$ core frags) & 3.57 & 1.97 & 1.04 & 0.57 & 1.12 & 0.88 & 3.27 & 1.35 & 2.94 & 1.01 & 1.06 & 3.74 & 0.42 & 1.07 & 0.3 & 1.21 & 1.98 & 1.09 & 1.57 & 2 \\
\hline Rejuvenation pieces & 0.88 & 1.81 & 0.54 & 0.1 & 0.16 & 0.18 & 0.53 & 0.11 & & 0 & & 0.47 & 0.14 & 0.31 & 0.1 & 0.06 & 0.05 & 0 & 0.78 & 2 \\
\hline Crested blades & 0.05 & 0.16 & & & & 0.05 & 0.36 & 0.17 & 0.25 & & & & & 0 & & 0.01 & 0.05 & & 0.05 & 0 \\
\hline Blades & 5.11 & 7.39 & 5.39 & 3.5 & 10.91 & 2.43 & 7.98 & 6.71 & 3.11 & 11.18 & 15.28 & 9.34 & 5.62 & 13.52 & 4.8 & 5.59 & 5.51 & 5.47 & 2.11 & 2 \\
\hline Flakes & 19.24 & 28.41 & 44.02 & 44.29 & 59.23 & 20.77 & 45.47 & 39.64 & 21.66 & 46.05 & 46.29 & 57.01 & 41.07 & 56.84 & 6.6 & 5.27 & 5.72 & 34.43 & 15.48 & 32 \\
\hline Burin spalls & 0.88 & 0.82 & & & & 1.36 & 0.5 & 0.87 & 0.53 & 1.01 & 0.41 & 0 & 0.84 & 2.78 & 0.5 & 0.96 & 0.31 & 1.09 & 1.47 & 2 \\
\hline Axe prep flakes & 0.02 & 0 & 0.14 & 0.26 & 0.16 & 0.21 & 0.31 & 0.57 & & & & & & 0 & & 0.03 & 0 & & 0 & 0 \\
\hline Thinning flakes & 1.79 & 4.6 & & & & $* *$ & 4.35 & 6.48 & 0.03 & 4.62 & 6.8 & 0 & 2.81 & & 15.4 & 8.66 & 5.91 & 2.19 & 1.76 & 0 \\
\hline Other debitage & 10.29 & 12.15 & 0.05 & 0.45 & 1.28 & 5.24 & 1.3 & 0.77 & 9.64 & & 1.39 & & & 2.98 & & & & & 9.41 & 12 \\
\hline Debris* & 47.65 & 29.72 & 40.8 & 47.91 & 19.42 & 63.77 & 29.36 & 37.21 & 54.99 & 25.12 & 20.42 & 21.96 & 44.17 & 9.33 & 68.6 & 75.53 & 77.41 & 50.82 & 57.4 & 14 \\
\hline TOTAL & 100.01 & 100 & 100 & 99.99 & 99.98 & 97.39 & 99.99 & 100.01 & 100 & 100 & 100 & 100 & 99.99 & 99.99 & 99.9 & 99.99 & 99.95 & 100.01 & 100.02 & 100 \\
\hline
\end{tabular}


Table 6.6. Counts of bifacial tool types at Naqada period Nile valley settlement sites. $\bullet=$ Present. ${ }^{*}$ Including preforms $\&$ choppers. + The bifacial tools were not specifically identified in the article, 3 could be identified from drawings, the rest were put in this category. $\uparrow+$ This total does not include piece esquilles,

\begin{tabular}{|c|c|c|c|c|c|c|c|c|c|c|c|c|c|c|c|c|c|c|c|c|}
\hline Publication & $\frac{\varrho}{\frac{0}{I}}$ & 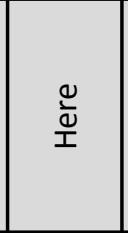 & $\begin{array}{l}\text { o } \\
\text { ने } \\
\breve{u} \\
\stackrel{\mathscr{\varepsilon}}{0} \\
\text { ำ }\end{array}$ & 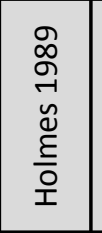 & 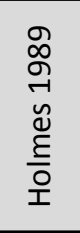 & 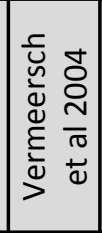 & 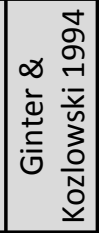 & 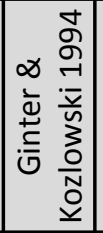 & 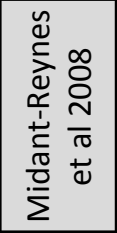 & 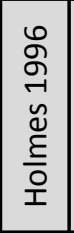 & 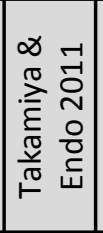 & 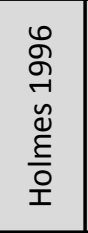 & 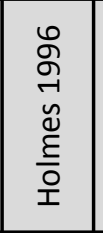 & 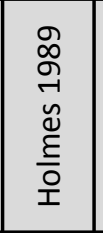 & 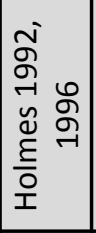 & 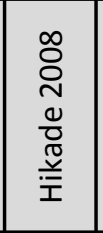 & 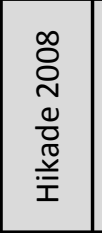 & 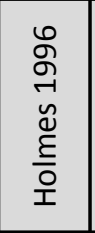 & $\begin{array}{l}\frac{0}{\dddot{U}} \\
\frac{1}{1}\end{array}$ & $\frac{\dddot{\Xi}}{\dddot{亠}}$ \\
\hline Site group & Mahâsna & Abydos & \multicolumn{4}{|c|}{ Naqada } & \multicolumn{2}{|c|}{\begin{tabular}{|l|} 
Armant \\
\end{tabular}} & Adaïma & \multicolumn{9}{|c|}{ Hierakonpolis } & \multicolumn{2}{|c|}{ Aswan } \\
\hline Site & 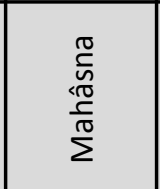 & 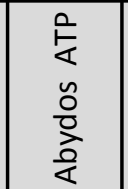 & 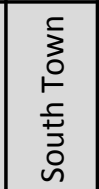 & $\begin{array}{ll}\frac{\pi}{0} & \infty \\
\frac{\pi}{0} & \frac{m}{T} \\
\frac{\pi}{2} & \frac{1}{2} \\
\frac{1}{2} & \end{array}$ & 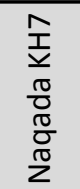 & 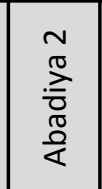 & 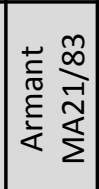 & 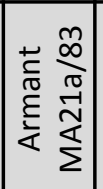 & $\frac{\frac{\pi}{5}}{\frac{\pi}{\pi}}$ & 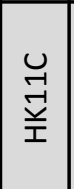 & 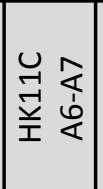 & $\underset{⿱}{\vec{I}}$ & 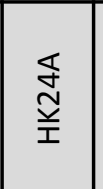 & 空 & $\begin{array}{l}\text { 晜 } \\
\text { 竎 }\end{array}$ & $\begin{array}{l}\text { Фิ } \\
\text { ָे }\end{array}$ & 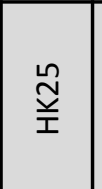 & 怘 & 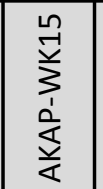 & 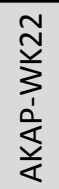 \\
\hline Time period & $\frac{\frac{u}{\bar{U}}}{\frac{u}{z}}$ & 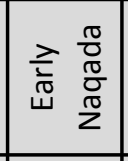 & $\begin{array}{l}\text { O } \\
\text { O } \\
\text { m }\end{array}$ & $\begin{array}{cc}\infty & u \\
\vdots & 0 \\
\overline{1} & 0 \\
\bar{z} & 0 \\
m\end{array}$ & $\frac{\infty}{\frac{1}{\Sigma}}$ & $\frac{\frac{m}{1}}{\frac{1}{z}}$ & $\overline{\bar{z}}$ & $\overline{\overline{\bar{z}}}$ & $\begin{array}{l}\sum_{\overline{\bar{j}}} \\
\frac{\dot{U}}{z}\end{array}$ & $\begin{array}{l}u \\
\infty \\
\text { on } \\
\text { ñ } \\
m\end{array}$ & 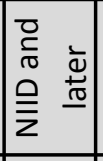 & $\begin{array}{l}u \\
\infty \\
\tilde{\omega} \\
0 \\
n\end{array}$ & 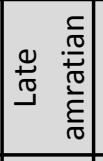 & 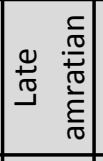 & 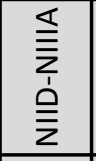 & 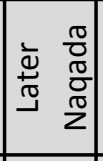 & $\frac{\text { Ù }}{\bar{z}}$ & 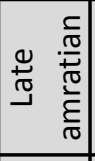 & $\frac{\stackrel{\infty}{\leftrightarrows}}{\overline{\bar{u}}}$ & 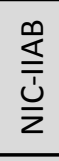 \\
\hline Total \# aftifacts & 6276 & 609 & 2206 & 5055 & 623 & 21090 & 11326 & 19831 & 12023 & \begin{tabular}{|c|}
119 \\
0 \\
\end{tabular} & 3677 & 214 & 711 & 3557 & \begin{tabular}{|c|}
54, \\
000 \\
\end{tabular} & 10451 & 7481 & 183 & 2042 & 50 \\
\hline Total \#tools & 660 & 79 & 177 & 194 & 48 & 546 & 743 & 1206 & 826 & 131 & 307 & 16 & 35 & 468 & ح1944 & 279 & 225 & 9 & 204 & 17 \\
\hline RFK & 0 & 0 & 0 & 0 & 0 & 0 & \begin{tabular}{|l|}
0 \\
\end{tabular} & \begin{tabular}{|l|}
0 \\
\end{tabular} & 0 & \begin{tabular}{|l|}
0 \\
\end{tabular} & 0 & 0 & 0 & \begin{tabular}{l|l|}
0 \\
\end{tabular} & & 0 & 0 & 0 & 0 & 0 \\
\hline Rhomboid & 0 & 0 & 0 & 0 & 0 & 0 & 0 & 0 & 0 & 0 & 0 & 0 & 0 & 0 & & 0 & 0 & 0 & 0 & 0 \\
\hline Fishtail & 0 & 0 & 0 & 0 & 0 & 0 & 0 & 0 & 0 & 0 & 0 & 0 & 0 & 0 & & 0 & 0 & 0 & 0 & 0 \\
\hline Concave-Base PP & 2 & 0 & 1 & 0 & 0 & 0 & 0 & 1 & 1 & 0 & 0 & 0 & 0 & 0 & $\bullet$ & 0 & 0 & 0 & 0 & 0 \\
\hline Eccentric & 0 & 0 & 0 & 0 & 0 & 0 & 0 & 0 & 0 & 0 & 0 & 0 & 0 & 0 & & 0 & 0 & 0 & 0 & 0 \\
\hline Other Fine Bifacials & 1 & 0 & 1 & 3 & 1 & 0 & 0 & 0 & 0 & 2 & 3 & 0 & 0 & 2 & - & 0 & 0 & 0 & 0 & 0 \\
\hline Bifacial Knife & 1 & 0 & 2 & 0 & 0 & 0 & 1 & 0 & 14 & 0 & 0 & 0 & 0 & 1 & - & 0 & 0 & 1 & 1 & 0 \\
\hline Bifacial Sickle & 0 & 0 & 0 & 0 & 0 & 2 & 3 & 1 & 3 & 0 & 0 & 0 & 0 & 0 & & 0 & 0 & 0 & 1 & 0 \\
\hline Bifacial Plane & 1 & 0 & 0 & 0 & 0 & 0 & 0 & 0 & 0 & 0 & 0 & 0 & 0 & 0 & & 0 & 0 & 0 & 0 & 0 \\
\hline Axe & 3 & 0 & 0 & 7 & 1 & 40 & 22 & 25 & 0 & 0 & 0 & 0 & 0 & 0 & & 0 & 0 & 0 & 0 & 0 \\
\hline $\begin{array}{l}\text { Drills: winged/ } \\
\text { crescent/cylindrical }\end{array}$ & 0 & 0 & 0 & 1 & 0 & 0 & 0 & 0 & 0 & 0 & 3 & 0 & 0 & 14 & - & 0 & 0 & 0 & 0 & 0 \\
\hline Pic/Grand Perçoir & 0 & 0 & 0 & 1 & 0 & 1 & 12 & 13 & 0 & 0 & 0 & 0 & 0 & 0 & & 0 & 0 & 0 & 0 & 0 \\
\hline $\begin{array}{l}\text { Non-standard, \& } \\
\text { Unidentifiable* }\end{array}$ & 16 & 1 & 4 & 15 & 2 & 13 & 54 & 43 & 1 & 1 & $7+$ & 0 & 1 & 18 & - & 12 & 13 & 0 & 0 & 0 \\
\hline Axe Preform & 0 & 0 & 0 & 0 & 0 & 0 & 22 & 9 & 0 & 0 & 0 & 0 & 0 & 0 & & 0 & 0 & 0 & 0 & 0 \\
\hline Total \# Bifacial Tools & 24 & 1 & 8 & 27 & 4 & 56 & 92 & 83 & 19 & 3 & 13 & 0 & 1 & 35 & ? & 12 & 13 & 1 & $2++$ & 0 \\
\hline
\end{tabular}


Table 6.7. Percentages of bifacial tool types at Naqada period Nile valley settlement sites. Sample sizes smaller than 30 are grayed-out. See Table 6.6 for references, specific counts, and notes.

\begin{tabular}{|c|c|c|c|c|c|c|c|c|c|c|c|c|c|c|c|c|c|c|c|c|}
\hline Site group & Mahâsna & Abydos & & $\mathrm{Naq}$ & ada & & Arm & nant & Adaïma & & & & Hie & erakon & polis & & & & Asv & \\
\hline Site & $\begin{array}{l}\frac{\pi}{5} \\
\frac{n}{\pi} \\
\frac{1}{\pi} \\
\frac{\pi}{\pi} \\
\sum\end{array}$ & 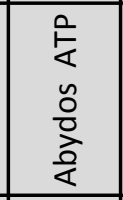 & 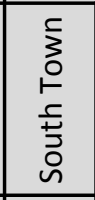 & $\begin{array}{l}\frac{\pi}{\delta} \\
\frac{0}{\sigma} \\
\frac{m}{I} \\
\frac{\pi}{z}\end{array}$ & 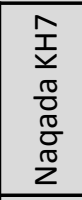 & 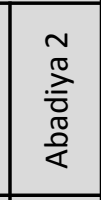 & 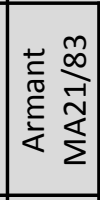 & 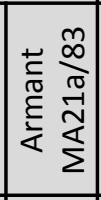 & $\frac{\overparen{\pi}}{: \frac{\delta}{\pi}}$ & $\begin{array}{l}\cup \\
\underset{ }{ت} \\
\text { I }\end{array}$ & 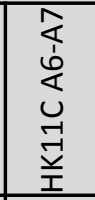 & $\underset{⿱ 亠 䒑 十}{\stackrel{⿱ 亠 乂}{I}}$ & 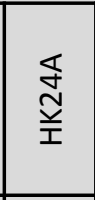 & 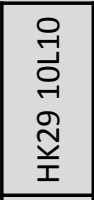 & 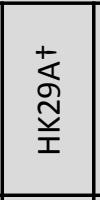 & $\begin{array}{l}\text { Фิ } \\
\text { ㅍ }\end{array}$ & 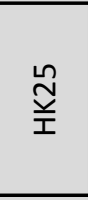 & 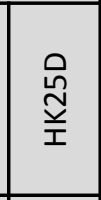 & 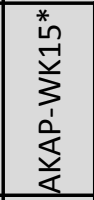 & $\begin{array}{l}\text { Nָ} \\
\stackrel{3}{3} \\
\vdots \\
\frac{1}{4} \\
\frac{1}{4}\end{array}$ \\
\hline Time period & $\frac{\underline{\bar{u}}}{z}$ & 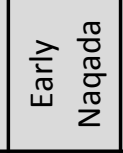 & $\begin{array}{l}\text { U } \\
0 \\
\stackrel{+}{+} \\
m\end{array}$ & 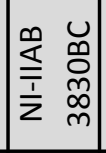 & $\frac{\infty}{\overline{\frac{1}{z}}}$ & $\frac{\frac{m}{1}}{\sum}$ & $\frac{\overline{\overline{1}}}{\bar{z}}$ & $\frac{\overline{\overline{1}}}{\bar{z}}$ & $\begin{array}{l}\text { 을 } \\
\text { 定 } \\
z\end{array}$ & $\begin{array}{l}\text { U } \\
\text { O } \\
\text { ํㅓ } \\
\text { ஸn }\end{array}$ & 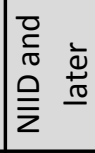 & 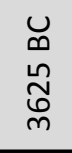 & 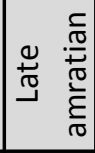 & 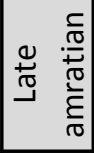 & 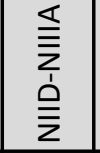 & 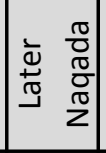 & $\frac{\text { Ù }}{\bar{z}}$ & 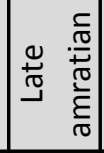 & $\frac{m}{\frac{\underline{U}}{z}}$ & $\frac{\varrho}{\frac{\varrho}{u}}$ \\
\hline Total \# artifacts & 6276 & 609 & 2206 & 5055 & 623 & 21090 & 11326 & 19831 & 12023 & $\begin{array}{c}119 \\
0\end{array}$ & 3677 & 214 & 711 & 3557 & $\begin{array}{c}\sim 5400 \\
0\end{array}$ & 10451 & 7481 & 183 & 2042 & 50 \\
\hline Total \#tools & 660 & 79 & 177 & 194 & 48 & 546 & 743 & 1206 & 826 & 131 & 307 & 16 & 35 & 468 & 1944 & 279 & 225 & 9 & 204 & 17 \\
\hline RFK & 0 & 0 & 0 & 0 & 0 & 0 & 0 & 0 & 0 & 0 & 0 & 0 & 0 & 0 & 0 & 0 & 0 & 0 & 0 & 0 \\
\hline Rhomboid & 0 & 0 & 0 & 0 & 0 & 0 & 0 & 0 & 0 & 0 & 0 & 0 & 0 & 0 & 0 & 0 & 0 & 0 & 0 & 0 \\
\hline Fishtail & 0 & 0 & 0 & 0 & 0 & 0 & 0 & 0 & 0 & 0 & 0 & 0 & 0 & 0 & 0 & 0 & 0 & 0 & 0 & 0 \\
\hline Concave-Base PP & 0.3 & 0 & 0.56 & 0 & 0 & 0 & 0 & 0.08 & 0.12 & 0 & 0 & 0 & 0 & 0 & 0.3 & 0 & 0 & 0 & 0 & 0 \\
\hline Eccentric & 0 & 0 & 0 & 0 & 0 & 0 & 0 & 0 & 0 & 0 & 0 & 0 & 0 & 0 & 0 & 0 & 0 & 0 & 0 & 0 \\
\hline Other Fine Bifacials & 0.15 & 0 & 0.56 & 1.55 & 2.08 & 0 & 0 & 0 & 0 & $\begin{array}{c}1.5 \\
3 \\
\end{array}$ & 0.98 & 0 & 0 & 0.43 & 1.1 & 0 & 0 & 0 & 0 & 0 \\
\hline Bifacial Knife & 0.15 & 0 & 1.13 & 0 & 0 & 0 & 0.13 & 0 & 1.69 & 0 & 0 & 0 & 0 & 0.21 & 0 & 0 & 0 & 11.11 & 0.49 & 0 \\
\hline Bifacial Sickle & 0 & 0 & 0 & 0 & 0 & 0.37 & 0.4 & 0.08 & 0.36 & 0 & 0 & 0 & 0 & 0 & 0 & 0 & 0 & 0 & 0.49 & 0 \\
\hline Bifacial Plane & 0.15 & 0 & 0 & 0 & 0 & 0 & 0 & 0 & 0 & 0 & 0 & 0 & 0 & 0 & 0 & 0 & 0 & 0 & 0 & 0 \\
\hline Axe & 0.45 & 0 & 0 & 3.61 & 2.08 & 7.33 & 2.96 & 2.07 & 0 & 0 & 0 & 0 & 0 & 0 & 0 & 0 & 0 & 0 & 0 & 0 \\
\hline $\begin{array}{l}\text { Drills: winged/ } \\
\text { crescent/cylindrical }\end{array}$ & 0 & 0 & 0 & 0.52 & 0 & 0 & 0 & 0 & 0 & 0 & 0.98 & 0 & 0 & 2.99 & 0.1 & 0 & 0 & 0 & 0 & 0 \\
\hline Pic/Grand Perçoir & 0 & 0 & 0 & 0.52 & 0 & 0.18 & 1.62 & 1.08 & 0 & 0 & 0 & 0 & 0 & 0 & 0 & 0 & 0 & 0 & 0 & 0 \\
\hline $\begin{array}{l}\text { Non-standard, \& } \\
\text { Unidentifiable }\end{array}$ & 2.42 & 1.27 & 2.26 & 7.73 & 4.17 & 2.38 & 7.27 & 3.57 & 0.12 & $\begin{array}{c}0.7 \\
6 \\
\end{array}$ & 2.28 & 0 & 2.86 & 3.85 & 0.5 & 4.3 & 5.78 & 0 & 0 & 0 \\
\hline Axe Preform & 0 & 0 & 0 & 0 & 0 & 0 & 2.96 & 0.75 & 0 & 0 & 0 & 0 & 0 & 0 & 0 & 0 & 0 & 0 & 0 & 0 \\
\hline Total Bifacial Tools (\%) & 3.62 & 1.27 & 4.51 & 13.93 & 8.33 & 10.26 & 12.38 & 6.88 & 2.29 & $\begin{array}{c}2.2 \\
9\end{array}$ & 4.24 & 0 & 2.86 & 7.48 & 2 & 4.3 & 5.78 & 11.11 & 0.98 & 0 \\
\hline
\end{tabular}


Table 6.8. Counts of bifacial tool types found in other related areas of the sites studies here, but not from the above statistically comparable samples. -=Present. *Spurrell (1896) described one of these with material from the cemeteries, but Holmes (1989) thinks it is from the settlement. **Harlan (1985) reported 32 bifacial tools but did not give specific types.

\begin{tabular}{|c|c|c|c|c|c|c|c|c|c|c|c|c|c|c|c|c|c|c|c|c|c|c|}
\hline Site group & & Mahas & & Abydos & & & Naqa & & & & & daïn & & & & & erak & onpoli & & & & Aswan \\
\hline Site & $\stackrel{1}{\frac{1}{2}}$ & 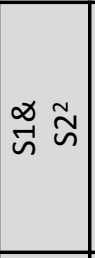 & 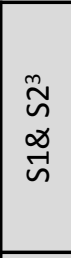 & $\begin{array}{l}\text { 足 } \\
\frac{1}{4} \\
n \\
0 \\
\frac{0}{0} \\
\frac{0}{4}\end{array}$ & $\begin{array}{l}\sum_{3}^{n} \\
0 \\
+1 \\
\frac{1}{1} \\
0 \\
Z\end{array}$ & 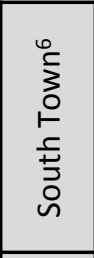 & $\stackrel{\stackrel{n}{Y}}{\underset{y}{\underline{Y}}}$ & $\begin{array}{l}\stackrel{n}{\stackrel{1}{T}} \\
\stackrel{T}{\underline{y}}\end{array}$ & $\begin{array}{l}\hat{m} \\
\stackrel{m}{I}\end{array}$ & $\begin{array}{l}\frac{n}{x} \\
x \\
m \\
\frac{m}{y}\end{array}$ & $\begin{array}{l}\stackrel{\infty}{0} \\
: \frac{E}{\pi} \\
\frac{\pi}{0}\end{array}$ & 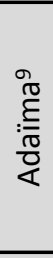 & 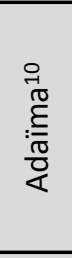 & $\begin{array}{l}\vec{\beth} \\
\stackrel{-}{-1} \\
\stackrel{\vec{I}}{I}\end{array}$ & $\begin{array}{l}\underset{7}{7} \\
\overrightarrow{-} \\
\underset{I}{I}\end{array}$ & $\begin{array}{l}m \\
\text { in } \\
0 \\
0 \\
\dot{y} \\
\infty \\
u \\
-1 \\
ت \vec{y} \\
\text { İ }\end{array}$ & 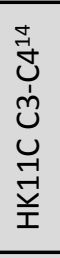 & 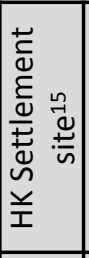 & 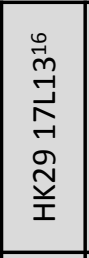 & 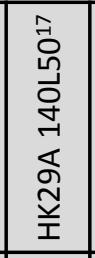 & 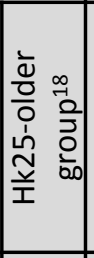 & 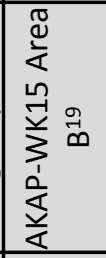 \\
\hline $\begin{array}{c}\text { Total \# of Lithic } \\
\text { artifacts }\end{array}$ & 23 & 44 & $\mathrm{n} / \mathrm{a}$ & 74 & 1031 & $124+$ & 6342 & $?$ & $?$ & $5388+$ & $?$ & $?$ & 106 & 8793 & 2601 & 1714 & $?$ & 61 & 2758 & 9000 & ? & 2595 \\
\hline total \# of tools & 22 & 42 & $\mathrm{n} / \mathrm{a}$ & 28 & 59 & $117+$ & 178 & $?$ & ? & 407 & $?$ & ? & 106 & $?$ & 287 & 226 & 48 & 61 & 240 & $?$ & 776 & $\begin{array}{l}\sim 155 \\
(6 \%)\end{array}$ \\
\hline RFK & 0 & 0 & 0 & 0 & 0 & 0 & 0 & & & 0 & 0 & 0 & 0 & & 0 & 0 & & 0 & 0 & & & \\
\hline Rhomboid & 0 & 0 & 0 & 0 & 0 & 0 & 0 & & & 0 & 0 & 0 & 0 & & 0 & 0 & & 2 & 0 & & 3 & \\
\hline Fishtail & 1 & 0 & 1 & 0 & 0 & 0 & 0 & & & 0 & 1 & 0 & 1 & & 0 & 0 & & 0 & 0 & & 24 & \\
\hline Concave-Base PP & 1 & 0 & 3 & 1 & 0 & 3* & 1 & $\bullet$ & $\bullet$ & 0 & 0 & 0 & 0 & & 0 & 0 & & 5 & 0 & & 15 & \\
\hline Eccentric & 0 & 0 & 0 & 0 & 0 & 0 & 0 & & & 0 & 0 & 0 & 0 & & 2 & 0 & & 1 & 0 & & & \\
\hline Other Fine Bifacials & 0 & 3 & 0 & 1 & 0 & 3 & 5 & & 5 & 0 & 0 & 0 & 1 & & 0 & 0 & & 0 & 0 & & & \\
\hline Bifacial Knife & 1 & 1 & 3 & 0 & 0 & 8 & 0 & & & 0 & 2 & 6 & 4 & & 6 & 4 & & 5 & 0 & 1 & 17 & \\
\hline Bifacial Sickle & 1 & 0 & 1 & 3 & 0 & 1 & 0 & & & 0 & 4 & 3 & 4 & & 0 & 0 & & 0 & 0 & & & \\
\hline Bifacial Plane & 0 & 0 & 0 & 0 & 0 & 1 & 0 & & & 1 & 1 & 0 & 0 & & 0 & 0 & & 0 & 0 & & & \\
\hline Axe & 0 & 12 & 6 & 0 & 4 & 29 & 12 & & 1 & 26 & 2 & 0 & 1 & & 0 & 0 & & 11 & 0 & & & \\
\hline $\begin{array}{l}\text { Drills: winged/ } \\
\text { crescent/cylindrical }\end{array}$ & 0 & 0 & 0 & 0 & 0 & 1 & 0 & & & 0 & 1 & 0 & 0 & & 0 & 0 & & 4 & 4 & & & \\
\hline Pic/Grand Perçoir & 0 & 0 & 0 & 0 & 0 & 0 & 2 & & & 2 & 0 & 0 & 0 & & 0 & 0 & & 0 & 0 & & & \\
\hline $\begin{array}{l}\text { Non-standard, \& } \\
\text { Unidentifiable }\end{array}$ & 1 & 0 & 6 & 1 & 2 & 5 & 7 & & & 22 & 2 & 0 & 1 & $32 * *$ & 0 & 0 & $\bullet$ & 2 & 3 & & & 1 \\
\hline $\begin{array}{l}\text { Unfinished Concave- } \\
\text { Base PP }\end{array}$ & 0 & 0 & 0 & 0 & 0 & 0 & 3 & & & 0 & 0 & 0 & 0 & & 0 & 0 & & 0 & 0 & & & \\
\hline Unfinished Knife & 0 & 1 & 0 & 0 & 0 & 0 & 0 & & & 0 & 0 & 0 & 0 & & 0 & 0 & & 0 & 0 & & & \\
\hline $\begin{array}{l}\text { Preform/ Unfinished } \\
\text { Axe }\end{array}$ & 0 & 0 & 0 & 0 & 0 & 1 & 0 & & & 0 & 0 & 0 & 0 & & 0 & 0 & & 0 & 0 & & & \\
\hline Axe Prep. Flake & 0 & 0 & 0 & 0 & 0 & 0 & 1 & & & 2 & & & & & & 0 & & 0 & 0 & & & 1 \\
\hline
\end{tabular}




\section{References for Table 6.8}

${ }^{1}$ Anderson's (2006) excavations: surface, blocks $2 \& 5$. Materials analyzed as part of this dissertation research.

2University of Pennsylvania Museum collection, from Garstang's (1903) excavations.

${ }^{3}$ Artifacts reported in Garstang (1903) but not found in the University of Pennsylvania Museum collection.

${ }^{4}$ Harvey (1998) excavations: Material recovered from other Ops besides $8 \& 19$. Materials analyzed as part of this dissertation research.

5 Prehistoric of Naqada Project (Holmes 1989:201-258).

${ }^{6}$ Petrie's South Town excavations (Baumgartel 1960:28, PI1; Holmes 1989:258-276; Petrie \& Quibell 1896:PL LXXI).

7Prehistoric of Naqada Project. Material from other areas of KH3 besides area B or X/XI (Holmes 1989:201-258).

8 Material from excavations by Midant-Reynes and others, from other parts of the settlement, outside of block 1001 mainly surface (Midant-Reynes \& Prost 2002:336-369).

${ }^{9}$ Material in the St-Germain-en-Laye Museum collection, mainly excavated by Morgan, and reported in Midant-Reynes \& Prost (2002:336-369).

${ }^{10}$ Brooklyn Museum Collection, from Morgan's excavations (Midant-Reynes \& Prost 2002:336-369; Needler 1984:83-87).

${ }^{11}$ Middens, kiln, and domestic areas of Hk11 (Harlan 1985: 103-104, 269).

12 Watrall's excavations in HK11 Square G (Friedman et al. 2002; Watrall 2000:12; Watrall 2001:8-9).

${ }^{13} 2008$ \& 2009 excavation material analyzed by Nagaya (Friedman et al. 2011:148-149).

142013 excavations (Nagaya 2013:14-15).

15 Brooklyn Museum collection from Morgan's excavations (Needler 1984).

16 House remains excavated by Hoffman, and analyzed by Holmes (1996).

${ }^{17}$ Material from 1986 excavations, find 4, analyzed by Takamiya \& Endo (2008:7-8).

${ }^{18}$ Friedman \& Nagaya's (2013:21-22) re-analysis of the older burnt bifacial group of material from HK25.

19 Usai's analysis of material from WK 15, Area B, in Gatto et al. (2009b:201-203); Usai report 2012.

\section{References for Table 6.9}

${ }^{1}$ Ayrton \& Loat 1911

${ }^{2}$ Garstang 1903

${ }^{3}$ Naville 1914; Peet 1914

${ }^{4}$ Hikade 1998, 2000; Date \& grave count: Hartmann 2011

${ }^{5}$ Baumgartel 1970; Hendrickx 2002:283-284; Holmes 1989:274, 276-280; Petrie \& Quibell plates LXXII; LXXIII; LXXIV; LXXVI; Spurrel 1896:56; Dates: Hendrickx \& van den Brink 2002:360

${ }^{6}$ Mond \& Meyers 1937; Dates: Hendrickx \& Van den Brink 2002:361

${ }^{7}$ Crubezy et al. 2002; Midant-Reynes \& Prost 2002:353-354

${ }^{8}$ Friedman 2003:18-19, 2004a:5, 2004b:8-9; Friedman et al. 1999.
${ }^{9}$ Adams 2000a:24,26-27,54,60,70,72,83-93,148,265,269,270,299-301photo268,drawings-299,300,PL- XXXa\&b, Fig 13, Fig 14, Adams 2000b:5, Adams 2001:5-6; Droux 2008:20, Droux 2011:16-17; Droux \& Friedman 2014: 6, 2007: 7-9; Friedman 2000b:14, 2005:4-6, 2009:7, 2010:68-71, 2011a:4-6, 2011b:13, 2012:6-7 , 2013:7,16; Friedman et al. 2008:90-91; Friedman et al. 2009: 194; Friedman et al. 2011:159-160, 168-169; Friedman et al. 2011:120, 134; Friedman et al. 2011:143,145; McNamara \& Droux 2006:9-10, 16; Nekhen News inset box 2012:7; Van Neer \& Linselle 2008:12-13 
Table 6.9. Counts of bifacial tool types found cemeteries associated with the sites studied here. References on previous page. Note that lithic artifacts were collected from the Nag el-Qarmila cemetery, but they have not been analyzed. No comment or description was given in the excavation notes so it is unlikely that they are bifacial tools (personal observation; Gatto, pers. comm.). *Including preforms \& choppers. **This RFK, sold to Morgan, probably came from the cemetery (Needler 1984:271). †Painted tomb cemetery (Quibell and Green 1902:48, PI60, 17).

\begin{tabular}{|c|c|c|c|c|c|c|c|c|c|}
\hline Site group & \multicolumn{2}{|c|}{ el-Mahâsna } & \multicolumn{2}{|c|}{ Abydos } & \multirow{2}{*}{\begin{tabular}{|c|} 
Naqada \\
Naqada- \\
main, B \& $\mathrm{T}^{5}$
\end{tabular}} & \multirow{2}{*}{\begin{tabular}{c|} 
Armant \\
Armant- 1200, \\
$1300,1400-1500^{6}$
\end{tabular}} & \multirow{2}{*}{$\begin{array}{c}\text { Adaïma- } \\
\text { Adaïma- East , } \\
\text { West } \\
\text { cemeteries }^{7}\end{array}$} & \multicolumn{2}{|c|}{ Hierakonpolis } \\
\hline Site & $\begin{array}{c}\text { Mahâsna } \\
\text { Cemetery } \mathrm{H}^{1}\end{array}$ & $\begin{array}{c}\text { Mahâsna- } \\
\text { Alawaniyeh }^{2}\end{array}$ & $\begin{array}{c}\text { Abydos- } \\
\text { cemetery } E^{3}\end{array}$ & \begin{tabular}{|c|} 
Abydos- \\
Cemetery $U^{4}$
\end{tabular} & & & & HK438 & HK6 9 \\
\hline Date & $|A-||| B$ & $\begin{array}{l}\text { SD 36-38/ } \\
\text { NIC-II }\end{array}$ & Predynastic & NIA-IID2; NIII & NIA-IIID1 & $\begin{array}{c}\text { NIC-NIIIA1; NIIIC- } \\
\text { NIIID }\end{array}$ & NIC-NIIIA1 & NIIA-C & $\begin{array}{l}\text { NIC-IIB; } \\
\text { NIIIA-C }\end{array}$ \\
\hline $\begin{array}{l}\text { \# of total graves / } \\
\text { \# of graves described }\end{array}$ & 600/ 135 & $45 / 20$ & $\sim 200 / 36$ & $\sim 600 /$ & $\begin{array}{l}\sim 2000 / \\
\sim 1300\end{array}$ & $? / 210$ & $211+/ 205$ & $462+/$ & $\begin{array}{l}72+(\text { not all } \\
\text { human)/ }\end{array}$ \\
\hline RFK & 0 & 0 & 2 & 2 & 7 & 3 & $1^{* *}$ & 0 & 0 \\
\hline Rhomboid & 0 & 0 & 0 & 1 & 6 & 1 & 0 & 0 & 2 \\
\hline Fishtail & 3 & 1 & 1 & 8 & 30 & 2 & 0 & 1 & 2 \\
\hline Fishtail model & 1 & 0 & 0 & 0 & 0 & 0 & 0 & 0 & 1 \\
\hline Concave-Base PP & 0 & 0 & 0 & 1 & 0 & 0 & 0 & 0 & $8+$ \\
\hline Model Concave-Base PP & 0 & 3 & 0 & 0 & 0 & 0 & 0 & 0 & 0 \\
\hline Eccentric & 0 & 0 & 0 & 0 & $(1)$ & 0 & 0 & 0 & $12+$ \\
\hline Other Fine Bifacials & 1 & 0 & 0 & 2 & 7 & 2 & 0 & 0 & 6 \\
\hline Bifacial Knife & 2 & 0 & 0 & 6 & 13 & 0 & 0 & 0 & 10 \\
\hline Model Knife & 0 & 0 & 0 & 0 & 0 & 0 & 1 & 0 & 0 \\
\hline Bifacial Sickle & 0 & 0 & 0 & 0 & 2 & 1 & 1 & 0 & 2 \\
\hline Bifacial Plane & 0 & 0 & 0 & 0 & 0 & 0 & 0 & 0 & 0 \\
\hline Axe & 0 & 0 & 0 & 0 & $3-4$ & $1-2$ & 0 & $1+$ & 0 \\
\hline Drills: winged/crescent/cylindrical & 0 & 0 & 0 & 0 & 0 & 0 & 0 & 0 & 0 \\
\hline Pic/Grand Perçoir & 0 & 0 & 0 & 0 & 0 & 1 & 0 & 0 & 0 \\
\hline Non-standard, \& Unidentifiable* & 0 & 0 & 0 & 0 & 2 & 1 & 0 & 0 & 0 \\
\hline Axe Preform & 0 & 0 & 0 & 0 & 0 & 0 & 0 & 0 & 0 \\
\hline
\end{tabular}


Table 6.10. Presence absence of Ripple-flaked knives and production remains across Nile valley settlement sites. Note that the Naqada and Hierakonpolis sites have each been collapsed for easier comparison. n/a mans the cemetery is not of the right date for RFKs.

\begin{tabular}{|l|c|c|c|c|c|c|c|}
\hline \multicolumn{1}{|c|}{ RFKs } & Mahâsna & Abydos & Naqada & Armant & Adaïma & $\begin{array}{c}\text { Hiera- } \\
\text { konpolis }\end{array}$ & Aswan \\
\hline RFKs- cemetery & $\backslash$ & $\bullet$ & $\bullet$ & $\bullet$ & $\backslash$ & $\mathrm{n} / \mathrm{a}$ & $\mathrm{n} / \mathrm{a}$ \\
\hline RFKs- settlement & $\backslash$ & $\backslash$ & $\backslash$ & $\backslash$ & $\backslash$ & $\backslash$ & $\backslash$ \\
\hline Unfinished RFKs- settlement & $\backslash$ & $\backslash$ & $\backslash$ & $\backslash$ & $\backslash$ & $?$ & $\backslash$ \\
\hline $\begin{array}{l}\text { Bifacial thinning flakes- } \\
\text { settlement }\end{array}$ & $\bullet$ & $\bullet$ & $\bullet$ & $\bullet$ & $\bullet$ & $\bullet$ & $\bullet$ \\
\hline
\end{tabular}

Table 6.11. Presence absence of rhomboid tools and production remains across Nile valley settlement sites. Note that the Naqada and Hierakonpolis sites have each been collapsed for easier comparison.

\begin{tabular}{|l|c|c|c|c|c|c|c|}
\hline \multicolumn{1}{|c|}{ Rhomboid Tools } & Mahâsna & Abydos & Naqada & Armant & Adaïma & $\begin{array}{c}\text { Hiera- } \\
\text { konpolis }\end{array}$ & Aswan \\
\hline Rhomboids- cemetery & $\backslash$ & $\bullet$ & $\bullet$ & $\bullet$ & $\backslash$ & $\bullet$ & $\backslash$ \\
\hline Rhomboids- settlement & $\backslash$ & $\backslash$ & $\backslash$ & $\backslash$ & $\backslash$ & $\bullet$ & $\backslash$ \\
\hline $\begin{array}{l}\text { Unfinished Rhomboids- } \\
\text { settlement }\end{array}$ & $\backslash$ & $\backslash$ & $\backslash$ & $\backslash$ & $\backslash$ & $\backslash$ & $\backslash$ \\
\hline $\begin{array}{l}\text { Bifacial thinning flakes- } \\
\text { settlement }\end{array}$ & $\bullet$ & $\bullet$ & $\bullet$ & $\bullet$ & $\bullet$ & $\bullet$ & $\bullet$ \\
\hline
\end{tabular}

Table 6.12. Presence absence of fishtails and production remains across Nile valley settlement sites. Note that the Naqada and Hierakonpolis sites have each been collapsed for easier comparison.

\begin{tabular}{|l|c|c|c|c|c|c|c|}
\hline \multicolumn{1}{|c|}{ Fishtails } & Mahâsna & Abydos & Naqada & Armant & Adaïma & $\begin{array}{c}\text { Hiera- } \\
\text { konpolis }\end{array}$ & Aswan \\
\hline Fishtails- cemetery & $\bullet$ & $\bullet$ & $\bullet$ & $\bullet$ & $\backslash$ & $\bullet$ & $\backslash$ \\
\hline Fishtails- settlement & $\bullet$ & $\backslash$ & $\backslash$ & $\backslash$ & $\bullet$ & $\bullet$ & $\backslash$ \\
\hline Unfinished Fishtails- settlement & $\backslash$ & $\backslash$ & $\backslash$ & $\backslash$ & $\backslash$ & $\backslash$ & $\backslash$ \\
\hline $\begin{array}{l}\text { Bifacial thinning flakes- } \\
\text { settlement }\end{array}$ & $\bullet$ & $\bullet$ & $\bullet$ & $\bullet$ & $\bullet$ & $\bullet$ & $\bullet$ \\
\hline
\end{tabular}

Table 6.13. Presence absence of concave-base projectile points and production remains across Nile valley settlement sites. *A preform from el-Mahâsna looks like the right shape to be a CBPP. Note that the Naqada and Hierakonpolis sites have each been collapsed for easier comparison.

\begin{tabular}{|l|c|c|c|c|c|c|c|}
\hline \multicolumn{1}{|c|}{ Concave-Base PP Tools } & $\begin{array}{c}\text { Mahâsn } \\
\text { a }\end{array}$ & Abydos & Naqada & Armant & Adaïma & $\begin{array}{c}\text { Hiera- } \\
\text { konpolis }\end{array}$ & Aswan \\
\hline CBPPs- cemetery & $\backslash$ & $\bullet$ & $\backslash$ & $\backslash$ & $\backslash$ & $\bullet$ & $\backslash$ \\
\hline CBPPs- settlement & $\bullet$ & $\bullet$ & $\bullet$ & $\bullet$ & $\bullet$ & $\bullet$ & $\backslash$ \\
\hline Unfinished CBPPs - settlement & $\bullet *$ & $\backslash$ & $\bullet$ & $\backslash$ & $\backslash$ & $\bullet$ & $\backslash$ \\
\hline $\begin{array}{l}\text { Bifacial thinning flakes - } \\
\text { settlement }\end{array}$ & $\bullet$ & $\bullet$ & $\bullet$ & $\bullet$ & $\bullet$ & $\bullet$ & $\bullet$ \\
\hline
\end{tabular}


Table 6.14. Presence absence of figural eccentrics and production remains across Nile valley settlement sites. $(\bullet)$ indicates that the eccentric is probably an heirloom. Note that the Naqada and Hierakonpolis sites have each been collapsed for easier comparison.

\begin{tabular}{|l|c|c|c|c|c|c|c|}
\hline \multicolumn{1}{|c|}{ Figural Eccentrics } & Mahâsna & Abydos & Naqada & Armant & Adaïma & $\begin{array}{c}\text { Hiera- } \\
\text { konpolis }\end{array}$ & Aswan \\
\hline Eccentrics- cemetery & $\backslash$ & $?$ & $(\bullet)$ & $\backslash$ & $\backslash$ & $\bullet$ & $\backslash$ \\
\hline Eccentrics- settlement & $\backslash$ & $\bullet$ & $\backslash$ & $\backslash$ & $\backslash$ & $\bullet$ & $\backslash$ \\
\hline Unfinished Eccentrics- settlement & $\backslash$ & $\backslash$ & $\backslash$ & $\backslash$ & $\backslash$ & $\backslash$ & $\backslash$ \\
\hline $\begin{array}{l}\text { Bifacial thinning flakes- } \\
\text { settlement }\end{array}$ & $\bullet$ & $\bullet$ & $\bullet$ & $\bullet$ & $\bullet$ & $\bullet$ & $\bullet$ \\
\hline
\end{tabular}

Table 6.15. Presence absence of bifacial knives and production remains across Nile valley settlement sites. Note that the Naqada and Hierakonpolis sites have each been collapsed for easier comparison.

\begin{tabular}{|l|c|c|c|c|c|c|c|}
\hline \multicolumn{1}{|c|}{ Bifacial Knives } & Mahâsna & Abydos & Naqada & Armant & Adaïma & $\begin{array}{c}\text { Hiera- } \\
\text { konpolis }\end{array}$ & Aswan \\
\hline Knives- cemetery & $\bullet$ & $\bullet$ & $\bullet$ & $\backslash$ & $\backslash$ & $\bullet$ & $\backslash$ \\
\hline Knives- settlement & $\bullet$ & $\backslash$ & $\bullet$ & $\bullet$ & $\bullet$ & $\bullet$ & $\bullet$ \\
\hline Unfinished Knives- settlement & $\bullet$ & $\backslash$ & $\backslash$ & $\backslash$ & $\backslash$ & $\bullet$ & $\backslash$ \\
\hline Bifacial thinning flakes- settlement & $\bullet$ & $\bullet$ & $\bullet$ & $\bullet$ & $\bullet$ & $\bullet$ & $\bullet$ \\
\hline
\end{tabular}

Table 6.16. Presence absence of bifacial sickles and production remains across Nile valley settlement sites. Note that the Naqada and Hierakonpolis sites have each been collapsed for easier comparison.

\begin{tabular}{|l|c|c|c|c|c|c|c|}
\hline \multicolumn{1}{|c|}{ Bifacial Sickles } & Mahâsna & Abydos & Naqada & Armant & Adaïma & $\begin{array}{c}\text { Hiera- } \\
\text { konpolis }\end{array}$ & Aswan \\
\hline Bifacial Sickles- cemetery & $\backslash$ & $\backslash$ & $\bullet$ & $\bullet$ & $\bullet$ & $\bullet$ & $\backslash$ \\
\hline Bifacial Sickles- settlement & $\bullet$ & $\bullet$ & $\bullet$ & $\bullet$ & $\bullet$ & $\backslash$ & $\bullet$ \\
\hline Unfinished BFC Sickles- settlement & $\backslash$ & $\backslash$ & $\backslash$ & $\backslash$ & $\backslash$ & $\backslash$ & $\backslash$ \\
\hline Bifacial thinning flakes- settlement & $\bullet$ & $\bullet$ & $\bullet$ & $\bullet$ & $\bullet$ & $\bullet$ & $\bullet$ \\
\hline
\end{tabular}

Table 6.17. Presence absence of axes and production remains across Nile valley settlement sites. Note that the Naqada and Hierakonpolis sites have each been collapsed for easier comparison.

\begin{tabular}{|l|c|c|c|c|c|c|c|}
\hline \multicolumn{1}{|c|}{ Axes } & Mahâsna & Abydos & Naqada & Armant & Adaïma & $\begin{array}{c}\text { Hiera- } \\
\text { konpolis }\end{array}$ & Aswan \\
\hline Axes- cemetery & $\backslash$ & $\backslash$ & $\bullet$ & $\bullet$ & $\backslash$ & $\bullet$ & $\backslash$ \\
\hline Axes- settlement & $\bullet$ & $\backslash$ & $\bullet$ & $\bullet$ & $\bullet$ & $\bullet$ & $\backslash$ \\
\hline Unfinished Axes- settlement & $\backslash$ & $\backslash$ & $\bullet$ & $\bullet$ & $\backslash$ & $\backslash$ & $\backslash$ \\
\hline $\begin{array}{l}\text { Axe Preparation Flakes- } \\
\text { settlement }\end{array}$ & $\bullet$ & $\backslash$ & $\bullet$ & $\bullet$ & $\backslash$ & $\bullet$ & $\bullet$ \\
\hline Bifacial thinning flakes- settlement & $\bullet$ & $\bullet$ & $\bullet$ & $\bullet$ & $\bullet$ & $\bullet$ & $\bullet$ \\
\hline
\end{tabular}




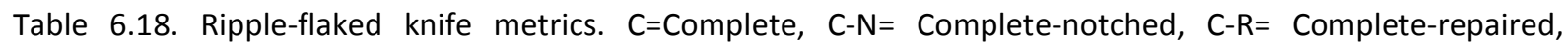
$\mathrm{MD}=$ Minor Damage. The reference for each entry is the relevant online museum database. Additional sources are noted. See Table 7.1 for museum list and abbreviations. *Measurement taken from drawing.

\begin{tabular}{|l|l|l|l|l|l|l|}
\hline \multicolumn{1}{|c|}{ ID } & \multicolumn{1}{|c|}{ Site } & $\begin{array}{c}\text { Preserv } \\
\text { ation }\end{array}$ & $\begin{array}{c}\text { Length } \\
(\mathbf{c m})\end{array}$ & $\begin{array}{c}\text { Width } \\
(\mathbf{c m})\end{array}$ & $\begin{array}{c}\text { Thick } \\
(\mathbf{c m})\end{array}$ & \multicolumn{1}{|c|}{ Reference } \\
\hline BM.EA.29286 & Unknown & C & 26.7 & 6 & 0.62 & Kelterborn 1984 \\
\hline BM.EA.29289 & Unknown & C & 23.5 & 5.4 & 0.6 & Kelterborn 1984 \\
\hline BM.EA.29290 & Abydos? & C & 21.4 & 5.4 & & \\
\hline BM.EA.32095 & Unknown & C & 22.9 & 5 & & \\
\hline BM.EA.32096 & Unknown & C & 25.3 & 5.3 & & \\
\hline BM.EA.32489 & Unknown & C-R & 23.5 & 6.3 & & \\
\hline BM.EA.59235 & Unknown & C & 23.1 & & & \\
\hline BMFA.03.1390 & Naqada & C-N & 21.5 & 5 & & \\
\hline BrM.09.889.120 & Abu Zaidan & C-MD & 24.6 & 5.9 & & Needler 1984:272-3 \\
\hline BrM.09.889.121 & Adaima & C-MD & 20 & 6 & 0.7 & Needler 1984:272-3 \\
\hline EM.Abydos.U.503 & Abydos-U & C & 14.9 & 5.8 & $0.5 *$ & Hikade 2013: PI1; 2003: 146, Fig 3 \\
\hline EM.R.536 & Tel el Farkha & C-R & 30.4 & 8.3 & 0.9 & Kabacinski 2012 \\
\hline FwM.E.GA.3193. & & & & & & \\
\hline 1943 & Unknown & C-R & 25.2 & & & \\
\hline LvpM.56.20.77or80 & Unknown & C-R & 22.8 & 5.3 & 0.7 & $1995: 76$, PI 117 \\
\hline MANStGL.84.087 & Unknown & C-R & 20.9 & 6 & 0.6 & Musée des antiquités 1982:100 \\
\hline MMA.07.228.105 & Unknown & C & 21.9 & & & \\
\hline MMA.11.167.1 & Unknown & C & 29.8 & & & \\
\hline OIM.10533 & Unknown & C & 23 & 5.8 & 0.7 & Teeter 2011 \\
\hline PR.1911.33.1 & Gerzeh & C-R & 25.5 & 6.3 & 0.7 & Petrie 1912; Stevenson 2009 \\
\hline UC.73354 & Abydos & C-R & 27.2 & 6.7 & & \\
\hline UC.73355 & Unknown & C-R & 18.4 & 4.6 & & \\
\hline UC.73364 & & & & \\
\hline
\end{tabular}


Table 6.19. Rhomboid metrics. $\mathrm{C}=$ Complete, $\mathrm{C}-\mathrm{R}=$ Complete-repaired, $\mathrm{MD}=$ Minor Damage, MF=Medial Fragment, PF=Proximal Fragment. The reference for each entry is the relevant online museum database. Additional sources are noted. See Table 7.1 for museum list and abbreviations. *Measurement taken from photo with scale.

\begin{tabular}{|c|c|c|c|c|c|c|}
\hline ID & Site & $\begin{array}{l}\text { Preser } \\
\text { vation } \\
\end{array}$ & $\begin{array}{c}\text { Length } \\
(\mathrm{cm})\end{array}$ & $\begin{array}{l}\text { With } \\
\text { (cm) }\end{array}$ & $\begin{array}{c}\text { Thick } \\
\text { (cm) }\end{array}$ & Reference \\
\hline Abydos.U.220 & Abydos & C-R & $22.6^{*}$ & & & Hikade 2000 \\
\hline Ash.1895.1020 & Naqada & C-R & 37.4 & 5.3 & & Payne 1993:167(1385) \\
\hline Ash.1927.3069 & Unknown & PF & $23+$ & 5.5 & & Payne 1993:167(1389) \\
\hline BM.EA.34297 & Unknown & $C-R$ & 22 & 4.3 & 0.6 & \\
\hline BM.EA.49723 & Unknown & $C-R$ & 35.5 & 5.6 & 0.8 & \\
\hline BM.EA.52848 & Unknown & $C$ & 34.5 & 5.2 & 0.9 & \\
\hline BMFA.13.3766 & Mesaed & $C-R$ & 23 & 4 & 0.3 & \\
\hline BMFA.17.83.R & $\begin{array}{l}\text { Khor Bahan, } \\
\text { Nubia }\end{array}$ & $\mathrm{MF}$ & $6.8+$ & $>=3.9$ & 0.6 & \\
\hline BrM.09.889.126 & Hierakonpolis & $C-R$ & 41 & 6.4 & 0.65 & Needler :114, 265 \\
\hline CMA.1914.672 & Unknown & C-MD & & 4 & & \\
\hline $\begin{array}{l}\text { FwM.E.GA.4218. } \\
1943\end{array}$ & Unknown & C-MD & 18.25 & & & \\
\hline $\begin{array}{l}\text { FwM.E.GA.4224. } \\
1943\end{array}$ & Unknown & C-R & 29 & & & \\
\hline MMA.16.2.11 & Unknown & $C$ & $18.5^{*}$ & & & \\
\hline MMA.7.228.106 & Unknown & $C-R$ & 21.5 & & & \\
\hline OIM.E.11226 & Saghel el-Baglieh & $C-R$ & 22.2 & 4.3 & 0.7 & \\
\hline UC. 4130 & Naqada & C-MD & 37.1 & 5.3 & & Baumgartel 1970; Payne 1987 \\
\hline UC.4389 & Naqada & C-R & 18.5 & 3.6 & & \\
\hline UC. 4828 & Naqada/Ballas & C-MD & 19.2 & 3.5 & & \\
\hline
\end{tabular}


Table 6.20 (part 1). Fishtail metrics.

\begin{tabular}{|c|c|c|c|c|c|c|c|}
\hline ID & Site & \begin{tabular}{|c|}
$\begin{array}{c}\text { Shape } \\
\text { type }\end{array}$ \\
\end{tabular} & \begin{tabular}{|c} 
Preserv- \\
ation
\end{tabular} & $\begin{array}{c}\text { Length } \\
(\mathrm{cm})\end{array}$ & $\begin{array}{c}\text { Width } \\
(\mathrm{cm})\end{array}$ & \begin{tabular}{|l}
$\begin{array}{c}\text { Thick } \\
(\mathrm{cm})\end{array}$ \\
\end{tabular} & Reference \\
\hline A.U.178 & Abydos-U & 1 & TTM & 15.5 & $7.6+$ & $0.8^{*}$ & Hikade 2003: 144 \\
\hline A.U.395 & Abydos-U & 3 & $C-R$ & 12.9 & 5.7 & $0.5^{*}$ & Hikade 2003: 143 \\
\hline Ash.1895.1000 & Naqada/Ballas & 1 & C & 10.1 & 7.3 & & Payne 1993:170 (1401) \\
\hline Ash.1895.1001 & Naqada & 1 & C & 13.4 & 9.3 & & Payne 1993: 170 (1400) \\
\hline Ash.1895.1023 & Naqada & 3 & C & 17.2 & 5.6 & & Payne 1993:170 (1407) \\
\hline Ash.1927.3065 & Unknown & 2 & C-R & 15.8 & 4.7 & & Payne 1993:171 (1410) \\
\hline Ash.1927.3066 & Unknown & 2 & C-R & 17 & 5.2 & & Payne 1993:171 (1409) \\
\hline Ash.1927.3067 & Unknown & 1 & C & 7.7 & 5.9 & & Payne 1993:170 (1405) \\
\hline BM.EA.32496 & Unknown & 3 & C & 11.2 & 5.2 & 0.4 & \\
\hline BM.EA.59240 & Unknown & $1 a$ & C & 14.2 & 6.6 & 0.8 & \\
\hline BM.EA.59241 & Unknown & 1 & C & 11.6 & 8.4 & 0.8 & \\
\hline BMFA.03.1386 & Abadiya & 1 & C & 13.5 & & 0.7 & \\
\hline BMFA.03.1387 & Abadiya & 1 & C & 11.5 & & & \\
\hline BMFA.03.1391 & Unknown & 1 & TTM & 13.5 & & & \\
\hline BMFA.11.247 & Mesaeed & 1 & TTM & 19.5 & $6+$ & 0.8 & \\
\hline BMFA.11.250 & Mesaeed & 3 & TTM & 15.6 & $4.8+$ & 0.5 & \\
\hline BMFA.11.257 & Mesaeed & 1 & $\mathrm{C}-\mathrm{N}$ & 9.5 & 4.5 & & \\
\hline BMFA.11.259 & Mesaeed & 3 & C & 14.6 & 4.9 & 0.4 & \\
\hline BMFA.13.3915 & Mesaeed & 1 & C & 13.5 & 7.5 & & \\
\hline BrM.07.447.866 & & 1 & $\mathrm{C}-\mathrm{N}$ & 12.22 & 5.87 & & \\
\hline BrM.07.447.870 & el-Ma'mariya & $1 a$ & $C-R$ & 12.5 & 6.5 & & Needler 1984:266-267 \\
\hline CMA.1914.673 & Unknown & 1 & $C$ & 15 & 10 & & \\
\hline CMA.1914.674 & Unknown & $1 a$ & C & 11 & 6 & & \\
\hline CMA.1914.717 & Unknown & 2 & C & 18.5 & 6.5 & & \\
\hline EM.34831 & Unknown & 2 & C-MDTT & 16 & & & Currelly 1913: 272 \\
\hline FwM.E.214.1932 & Unknown & 2 & C-MDTT & 16.5 & & & \\
\hline FwM.E.49.1899 & Unknown & 1 & C-MDTT & 10.5 & & & \\
\hline FwM.E.50.1899 & Unknown & 1 & $C-R$ & 11 & & & \\
\hline FwM.E.51.1899 & Diospolis Parva & 1 & C-MDTT & 12 & & & \\
\hline Fwm.E.52.1899 & Unknown & 2 & C & 16 & & & \\
\hline FwM.E.GA.4144.1943 & unknown & $1 a$ & C & 12.3 & & & \\
\hline FwM.E.GA.4148.1943 & Unknown & 2 & TTM & 16.5 & & & \\
\hline FwM.E.Misc.94 & Unknown & $1 a$ & $C-R$ & 12 & & & \\
\hline FwM.GA.3162.1943 & Unknown & 2 & C & 14.9 & 5 & 0.4 & \\
\hline FwM.GA.3174.1943 & Unknown & $1 a$ & C-R-MD & 10 & & & \\
\hline HK.6.2006.2 & HK6 & $1 a$ & C & 7.9 & 4.4 & 0.6 & $\begin{array}{l}\text { Friedman2006:7; Nagaya } \\
2011\end{array}$ \\
\hline LACMA.1998.94.1 & Unknown & 2 & C & 17.15 & 5.27 & 0.32 & \\
\hline LvpM.1973.2.254 & Abydos? & 3 & C-R & 14 & 4.8 & 0.5 & Bienkowski \& Tooley 1995:48 \\
\hline LvpM.56.20.42 & Naqada? & 2 & C & 10.5 & 4.4 & 0.5 & \\
\hline MAP.301.1 & MAP & $1 a$ & $\mathrm{MF}$ & $7.76+$ & $4.05+$ & 0.61 & \\
\hline MMA.10.176.96 & Unknown & $1 a$ & C & 15.2 & 4 & & \\
\hline MMA.16.2.4 & Unknown & 2 & C & 15.7 & 5.8 & 0.8 & \\
\hline MMA.16.2.8 & Unknown & 1 & C & 9.5 & & & \\
\hline MMA.17.6.25 & & 2 & DF & 99.7 & 5.6 & & \\
\hline MMA.20.5 & Unknown & 3 & C & 12 & 5 & & \\
\hline OIM.11250 & Unknown & 2 & C & 18.3 & 6.1 & 0.8 & \\
\hline OIM.11252 & Unkown & 1 & C & 11.5 & 7.9 & 0.6 & \\
\hline OIM.11253 & Unknown & $1 a$ & C-R & 11.8 & 6.9 & 0.9 & \\
\hline
\end{tabular}


Table 6.20 (part 2). Fishtail metrics. This survey did not include Fishtails that were clearly re-worked, nor fishtails that had handles where it was not clear if the handles were included in the length measurement). $\mathrm{C}=$ Complete, $\mathrm{C}-\mathrm{MDTT}=$ Complete- minor damage on 1 tang tip, $\mathrm{C}-\mathrm{N}=$ Complete-notched, C-R= Completerepaired, DF=Distal Fragment, MD=Minor Damage, MF=Medial Fragment, TTM=Tang Tip Missing. The reference for each entry is the relevant online museum database. Additional sources are noted. See Table 7.1 for museum list and abbreviations. *Thickness measured from drawing.

\begin{tabular}{|c|c|c|c|c|c|c|c|}
\hline ID & Site & $\begin{array}{c}\text { Shape } \\
\text { type }\end{array}$ & $\begin{array}{c}\text { Preserv } \\
\text { ation }\end{array}$ & $\begin{array}{c}\text { Length } \\
(\mathrm{cm})\end{array}$ & $\begin{array}{c}\text { Width } \\
(\mathrm{cm})\end{array}$ & \begin{tabular}{|l} 
Thick \\
$(\mathrm{cm})$
\end{tabular} & Reference \\
\hline OIM.11254 & Unknown & 1 & C-N-MD & 11.6 & 5.5 & 0.6 & \\
\hline PR.1900.42.1 & Abydos-G & 2 & TTM & 16 & 5.3 & .5 & Petrie 1902 \\
\hline PR.1901.42.107 & Mahasna & $1 a$ & C & 14.6 & 5.3 & 0.7 & Garstang 1903: PI5 (6) \\
\hline PR.1901.42.114 & Mahasna & $1 a$ & DF & $>9.5$ & 4.7 & 0.8 & Garstang 1903:5,8 \\
\hline RMAH.5663 & El-Haraga & 2 & $C$ & 20 & 4.5 & & \\
\hline $\begin{array}{l}\text { RVFAM. } \\
\text { E1.01.015.1998 }\end{array}$ & Unknown & 2 & C & 16.5 & 5.1 & 0.32 & Kaplan 2005:124 \\
\hline SNHM.A328574.0 & Unkown & 1 & $c$ & 13 & & & \\
\hline UC. 4133 & Naqada & 1 & $C-R$ & 13.3 & 7.5 & & $\begin{array}{l}\text { Petrie \& Quibell } \\
\text { 1896:PI73(66) }\end{array}$ \\
\hline UC.4133a & Naqada & $1 a$ & $C-R$ & 12.4 & 7 & & $\begin{array}{l}\text { Petrie \& Quibell } \\
\text { 1896:PI73(66) }\end{array}$ \\
\hline UC.42817 & Abydos? & 2 & $C$ & 16.4 & 5.4 & & \\
\hline UC.4429 & Naqada & 2 & $c$ & 17.5 & 6.6 & & \\
\hline UC.4527 & Naqada & 1 & $c$ & 13.2 & 5.6 & & \\
\hline UC. 4528 & Naqada & 1 & TTM & 9 & $4.3+$ & & \\
\hline UC. 4827 & Naqada & $1 a$ & $C-R$ & 13.5 & 6.4 & & \\
\hline UC.4919 & Naqada & $1 a$ & $C$ & 9 & 3.2 & & \\
\hline UC.5405 & Naqada & 1 & C-R & 12.5 & 8.8 & & \\
\hline UC. 6070 & Armant & $1 a$ & TTM & 11.4 & & & \\
\hline UC. 8965 & Unknown & 2 & c & 11.7 & 5.7 & & \\
\hline
\end{tabular}

Table 6.21. Metric data for the lengths of Fishtails showing that they became longer and less variable over time.

\begin{tabular}{|l|r|r|r|r|}
\hline & Type 1 & Type 1a & Type 3 & Type 2 \\
\hline $\mathrm{N}$ & 24 & 14 & 7 & 18 \\
\hline Mean (cm) & 12.23 & 11.99 & 13.93 & 16.16 \\
\hline $\begin{array}{l}\text { Standard } \\
\text { deviation }\end{array}$ & 2.44 & 2.07 & 2.09 & 2.2 \\
\hline $\mathrm{CV}$ & $19.94 \%$ & $17.28 \%$ & $14.99 \%$ & $13.63 \%$ \\
\hline
\end{tabular}

Table 6.22. Metric data for Concave base projectile points.

\begin{tabular}{|l|r|r|r|}
\hline & \multicolumn{1}{|c|}{$\begin{array}{l}\text { Length } \\
\text { (cm) }\end{array}$} & \multicolumn{1}{c|}{$\begin{array}{l}\text { Width } \\
\text { (cm) }\end{array}$} & \multicolumn{1}{c|}{$\begin{array}{l}\text { Thick } \\
\text { (cm) }\end{array}$} \\
\hline $\mathrm{N}$ & 11 & 18 & 15 \\
\hline Mean (cm) & 6.45 & 2.49 & 0.597 \\
\hline $\begin{array}{l}\text { Standard } \\
\text { deviation }\end{array}$ & 1.89 & 0.315 & 0.092 \\
\hline CV & $29.3 \%$ & $12.65 \%$ & $15.41 \%$ \\
\hline
\end{tabular}


Table 6.23. Concave-base projectile point metrics. $C=$ Complete, $C-R=$ Complete-repaired, DF=Distal Fragment, $\mathrm{MD}=$ Minor Damage, MF=Medial Fragment, 1TB= One Tang Broken. The reference for each entry is the relevant online museum database. Additional sources are noted. See Table 7.1 for museum list and abbreviations. *Thickness taken from photo with scale.

\begin{tabular}{|c|c|c|c|c|c|c|}
\hline ID & Site & $\begin{array}{l}\text { Preser } \\
\text { vation }\end{array}$ & $\begin{array}{l}\text { Lengt } \\
\mathrm{h}(\mathrm{cm})\end{array}$ & $\begin{array}{l}\text { Width } \\
(\mathrm{cm})\end{array}$ & \begin{tabular}{|l} 
Thick \\
$(\mathrm{cm})$
\end{tabular} & Reference \\
\hline Ad.409 & Adaima & $1 \mathrm{~TB}$ & 4.7 & 1.7 & 0.6 & Midant-Reynes \& Prost 2002: 366(409) \\
\hline AMC.12.48 & Abydos & DF & $6.57+$ & 2.53 & 0.53 & \\
\hline Ash.E.1612 & Mahasna & $1 \mathrm{~TB}$ & 7.5 & & & Payne 1993: 180 (1479) \\
\hline Ash.E.1613 & Hierakonpolis-Nekhen & DF & $5.2+$ & 3 & & Payne 1993: 180 (1478) \\
\hline BrM.09.889.127 & Hierakonpolis & $C-R$ & $8.3^{*}$ & $2.6^{*}$ & $0.5^{*}$ & Needler 1984:263 \\
\hline BrM.09.889.128 & Hierakonpolis & $1 \mathrm{~TB}$ & 6.7 & & & Needler 1984:263 \\
\hline EK.2012.3.38 & Elkab & DF & $5.9+$ & $p=2.52$ & 0.6 & Claes et al. 2014:85 \\
\hline HK.6.str7.363 & Hierakonpolis-HK6 & C & 10 & & & $\begin{array}{l}\text { Friedman2010:69; } \\
\text { Droux\&Friedman2007 }\end{array}$ \\
\hline KH4.C & Naqada-KH4 & $\mathrm{MF}$ & $3.6+$ & 2.8 & 0.8 & Holmes 1989:248-249 \\
\hline MAP.2528.1 & el-Mahasna & $1 \mathrm{~TB}$ & 7.4 & 2.55 & 0.58 & \\
\hline MAP.3029.1 & el-Mahasna & $1 \mathrm{~TB}$ & 4.22 & 2.46 & 0.6 & \\
\hline MAP.395.1 & el-Mahasna & MF & $4.85+$ & 2.21 & 0.54 & \\
\hline UC.10028 & Hemmamiya North spur & DF & $3.2+$ & 2.5 & 0.5 & Holmes 1989:83-84 \\
\hline UC. 10345 & Hemmamiya North spur & $1 T B$ & 3.4 & 2.5 & 0.5 & Holmes 1989:83-84 \\
\hline UC.27229 & Badari 3000/10 & $1 \mathrm{~TB}$ & $4.4+$ & 3.1 & 0.4 & Holmes 1989:163 \\
\hline UC.5343 & Naqada-South Town & C & 6.5 & 2.4 & 0.8 & Holmes 1989:274 \\
\hline UC. 5352 & Naqada-South Town & C & 5.8 & 2.3 & 0.5 & Holmes 1989:274-275 \\
\hline UC. 6230 & Naqada & $1 \mathrm{~TB}$ & $6.5^{*}$ & $2.5^{*}$ & & \\
\hline UC.9433 & Badari 3000/3 & DF & $6.2+$ & 2.6 & 0.6 & Holmes 1989:163 \\
\hline UC.9434 & Badari 3000/3 & DF & $5.4+$ & 2.6 & 0.6 & Holmes 1989:163 \\
\hline UC.9436 & Badari 3000/6 & DF & $6+$ & 2.2 & 0.5 & Holmes 1989:163 \\
\hline UC.9437 & Badari 3000/6 & $\mathrm{MF}$ & $4.4+$ & 2.2 & 0.6 & Holmes 1989:163 \\
\hline UC.9438 & Badari 3000/6 & DF & $4.7+$ & $2+$ & 0.6 & Holmes 1989:163 \\
\hline
\end{tabular}

Table 6.24. Metric data for Bifacial sickles.

\begin{tabular}{|l|r|r|}
\hline & \multicolumn{1}{|c|}{$\begin{array}{l}\text { Width } \\
(\mathbf{c m})\end{array}$} & \multicolumn{1}{c|}{$\begin{array}{l}\text { Thick } \\
(\mathbf{c m})\end{array}$} \\
\hline $\mathrm{N}$ & 22 & 19 \\
\hline Mean $(\mathrm{cm})$ & 2.87 & 0.79 \\
\hline $\begin{array}{l}\text { Standard } \\
\text { deviation }\end{array}$ & 0.713 & 0.235 \\
\hline $\mathrm{CV}$ & $24.84 \%$ & $29.74 \%$ \\
\hline
\end{tabular}

Table 6.26. Metric data on the lengths of axes. Data on the Naqada sites is from Holmes (1990).

\begin{tabular}{|l|r|r|r|r|r|}
\hline \multicolumn{1}{|c|}{ Site } & $\begin{array}{c}\text { Mahasn } \\
\text { a }\end{array}$ & Armant & $\begin{array}{c}\text { Naqada- } \\
\text { KH3 }\end{array}$ & $\begin{array}{r}\text { Naqada- } \\
\text { KH4 }\end{array}$ & \multicolumn{1}{c|}{ HK } \\
\hline $\mathrm{N}$ & 12 & 31 & 34 & 12 & 10 \\
\hline $\begin{array}{l}\text { Mean } \\
\text { (cm) }\end{array}$ & 6.79 & 7.49 & 6.18 & 6.62 & 7.79 \\
\hline $\begin{array}{l}\text { Standard } \\
\text { deviation }\end{array}$ & 1.51 & 1.86 & 1.03 & 1.84 & 1.65 \\
\hline CV & $22.24 \%$ & $24.83 \%$ & $16.67 \%$ & $27.79 \%$ & $21.18 \%$ \\
\hline
\end{tabular}


Table 6.25. Bifacial sickle metrics. $\mathrm{C}=$ Complete, $\mathrm{EF}=\mathrm{End}$ Fragment, MF=Medial Fragment. The reference for each entry is the relevant online museum database. Additional sources are noted. See Table 7.1 for museum list and abbreviations. * Measurement taken from photo or drawing with scale.

\begin{tabular}{|c|c|c|c|c|c|c|c|}
\hline ID & Site & \begin{tabular}{|c} 
\# of \\
points \\
extant
\end{tabular} & $\begin{array}{l}\text { Preser } \\
\text { vation }\end{array}$ & $\begin{array}{c}\text { Length } \\
(\mathrm{cm})\end{array}$ & $\begin{array}{l}\text { Width } \\
(\mathrm{cm})\end{array}$ & $\begin{array}{l}\text { Thick } \\
\text { (cm) }\end{array}$ & Reference \\
\hline Ab2.Fig22.11 & Abadiya 2 & 1 & EF & $3.54+$ & 1.3 & & $\begin{array}{l}\text { Vermeersch et al. } \\
\text { 2004:234,237 }\end{array}$ \\
\hline Ad.256 & Adaima & 1 & EF & $6.5+$ & 2.1 & 0.6 & $\begin{array}{l}\text { Midant-Reynes \& Prost } \\
\text { 2002:354 }\end{array}$ \\
\hline Ad.258 & Adaima & 1 & c & 8 & 2.4 & 0.8 & $\begin{array}{l}\text { Midant-Reynes \& Prost } \\
\text { 2002:354 }\end{array}$ \\
\hline Ad.259 & Adaima & 1 & EF & $8.8+$ & 2.7 & 0.5 & $\begin{array}{l}\text { Midant-Reynes \& Prost } \\
\text { 2002:354 }\end{array}$ \\
\hline Ad.261 & $\begin{array}{l}\text { Adaima- West } \\
\text { cemetery }\end{array}$ & 2 & c & 16.3 & 3.1 & 1.1 & $\begin{array}{l}\text { Midant-Reynes \& Prost } \\
\text { 2002:354 }\end{array}$ \\
\hline AKAP.1552.1 & Nag El-Qarmila & 1 & EF & $5.76+$ & 2.44 & 0.77 & \\
\hline ATP.3418 & Abydos & 2 & $c$ & 16 & & & \\
\hline ATP.3419 & Abydos & 2 & c & 17.6 & 4.24 & $1.31 *$ & \\
\hline ATP.3420 & Abydos & 2 & c & 22 & & & \\
\hline BrM.07.447.802 & Adaima & 1 & EF & $15.6+$ & 3.7 & 0.9 & Needler 1984:286 \\
\hline BrM.07.447.806 & Adaima & 0 & $E F$ & $7.3+$ & 3.6 & 0.5 & Needler 1984:85-86 (72) \\
\hline BrM.07.447.807 & Adaima & 0 & $\mathrm{MF}$ & $7.3+$ & 3.6 & $1 *$ & Needler 1984:85-86 (71) \\
\hline EK.TP4.1 & Elkab & 0 & $\mathrm{MF}$ & $9.08+$ & 3.84 & 0.91 & Claes et al. 2014:85 \\
\hline MA2183.Y.S.PI12.4 & Armant & 0 & MF & $5.9+$ & 2.86 & 1.14 & Ginter \& Kozlowski 1994:61 \\
\hline MAP.2861.1 & Mahasna & 1 & $\mathrm{EF}$ & 8 & 3.8 & 0.86 & \\
\hline UC.10525 & $\begin{array}{l}\text { Hemmamiya, North } \\
\text { Spur }\end{array}$ & 1 & EF & 5.6 & 2.4 & 0.5 & Holmes 1989:81-82 \\
\hline UC.10619 & Hemmamiya, 1900 & 1 & $\mathrm{EF}$ & $7+$ & 2.7 & 0.6 & Holmes 1989:162 \\
\hline UC.10620 & Hemmamiya, 1900 & 0 & $\mathrm{MF}$ & $4.6+$ & 2.3 & 0.6 & Holmes 1989:162 \\
\hline UC.26823 & Badari, 3000/3 & 0 & EF & $4.2+$ & 2.6 & 0.6 & Holmes 1989:162 \\
\hline UC.4609 & Naqada & 1 & EF & 11.7* & 2.6 & & \\
\hline UC.5332 & Naqada-South Town & 1 & $E F$ & 7.6 & 2.9 & 0.7 & Holmes 1989:267 \\
\hline UC.9618 & Badari, 3000/3 & 0 & $\mathrm{MF}$ & $4.5+$ & 3 & 0.9 & \\
\hline UC.9653 & Badari 3000/6 & 0 & MF & $3.9+$ & 1.9 & 0.7 & Holmes 1989:162 \\
\hline UC.9879a & Hemmamiya, 1700 & 2 & $C$ & $\sim 11.7$ & 3 & & \\
\hline
\end{tabular}

Table 6.27. Metric data on the widths of axes. Data on the Naqada sites is from Holmes (1990).

\begin{tabular}{|l|r|r|r|r|}
\hline \multicolumn{1}{|c|}{ Site } & Mahasna & Armant & $\begin{array}{c}\text { Naqada- } \\
\text { KH3 }\end{array}$ & $\begin{array}{c}\text { Naqada- } \\
\text { KH4 }\end{array}$ \\
\hline $\mathrm{N}$ & 15 & 31 & 34 & 12 \\
\hline Mean (cm) & 5.44 & 5.27 & 4.38 & 4.78 \\
\hline $\begin{array}{l}\text { Standard } \\
\text { deviation }\end{array}$ & 1.095 & 1.08 & 0.791 & 0.98 \\
\hline CV & $20.12 \%$ & $20.49 \%$ & $18.06 \%$ & $20.5 \%$ \\
\hline
\end{tabular}

Table 6.28. Metric data on axe thickness. Data on the Naqada sites is from Holmes (1990).

\begin{tabular}{|l|r|r|r|r|}
\hline \multicolumn{1}{|c|}{ Site } & Mahasna & Armant* & $\begin{array}{c}\text { Naqada- } \\
\text { KH3 }\end{array}$ & \multicolumn{1}{c|}{$\begin{array}{c}\text { Naqada- } \\
\text { KH4 }\end{array}$} \\
\hline $\mathrm{N}$ & 15 & 10 & 34 & 12 \\
\hline Mean (cm) & 2.26 & 2.64 & 1.935 & 2.175 \\
\hline $\begin{array}{l}\text { Standard } \\
\text { deviation }\end{array}$ & 0.456 & 0.45 & 0.485 & 0.436 \\
\hline CV & $20.15 \%$ & $17.04 \%$ & $25.06 \%$ & $20.05 \%$ \\
\hline
\end{tabular}


Table 6.29 (part 1). Axe metrics. C=Complete, DF=Distal Fragment.

\begin{tabular}{|c|c|c|c|c|c|c|}
\hline ID & Site & $\begin{array}{c}\text { Preserv } \\
\text { ation }\end{array}$ & $\begin{array}{c}\text { Length } \\
(\mathrm{cm})\end{array}$ & $\begin{array}{l}\text { Width } \\
\text { (cm) }\end{array}$ & $\begin{array}{l}\text { Thick } \\
(\mathrm{cm})\end{array}$ & Reference \\
\hline BrM.07.447.1008 & Hierakonpolis & $c$ & 9.3 & & & Needler 1984:117 \\
\hline BrM.07.447.1009 & Hierakonpolis & $c$ & 8.9 & & & Needler 1984:279 \\
\hline BrM.07.447.1011 & Hierakonpolis & $c$ & 7.1 & & & Needler 1984:279 \\
\hline BrM.07.447.1018 & Hierakonpolis & c & 9.9 & & & Needler 1984:117 \\
\hline BrM.07.447.1019 & Hierakonpolis & $c$ & 8.3 & & & Needler 1984:114 \\
\hline BrM.07.447.1020 & Hierakonpolis & $c$ & 5.7 & & & Needler 1984:117 \\
\hline BrM.07.447.1022 & Hierakonpolis & $c$ & 8.5 & & & Needler 1984:117 \\
\hline BrM.07.447.990 & Hierakonpolis & C & 8.2 & & & Needler 1984:117 \\
\hline BrM.07.447.994 & Hierakonpolis & $c$ & 4.5 & & & Needler 1984:117 \\
\hline BrM.07.447.998 & Hierakonpolis & $c$ & 7.5 & & & Needler 1984:279 \\
\hline Man.23428 & Armant & $c$ & 8.8 & 5.4 & 2.3 & \\
\hline ManM.23382 & Armant & $c$ & 10.2 & 7.9 & & Mond \& Meyers 1937: 233, PI 62 (12) \\
\hline ManM.23383 & Armant & $c$ & 10.5 & 6.4 & 3 & Mond \& Meyers 1937: 234,243, PI57,62 \\
\hline ManM.23384 & Armant & $C$ & 8.8 & 5.7 & & \\
\hline ManM.23385 & Armant & $\mathrm{DF}$ & $8.5+$ & 5.9 & & \\
\hline ManM.23386 & Armant & $c$ & 7.7 & 5.5 & & \\
\hline ManM.23387 & Armant & $c$ & 9.1 & 6.2 & & \\
\hline ManM.23388 & Armant & $c$ & 9.5 & 5.6 & & \\
\hline ManM.23389 & Armant & $c$ & 8.4 & 5.8 & 3 & Mond \& Meyers 1937: 243, PI62 (15) \\
\hline ManM.23390 & Armant & $c$ & 9.8 & 6.4 & & Mond \& Meyers 1937:233, PI 57 (17) \\
\hline ManM.23391 & Armant & c & 8.8 & 5.2 & 2.3 & Mond \& Meyers 1937:234,244,P157 (19) \\
\hline ManM.23392 & Armant & $c$ & 8.3 & 5.4 & & Mond \& Meyers 1937:133, PI 57 (18) \\
\hline ManM.23393 & Armant & $c$ & 9.4 & 6.2 & 2.2 & Mond \& Meyers 1937:244, PI 62 (17) \\
\hline ManM.23394 & Armant & $c$ & 9.2 & 6.2 & 2.9 & Mond \& Meyers 1937: 244, PI 62 (21) \\
\hline ManM.23396 & Armant & C & 8.5 & 5.7 & & Mond \& Meyers 1937:243, PI 62 (14) \\
\hline ManM.23397 & Armant & c & 8 & 6.9 & & Mond \& Meyers 1937:234, PI 57 (23) \\
\hline ManM.23399 & Armant & $c$ & 8.9 & 6.2 & & \\
\hline ManM.23405 & Armant & $c$ & 5.3 & 4.7 & 3 & Mond \& Meyers 1937:244, PI 62 (18) \\
\hline ManM.23405 & Armant & $c$ & 5.4 & 4.8 & 3.1 & Mond \& Meyers 1937:244, PI 62 (18) \\
\hline ManM.23412 & Armant & $c$ & 4.9 & 4.5 & & Mond \& Meyers 1937:233, PI 57 (5) \\
\hline ManM.23644 & Armant & c & 7.6 & 5 & & \\
\hline ManM.23645 & Armant & c & 7.5 & 5.1 & & \\
\hline ManM.23658 & Armant & $c$ & 6.7 & 6.5 & & Mond \& Meyers 1937:243, PI 61 (11) \\
\hline ManM.23659 & Armant & $c$ & 4.7 & 5 & & \\
\hline ManM.23660 & Armant & $c$ & 5.4 & 4.7 & & \\
\hline ManM.23692 & Armant & DF & $6.5+$ & 5.9 & & \\
\hline ManM.23704 & Armant & $c$ & 5.5 & 3.7 & & \\
\hline ManM.23705 & Armant & c & 4.9 & 3.3 & & \\
\hline ManM.23706 & Armant & $c$ & 5.1 & 3.3 & & \\
\hline ManM.23707 & Armant & $c$ & 4.2 & 3.7 & & \\
\hline ManM.23848 & Armant & c & 5.8 & 3.7 & & \\
\hline ManM.23859 & Armant & $c$ & 7.1 & 4.9 & 1.8 & Mond \& Meyers 1937:48, PI 16 (1) \\
\hline ManM.23862 & Armant & $c$ & 8.2 & 4.4 & 2.8 & Mond \& Meyers 1937:48 \\
\hline MAP.1973.30 & el-Mahasna & $c$ & 7.11 & 4.74 & 2.5 & \\
\hline MAP.2522.26 & el-Mahasna & $\mathrm{DF}$ & $4.73+$ & 5.29 & 1.64 & \\
\hline MAP.2522.31 & el-Mahasna & $c$ & 8.06 & 6 & 2.33 & \\
\hline PM.E.9633 & el-Mahasna & $\mathrm{DF}$ & $10.02+$ & 8.07 & 2.34 & \\
\hline
\end{tabular}


Table 6.29 (part 2). Axe metrics. C=Complete, DF=Distal Fragment. The artifacts from el-Mahasna were analyzed by the author. The reference for each entry is the relevant online museum database. Additional sources are noted. See Table 7.1 for museum list and abbreviations.

\begin{tabular}{|c|c|c|c|c|c|c|}
\hline ID & Site & Preservation & $\begin{array}{l}\text { Length } \\
(\mathrm{cm})\end{array}$ & $\begin{array}{c}\text { Width } \\
(\mathrm{cm})\end{array}$ & $\begin{array}{l}\text { Thick } \\
\text { (cm) }\end{array}$ & Reference \\
\hline PM.E.9634 & el-Mahasna & C & 9.14 & 6.96 & 2.9 & \\
\hline PM.E.9653 & el-Mahasna & DF & $6.79+$ & 3.97 & 1.72 & \\
\hline PM.E.9654 & el-Mahasna & $C$ & 4.96 & 5.98 & 2.7 & \\
\hline PM.E.9655 & el-Mahasna & C & 5.96 & 4.75 & 2.35 & \\
\hline PM.E.9656 & el-Mahasna & $C$ & 6.08 & 4.96 & 2.81 & \\
\hline PM.E.9681 & el-Mahasna & C & 7.26 & 6.08 & 1.69 & \\
\hline PM.E.9682 & el-Mahasna & C & 9.22 & 5.13 & 2.05 & \\
\hline PM.E.9687 & el-Mahasna & C & 4.98 & 4.36 & 2.18 & \\
\hline PM.E.9689 & el-Mahasna & C & 5.05 & 4.23 & 2.67 & \\
\hline PM.E.9690 & el-Mahasna & C & 6.16 & 5.12 & 2.58 & \\
\hline PM.E.9692 & el-Mahasna & $C$ & 7.49 & 6.02 & 1.48 & \\
\hline
\end{tabular}

Table 6.30. Comparison of reduction categories in different blocks at el-Mahâsna.

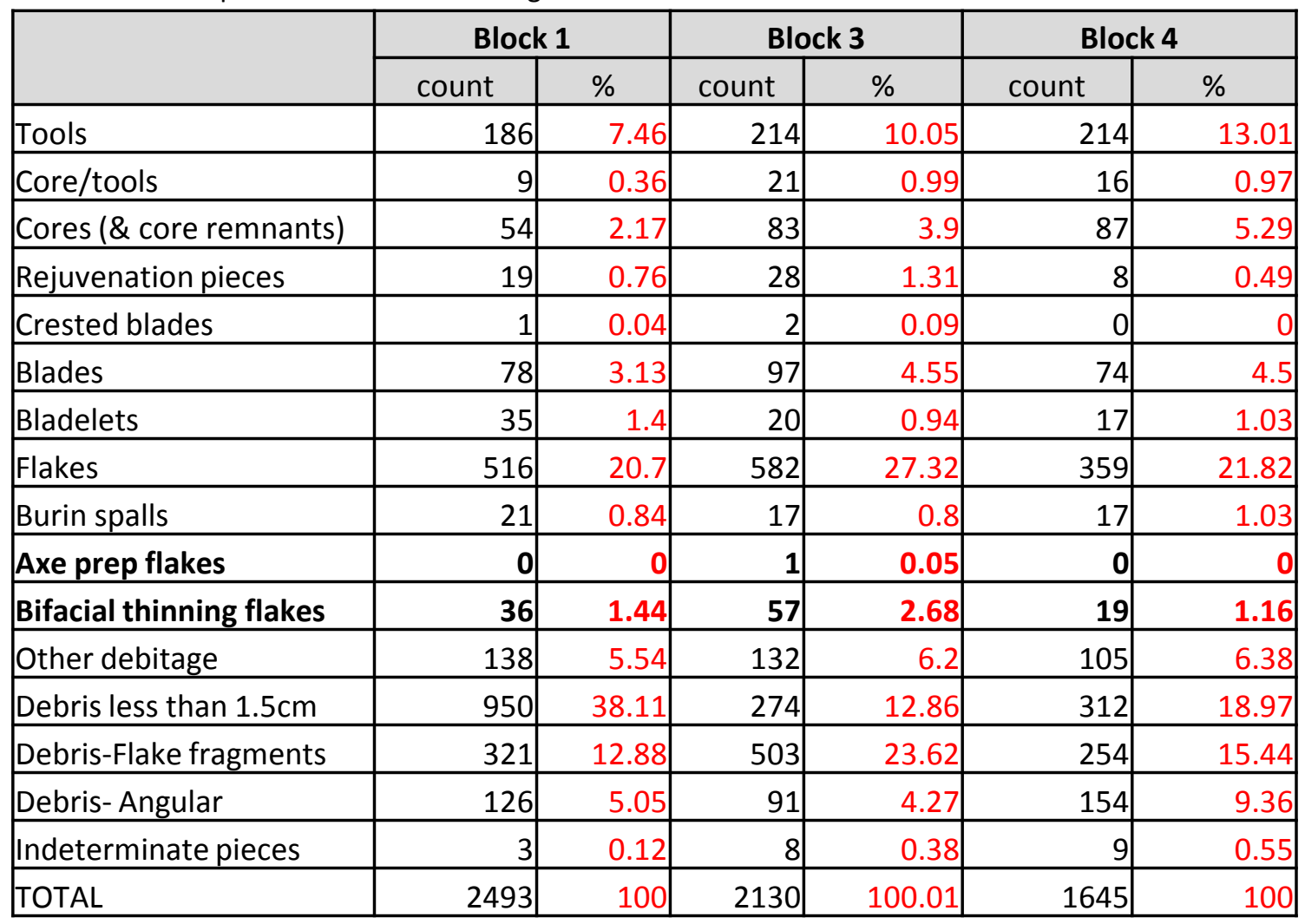


Table 6.31. The total percentage of bifacial tools in each block. And the $95 \%$ binomial confidence limits. The intervals overlap significantly showing that although the percentage in Block 3 appears higher, it could be due to chance or sample size.

\begin{tabular}{|l|r|r|r|}
\hline All Bifacial Tools & Block 1 & Block 3 & Block 4 \\
\hline UCL (95\%) & 4.43 & 9.79 & 6.74 \\
\hline$\%$ & 1.54 & 5.97 & 3.48 \\
\hline LCL (95\%) & 0.56 & 3.62 & 1.81 \\
\hline
\end{tabular}

Table 6.32 . The percentage of only the standardized identifiable bifacial tools in each block and the $95 \%$ binomial confidence limits. The intervals overlap significantly showing that the higher percentage in Block 3 could be due to chance or sample size.

\begin{tabular}{|l|r|r|r|}
\hline $\begin{array}{c}\text { Standardized } \\
\text { Bifacials }\end{array}$ & Block 1 & Block 3 & Block 4 \\
\hline UCL & 2.82 & 6.6 & 1.29 \\
\hline$\%$ & 0.51 & 2.99 & 0 \\
\hline$L C L$ & 0.13 & 1.77 & 0 \\
\hline
\end{tabular}

Table 6.33. Comparison of bifacial tool types in different blocks at el-Mahâsna.

\begin{tabular}{|c|c|c|c|c|c|c|}
\hline \multirow{2}{*}{ Bifacial Tool Type } & \multicolumn{2}{|c|}{ Block 1} & \multicolumn{2}{|c|}{ Block 3} & \multicolumn{2}{|c|}{ Block 4} \\
\hline & count & $\%$ & count & $\%$ & count & $\%$ \\
\hline RFK & 0 & 0 & 0 & 0 & 0 & 0 \\
\hline Fishtail & 0 & 0 & 0 & 0 & 0 & 0 \\
\hline Rhomboid & 0 & 0 & 0 & 0 & 0 & $\underline{0}$ \\
\hline Concave-Base PP & 0 & 0 & 2 & 0.85 & 0 & 0 \\
\hline Figural eccentric & 0 & 0 & 0 & 0 & 0 & 0 \\
\hline Other Fine Bifacials & 0 & 0 & 1 & 0.43 & 0 & 0 \\
\hline Bifacial Knife & 0 & 0 & 1 & 0.43 & 0 & 0 \\
\hline Bifacial Sickle & 0 & 0 & 0 & 0 & 0 & 0 \\
\hline Bifacial Plane & 1 & 0.51 & 0 & 0 & 0 & 0 \\
\hline Axe & 0 & 0 & 3 & 1.28 & 0 & 0 \\
\hline Drills:winged/crescent/cylindrical & 0 & 0 & 0 & 0 & 0 & 0 \\
\hline Pic/Grand Perçoir & 0 & 0 & 0 & 0 & 0 & 0 \\
\hline Non-standard, \& Unidentifiable & 2 & 1.03 & 6 & 2.55 & 8 & 3.48 \\
\hline Unfinished BFC & 0 & 0 & 1 & 0.43 & 0 & 0 \\
\hline Unfinished Concave-Base PP & 0 & 0 & 0 & 0 & 0 & 0 \\
\hline Unfinished Knife & 0 & 0 & 0 & 0 & 0 & 0 \\
\hline Preform/ Unfinished Axe & 0 & 0 & 0 & 0 & 0 & 0 \\
\hline $\begin{array}{l}\text { Other Tools (Bifacially Edged \& } \\
\text { Unifacial) }\end{array}$ & 192 & 98.46 & 221 & 94.04 & 222 & 96.52 \\
\hline total $\mathrm{N}$ of tools & 195 & 100 & 235 & 100.01 & 230 & 100 \\
\hline
\end{tabular}

Table 6.34. Percentages of bifacial production flakes and tranchet flakes in different sectors of Armant MA 21/83. Note that these counts do not include artifacts from features.

\begin{tabular}{|l|r|r|r|r|}
\hline & \multicolumn{2}{|c|}{ Northern part } & \multicolumn{2}{c|}{ Southern Part } \\
\hline Total \# of artifacts & \multicolumn{2}{|c|}{2818} & \multicolumn{2}{c|}{6691} \\
\hline count/\% & \multicolumn{1}{|c|}{ count } & \multicolumn{1}{c|}{$\%$} & count & \multicolumn{1}{c|}{$\%$} \\
\hline & & & & \\
Thinning flakes & 68 & 2.41 & 306 & 4.57 \\
\hline Axe prep flakes & 8 & 0.28 & 25 & 0.37 \\
\hline
\end{tabular}


Table 6.35. Percentages of bifacial tool types in different sectors of Armant MA 21/83.

\begin{tabular}{|c|c|c|c|c|}
\hline \multirow{2}{*}{ Bifacial Tools } & \multicolumn{2}{|c|}{ Northern part } & \multicolumn{2}{|c|}{ Southern Part } \\
\hline & count & $\%$ & count & $\%$ \\
\hline RFK & 0 & 0 & 0 & 0 \\
\hline Rhomboid & 0 & 0 & 0 & 0 \\
\hline Fishtail & 0 & 0 & 0 & 0 \\
\hline Concave-Base PP & 0 & 0 & 0 & 0 \\
\hline Figure & 0 & 0 & 0 & 0 \\
\hline Other Fine Bifacials & 0 & 0 & 0 & 0 \\
\hline Knife & 0 & 0 & 0 & 0 \\
\hline Bifacial Sickle & 0 & 0 & 2 & 0.48 \\
\hline Bifacial Plane & 3 & 1.43 & 4 & 0.97 \\
\hline Axe & 8 & 3.81 & 11 & 2.66 \\
\hline Drills: winged/crescent/cylindrical & 0 & 0 & 0 & 0 \\
\hline Pic/Grand Perçoir & 2 & 0.95 & 9 & 2.18 \\
\hline Non-standard, \& Unidentifiable & 0 & 0 & 0 & 0 \\
\hline Axe preform & 4 & 1.9 & 12 & 2.91 \\
\hline Bifacial tool preform & 6 & 2.86 & 40 & 9.69 \\
\hline $\begin{array}{l}\text { Other Tools (Bifacially Edged \& } \\
\text { Unifacial) }\end{array}$ & 187 & 89.05 & 335 & 81.11 \\
\hline Total \# of tools & 210 & 100 & 413 & 100 \\
\hline
\end{tabular}

Table 6.36. 95\% binomial confidence intervals for proportions of bifacial tools in different sectors of Armant MA 21/83.

\begin{tabular}{|l|r|r|}
\hline & Northern & Southern \\
\hline UCL (95\%) & 10.35 & 9.09 \\
\hline Bifacial tools & 6.19 & 6.29 \\
\hline LCL (95\%) & 3.69 & 4.35 \\
\hline
\end{tabular}

Table 6.37. Summary of the organization of production for bifacial tool types.

\begin{tabular}{|l|c|l|}
\hline \multicolumn{1}{|c|}{ Tool Type } & $\begin{array}{c}\text { Inter-site } \\
\text { specialization }\end{array}$ & $\begin{array}{c}\text { Intra- site } \\
\text { specialization }\end{array}$ \\
\hline RFKs & $\bullet$ & \\
\hline Fishtails & $\bullet$ & \\
\hline Rhomboids & $\bullet$ & \\
\hline CBPPs & $?$ & $\bullet$ \\
\hline Figural eccentrics & No & \\
\hline Bifacial Knives & Some & \\
\hline Bifacial Sickles & $\bullet$ & \\
\hline Axes & No & \\
\hline
\end{tabular}


Table 6.38. Counts and percentages of bifacial tool types at Hierakonpolis localities. $\bullet=$ Present. Sample sizes smaller than 30 are grayed-out.

\begin{tabular}{|c|c|c|c|c|c|c|c|c|c|c|c|c|c|c|c|c|c|c|}
\hline Site & \multicolumn{2}{|c|}{ HK11C } & \multicolumn{2}{|c|}{$\begin{array}{l}\text { HK11C } \\
\text { (A6-A7) }\end{array}$} & \multicolumn{2}{|c|}{ НК14 } & \multicolumn{2}{|c|}{ HK24A } & \multicolumn{2}{|c|}{$\begin{array}{c}\text { HK29 } \\
\text { (10L10) } \\
\end{array}$} & \multicolumn{2}{|c|}{ HK29At } & \multicolumn{2}{|c|}{ HK29B } & \multicolumn{2}{|c|}{ НК25 } & \multicolumn{2}{|c|}{ HK25D } \\
\hline Date & \multicolumn{2}{|c|}{$\begin{array}{c}\text { NIID and } \\
\text { later }\end{array}$} & \multicolumn{2}{|c|}{$3550 \mathrm{BC}$} & \multicolumn{2}{|c|}{3625 BC } & \multicolumn{2}{|c|}{$\begin{array}{c}\text { Late } \\
\text { Amratian }\end{array}$} & \multicolumn{2}{|c|}{$\begin{array}{c}\text { 3500BC Late } \\
\text { Amratian }\end{array}$} & \multicolumn{2}{|c|}{ NIID-NIIIA+† } & \multicolumn{2}{|c|}{$\begin{array}{c}\text { 2nd half 4th } \\
\text { mill BC }\end{array}$} & \multicolumn{2}{|c|}{ NIIC-D } & \multicolumn{2}{|c|}{$\begin{array}{c}\text { Late } \\
\text { Amratian }\end{array}$} \\
\hline Total \# of artifacts & \multicolumn{2}{|c|}{1190} & \multicolumn{2}{|c|}{3677} & \multicolumn{2}{|c|}{214} & \multicolumn{2}{|c|}{711} & \multicolumn{2}{|c|}{3557} & \multicolumn{2}{|c|}{$\sim 54000$} & \multicolumn{2}{|c|}{10451} & \multicolumn{2}{|c|}{7481} & \multicolumn{2}{|c|}{183} \\
\hline Total \# of tools & \multicolumn{2}{|c|}{131} & \multicolumn{2}{|c|}{307} & \multicolumn{2}{|c|}{16} & 35 & & 46 & & $\sim 19$ & & 27 & & 22 & 25 & 9 & \\
\hline & Count & $\%$ & Count & $\%$ & Count & $\%$ & Count & $\%$ & Count & $\%$ & Count & $\%$ & Count & $\%$ & Count & $\%$ & Count & $\%$ \\
\hline RFK & 0 & 0 & 0 & 0 & 0 & 0 & 0 & 0 & 0 & 0 & & 0 & 0 & 0 & 0 & 0 & 0 & 0 \\
\hline Rhomboid & 0 & 0 & 0 & 0 & 0 & 0 & 0 & 0 & 0 & 0 & & 0 & 0 & 0 & 0 & 0 & 0 & 0 \\
\hline Fishtail & 0 & 0 & 0 & 0 & 0 & 0 & 0 & 0 & 0 & 0 & & 0 & 0 & 0 & 0 & 0 & 0 & 0 \\
\hline Concave-Base PP & 0 & 0 & 0 & 0 & 0 & 0 & 0 & 0 & 0 & 0 & $\bullet$ & 0.3 & 0 & 0 & 0 & 0 & 0 & 0 \\
\hline Eccentric & 0 & 0 & 0 & 0 & 0 & 0 & 0 & 0 & 0 & 0 & & 0 & 0 & 0 & 0 & 0 & 0 & 0 \\
\hline Other Fine Bifacials & 2 & 1.53 & 3 & 0.98 & 0 & 0 & 0 & 0 & 2 & 0.43 & $\bullet$ & 1.1 & 0 & 0 & 0 & 0 & 0 & 0 \\
\hline Bifacial Knife & 0 & 0 & 0 & 0 & 0 & 0 & 0 & 0 & 1 & 0.21 & $\bullet$ & 0 & 0 & 0 & 0 & 0 & 1 & 11.11 \\
\hline Bifacial Sickle & 0 & 0 & 0 & 0 & 0 & 0 & 0 & 0 & 0 & 0 & & 0 & 0 & 0 & 0 & 0 & 0 & 0 \\
\hline Bifacial Plane & 0 & 0 & 0 & $\underline{0}$ & 0 & 0 & 0 & 0 & 0 & 0 & & 0 & 0 & 0 & 0 & 0 & 0 & 0 \\
\hline Axe & 0 & 0 & 0 & 0 & 0 & 0 & 0 & 0 & 0 & 0 & & 0 & 0 & 0 & 0 & 0 & 0 & 0 \\
\hline Drills: winged/crescent/cylindrical & 0 & 0 & 3 & 0.98 & 0 & 0 & 0 & 0 & 14 & 2.99 & $\bullet$ & 0.1 & 0 & 0 & 0 & 0 & 0 & 0 \\
\hline Pic/Grand Perçoir & 0 & 0 & 0 & 0 & 0 & 0 & 0 & 0 & 0 & 0 & & 0 & 0 & 0 & 0 & 0 & 0 & 0 \\
\hline Non-standard, \& Unidentifiable & 1 & 0.76 & 7 & 2.28 & 0 & 0 & 1 & 2.86 & 18 & 3.85 & $\bullet$ & 0.5 & 12 & 4.3 & 13 & 5.78 & 0 & 0 \\
\hline Other Tools (Bifacially Edged \& Unifacial) & 128 & 97.71 & 294 & 95.77 & 16 & 100 & 34 & 97.14 & 433 & 92.52 & $?$ & $?$ & 267 & 95.7 & 212 & 94.22 & 8 & 88.89 \\
\hline Total & 131 & 100 & 307 & 100.01 & 16 & 100 & 35 & 100 & 468 & 100 & 0 & & 279 & 100 & 225 & 100 & 9 & 100 \\
\hline
\end{tabular}




\section{Chapter 7 Tables}

Table 7.1. List of museums, excavation projects, or other entities, and their abbreviations.

\begin{tabular}{|c|c|c|}
\hline Abbreviation & Museum or Responsible entity & Location \\
\hline ÄM & Ägyptisches Museum & Berlin, Germany \\
\hline Ash & Ashmolean Museum & Oxford, UK \\
\hline BM & British Museum & London, UK \\
\hline BMFA & Boston Museum of Fine arts & Boston, MA, USA \\
\hline BrM & Brooklyn Museum & Brooklyn, NY, USA \\
\hline CMA & Clevland Museum of Art & Clevland, OH, USA \\
\hline EM & Egyptian Museum & Cairo, Egypt \\
\hline FwM & Fitzwiliam Museum & Cambridge, UK \\
\hline Hild & Roemer- und Pelizaeus-Museum Hildesheim & Hildesheim, Germany \\
\hline Kassel & Staatliche Museen Kassel & Kassel, Germany \\
\hline KhM & Kunsthistorisches Museum (Art History Museum) & Vienna, Austria \\
\hline LACMA & Los Angeles County Museum of Art & Los Angeles, USA \\
\hline \begin{tabular}{|l|l}
$\mathrm{LV}$ \\
\end{tabular} & Louvre & Paris, France \\
\hline LvpM & World Museum of Liverpool & Liverpool, UK \\
\hline ManM & Manchester Museum & Manchester, UK \\
\hline MANStGL & Musée Archéologie Nationale & St. Germain-en-Laye, France \\
\hline MCCM & Michael C. Carlos Museum & Atlanta, GA, USA \\
\hline MdO & Museo delle Origine & Rome, Italy \\
\hline MMA & Metropolitan Museum of Art & New York, NY, USA \\
\hline MRM & Musée Royal de Mariemont & Morlanwelz, Belgium \\
\hline NMI & National Museum of Ireland & Dublin, Ireland \\
\hline NMA & Nubian Museum & Aswan, Egypt \\
\hline OIM & Oriental Institute Museum & Chicago, IL, USA \\
\hline PAHMA & Phoebe A. Hearst Museum of Anthropology & Berkeley, CA, USA \\
\hline PM & University of Pennsylvania Museum & Philadelphia, PA, USA \\
\hline PR & Pitt Rivers Museum & Dxford, UK \\
\hline RISD & Rhode Island School of Design Museum & Providence, RI, USA \\
\hline RVFAM & Robert V. Fullerton Art Museum & San Bernadino, CA, USA \\
\hline RMAH & Royal Museums of Art and History & Brussels, Belgium \\
\hline SNHM & Smithsonian Natural History Museum & Washington, DC, USA \\
\hline UC & Petrie Museum (University College London) & London, UK \\
\hline \multirow[t]{2}{*}{\begin{tabular}{|l|l|} 
YPM \\
\end{tabular}} & Yale Peabody Museum & New Haven, CT, USA \\
\hline & Project/Site/Responsible entity & \\
\hline Abydos / A.U & Abydos & German dig house at Abydos \\
\hline $\mathrm{AMC}$ & Abydos Middle Cemetery & American dig house at Abydos \\
\hline Ad & Adaima & French storeroom for Adaima \\
\hline AKAP & Aswan-Kom-Ombo Archaeological Project & Government store rooms in Kom Ombo \\
\hline $\mathrm{CAH}$ & Christie's Auction House & Location of items unknown \\
\hline EK & Elkab & Belgian Dig house at Elkab \\
\hline FGC & Former Garstang Collection & Location of items unknown \\
\hline FMcGC & Former McGregor Collection & Location of items unknown \\
\hline Gnefer & Galerie Nefer & Location of items unknown \\
\hline HK & Hierakonpolis & Storerooms for Hierakonpolis \\
\hline KAS & Kom al-Ahmar /Sharuna & Storerooms for Kom al-Ahmar /Sharuna \\
\hline MAP & El-Mahâsna & American dig house at Abydos \\
\hline $\mathrm{MbC}$ & Mildenberg Collection & Location of items unknown \\
\hline
\end{tabular}


Table 7.2 (part 1). Ripple-flaked knives with identifiable raw materials.

\begin{tabular}{|c|c|c|c|c|c|}
\hline Site & ID & RM & $\begin{array}{l}\text { Light/ } \\
\text { Dark }\end{array}$ & Notes & Publication \\
\hline Unknown & BM.EA.29286 & Other:VTO & Light & & \\
\hline Unknown & BM.EA.29289 & $1,2,4$ & Light & & \\
\hline Abydos? & BM.EA.29290 & 4 & Light & & \\
\hline Unknown & BM.EA.32095 & $1,2,4$ & Light & & \\
\hline Unknown & BM.EA.32096 & Other:VTO & Light & & \\
\hline Unknown & BM.EA.32489 & 7 & Dark & & \\
\hline Unknown & BM.EA.59235 & $1,2,4$ & Light & & \\
\hline Unknown & BM.EA.68512 & 4 & Light & $\begin{array}{l}\text { "Pitt-rivers Knife". Ivory } \\
\text { handle. Purchased. }\end{array}$ & \\
\hline Naqada & BMFA.03.1390 & Other:VTO & Light & & \\
\hline Abu Zaidan & BrM.09.889.118 & 2,4 & Light & $\begin{array}{l}\text { "Abu Zaidan Knife." Ivory } \\
\text { handle. Tomb } 32 .\end{array}$ & $\begin{array}{l}\text { Midant-Reynes 1987; } \\
\text { Needler 1984:268-271 }\end{array}$ \\
\hline Abu Zaidan & BrM.09.889.120 & Other:VTO & Light & Tomb 32. & Needler 1984:272-3 \\
\hline Adaima & BrM.09.889.121 & $1,2,4$ & Light & & Needler 1984:271 \\
\hline Harageh & CMA.1915.30 & 4 & Light & Cemetery G Tomb 413. & \\
\hline Abydos & $\begin{array}{l}\text { EM.Abydos.U.50 } \\
3\end{array}$ & 4 & Light & Ivory Handle. Tomb 503. & $\begin{array}{l}\text { Hikade 2013: PI1; Hikade } \\
\text { 2003:Fig } 3\end{array}$ \\
\hline Gebel Tarif? & EM.Gebel Tarif & 4 & Light & $\begin{array}{l}\text { "Gebel Tarif knife." Gold } \\
\text { handle. }\end{array}$ & \\
\hline Tel el Farkha & EM.R.536 & 5 & Dark & & \\
\hline Unknown & FwM.E.204.1939 & 4 & Light & & \\
\hline Unknown & FwM.E.48.1899 & 4 & Light & & \\
\hline Unknown & $\begin{array}{l}\text { FwM.E.GA.4193. } \\
1943\end{array}$ & 4 & Light & & \\
\hline Unknown & KhM.8017 & $1,2,4$ & Light & & \\
\hline Gebel Arak & LV.E 11517 & 4 & Light & $\begin{array}{l}\text { "Gebel Arak Knife." Ivory } \\
\text { Handle. }\end{array}$ & Delange 2009; Dreyer 1999 \\
\hline Unknown & ManM.38160 & Other:VTO & Light & & \\
\hline Gerzeh & ManM.5305.a-e & Other:VTO & Light & & \\
\hline Unknown & $\begin{array}{l}\text { MCCM.L.2012.28 } \\
.1\end{array}$ & 4 & Light & & \\
\hline Unknown & MMA.11.167.1 & 4 & Light & & \\
\hline Unknown & MMA.16.2.22 & Other:VTO & Light & & \\
\hline Unknown & MMA.26.241.1 & $\begin{array}{l}\text { Other: } \\
\text { Chocolate }\end{array}$ & Dark & $\begin{array}{l}\text { "Carter knife" or the "New } \\
\text { York knife." Ivory Handle. }\end{array}$ & \\
\hline Unknown & MMA.7228.105 & 2,4 & Light & & \\
\hline Qushtamna & NMA.187 & 2,4 & Light & & $\begin{array}{l}\text { Gaballa N.D. Firth 1912:9, } \\
\text { PI 38a }\end{array}$ \\
\hline Unknown & OIM.10533 & 4 & Light & & \\
\hline Unknown & OIM.9390 & 4 & Light & $\begin{array}{l}\text { registration number:E } 9390 \\
\text { A\&B, Accession number: } \\
138\end{array}$ & \\
\hline Unknown & OIM.9391a-b & 5 & ? & & \\
\hline Naga-ed-Deir & PAHMA.6.4752 & Other:VTO & Light & Cemetery 7000 Tomb 151 & \\
\hline Abydos & PR.1900.42.2 & Other:VTO & Light & Cemetery G & Petrie 1902 \\
\hline Abydos & PR.1901.40.23 & 4 & Light & Um el Gaab- Tomb of Djer & Petrie 1901 \\
\hline Gerzeh & PR.1911.33.1 & Other:VTO & Light & Grave 25 & $\begin{array}{l}\text { Petrie. 1912; Stevenson } \\
2009\end{array}$ \\
\hline Unknown & RISD.23.002 & Other:VTO & Light & & \\
\hline Fayoum & RMAH.E.01236 & $1,2,4$ & Light & & Global Egyptian Museum \\
\hline
\end{tabular}


Table 7.2 (part 2). Ripple-flaked knives with identifiable raw materials. In all cases, especially when no publication is given, the publication reference is the online collection database of the museum for that item. See Table 7.1 for museum list and abbreviations. "Other:VTO" = "Other: Variable Translucent - Opaque."

\begin{tabular}{|l|l|l|l|l|l|}
\hline \multicolumn{1}{|c|}{ Site } & \multicolumn{1}{|c|}{ ID } & \multicolumn{1}{c|}{ RM } & $\begin{array}{l}\text { Light/ } \\
\text { Dark }\end{array}$ & \multicolumn{1}{c|}{ Notes } & \multicolumn{1}{c|}{ Publication } \\
\hline Unknown & UC.16294 & $\begin{array}{l}\text { Other: } \\
\text { Chocolate }\end{array}$ & Dark & $\begin{array}{l}\text { “London knife” or “UCL } \\
\text { knife." Ivory handle. }\end{array}$ & \\
\hline Abydos & UC.35723 & $\begin{array}{l}\text { Other: } \\
\text { Chocolate }\end{array}$ & Dark & Um el Gaab- Tomb of Djer & \\
\hline Ballas/Naqada & UC.6147 & 2,4 & Light & & \\
\hline Abydos? & UC.73354 & $1,2,4$ & Light & & Petrie \& Quibell 1896: PI 86. \\
\hline Abydos? & UC.73355 & 2,4 & Light & & Petrie \& Quibell 1896: PI 86. \\
\hline Unknown & UC.73364 & 5 & Dark? & & Petrie \& Quibell 1896: PI 86. \\
\hline
\end{tabular}

Table 7.3. Raw material types of different specialist produced tool types. Blanks $=0$. New raw material groups include: 'Other: variable translucent-opaque', which is light in color ranging from beiges to light or medium yellowish browns, with opaque and translucent patches that occurred in swirls or large bands; 'Other: chocolate' which is homogeneous dark brown, fine grained, and with chalky white cortex, it corresponds to a raw material described by Hikade (2013:23, PI 2c-d) as looking like chocolate.

\begin{tabular}{|c|c|c|c|c|c|c|c|c|c|c|c|c|c|c|c|c|c|}
\hline \multirow[b]{2}{*}{ Raw material } & \multirow[b]{2}{*}{$\begin{array}{l}\text { Light/ } \\
\text { Dark }\end{array}$} & \multicolumn{2}{|c|}{ RFKs } & \multicolumn{2}{|c|}{ Rhomboids } & \multicolumn{2}{|c|}{ Fishtails } & \multicolumn{2}{|c|}{ CBPPs } & \multicolumn{2}{|c|}{$\begin{array}{l}\text { Bifacial } \\
\text { sickles }\end{array}$} & \multicolumn{2}{|c|}{ Axes } & \multicolumn{2}{|c|}{$\begin{array}{l}\text { Large blade } \\
\text { knives }\end{array}$} & \multicolumn{2}{|c|}{$\begin{array}{c}\text { Micro- } \\
\text { endscrapers }\end{array}$} \\
\hline & & Count & $\%$ & Count & $\%$ & Count & $\%$ & Count & $\%$ & Count & $\%$ & Count & $\%$ & Count & $\%$ & Count & $\%$ \\
\hline Agate (?) & Light & & & & & 1 & .99 & & & & & & & & & & \\
\hline Rock Crystal & & & & & & 1 & .99 & & & & & & & & & & \\
\hline 1. Indistinct Beige & Light & & & & & & & & & & & 15 & 18.29 & 10 & 14.08 & & \\
\hline 2. Beige (Fine) & Light & & & & & & & & & & & 4 & 4.88 & 15 & 21.13 & & \\
\hline 4. Beige $w /$ Pink Bands & Light & 14 & 31.82 & & & 15 & 14.85 & 1 & 3.23 & 1 & 5 & 2 & 2.44 & & & & \\
\hline 1,2,4 Indistinguishable & Light & 12 & 27.27 & 5 & 25 & 20 & 19.8 & 4 & 12.9 & 4 & 20 & 34 & 41.46 & 29 & 40.85 & & \\
\hline $\begin{array}{l}\text { 8. Brown Translucent } \\
\text { with White Mottles }\end{array}$ & Light & & & & & 9 & 8.91 & 1 & 3.23 & 3 & 15 & 8 & 9.76 & 2 & 2.82 & 11 & 44 \\
\hline $\begin{array}{l}\text { 10. Pink-Grey } \\
\text { Translucent }\end{array}$ & Light & & & & & 1 & 99 & & & 1 & 5 & 2 & 2.44 & & & 2 & 8 \\
\hline 11. Pink-Purple-Red & Light & & & & & & & 1 & 3.23 & & & & & & & 6 & 24 \\
\hline 13. Caramel & Light & & & & & & & 6 & 19.35 & & & & & 1 & 1.41 & & \\
\hline Other: VTO & Light & 11 & 25 & 3 & 15 & 2 & 1.98 & & & & & & & & & & \\
\hline Other Light Cherts & Light & & & & & 3 & 2.97 & 2 & 6.45 & & & 8 & 9.76 & 3 & 4.23 & 6 & 24 \\
\hline $\begin{array}{l}\text { Other Indeterminate or } \\
\text { light \& dark mixed }\end{array}$ & Both & & & & & & & & & & & 1 & 1.22 & 2 & 2.82 & & \\
\hline $\begin{array}{l}5,6,7,8 \text { Indeterminate } \\
\text { Mid-Browns }\end{array}$ & Dark? & & & & & 4 & 3.96 & & & 1 & 5 & 4 & 4.88 & 4 & 5.63 & & \\
\hline $\begin{array}{l}\text { 5. Medium Brown } \\
\text { Homogeneous }\end{array}$ & Dark & 3 & 6.82 & & & & & & & & & & & & & & \\
\hline $\begin{array}{l}\text { 6. Brown w/ } \\
\text { Foraminifera }\end{array}$ & Dark & & & & & & & & & & & & & & & & \\
\hline 7. Dark Grey and Brown & Dark & 1 & 2.27 & 11 & 55 & 35 & 34.65 & 14 & 45.16 & 8 & 40 & 1 & 1.22 & 4 & 5.63 & & \\
\hline $\begin{array}{l}\text { Other Dark Grey or } \\
\text { Black Cherts }\end{array}$ & Dark & & & 1 & 5 & 8 & 7.92 & 2 & 6.45 & 2 & 10 & 3 & 3.66 & 1 & 1.41 & & \\
\hline Other: Chocolate & Dark & 3 & 6.82 & & & & & & & & & & & & & & \\
\hline Obsidian & Dark & & & & & 2 & 1.98 & & & & & & & & & & \\
\hline TOTAL & & 44 & 100 & 20 & 100 & 101 & 99.99 & 31 & 100 & 20 & 100 & 82 & 100 & 71 & 100 & 25 & 100 \\
\hline
\end{tabular}


Table 7.4. Frequencies of dark and light chert varieties by tool type.

\begin{tabular}{|c|c|c|c|c|c|c|c|c|c|c|c|c|c|c|c|c|c|c|}
\hline & 늠 & $\frac{u}{x}$ & $\begin{array}{l}\frac{u}{c} \\
\frac{c}{c} \\
\frac{\varepsilon}{c} \\
\frac{c}{\alpha}\end{array}$ & & $\frac{\lambda}{\frac{2}{\pi}}$ & $\begin{array}{l}\frac{n}{\sqrt{0}} \\
\frac{c}{\frac{n}{4}}\end{array}$ & & $\begin{array}{l}\frac{n}{\sqrt{\pi}} \\
\frac{c}{\frac{n}{4}}\end{array}$ & 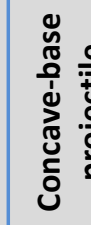 & $\frac{0}{0}$ & $\begin{array}{l}\bar{\pi} \\
\frac{\pi}{0} \\
\frac{\pi}{\infty}\end{array}$ & $\frac{\mathscr{y}}{\frac{u}{u}}$ & y & $\begin{array}{l}\text { yx } \\
x \\
x\end{array}$ & & $\frac{\text { }}{\frac{2}{2}}$ & & 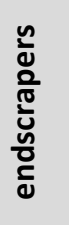 \\
\hline & Count & $\%$ & Count & $\%$ & Count & $\%$ & Count & $\%$ & Count & $\%$ & Count & $\%$ & Count & $\%$ & Count & $\%$ & Count & $\%$ \\
\hline $\begin{array}{l}\text { Light } d s \\
\quad \underline{h d}\end{array}$ & 37 & 84.09 & 8 & 40 & 19 & 35.19 & 28 & 77.78 & 15 & 48.39 & 8 & 40 & 73 & 89.02 & 60 & 84.51 & 25 & 100 \\
\hline $\begin{array}{c}\text { Dark ds } \\
\text { km }\end{array}$ & 7 & 15.91 & 12 & 60 & 31 & 57.41 & 8 & 22.22 & 16 & 51.61 & 11 & 55 & 8 & 9.76 & 9 & 12.68 & 0 & 0 \\
\hline $\begin{array}{c}\text { In- } \\
\text { determi } \\
\text { nate }\end{array}$ & 0 & 0 & 0 & 0 & 4 & 7.41 & 0 & 0 & 0 & 0 & 1 & 5 & 1 & 1.22 & 2 & 2.82 & 0 & 0 \\
\hline TOTAL & 44 & 100 & 20 & 100 & 54 & 100.01 & 36 & 100 & 31 & 100 & 20 & 100 & 82 & 100 & 71 & $\begin{array}{c}100.0 \\
1\end{array}$ & 25 & 100 \\
\hline
\end{tabular}

Table 7.5. Locations of provenienced RFKs, by raw material type. Note that "Other:VTO" is an abbreviation for "Other: Variable Translucent - Opaque."

\begin{tabular}{|l|r|r|r|r|r|}
\hline \multicolumn{1}{|c|}{ Site } & Type 4 & \multicolumn{1}{c|}{$\begin{array}{c}\text { Type } \\
\mathbf{1 , 2 , 4}\end{array}$} & $\begin{array}{c}\text { Type } \\
\text { Other: } \\
\text { VTO }\end{array}$ & Type 5 & $\begin{array}{c}\text { Type } \\
\text { Other: } \\
\text { Chocolate }\end{array}$ \\
\hline Tel El Farkha & & & & 1 & \\
\hline Gerzeh & & & 2 & & \\
\hline Faiyum & & 1 & & & \\
\hline Haragah & 1 & & & & \\
\hline Naga ed-deir & & & 1 & & \\
\hline Abydos & 3 & 2 & 1 & & \\
\hline Gebel Tarif & 1 & & & & \\
\hline Ballas & & 1 & & & \\
\hline Naqada & & & & & \\
\hline Adaima & & 1 & & & \\
\hline Abu Zaidan & & 1 & 1 & & \\
\hline Qushtamna & & 1 & & & \\
\hline
\end{tabular}


Table 7.6. Rhomboid tools with identifiable raw materials. Note that "Other:VTO" is an abbreviation for "Other: Variable Translucent - Opaque." In all cases, especially when no publication is given, the publication reference is the online collection database of the museum for that item. See Table 7.1 for the list of museums and their abbreviations.

\begin{tabular}{|c|c|c|c|c|c|}
\hline Site & ID & RM & Light/ Dark & Notes & Reference \\
\hline Abydos & A.U. 220 & 7 & Dark & Tomb U220 & $\begin{array}{l}\text { Personal observation; } \\
\text { Hikade } 2000\end{array}$ \\
\hline Unknown & BM.EA.34297 & 7 & Dark & & \\
\hline Unknown & BM.EA.49723 & Other: VTO & Light & & \\
\hline Unknown & BM.EA.52848 & 2,4 & Light & & \\
\hline Mesaeed & BMFA.13.3766 & 7 & Dark & & \\
\hline Unknown & CMA.1914.672 & 2 & Light & & \\
\hline Unknown & EM.52852 & 7 & Dark & & \\
\hline Unknown & FwM.E.GA.4140.1943 & 7 & Dark & & \\
\hline Unknown & FwM.E.GA.4224.1943 & Other: VTO & Light & & \\
\hline HK6/25 & HK6.25.unknown & $\begin{array}{l}\text { Other: Murky } \\
\text { Black }\end{array}$ & Dark & $\begin{array}{l}\text { From "pillared halls", } \\
\text { so HK25 or HK6 }\end{array}$ & Nagaya 2011 \\
\hline HK6 & HK6.2009.30 & 7 & Dark & & Droux 2009:15 \\
\hline HK6 & HK6.2014.T72.439 & $1,2,4$ & Light & Tomb 72 & Droux 2014:4-7, 18-19 \\
\hline Naqada & ManM.2428 & Other: VTO & Light & & $\begin{array}{l}\text { Petrie \& Quibell } \\
\text { 1896:PI 72, no. } 52\end{array}$ \\
\hline Unknown & MMA.16.2.11 & 7 & Dark & & \\
\hline Unknown & MMA.16.2.17 & $1,2,4$ & Light & & \\
\hline Unknown & MMA.7.228.106 & 7 & Dark & & \\
\hline Naqada & RMAH.E.06185c & 7 & Dark & Royal tomb & \\
\hline Naqada & UC.4130 & 2,4 & Light & Tomb 1437 & $\begin{array}{l}\text { Baumgartel 1970; } \\
\text { Payne 1987 }\end{array}$ \\
\hline Naqada & UC.4389 & 7 & Dark & Tomb 1241 & \\
\hline Naqada/Ballas & UC.4828 & 7 & Dark & Tomb 1898 & \\
\hline
\end{tabular}

Table 7.7. Locations of provenienced rhomboid tools, by raw material type. Note that "Other: VTO" is an abbreviation for "Other: Variable Translucent Opaque."

\begin{tabular}{|l|c|c|c|c|}
\hline \multirow{2}{*}{\multicolumn{1}{|c|}{ Site }} & \multicolumn{2}{c|}{ Light } & \multicolumn{2}{c|}{ Dark } \\
\cline { 2 - 5 } & $\begin{array}{c}\text { Type } \\
\mathbf{1 , 2 , 4}\end{array}$ & $\begin{array}{c}\text { Type } \\
\text { Other: VTO }\end{array}$ & Type 7 & $\begin{array}{c}\text { Type } \\
\text { Murky } \\
\text { Black }\end{array}$ \\
\hline Mesaeed & & & 1 & \\
\hline Abydos & & & 1 & \\
\hline Naqada & 1 & 1 & 3 & \\
\hline HK6 & 1 & & 1 & 1 \\
\hline
\end{tabular}


Table 7.8 (part 1): Fishtails.

\begin{tabular}{|c|c|c|c|c|c|c|}
\hline Site & ID & RM & $\begin{array}{l}\text { Light/ } \\
\text { Dark }\end{array}$ & $\begin{array}{l}\text { Shape } \\
\text { Type }\end{array}$ & Notes & Reference \\
\hline Abydos & A.U.127 & 4 & Light & 2 & Tomb U 127 & $\begin{array}{l}\text { Hikade 1998, 2001, } \\
\text { 2003: } 145\end{array}$ \\
\hline Abydos & A.U.178 & $\begin{array}{l}\text { Other: } \\
\text { Dark Gray }\end{array}$ & Dark & 1 & Tomb U 178 & $\begin{array}{l}\text { Hikade 2003:144; } \\
\text { personal observation }\end{array}$ \\
\hline Abydos & A.U.211 & 7 & Dark & 1 & Tomb U 211 & Hikade 2003: 144 \\
\hline Abydos & A.U. 279 & 7 & Dark & 1 & Tomb U 279 & $\begin{array}{l}\text { Hikade 2003: 145; } \\
2013 \text { Pl1 }\end{array}$ \\
\hline Abydos & A.U.381a? & 8 & Light & 3 & Tomb U381a & $\begin{array}{l}\text { Hikade 2003:144; } \\
\text { personal observation }\end{array}$ \\
\hline Abydos & A.U.395 & 4 & Light & 3 & Tomb U 395 & $\begin{array}{l}\text { Hikade 2003:143; } \\
\text { personal observation }\end{array}$ \\
\hline Unknown & BM.EA.32098 & 2,4 & Light & 2 & & \\
\hline Unknown & BM.EA.32496 & 7 & Dark & 3 & & \\
\hline Abydos & BM.EA.37279 & 8 & Light & Other & From Osiris temple & $\begin{array}{l}\text { Petrie 1902:24, PI 51, } \\
\text { no.22 }\end{array}$ \\
\hline Unknown & BM.EA.59240 & 8 & Light & $1 a$ & & \\
\hline Unknown & BM.EA.59241 & 7 & Dark & 1 & & \\
\hline Abadiya & BMFA.03.1386 & $\begin{array}{l}\text { Other IND } \\
\text { Light }\end{array}$ & Light & 1 & & \\
\hline Abadiya & BMFA.03.1387 & 7 & Dark & 1 & & \\
\hline Unkown & BMFA.03.1391 & 4 & Light & 1 & & \\
\hline Mesaeed & BMFA.11.247 & 8 & Light & 1 & Tomb 26.1 & \\
\hline Mesaeed & $\begin{array}{l}\text { BMFA.11.248 } \\
\& 11.249\end{array}$ & 7 & Dark & Frag. & $\begin{array}{l}\text { Tomb 40.2. RM designation } \\
\text { based on description }\end{array}$ & \\
\hline Mesaeed & BMFA.11.250 & 4 & Light & 3 & Tomb 123.13 & \\
\hline Mesaeed & $\begin{array}{l}\text { BMFA.11.251\&2 } \\
52\end{array}$ & 4 & Light & 1/1a Frag. & Tomb 26.5a\&b & \\
\hline Mesaeed & BMFA.11.257 & 8 & Light & 1 & Tomb 123.14 & \\
\hline Mesaeed & BMFA.11.259 & 8 & Light & 3 & Tomb 123.15 & \\
\hline Giza & BMFA.11.765 & 7 & Dark & & $\begin{array}{l}\text { Ground Fishtail. Inscribed. } \\
\text { Menkaure valley temple. }\end{array}$ & \\
\hline Giza & BMFA.11.766 & 7 & Dark & & $\begin{array}{l}\text { Ground Fishtail. Inscribed. } \\
\text { Menkaure valley temple. }\end{array}$ & \\
\hline Mesaeed & BMFA.13.3915 & 2,4 & Light & 1 & Tomb 825 & \\
\hline $\begin{array}{l}\text { el- } \\
\text { Ma'mariya }\end{array}$ & BrM.07.447.870 & 5,7 & ? & $1 a$ & Tomb 83 & $\begin{array}{l}\text { Needler 1984:266- } \\
267\end{array}$ \\
\hline $\begin{array}{l}\text { Unknown- } \\
\text { Akhmim? }\end{array}$ & BrM.35.1445 & Obsidian & Dark & Other & $\begin{array}{l}\text { Purchased, said to come from } \\
\text { Akhmim }\end{array}$ & $\begin{array}{l}\text { Needler 1984:274- } \\
275\end{array}$ \\
\hline Unknown & CAH.1032.4-1 & $\begin{array}{l}\text { Other IND } \\
\text { Light }\end{array}$ & Light & $1 a$ & & \\
\hline Unknown & САН.1032.4-2 & 7 & Dark & 1 & & \\
\hline Unknown & САН.1691.4 & $\begin{array}{l}\text { Other: } \\
\text { Dark Gray }\end{array}$ & Dark & 3 & Wrapped gold handle. & \\
\hline Unknown & CAH.unknown & $\begin{array}{l}\text { Other: } \\
\text { Dark Gray }\end{array}$ & Dark & 3 & Wrapped gold handle. & \\
\hline Unknown & CMA.1914.673 & 7 & Dark & 1 & & \\
\hline Unknown & CMA.1914.674 & $\begin{array}{l}\text { Other: } \\
\text { Dark Gray }\end{array}$ & Dark & $1 a$ & & \\
\hline Unknown & CMA.1914.717 & $1,2,4$ & Light & 2 & & \\
\hline
\end{tabular}


Table 7.8 (part 2): Fishtails.

\begin{tabular}{|c|c|c|c|c|c|c|}
\hline Site & ID & RM & $\begin{array}{l}\text { Light/ } \\
\text { Dark }\end{array}$ & $\begin{array}{l}\text { Shape } \\
\text { Type }\end{array}$ & Notes & Reference \\
\hline Unknown & EM.34210 & 4 & Light & 2 & $\begin{array}{l}\text { Elaborate gold handle, fake? } \\
\text { (Additional reference number: } \\
64868 \text { ) }\end{array}$ & $\begin{array}{l}\text { Currelley 1913:272, } \\
\text { Pl47 }\end{array}$ \\
\hline Unknown & EM.34813 & $1,2,4$ & Light & 2 & & \\
\hline Unknown & EM.67932 & $\begin{array}{l}\text { Rock } \\
\text { crystal }\end{array}$ & Light & Other & & \\
\hline Unknown & FwM.E.214.1932 & $\begin{array}{l}\text { Other IND } \\
\text { Light }\end{array}$ & Light & 2 & & \\
\hline Unknown & FwM.E.49.1899 & 8 & Light & 1 & & \\
\hline Unknown & FwM.E.50.1899 & 7 & Dark & 1 & & \\
\hline $\begin{array}{l}\text { Diospolis } \\
\text { Parva }\end{array}$ & FwM.E.51.1899 & 7 & Dark & 1 & Burial 86 & \\
\hline Unknown & FwM.E.52.1899 & 2,4 & Light & 2 & & \\
\hline Unknown & $\begin{array}{l}\text { FwM.E.GA.4144.194 } \\
3\end{array}$ & Agate? & Light & $1 a$ & & \\
\hline Unknown & $\begin{array}{l}\text { FwM.E.GA.4146.194 } \\
3\end{array}$ & 4 & Light & 1 & & \\
\hline Unknown & $\begin{array}{l}\text { FwM.E.GA.4148.194 } \\
3\end{array}$ & 4 & Light & 2 & & \\
\hline Unknown & FwM.E.Misc.94 & 4 & Light & $1 a$ & & \\
\hline Unknown & FwM.GA.3162.1943 & 4 & Light & 2 & & \\
\hline Unknown & FwM.GA.3174.1943 & $5,6,8$ & $?$ & $1 a$ & & \\
\hline Unknown & Hild.5106 & $1,2,4$ & Light & 2 & & \\
\hline HK43 & HK.43.2004 & $\begin{array}{l}\text { Other: } \\
\text { Dark Gray }\end{array}$ & Dark & 3 & Burial 412 & Friedman 2004:8-9 \\
\hline HK6 & HK6.2006.2 & $\begin{array}{l}\text { Other: } \\
\text { Dark Gray }\end{array}$ & Dark & $1 a$ & Structure E8 & $\begin{array}{l}\text { Friedman 2006:7-8; } \\
\text { Nagaya } 2011\end{array}$ \\
\hline Unknown & LACMA.1998.94.1 & 4 & Light & 2 & & \\
\hline Abydos? & LvpM.1973.2.254 & 2,4 & Light & 3 & $\begin{array}{l}\text { Also listed as number } \\
56.20 .23\end{array}$ & $\begin{array}{l}\text { Global Egyptian } \\
\text { Museum }\end{array}$ \\
\hline Naqada & LvpM.56.20.42 & 7 & Dark & 2 & $\begin{array}{l}\text { Additional reference number } \\
\text { 1978.291.266 }\end{array}$ & \\
\hline Naqada & ManM.2424 & 4 & Light & 2 & & $\begin{array}{l}\text { Petrie \& Quibell 1896: } \\
\text { PI. } 73 \text { no. } 63\end{array}$ \\
\hline Unknown & ManM.5576 & 7 & Dark & 1 & & \\
\hline Naqada & ManM.5577 & 7 & Dark & 3 & & $\begin{array}{l}\text { Petrie \& Quibell 1896: } \\
\text { PI. } 73 \text { no. } 62\end{array}$ \\
\hline Naqada & ManM.5579 & 7 & Dark & 1 & & $\begin{array}{l}\text { Petrie \& Quibell 1896: } \\
\text { PI. } 72 \text { no. } 66\end{array}$ \\
\hline Naqada & ManM.5581.a-b & $1,2,4$ & Light & $\begin{array}{l}\text { 1/1a } \\
\text { Frag. }\end{array}$ & & $\begin{array}{l}\text { Petrie \& Quibell 1896: } \\
\text { PI. } 73 \text { no. } 66\end{array}$ \\
\hline MAP & MAP.301.1 & 7 & Dark & $1 a$ & & Here \\
\hline Unknown & MMA.10.130.1222 & 2,4 & Light & 2 & & \\
\hline Unknown & MMA.10.130.1223 & 8 & Light & 1 & & \\
\hline Unknown & MMA.10.176.96 & 7 & Dark & $1 a$ & & \\
\hline Unknown & MMA.16.2.4 & 2,4 & Light & 2 & & \\
\hline Unknown & MMA.16.2.8 & 7 & Dark & 1 & & \\
\hline Unknown & MMA.20.5 & 4 & Light & 3 & $\begin{array}{l}\text { Found with wrapping over the } \\
\text { forked end }\end{array}$ & \\
\hline
\end{tabular}


Table 7.8 (part 3). Fishtails with identifiable raw materials.

\begin{tabular}{|c|c|c|c|c|c|c|}
\hline Site & ID & RM & $\begin{array}{l}\text { Light/ } \\
\text { Dark }\end{array}$ & $\begin{array}{l}\text { Shape } \\
\text { Type }\end{array}$ & Notes & Reference \\
\hline Abydos? & MMA.24.2.13 & Obsidian & Dark & $2 a$ & & \\
\hline Unknown & MMA.7.228.162 & 2,4 & Light & 1 & & \\
\hline \begin{tabular}{|l|} 
Khor \\
Bahan
\end{tabular} & NMA.133 & $\begin{array}{l}\text { Other: } \\
\text { Dark Gray }\end{array}$ & Dark & 1 & & Gaballa N.D. \\
\hline Unknown & NMA.unknown & 7 & Dark & 1a & & Gaballa N.D. \\
\hline $\begin{array}{l}\text { Hu/Diospol } \\
\text { is Parva }\end{array}$ & NMI.1901.803 & 7 & Dark & 1 & & Global Egyptian Museum \\
\hline $\begin{array}{l}\text { Hu/Diospol } \\
\text { is Parva }\end{array}$ & NMI.1901.804 & 5,8 & ? & 1 & & Global Egyptian Museum \\
\hline Unknown & OIM.11250 & 4 & Light & 2 & & \\
\hline Unknown & OIM.11252 & 7 & Dark & 1 & & \\
\hline $\begin{array}{l}\text { West } \\
\text { Theban } \\
\text { Plateau }\end{array}$ & OIM.12333.570 & 7 & Dark & 1 & & \\
\hline Unknown & PAHMA.6.17270 & 7 & Dark & 2 & & \\
\hline Unknown & PAHMA.6.17272 & 7 & $\begin{array}{l}\text { other- } \\
\text { both }\end{array}$ & $1 a$ & & \\
\hline $\begin{array}{l}\text { Naga-ed- } \\
\text { Deir }\end{array}$ & PAHMA.6.4751 & 7 & Dark & $1 \mathrm{a}$ & $\begin{array}{l}\text { Cemetery } 7000 \\
\text { Tomb } 14\end{array}$ & \\
\hline Abydos & PR.1900.42.1 & 10 & Light & 2 & Cemetery G & Petrie 1902 \\
\hline Mahasna & PR.1901.42.107 & 7 & Dark & $1 \mathrm{a}$ & $\begin{array}{l}\text { Found in Garstang's } \\
\text { "settlement 2" }\end{array}$ & \\
\hline Mahasna & PR.1901.42.114 & $1,2,4$ & Light & $1 a$ & From cemetery & Garstang 1903:5,8 \\
\hline Harageh & RMAH.05663 & $1,2,4$ & Light & 2 & Tomb 537 & \\
\hline Unknown & $\begin{array}{l}\text { RVFAM.E1.01.015.1 } \\
998\end{array}$ & 4 & Light & 2 & & Kaplan 2005 \\
\hline Unknown & SNHM.A328574.0 & 7 & Dark & 1 & & \\
\hline $\begin{array}{l}\text { Hemmami } \\
\text { a North } \\
\text { Spur }\end{array}$ & UC.10244 & 2,4 & Light & 2 & E:216 at 2'6" & $\begin{array}{l}\text { Brunton\&Caton-Thompson } \\
\text { 1928:Pl80no.72,PI72no. } 98\end{array}$ \\
\hline Naqada & UC.4133 & $\begin{array}{l}\text { Other: } \\
\text { Dark Gray }\end{array}$ & Dark & 1 & Tomb 1676 & $\begin{array}{l}\text { Baumgartel 1970; Petrie \& } \\
\text { Quibell 1896: PI. } 73 \text { no. } 66\end{array}$ \\
\hline Naqada & UC.4133a & 8 & Light & $1 a$ & Tomb 1676 & $\begin{array}{l}\text { Baumgartel 1970; Petrie \& } \\
\text { Quibell 1896: PI. } 73 \text { no. } 66\end{array}$ \\
\hline Naqada & UC.4273 & 7 & Dark & 1 & Tomb 1412 & \\
\hline Abydos? & UC.42817 & $\begin{array}{l}\text { Other: } \\
\text { VTO }\end{array}$ & Light & 2 & & \\
\hline Naqada & UC.4429 & 2,4 & Light & 2 & Tomb 430 & \\
\hline Naqada & UC. 4527 & 5,8 & $?$ & 1 & Tomb 211 & \\
\hline Naqada & UC.4528 & 7 & Dark & 1 & Tomb 211 & \\
\hline Naqada & UC.4564 \& 4955 & $1,2,4$ & Light & Frag. & Tomb 1660 & \\
\hline Naqada & UC.4826 & 7 & Dark & Frag. & Tomb 1856 & \\
\hline Naqada & UC.4827 & $\begin{array}{l}\text { Other: } \\
\text { VTO }\end{array}$ & Light & $1 a$ & Tomb 1856 & \\
\hline $\begin{array}{l}\text { Naqada/ } \\
\text { Ballas }\end{array}$ & UC.4919 & 7 & Dark & $1 a$ & Tomb 663 & \\
\hline Naqada & UC.5369 & 7 & Dark & Frag. & Tomb 260 & \\
\hline Naqada & UC.5404 & $1,2,4$ & Light & Frag. & Tomb 100 & \\
\hline
\end{tabular}


Table 7.8 (part 4). Fishtails with identifiable raw materials. Note that “Other:VTO" is an abbreviation for "Other: Variable Translucent - Opaque." In all cases, especially when no publication is given, the publication reference is the online collection database of the museum for that item. See Table 7.1 for the list of museums and their abbreviations.

\begin{tabular}{|l|l|l|l|l|l|l|}
\hline \multicolumn{1}{|c|}{ Site } & \multicolumn{1}{c|}{ ID } & RM & $\begin{array}{c}\text { Light/ } \\
\text { Dark }\end{array}$ & $\begin{array}{c}\text { Shape } \\
\text { Type }\end{array}$ & \multicolumn{1}{c|}{ Notes } & Reference \\
\hline Naqada & UC.5405 & 7 & Dark & 1 & Tomb 100 & \\
\hline Naqada & UC. 5918 & 2,4 & Light & Frag. & Tomb 1332 & \\
\hline Armant & UC.6070 & $1,2,4$ & Light & 1 a & & \\
\hline Unknown & UC.8965 & 2,4 & Light & 2 & & \\
\hline Mahasna & YPM.ANT.006807 & 7 & Dark & $1 \mathrm{a}$ & Tomb H & \\
\hline
\end{tabular}

Table 7.9. Frequencies of fishtail raw materials by fishtail shape type.

\begin{tabular}{|c|c|c|c|c|c|c|c|c|c|}
\hline \multirow{2}{*}{ Raw Material } & \multirow{2}{*}{ Light/Dark } & \multicolumn{2}{|c|}{ Type 1} & \multicolumn{2}{|c|}{ Type 1a } & \multicolumn{2}{|c|}{ Type 3} & \multicolumn{2}{|c|}{ Type 2} \\
\hline & & Count & $\%$ & Count & $\%$ & Count & $\%$ & Count & $\%$ \\
\hline Agate(?) & Light & 0 & 0 & 1 & 4.55 & 0 & 0 & 0 & 0 \\
\hline 1. Indistinct Beige & Light & & & & & & & & \\
\hline 2. Beige (Fine) & Light & & & & & & & & \\
\hline 4. Beige with Pink Bands & Light & 2 & 6.25 & 2 & 9.09 & 3 & 27.27 & 8 & 32 \\
\hline 1,2,4 Indistinguishable & Light & 2 & 6.25 & 3 & 13.64 & 1 & 9.09 & 11 & 44 \\
\hline 8. Brown Translucent w/ White Mottles & Light & 4 & 12.5 & 2 & 9.09 & 2 & 18.18 & 0 & 0 \\
\hline 10. Pink-Grey Translucent & Light & 0 & 0 & 0 & 0 & 0 & 0 & 1 & 4 \\
\hline 11. Pink-Purple-Red Group & Light & & & & & & & & \\
\hline 13. Caramel & Light & & & & & & & & \\
\hline Other: Variable Translucent and Opaque & Light & 0 & 0 & 1 & 4.55 & 0 & 0 & 1 & 4 \\
\hline Other Light Cherts & Light & 1 & 3.13 & 1 & 4.55 & 0 & 0 & 1 & 4 \\
\hline Other Indeterminate or Light \& Dark Mixed & ?/Both & & & & & & & & \\
\hline $5,6,7,8$ Indeterminate Mid-Browns & ? & 2 & 6.25 & 2 & 9.09 & 0 & 0 & 0 & 0 \\
\hline 5. Medium Brown Homogeneous & Dark & & & & & & & & \\
\hline 6. Brown with Foraminifera & Dark & & & & & & & & \\
\hline 7. Dark Grey and Brown & Dark & 18 & 56.25 & 8 & 36.36 & 2 & 18.18 & 2 & 8 \\
\hline Other Dark Grey or Black Cherts & Dark & 3 & 9.38 & 2 & 9.09 & 3 & 27.27 & 0 & 0 \\
\hline Other: Chocolate & Dark & & & & & & & & \\
\hline Obsidian & Dark & 0 & 0 & 0 & 0 & 0 & 0 & 1 & 4 \\
\hline Total & & 32 & 100.01 & 22 & 100.01 & 11 & 99.99 & 25 & 100 \\
\hline
\end{tabular}


Table 7.10. Raw material types of Early fishtails (shape types $1 \& 1$ a) by site.

\begin{tabular}{|c|c|c|c|c|c|c|c|c|c|}
\hline \multirow{2}{*}{ Site } & \multicolumn{5}{|c|}{ Light } & $?$ & \multicolumn{3}{|c|}{ Dark } \\
\hline & Type 4 & $\begin{array}{l}\text { Type } \\
1,2,4\end{array}$ & $\begin{array}{c}\text { Type } \\
10 \\
\end{array}$ & $\begin{array}{c}\text { Other } \\
\text { light }\end{array}$ & Type 8 & $\begin{array}{l}\text { Type } \\
5,6,8 \\
\end{array}$ & Type 7 & \begin{tabular}{|l|}
$\begin{array}{l}\text { Other } \\
\text { grays }\end{array}$ \\
\end{tabular} & Obsidian \\
\hline \multicolumn{10}{|l|}{ Harageh } \\
\hline \multicolumn{10}{|l|}{ Hemmamiya } \\
\hline Mesaeed & 1 & 1 & & & 2 & & & & \\
\hline Naga d-Deir & & & & & & & 1 & & \\
\hline Mahasna & & 1 & & & & & 3 & & \\
\hline Abydos & & & & & & & 2 & 1 & \\
\hline $\begin{array}{l}\text { Abadiya/Hu/ } \\
\text { Diospolis parva }\end{array}$ & & & & 1 & & 1 & 3 & & \\
\hline Naqada/Ballas & & 1 & & 1 & 1 & 1 & 5 & 1 & \\
\hline West Theban Plateau & & & & & & & 1 & & \\
\hline Armant & & 1 & & & & & & & \\
\hline \multicolumn{10}{|l|}{ Adaïma } \\
\hline Hierakonpolis & & & & & & 1 & & & \\
\hline el-Ma'mariya & & & & & & 1 & & & \\
\hline Khor Bahan & & & & & & 1 & & & \\
\hline
\end{tabular}

Table 7.11. Raw material types of Later fishtails (shape type 2) by site.

\begin{tabular}{|c|c|c|c|c|c|c|c|c|c|}
\hline \multirow{2}{*}{ Site } & \multicolumn{5}{|c|}{ Light } & $?$ & \multicolumn{3}{|c|}{ Dark } \\
\hline & Type 4 & $\begin{array}{l}\text { Type } \\
1,2,4\end{array}$ & $\begin{array}{c}\text { Type } \\
10\end{array}$ & $\begin{array}{l}\text { Other } \\
\text { light }\end{array}$ & Type 8 & $\begin{array}{l}\text { Type } \\
5,6,8\end{array}$ & Type 7 & $\begin{array}{l}\text { Other } \\
\text { grays }\end{array}$ & Obsidian \\
\hline Harageh & & 1 & & & & & & & \\
\hline Hemmamiya & & 1 & & & & & & & \\
\hline Mesaeed & 1 & & & & 1 & & & & \\
\hline \multicolumn{10}{|l|}{ Naga d-Deir } \\
\hline \multicolumn{10}{|l|}{ Mahasna } \\
\hline Abydos & 2 & 1 & 1 & 1 & 1 & & & & 1 \\
\hline \multicolumn{10}{|l|}{$\begin{array}{l}\text { Abadiya/Hu/ } \\
\text { Diospolis parva }\end{array}$} \\
\hline Naqada/Ballas & 1 & 1 & & & & & 2 & & \\
\hline \multicolumn{10}{|c|}{ West Theban Plateau } \\
\hline \multicolumn{10}{|l|}{ Armant } \\
\hline \multicolumn{10}{|l|}{ Adaima } \\
\hline Hierakonpolis & & & & & & & & 2 & \\
\hline \multicolumn{10}{|l|}{ el-Ma'mariya } \\
\hline Khor Bahan & & & & & & & & & \\
\hline
\end{tabular}


Table 7.12 (part 1). Concave Base Projectile Points with identifiable raw materials.

\begin{tabular}{|c|c|c|c|c|c|}
\hline Site & ID & RM & $\begin{array}{l}\text { Light/ } \\
\text { Dark }\end{array}$ & Notes & Publication \\
\hline Abydos & AMC.12.48 & 7 & Dark & $\begin{array}{l}\text { From Abydos Middle } \\
\text { Cemetery (Cem. E/G) }\end{array}$ & Here \\
\hline Elkab & EK.2012.3.38 & 7 & Dark & & Claes et al. 2014:85 \\
\hline HK6 & HK.6.str7-1 & 7 & Dark & $\begin{array}{l}\text { Photo in Friedman } \\
\text { 2011c:42 }\end{array}$ & $\begin{array}{l}\text { Friedman 2010:69-70; Friedman et al. } \\
\text { 2009: 194; Droux \& Friedman 2007:7-9 }\end{array}$ \\
\hline HK6 & HK.6.str7-2 & 7 & Dark & $\begin{array}{l}\text { Photo in Friedman } \\
\text { 2011c:42 }\end{array}$ & $\begin{array}{l}\text { Friedman 2010:69-70; Friedman et al. } \\
\text { 2009: 194; Droux \& Friedman 2007:7-9 }\end{array}$ \\
\hline HK6 & HK.6.str7-3 & 7 & Dark & $\begin{array}{l}\text { Photo in Friedman } \\
\text { 2011c:42 }\end{array}$ & $\begin{array}{l}\text { Friedman 2010:69-70; Friedman et al. } \\
\text { 2009: 194; Droux \& Friedman 2007:7-9 }\end{array}$ \\
\hline HK6 & HK.6.str7.363 & $1,2,4$ & Light & $\begin{array}{l}\text { Photo in Friedman } \\
\text { 2011c:42 }\end{array}$ & $\begin{array}{l}\text { Friedman 2010:69-70; Friedman et al. } \\
\text { 2009: 194; Droux \& Friedman 2007: 7-9 }\end{array}$ \\
\hline HK6 & HK.6.str7-4 & 7 & Dark & $\begin{array}{l}\text { Photo in Friedman } \\
\text { 2011c: } 42\end{array}$ & $\begin{array}{l}\text { Friedman 2010:69-70; Friedman et al. } \\
\text { 2009: 194; Droux \& Friedman 2007:7-9 }\end{array}$ \\
\hline HK6 & HK.6.str7-5 & 7 & Dark & $\begin{array}{l}\text { Photo in Friedman } \\
\text { 2011c: } 42\end{array}$ & $\begin{array}{l}\text { Friedman 2010:69-70; Friedman et al. } \\
\text { 2009:194; Droux \& Friedman 2007:7-9 }\end{array}$ \\
\hline K6 & HK.6.str7.648 & 7 & Dark & $\begin{array}{l}\text { Photo in Friedman } \\
\text { 2011c:42 }\end{array}$ & $\begin{array}{l}\text { Friedman 2010:69-70; Friedman et al. } \\
\text { 2009: 194; Droux \& Friedman 2007:7-9 }\end{array}$ \\
\hline HK6 & $\begin{array}{l}\text { HK6.2006.str8 } \\
-1\end{array}$ & 13. Caramel & Light & Photo in NN 18 (2006) & $\begin{array}{l}\text { Friedman 2010:71; Friedman et al. } \\
\text { 2008:90 }\end{array}$ \\
\hline HK6 & $\begin{array}{l}\text { HK6.2006.str8 } \\
-2\end{array}$ & $1,2,4$ & Light & Photo in NN 18 (2006) & $\begin{array}{l}\text { Friedman 2010:71; Friedman et al. } \\
\text { 2008:90 }\end{array}$ \\
\hline HK6 & HK6.2014 & 7 & Dark & Tomb 72 & Droux 2014:4-7, 18-19 \\
\hline HK6 & HK6.2015.75-1 & 77 & Dark & & Friedman \& Droux 2015:4-6 \\
\hline Qushtamna & $\begin{array}{l}\text { LvpM.49.47.5 } \\
\text { 86A-D }\end{array}$ & 8 & Light & & Global Egyptian Museum \\
\hline Qushtamna & $\begin{array}{l}\text { LvpM.49.47.5 } \\
\text { 86A-D }\end{array}$ & $\begin{array}{l}\text { Other: } \\
\text { Dark Grays }\end{array}$ & Dark & & Global Egyptian Museum \\
\hline Mostagedda & ManM.8732 & 13. Caramel & Light & & \\
\hline Mahasna & MAP.2528.1 & 7 & Dark & & Here \\
\hline Mahasna & MAP.3029.1 & 13. Caramel & Light & & Here \\
\hline $\begin{array}{l}\text { Hemmamiya, } \\
\text { North Spur }\end{array}$ & UC.10345 & 11 & Dark & $\begin{array}{l}\text { A3:60 at } 1 ' 6 " \text { but } \\
\text { possibly not in situ }\end{array}$ & $\begin{array}{l}\text { Brunton \& Caton-Thompson 1928:78, } \\
\text { PL79, no41 }\end{array}$ \\
\hline \begin{tabular}{|l|} 
Badari \\
$3000 / 10$
\end{tabular} & UC. 27229 & $\begin{array}{l}\text { Other Light } \\
\text { IND }\end{array}$ & Light & & Holmes 1989:163 \\
\hline $\begin{array}{l}\text { Naqada- } \\
\text { South Town }\end{array}$ & UC.5343 & 7 & Dark? & & $\begin{array}{l}\text { Holmes 1989:274; Petrie \& Quibell } \\
\text { 1896: PI55 }\end{array}$ \\
\hline $\begin{array}{l}\text { Naqada- } \\
\text { South Town }\end{array}$ & UC.5352 & a & Light & & $\begin{array}{l}\text { Holmes 1989:274-275; Petrie \& Quibell } \\
\text { 1896: PI 72, no } 57\end{array}$ \\
\hline $\begin{array}{l}\text { Unknown- } \\
\text { Abydos? }\end{array}$ & UC.6144 & $\begin{array}{l}\text { Other Light } \\
\text { IND }\end{array}$ & Light & Purchased at Abydos & \\
\hline Naqada & UC.6230 & 7 & Light & & \\
\hline $\begin{array}{l}\text { Badari } \\
3000 / 3\end{array}$ & UC.9433 & 13 & Light & & Holmes 1989:163 \\
\hline $\begin{array}{l}\text { Badari } \\
3000 / 3\end{array}$ & UC.9434 & 13 & Light & & Holmes 1989:163 \\
\hline $\begin{array}{l}\text { Badari } \\
3000 / 6\end{array}$ & UC.9436 & $1,2,4$ & Light & & Holmes 1989:163 \\
\hline
\end{tabular}


Table 7.12 (part 2). Concave Base Projectile Points with identifiable raw materials. In all cases, especially when no publication is given, the publication reference is the online collection database of the museum for that item. See Table 7.1 for the list of museums and their abbreviations.

\begin{tabular}{|l|l|l|l|l|l|}
\hline \multicolumn{1}{|c|}{ Site } & \multicolumn{1}{|c|}{ ID } & RM & $\begin{array}{l}\text { Light/ } \\
\text { Dark }\end{array}$ & \multicolumn{1}{c|}{ Notes } & Publication \\
\hline $\begin{array}{l}\text { Badari } \\
3000 / 6\end{array}$ & UC.9437 & $1,2,4$ & Light & & Holmes 1989:163 \\
\hline $\begin{array}{l}\text { Badari } \\
3000 / 6\end{array}$ & UC.9438 & $\begin{array}{l}\text { Other: } \\
\text { Dark Grays }\end{array}$ & Dark & & Holmes 1989:163 \\
\hline $\begin{array}{l}\text { Badari } \\
3000 / 6\end{array}$ & UC.9442 & 7 & Dark & Wing fragment & \\
\hline $\begin{array}{l}\text { Hemmamiya, } \\
1700\end{array}$ & UC.9443 & 13 & Light & From cemetery 1700 & \\
\hline
\end{tabular}

Table 7.13. Raw material types of concave base projectile points by site.

\begin{tabular}{|c|c|c|c|c|c|c|c|c|}
\hline \multirow[b]{2}{*}{ Site } & \multicolumn{6}{|c|}{ Light } & \multicolumn{2}{|c|}{ Dark } \\
\hline & $\begin{array}{c}\text { Type } \\
1 / 2 / 4\end{array}$ & Type 4 & Type 8 & $\begin{array}{c}\text { Type } \\
11\end{array}$ & $\begin{array}{c}\text { Type } \\
13 \\
\end{array}$ & $\begin{array}{l}\text { Other } \\
\text { Light }\end{array}$ & Type 7 & $\begin{array}{l}\text { Other } \\
\text { Dark }\end{array}$ \\
\hline Mostagedda & & & & & 1 & & & \\
\hline Badari & 2 & & & & 3 & 1 & 1 & 1 \\
\hline Hemmamiya & & & & 1 & & & & \\
\hline Mahasna & & & & & 1 & & 1 & \\
\hline Abydos & & & & & & 1 & 1 & \\
\hline Naqada & & 1 & & & & & 2 & \\
\hline HK6 & 2 & & & & 1 & & 8 & \\
\hline Elkab & & & & & & & 1 & \\
\hline Qushtamna & & & 1 & & & & & 1 \\
\hline
\end{tabular}


Table 7.14. Bifacial sickles with identifiable raw materials. In all cases, especially when no publication is given, the publication reference is the online collection database of the museum for that item. See Table 7.1 for the list of museums and their abbreviations.

\begin{tabular}{|c|c|c|c|c|c|}
\hline Site & ID & RM & $\begin{array}{l}\text { Light } \\
\text { /Dark }\end{array}$ & Notes & Publication \\
\hline $\begin{array}{l}\text { 1Nag El- } \\
\text { Qarmila }\end{array}$ & AKAP.1552.1 & 7 & Dark & Settlement & \\
\hline 4Abydos & ATP.3418 & 7 & Dark & Settlement & Here \\
\hline 3Abydos & ATP.3419 & 7 & Dark & Settlement & Here \\
\hline 2Abydos & ATP.3420 & $1,2,4$ & Light & Settlement & Here \\
\hline 5Adaima & $\begin{array}{l}\text { BrM.07.447.80 } \\
2\end{array}$ & $1,2,4$ & Light & Settlement, excavated, sickle gloss & Needler 1984:286 \\
\hline 6Elkab & EK.2012.4.1 & 7 & Dark & Settlement & Claes et al. 2014:85 \\
\hline 7HK6 & HK6.14.440 & 7 & Dark & Tomb 72; used & Droux 2014:4-7, 18-19 \\
\hline $8 \mathrm{HK} 6$ & HК6.14.441 & 8 & Light & Tomb 72; used & Droux 2014:4-7, 18-19 \\
\hline 9Armant & ManM.23814 & 7 & Dark & $\begin{array}{l}\text { Sickle gloss; Settlement level: I.O.10, } \\
14 a, 22 A\end{array}$ & \\
\hline 10Armant & ManM.23824 & 5,8 & Dark & $\begin{array}{l}\text { Settlement level:14a, Area } 31 \text { (1000), } \\
\text { 22J }\end{array}$ & \\
\hline 11Armant & ManM.23832 & 7 & Dark & $\begin{array}{l}\text { Sickle gloss; Settlement, unstratified } \\
14 a\end{array}$ & \\
\hline 12Mahasna & MAP.2861.1 & 10 & Light & Settlement & \\
\hline $\begin{array}{l}\text { 13Hemmamiya, } \\
\text { North Spur }\end{array}$ & UC.10525 & 4 & Light & Under a Naqada Period hut curcle & \\
\hline $\begin{array}{l}\text { 14Hemmamia, } \\
1900\end{array}$ & UC.10619 & 7 & Dark & Settlement & Holmes 1989:162 \\
\hline $\begin{array}{l}\text { 15Hemmamia, } \\
1900\end{array}$ & UC.10620 & $\begin{array}{l}\text { Other } \\
\text { dark } \\
\text { grays }\end{array}$ & Dark & Settlement & \\
\hline $\begin{array}{l}\text { 16Badari, } \\
3000 / 3\end{array}$ & UC. 26823 & 8 & Light & Settlement & Holmes 1989:162 \\
\hline 17Naqada & UC.4609 & $1,2,4$ & Light & Tomb 1906 & \\
\hline $\begin{array}{l}\text { 18Naqada, } \\
\text { South Town }\end{array}$ & UC.5332 & 8 & ? & Settlement & \\
\hline $\begin{array}{l}\text { 19Badari, } \\
3000 / 3\end{array}$ & UC.9618 & $\begin{array}{l}\text { Other } \\
\text { dark } \\
\text { grays }\end{array}$ & Dark & Settlement & \\
\hline $20 \mathrm{Hemmamia}$ & UC.9879a & $1,2,4$ & Light & Cemetery 24/1700 & \\
\hline
\end{tabular}


Table 7.15. Raw material types of bifacial sickles by site.

\begin{tabular}{|l|c|c|c|c|c|c|c|}
\hline \multirow{2}{*}{ Site } & \multicolumn{4}{c|}{ Light } & \multicolumn{3}{c|}{ Dark } \\
\cline { 2 - 8 } & $\begin{array}{c}\text { Type } \\
\mathbf{4}\end{array}$ & $\begin{array}{c}\text { Type } \\
\mathbf{1 , 2 , 4}\end{array}$ & $\begin{array}{c}\text { Type } \\
\mathbf{8}\end{array}$ & $\begin{array}{c}\text { Type } \\
\mathbf{1 0}\end{array}$ & $\begin{array}{c}\text { Type } \\
\mathbf{5 , 6 , 7} \\
\mathbf{8}\end{array}$ & $\begin{array}{c}\text { Type } \\
\mathbf{7}\end{array}$ & $\begin{array}{c}\text { Other } \\
\text { Dark } \\
\text { Grays }\end{array}$ \\
\hline Badari & & & 1 & & & & 1 \\
\hline Hemmamiya & 1 & 1 & & & & 1 & 1 \\
\hline Mahasna & & & & 1 & & & \\
\hline Abydos & & 1 & & & & 2 & \\
\hline Naqada & & 1 & 1 & & & & \\
\hline Armant & & & & & 1 & 2 & \\
\hline Adaima & & 1 & & & & & \\
\hline HK6 & & & 1 & & & 1 & \\
\hline Elkab & & & & & & 1 & \\
\hline Nag el Qarmila & & & & & & 1 & \\
\hline
\end{tabular}


Table 7.16 (part 1). Axes with identifiable raw materials.

\begin{tabular}{|c|c|c|c|c|c|}
\hline Site & ID & RM & $\begin{array}{l}\text { Light/ } \\
\text { Dark }\end{array}$ & Notes & Reference \\
\hline Abydos & BM.EA.37268 & 2,4 & Light & & Petrie 1902 \\
\hline Naqada & FWM.E.4.1895 & $\begin{array}{l}\text { Other light- } \\
1 / 8\end{array}$ & Light & & \\
\hline Naqada & FWM.E.5.1895 & 1,2 & Light & & \\
\hline Elkab & $\begin{array}{l}\text { FWM.E.74.190 } \\
2\end{array}$ & 1 & Light & & \\
\hline Armant & ManM.23382 & $1,2,4$ & Light & Area $31,1000.21 \mathrm{~F}$ & Mond \& Meyers 1937:233 (12); PI 62 (12) \\
\hline Armant & ManM.23383 & $\begin{array}{l}\text { Other light: } \\
\text { Beige w/ } \\
\text { dark bands }\end{array}$ & Light & $\begin{array}{l}\text { Level: } 3 \text { N } 12 \text { (1081), } 24 \\
\text { B }\end{array}$ & $\begin{array}{l}\text { Mond \& Meyers 1937:234 (21), } 243 \text { (16), } \\
\text { PI } 57 \text { (21), } 62 \text { (16) }\end{array}$ \\
\hline Armant & ManM.23384 & $1,2,4$ & Light & Level II M 11a, 24 P & \\
\hline Armant & ManM.23385 & 1 & Light & Level: 2 J 14, 24 & \\
\hline Armant & ManM.23386 & $1,2,4$ & Light & Level: I J 10 (1010), $24 Q$ & \\
\hline Armant & ManM.23387 & 1 & Light & Level: I J 10 (1013), 24N & \\
\hline Armant & ManM.23388 & $\begin{array}{l}\text { Other light- } \\
1 / 8\end{array}$ & Light & Level: I L 12 (1056), 24F & \\
\hline Armant & ManM.23389 & 1,2 & Light & Level: I O 5, 24E & Mond \& Meyers 1937:243 (15), PI 62 (15) \\
\hline Armant & ManM.23390 & 1 & Light & Level: I J 12 (1035), 24C & Mond \& Meyers 1937:233 (17), PI 57 (17) \\
\hline Armant & ManM.23391 & $\begin{array}{l}\text { Other light: } \\
\text { Beige w/ } \\
\text { dark bands }\end{array}$ & Light & Level: I L 12 (1061), 24 L & $\begin{array}{l}\text { Mond \& Meyers 1937:244 (19), } 234 \text { (19), } \\
\text { PI } 57 \text { (19), } 62 \text { (19) }\end{array}$ \\
\hline Armant & ManM.23392 & $1,2,4$ & Light & Level: I M 8, 24K & Mond \& Meyers 1937:133 (18), PI 57 (18) \\
\hline Armant & ManM.23393 & 7 & Dark & Level: I J 12 (1034) 24 R & Mond \& Meyers 1937:244 (17), PI 62 (17) \\
\hline Armant & ManM.23394 & $1,2,4$ & Light & Level: I K 11 (1019), 240 & Mond \& Meyers 1937:244 (25), PI 62 (25) \\
\hline Armant & ManM.23396 & $1,2,4$ & Light & $\begin{array}{l}\text { Unstratified (cultivation) } \\
6 \mathrm{~A}\end{array}$ & Mond \& Meyers 1937:243 (14), PI 62 (14) \\
\hline Armant & ManM.23397 & $\begin{array}{ll}1,2,4 \\
1,2\end{array}$ & Light & Unstratified (cultivation) & Mond \& Meyers 1937:234 (23), PI 57 (23) \\
\hline Armant & ManM.23399 & 1 & Light & II.L.7 30J & \\
\hline Armant & ManM.23405 & $1,2,4$ & Light & Level 3 K 13, 29 L & Mond \& Meyers 1937:244 (18), PI 62 (18) \\
\hline Armant & ManM.23412 & $1,2,4$ & Light & Level I L 14, 29 C & Mond \& Meyers 1937:233 (5), PI 57 (5) \\
\hline Armant & ManM.23644 & $1,2,4$ & Light & Level I.H.12 (1036), 31A & \\
\hline Armant & ManM.23645 & 1 & Light & $\begin{array}{l}\text { Level i.F.4, Area 30-31, } \\
\text { 37M }\end{array}$ & \\
\hline Armant & ManM.23658 & 4 & Light & Level: III.C.1, 4, 37 & Mond \& Meyers 1937:243 (11) PI 61 (11) \\
\hline Armant & ManM.23659 & 1,4 & Light & Level: III.K.14, 32B & \\
\hline Armant & ManM.23660 & 1,2 & Light & Level: I.K.10 (1003), 32E & \\
\hline Armant & ManM.23692 & $1,2,4$ & Light & Level: I.G.8 & \\
\hline Armant & ManM.23704 & $1,2,4$ & Light & & \\
\hline Armant & ManM.23705 & 5,7 & Dark & Level: II.K.8, 14Q & \\
\hline Armant & ManM.23706 & 1,2 & Light & Level: I.M.14, 8 & \\
\hline Armant & ManM.23707 & $1,2,4$ & Light & Level: I.J.14, 8 & \\
\hline Armant & ManM.23848 & $\begin{array}{l}\text { Other: Light } \\
\& \text { Dark }\end{array}$ & Both & Level: III.K.8, 38b & \\
\hline Armant & ManM.23859 & $\begin{array}{l}\text { Other light- } \\
1 / 8\end{array}$ & Light & Cemetery 1300 & Mond \& Meyers 1937:48, PI 16 (1) \\
\hline Armant & ManM.23862 & 1,2 & Light & Cemetery 700 & Mond \& Meyers 1937:48 \\
\hline Mahasna & MAP.1973.30 & 1 & Light & & Here \\
\hline Mahasna & MAP.2522.26 & 2 & Light & & Here \\
\hline
\end{tabular}


Table 7.16 (part 2). Axes with identifiable raw materials.

\begin{tabular}{|c|c|c|c|c|c|}
\hline Site & ID & RM & $\begin{array}{c}\text { Light/ } \\
\text { Dark }\end{array}$ & Notes & Reference \\
\hline Mahasna & MAP.2522.31 & 1 & Light & & Here \\
\hline Mahasna & PM.E9633 & 1 & Light & & \\
\hline Mahasna & PM.E9634 & 1 & Light & & \\
\hline Mahasna & PM.E9653 & 1 & Light & & \\
\hline Mahasna & PM.E9654 & 1 & Light & & \\
\hline Mahasna & PM.E9655 & 4 & Light & & \\
\hline Mahasna & PM.E9656 & Other light- $1 / 8$ & Light & & \\
\hline Mahasna & PM.E9681 & 8 & Light & & \\
\hline Mahasna & PM.E9682 & 1 & Light & & \\
\hline Mahasna & PM.E9687 & $\begin{array}{l}\text { Other Dark } \\
\text { grays }\end{array}$ & Dark & & \\
\hline Mahasna & PM.E9689 & 10 & Light & & \\
\hline Mahasna & PM.E9690 & 10 & Light & & \\
\hline Mahasna & PM.E9692 & 2,4 & Light & & \\
\hline Mahasna & PR.1901.42.87 & 8 & Light & & \\
\hline Naqada & UC.4953 & 1,2 & Light & Tomb 1578 & \\
\hline Naqada & UC.5357 & 5 & Dark & & \\
\hline Naqada & UC.5358 & 8 & Light & & \\
\hline $\begin{array}{l}\text { Naqada South } \\
\text { Town }\end{array}$ & UC.5510 & 1,2 & Light & & \\
\hline $\begin{array}{l}\text { Naqada South } \\
\text { Town }\end{array}$ & UC.5511 & $1,2,4$ & Light & & \\
\hline $\begin{array}{l}\text { Naqada South } \\
\text { Town }\end{array}$ & UC. 5530 & 1,2 & Light & & \\
\hline $\begin{array}{l}\text { Naqada South } \\
\text { Town }\end{array}$ & UC.5531 & 8 & Light & & \\
\hline $\begin{array}{l}\text { Naqada South } \\
\text { Town }\end{array}$ & UC.5532 & 1,2 & Light & & \\
\hline $\begin{array}{l}\text { Naqada South } \\
\text { Town }\end{array}$ & UC. 5533 & 1 & Light & & \\
\hline $\begin{array}{l}\text { Naqada South } \\
\text { Town }\end{array}$ & UC.5534 & 6,7 & Dark & & \\
\hline Naqada & UC.5541 & Other light- $1 / 8$ & Light & & \\
\hline Naqada & UC. 5542 & Other light- $1 / 8$ & Light & & \\
\hline Naqada & UC.5543 & 2 & Light & & \\
\hline Naqada & UC. 5544 & $\begin{array}{l}\text { Other Dark } \\
\text { grays }\end{array}$ & Dark & & \\
\hline Naqada & UC.5546 & 8 & Light & & \\
\hline Naqada & UC. 5547 & 8 & Light & & \\
\hline Naqada & UC.5551 & 1,2 & Light & & \\
\hline Naqada & UC.5552 & 2 & Light & & \\
\hline Naqada & UC.5555 & 1 & Light & & \\
\hline Naqada & UC.5558 & 1,2 & Light & & \\
\hline Naqada & UC.5605 & 2,4 & Light & Tomb 350 fill & \\
\hline Naqada & UC.6229 & $1,2,4$ & Light & Cemetery B surface & \\
\hline Badari & UC.9745 & 1,2 & Light & From Area 3000/6 & \\
\hline
\end{tabular}


Table 7.16 (part 3). Axes with identifiable raw materials. In all cases, especially when no publication is given, the publication reference is the online collection database of the museum for that item. See Table 7.1 for the list of museums and their abbreviations.

\begin{tabular}{|c|c|c|c|c|c|}
\hline Site & ID & RM & $\begin{array}{l}\text { Light/ } \\
\text { Dark }\end{array}$ & Notes & Reference \\
\hline Badari & UC.9760 & 5 & Dark & $\begin{array}{l}\text { Refashioned from an older } \\
\text { (patinated) piece }\end{array}$ & $\begin{array}{l}\text { Brunton \& Caton- } \\
\text { Thompson 1928:PI 57(4) }\end{array}$ \\
\hline Badari & UC.9762 & 2 & Light & From Area 3000/10 & \\
\hline Badari & UC.9839 & 8 & Light & Cemetery 25/5500 & \\
\hline Badari & UC.9865 & 8 & Light & & \\
\hline
\end{tabular}

Table 7.17. Raw material types of axes by site.

\begin{tabular}{|c|c|c|c|c|c|c|c|c|c|c|}
\hline \multirow{2}{*}{ Site } & \multicolumn{7}{|c|}{ Light } & \multicolumn{3}{|c|}{ Dark } \\
\hline & Type 1 & Type 2 & Type 4 & $\begin{array}{l}\text { Type } \\
1,2,4\end{array}$ & Type 8 & $\begin{array}{c}\text { Type } \\
10\end{array}$ & $\begin{array}{c}\text { Other } \\
\text { light }\end{array}$ & $\begin{array}{c}\text { Type } \\
5,6,7,8\end{array}$ & Type 7 & $\begin{array}{l}\text { Other } \\
\text { grays }\end{array}$ \\
\hline Badari & & 1 & & 1 & 2 & & & 1 & & \\
\hline Mahasna & 7 & 1 & 1 & 1 & 2 & 2 & 1 & & & 1 \\
\hline Abydos & & & & 1 & & & & & & \\
\hline Naqada & 2 & 2 & & 13 & 4 & & 3 & 2 & & 2 \\
\hline Armant & 5 & & 1 & 18 & & & 4 & 1 & 1 & \\
\hline Elkab & 1 & & & & & & & & & \\
\hline
\end{tabular}


Table 7.18 (part 1). Large blade knives with identifiable raw materials.

\begin{tabular}{|c|c|c|c|c|c|}
\hline Site & ID & RM & Light /Dark & Notes & Publication \\
\hline Abydos & ATP.1990.36 & 13 & Light & & Here \\
\hline Mahasna & BM.EA.49002 & $\begin{array}{l}\text { Other: Dark } \\
\text { unknown }\end{array}$ & Dark & Tomb H23 & \\
\hline Mesaeed & BMFA.11.781 & 1,2 & Light & Tomb 21 (M/21/8) & \\
\hline Mesaeed & BMFA.11.783 & 1,2 & Light & Tomb 4 number 4 & \\
\hline Unknown & FWM. E.56.1899 & 1,2 & Light & $\begin{array}{l}\text { Ripple-flaking on the } \\
\text { ventral }\end{array}$ & \\
\hline Unknown & FWM.E.416.1982 & 7 & Dark & & \\
\hline Unknown & FWM.E.46.1899 & 2,4 & Light & & \\
\hline Unknown & FWM.E.700.1954 & 2,4 & Light & & \\
\hline Naqada & ManM.5570 & 1,2 & Light & & $\begin{array}{l}\text { Petrie \& Quibell 1896:PI. } \\
73 \text { (71) }\end{array}$ \\
\hline Naqada & ManM.5571 & Other IND & Both & & \\
\hline Naqada & ManM.5572 & 1,2 & Light & & \\
\hline Hemmamiya & MANM.7637 & 1,2 & Light & & \\
\hline Naqada/Ballas & ManM.8382a & 6,7 & Dark & & \\
\hline Naqada/Ballas & ManM.8382b & 2 & Light & & \\
\hline Mahasna & MAP.2176 & 2 & Light & & Here \\
\hline Gerzeh & RMAH.E.05017a & $1,2,4$ & Light & Tomb 97? & \\
\hline Naqada & UC.4235 & 1 & Light & Tomb 1345 & \\
\hline Naqada & UC. 4236 & 1 & Light & Tomb 1345 & \\
\hline Naqada & UC. 4268 & 1 & Light & Tomb 1866 & \\
\hline Naqada & UC.4315 & 2 & Light & Tomb 108 & \\
\hline Naqada & UC. 4408 & 2,4 & Light & Tomb 370 & $\begin{array}{l}\text { Holmes 1989:280; Petrie } \\
\text { \& Quibell 1896: PI } 74 \text { (81) }\end{array}$ \\
\hline Naqada & UC. 4420 & $1,2,4$ & Light & Tomb 1203 & \\
\hline Naqada & UC. 4474 & 2 & Light & Tomb 294 & \\
\hline Naqada & UC. 4534 & 2 & Light & Tomb 162 & \\
\hline Naqada & UC.4779 & 2,4 & Light & Tomb 1791 & \\
\hline Naqada & UC.4779a & 2 & Light & Tomb 1791 & \\
\hline Naqada & UC.4793 & 1 & Light & Tomb 456 & \\
\hline Naqada & UC. 4813 & 2 & Light & Tomb 653 & \\
\hline Naqada & UC. 4821 & 8 & Light & Tomb 1298 & \\
\hline Naqada & UC. 4830 & 7 & Dark & Cemetery B & \\
\hline Naqada & UC.4845 & 1 & Light & Tomb 1233 & \\
\hline Naqada & UC. 4848 & 1 & Light & Tomb 1233 & \\
\hline Naqada & UC.4879 & 1,2 & Light & Tomb 379 & \\
\hline Naqada & UC.4881 & 2 & Light & Tomb 394 & \\
\hline Naqada & UC.4883 & 1 & Light & $\begin{array}{l}\text { Tomb 436. Unusual } \\
\text { example }\end{array}$ & \\
\hline Naqada & UC.4892 & 1,2 & Light & Tomb 458 & \\
\hline Naqada & UC.4923 & 1 & Light & Tomb 675 & \\
\hline Naqada & UC.4951 & 2 & Light & Tomb 1434 & \\
\hline Naqada & UC.4956 & 1,2 & Light & Tomb 1692 & \\
\hline Naqada & UC.4958 & 1,2 & Light & Tomb 1796 & \\
\hline Naqada & UC.4959 & 2 & Light & Tomb 1796 & \\
\hline $\begin{array}{l}\text { Naqada- } \\
\text { South Town }\end{array}$ & UC.5330 & 1,2 & Light & & \\
\hline $\begin{array}{l}\text { Naqada- } \\
\text { South Town }\end{array}$ & UC.5339 & 2 & Light & & $\begin{array}{l}\text { Holmes 1989:265, } \\
\text { Baumgartel 1960:40 }\end{array}$ \\
\hline
\end{tabular}


Table 7.18 (part 2). Large blade knives with identifiable raw materials. In all cases, especially when no publication is given, the publication reference is the online collection database of the museum for that item. See Table 7.1 for the list of museums and their abbreviations.

\begin{tabular}{|c|c|c|c|c|c|}
\hline Site & ID & RM & $\begin{array}{l}\text { Light } \\
\text { /Dark }\end{array}$ & Notes & Publication \\
\hline $\begin{array}{l}\text { Naqada- } \\
\text { South Town }\end{array}$ & UC. 5340 & 2 & Light & & \\
\hline $\begin{array}{l}\text { Naqada- } \\
\text { South Town }\end{array}$ & UC. 5518 & $1,2,4$ & Light & & \\
\hline $\begin{array}{l}\text { Naqada- } \\
\text { uth Town }\end{array}$ & UC. 5520 & 2 & Light & & \\
\hline Naqada & UC.5919 & 1 & Light & tomb 1385 & \\
\hline Naqada & UC.5920 & Other IND & Both & Tomb 1385 & \\
\hline Abydos? & UC.6145 & 2 & Light & & \\
\hline Naqada & UC.6234a & 1,2 & Light & Tomb 1849 & \\
\hline Naqada & UC.6234b & 1,2 & Light & Tomb 1849 & \\
\hline Naqada & UC.6234c & 1,2 & Light & Tomb 1849 & \\
\hline Naqada & UC.6235a & 1 & Light & Tomb 1849 & \\
\hline Naqada & UC.73366 & 1,2 & Light & Cemetery? & $\begin{array}{l}\text { Petrie \& Quibell 1896:51, PI } \\
72\end{array}$ \\
\hline Badari & UC.9655 & 8 & Light & & Holmes 1988 \\
\hline Badari, 3000/6 & UC.9656 & $1,2,4$ & Light & Settlement & Holmes 1989:154-155 \\
\hline Badari, $3000 / 6$ & UC.9657 & 7 & Dark & Settlement & \\
\hline Badari, 3000/6 & UC.9658 & $1,2,4$ & Light & Settlement & Holmes 1989:154-155 \\
\hline Badari, 3000/6 & UC.9659 & 2 & Light & Settlement & \\
\hline \begin{tabular}{|l|l} 
Badari & I
\end{tabular} & UC.9660 & 1,2 & Light & & \\
\hline Badari, 3000/6 & UC.9662 & $1,2,4$ & Light & Settlement & Holmes 1989:154-155 \\
\hline Badari, 3000/6 & UC.9664 & $\begin{array}{l}\text { Other light } \\
\text { semi } \\
\text { translucent }\end{array}$ & Light & Settlement & Holmes 1989:154-155 \\
\hline Badari, 3000/6 & UC.9669 & $\begin{array}{l}\text { Other light } \\
\text { semi } \\
\text { translucent }\end{array}$ & Light & Settlement & Holmes 1989:154-155 \\
\hline Badari, 3000/6 & UC.9685 & $1,2,4$ & Light & Settlement & Holmes 1989:154-155 \\
\hline Badari, 3000/6 & UC.9692 & $5 ?$ & ? & Settlement & Holmes 1989:154-155 \\
\hline \begin{tabular}{|l|l} 
Badari \\
\end{tabular} & UC.9801 & $5 ?$ & Dark & Cemetery 3800 & \\
\hline Badari & UC.9802 & $5 ?$ & Dark & Cemetery 3800 & \\
\hline $\begin{array}{l}\text { Hemmamia, } \\
\text { North Spur }\end{array}$ & UC.10308 & $1,2,4$ & Light & $\begin{array}{l}\text { Shaped haft; Level } \\
\text { 1'6"';inside a pot w/ other } \\
\text { blades }\end{array}$ & $\begin{array}{l}\text { Brunton \& Caton-Thompson } \\
\text { 1928: PI71, no 85a; Holmes } \\
\text { 1989:75 }\end{array}$ \\
\hline $\begin{array}{l}\text { Hemmamia, } \\
\text { North Spur }\end{array}$ & UC.10309 & $1,2,4$ & Light & $\begin{array}{l}\text { Found in a pot w other } \\
\text { blades }\end{array}$ & $\begin{array}{l}\text { Brunton \& Caton-Thompson } \\
\text { 1928: PI71, no 85b }\end{array}$ \\
\hline $\begin{array}{l}\text { Hemmamia, } \\
\text { North Spur }\end{array}$ & UC.10339 & 7 & Dark & Shaped handle; 1'-1'6" & $\begin{array}{l}\text { Brunton \& Caton-Thompson } \\
\text { 1928: PI71, no 57/50 }\end{array}$ \\
\hline $\begin{array}{l}\text { Hemmamia, } \\
\text { North Spur }\end{array}$ & UC.10341 & $\begin{array}{l}\text { Other light } \\
\text { semi } \\
\text { translucent }\end{array}$ & Light & $\begin{array}{l}\text { Posibly Heat-treated; } \\
\text { Level } 1 f^{\prime}-1 ' 6 "\end{array}$ & $\begin{array}{l}\text { Brunton \& Caton-Thompson } \\
\text { 1928: PI71(56/74); Holmes } \\
\text { 1989:75 }\end{array}$ \\
\hline
\end{tabular}


Table 7.19. Raw material types of large blade knives by site.

\begin{tabular}{|l|c|c|c|c|c|c|c|c|c|c|}
\hline \multirow{2}{*}{ Site } & \multicolumn{5}{|c|}{ Light } & \multicolumn{3}{c|}{ Dark } \\
\cline { 2 - 12 } & Type 1 Type 2 & $\begin{array}{c}\text { Type } \\
\mathbf{1 , 2 , 4}\end{array}$ & Type 8 & $\begin{array}{c}\text { Type } \\
\mathbf{1 3}\end{array}$ & $\begin{array}{c}\text { Other } \\
\text { Light }\end{array}$ & IND & $\begin{array}{c}\text { Type } \\
\mathbf{5 , 6 , 7}\end{array}$ & Type 7 $\begin{array}{l}\text { Other } \\
\text { Grays }\end{array}$ \\
\hline Gerzeh & & & 1 & & & & & & & \\
\hline Badari & & 1 & 5 & 1 & & 2 & & 3 & 1 & \\
\hline Hemmamia & & & 3 & & & 1 & & & 1 & \\
\hline Mesaeed & & & 2 & & & & & & & \\
\hline Mahasna & & 1 & & & & & & & & 1 \\
\hline Abydos & & 1 & & & 1 & & & & & \\
\hline Naqada & 10 & 12 & 15 & 1 & & & 2 & 1 & 1 & \\
\hline
\end{tabular}

Table 7.21. Microendscrapers with identifiable raw materials organized by site.

\begin{tabular}{|l|r|r|r|r|}
\hline \multirow{2}{*}{\multicolumn{1}{|c|}{ Site }} & \multicolumn{5}{c|}{ Light } \\
\cline { 2 - 5 } & Type 8 & Type 10 & $\begin{array}{c}\text { Type 8 or } \\
10\end{array}$ & Type 11 \\
\hline Badari & & & 1 & \\
\hline Hemmamiya & 1 & & & \\
\hline Mahasna & 3 & & & 6 \\
\hline Abydos & & & 1 & \\
\hline Naqada & 3 & & 4 & \\
\hline Nag el-Qarmila & 2 & & & \\
\hline Khor Bahan & 1 & & & \\
\hline
\end{tabular}


Table 7.20. Microendscrapers with identifiable raw materials. When no publication is given, the reference is the online collection database of the museum for that item. See Table 7.1 for the list of museums and their abbreviations.

\begin{tabular}{|c|c|c|c|c|c|c|}
\hline Site & ID & blank & RM & $\begin{array}{l}\text { Light/ } \\
\text { Dark }\end{array}$ & Notes & Reference \\
\hline Abydos-U & A.U.127 (20) & $\begin{array}{l}\text { Heat-treated } \\
\text { Bladelets }\end{array}$ & $\begin{array}{l}8 \text { or } \\
10\end{array}$ & Light & $\begin{array}{l}\text { Tomb U-127; } 20 \\
\text { examples from the same } \\
\text { core }\end{array}$ & Hikade 1996 \\
\hline $\begin{array}{l}\text { Nag el- } \\
\text { Qarmila }\end{array}$ & AKAP.1555.1 & Bladelet & 10 & Light & & Here \\
\hline $\begin{array}{l}\text { Nag el- } \\
\text { Qarmila }\end{array}$ & AKAP.1756.2 & Heat-treated Bladelet & 10 & Light & & Here \\
\hline $\begin{array}{l}\text { Nag el- } \\
\text { Qarmila }\end{array}$ & AKAP.1808.18 & Heat-treated Bladelet & 8 & Light & & Here \\
\hline $\begin{array}{l}\text { Nag el- } \\
\text { Qarmila }\end{array}$ & AKAP.1890.4 & Bladelet & 8 & Light & & Here \\
\hline Khor Bahan & BMFA.17.58 & $\begin{array}{l}\text { Bladelet (HT } \\
\text { Indeterminate) }\end{array}$ & 8 & Light & $\begin{array}{l}\text { Possible notch right } \\
\text { lateral }\end{array}$ & \\
\hline Mahasna & MAP.1302.33 & Heat-treated Bladelet & 8 & Light & & Here \\
\hline Mahasna & MAP.1307.10 & Heat-treated Bladelet & 11 & Light & & Here \\
\hline Mahasna & MAP.1595.1 & Heat-treated Bladelet & 11 & Light & & Here \\
\hline Mahasna & MAP.1595.12 & Heat-treated Bladelet & 11 & Light & & Here \\
\hline Mahasna & MAP.1872.30 & Heat-treated Bladelet & 11 & Light & & Here \\
\hline Mahasna & $\begin{array}{l}\text { MAP.2317.19 \& } \\
64\end{array}$ & Bladelet & 8 & Light & & Here \\
\hline Mahasna & MAP.2387.52 & Heat-treated Bladelet & 11 & Light & & Here \\
\hline Mahasna & MAP.2439.98 & Heat-treated Bladelet & 11 & Light & & Here \\
\hline Mahasna & MAP.2484.124 & Heat-treated Bladelet & 8 & Light & & Here \\
\hline Hemmamiya & UC.10184 & $\begin{array}{l}\text { Bladelet (HT } \\
\text { Probable) }\end{array}$ & 8 & Light & North Spur, H:319 at 3' & $\begin{array}{l}\text { Brunton \& Caton- } \\
\text { Thompson:114 Pl80(83) }\end{array}$ \\
\hline Naqada & $\begin{array}{l}\text { UC.4614; 4615; } \\
4620 ; 4622 ; \\
4629\end{array}$ & $\begin{array}{l}\text { Heat-treated } \\
\text { Bladelets }\end{array}$ & 8 & Light & $\begin{array}{l}\text { Tomb } 144 ; 5 \text { examples } \\
\text { probably from the same } \\
\text { core }\end{array}$ & \\
\hline Naqada & UC.4619 & Bladelet & $\begin{array}{l}8 \text { or } \\
10\end{array}$ & Light & Tomb 144 & \\
\hline Naqada & $\begin{array}{l}\text { UC.4621; } \\
\text { 4624(?) }\end{array}$ & $\begin{array}{l}\text { Bladelet (HT } \\
\text { Indeterminate) }\end{array}$ & $\begin{array}{l}8 \text { or } \\
10\end{array}$ & Light & $\begin{array}{l}\text { Tomb 144; } 2 \text { examples } \\
\text { probably from the same } \\
\text { core }\end{array}$ & \\
\hline Naqada & UC. $4628 ; 4634$ & Bladelet & 8 & Light & $\begin{array}{l}\text { Tomb 144; } 2 \text { examples } \\
\text { probably from the same } \\
\text { core }\end{array}$ & \\
\hline Naqada & UC. $4802 ; 4804$ & $\begin{array}{l}\text { Bladelet (HT } \\
\text { Probable) }\end{array}$ & $\begin{array}{l}8 \text { or } \\
10\end{array}$ & Light & $\begin{array}{l}\text { Tomb } 471 ; 2 \text { examples } \\
\text { probably from the same } \\
\text { core }\end{array}$ & \\
\hline Naqada & UC. 4857 & $\begin{array}{l}\text { Bladelet (HT } \\
\text { Indeterminate) }\end{array}$ & 8 & Light & Tomb 1233 & \\
\hline Naqada & UC.4931 & Heat-treated Bladelet & 8 & Light & Tomb 1041 & \\
\hline Naqada & UC.4968; 4970 & Bladelet & $\begin{array}{l}8 \text { or } \\
10 \\
\end{array}$ & Light & $\begin{array}{l}\text { Tomb 1786; } 2 \text { examples } \\
\text { probably from the same } \\
\text { core }\end{array}$ & \\
\hline $\begin{array}{l}\text { Badari, } \\
3000 / 6\end{array}$ & UC.9694 & $\begin{array}{l}\text { Bladelet (HT } \\
\text { Indeterminate) }\end{array}$ & $\begin{array}{l}8 \text { or } \\
10\end{array}$ & Light & Small notch right lateral & \\
\hline
\end{tabular}


Table 7.22 (part 1). Summary of findings relative to the expectations for the ritual production model, by tool class. Continued on next page.

\begin{tabular}{|c|c|c|c|c|c|}
\hline Tool & $\begin{array}{l}\text { Raw materials: Symbolic } \\
\text { meanings associated w/ RM } \\
\text {-Association between tool type } \\
\text { (or color) \& RM }\end{array}$ & $\begin{array}{l}\text { Production: } \\
\text { Ritual production } \\
\text { contexts, part time } \\
\text { production }\end{array}$ & $\begin{array}{l}\text { Context: } \\
\text { Ritual \& } \\
\text { Domestic } \\
\text { contexts? }\end{array}$ & $\begin{array}{l}\text { Context } \\
\text { Widespread distribution? }\end{array}$ & $\begin{array}{l}\text { Best fitting } \\
\text { model }\end{array}$ \\
\hline 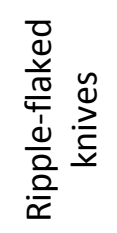 & $\begin{array}{l}\text { No. } \\
\text { Preference for type } 4 \text { may be due } \\
\text { to prod. location, and the RM } \\
\text { changes over time. }\end{array}$ & $\begin{array}{l}\text { Unknown. Possibly } \\
\text { associated with Ritual, } \\
\text { contexts, but } \\
\text { sponsorship and/or full- } \\
\text { time production likely. }\end{array}$ & $\begin{array}{l}\text { No. } \\
\text { Only ritual } \\
\text { contexts. }\end{array}$ & $\begin{array}{l}\text { Partially. } \\
\text { Found in cemeteries of all scale, } \\
\text { most common in highest order } \\
\text { sites like Abydos. Tombs of } \\
\text { upper or middle status/wealth. }\end{array}$ & $\begin{array}{l}\text { Prestige } \\
\text { goods model }\end{array}$ \\
\hline $\begin{array}{l}\frac{0}{0} \\
\frac{0}{\varepsilon} \\
\frac{0}{0} \\
\frac{0}{\infty}\end{array}$ & $\begin{array}{l}\text { No. } \\
\text { Functional considerations } \\
\text { dominant, no color preference. }\end{array}$ & $\begin{array}{l}\text { YES } \\
\text { Production in at least } 1 \\
\text { ritual area. Possibly part } \\
\text { time production. }\end{array}$ & $\begin{array}{l}\text { No. } \\
\text { Only ritual } \\
\text { contexts. }\end{array}$ & $\begin{array}{l}\text { Partially. Found in cemeteries of } \\
\text { all scale, most common in } \\
\text { highest order sites like Nagada. } \\
\text { Definitely in wealthy graves. }\end{array}$ & $\begin{array}{l}\text { Prestige } \\
\text { goods model }\end{array}$ \\
\hline 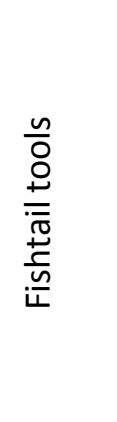 & $\begin{array}{l}\text { No. No correlation between Rm } \\
\text { type and tool type, but change } \\
\text { toward prevalence of one type } \\
\text { which may relate to changes in } \\
\text { production } \\
\text { Also changed to include use of } \\
\text { rare materials. }\end{array}$ & $\begin{array}{l}\text { YES } \\
\text { Multiple production } \\
\text { locations, at least some } \\
\text { in ritual contexts, } \\
\text { probably part-time } \\
\text { production. Then } \\
\text { changing to fewer } \\
\text { production locations and } \\
\text { full time production. }\end{array}$ & $\begin{array}{l}\text { YES } \\
\text { Ritual \& } \\
\text { habitation } \\
\text { contexts. }\end{array}$ & $\begin{array}{l}\text { YES. Cemeteries of all scales, } \\
\text { multiple locations in settlements, } \\
\text { cemeteries and graves of } \\
\text { different status/wealth. }\end{array}$ & $\begin{array}{l}\text { Both. Change } \\
\text { over time. } \\
\text { Ritual } \\
\text { production } \\
\text { then Prestige } \\
\text { goods }\end{array}$ \\
\hline 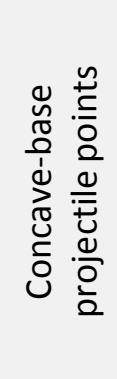 & $\begin{array}{l}\text { No. No correlation between RM } \\
\text { and tool type. Many materials } \\
\text { used. May indicate multiple prod } \\
\text { locations. }\end{array}$ & $\begin{array}{l}\text { YES. } \\
\text { Production in both } \\
\text { ritual \& non-ritual } \\
\text { contexts. } \\
\text { Probably part time. }\end{array}$ & $\begin{array}{l}\text { YES } \\
\text { Ritual \& } \\
\text { habitation } \\
\text { contexts. }\end{array}$ & $\begin{array}{l}\text { YES } \\
\text { (For the most part) Settlements } \\
\text { \& cemeteries of all scales. } \\
\text { Graves of different } \\
\text { wealth/status. At Mahâsna } \\
\text { found in multiple settlement } \\
\text { contexts, but at HK concentrated } \\
\text { in ritual contexts. }\end{array}$ & $\begin{array}{l}\text { Both, change } \\
\text { over time or } \\
\text { regional } \\
\text { differences. }\end{array}$ \\
\hline
\end{tabular}


Table 7.22 (part 2). Summary of findings relative to the expectations for the ritual production model, by tool class.

\begin{tabular}{|c|c|c|c|c|c|}
\hline Tool & $\begin{array}{l}\text { Raw materials: Symbolic } \\
\text { meanings associated w/ RM } \\
\text {-Association between tool type } \\
\text { (or color) \& RM }\end{array}$ & $\begin{array}{l}\text { Production: } \\
\text { Ritual production } \\
\text { contexts, part time } \\
\text { production }\end{array}$ & $\begin{array}{l}\text { Context: } \\
\text { Ritual \& } \\
\text { Domestic } \\
\text { contexts? }\end{array}$ & $\begin{array}{l}\text { Context } \\
\text { Widespread distribution? }\end{array}$ & $\begin{array}{l}\text { Best fitting } \\
\text { model }\end{array}$ \\
\hline 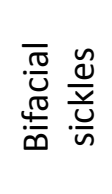 & $\begin{array}{l}\text { No. No correlation between RM } \\
\text { and tool type. Many materials } \\
\text { used. May indicate multiple prod } \\
\text { locations. }\end{array}$ & Indeterminate. & $\begin{array}{l}\text { Yes. } \\
\text { Burials \& } \\
\text { habitation } \\
\text { contexts. }\end{array}$ & $\begin{array}{l}\text { YES. Settlements \& cemeteries of } \\
\text { all scales. Multiple } \mathrm{HH} \text { contexts } \\
\text { within settlements, graves of } \\
\text { different status/wealth. }\end{array}$ & IND \\
\hline$\stackrel{\mathscr{x}}{x}$ & $\begin{array}{l}\text { Yes. Preference for one color, not } \\
\text { explainable by functional } \\
\text { considerations or available } \\
\text { materials. }\end{array}$ & $\begin{array}{l}\text { YES- Both ritual \& non- } \\
\text { ritual contexts }\end{array}$ & $\begin{array}{l}\text { Yes, } \\
\text { Some ritual } \\
\text { contexts \& } \\
\text { many } \\
\text { habitation } \\
\text { contexts }\end{array}$ & $\begin{array}{l}\text { Yes. Settlements \& cemeteries of } \\
\text { all scales. Multiple HH contexts } \\
\text { in settlements. }\end{array}$ & $\begin{array}{l}\text { YES-ritual } \\
\text { production } \\
\text { model (but } \\
\text { the ritual } \\
\text { contexts are } \\
\text { not strong }\end{array}$ \\
\hline 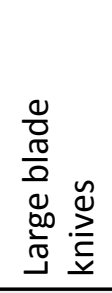 & $\begin{array}{l}\text { Yes. Preference for one color. } \\
\text { Functional explanations and local } \\
\text { material indetermiante. }\end{array}$ & $\begin{array}{l}\text { Indeterminate. } \\
\text { (possible) }\end{array}$ & $\begin{array}{l}\text { Yes. } \\
\text { Burials \& } \\
\text { habitation } \\
\text { contexts. }\end{array}$ & $\begin{array}{l}\text { Yes. Cemeteries of all scales, } \\
\text { upper \& mid level settlements. } \\
\text { Multiple contexts in settlements, } \\
\text { graves of different } \\
\text { wealth/status. }\end{array}$ & $\begin{array}{l}\text { YES- mainly } \\
\text { for burials, } \\
\text { but had by } \\
\text { all, and } \\
\text { special RM } \\
\text { selection. }\end{array}$ \\
\hline 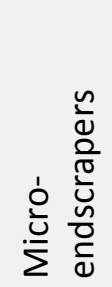 & $\begin{array}{l}\text { Yes. Correlations between tool } \\
\text { type and raw material type, } \\
\text { which is not explainable by } \\
\text { functional considerations or easy } \\
\text { access to local resources. }\end{array}$ & $\begin{array}{l}\text { YES } \\
\text { Production in both ritual } \\
\text { \& non-ritual contexts. } \\
\text { Part time, possibly } \\
\text { eventually transitioning } \\
\text { to full-time. }\end{array}$ & $\begin{array}{l}\text { YES. } \\
\text { Ritual \& } \\
\text { habitation } \\
\text { contexts. }\end{array}$ & $\begin{array}{l}\text { Yes } \\
\text { Settlements of all scales \& some } \\
\text { cemeteries. Multiple HH } \\
\text { contexts in settlements, graves } \\
\text { of different status. }\end{array}$ & $\begin{array}{l}\text { YES-ritual } \\
\text { production } \\
\text { model }\end{array}$ \\
\hline
\end{tabular}




\section{Chapter 1 Figures}

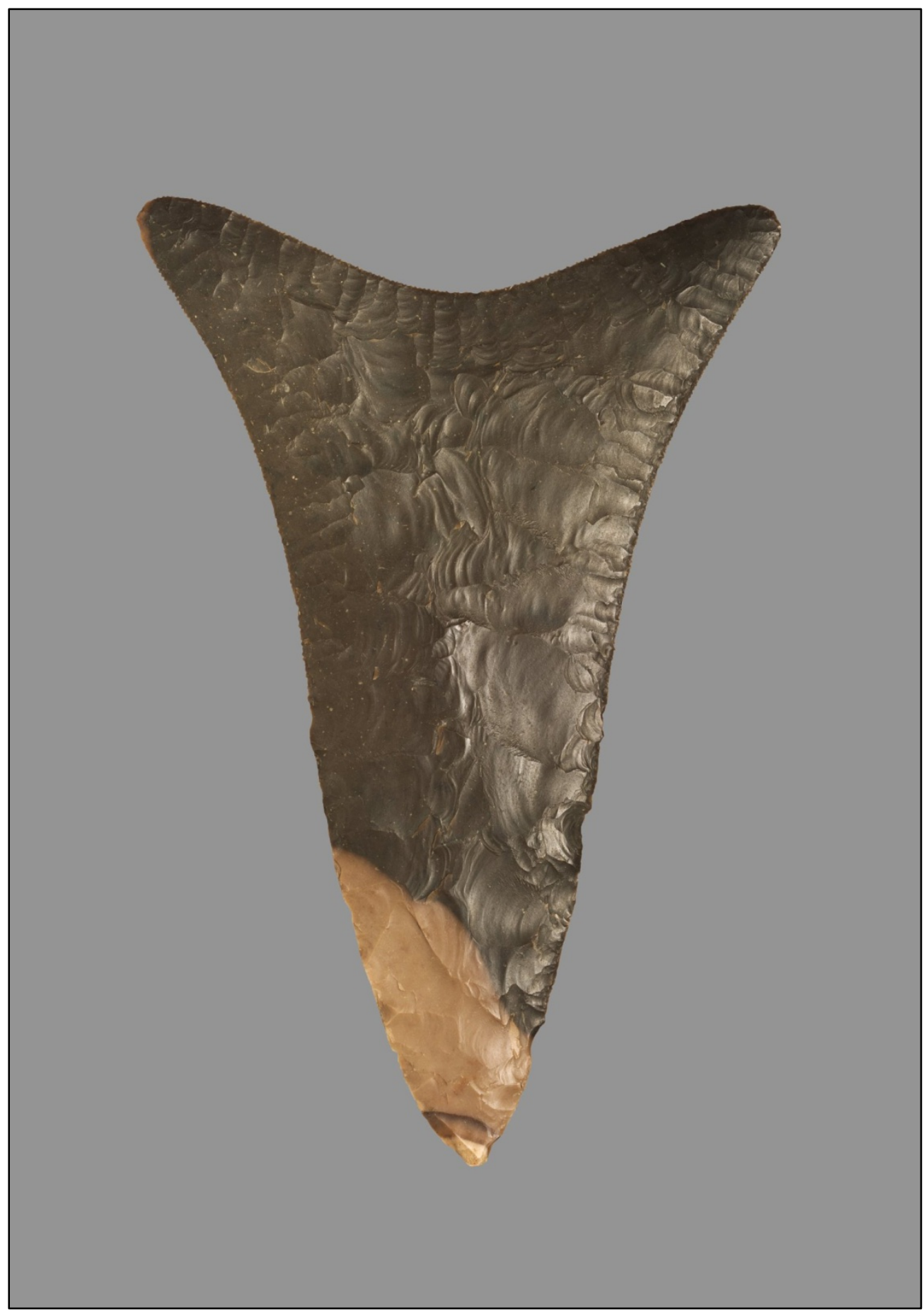

Figure 1.1. Fishtail knife. Egypt, Predynastic Period, Naqada la-lla periods. Dark brownto dark green-colored flint; overall: $10.00 \mathrm{~cm}$ (3 7/8 inches). Image credit: The Cleveland Museum of Art, Gift of the John Huntington Art and Polytechnic Trust 1914.673 . 


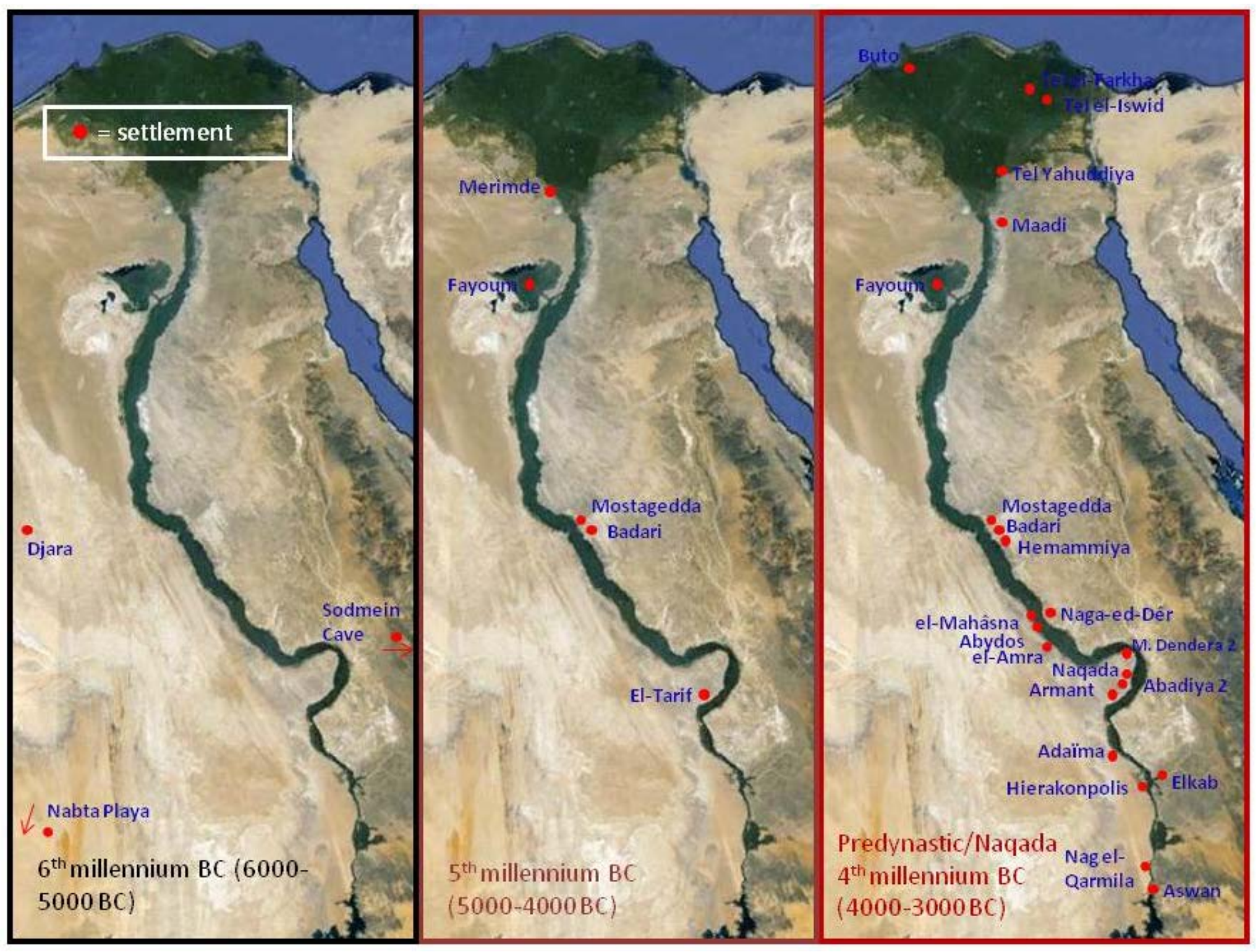

Figure 1.2. Settlement sites in the Nile Valley during the process of aridificaiton. 


\section{Chapter 2 Figures}

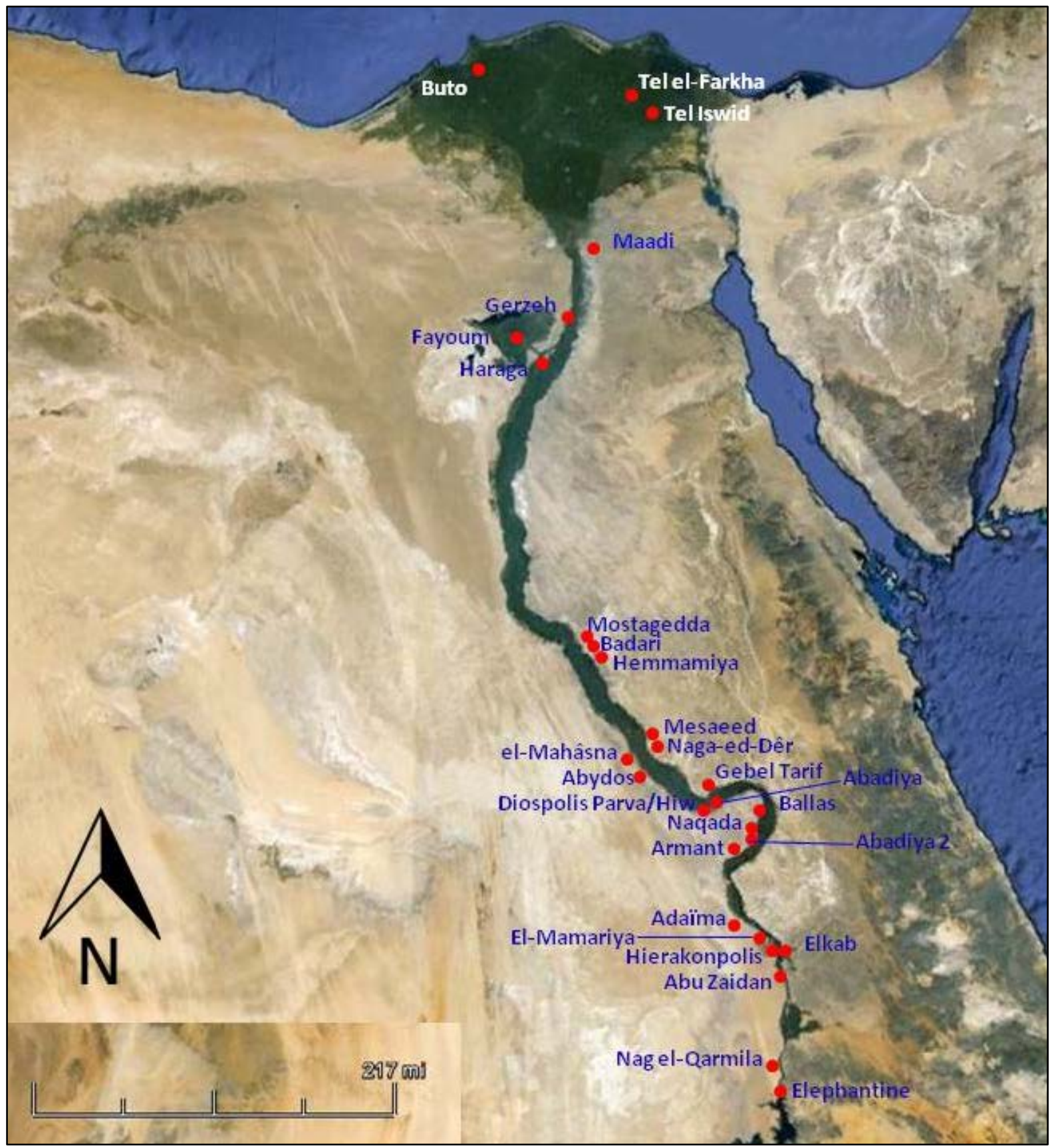

Figure 2.2. Map of sites Mentioned in this study. Not pictured are Khor Bahan, and Qushtamna, in Nubia. Base map form Google Earth. 


\section{Chapter 3 Figures}

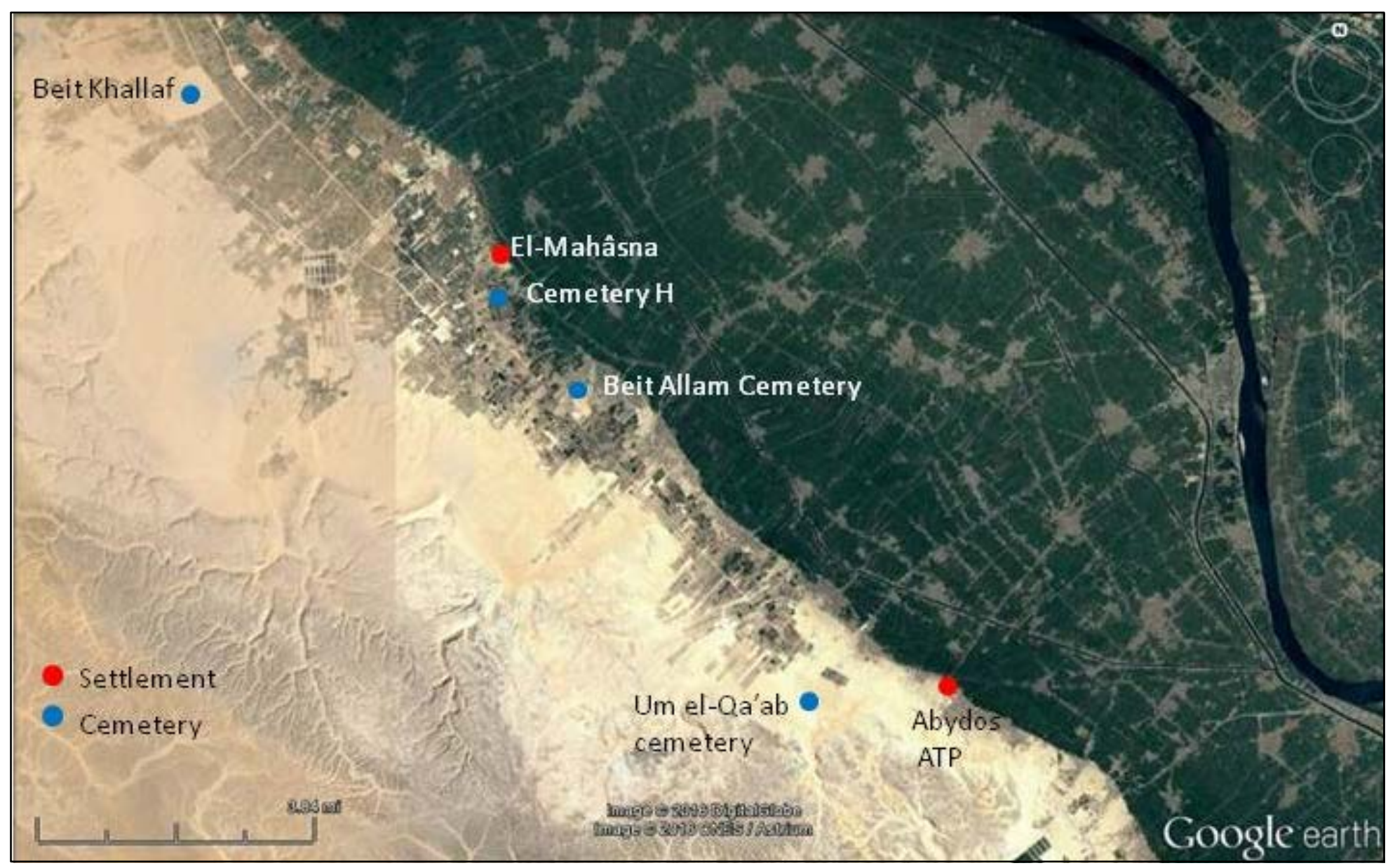

Figure 3.1 Overview of the Abydos-Mahâsna Area. See Figure 3.8 for more sites near Abydos. 


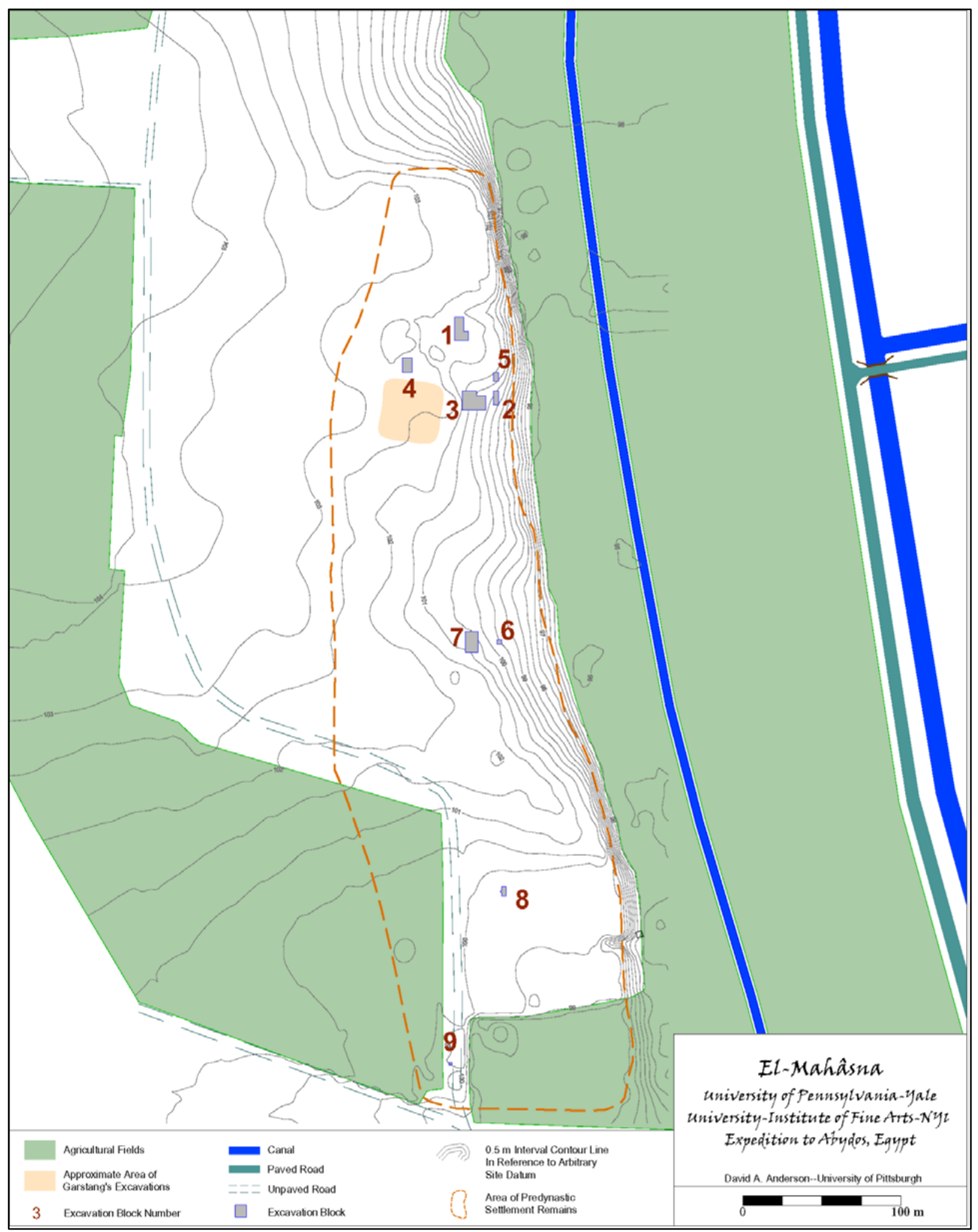

Figure 3.2. Map of the Blocks excavated by Anderson (2006). Image credit: David Anderson. 


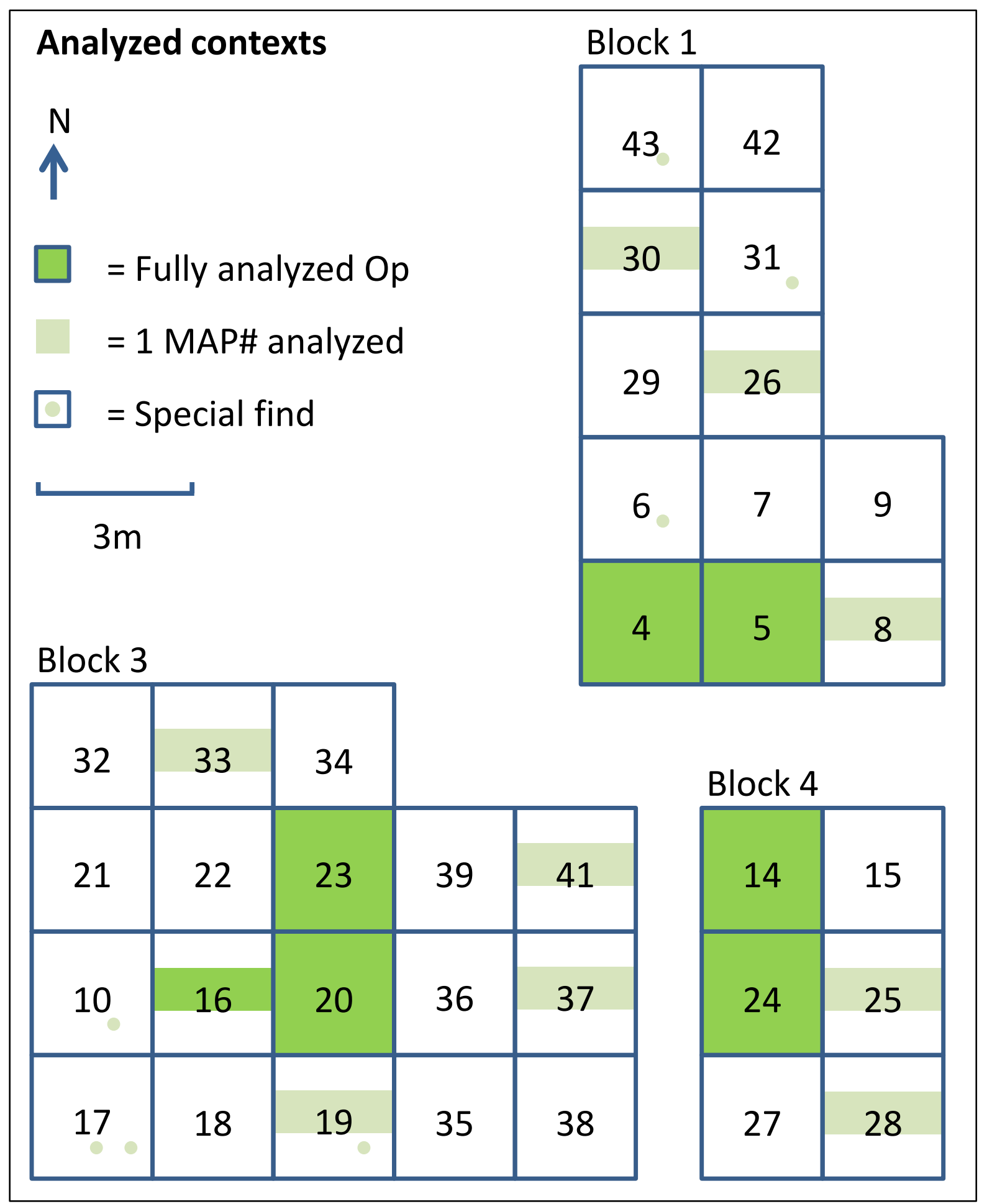

Figure 3.3. Locations and amounts of analyzed material from each excavation block at el-Mahâsna. 


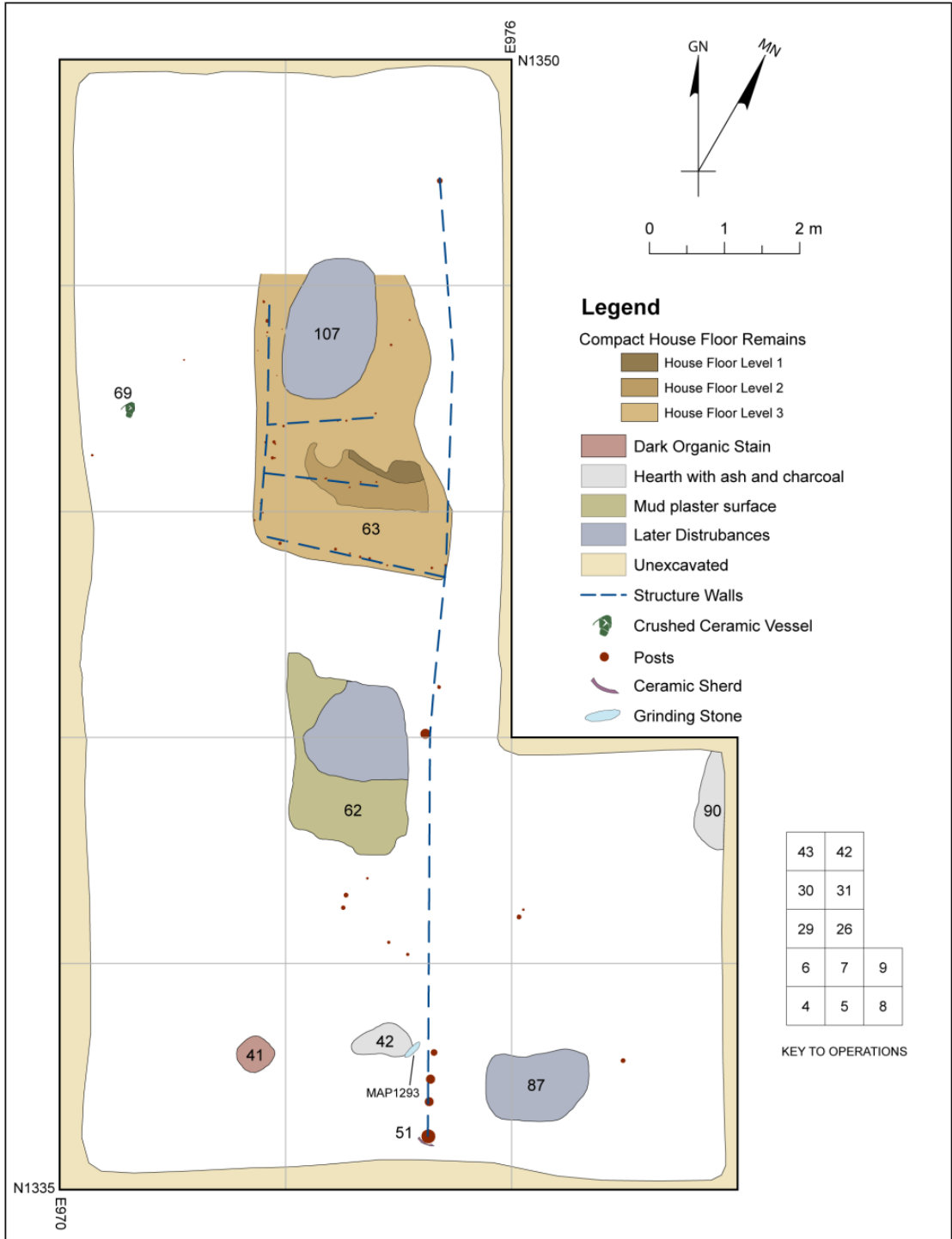

Figure 3.4. Features in el-Mahâsna Block 1 (Anderson 2006). Image credit: David Anderson.

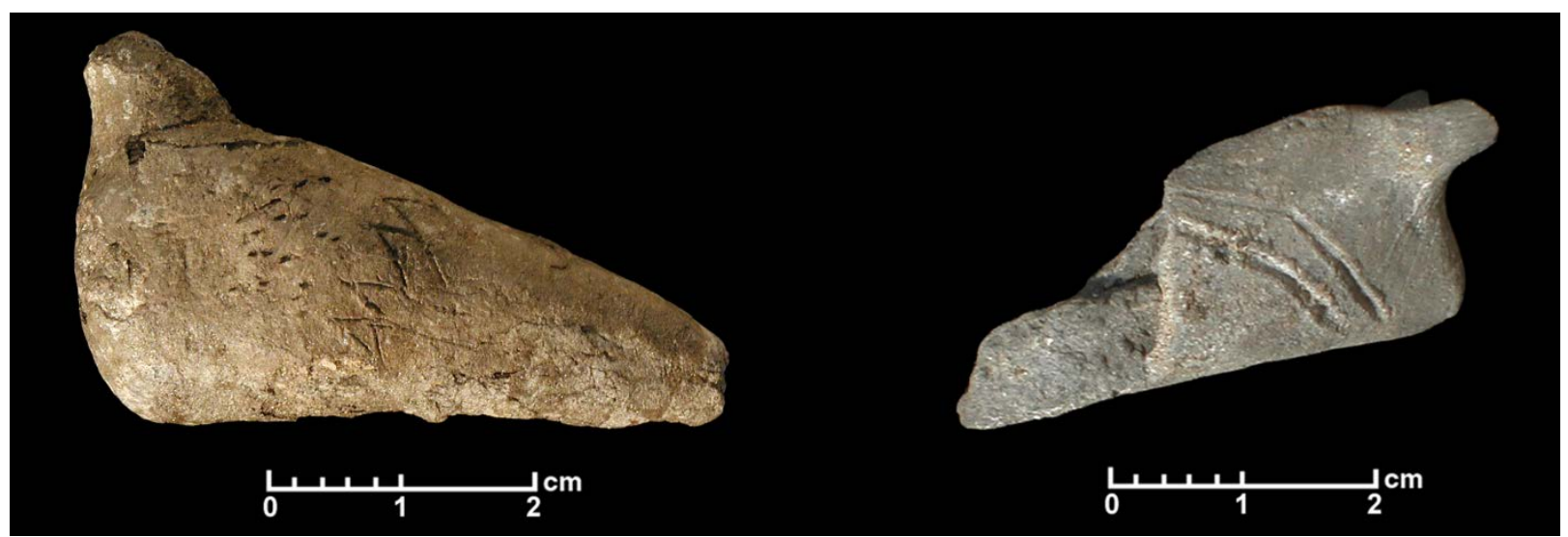

Figure 3.5. Figurines from el-Mahâsna Block 3. Left: fragments of a seated female figurine. Right fragment of a cattle figurine with incisions at the neck. Image credits: David Anderson. 


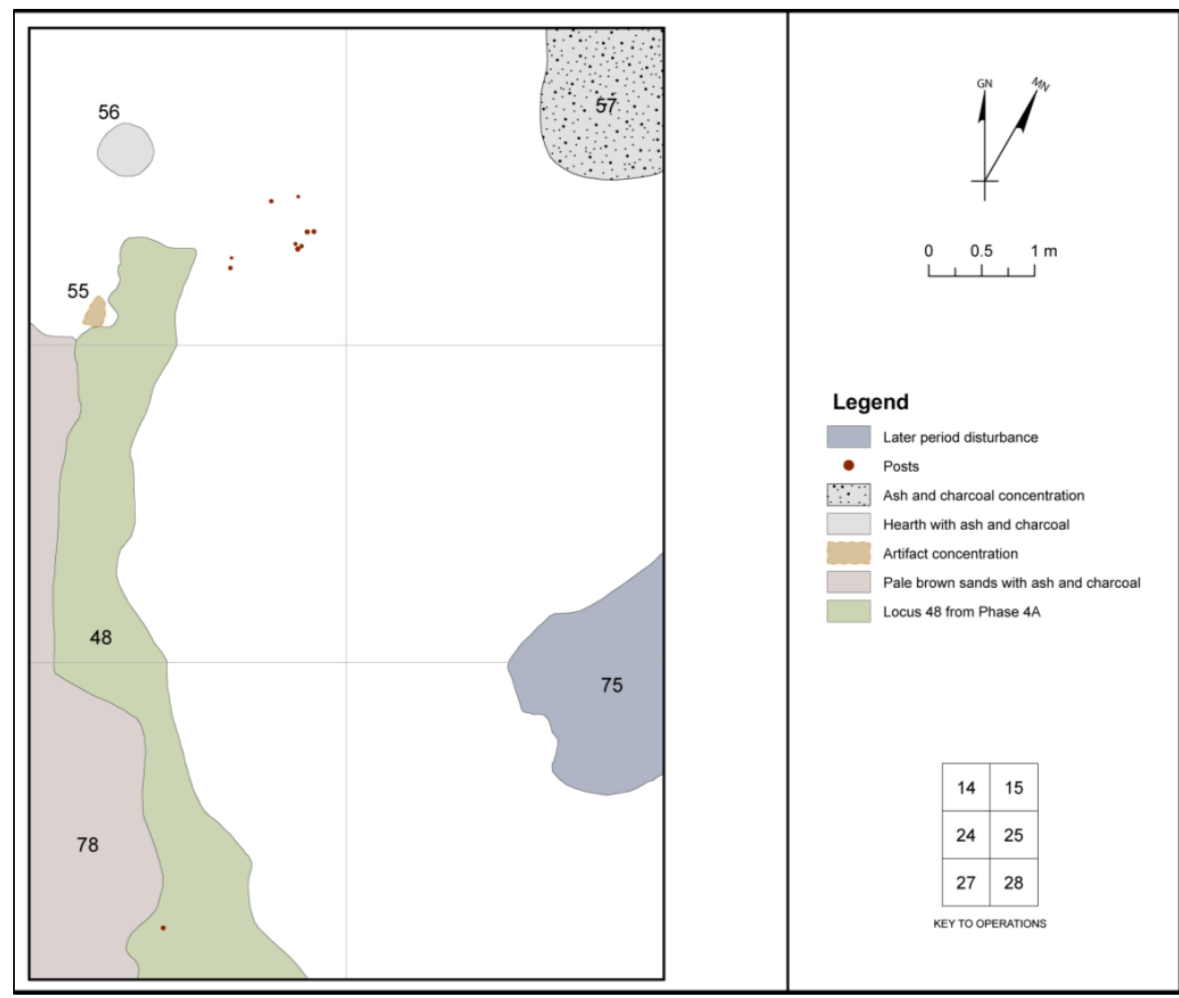

Figure 3.6. Features in el-Mahâsna Block 4. From Anderson (2006).

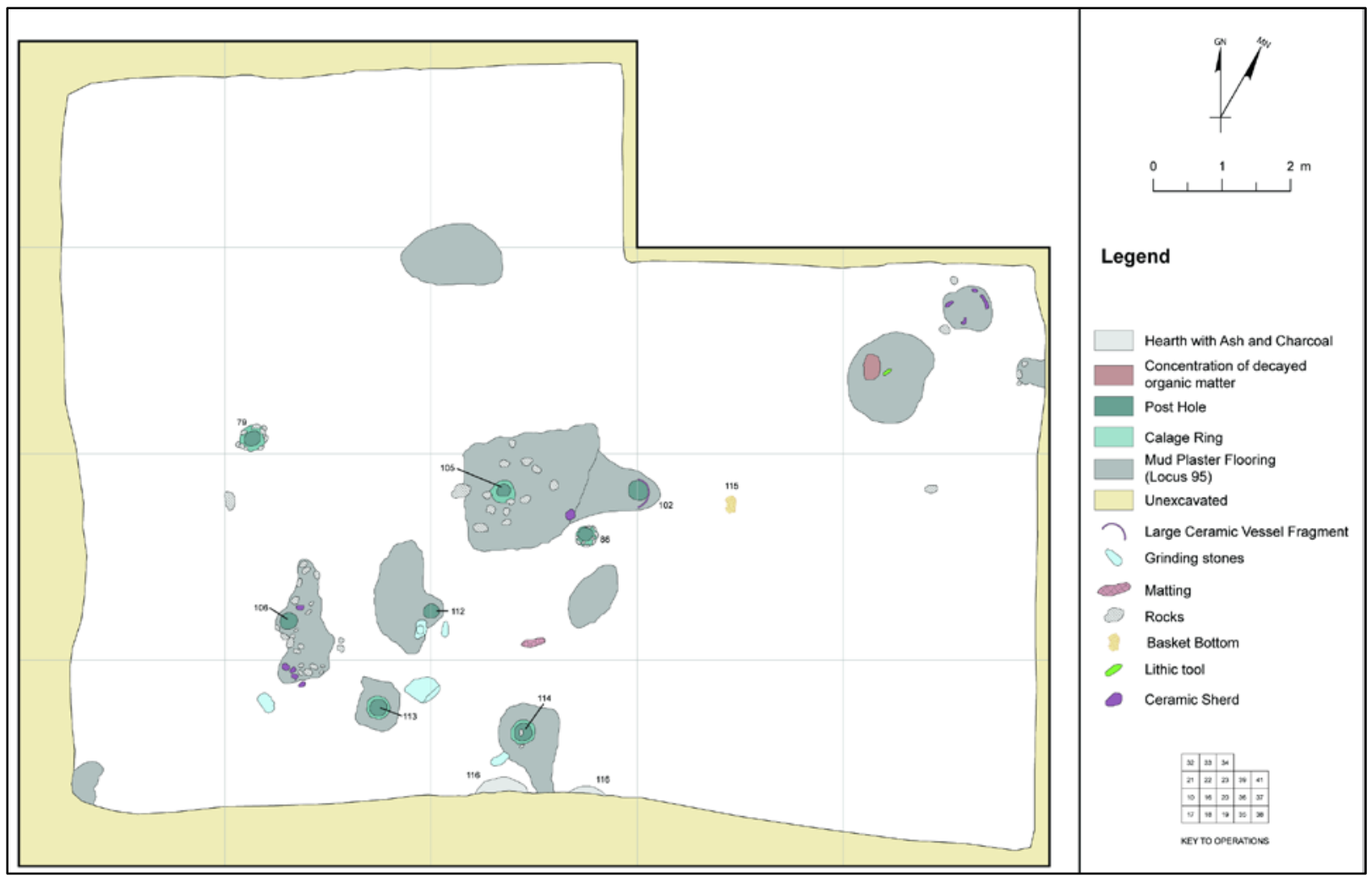

Figure 3.7. Features in el-Mahâsna Block 3. From Anderson (2006). 


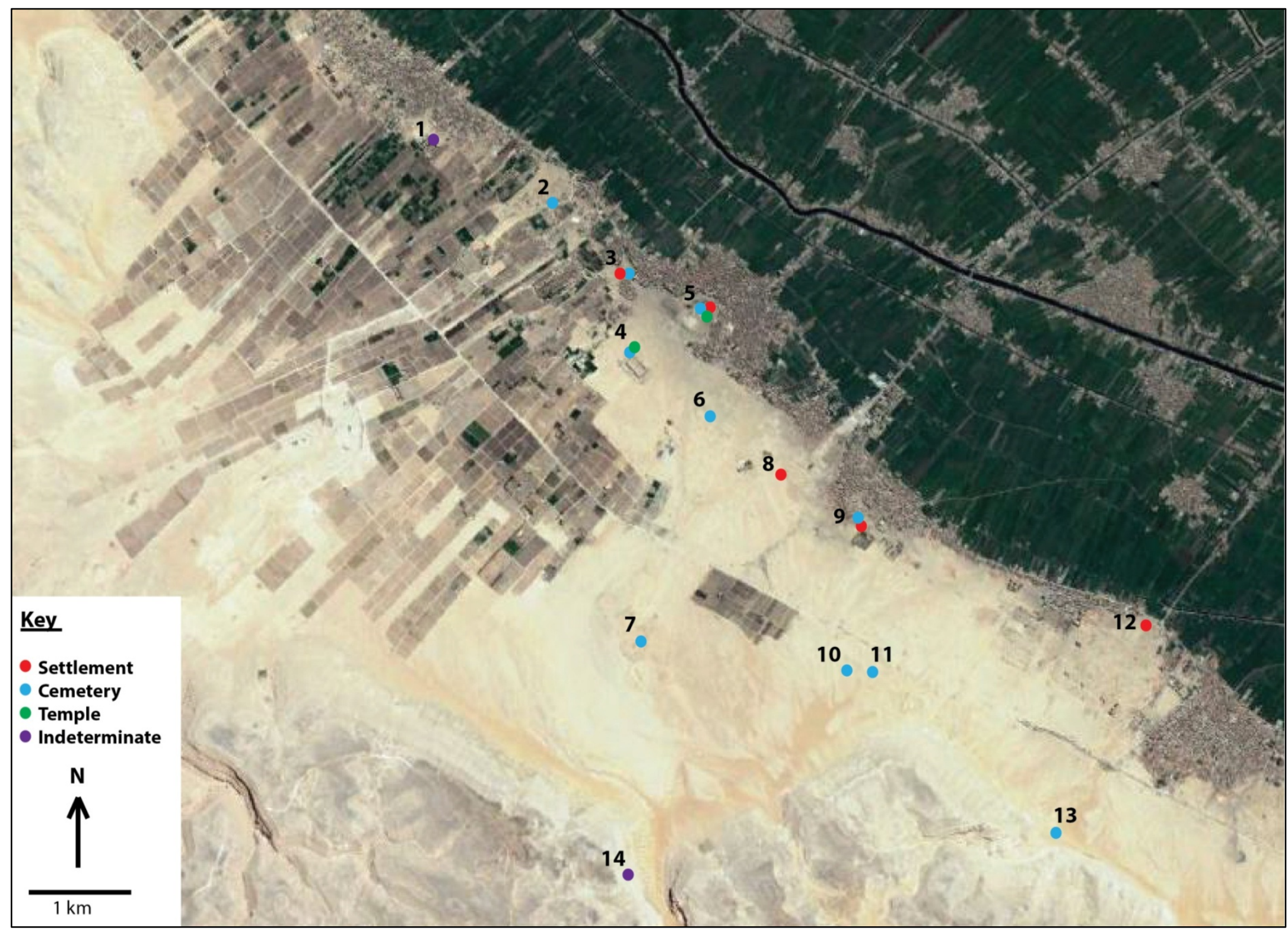

Figure 3.8. Location of the Predyastic-Early Dynastic remains in the Abydos Area. See table on the next page for site information. 


\begin{tabular}{|c|c|c|c|c|c|}
\hline$\#$ & Site & Site type & Patch \# & Date & Publication \\
\hline 1 & S83-54 & ? & S83-54 & & Patch 1991:426 \\
\hline 2 & Salmani & Cemetery & S83-53 & NIC-NIIIA2 & el-Sayed 1979; Patch 1991:424-425 \\
\hline 3 & $\mathrm{D}$ & $\begin{array}{l}\text { Beer kilns \& } \\
\text { Cemetery }\end{array}$ & $\mathrm{n} / \mathrm{a}$ & $\begin{array}{l}\text { Ild-IIla1 (Kilns); } \\
\text { E D (tombs) }\end{array}$ & Peet 1914; Peet \& Loat 1913 \\
\hline 4 & $\begin{array}{l}\text { S/ Funerary } \\
\text { enclosures }\end{array}$ & $\begin{array}{l}\text { Temple \& } \\
\text { cemetery }\end{array}$ & S83-59 & Early Dynastic & $\begin{array}{l}\text { D. Adams \& O’Connor 2003; Bestock 2008, 2009; O'Connor 1999:102, 2009:158- } \\
\text { 181; Patch 1991:434-435; Peet 1914:30-34; Petrie } 1925\end{array}$ \\
\hline 5 & $\begin{array}{l}\text { Kom es- } \\
\text { Sultan }\end{array}$ & $\begin{array}{l}\text { Settlement } \\
\text { \& Temple }\end{array}$ & $\begin{array}{l}\text { NIII/ } \\
\text { D.0- } 2\end{array}$ & Early Dynastic & D. Adams 1999:108; Petrie 1902, 1903 \\
\hline 5 & $\begin{array}{l}\text { M (Kom es- } \\
\text { Sultan) }\end{array}$ & Cemetery & S83-56 & NIIIC1/D 1 & Petrie 1900: 27-28; 1901:36-37; 1902: 14-22; Patch 1991:428-429 \\
\hline 6 & $\mathrm{E} / \mathrm{G}(\mathrm{AMC})$ & Cemetery & $\begin{array}{l}\text { S83- } \\
46 / 47\end{array}$ & NIA-IIIA2 & Naville 1914:12-17; Patch 1991: 415-418; Peet 1914:17-19; Petrie 1902:34-35 \\
\hline & Frankfort & Cemetery & N/A & NIIC-NIID2 & Frankfort 1930:213-215: On the high ground south of the northern expedition house. \\
\hline 7 & $\begin{array}{l}\text { Umm el- } \\
\text { Qa'ab }\end{array}$ & Cemetery & & $\begin{array}{l}\text { NI-Early } \\
\text { Dynastic }\end{array}$ & $\begin{array}{l}\text { Amelineau 1899; Dreyer et al. } 1996 \text { - 2011; Hartmann 2011; Naville 1914; Peet } \\
\text { 1914; Petrie 1900, 1901, } 1902\end{array}$ \\
\hline 8 & $\begin{array}{l}\text { Seti temple } \\
\text { breweries }\end{array}$ & $\begin{array}{l}\text { Settlement } \\
\text { \& Beer kilns }\end{array}$ & S83-61 & NIId-IIla1 & Patch 1991:437; Peet 1914; Peet \& Loat 1913 \\
\hline 9 & $\begin{array}{l}\text { Habachi \& } \\
\text { Others }\end{array}$ & $\begin{array}{l}\text { Cemetery \& } \\
\text { Settlement }\end{array}$ & S83-72 & $\begin{array}{l}1^{\text {st }} \text { dynasty/ } \\
\text { NIIC2? }\end{array}$ & el-Aref 2016; Habachi 1939; Hossein 2011; Patch 1991:414, 448; \\
\hline 10 & $x / \mathrm{X} / \mathrm{B}$ & Cemetery & S83-43 & NIID1- NIIIB & Randall-Maclver \& Mace 1902:53-55; Patch 1991:411-412; Location approximate \\
\hline 11 & $\phi / C$ & Cemetery & S83-44 & NI-Ila or IC? ${ }^{2}$ & Randall-Maclver \& Mace 1902:51-53; Patch 1991:413. Location approximate \\
\hline 12 & Abydos ATP & Settlement & S83-3 & NIB-NIC/IIA & Harvey 1998:146-147; Randall-Maclver \&Mace 1902:76; Patch 1991:376-377 \\
\hline 13 & S83-16 & Cemetery & S83-16 & Predynastic & Patch 1991:384-385; Ayrton et al. 1904:PI. LXI \\
\hline \multirow[t]{2}{*}{14} & S83-52 & Workshop? & S83-52 & Predynastic? & Patch 1991: 423 ; Location approximate \\
\hline & Hawashim & Cemetery & S83-71 & IIC-III? & el-Sayed 1979:259-60; Patch 1991:447:"west" of Hawashim, $2 \mathrm{~km}$ south of Ramses temple \\
\hline
\end{tabular}

Figure 3.8 references 


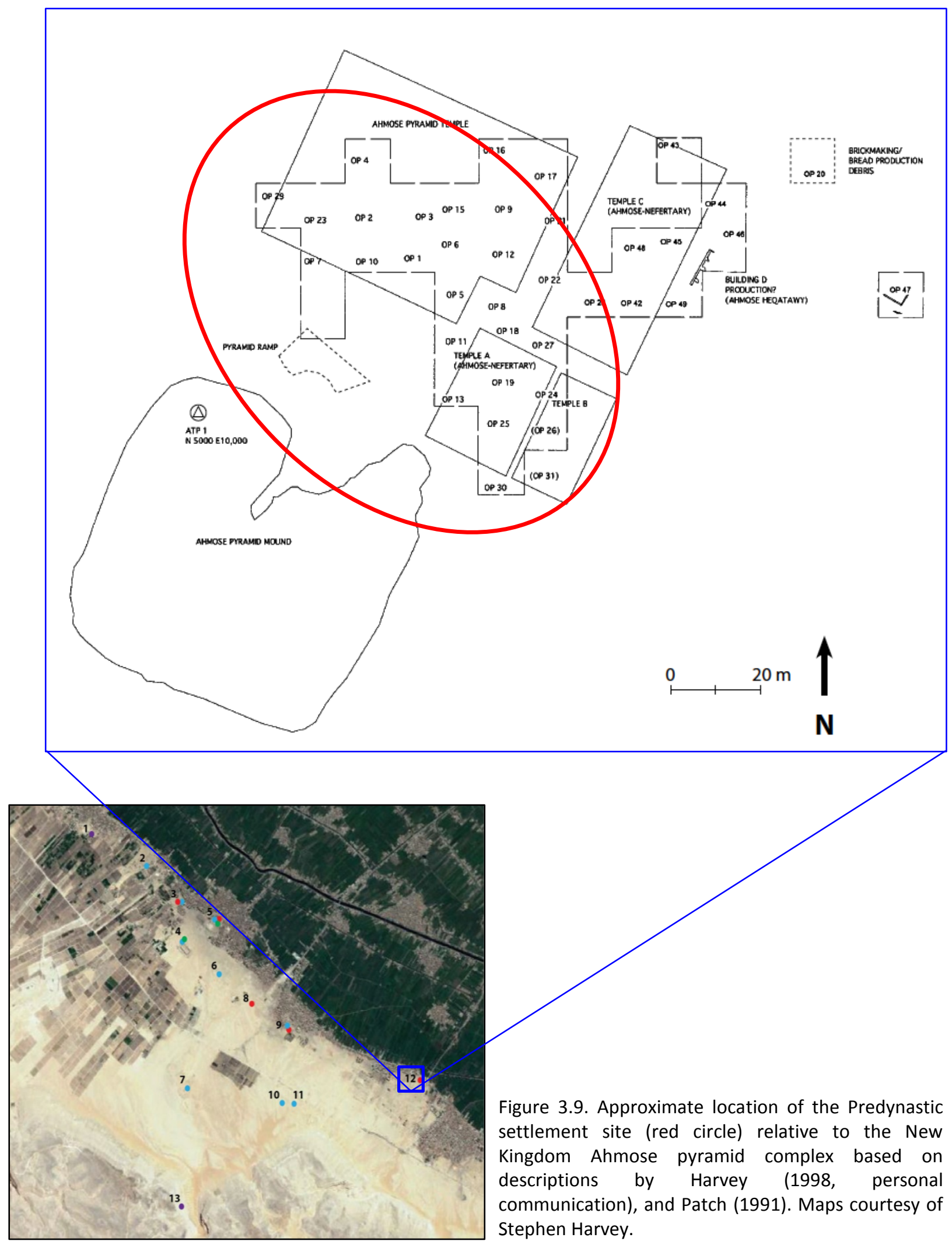




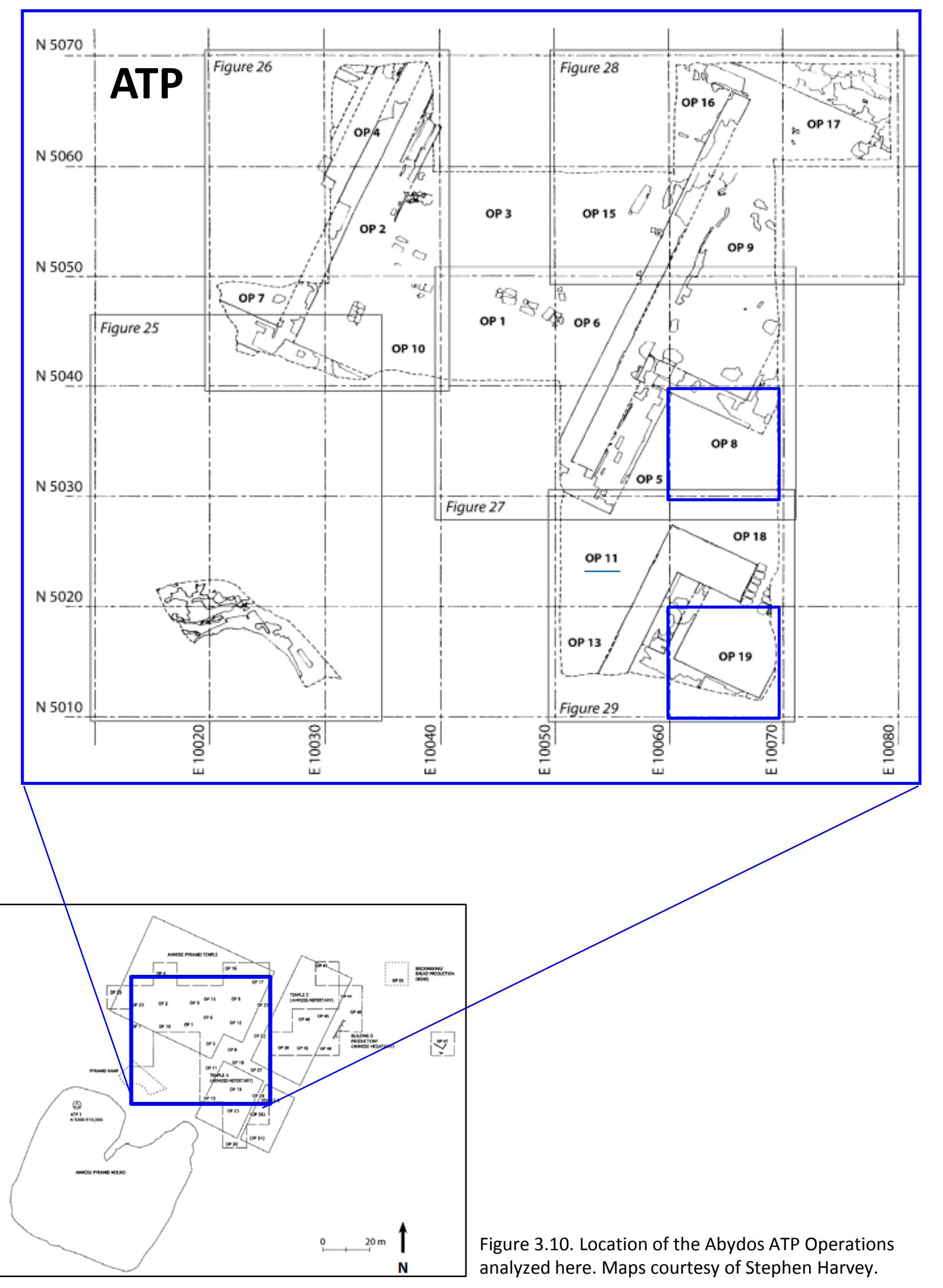




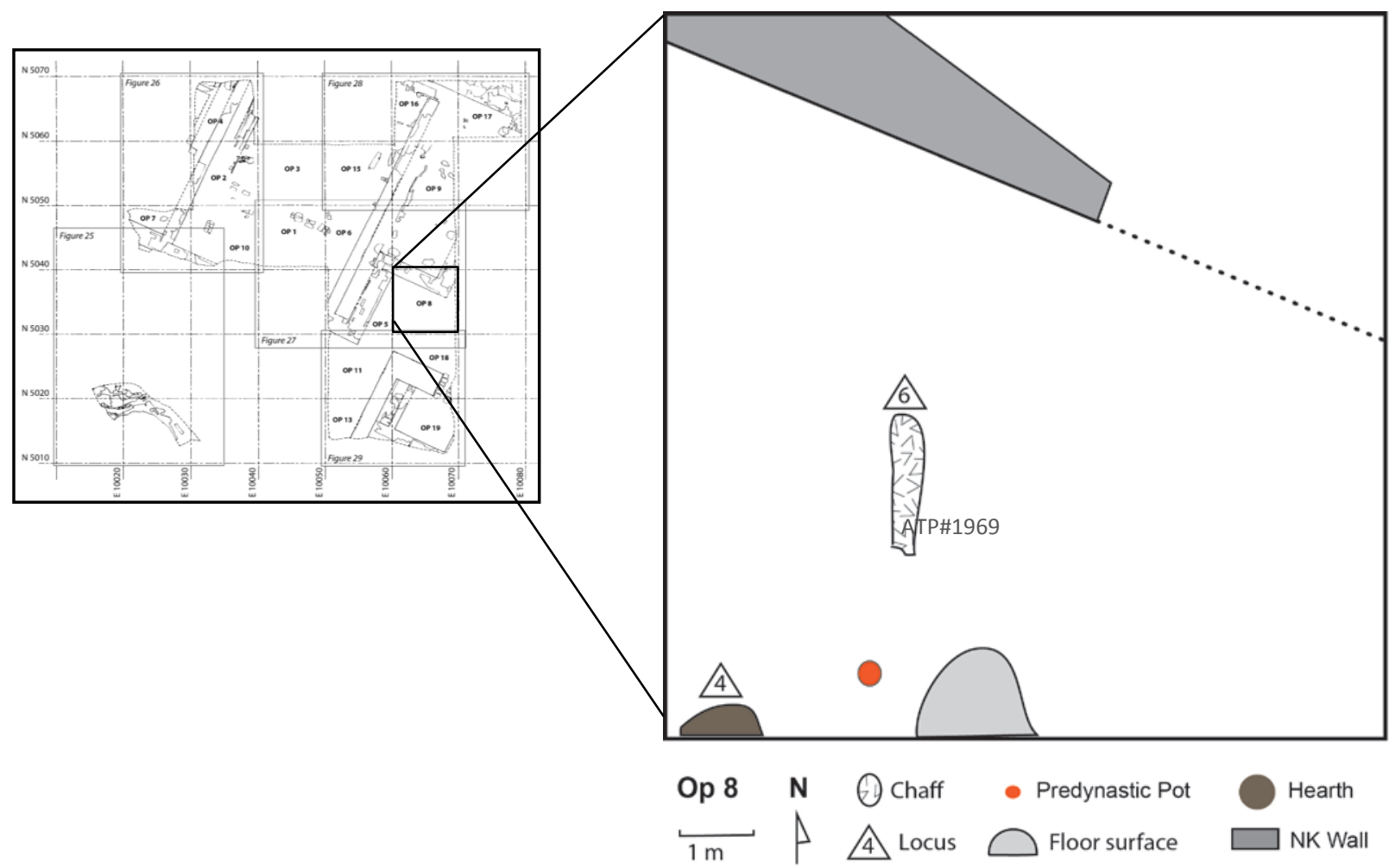

Figure 3.11. The uppermost features in the Predynastic layer of Op 8. NK=New Kingdom.

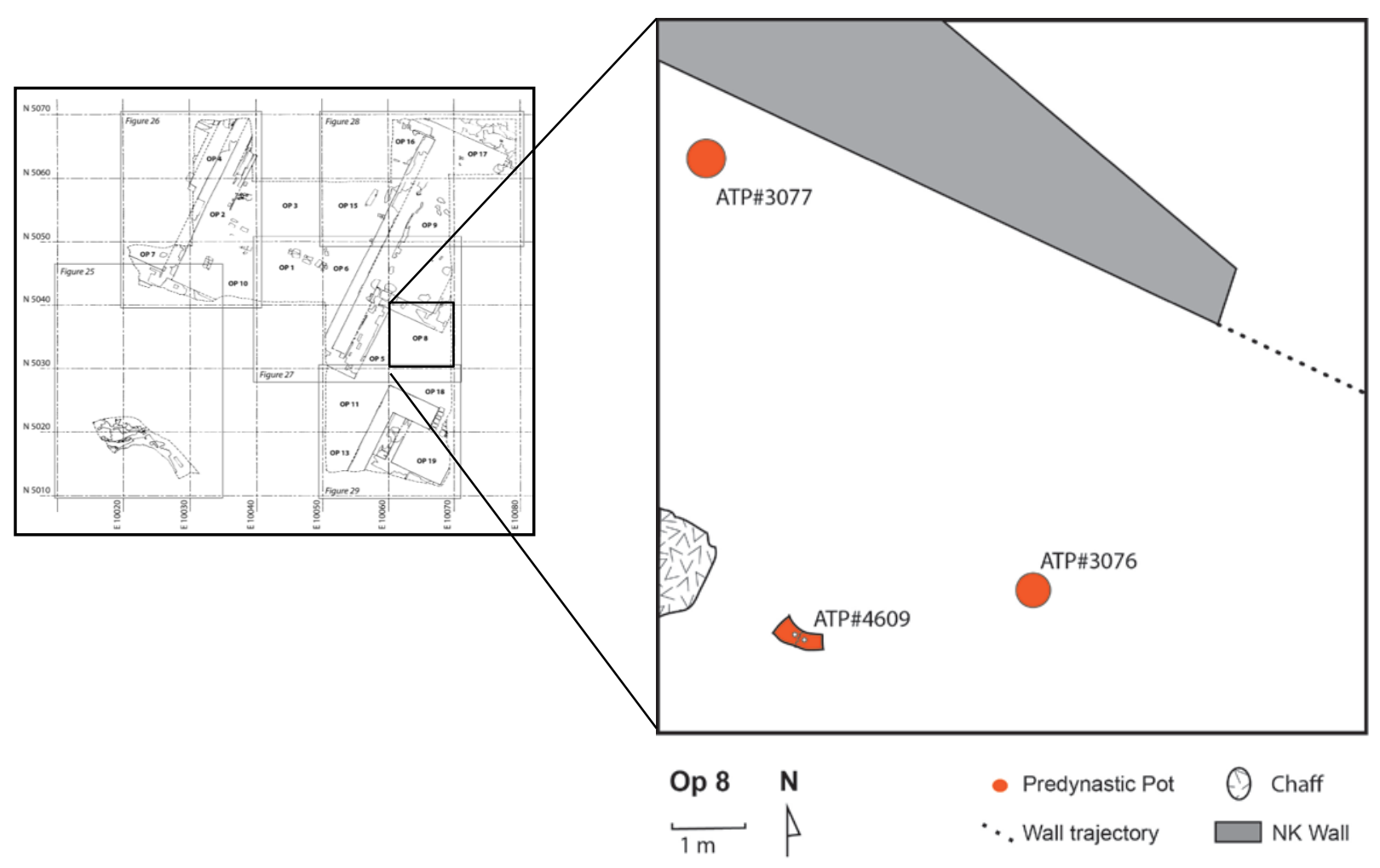

Figure 3.12. The lower features in the Predynastic layer of Op 8. NK=New Kingdom. 


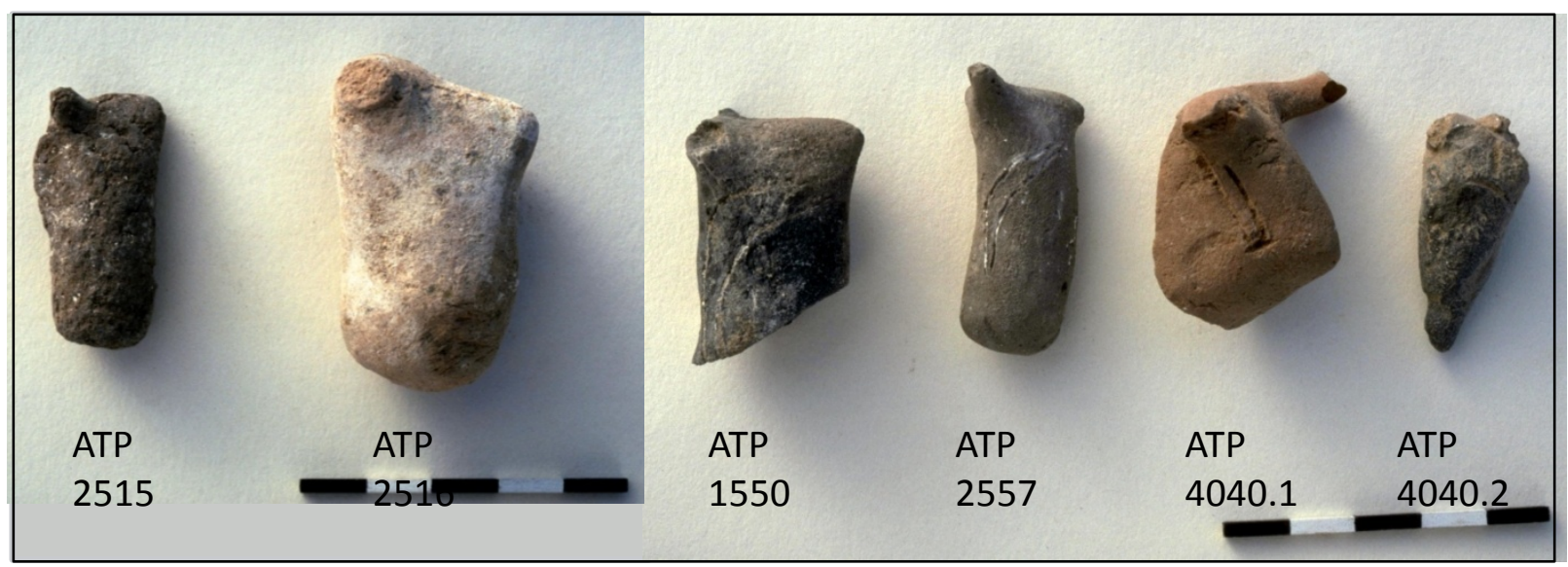

Figure 3.13. Figurine fragments in the shape of cattle. Left: ATP.2515 \&2516-Op 8; Right: ATP.1550- Op 4, ATP.2577-Op11, ATP.4040- Op 19. Image credits: Ahmose-Tetisheri Project.

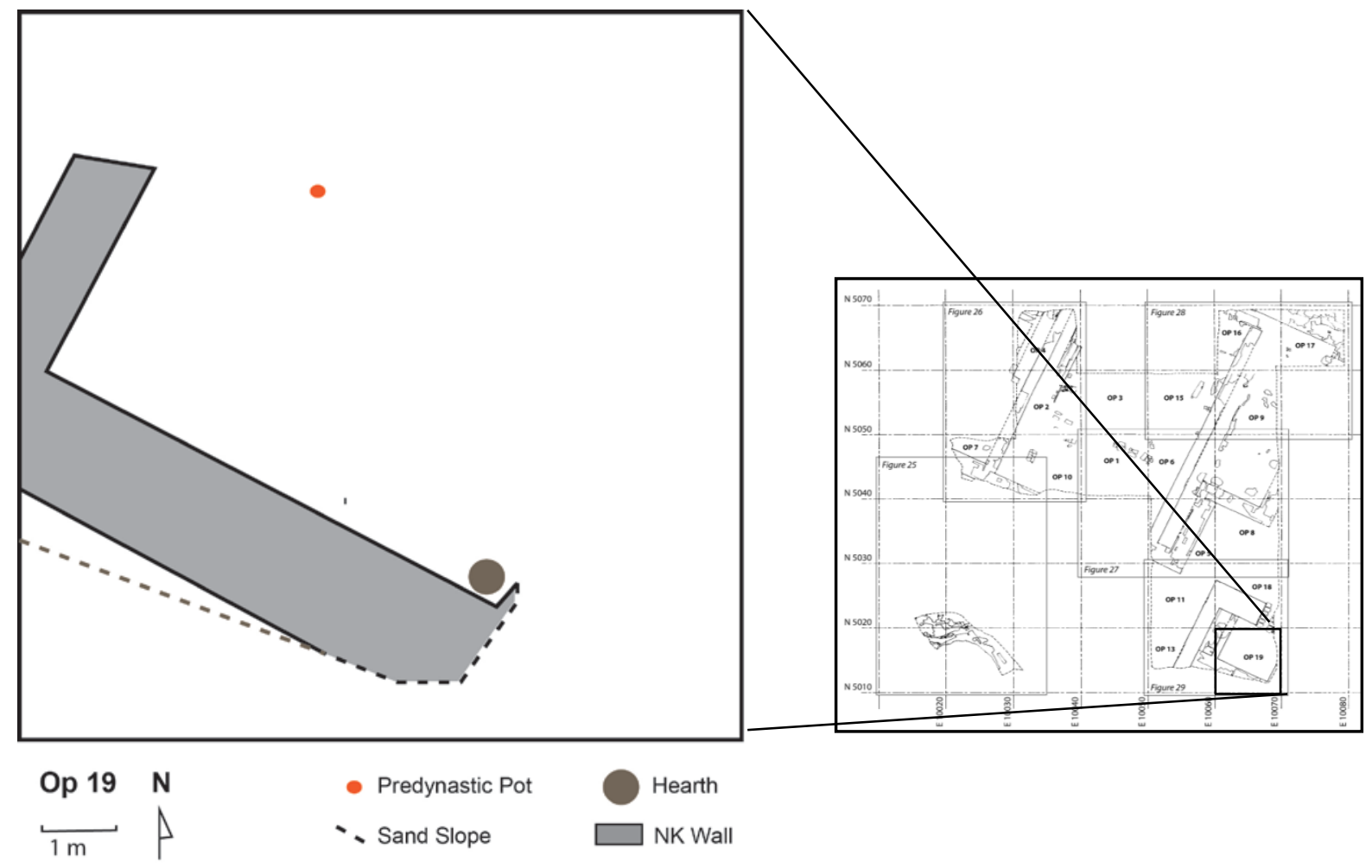

Figure 3.14. The Predynastic remains in Op 19. NK=New Kingdom. 


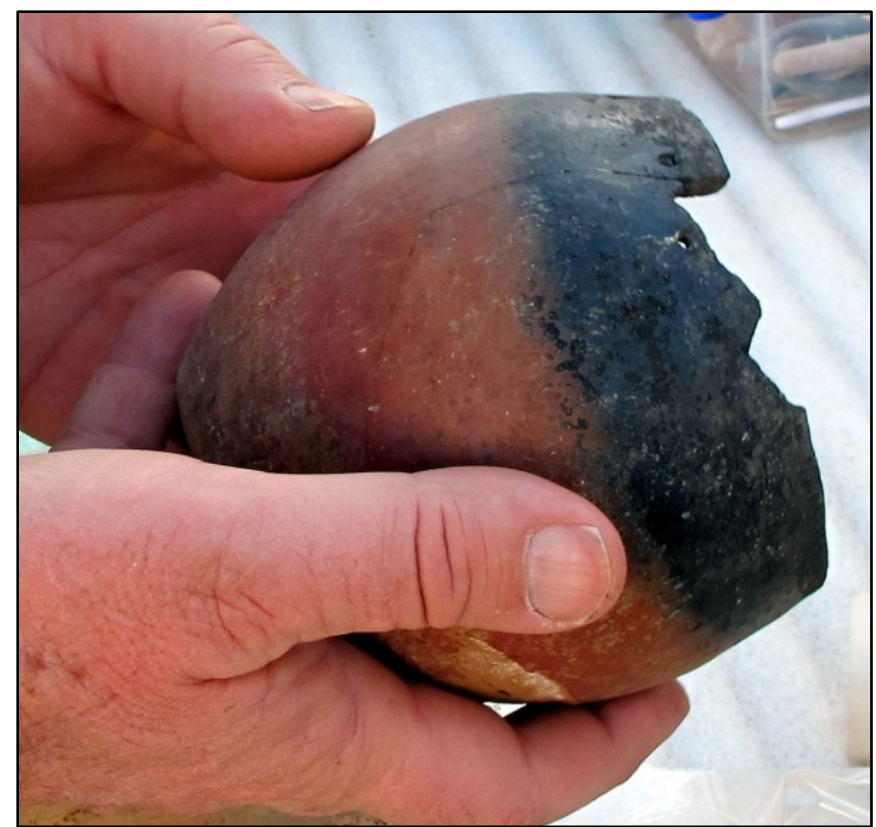

Figure 3.15a. Black-topped ceramic vessel with repair holes. ATP4615- Op11.

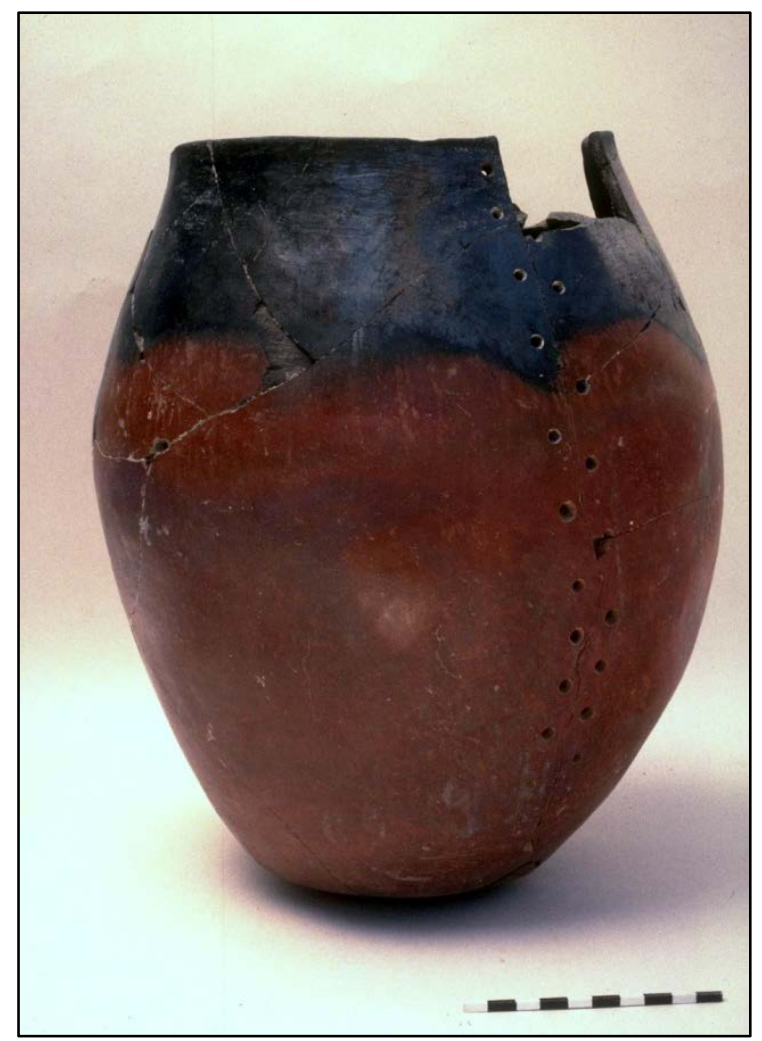

Figure 3.15b. Black-topped ceramic vessel with repair holes. ATP1969- Op 8. Image credit: Ahmose-Tetisheri Project.

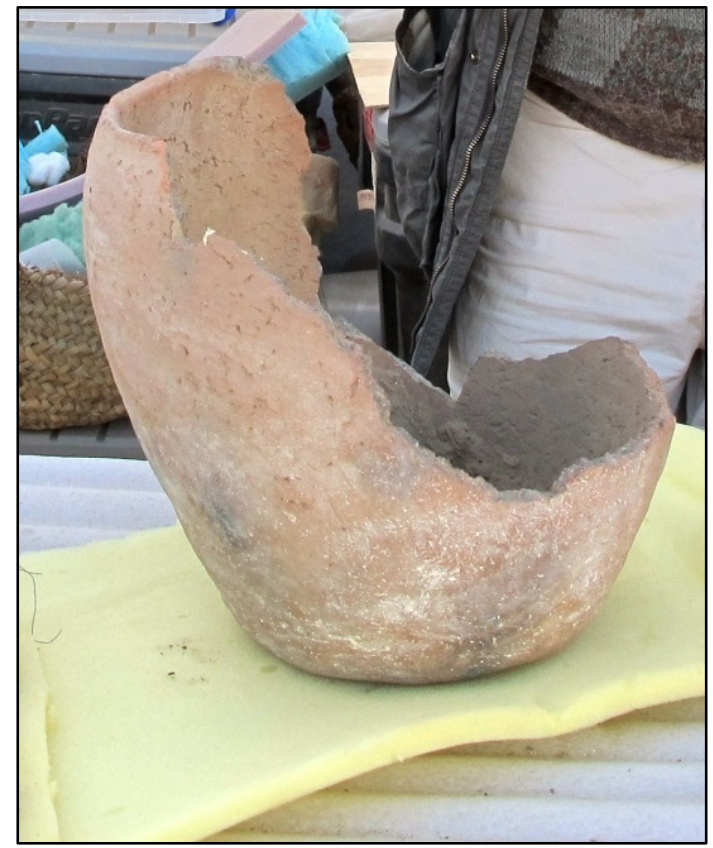

Figure 3.16a. Large open-mouthed jar.

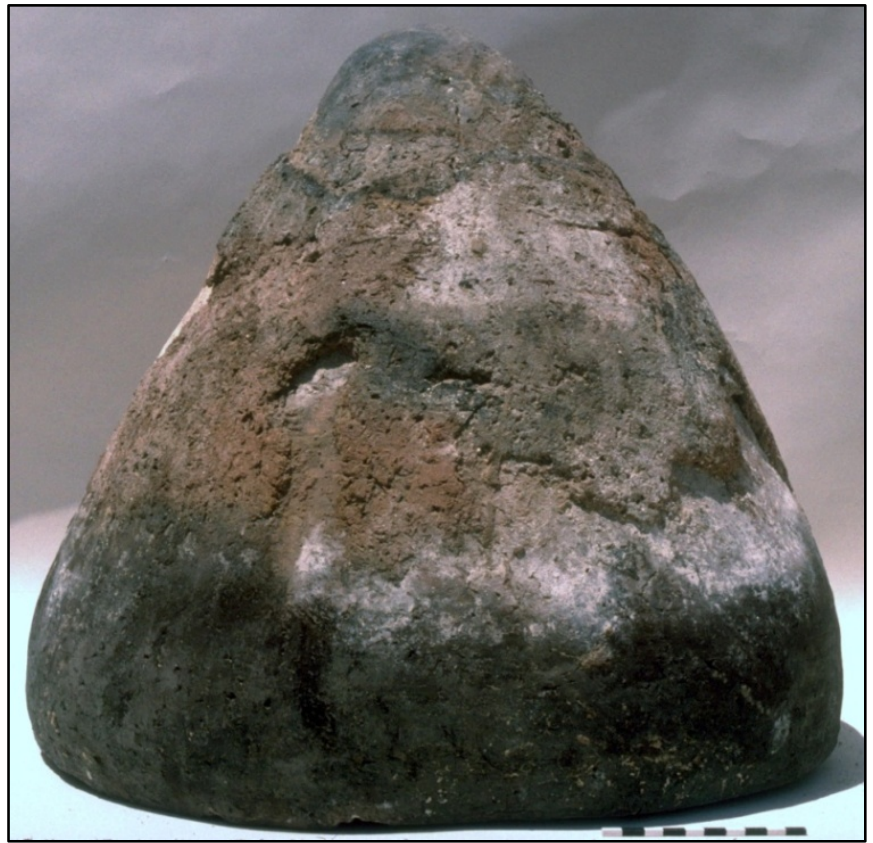

Figure 3.16b. Large open-mouthed jar. ATP3076- Op8. Image credit: Ahmose-Tetisheri Project. 

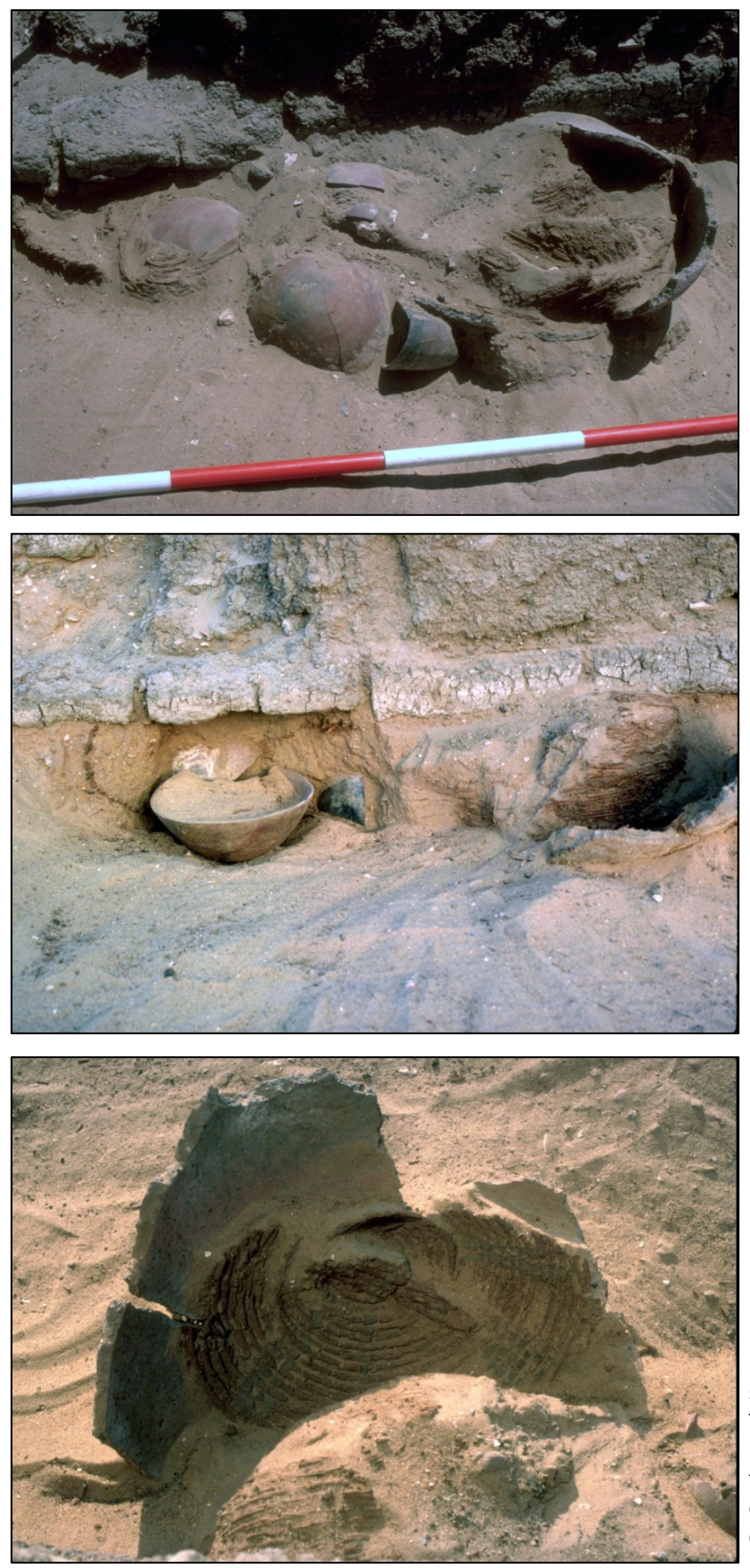

Figure 3.17a-c. Cache of pottery and baskets in Op 11. Top: Cache in situ. Middle: Detail after removal of some piece showing parts of the cache underneath a New Kingdom structure. Bottom: Detail of Basketry. The Basketry does seem to be Predynastic in date since it compares favorably to known Predynastic styles of basketry (Wendrich 2000). Image credits: Ahmose-Tetisheri Project. 


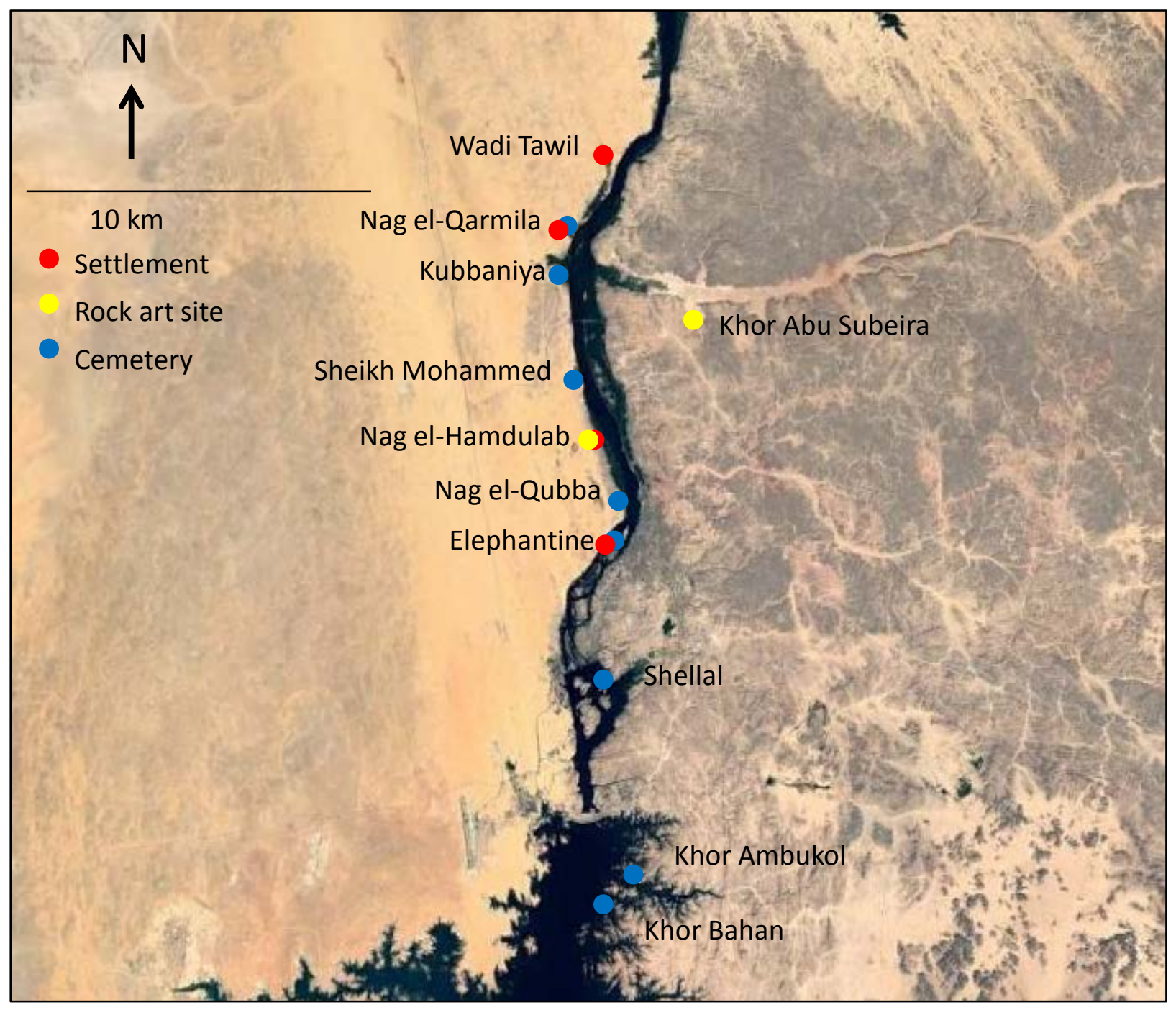

Figure 3.18. Predynastic sites in the Aswan area. 


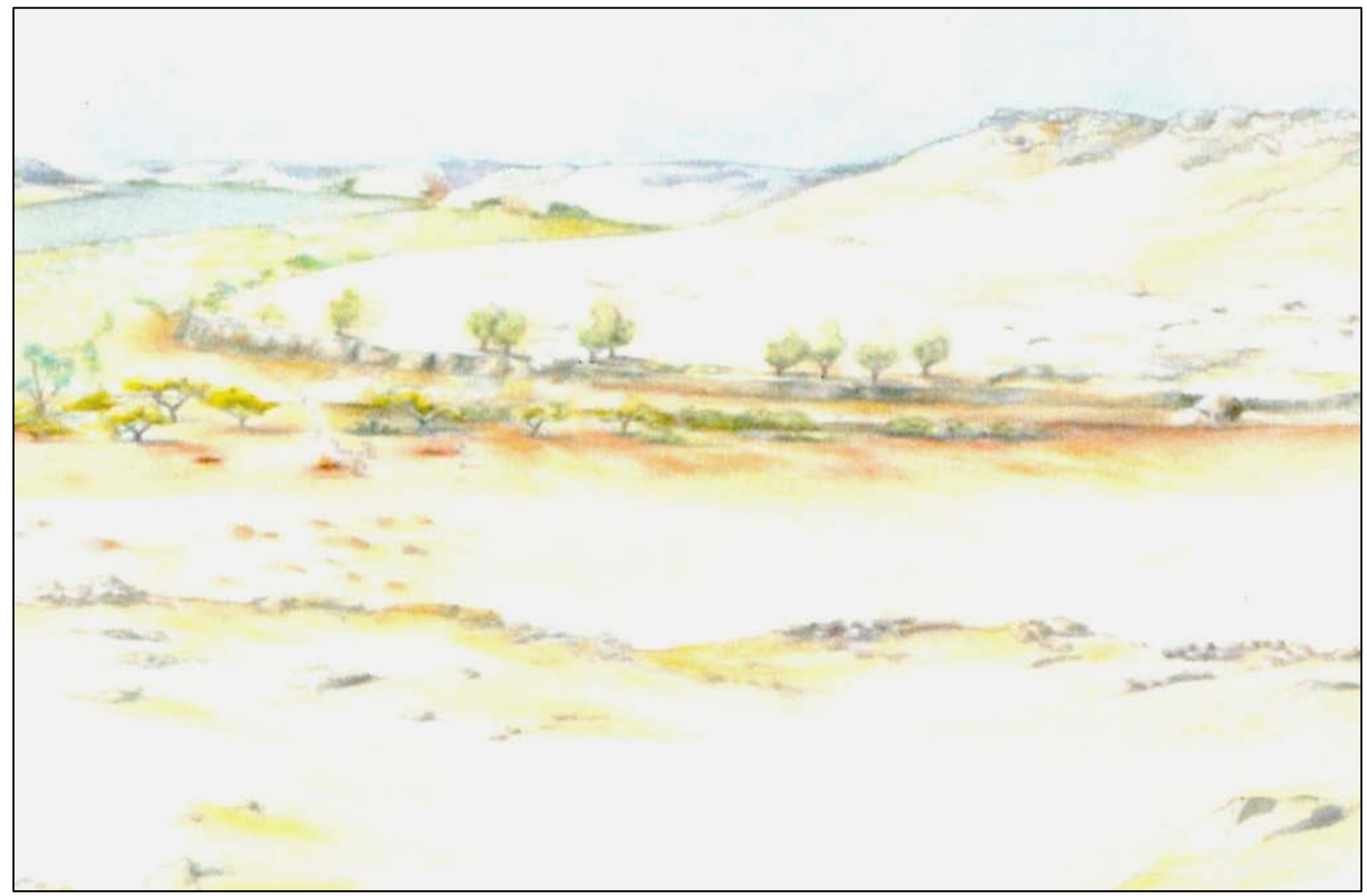

Figure 3.19: Artist's reconstruction of the Nag el-Qarmila landscape, from Gatto et al. (2009c). 


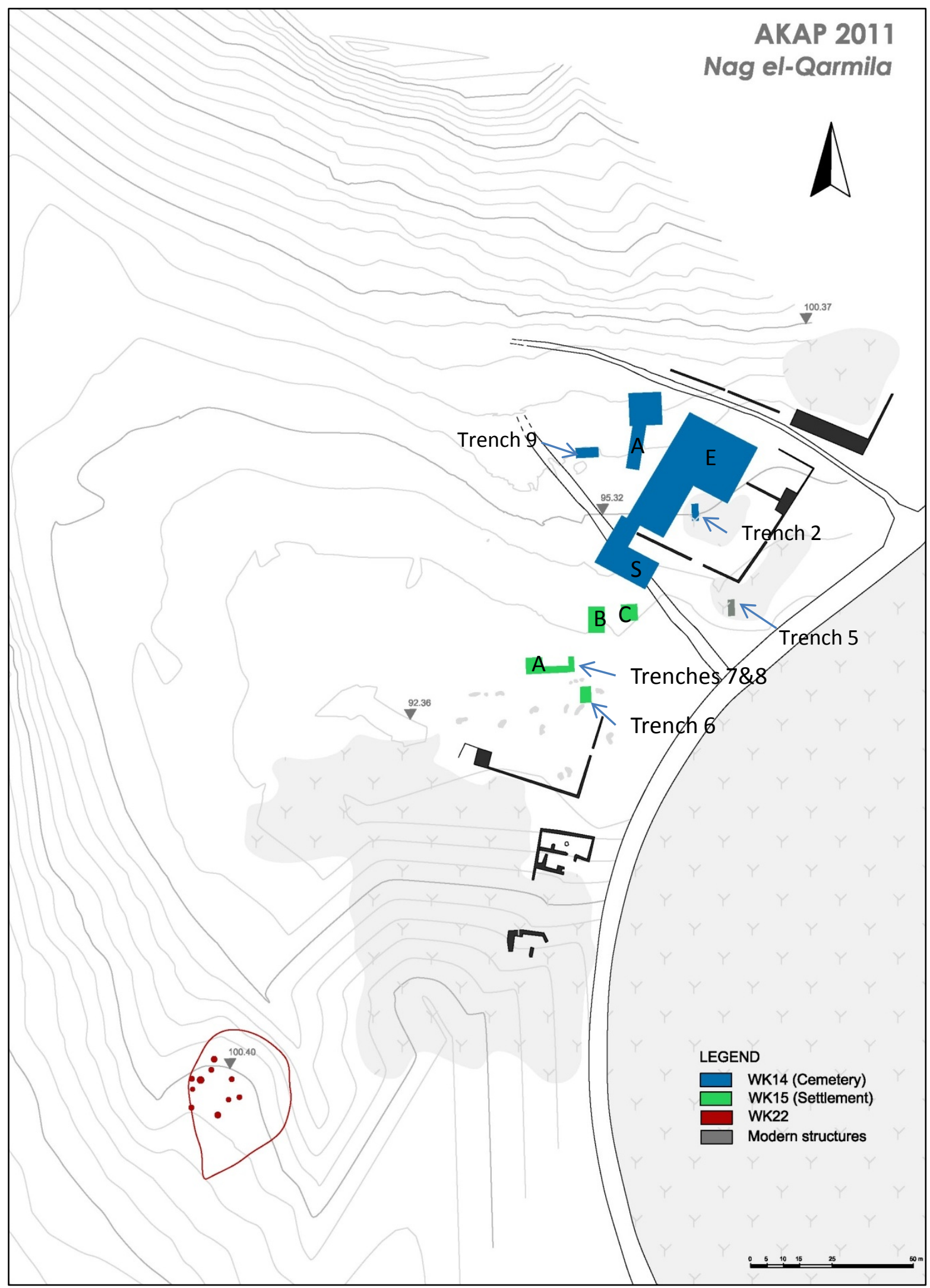

Figure 3.20. Nag el-Qarmila. Image credit: Aswan-Kom Ombo Archaeological Project. 


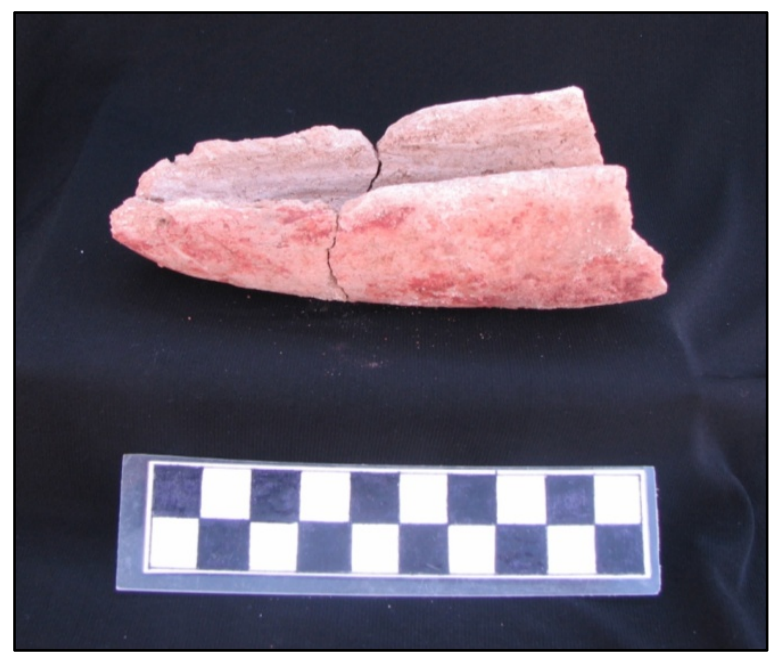

Figure 3.21. Fragment of a model boat with red ochre from Nag el-Qarmila (AKAP WK15). Image credit: Aswan-Kom Ombo Archaeological Project.

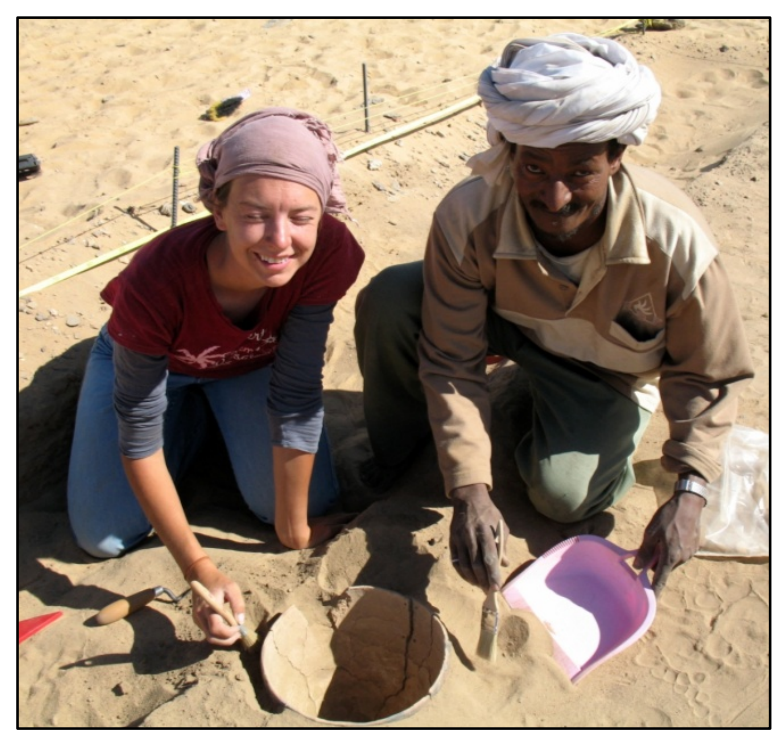

Figure 3.22. Excavation of a cracked in-situ pot with repair holes Nag el-Qarmila (AKAP WK15).

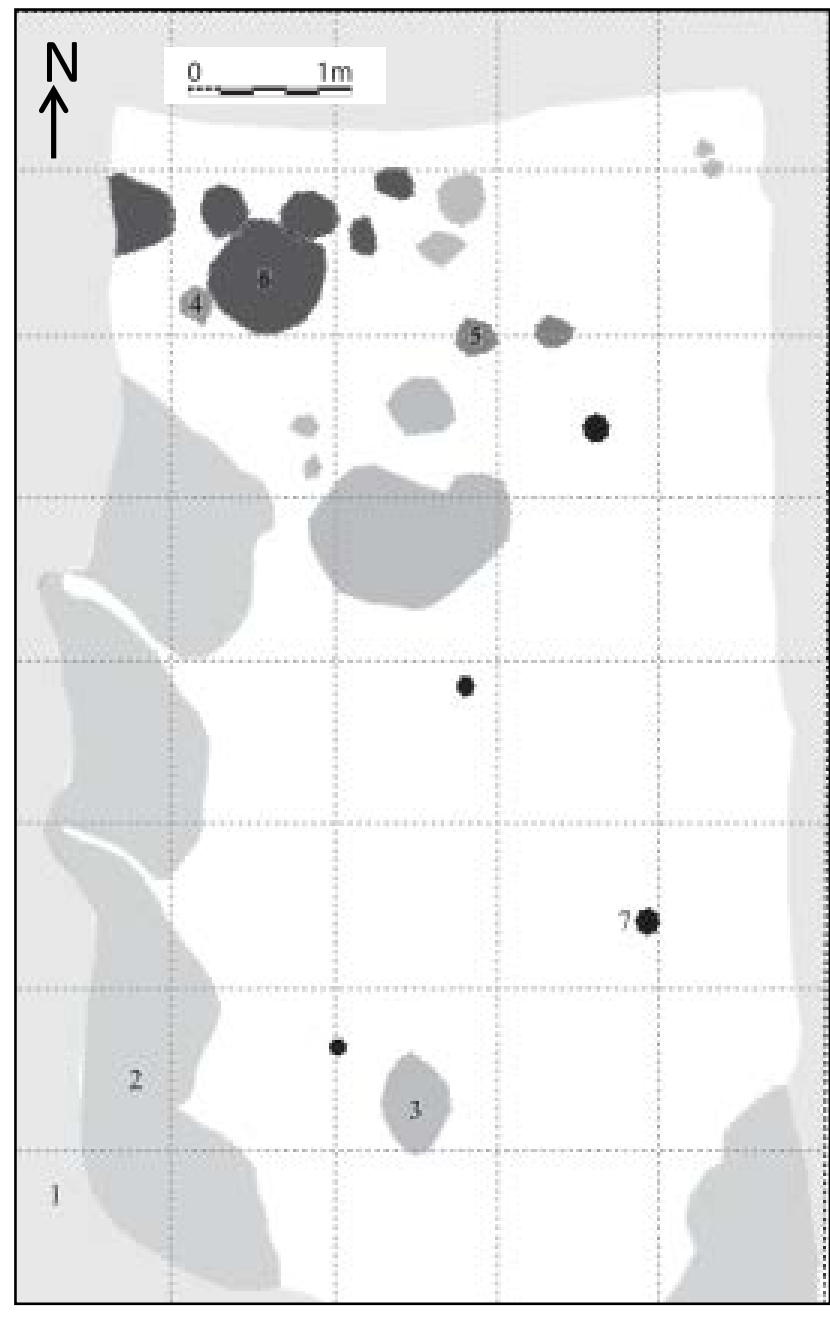

Figure 3.23. Nag el-Qarmila, WK15 Area B, Lowest level. 1)Sand; 2)Silt extraction pits; 3) Depressions; 4) Pot emplacements; 5) Mud-lined pits; 6) Hearths; 7) Post holes. Image credit: Aswan-Kom Ombo Archaeological Project. 


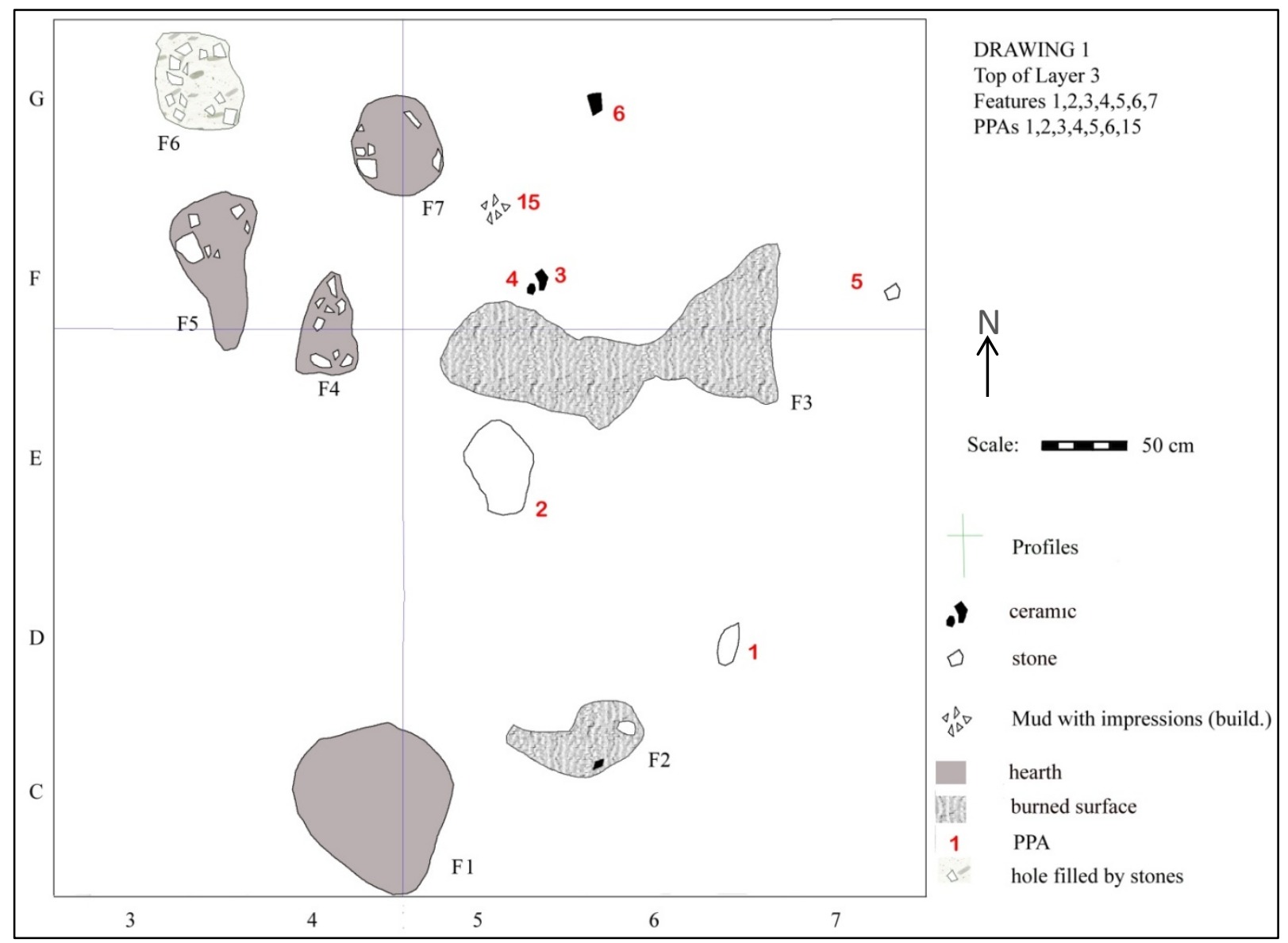

Figure 3.24. Nag el-Qarmila, WK15 Area A, Layer 3. 1=upper grinder, $2=$ mortar, $3=$ =ceramic sherd with repair hole, 4=sherd, 5=hammerstone, 6=incised ceramic sherd. Image credit: Aswan-Kom Ombo Archaeological Project.

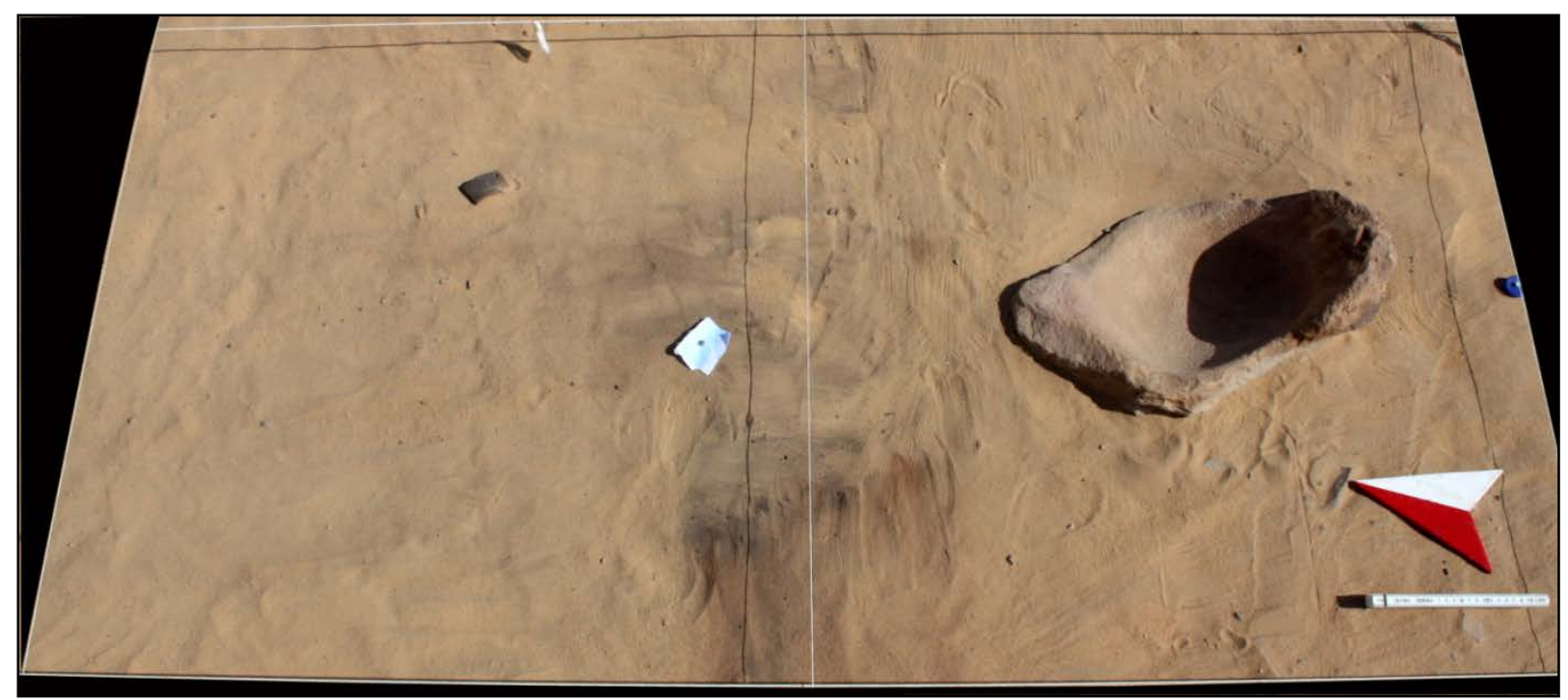

Figure 3.25. Nag el-Qarmila, WK15 Area A, in-situ stone mortar. Image credit: Aswan-Kom Ombo Archaeological Project. 


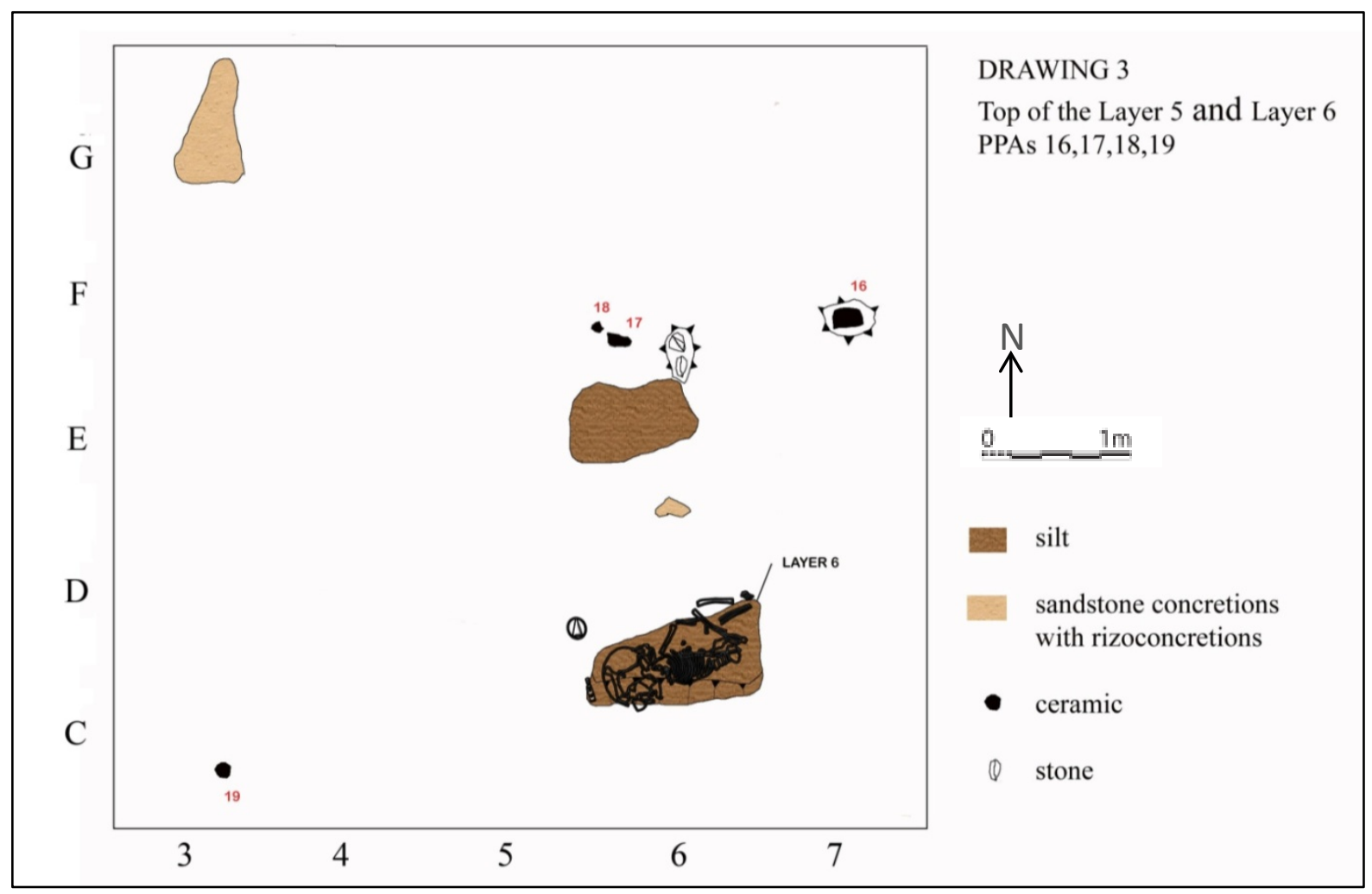

Figure 3.26. Nag el-Qarmila, WK15 Area A, lowest layer with child burial. Image credit: Aswan-Kom Ombo Archaeological Project.

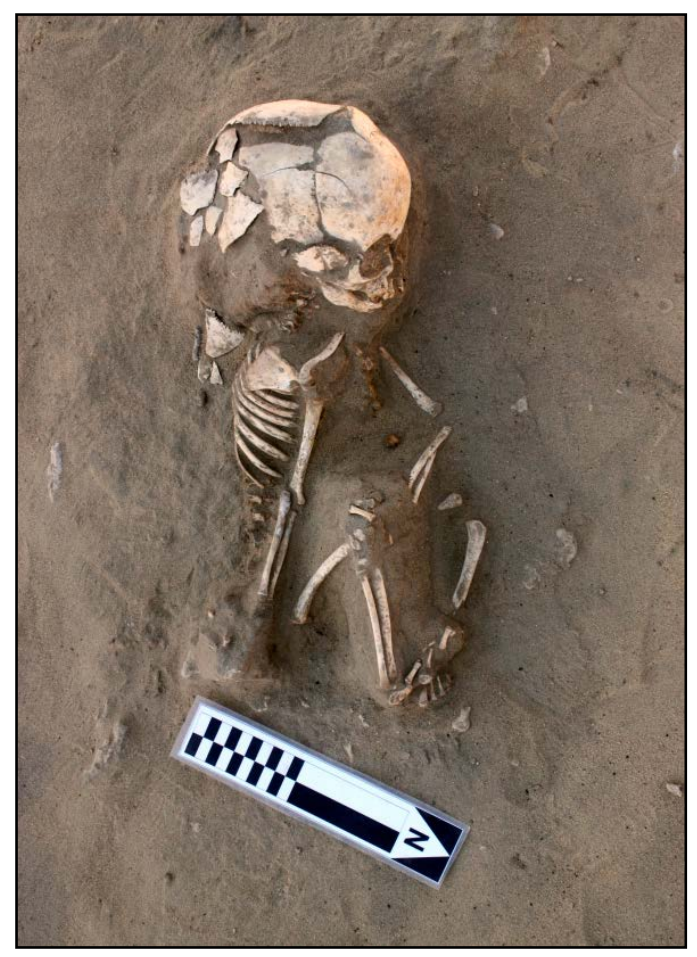

Figure 3.27. Child burial in Nag el-Qarmila, WK15 Area A. Image credit: Aswan-Kom Ombo Archaeological Project. 


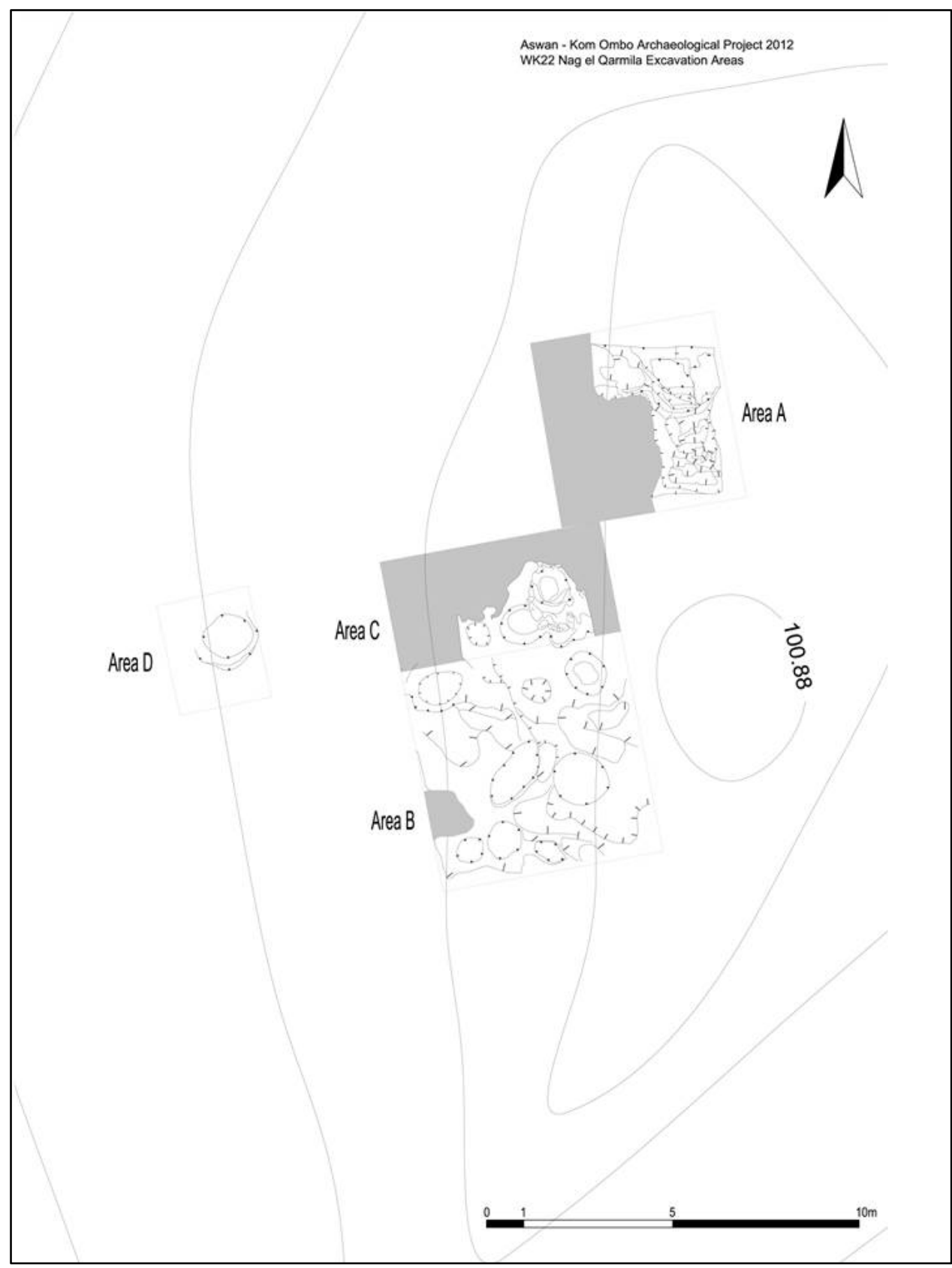

Figure 3.28. Plan of features in Nag el-Qarmila, WK22. Image credit: Aswan-Kom Ombo Archaeological Project. 




Figure 3.29. Hierakonpolis map. Courtesy of the Hierakonpolis Expedition, cartography by Joel Paulson. 


\section{Chapter 4 Figures}

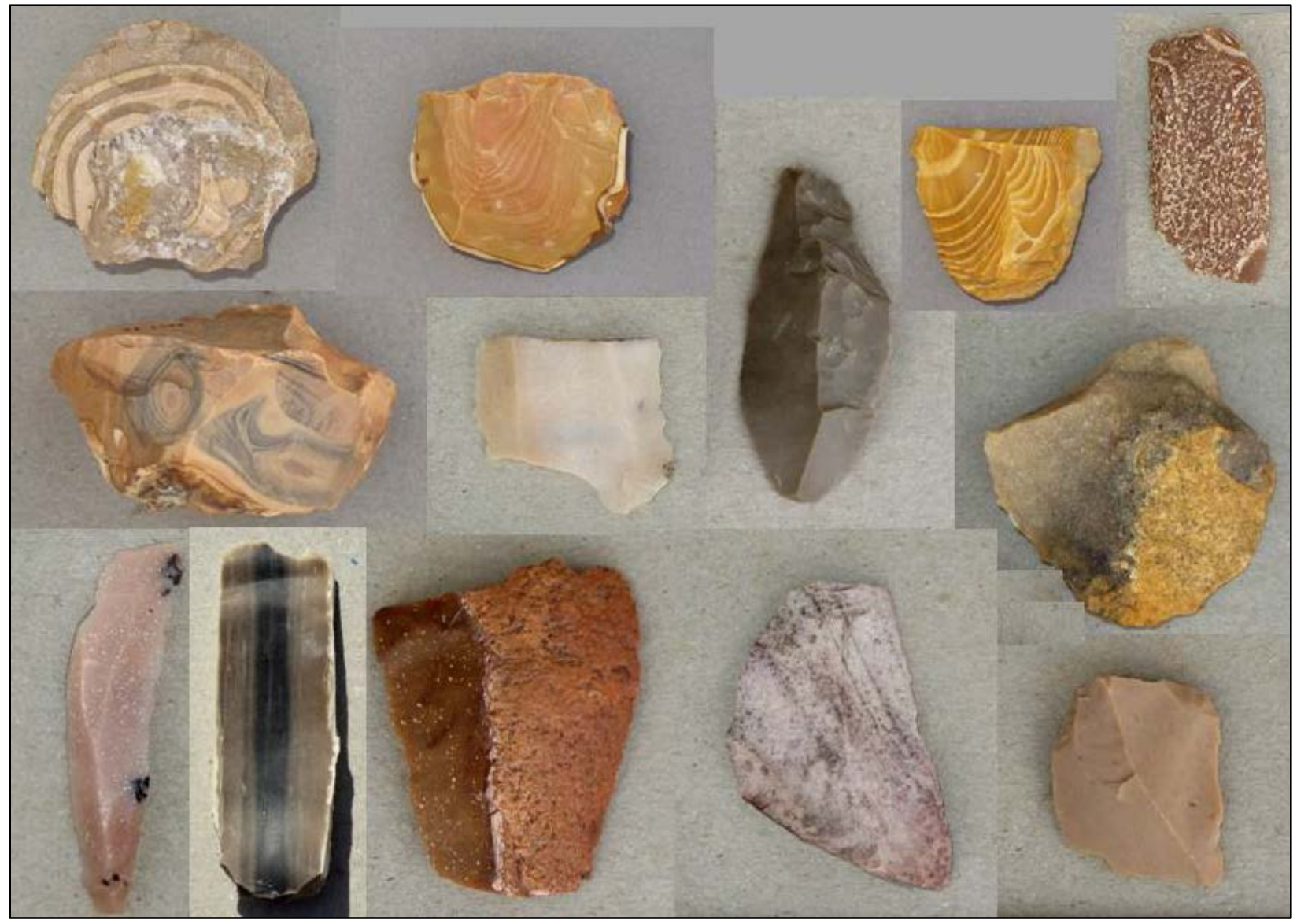

Figure 4.1. Variability in microcrystaline quartz rocks (chert) in Egypt.

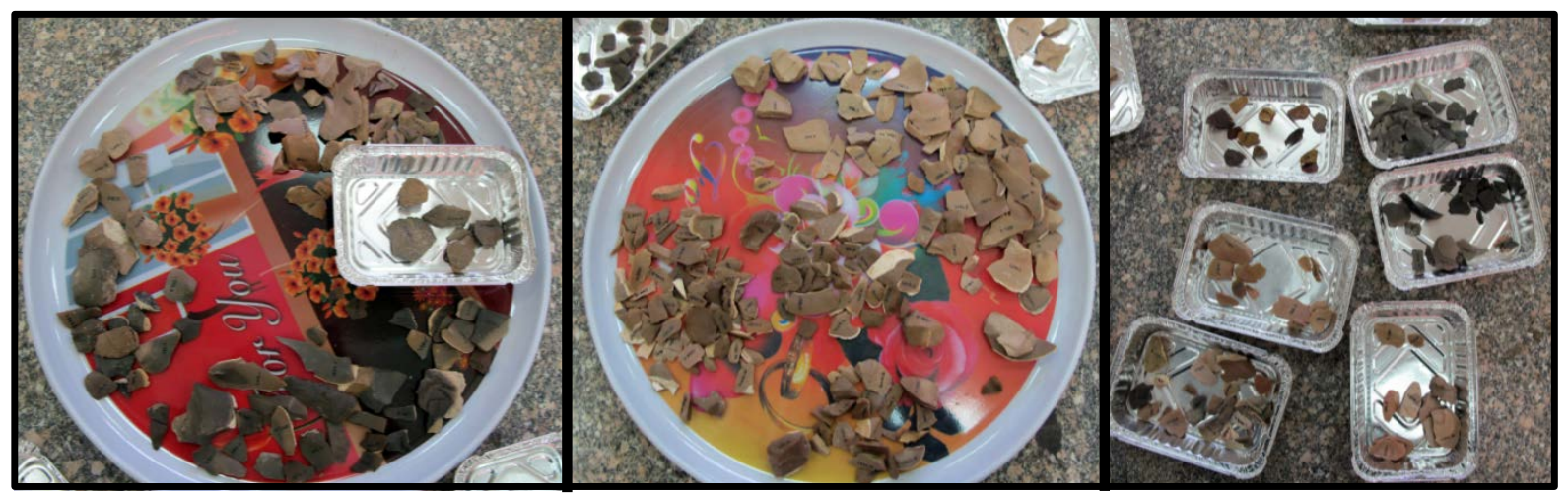

Figure 4.2. Forming groups of chert varieties based on macroscopic properties of cortex type, structures, color, luster, translucence, and texture. 


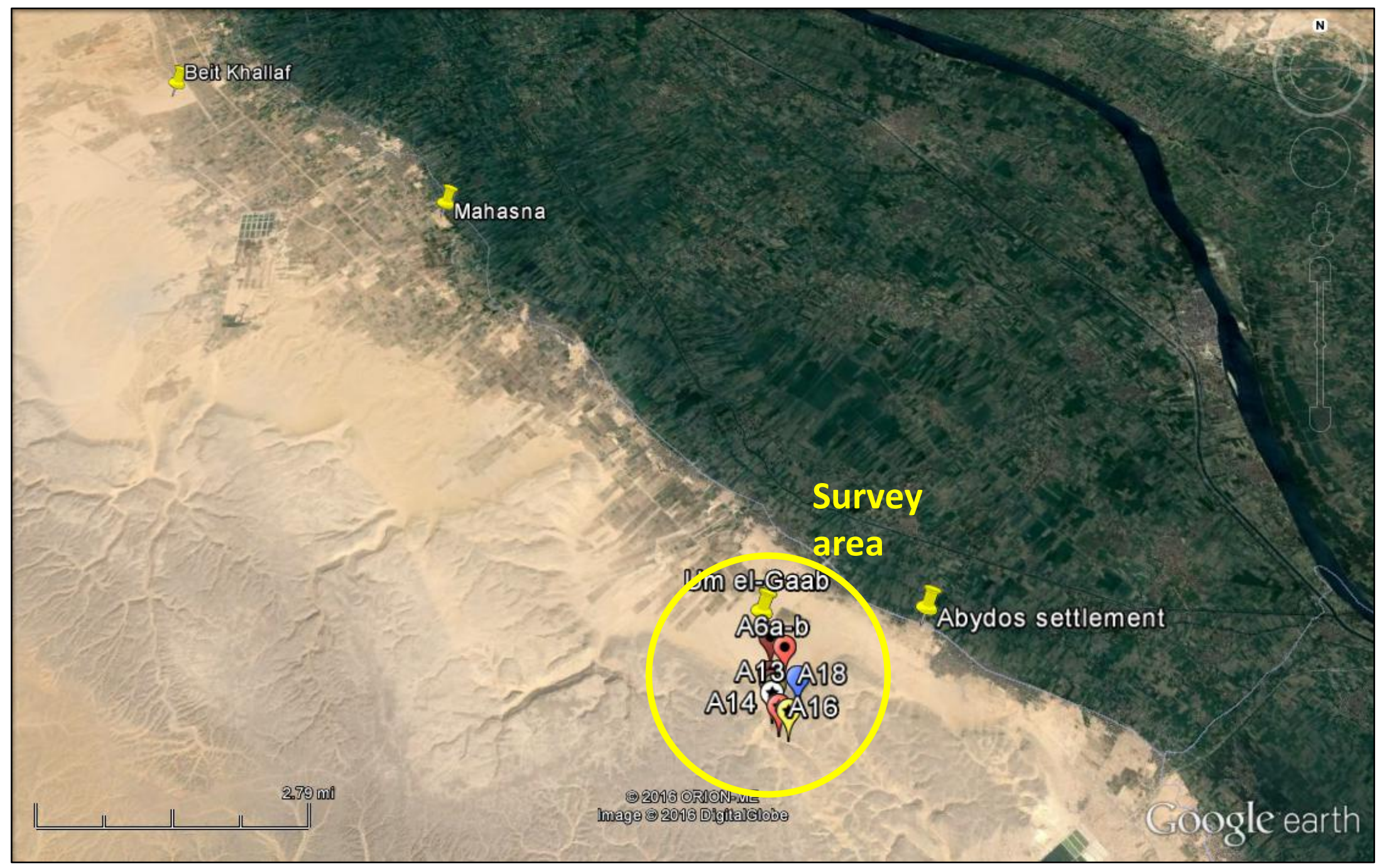

Figure 4.3. Overview of Raw material survey sites in the Abydos region. 


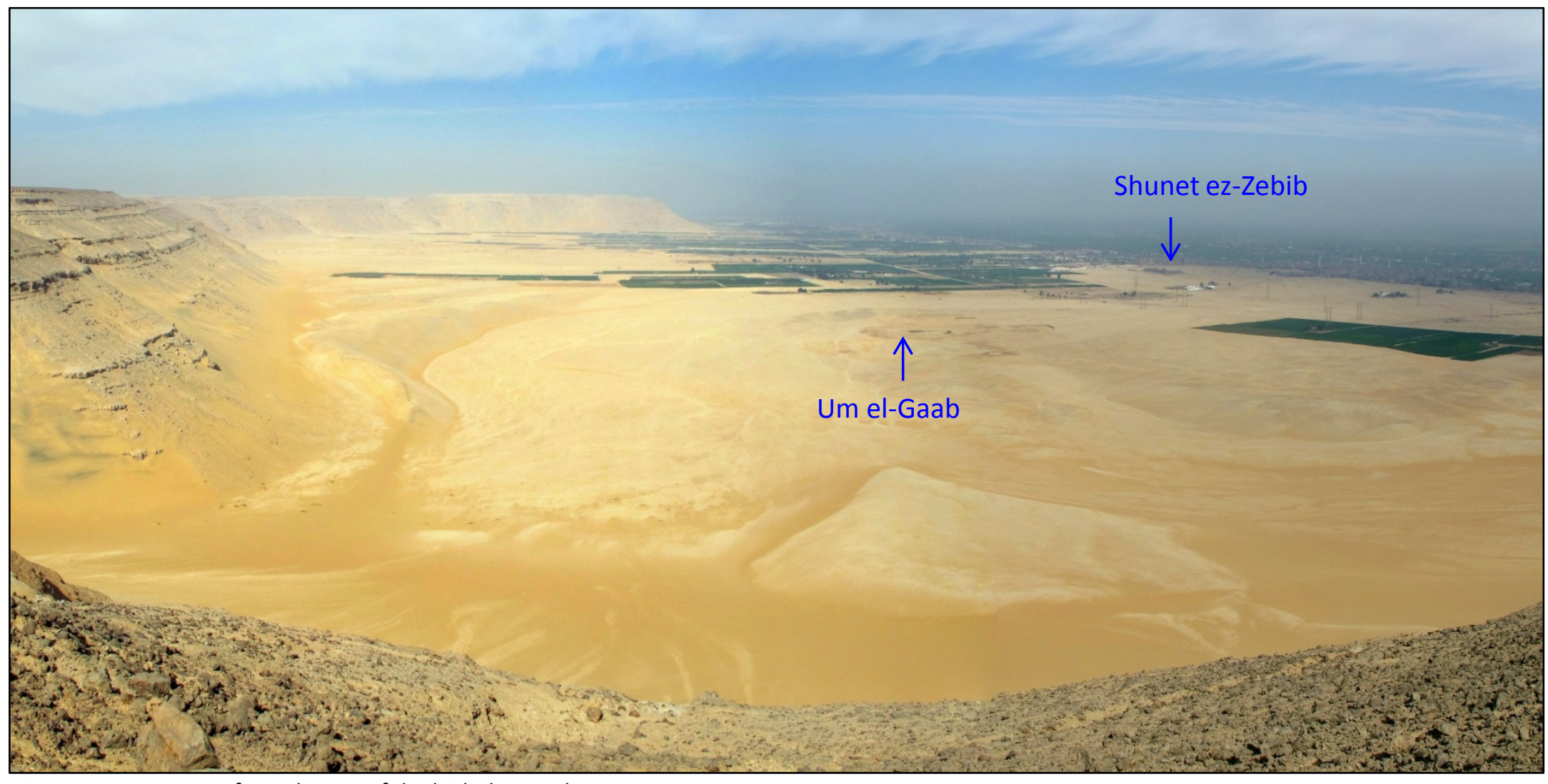

Figure 4.4. Panorama from the top of the high desert plateau. 


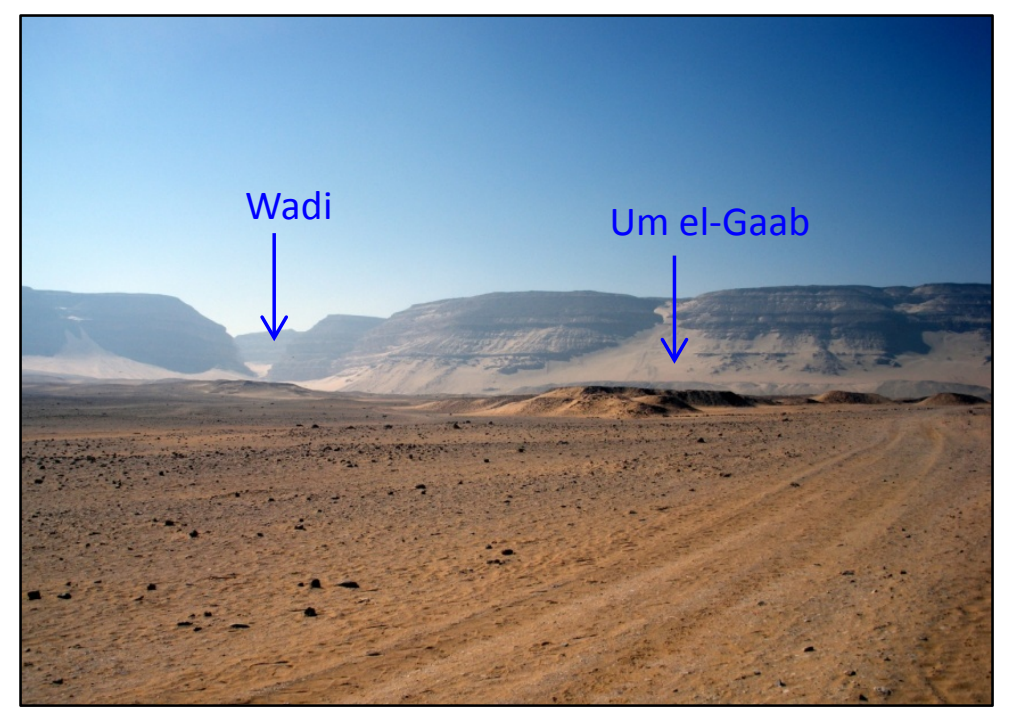

Figure X4.5. High desert plateaus, low desert surface, Um el-Gaab cemetery in front of a wadi leading up into the high desert.

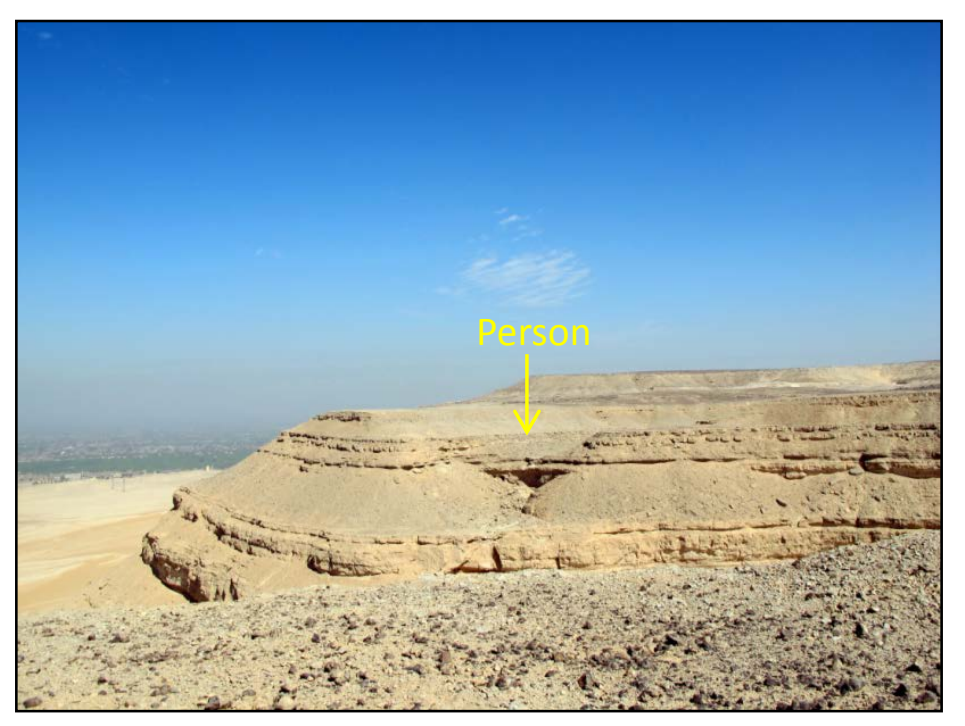

Figure 4.7. High desert plateau in survey area. For a sense of scale, there is a person just below the arrow, barely visible.

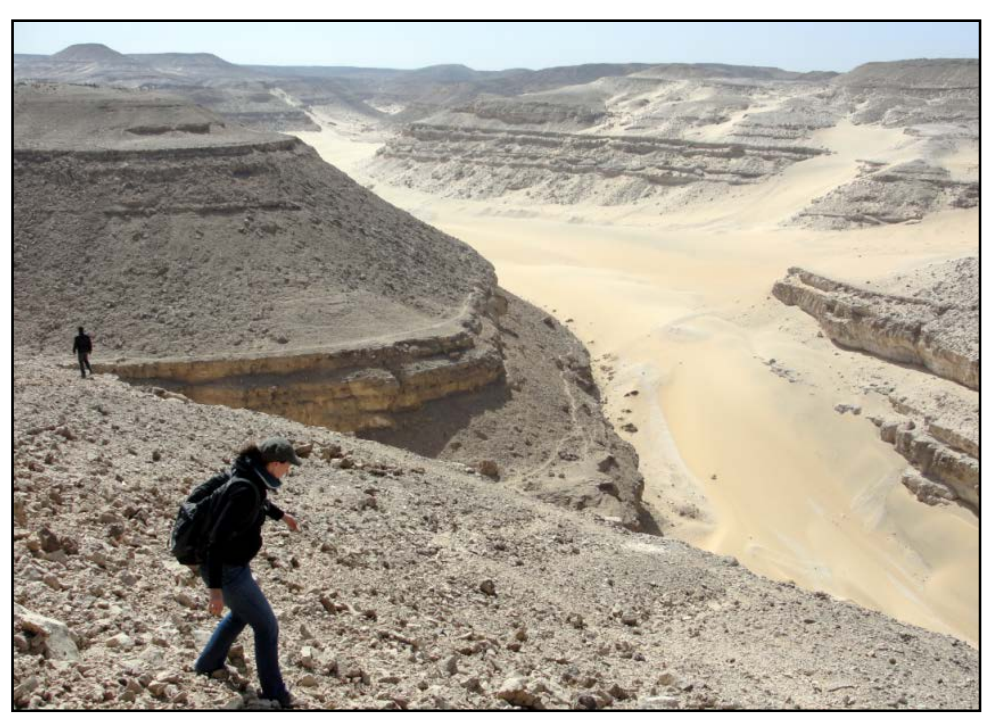

Figure 4.6. The wadi leading up to the high desert, along which raw materials were observed.

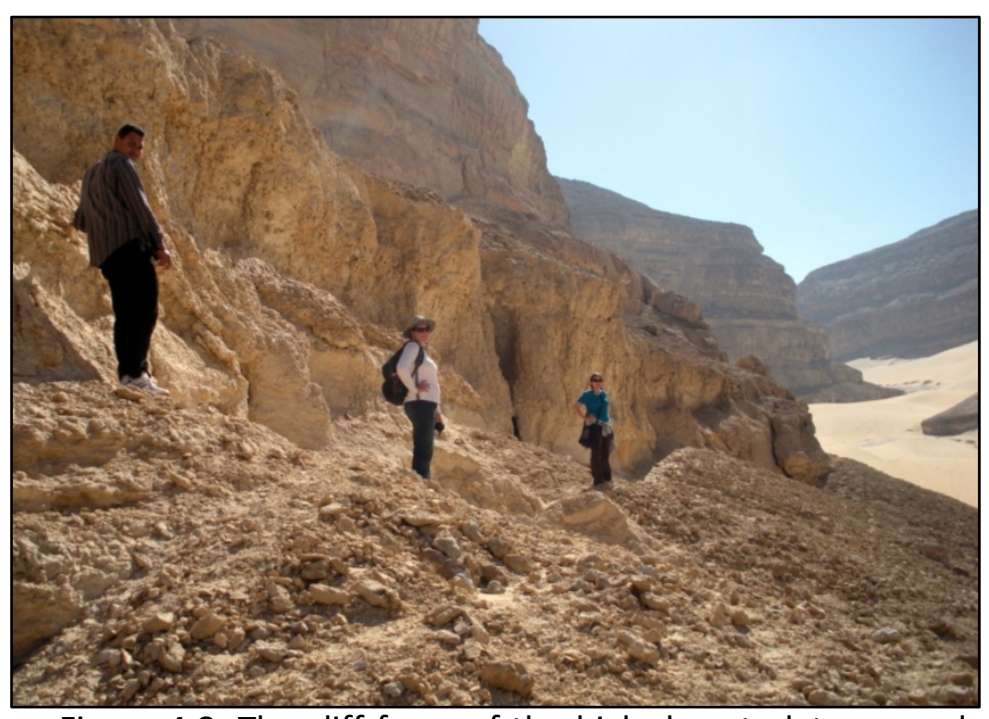

Figure 4.8. The cliff faces of the high desert plateaus and the lag deposits and slopes in front of them. 


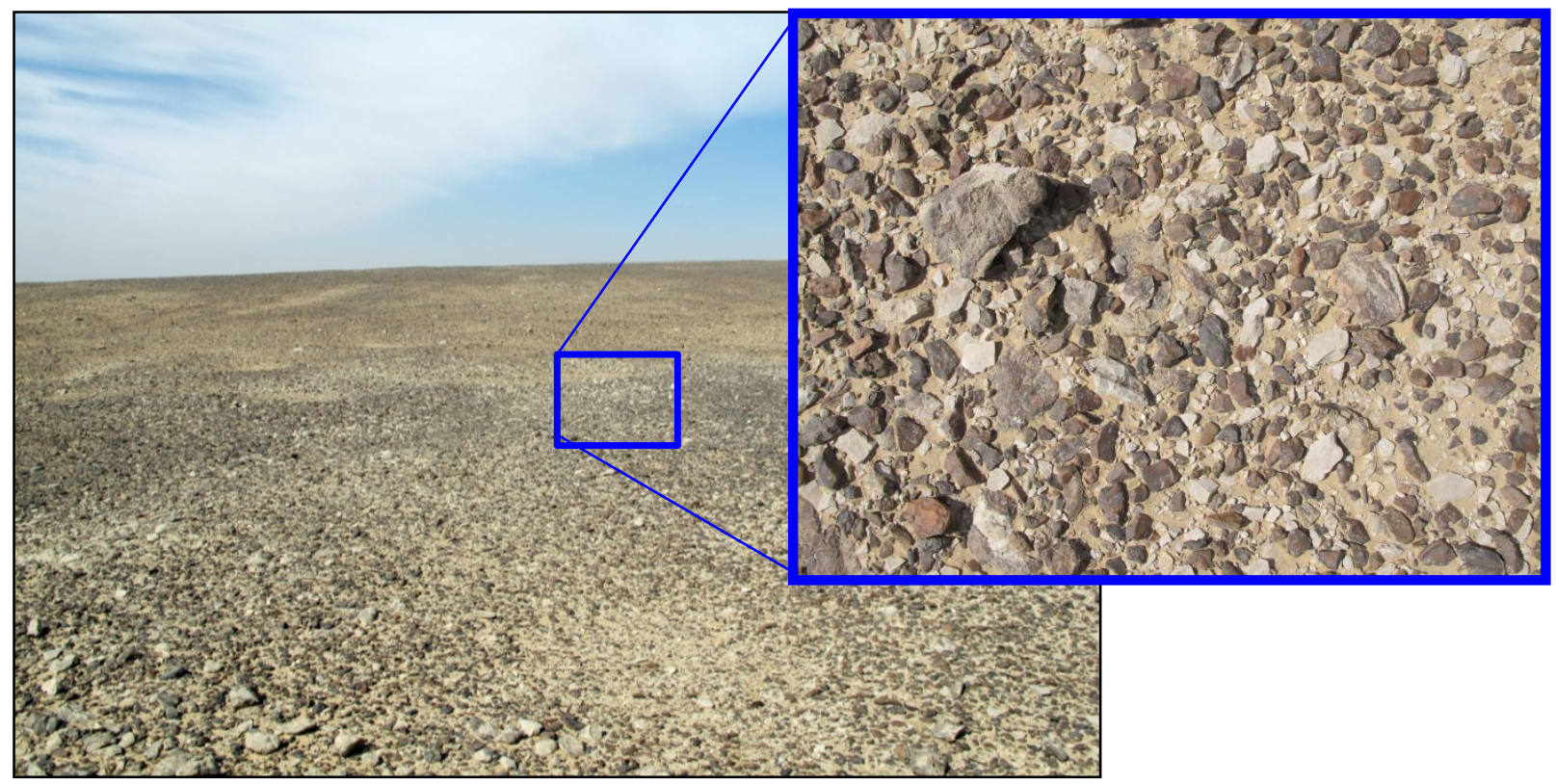

Figure 4.9. High desert surface with chert pavement, \& close up showing scatter of natural chert fragments and artifacts. Approximate average size of pieces in the close up is $\sim 3 \mathrm{~cm}$.

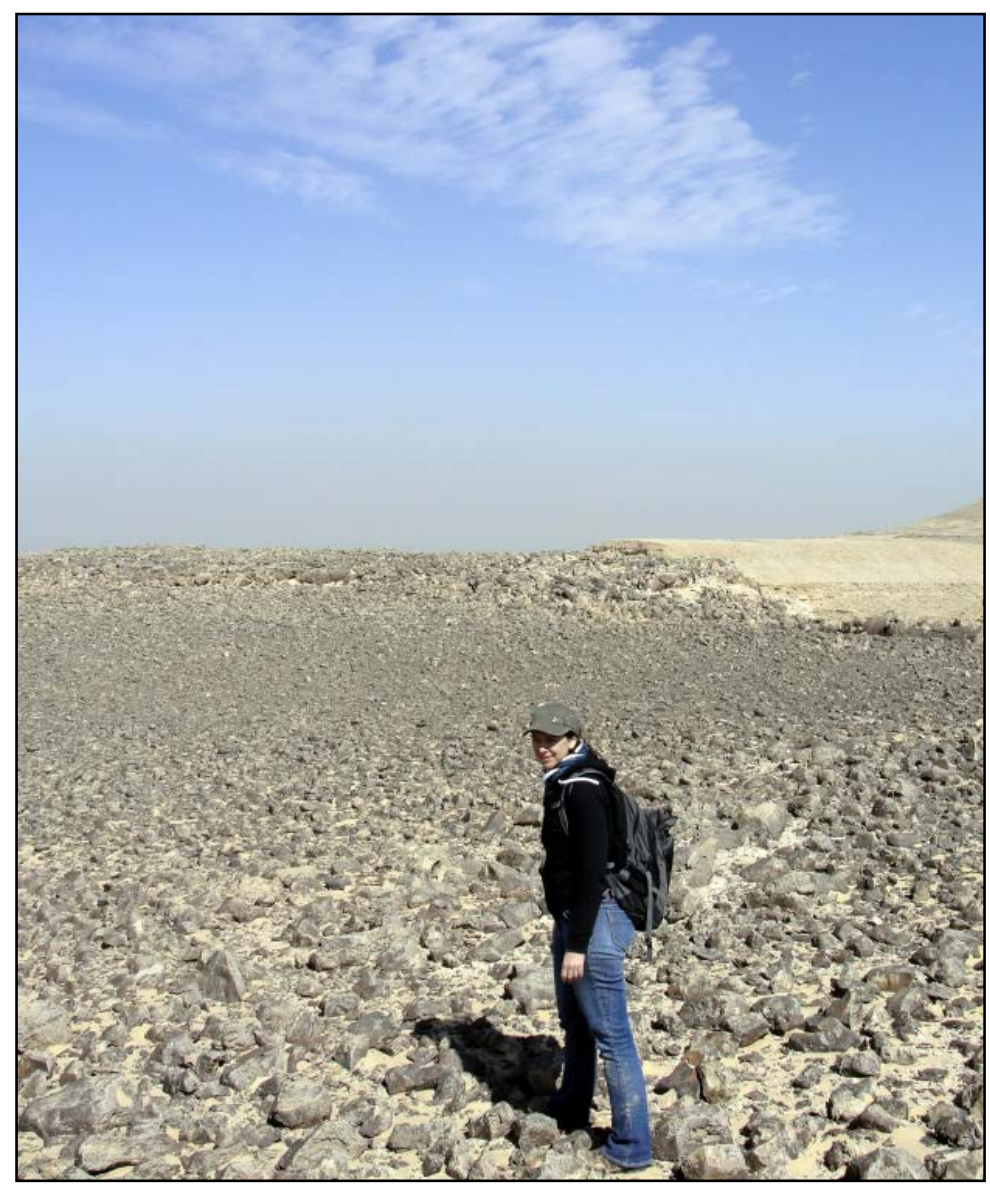

Figure 4.10. High desert surface in an area with larger stone fragments. 


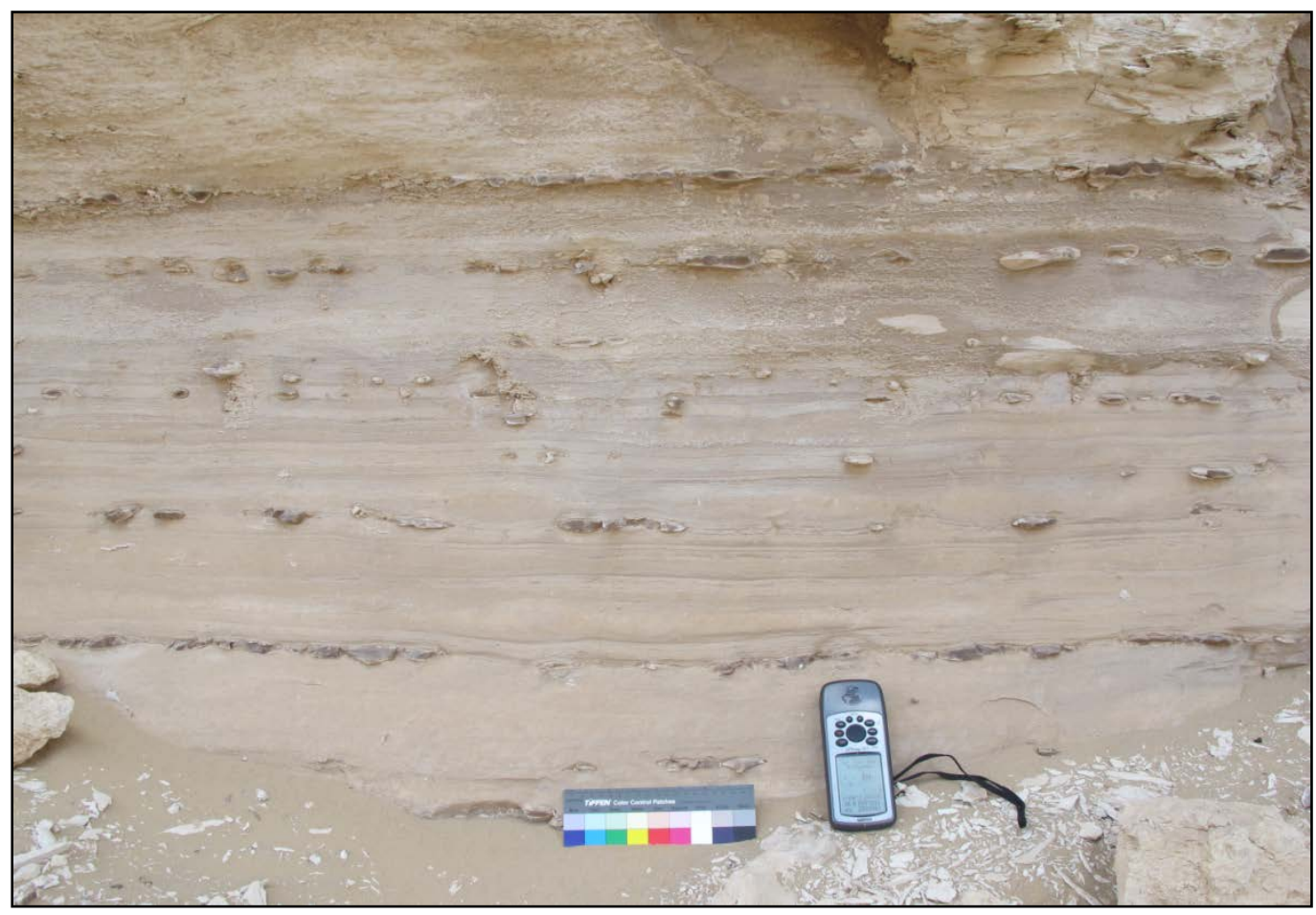

Figure 4.11. Exposed thin chert nodules in primary context. Nodule A 10.

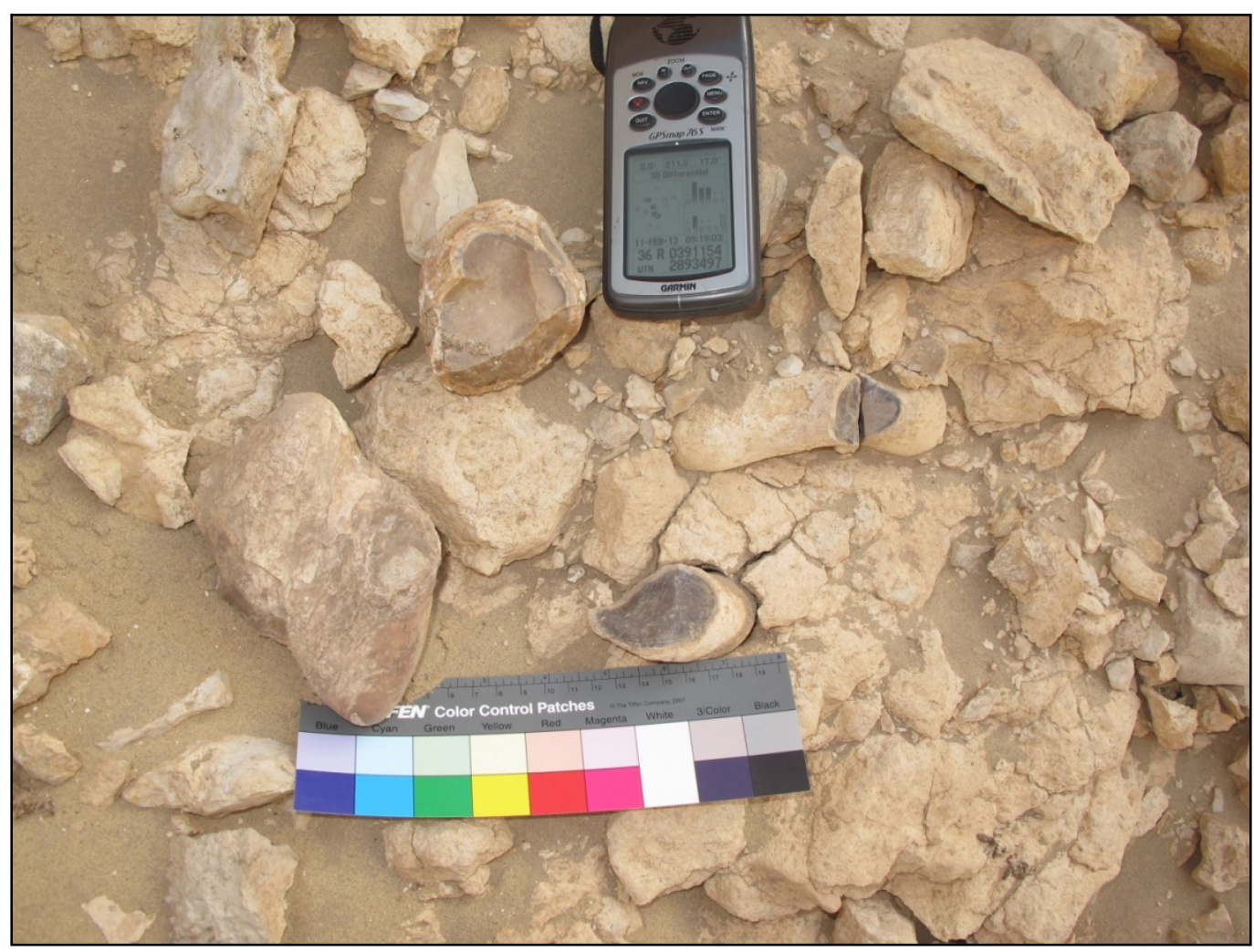

Figure 4.12. Exposed thin chert nodules in primary context. Nodule A 14. 


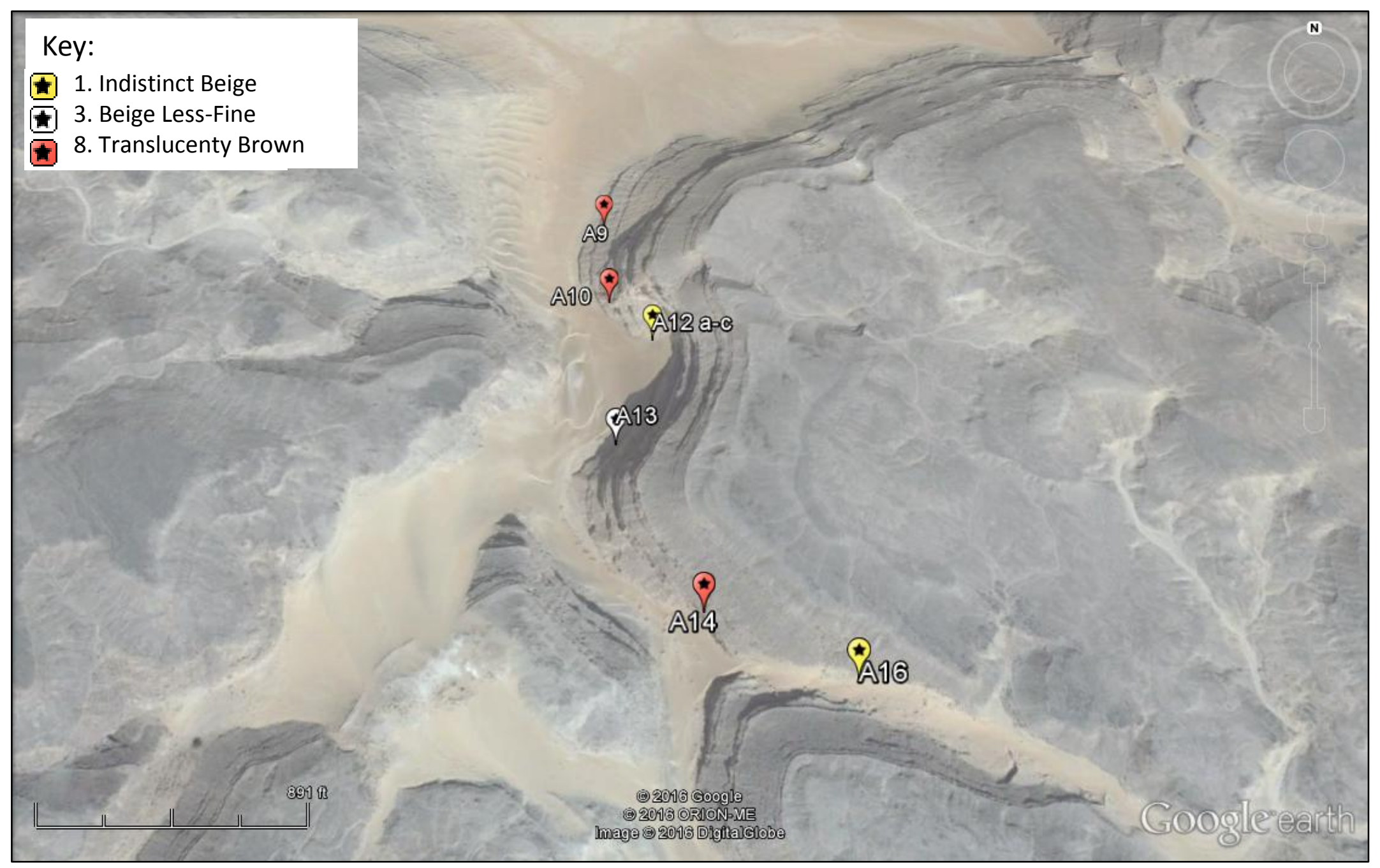

Figure 4.13. Map showing locations of primary contexts for chert varieties. 


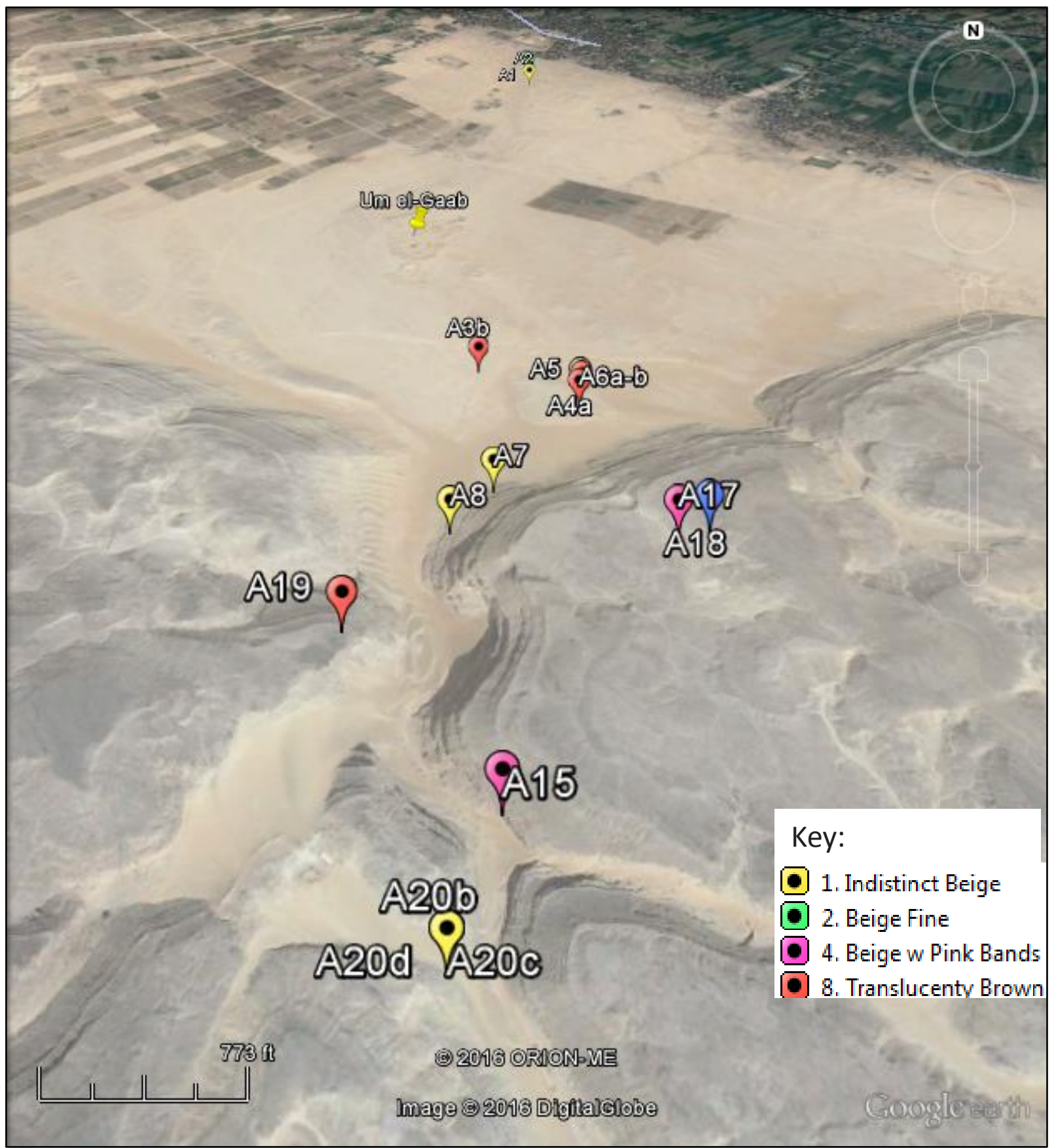

Figure 4.14. Map showing locations of chert varieties found in secondary context. 


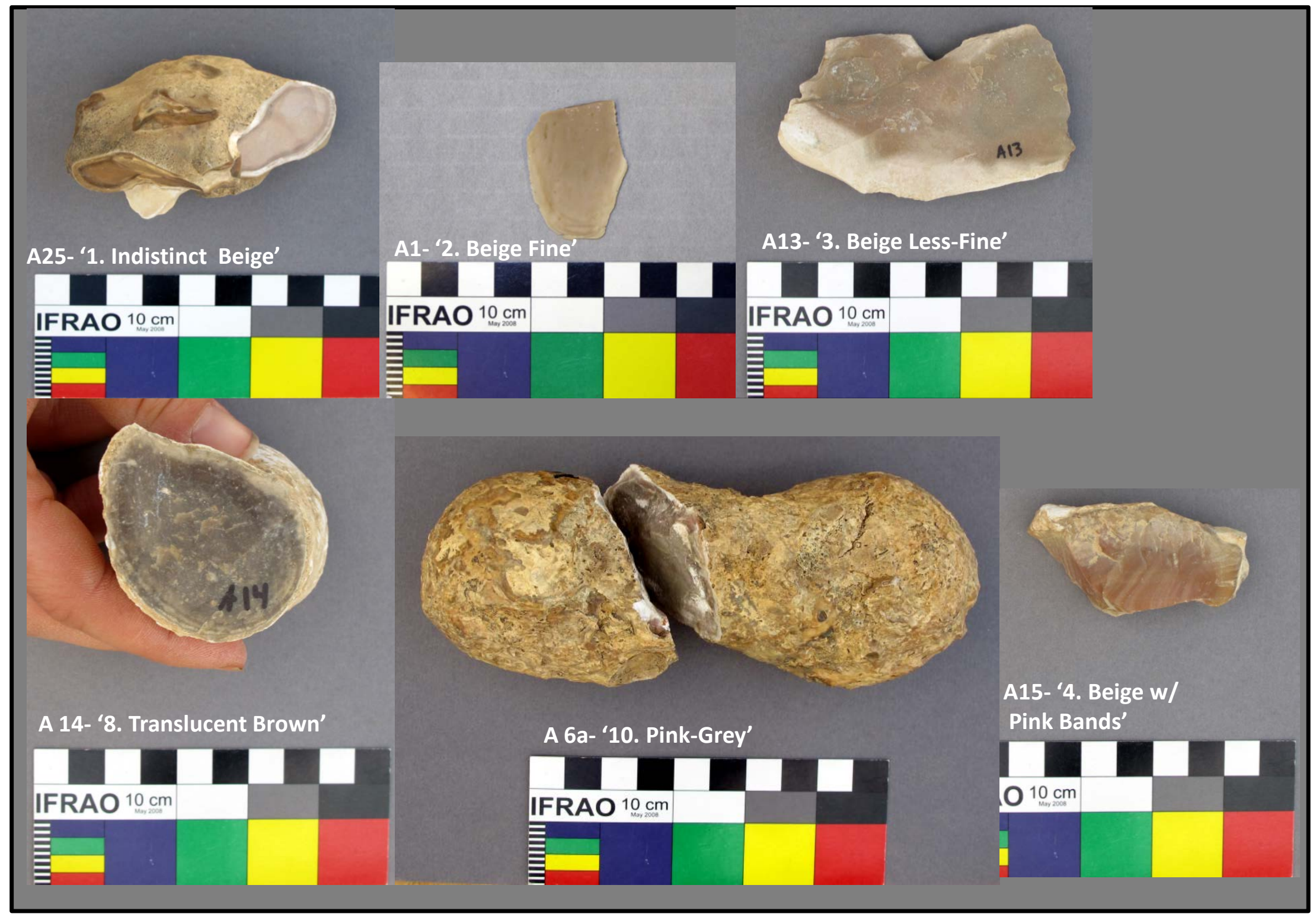

Figure 4.15. Examples of raw materials identified during the raw material survey in the Abydos area. 


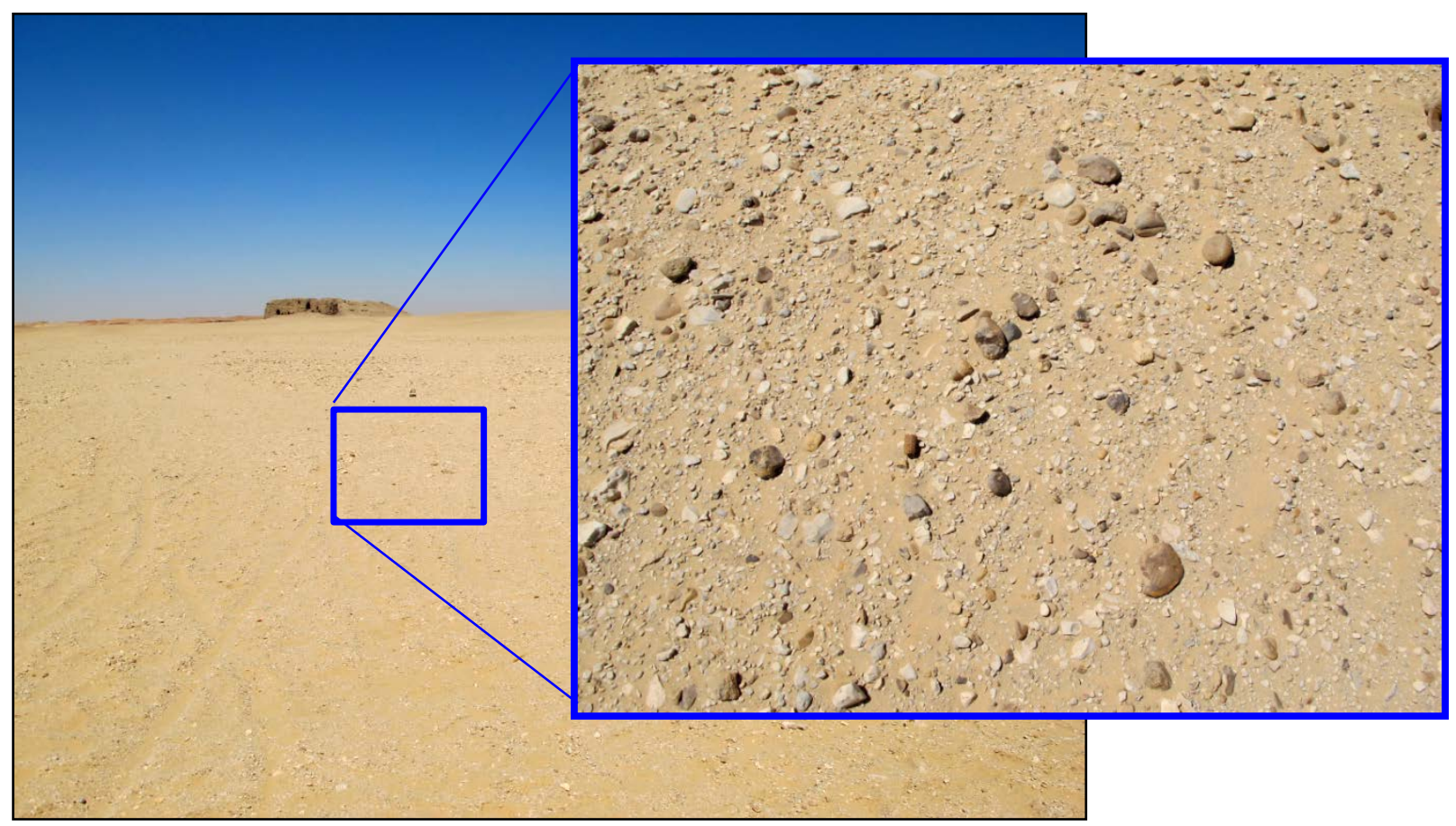

Figure 4.16. Desert surface around Beit Khallaf.

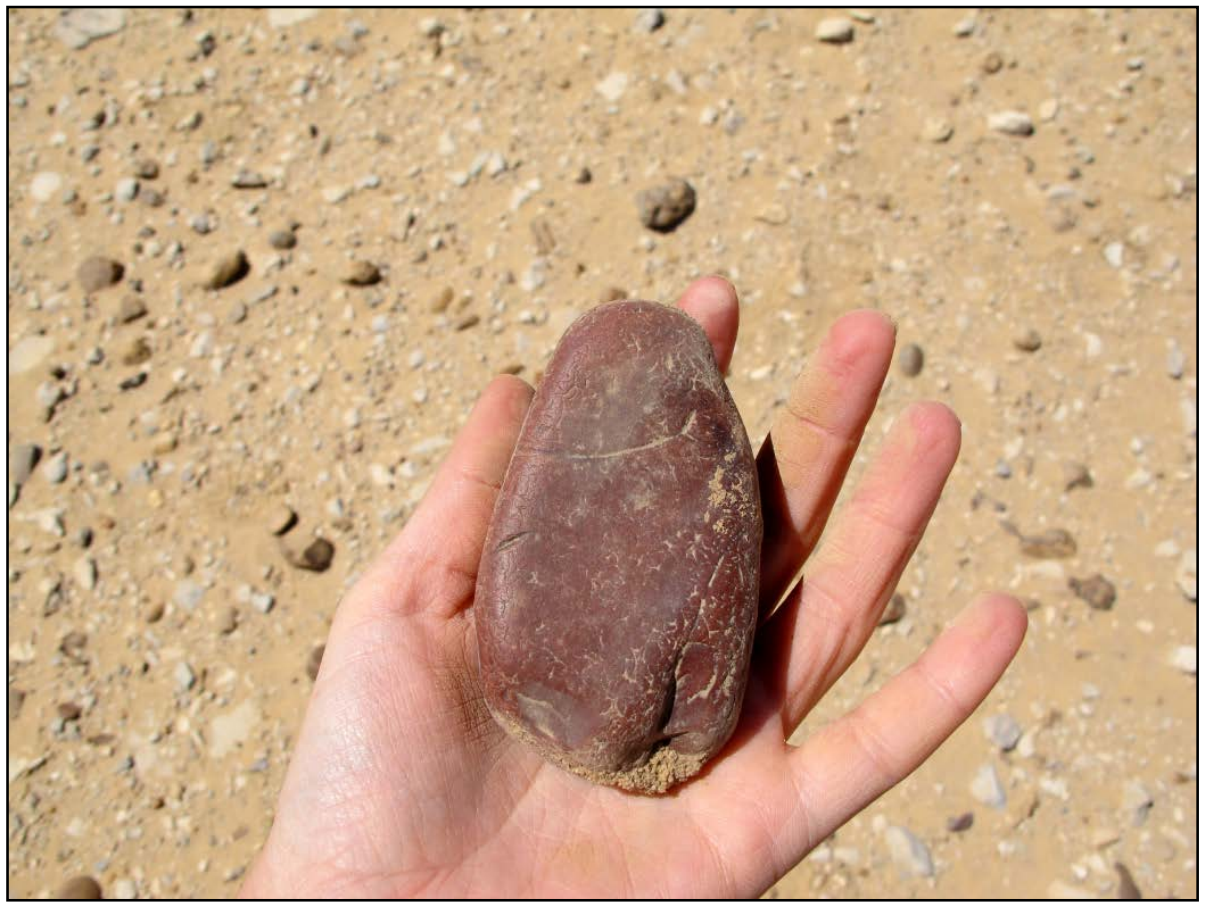

Figure 4.17. Chert variety 9.Translucent Brown with pink gravel cortex. 


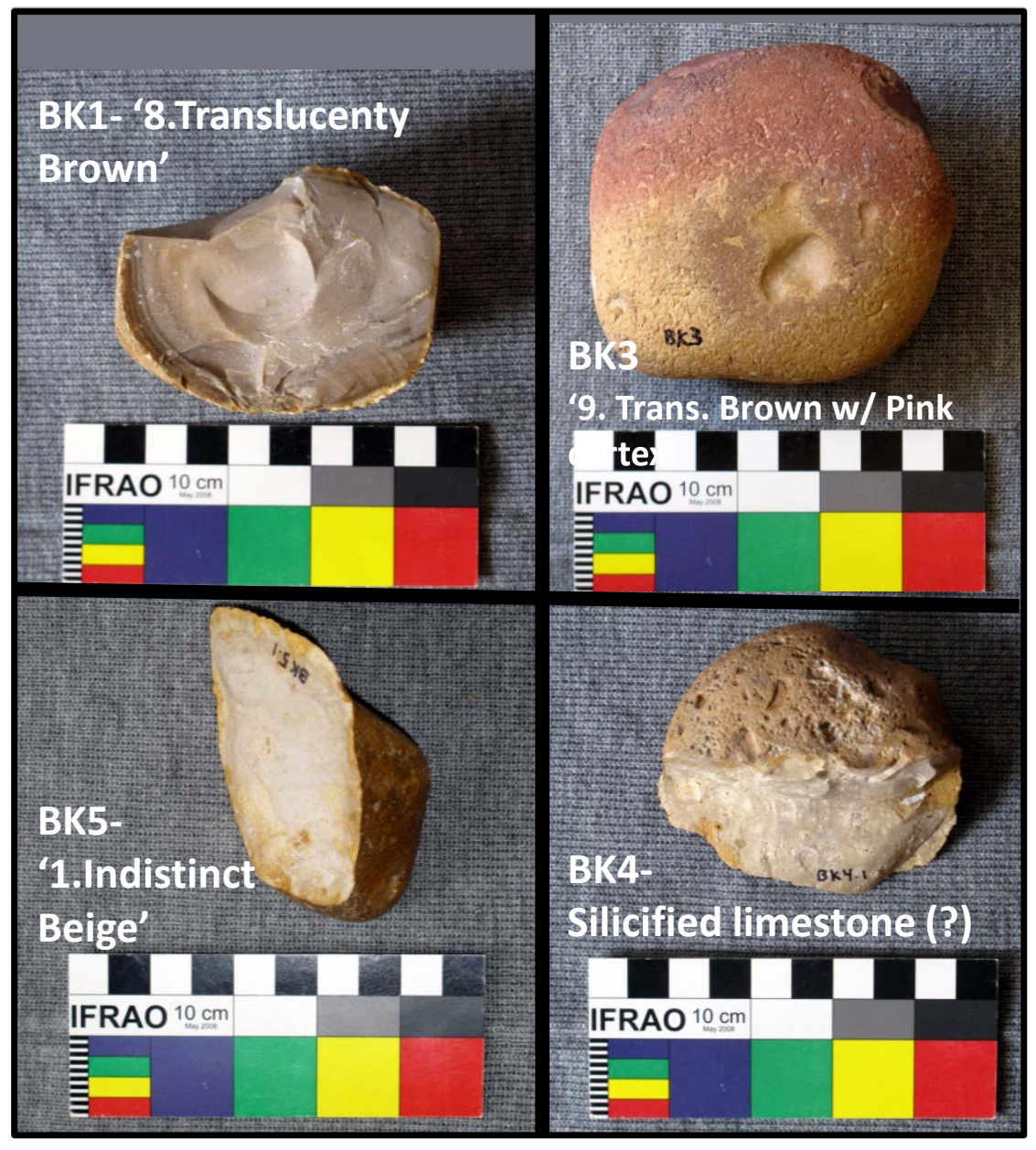

Figure 4.18. Raw material types identified at Beit Khallaf.

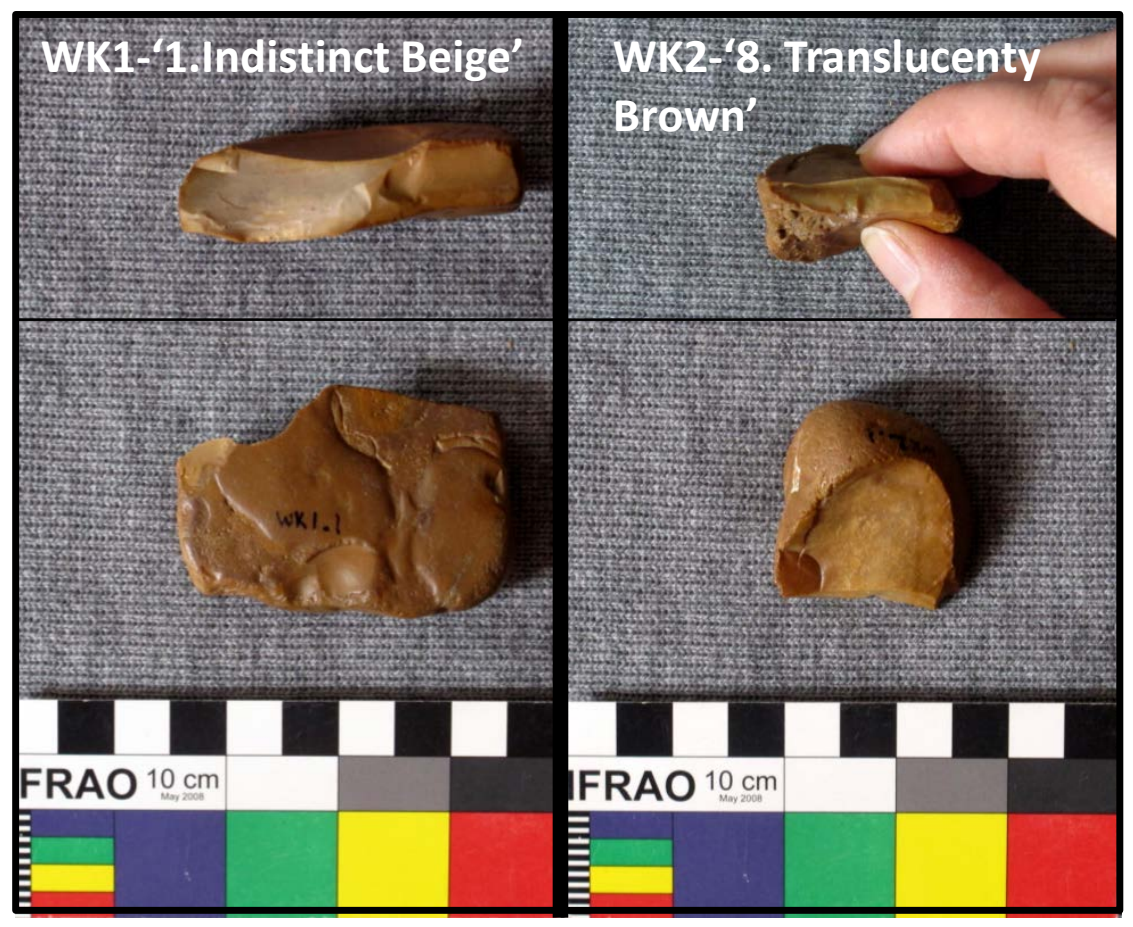

Figure 4.19. Two views of each of the raw material types identified in the vicinity of Nag el-Qarmila. 


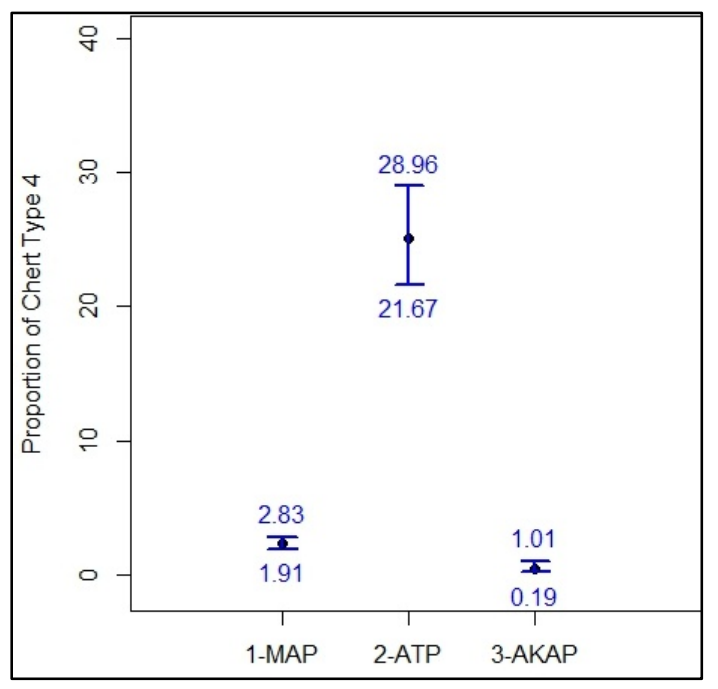

Figure 4.20. 95\% binomial confidence intervals for chert type '4. Beige with Pink Bands' at elMahâsna, Abydos and Nag el-Qarmila.

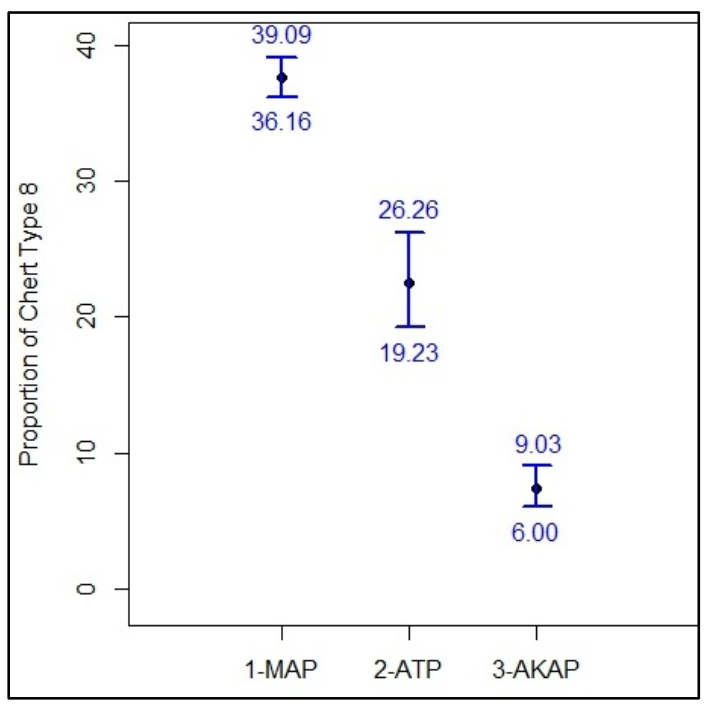

Figure 4.22. 95\% binomial confidence intervals for chert type '8. Translucenty Brown' at el-Mahâsna, Abydos and Nag elQarmila.

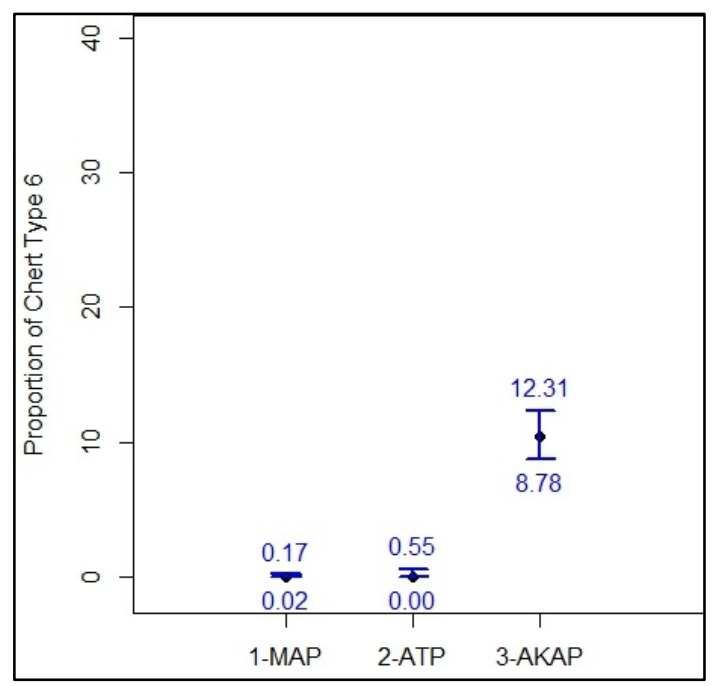

Figure 4.21. 95\% binomial confidence intervals for chert type '6. Brown Fossil' at el-Mahâsna, Abydos and Nag el-Qarmila.

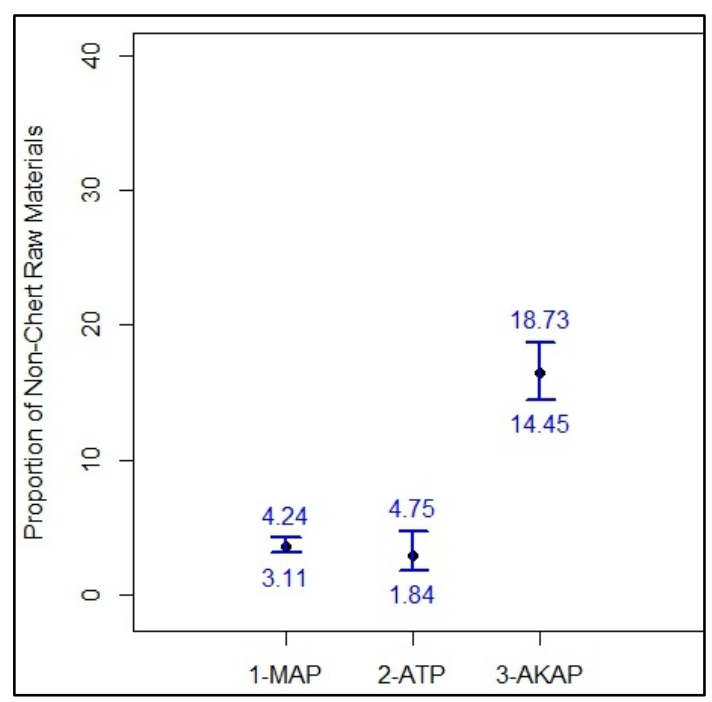

Figure 4.23. 95\% binomial confidence intervals for the proportions of Non-Chert materials at el-Mahâsna, Abydos and Nag elQarmila. 


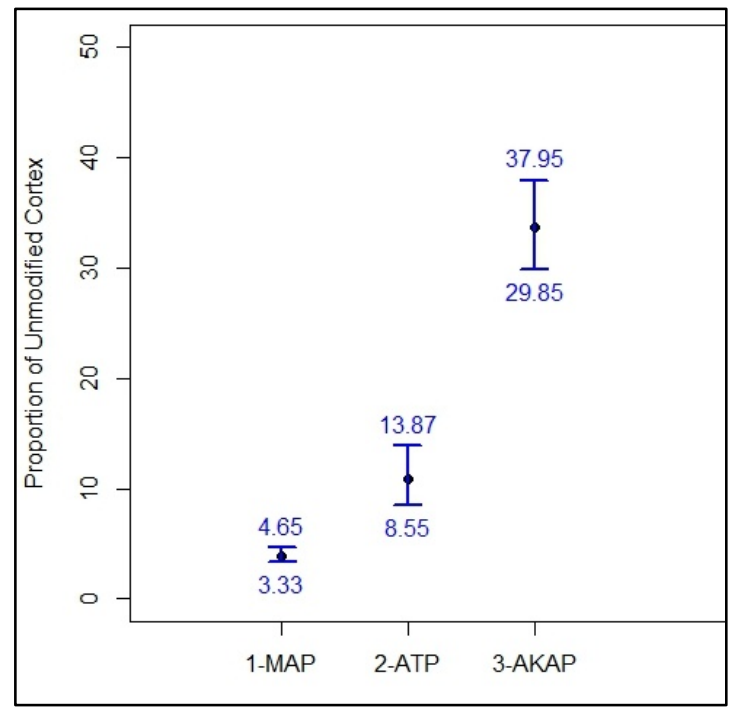

Figure 4.24. Proportions of unmodified cortex at el- Mahâsna, Abydos and Nag el-Qarmila, with the $95 \%$ binomial confidence intervals.

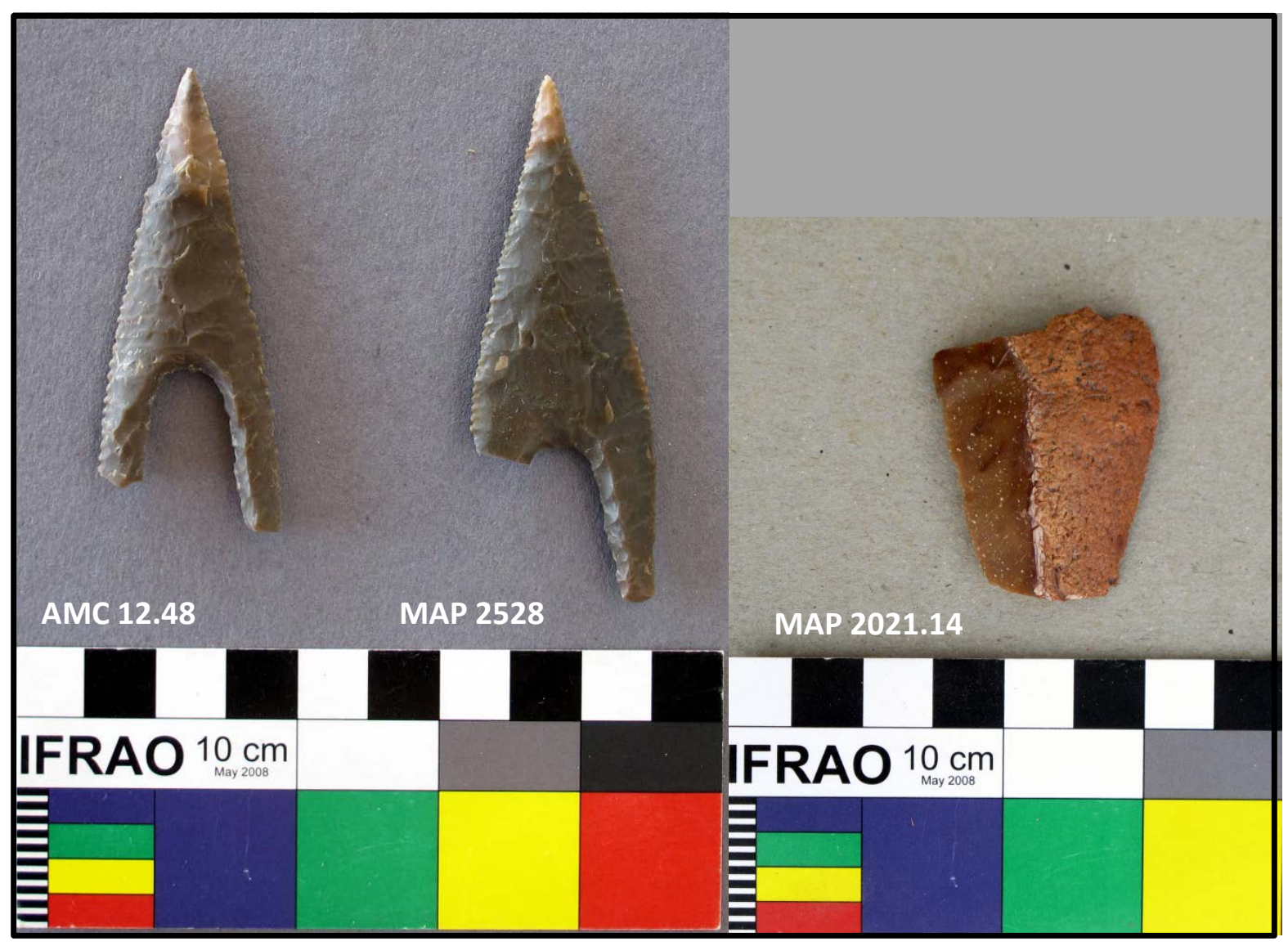

Figure 4.25. Three artifacts analyzed with X-ray Fluorescence. 


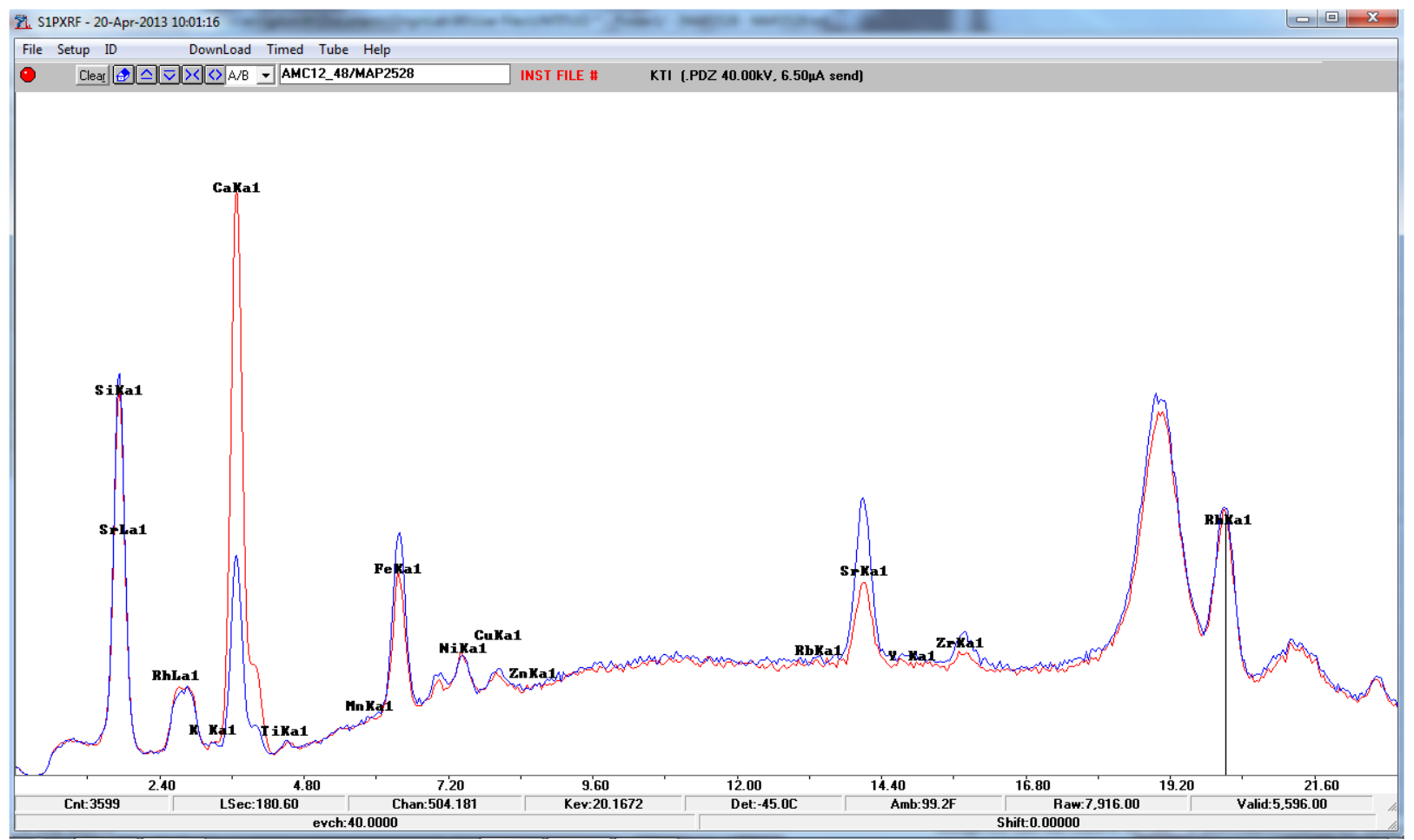

Figure 4.26. X-ray Fluorescence results comparing AMC 12.48 and MAP 2528, showing peaks in the same places. Note that the $\mathrm{Rh}, \mathrm{Cu}$, and $\mathrm{Ni}$ peaks are due to the instrument. Normalized to Rh Ka1 peak. Bruker Tracer III-V, $40 \mathrm{keV}, 6.5 \mathrm{uA}, 180 \mathrm{sec}$, Rh anode, no filter, no vacuum.

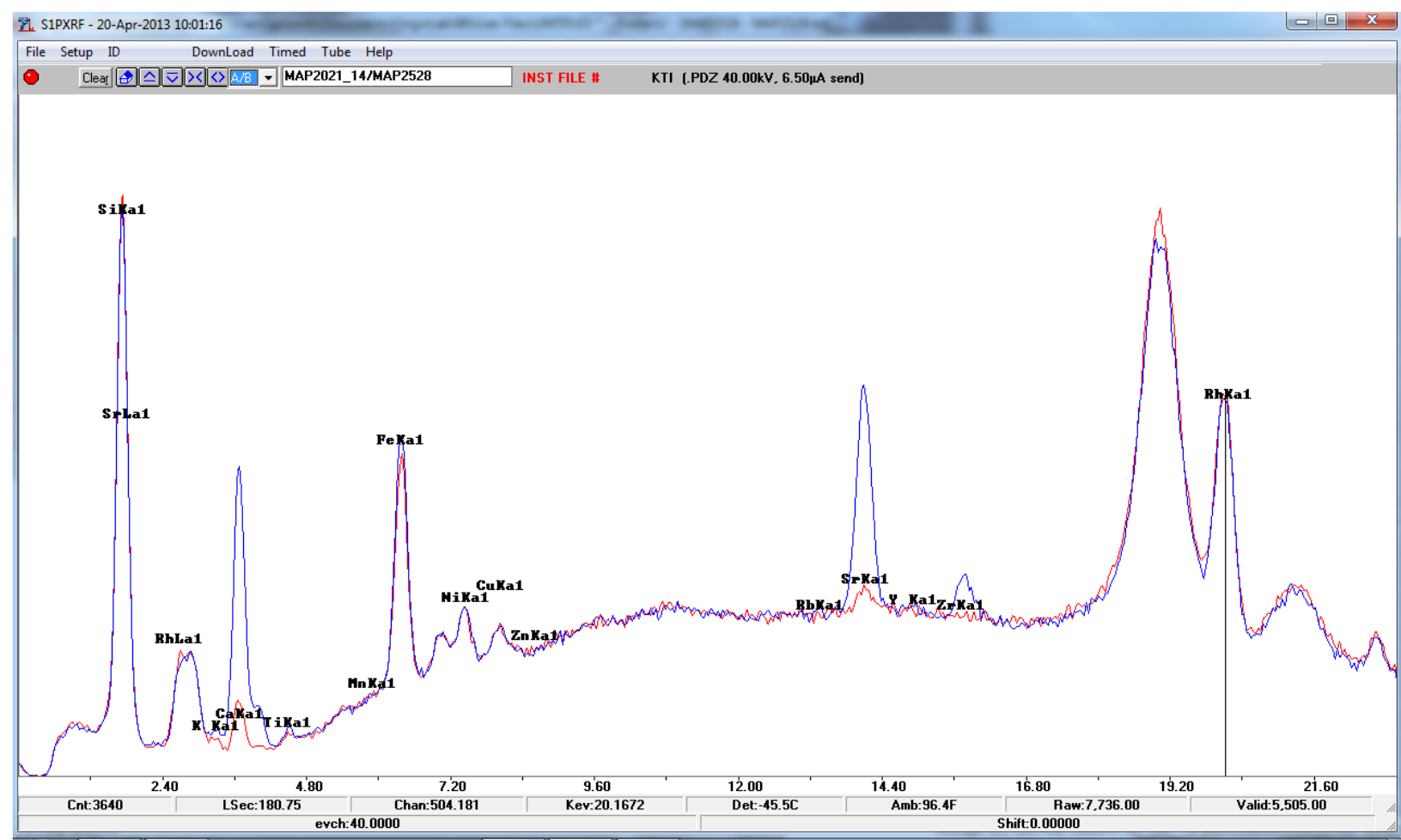

Figure 4.27. X-ray Fluorescence results comparing MAP 2021.14 and MAP 2528 showing peaks in different places. Note that the $\mathrm{Rh}, \mathrm{Cu}$, and $\mathrm{Ni}$ peaks are due to the instrument. Normalized to Rh Ka1 peak. Bruker Tracer III-V, $40 \mathrm{keV}, 6.5 \mathrm{uA}, 180 \mathrm{sec}$, Rh anode, no filter, no vacuum. 


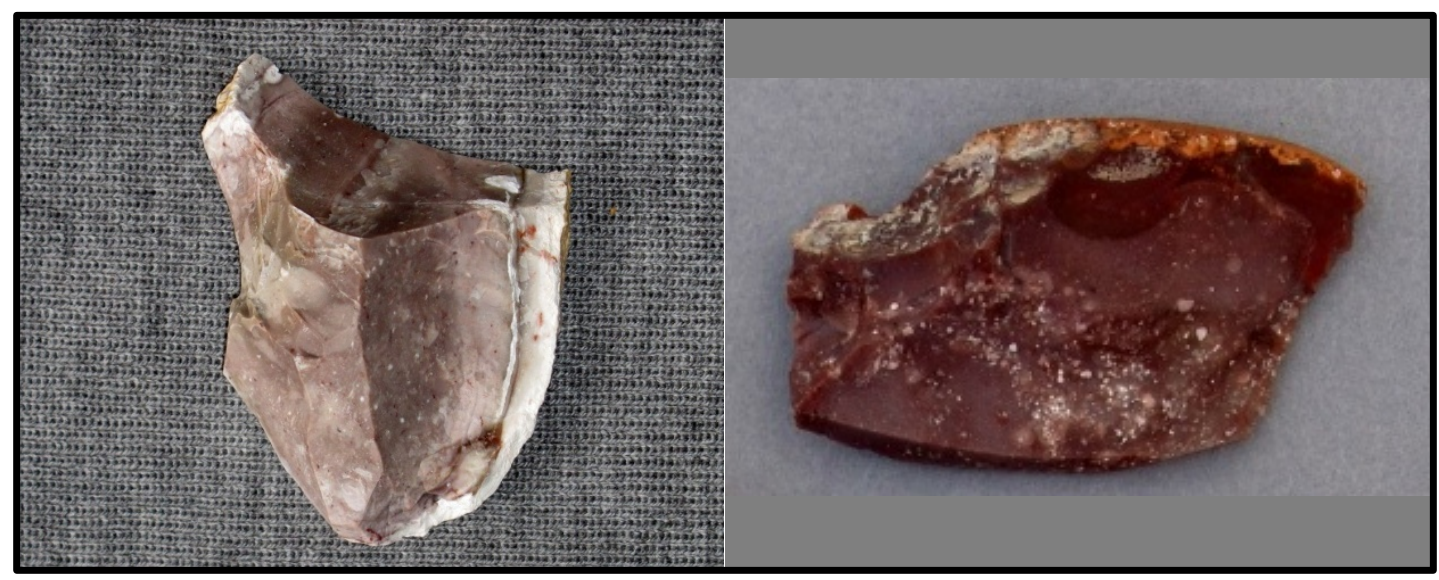

Figure 4.28. Left: Pinkish raw material sample with light speckling. Right: Heat treated artifact. Is it only the pinkish variety of chert that turns so red during heat treatment?

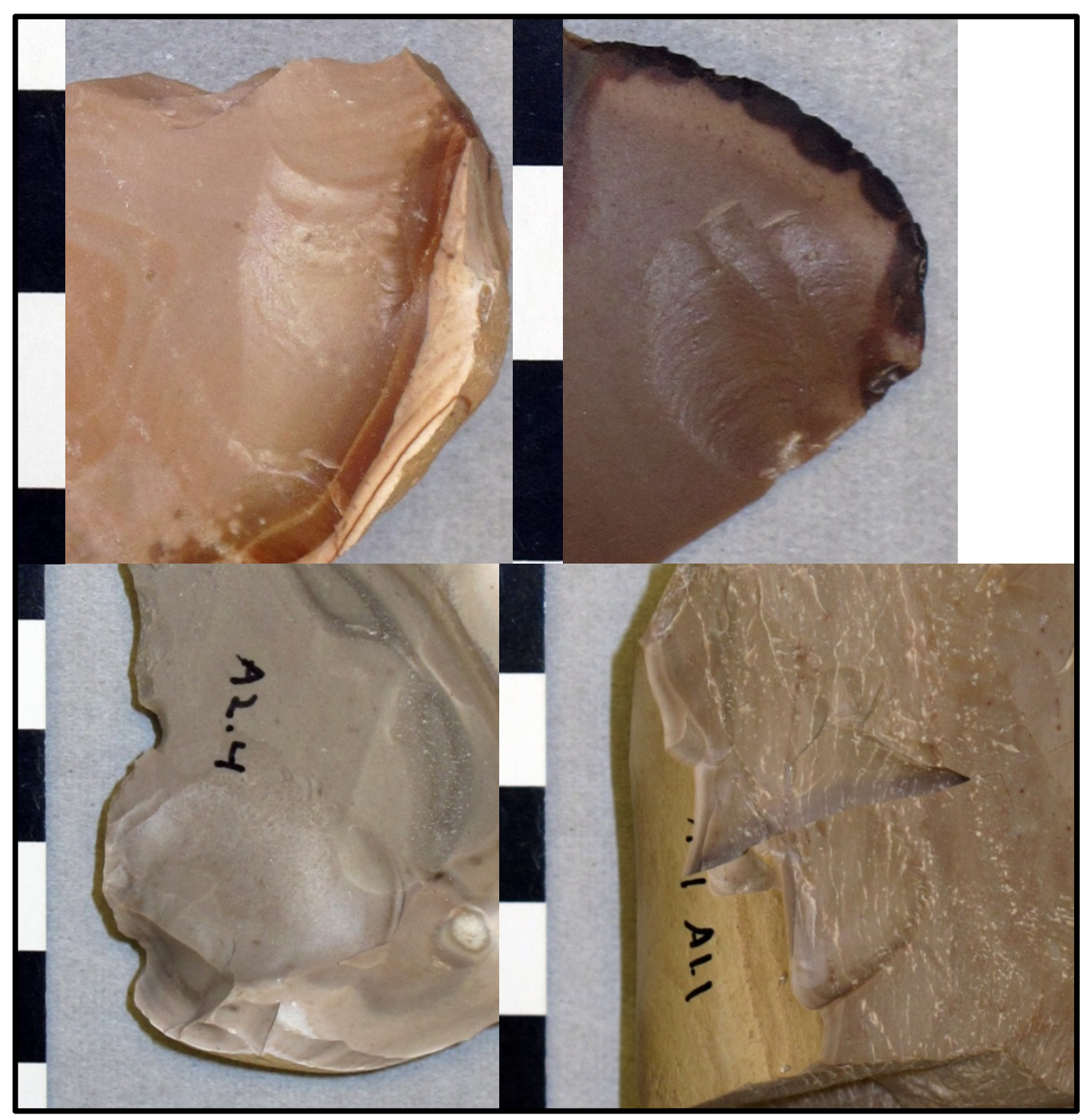

Figure 4.29. Heat treated pieces with glossy scars of flakes removed after heating. The surface exposed during heating is more matte than the fresher scar. 


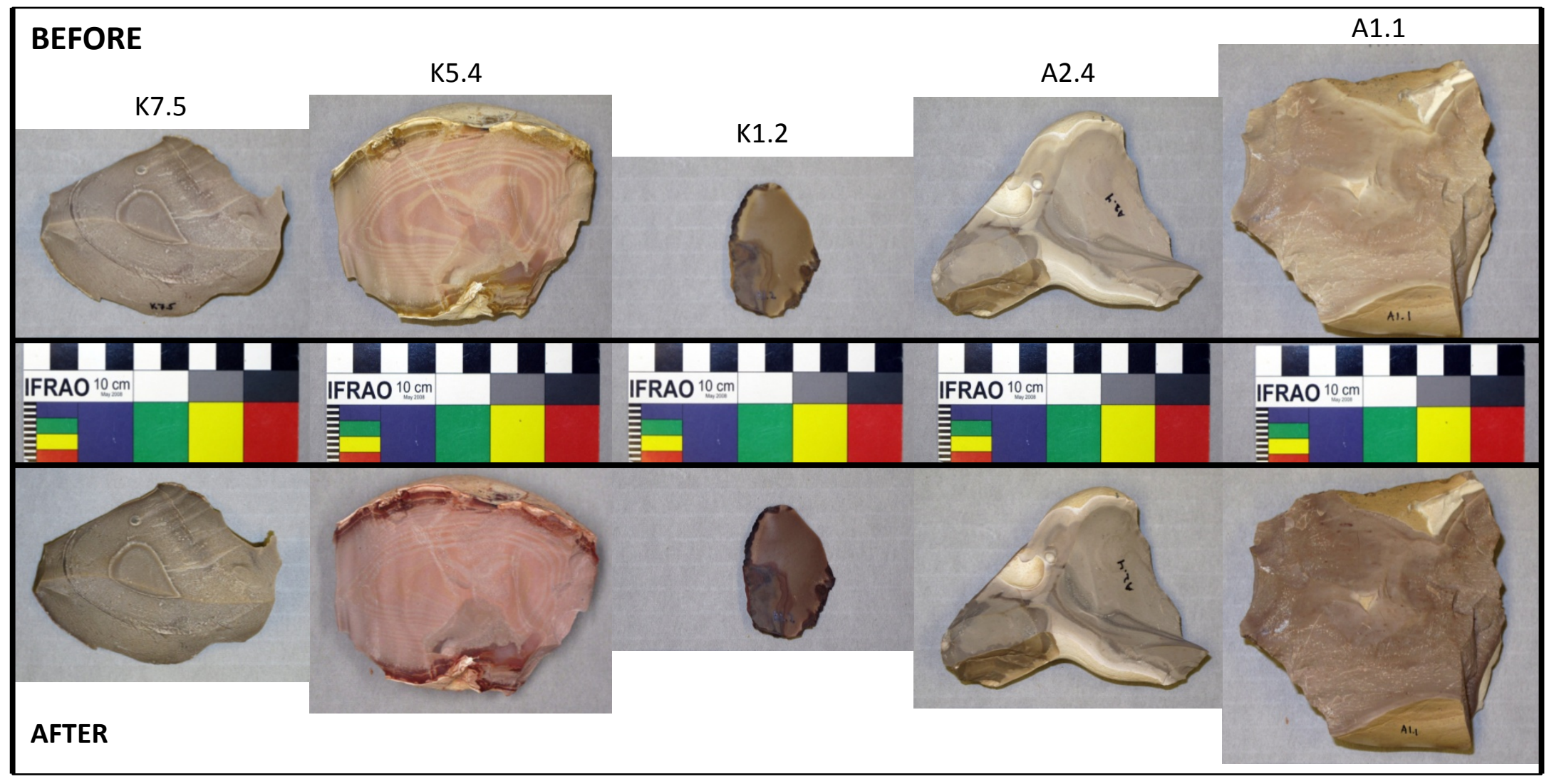

Figure 4.30. Examples of stones before and after heat-treatment. 


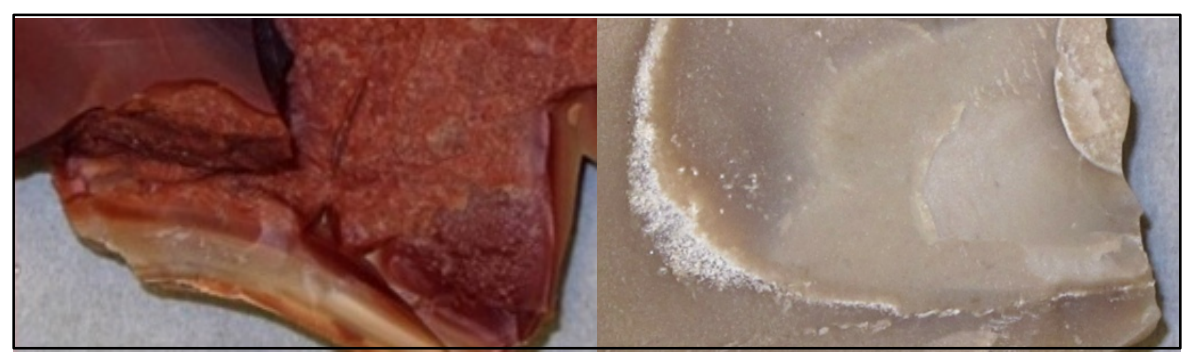

Figure 4.31. Comparison of two pieces heated to the same temperature. Left: $\mathrm{K} 3,350^{\circ}$, burnt. Right: $\mathrm{K} 7,350^{\circ}$, perfect.

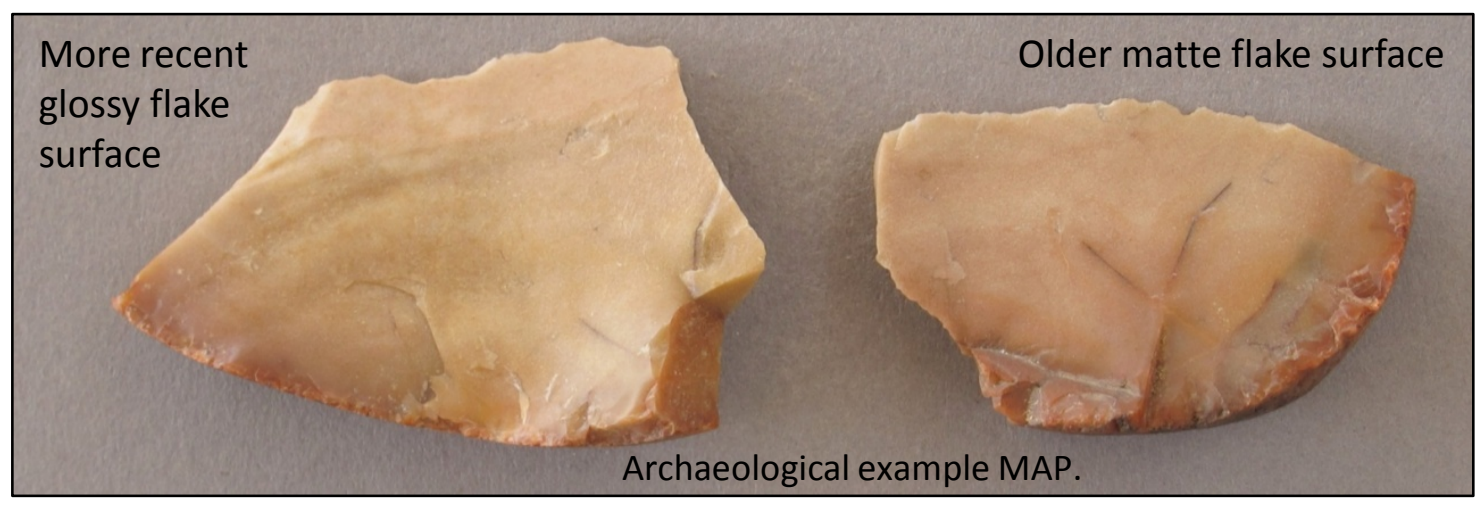

Figure 4.32. Examples of differential luster of flake scars before and after heating. In the archaeological example, upper left, the flake was heated causing the exposed surface to become matte, then a flake was removed, leaving a glossier scar. The two flakes In the archaeological example refit. The outermost surface of the outermost flake (lower right) is matte, while the ventral surface of that flake (not shown), and the surface of the refitting flake (lower right,) is glossy. Note that with patinas you get the opposite effect- the older surfaces are glossy and the fresher scars reveal a more matte material underneath.

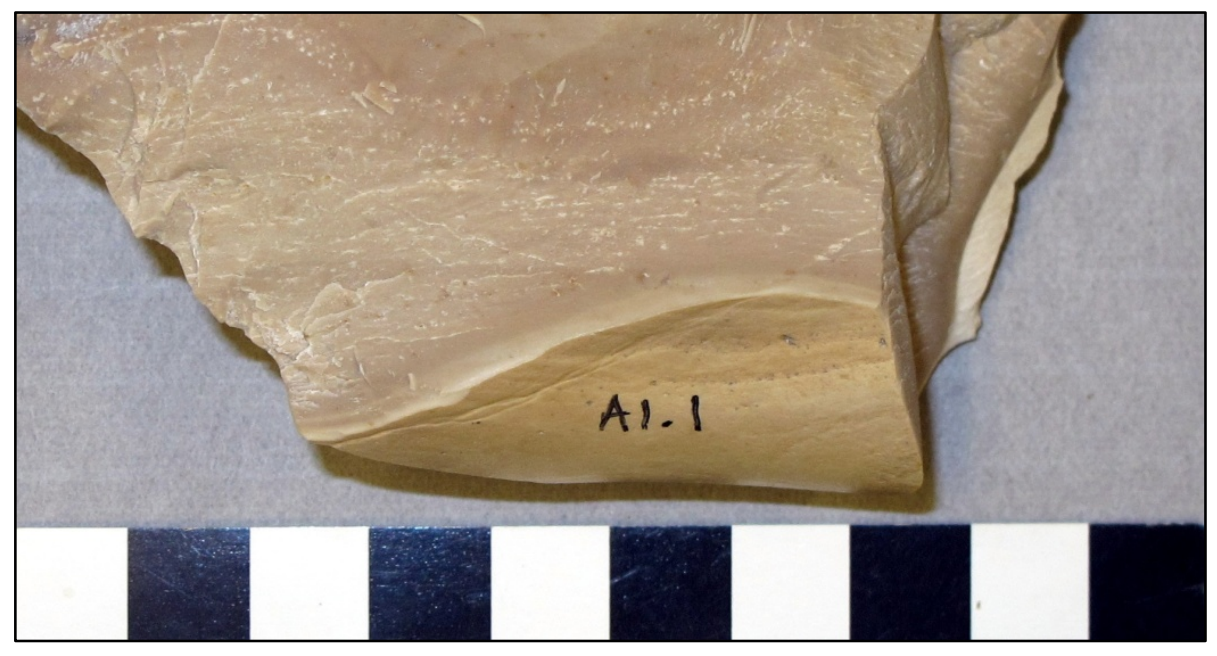

Figure 4.33. Raw material sample showing natural white speckling. 


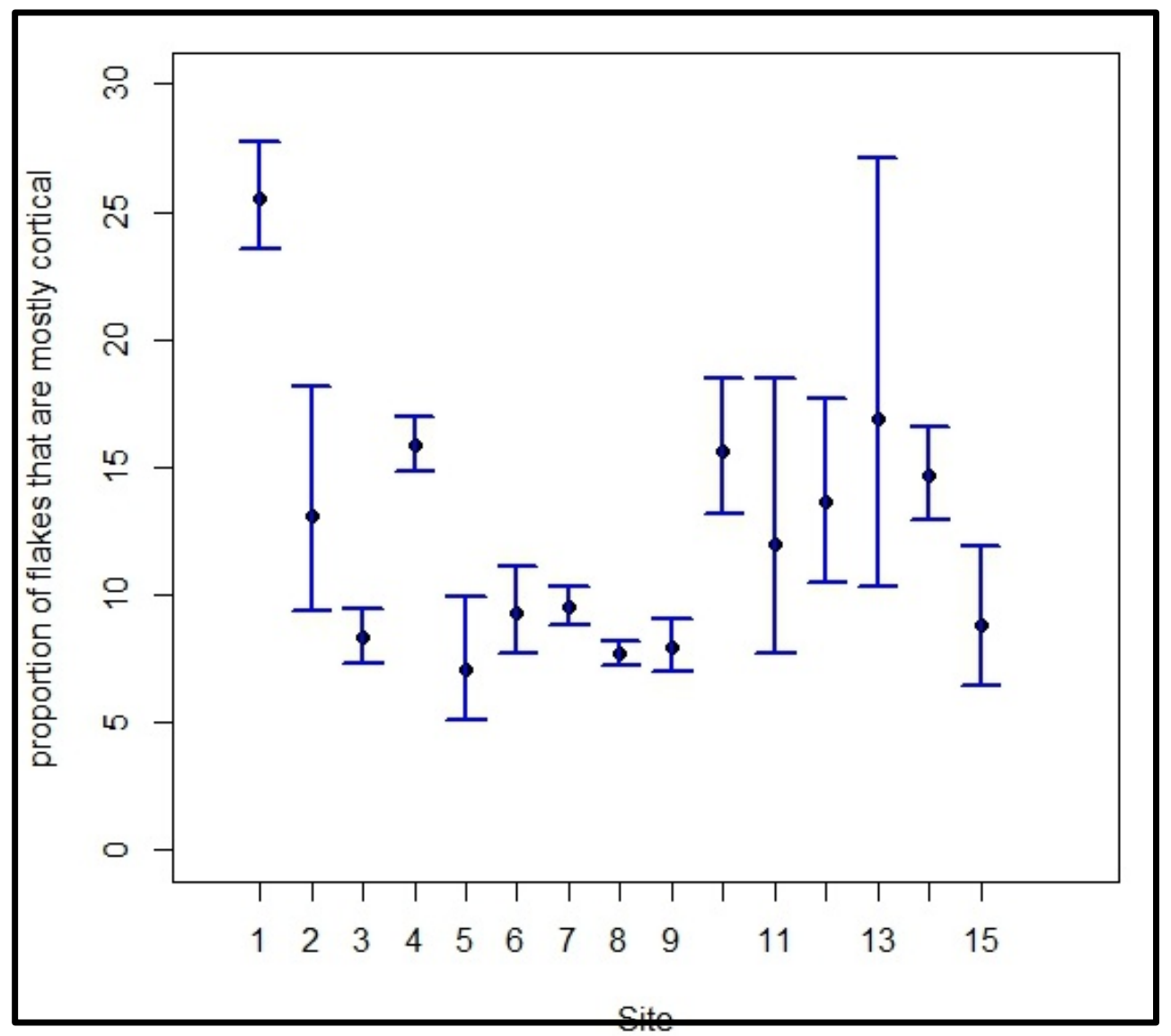

Figure 4.34. Comparison of the percentage of flakes with more than $50 \%$ cortex, showing the $95 \%$ confidence limits for the proportions. For site numbers see Table 4.19. 


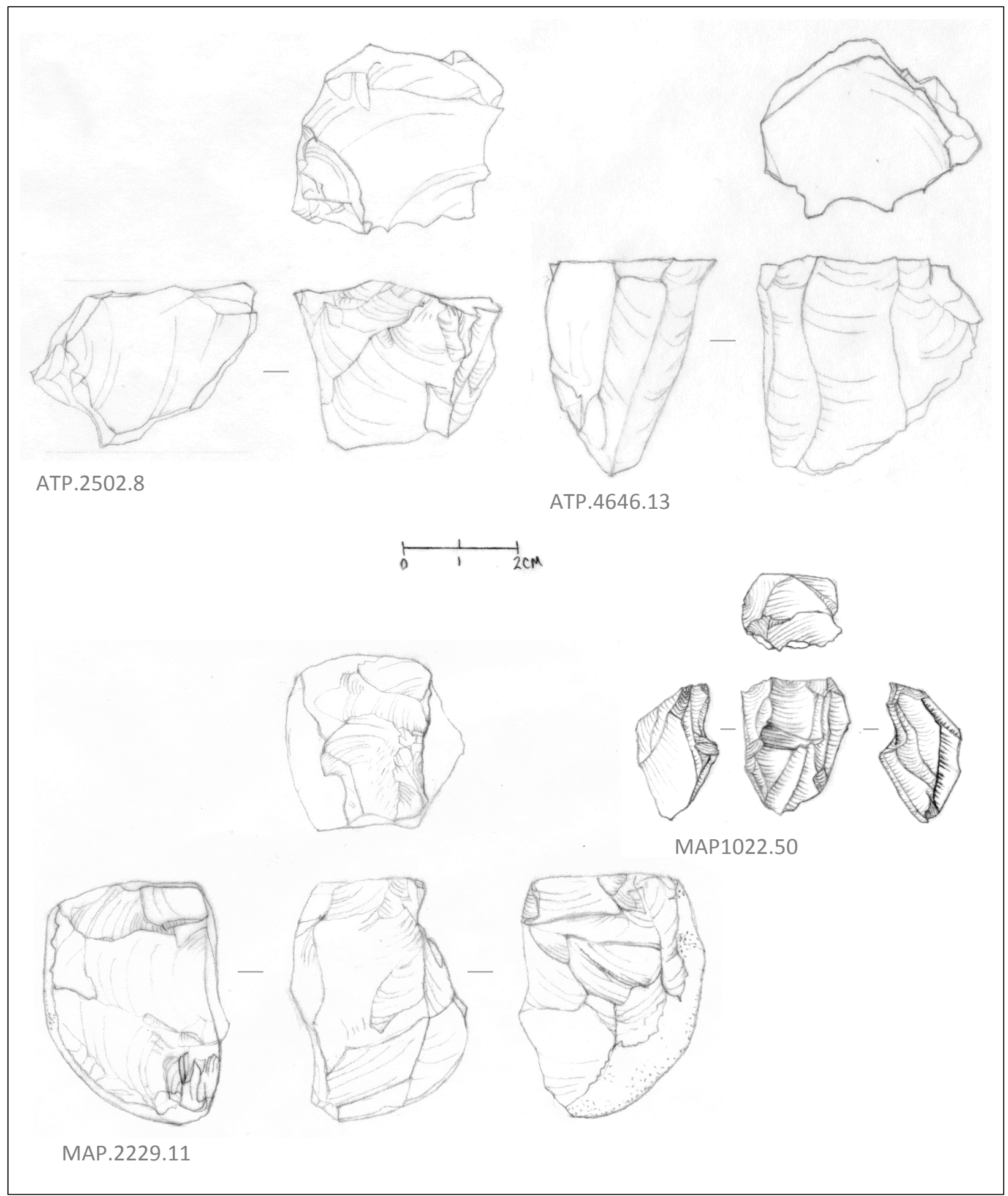

Figure 4.35. Cores. ATP.2502.8 Multi-platform flake core. LDH; ATP.4646.13. Single platform blade core. LDH; MAP.2229.11. Flake core with 90 degree rotated platforms. LDH; MAP1022.50. bladelet core. 


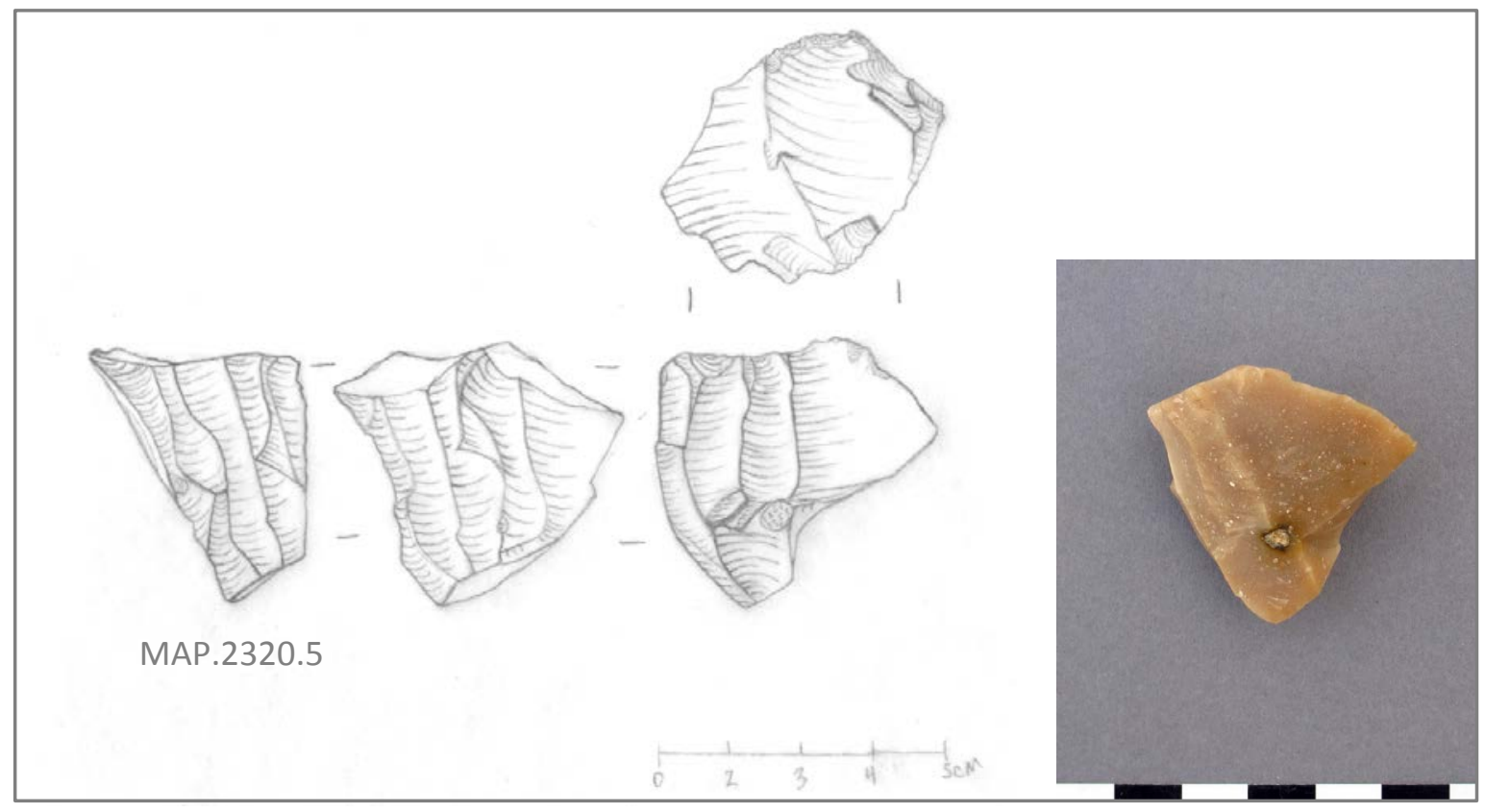

Figure 4.36. MAP.2320.5. Bladelet core. MC.

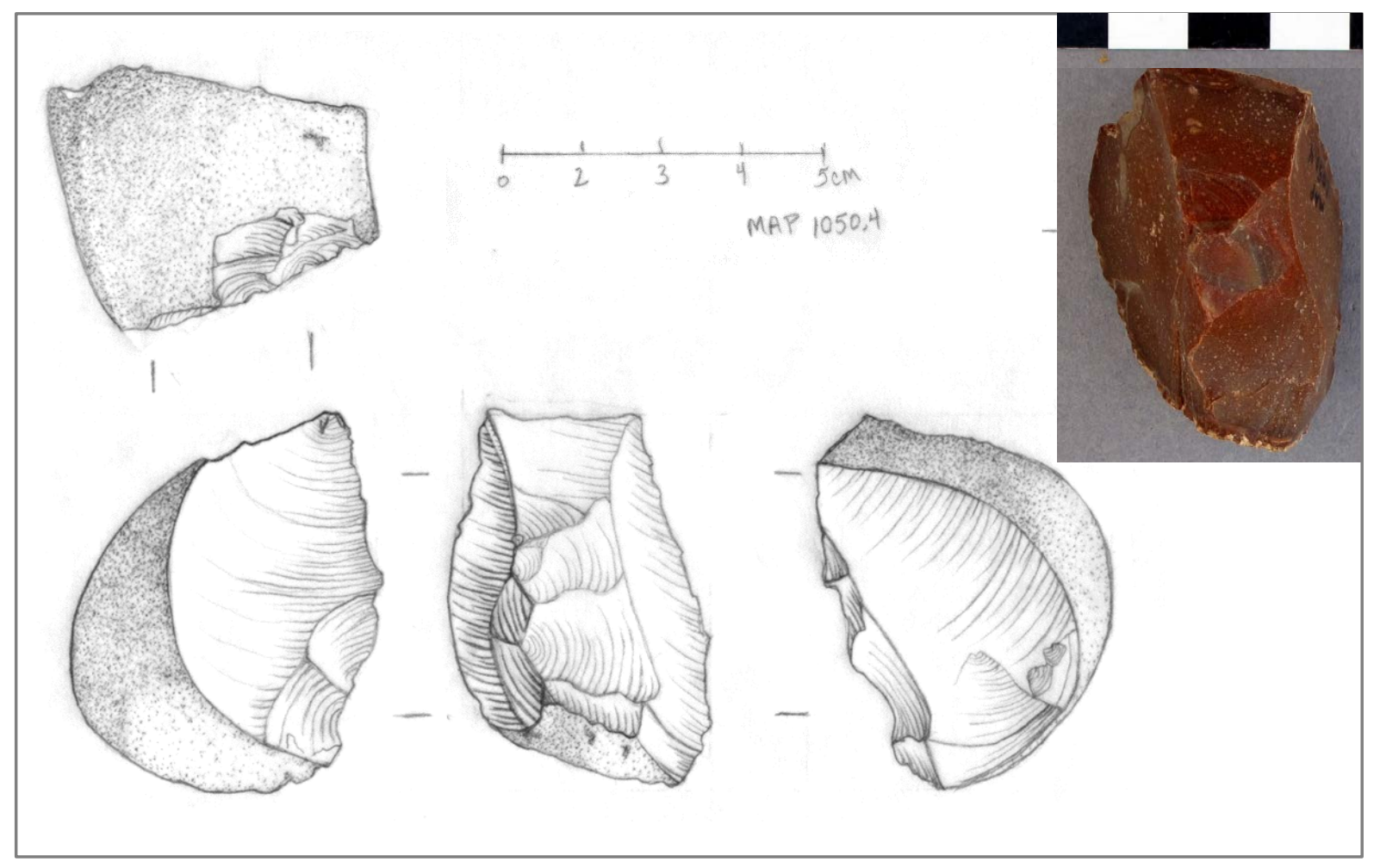

Figure 4.37. MAP.1050.4 Heat-treated core with a prepared crest, probably for bladelet production. MC. 


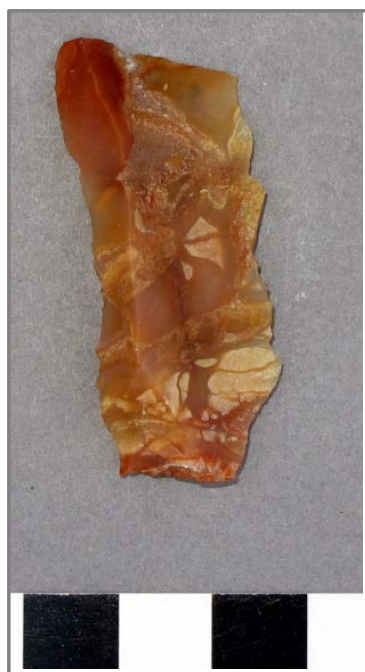

ATP. 2502.15

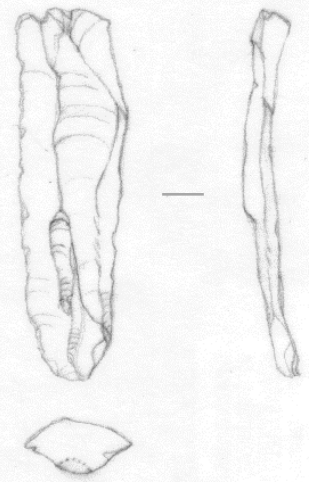

MAP.1317.27
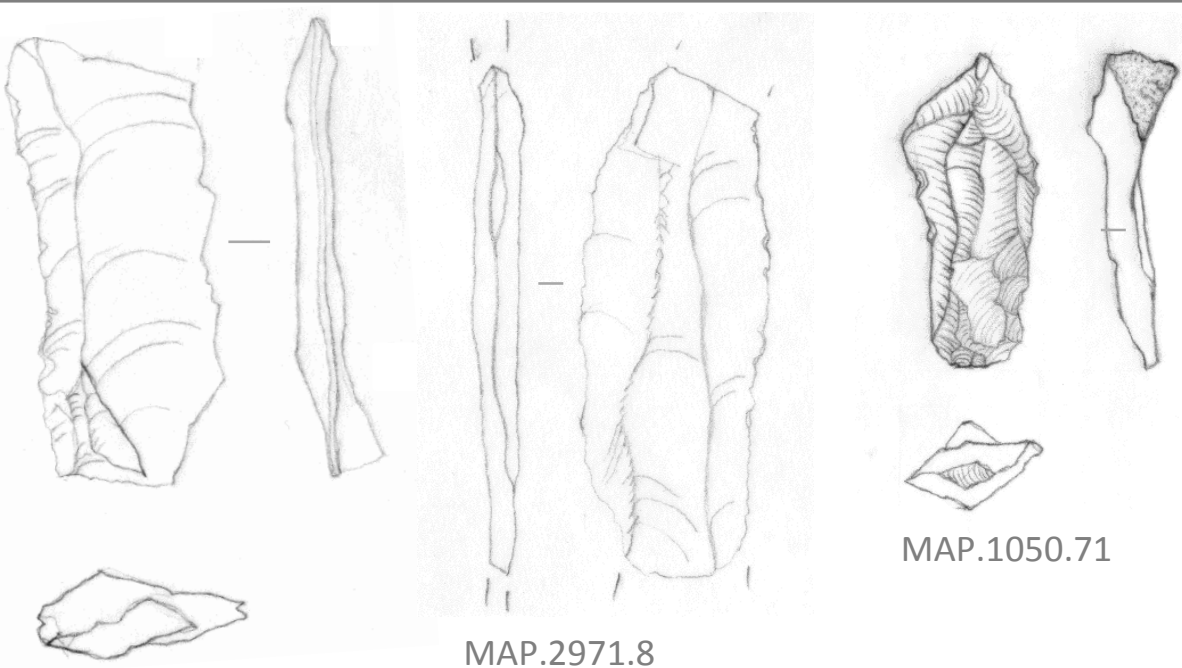

MAP.2971.8

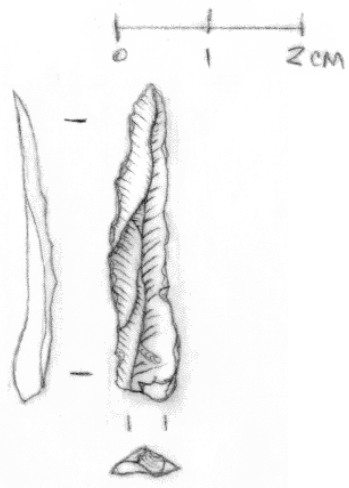

MAP.2386.40
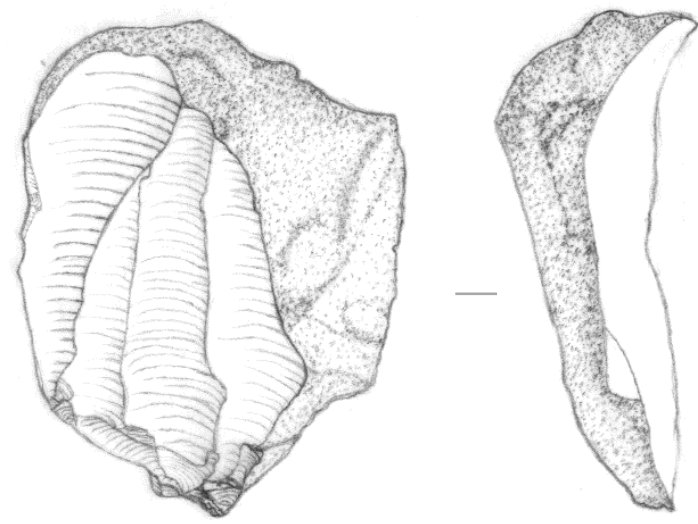

Figure 4.38. Blade debitage. ATP.2502.15. Agate Blade. LDH; MAP.2971.8. Medial blade fragment. LDH; MAP.1050.71. Secondary crested blade. MC; MAP.1317.27. Bladelet. LDH; MAP.2386.40. Bladelet. MC; ATP.4289.1. Flake from a blade core.

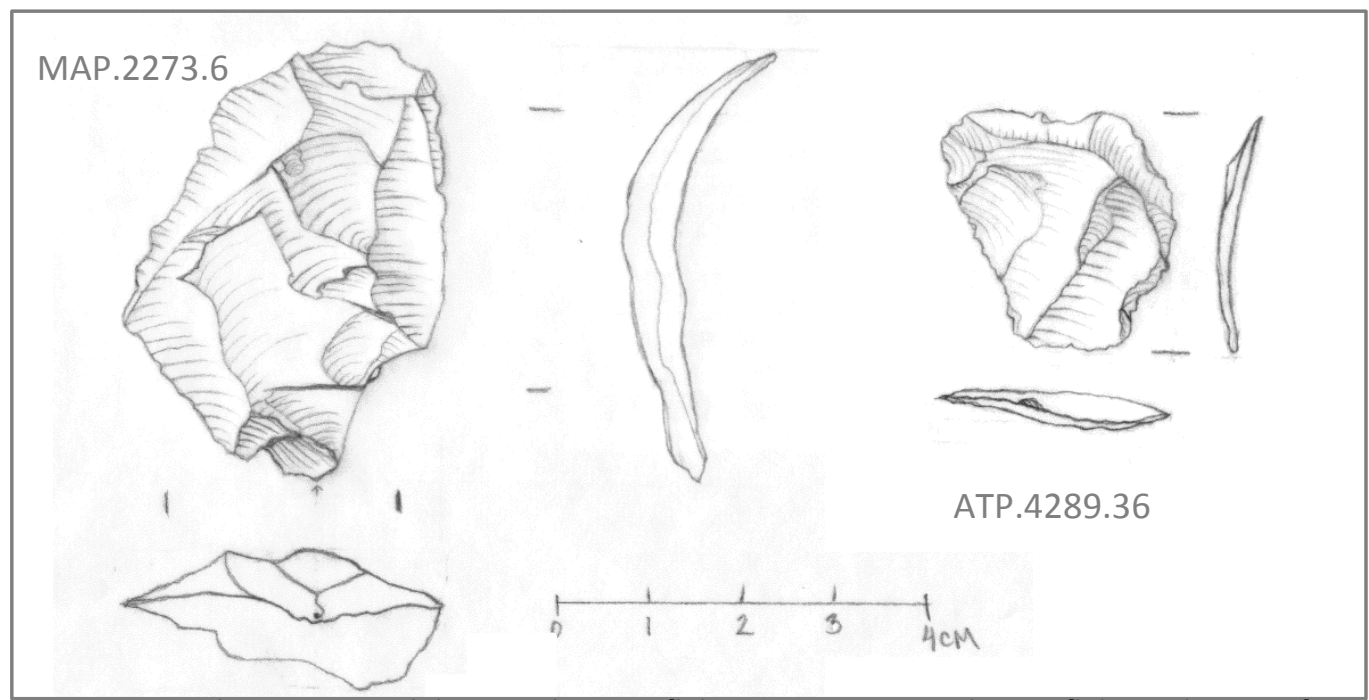

Figure 4.39. Characteristic debitage, thinning flakes. MAP.2273. 6.Thining flake with punctiform platform; ATP.4289.36. Thinning flake. With punctiform platform. MC. 


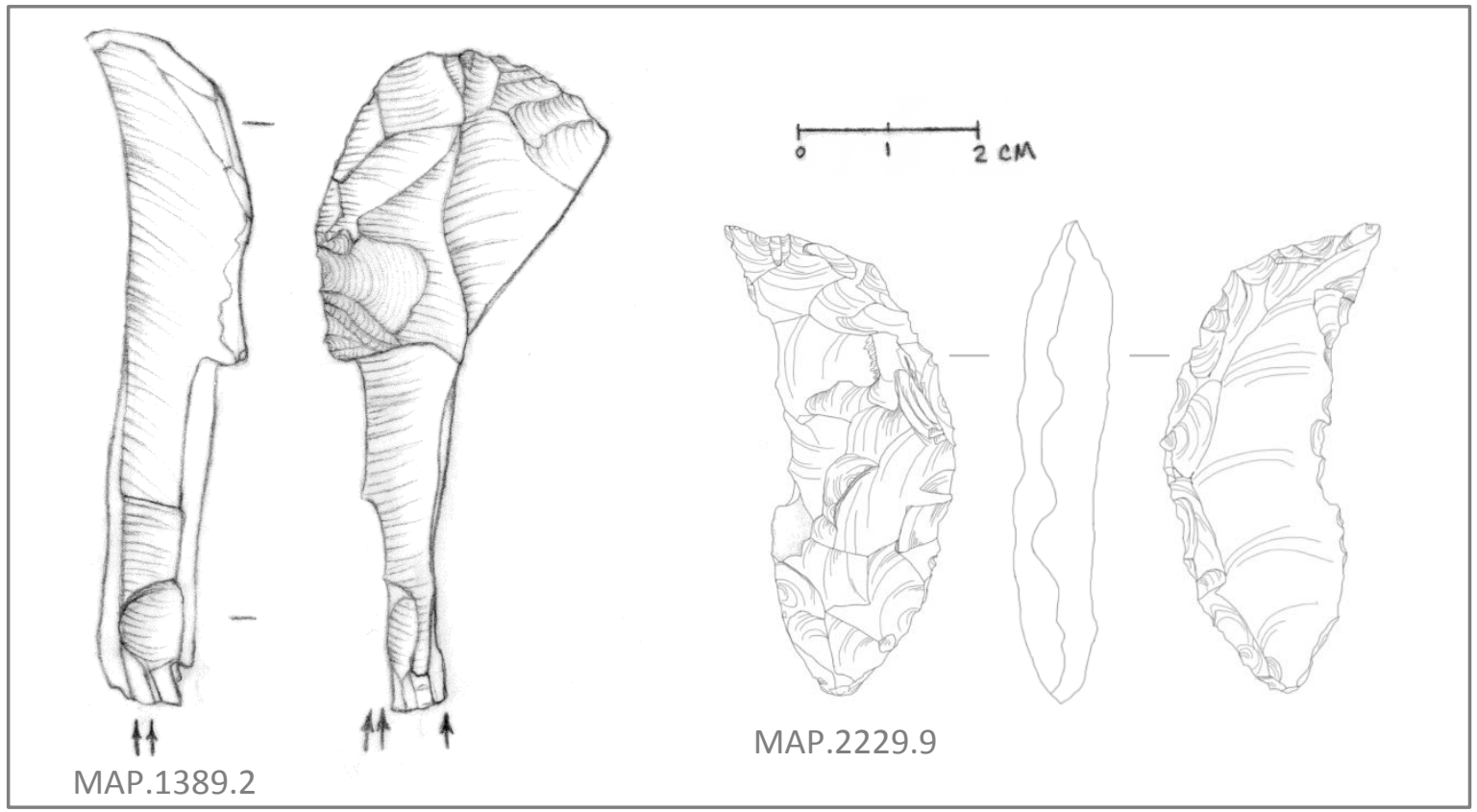

Figure 4.40. Characteristic debitage. MAP.1389.2. Plunging burin spall off of a scraper with previous burin spall scars. MC. MAP.2229.9. Axe preparation flake (tranchet). Digital drawing LDH. 

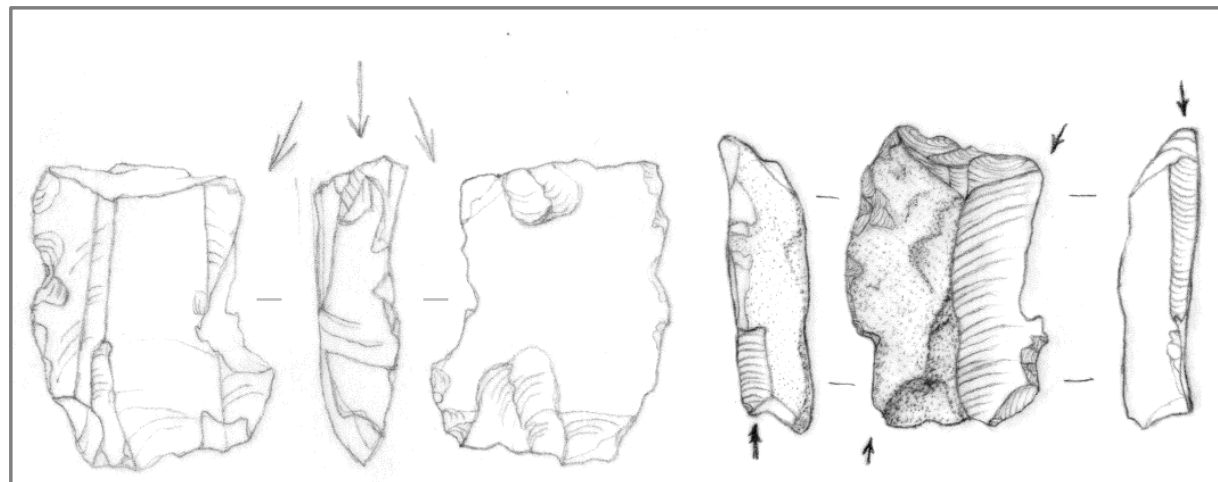

MAP.2173.22

MAP.1022.54

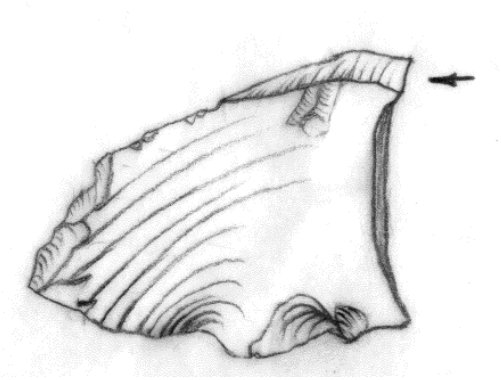

ATP.1990.37
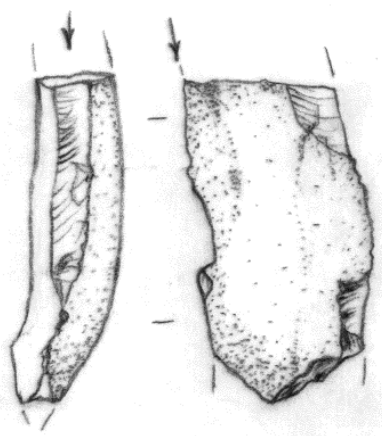

ATP.1990.92.

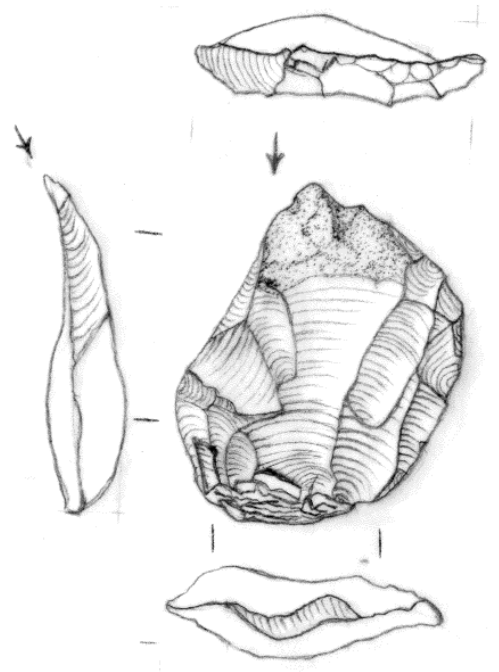

MAP.2931.15
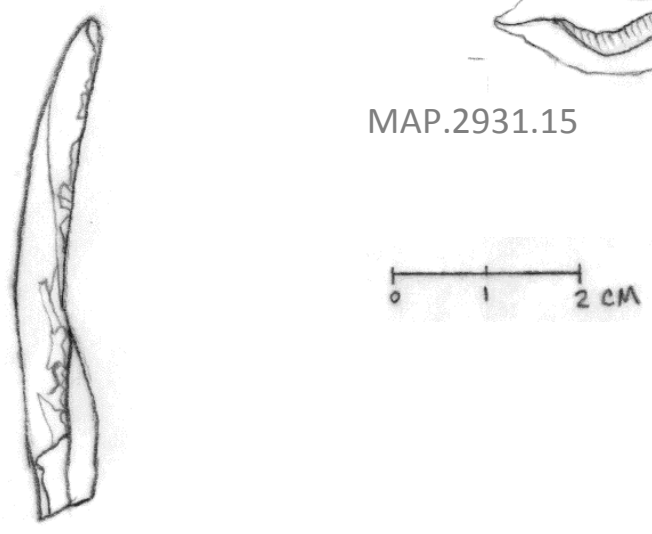

Figure 4.41. Burins. MAP.2173.22. Burin. LDH; MAP.1022.54. Multiple Burin on a truncation. MC; ATP.1990.37. Burin. MC; ATP.1990.92. Burin. MC; MAP.2931.15. Burin on a scraper. MC; ATP.1990.112. Burin. MC. 


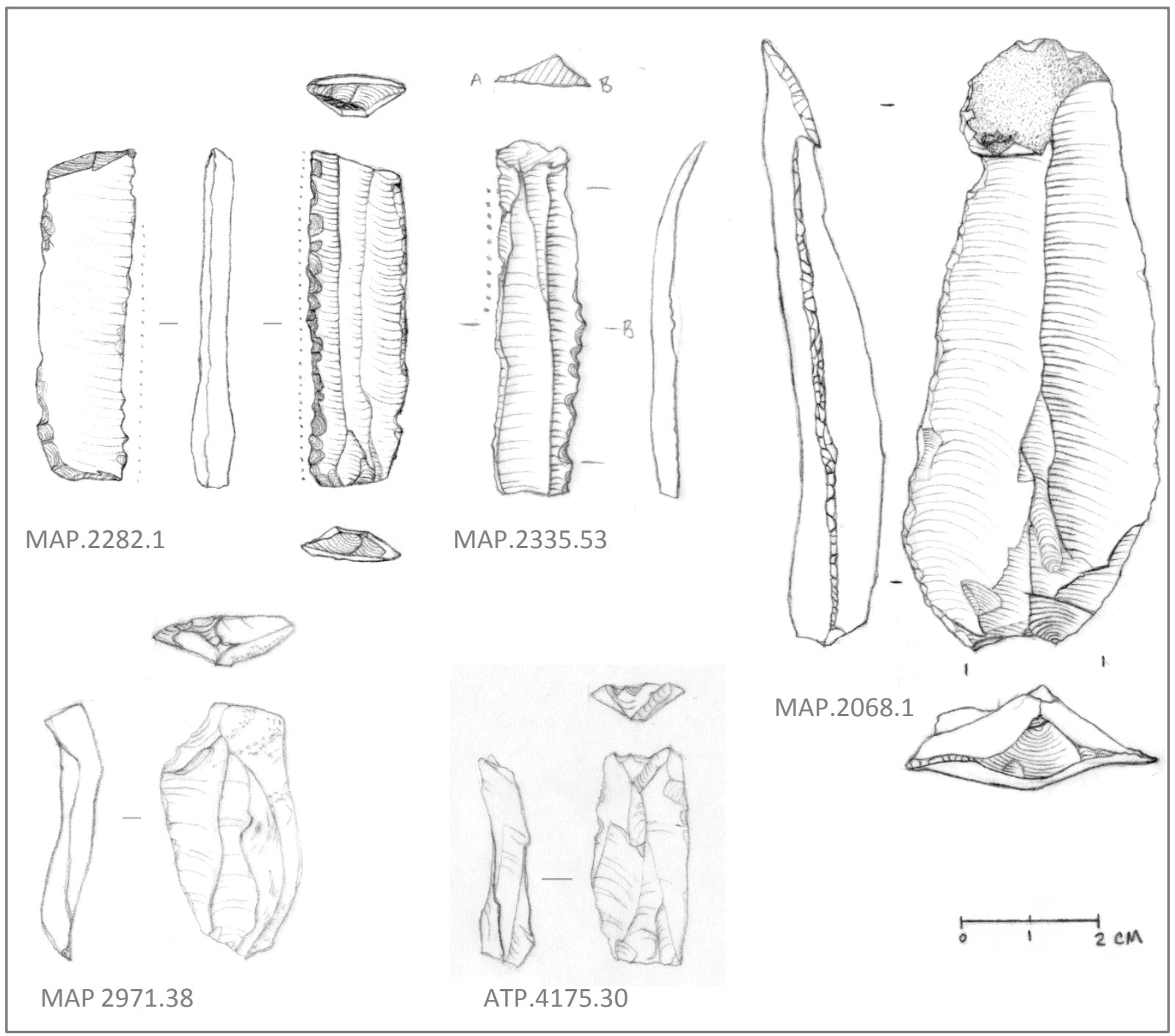

Figure 4.42. Unifacial blade tools. MAP.2282.1. Double-truncated Sickle blade. MC; MAP.2335.53. Sickle blade. MC; MAP.2068.1 Backed blade. MC; MAP 2971.38. Truncation. DH; ATP.4175.30. Truncation on a blade. LDH.

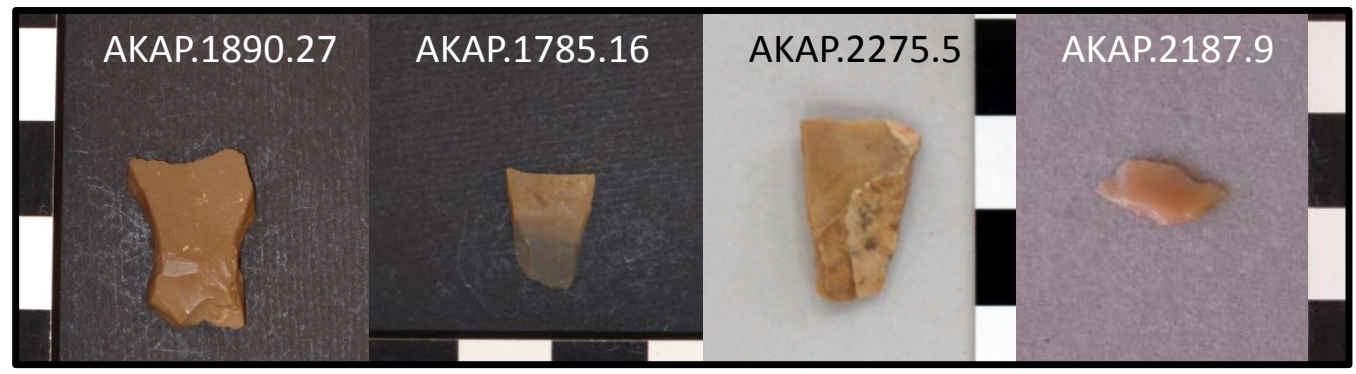

Figure 4.43. Unifacial tools: projectile points from Nag el-Qarmila. AKAP.189027, AKAP1785.16, AKAP2275.5, transverse arrowheads. AKAP.2187.9 Lunate. 


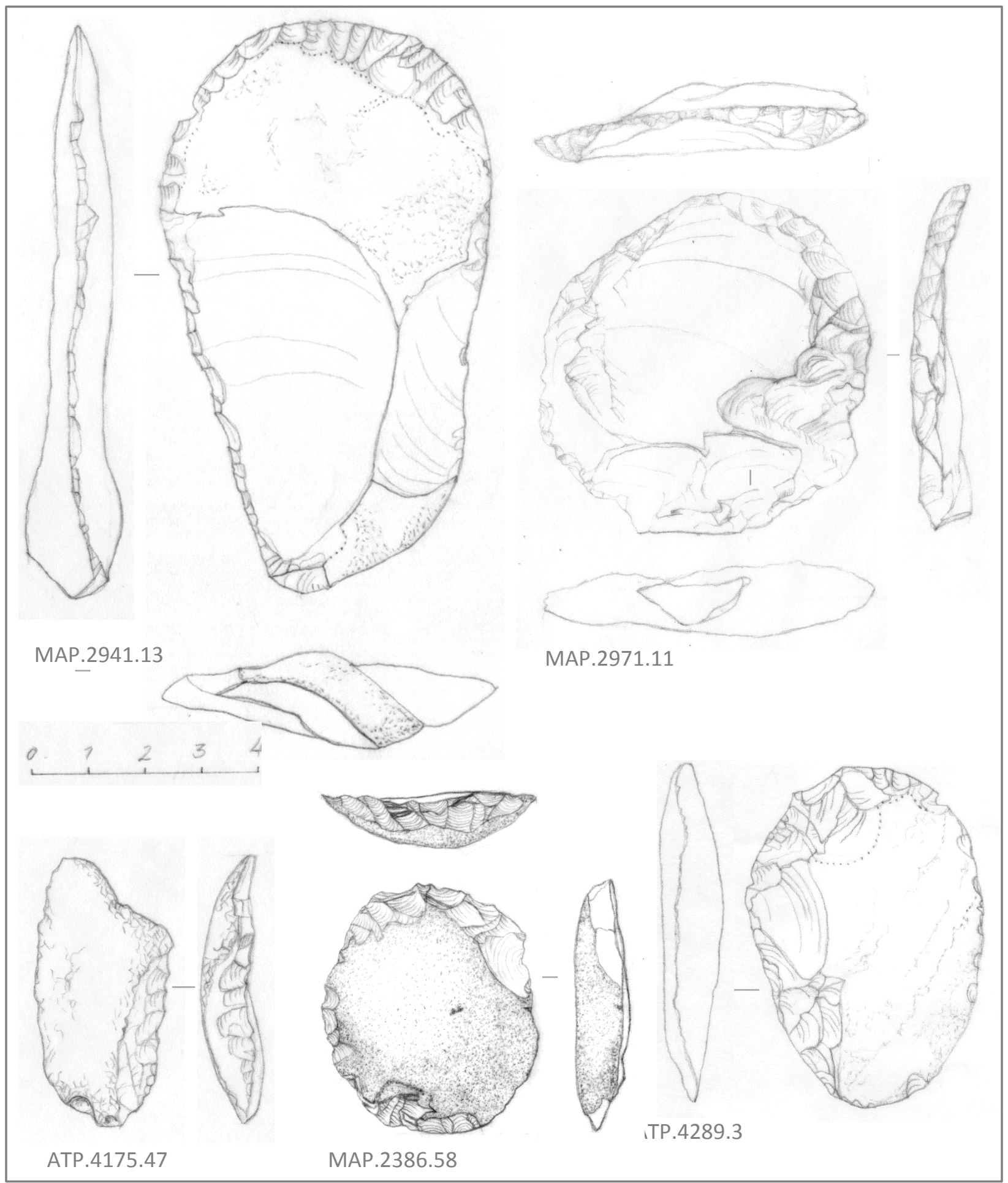

Figure 4.44. Unifacial tools: scrapers. MAP.2941.13. Endscraper. LDH; MAP.2971.11. Endscraper. LDH; ATP.4175.47. Side scraper. LDH; MAP.2386.58. Endscraper. MC; ATP.4289.3. Endscraper. LDH. 


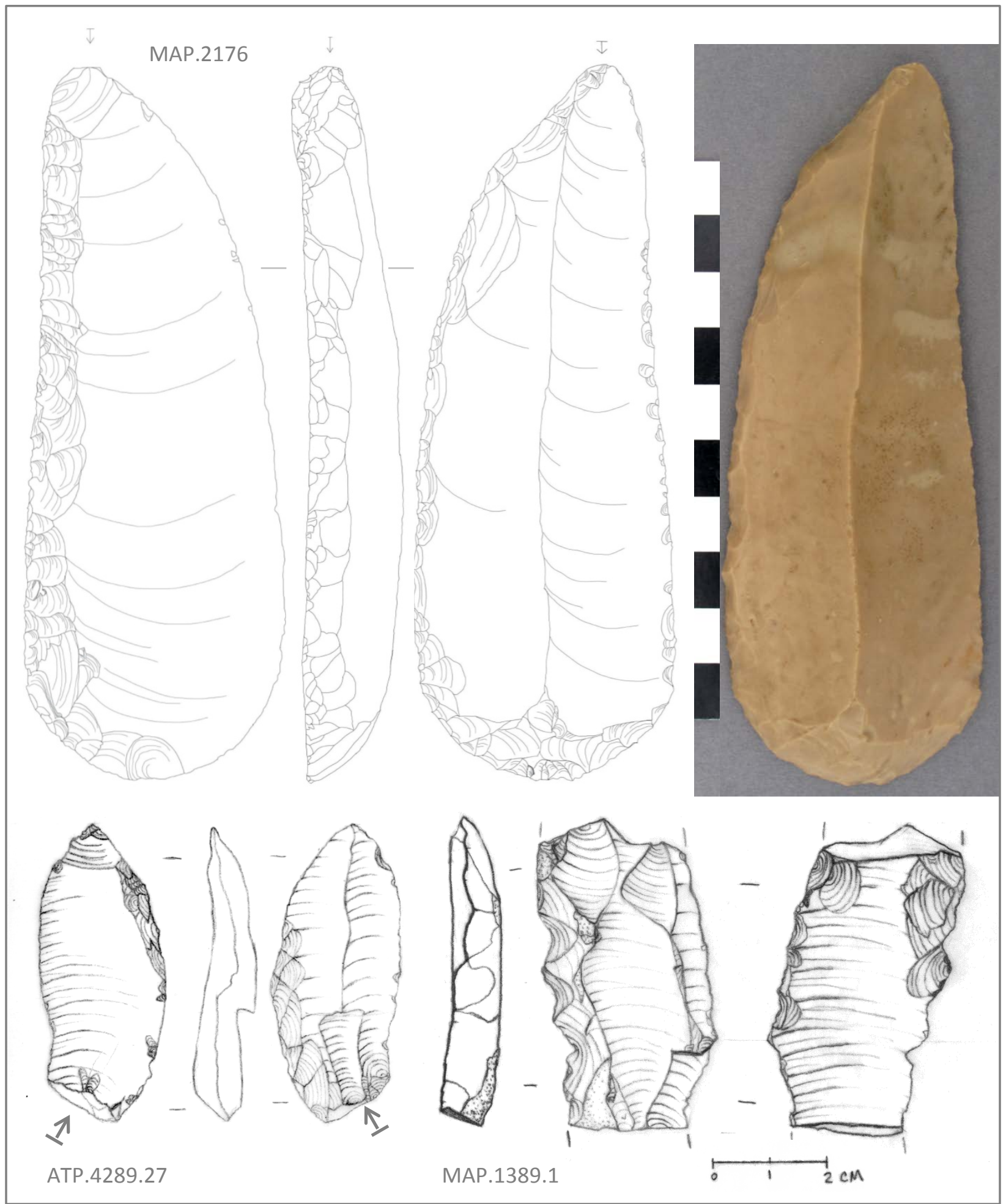

Figure 4.45. Edge retouched tools. MAP.2176. Endscraper knife; ATP.4289.27 knife on a blade-like flake; MAP.1389.1. Denticulated blade. MC. 


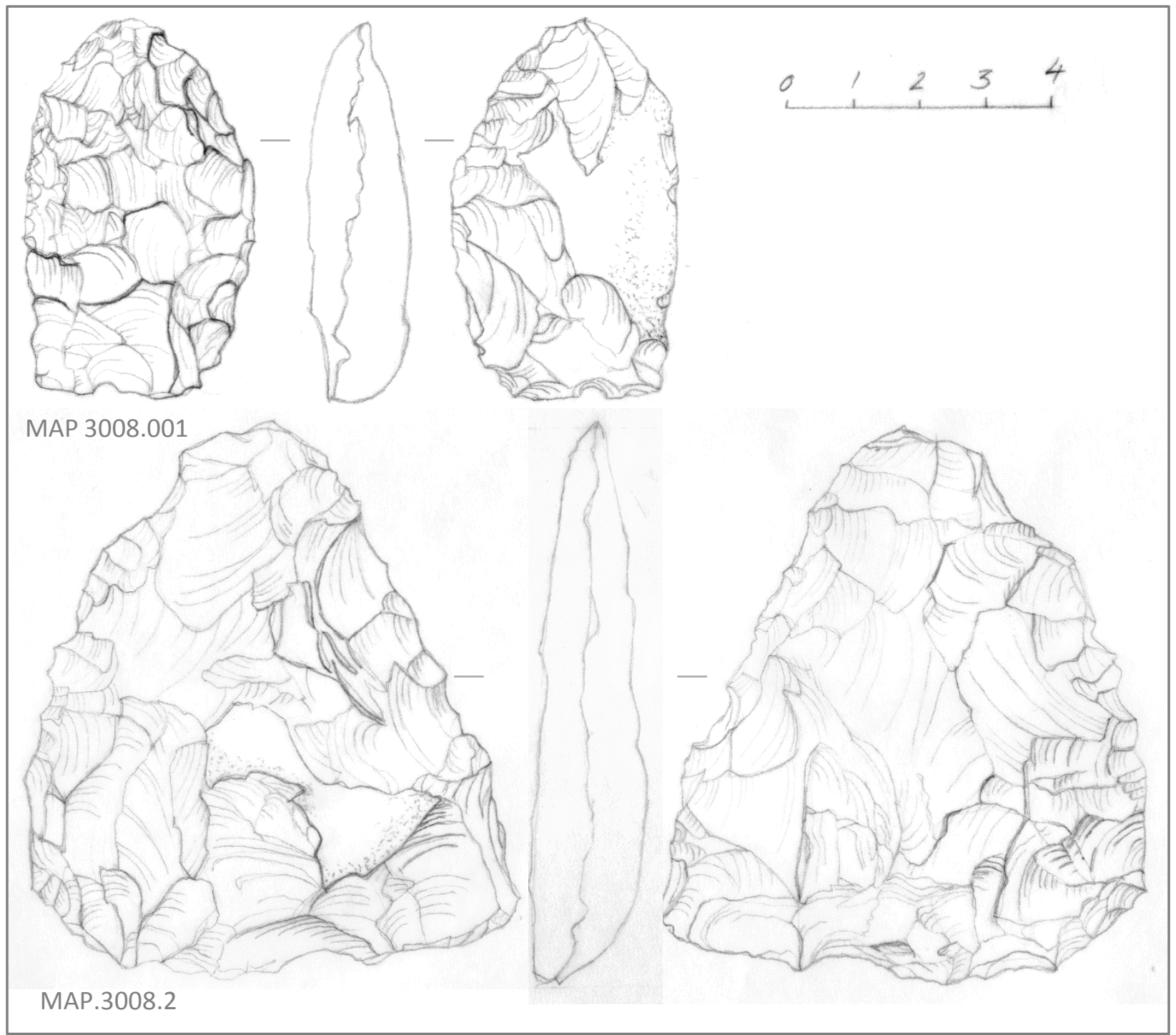

Figure 4.46. Bifacial tools. Preforms. MAP 3008.001. Preform. LDH; MAP.3008.2 Bifacial triangle. (Preform?)

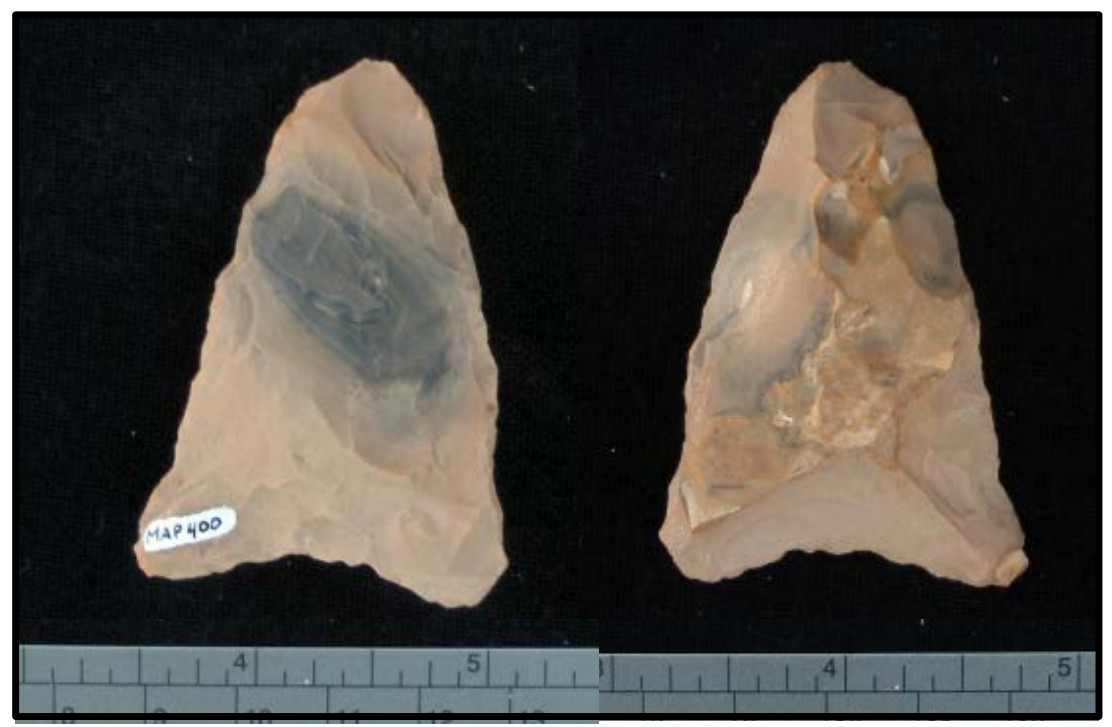

Figure 4.47. Bifacial tools.

Preforms. MAP 400. Probably preform for a concave base projectile point. 


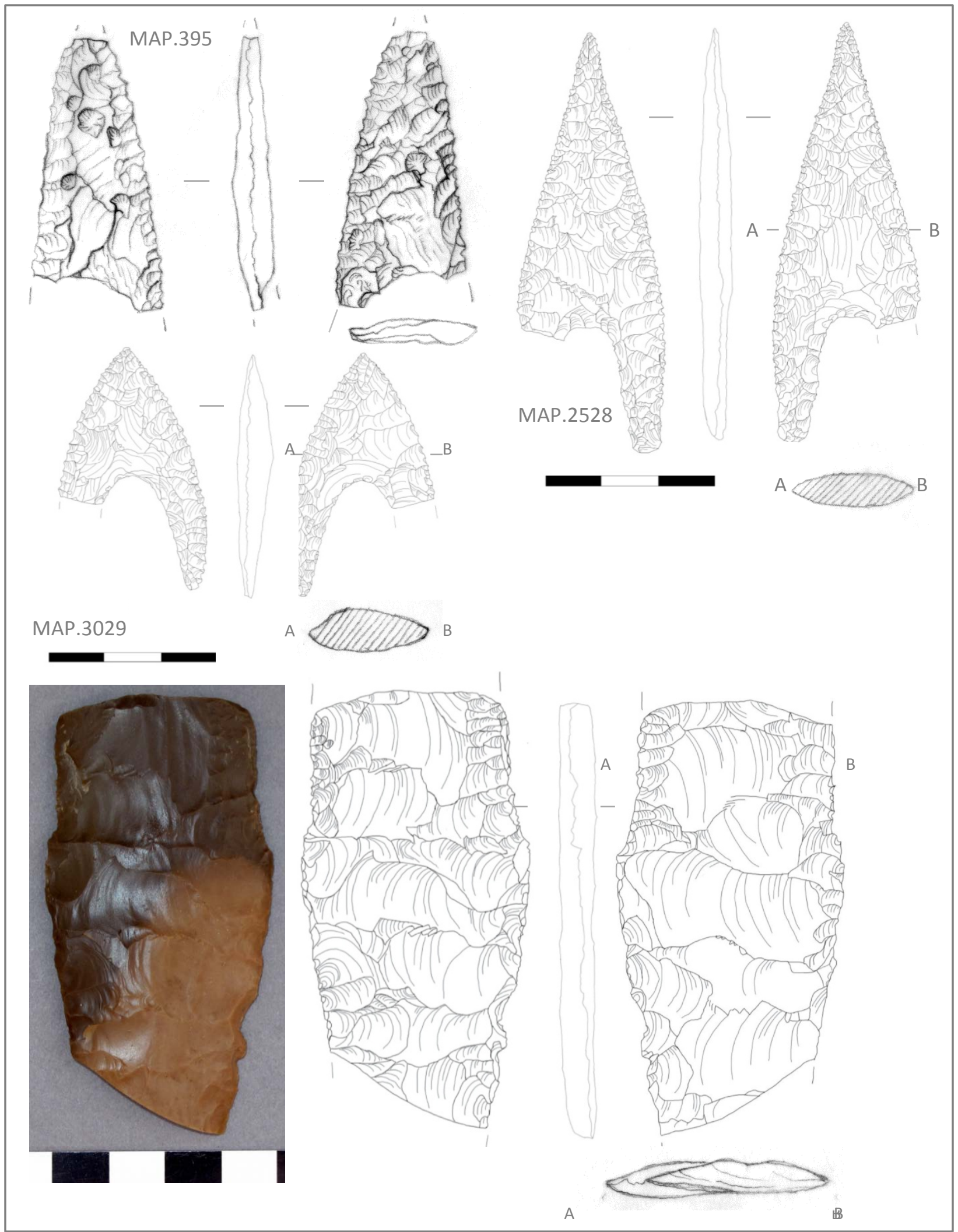

Figure 4.48. Bifacial tools. MAP.395. Burnt concave-base projectile point fragment. LDH; MAP.2528. Concave base projectile point. LDH. Photos Figures 4.25, 6.14, 7.4; MAP.3029. Concave base projectile point. LDH. Photos Figures 6.14, 7.4; MAP.301 Fishtail knife fragment. LDH. 


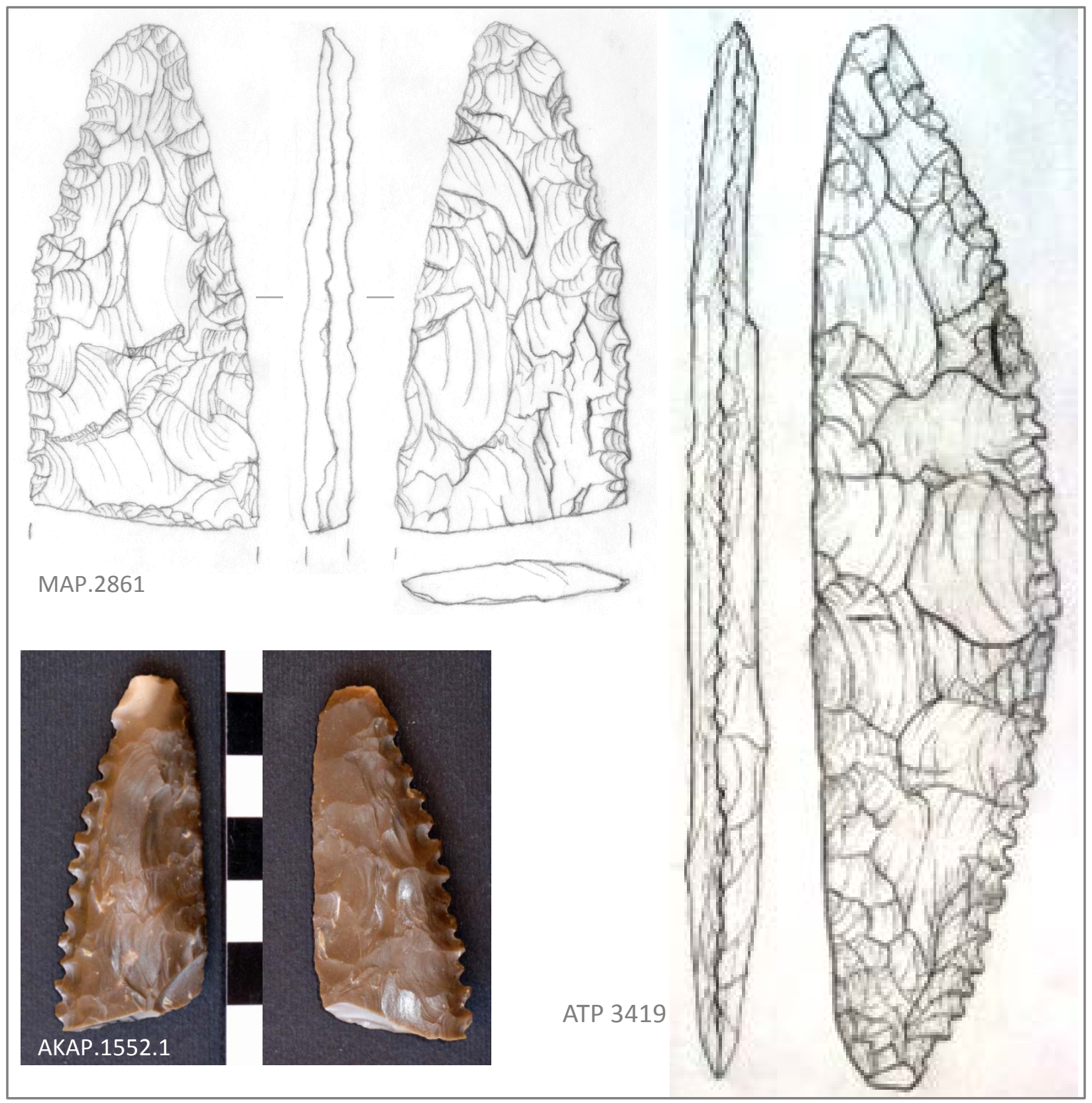

Figure 4.49. Bifacial sickles. MAP.2861. LDH. Photo Figure 7.5; AKAP.1552.1; ATP.3419. Drawing by Matthew Loeser. Photo Figure 7.5.

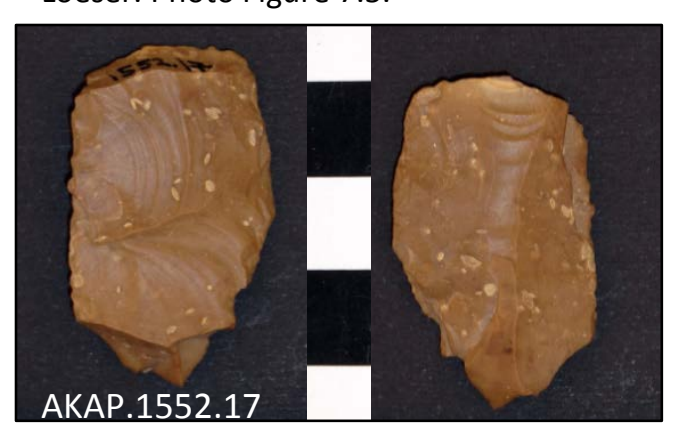

Figure 4.50. Bifacial knife fragments, above, right.

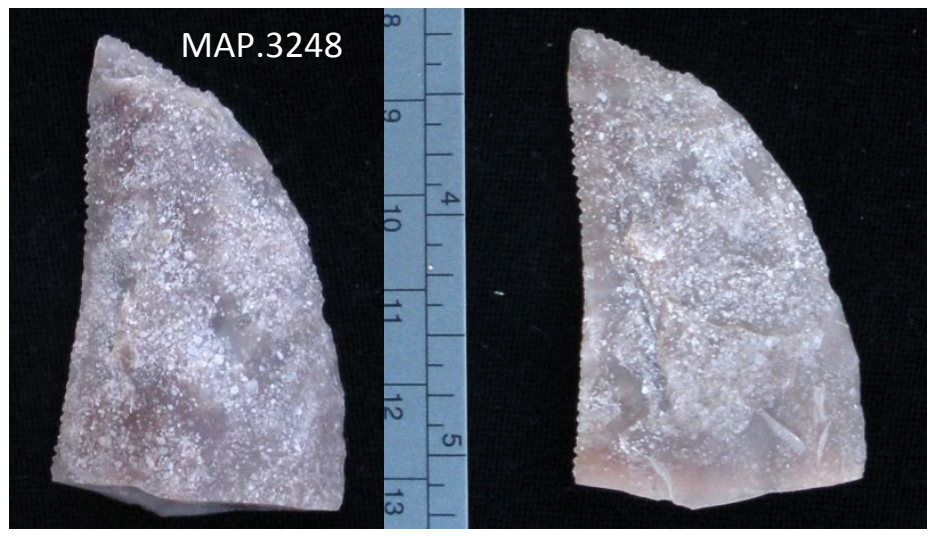




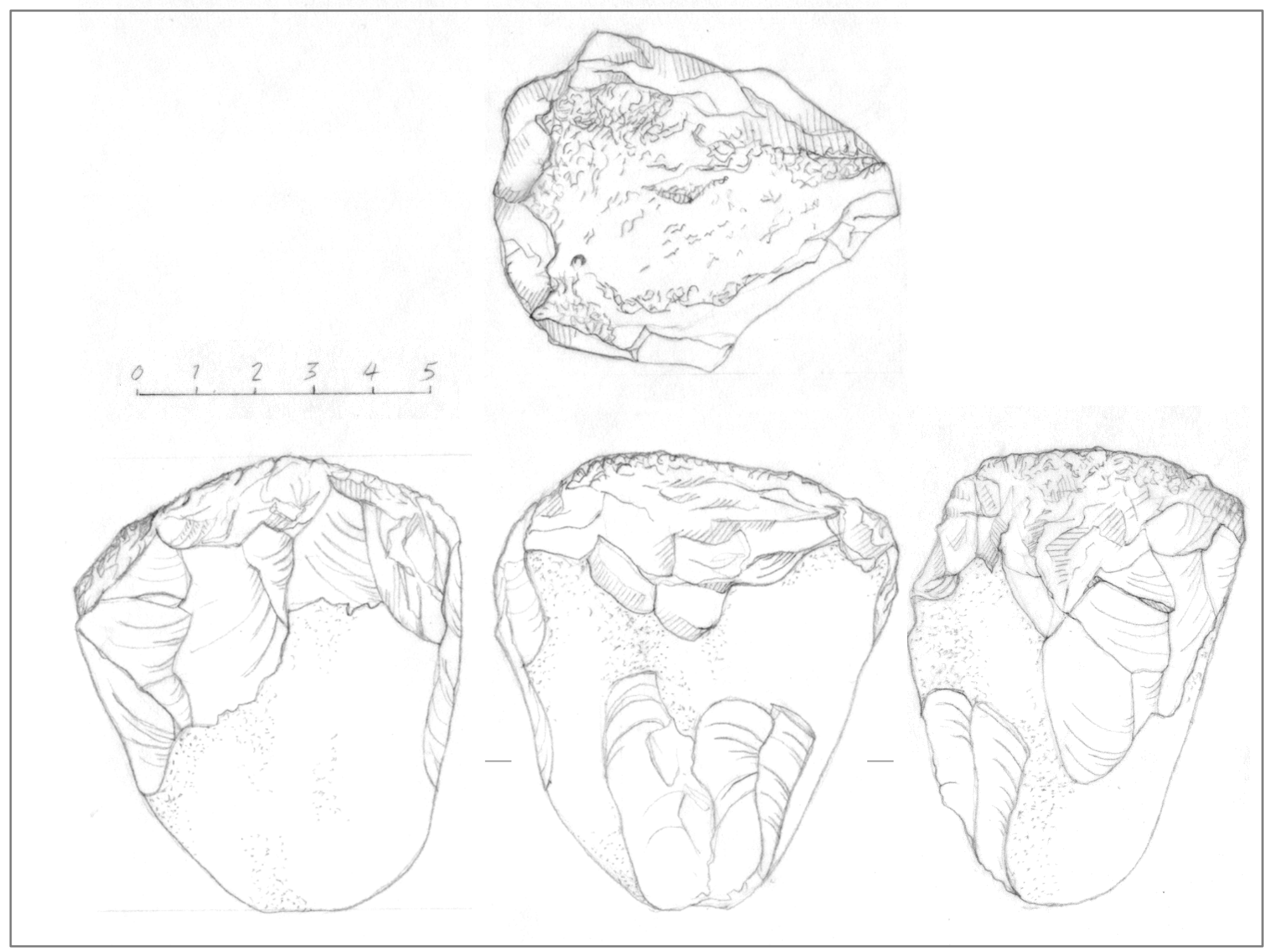

Figure 4.51. Pounder/pestle. MAP.2173.1. LDH. 


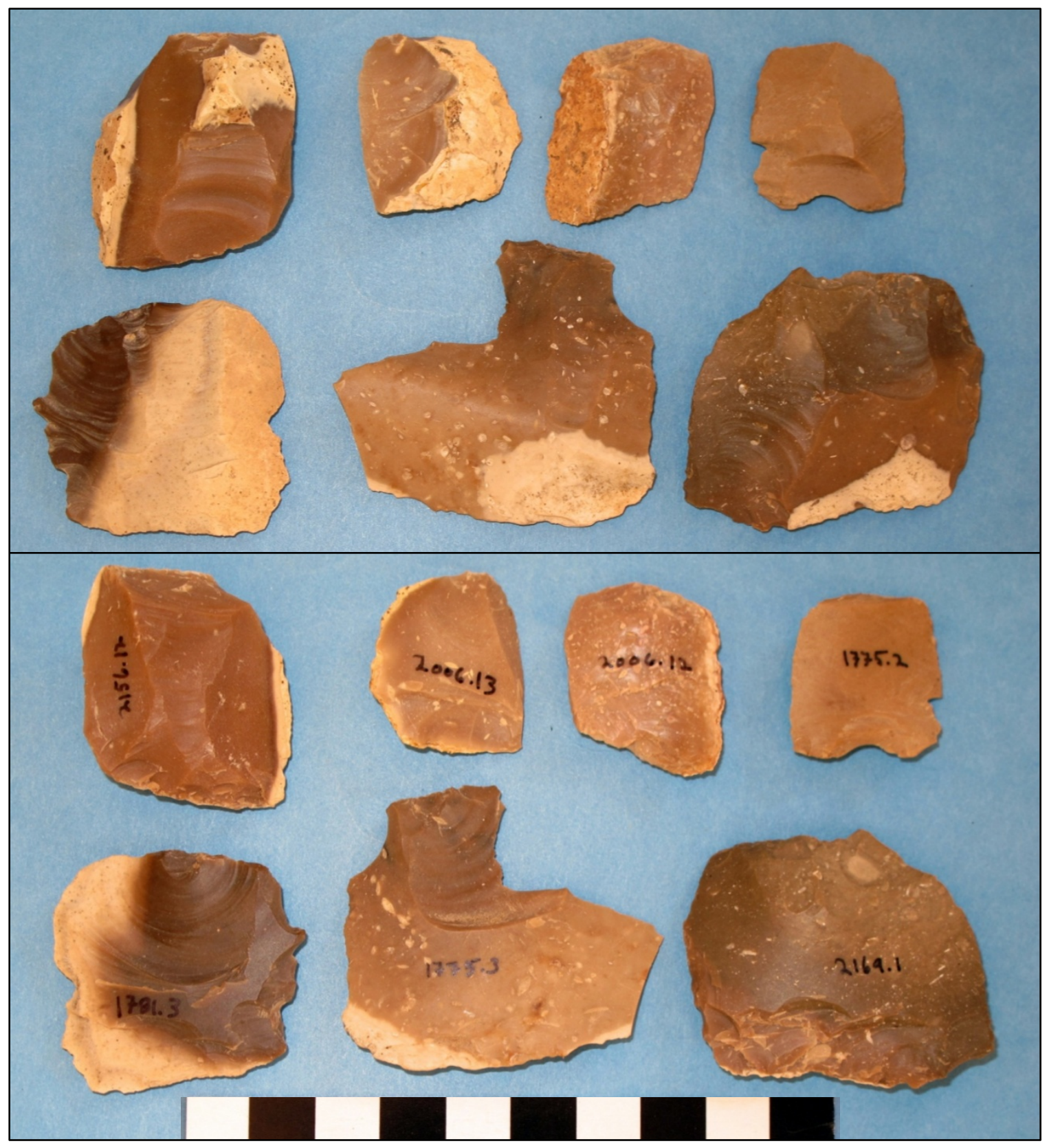

Figure 4.52. Piece Esquilles from Nag el-Qarmila (AKAP).Dorsal and ventral views. 


\section{Chapter 5 Figures}

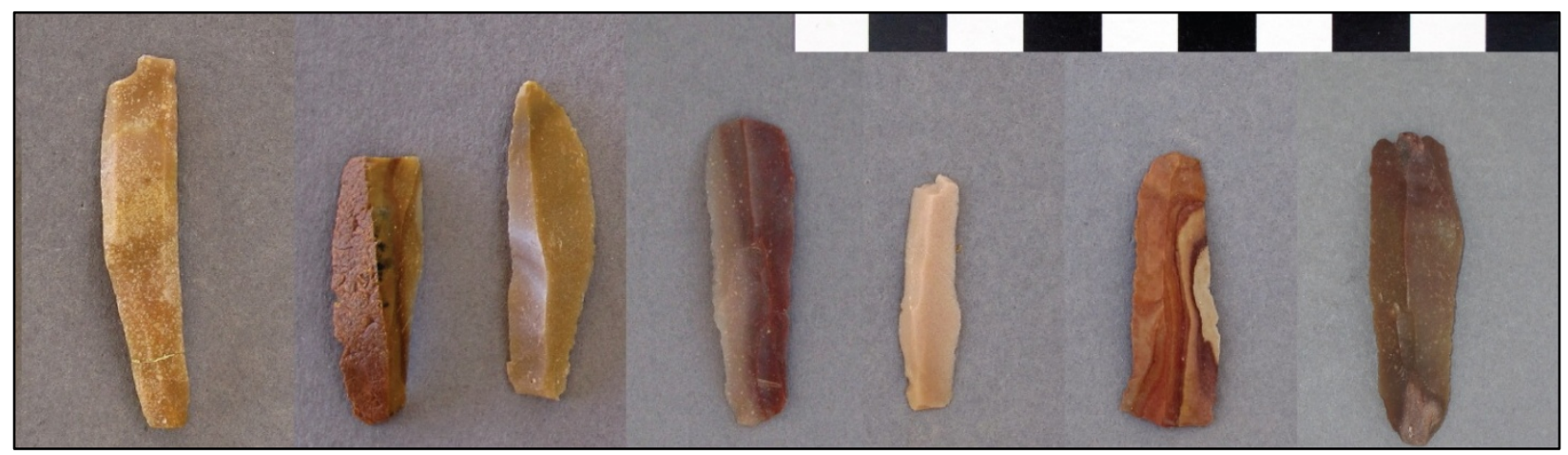

Figure 5.1. Examples of bladelets (el-Mahâsna MAP 1030).

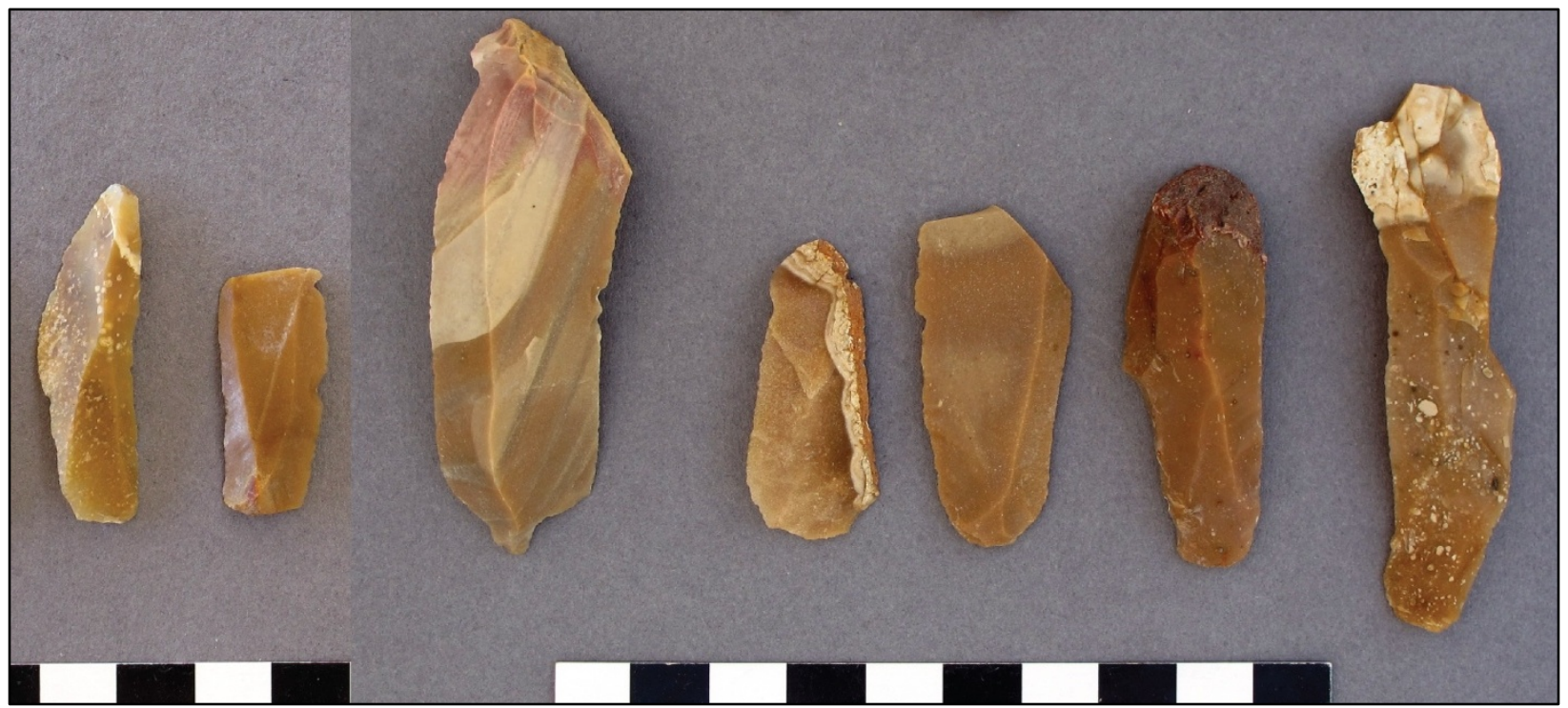

Figure 5.2. Examples of medium blades (el-Mahâsna).

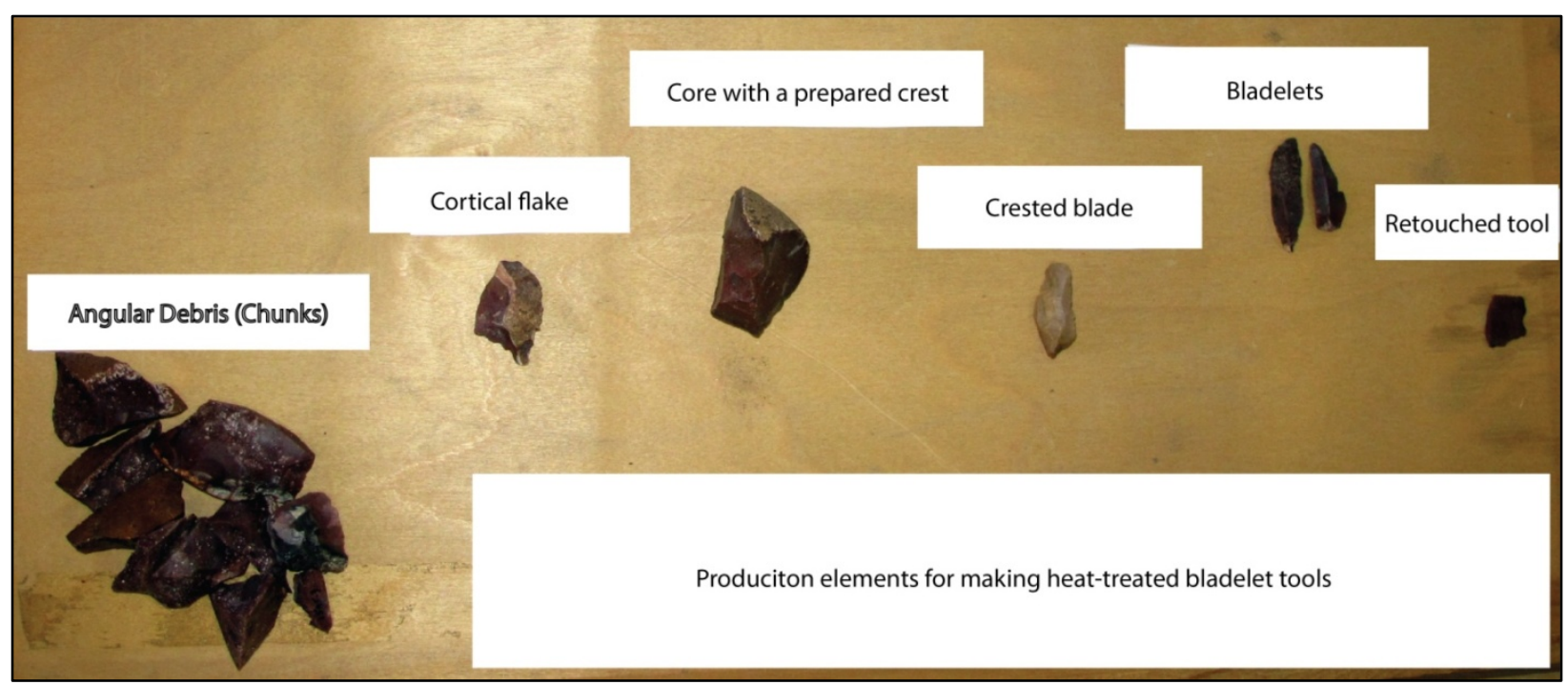

Figure 5.3. A full sequence of production remains from making heat-treated bladelet tools. From el-Mahasna. 


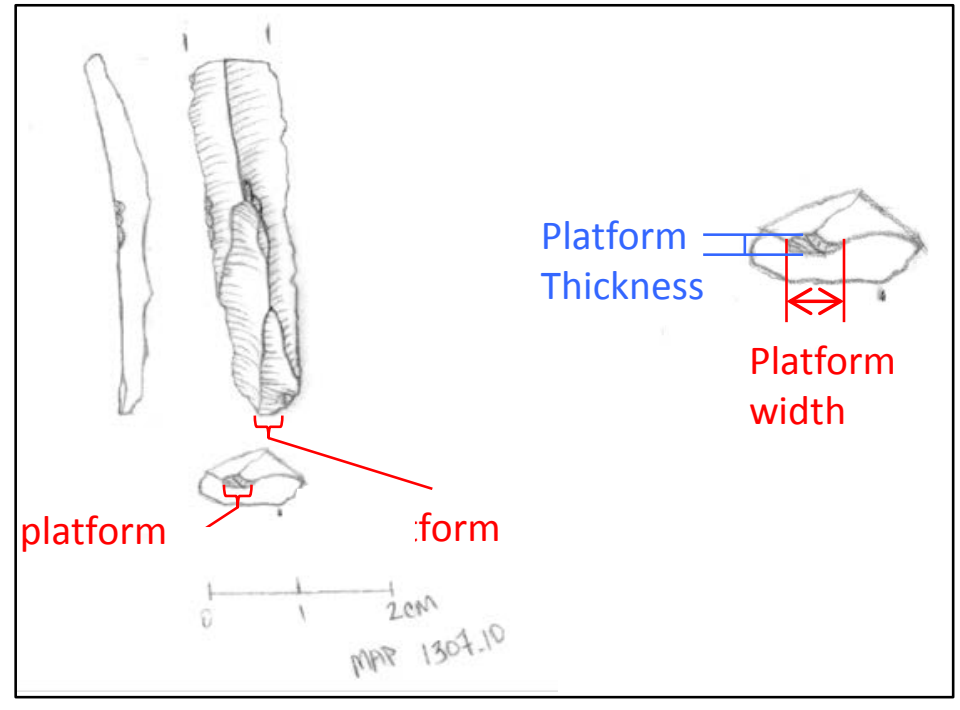

Figure 5.4. Left: Location of the platform (shown on MAP 1307.10). Right: Platform width and thickness measurements, following Debenath \& Dibble (1994:17) (not to scale).

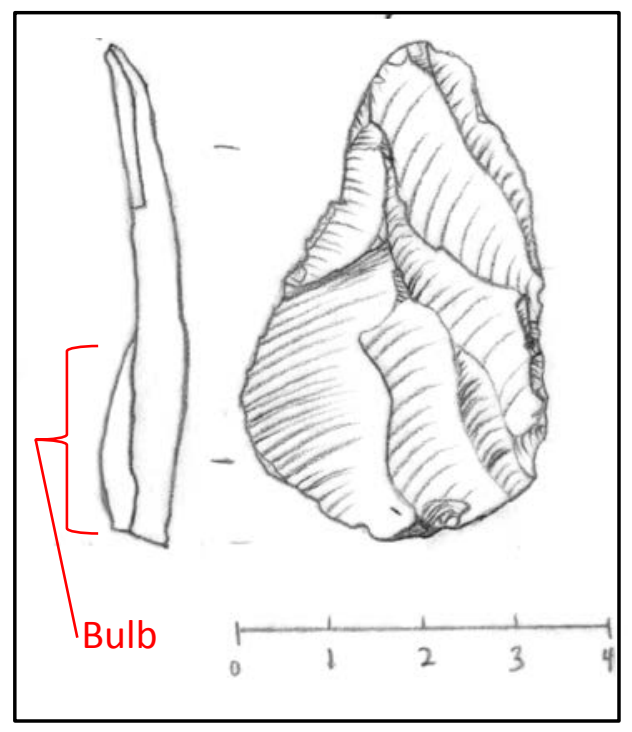

Figure 5.5. Bulb of percussion on a retouched flake (ATP 1990.112).

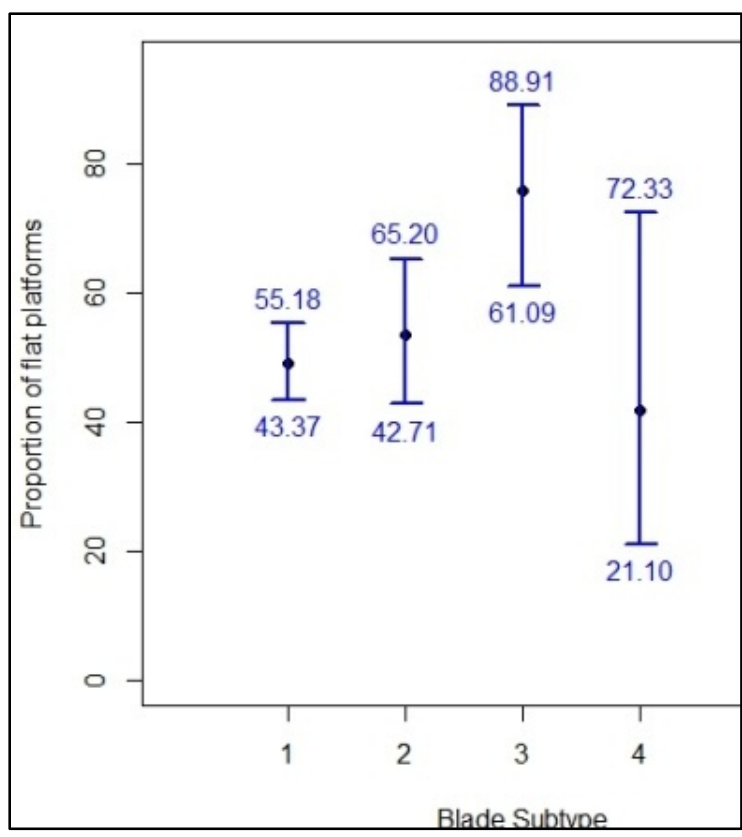

Figure 5.6. Frequency of flat platforms of four blade subtypes, and their $95 \%$ binomial confidence intervals. 1=medium blades, 2=bladelets, 3=twisted medium blades, 4=twisted bladelets.

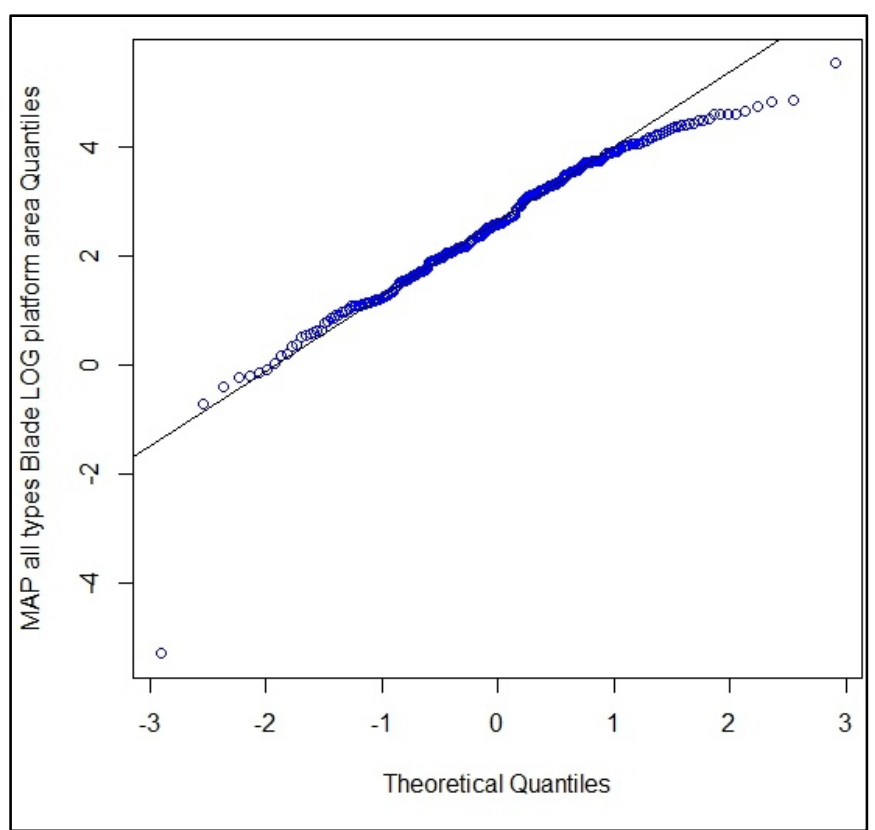

Figure 5.7. QQ plot of the log transformed platform areas showing that the data are normally distributed. 


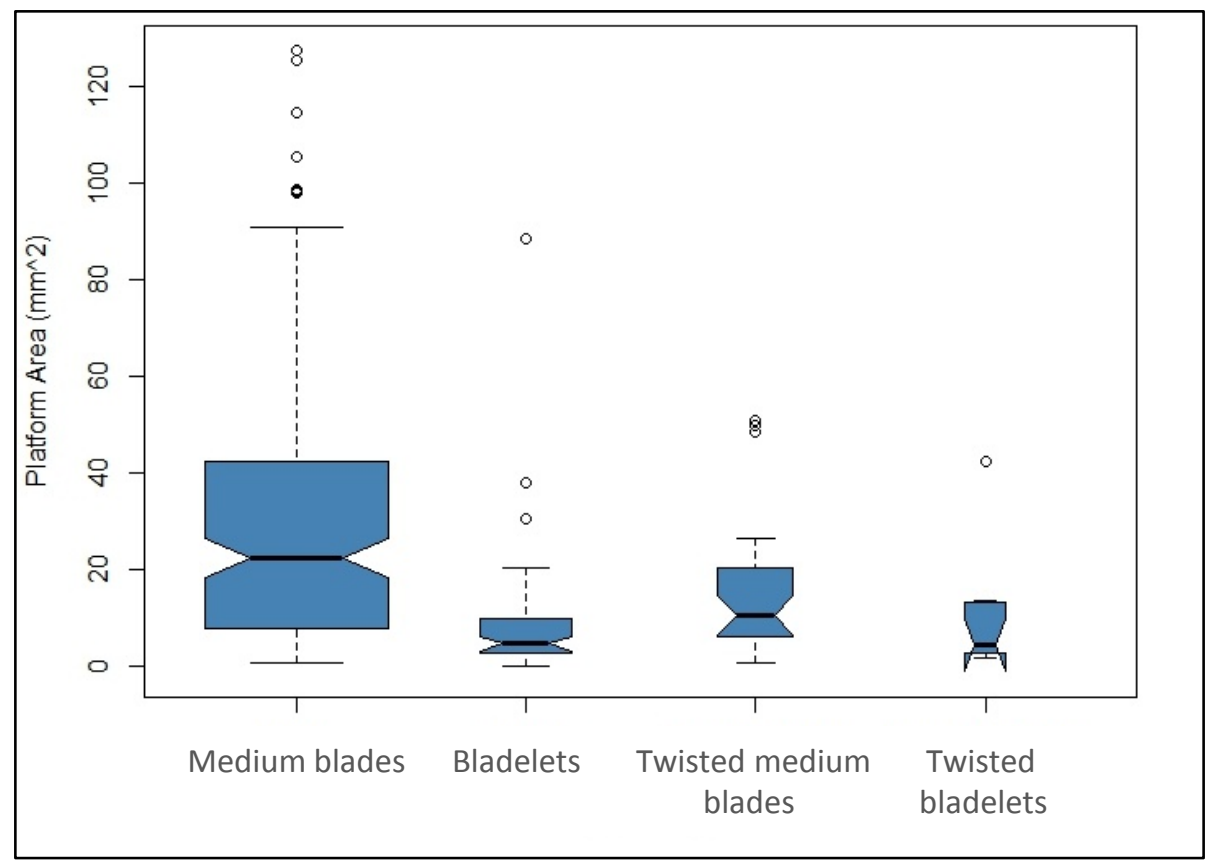

Figure 5.8. Box plot of the platform areas of blade types. One outlier from elMahâsna with a platform area of 250 is not shown.

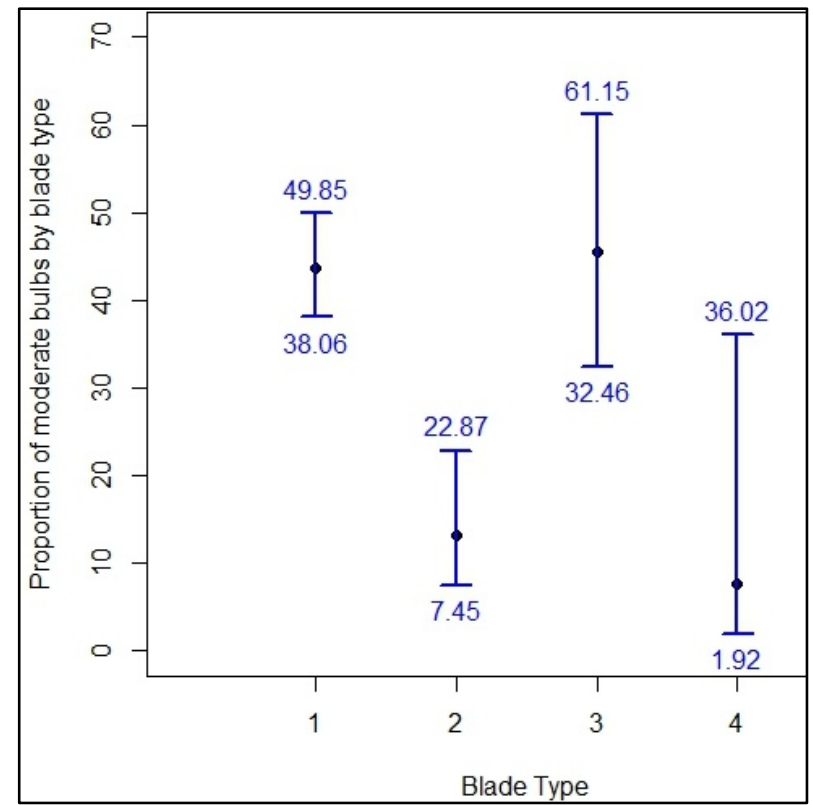

Figure 5.9. Percentages and $95 \%$ binomial confidence intervals for moderate bulbs by blade types. 1:Medium blades, 2:Bladelets, 3:Twisted medium blades, 4:Twisted bladelets. The confidence intervals show two separate groups: medium blades and twisted medium blades vs. bladelets and twisted bladelets.

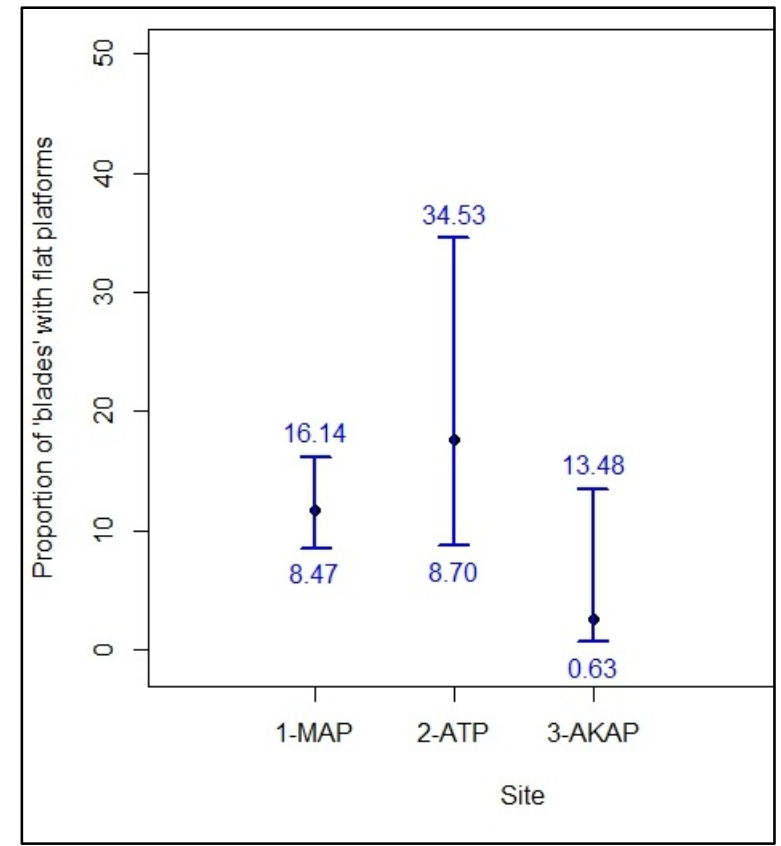

Figure 5.10. The $95 \%$ binomial confidence intervals for the frequencies of medium blades with cortical platforms at each of the three sites. 


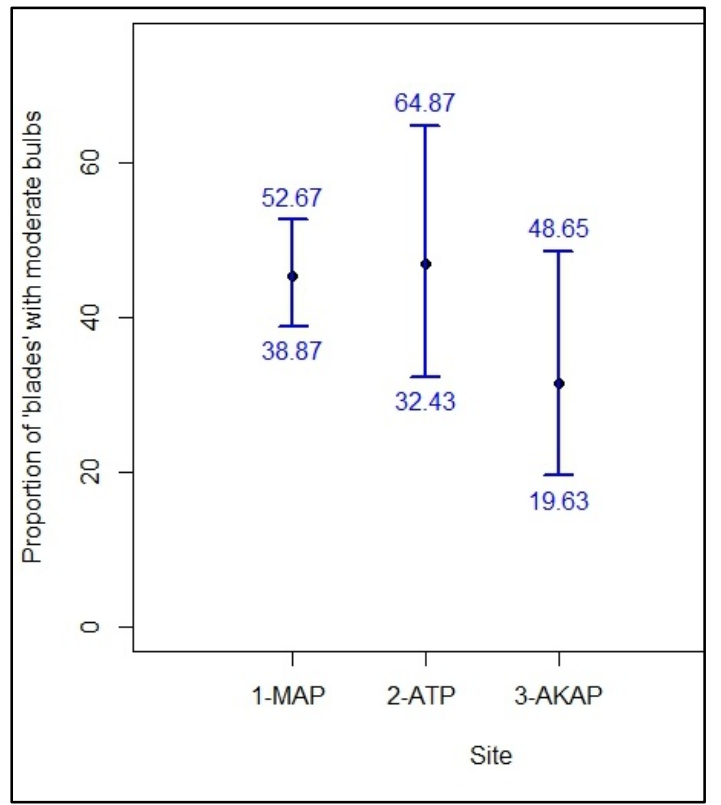

Figure 5.11. The $95 \%$ binomial confidence intervals for the frequencies of medium blades with moderate bulbs at each of the three sites.

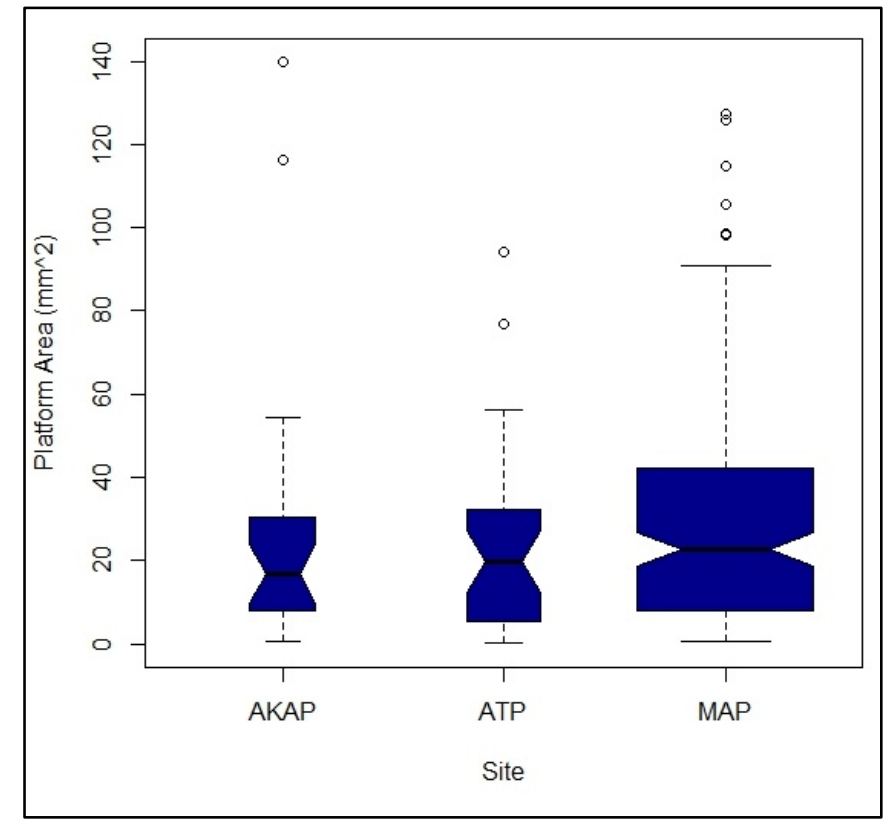

Figure 5.12. Box plot of the platform areas of medium blades from three sites. Mean platform areas are as follows: AKAP- 25.67 ( $N=27)$; ATP- 24.36 ( $N=31)$; MAP$30.17(\mathrm{~N}=188)$.

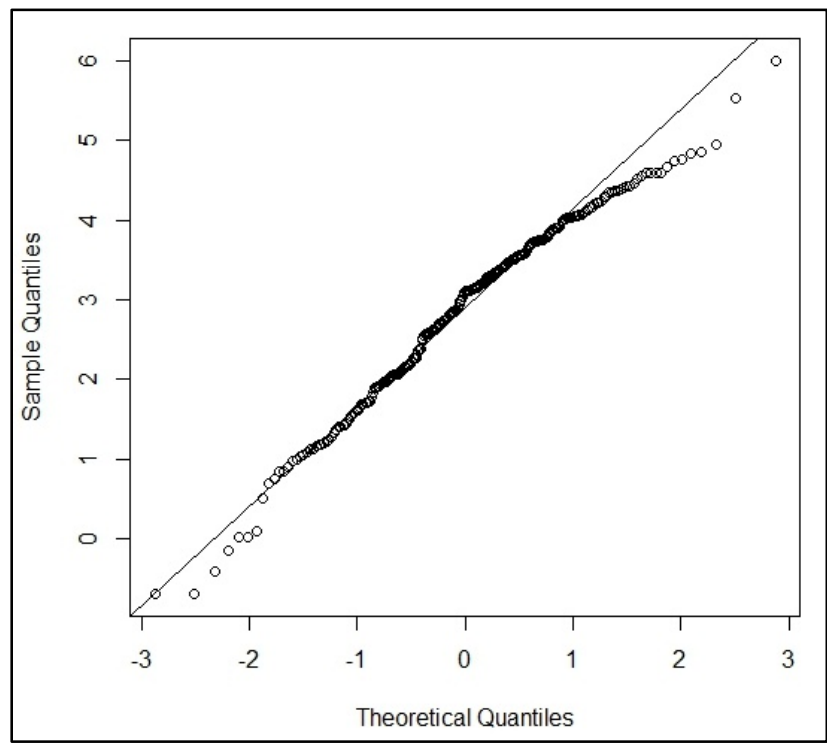

Figure 5.13. A qq plot of the Log transformed platform areas of medium blades from all three sites showing that the data are normally distributed. A Bartlett's test of the homogeneity of the log transformed variances returned a $p$-value of 0.1869 , so the null hypothesis that the variances are equal should not be rejected.

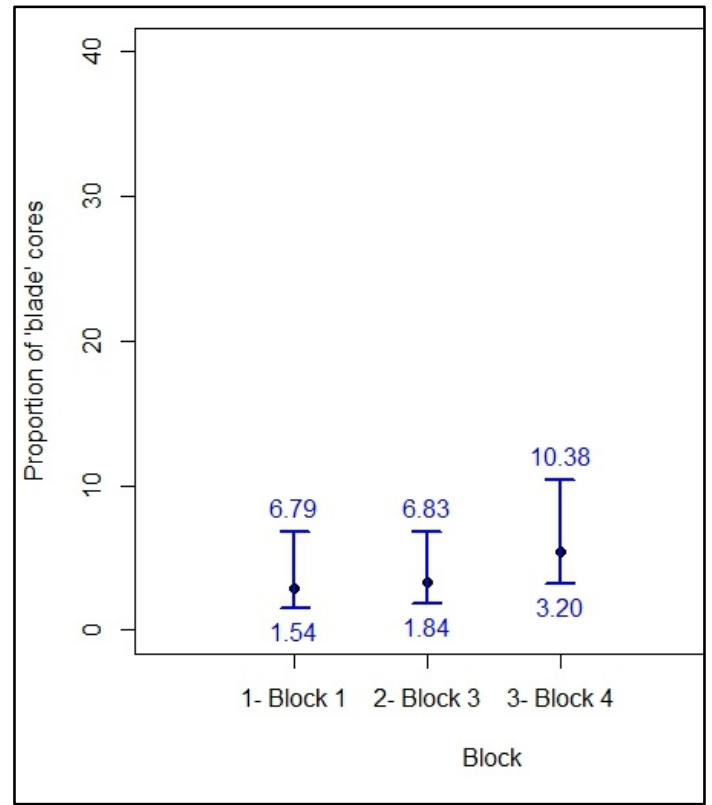

Figure 5.14. The $85 \%$ binomial confidence intervals for the proportions of medium blade cores among all medium blade debitage and tools at el-Mahasna. Note that the $85 \%$ confidence intervals are shown rather than the 95\% intervals to demonstrate that even at a smaller confidence interval there is still significant overlap. 


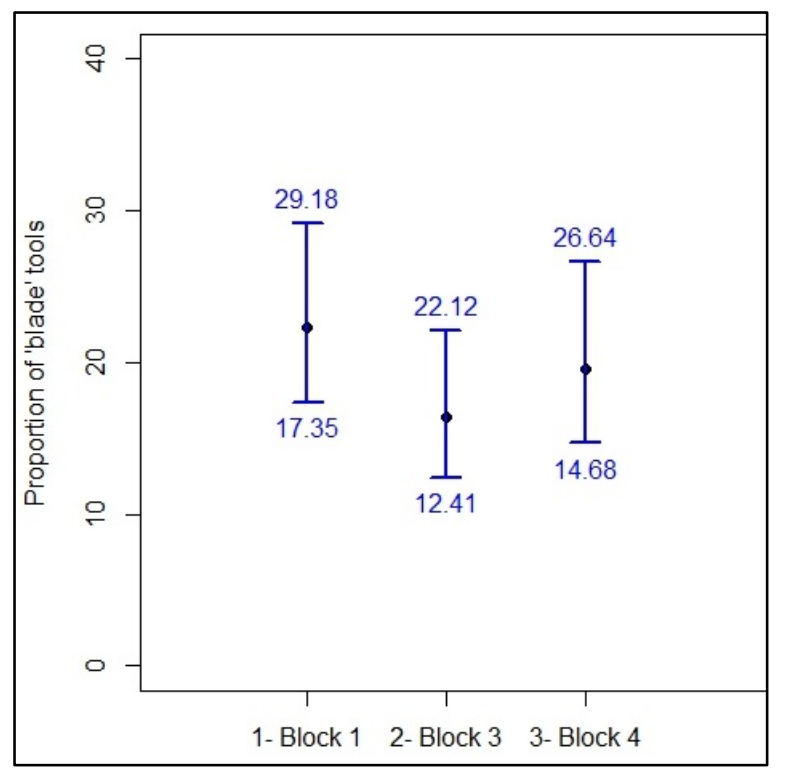

Figure 5.15 . The $85 \%$ binomial confidence intervals for the proportions of medium blade tools among all medium blade debitage and tools at el-Mahasna. Note that the $85 \%$ confidence intervals are shown rather than the $95 \%$ intervals to demonstrate that even at a smaller confidence interval there is still significant overlap.

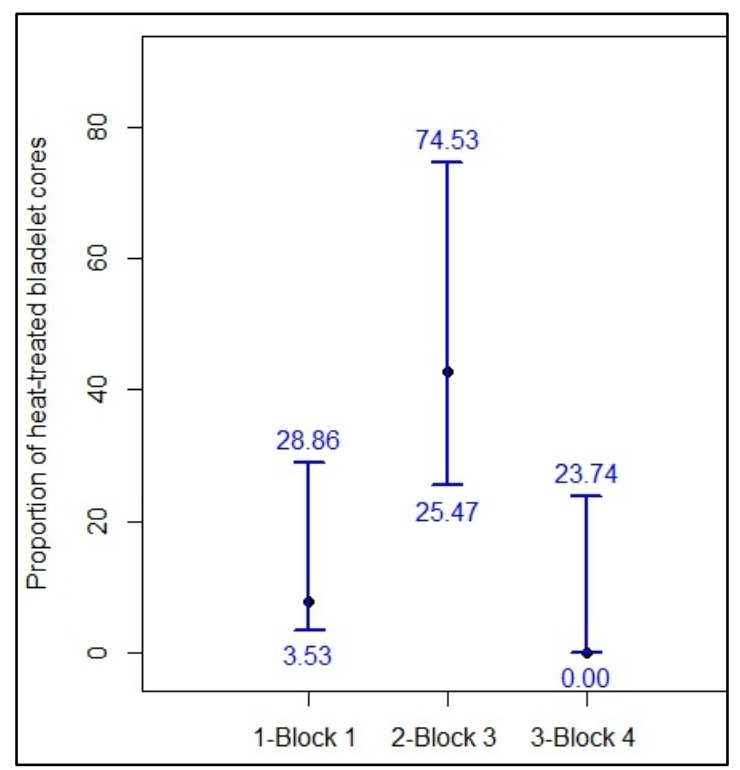

Figure 5.17. The $85 \%$ binomial confidence intervals for the proportions of heat-treated bladelet cores among all heat-treated-bladelet debitage and tools at el-Mahasna. Note that the $85 \%$ confidence intervals are shown rather than the $95 \%$ intervals because the sample size is so small. It is not until the $80 \%$ confidence level that the proportion of cores from Block 3 can be differentiated from the rest.

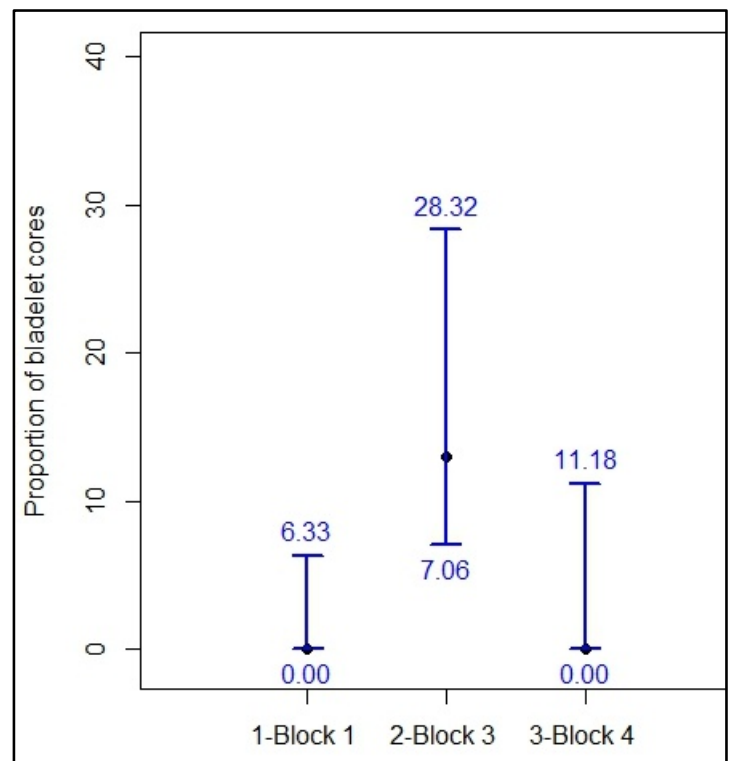

Figure 5.16. The $85 \%$ binomial confidence intervals for the proportions of non-heat-treated bladelet cores among all non-heat-treatedbladelet debitage and tools at el-Mahasna. Note that the $85 \%$ confidence intervals are shown since the sample size is so small. It is not until the $80 \%$ confidence level that the proportion of cores from Block 3 can be differentiated from the rest.

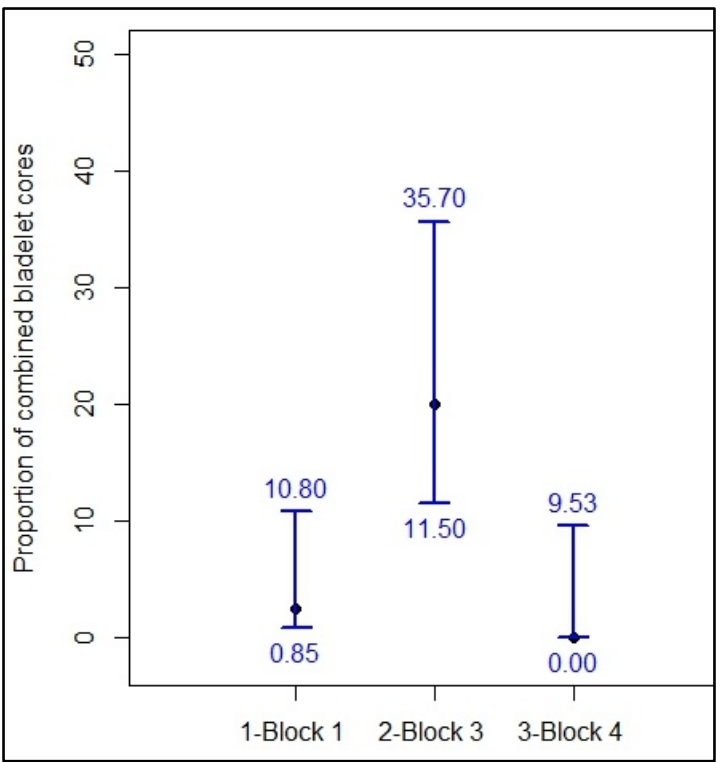

Figure 5.18. The $90 \%$ binomial confidence intervals for the proportions of heat-treated and non-heat-treated bladelet cores combined among all the heat-treated and non-heat-treated bladelet debitage and tools at el-Mahasna. 


\section{Chapter 6 Figures}

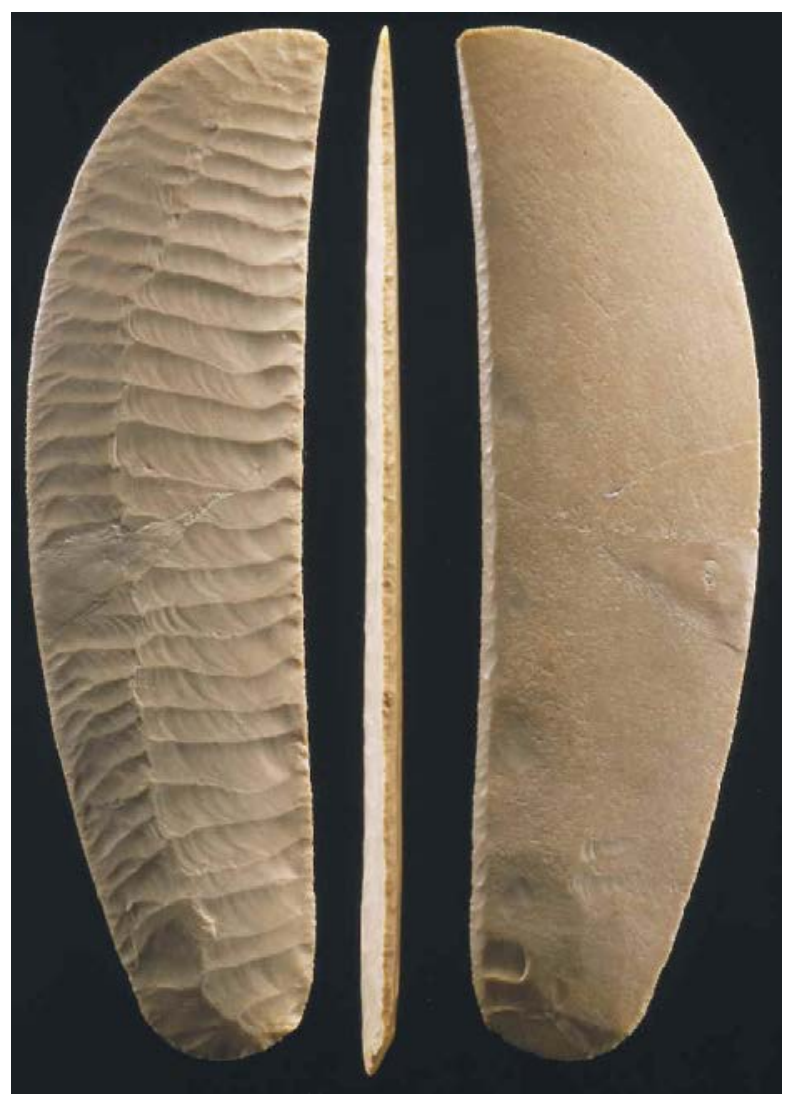

Figure 6.1. Ripple Flaked knife. Image Credit: Pete Bostrum . http://lithiccastinglab.com/gallerypages/2012septemberpredynasticknivespage1.htm

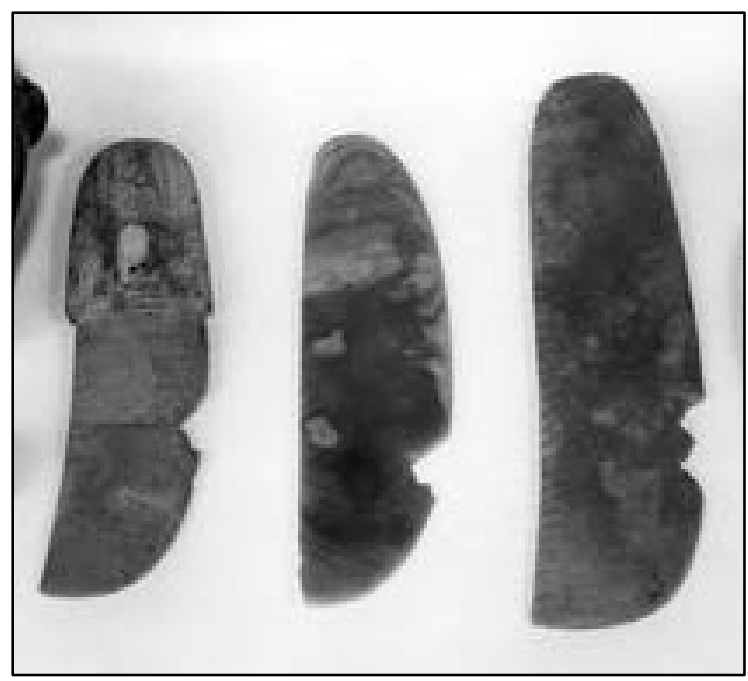

Figure 6.2. Three ripple-flaked knives from Abu Zaidan, broken in the same way. Image credit: Brooklyn Museum, Charles Edwin Wilbour Fund, 09.889.118. CC-BY 3.0.

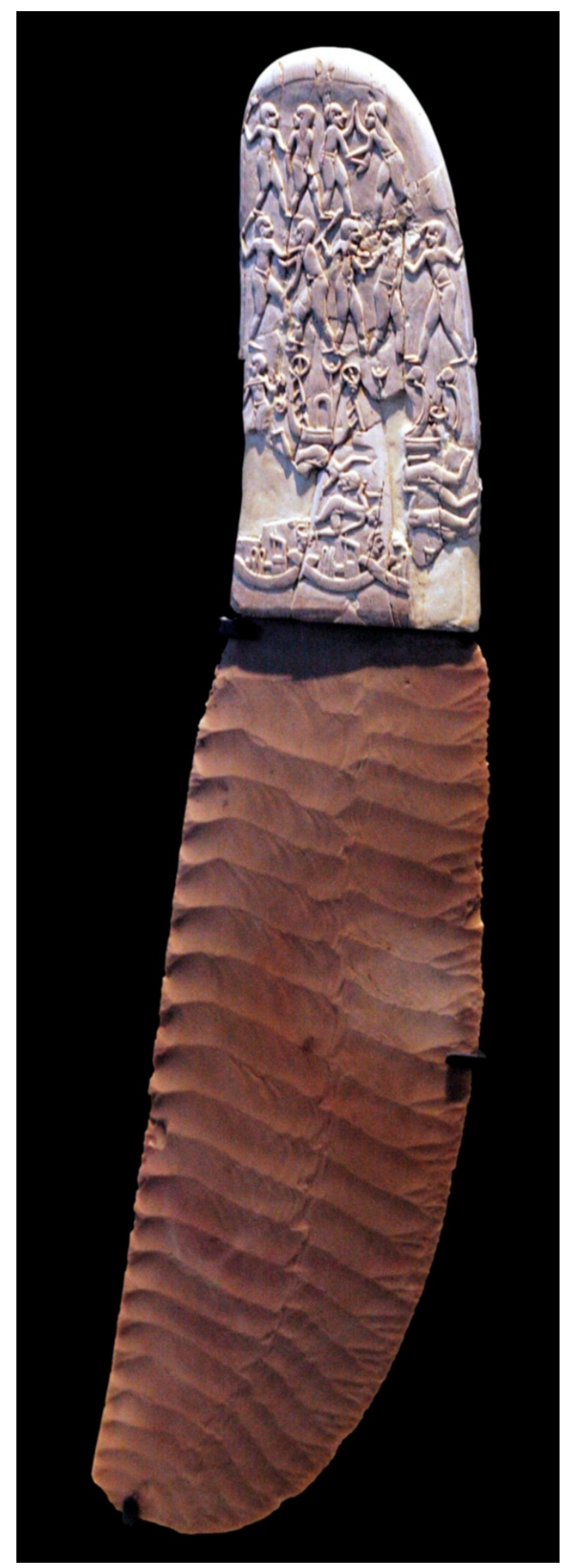

Figure 6.3. Ripple-flaked knife with decorated ivory handle from Gebel Arak . Image credit: User:Rama/ Wikimedia/ CC-BY-SA- 2.0 FR 


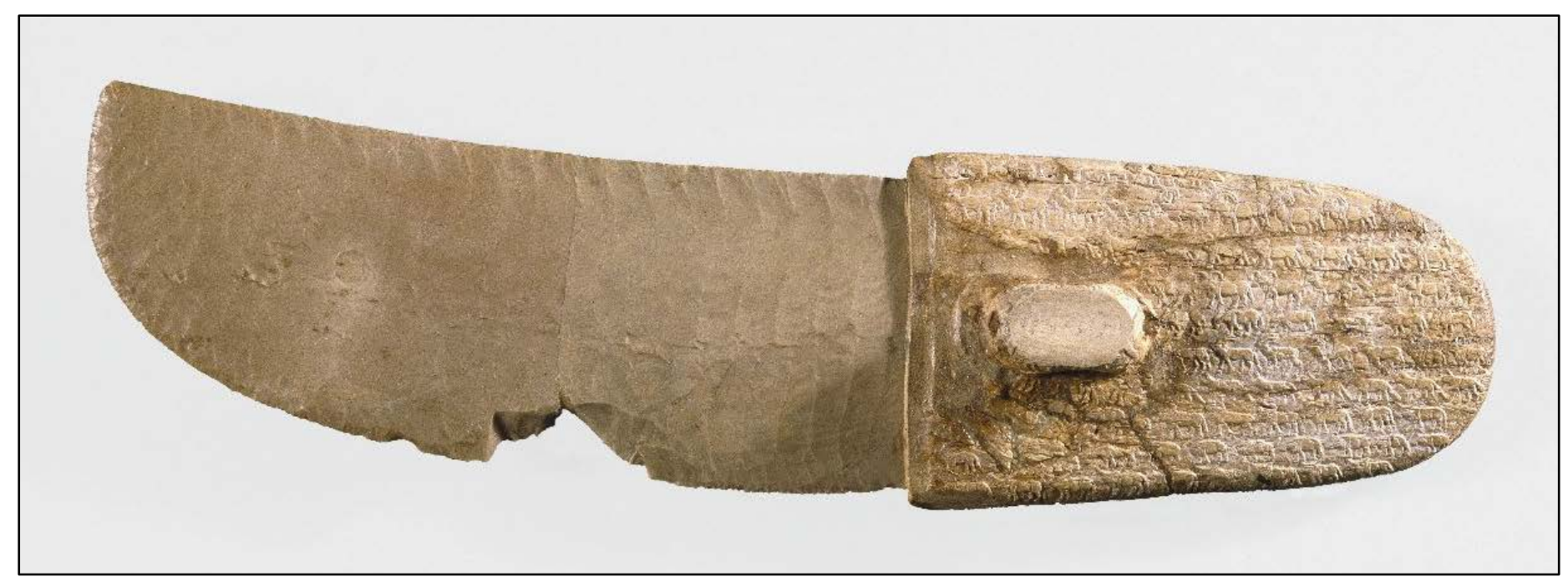

Figure 6.4. Ripple-flaked knife and handle from Abu Zaidan. Image credit: Brooklyn Museum, Charles Edwin Wilbour Fund, 09.889.118. CC-BY 3.0.

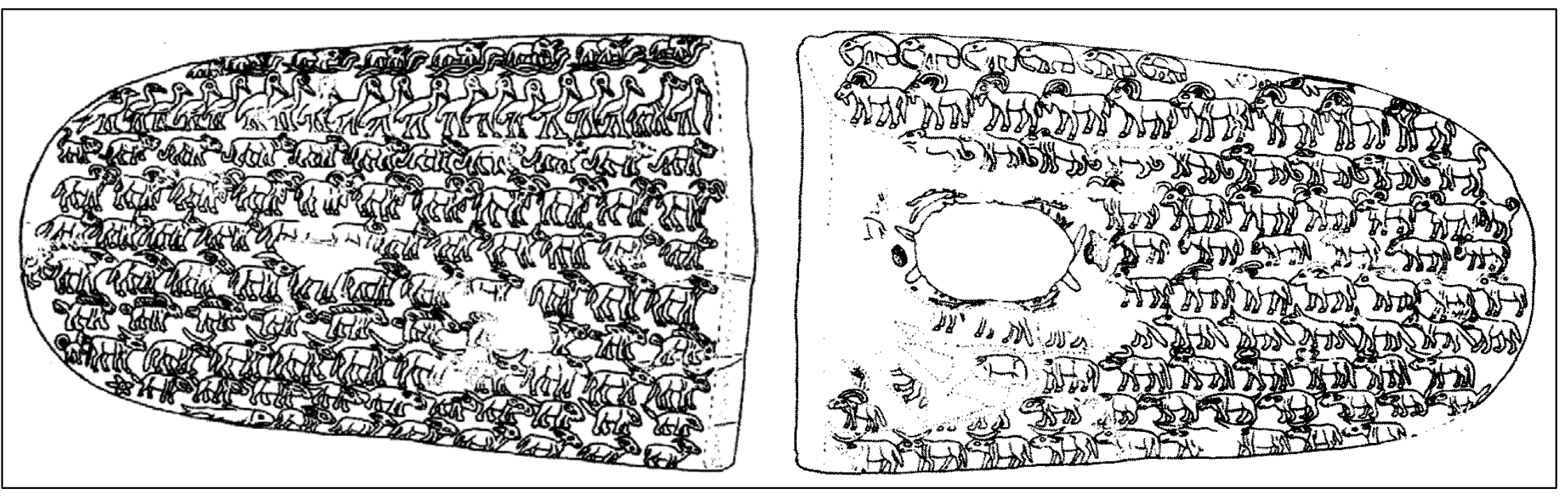

Figure 6.5. Drawing of the Abu Zaidan knife handle. Image credit: Brooklyn Museum, Charles Edwin Wilbour Fund, 09.889.118. CC-BY 3.0. 


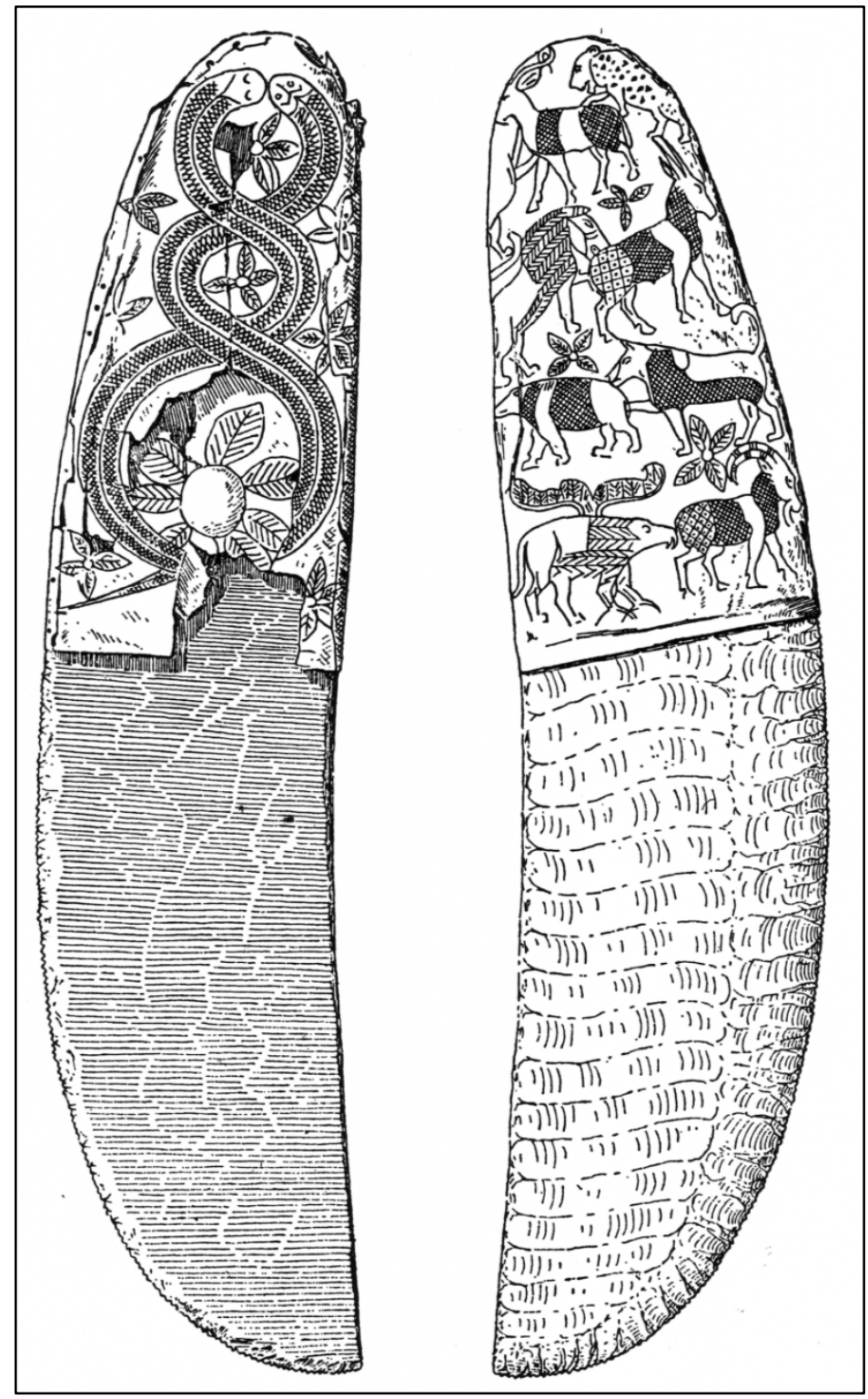

Figure 6. 6. Drawing of the Gebel Tarif ripple-flaked knife with gold embossed handle from Quibell (1905). Note that the knife face in the handle has been flipped since the drawing was made.

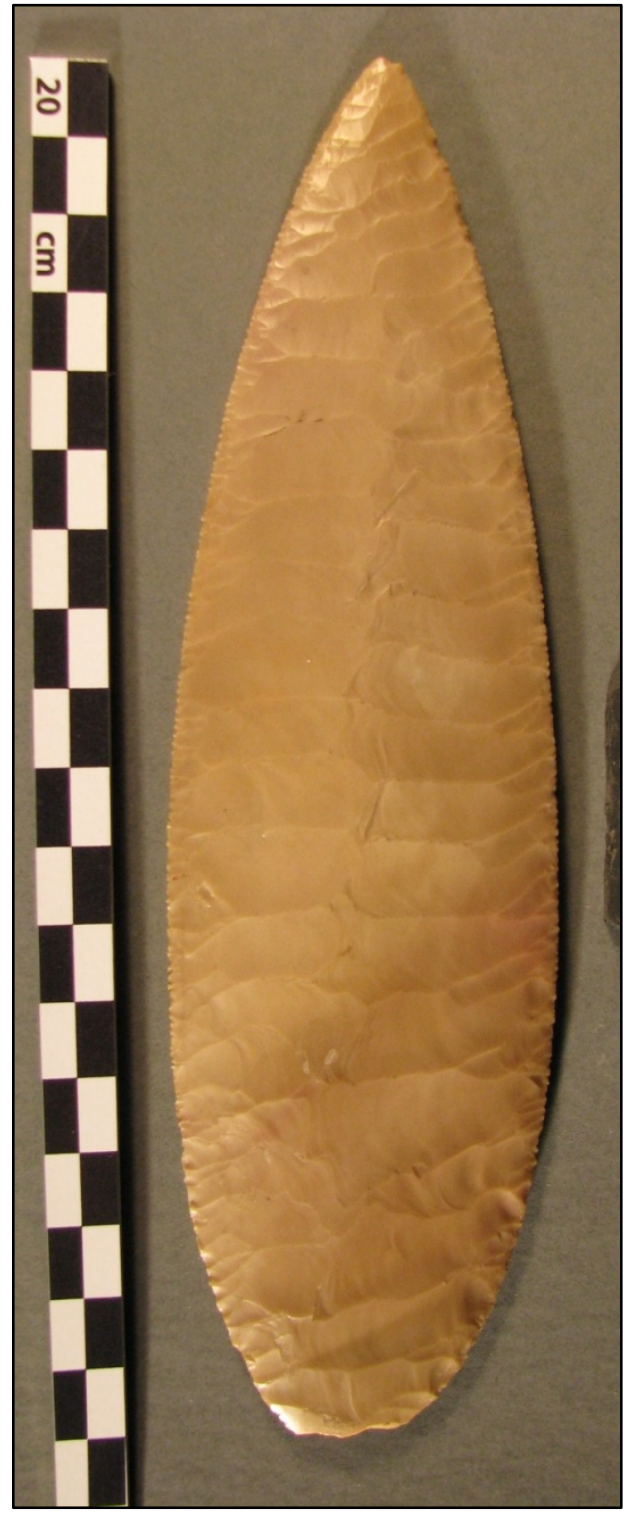

Figure 6.7. Dagger/lance with Ripple flaking that is not a ripple-flaked knife. Image credit: University of Pennsylvania Museum E.1114. 


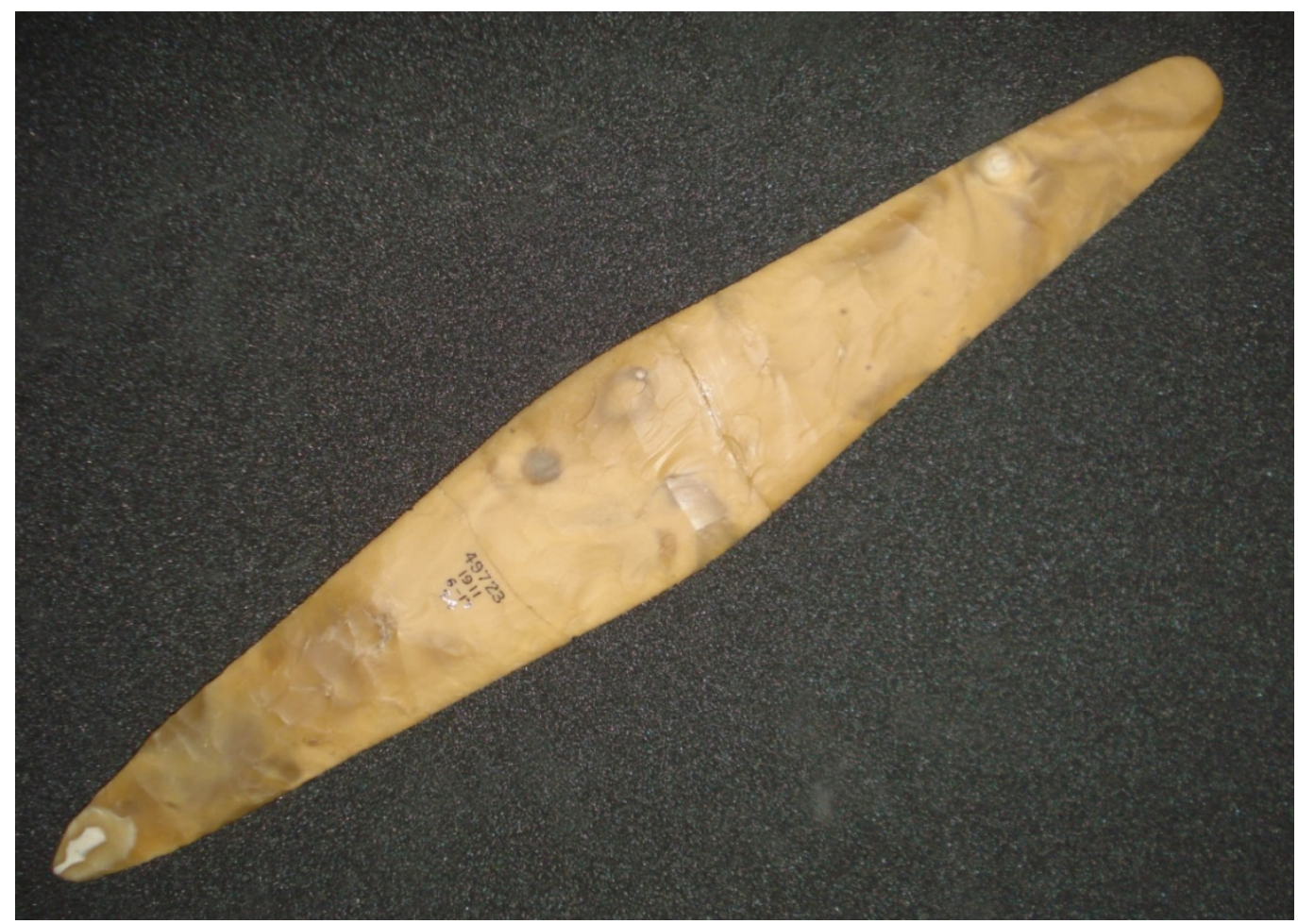

Figure 6.8. Rhomboid tool. BM.EA.49723. Image credit: (c) Trustees of the British Museum. CC BY-NC-SA 4.0.

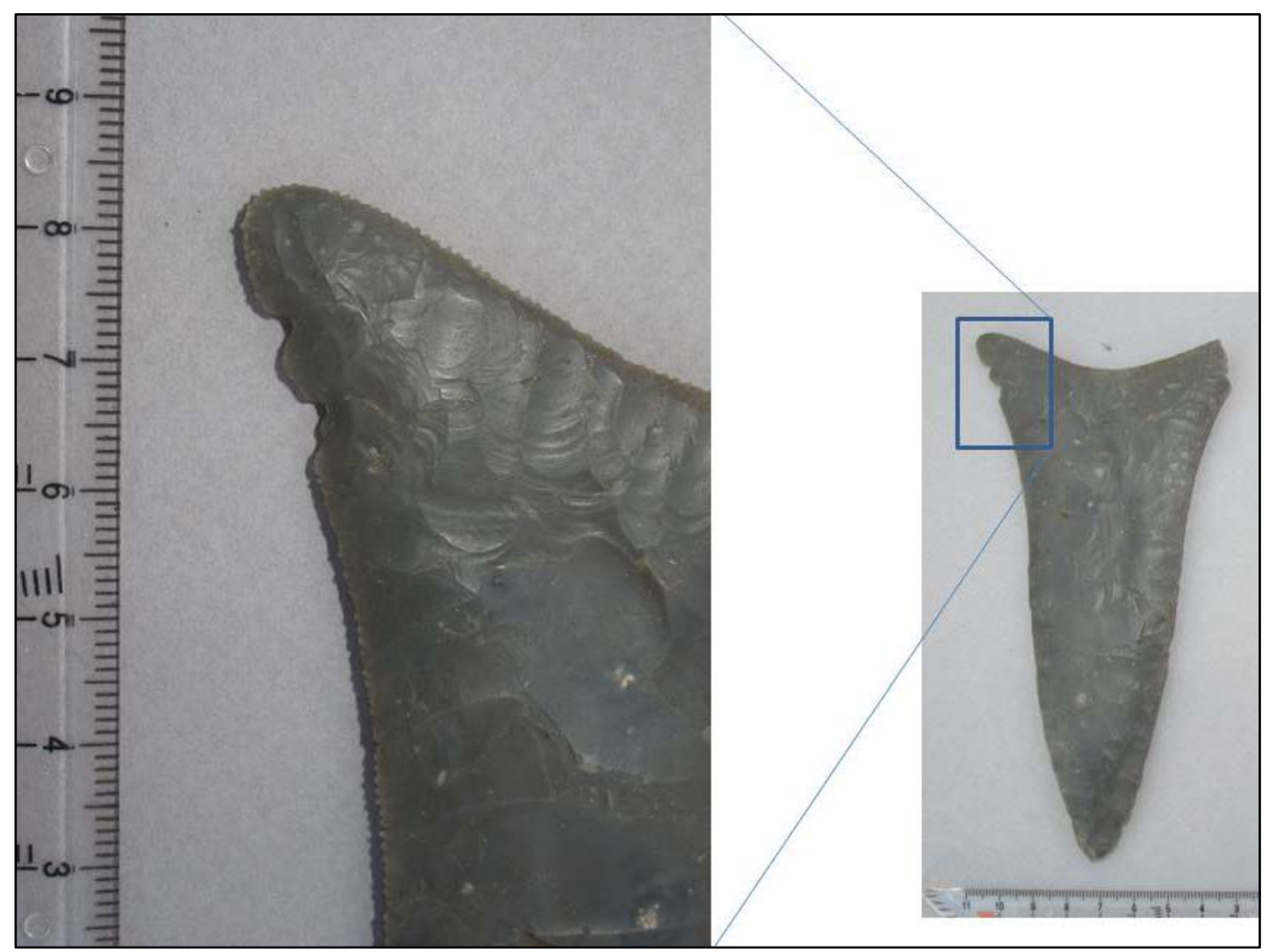

Figure 6.9. Detail of a fishtail knife showing microserrations and notches. Abydos cemetery $\mathrm{U}$, tomb 178. Image taken with permission of the German Archaeological Mission to Abydos. 


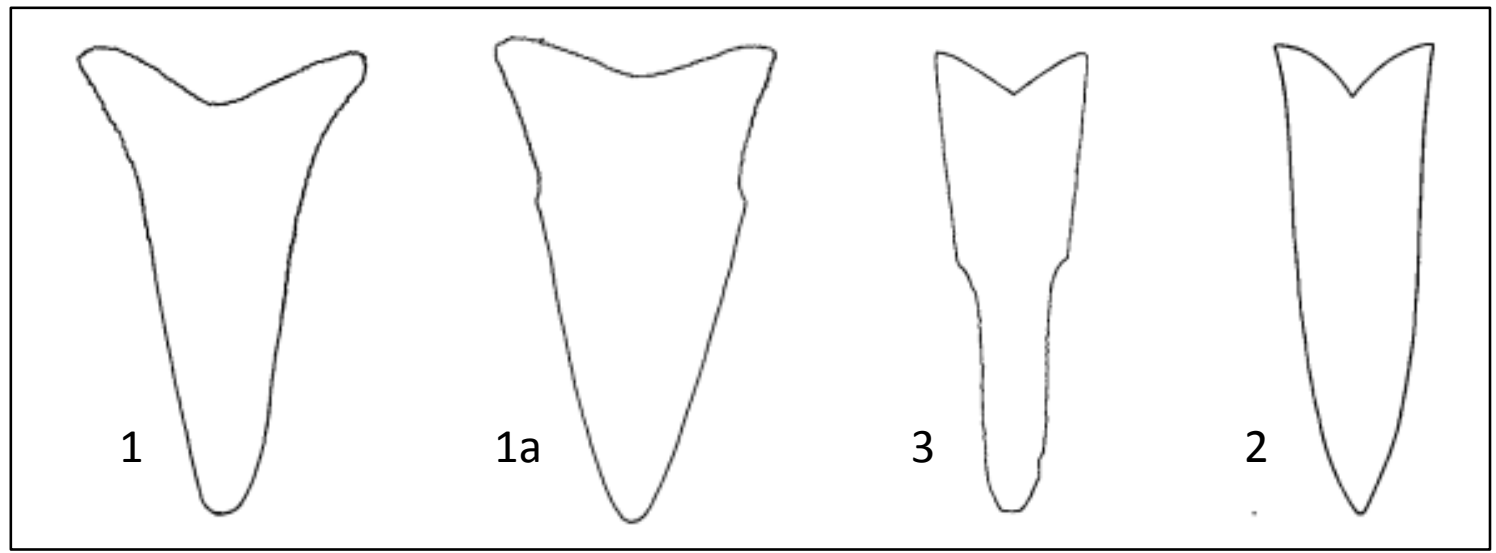

Figure 6.10. Fishtail shapes (from Massoulard 1936; van Walsem1978:237-242). The shape of the fishtails varied over time. See Ch 7.1.

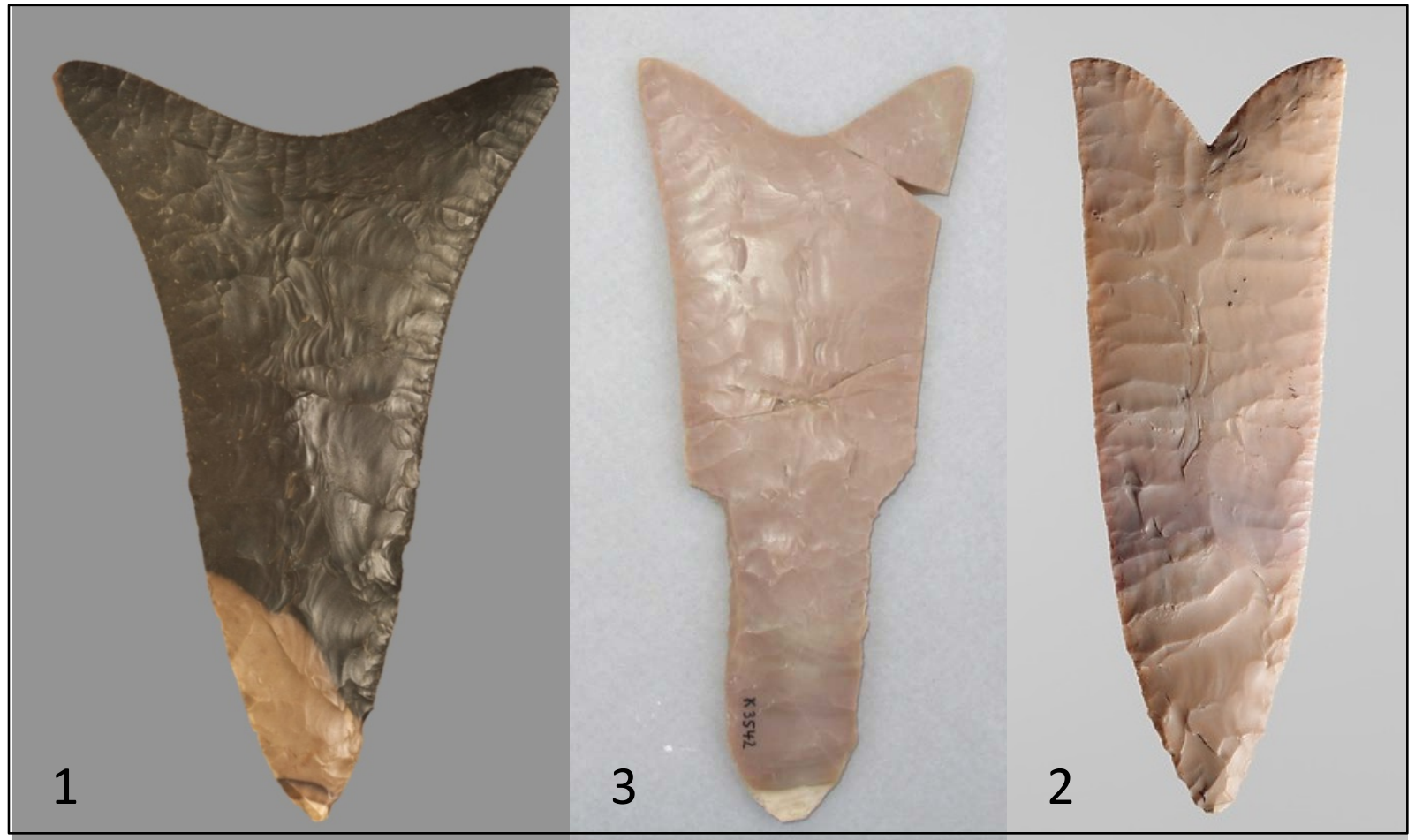

Figure 6.11. Three stylistic shapes of fishtails (Massoulard 1936; van Walsem 1978). Images not to scale. Image 1 credit: Egypt, Predynastic Period, Naqada la-lla periods. Dark brown- to dark green-colored flint; overall: $10.00 \mathrm{~cm}$ (3 7/8 inches). The Cleveland Museum of Art, Gift of the John Huntington Art and Polytechnic Trust 1914.673 Image 3 credit: Abydos U tomb 395. Image taken with permission of the German Archaeological Mission to Abydos. Image 2 credit: MMA.16.2.4 The Metropolitan Museum of Art, Rogers Fund, 1916. www.metmuseum.org. 


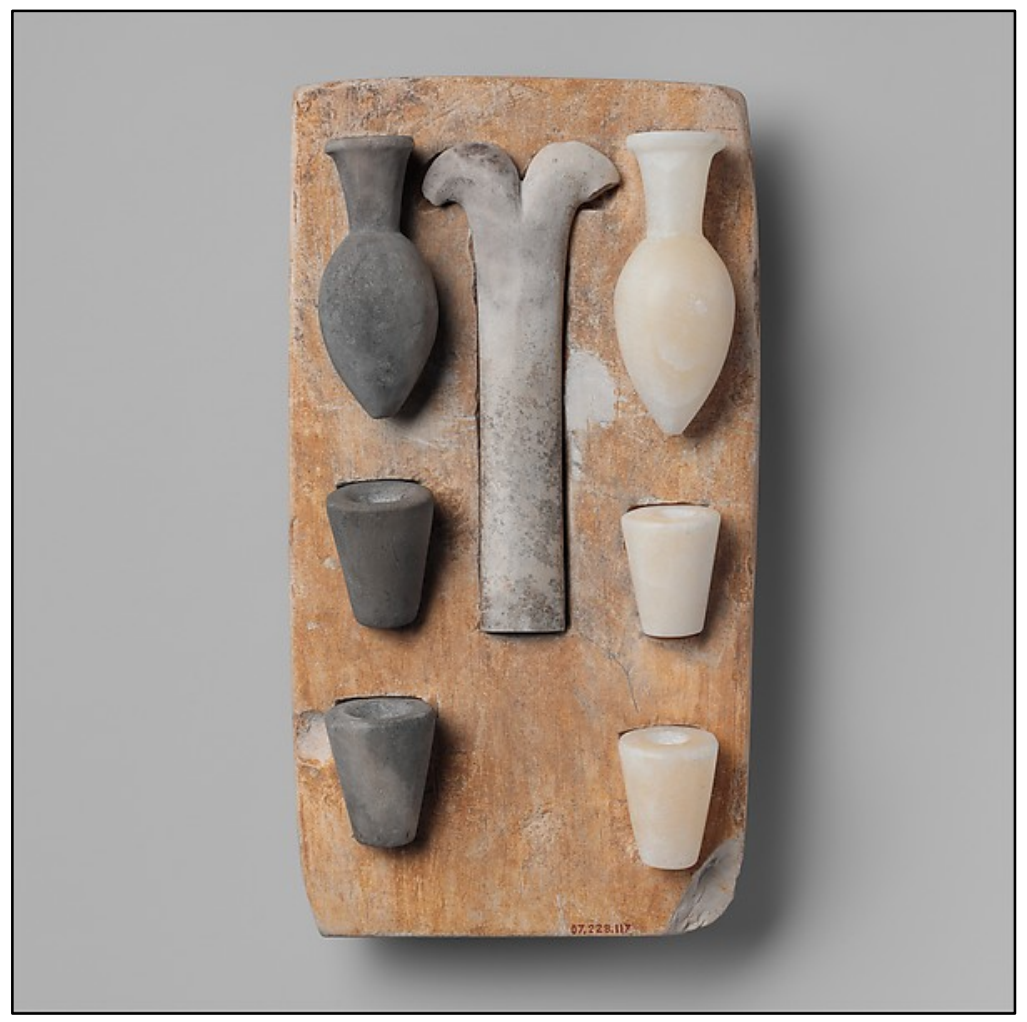

Figure 6.12. A Psš-kf set for use in the opening of the mouth reanimation ceremony. Image credit: The Metropolitan Museum of Art, Rogers Fund 1907. www.metmuseum.org

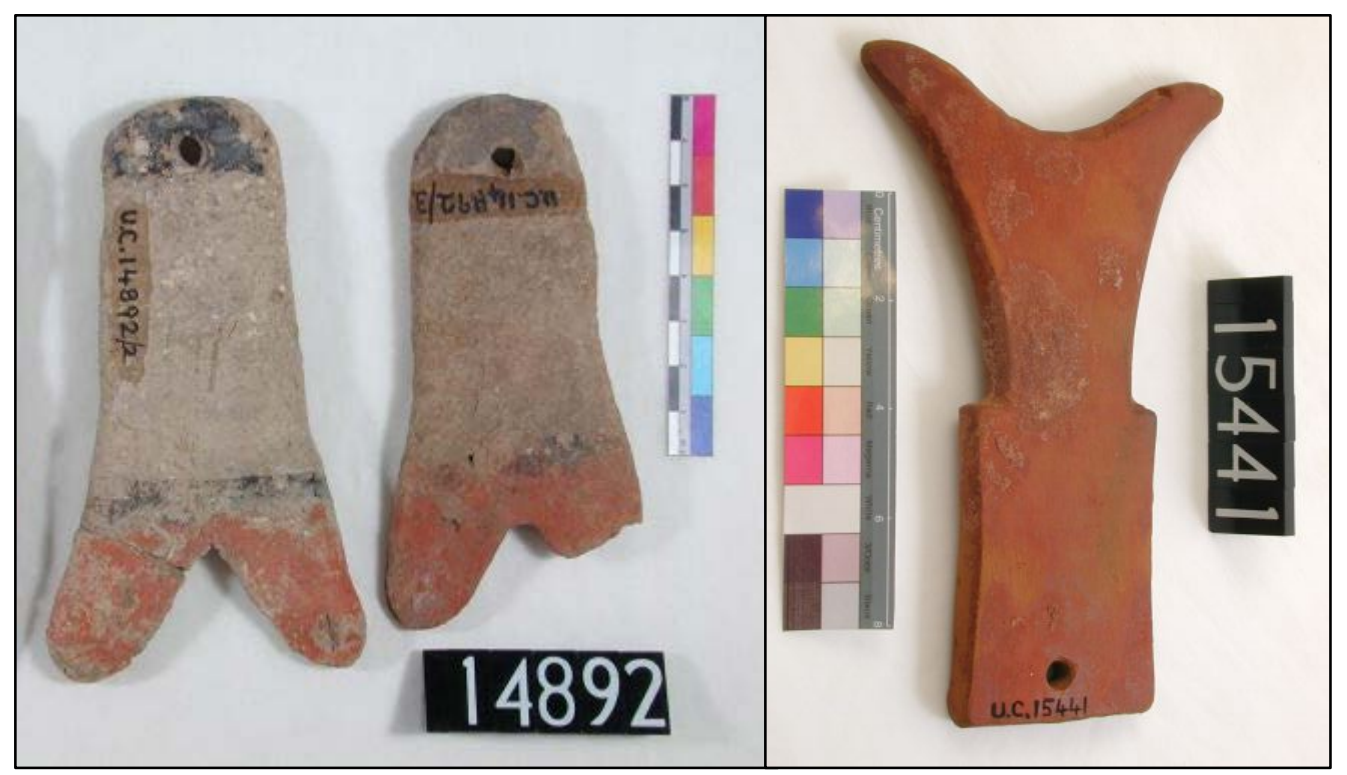

Figure 6.13. Model fishtails with hafts. Image credits: The Petrie Museum of Egyptian Archaeology UCL. CC BT-NC-SA 3.0. 


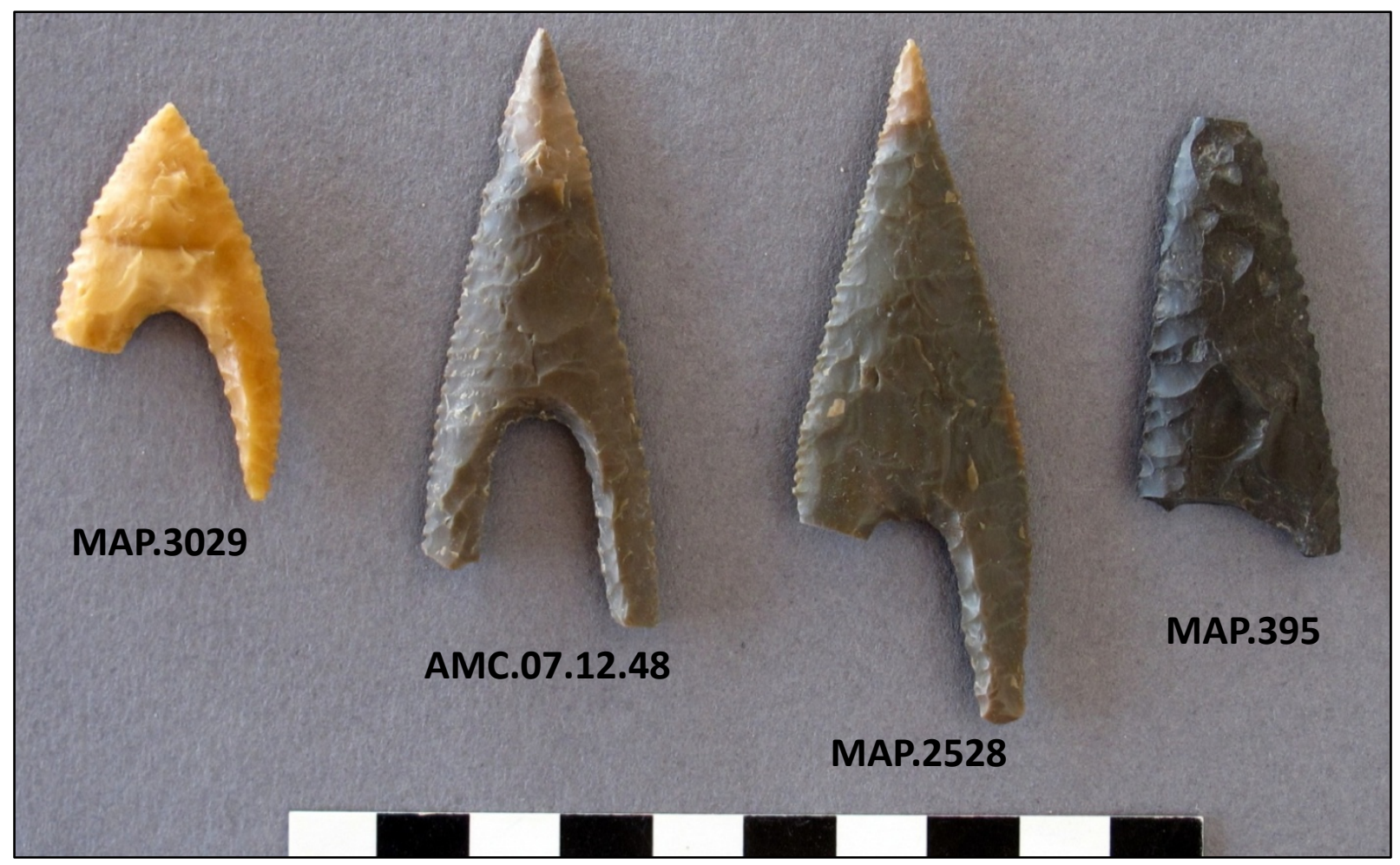

Figure 6.14. Concave-base projectile points from el-Mahasna and the Abydos Middle Cemetery.

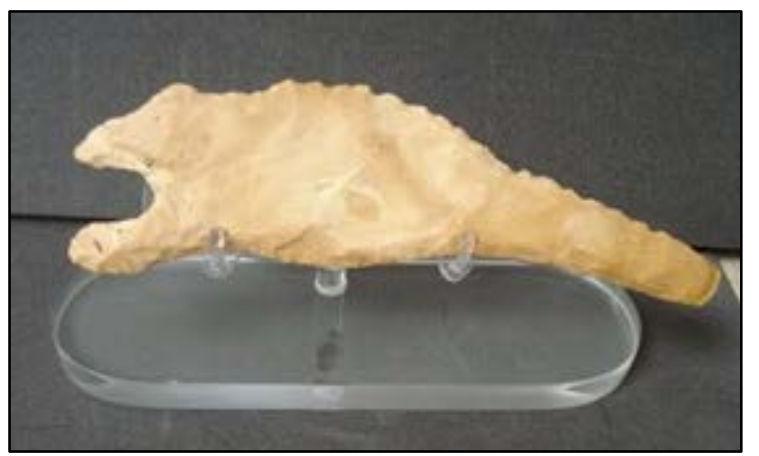

Figure 6.15. Crocodile eccentric BM.EA.37269. Image credit: (C) Trustees of the British Museum. CC BY-NC-SA 4.0

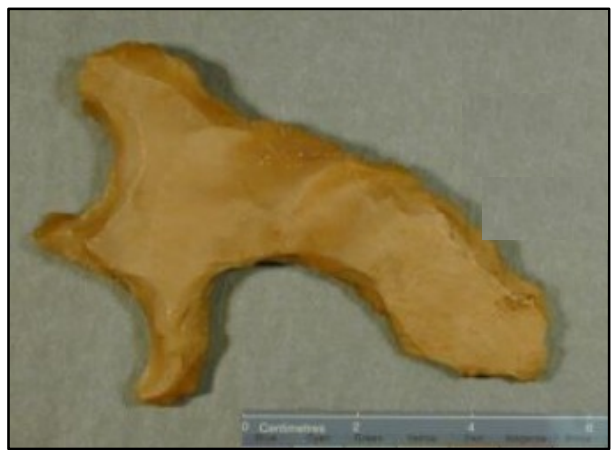

Figure 6.16. Crocodile(?) eccentric UC.42705a Image credit: The Petrie Museum of Egyptian Archaeology UCL. CC BT-NC-SA 3.0.

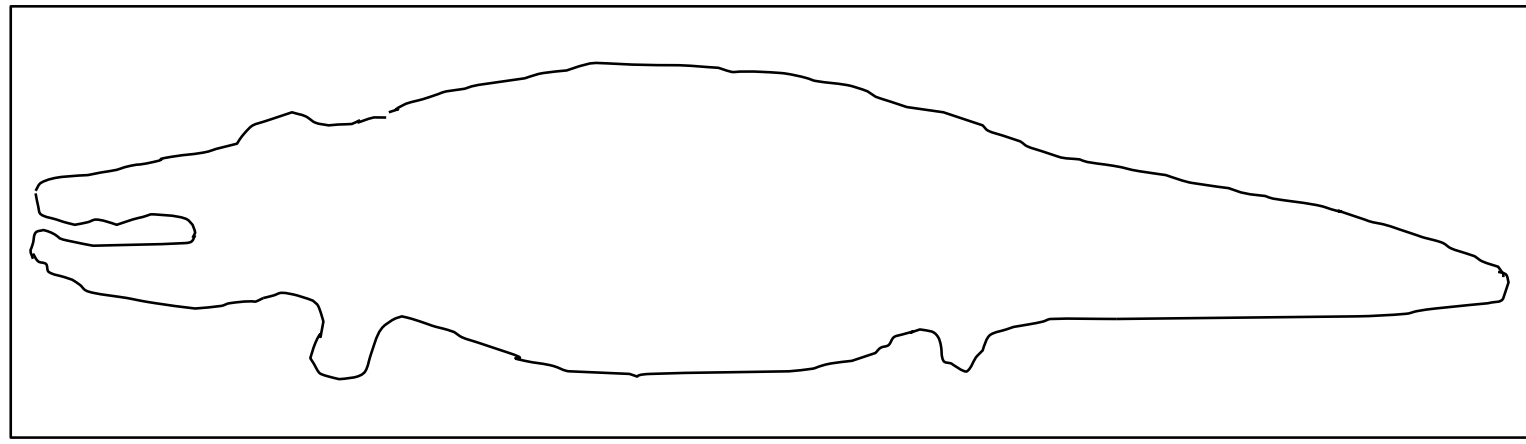

Figure 6.17. Approximate outline of the crocodile eccentric in Cahn 2005 (no.4), drawn from photo. Not intended to be a technical drawing, only to convey the basic shape. 

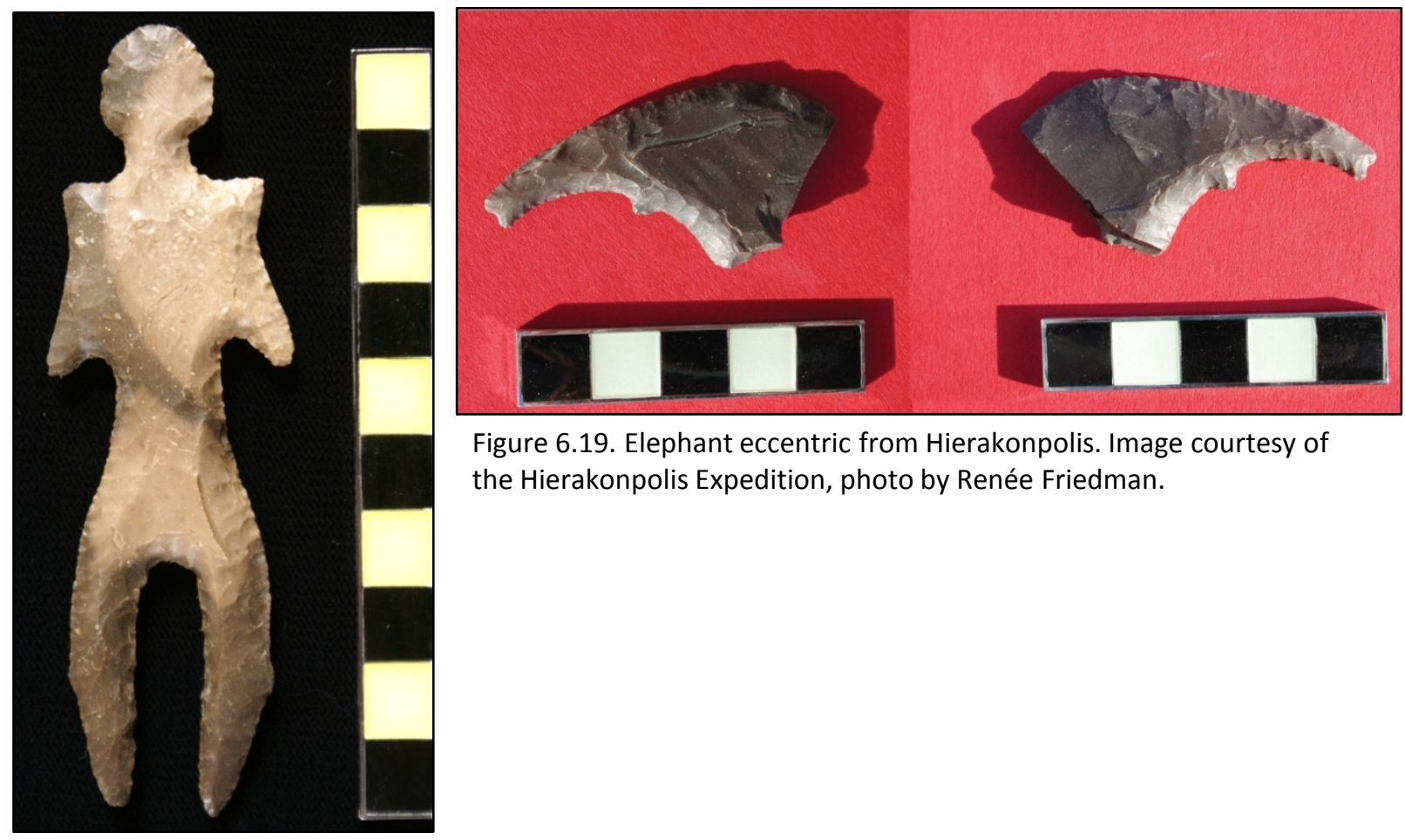

Figure 6.19. Elephant eccentric from Hierakonpolis. Image courtesy of the Hierakonpolis Expedition, photo by Renée Friedman.

Figure 6.18. Figural eccentric showing a human, possibly a dwarf. Image courtesy of the Hierakonpolis expedition, photo by Renée Friedman.

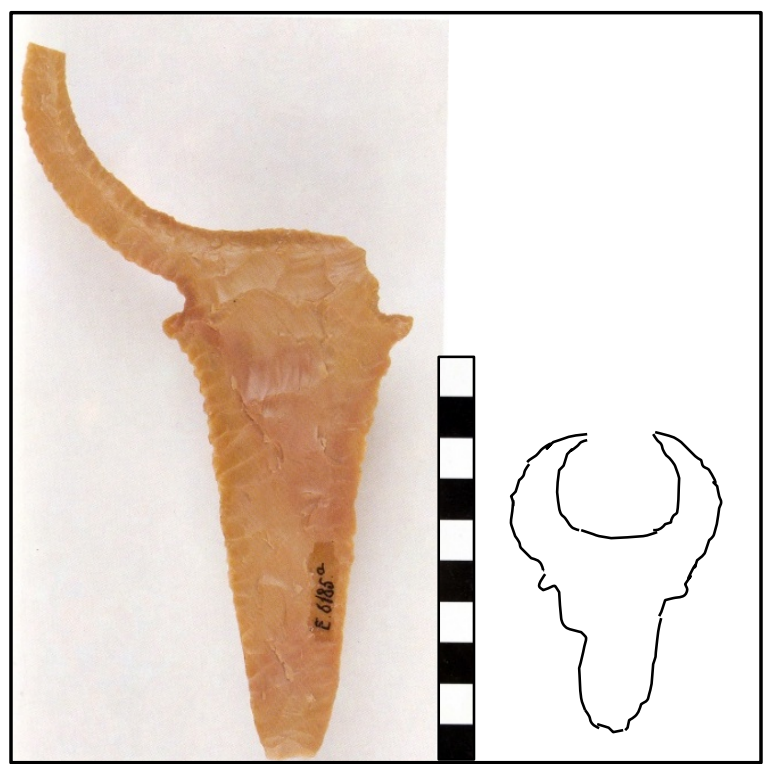

Figure 6.20. Bull head figural eccentrics. Left RMAH.E.6185a. Image credit: Royal Museums of Art and History, Brussels. Right: BM.EA.32124. Right: BM.EA.32124. drawn from personal photo. Not intended to be a technical drawing, only to convey the basic shape. Images approximately to scale.

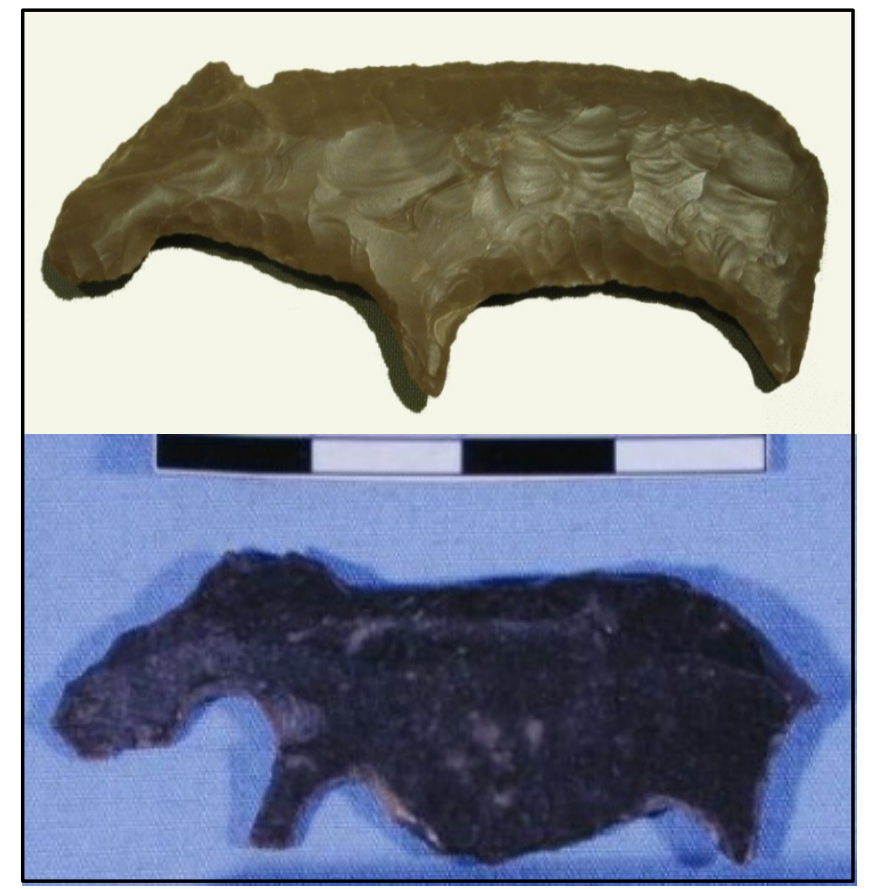

Figure 6.21. Hippopotamus eccentrics (images not to scale). Top: Hierakonpolis, image courtesy of the Hierakonpolis Expedition, photo by Renée Friedman. Middle: UC.16780. The Petrie Museum of Egyptian Archaeology UCL. CC BT-NC-SA 3.0. 


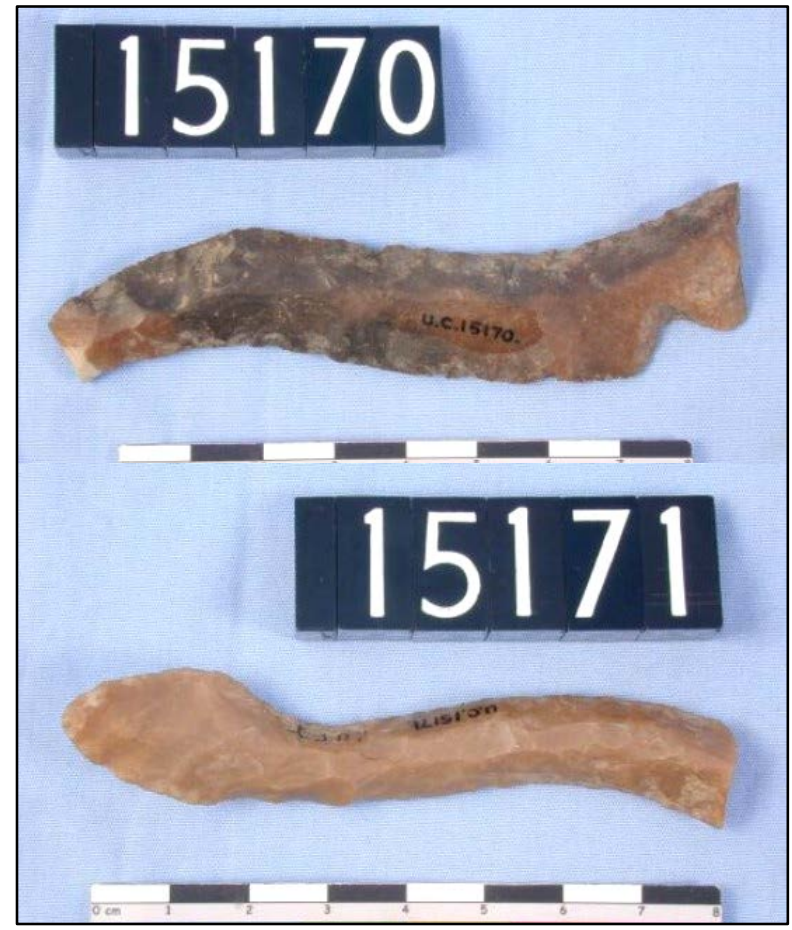

Figure 6.22. Snake figural eccentrics, UC15170 \& 15171. Image credit: The Petrie Museum of Egyptian Archaeology UCL. CC BT-NC-SA 3.0.

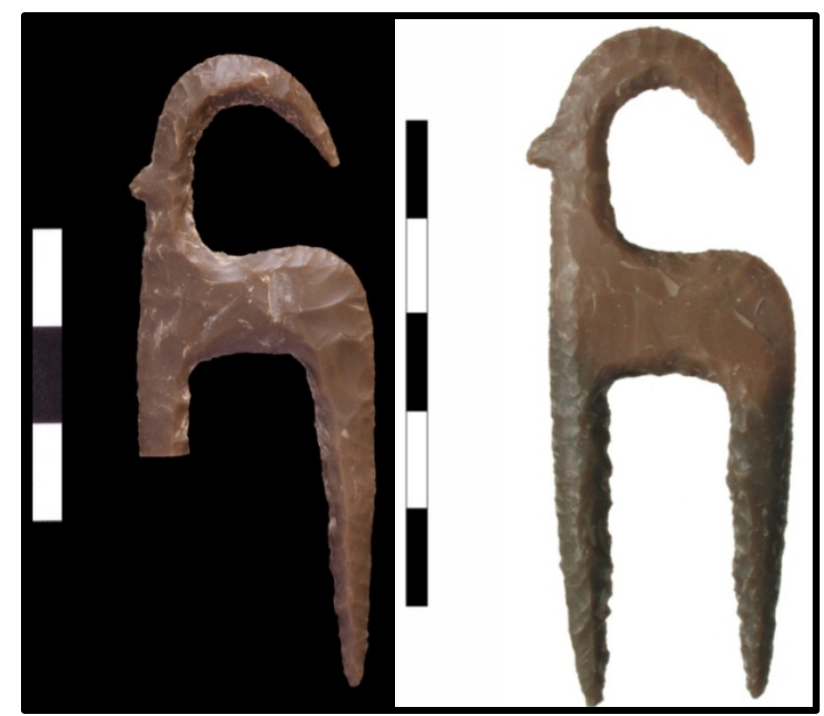

Figure 6.23. Ibex eccentrics from Hierakonpolis. Images courtesy of the Hierakonpolis expedition, left photo by James Rossiter, right photo by Laurent Bavay.

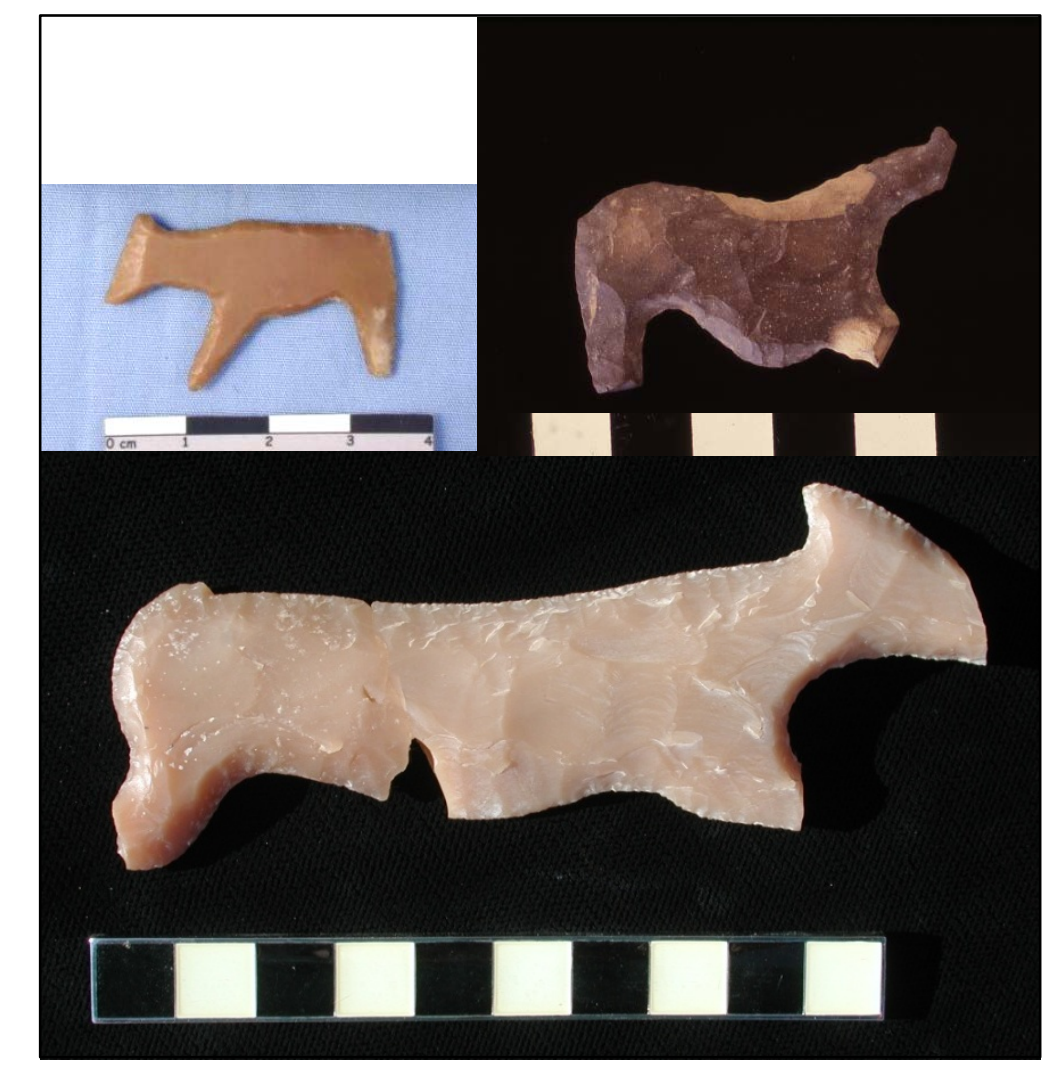

Figure 6.24. Dog and quadruped eccentrics from Hierakonpolis. Upper left: UC.1567 Image credit: The Petrie Museum of Egyptian Archaeology UCL. CC BT-NC-SA 3.0. Upper right and lower: Images courtesy of the Hierakonpolis expedition, photos by Renée Friedman. 


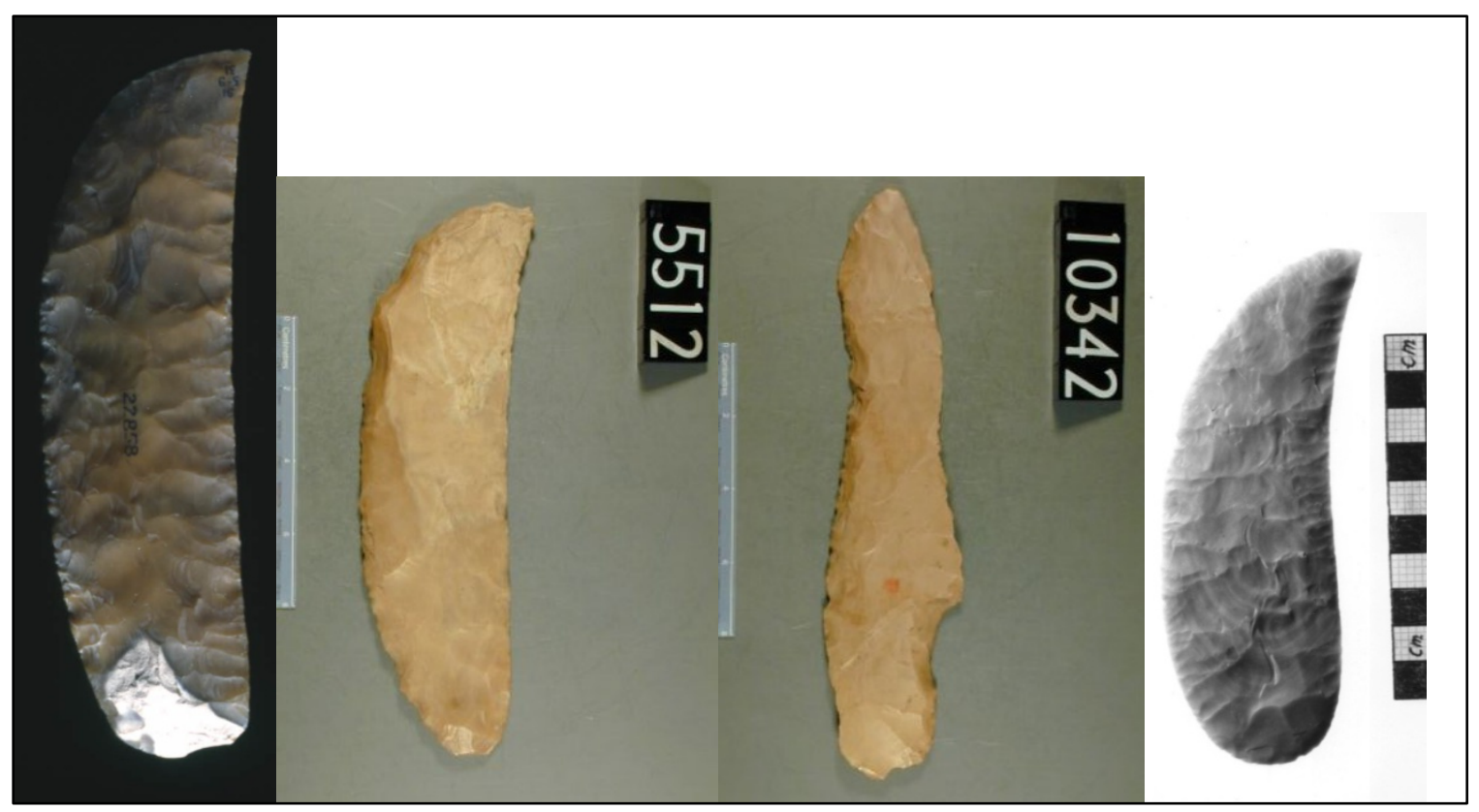

Figure 6.25. Bifacial knives. Images approximately to scale. From left: BM.22858, Image credit: (C) Trustees of the British Museum. CC BY-NC-SA 4.0; UC.5512 \& UC.10342, Image credits: The Petrie Museum of Egyptian Archaeology UCL. CC BT-NC-SA 3.0; BrM.09.889.122, Image Credit: Brooklyn Museum, Charles Edwin Wilbour Fund, CC-BY 3.0.

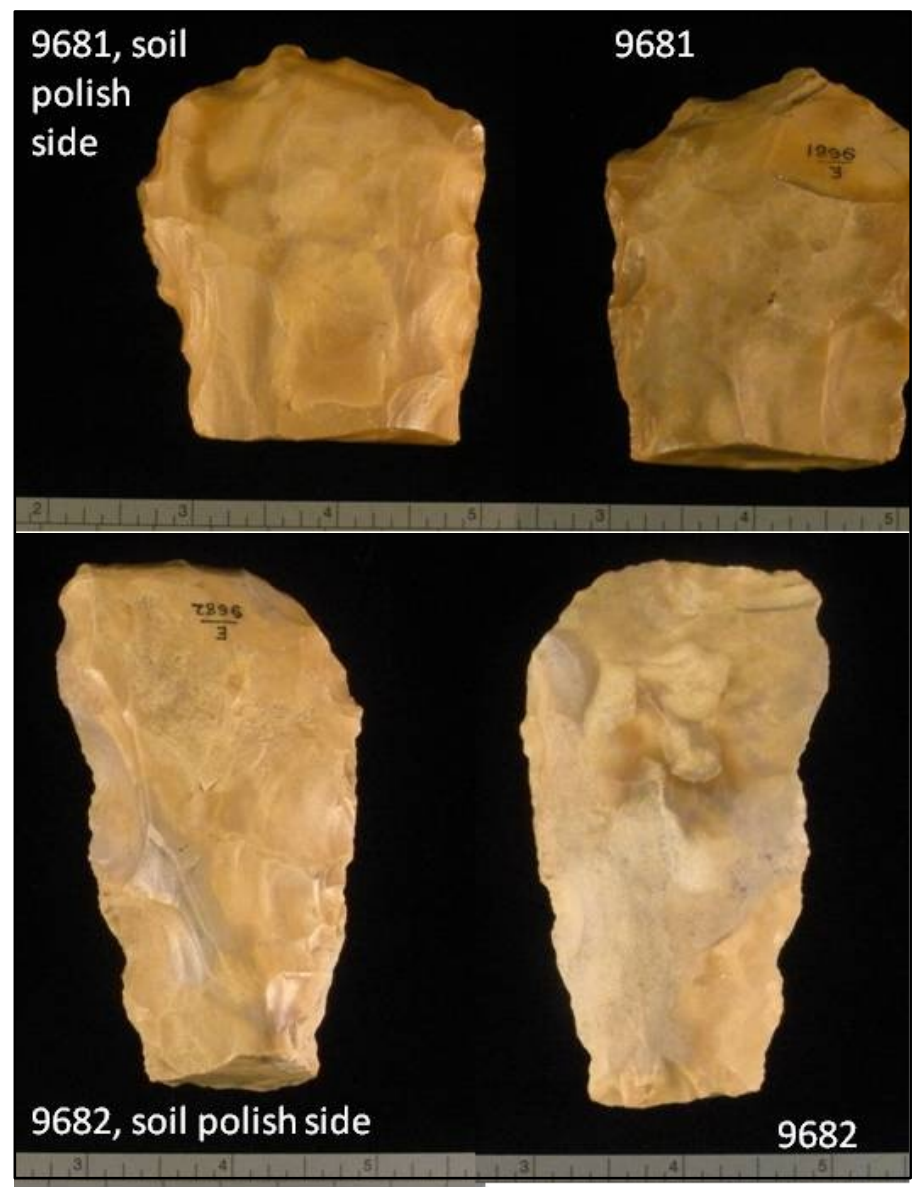

Figure 6.26. Axes with soil polish. University of Pennsylvania Museum (E.9681, E.9682). 


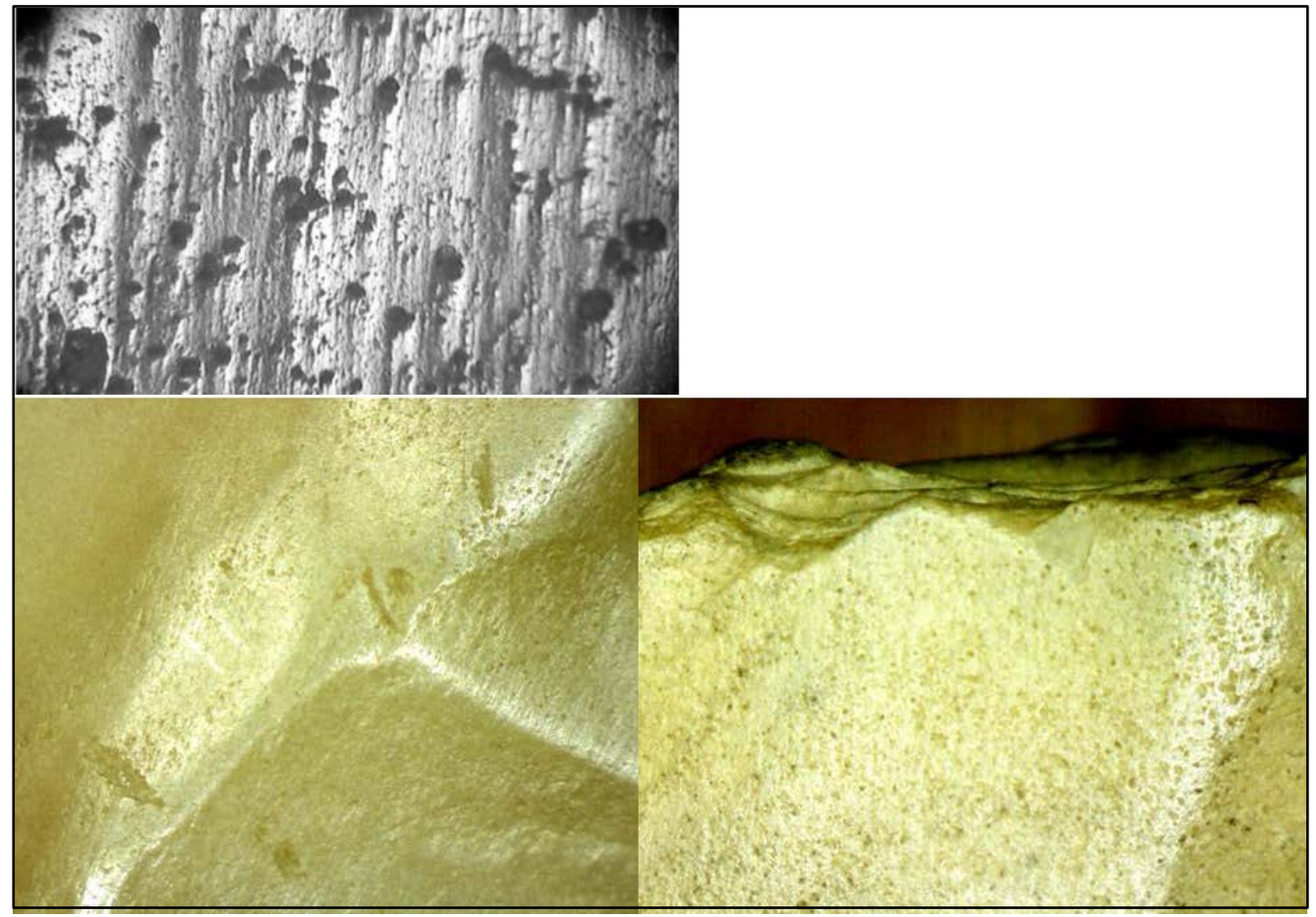

Figure 6.27 Axes with soil polish. Upper: From Yerkes et al. (2003:1056, fig 4). Width of photomicrograph is $1.3 \mathrm{~mm}$. Bottom: el-Mahâsna artifacts from the University of Pennsylvania Museum, Left E.9681, Right E.9682, both photographs taken at 30x magnification. Note that the upper photograph has a higher magnification.

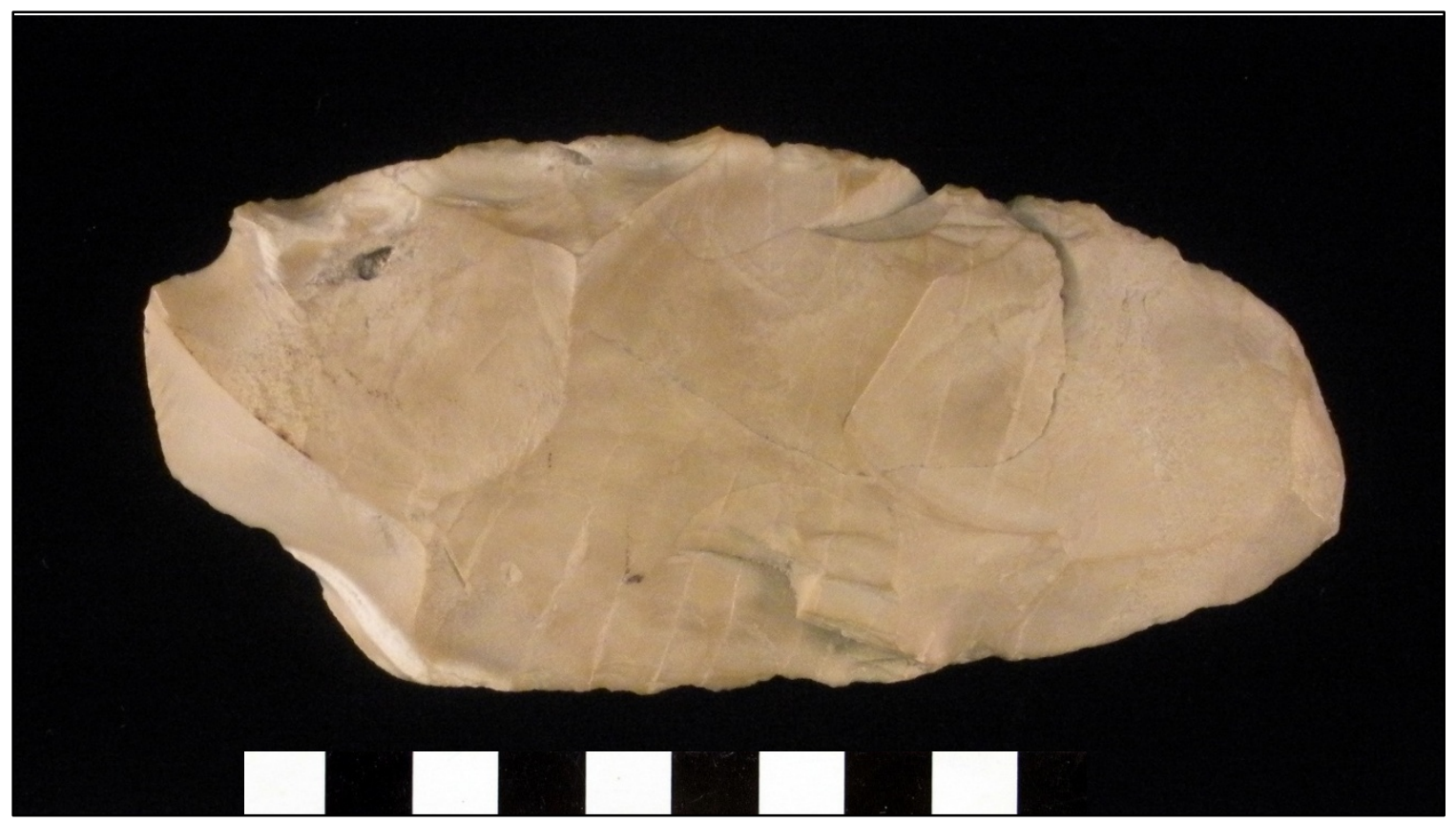

Figure 6.28. Bifacial tool preform from the the ' $\mathrm{M} 2$ ' (S2) portion of the el-Mahasna settlement. A flake has been initiated but did not fully detach, showing that the tool was unfinished. University of Pennsylvania Museum E.9632. 


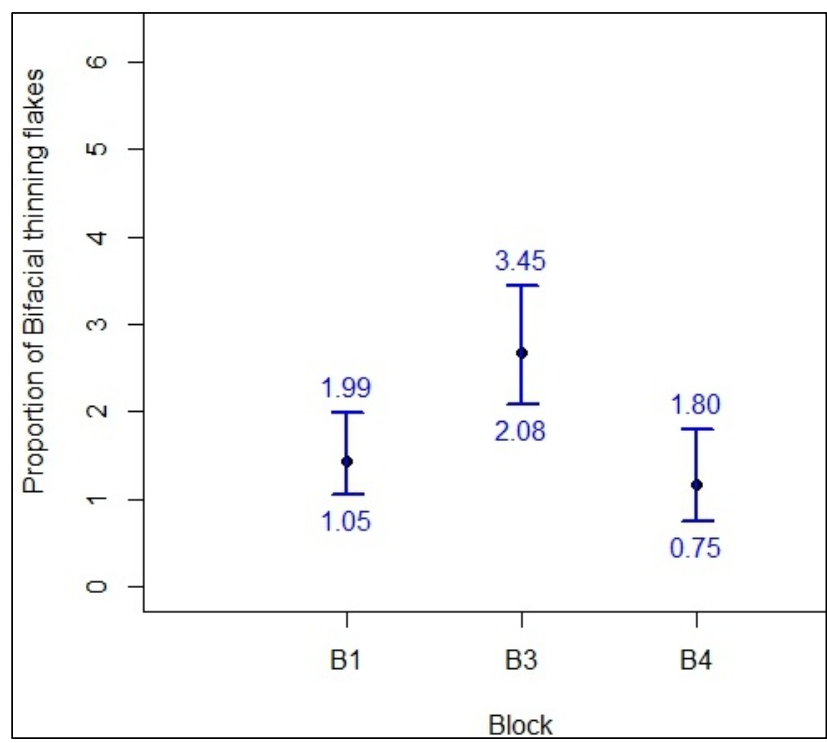

Figure 6.29. Percentages of bifacial thinning flakes in different blocks at el-Mahâsna, with the 95\% upper and lower binomial confidence intervals, showing that the higher amount in Block 3 is differentiable from the other blocks. See also Table 6.30.

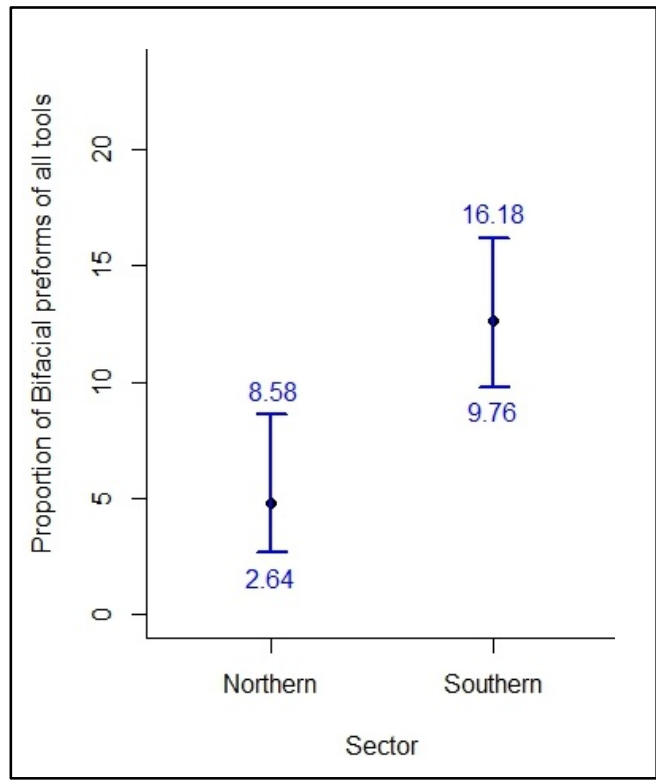

Figure 6.31. 95\% binomial confidence intervals for the proportions of bifacial preforms in two sectors of Armant MA 21/83. Bifacial preforms constituted $4.76 \%$ of the tools in the northern sector, and $12.6 \%$ in the southern sector.

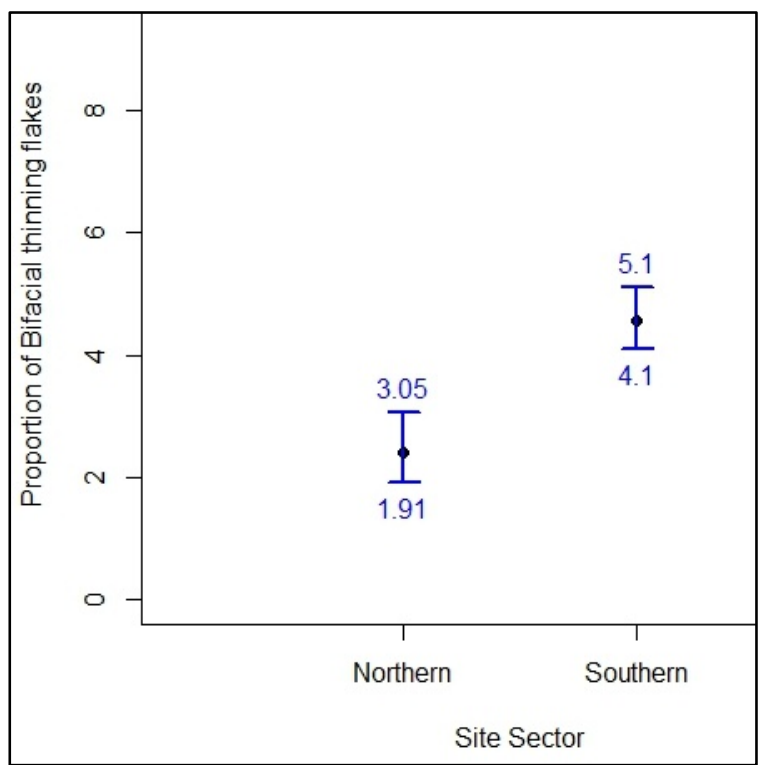

Figure 6.30. Percentages of bifacial thinning flakes in different parts of Armant MA21/83, with the 95\% upper and lower binomial confidence intervals, showing a clearly higher amount in the southern part of the site.

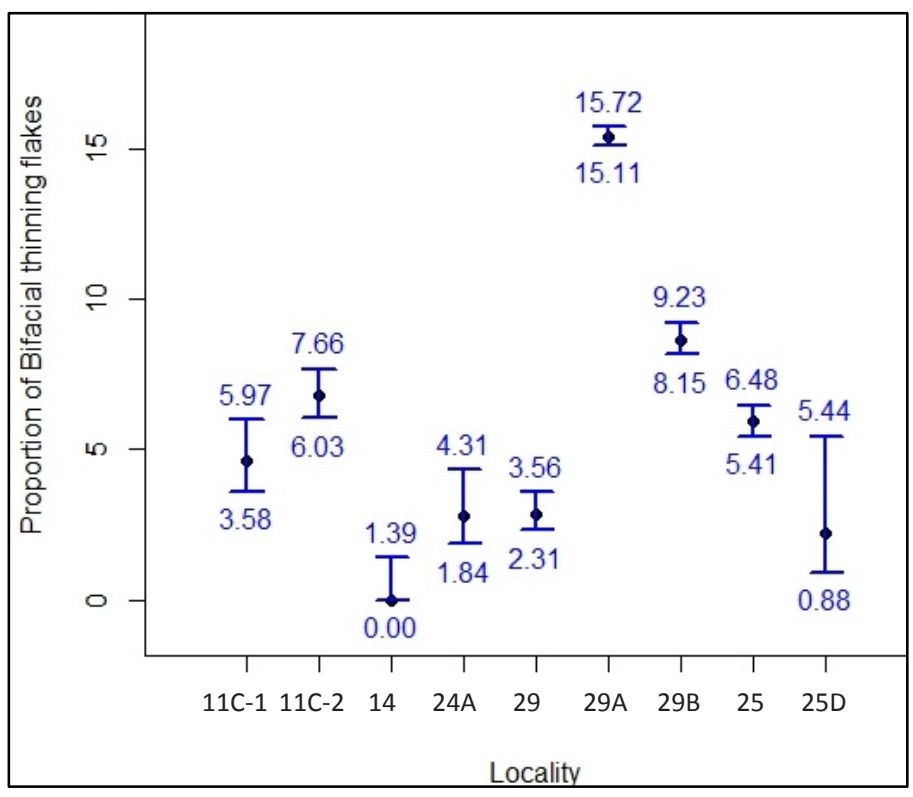

Figure 6.32. Percentages of bifacial thinning flakes in different Hierakonpolis localities, and the 95\% binomial confidence intervals. HK11C-1 (Holmes 1996); HK11C-2 (Takamiya \& Endo 2011); HK29 17L13 (Holmes 1996). For other references see table 6.5. 


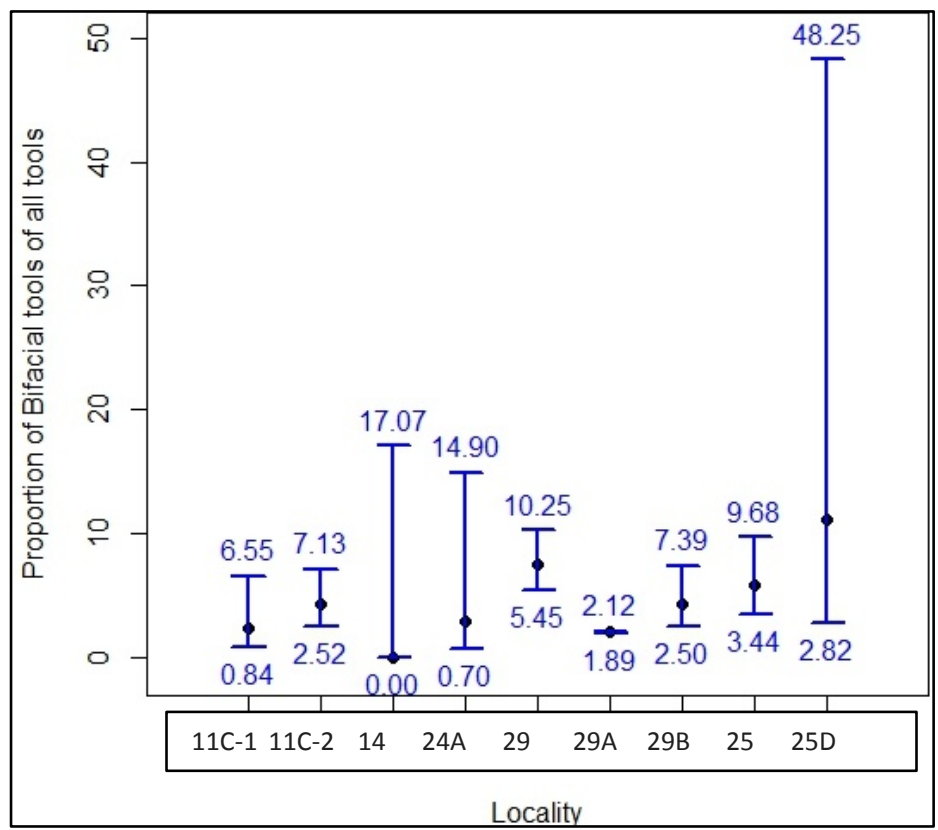

Figure 6.34. Percentages of bifacial tools in Hierakonpolis localities, and the 95\% binomial confidence intervals. HK11C-1 (Holmes 1996); HK11C-2 (Takamiya \& Endo 2011); HK29 10L10 (Holmes 1996). For other references see table 6.5 .

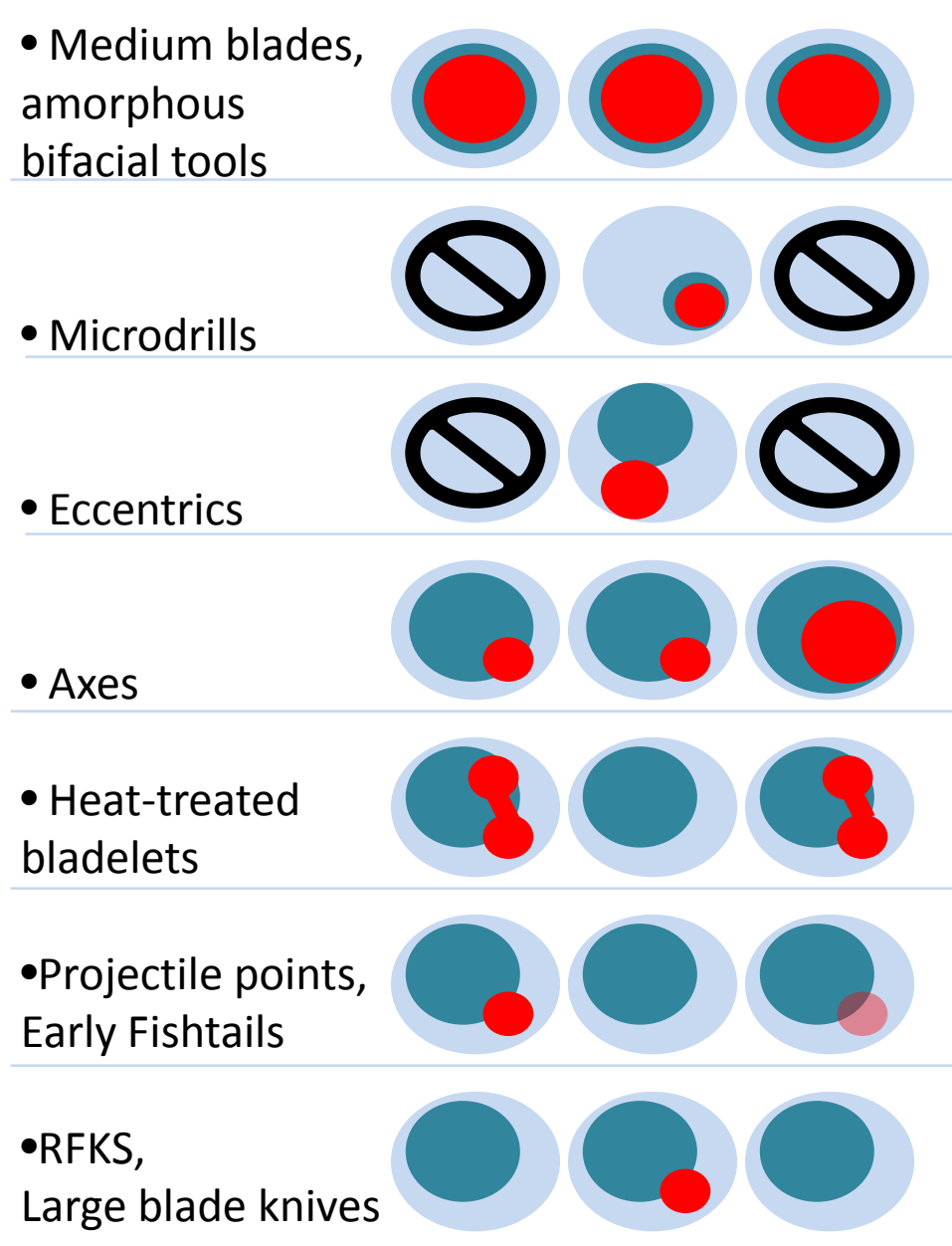

Key:

Settlement

Use area

Production area

Figure 6.35. Visual representation of differences in the distribution of production remains and tools for different tool classes, across hypothetical settlements. 


\section{Chapter 7 Figures}
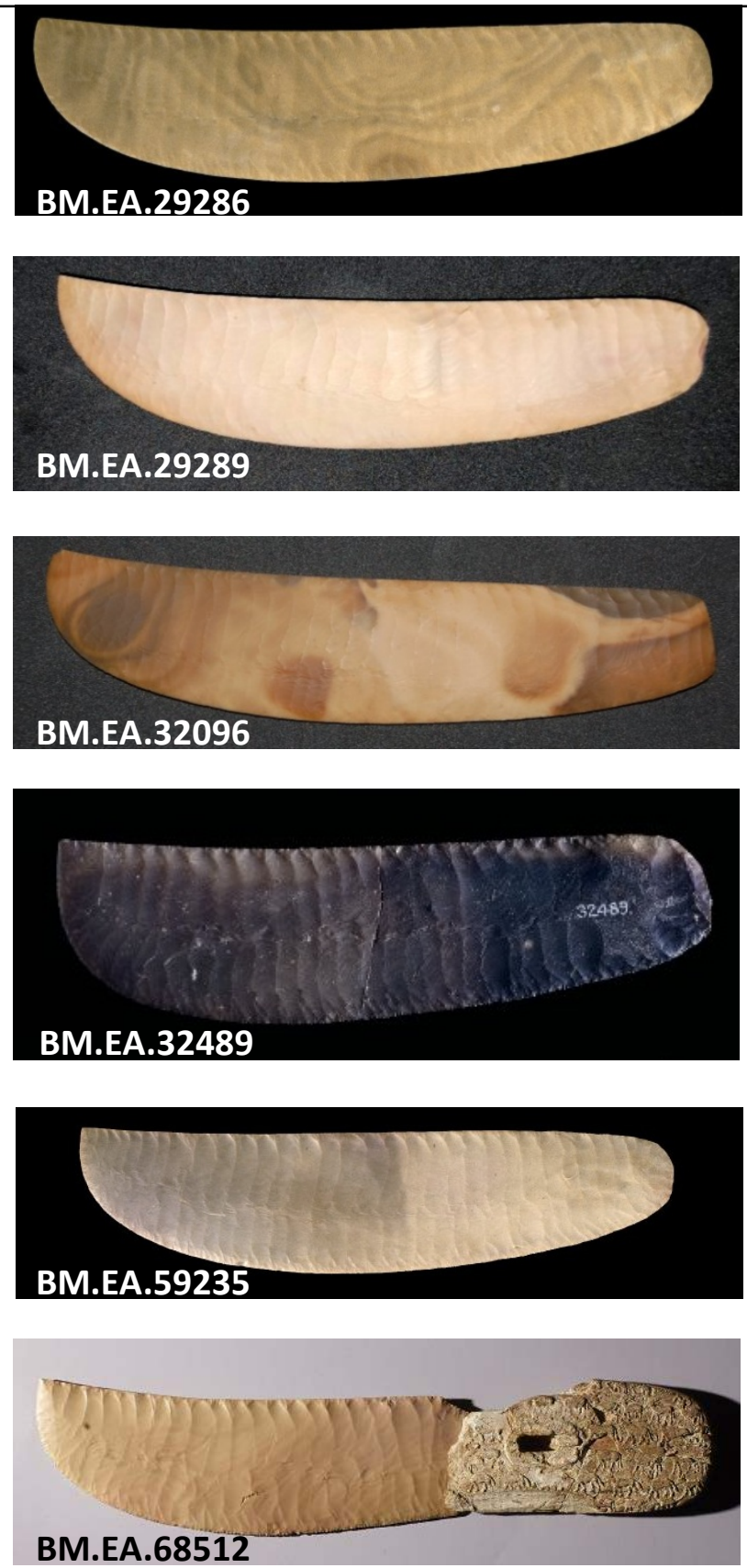
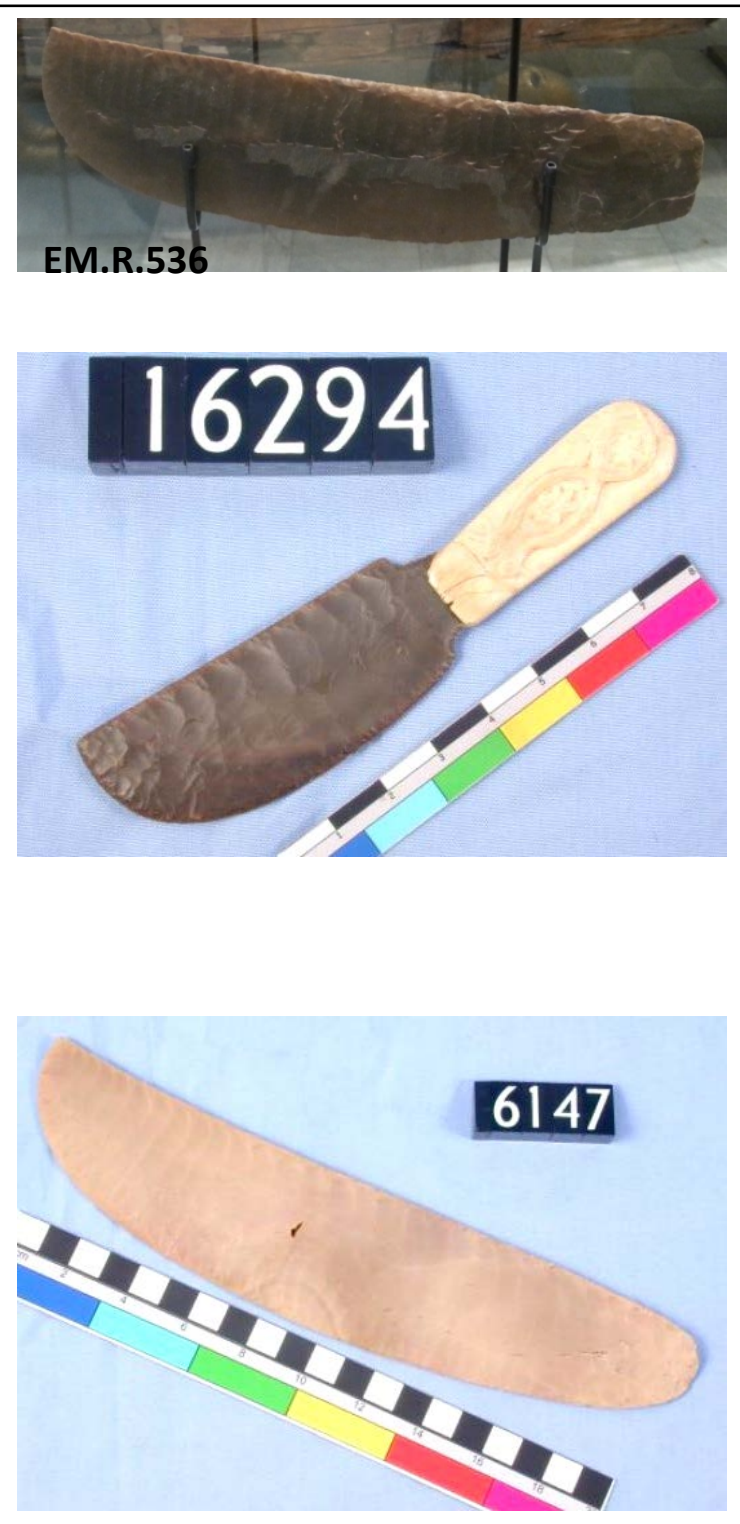

Figure 7.1. Ripple flaked knives. Images not to scale. See also Figures 6.1-6.6 Left, from top:

-BM.EA.29286 RM type Other:Variable TranslucentOpaque.

-BM.EA.29289 RM type 1/2/4;

-BM.EA.32096 RM type Other: Variable TranslucentOpaque;

-BM.EA.32489 RM type 7;

-BM.EA.59235 RM type 1/2/4;
-BM.EA.68512 Pitt Rivers Knife RM type 4;

British museum images credit: (C)Trustees of the British Museum. CC BY-NC-SA 4.0;

Right from top:

-EM.R.536 RM type 5;

-UC.16294 RM type Other: Chocolate;

-UC.6147 RM type 2/4;

UC image credits: The Petrie Museum of Egyptian Archaeology UCL. CC BT-NC-SA 3.0. 


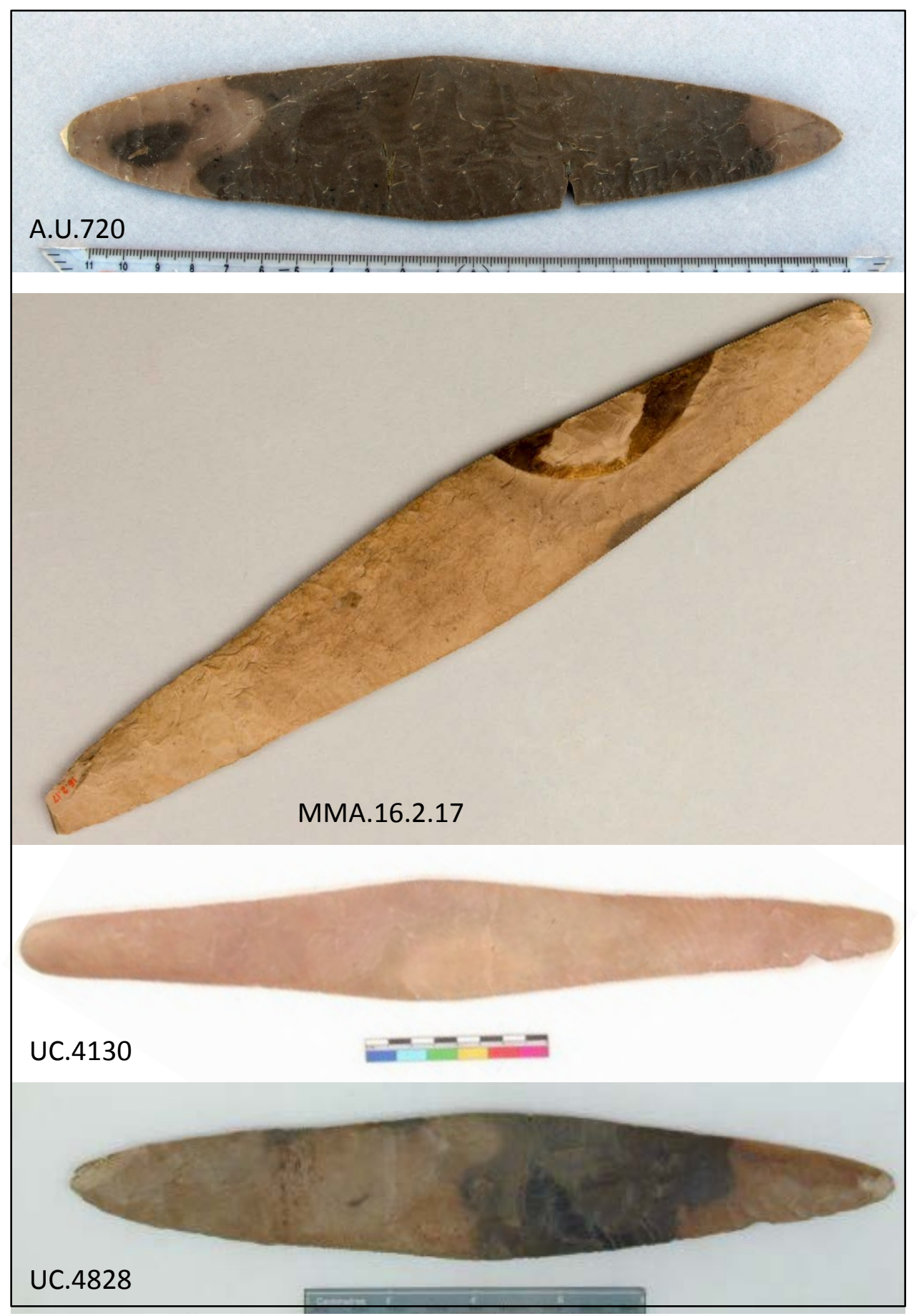

Figure 7.2. Rhomboid tools. Images not to scale. From top.

-A.U.720 RM type 7; Image taken with permission of the Gernıan Archaeological Mission to Abydos.

-MMA.16.2.17 RM type 1/2/4; Image credit: The Metropolitan Museum of Art, Rogers Fund, 1916. www.metmuseum.org;

-UC.4130 RM type1/2/4;

-UC 4828 RM type 7;

Petrie Museum image credits: The Petrie Museum of Egyptian Archaeology UCL CC BY-NCSA 3.0. 


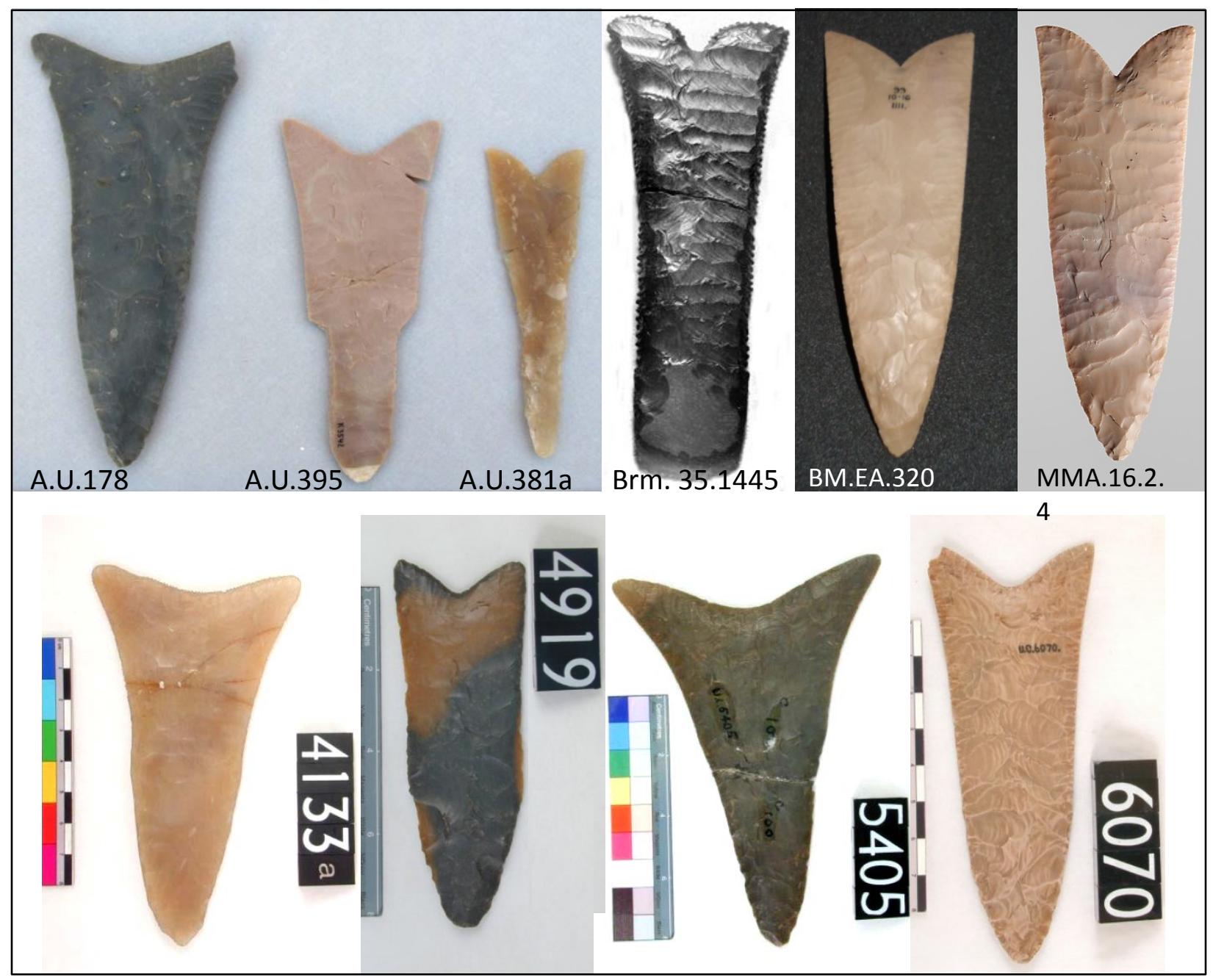

Figure 7.3. Fishtails. Images not to scale. Top row from left:

-A.U.178 RM type Other Dark Grays;

-MMA. 16.2.4 RM Type 2/4; Image credit: The

-A.U.395 RM type 4;

-A.U.381a RM type 8;

Abydos fishtail image taken with permission of the German Archaeological Mission to Abydos.

Metropolitan Museum of Art, Rogers Fund, 1916.

metmuseum.org.

Bottom row from left:

-UC.4133a RM Type 8;

-UC.4919 RM Type 7;

-BrM.35.1445 RM type obsidian; Image credit: Brooklyn-UC.5405 RM Type 7;

Museum, Charles Edwin Wilbour Fund, CC-BY 3.0

-BM.EA.32098 RM type 1/2/4; Image credit: (C)

-UC.6070 RM Type 1/2/4;

Trustees of the British Museum. CC BY-NC-SA 4.0

Petrie Museum image credits: The Petrie Museum of Egyptian Archaeology UCL CC BY-NC-SA 3.0. 


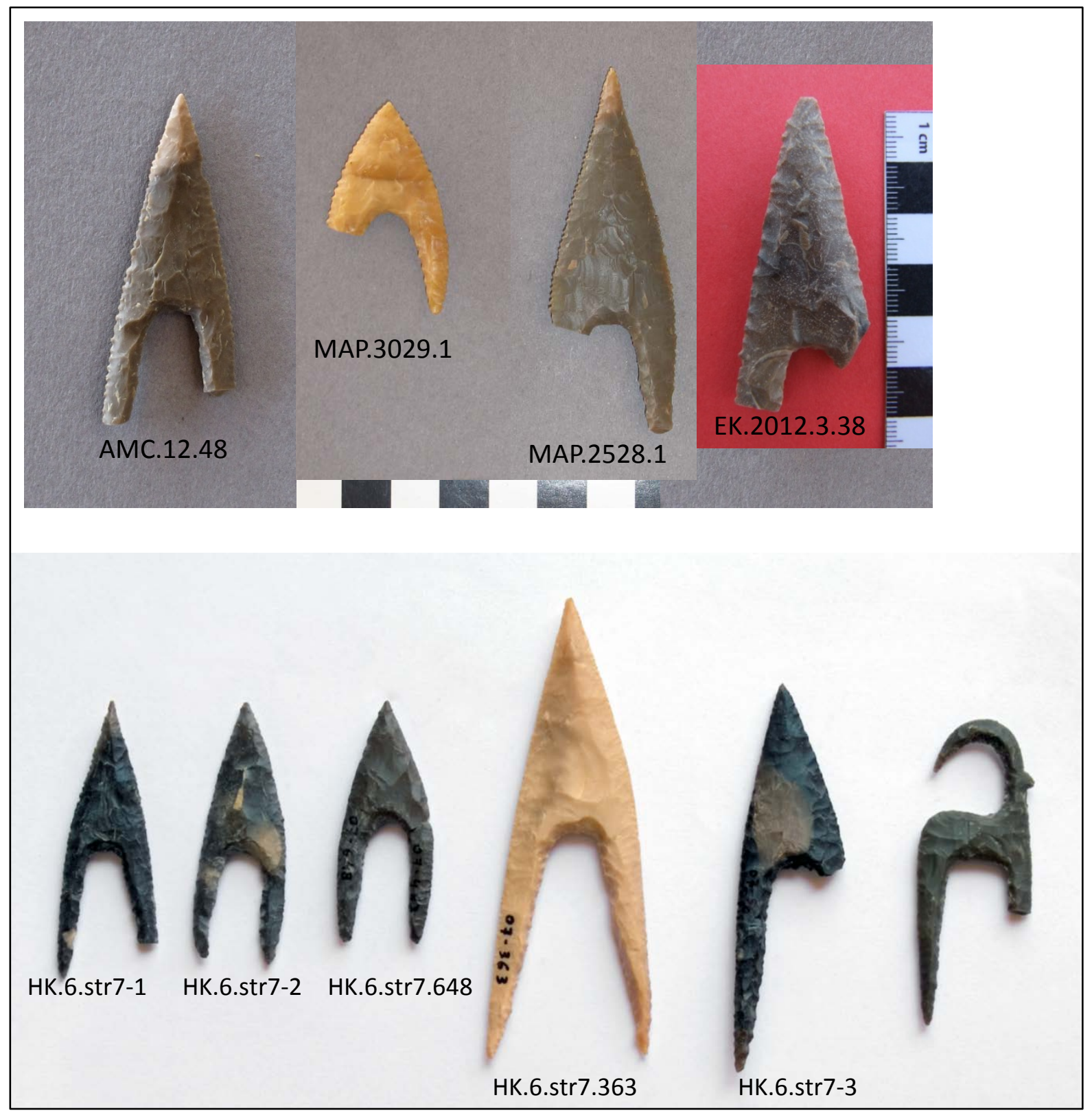

Figure 7.4. Concave Base Projectile Points \& gazelle-shaped eccentric. Top row from left: -AMC.12.48 RM type 7; -MAP.3029.1 RM type 13;

-MAP.2528.1 RM type 7;

-EK.2012.3.38 RM type 7 (Image courtesy of the Belgian Archaeological Mission to Elkab);

Bottom row from left (Image courtesy of the Hierakonpolis Expedition, photo by James Rossiter. Not to scale with top row images. ID numbers refer to table 7.12):

-HK.6.str7-1 RM type 7;

-HK.6.str7-2 RM type 7;

-HK.6.str7.648 RM type 7;

-HK.6.str7.363 RM type 1/2/4;

-HK.6.str7-3 RM type 7. 


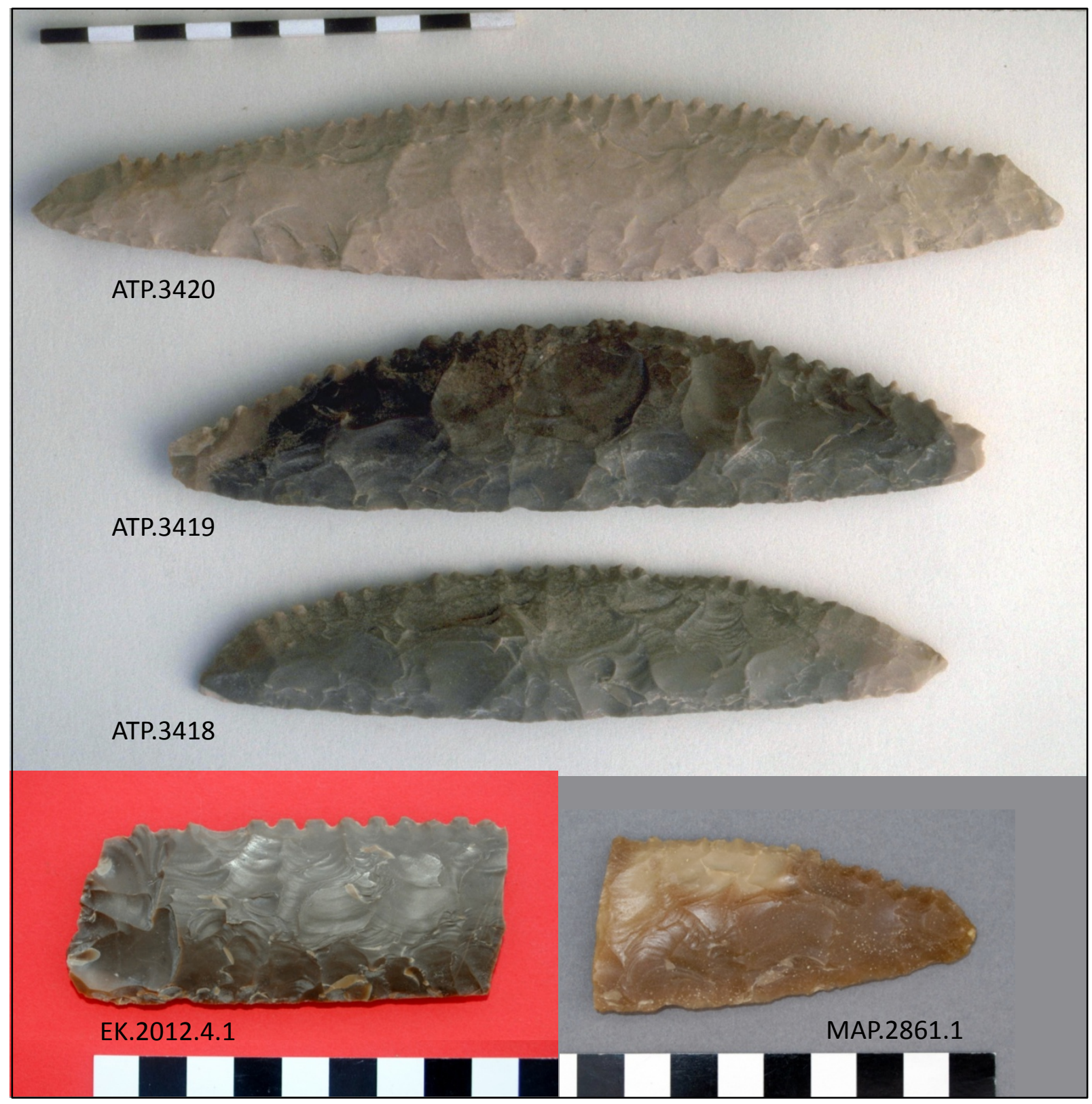

Figure 7.5. Bifacial sickles. Images approximately to scale. Top, from top: -ATP.3420 RM type, 1/2/4;

-ATP.3419 RM type 7;

-ATP.3418 RM Type 7; (Image courtesy of the Ahmose-Tetisheri Project)

Bottom row, from left:

-EK.2012.4.1 RM type 7 (Image courtesy of the Belgian Archaeological Mission to Elkab);

-MAP.2861.1 RM type 10; 


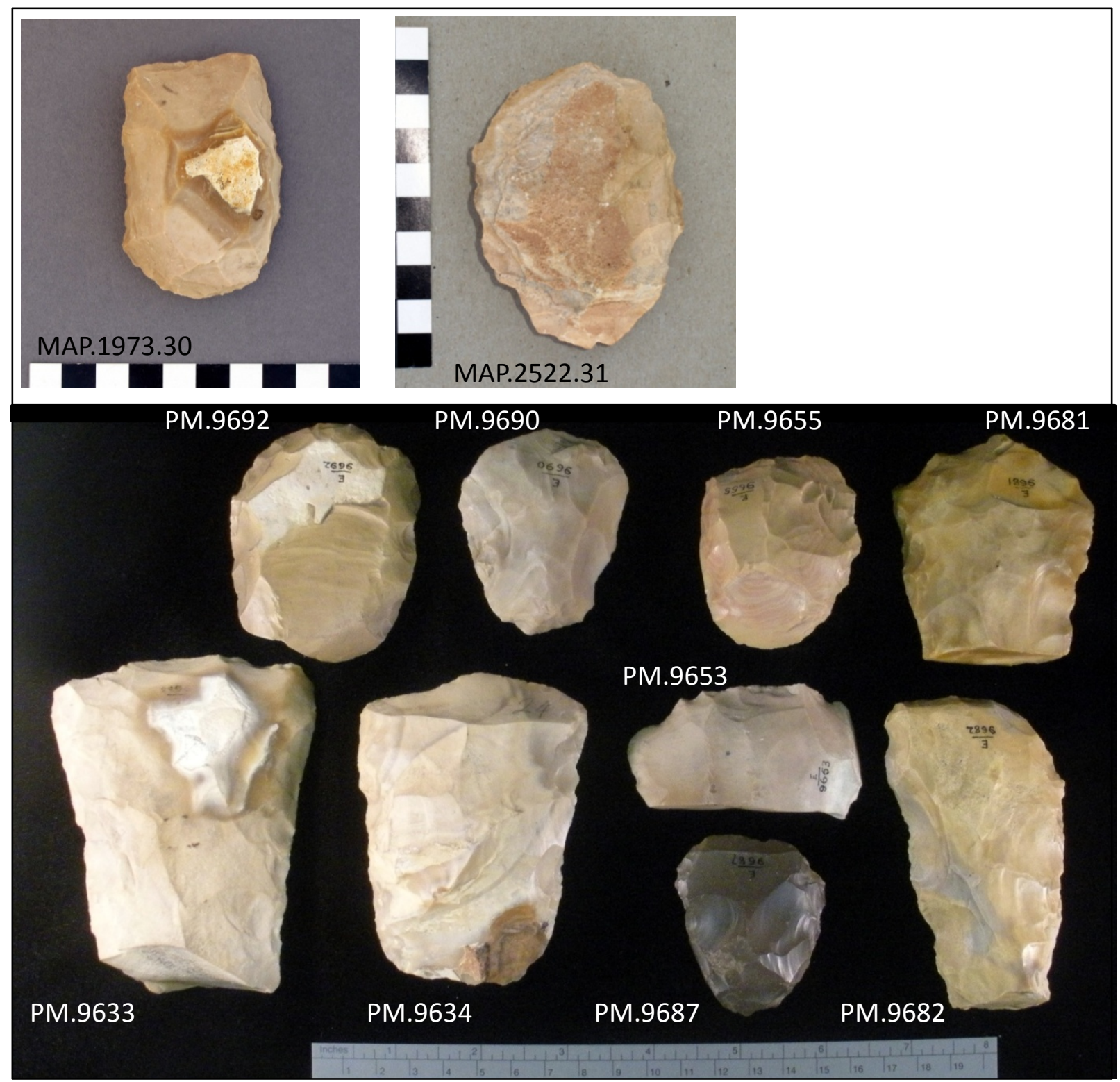

Figure 7.6. Axes. Images to scale. From top left:

-MAP. 1973.30 RM type 1;

-MAP.2522.31 RM type 1;

-PM.9692 RM type 2/4;

-PM.9690 RM type 10;

-PM.9655 RM type 4;

-PM.9681 RM type 8;

-PM.9653 RM type 1;

-PM.9633 RM type 1;

-PM.9634 RM type 1;

-PM.9687 RM type Other Dark Gray;

-PM.9682 RM type 1. 


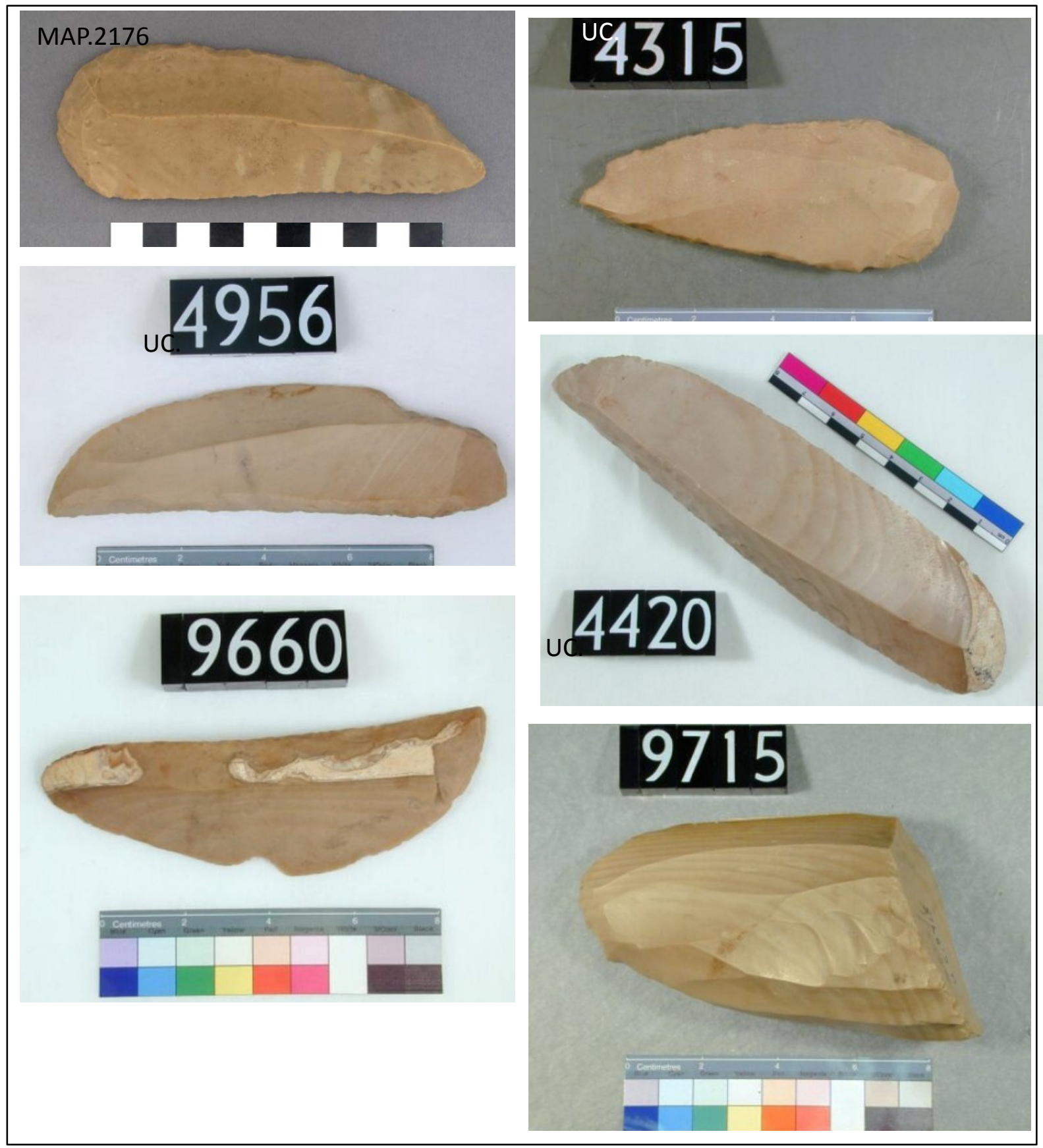

Figure 7.7. Large blade knives. Images not to scale. From top left and across:

-MAP.2176 RM type 2;

-UC.4315 RM type 2;

-UC.4956 RM type 1,2;

-UC.4420 RM type 1,2;

-UC.9660 RM type 1,2;

-UC.9715 possible core for large blades from Badari 3000/6.

Petrie Museum Image credits: The Petrie Museum of Egyptian Archaeology UCL. CC BT-NC-SA 3.0. 


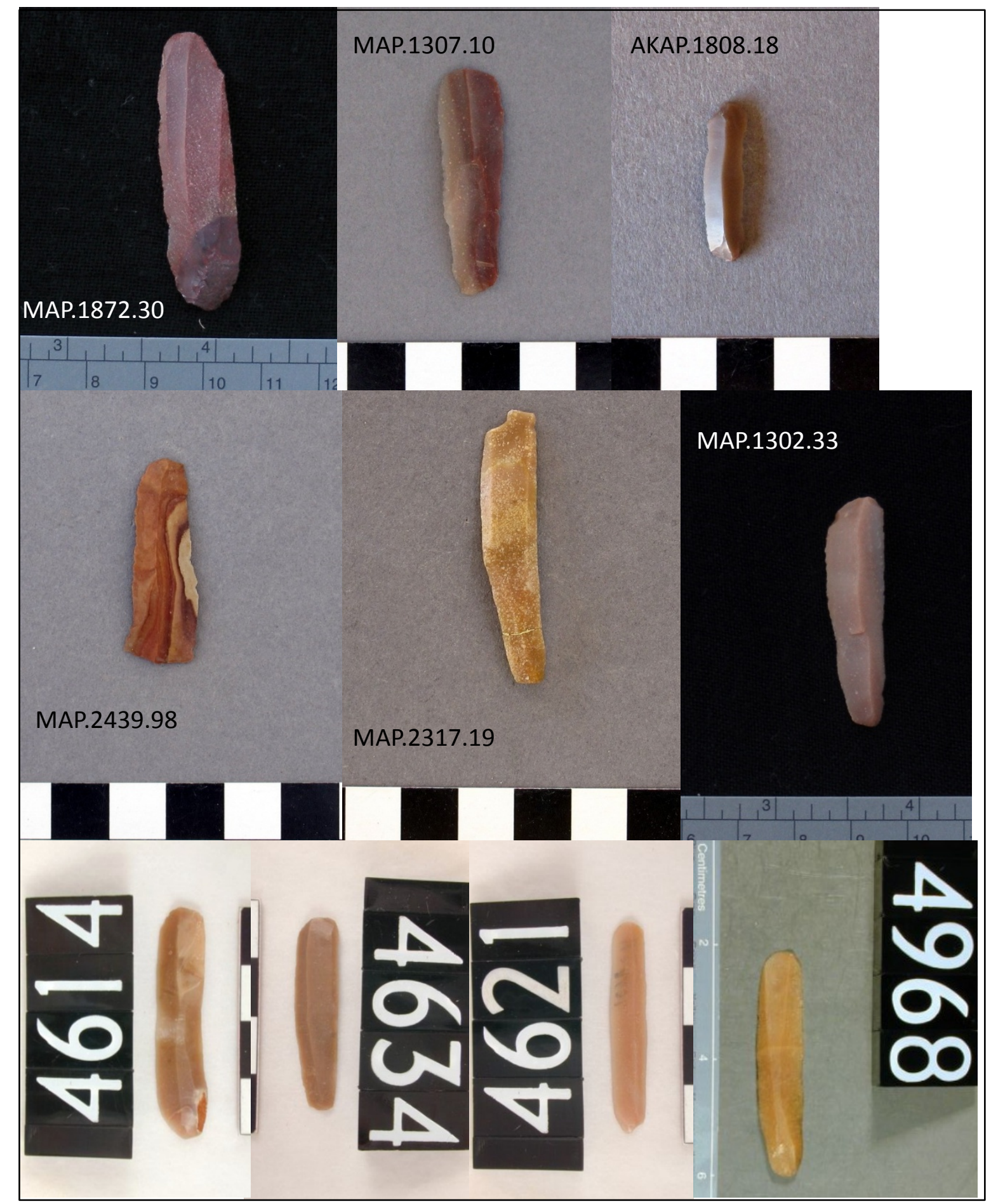

Figure 7.8. Microendscrapers / Glossy bladelet tools. Images to scale. From top left and across: -MAP.1872.30 RM type 11; -MAP.1307.10 RM type 11; -AKAP.1808.18 RM type 8; -MAP.2439.98 RM type 11; -MAP2317.19\&64 RM type 8 (2 fragments that refit together);

-MAP.1302.33 RM type 8; -UC.4614 RM type 8; -UC.4634 RM type 8; -UC.4621 RM type 8 or 10 ; -UC.4968 RM type 8 or 10.

Petrie Museum Image credits: The Petrie Museum of Egyptian Archaeology UCL. CC BT-NC-SA 3.0. 


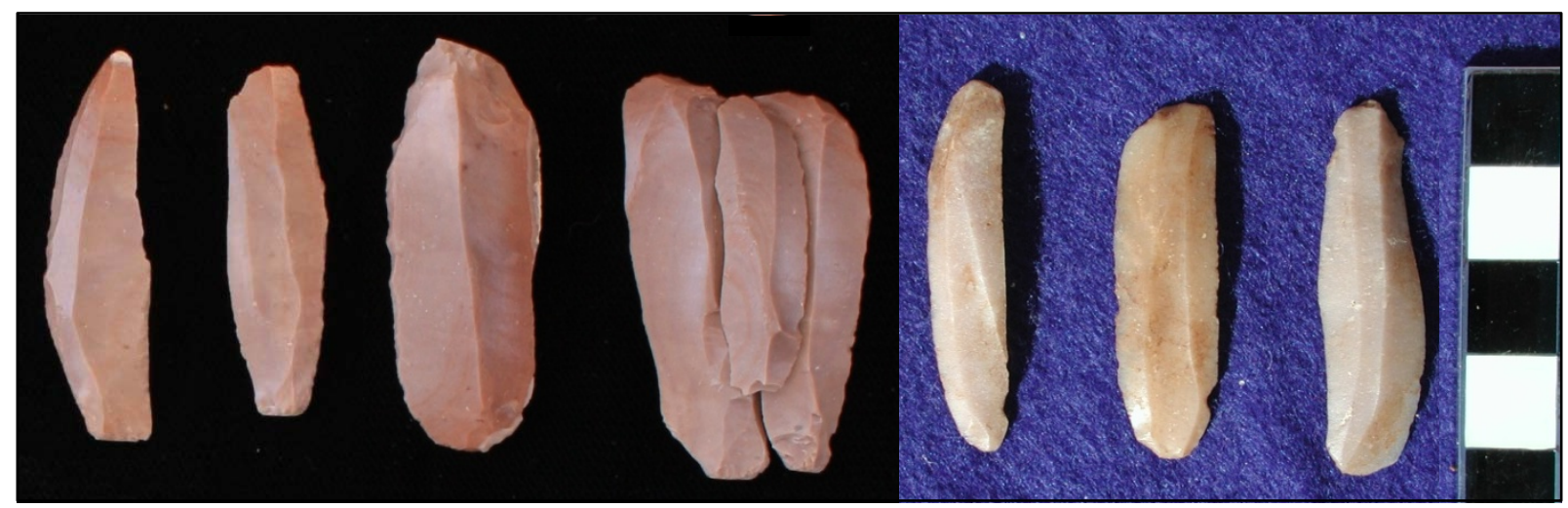

Figure 7.9. Bladelets \& bladelet tools including microendscrapers from Hierakonpolis. Left: HK6 Tomb 72 Right: HK43 Tomb 333. These items show that similar raw materials were used to make bladelets and microendscrapers at Hierakonpolis as at el-Mahâsna, Nag el-Qarmila, and Naqada (compare to Figure 7.8). Images courtesy of the Hierakonpolis expedition. Left photo by Xavier Droux, right photo by Renée Friedman.

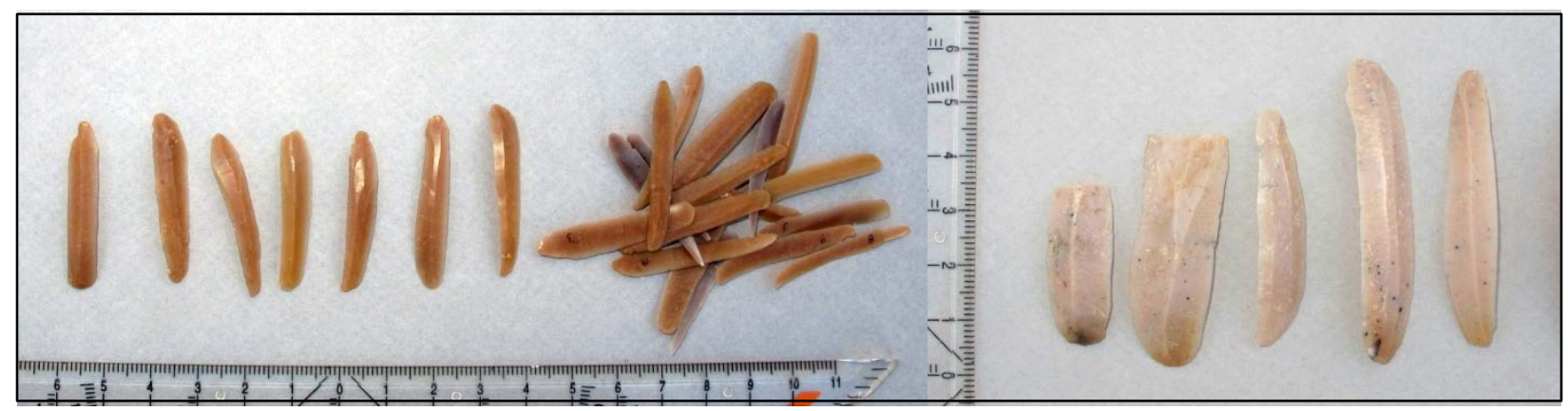

Figure 7.10. Bladelets from Abydos cemetery U. Hikade (1998) reported that at least $33 \%$ of bladelets were retouched into microendscrapers. These items show that similar raw materials were used for the Cemetery $U$ bladelets as at el-Mahâsna, Nag el Qarmila, Naqada, and Hierakonpolis (compare to Figures 7.8 \& 7.9)., and that bladelets were struck form the same core, with some turned into microendscrapers. Image taken with permission of the German Archaeological Mission to Abydos.

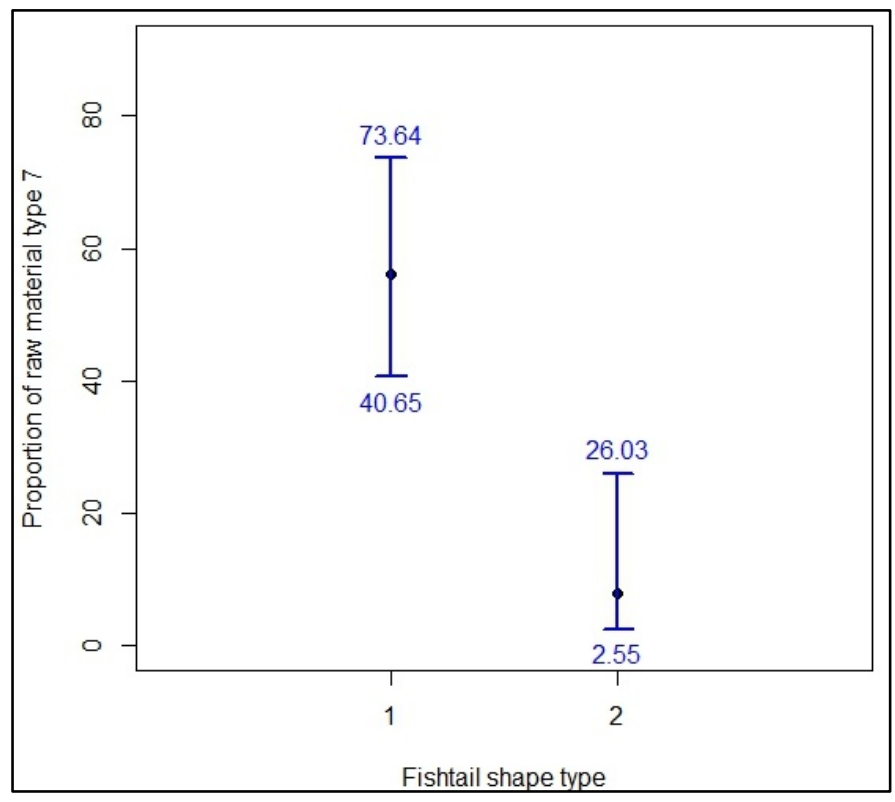

Figure 7.11. The $95 \%$ binomial confidence limits for the proportion of raw material type 7. Dark gray and brown among fishtails of shape types 1 and 2 . 


\section{Chapter 8 Figures}

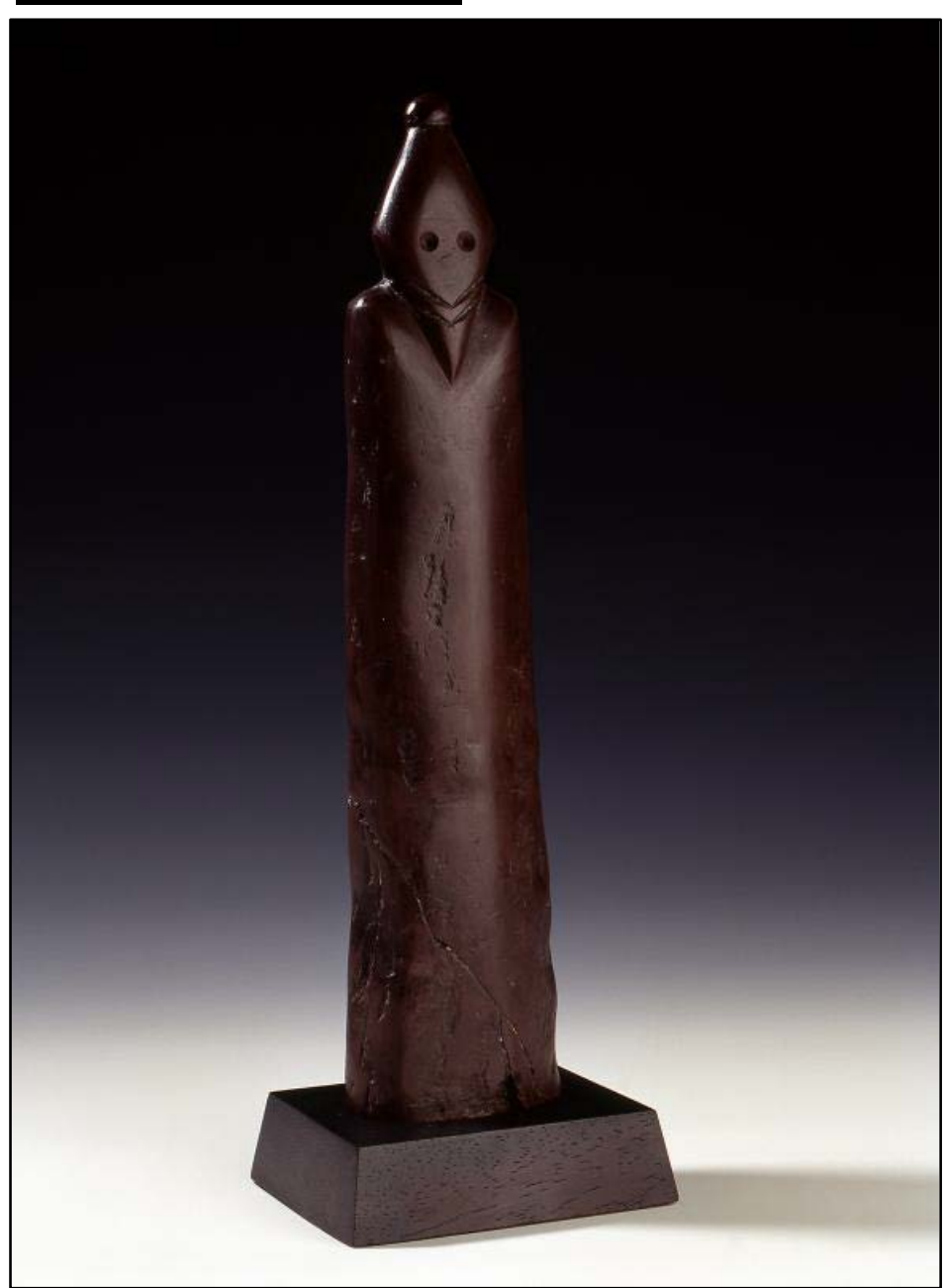

Figure 8.1. Statuette depicting a bearded man with conical headware that forms a rhomboid shape. From Gebelein. Musee des Confluences 90000172. Image credit: Museum of Confluences (Lyon, France), CC BY-SA 


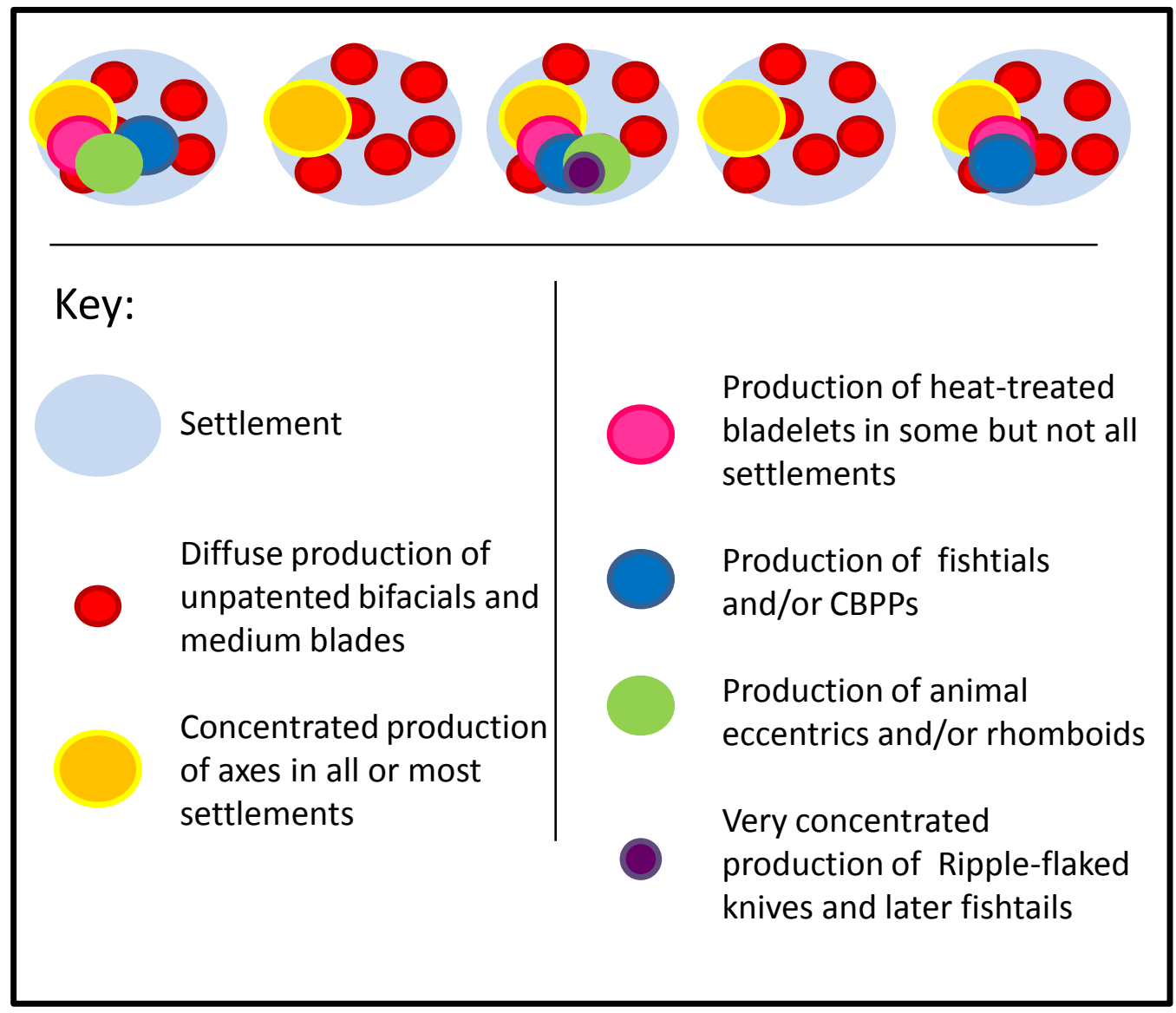

Figure 8.2. Visual representation of differences in production of tool types across hypothetical settlements. Even this does not capture the full variability since there are additional differences in the distribution of different tool types. See Ch. 6. 